Supporting Information

For

\title{
Using Bases as the Initiator to Isomerize Allylic Alcohols: Insights from Density Functional Theory Studies
}

Lin Zhang, ${ }^{a}$ Bin Wang, ${ }^{c}$ Yaqi Zhao, ${ }^{a}$ Min Pu, ${ }^{a}$ Shubin Liu, ${ }^{*}$ Ming Lei ${ }^{a *}$

\author{
${ }^{a}$ State Key Laboratory of Chemical Resource Engineering, Institute of Computational \\ Chemistry, College of Chemistry, Beijing University of Chemical Technology, Beijing, \\ 100029, China \\ b Research Computing Center, University of North Carolina, Chapel Hill, North \\ Carolina, 27599-3420, USA \\ ${ }^{c}$ Department of Chemistry, College of Chemistry and Chemical Engineering, Hunan \\ Normal University, Changsha, 410000, China
}

*Email: 1eim@mail.buct.edu.cn (Ming Lei), shubin@email.unc.edu (Shubin Liu) 


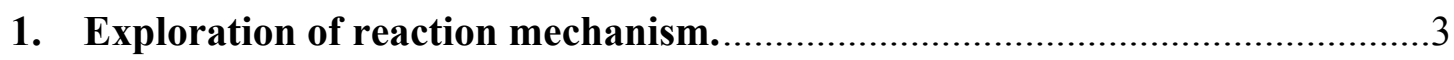

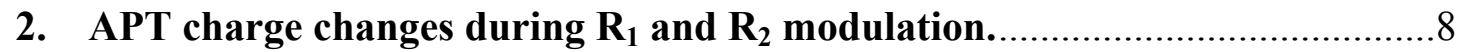

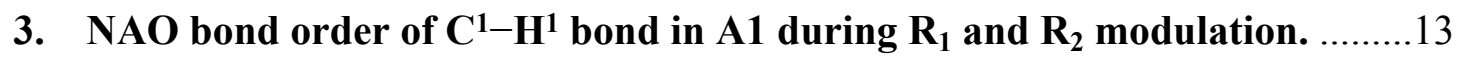

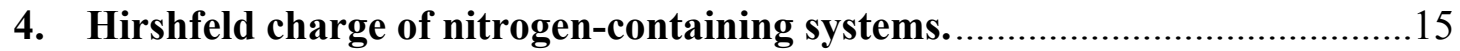

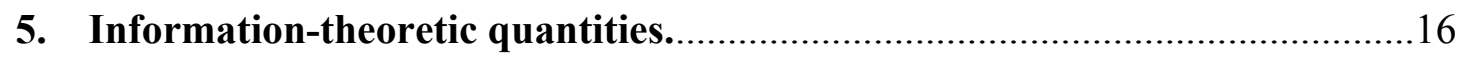

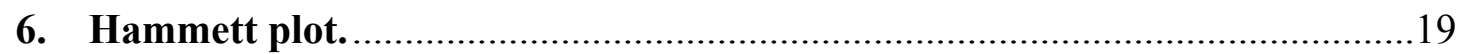

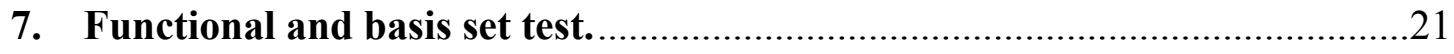

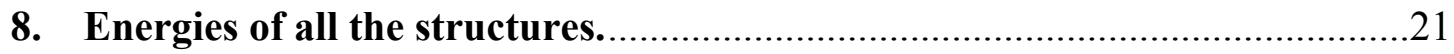

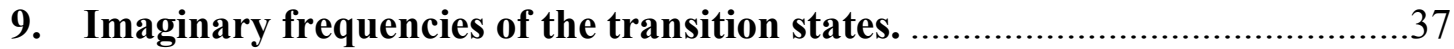

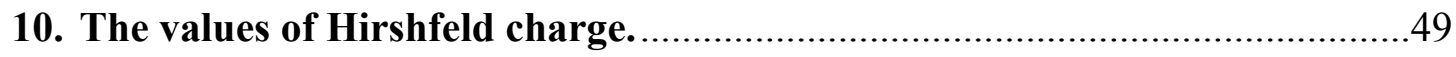

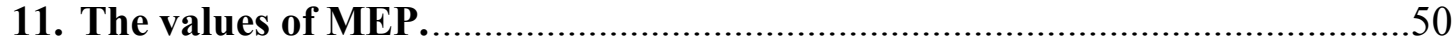

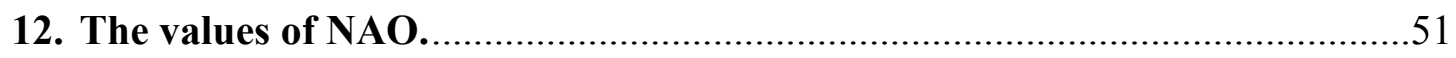

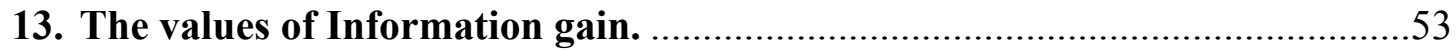

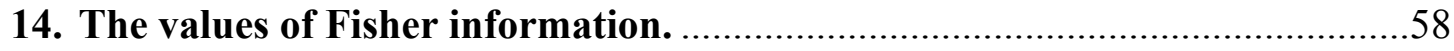

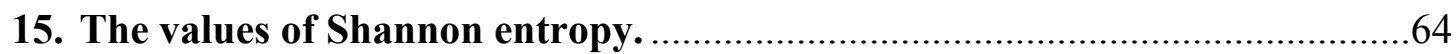

16. Optimized geometric structures and parameters of key transition states......69

17. Effects of different metal cations on the isomerization of allylic alcohol. ......73

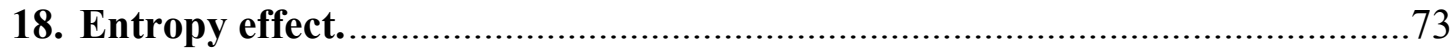

19. Related calculated results about the JACS paper in 2016 ...........................74

20. Atomic cartesian coordinates of intermediates and transition states (presented in $\AA$ ). . .76 


\section{Exploration of reaction mechanism.}

\subsection{Catalyzed by $t$ BuONa.}

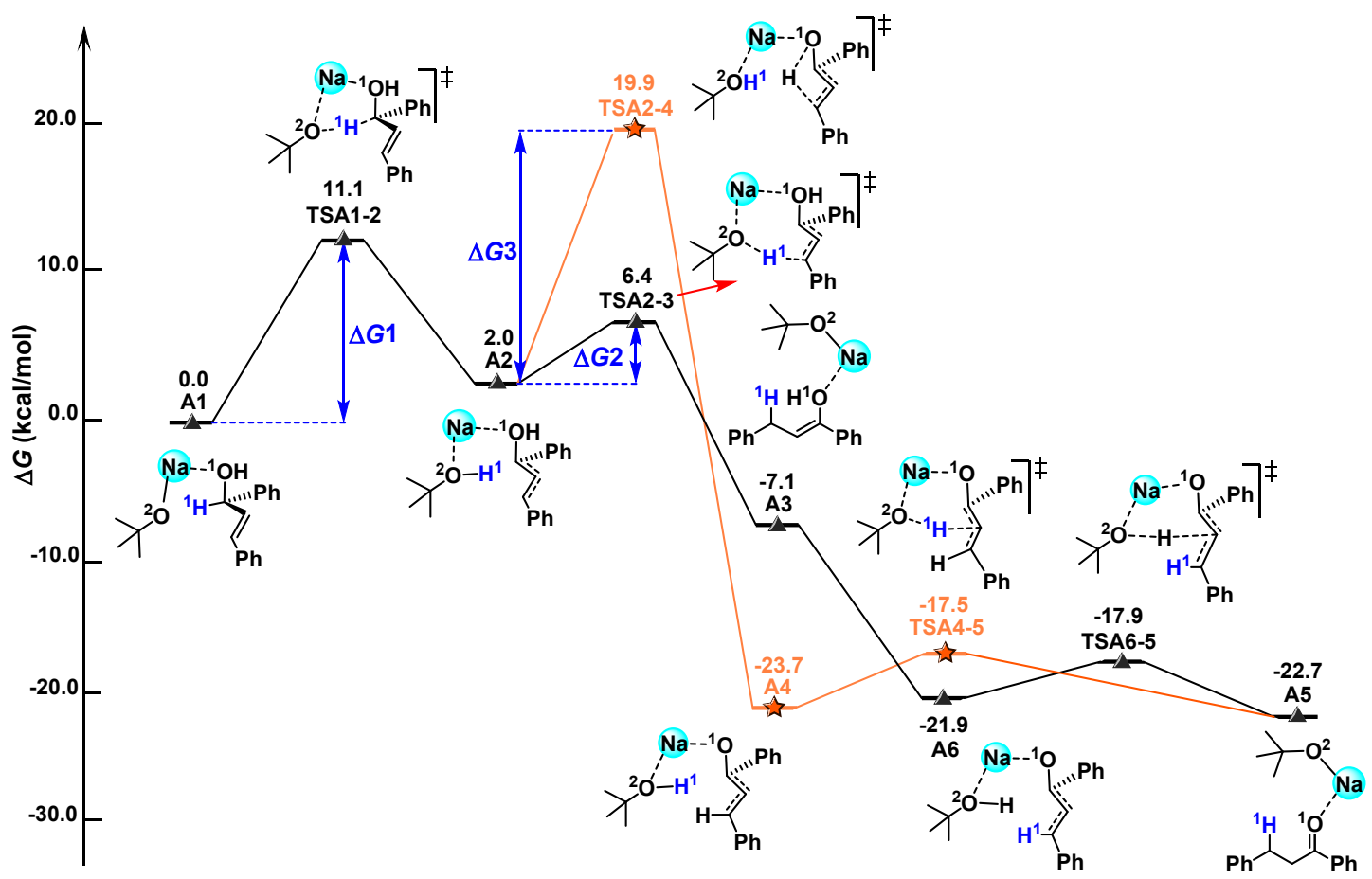

Figure S1. Free energy profiles of the isomerization of allylic alcohol to ketone catalyzed by tBuONa (unit: kcal/mol). Key bond lengths are given in $\AA$.

In path B, initially, B1 is converted to intermediate B2 through TSB1-2 with a free energy barrier of $15.7 \mathrm{kcal} / \mathrm{mol}$, which is $4.6 \mathrm{kcal} / \mathrm{mol}$ higher than that of the $\Delta G 1(11.1$ $\mathrm{kcal} / \mathrm{mol}$ ) in path $\mathrm{A}$. Then, the $\mathrm{H}^{1}$ atom of $\mathbf{B} 2$ is transferred to the $\mathrm{C}^{3}$ atom to form the enolate anion intermediate B3 via TSB2-3, which is a barrier-free process. Obviously, path B is less favorable than path A. 


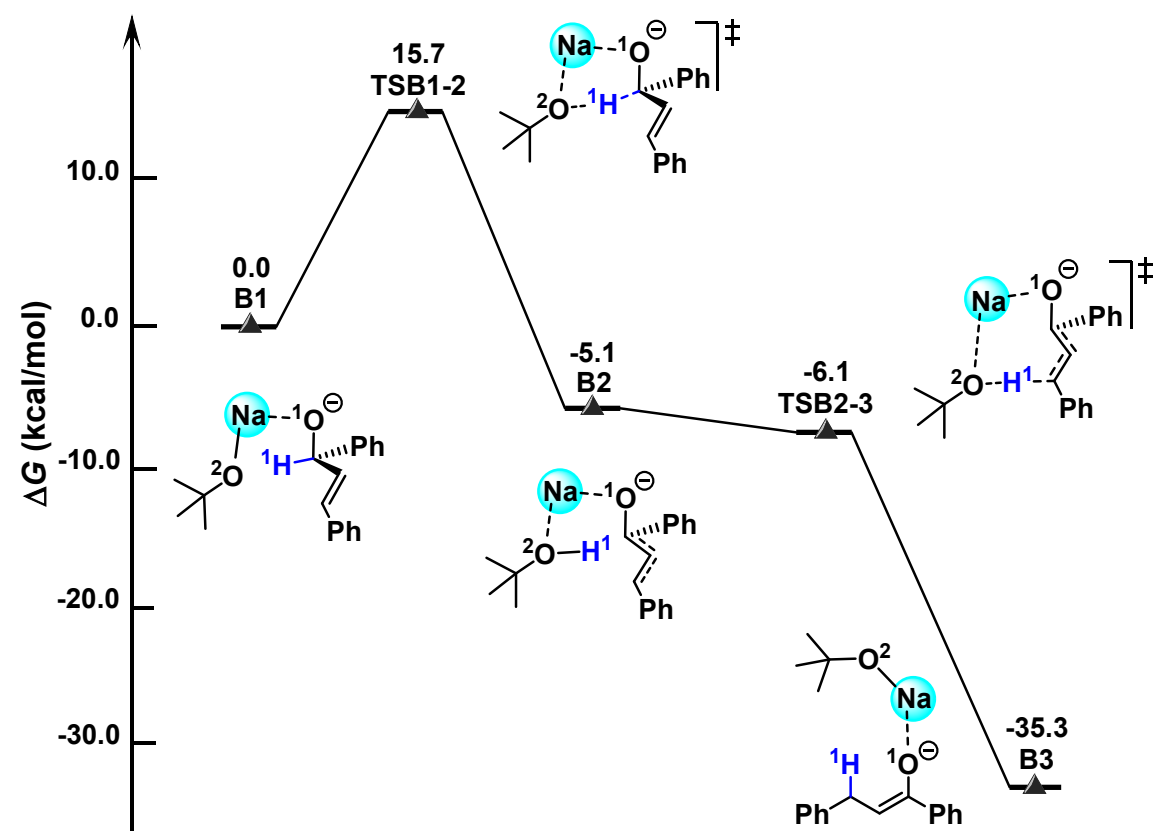

Figure S2. Free energy profile of the isomerization of allylic alcohol to the enolate anion intermediate catalyzed by $\mathrm{tBuONa}$ (unit: $\mathrm{kcal} / \mathrm{mol}$ ).

In path $\mathrm{C}$, there are 2 equiv of $t \mathrm{BuONa}$ to assist the isomerization of allylic alcohol anion. One $t \mathrm{BuONa}$ molecule removes the proton from the allylic alcohol at first, which is a barrier-free process. The release of $t \mathrm{BuOH}$ is an endergonic process by 16.4 $\mathrm{kcal} / \mathrm{mol}$ from $\mathbf{C 2}$ to $\mathbf{C 3}$. Then, the second $t \mathrm{BuONa}$ molecule interacts with $\mathbf{C} 3$ to generate C4. Subsequently, the $\mathrm{C}-\mathrm{H}$ bond in $\mathbf{C 4}$ is activated via TSC4-5, with a free energy barrier of $7.3 \mathrm{kcal} / \mathrm{mol}$. Finally, the $\mathrm{H}^{1}$ atom is transferred to $\mathrm{C}^{3}$ atom form intermediate C6 via TSC5-6, with a low free energy barrier of $1.3 \mathrm{kcal} / \mathrm{mol}$. The calculated results show that the release of $t \mathrm{BuOH}$ is the rate-determining step in this pathway. 


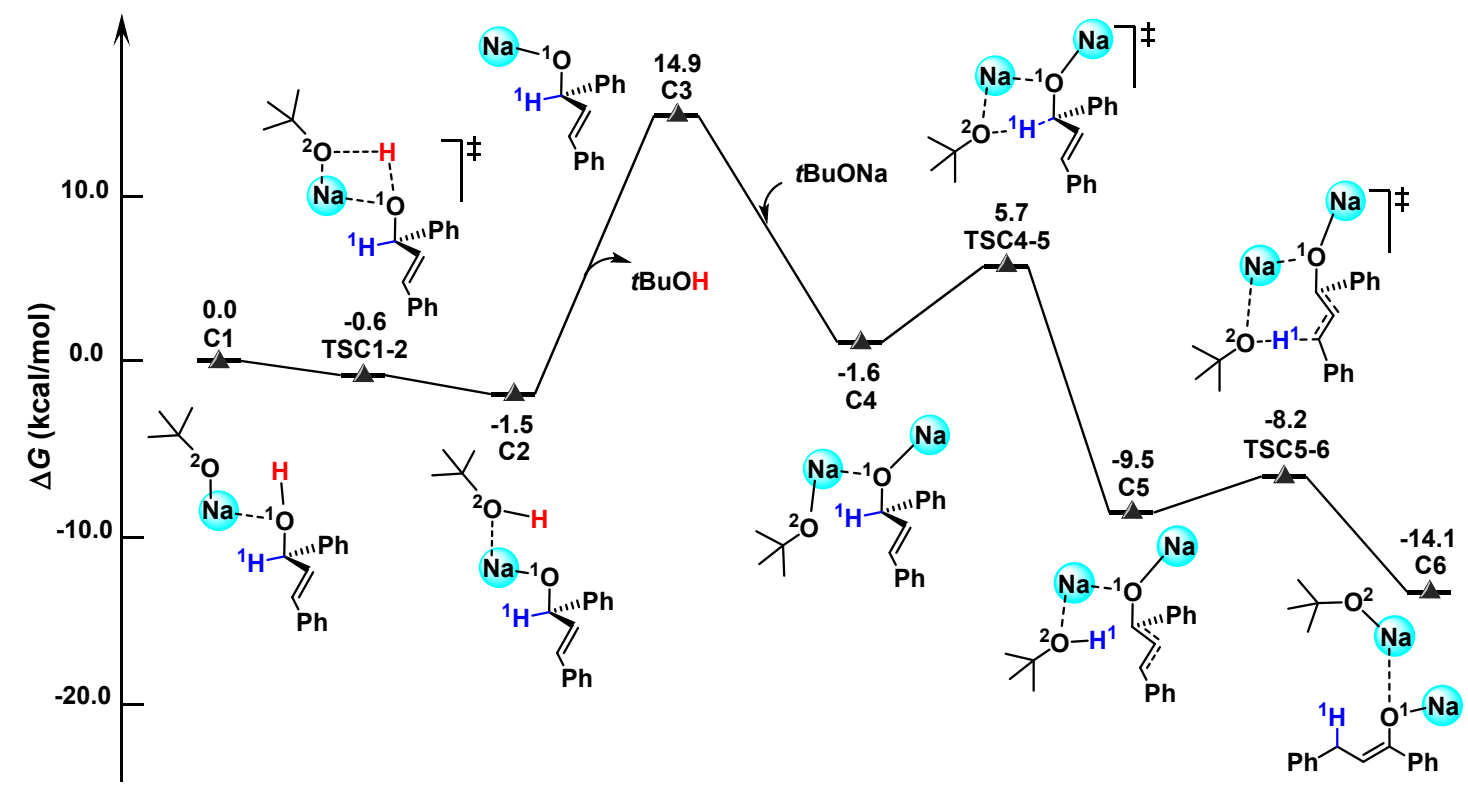

Figure S3. Free energy profile of the isomerization of allylic alcohol anion catalyzed by 2 equiv tBuONa (unit: $\mathrm{kcal} / \mathrm{mol}$ ).

In path $\mathrm{D}$, we have explored the isomerization of allylic alcohol catalyzed by $t \mathrm{BuO}^{-}$. The calculated results show that the rate-determining step (RDS) is hydrogen transfer, and the free energy barrier is $14.7 \mathrm{kcal} / \mathrm{mol}$. Compared with Path $\mathrm{A}$ with the participation of cations (the energy barrier of RDS is $11.1 \mathrm{kcal} / \mathrm{mol}$ ), it is unfavorable. Therefore, it is proved that the metal cation plays an important role in the isomerization process of allylic alcohol. 


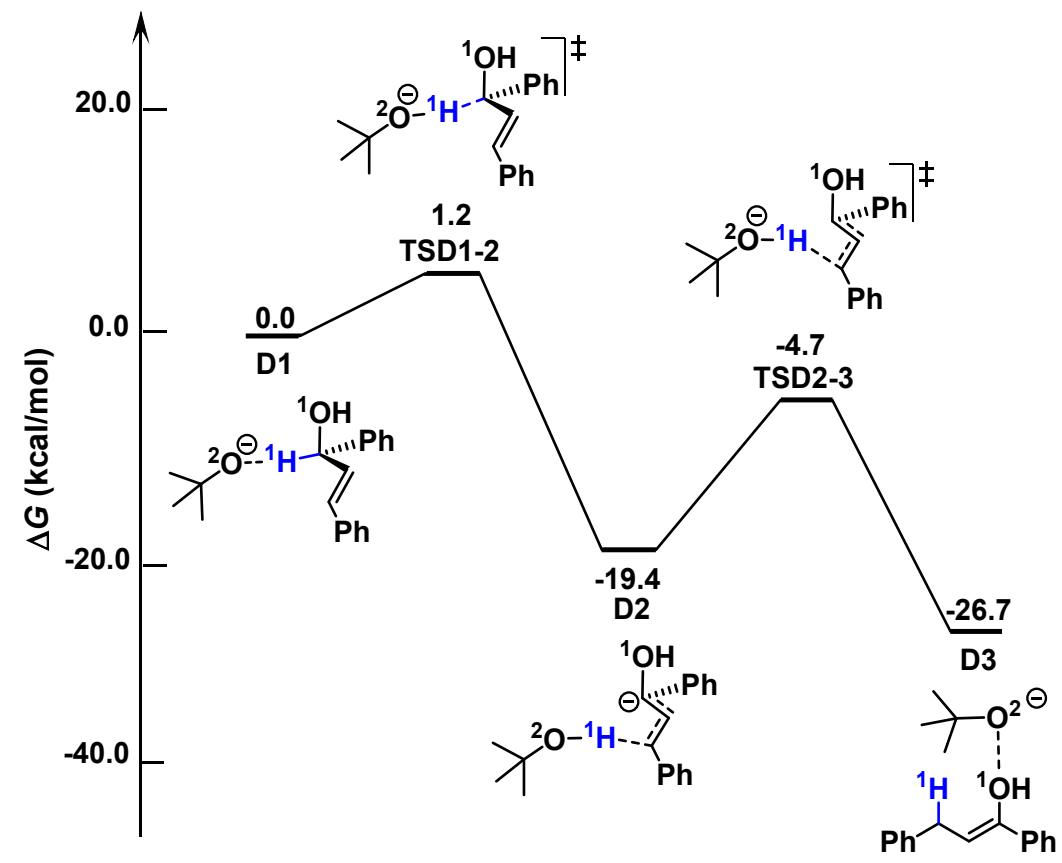

Figure S4: Free energy profile of the isomerization of allylic alcohol catalyzed by tBuO- (unit: kcal/mol).

\subsection{Catalyzed by TBD (1, 5, 7-triazabicyclo[4.4.0]dec-5-ene).}

In path B, initially, intermediate b1 is converted to intermediate b2 through TSb12 with a free energy barrier of $22.6 \mathrm{kcal} / \mathrm{mol}$. Then, while the $\mathrm{H}^{1}$ atom transfers to the $\mathrm{C}^{3}$ atom, the proton on the $\mathrm{O}^{1}$ transfers to the $\mathrm{N}$ atom via TSb2-3 with a free energy barrier of $8.0 \mathrm{kcal} / \mathrm{mol}$. Obviously, path B is less favorable than path A. 


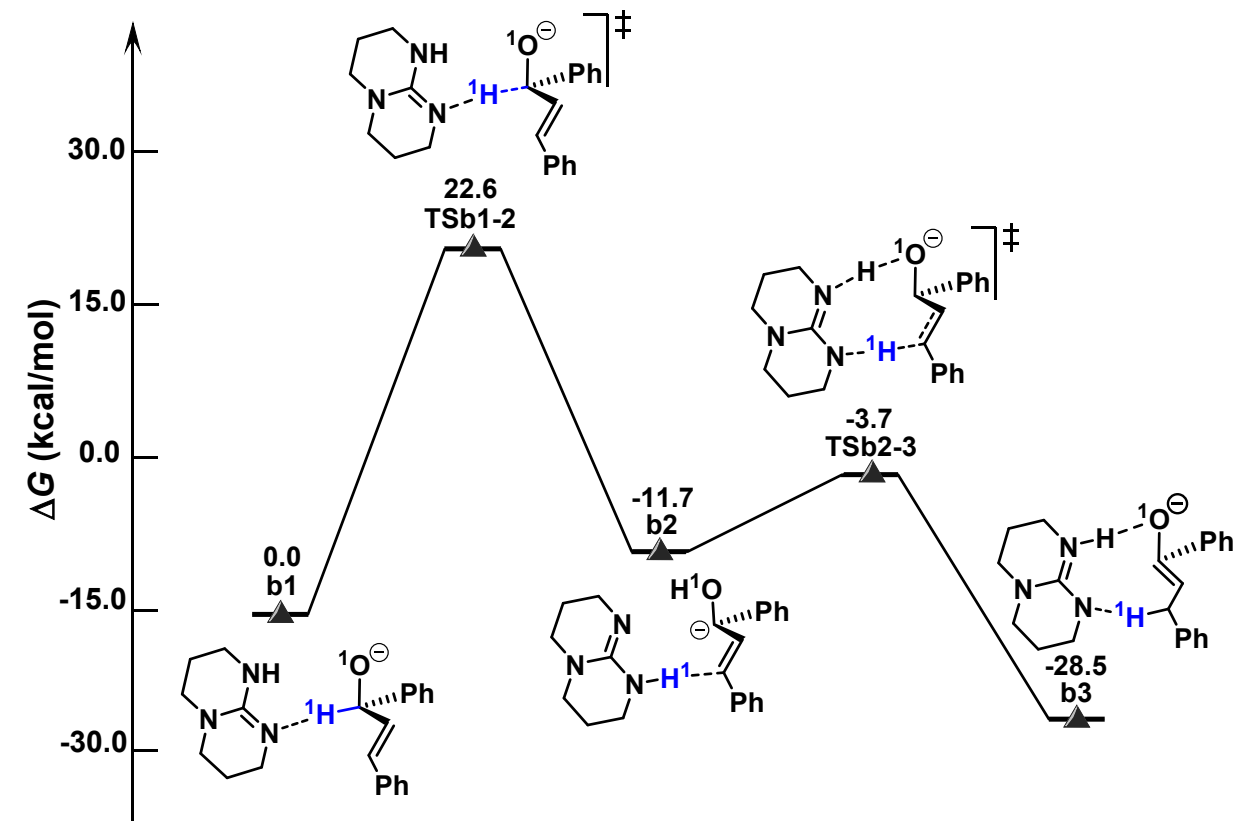

Figure S5. Free energy profile of the isomerization of allylic alcohol anion to the enolate anion intermediate catalyzed by TBD (unit: kcal/mol). 


\section{APT charge changes during $R_{1}$ and $R_{2}$ modulation.}

During the modulation of the $\mathrm{R}_{1}$ and $\mathrm{R}_{2}$ substituents, the changes in the APT charges of the $\mathrm{C}^{1}$ and $\mathrm{H}^{1}$ atoms in the intermediate $\mathbf{A 1}$ are shown in Figures S6-S9. It can be seen that the APT charge of the $\mathrm{C}^{1}$ atom is larger than the APT charge of the $\mathrm{H}^{1}$ atom, indicating that the $\mathrm{C}^{1}$ atom is positively charged with respect to the $\mathrm{H}^{1}$ atom and has electrophilicity. In addition, the calculated results show that during the change from the electron-donating group to the electron-withdrawing group, the APT charge on the $\mathrm{C}^{1}$ atom gradually decreases, indicating that its electrophilicity is gradually weakened; while the APT charge of the $\mathrm{H}^{1}$ atom in gradually increases, indicating that its nucleophilicity gradually decreases. All of these indicate that the $\mathrm{C}-\mathrm{H}$ bond is gradually weakened. Thus, this well explains why the energy barrier for $\mathrm{C}-\mathrm{H}$ cleavage is reduced during the change of substituents from electron-donating groups to electronwithdrawing groups. Thus, the energy barrier for $\mathrm{C}-\mathrm{H}$ bond cleavage gradually decreases, which is consistent with the result of the modulation of the substituents. 

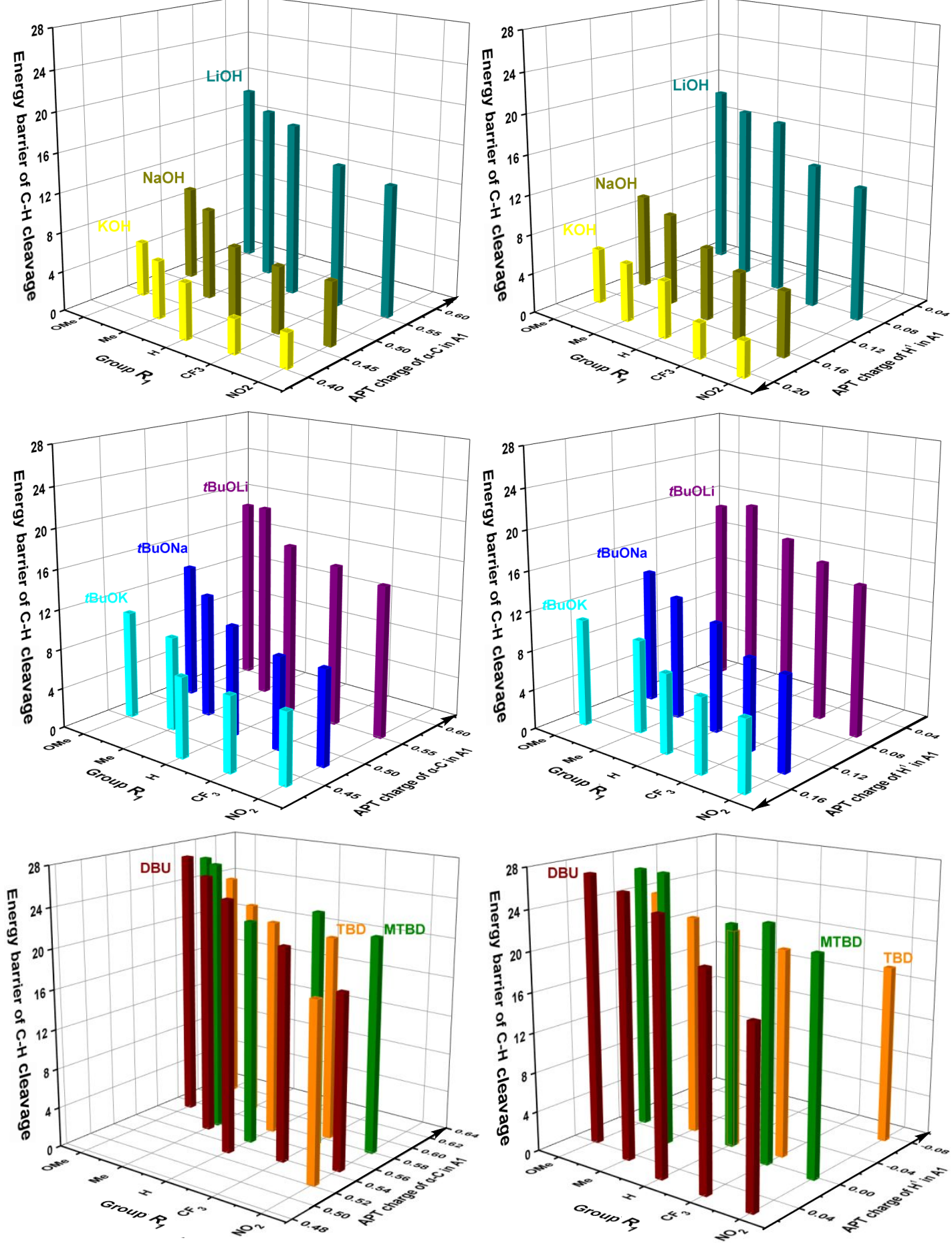

Figure S6. The change of APT charge (unit: a.u.) on $C^{1}$ and $H^{1}$ atoms in the intermediate $A 1$ during the modulation of $R_{1}$ substituent. 

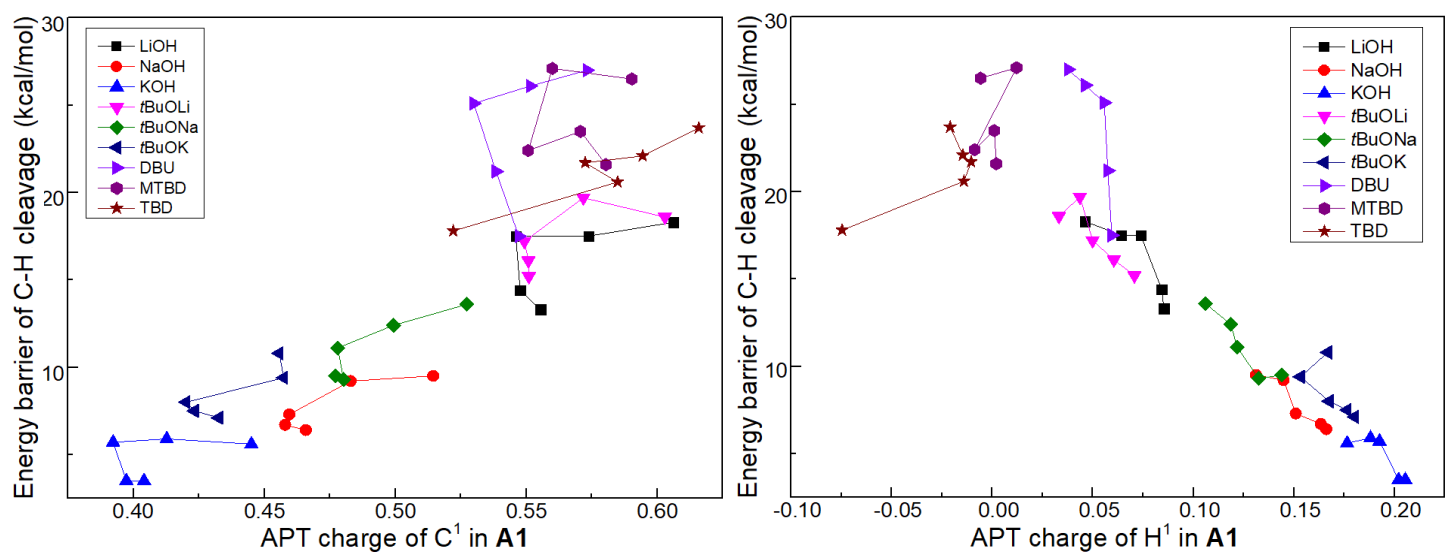

Figure S7. The correlation between the APT charge (unit: a.u.) on $C^{1}$ and $H^{1}$ atoms in intermediate $\boldsymbol{A} \mathbf{1}$ and the energy barrier of the $C-H$ cleavage during the modulation of $R_{1}$ substituent. 

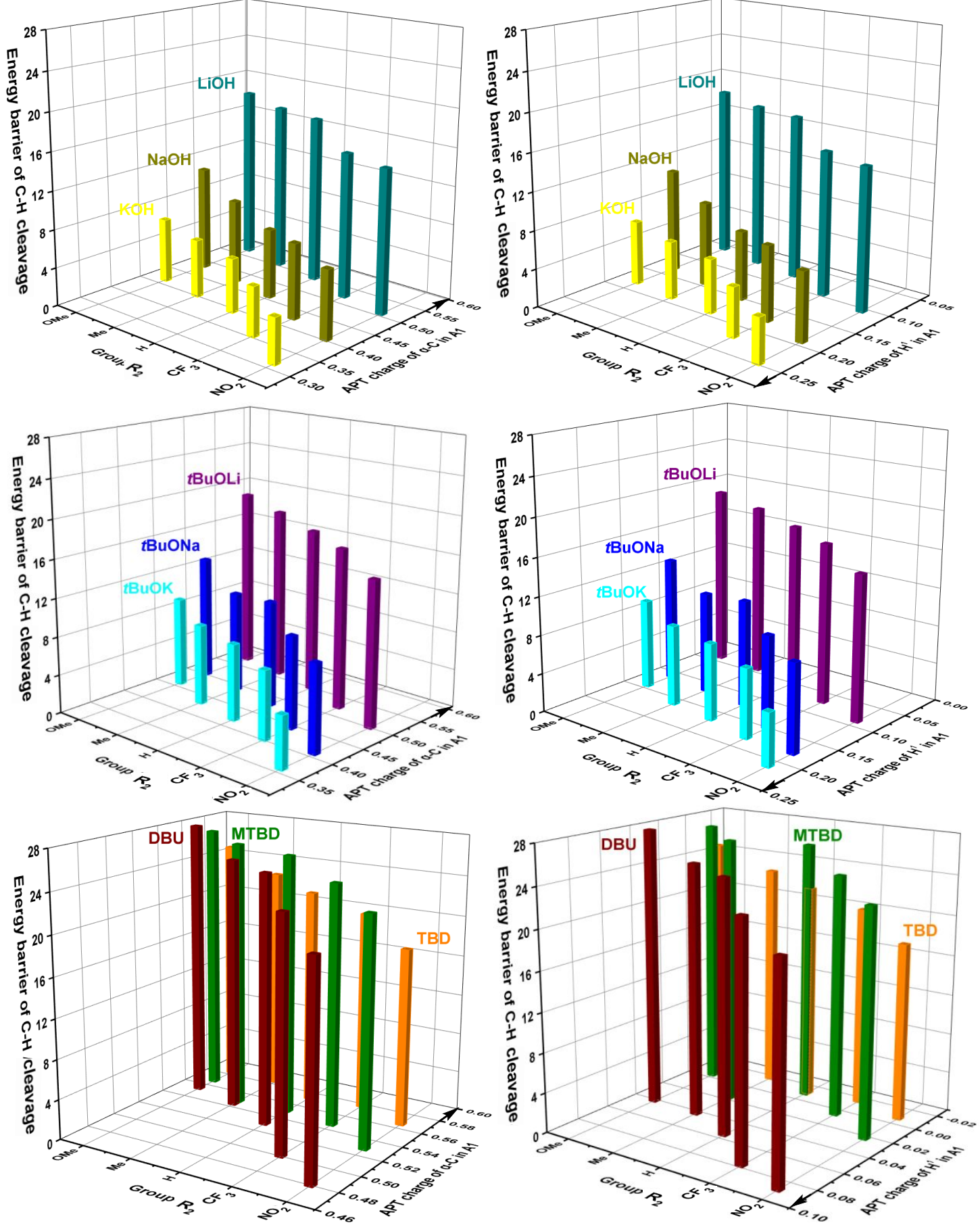

Figure S8. The change of APT charge (unit: a.u.) on $C^{1}$ and $H^{1}$ atoms in the intermediate $\boldsymbol{A 1}$ during the modulation of $R_{2}$ substituent. 

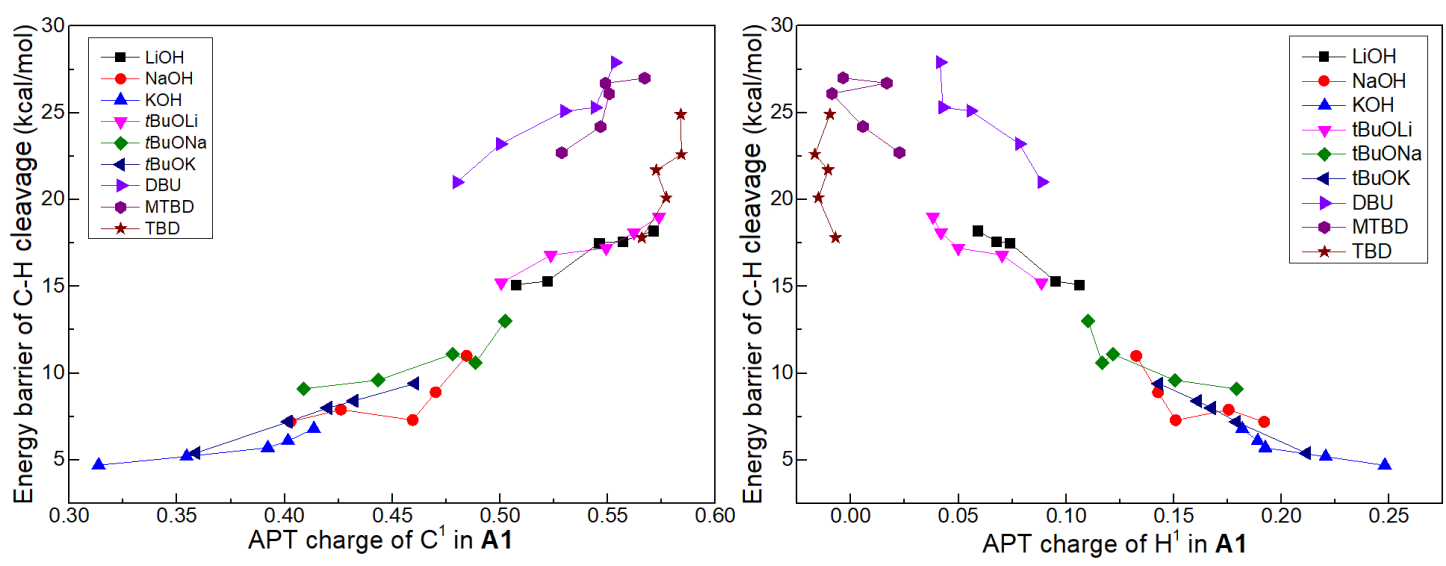

Figure S9. The correlation between the APT charge (unit: a.u.) on $C^{1}$ and $H^{1}$ atoms in intermediate $\boldsymbol{A 1}$ and the energy barrier of the $C-H$ cleavage during the modulation of $R_{2}$ substituent. 


\section{NAO bond order of $C^{1-}-H^{1}$ bond in $A 1$ during $R_{1}$ and $R_{2}$ modulation.}

In order to verify the accuracy of APT charge analysis results, we conducted further analysis and verification in the perspective of NAO bond order. The NBO method was employed to calculate the NAO bond order of $\mathrm{C}^{1}-\mathrm{H}^{1}$ bond in intermediate A1 during the modulation of substituents (Figure S10-S11). The results show that during the transition of the substituent from the electron-donating group to the electronwithdrawing group, the bond order of the $\mathrm{C}^{1}-\mathrm{H}^{1}$ bond gradually decreases, and the cleavage energy barrier also decreases accordingly.

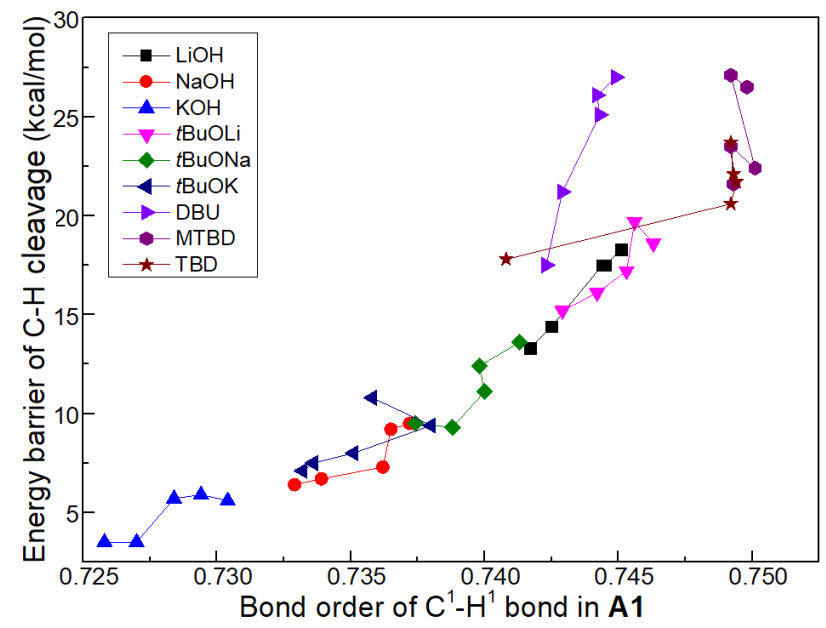

Figure S10. The relationship between the bond order (unit: a.u.) of $C^{1-} H^{1}$ bond in

A1 and the energy barrier of the $C-H$ cleavage during the modulation of substituent $R_{1}$. 


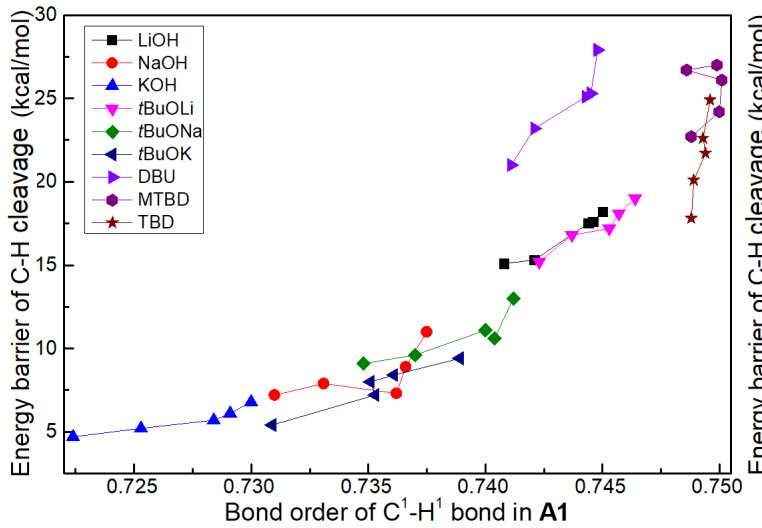

(a)

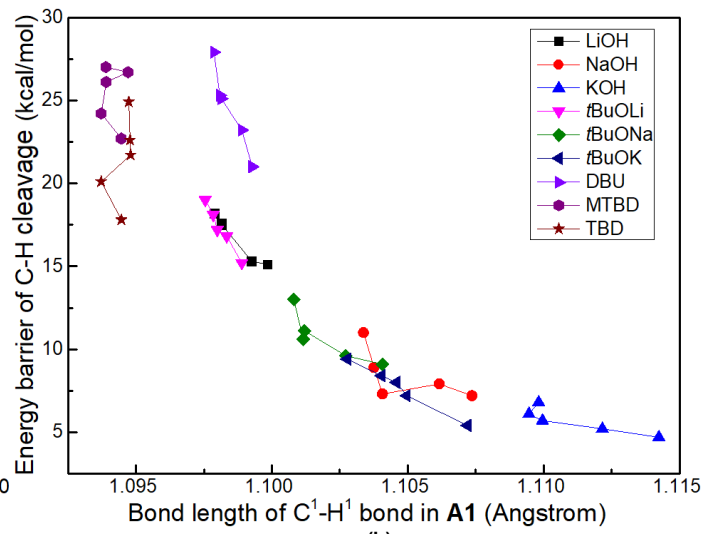

(b)

Figure S11. The relationship between the bond order (unit: a.u.) and the bond length of $C^{1-} H^{1}$ in $A 1$ and the energy barrier of the $C-H$ cleavage during the modulation of substituent $R_{2}$. 


\section{Hirshfeld charge of nitrogen-containing systems.}

As shown in Figure S13, we explored the relationship between the Hirshfeld charges of $\mathrm{C}^{1}$ and $\mathrm{H}^{1}$ atoms and the $\mathrm{C}-\mathrm{H}$ cleavage energy barrier in the nitrogencontaining system, and the calculated results show that there is no correlation between of them, taking the modulation of substituent $\mathrm{R}_{2}$ as an example. In other words, Hirshfeld charge is a descriptor of oxygen-containing systems but not nitrogencontaining systems. In addition, in order to increase the reference point of the nitrogencontaining system, we have modified the substituents of bases TBD and DBU (Figure S12).

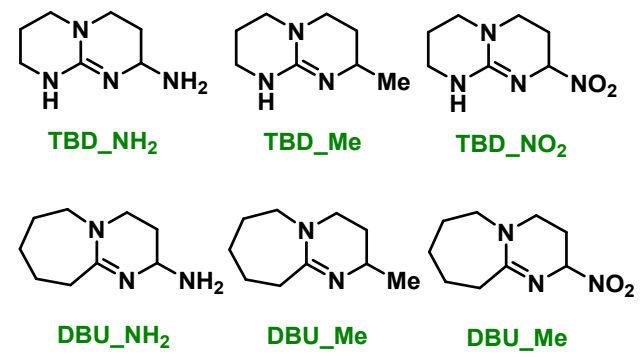

Figure S12: The modulation of nitrogen-containing bases.
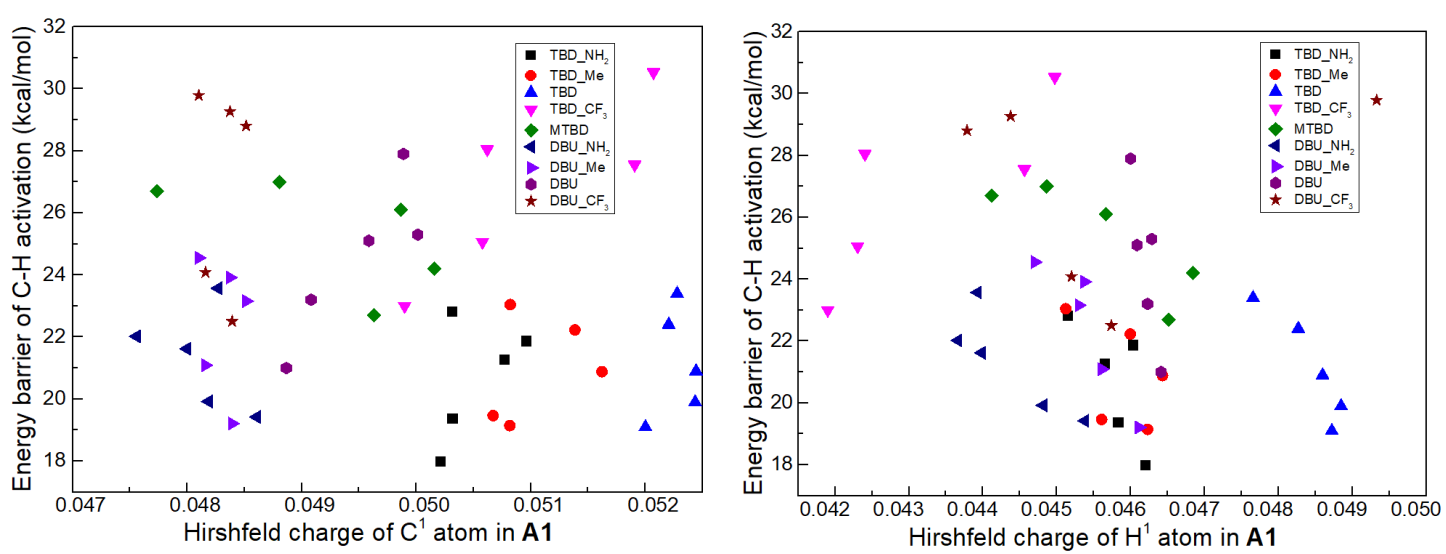

Figure S13: The correlation between the Hirshfeld charge (unit: a.u.) of $C^{1}$ and $H^{1}$ atoms in $\mathrm{Al}$ and the energy barrier of the $C-H$ cleavage. In each nitrogen-containing system, five substituting groups with $\mathrm{R}_{2}=\mathrm{OMe}, \mathrm{Me}, \mathrm{H}, \mathrm{CF}_{3}, \mathrm{NO}_{2}$ were considered. 
5. Information-theoretic quantities.

\subsection{Shannon Entropy}
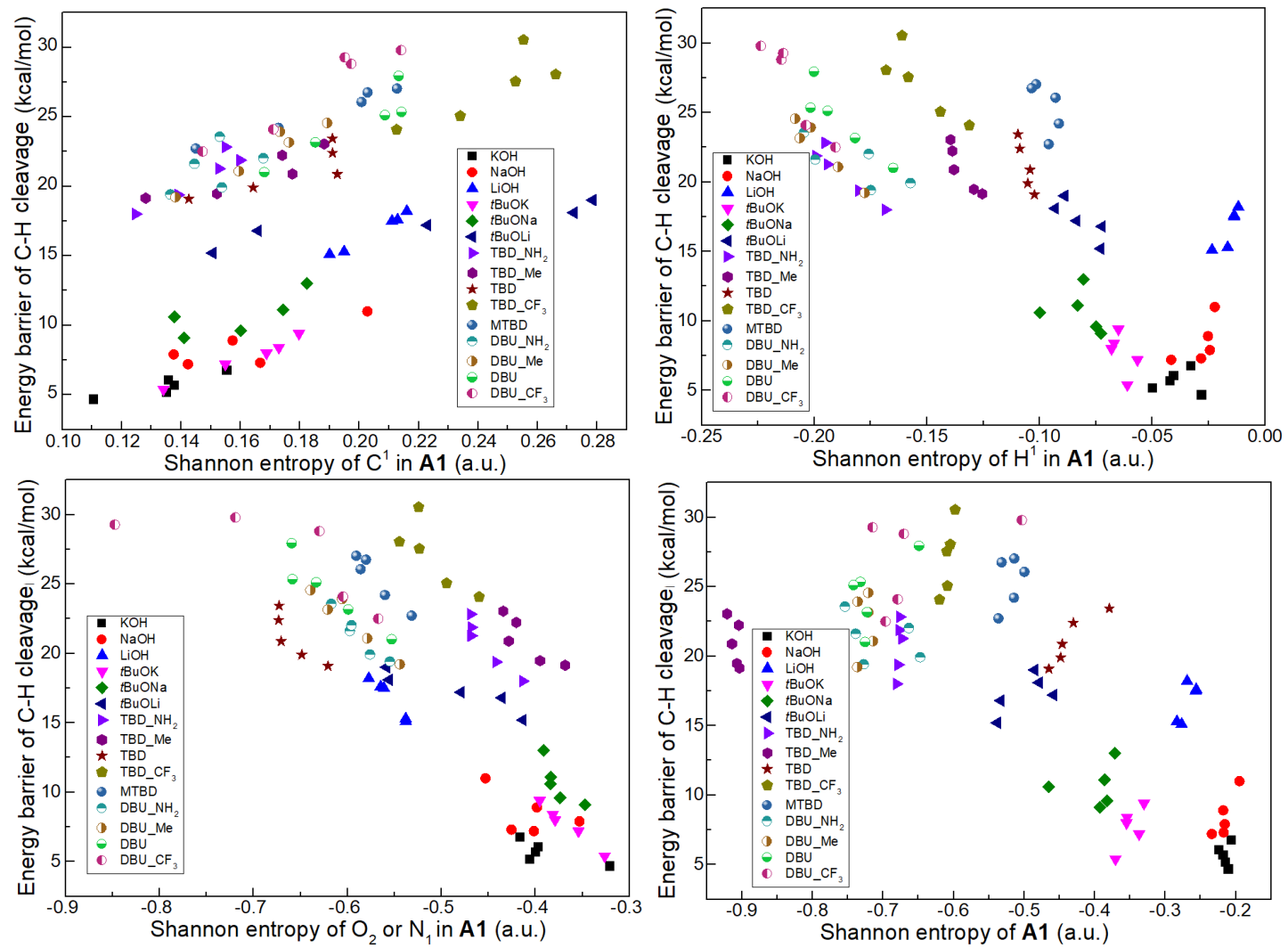

Figure S14: The correlation between the Shannon entropy of the key atoms in A1 and the energy barrier of the $C-H$ cleavage step for both oxygen and nitrogen containing systems. 


\subsection{Information Gain $\left(I_{\mathrm{G}}\right)$}
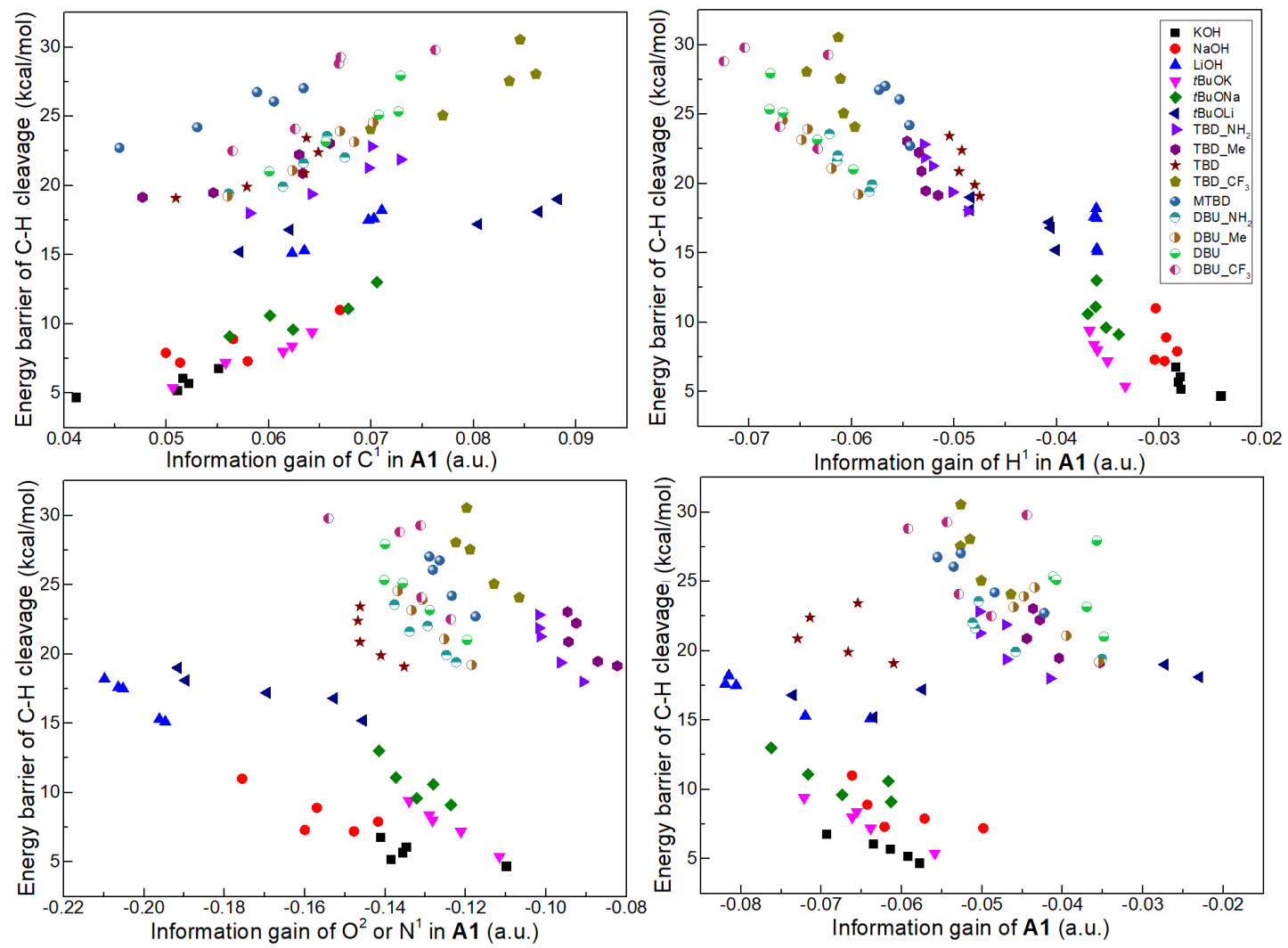

Figure S15: The correlation between the $I_{G}$ of the key atoms in $\mathbf{A 1}$ and the energy barrier of the $C-H$ cleavage step for both oxygen and nitrogen containing systems. 


\subsection{Fisher Information}
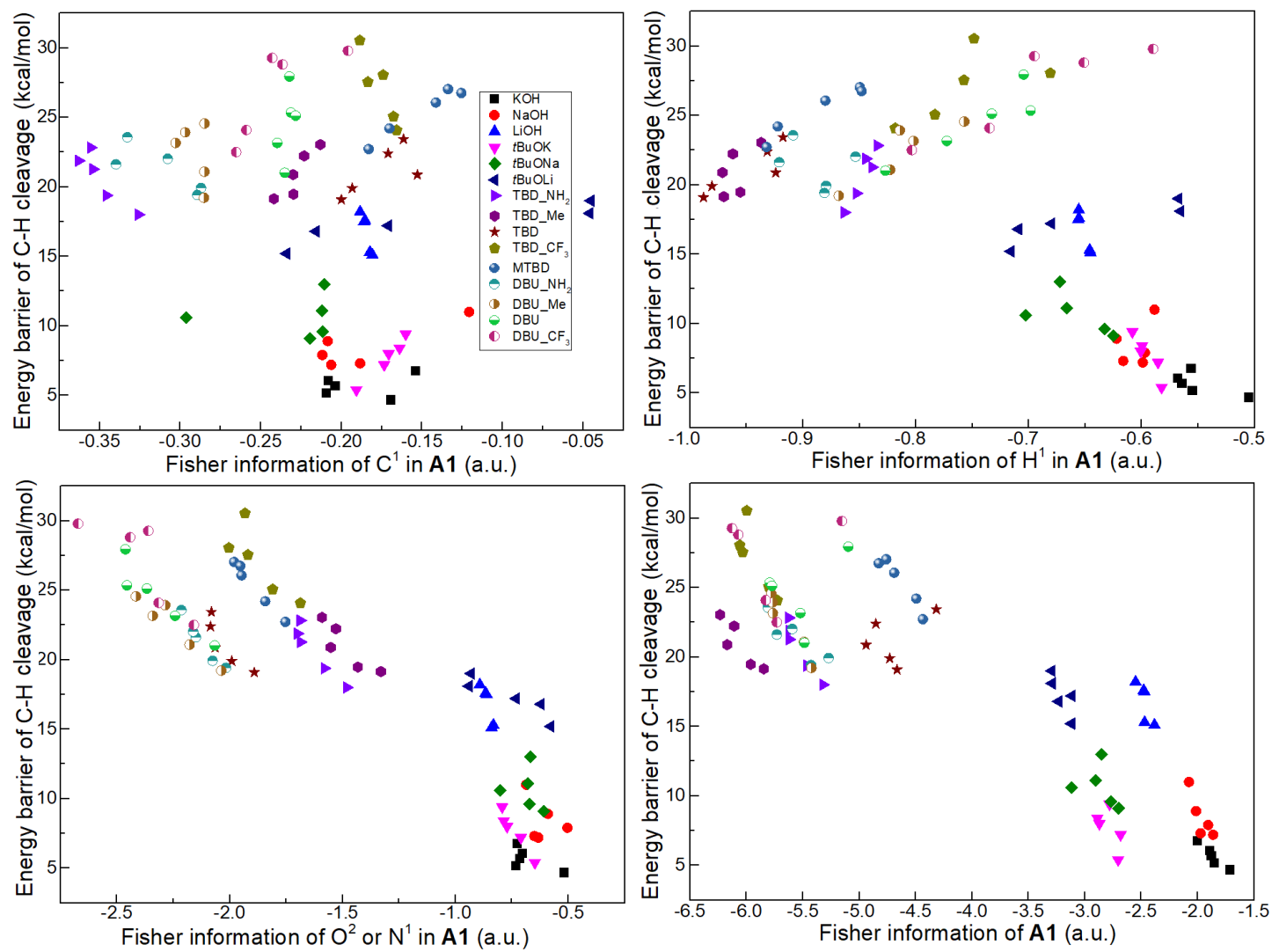

Figure S16: The correlation between the Fisher information of the key atoms in A1 and the energy barrier of the $C-H$ cleavage step for both oxygen and nitrogen containing systems. 


\section{Hammett plot.}
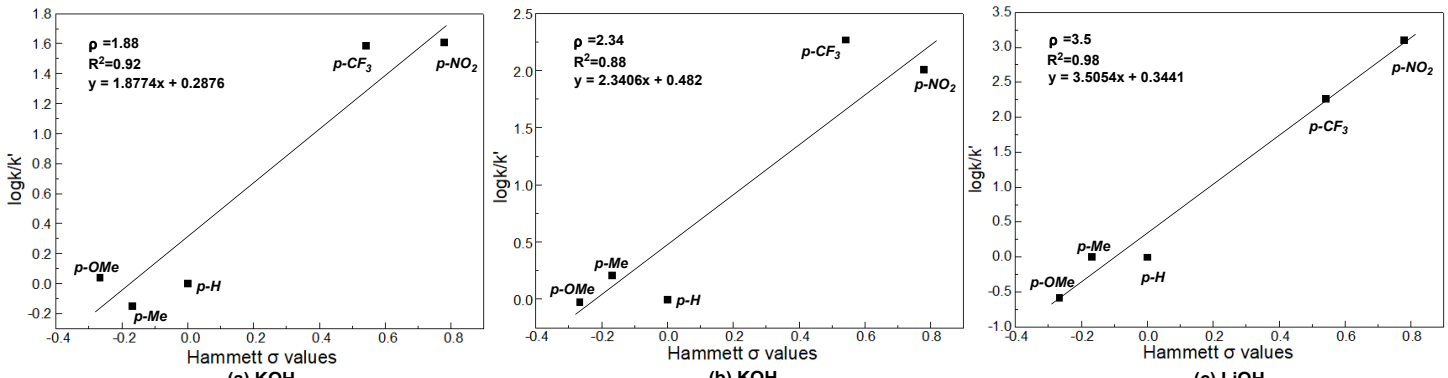

(a) $\mathrm{KOH}$
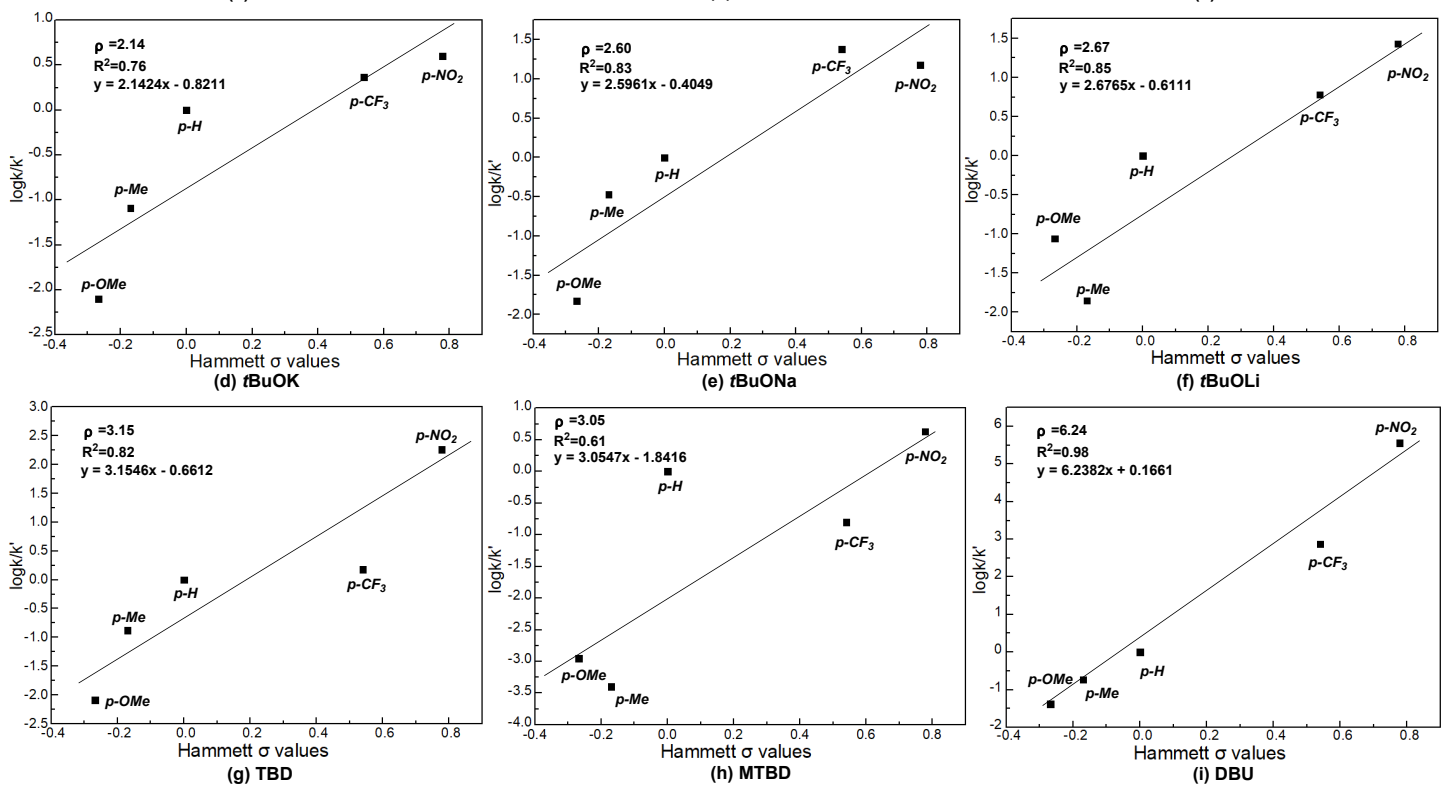

Figure S17: Plots of the Hammett competition studies on modulation of $R_{1}$ substituent. 

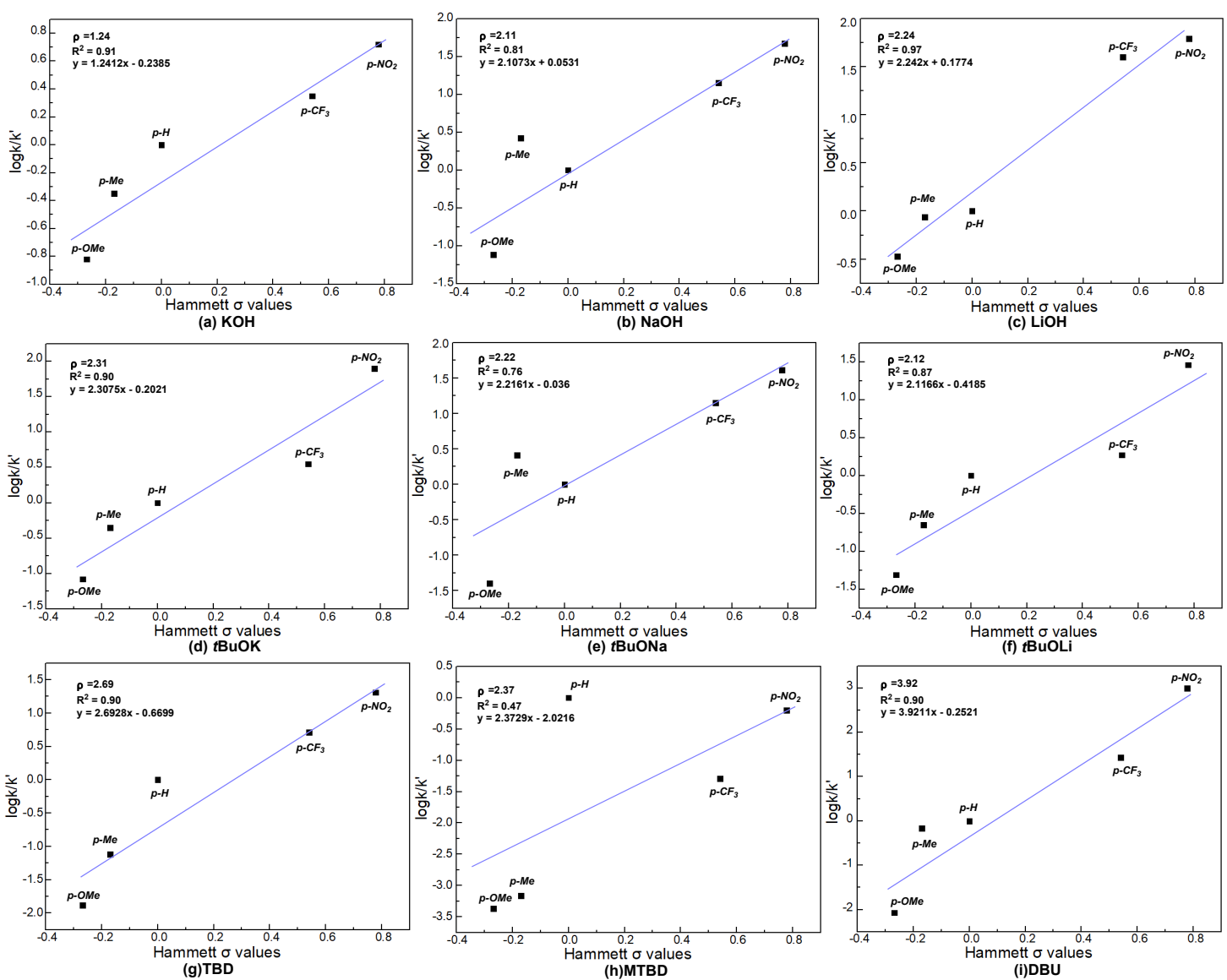

Figure S18: Plots of the Hammett competition studies on modulation of $R_{2}$ substituent. 


\section{Functional and basis set test.}

Table S1. The relative Gbbis free energies (in kcal/mol) of key intermediates in the isomerization of allylic alcohol catalyzed by tBuOK at $\omega B 97 X-D / 6-31 G^{*}, B 3 L Y P / 6-$ $31 G^{*}$ and $B 3 L Y P-D 3 / 6-31 G^{*}$ levels.

\begin{tabular}{cccc}
\hline Compounds & $\omega$ B97X-D & B3LYP & B3LYP-D3 \\
\hline A1 & 0.0 & 0.0 & 0.0 \\
TSA1-2 & 8.0 & 8.9 & 7.6 \\
A2 & -2.4 & -1.3 & -2.9 \\
TSA2-3 & 3.5 & 5.5 & 3.0 \\
\hline
\end{tabular}

Table S2: Other basis sets such as $6-31++G(d, p)$ and $6-311+G(d)$ were tested but no qualitatively different result was obtained.

\begin{tabular}{cccc}
\hline$\Delta G 1(\mathrm{kcal} / \mathrm{mol})$ & $6-31 \mathrm{G}(\mathrm{d})$ & $6-31++\mathrm{G}(\mathrm{d}, \mathrm{p})$ & $6-311+\mathrm{G}(\mathrm{d})$ \\
\hline$\Delta G 1_{\mathrm{KOH}}$ & 5.7 & 6.3 & 8.8 \\
$\Delta G 1_{\mathrm{NaOH}}$ & 9.5 & 9.0 & 11.5 \\
$\Delta G 1_{\mathrm{LiOH}}$ & 17.5 & 18.0 & 19.8 \\
$\Delta G 1_{t \mathrm{BuOK}}$ & 8.0 & 7.7 & 10.6 \\
$\Delta G 1_{t \mathrm{BuONa}}$ & 11.1 & 9.3 & 12.3 \\
$\Delta G 1_{t \mathrm{BuOLi}}$ & 17.2 & 15.7 & 18.3 \\
$\Delta G 1_{\mathrm{TBD}}$ & 20.9 & 19.4 & 20.5 \\
$\Delta G 1_{\mathrm{MTBD}}$ & 22.4 & 21.5 & 24.5 \\
$\Delta G 1_{\mathrm{DBU}}$ & 25.1 & 22.1 & 24.8 \\
\hline
\end{tabular}

\section{Energies of all the structures.}

Table S3. The calculated absolute electronic energies ( $E$, in a.u.), thermal free energies ( $G$, in a.u.), and relative Gibbs energies $(\Delta G$, in $\mathrm{kcal} / \mathrm{mol}$ ) (Calculated at $298.15 \mathrm{~K}$ and $1 \mathrm{~atm})$.

\begin{tabular}{llll}
\hline Complex & $\boldsymbol{E}$ & $\boldsymbol{G}$ & $\Delta G$ \\
\hline
\end{tabular}




$$
R_{1}=R_{2}=H
$$

$\mathrm{A} 1_{(\mathrm{KOH}, \mathrm{R} 1=\mathrm{R} 2=\mathrm{H})}$

$\mathrm{TSA}_{\left.1-\mathbf{2}_{(\mathrm{KOH}, \mathrm{R} 1=\mathrm{R} 2}=\mathrm{H}\right)}$

$A 2_{(\mathrm{KOH}, \mathrm{R} 1=\mathrm{R} 2=\mathrm{H})}$

$\mathrm{TSA}_{2}-3_{(\mathrm{KOH}, \mathrm{R} 1=\mathrm{R} 2=\mathrm{H})}$

$\mathrm{A3}_{(\mathrm{KOH}, \mathrm{R} 1=\mathrm{R} 2=\mathrm{H})}$

$A 1_{(\mathrm{NaOH}, \mathrm{R} 1=\mathrm{R} 2=\mathrm{H})}$

$\operatorname{TSA1}^{\left.-2_{(\mathrm{NaOH}}, \mathrm{R} 1=\mathrm{R} 2=\mathrm{H}\right)}$

$\mathrm{A} 2_{(\mathrm{NaOH}, \mathrm{R} 1=\mathrm{R} 2=\mathrm{H})}$

$\mathrm{TSA}_{2}-3_{(\mathrm{NaOH}, \mathrm{R} 1=\mathrm{R} 2=\mathrm{H})}$

$\mathrm{A3}_{(\mathrm{NaOH}, \mathrm{R} 1=\mathrm{R} 2=\mathrm{H})}$

$\mathbf{A} 1_{(\mathrm{LiOH}, \mathrm{R} 1=\mathrm{R} 2=\mathrm{H})}$

TSA1-2 $_{(\mathrm{LiOH}, \mathrm{R} 1=\mathrm{R} 2=\mathrm{H})}$

$A 2_{(\mathrm{LiOH}, \mathrm{R} 1=\mathrm{R} 2=\mathrm{H})}$

TSA2-3 $_{(\mathrm{LiOH}, \mathrm{R} 1=\mathrm{R} 2=\mathrm{H})}$

$\mathrm{A3}_{(\mathrm{LiOH}, \mathrm{R} 1=\mathrm{R} 2=\mathrm{H})}$

$\mathrm{A1}_{(t \mathrm{BuOK}, \mathrm{R} 1=\mathrm{R} 2=\mathrm{H})}$

TSA1-2 ${ }_{(t \mathrm{BuOK}, \mathrm{R} 1=\mathrm{R} 2=\mathrm{H})}$

$\mathrm{A} 2_{(\mathrm{BuOK}, \mathrm{R} 1=\mathrm{R} 2=\mathrm{H})}$

$\mathrm{TSA}_{\left.2-3_{(t \mathrm{BuOK}}, \mathrm{R} 1=\mathrm{R} 2=\mathrm{H}\right)}$

$\mathrm{A3}_{(\mathrm{tBuOK}, \mathrm{R} 1=\mathrm{R} 2=\mathrm{H})}$

$\mathbf{A} 1_{(t \mathrm{BuONa}, \mathrm{R} 1=\mathrm{R} 2=\mathrm{H})}$

TSA1-2 $_{(t \mathrm{BuONa}, \mathrm{R} 1=\mathrm{R} 2=\mathrm{H})}$

$\mathrm{A} \mathbf{2}_{(t \mathrm{BuONa}, \mathrm{R} 1=\mathrm{R} 2=\mathrm{H})}$

TSA2-3 $\left.{ }_{(t \mathrm{BuONa}}, \mathrm{R} 1=\mathrm{R} 2=\mathrm{H}\right)$

$\mathrm{A3}_{(\mathrm{tBuONa}, \mathrm{R} 1=\mathrm{R} 2=\mathrm{H})}$

TSA2-4 ${ }_{(t \mathrm{BuONa}, \mathrm{R} 1=\mathrm{R} 2=\mathrm{H})}$

$\mathrm{A4}_{(t \mathrm{BuONa}, \mathrm{R} 1=\mathrm{R} 2=\mathrm{H})}$

TSA4-5 $_{(t \mathrm{BuONa}, \mathrm{R} 1=\mathrm{R} 2=\mathrm{H})}$
$-1330.72476909$

$-1330.71194577$

$-1330.73119248$

$-1330.71734622$

$-1330.73791700$

$-893.11218722$

$-893.09415635$

$-893.11006883$

$-893.10247537$

$-893.12425034$

$-738.38746201$

$-738.35786185$

$-738.37074113$

$-738.36698496$

$-738.39838439$

$-1487.94929270$

$-1487.93252288$

$-1487.95351477$

$-1487.93871926$

$-1487.96374680$

$-1050.33604906$

$-1050.31359548$

$-1050.33084658$

$-1050.32308620$

$-1050.34989126$

$-1050.30034329$

$-1050.37514389$

$-1050.36123437$
$-1330.510708$

0.0

$-1330.501690$

5.7

$-1330.518114$

$-4.6$

$-1330.506024$

2.9

$-1330.518958$

$-5.2$

$-892.896595$

0.0

$-892.881524$

9.5

$-892.894632$

1.2

$-892.891093$

3.5

$-892.906460$

$-6.2$

$-738.169257$

0

$-738.141350$

17.5

$-738.151949$

10.9

$-738.151608$

11.1

$-738.179413$

$-6.4$

$-1487.626886$

0.0

$-1487.614193$

8.0

$-1487.630676$

$-2.4$

$-1487.621347$

3.5

$-1487.637260$

$-6.5$

$-1050.011928$

0.0

$-1049.994187$

11.1

$-1050.008757$

2.0

$-1050.001761$

6.4

$-1050.023246$

$-7.1$

$-1049.980207$

19.9

$-1050.049703$

$-23.7$

$-1050.039851$ 


\begin{tabular}{|c|c|c|c|}
\hline $\mathbf{A 5}_{(t \mathrm{BuONa}, \mathrm{R} 1=\mathrm{R} 2=\mathrm{H})}$ & -1050.37524885 & -1050.048203 & -22.5 \\
\hline $\mathrm{B} 1_{(t \mathrm{BuONa}, \mathrm{R} 1=\mathrm{R} 2=\mathrm{H})}$ & -1049.76141247 & -1049.450285 & 0.0 \\
\hline TSB1-2 $(t \mathrm{BuONa}, \mathrm{R} 1=\mathrm{R} 2=\mathrm{H})$ & -1049.73240884 & -1049.42530 & 15.7 \\
\hline $\mathrm{B} 2_{(t \mathrm{BuONa}, \mathrm{R} 1=\mathrm{R} 2=\mathrm{H})}$ & -1049.76966910 & -1049.458427 & -5.1 \\
\hline $\left.\mathrm{TSB2}^{-3} 3_{(t \mathrm{BuONa}}, \mathrm{R} 1=\mathrm{R} 2=\mathrm{H}\right)$ & -1049.76842728 & -1049.459936 & -6.1 \\
\hline $\mathrm{B3}_{(t \mathrm{BuONa}, \mathrm{R} 1=\mathrm{R} 2=\mathrm{H})}$ & -1049.81572856 & -1049.506598 & -35.3 \\
\hline$C 1_{(t \mathrm{BuONa}, \mathrm{R} 1=\mathrm{R} 2=\mathrm{H})}$ & -1050.35522593 & -1050.029495 & 0.0 \\
\hline $\left.\operatorname{TSC1} 1-2_{(t \mathrm{BuONa},} \mathrm{R} 1=\mathrm{R} 2=\mathrm{H}\right)$ & -1050.35315819 & -1050.030397 & -0.6 \\
\hline $\mathrm{C} 2_{(t \mathrm{BuONa}, \mathrm{R} 1=\mathrm{R} 2=\mathrm{H})}$ & -1050.35788145 & -1050.031964 & -1.5 \\
\hline $\mathrm{C3}_{(t \mathrm{BuONa}, \mathrm{R} 1=\mathrm{R} 2=\mathrm{H})}$ & -816.70720382 & -816.512872 & 14.9 \\
\hline $\mathrm{C4}_{(t \mathrm{BuONa}, \mathrm{R} 1=\mathrm{R} 2=\mathrm{H})}$ & -1212.04500034 & -1211.735169 & -1.6 \\
\hline $\mathrm{TSC}_{4}-5_{(\mathrm{tBuONa}, \mathrm{R} 1=\mathrm{R} 2=\mathrm{H})}$ & -1212.03140851 & -1211.723504 & 5.7 \\
\hline $\mathrm{C5}_{(t \mathrm{BuONa}, \mathrm{R} 1=\mathrm{R} 2=\mathrm{H})}$ & -1212.06030041 & -1211.747662 & -9.5 \\
\hline $\operatorname{TSC}_{-6} \mathbf{6}_{(t \mathrm{BuONa}, \mathrm{R} 1=\mathrm{R} 2=\mathrm{H})}$ & -1212.05070573 & -1211.745704 & -8.2 \\
\hline $\mathrm{C6}_{(t \mathrm{BuONa}, \mathrm{R} 1=\mathrm{R} 2=\mathrm{H})}$ & -1212.06551308 & -1211.755000 & -14.1 \\
\hline $\left.\mathbf{A} 1_{(t \mathrm{BuOLi}}, \mathrm{R} 1=\mathrm{R} 2=\mathrm{H}\right)$ & -895.60824286 & -895.280019 & 0.0 \\
\hline $\left.\operatorname{TSA}_{1-2}{ }_{(t \mathrm{BuOLi}}, \mathbf{R} 1=\mathbf{R} 2=\mathrm{H}\right)$ & -895.57331785 & -895.252621 & 17.2 \\
\hline $\left.\mathbf{A} \mathbf{2}_{(t \mathrm{BuOLi}} \mathrm{R} 1=\mathrm{R} 2=\mathrm{H}\right)$ & -895.59403696 & -895.266278 & 8.6 \\
\hline $\left.\operatorname{TSA}_{2}-3_{(t \mathrm{BuOLi}}, \mathbf{R} 1=\mathrm{R} 2=\mathrm{H}\right)$ & -895.58664826 & -895.263693 & 10.2 \\
\hline $\left.\mathbf{A} 3_{(t \mathrm{BuOLi}}, \mathrm{R} 1=\mathrm{R} 2=\mathrm{H}\right)$ & -895.62048472 & -895.291488 & -7.2 \\
\hline 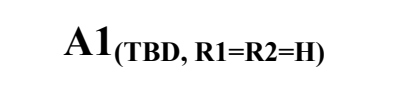 & -1093.71931517 & -1093.315075 & 0.0 \\
\hline TSA1-2 $_{(\mathrm{TBD}, \mathrm{R} 1=\mathrm{R} 2=\mathrm{H})}$ & -1093.68705795 & -1093.281824 & 20.9 \\
\hline $\mathbf{A} \mathbf{2}_{(\mathrm{TBD}, \mathrm{R} 1=\mathrm{R} 2=\mathrm{H})}$ & -1093.69547408 & -1093.286978 & 17.6 \\
\hline $\mathrm{TSA}_{2}-3_{(\mathrm{TBD}, \mathrm{R} 1=\mathrm{R} 2=\mathrm{H})}$ & -1093.69057326 & -1093.287577 & 17.3 \\
\hline 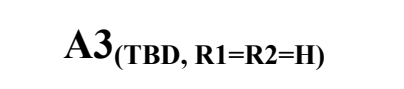 & -1093.72889332 & -1093.322188 & -4.5 \\
\hline 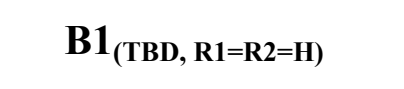 & -1093.12291719 & -1092.732178 & 0.0 \\
\hline $\mathrm{TSB}_{1-2} \mathbf{2}_{(\mathrm{TBD}, \mathrm{R} 1=\mathrm{R} 2=\mathrm{H})}$ & -1093.08461370 & -1092.696199 & 22.6 \\
\hline 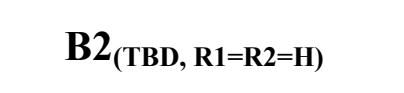 & -1093.14594982 & -1092.750836 & -11.7 \\
\hline $\mathrm{TSB}^{-}-3_{(\mathrm{TBD}, \mathrm{R} 1=\mathrm{R} 2=\mathrm{H})}$ & -1093.12604534 & -1092.738111 & -3.7 \\
\hline
\end{tabular}




\begin{tabular}{|c|c|c|c|}
\hline $\mathrm{B3}_{(\mathrm{TBD}, \mathrm{R} 1=\mathrm{R} 2=\mathrm{H})}$ & -1093.17106678 & -1092.777586 & -28.5 \\
\hline $\mathbf{A} 1_{(\mathbf{M T B D}, \mathrm{R} 1=\mathrm{R} 2=\mathrm{H})}$ & -1133.01532316 & -1132.582780 & 0.0 \\
\hline $\left.\operatorname{TSA1} \mathbf{2}_{(\mathrm{MTBD}}, \mathrm{R} 1=\mathrm{R} 2=\mathrm{H}\right)$ & -1132.97884231 & -1132.547027 & 22.4 \\
\hline $\mathbf{A} 2_{(\mathrm{MTBD}, \mathrm{R} 1=\mathrm{R} 2=\mathrm{H})}$ & -1132.98855661 & -1132.551912 & 19.4 \\
\hline TSA2-3 & -1132.98439614 & -1132.552665 & 18.9 \\
\hline 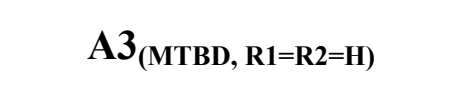 & -1133.02397326 & -1132.591489 & -5.5 \\
\hline $\mathbf{A} \mathbf{1}_{(\mathrm{DBU}, \mathrm{R} 1=\mathbf{R} \mathbf{2}=\mathbf{H})}$ & -1116.97437202 & -1116.532911 & 0.0 \\
\hline $\operatorname{TSA}_{1-2}(\mathrm{DBU}, \mathbf{R} 1=\mathbf{R} 2=\mathrm{H})$ & -1116.93657733 & -1116.492896 & 25.1 \\
\hline $\mathrm{A} \mathbf{2}_{(\mathrm{DBU}, \mathrm{R} 1=\mathrm{R} 2=\mathrm{H})}$ & -1116.94854026 & -1116.500379 & 20.4 \\
\hline $\mathrm{TSA}_{\left.2-3_{(\mathrm{DBU}}, \mathrm{R} 1=\mathrm{R} 2=\mathrm{H}\right)}$ & -1116.94582350 & -1116.501059 & 20.0 \\
\hline $\mathbf{A} 3_{(\mathrm{DBU}, \mathrm{R} 1=\mathrm{R} 2=\mathrm{H})}$ & -1116.98951938 & -1116.543113 & -6.4 \\
\hline \multicolumn{4}{|c|}{$R_{1}=O M e, R_{2}=H$} \\
\hline $\mathbf{A} 1_{(\mathrm{KOH}, \mathrm{R} 1=\mathrm{OMe}, \mathrm{R} 2=\mathrm{H})}$ & -1445.21411122 & -1444.967967 & 0.0 \\
\hline TSA1-2 $(\mathrm{KOH}, \mathrm{R} 1=\mathrm{OMe}, \mathrm{R} 2=\mathrm{H})$ & -1445.19995901 & -1444.959045 & 5.6 \\
\hline$A 2_{(\mathrm{KOH}, \mathrm{R} 1=\mathrm{OMe}, \mathrm{R} 2=\mathrm{H})}$ & -1445.21461427 & -1444.972752 & -3.0 \\
\hline $\mathrm{TSA}^{-}-3_{(\mathrm{KOH}, \mathrm{R} 1=\mathrm{OMe}, \mathrm{R} 2=\mathrm{H})}$ & -1445.20549449 & -1444.964780 & 2.0 \\
\hline $\mathbf{A} 3_{(\mathrm{KOH}, \mathrm{R} 1=\mathrm{OMe}, \mathrm{R} 2=\mathrm{H})}$ & -1445.22719885 & -1444.978753 & -6.8 \\
\hline$A 1_{(\mathrm{NaOH}, \mathrm{R} 1=\mathrm{OMe}, \mathrm{R} 2=\mathrm{H})}$ & -1007.60162237 & -1007.354731 & 0.0 \\
\hline TSA1-2 $_{(\mathrm{NaOH}, \mathrm{R} 1=\mathrm{OMe}, \mathrm{R} 2=\mathrm{H})}$ & -1007.58221889 & -1007.339627 & 9.5 \\
\hline $\mathrm{A} \mathbf{2}_{(\mathrm{NaOH}, \mathrm{R} 1=\mathrm{OMe}, \mathrm{R} 2=\mathrm{H})}$ & -1007.59318063 & -1007.351167 & 2.2 \\
\hline $\mathrm{TSA} 2-3_{(\mathrm{NaOH}, \mathrm{R} 1=\mathrm{OMe}, \mathrm{R} 2=\mathrm{H})}$ & -1007.59053019 & -1007.349234 & 3.4 \\
\hline $\mathrm{A3}_{(\mathrm{NaOH}, \mathrm{R} 1=\mathrm{OMe}, \mathrm{R} 2=\mathrm{H})}$ & -1007.61345586 & -1007.365173 & -6.6 \\
\hline $\mathbf{A} 1_{(\mathrm{LiOH}, \mathrm{R} 1=\mathrm{OMe}, \mathrm{R} 2=\mathrm{H})}$ & -852.87718537 & -852.629282 & 0.0 \\
\hline TSA1-2 $_{(\mathrm{LiOH}, \mathrm{R} 1=\mathrm{OMe}, \mathrm{R} 2=\mathrm{H})}$ & -852.84557588 & -852.600120 & 18.3 \\
\hline$A 2_{(\mathrm{LiOH}, \mathrm{R} 1=\mathrm{OMe}, \mathrm{R} 2=\mathrm{H})}$ & -852.85385062 & -852.607202 & 13.9 \\
\hline TSA2-3 $(\mathrm{LiOH}, \mathrm{R} 1=\mathrm{OMe}, \mathrm{R} 2=\mathrm{H})$ & -852.85566466 & -852.610488 & 11.8 \\
\hline $\mathbf{A} \mathbf{3}_{(\mathrm{LiOH}, \mathrm{R} 1=\mathrm{OMe}, \mathrm{R} 2=\mathrm{H})}$ & -852.88767371 & -852.638014 & -5.5 \\
\hline $\mathrm{A1}_{(t \mathrm{BuOK}, \mathrm{R} 1=\mathrm{OMe}, \mathrm{R} 2=\mathrm{H})}$ & -1602.43830787 & -1602.085901 & 0.0 \\
\hline TSA1-2 $\left.{ }_{(t \mathrm{BuOK},} \mathrm{R} 1=\mathrm{OMe}, \mathrm{R} 2=\mathrm{H}\right)$ & -1602.41634838 & -1602.068643 & 10.8 \\
\hline
\end{tabular}




\begin{tabular}{|c|c|c|c|}
\hline $\left.\mathbf{A} 2_{(t \mathrm{BuOK}}, \mathbf{R} 1=\mathrm{OMe}, \mathbf{R} 2=\mathrm{H}\right)$ & -1602.43698716 & -1602.084376 & 1.0 \\
\hline $\mathrm{TSA2}_{\left.2-3_{(t \mathrm{BuOK}}, \mathrm{R} 1=\mathrm{OMe}, \mathrm{R} 2=\mathrm{H}\right)}$ & -1602.42707703 & -1602.078379 & 4.7 \\
\hline $\mathbf{A} 3_{(t \mathrm{BuOK}, \mathrm{R} 1=\mathrm{OMe}, \mathbf{R 2}=\mathbf{H})}$ & -1602.45398018 & -1602.098799 & -8.1 \\
\hline $\mathbf{A} \mathbf{1}_{(t \mathrm{BuONa}, \mathrm{R} 1=\mathrm{OMe}, \mathrm{R} 2=\mathrm{H})}$ & -1164.82578284 & -1164.471319 & 0.0 \\
\hline TSA1-2 $(t \mathrm{BuONa}, \mathrm{R} 1=\mathrm{OMe}, \mathrm{R} 2=\mathrm{H})$ & -1164.80044242 & -1164.449601 & 13.6 \\
\hline $\mathrm{A} 2_{(t \mathrm{BuONa}, \mathrm{R} 1=\mathrm{OMe}, \mathrm{R} 2=\mathrm{H})}$ & -1164.81675264 & -1164.462833 & 5.3 \\
\hline $\mathrm{TSA}-3_{(\mathrm{tBuONa}, \mathrm{R} 1=\mathrm{OMe}, \mathrm{R} 2=\mathrm{H})}$ & -1164.81039218 & -1164.460882 & 6.5 \\
\hline $\mathrm{A3}_{(t \mathrm{BuONa}, \mathrm{R} 1=\mathrm{OMe}, \mathrm{R} 2=\mathrm{H})}$ & -1164.84042871 & -1164.484100 & -8.0 \\
\hline $\left.\mathrm{A1}_{(t \mathrm{BuOLi}}, \mathrm{R} 1=\mathrm{OMe}, \mathrm{R} 2=\mathrm{H}\right)$ & -1010.09806916 & -1009.740919 & 0.0 \\
\hline $\left.\operatorname{TSA}_{1-2}{ }_{(t \mathrm{BuOLi}}, \mathrm{R} 1=\mathrm{OMe}, \mathrm{R} 2=\mathrm{H}\right)$ & -1010.06392242 & -1009.711214 & 18.6 \\
\hline $\mathrm{A} 2_{(t \mathrm{BuOLi}, \mathrm{R} 1=\mathrm{OMe}, \mathrm{R} 2=\mathrm{H})}$ & -1010.07699374 & -1009.720725 & 12.7 \\
\hline $\operatorname{TSA}_{\left.2-3_{(t \mathrm{BuOLi}}, \mathrm{R} 1=\mathrm{OMe}, \mathrm{R} 2=\mathrm{H}\right)}$ & -1010.07479230 & -1009.721604 & 12.1 \\
\hline $\mathbf{A} 3_{(t \mathrm{BuOLi}, \mathrm{R} 1=\mathrm{OMe}, \mathrm{R} 2=\mathrm{H})}$ & -1010.11092308 & -1009.754119 & -8.3 \\
\hline 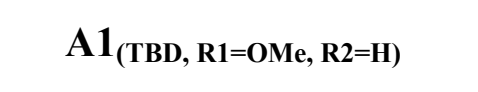 & -1208.21217442 & -1207.775208 & 0.0 \\
\hline $\left.\mathrm{TSA}_{1-2} \mathbf{2}_{(\mathrm{TBD}}, \mathbf{R} 1=\mathrm{OMe}, \mathbf{R} 2=\mathrm{H}\right)$ & -1208.17357969 & -1207.737427 & 23.7 \\
\hline $\mathrm{A} 2_{(\mathrm{TBD}, \mathrm{R} 1=\mathrm{OMe}, \mathrm{R} 2=\mathrm{H})}$ & -1208.17936933 & -1207.742884 & 20.3 \\
\hline $\left.\mathrm{TSA}_{2}-\mathbf{3}_{(\mathrm{TBD}}, \mathrm{R} 1=\mathrm{OMe}, \mathrm{R} 2=\mathrm{H}\right)$ & -1208.17837461 & -1207.744219 & 19.4 \\
\hline 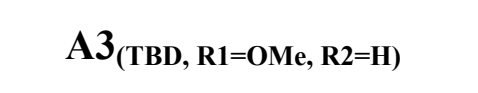 & -1208.21786869 & -1207.780417 & -3.3 \\
\hline 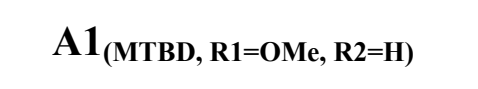 & -1247.51020546 & -1247.045765 & 0.0 \\
\hline 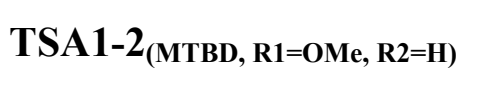 & -1247.46563429 & -1247.003595 & 26.5 \\
\hline $\mathbf{A} 2_{(\mathbf{M T B D}, \mathbf{R} 1=\mathrm{OMe}, \mathrm{R} 2=\mathrm{H})}$ & -1247.47462267 & -1247.008058 & 23.7 \\
\hline TSA2-3 $_{(M T B D,}$ R1=OMe, R2=H) & -1247.47209718 & -1247.010305 & 22.3 \\
\hline $\mathbf{A} \mathbf{3}_{(\mathbf{M T B D}, \mathbf{R} 1=\mathbf{O M e}, \mathbf{R} 2=\mathbf{H})}$ & -1247.51300621 & -1247.049803 & -2.5 \\
\hline 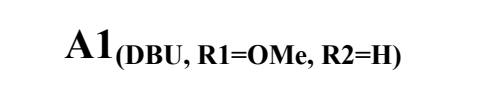 & -1231.46351985 & -1230.990041 & 0.0 \\
\hline $\left.\operatorname{TSA}^{1-2}{ }_{(\mathrm{DBU}}, \mathrm{R} 1=\mathrm{OMe}, \mathrm{R} 2=\mathrm{H}\right)$ & -1231.42272625 & -1230.947036 & 27.0 \\
\hline$A 2_{(\mathrm{DBU}, \mathrm{R} 1=\mathrm{OMe}, \mathrm{R} 2=\mathrm{H})}$ & -1231.43265417 & -1230.954459 & 22.3 \\
\hline TSA2-3 $(\mathrm{DBU}, \mathrm{R} 1=\mathrm{OMe}, \mathrm{R} 2=\mathrm{H})$ & -1231.43404793 & -1230.957025 & 20.7 \\
\hline \multirow[t]{2}{*}{$\mathbf{A} \mathbf{3}_{(\mathbf{D B U}, \mathbf{R} 1=\mathbf{O M e}, \mathbf{R} 2=\mathbf{H})}$} & -1231.47896811 & -1231.002081 & -7.6 \\
\hline & $R_{1}=M e, R_{2}=H$ & & \\
\hline
\end{tabular}




\begin{tabular}{|c|c|c|c|}
\hline $\mathbf{A} 1_{(\mathrm{KOH}, \mathrm{R} 1=\mathrm{Me}, \mathrm{R} 2=\mathrm{H})}$ & -1370.03172330 & -1369.791068 & 0.0 \\
\hline TSA1-2 $_{(\mathrm{KOH}, \mathrm{R} 1=\mathrm{Me}, \mathrm{R} 2=\mathrm{H})}$ & -1370.01864400 & -1369.781714 & 5.6 \\
\hline $\mathbf{A} 2_{(\mathrm{KOH}, \mathrm{R} 1=\mathrm{Me}, \mathrm{R} 2=\mathrm{H})}$ & -1370.03727714 & -1369.798325 & -4.6 \\
\hline TSA2-3 & -1370.02405745 & -1369.788124 & 1.8 \\
\hline $\mathrm{A3}_{(\mathrm{KOH}, \mathrm{R} 1=\mathrm{Me}, \mathrm{R} 2=\mathrm{H})}$ & -1370.04514679 & -1369.800622 & -6.0 \\
\hline $\mathbf{A} \mathbf{1}_{(\mathbf{N a O H}, \mathbf{R} 1=\mathrm{Me}, \mathrm{R} 2=\mathbf{H})}$ & -932.41908482 & -932.177638 & 0.0 \\
\hline $\operatorname{TSA}_{1-2}(\mathrm{NaOH}, \mathrm{R} 1=\mathrm{Me}, \mathrm{R} 2=\mathrm{H})$ & -932.40079098 & -932.163017 & 9.2 \\
\hline $\mathrm{A} 2_{(\mathrm{NaOH}, \mathrm{R} 1=\mathrm{Me}, \mathrm{R} 2=\mathrm{H})}$ & -932.41633053 & -932.175516 & 1.3 \\
\hline $\mathrm{TSA}_{\left.2-3_{(\mathrm{NaOH}}, \mathrm{R} 1=\mathrm{Me}, \mathrm{R} 2=\mathrm{H}\right)}$ & -932.40954647 & -932.171341 & 4.0 \\
\hline $\mathrm{A3}_{(\mathrm{NaOH}, \mathrm{R} 1=\mathrm{Me}, \mathrm{R} 2=\mathrm{H})}$ & -932.43139856 & -932.188168 & -6.6 \\
\hline$A 1_{(\mathrm{LiOH}, \mathrm{R} 1=\mathrm{Me}, \mathrm{R} 2=\mathrm{H})}$ & -777.69455733 & -777.450440 & 0.0 \\
\hline TSA1-2 $_{(\mathrm{LiOH}, \mathrm{R} 1=\mathrm{Me}, \mathrm{R} 2=\mathrm{H})}$ & -777.66446827 & -777.422545 & 17.5 \\
\hline $\left.\mathbf{A} 2_{(\mathrm{LiOH}, \mathrm{R}} \mathbf{1}=\mathrm{Me}, \mathrm{R} 2=\mathrm{H}\right)$ & -777.67728275 & -777.433028 & 10.93 \\
\hline TSA2-3 $3_{(\mathrm{LiOH}, \mathrm{R} 1=\mathrm{Me}, \mathrm{R} 2=\mathrm{H})}$ & -777.67440522 & -777.433052 & 10.91 \\
\hline $\mathbf{A} \mathbf{3}_{(\mathrm{LiOH}, \mathrm{R} 1=\mathrm{Me}, \mathrm{R} 2=\mathrm{H})}$ & -777.70567803 & -777.460053 & -6.0 \\
\hline $\mathrm{A1}_{(t \mathrm{BuOK}, \mathrm{R} 1=\mathrm{Me}, \mathrm{R} 2=\mathrm{H})}$ & -1527.25688106 & -1526.908910 & 0.0 \\
\hline $\operatorname{TSA1}_{1-2}(t \mathrm{BuOK}, \mathrm{R} 1=\mathrm{Me}, \mathrm{R} 2=\mathrm{H})$ & -1527.23901926 & -1526.893851 & 9.4 \\
\hline $\mathbf{A} 2_{(t \mathrm{BuOK}, \mathrm{R} 1=\mathrm{Me}, \mathrm{R} 2=\mathrm{H})}$ & -1527.25972636 & -1526.910578 & -1.0 \\
\hline $\operatorname{TSA}-3_{(t \mathrm{BuOK}, \mathrm{R} 1=\mathrm{Me}, \mathrm{R} 2=\mathrm{H})}$ & -1527.24569954 & -1526.901673 & 4.5 \\
\hline $\mathrm{A} \mathbf{3}_{(t \mathrm{BuOK}, \mathrm{R} 1=\mathrm{Me}, \mathrm{R} 2=\mathrm{H})}$ & -1527.27123090 & -1526.921053 & -7.6 \\
\hline $\mathrm{A1}_{(t \mathrm{BuONa}, \mathrm{R} 1=\mathrm{Me}, \mathrm{R} 2=\mathrm{H})}$ & -1089.64310925 & -1089.294468 & 0.0 \\
\hline $\left.\operatorname{TSA}_{1-2}{ }_{(t \mathrm{BuONa}}, \mathrm{R} 1=\mathrm{Me}, \mathrm{R} 2=\mathrm{H}\right)$ & -1089.62239076 & -1089.274729 & 12.4 \\
\hline$A 2_{(t \mathrm{BuONa}, \mathrm{R} 1=\mathrm{Me}, \mathrm{R} 2=\mathrm{H})}$ & -1089.63992127 & -1089.288915 & 3.5 \\
\hline $\mathrm{TSA}_{\left.2-3_{(\mathrm{BuONa}}, \mathrm{R} 1=\mathrm{Me}, \mathrm{R} 2=\mathrm{H}\right)}$ & -1089.62896136 & -1089.284984 & 6.0 \\
\hline $\left.\mathbf{A} 3_{(t \mathrm{BuONa}}, \mathrm{R} 1=\mathrm{Me}, \mathrm{R} 2=\mathrm{H}\right)$ & -1089.65716835 & -1089.306999 & -7.9 \\
\hline $\mathbf{A} \mathbf{1}_{(t \mathrm{BuOLi}, \mathrm{R} 1=\mathrm{Me}, \mathrm{R} 2=\mathrm{H})}$ & -934.91535941 & -934.564635 & 0.0 \\
\hline TSA1-2 $(t \mathrm{BuOLi}, \mathrm{R} 1=\mathrm{Me}, \mathbf{R 2}=\mathrm{H})$ & -934.88278515 & -934.533205 & 19.7 \\
\hline $\left.\mathbf{A} 2_{(t \mathrm{BuOLi}}, \mathrm{R} 1=\mathrm{Me}, \mathbf{R} 2=\mathrm{H}\right)$ & -934.90048755 & -934.546190 & 11.6 \\
\hline $\left.\operatorname{TSA}-3_{(t \mathrm{BuOLi}}, \mathrm{R} 1=\mathrm{Me}, \mathrm{R} 2=\mathrm{H}\right)$ & -934.89354533 & -934.544665 & 12.5 \\
\hline
\end{tabular}




\begin{tabular}{|c|c|c|c|}
\hline $\left.\mathbf{A} 3_{(t \mathrm{BuOLi}}, \mathbf{R} 1=\mathrm{Me}, \mathbf{R} 2=\mathrm{H}\right)$ & -934.92792217 & -934.574160 & -6.0 \\
\hline 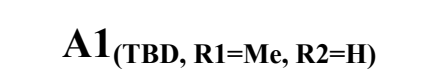 & -1133.03005444 & -1132.597640 & 0.0 \\
\hline $\left.\operatorname{TSA1} \mathbf{2}_{(\mathrm{TBD}}, \mathrm{R} 1=\mathrm{Me}, \mathrm{R} 2=\mathrm{H}\right)$ & -1132.99345369 & -1132.562477 & 22.1 \\
\hline $\mathbf{A} \mathbf{2}_{(\mathrm{TBD}, \mathrm{R} 1=\mathrm{Me}, \mathrm{R} 2=\mathrm{H})}$ & -1133.00183135 & -1132.567905 & 18.7 \\
\hline $\mathrm{TSA}^{\left.2-3_{(\mathrm{TBD}}, \mathrm{R} 1=\mathrm{Me}, \mathrm{R} 2=\mathrm{H}\right)}$ & -1132.99729685 & -1132.568860 & 18.1 \\
\hline $\mathbf{A} \mathbf{3}_{(\mathrm{TBD}, \mathrm{R} 1=\mathrm{Me}, \mathrm{R} 2=\mathrm{H})}$ & -1133.03603768 & -1132.603608 & -3.7 \\
\hline $\mathbf{A} 1_{(\mathbf{M T B D}, \mathbf{R} 1=\mathrm{Me}, \mathbf{R} 2=\mathbf{H})}$ & -1172.32817719 & -1171.870006 & 0.0 \\
\hline 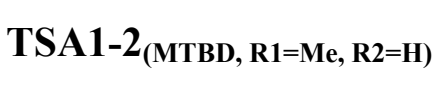 & -1172.28515331 & -1171.826866 & 27.1 \\
\hline $\left.\mathbf{A} 2_{(M T B D}, \mathrm{R} 1=\mathrm{Me}, \mathrm{R} 2=\mathrm{H}\right)$ & -1172.29471874 & -1171.832025 & 23.8 \\
\hline $\mathrm{TSA}_{\left.2-3_{(\mathrm{MTBD}}, \mathrm{R} 1=\mathrm{Me}, \mathrm{R} 2=\mathrm{H}\right)}$ & -1172.29099226 & -1171.832569 & 23.5 \\
\hline $\mathbf{A} 3_{(\mathbf{M T B D}, \mathrm{R} 1=\mathrm{Me}, \mathrm{R} 2=\mathrm{H})}$ & -1172.33117478 & -1171.871766 & -1.1 \\
\hline $\mathbf{A} 1_{(\mathbf{D B U}, \mathbf{R} 1=\mathrm{Me}, \mathbf{R} 2=\mathbf{H})}$ & -1156.28137356 & -1155.814891 & 0.0 \\
\hline TSA1-2 $_{(\mathrm{DBU}, \mathrm{R} 1=\mathrm{Me}, \mathrm{R} 2=\mathrm{H})}$ & -1156.24279605 & -1155.773289 & 26.1 \\
\hline$\left.A 2_{(\mathrm{DBU},} \mathbf{R} 1=\mathrm{Me}, \mathbf{R} 2=\mathrm{H}\right)$ & -1156.25500902 & -1155.779796 & 22.0 \\
\hline $\left.\mathrm{TSA2}_{2} \mathbf{3}_{(\mathrm{DBU}}, \mathrm{R} 1=\mathrm{Me}, \mathrm{R} 2=\mathrm{H}\right)$ & -1156.25273847 & -1155.782824 & 20.1 \\
\hline $\mathbf{A} 3_{(\mathbf{D B U}, \mathbf{R} 1=\mathrm{Me}, \mathbf{R} 2=\mathbf{H})}$ & -1156.29679826 & -1155.824728 & -6.2 \\
\hline \multicolumn{4}{|c|}{$R_{1}=C F_{3}, R_{2}=H$} \\
\hline $\mathbf{A} 1_{(\mathrm{KOH}, \mathrm{R} 1=\mathrm{CF} 3, \mathrm{R} 2=\mathrm{H})}$ & -1667.68115699 & -1667.465579 & 0.0 \\
\hline TSA1-2 $(\mathrm{KOH}, \mathrm{R} 1=\mathrm{CF} 3, \mathrm{R} 2=\mathrm{H})$ & -1667.66946095 & -1667.460023 & 3.5 \\
\hline $\mathrm{A} 2_{(\mathrm{KOH}, \mathrm{R} 1=\mathrm{CF} 3, \mathrm{R} 2=\mathrm{H})}$ & -1667.69117572 & -1667.479448 & -8.7 \\
\hline $\mathrm{TSA2}^{\left.-3_{(\mathrm{KOH}, \mathrm{R}}=\mathrm{CF} 3, \mathrm{R} 2=\mathrm{H}\right)}$ & -1667.67337823 & -1667.464667 & 0.6 \\
\hline $\mathbf{A} 3_{(\mathrm{KOH}, \mathrm{R} 1=\mathrm{CF} 3, \mathrm{R} 2=\mathrm{H})}$ & -1667.69438005 & -1667.476619 & -6.9 \\
\hline $\mathbf{A} \mathbf{1}_{(\mathrm{NaOH}, \mathrm{R} 1=\mathrm{CF} 3, \mathrm{R} 2=\mathrm{H})}$ & -1230.06790531 & -1229.851683 & 0.0 \\
\hline TSA1-2 $(\mathrm{NaOH}, \mathrm{R} 1=\mathrm{CF} 3, \mathrm{R} 2=\mathrm{H})$ & -1230.05149889 & -1229.841550 & 6.4 \\
\hline $\mathbf{A} 2_{(\mathrm{NaOH}, \mathrm{R} 1=\mathrm{CF} 3, \mathrm{R} 2=\mathrm{H})}$ & -1230.06928672 & -1229.855195 & -2.2 \\
\hline $\left.\mathrm{TSA}_{2}-3_{(\mathrm{NaOH}, \mathrm{R} 1=\mathrm{CF}}, \mathrm{R} 2=\mathrm{H}\right)$ & -1230.05843812 & -1229.846299 & 3.4 \\
\hline $\mathrm{A3}_{(\mathrm{NaOH}, \mathrm{R} 1=\mathrm{CF} 3, \mathrm{R} 2=\mathrm{H})}$ & -1230.08033886 & -1229.863060 & -7.1 \\
\hline $\mathbf{A 1}_{(\mathbf{L i O H}, \mathbf{R} 1=\mathrm{CF} 3, \mathbf{R} 2=\mathrm{H})}$ & -1075.34240277 & -1075.123965 & 0.0 \\
\hline TSA1-2 $_{(\mathrm{LiOH}, \mathrm{R} 1=\mathrm{CF} 3, \mathrm{R} 2=\mathrm{H})}$ & -1075.31508778 & -1075.100989 & 14.4 \\
\hline
\end{tabular}




\begin{tabular}{|c|c|c|c|}
\hline $\mathbf{A} 2_{(\mathrm{LiOH}, \mathrm{R} 1=\mathrm{CF} 3, \mathrm{R} 2=\mathrm{H})}$ & -1075.32896666 & -1075.110189 & 8.6 \\
\hline TSA2-3 $(\mathrm{LiOH}, \mathrm{R} 1=\mathrm{CF} 3, \mathrm{R} 2=\mathrm{H})$ & -1075.32323106 & -1075.109435 & 9.1 \\
\hline $\mathbf{A} \mathbf{3}_{(\mathrm{LiOH}, \mathrm{R} 1=\mathrm{CF} 3, \mathbf{R} 2=\mathrm{H})}$ & -1075.35374873 & -1075.135246 & -7.1 \\
\hline $\mathbf{A} 1_{(t \mathrm{BuOK}, \mathrm{R} 1=\mathrm{CF} 3, \mathrm{R} 2=\mathrm{H})}$ & -1824.90498614 & -1824.584316 & 0.0 \\
\hline $\operatorname{TSA}_{1-2}(t \mathrm{BuOK}, \mathrm{R} 1=\mathrm{CF} 3, \mathrm{R} 2=\mathrm{H})$ & -1824.89001859 & -1824.572426 & 7.5 \\
\hline $\mathrm{A} \mathbf{2}_{(t \mathrm{BuOK}, \mathrm{R} 1=\mathrm{CF} 3, \mathrm{R} 2=\mathrm{H})}$ & -1824.91354251 & -1824.591112 & -4.3 \\
\hline $\left.\mathrm{TSA}_{2}-3_{(t \mathrm{BuOK},} \mathrm{R} 1=\mathrm{CF} 3, \mathrm{R} 2=\mathrm{H}\right)$ & -1824.89921953 & -1824.579205 & 3.2 \\
\hline $\mathrm{A3}_{(t \mathrm{BuOK}, \mathrm{R} 1=\mathrm{CF} 3, \mathrm{R} 2=\mathrm{H})}$ & -1824.91930603 & -1824.595610 & -7.1 \\
\hline $\mathrm{A1}_{(t \mathrm{BuONa}, \mathrm{R} 1=\mathrm{CF} 3, \mathrm{R} 2=\mathrm{H})}$ & -1089.64310925 & -1089.294468 & 0.0 \\
\hline TSA1-2 $(t \mathrm{BuONa}, \mathrm{R} 1=\mathrm{CF} 3, \mathbf{R} 2=\mathrm{H})$ & -1089.62239076 & -1089.274729 & 9.3 \\
\hline $\mathrm{A} 2_{(t \mathrm{BuONa}, \mathrm{R} 1=\mathrm{CF} 3, \mathrm{R} 2=\mathrm{H})}$ & -1089.63992127 & -1089.288915 & -0.4 \\
\hline $\mathrm{TSA}_{2}-3_{(t \mathrm{BuONa}, \mathrm{R} 1=\mathrm{CF} 3, \mathrm{R} 2=\mathrm{H})}$ & -1089.62896136 & -1089.284984 & 6.2 \\
\hline $\left.\mathrm{A}_{(t \mathrm{BuONa}}, \mathrm{R} 1=\mathrm{CF} 3, \mathrm{R} 2=\mathrm{H}\right)$ & -1089.65716835 & -1089.306999 & -8.1 \\
\hline $\left.\mathrm{A1}_{(t \mathrm{BuOLi}}, \mathrm{R} 1=\mathrm{CF} 3, \mathrm{R} 2=\mathrm{H}\right)$ & -1232.56263046 & -1232.237276 & 0.0 \\
\hline $\operatorname{TSA}_{1-2}(t \mathrm{BuOLi}, \mathrm{R} 1=\mathrm{CF} 3, \mathrm{R} 2=\mathrm{H})$ & -1232.53362704 & -1232.211566 & 16.1 \\
\hline $\left.\mathrm{A} 2_{(t \mathrm{BuOLi}}, \mathrm{R} 1=\mathrm{CF} 3, \mathrm{R} 2=\mathrm{H}\right)$ & -1232.55252752 & -1232.225696 & 7.3 \\
\hline $\mathrm{TSA}_{\left.2-3_{(t \mathrm{BuOLi}}, \mathrm{R} 1=\mathrm{CF} 3, \mathrm{R} 2=\mathrm{H}\right)}$ & -1232.54246677 & -1232.220903 & 10.3 \\
\hline $\left.\mathbf{A} 3_{(t \mathrm{BuOLi}}, \mathrm{R} 1=\mathrm{CF} 3, \mathrm{R} 2=\mathrm{H}\right)$ & -1232.57539726 & -1232.247985 & -6.7 \\
\hline 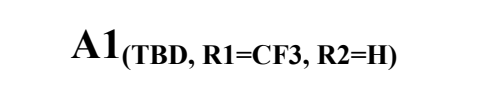 & -1430.67836154 & -1430.274355 & 0.0 \\
\hline 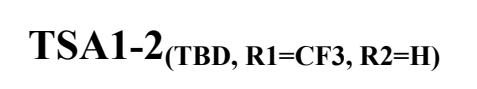 & -1430.64633854 & -1430.241503 & 20.6 \\
\hline $\mathbf{A} 2_{(\mathrm{TBD}, \mathrm{R} 1=\mathrm{CF} 3, \mathbf{R} 2=\mathbf{H})}$ & -1430.65639970 & -1430.249096 & 15.9 \\
\hline 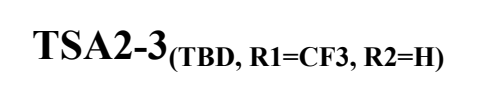 & -1430.64864913 & -1430.245956 & 17.8 \\
\hline $\mathbf{A} 3_{(\mathrm{TBD}, \mathrm{R} 1=\mathrm{CF} 3, \mathrm{R} 2=\mathrm{H})}$ & -1430.68329994 & -1430.276866 & -1.6 \\
\hline$\left.A 1_{(M T B D}, R 1=C F 3, R 2=H\right)$ & -1469.97657705 & -1469.54429 & 0.0 \\
\hline $\left.\mathrm{TSA}_{1-2}{ }_{(\mathrm{MTBD}}, \mathrm{R} 1=\mathrm{CF} 3, \mathrm{R} 2=\mathrm{H}\right)$ & -1469.93824169 & -1469.506806 & 23.5 \\
\hline$\left.A 2_{(\mathrm{MTBD}, \mathrm{R} 1=\mathrm{CF}}, \mathrm{R} 2=\mathrm{H}\right)$ & -1469.95039113 & -1469.515147 & 18.3 \\
\hline 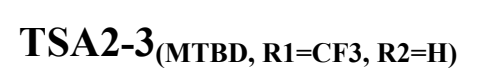 & -1469.94297833 & -1469.511483 & 20.6 \\
\hline $\left.\mathbf{A} 3_{(M T B D}, R 1=C F 3, R 2=H\right)$ & -1469.97890054 & -1469.545455 & -0.7 \\
\hline $\left.\mathbf{A} 1_{(\mathbf{D B U}, \mathrm{R} 1=\mathrm{CF}}, \mathrm{R} 2=\mathrm{H}\right)$ & -1453.93044841 & -1453.489103 & 0.0 \\
\hline
\end{tabular}




\begin{tabular}{|c|c|c|c|}
\hline TSA1-2 $(\mathrm{DBU}, \mathrm{R} 1=\mathrm{CF} 3, \mathrm{R} 2=\mathrm{H})$ & -1453.89884401 & -1453.455332 & 21.2 \\
\hline $\left.\mathbf{A} 2_{(\mathrm{DBU}}, \mathbf{R} 1=\mathrm{CF} 3, \mathrm{R} 2=\mathrm{H}\right)$ & -1453.91116554 & -1453.461691 & 17.2 \\
\hline TSA2-3 $(\mathrm{DBU}, \mathrm{R} 1=\mathrm{CF} 3, \mathrm{R} 2=\mathrm{H})$ & -1453.90420453 & -1453.461006 & 17.6 \\
\hline $\mathbf{A} 3_{(\mathbf{D B U}, \mathbf{R} 1=\mathbf{C F} 3, \mathbf{R} 2=\mathbf{H})}$ & -1453.94527471 & -1453.498707 & -6.0 \\
\hline \multicolumn{4}{|c|}{$\mathrm{R}_{1}=\mathrm{NO}_{2}, \mathrm{R}_{2}=\mathrm{H}$} \\
\hline $\mathrm{A1}_{(\mathrm{KOH}, \mathrm{R} 1=\mathrm{NO} 2, \mathrm{R} 2=\mathrm{H})}$ & -1535.16532554 & -1534.949607 & 0.0 \\
\hline TSA1-2 $(\mathrm{KOH}, \mathrm{R} 1=\mathrm{NO} 2, \mathrm{R} 2=\mathrm{H})$ & -1535.15518095 & -1534.944078 & 3.5 \\
\hline $\mathrm{A} \mathbf{2}_{(\mathrm{KOH}, \mathrm{R} 1=\mathrm{NO} 2, \mathrm{R} 2=\mathrm{H})}$ & -1535.18056040 & -1534.967784 & -11.4 \\
\hline TSA2-3 $(\mathrm{KOH}, \mathrm{R} 1=\mathrm{NO} 2, \mathrm{R} 2=\mathrm{H})$ & -1535.15776431 & -1534.950124 & -0.3 \\
\hline $\mathrm{A3}_{(\mathrm{KOH}, \mathrm{R} 1=\mathrm{NO} 2, \mathrm{R} 2=\mathrm{H})}$ & -1535.17857884 & -1534.961023 & -7.2 \\
\hline$A 1_{(\mathrm{NaOH}, \mathrm{R} 1=\mathrm{NO} 2, \mathrm{R} 2=\mathrm{H})}$ & -1097.55174241 & -1097.336314 & 0.0 \\
\hline $\mathrm{TSA}_{\left.1-2_{(\mathrm{NaOH}}, \mathrm{R} 1=\mathrm{NO} 2, \mathrm{R} 2=\mathrm{H}\right)}$ & -1097.53706264 & -1097.325619 & 6.7 \\
\hline $\mathbf{A} 2_{(\mathrm{NaOH}, \mathrm{R} 1=\mathrm{NO} 2, \mathrm{R} 2=\mathrm{H})}$ & -1097.55791150 & -1097.342978 & -4.2 \\
\hline $\left.\mathrm{TSA}_{2}-3_{(\mathrm{NaOH}}, \mathrm{R} 1=\mathrm{NO} 2, \mathrm{R} 2=\mathrm{H}\right)$ & -1097.54229966 & -1097.330547 & 3.6 \\
\hline $\mathbf{A 3}_{(\mathrm{NaOH}, \mathrm{R} 1=\mathrm{NO} 2, \mathrm{R} 2=\mathrm{H})}$ & -1097.56456034 & -1097.347283 & -6.9 \\
\hline $\mathbf{A} 1_{(\mathrm{LiOH}, \mathrm{R} 1=\mathrm{NO} 2, \mathrm{R} 2=\mathrm{H})}$ & -942.82578361 & -942.607092 & 0.0 \\
\hline $\mathrm{TSA1}_{1-2}(\mathrm{LiOH}, \mathrm{R} 1=\mathrm{NO} 2, \mathrm{R} 2=\mathrm{H})$ & -942.80079282 & -942.585937 & 13.3 \\
\hline $\mathbf{A} 2_{(\mathrm{LiOH}, \mathrm{R} 1=\mathrm{NO} 2, \mathrm{R} 2=\mathrm{H})}$ & -942.81665399 & -942.5985 & 5.4 \\
\hline $\mathrm{TSA2}_{2} \mathbf{3}_{(\mathrm{LiOH}, \mathrm{R} 1=\mathrm{NO} 2, \mathrm{R} 2=\mathrm{H})}$ & -942.80772284 & -942.594729 & 7.8 \\
\hline $\mathrm{A}_{(\mathrm{LiOH}, \mathrm{R} 1=\mathrm{NO} 2, \mathrm{R} 2=\mathrm{H})}$ & -942.83758852 & -942.619011 & -7.5 \\
\hline $\mathrm{A1}_{(t \mathrm{BuOK}, \mathrm{R} 1=\mathrm{NO} 2, \mathrm{R} 2=\mathrm{H})}$ & -1692.38849784 & -1692.068076 & 0.0 \\
\hline $\left.\operatorname{TSA}_{1-2}{ }_{(t \mathrm{BuOK}}, \mathrm{R} 1=\mathrm{NO} 2, \mathrm{R} 2=\mathrm{H}\right)$ & -1692.37551560 & -1692.056694 & 7.1 \\
\hline $\mathbf{A} 2_{(t \mathrm{BuOK}, \mathrm{R} 1=\mathrm{NO} 2, \mathrm{R} 2=\mathrm{H})}$ & -1692.40307288 & -1692.081635 & -8.5 \\
\hline 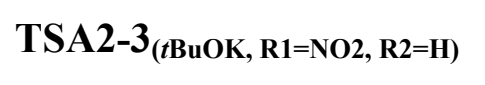 & -1692.37876671 & -1692.060413 & 4.8 \\
\hline $\mathrm{A3}_{(t \mathrm{BuOK}, \mathrm{R} 1=\mathrm{NO} 2, \mathrm{R} 2=\mathrm{H})}$ & -1692.40275737 & -1692.078486 & -6.5 \\
\hline $\mathbf{A 1}_{(t \mathrm{BuONa}, \mathrm{R} 1=\mathrm{NO} 2, \mathrm{R} 2=\mathrm{H})}$ & -1254.77431111 & -1254.453124 & 0.0 \\
\hline $\left.\operatorname{TSA}_{1-2}{ }_{(t \mathrm{BuONa}}, \mathrm{R} 1=\mathrm{NO} 2, \mathrm{R} 2=\mathrm{H}\right)$ & -1254.75610739 & -1254.43794 & 9.5 \\
\hline $\mathrm{A2}_{(t \mathrm{BuONa}, \mathrm{R} 1=\mathrm{NO} 2, \mathrm{R} 2=\mathrm{H})}$ & -1254.78158191 & -1254.458406 & -3.3 \\
\hline TSA2-3 $(t \mathrm{BuONa}, \mathrm{R} 1=\mathrm{NO} 2, \mathrm{R} 2=\mathrm{H})$ & -1254.76317582 & -1254.442636 & 6.6 \\
\hline
\end{tabular}




\begin{tabular}{|c|c|c|c|}
\hline $\mathrm{A} 3_{(t \mathrm{BuONa}, \mathrm{R} 1=\mathrm{NO} 2, \mathrm{R} 2=\mathrm{H})}$ & -1254.78824567 & -1254.466868 & -8.6 \\
\hline $\left.\mathrm{A1}_{(t \mathrm{BuOLi}}, \mathrm{R} 1=\mathrm{NO} 2, \mathrm{R} 2=\mathrm{H}\right)$ & -1100.04537392 & -1099.72091 & 0.0 \\
\hline TSA1-2 $(t$ BuOLi, R1=NO2, R2=H) & -1100.01930982 & -1099.69662 & 15.2 \\
\hline $\left.\mathrm{A} 2_{(t \mathrm{BuOLi}}, \mathrm{R} 1=\mathrm{NO} 2, \mathrm{R} 2=\mathrm{H}\right)$ & -1100.04023619 & -1099.71371 & 4.5 \\
\hline $\left.\mathrm{TSA}_{2}-3_{(t \mathrm{BuOLi}}, \mathrm{R} 1=\mathrm{NO} 2, \mathrm{R} 2=\mathrm{H}\right)$ & -1100.02685868 & -1099.7052 & 10.0 \\
\hline $\left.\mathrm{A3}_{(t \mathrm{BuOLi}}, \mathrm{R} 1=\mathrm{NO} 2, \mathrm{R} 2=\mathrm{H}\right)$ & -1100.05856375 & -1099.73182 & -6.8 \\
\hline 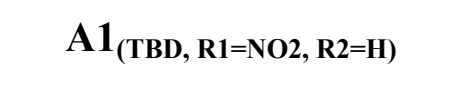 & -1298.16466913 & -1297.7575 & 0.0 \\
\hline $\mathrm{TSA}_{1-2}(\mathrm{TBD}, \mathrm{R} 1=\mathrm{NO} 2, \mathrm{R} 2=\mathrm{H})$ & -1298.13105758 & -1297.7292 & 17.8 \\
\hline 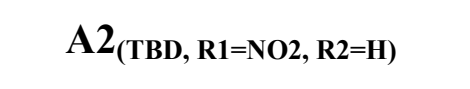 & -1298.14659655 & -1297.7395 & 11.3 \\
\hline $\mathrm{TSA}_{2-3}(\mathrm{TBD}, \mathrm{R} 1=\mathrm{NO} 2, \mathrm{R} 2=\mathrm{H})$ & -1298.13450115 & -1297.7323 & 15.8 \\
\hline $\mathrm{A3}_{(\mathrm{TBD}, \mathrm{R} 1=\mathrm{NO} 2, \mathrm{R} 2=\mathrm{H})}$ & -1298.16634642 & -1297.7613 & -2.4 \\
\hline $\mathbf{A} 1_{(\mathrm{MTBD}, \mathrm{R} 1=\mathrm{NO} 2, \mathrm{R} 2=\mathrm{H})}$ & -1337.46008123 & -1337.028989 & 0.0 \\
\hline $\mathrm{TSA}_{1-2} \mathbf{( M T B D}_{\mathrm{R} 1=\mathrm{NO} 2, \mathrm{R} 2=\mathrm{H})}$ & -1337.42542580 & -1336.992792 & 22.7 \\
\hline $\mathrm{A} 2_{(\mathrm{MTBD}, \mathrm{R} 1=\mathrm{NO} 2, \mathrm{R} 2=\mathrm{H})}$ & -1337.44200021 & -1337.003182 & 16.2 \\
\hline TSA2-3 ${ }_{(\mathrm{MTBD}, \mathrm{R} 1=\mathrm{NO} 2, \mathrm{R} 2=\mathrm{H})}$ & -1337.42916134 & -1337.001232 & 17.4 \\
\hline $\mathbf{A} 3_{(\mathrm{MTBD}, \mathrm{R} 1=\mathrm{NO} 2, \mathrm{R} 2=\mathrm{H})}$ & -1337.46212409 & -1337.034575 & -3.5 \\
\hline$A 1_{(\mathrm{DBU}, \mathrm{R} 1=\mathrm{NO} 2, \mathrm{R} 2=\mathrm{H})}$ & -1321.41430018 & -1320.972776 & 0.0 \\
\hline $\mathrm{TSA}_{1-2}(\mathrm{DBU}, \mathrm{R} 1=\mathrm{NO} 2, \mathrm{R} 2=\mathrm{H})$ & -1321.38610102 & -1320.94485 & 17.5 \\
\hline $\mathrm{A} 2_{(\mathrm{DBU}, \mathrm{R} 1=\mathrm{NO} 2, \mathrm{R} 2=\mathrm{H})}$ & -1321.40096709 & -1320.952132 & 13.0 \\
\hline $\mathrm{TSA}_{2}-3_{(\mathrm{DBU}, \mathrm{R} 1=\mathrm{NO} 2, \mathrm{R} 2=\mathrm{H})}$ & -1321.39037893 & -1320.946732 & 16.3 \\
\hline $\mathbf{A} 3_{(\mathrm{DBU}, \mathrm{R} 1=\mathrm{NO} 2, \mathrm{R} 2=\mathrm{H})}$ & -1321.42878395 & -1320.981492 & -5.5 \\
\hline \multicolumn{4}{|c|}{$R_{1}=H, R_{2}=O M e$} \\
\hline $\mathbf{A} 1_{(\mathrm{KOH}, \mathrm{R} 1=\mathrm{H}, \mathrm{R} 2=\mathrm{OMe})}$ & -1445.21341856 & -1444.96842 & 0.0 \\
\hline TSA1-2 $_{(\mathrm{KOH}, \mathrm{R} 1=\mathrm{H}, \mathrm{R} 2=\mathrm{OMe})}$ & -1445.19867871 & -1444.957623 & 6.8 \\
\hline $\mathbf{A} 2_{(\mathrm{KOH}, \mathrm{R} 1=\mathrm{H}, \mathrm{R} 2=\mathrm{OMe})}$ & -1445.21743033 & -1444.97451 & -3.8 \\
\hline 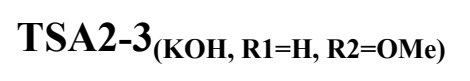 & -1445.20871016 & -1444.965858 & 1.6 \\
\hline $\mathbf{A} \mathbf{3}_{(\mathrm{KOH}, \mathrm{R} 1=\mathrm{H}, \mathrm{R} 2=\mathrm{OMe})}$ & -1445.22648593 & -1444.978381 & -6.3 \\
\hline $\mathbf{A} 1_{(\mathrm{NaOH}, \mathrm{R} 1=\mathrm{H}, \mathrm{R} 2=\mathrm{OMe})}$ & -1007.60096121 & -1007.355444 & 0.0 \\
\hline TSA1-2 $_{(\mathrm{NaOH}, \mathrm{R} 1=\mathrm{H}, \mathrm{R} 2=\mathrm{OMe})}$ & -1007.58115298 & -1007.337933 & 11.0 \\
\hline
\end{tabular}




\begin{tabular}{|c|c|c|c|}
\hline $\mathbf{A} 2_{(\mathrm{NaOH}, \mathrm{R} 1=\mathrm{H}, \mathrm{R} 2=\mathrm{OMe})}$ & -1007.59659015 & -1007.350424 & 3.2 \\
\hline TSA2 $^{-3}{ }_{(\mathrm{NaOH}, \mathrm{R} 1=\mathrm{H}, \mathrm{R} 2=\mathrm{OMe})}$ & -1007.58934530 & -1007.347511 & 5.0 \\
\hline $\mathbf{A} 3_{(\mathrm{NaOH}, \mathrm{R} 1=\mathrm{H}, \mathrm{R} 2=\mathrm{OMe})}$ & -1007.61322903 & -1007.364744 & -5.8 \\
\hline $\mathbf{A} 1_{(\mathrm{LiOH}, \mathrm{R} 1=\mathrm{H}, \mathrm{R} 2=\mathrm{OMe})}$ & -852.87649215 & -852.62839 & 0.0 \\
\hline TSA1-2 $_{(\mathrm{LiOH}, \mathrm{R} 1=\mathrm{H}, \mathrm{R} 2=\mathrm{OMe})}$ & -852.84578284 & -852.599452 & 18.2 \\
\hline $\mathbf{A} \boldsymbol{2}_{(\mathrm{LiOH}, \mathrm{R} 1=\mathrm{H}, \mathrm{R} 2=\mathrm{OMe})}$ & -852.85744405 & -852.608699 & 12.4 \\
\hline $\mathrm{TSA2}_{(\mathrm{LiOH}, \mathrm{R} 1=\mathrm{H}, \mathrm{R} 2=\mathrm{OMe})}$ & -852.85422840 & -852.608522 & 12.5 \\
\hline $\mathrm{A3}_{(\mathrm{LiOH}, \mathrm{R} 1=\mathrm{H}, \mathrm{R} 2=\mathrm{OMe})}$ & -852.88779425 & -852.638157 & -6.1 \\
\hline $\mathrm{A} 1_{(t \mathrm{BuOK}, \mathrm{R} 1=\mathrm{H}, \mathrm{R} 2=\mathrm{OMe})}$ & -1602.43852476 & -1602.086088 & 0.0 \\
\hline $\left.\operatorname{TSA1}^{-2}{ }_{(t \mathrm{BuOK}}, \mathrm{R} 1=\mathrm{H}, \mathrm{R} 2=\mathrm{OMe}\right)$ & -1602.42034915 & -1602.071049 & 9.4 \\
\hline $\mathrm{A} 2_{(t \mathrm{BuOK}, \mathrm{R} 1=\mathrm{H}, \mathrm{R} 2=\mathrm{OMe})}$ & -1602.43693758 & -1602.085419 & 0.4 \\
\hline $\mathrm{TSA}^{-2} \mathbf{3}_{(\mathrm{tBuOK}, \mathrm{R} 1=\mathrm{H}, \mathrm{R} 2=\mathrm{OMe})}$ & -1602.42562833 & -1602.076372 & 6.1 \\
\hline $\mathbf{A} 3_{(t \mathrm{BuOK}, \mathrm{R} 1=\mathrm{H}, \mathrm{R} 2=\mathrm{OMe})}$ & -1602.45321201 & -1602.097687 & -8.2 \\
\hline $\mathbf{A} 1_{(t \mathrm{BuONa}, \mathrm{R} 1=\mathrm{H}, \mathrm{R} 2=\mathrm{OMe})}$ & -1164.82503911 & -1164.472131 & 0.0 \\
\hline TSA1-2 $(t$ BuONa, R1=H, R2=OMe $)$ & -1164.80155328 & -1164.451354 & 13.0 \\
\hline $\mathrm{A} 2_{(t \mathrm{BuONa}, \mathrm{R} 1=\mathrm{H}, \mathrm{R} 2=\mathrm{OMe})}$ & -1164.81702838 & -1164.463401 & 5.5 \\
\hline $\mathrm{TSA}_{\left.2-3_{(t \mathrm{BuONa}}, \mathrm{R} 1=\mathrm{H}, \mathrm{R} 2=\mathrm{OMe}\right)}$ & -1164.80945022 & -1164.45878 & 8.4 \\
\hline $\mathbf{A} 3_{(t \mathrm{BuONa}, \mathrm{R} 1=\mathrm{H}, \mathrm{R} 2=\mathrm{OMe})}$ & -1164.83988296 & -1164.485031 & -8.1 \\
\hline $\left.\mathbf{A} 1_{(t \mathrm{BuOLi}}, \mathrm{R} 1=\mathrm{H}, \mathrm{R} 2=\mathrm{OMe}\right)$ & -1010.09743212 & -1009.739826 & 0.0 \\
\hline $\left.\operatorname{TSA}_{1-2}{ }_{(t \mathrm{BuOLi}}, \mathrm{R} 1=\mathrm{H}, \mathrm{R} 2=\mathrm{OMe}\right)$ & -1010.06232463 & -1009.709587 & 19.0 \\
\hline $\left.\mathrm{A} 2_{(t \mathrm{BuOLi}}, \mathrm{R} 1=\mathrm{H}, \mathrm{R} 2=\mathrm{OMe}\right)$ & -1010.07742473 & -1009.720277 & 12.3 \\
\hline $\left.\operatorname{TSA}-3_{(t \mathrm{BuOLi}}, \mathrm{R} 1=\mathrm{H}, \mathrm{R} 2=\mathrm{OMe}\right)$ & -1010.07009930 & -1009.717056 & 14.3 \\
\hline $\left.\mathrm{A3}_{(t \mathrm{BuOLi}}, \mathrm{R} 1=\mathrm{H}, \mathrm{R} 2=\mathrm{OMe}\right)$ & -1010.11108455 & -1009.753977 & -8.9 \\
\hline 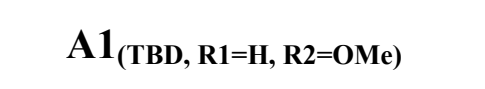 & -1208.20805396 & -1207.774033 & 0.0 \\
\hline TSA1-2 $_{(\mathrm{TBD}, \mathrm{R} 1=\mathrm{H}, \mathrm{R} 2=\mathrm{OMe})}$ & -1208.17096845 & -1207.736693 & 23.4 \\
\hline 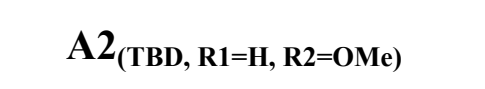 & -1208.17898337 & -1207.742751 & 19.6 \\
\hline 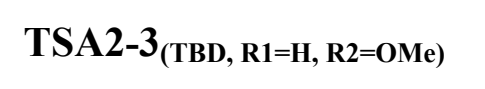 & -1208.17666953 & -1207.743824 & 19.0 \\
\hline 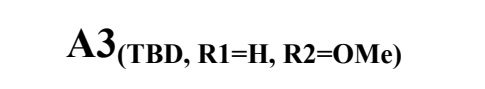 & -1208.21775944 & -1207.782332 & -5.2 \\
\hline 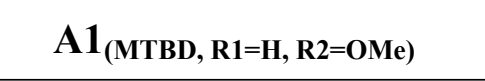 & -1247.51008657 & -1247.046434 & 0.0 \\
\hline
\end{tabular}




\begin{tabular}{|c|c|c|c|}
\hline 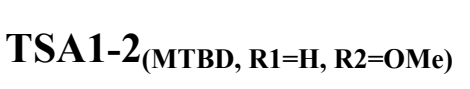 & -1247.46604384 & -1247.003366 & 27.0 \\
\hline 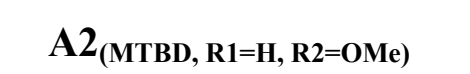 & -1247.47410628 & -1247.008098 & 24.1 \\
\hline 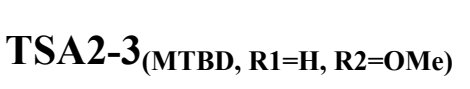 & -1247.47045031 & -1247.007722 & 24.3 \\
\hline $\mathbf{A} 3_{(\mathrm{MTBD}, \mathrm{R} 1=\mathrm{H}, \mathrm{R} 2=\mathrm{OMe})}$ & -1247.51300935 & -1247.049094 & -1.7 \\
\hline $\mathrm{A1}_{(\mathrm{DBU}, \mathrm{R} 1=\mathrm{H}, \mathrm{R} 2=\mathrm{OMe})}$ & -1231.46328313 & -1230.991731 & 0.0 \\
\hline TSA1-2 $_{(\mathrm{DBU}, \mathrm{R} 1=\mathrm{H}, \mathrm{R} 2=\mathrm{OMe})}$ & -1231.42033821 & -1230.947212 & 27.9 \\
\hline $\left.\mathbf{A} 2_{(\mathrm{DBU},}, \mathbf{R} 1=\mathrm{H}, \mathbf{R} 2=\mathrm{OMe}\right)$ & -1231.43196187 & -1230.954367 & 23.4 \\
\hline $\mathrm{TSA}^{\left.2-3_{(\mathrm{DBU}}, \mathrm{R} 1=\mathrm{H}, \mathrm{R} 2=\mathrm{OMe}\right)}$ & -1231.42932842 & -1230.955596 & 22.7 \\
\hline 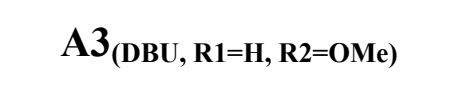 & -1231.47836069 & -1231.001012 & -5.8 \\
\hline \multicolumn{4}{|c|}{$R_{1}=H, R_{2}=M e$} \\
\hline $\mathbf{A} 1_{(\mathrm{KOH}, \mathrm{R} 1=\mathrm{H}, \mathrm{R} 2=\mathrm{Me})}$ & -1370.03174371 & -1369.791751 & 0.0 \\
\hline $\operatorname{TSA}_{1-2}(\mathrm{KOH}, \mathrm{R} 1=\mathrm{H}, \mathrm{R} 2=\mathrm{Me})$ & -1370.01864819 & -1369.781978 & 6.2 \\
\hline 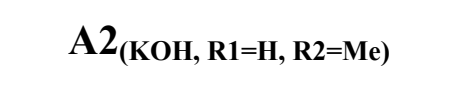 & -1370.03736908 & -1369.799793 & -5.0 \\
\hline 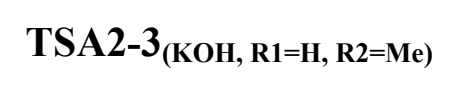 & -1370.02844238 & -1369.790298 & 0.9 \\
\hline $\mathbf{A 3}_{(\mathrm{KOH}, \mathrm{R} 1=\mathrm{H}, \mathrm{R} 2=\mathrm{Me})}$ & -1370.04481073 & -1369.801072 & -5.8 \\
\hline $\mathbf{A} 1_{(\mathrm{NaOH}, \mathrm{R} 1=\mathrm{H}, \mathrm{R} 2=\mathrm{Me})}$ & -932.41920785 & -932.177731 & 0.0 \\
\hline $\mathrm{TSA}_{1-2} \mathbf{( N a O H}_{\mathrm{R} 1=\mathrm{H}, \mathrm{R} 2=\mathrm{Me})}$ & -932.40194413 & -932.163577 & 8.9 \\
\hline $\mathbf{A} 2_{(\mathrm{NaOH}, \mathrm{R} 1=\mathrm{H}, \mathrm{R} 2=\mathrm{Me})}$ & -932.41632392 & -932.175011 & 1.7 \\
\hline $\mathrm{TSA}_{2}-3_{(\mathrm{NaOH}, \mathrm{R} 1=\mathrm{H}, \mathrm{R} 2=\mathrm{Me})}$ & -932.40880545 & -932.171252 & 4.1 \\
\hline $\mathbf{A} \mathbf{3}_{(\mathrm{NaOH}, \mathrm{R} 1=\mathrm{H}, \mathrm{R} 2=\mathrm{Me})}$ & -932.43141270 & -932.188451 & -6.7 \\
\hline $\mathbf{A} \mathbf{1}_{(\mathrm{LiOH}, \mathrm{R} 1=\mathrm{H}, \mathrm{R} 2=\mathrm{Me})}$ & -777.69459112 & -777.450815 & 0.0 \\
\hline $\operatorname{TSA}_{1-2}(\mathrm{LiOH}, \mathrm{R} 1=\mathrm{H}, \mathrm{R} 2=\mathrm{Me})$ & -777.66466040 & -777.422787 & 17.6 \\
\hline $\mathbf{A} \mathbf{2}_{(\mathrm{LiOH}, \mathrm{R} 1=\mathrm{H}, \mathrm{R} 2=\mathrm{Me})}$ & -777.67699703 & -777.433563 & 10.8 \\
\hline 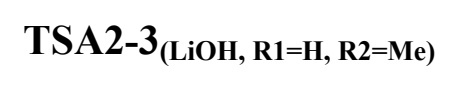 & -777.67365188 & -777.432757 & 11.3 \\
\hline $\mathbf{A} 3_{(\mathrm{LiOH}, \mathrm{R} 1=\mathrm{H}, \mathrm{R} 2=\mathrm{Me})}$ & -777.70607197 & -777.460677 & -6.2 \\
\hline $\mathrm{A1}_{(t \mathrm{BuOK}, \mathrm{R} 1=\mathrm{H}, \mathrm{R} 2=\mathrm{Me})}$ & -1527.25644516 & -1526.90765 & 0.0 \\
\hline $\operatorname{TSA1}^{-2} \mathbf{( t B u O K}_{\mathbf{R} 1=\mathrm{H}, \mathrm{R} 2=\mathrm{Me})}$ & -1527.23923207 & -1526.894198 & 8.4 \\
\hline $\mathrm{A} 2_{(t \mathrm{BuOK}, \mathrm{R} 1=\mathrm{H}, \mathrm{R} 2=\mathrm{Me})}$ & -1527.25983703 & -1526.910521 & -1.8 \\
\hline $\mathrm{TSA}_{\left.2-3_{(\mathrm{BuOK}}, \mathrm{R} 1=\mathrm{H}, \mathrm{R} 2=\mathrm{Me}\right)}$ & -1527.24518192 & -1526.901739 & 3.7 \\
\hline
\end{tabular}




\begin{tabular}{|c|c|c|c|}
\hline $\mathbf{A} 3_{(t \mathrm{BuOK}, \mathrm{R} 1=\mathrm{H}, \mathrm{R} 2=\mathrm{Me})}$ & -1527.27085030 & -1526.920686 & -8.2 \\
\hline$A 1_{(t \mathrm{BuONa}, \mathrm{R} 1=\mathrm{H}, \mathrm{R} 2=\mathrm{Me})}$ & -1089.64309604 & -1089.293563 & 0.0 \\
\hline TSA1-2 $(t$ BuONa, R1=H, R2=Me) & -1089.62265400 & -1089.27672 & 10.6 \\
\hline $\mathbf{A} 2_{(t \mathrm{BuONa}, \mathrm{R} 1=\mathrm{H}, \mathrm{R} 2=\mathrm{Me})}$ & -1089.64001385 & -1089.289014 & 2.9 \\
\hline TSA2-3 $(t \mathrm{BuONa}, \mathrm{R} 1=\mathrm{H}, \mathrm{R} 2=\mathrm{Me})$ & -1089.62912944 & -1089.283924 & 6.0 \\
\hline $\mathrm{A3}_{(t \mathrm{BuONa}, \mathrm{R} 1=\mathrm{H}, \mathrm{R} 2=\mathrm{Me})}$ & -1089.65732778 & -1089.304861 & -7.1 \\
\hline $\mathrm{A1}_{(t \mathrm{BuOLi}, \mathrm{R} 1=\mathrm{H}, \mathrm{R} 2=\mathrm{Me})}$ & -934.91536877 & -934.561571 & 0.0 \\
\hline $\operatorname{TSA}_{1-2}(t \mathrm{BuOLi}, \mathrm{R} 1=\mathrm{H}, \mathrm{R} 2=\mathrm{Me})$ & -934.88120646 & -934.532758 & 18.1 \\
\hline $\mathbf{A} 2_{(t \mathrm{BuOLi}, \mathrm{R} 1=\mathrm{H}, \mathrm{R} 2=\mathrm{Me})}$ & -934.90040970 & -934.546592 & 9.4 \\
\hline $\left.\operatorname{TSA}^{2-3}{ }_{(t \mathrm{BuOLi}}, \mathrm{R} 1=\mathrm{H}, \mathrm{R} 2=\mathrm{Me}\right)$ & -934.89304123 & -934.544697 & 10.6 \\
\hline $\left.\mathbf{A} 3_{(t \mathrm{BuOLi}}, \mathbf{R} 1=\mathrm{H}, \mathbf{R 2}=\mathrm{Me}\right)$ & -934.92873406 & -934.57509 & -8.5 \\
\hline $\mathbf{A} 1_{(\mathrm{TBD}, \mathrm{R} 1=\mathrm{H}, \mathrm{R} 2=\mathrm{Me})}$ & -1133.02630624 & -1132.598044 & 0.0 \\
\hline 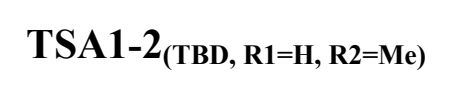 & -1132.99369548 & -1132.562356 & 22.6 \\
\hline 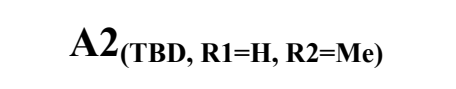 & -1133.00143868 & -1132.567504 & 19.4 \\
\hline 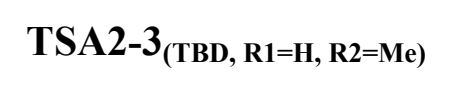 & -1132.99665300 & -1132.566947 & 19.7 \\
\hline 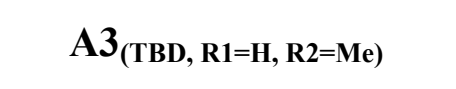 & -1133.03622026 & -1132.60236 & -2.5 \\
\hline 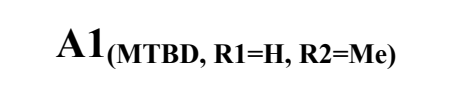 & -1172.32828517 & -1171.870509 & 0.0 \\
\hline $\operatorname{TSA1}_{(\mathrm{MTBD}, \mathrm{R} 1=\mathrm{H}, \mathrm{R} 2=\mathrm{Me})}$ & -1172.28544880 & -1171.827888 & 26.7 \\
\hline 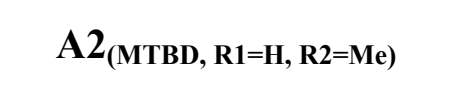 & -1172.29449379 & -1171.830763 & 24.9 \\
\hline $\left.\operatorname{TSA}_{2-3}{ }_{(M T B D,} \mathrm{R} 1=\mathrm{H}, \mathrm{R} 2=\mathrm{Me}\right)$ & -1172.29050706 & -1171.832321 & 24.0 \\
\hline $\mathbf{A} \mathbf{3}_{(\mathrm{MTBD}, \mathrm{R} 1=\mathrm{H}, \mathrm{R} 2=\mathrm{Me})}$ & -1172.33160214 & -1171.872805 & -1.4 \\
\hline 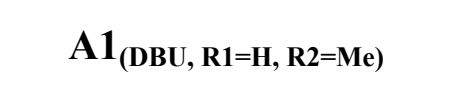 & -1156.28134495 & -1155.813459 & 0.0 \\
\hline TSA1-2 $_{(\mathrm{DBU}, \mathrm{R} 1=\mathrm{H}, \mathrm{R} 2=\mathrm{Me})}$ & -1156.24286846 & -1155.77308 & 25.3 \\
\hline 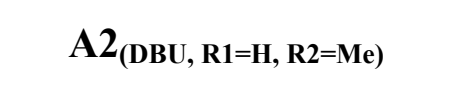 & -1156.25459020 & -1155.779547 & 21.3 \\
\hline $\mathrm{TSA}_{\left.2-3_{(\mathrm{DBU}}, \mathrm{R} 1=\mathrm{H}, \mathrm{R} 2=\mathrm{Me}\right)}$ & -1156.25193882 & -1155.781472 & 20.1 \\
\hline $\mathbf{A} 3_{(\mathbf{D B U}, \mathbf{R} 1=\mathrm{H}, \mathbf{R} 2=\mathrm{Me})}$ & -1156.29652802 & -1155.822745 & -5.8 \\
\hline \multicolumn{4}{|c|}{$R_{1}=H, R_{2}=C F_{3}$} \\
\hline $\mathbf{A} 1_{(\mathrm{KOH}, \mathrm{R} 1=\mathrm{H}, \mathrm{R} 2=\mathrm{CF} 3)}$ & -1667.68061993 & -1667.46677 & 0.0 \\
\hline $\mathrm{TSA1}-2_{(\mathrm{KOH}, \mathrm{R} 1=\mathrm{H}, \mathrm{R} 2=\mathrm{CF} 3)}$ & -1667.66882712 & -1667.458519 & 5.2 \\
\hline
\end{tabular}




\begin{tabular}{|c|c|c|c|}
\hline $\mathbf{A} 2_{(\mathrm{KOH}, \mathrm{R} 1=\mathrm{H}, \mathrm{R} 2=\mathrm{CF} 3)}$ & -1667.69138544 & -1667.479225 & -7.8 \\
\hline TSA2-3 ${ }_{(\mathrm{KOH}, \mathrm{R} 1=\mathrm{H}, \mathrm{R} 2=\mathrm{CF} 3)}$ & -1667.68255460 & -1667.470708 & -2.5 \\
\hline $\mathbf{A} 3_{(\mathrm{KOH}, \mathrm{R} 1=\mathrm{H}, \mathrm{R} 2=\mathrm{CF} 3)}$ & -1667.69479818 & -1667.477269 & -6.6 \\
\hline $\mathbf{A} 1_{(\mathrm{NaOH}, \mathrm{R} 1=\mathrm{H}, \mathrm{R} 2=\mathrm{CF} 3)}$ & -1230.06768241 & -1229.852206 & 0.0 \\
\hline $\mathrm{TSA}_{1-2}(\mathrm{NaOH}, \mathrm{R} 1=\mathrm{H}, \mathrm{R} 2=\mathrm{CF} 3)$ & -1230.05142761 & -1229.839643 & 7.9 \\
\hline$A 2_{(\mathrm{NaOH}, \mathrm{R} 1=\mathrm{H}, \mathrm{R} 2=\mathrm{CF} 3)}$ & -1230.06971506 & -1229.855357 & -2.0 \\
\hline $\mathrm{TSA} 2-3_{(\mathrm{NaOH}, \mathrm{R} 1=\mathrm{H}, \mathrm{R2}=\mathrm{CF} 3)}$ & -1230.06165036 & -1229.849827 & 1.5 \\
\hline $\mathrm{A} 3_{(\mathrm{NaOH}, \mathrm{R} 1=\mathrm{H}, \mathrm{R} 2=\mathrm{CF} 3)}$ & -1230.07944154 & -1229.861925 & -6.1 \\
\hline $\mathrm{A1}_{(\mathrm{LiOH}, \mathrm{R} 1=\mathrm{H}, \mathrm{R} 2=\mathrm{CF} 3)}$ & -1075.34259244 & -1075.124459 & 0.0 \\
\hline $\operatorname{TSA}_{1-2}(\mathrm{LiOH}, \mathrm{R} 1=\mathrm{H}, \mathrm{R} 2=\mathrm{CF} 3)$ & -1075.31387277 & -1075.100039 & 15.3 \\
\hline $\mathbf{A} 2_{(\mathrm{LiOH}, \mathrm{R} 1=\mathrm{H}, \mathrm{R} 2=\mathrm{CF} 3)}$ & -1075.32956574 & -1075.111894 & 7.9 \\
\hline TSA2 $_{-3}(\mathrm{LiOH}, \mathrm{R} 1=\mathrm{H}, \mathrm{R} 2=\mathrm{CF} 3)$ & -1075.32567255 & -1075.111565 & 8.1 \\
\hline $\mathbf{A} 3_{(\mathrm{LiOH}, \mathrm{R} 1=\mathrm{H}, \mathrm{R} 2=\mathrm{CF} 3)}$ & -1075.35187327 & -1075.133546 & -5.7 \\
\hline $\mathrm{A1}_{(t \mathrm{BuOK}, \mathrm{R} 1=\mathrm{H}, \mathrm{R} 2=\mathrm{CF} 3)}$ & -1824.90544717 & -1824.583603 & 0.0 \\
\hline $\operatorname{TSA}_{1-2}(t \mathrm{BuOK}, \mathrm{R} 1=\mathrm{H}, \mathbf{R} 2=\mathrm{CF} 3)$ & -1824.88990529 & -1824.572095 & 7.2 \\
\hline $\mathbf{A} 2_{(t \mathrm{BuOK}, \mathrm{R} 1=\mathrm{H}, \mathrm{R} 2=\mathrm{CF} 3)}$ & -1824.91360342 & -1824.591277 & -4.8 \\
\hline $\left.\operatorname{TSA}_{2}-3_{(t \mathrm{BuOK},} \mathrm{R} 1=\mathrm{H}, \mathrm{R} 2=\mathrm{CF} 3\right)$ & -1824.89749111 & -1824.579746 & 2.4 \\
\hline $\mathrm{A3}_{(t \mathrm{BuOK}, \mathrm{R} 1=\mathrm{H}, \mathrm{R} 2=\mathrm{CF} 3)}$ & -1824.92093830 & -1824.596671 & -8.2 \\
\hline $\mathrm{A1}_{(t \mathrm{BuONa}, \mathrm{R} 1=\mathrm{H}, \mathrm{R} 2=\mathrm{CF} 3)}$ & -1387.29112644 & -1386.967859 & 0.0 \\
\hline TSA1-2 ${ }_{(t \mathrm{BuONa}, \mathrm{R} 1=\mathrm{H}, \mathrm{R} 2=\mathrm{CF} 3)}$ & -1387.27082962 & -1386.95262 & 9.6 \\
\hline $\mathrm{A} 2_{(t \mathrm{BuONa}, \mathrm{R} 1=\mathrm{H}, \mathrm{R} 2=\mathrm{CF} 3)}$ & -1387.29311830 & -1386.968797 & -0.6 \\
\hline $\mathrm{TSA}_{2}-3_{(t \mathrm{BuONa}, \mathrm{R} 1=\mathrm{H}, \mathrm{R} 2=\mathrm{CF} 3)}$ & -1387.28070995 & -1386.962285 & 3.5 \\
\hline $\mathrm{A3}_{(t \mathrm{BuONa}, \mathrm{R} 1=\mathrm{H}, \mathrm{R} 2=\mathrm{CF} 3)}$ & -1387.30493154 & -1386.982115 & -8.9 \\
\hline $\left.\mathrm{A} 1_{(t \mathrm{BuOLi}}, \mathrm{R} 1=\mathrm{H}, \mathrm{R} 2=\mathrm{CF} 3\right)$ & -1232.56293676 & -1232.236428 & 0.0 \\
\hline TSA1-2 $(t \mathrm{BuOLi}, \mathrm{R} 1=\mathrm{H}, \mathrm{R} 2=\mathrm{CF} 3)$ & -1232.53239468 & -1232.209608 & 16.8 \\
\hline $\left.\mathrm{A} 2_{(t \mathrm{BuOLi}}, \mathrm{R} 1=\mathrm{H}, \mathrm{R} 2=\mathrm{CF} 3\right)$ & -1232.55278535 & -1232.224979 & 7.2 \\
\hline $\left.\mathrm{TSA} 2-3_{(t \mathrm{BuOLi}}, \mathrm{R} 1=\mathrm{H}, \mathrm{R} 2=\mathrm{CF} 3\right)$ & -1232.54506076 & -1232.223046 & 8.4 \\
\hline $\left.\mathbf{A} 3_{(t \mathrm{BuOLi}}, \mathrm{R} 1=\mathrm{H}, \mathrm{R} 2=\mathrm{CF} 3\right)$ & -1232.57454353 & -1232.247004 & -6.6 \\
\hline 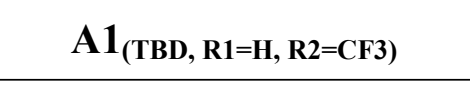 & -1430.67499527 & -1430.272757 & 0.0 \\
\hline
\end{tabular}




\begin{tabular}{|c|c|c|c|}
\hline $\left.\mathrm{TSA1}-2_{(\mathrm{TBD}}, \mathrm{R} 1=\mathrm{H}, \mathrm{R} 2=\mathrm{CF} 3\right)$ & -1430.64501782 & -1430.241054 & 19.9 \\
\hline 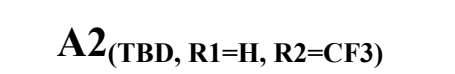 & -1430.65697039 & -1430.249381 & 14.7 \\
\hline $\mathrm{TSA}_{\left.2-3_{(\mathrm{TBD}}, \mathrm{R} 1=\mathrm{H}, \mathrm{R} 2=\mathrm{CF} 3\right)}$ & -1430.65041292 & -1430.248498 & 15.2 \\
\hline $\mathbf{A} 3_{(\mathrm{TBD}, \mathrm{R} 1=\mathrm{H}, \mathrm{R} 2=\mathrm{CF} 3)}$ & -1430.68908873 & -1430.280128 & -4.6 \\
\hline $\mathbf{A} 1_{(\mathrm{MTBD}, \mathrm{R} 1=\mathrm{H}, \mathrm{R} 2=\mathrm{CF} 3)}$ & -1469.97653448 & -1469.544737 & 0.0 \\
\hline 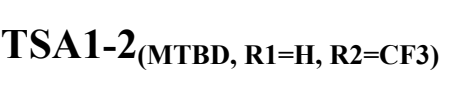 & -1469.93697129 & -1469.506177 & 24.2 \\
\hline 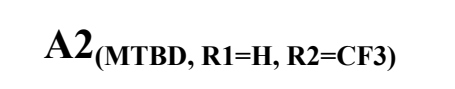 & -1469.95014172 & -1469.513101 & 19.9 \\
\hline 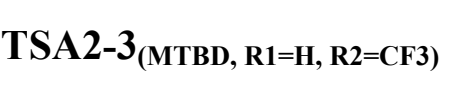 & -1469.94455885 & -1469.513676 & 19.5 \\
\hline $\left.\mathbf{A} 3_{(M T B D}, R 1=H, R 2=C F 3\right)$ & -1469.98361089 & -1469.550545 & -3.6 \\
\hline $\mathbf{A} 1_{(\mathbf{D B U}, \mathbf{R} 1=\mathrm{H}, \mathrm{R} 2=\mathrm{CF} 3)}$ & -1453.93033653 & -1453.489971 & 0.0 \\
\hline $\operatorname{TSA}_{1-2}(\mathrm{DBU}, \mathrm{R} 1=\mathrm{H}, \mathrm{R} 2=\mathrm{CF} 3)$ & -1453.89596382 & -1453.453067 & 23.2 \\
\hline $\mathbf{A} \mathbf{2}_{(\mathrm{DBU}, \mathrm{R} 1=\mathrm{H}, \mathrm{R} 2=\mathrm{CF} 3)}$ & -1453.90978519 & -1453.46113 & 18.1 \\
\hline $\mathrm{TSA}_{\left.2-3_{(\mathrm{DBU}}, \mathrm{R} 1=\mathrm{H}, \mathrm{R} 2=\mathrm{CF} 3\right)}$ & -1453.90586033 & -1453.46231 & 18.4 \\
\hline 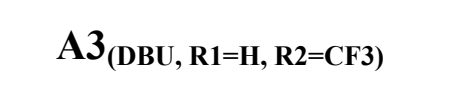 & -1453.94600675 & -1453.499125 & -5.7 \\
\hline \multicolumn{4}{|c|}{$R_{1}=H, R_{2}=N O_{2}$} \\
\hline $\mathbf{A} 1_{(\mathrm{KOH}, \mathrm{R} 1=\mathrm{H}, \mathrm{R} 2=\mathrm{NO} 2)}$ & -1535.16476052 & -1534.951001 & 0.0 \\
\hline $\mathrm{TSA}_{1-2}(\mathrm{KOH}, \mathrm{R} 1=\mathrm{H}, \mathrm{R} 2=\mathrm{NO} 2)$ & -1535.15358005 & -1534.943549 & 4.7 \\
\hline $\mathrm{A} 2_{(\mathrm{KOH}, \mathrm{R} 1=\mathrm{H}, \mathrm{R} 2=\mathrm{NO} 2)}$ & -1535.18028457 & -1534.967018 & -10.1 \\
\hline $\mathrm{TSA}^{2-3_{(\mathrm{KOH}, \mathrm{R} 1=\mathrm{H}, \mathrm{R} 2=\mathrm{NO} 2)}}$ & -1535.17072032 & -1534.95851 & -4.7 \\
\hline $\mathrm{A3}_{(\mathrm{KOH}, \mathrm{R} 1=\mathrm{H}, \mathrm{R} 2=\mathrm{NO} 2)}$ & -1535.17456102 & -1534.958707 & -4.8 \\
\hline$A 1_{(\mathrm{NaOH}, \mathrm{R} 1=\mathrm{H}, \mathrm{R} 2=\mathrm{NO} 2)}$ & -1097.55152607 & -1097.335875 & 0.0 \\
\hline TSA1-2 $(\mathrm{NaOH}, \mathrm{R} 1=\mathrm{H}, \mathrm{R} 2=\mathrm{NO} 2)$ & -1097.53547411 & -1097.324424 & 7.2 \\
\hline $\mathbf{A} 2_{(\mathrm{NaOH}, \mathrm{R} 1=\mathrm{H}, \mathrm{R} 2=\mathrm{NO} 2)}$ & -1097.55792377 & -1097.343012 & -4.5 \\
\hline TSA2-3 ${ }_{(\mathrm{NaOH}, \mathrm{R} 1=\mathrm{H}, \mathrm{R} 2=\mathrm{NO} 2)}$ & -1097.54891559 & -1097.335945 & -0.04 \\
\hline $\mathrm{A3}_{(\mathrm{NaOH}, \mathrm{R} 1=\mathrm{H}, \mathrm{R} 2=\mathrm{NO} 2)}$ & -1097.55931274 & -1097.342307 & -4.3 \\
\hline $\mathrm{A1}_{(\mathrm{LiOH}, \mathrm{R} 1=\mathrm{H}, \mathrm{R} 2=\mathrm{NO} 2)}$ & -942.82612807 & -942.607847 & 0.0 \\
\hline TSA1-2 $(\mathrm{LiOH}, \mathrm{R} 1=\mathrm{H}, \mathrm{R} 2=\mathrm{NO} 2)$ & -942.79866278 & -942.583833 & 15.1 \\
\hline$A 2_{(\mathrm{LiOH}, \mathrm{R} 1=\mathrm{H}, \mathrm{R} 2=\mathrm{NO} 2)}$ & -942.81695864 & -942.59991 & 5.0 \\
\hline TSA2-3 $_{(\mathrm{LiOH}, \mathrm{R} 1=\mathrm{H}, \mathrm{R} 2=\mathrm{NO} 2)}$ & -942.81219604 & -942.597162 & 6.7 \\
\hline
\end{tabular}




\begin{tabular}{|c|c|c|c|}
\hline $\mathbf{A} 3_{(\mathrm{LiOH}, \mathrm{R} 1=\mathrm{H}, \mathrm{R} 2=\mathrm{NO} 2)}$ & -942.83254461 & -942.615332 & -4.7 \\
\hline $\mathbf{A} 1_{(t \mathrm{BuOK}, \mathrm{R} 1=\mathrm{H}, \mathrm{R} 2=\mathrm{NO} 2)}$ & -1692.38887601 & -1692.06729 & 0.0 \\
\hline $\operatorname{TSA1}^{-2} 2_{(\mathrm{BuOK}, \mathrm{R} 1=\mathrm{H}, \mathrm{R} 2=\mathrm{NO} 2)}$ & -1692.37544712 & -1692.058729 & 5.4 \\
\hline $\mathrm{A} 2_{(t \mathrm{BuOK}, \mathrm{R} 1=\mathrm{H}, \mathrm{R} 2=\mathrm{NO} 2)}$ & -1692.40252248 & -1692.079596 & -7.7 \\
\hline $\left.\mathrm{TSA}^{-2} \mathbf{3}_{(\mathrm{BBOOK}}, \mathrm{R} 1=\mathrm{H}, \mathrm{R} 2=\mathrm{NO} 2\right)$ & -1692.38386258 & -1692.065779 & 0.9 \\
\hline $\mathrm{A3}_{(t \mathrm{BuOK}, \mathrm{R} 1=\mathrm{H}, \mathrm{R} 2=\mathrm{NO} 2)}$ & -1692.40041210 & -1692.078188 & -6.8 \\
\hline $\mathrm{A1}_{(t \mathrm{BuONa}, \mathrm{R} 1=\mathrm{H}, \mathrm{R} 2=\mathrm{NO} 2)}$ & -1254.77472990 & -1254.451762 & 0.0 \\
\hline $\operatorname{TSA}_{1-2}(t \mathrm{BuONa}, \mathrm{R} 1=\mathrm{H}, \mathrm{R} 2=\mathrm{NO} 2)$ & -1254.75613810 & -1254.43727 & 9.1 \\
\hline $\mathrm{A} 2_{(t \mathrm{BuONa}, \mathrm{R} 1=\mathrm{H}, \mathrm{R} 2=\mathrm{NO} 2)}$ & -1254.78147527 & -1254.457077 & -3.3 \\
\hline 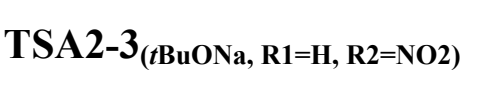 & -1254.76907782 & -1254.448824 & 1.8 \\
\hline $\left.\mathrm{A}_{(t \mathrm{BuONa}}, \mathrm{R} 1=\mathrm{H}, \mathrm{R} 2=\mathrm{NO} 2\right)$ & -1254.78427134 & -1254.462106 & -6.5 \\
\hline $\left.\mathbf{A 1}_{(t \mathrm{BuOLi}} \mathrm{R} \mathbf{1}=\mathrm{H}, \mathrm{R} 2=\mathrm{NO} 2\right)$ & -1100.04624907 & -1099.720225 & 0.0 \\
\hline $\left.\mathrm{TSA}_{1-2}{ }_{(t \mathrm{BuOLi}}, \mathrm{R} 1=\mathrm{H}, \mathrm{R} 2=\mathrm{NO} 2\right)$ & -1100.01677942 & -1099.696008 & 15.2 \\
\hline $\left.\mathrm{A} 2_{(t \mathrm{BuOLi}}, \mathrm{R} 1=\mathrm{H}, \mathrm{R} 2=\mathrm{NO} 2\right)$ & -1100.04034353 & -1099.712964 & 4.6 \\
\hline $\operatorname{TSA}_{\left.2-3_{(t \mathrm{BuOLi}}, \mathrm{R} 1=\mathrm{H}, \mathrm{R} 2=\mathrm{NO} 2\right)}$ & -1100.03139400 & -1099.708029 & 7.7 \\
\hline $\left.\mathrm{A3}_{(t \mathrm{BuOLi}}, \mathrm{R} 1=\mathrm{H}, \mathrm{R} 2=\mathrm{NO} 2\right)$ & -1100.05436453 & -1099.72766 & -4.7 \\
\hline 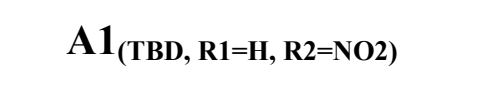 & -1298.15904607 & -1297.758122 & 0.0 \\
\hline $\mathrm{TSA}_{\left.1-2_{(\mathrm{TBD}}, \mathrm{R} 1=\mathrm{H}, \mathrm{R} 2=\mathrm{NO} 2\right)}$ & -1298.13105028 & -1297.72771 & 19.1 \\
\hline 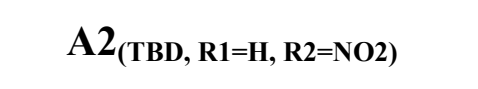 & -1298.14757388 & -1297.739801 & 11.5 \\
\hline $\mathrm{TSA}_{2-3_{(\mathrm{TBD}, \mathrm{R} 1=\mathrm{H}, \mathrm{R} 2=\mathrm{NO} 2)}}$ & -1298.13819716 & -1297.737421 & 13.0 \\
\hline $\mathrm{A}_{(\mathrm{TBD}, \mathrm{R} 1=\mathrm{H}, \mathrm{R} 2=\mathrm{NO} 2)}$ & -1298.17261672 & -1297.76401 & -3.7 \\
\hline 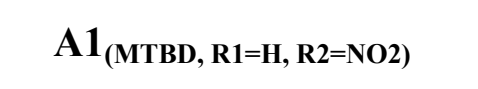 & -1337.45990886 & -1337.028989 & 0.0 \\
\hline TSA1-2 $(\mathrm{MTBD}, \mathrm{R} 1=\mathrm{H}, \mathrm{R} 2=\mathrm{NO} 2)$ & -1337.42323808 & -1336.992792 & 22.7 \\
\hline $\mathrm{A} 2_{(\mathrm{MTBD}, \mathrm{R} 1=\mathrm{H}, \mathrm{R} 2=\mathrm{NO} 2)}$ & -1337.44110218 & -1337.003182 & 16.2 \\
\hline $\left.\mathrm{TSA2}^{-3} \mathbf{3}_{(\mathrm{MTBD}}, \mathrm{R} 1=\mathrm{H}, \mathrm{R} 2=\mathrm{NO} 2\right)$ & -1337.43271661 & -1337.001232 & 17.4 \\
\hline 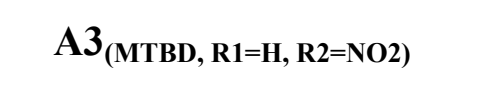 & -1337.46740583 & -1337.034575 & -3.5 \\
\hline $\mathbf{A} 1_{(\mathbf{D B U}, \mathbf{R} 1=\mathrm{H}, \mathrm{R} 2=\mathrm{NO} 2)}$ & -1321.41455121 & -1320.974004 & 0.0 \\
\hline TSA1-2 $(\mathrm{DBU}, \mathrm{R} 1=\mathrm{H}, \mathrm{R} 2=\mathrm{NO} 2)$ & -1321.38320604 & -1320.940518 & 21.0 \\
\hline $\left.\mathbf{A} 2_{(\mathrm{DBU}}, \mathrm{R} 1=\mathrm{H}, \mathrm{R} 2=\mathrm{NO} 2\right)$ & -1321.40042630 & -1320.951404 & 14.2 \\
\hline
\end{tabular}




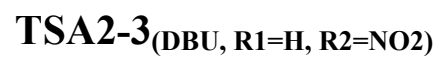

\section{Imaginary frequencies of the transition states.}

Table S4. Calculated imaginary frequencies of transition states at $\omega \mathrm{B} 97 \mathrm{X}-\mathrm{D} / 6-31 \mathrm{G}(\mathrm{d})$ level.

\begin{tabular}{|c|c|}
\hline \multicolumn{2}{|c|}{$R_{1}=R_{2}=H$} \\
\hline $\operatorname{TSA}_{1-2}(\mathrm{KOH}, \mathrm{R} 1=\mathrm{R} 2=\mathrm{H})$ & $1005.72 i$ \\
\hline $\mathrm{TSA}-\mathbf{3}_{(\mathrm{KOH}, \mathrm{R} 1=\mathrm{R} 2=\mathrm{H})}$ & $1033.14 i$ \\
\hline TSA1-2 $(\mathrm{NaOH}, \mathrm{R} 1=\mathrm{R} 2=\mathrm{H})$ & $1132.43 i$ \\
\hline TSA2-3 $(\mathrm{NaOH}, \mathrm{R} 1=\mathrm{R} 2=\mathrm{H})$ & $1078.15 i$ \\
\hline TSA1-2 $(\mathrm{LiOH}, \mathrm{R} 1=\mathrm{R} 2=\mathrm{H})$ & $1183.67 i$ \\
\hline $\mathrm{TSA}-\mathbf{3}_{(\mathrm{LiOH}, \mathrm{R} 1=\mathrm{R} 2=\mathrm{H})}$ & $1000.27 i$ \\
\hline 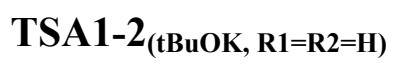 & $1260.28 i$ \\
\hline $\mathrm{TSA} 2-3_{(\mathrm{tBuOK}, \mathrm{R} 1=\mathrm{R} 2=\mathrm{H})}$ & $1211.59 i$ \\
\hline $\operatorname{TSA}_{\left.1-2_{(\mathrm{tBuONa}}, \mathrm{R} 1=\mathrm{R} 2=\mathrm{H}\right)}$ & $1208.41 i$ \\
\hline $\mathrm{TSA}_{\left.2-3_{(\mathrm{tBuONa}}, \mathrm{R} 1=\mathrm{R} 2=\mathrm{H}\right)}$ & $1171.79 i$ \\
\hline $\left.\mathrm{TSA}-4_{(\mathrm{tBuONa}}, \mathrm{R} 1=\mathrm{R} 2=\mathrm{H}\right)$ & $1272.36 i$ \\
\hline TSA5-6 $_{(\mathrm{tBuONa}, \mathrm{R} 1=\mathrm{R} 2=\mathrm{H})}$ & $1123.71 i$ \\
\hline $\mathrm{TSB}_{1-2}(\mathrm{tBuONa}, \mathrm{R} 1=\mathrm{R} 2=\mathrm{H})$ & $1445.27 i$ \\
\hline $\left.\mathrm{TSB}_{2}-3_{(\mathrm{tBuONa}}, \mathrm{R} 1=\mathrm{R} 2=\mathrm{H}\right)$ & $712.06 i$ \\
\hline $\operatorname{TSC}_{\left.1-2_{(\mathrm{tBuONa}}, \mathrm{R} 1=\mathrm{R} 2=\mathrm{H}\right)}$ & $929.50 i$ \\
\hline $\mathrm{TSC} 4-5_{(\mathrm{tBuONa}, \mathrm{R} 1=\mathrm{R} 2=\mathrm{H})}$ & $1330.40 i$ \\
\hline $\operatorname{TSC}^{-6} 6_{(\mathrm{tBuONa}, \mathrm{R} 1=\mathrm{R} 2=\mathrm{H})}$ & $1229.56 i$ \\
\hline 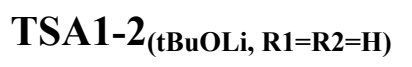 & $1072.72 i$ \\
\hline TSA2 $\left.-3_{(\mathrm{tBuOLi}}, \mathrm{R} 1=\mathrm{R} 2=\mathrm{H}\right)$ & $1184.33 i$ \\
\hline $\operatorname{TSA}_{1-2} \mathbf{T B D}_{(\mathrm{R} 1=\mathrm{R} 2=\mathrm{H})}$ & $1154.42 i$ \\
\hline $\mathrm{TSA}_{\left.2-3_{(\mathrm{TBD}}, \mathrm{R} 1=\mathrm{R} 2=\mathrm{H}\right)}$ & $1143.30 i$ \\
\hline $\mathrm{TSC}_{1-2} \mathbf{T}_{(\mathrm{TBD}, \mathrm{R} 1=\mathrm{R} 2=\mathrm{H})}$ & $1113.54 i$ \\
\hline
\end{tabular}


$\mathrm{TSC}_{2}-3_{(\mathrm{TBD}, \mathrm{R} 1=\mathrm{R} 2=\mathrm{H})}$

$1402.82 i$

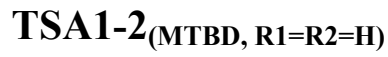

$1123.92 i$

$\mathrm{TSA}_{2}-3_{(\mathrm{MTBD}, \mathrm{R} 1=\mathrm{R} 2=\mathrm{H})}$

$978.57 i$

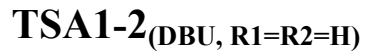

$265.17 i$

$\mathrm{TSA2}_{\mathbf{( D B U}, \mathrm{R} 1=\mathrm{R} 2=\mathrm{H})}$

$677.87 i$

$$
R_{1}=\mathrm{OMe}, \mathrm{R}_{2}=\mathrm{H}
$$

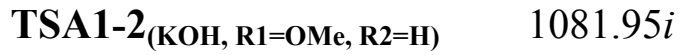

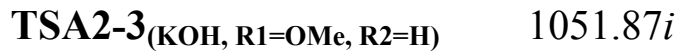

TSA1-2 $(\mathbf{N a O H}, \mathbf{R 1}=\mathbf{O M e}, \mathbf{R 2}=\mathbf{H}) \quad 1113.36 i$

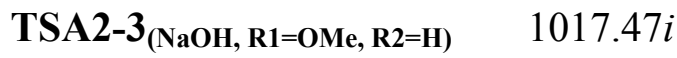

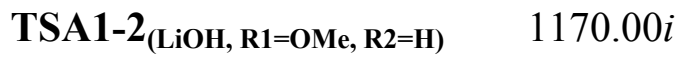

$\mathbf{T S A 2}^{\left.-3_{(\mathrm{LiOH}, ~ R 1}=\mathbf{O M e}, \mathbf{R 2}=\mathbf{H}\right)} \quad 839.17 i$

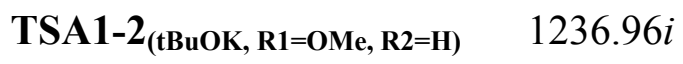

$\mathbf{T S A 2}_{\mathbf{( t B u O K}, \mathbf{R} 1=\mathbf{O M e}, \mathbf{R 2}=\mathbf{H}) \quad 1241.92 i}$

TSA1-2 $\left.{ }_{(\text {tBuONa, }} \mathbf{R 1}=\mathbf{O M e}, \mathbf{R} 2=\mathbf{H}\right) \quad 1170.88 i$

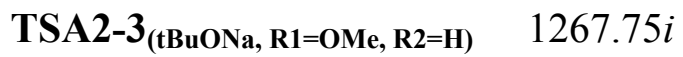

TSA1-2 $\left.{ }_{(\text {tBuOLi, }} \mathbf{R} 1=\mathbf{O M e}, \mathbf{R 2}=\mathbf{H}\right) \quad 1251.53 i$

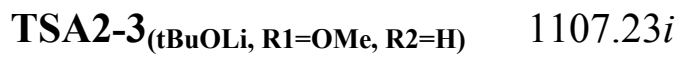

$\mathbf{T S A 1}_{\mathbf{( T B D}, \mathbf{R} 1=\mathbf{O M e}, \mathbf{R 2}=\mathbf{H}) \quad 1071.95 i}$

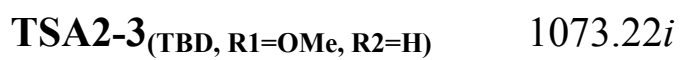

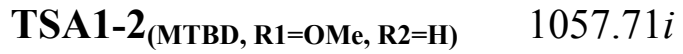

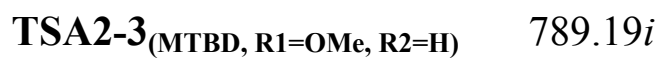

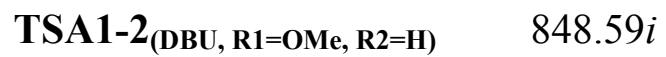

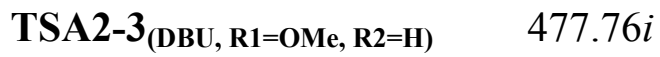

$$
R_{1}=M e, R_{2}=H
$$

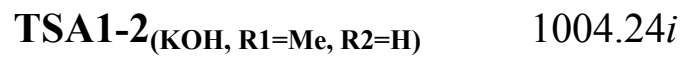

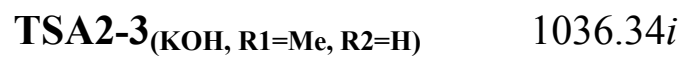

TSA1-2 $(\mathbf{N a O H}, \mathbf{R} 1=\mathrm{Me}, \mathbf{R 2}=\mathbf{H}) \quad 1116.52 i$

$\mathbf{T S A 2}_{\mathbf{3}} \mathbf{3}_{(\mathrm{NaOH}, \mathbf{R} 1=\mathrm{Me}, \mathbf{R 2}=\mathbf{H})} \quad 1059.54 i$ 


$$
\begin{aligned}
& \text { TSA1-2 }_{(\mathbf{L i O H}, \mathbf{R} 1=M e, ~ R 2=H)} \quad 1186.67 i
\end{aligned}
$$

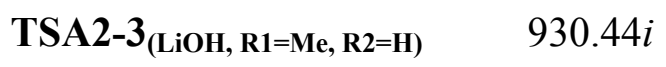

$$
\begin{aligned}
& \mathbf{T S A 1}_{\mathbf{( t B u O K}, \mathbf{R} 1=\mathbf{M e}, \mathbf{R} 2=\mathbf{H})} \quad 1205.46 i
\end{aligned}
$$

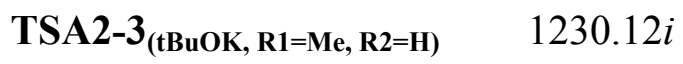

$$
\begin{aligned}
& \text { TSA1-2 } \text { (tBuONa, }_{\text {R1 }=\text { Me, }} \text { R2=H) } \quad 1236.34 i \\
& \text { TSA2-3 } \left.\text { (tBuONa, }_{\text {R1 }}=\mathbf{M e}, \mathbf{R} 2=\mathbf{H}\right) \quad 1273.40 i \\
& \text { TSA1-2 } \left.{ }_{(\text {tBuOLi, }} \mathbf{R 1}=\mathbf{M e}, \mathbf{R 2}=\mathbf{H}\right) \quad 1277.08 i \\
& \left.\mathbf{T S A 2}_{\mathbf{( t B u O L i}} \mathbf{R} 1=\mathbf{M e}, \mathbf{R 2}=\mathbf{H}\right) \quad 1136.36 i
\end{aligned}
$$

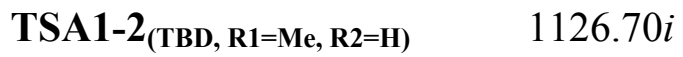

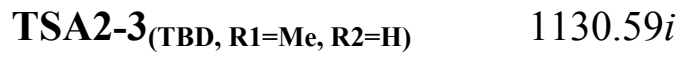

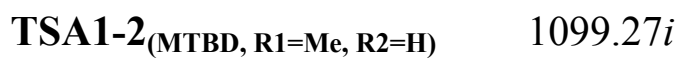

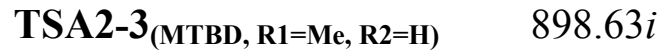

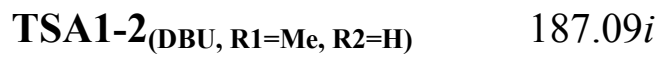

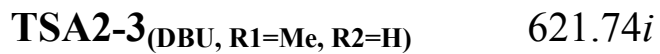

$$
\begin{aligned}
& R_{1}=C F_{3}, R_{2}=H
\end{aligned}
$$

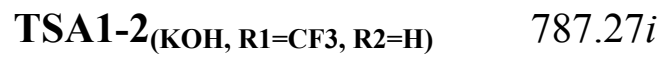

$\mathbf{T S A 2}_{\mathbf{( K O H}, \mathbf{R} 1=\mathbf{C F 3}, \mathbf{R} 2=\mathbf{H})} \quad 936.21 i$

TSA1-2 $_{(\mathrm{NaOH}, \mathbf{R} 1=\mathbf{C F 3}, \mathbf{R} 2=\mathbf{H})} \quad 1066.40 i$

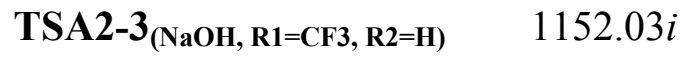

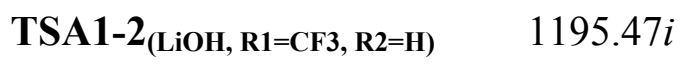

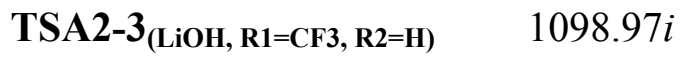

TSA1-2 ${ }_{(\mathbf{t B u O K}, \mathbf{R} 1=\mathbf{C F} 3, \mathbf{R} 2=\mathbf{H}) \quad 1214.46 i}$

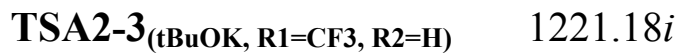

TSA1-2 $\left._{(\text {tBuONa, }} \mathbf{R 1}=\mathbf{C F} 3, \mathbf{R} 2=\mathbf{H}\right) \quad 1177.32 i$

TSA2-3 (tBuONa, $\left._{\text {R1 }}=\mathbf{C F 3}, \mathbf{R 2}=\mathbf{H}\right) \quad 1252.04 i$

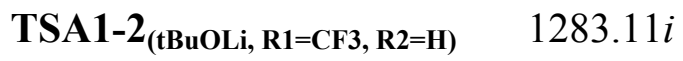

$\left.\mathbf{T S A 2}_{\mathbf{( t B u O L i}} \mathbf{R} \mathbf{1}=\mathbf{C F 3}, \mathbf{R} 2=\mathbf{H}\right) \quad 1240.24 i$

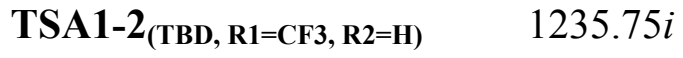

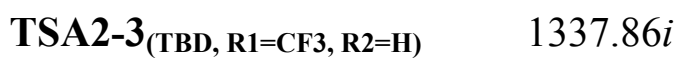




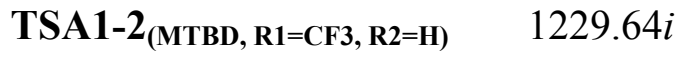

$$
\begin{aligned}
& \text { TSA2-3 } \text { (MTBD, R1=CF3, R2=H) } \quad 1193.18 i \\
& \mathbf{T S A 1}_{(\mathbf{D B U}, \mathbf{R} 1=\mathbf{C F} 3, \mathbf{R} 2=\mathbf{H}) \quad 785.03 i}
\end{aligned}
$$

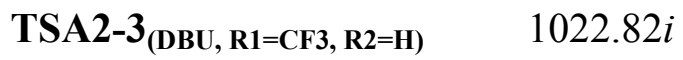

$$
\begin{aligned}
& R_{1}=N_{2}, R_{2}=H \\
& R_{1}=H, R_{2}=O M e
\end{aligned}
$$

TSA1-2 $($ КОН, R1=H, R2=OMe) $1015.51 i$

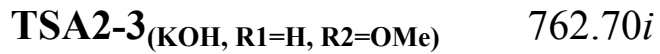

TSA1-2 $(\mathbf{N a O H}, \mathbf{R} 1=\mathbf{H}, \mathbf{R 2}=\mathbf{O M e}) \quad 1078.27 i$

$\mathbf{T S A 2}_{(\mathbf{N a O H}, \mathbf{R} 1=\mathbf{H}, \mathbf{R} 2=\mathbf{O M e}) \quad 1057.20 i}$

TSA1-2 (LiOH, R1=H, R2=OMe) $\quad 1172.45 i$ 


\begin{tabular}{|c|c|}
\hline $\mathrm{OH}, \mathbf{R} 1=\mathbf{H}, \mathbf{R} 2=\mathrm{OMe})$ & $20.68 i$ \\
\hline uOK, R1=H, R2=OMe) & 213.93 \\
\hline SA2-3 (tBuOK, R1=H, R2=OMe) & $1248.77 t$ \\
\hline$A 1-2_{(\text {BuONa, }}$ R1=H, R2=OMe) & 196.90 \\
\hline uONa, R1=H, R2=OMe) & 76 \\
\hline$(\mathrm{tBuOLi}, \mathbf{R} 1=\mathrm{H}, \mathbf{R 2}=\mathrm{OMe})$ & $5 i$ \\
\hline uOLi, R1=H, R2=OMe) & .83 \\
\hline 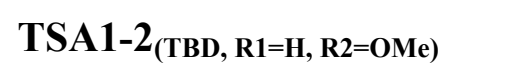 & .89 \\
\hline TBD, R1=H, R2=OMe) & \\
\hline $\mathrm{TBD}, \mathrm{R} 1=\mathrm{H}, \mathrm{R} 2=\mathrm{OMe})$ & $\begin{array}{lll} & \\
\end{array}$ \\
\hline BD, R1=H, R2=OMe) & $4 i$ \\
\hline SA1-2 $($ DBU, R1=H, R2=OMe) & $66.62 i$ \\
\hline $\mathbf{U}, \mathbf{R} \mathbf{1}=\mathbf{H}, \mathbf{R} 2=\mathbf{O M e})$ & \\
\hline \multicolumn{2}{|l|}{$R_{1}=H, R_{2}=M e$} \\
\hline $\mathrm{H}, \mathrm{R} 1=\mathrm{H}, \mathbf{R} 2=\mathrm{Me})$ & \\
\hline $\mathbf{R} 1=\mathbf{H}, \mathbf{R} 2=\mathbf{M e})$ & $792.66 i$ \\
\hline $\mathrm{NaOH}, \mathbf{R} 1=\mathrm{H}, \mathrm{R} 2=\mathrm{Me})$ & 70 \\
\hline $\mathrm{OH}, \mathbf{R} 1=\mathrm{H}, \mathbf{R} 2=\mathrm{Me})$ & 1082.34 \\
\hline SA1-2 $(\mathrm{LiOH}, \mathrm{R} 1=\mathrm{H}, \mathrm{R} 2=\mathrm{Me})$ & 35.49 \\
\hline $\mathrm{OH}, \mathbf{R} 1=\mathrm{H}, \mathbf{R} 2=\mathbf{M e})$ & $+5 l$ \\
\hline $\mathrm{tBuOK}, \mathbf{R} 1=\mathrm{H}, \mathbf{R 2}=\mathrm{Me})$ & 0.12 \\
\hline$\Gamma S A 2-3_{(\mathrm{tBuOK}, \mathrm{R} 1=\mathrm{H}, \mathrm{R} 2=\mathrm{Me})}$ & $2.37 i$ \\
\hline $\left.\mathrm{SA} 1-\mathbf{2}_{(\mathrm{tBuONa}}, \mathrm{R} 1=\mathrm{H}, \mathrm{R} 2=\mathrm{Me}\right)$ & 0.14 \\
\hline TSA2-3 $(\mathrm{tBuONa}, \mathrm{R} 1=\mathrm{H}, \mathrm{R} 2=\mathrm{Me})$ & $217.19 i$ \\
\hline$\Gamma S A 1-2_{(\text {tBuOLi, }}$ R1=H, R2=Me) & $606.86 i$ \\
\hline TSA2-3 $\left.{ }_{(\mathrm{tBuOLi}}, \mathrm{R} 1=\mathrm{H}, \mathrm{R} 2=\mathrm{Me}\right)$ & 1167.68 \\
\hline$\Gamma \mathrm{SA} 1-2_{(\mathrm{TBD}, \mathrm{R} 1=\mathrm{H}, \mathrm{R} 2=\mathrm{Me})}$ & 1100.64 \\
\hline $\mathrm{D}, \mathbf{R} 1=\mathbf{H}, \mathbf{R} 2=\mathrm{Me})$ & 1146 \\
\hline $\mathrm{SAI-2}(\mathrm{N}$ & 10 \\
\hline
\end{tabular}




$$
\begin{array}{cc}
\text { TSA2-3 }_{(\text {MTBD, R1=H, R2=Me) }} & 942.87 i \\
\text { TSA1-2 }_{(\mathbf{D B U}, \mathbf{R} 1=\mathrm{H}, \mathbf{R} 2=\mathrm{Me})} & 158.56 i \\
\text { TSA2-3 }_{(\mathrm{DBU}, \mathbf{R} 1=\mathrm{H}, \mathbf{R} 2=\mathrm{Me})} & 671.47 i \\
\boldsymbol{R}_{\mathbf{1}}=\boldsymbol{H}, \boldsymbol{R}_{\mathbf{2}}=\boldsymbol{C F}_{3} &
\end{array}
$$

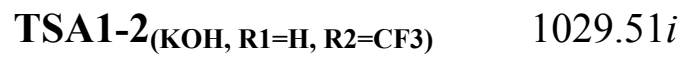

$\mathbf{T S A 2}_{\mathbf{( K O H}, \mathbf{R} 1=\mathbf{H}, \mathbf{R 2}=\mathbf{C F} 3)} \quad 889.59 i$

TSA1-2 $(\mathbf{N a O H}, \mathbf{R} 1=\mathbf{H}, \mathbf{R 2}=\mathbf{C F 3}) \quad 1039.51 i$

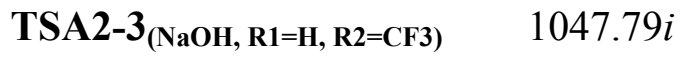

TSA1-2 ${ }_{(\mathbf{L i O H}, \mathbf{R} 1=\mathbf{H}, \mathbf{R 2}=\mathbf{C F 3})} \quad 1218.39 i$

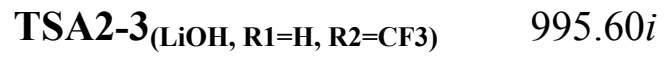

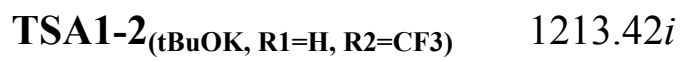

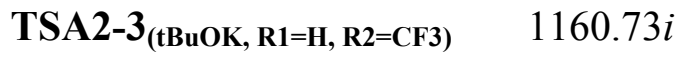

TSA1-2 ${ }_{(\mathbf{t B u O N a}, \mathbf{R} 1=\mathbf{H}, \mathbf{R} 2=\mathbf{C F} 3)} 1214.11 i$

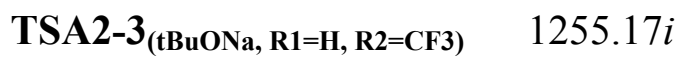

TSA1-2 $\left._{(\mathbf{t B u O L i}}, \mathbf{R} 1=\mathbf{H}, \mathbf{R} 2=\mathbf{C F 3}\right) \quad 1322.12 i$

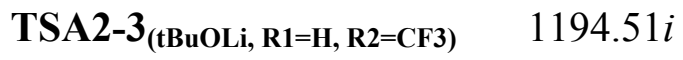

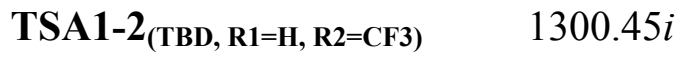

$\mathbf{T S A 2}_{\mathbf{3}} \mathbf{3}_{(\mathbf{T B D}, \mathbf{R} 1=\mathbf{H}, \mathbf{R} 2=\mathbf{C F} 3) \quad 1248.26 i}$

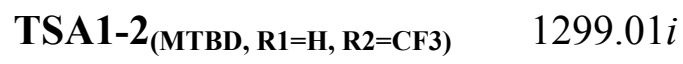

$\mathbf{T S A 2}_{\mathbf{( M T B D}, \mathbf{R} 1=\mathbf{H}, \mathbf{R 2}=\mathbf{C F} 3)} \quad 1098.00 i$

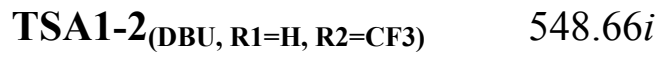

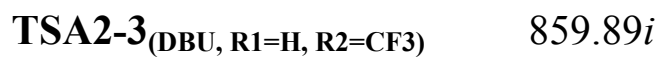

$$
\mathrm{R}_{1}=\mathrm{H}, \mathrm{R}_{2}=\mathrm{NO}_{2}
$$

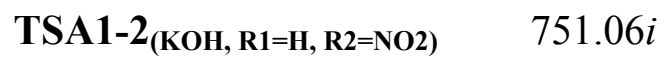

TSA2-3 $($ KOH, R1=H, R2=NO2) $\quad 1005.87 i$

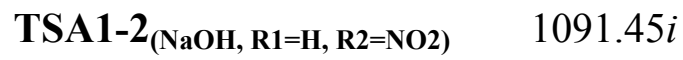

TSA2-3 (NaOH, R1=H, R2=NO2) $_{1021.32 i}$

TSA1-2 $_{(\mathrm{LiOH}, \mathbf{R} 1=H, ~ R 2=N O 2)} \quad 1221.96 i$

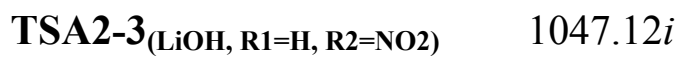




\begin{tabular}{|c|c|}
\hline 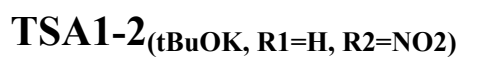 & $1214.38 i$ \\
\hline $\mathrm{TSA2}_{\mathbf{( t B u O K}, \mathrm{R} 1=\mathrm{H}, \mathrm{R} 2=\mathrm{NO} 2)}$ & $1035.09 i$ \\
\hline $\mathrm{TSA}_{1-2}$ (tBuONa, $\left.\mathrm{R} 1=\mathrm{H}, \mathrm{R} 2=\mathrm{NO} 2\right)$ & $1215.56 i$ \\
\hline $\mathrm{TSA}_{2}-3_{(\mathrm{tBuONa}, \mathrm{R} 1=\mathrm{H}, \mathrm{R} 2=\mathrm{NO} 2)}$ & $1217.72 i$ \\
\hline $\mathrm{TSA}_{1-2}(\mathrm{tBuOLi}, \mathrm{R} 1=\mathrm{H}, \mathrm{R} 2=\mathrm{NO} 2)$ & $1347.15 i$ \\
\hline $\mathrm{TSA2}_{(\mathrm{tBuOLi}, \mathrm{R} 1=\mathrm{H}, \mathrm{R} 2=\mathrm{NO} 2)}$ & $1238.12 i$ \\
\hline $\mathrm{TSA}_{1-2}(\mathrm{TBD}, \mathrm{R} 1=\mathrm{H}, \mathrm{R} 2=\mathrm{NO} 2)$ & $1419.86 i$ \\
\hline $\mathrm{TSA}_{2-3}(\mathrm{TBD}, \mathrm{R} 1=\mathrm{H}, \mathrm{R} 2=\mathrm{NO} 2)$ & $1375.12 i$ \\
\hline TSA1-2 (MTBD, R1=H, R2=NO2) & $1439.86 i$ \\
\hline $\mathrm{TSA}_{2-3}$ (MTBD, R1=H, R2=NO2) & $1262.65 i$ \\
\hline TSA1-2 ${ }_{(\mathrm{DBU}, \mathrm{R} 1=\mathrm{H}, \mathrm{R} 2=\mathrm{NO} 2)}$ & $919.26 i$ \\
\hline $\mathrm{TSA}_{2-3}{ }_{(\mathrm{DBU}, \mathrm{R} 1=\mathrm{H}, \mathrm{R} 2=\mathrm{NO} 2)}$ & $1089.64 i$ \\
\hline
\end{tabular}


10. The values of Hirshfeld charge.

Table S5: Hirshfeld charge of $\mathrm{C}^{1}$ atom in $\mathbf{A 1}$ (unit: a.u.).

\begin{tabular}{cccccc}
\hline & OMe & $\mathbf{M e}$ & $\mathbf{H}$ & $\mathbf{C F}_{\mathbf{3}}$ & $\mathbf{N O}_{\mathbf{2}}$ \\
\hline KOH & 0.04896 & 0.04888 & 0.04877 & 0.04809 & 0.04748 \\
NaOH & 0.05504 & 0.05484 & 0.05482 & 0.05378 & 0.05345 \\
LiOH & 0.06815 & 0.06782 & 0.0679 & 0.06704 & 0.06689 \\
$\boldsymbol{t B u O K}$ & 0.05193 & 0.05094 & 0.05101 & 0.05069 & 0.05009 \\
$\boldsymbol{t B u O N a}$ & 0.057 & 0.0569 & 0.05668 & 0.05552 & 0.05479 \\
$\boldsymbol{t B u O L i}$ & 0.06889 & 0.0687 & 0.06849 & 0.06773 & 0.06724 \\
TBD_NH & 0.05096 & 0.05031 & 0.05077 & 0.05031 & 0.05021 \\
TBD_Me & 0.05082 & 0.05162 & 0.05139 & 0.05067 & 0.05082 \\
TBD & 0.05228 & 0.05221 & 0.05244 & 0.05243 & 0.052 \\
TBD_CF 3 & 0.05062 & 0.05207 & 0.05191 & 0.05058 & 0.0499 \\
MTBD & 0.04881 & 0.04774 & 0.04987 & 0.05016 & 0.04963 \\
DBU_NH & 0.04827 & 0.048 & 0.04756 & 0.04819 & 0.04861 \\
DBU_Me & 0.0481 & 0.04852 & 0.04837 & 0.04816 & 0.04839 \\
DBU & 0.04989 & 0.05001 & 0.04959 & 0.04908 & 0.04887 \\
DBU_CF & 0.0481 & 0.04852 & 0.04837 & 0.04816 & 0.04839 \\
\hline & & & & & \\
\hline
\end{tabular}

Table S6: Hirshfeld charge of $\mathrm{H}^{1}$ atom in $\mathbf{A 1}$ (unit: a.u.).

\begin{tabular}{ccccccc}
\hline OMe & Me & & H & $\mathrm{CF}_{3}$ & $\mathrm{NO}_{\mathbf{2}}$ \\
\hline
\end{tabular}




\begin{tabular}{cccccc} 
KOH & 0.04108 & 0.04108 & 0.04122 & 0.04086 & 0.04099 \\
NaOH & 0.04809 & 0.04825 & 0.04806 & 0.04729 & 0.04725 \\
LiOH & 0.06017 & 0.05952 & 0.05963 & 0.05873 & 0.05863 \\
$\boldsymbol{t B u O K}$ & 0.04416 & 0.04425 & 0.04449 & 0.04403 & 0.04452 \\
$\boldsymbol{t}$ BuONa & 0.05001 & 0.0497 & 0.04954 & 0.04806 & 0.04761 \\
$\boldsymbol{t B u O L i}$ & 0.06114 & 0.0603 & 0.05983 & 0.05844 & 0.05792 \\
TBD_NH2 & 0.04603 & 0.04515 & 0.04565 & 0.04583 & 0.0462 \\
TBD_Me & 0.04512 & 0.04643 & 0.046 & 0.04561 & 0.04623 \\
TBD & 0.04766 & 0.04827 & 0.0486 & 0.04885 & 0.04872 \\
TBD_CF 3 & 0.0424 & 0.04497 & 0.04456 & 0.04231 & 0.0419 \\
MTBD & 0.04486 & 0.04412 & 0.04566 & 0.04685 & 0.04651 \\
DBU_NH2 & 0.04393 & 0.04398 & 0.04367 & 0.04482 & 0.04538 \\
DBU_Me & 0.04471 & 0.0453 & 0.04537 & 0.04561 & 0.04611 \\
DBU & 0.046 & 0.04629 & 0.04609 & 0.04623 & 0.04641 \\
DBU_CF 3 & 0.04933 & 0.04379 & 0.04438 & 0.0452 & 0.04574 \\
\hline & & & & &
\end{tabular}

11. The values of MEP.

Table S7: MEP of $\mathrm{C}^{1}$ atom in $\mathbf{A 1}$ (unit: a.u.).

\begin{tabular}{cccccc}
\hline & OMe & Me & H & $\mathbf{C F}_{3}$ & NO $_{2}$ \\
\hline KOH & -14.70496 & -14.70418 & -14.70309 & -14.69779 & -14.6934 \\
NaOH & -14.69247 & -14.69175 & -14.69084 & -14.68628 & -14.68195 \\
LiOH & -14.67608 & -14.67563 & -14.67454 & -14.67 & -14.66557 \\
$\boldsymbol{t B u O K}$ & -14.69455 & -14.69344 & -14.69274 & -14.68754 & -14.68281 \\
$\boldsymbol{t B u O N a}$ & -14.68184 & -14.68107 & -14.68007 & -14.67569 & -14.6721 \\
$\boldsymbol{t B u O L i}$ & -14.66653 & -14.66585 & -14.66504 & -14.66017 & -14.65645 \\
TBD_NH & -14.69838 & -14.69808 & -14.69673 & -14.69127 & -14.68678 \\
TBD_Me & -14.69669 & -14.69506 & -14.69442 & -14.68858 & -14.6836 \\
TBD & -14.69562 & -14.6948 & -14.69341 & -14.68732 & -14.6832 \\
\hline
\end{tabular}




\begin{tabular}{cccccc} 
TBD_CF 3 & -14.69723 & -14.69521 & -14.69411 & -14.68937 & -14.6854 \\
MTBD & -14.69413 & -14.69366 & -14.6908 & -14.68516 & -14.68097 \\
DBU_NH2 & -14.70222 & -14.70179 & -14.70136 & -14.6938 & -14.68873 \\
DBU_Me & -14.70355 & -14.70227 & -14.70157 & -14.6954 & -14.69044 \\
DBU & -14.70199 & -14.70079 & -14.70041 & -14.69477 & -14.69043 \\
DBU_CF 3 & -14.701 & -14.69927 & -14.69959 & -14.69056 & -14.68581 \\
\hline
\end{tabular}

Table S8: MEP of $\mathrm{H}^{1}$ atom in A1 (unit: a.u.).

\begin{tabular}{cccccc}
\hline & OMe & $\mathbf{M e}$ & $\mathbf{H}$ & $\mathbf{C F}_{\mathbf{3}}$ & $\mathbf{N O}_{\mathbf{2}}$ \\
\hline KOH & -1.13572 & -1.13436 & -1.13297 & -1.12616 & -1.12061 \\
NaOH & -1.12575 & -1.12444 & -1.12323 & -1.11722 & -1.11206 \\
LiOH & -1.11238 & -1.11146 & -1.11024 & -1.10463 & -1.09969 \\
$\boldsymbol{t B u O K}$ & -1.12603 & -1.12391 & -1.1229 & -1.11666 & -1.11085 \\
$\boldsymbol{t B u O N a}$ & -1.11547 & -1.11402 & -1.11276 & -1.10678 & -1.10208 \\
$\boldsymbol{t B u O L i}$ & -1.10243 & -1.10122 & -1.10014 & -1.09439 & -1.08996 \\
TBD_NH & -1.13513 & -1.13391 & -1.13248 & -1.12595 & -1.12078 \\
TBD_Me & -1.13295 & -1.1312 & -1.13036 & -1.1232 & -1.11759 \\
TBD & -1.13247 & -1.13116 & -1.12962 & -1.12288 & -1.11793 \\
TBD_CF & -1.13327 & -1.13152 & -1.13012 & -1.12414 & -1.11919 \\
MTBD & -1.12928 & -1.12814 & -1.12564 & -1.11993 & -1.11543 \\
DBU_NH & -1.13764 & -1.13663 & -1.13567 & -1.12762 & -1.12221 \\
DBU_Me & -1.13884 & -1.13725 & -1.13618 & -1.1292 & -1.12373 \\
DBU & -1.13826 & -1.13656 & -1.13577 & -1.12905 & -1.12395 \\
DBU_CF & -1.13394 & -1.13301 & -1.13367 & -1.12373 & -1.1184 \\
\hline
\end{tabular}

12. The values of NAO.

Table S9: NAO of $\mathrm{C}^{1}$ atom in $\mathbf{A 1}$ (unit: a.u.).

\begin{tabular}{lllllll}
\hline OMe & $\mathrm{Me}$ & $\mathrm{H}$ & $\mathrm{CF}_{3}$ & $\mathrm{NO}_{2}$ \\
\hline
\end{tabular}




\begin{tabular}{cccccc} 
KOH & -0.36683 & -0.37103 & -0.37648 & -0.4013 & -0.4219 \\
NaOH & -0.41278 & -0.4172 & -0.42177 & -0.44466 & -0.46459 \\
LiOH & -0.46366 & -0.4678 & -0.47271 & -0.49572 & -0.51571 \\
$\boldsymbol{t}$ BuOK & -0.39837 & -0.40579 & -0.40938 & -0.43342 & -0.45543 \\
$\boldsymbol{t B u O N a}$ & -0.44436 & -0.44855 & -0.45344 & -0.47495 & -0.49282 \\
$\boldsymbol{t B u O L i}$ & -0.49459 & -0.49857 & -0.50297 & -0.52522 & -0.5432 \\
TBD_NH & -0.35486 & -0.35786 & -0.36314 & -0.38837 & -0.40847 \\
TBD_Me & -0.36311 & -0.37003 & -0.37319 & -0.39941 & -0.42142 \\
TBD & -0.36721 & -0.37228 & -0.37855 & -0.40508 & -0.42391 \\
TBD_CF & -0.35999 & -0.36729 & -0.37268 & -0.39506 & -0.41402 \\
MTBD & -0.36167 & -0.36777 & -0.37377 & -0.39799 & -0.41898 \\
DBU_NH & -0.34033 & -0.34362 & -0.34686 & -0.37814 & -0.40005 \\
DBU_Me & -0.33563 & -0.34136 & -0.34527 & -0.37179 & -0.3935 \\
DBU & -0.3403 & -0.34593 & -0.34855 & -0.374 & -0.3936 \\
DBU_CF 3 & -0.3512 & -0.3521 & -0.35222 & -0.38907 & -0.4097 \\
\hline & & & & & \\
\hline & & & & & \\
\hline
\end{tabular}

Table S10: NAO of $\mathrm{H}^{1}$ atom in A1 (unit: a.u.).

\begin{tabular}{cccccc}
\hline & OMe & Me & $\mathbf{H}$ & $\mathbf{C F}_{\mathbf{3}}$ & $\mathbf{N O}_{\mathbf{2}}$ \\
\hline KOH & 0.17567 & 0.17466 & 0.17376 & 0.16838 & 0.16451 \\
NaOH & 0.1697 & 0.16953 & 0.16873 & 0.16552 & 0.1618 \\
LiOH & 0.15194 & 0.15212 & 0.15173 & 0.14942 & 0.14608 \\
$\boldsymbol{t B u O K}$ & 0.16698 & 0.1639 & 0.16439 & 0.16142 & 0.15492 \\
$\boldsymbol{t B u O N a}$ & 0.1553 & 0.15406 & 0.15339 & 0.14856 & 0.14576 \\
$\boldsymbol{t B u O L i}$ & 0.13677 & 0.13536 & 0.13481 & 0.13079 & 0.12835 \\
TBD_NH & 0.17199 & 0.17125 & 0.1706 & 0.16734 & 0.16395 \\
TBD_Me & 0.16586 & 0.16543 & 0.16525 & 0.16034 & 0.15638 \\
TBD & 0.1664 & 0.16727 & 0.16595 & 0.16147 & 0.15914 \\
TBD_CF & 0.16797 & 0.16731 & 0.1661 & 0.1621 & 0.15919 \\
MTBD & 0.15392 & 0.15519 & 0.14962 & 0.14652 & 0.1449 \\
\hline
\end{tabular}




\begin{tabular}{cccccc} 
DBU_NH & 0.17353 & 0.17377 & 0.17354 & 0.16812 & 0.16399 \\
DBU_Me & 0.17685 & 0.17544 & 0.17492 & 0.17063 & 0.16668 \\
DBU & 0.17276 & 0.17138 & 0.17223 & 0.16977 & 0.16674 \\
DBU_CF 3 & 0.17497 & 0.17256 & 0.1728 & 0.1648 & 0.16095 \\
\hline
\end{tabular}

13. The values of Information gain.

Table S11: Information gain of A1 (unit: a.u.).

\begin{tabular}{|c|c|c|c|c|}
\hline & $\mathbf{C}^{1}$ & $\mathbf{H}^{1}$ & $\mathbf{O}^{2}$ & Total \\
\hline $\mathrm{KOH}_{\mathbf{R} 2=\mathrm{OMe}}$ & 0.00749 & 0.0047958 & 0.6636126 & 2.13603204 \\
\hline $\mathrm{KOH}_{\mathrm{R} 2=\mathrm{Me}}$ & 0.007614 & 0.0046238 & 0.6624986 & 2.07555493 \\
\hline $\mathrm{KOH}_{\mathrm{R} 2=\mathrm{H}}$ & 0.007773 & 0.0043522 & 0.6614008 & 1.94637822 \\
\hline $\mathrm{KOH}_{\mathrm{R} 2=\mathrm{CF} 3}$ & 0.008489 & 0.0042073 & 0.6575902 & 2.02034287 \\
\hline $\mathrm{KOH}_{\mathrm{R} 2=\mathrm{NO} 2}$ & 0.008998 & 0.0036494 & 0.6543311 & 2.03170984 \\
\hline $\mathrm{NaOH}_{\mathrm{R} 2=\mathrm{OMe}}$ & 0.00061 & -0.001078 & 0.6415056 & 2.07839095 \\
\hline $\mathrm{NaOH}_{\mathrm{R} 2=\mathrm{Me}}$ & 0.000986 & -0.001394 & 0.6402508 & 2.01754283 \\
\hline $\mathrm{NaOH}_{\mathrm{R} 2=\mathrm{H}}$ & 0.001099 & -0.001422 & 0.6393289 & 1.88995418 \\
\hline $\mathrm{NaOH}_{\mathrm{R} 2=\mathrm{CF} 3}$ & 0.002236 & -0.001424 & 0.6337881 & 1.96240101 \\
\hline $\mathrm{NaOH}_{\mathrm{R} 2=\mathrm{NO} 2}$ & 0.002518 & -0.001844 & 0.6314318 & 1.9758707 \\
\hline $\mathrm{LiOH}_{\mathrm{R} 2=\mathrm{OMe}}$ & -0.00995 & -0.007777 & 0.634265 & 2.03930058 \\
\hline $\mathrm{LiOH}_{\mathrm{R} 2=\mathrm{Me}}$ & -0.00947 & -0.007556 & 0.6328059 & 1.97944519 \\
\hline $\mathrm{LiOH}_{\mathrm{R} 2=\mathrm{H}}$ & -0.00943 & -0.007794 & 0.6322662 & 1.85168554 \\
\hline $\mathrm{LiOH}_{\mathrm{R} 2=\mathrm{CF} 3}$ & -0.00842 & -0.007828 & 0.6285066 & 1.92381794 \\
\hline $\mathrm{LiOH}_{\mathrm{R} 2=\mathrm{NO} 2}$ & -0.0083 & -0.008189 & 0.6267875 & 1.93695563 \\
\hline $\mathrm{tBuOK}_{\mathrm{R} 2=\mathrm{OMe}}$ & 0.006054 & 0.0015831 & 0.5174738 & 2.61047282 \\
\hline $\mathrm{tBuOK}_{\mathrm{R} 2=\mathrm{Me}}$ & 0.007058 & 0.0011769 & 0.5136022 & 2.54231107 \\
\hline $\mathrm{tBuOK}_{\mathrm{R} 2=\mathrm{H}}$ & 0.007081 & 0.0009003 & 0.5139367 & 2.41504474 \\
\hline $\mathrm{tBuOK}_{\mathrm{R} 2=\mathrm{CF} 3}$ & 0.007611 & 0.0008598 & 0.5125227 & 2.48979516 \\
\hline $\mathrm{tBuOK}_{\mathrm{R} 2=\mathrm{NO} 2}$ & 0.00816 & $-3.65 \mathrm{E}-05$ & 0.508414 & 2.49762701 \\
\hline $\mathrm{tBuONa}_{\mathrm{R} 2=\mathrm{OMe}}$ & -0.00022 & -0.002546 & 0.4996202 & 2.5600063 \\
\hline
\end{tabular}




\begin{tabular}{|c|c|c|c|c|}
\hline $\mathrm{tBuONa}_{\mathrm{R} 2=\mathrm{Me}}$ & $2.25 \mathrm{E}-05$ & -0.002536 & 0.4989692 & 2.49986471 \\
\hline $\mathrm{tBuONa}_{\mathrm{R} 2=\mathrm{H}}$ & 0.000294 & -0.002594 & 0.4986683 & 2.37087601 \\
\hline $\mathrm{tBuONa}_{\mathrm{R} 2=\mathrm{CF} 3}$ & 0.001668 & -0.002648 & 0.4982682 & 2.44594993 \\
\hline $\mathrm{tBuONa}_{\mathrm{R} 2=\mathrm{NO} 2}$ & 0.002489 & -0.002855 & 0.4958948 & 2.45728095 \\
\hline $\mathrm{tBuOLi}_{\mathrm{R} 2=\mathrm{OMe}}$ & -0.00972 & -0.007874 & 0.5037856 & 2.51634733 \\
\hline $\mathrm{tBuOLi}_{\mathrm{R} 2=\mathrm{Me}}$ & -0.00941 & -0.007551 & 0.5029997 & 2.45722889 \\
\hline $\mathrm{tBuOLi}_{\mathrm{R} 2=\mathrm{H}}$ & -0.00913 & -0.007475 & 0.5023835 & 2.32908692 \\
\hline $\mathrm{tBuOLi}_{\mathrm{R} 2=\mathrm{CF} 3}$ & -0.00811 & -0.007623 & 0.5017782 & 2.40160036 \\
\hline $\mathbf{t B u O L i} i_{\mathrm{R} 2=\mathrm{NO} 2}$ & -0.0075 & -0.007773 & 0.5001888 & 2.41294205 \\
\hline TBD_NH2 $2=O M e$ & 0.004896 & 0.0062123 & 0.2809501 & 2.85048312 \\
\hline TBD_NH2 $2=M e$ & 0.005829 & 0.0067567 & 0.282246 & 2.79028341 \\
\hline TBD_NH2 $22=\mathrm{H}$ & 0.005289 & 0.0061961 & 0.2824897 & 2.66216245 \\
\hline TBD_NH2 2 2 $=$ CF3 & 0.006111 & 0.0058973 & 0.282744 & 2.73483806 \\
\hline TBD_NH2 $2_{\mathrm{R} 2=\mathrm{NO} 2}$ & 0.006327 & 0.0055119 & 0.2820208 & 2.74545828 \\
\hline TBD_Me $e_{\mathrm{R} 2=\mathrm{OMe}}$ & 0.005126 & 0.0073417 & 0.2845021 & 2.86154607 \\
\hline TBD_Me $\mathbf{R}_{\mathrm{R} 2=\mathrm{Me}}$ & 0.004495 & 0.0063847 & 0.2858986 & 2.79897185 \\
\hline TBD_Me $e_{R 2=H}$ & 0.004746 & 0.0066537 & 0.2854256 & 2.67029168 \\
\hline TBD_Me ${ }_{\mathrm{R} 2=\mathrm{CF} 3}$ & 0.005881 & 0.0065953 & 0.2843208 & 2.74297969 \\
\hline TBD_Me $\mathrm{R} 2=\mathrm{NO} 2$ & 0.005848 & 0.0059657 & 0.2842457 & 2.75359471 \\
\hline $\mathrm{TBD}_{\mathrm{R} 2=\mathrm{OMe}}$ & 0.004334 & 0.0052289 & 0.3329607 & 2.76322976 \\
\hline $\mathrm{TBD}_{\mathrm{R} 2=\mathrm{Me}}$ & 0.003094 & 0.0039089 & 0.3327175 & 2.69928579 \\
\hline $\mathrm{TBD}_{\mathrm{R} 2=\mathrm{H}}$ & 0.003555 & 0.0042719 & 0.3335991 & 2.57247848 \\
\hline $\mathrm{TBD}_{\mathrm{R} 2=\mathrm{CF} 3}$ & 0.002702 & 0.0033167 & 0.3327213 & 2.64222451 \\
\hline $\mathrm{TBD}_{\mathrm{R} 2=\mathrm{NO} 2}$ & 0.003031 & 0.0034391 & 0.3317093 & 2.65246633 \\
\hline TBD_CF3 $3_{\mathrm{R} 2=\mathrm{OMe}}$ & 0.00522 & 0.0110422 & 0.2934647 & 2.81230594 \\
\hline TBD_CF $3_{\mathrm{R} 2=\mathrm{Me}}$ & 0.004137 & 0.0092008 & 0.2936444 & 2.74875931 \\
\hline TBD_CF3 ${ }_{\mathrm{R} 2=\mathrm{H}}$ & 0.004248 & 0.0094448 & 0.2939094 & 2.62096555 \\
\hline TBD_CF3 $3_{\mathrm{R} 2=\mathrm{CF} 3}$ & 0.006054 & 0.0110446 & 0.2929406 & 2.69393515 \\
\hline TBD_CF3 $3_{\mathrm{R} 2=\mathrm{NO} 2}$ & 0.006936 & 0.0112782 & 0.2916838 & 2.70504937 \\
\hline
\end{tabular}




\begin{tabular}{|c|c|c|c|c|}
\hline $\mathrm{MTBD}_{\mathrm{R} 2=\mathrm{OMe}}$ & 0.006532 & 0.0045327 & 0.3092407 & 2.8747657 \\
\hline $\operatorname{MTBD}_{\mathrm{R} 2=\mathrm{Me}}$ & 0.007809 & 0.0053233 & 0.3084956 & 2.81400095 \\
\hline $\operatorname{MTBD}_{\mathrm{R} 2=\mathrm{H}}$ & 0.005494 & 0.0034421 & 0.3101831 & 2.68424852 \\
\hline $\operatorname{MTBD}_{\mathrm{R} 2=\mathrm{CF} 3}$ & 0.005913 & 0.0029139 & 0.3094576 & 2.75431124 \\
\hline $\mathrm{MTBD}_{\mathrm{R} 2=\mathrm{NO} 2}$ & 0.006579 & 0.0033903 & 0.3078358 & 2.76496228 \\
\hline $\mathrm{DBU}{ }_{-} \mathrm{NH} 2_{\mathrm{R} 2=\mathrm{OMe}}$ & 0.00732 & 0.00924 & 0.2649558 & 3.04298573 \\
\hline DBU_NH2 $2_{\mathrm{R} 2=M e}$ & 0.007683 & 0.009145 & 0.2634201 & 2.98106456 \\
\hline DBU_NH2 $2_{\mathrm{R} 2=\mathrm{H}}$ & 0.008201 & 0.0093575 & 0.2613739 & 2.85217541 \\
\hline DBU_NH2 2 & 0.007939 & 0.0082153 & 0.2652762 & 2.92453265 \\
\hline $\mathrm{DBU}{ }_{-} \mathrm{NH} 2_{\mathrm{R} 2=\mathrm{NO} 2}$ & 0.007662 & 0.0077294 & 0.2644628 & 2.93422412 \\
\hline $\mathrm{DBU}_{-} \mathrm{Me}_{\mathrm{R} 2=\mathrm{OMe}}$ & 0.007708 & 0.0087038 & 0.2550108 & 3.0535334 \\
\hline DBU_Me $\mathbf{R}_{\mathrm{R} 2=\mathrm{Me}}$ & 0.007387 & 0.0082063 & 0.2542033 & 2.99082044 \\
\hline $\mathrm{DBU}_{-} \mathrm{Me}_{\mathrm{R} 2=\mathrm{H}}$ & 0.007567 & 0.0081004 & 0.2526993 & 2.8620686 \\
\hline $\mathrm{DBU}_{-} \mathrm{Me}_{\mathrm{R} 2=\mathrm{CF} 3}$ & 0.008062 & 0.0078082 & 0.2524242 & 2.93334957 \\
\hline $\mathrm{DBU}_{-} \mathrm{Me}_{\mathrm{R} 2=\mathrm{NO} 2}$ & 0.007996 & 0.0073095 & 0.251799 & 2.94375741 \\
\hline $\mathbf{D B U}_{\mathbf{R 2}=\mathrm{OMe}}$ & 0.005331 & 0.0075308 & 0.260761 & 2.92037138 \\
\hline $\mathrm{DBU}_{\mathrm{R} 2=\mathrm{Me}}$ & 0.005371 & 0.0072499 & 0.2604561 & 2.85844849 \\
\hline $\mathrm{DBU}_{\mathbf{R} 2=\mathrm{H}}$ & 0.005865 & 0.0074525 & 0.2586644 & 2.72972549 \\
\hline $\mathrm{DBU}_{\mathbf{R} 2=\mathrm{CF} 3}$ & 0.006984 & 0.0072605 & 0.2570524 & 2.80108747 \\
\hline $\mathrm{DBU}_{\mathrm{R} 2=\mathrm{NO} 2}$ & 0.00738 & 0.0070198 & 0.2551214 & 2.81159498 \\
\hline $\mathrm{DBU}_{-} \mathrm{CF}_{\mathrm{R} 2=\mathrm{OMe}}$ & 0.002432 & 0.0043037 & 0.2758396 & 2.99512118 \\
\hline DBU_CF $3_{\mathrm{R} 2=\mathrm{Me}}$ & 0.009589 & 0.0095865 & 0.2626193 & 2.94441442 \\
\hline DBU_CF3 $3_{\mathrm{R} 2=\mathrm{H}}$ & 0.00858 & 0.0002218 & 0.2587815 & 2.81183101 \\
\hline $\mathrm{DBU} \mathrm{CF}_{\mathrm{R} 2=\mathrm{CF} 3}$ & 0.008914 & 0.0082398 & 0.2636915 & 2.88572729 \\
\hline DBU_CF3 $3_{\mathrm{R} 2=\mathrm{NO} 2}$ & 0.008956 & 0.0076985 & 0.2633078 & 2.89628706 \\
\hline
\end{tabular}

Table S12: Information gain of TSA1-2 (unit: a.u.).

\begin{tabular}{lllll}
\hline $\mathbf{C}^{1}$ & $\mathbf{H}^{1}$ & $\mathbf{O}^{2}$ & Total \\
\hline
\end{tabular}




\begin{tabular}{|c|c|c|c|c|}
\hline $\mathrm{KOH}_{\mathrm{R} 2=\mathrm{OMe}}$ & 0.06256 & -0.02359 & 0.5224071 & 2.0666551 \\
\hline $\mathrm{KOH}_{\mathrm{R} 2=\mathrm{Me}}$ & 0.059211 & -0.02334 & 0.5277064 & 2.0119792 \\
\hline $\mathrm{KOH}_{\mathrm{R} 2=\mathrm{H}}$ & 0.05995 & -0.02381 & 0.5256733 & 1.8849507 \\
\hline $\mathrm{KOH}_{\mathrm{R} 2=\mathrm{CF} 3}$ & 0.059535 & -0.02369 & 0.5190189 & 1.9610843 \\
\hline $\mathrm{KOH}_{\mathrm{R} 2=\mathrm{NO} 2}$ & 0.050155 & -0.02034 & 0.5444287 & 1.9739217 \\
\hline $\mathrm{NaOH}_{\mathrm{R} 2=\mathrm{OMe}}$ & 0.067566 & -0.03141 & 0.465931 & 2.0122165 \\
\hline $\mathrm{NaOH}_{\mathrm{R} 2=\mathrm{Me}}$ & 0.057511 & -0.03071 & 0.4832629 & 1.9532764 \\
\hline $\mathrm{NaOH}_{\mathrm{R} 2=\mathrm{H}}$ & 0.059048 & -0.03187 & 0.4793445 & 1.8278162 \\
\hline $\mathrm{NaOH}_{\mathrm{R} 2=\mathrm{CF} 3}$ & 0.05219 & -0.02967 & 0.492023 & 1.9052546 \\
\hline $\mathrm{NaOH}_{\mathrm{R} 2=\mathrm{NO} 2}$ & 0.053859 & -0.03132 & 0.4836936 & 1.9260226 \\
\hline $\mathrm{LiOH}_{\mathrm{R} 2=\mathrm{OMe}}$ & 0.061096 & -0.04391 & 0.4244685 & 1.9578106 \\
\hline $\mathrm{LiOH}_{\mathrm{R} 2=\mathrm{Me}}$ & 0.060799 & -0.04389 & 0.4264334 & 1.8975202 \\
\hline $\mathrm{LiOH}_{\mathrm{R} 2=\mathrm{H}}$ & 0.060305 & -0.0439 & 0.4270758 & 1.771099 \\
\hline $\mathrm{LiOH}_{\mathrm{R} 2=\mathrm{CF} 3}$ & 0.055067 & -0.0439 & 0.4323655 & 1.8518342 \\
\hline $\mathrm{LiOH}_{\mathrm{R} 2=\mathrm{NO} 2}$ & 0.053989 & -0.04421 & 0.4320836 & 1.8730488 \\
\hline $\mathrm{tBuOK}_{\mathrm{R} 2=\mathrm{OMe}}$ & 0.07028 & -0.03522 & 0.3833857 & 2.5383422 \\
\hline $\mathrm{tBuOK}_{\mathrm{R} 2=\mathrm{Me}}$ & 0.069361 & -0.03518 & 0.3846019 & 2.4767245 \\
\hline $\mathrm{tBuOK} K_{\mathrm{R} 2=\mathrm{H}}$ & 0.068483 & -0.03515 & 0.3857161 & 2.3488817 \\
\hline $\mathrm{tBuOK}_{\mathrm{R} 2=\mathrm{CF} 3}$ & 0.063407 & -0.03418 & 0.3913521 & 2.4259428 \\
\hline $\mathrm{tBuOK}_{\mathrm{R} 2=\mathrm{NO} 2}$ & 0.058782 & -0.03334 & 0.3968508 & 2.4417403 \\
\hline $\mathrm{tBuONa}_{\mathrm{R} 2=\mathrm{OMe}}$ & 0.070344 & -0.03867 & 0.3580509 & 2.4837916 \\
\hline $\mathrm{tBuONa}_{\mathrm{R} 2=\mathrm{Me}}$ & 0.060156 & -0.03951 & 0.3709969 & 2.4381843 \\
\hline $\mathrm{tBuONa}_{\mathrm{R} 2=\mathrm{H}}$ & 0.068078 & -0.0388 & 0.3612952 & 2.2992467 \\
\hline $\mathrm{tBuONa}_{\mathrm{R} 2=\mathrm{CF} 3}$ & 0.06404 & -0.03784 & 0.3660733 & 2.3785463 \\
\hline $\mathrm{tBuONa}_{\mathrm{R} 2=\mathrm{NO} 2}$ & 0.058687 & -0.03682 & 0.3722887 & 2.3959715 \\
\hline $\mathrm{tBuOLi}_{\mathrm{R} 2=\mathrm{OMe}}$ & 0.078516 & -0.05627 & 0.3122771 & 2.489069 \\
\hline $\mathrm{tBuOLi}_{\mathrm{R} 2=\mathrm{Me}}$ & 0.076952 & -0.05602 & 0.3134026 & 2.4342642 \\
\hline $\mathrm{tBuOLi}_{\mathrm{R} 2=\mathrm{H}}$ & 0.071345 & -0.04819 & 0.3329538 & 2.2716631 \\
\hline $\mathrm{tBuOLi}_{\mathrm{R} 2=\mathrm{CF} 3}$ & 0.053959 & -0.04819 & 0.3490324 & 2.3280507 \\
\hline
\end{tabular}




\begin{tabular}{|c|c|c|c|c|}
\hline $\mathrm{tBuOLi}_{\mathrm{R} 2=\mathrm{NO} 2}$ & 0.049658 & -0.04785 & 0.3546396 & 2.3494365 \\
\hline TBD_NH2 $2_{\mathrm{R} 2=\mathrm{OMe}}$ & 0.077784 & -0.04665 & 0.1791616 & 2.8034047 \\
\hline TBD_NH2 $2_{\mathrm{R} 2=\mathrm{Me}}$ & 0.075895 & -0.0462 & 0.1804864 & 2.7399346 \\
\hline TBD_NH2 $2_{\mathrm{R} 2=\mathrm{H}}$ & 0.075014 & -0.04592 & 0.1810542 & 2.6118975 \\
\hline TBD_NH2 2 R2=CF3 & 0.070302 & -0.04428 & 0.1863951 & 2.6878832 \\
\hline TBD_NH2 2 R2=NO2 & 0.064486 & -0.04315 & 0.1912051 & 2.7039038 \\
\hline TBD_Me $\mathbf{M}_{\mathrm{R} 2=\mathrm{OMe}}$ & 0.071083 & -0.04725 & 0.1898592 & 2.8179173 \\
\hline TBD_Me $\mathbf{R} 2=M e$ & 0.067811 & -0.0468 & 0.1915316 & 2.7545411 \\
\hline $\mathrm{TBD}_{\mathbf{M}} \mathrm{Me}_{\mathrm{R} 2=\mathrm{H}}$ & 0.067715 & -0.0468 & 0.1929492 & 2.6274724 \\
\hline TBD_Me $\mathrm{R}_{\mathrm{R} 2=\mathrm{CF} 3}$ & 0.060469 & -0.04617 & 0.1972466 & 2.70257 \\
\hline TBD_Me $\mathrm{R} 2=\mathrm{NO} 2$ & 0.053519 & -0.04561 & 0.201966 & 2.718247 \\
\hline $\mathrm{TBD}_{\mathrm{R} 2=\mathrm{OMe}}$ & 0.068025 & -0.04524 & 0.18673 & 2.6977909 \\
\hline $\mathrm{TBD}_{\mathrm{R} 2=\mathrm{Me}}$ & 0.067938 & -0.04535 & 0.185953 & 2.6278796 \\
\hline $\mathrm{TBD}_{\mathrm{R} 2=\mathrm{H}}$ & 0.067022 & -0.04524 & 0.1873466 & 2.4995449 \\
\hline $\mathrm{TBD}_{\mathrm{R} 2=\mathrm{CF} 3}$ & 0.060572 & -0.04468 & 0.191669 & 2.575586 \\
\hline $\mathrm{TBD}_{\mathrm{R} 2=\mathrm{NO} 2}$ & 0.053958 & -0.04408 & 0.1964316 & 2.5914653 \\
\hline TBD_CF3 $3_{\mathrm{R} 2=\mathrm{OMe}}$ & 0.091326 & -0.05335 & 0.171063 & 2.7608073 \\
\hline TBD_CF $3_{\mathrm{R} 2=\mathrm{Me}}$ & 0.088696 & -0.0521 & 0.1739418 & 2.6961111 \\
\hline TBD_CF3 $3_{\mathrm{R} 2=\mathrm{H}}$ & 0.087756 & -0.05165 & 0.1750313 & 2.5682741 \\
\hline TBD_CF3 $3_{\mathrm{R} 2=\mathrm{CF} 3}$ & 0.083061 & -0.04974 & 0.1800341 & 2.643833 \\
\hline TBD_CF3 $3_{\mathrm{R} 2=\mathrm{NO} 2}$ & 0.076902 & -0.04841 & 0.1850541 & 2.6586229 \\
\hline $\mathrm{MTBD}_{\mathrm{R} 2=\mathrm{OMe}}$ & 0.069941 & -0.05219 & 0.180242 & 2.822123 \\
\hline $\mathrm{MTBD}_{\mathrm{R} 2=\mathrm{Me}}$ & 0.066672 & -0.052 & 0.1820217 & 2.7584548 \\
\hline $\operatorname{MTBD}_{\mathrm{R} 2=\mathrm{H}}$ & 0.066012 & -0.0519 & 0.1820579 & 2.6307149 \\
\hline $\operatorname{MTBD}_{\mathrm{R} 2=\mathrm{CF} 3}$ & 0.058928 & -0.05146 & 0.1860263 & 2.7058704 \\
\hline $\mathrm{MTBD}_{\mathrm{R} 2=\mathrm{NO} 2}$ & 0.051999 & -0.05091 & 0.1903084 & 2.7227091 \\
\hline DBU_NH2 $2=$ OMe & 0.073031 & -0.05293 & 0.1272632 & 2.9925605 \\
\hline DBU_NH2 $2=M e$ & 0.07109 & -0.05229 & 0.1294879 & 2.9302288 \\
\hline DBU_NH2 $2=H$ & 0.075632 & -0.052 & 0.1319394 & 2.8010002 \\
\hline
\end{tabular}




\begin{tabular}{|c|c|c|c|c|}
\hline DBU_NH2 2 R2=CF3 & 0.069316 & -0.04981 & 0.140513 & 2.8786983 \\
\hline $\mathrm{DBU} \mathrm{NH}_{\mathrm{R} 2=\mathrm{NO} 2}$ & 0.063767 & -0.05054 & 0.1421528 & 2.8991249 \\
\hline $\mathrm{DBU}_{-} \mathrm{Me}_{\mathrm{R} 2=\mathrm{OMe}}$ & 0.077924 & -0.05803 & 0.1181564 & 3.0100624 \\
\hline DBU_Me $\mathbf{R}_{\mathrm{R} 2=\mathrm{Me}}$ & 0.075737 & -0.05669 & 0.1207907 & 2.9447524 \\
\hline $\mathrm{DBU}_{-} \mathrm{Me}_{\mathrm{R} 2=\mathrm{H}}$ & 0.074543 & -0.0562 & 0.1219328 & 2.8172719 \\
\hline $\mathrm{DBU}_{-} \mathrm{Me}_{\mathrm{R} 2=\mathrm{CF} 3}$ & 0.070379 & -0.05416 & 0.1271041 & 2.8938372 \\
\hline $\mathrm{DBU} \_\mathrm{Me}_{\mathrm{R} 2=\mathrm{NO} 2}$ & 0.063972 & -0.05204 & 0.1332919 & 2.9082918 \\
\hline $\mathrm{DBU}_{\mathrm{R} 2=\mathrm{OMe}}$ & 0.078212 & -0.06041 & 0.1207343 & 2.8846409 \\
\hline $\operatorname{DBU}_{\mathrm{R} 2=\mathrm{Me}}$ & 0.078052 & -0.06076 & 0.1202251 & 2.8173003 \\
\hline $\mathbf{D B U}_{\mathbf{R} 2=\mathrm{H}}$ & 0.076627 & -0.05925 & 0.1230642 & 2.6889774 \\
\hline $\mathrm{DBU}_{\mathrm{R} 2=\mathrm{CF} 3}$ & 0.072568 & -0.05609 & 0.1281347 & 2.7641134 \\
\hline $\mathrm{DBU}_{\mathrm{R} 2=\mathrm{NO} 2}$ & 0.067424 & -0.05283 & 0.1354942 & 2.7767327 \\
\hline DBU_CF3 $3_{\mathrm{R} 2=\mathrm{OMe}}$ & 0.0787 & -0.06616 & 0.1217403 & 2.950686 \\
\hline DBU_CF $3_{\mathrm{R} 2=\mathrm{Me}}$ & 0.076457 & -0.0629 & 0.1262189 & 2.8852264 \\
\hline DBU_CF $3_{\mathrm{R} 2=\mathrm{H}}$ & 0.075616 & -0.06209 & 0.1275605 & 2.7574474 \\
\hline DBU_CF $3_{\mathrm{R} 2=\mathrm{CF} 3}$ & 0.07149 & -0.05878 & 0.1326895 & 2.8328485 \\
\hline DBU_CF3 $3_{\mathrm{R} 2=\mathrm{NO} 2}$ & 0.06541 & -0.05563 & 0.1396373 & 2.8474163 \\
\hline
\end{tabular}

14. The values of Fisher information.

Table S13: Fisher information of A1 (unit: a.u.).

\begin{tabular}{crrrr}
\hline & \multicolumn{1}{c}{$\mathbf{C}^{\mathbf{1}}$} & \multicolumn{1}{c}{$\mathbf{H}^{\mathbf{1}}$} & \multicolumn{1}{c}{$\mathbf{O}^{\mathbf{2}}$} & \multicolumn{1}{c}{ Total } \\
\hline $\mathbf{K O H}_{\mathbf{R} 2=\mathbf{O M e}}$ & 243.789 & 3.812596 & 448.8508 & 8074.875 \\
$\mathbf{K O H}_{\mathbf{R} 2=\mathbf{M e}}$ & 243.7839 & 3.80564 & 448.8453 & 7626.257 \\
$\mathbf{K O H}_{\mathbf{R} 2=\mathbf{H}}$ & 243.7801 & 3.800131 & 448.8381 & 7375.55 \\
$\mathbf{K O H}_{\mathbf{R} 2=\mathbf{C F 3}}$ & 243.7696 & 3.776533 & 448.8168 & 9344.78 \\
$\mathbf{K O H}_{\mathbf{R} 2=\mathbf{N} 2}$ & 243.7591 & 3.75706 & 448.7999 & 8610.356 \\
$\mathbf{N a O H}_{\mathbf{R} 2=\mathbf{O M e}}$ & 243.8101 & 3.890244 & 448.715 & 6198.386 \\
$\mathbf{N a O H}_{\mathbf{R} 2=\mathbf{M e}}$ & 243.8047 & 3.884398 & 448.7079 & 5749.783 \\
\hline & & 558 & &
\end{tabular}




\begin{tabular}{|c|c|c|c|c|}
\hline $\mathrm{NaOH}_{\mathrm{R} 2=\mathrm{H}}$ & 243.8032 & 3.878968 & 448.7007 & 5499.064 \\
\hline $\mathrm{NaOH}_{\mathrm{R} 2=\mathrm{CF} 3}$ & 243.7894 & 3.851282 & 448.6595 & 7468.274 \\
\hline $\mathrm{NaOH}_{\mathrm{R} 2=\mathrm{NO} 2}$ & 243.7783 & 3.835688 & 448.6385 & 6733.864 \\
\hline $\mathrm{LiOH}_{\mathrm{R} 2=\mathrm{OMe}}$ & 243.8136 & 3.961751 & 448.5572 & 5371.958 \\
\hline $\mathrm{LiOH}_{\mathrm{R} 2=\mathrm{Me}}$ & 243.8066 & 3.951553 & 448.5464 & 4923.342 \\
\hline $\mathrm{LiOH}_{\mathrm{R} 2=\mathrm{H}}$ & 243.8057 & 3.94948 & 448.5423 & 4672.641 \\
\hline $\mathrm{LiOH}_{\mathrm{R} 2=\mathrm{CF} 3}$ & 243.7877 & 3.92315 & 448.5105 & 6641.852 \\
\hline $\mathrm{LiOH}_{\mathrm{R} 2=\mathrm{NO} 2}$ & 243.7772 & 3.91084 & 448.4937 & 5907.447 \\
\hline $\mathrm{tBuOK}_{\mathrm{R} 2=\mathrm{OMe}}$ & 243.7676 & 3.765187 & 447.4228 & 9078.773 \\
\hline $\mathrm{tBuOK}_{\mathrm{R} 2=\mathrm{Me}}$ & 243.7612 & 3.75387 & 447.4177 & 8630.277 \\
\hline $\mathrm{tBuOK}_{\mathrm{R} 2=\mathrm{H}}$ & 243.7624 & 3.751653 & 447.4145 & 8379.563 \\
\hline $\mathrm{tBuOK}_{\mathrm{R} 2=\mathrm{CF} 3}$ & 243.7422 & 3.725535 & 447.3915 & 10348.7 \\
\hline $\mathrm{tBuOK} K_{\mathrm{R} 2=\mathrm{NO} 2}$ & 243.737 & 3.713197 & 447.3821 & 9614.382 \\
\hline $\mathrm{tBuONa}_{\mathrm{R} 2=\mathrm{OMe}}$ & 243.8191 & 3.865452 & 447.2611 & 7202.401 \\
\hline $\mathrm{tBuONa}_{\mathrm{R} 2=\mathrm{Me}}$ & 243.8134 & 3.855366 & 447.2623 & 6753.796 \\
\hline $\mathrm{tBuONa}_{\mathrm{R} 2=\mathrm{H}}$ & 243.8067 & 3.846202 & 447.2593 & 6503.095 \\
\hline $\mathrm{tBuONa}_{\mathrm{R} 2=\mathrm{CF} 3}$ & 243.7849 & 3.798388 & 447.2587 & 8472.28 \\
\hline $\mathrm{tBuONa}_{\mathrm{R} 2=\mathrm{NO} 2}$ & 243.7722 & 3.774673 & 447.2452 & 7737.864 \\
\hline $\mathrm{tBuOLi}_{\mathrm{R} 2=\mathrm{OMe}}$ & 243.8239 & 3.948037 & 447.0471 & 6375.846 \\
\hline $\mathrm{tBuOLi}_{\mathrm{R} 2=\mathrm{Me}}$ & 243.8172 & 3.934904 & 447.043 & 5927.226 \\
\hline $\mathrm{tBuOLi}_{\mathrm{R} 2=\mathrm{H}}$ & 243.8124 & 3.923585 & 447.04 & 5676.534 \\
\hline $\mathrm{tBuOLi} \mathrm{i}_{\mathrm{R} 2=\mathrm{CF} 3}$ & 243.7926 & 3.882869 & 447.0344 & 7645.693 \\
\hline $\mathrm{tBuOLi}_{\mathrm{R} 2=\mathrm{NO} 2}$ & 243.7808 & 3.862689 & 447.0242 & 6911.287 \\
\hline TBD_NH2 2 R2=OMe & 243.807 & 3.95722 & 336.6874 & 7966.372 \\
\hline TBD_NH2 $2_{\mathrm{R} 2=\mathrm{Me}}$ & 243.7949 & 3.931547 & 336.6988 & 7517.784 \\
\hline TBD_NH2 2 & 243.7923 & 3.930501 & 336.7056 & 7267.082 \\
\hline TBD_NH2 $2_{\mathrm{R} 2=\mathrm{CF} 3}$ & 243.779 & 3.903531 & 336.6946 & 9236.363 \\
\hline TBD_NH2 2 R2=NO2 & 243.7662 & 3.885575 & 336.6757 & 8501.923 \\
\hline TBD_Me ${ }_{\mathrm{R} 2=\mathrm{OMe}}$ & 243.8066 & 3.957575 & 336.7664 & 7876.064 \\
\hline
\end{tabular}




\begin{tabular}{|c|c|c|c|c|}
\hline TBD_Me $\mathbf{R}_{\mathrm{R} 2=\mathrm{Me}}$ & 243.8106 & 3.969826 & 336.777 & 7427.466 \\
\hline TBD_Me $_{\mathrm{R} 2=\mathrm{H}}$ & 243.8035 & 3.956714 & 336.7615 & 7176.72 \\
\hline$T_{B D}{ }_{M e} e_{R 2=C F 3}$ & 243.7841 & 3.91701 & 336.7419 & 9145.959 \\
\hline TBD_Me ${ }_{\mathrm{R} 2=\mathrm{NO} 2}$ & 243.7732 & 3.902286 & 336.729 & 8411.524 \\
\hline $\mathrm{TBD}_{\mathrm{R} 2=\mathrm{OMe}}$ & 243.7892 & 4.005328 & 337.3209 & 7624.565 \\
\hline $\mathbf{T B D}_{\mathrm{R} 2=\mathrm{Me}}$ & 243.7921 & 4.022956 & 337.3118 & 7175.84 \\
\hline $\mathrm{TBD}_{\mathrm{R} 2=\mathrm{H}}$ & 243.7865 & 4.008584 & 337.318 & 6925.207 \\
\hline $\mathrm{TBD}_{\mathrm{R} 2=\mathrm{CF} 3}$ & 243.7913 & 4.02914 & 337.3155 & 8894.402 \\
\hline $\mathrm{TBD}_{\mathrm{R} 2=\mathrm{NO} 2}$ & 243.7814 & 4.007927 & 337.3064 & 8160.027 \\
\hline TBD_CF3 $3_{\mathrm{R} 2=\mathrm{OMe}}$ & 243.8179 & 3.998214 & 336.7417 & 9594.209 \\
\hline TBD_CF 3 $3_{\mathrm{R} 2=\mathrm{Me}}$ & 243.8268 & 4.016647 & 336.7269 & 9145.575 \\
\hline TBD_CF3 $3_{\mathrm{R} 2=\mathrm{H}}$ & 243.819 & 4.00794 & 336.734 & 8894.904 \\
\hline TBD_CF3 $3_{\mathrm{R} 2=\mathrm{CF} 3}$ & 243.7963 & 3.960908 & 336.7122 & 10864.06 \\
\hline TBD_CF3 $3_{\mathrm{R} 2=\mathrm{NO} 2}$ & 243.7807 & 3.93388 & 336.683 & 10129.62 \\
\hline $\mathrm{MTBD}_{\mathrm{R} 2=\mathrm{OMe}}$ & 243.7514 & 3.903223 & 337.0266 & 7874.827 \\
\hline $\operatorname{MTBD}_{\mathrm{R} 2=\mathrm{Me}}$ & 243.7348 & 3.874406 & 337.0367 & 7426.286 \\
\hline $\operatorname{MTBD}_{\mathrm{R} 2=\mathrm{H}}$ & 243.7548 & 3.905376 & 337.038 & 7175.472 \\
\hline $\operatorname{MTBD}_{\mathrm{R} 2=\mathrm{CF} 3}$ & 243.755 & 3.904324 & 337.0113 & 9144.646 \\
\hline $\mathrm{MTBD}_{\mathrm{R} 2=\mathrm{NO} 2}$ & 243.7358 & 3.876959 & 337.0087 & 8410.316 \\
\hline DBU_NH2 $2_{\text {R2 }=O M e}$ & 243.8071 & 3.973629 & 337.0359 & 8129.112 \\
\hline DBU_NH2 2 2=Me & 243.8026 & 3.963882 & 337.0159 & 7680.513 \\
\hline DBU_NH2 $2_{\mathrm{R} 2=\mathrm{H}}$ & 243.7977 & 3.952523 & 336.9839 & 7429.838 \\
\hline $\mathrm{DBU}{ }_{-} \mathrm{NH} 2_{\mathrm{R} 2=\mathrm{CF} 3}$ & 243.7863 & 3.931087 & 337.0257 & 9398.963 \\
\hline DBU_NH2 2 R2=NO2 & 243.7803 & 3.926349 & 336.9996 & 8664.562 \\
\hline DBU_Me $\mathbf{M e}_{\mathrm{R}=\mathrm{OMe}}$ & 243.808 & 3.962324 & 337.1224 & 8038.624 \\
\hline DBU_Me $\mathbf{R}_{\mathrm{R}=\mathrm{Me}}$ & 243.8053 & 3.965554 & 337.1064 & 7590.019 \\
\hline $\mathrm{DBU}_{-} \mathrm{Me}_{\mathrm{R} 2=\mathrm{H}}$ & 243.8015 & 3.959901 & 337.0813 & 7339.34 \\
\hline DBU_Me $\mathrm{Me}_{\mathrm{R} 2=\mathrm{CF} 3}$ & 243.7871 & 3.934332 & 337.0457 & 9308.491 \\
\hline $\mathrm{DBU}_{-} \mathrm{Me}_{\mathrm{R} 2=\mathrm{NO} 2}$ & 243.7793 & 3.923464 & 337.0222 & 8574.083 \\
\hline
\end{tabular}




\begin{tabular}{|c|c|c|c|c|}
\hline $\mathrm{DBU}_{\mathrm{R} 2=\mathrm{OMe}}$ & 243.8068 & 3.994376 & 337.2439 & 7787.872 \\
\hline $\mathbf{D B U}_{\mathbf{R} 2=\mathrm{Me}}$ & 243.8042 & 3.991159 & 337.2314 & 7339.263 \\
\hline $\operatorname{DBU}_{\mathbf{R} 2=\mathrm{H}}$ & 243.8016 & 3.982327 & 337.2035 & 7088.574 \\
\hline $\mathrm{DBU}_{\mathrm{R} 2=\mathrm{CF} 3}$ & 243.7896 & 3.952373 & 337.1737 & 9057.75 \\
\hline $\mathrm{DBU}_{\mathrm{R} 2=\mathrm{NO} 2}$ & 243.7796 & 3.935763 & 337.1304 & 8323.337 \\
\hline DBU_CF3 $3_{\mathrm{R} 2=\mathrm{OMe}}$ & 243.7585 & 3.950878 & 337.1985 & 9755.675 \\
\hline $\mathrm{DBU} \mathbf{C F}_{\mathrm{R} 2=\mathrm{Me}}$ & 243.7835 & 3.929831 & 337.067 & 9308.102 \\
\hline DBU_CF3 $3_{\mathrm{R} 2=\mathrm{H}}$ & 243.7905 & 3.955338 & 337.0133 & 9057.492 \\
\hline DBU_CF $3_{\mathrm{R} 2=\mathrm{CF} 3}$ & 243.7706 & 3.921196 & 337.0616 & 11026.6 \\
\hline $\mathrm{DBU}_{-} \mathrm{CF}_{\mathrm{R} 2=\mathrm{NO} 2}$ & 243.7626 & 3.911075 & 337.0384 & 10292.2 \\
\hline
\end{tabular}

Table S14: Fisher information of TSA1-2 (unit: a.u.).

\begin{tabular}{|c|c|c|c|c|}
\hline & $\mathbf{C}^{1}$ & $\mathbf{H}^{1}$ & $\mathbf{O}^{2}$ & Total \\
\hline $\mathrm{KOH}_{\mathrm{R} 2=\mathrm{OMe}}$ & 243.63493 & 3.255932 & 448.1228 & 8072.8698 \\
\hline $\mathrm{KOH}_{\mathrm{R} 2=\mathrm{Me}}$ & 243.5755 & 3.2371853 & 448.1399 & 7624.3615 \\
\hline $\mathrm{KOH}_{\mathrm{R} 2=\mathrm{H}}$ & 243.5759 & 3.2353524 & 448.1229 & 7373.6684 \\
\hline $\mathrm{KOH}_{\mathrm{R} 2=\mathrm{CF} 3}$ & 243.56014 & 3.2212121 & 448.0838 & 9342.9266 \\
\hline $\mathrm{KOH}_{\mathrm{R} 2=\mathrm{NO} 2}$ & 243.58965 & 3.251701 & 448.279 & 8608.6406 \\
\hline $\mathrm{NaOH}_{\mathrm{R} 2=\mathrm{OMe}}$ & 243.68946 & 3.301634 & 448.0287 & 6196.3105 \\
\hline $\mathrm{NaOH}_{\mathrm{R} 2=\mathrm{Me}}$ & 243.59627 & 3.2621213 & 448.1177 & 5747.7694 \\
\hline $\mathrm{NaOH}_{\mathrm{R} 2=\mathrm{H}}$ & 243.6149 & 3.2626027 & 448.0508 & 5497.0901 \\
\hline $\mathrm{NaOH}_{\mathrm{R} 2=\mathrm{CF} 3}$ & 243.57765 & 3.2541279 & 448.156 & 7466.3671 \\
\hline $\mathrm{NaOH}_{\mathrm{R} 2=\mathrm{NO} 2}$ & 243.57206 & 3.2367035 & 448.006 & 6732.0018 \\
\hline $\mathrm{LiOH}_{\mathrm{R} 2=\mathrm{OMe}}$ & 243.62531 & 3.3060541 & 447.6656 & 5369.4081 \\
\hline $\mathrm{LiOH}_{\mathrm{R} 2=\mathrm{Me}}$ & 243.62136 & 3.2959638 & 447.6804 & 4920.8644 \\
\hline $\mathrm{LiOH}_{\mathrm{R} 2=\mathrm{H}}$ & 243.61977 & 3.2932916 & 447.6806 & 4670.1698 \\
\hline $\mathrm{LiOH}_{\mathrm{R} 2=\mathrm{CF} 3}$ & 243.60546 & 3.2770532 & 447.6787 & 6639.3825 \\
\hline $\mathrm{LiOH}_{\mathrm{R} 2=\mathrm{NO} 2}$ & 243.59649 & 3.2654032 & 447.6542 & 5905.0654 \\
\hline & & & & \\
\hline
\end{tabular}




\begin{tabular}{|c|c|c|c|c|}
\hline $\mathrm{tBuOK}_{\mathrm{R} 2=\mathrm{OMe}}$ & 243.60756 & 3.1569224 & 446.6303 & 9075.9927 \\
\hline $\mathrm{tBuOK}_{\mathrm{R} 2=\mathrm{Me}}$ & 243.59727 & 3.1543174 & 446.6318 & 8627.3897 \\
\hline $\mathrm{tBuOK}_{\mathrm{R} 2=\mathrm{H}}$ & 243.59184 & 3.1508945 & 446.6435 & 8376.6918 \\
\hline $\mathrm{tBuOK}_{\mathrm{R} 2=\mathrm{CF} 3}$ & 243.56881 & 3.1400424 & 446.6814 & 10346.018 \\
\hline $\mathrm{tBuOK}_{\mathrm{R} 2=\mathrm{NO} 2}$ & 243.54637 & 3.1307662 & 446.7345 & 9611.6776 \\
\hline $\mathrm{tBuONa}_{\mathrm{R} 2=\mathrm{OMe}}$ & 243.60848 & 3.1929167 & 446.5946 & 7199.5501 \\
\hline $\mathrm{tBuONa}_{\mathrm{R} 2=\mathrm{Me}}$ & 243.51716 & 3.1525799 & 446.4617 & 6750.6807 \\
\hline $\mathrm{tBuONa}_{\mathrm{R} 2=\mathrm{H}}$ & 243.59477 & 3.1797716 & 446.5795 & 6500.1921 \\
\hline $\mathrm{tBuONa}_{\mathrm{R} 2=\mathrm{CF} 3}$ & 243.57328 & 3.1654915 & 446.5867 & 8469.5123 \\
\hline $\mathrm{tBuONa}_{\mathrm{R} 2=\mathrm{NO} 2}$ & 243.5526 & 3.1494874 & 446.6363 & 7735.1638 \\
\hline $\mathrm{tBuOLi}_{\mathrm{R} 2=\mathrm{OMe}}$ & 243.7787 & 3.3807577 & 446.1164 & 6372.5535 \\
\hline $\mathrm{tBuOLi}_{\mathrm{R} 2=\mathrm{Me}}$ & 243.7713 & 3.3693837 & 446.1035 & 5923.9354 \\
\hline $\mathrm{tBuOLi}_{\mathrm{R} 2=\mathrm{H}}$ & 243.64145 & 3.2436526 & 446.3097 & 5673.4183 \\
\hline $\mathrm{tBuOLi} \mathbf{i}_{\mathrm{R} 2=\mathrm{CF} 3}$ & 243.57688 & 3.1737159 & 446.4136 & 7642.4609 \\
\hline $\mathrm{tBuOLi}_{\mathrm{R} 2=\mathrm{NO} 2}$ & 243.54681 & 3.1468066 & 446.4465 & 6908.1685 \\
\hline TBD_NH2 2 R2=OMe & 243.44398 & 3.113146 & 334.9876 & 7960.7547 \\
\hline TBD_NH2 2 R $=\mathrm{Me}$ & 243.43945 & 3.0976345 & 335.0109 & 7512.1594 \\
\hline TBD_NH2 2 R2=H & 243.43825 & 3.0918182 & 335.02 & 7261.4633 \\
\hline TBD_NH2 $2_{\mathrm{R} 2=\mathrm{CF} 3}$ & 243.43327 & 3.0509746 & 335.1143 & 9230.9013 \\
\hline TBD_NH2 $2_{\mathrm{R} 2=\mathrm{NO} 2}$ & 243.44027 & 3.0224345 & 335.1943 & 8496.602 \\
\hline TBD_Me $\mathrm{M} 2=\mathrm{OMe}$ & 243.5936 & 3.0206133 & 335.1752 & 7869.8307 \\
\hline TBD_Me $2=M e$ & 243.58069 & 2.9986001 & 335.2247 & 7421.2959 \\
\hline$T_{B D} M_{R 2}=H$ & 243.58039 & 2.9946809 & 335.2322 & 7170.6115 \\
\hline TBD_Me $\mathrm{R}_{\mathrm{R} 2=\mathrm{CF} 3}$ & 243.5545 & 2.9615696 & 335.3101 & 9139.997 \\
\hline TBD_Me $\mathrm{Me}_{\mathrm{R} 2=\mathrm{NO} 2}$ & 243.53139 & 2.9322786 & 335.399 & 8405.6786 \\
\hline $\mathrm{TBD}_{\mathrm{R} 2=\mathrm{OMe}}$ & 243.62778 & 3.0877339 & 335.2403 & 7620.2483 \\
\hline $\mathrm{TBD}_{\mathrm{R} 2=\mathrm{Me}}$ & 243.62113 & 3.0913416 & 335.2266 & 7170.9868 \\
\hline $\mathrm{TBD}_{\mathrm{R} 2=\mathrm{H}}$ & 243.63372 & 3.0844269 & 335.2533 & 6920.2682 \\
\hline $\mathrm{TBD}_{\mathrm{R} 2=\mathrm{CF} 3}$ & 243.59807 & 3.0485927 & 335.3251 & 8889.6731 \\
\hline
\end{tabular}




\begin{tabular}{|c|c|c|c|c|}
\hline $\mathrm{TBD}_{\mathrm{R} 2=\mathrm{NO} 2}$ & 243.58145 & 3.0199083 & 335.4154 & 8155.3627 \\
\hline TBD_CF3 $3_{\mathrm{R} 2=\mathrm{OMe}}$ & 243.64379 & 3.3173307 & 334.7379 & 9588.1507 \\
\hline TBD_CF3 $3_{\mathrm{R} 2=\mathrm{Me}}$ & 243.6383 & 3.2681953 & 334.7948 & 9139.5779 \\
\hline TBD_CF3 $3_{\mathrm{R} 2=\mathrm{H}}$ & 243.63543 & 3.2505837 & 334.8149 & 8888.8718 \\
\hline TBD_CF3 ${ }_{\mathrm{R} 2=\mathrm{CF} 3}$ & 243.62868 & 3.1777524 & 334.9026 & 10858.257 \\
\hline TBD_CF3 $3_{\mathrm{R} 2=\mathrm{NO} 2}$ & 243.61501 & 3.1155801 & 334.9964 & 10123.895 \\
\hline $\mathrm{MTBD}_{\mathrm{R} 2=\mathrm{OMe}}$ & 243.61763 & 3.0536115 & 335.0462 & 7870.0675 \\
\hline $\operatorname{MTBD}_{\mathrm{R} 2=\mathrm{Me}}$ & 243.60923 & 3.0263617 & 335.0834 & 7421.459 \\
\hline $\operatorname{MTBD}_{\mathrm{R} 2=\mathrm{H}}$ & 243.6135 & 3.0252431 & 335.0904 & 7170.7826 \\
\hline $\mathrm{MTBD}_{\mathrm{R} 2=\mathrm{CF} 3}$ & 243.58493 & 2.9820862 & 335.1687 & 9140.1521 \\
\hline $\mathrm{MTBD}_{\mathrm{R} 2=\mathrm{NO} 2}$ & 243.55278 & 2.9446991 & 335.2554 & 8405.8813 \\
\hline $\mathrm{DBU}{ }_{-} \mathrm{NH} 2_{\mathrm{R} 2=\mathrm{OMe}}$ & 243.47433 & 3.0648351 & 334.8219 & 8123.3015 \\
\hline DBU_NH2 $2=M e$ & 243.46278 & 3.0429399 & 334.8662 & 7674.7826 \\
\hline $\mathrm{DBU} \mathbf{N H}_{\mathbf{R} 2=\mathrm{H}}$ & 243.49001 & 3.0990727 & 334.8218 & 7424.2448 \\
\hline DBU_NH2 2 2=CF3 & 243.49929 & 3.0516427 & 334.9499 & 9393.6927 \\
\hline DBU_NH2 2 R2=NO2 & 243.49094 & 3.0453364 & 334.9833 & 8659.1357 \\
\hline DBU_Me $\mathbf{R}_{\mathrm{R}=\mathrm{OMe}}$ & 243.52309 & 3.2052161 & 334.7072 & 8032.8441 \\
\hline DBU_Me $\mathbf{M}_{\mathrm{R} 2=\mathrm{Me}}$ & 243.5025 & 3.1635883 & 334.7658 & 7584.254 \\
\hline $\mathrm{DBU}_{-} \mathrm{Me}_{\mathrm{R} 2=\mathrm{H}}$ & 243.50472 & 3.1457109 & 334.7956 & 7333.5717 \\
\hline $\mathrm{DBU}_{-} \mathrm{Me}_{\mathrm{R} 2=\mathrm{CF} 3}$ & 243.50218 & 3.1116382 & 334.8679 & 9303.0016 \\
\hline $\mathrm{DBU}_{-} \mathrm{Me}_{\mathrm{R} 2=\mathrm{NO} 2}$ & 243.49409 & 3.0552949 & 334.9853 & 8568.6597 \\
\hline $\mathrm{DBU}_{\mathrm{R} 2=\mathrm{OMe}}$ & 243.57465 & 3.2898594 & 334.7821 & 7782.7748 \\
\hline $\mathrm{DBU}_{\mathrm{R} 2=\mathrm{Me}}$ & 243.57293 & 3.2927322 & 334.7761 & 7333.4699 \\
\hline $\mathbf{D B U}_{\mathbf{R} 2=\mathrm{H}}$ & 243.5732 & 3.2495954 & 334.8365 & 7082.8 \\
\hline $\mathrm{DBU}_{\mathrm{R} 2=\mathrm{CF} 3}$ & 243.54987 & 3.1798187 & 334.9319 & 9052.2282 \\
\hline $\mathrm{DBU}_{\mathrm{R} 2=\mathrm{NO} 2}$ & 243.54455 & 3.1087038 & 335.0642 & 8317.8504 \\
\hline DBU_CF3 $3_{\mathrm{R} 2=\mathrm{OMe}}$ & 243.56257 & 3.3609162 & 334.5262 & 9750.521 \\
\hline DBU_CF $3_{\mathrm{R} 2=\mathrm{Me}}$ & 243.54688 & 3.2786244 & 334.6255 & 9302.0303 \\
\hline DBU_CF3 $3_{\mathrm{R} 2=\mathrm{H}}$ & 243.54753 & 3.2602168 & 334.6527 & 9051.3642 \\
\hline
\end{tabular}




\begin{tabular}{|c|c|c|c|c|}
\hline DBU_CF3 $3_{\mathrm{R} 2=\mathrm{CF} 3}$ & 243.51159 & 3.1864339 & 334.7465 & 11020.776 \\
\hline DBU_CF3 $3_{\mathrm{R} 2=\mathrm{NO} 2}$ & 243.49728 & 3.1075578 & 334.8792 & 10286.47 \\
\hline
\end{tabular}

15. The values of Shannon entropy.

Table S15: Shannon entropy of A1 (unit: a.u.).

\begin{tabular}{|c|c|c|c|c|}
\hline & $\mathbf{C}^{1}$ & $\mathbf{H}^{1}$ & $\mathbf{O}^{2}$ & Total \\
\hline $\mathrm{KOH}_{\mathrm{R} 2=\mathrm{OMe}}$ & 4.12673072 & 2.7230252 & 3.4462856 & 110.5116614 \\
\hline $\mathrm{KOH}_{\mathrm{R} 2=\mathrm{Me}}$ & 4.12533469 & 2.722676 & 3.4428001 & 109.0919838 \\
\hline $\mathrm{KOH}_{\mathrm{R} 2=\mathrm{H}}$ & 4.12455377 & 2.7218755 & 3.4387416 & 98.97429175 \\
\hline $\mathrm{KOH}_{\mathrm{R} 2=\mathrm{CF} 3}$ & 4.12267201 & 2.7224042 & 3.4267887 & 98.45080282 \\
\hline $\mathrm{KOH}_{\mathrm{R} 2=\mathrm{NO} 2}$ & 4.1210757 & 2.7219924 & 3.4163512 & 102.9918689 \\
\hline $\mathrm{NaOH}_{\mathrm{R} 2=\mathrm{OMe}}$ & 4.10799981 & 2.7025562 & 3.3925909 & 122.7970923 \\
\hline $\mathrm{NaOH}_{\mathrm{R} 2=\mathrm{Me}}$ & 4.10695673 & 2.7023564 & 3.3885763 & 121.3844104 \\
\hline $\mathrm{NaOH}_{\mathrm{R} 2=\mathrm{H}}$ & 4.10628012 & 2.7019649 & 3.3849636 & 111.2642764 \\
\hline $\mathrm{NaOH}_{\mathrm{R} 2=\mathrm{CF} 3}$ & 4.10479098 & 2.702169 & 3.3653591 & 110.7375947 \\
\hline $\mathrm{NaOH}_{\mathrm{R} 2=\mathrm{NO} 2}$ & 4.10265781 & 2.7014581 & 3.3568457 & 115.2827521 \\
\hline $\mathrm{LiOH}_{\mathrm{R} 2=} \mathrm{OMe}$ & 4.08288426 & 2.6658204 & 3.3684489 & 131.3393908 \\
\hline $\mathrm{LiOH}_{\mathrm{R} 2=\mathrm{Me}}$ & 4.08138634 & 2.6662536 & 3.3624446 & 129.9184825 \\
\hline $\mathrm{LiOH}_{\mathrm{R} 2=\mathrm{H}}$ & 4.08081462 & 2.6658628 & 3.3598656 & 119.8027424 \\
\hline $\mathrm{LiOH}_{\mathrm{R} 2=\mathrm{CF} 3}$ & 4.0775592 & 2.6667508 & 3.3452167 & 119.2860605 \\
\hline $\mathrm{LiOH}_{\mathrm{R} 2=\mathrm{NO} 2}$ & 4.07517073 & 2.6666393 & 3.3381326 & 123.8344946 \\
\hline $\mathrm{tBuOK}_{\mathrm{R} 2=\mathrm{OMe}}$ & 4.11135365 & 2.682306 & 2.8146756 & 151.2290267 \\
\hline $\mathrm{tBuOK}_{\mathrm{R} 2=\mathrm{Me}}$ & 4.11287974 & 2.6846144 & 2.8026656 & 149.8438561 \\
\hline $\mathrm{tBuOK}_{\mathrm{R} 2=\mathrm{H}}$ & 4.11265138 & 2.6855179 & 2.804107 & 139.7261557 \\
\hline $\mathrm{tBuOK}_{\mathrm{R} 2=\mathrm{CF} 3}$ & 4.10564395 & 2.6755259 & 2.7934416 & 139.1718184 \\
\hline $\mathrm{tBuOK}_{\mathrm{R} 2=\mathrm{NO} 2}$ & 4.1069109 & 2.6808288 & 2.7832815 & 143.7443646 \\
\hline $\mathrm{tBuONa}_{\mathrm{R2}=\mathrm{OMe}}$ & 4.10660117 & 2.6890656 & 2.7863429 & 163.566173 \\
\hline $\mathrm{tBuONa}_{\mathrm{R} 2=\mathrm{Me}}$ & 4.10533533 & 2.6890421 & 2.7849014 & 162.1485971 \\
\hline
\end{tabular}




\begin{tabular}{|c|c|c|c|c|}
\hline $\mathrm{tBuONa}_{\mathrm{R} 2=\mathrm{H}}$ & 4.10393405 & 2.6874676 & 2.7837814 & 152.031063 \\
\hline $\mathrm{tBuONa}_{\mathrm{R} 2=\mathrm{CF} 3}$ & 4.10086184 & 2.6810108 & 2.7816346 & 151.4983371 \\
\hline $\mathrm{tBuONa}_{\mathrm{R} 2=\mathrm{NO} 2}$ & 4.09885678 & 2.6789849 & 2.773336 & 156.0406526 \\
\hline $\mathrm{tBuOLi}_{\mathrm{R} 2=\mathrm{OMe}}$ & 4.08576258 & 2.6649289 & 2.7843436 & 172.0856219 \\
\hline $\mathrm{tBuOLi}_{\mathrm{R} 2=\mathrm{Me}}$ & 4.08469463 & 2.6659767 & 2.7818235 & 170.6616307 \\
\hline $\mathrm{tBuOLi}_{\mathrm{R} 2=\mathrm{H}}$ & 4.08402043 & 2.6659529 & 2.7796703 & 160.5470562 \\
\hline $\mathrm{tBuOLi} \mathrm{i}_{\mathrm{R} 2=\mathrm{CF} 3}$ & 4.0811226 & 2.6623853 & 2.7743775 & 160.0152835 \\
\hline $\mathrm{tBuOLi}_{\mathrm{R} 2=\mathrm{NO} 2}$ & 4.07882043 & 2.661574 & 2.7681669 & 164.5622692 \\
\hline TBD_NH2 $2_{\mathrm{R} 2=\mathrm{OMe}}$ & 4.12477605 & 2.7420114 & 3.8432682 & 204.821561 \\
\hline TBD_NH2 $2_{\mathrm{R} 2=\mathrm{Me}}$ & 4.12225947 & 2.7402869 & 3.8501349 & 203.4066017 \\
\hline TBD_NH2 $2_{\mathrm{R} 2=\mathrm{H}}$ & 4.12110886 & 2.7408417 & 3.8518116 & 193.2886291 \\
\hline TBD_NH2 2 2=CF3 & 4.11705987 & 2.7361525 & 3.8504382 & 192.7712594 \\
\hline TBD_NH2 2 2 $=\mathrm{NO} 2$ & 4.11377309 & 2.7333816 & 3.8437389 & 197.3124642 \\
\hline TBD_Me $\mathbf{R}_{\mathrm{R}=\mathrm{OMe}}$ & 4.12396461 & 2.7406351 & 3.8831974 & 209.0759929 \\
\hline$T_{B D \_M e_{R 2}=M e}$ & 4.12247723 & 2.7413612 & 3.8887101 & 207.6687555 \\
\hline TBD_Me $e_{\mathrm{R} 2=\mathrm{H}}$ & 4.12164813 & 2.7405 & 3.8843025 & 197.5448805 \\
\hline TBD_Me $e_{\mathrm{R} 2=\mathrm{CF} 3}$ & 4.1176388 & 2.7350285 & 3.8785123 & 197.0179818 \\
\hline TBD_Me $e_{\mathrm{R} 2=\mathrm{NO} 2}$ & 4.1141942 & 2.7327169 & 3.8760837 & 201.5607607 \\
\hline $\mathrm{TBD}_{\mathrm{R} 2=\mathrm{OMe}}$ & 4.12673041 & 2.7203183 & 4.1417141 & 198.6351028 \\
\hline $\mathrm{TBD}_{\mathrm{R} 2=\mathrm{Me}}$ & 4.12526426 & 2.7160131 & 4.1388004 & 197.2001522 \\
\hline $\mathrm{TBD}_{\mathrm{R} 2=\mathrm{H}}$ & 4.12433092 & 2.717882 & 4.142798 & 187.0937437 \\
\hline $\mathrm{TBD}_{\mathrm{R} 2=\mathrm{CF} 3}$ & 4.12179624 & 2.7154304 & 4.1400827 & 186.5743912 \\
\hline $\mathrm{TBD}_{\mathrm{R} 2=\mathrm{NO} 2}$ & 4.11933683 & 2.7154184 & 4.1351142 & 191.1383856 \\
\hline TBD_CF3 $3_{\mathrm{R} 2=\mathrm{OMe}}$ & 4.12336394 & 2.7569633 & 3.8954095 & 198.3691457 \\
\hline TBD_CF3 $3_{\mathrm{R} 2=\mathrm{Me}}$ & 4.12245281 & 2.7565995 & 3.889343 & 196.9487431 \\
\hline TBD_CF3 $3_{\mathrm{R} 2=\mathrm{H}}$ & 4.12017233 & 2.7559162 & 3.8935399 & 186.8386329 \\
\hline TBD_CF3 $3_{\mathrm{R} 2=\mathrm{CF} 3}$ & 4.11708148 & 2.7518328 & 3.8881851 & 186.3022853 \\
\hline TBD_CF3 $3_{\mathrm{R} 2=\mathrm{NO} 2}$ & 4.11361981 & 2.7470412 & 3.8782021 & 190.8397749 \\
\hline $\mathrm{MTBD}_{\mathrm{R} 2=\mathrm{OMe}}$ & 4.11779774 & 2.6965173 & 4.0080369 & 208.6899529 \\
\hline
\end{tabular}




\begin{tabular}{|c|c|c|c|c|}
\hline $\operatorname{MTBD}_{\mathrm{R} 2=\mathrm{Me}}$ & 4.11497708 & 2.6999225 & 4.0058857 & 207.2845322 \\
\hline $\operatorname{MTBD}_{\mathrm{R} 2=\mathrm{H}}$ & 4.11553088 & 2.6913394 & 4.0132415 & 197.1474485 \\
\hline $\operatorname{MTBD}_{\mathrm{R} 2=\mathrm{CF} 3}$ & 4.11326161 & 2.6899693 & 4.0060104 & 196.6253003 \\
\hline $\mathrm{MTBD}_{\mathrm{R} 2=\mathrm{NO} 2}$ & 4.10869977 & 2.6915228 & 3.9992893 & 201.198434 \\
\hline DBU_NH2 $2=0 M e$ & 4.13249509 & 2.7542105 & 3.8501131 & 219.2236023 \\
\hline DBU_NH2 $2_{\mathrm{R} 2=\mathrm{Me}}$ & 4.13104636 & 2.7523349 & 3.8418217 & 217.8084699 \\
\hline $\mathrm{DBU} \mathrm{NH}_{\mathbf{R} 2=\mathrm{H}}$ & 4.12990302 & 2.7513877 & 3.8312762 & 207.703048 \\
\hline DBU_NH2 $2_{22=C F 3}$ & 4.12567672 & 2.7466931 & 3.8488139 & 207.1459553 \\
\hline DBU_NH2 $2_{\mathrm{R} 2=\mathrm{NO} 2}$ & 4.1223638 & 2.7462635 & 3.8430437 & 211.7021216 \\
\hline DBU_Me $\mathbf{R}_{2=\mathrm{OMe}}$ & 4.13153282 & 2.7488389 & 3.8497961 & 223.435872 \\
\hline DBU_Me $\mathbf{M e}_{\mathbf{R} 2=\mathrm{Me}}$ & 4.12991135 & 2.7486801 & 3.8447304 & 222.0264887 \\
\hline $\mathrm{DBU}_{-} \mathrm{Me}_{\mathrm{R} 2=\mathrm{H}}$ & 4.12872478 & 2.7477361 & 3.8361008 & 211.9196914 \\
\hline DBU_Me $\mathrm{R}_{\mathrm{R} 2=\mathrm{CF} 3}$ & 4.12596997 & 2.7452816 & 3.829164 & 211.3778661 \\
\hline $\mathrm{DBU}_{-} \mathrm{Me}_{\mathrm{R} 2=\mathrm{NO} 2}$ & 4.12253598 & 2.7434455 & 3.8240747 & 215.9281918 \\
\hline $\mathbf{D B U}_{\mathbf{R} 2=\mathrm{OMe}}$ & 4.12842285 & 2.7505244 & 3.9084603 & 213.3681304 \\
\hline $\mathrm{DBU}_{\mathrm{R} 2=\mathrm{Me}}$ & 4.12695343 & 2.7501321 & 3.9056569 & 211.9553129 \\
\hline $\mathbf{D B U}_{\mathbf{R} 2=\mathrm{H}}$ & 4.12640794 & 2.7496823 & 3.8947458 & 201.8437794 \\
\hline $\mathrm{DBU}_{\mathbf{R} 2=\mathbf{C F} 3}$ & 4.12440205 & 2.7465891 & 3.8838722 & 201.3074782 \\
\hline $\mathrm{DBU}_{\mathrm{R} 2=\mathrm{NO} 2}$ & 4.12153275 & 2.7446516 & 3.8709622 & 205.8568125 \\
\hline $\mathrm{DBU}_{-} \mathrm{CF3}_{\mathrm{R} 2=\mathrm{OMe}}$ & 4.13046817 & 2.740624 & 3.918364 & 212.5257384 \\
\hline DBU_CF $3_{\mathrm{R} 2=M e}$ & 4.13209993 & 2.744254 & 3.8511765 & 211.2920824 \\
\hline DBU_CF3 $3_{\mathrm{R} 2=\mathrm{H}}$ & 4.13041624 & 2.7476577 & 3.8268244 & 201.2193609 \\
\hline $\mathrm{DBU} \mathbf{C F}_{\mathrm{R} 2=\mathrm{CF} 3}$ & 4.12635378 & 2.7424501 & 3.854303 & 200.6608361 \\
\hline DBU_CF3 $3_{\mathrm{R} 2=\mathrm{NO} 2}$ & 4.12334702 & 2.7405422 & 3.8488975 & 205.2103806 \\
\hline
\end{tabular}

Table S16: Shannon entropy of TSA1-2 (unit: a.u.).

\begin{tabular}{|c|c|c|c|c|}
\hline & $\mathbf{C}^{1}$ & $\mathbf{H}^{1}$ & $\mathbf{O}^{2}$ & Total \\
\hline $\mathrm{KOH}_{\mathrm{R} 2=\mathrm{OMe}}$ & 4.28196788 & 2.6899397 & 3.02950859 & 110.3049837 \\
\hline
\end{tabular}




\begin{tabular}{|c|c|c|c|c|}
\hline $\mathrm{KOH}_{\mathrm{R} 2=\mathrm{Me}}$ & 4.26094279 & 2.68196046 & 3.04556574 & 108.8676262 \\
\hline $\mathrm{KOH}_{\mathrm{R} 2=\mathrm{H}}$ & 4.26219326 & 2.67959736 & 3.03867555 & 98.75597104 \\
\hline $\mathrm{KOH}_{\mathrm{R} 2=\mathrm{CF} 3}$ & 4.25764189 & 2.67239606 & 3.02049344 & 98.23589888 \\
\hline $\mathrm{KOH}_{\mathrm{R} 2=\mathrm{NO} 2}$ & 4.23145934 & 2.69364612 & 3.09532458 & 102.7808213 \\
\hline $\mathrm{NaOH}_{\mathrm{R} 2=\mathrm{OMe}}$ & 4.31073859 & 2.68028379 & 2.93947781 & 122.6022167 \\
\hline $\mathrm{NaOH}_{\mathrm{R} 2=\mathrm{Me}}$ & 4.26428867 & 2.67706083 & 2.99027569 & 121.1665591 \\
\hline $\mathrm{NaOH}_{\mathrm{R} 2=\mathrm{H}}$ & 4.27291375 & 2.6735638 & 2.95946287 & 111.0472293 \\
\hline $\mathrm{NaOH}_{\mathrm{R} 2=\mathrm{CF} 3}$ & 4.24230362 & 2.67772753 & 3.01235713 & 110.5219902 \\
\hline $\mathrm{NaOH}_{\mathrm{R} 2=\mathrm{NO} 2}$ & 4.24497495 & 2.65983658 & 2.95524116 & 115.0489342 \\
\hline $\mathrm{LiOH}_{\mathrm{R} 2=\mathrm{OMe}}$ & 4.29889138 & 2.65400621 & 2.79125126 & 131.0705189 \\
\hline $\mathrm{LiOH}_{\mathrm{R} 2=\mathrm{Me}}$ & 4.29426404 & 2.65259624 & 2.79784944 & 129.6624328 \\
\hline $\mathrm{LiOH}_{\mathrm{R} 2=\mathrm{H}}$ & 4.29179977 & 2.65222615 & 2.79872349 & 119.5460589 \\
\hline $\mathrm{LiOH}_{\mathrm{R} 2=\mathrm{CF} 3}$ & 4.27247322 & 2.6502349 & 2.80726527 & 119.0027674 \\
\hline $\mathrm{LiOH}_{\mathrm{R} 2=\mathrm{NO} 2}$ & 4.26516418 & 2.64310893 & 2.80033697 & 123.5577927 \\
\hline $\mathrm{tBuOK}_{\mathrm{R} 2=\mathrm{OMe}}$ & 4.29105216 & 2.61730446 & 2.41928939 & 150.8990361 \\
\hline $\mathrm{tBuOK}_{\mathrm{R} 2=\mathrm{Me}}$ & 4.28578731 & 2.61765043 & 2.42129961 & 149.4897351 \\
\hline $\mathrm{tBuOK}_{\mathrm{R} 2=\mathrm{H}}$ & 4.28140913 & 2.61741618 & 2.42510266 & 139.3713948 \\
\hline $\mathrm{tBuOK}_{\mathrm{R} 2=\mathrm{CF} 3}$ & 4.26051778 & 2.61893736 & 2.43935643 & 138.8348085 \\
\hline $\mathrm{tBuOK}_{\mathrm{R} 2=\mathrm{NO} 2}$ & 4.24095954 & 2.61972578 & 2.45703822 & 143.3738583 \\
\hline $\mathrm{tBuONa}_{\mathrm{R} 2=\mathrm{OMe}}$ & 4.28887479 & 2.60845164 & 2.39510473 & 163.1948487 \\
\hline $\mathrm{tBuONa}_{\mathrm{R} 2=\mathrm{Me}}$ & 4.2431134 & 2.58906543 & 2.40069068 & 161.68305 \\
\hline $\mathrm{tBuONa}_{\mathrm{R} 2=\mathrm{H}}$ & 4.27829086 & 2.60431196 & 2.400041 & 151.6451966 \\
\hline $\mathrm{tBuONa}_{\mathrm{R} 2=\mathrm{CF} 3}$ & 4.2609515 & 2.60605423 & 2.4079465 & 151.1157922 \\
\hline $\mathrm{tBuONa}_{\mathrm{R} 2=\mathrm{NO} 2}$ & 4.23982769 & 2.60620557 & 2.42626324 & 155.6482105 \\
\hline $\mathrm{tBuOLi}_{\mathrm{R} 2=\mathrm{OMe}}$ & 4.36431092 & 2.57588325 & 2.22586672 & 171.6003401 \\
\hline $\mathbf{t B u O L i} \mathbf{i}_{\mathbf{R} 2=M e}$ & 4.35687729 & 2.57284509 & 2.22627744 & 170.1827819 \\
\hline $\mathrm{tBuOLi}_{\mathrm{R} 2=\mathrm{H}}$ & 4.30693602 & 2.58238824 & 2.30032401 & 160.0886395 \\
\hline $\mathrm{tBuOLi} \mathbf{i}_{\mathrm{R} 2=\mathrm{CF} 3}$ & 4.24707473 & 2.59006053 & 2.33870829 & 159.4821363 \\
\hline $\mathrm{tBuOLi}_{\mathrm{R} 2=\mathrm{NO} 2}$ & 4.22942767 & 2.58859847 & 2.35490641 & 164.0240402 \\
\hline
\end{tabular}




\begin{tabular}{|c|c|c|c|c|}
\hline TBD_NH2 $2_{\mathrm{R} 2=\mathrm{OMe}}$ & 4.28468766 & 2.54253043 & 3.3749806 & 204.1443686 \\
\hline TBD_NH2 2 R2=Me & 4.27722417 & 2.54517704 & 3.38117037 & 202.7304832 \\
\hline TBD_NH2 $2_{\mathrm{R} 2=\mathrm{H}}$ & 4.27390486 & 2.54662472 & 3.38345225 & 192.6156246 \\
\hline TBD_NH2 $2_{\mathrm{R} 2=\mathrm{CF} 3}$ & 4.25606742 & 2.5559274 & 3.40856513 & 192.0926185 \\
\hline TBD_NH2 $2_{\mathrm{R} 2=\mathrm{NO} 2}$ & 4.23849407 & 2.56490019 & 3.43033569 & 196.6316389 \\
\hline TBD_Me $\mathrm{Me}_{\mathrm{R} 2=\mathrm{OMe}}$ & 4.3121226 & 2.60110339 & 3.44906844 & 208.1549296 \\
\hline$T_{B D \_} M_{\mathrm{R} 2=M e}$ & 4.29994051 & 2.6032901 & 3.46038639 & 206.7545443 \\
\hline $\mathrm{TBD}_{\mathbf{N}} \mathrm{Me}_{\mathrm{R} 2=\mathrm{H}}$ & 4.29571443 & 2.60174711 & 3.46399397 & 196.6403444 \\
\hline TBD_Me $e_{\mathrm{R} 2=\mathrm{CF} 3}$ & 4.26976685 & 2.60582715 & 3.48360923 & 196.1108966 \\
\hline TBD_Me $\mathrm{M} 2=\mathrm{NO} 2$ & 4.24227687 & 2.60726715 & 3.50780437 & 200.6570348 \\
\hline $\mathrm{TBD}_{\mathrm{R} 2=\mathrm{OMe}}$ & 4.31768512 & 2.61067637 & 3.46904807 & 198.255851 \\
\hline $\mathrm{TBD}_{\mathrm{R} 2=\mathrm{Me}}$ & 4.31624686 & 2.60722584 & 3.4655905 & 196.7698809 \\
\hline $\mathrm{TBD}_{\mathrm{R} 2=\mathrm{H}}$ & 4.31693091 & 2.61359549 & 3.47231794 & 186.6478305 \\
\hline $\mathrm{TBD}_{\mathrm{R} 2=\mathrm{CF} 3}$ & 4.28607761 & 2.6101431 & 3.49150868 & 186.1262437 \\
\hline $\mathrm{TBD}_{\mathrm{R} 2=\mathrm{NO} 2}$ & 4.26193202 & 2.61309347 & 3.51442835 & 190.6733265 \\
\hline TBD_CF3 $3_{\mathrm{R} 2=\mathrm{OMe}}$ & 4.38947781 & 2.58880753 & 3.35072824 & 197.7646044 \\
\hline TBD_CF3 $3_{\mathrm{R} 2=\mathrm{Me}}$ & 4.37775974 & 2.59550267 & 3.3651263 & 196.3509396 \\
\hline TBD_CF3 $3_{\mathrm{R} 2=\mathrm{H}}$ & 4.37276881 & 2.59758704 & 3.37018239 & 186.2289757 \\
\hline TBD_CF3 $3_{\mathrm{R} 2=\mathrm{CF} 3}$ & 4.35109847 & 2.60779138 & 3.39368977 & 185.6935304 \\
\hline TBD_CF3 $3_{\mathrm{R} 2=\mathrm{NO} 2}$ & 4.32618979 & 2.61582529 & 3.4184022 & 190.2198142 \\
\hline $\mathrm{MTBD}_{\mathrm{R} 2=\mathrm{OMe}}$ & 4.33047659 & 2.59489993 & 3.41748223 & 208.1760722 \\
\hline $\operatorname{MTBD}_{\mathrm{R} 2=\mathrm{Me}}$ & 4.31778062 & 2.5963806 & 3.4256978 & 206.7526909 \\
\hline $\operatorname{MTBD}_{\mathrm{R} 2=\mathrm{H}}$ & 4.31631355 & 2.59834793 & 3.42726809 & 196.6478123 \\
\hline $\operatorname{MTBD}_{\mathrm{R} 2=\mathrm{CF} 3}$ & 4.28606642 & 2.59848747 & 3.44586287 & 196.1108073 \\
\hline $\mathrm{MTBD}_{\mathrm{R} 2=\mathrm{NO} 2}$ & 4.25362576 & 2.59554813 & 3.46769253 & 200.6620169 \\
\hline DBU_NH2 $2_{\mathrm{R} 2=\mathrm{OMe}}$ & 4.2854935 & 2.54964219 & 3.23292746 & 218.4695753 \\
\hline DBU_NH2 $2=M e$ & 4.27553681 & 2.5526909 & 3.24436956 & 217.0696134 \\
\hline $\mathrm{DBU} \mathbf{N H}_{\mathbf{R} 2=\mathrm{H}}$ & 4.29761393 & 2.57550457 & 3.23559231 & 207.0395794 \\
\hline $\mathrm{DBU} \mathrm{NH}_{\mathrm{R} 2=\mathrm{CF} 3}$ & 4.27940223 & 2.58936836 & 3.27279517 & 206.4984951 \\
\hline
\end{tabular}




\begin{tabular}{|c|c|c|c|c|}
\hline DBU_NH2 $2_{\mathrm{R} 2=\mathrm{NO} 2}$ & 4.25874801 & 2.57127002 & 3.28827306 & 210.9745427 \\
\hline $\mathrm{DBU}_{-} \mathrm{Me}_{\mathrm{R} 2=\mathrm{OMe}}$ & 4.32072731 & 2.5402972 & 3.21045699 & 222.7149035 \\
\hline DBU_Me $\mathbf{R}_{2=M e}$ & 4.3062502 & 2.54217584 & 3.22377645 & 221.3055783 \\
\hline $\mathrm{DBU}_{-} \mathrm{Me}_{\mathrm{R} 2=\mathrm{H}}$ & 4.30197474 & 2.54608887 & 3.2304229 & 211.1836188 \\
\hline $\mathrm{DBU}_{-} \mathrm{Me}_{\mathrm{R} 2=\mathrm{CF} 3}$ & 4.28540679 & 2.55581901 & 3.25026115 & 210.6635651 \\
\hline $\mathrm{DBU}_{-} \mathrm{Me}_{\mathrm{R} 2=\mathrm{NO} 2}$ & 4.26067898 & 2.56574584 & 3.27976678 & 215.1916517 \\
\hline $\mathrm{DBU}_{\mathrm{R} 2=\mathrm{OMe}}$ & 4.34167892 & 2.55030753 & 3.24906743 & 212.7191676 \\
\hline $\mathrm{DBU}_{\mathrm{R} 2=\mathrm{Me}}$ & 4.34114622 & 2.54839617 & 3.24707123 & 211.2232962 \\
\hline $\operatorname{DBU}_{\mathbf{R} 2=\mathrm{H}}$ & 4.33501223 & 2.55553883 & 3.26142769 & 201.10178 \\
\hline $\mathrm{DBU}_{\mathrm{R} 2=\mathrm{CF} 3}$ & 4.30961423 & 2.56462186 & 3.28468926 & 200.5847578 \\
\hline $\mathrm{DBU}_{\mathrm{R} 2=\mathrm{NO} 2}$ & 4.28956308 & 2.57962546 & 3.31792722 & 205.131352 \\
\hline DBU_CF3 $3_{\mathrm{R} 2=\mathrm{OMe}}$ & 4.34451765 & 2.51681363 & 3.19913408 & 212.0223197 \\
\hline DBU_CF $3_{\mathrm{R} 2=\mathrm{Me}}$ & 4.329336 & 2.5295782 & 3.22121777 & 210.6214918 \\
\hline DBU_CF $3_{\mathrm{R} 2=\mathrm{H}}$ & 4.32546939 & 2.53377495 & 2.97908962 & 200.5044107 \\
\hline $\mathrm{DBU} \mathrm{CF}_{\mathrm{R} 2=\mathrm{CF} 3}$ & 4.29736817 & 2.53862551 & 3.24939099 & 199.9813708 \\
\hline DBU_CF $3_{\mathrm{R} 2=\mathrm{NO} 2}$ & 4.27055258 & 2.54984361 & 3.28159462 & 204.5137451 \\
\hline
\end{tabular}

16. Optimized geometric structures and parameters of key transition states.

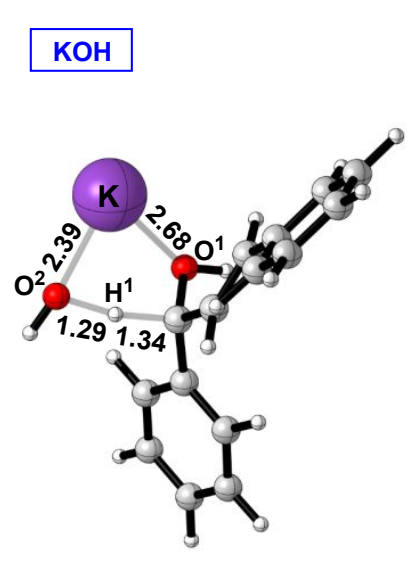

TSA1-2

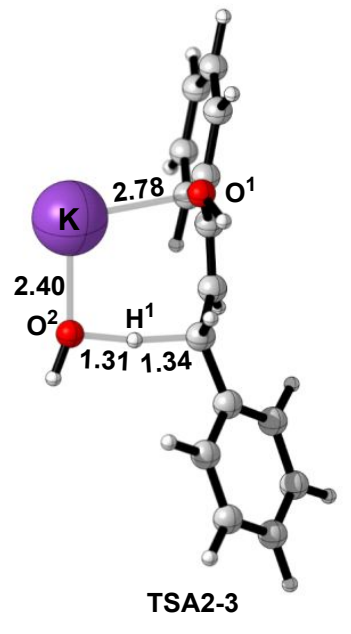



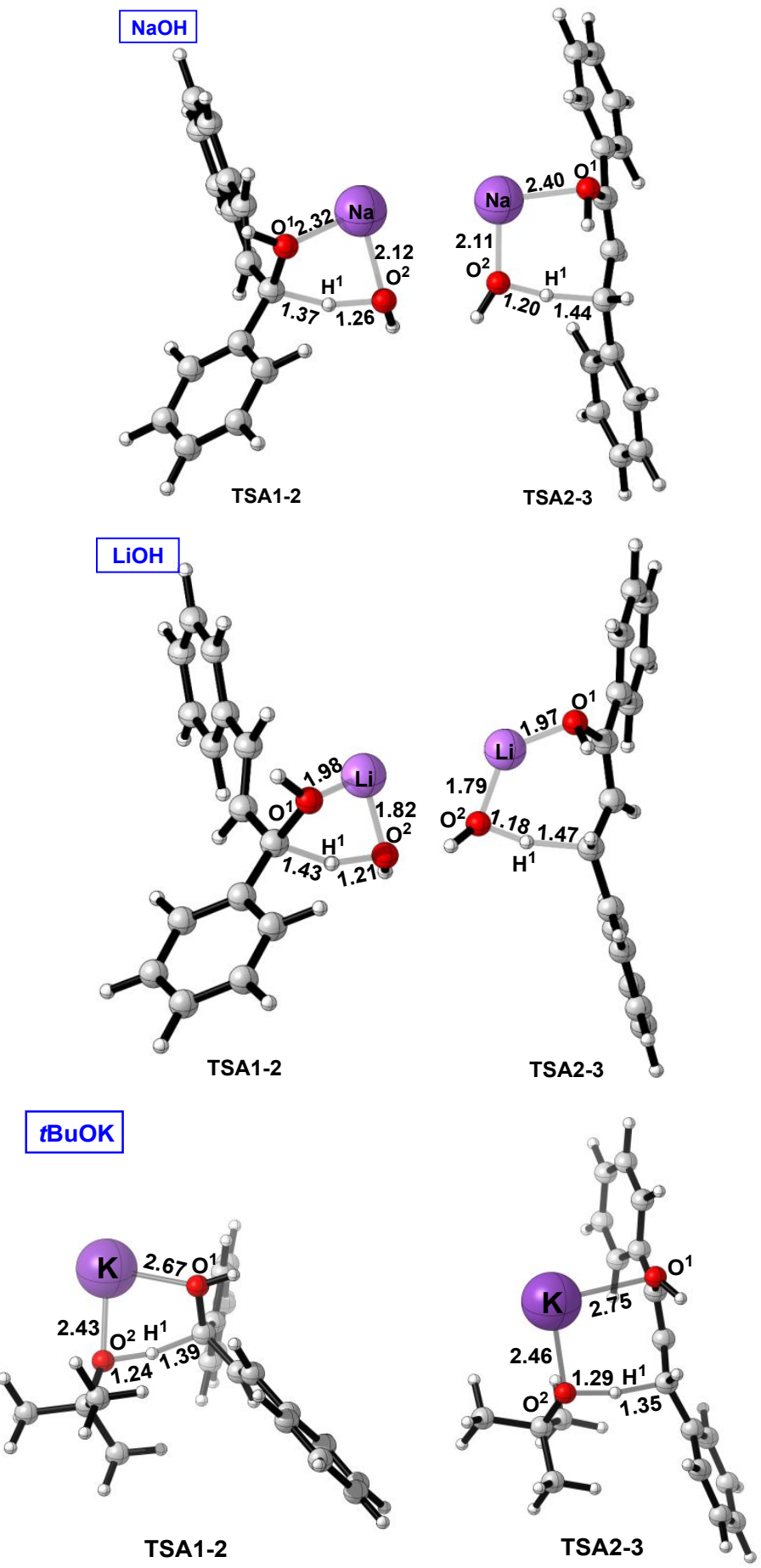

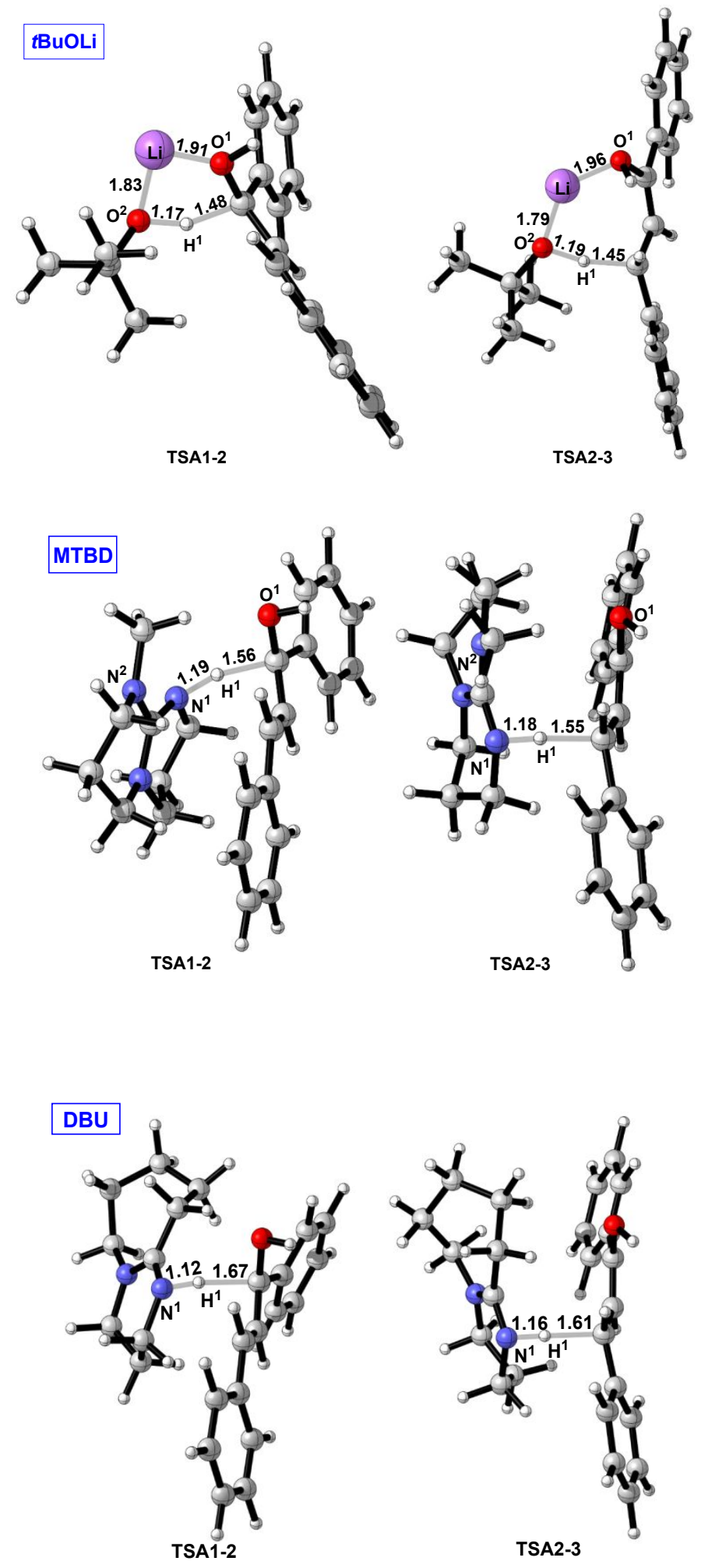
17. Effects of different metal cations on the isomerization of allylic alcohol.
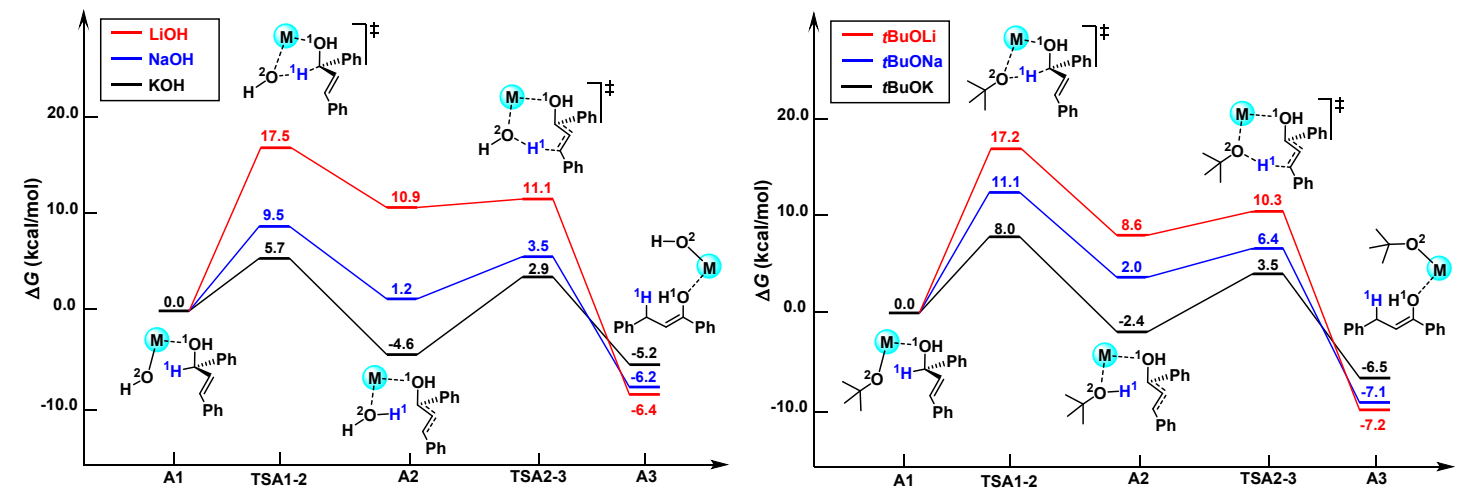

Figure S19: The isomerization of allylic alcohol catalyzed by Bases I and II.

In order to compare the effects of different cations, we calculated the isomerization patterns of allylic alcohol catalyzed by $\mathrm{KOH}, \mathrm{NaOH}, \mathrm{LiOH}$ (Base I) and $t \mathrm{BuOK}$, $t \mathrm{BuONa}, t \mathrm{BuOLi}$ (Base II) in Figure S18. The calculated results show that the isomerization patterns catalyzed by these six bases are consistent, and the efficiency order of the three cations is $\mathrm{K}^{+}>\mathrm{Na}^{+}>\mathrm{Li}^{+}$. Therefore, the reaction mechanisms of these six bases are not shown separately, but only summarized and shown in Figure 4 in the manuscript. 


\section{Entropy effect.}

According to our calculated results, the free energy of TSa2-3 is lower than that of $\mathbf{a} 2$ due to the entropy effect, but the electronic energy of TSa2-3 is higher than that of $\mathbf{a 2}$, so the transition state TSa2-3 is reasonable. The same is true for TSa4-5.

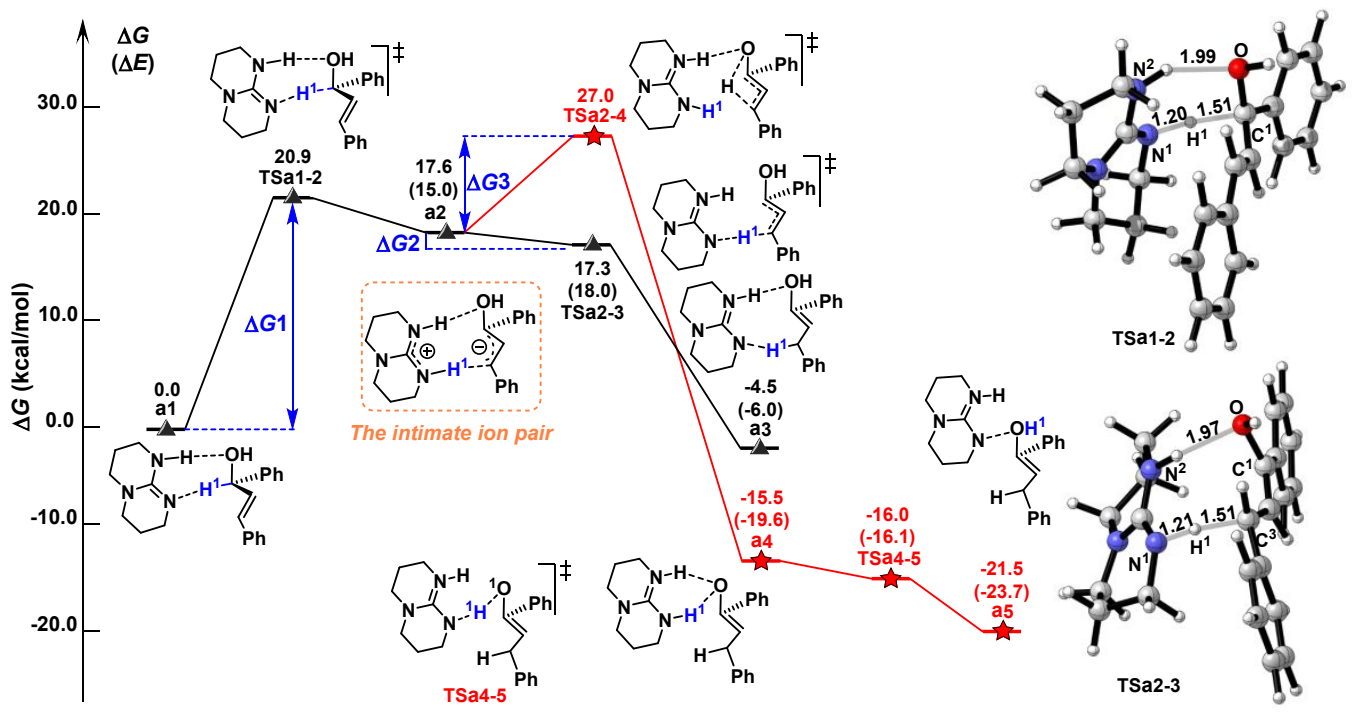

Figure S20: Free energy profiles of the isomerization of allylic alcohol to the enolate intermediate catalyzed by TBD (unit: $\mathrm{kcal} / \mathrm{mol}$ ). Key bond lengths are given in $A$.

Table S17: The relative electronic energies, thermal free energies, and entropy calculated at $298.15 \mathrm{~K}$.

\begin{tabular}{|c|c|c|c|}
\hline Complexes & $\Delta G$ (in $\mathrm{kcal} / \mathrm{mol})$ & $\Delta E($ in $\mathrm{kcal} / \mathrm{mol})$ & $\Delta S$ (in $\mathrm{kcal} / \mathrm{mol})$ \\
\hline $\mathbf{a 1}$ & 0.0 & 0.0 & 0.0 \\
\hline TSa1-2 & 20.9 & 20.2 & -0.013223687 \\
\hline $\mathbf{a 2}$ & 17.6 & 15.0 & -0.010199266 \\
\hline TSa2-3 & 17.3 & 18.0 & 0.165294143 \\
\hline $\mathbf{a 3}$ & -4.5 & -6.0 & 0.169208843 \\
\hline
\end{tabular}




\section{Related calculation results about the JACS paper in 2016.}

In order to verify the reliability of our works, we cost a lot of time to recalculate the TBD system proposed in this comment at the B3LYP-D3/aug-cc-pVDZ level in solvent instead of single point calculation as shown in Figure S21 below.

In Figure S21, we recalculated the structures mentioned by JACS paper (JACS. 2016, 138, 13408-13414) at B3LYP-D3/aug-cc-pVDZ level using Gaussian 16 program. This calculation is very time-consuming. The SMD polarizable continuum model was employed in toluene solvent. The calculated results show that the $\mathrm{C}-\mathrm{H}$ bond cleavage is the rate-determining step, and that the black path in hydrogen transfer step is favorable, which is consistent with our works at $\omega \mathrm{B} 97 \mathrm{X}-\mathrm{D} / 6-31 \mathrm{G}^{*}$ level. Therefore, we believe that the calculation in our manuscript jo-2020-02534p is reliable and convincing. Actually our works agree well with the experimental data by JACS, it is in that the $\mathrm{N}-\mathrm{H}$ and $\mathrm{N}-\mathrm{D}$ bonds of TBD moiety of ionic pair II are equivalent to each other leading to $48 \%$ of $\mathrm{D}$ was found at $\mathrm{C}_{3}$. The calculation method of free energies mentioned in the Supporting Information of the JACS paper is ambiguous, which could make the incorrect free energy reaction profiles.

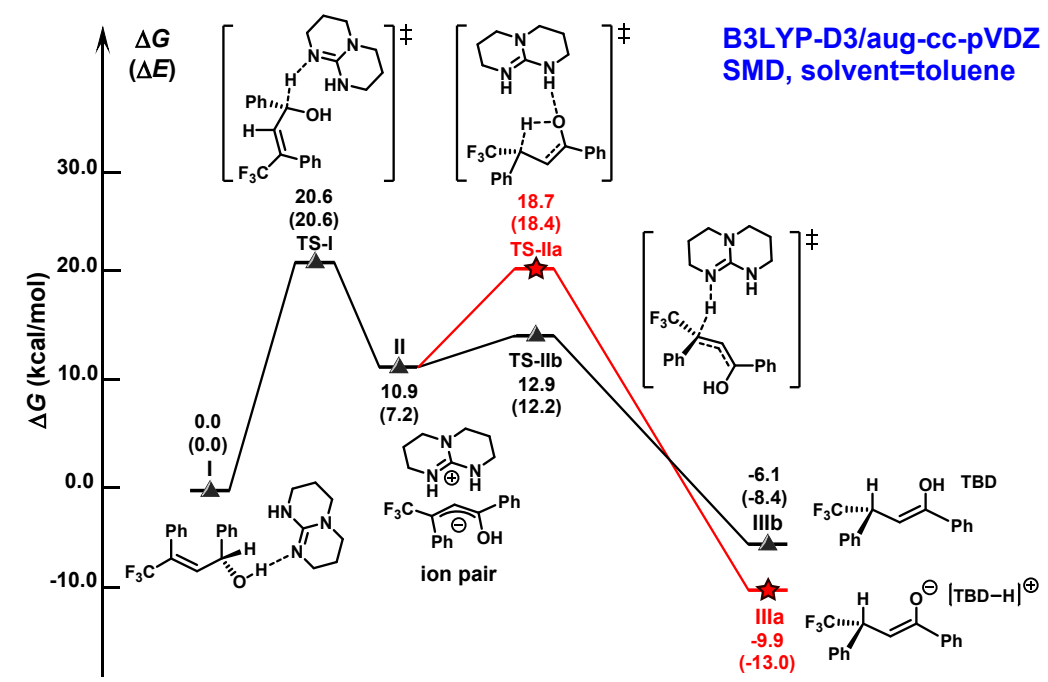


Figure S21: The calculated free energy profiles along reaction pathway of TBDcatalyzed allylic alcohol isomerization at B3LYP-D3/aug-cc-pVDZ level.

Table S18: The relative Gbbis free energies and electronic energies in solvent (in kcal/mol) at $\omega B 97 X-D / 6-31 G^{*}$ level (b), and B3LYP-D3/aug-cc-pVDZ level (a) calculated by this work and those calculated at B3LYP-D3/aug-cc-pVTZ//B3LYP-D3/6$31 G^{* *}$ level (c) by JACS paper.

\begin{tabular}{|c|c|c|c|c|c|c|}
\hline Complexes & $\Delta G_{(\mathrm{a})}$ & $\Delta E_{(\mathrm{a})}$ & $\Delta G_{(\mathrm{b})}$ & $\Delta E_{(\mathrm{b})}$ & $\Delta G_{(\mathrm{c})}$ & $\Delta E_{(\mathrm{c})}$ \\
\hline I & 0.0 & 0.0 & 0.0 & 0.0 & 0.0 & 0.0 \\
\hline TSI & 20.6 & 20.6 & 24.1 & 24.1 & 19.6 & 26.5 \\
\hline II & 10.9 & 7.2 & 18.0 & 14.6 & 13.2 & 10.7 \\
\hline TSIIa & 18.7 & 18.4 & 27.4 & 27.1 & 16.9 & 23.8 \\
\hline TSIIb & 12.9 & 12.2 & 15.2 & 16.2 & 17.1 & 16.6 \\
\hline IIIa & -9.9 & -13.0 & -8.4 & -12.1 & -6.1 & -8.0 \\
\hline IIIb & -6.1 & -8.4 & -9.4 & -11.0 & -2.3 & -7.1 \\
\hline
\end{tabular}




\section{Atomic cartesian coordinates of intermediates and transition states (presented}

in $\AA$ ).

(1) $\mathbf{R}_{1}=\mathbf{R}_{2}=H$

\begin{tabular}{|c|c|c|c|}
\hline \multicolumn{4}{|c|}{ А1 кон } \\
\hline & \multicolumn{3}{|c|}{ Coordinates (Angstroms) } \\
\hline & $\mathrm{X}$ & $\mathrm{Y}$ & Z \\
\hline$\overline{\mathrm{C}}$ & -2.118941000 & -3.125818000 & -0.818489000 \\
\hline $\mathrm{C}$ & -1.268229000 & -2.031527000 & -0.687397000 \\
\hline $\mathrm{C}$ & -1.680578000 & -0.898146000 & 0.018232000 \\
\hline $\mathrm{C}$ & -2.949410000 & -0.870420000 & 0.606500000 \\
\hline $\mathrm{C}$ & -3.794289000 & -1.970044000 & 0.475195000 \\
\hline $\mathrm{C}$ & -3.384888000 & -3.095101000 & -0.237810000 \\
\hline $\mathrm{H}$ & -1.790712000 & -4.005992000 & -1.364434000 \\
\hline $\mathrm{H}$ & -0.270864000 & -2.063548000 & -1.123066000 \\
\hline $\mathrm{H}$ & -3.225908000 & 0.036971000 & 1.162637000 \\
\hline $\mathrm{H}$ & -4.776915000 & -1.951967000 & 0.939458000 \\
\hline $\mathrm{H}$ & -4.047045000 & -3.951317000 & -0.333848000 \\
\hline $\mathrm{C}$ & -0.801718000 & 0.338467000 & 0.107253000 \\
\hline $\mathrm{C}$ & 0.638068000 & 0.014365000 & 0.376162000 \\
\hline $\mathrm{H}$ & 0.789610000 & -0.665748000 & 1.212948000 \\
\hline $\mathrm{O}$ & -0.958845000 & 1.130726000 & -1.092792000 \\
\hline $\mathrm{C}$ & 1.676727000 & 0.494412000 & -0.312652000 \\
\hline $\mathrm{H}$ & 1.468471000 & 1.172278000 & -1.139316000 \\
\hline $\mathrm{C}$ & 3.102949000 & 0.215648000 & -0.070698000 \\
\hline $\mathrm{C}$ & 4.047805000 & 0.672028000 & -0.997788000 \\
\hline $\mathrm{C}$ & 3.568202000 & -0.482909000 & 1.052135000 \\
\hline $\mathrm{C}$ & 5.406713000 & 0.434583000 & -0.820261000 \\
\hline $\mathrm{H}$ & 3.707893000 & 1.221415000 & -1.872726000 \\
\hline $\mathrm{C}$ & 4.924182000 & -0.721892000 & 1.231752000 \\
\hline $\mathrm{H}$ & 2.864927000 & -0.834157000 & 1.801208000 \\
\hline $\mathrm{C}$ & 5.851202000 & -0.265688000 & 0.296355000 \\
\hline $\mathrm{H}$ & 6.118870000 & 0.798781000 & -1.555160000 \\
\hline $\mathrm{H}$ & 5.261382000 & -1.262464000 & 2.111513000 \\
\hline $\mathrm{H}$ & 6.911262000 & -0.450778000 & 0.441772000 \\
\hline $\mathrm{H}$ & -1.222550000 & 0.981390000 & 0.908231000 \\
\hline $\mathrm{H}$ & -0.739496000 & 0.554264000 & -1.837202000 \\
\hline $\mathrm{O}$ & -2.793300000 & 1.975139000 & 1.719940000 \\
\hline $\mathrm{H}$ & -2.698239000 & 1.771341000 & 2.653891000 \\
\hline $\mathrm{K}$ & -3.033699000 & 2.713414000 & -0.438633000 \\
\hline
\end{tabular}

TSA1-2 


\begin{tabular}{|c|c|c|c|}
\hline \multicolumn{4}{|c|}{ Coordinates (Angstroms) } \\
\hline & $\mathrm{X}$ & Y & Z \\
\hline $\mathrm{C}$ & 3.495690000 & 2.638081000 & 0.158484000 \\
\hline $\mathrm{C}$ & 2.325689000 & 1.886096000 & 0.072389000 \\
\hline $\mathrm{C}$ & 2.374077000 & 0.498954000 & -0.103244000 \\
\hline $\mathrm{C}$ & 3.634178000 & -0.109748000 & -0.208117000 \\
\hline $\mathrm{C}$ & 4.799908000 & 0.641066000 & -0.141043000 \\
\hline $\mathrm{C}$ & 4.737005000 & 2.021602000 & 0.048510000 \\
\hline $\mathrm{H}$ & 3.431884000 & 3.713936000 & 0.297752000 \\
\hline $\mathrm{H}$ & 1.363853000 & 2.388321000 & 0.130165000 \\
\hline $\mathrm{H}$ & 3.679814000 & -1.188112000 & -0.332487000 \\
\hline $\mathrm{H}$ & 5.764715000 & 0.148264000 & -0.226723000 \\
\hline $\mathrm{H}$ & 5.649050000 & 2.608583000 & 0.107333000 \\
\hline $\mathrm{C}$ & 1.160049000 & -0.369924000 & -0.160421000 \\
\hline $\mathrm{C}$ & -0.147037000 & 0.234619000 & 0.082867000 \\
\hline $\mathrm{H}$ & -0.198827000 & 0.846235000 & 0.984326000 \\
\hline $\mathrm{O}$ & 1.167426000 & -1.221749000 & -1.327971000 \\
\hline $\mathrm{C}$ & -1.284498000 & -0.000726000 & -0.609958000 \\
\hline $\mathrm{H}$ & -1.207407000 & -0.537639000 & -1.557186000 \\
\hline $\mathrm{C}$ & -2.632792000 & 0.457473000 & -0.249241000 \\
\hline $\mathrm{C}$ & -3.636837000 & 0.495148000 & -1.228561000 \\
\hline $\mathrm{C}$ & -2.980189000 & 0.849299000 & 1.053535000 \\
\hline $\mathrm{C}$ & -4.924513000 & 0.925041000 & -0.929462000 \\
\hline $\mathrm{H}$ & -3.393831000 & 0.195879000 & -2.246138000 \\
\hline $\mathrm{C}$ & -4.265285000 & 1.285307000 & 1.352119000 \\
\hline $\mathrm{H}$ & -2.237844000 & 0.799849000 & 1.845389000 \\
\hline $\mathrm{C}$ & -5.246434000 & 1.327116000 & 0.363990000 \\
\hline $\mathrm{H}$ & -5.678783000 & 0.949382000 & -1.711018000 \\
\hline $\mathrm{H}$ & -4.504869000 & 1.584430000 & 2.368877000 \\
\hline $\mathrm{H}$ & -6.251430000 & 1.663259000 & 0.600831000 \\
\hline $\mathrm{H}$ & 1.324658000 & -1.275952000 & 0.814703000 \\
\hline $\mathrm{H}$ & 1.187110000 & -0.653396000 & -2.111086000 \\
\hline $\mathrm{O}$ & 1.384280000 & -2.336992000 & 1.546967000 \\
\hline $\mathrm{H}$ & 2.154459000 & -2.217661000 & 2.109221000 \\
\hline $\mathrm{K}$ & -0.313171000 & -3.012204000 & 0.008579000 \\
\hline
\end{tabular}

\begin{tabular}{|c|c|c|c|}
\hline \multicolumn{4}{|c|}{ A2 $2_{\mathrm{KOH}}$} \\
\hline & \multicolumn{3}{|c|}{ Coordinates (Angstroms) } \\
\hline & $X$ & $\mathrm{Y}$ & Z \\
\hline$\overline{\mathrm{C}}$ & -3.590709000 & -2.028001000 & 0.904074000 \\
\hline $\mathrm{C}$ & -2.361473000 & -1.586830000 & 0.442098000 \\
\hline $\mathrm{C}$ & -2.260203000 & -0.555847000 & -0.528859000 \\
\hline $\mathrm{C}$ & -3.482414000 & 0.009417000 & -0.974619000 \\
\hline
\end{tabular}




\begin{tabular}{lrrr}
\hline $\mathrm{C}$ & -4.709226000 & -0.439123000 & -0.493731000 \\
$\mathrm{C}$ & -4.783140000 & -1.459019000 & 0.449673000 \\
$\mathrm{H}$ & -3.619486000 & -2.826509000 & 1.641255000 \\
$\mathrm{H}$ & -1.460404000 & -2.047405000 & 0.836415000 \\
$\mathrm{H}$ & -3.450398000 & 0.782712000 & -1.735187000 \\
$\mathrm{H}$ & -5.621259000 & 0.013510000 & -0.876036000 \\
$\mathrm{H}$ & -5.741325000 & -1.808601000 & 0.820778000 \\
$\mathrm{C}$ & -1.000479000 & -0.011774000 & -0.966550000 \\
$\mathrm{C}$ & 0.269809000 & -0.401894000 & -0.574645000 \\
$\mathrm{H}$ & 0.326846000 & -1.354104000 & -0.051307000 \\
$\mathrm{O}$ & -1.122736000 & 1.225969000 & -1.649299000 \\
$\mathrm{C}$ & 1.459183000 & 0.301350000 & -0.758751000 \\
$\mathrm{H}$ & 1.428651000 & 1.239009000 & -1.313958000 \\
$\mathrm{C}$ & 2.792816000 & -0.171259000 & -0.416097000 \\
$\mathrm{C}$ & 3.902975000 & 0.670764000 & -0.637070000 \\
$\mathrm{C}$ & 3.063067000 & -1.427352000 & 0.165566000 \\
$\mathrm{C}$ & 5.193003000 & 0.290337000 & -0.294768000 \\
$\mathrm{H}$ & 3.736538000 & 1.645733000 & -1.091759000 \\
$\mathrm{C}$ & 4.354679000 & -1.804022000 & 0.510753000 \\
$\mathrm{H}$ & 2.252480000 & -2.129711000 & 0.340200000 \\
$\mathrm{C}$ & 5.434560000 & -0.952180000 & 0.288071000 \\
$\mathrm{H}$ & 6.018421000 & 0.971508000 & -0.486832000 \\
$\mathrm{H}$ & 4.519604000 & -2.782461000 & 0.955369000 \\
$\mathrm{H}$ & 6.443029000 & -1.252916000 & 0.555485000 \\
$\mathrm{H}$ & -0.516814000 & 1.206177000 & -2.400925000 \\
$\mathrm{O}$ & 0.821359000 & 1.833576000 & 1.688402000 \\
$\mathrm{H}$ & 1.551577000 & 1.864520000 & 2.314720000 \\
$\mathrm{~K}$ & -1.594877000 & 2.006443000 & 0.870989000 \\
$\mathrm{H}$ & 1.151541000 & 1.272371000 & 0.939306000 \\
\hline---150
\end{tabular}

TSA2-3 ${ }_{\mathrm{KOH}}$

\begin{tabular}{lccc} 
& \multicolumn{3}{c}{ Coordinates (Angstroms) } \\
$\mathrm{C}$ & $\mathrm{X}$ & $\mathrm{Y}$ & $\mathrm{Z}$ \\
$\mathrm{C}$ & 3.682107000 & -1.686477000 & -1.394855000 \\
$\mathrm{C}$ & 2.454647000 & -1.198833000 & -0.965066000 \\
$\mathrm{C}$ & 2.312122000 & -0.617019000 & 0.307556000 \\
$\mathrm{C}$ & 4.459282000 & -0.505880000 & 1.110546000 \\
$\mathrm{C}$ & 4.688178000 & -0.985885000 & 0.670395000 \\
$\mathrm{H}$ & 3.761874000 & -2.140226000 & -2.378658000 \\
$\mathrm{H}$ & 1.596323000 & -1.264243000 & -1.627918000 \\
$\mathrm{H}$ & 3.370625000 & -0.050392000 & 2.090741000 \\
$\mathrm{H}$ & 5.557439000 & -0.897919000 & 1.316326000
\end{tabular}




\begin{tabular}{cccc}
\hline H & 5.768024000 & -1.960661000 & -0.921509000 \\
$\mathrm{C}$ & 1.025421000 & -0.080962000 & 0.770428000 \\
$\mathrm{C}$ & -0.196791000 & -0.342301000 & 0.258841000 \\
$\mathrm{H}$ & -0.244097000 & -1.115097000 & -0.504623000 \\
$\mathrm{O}$ & 1.186731000 & 0.906089000 & 1.755228000 \\
$\mathrm{C}$ & -1.418952000 & 0.413895000 & 0.508412000 \\
$\mathrm{C}$ & -2.736959000 & -0.241488000 & 0.268491000 \\
$\mathrm{C}$ & -2.904300000 & -1.611168000 & 0.031122000 \\
$\mathrm{C}$ & -3.889563000 & 0.560698000 & 0.254126000 \\
$\mathrm{C}$ & -4.162537000 & -2.152794000 & -0.223271000 \\
$\mathrm{H}$ & -2.043484000 & -2.273900000 & 0.066316000 \\
$\mathrm{C}$ & -5.146891000 & 0.022433000 & 0.015913000 \\
$\mathrm{H}$ & -3.779368000 & 1.629870000 & 0.423889000 \\
$\mathrm{C}$ & -5.292285000 & -1.342109000 & -0.230754000 \\
$\mathrm{H}$ & -4.259443000 & -3.220558000 & -0.402322000 \\
$\mathrm{H}$ & -6.018761000 & 0.671706000 & 0.013465000 \\
$\mathrm{H}$ & -6.273457000 & -1.766845000 & -0.421703000 \\
$\mathrm{H}$ & 0.372869000 & 0.925746000 & 2.277119000 \\
$\mathrm{H}$ & -1.299406000 & 1.447651000 & -0.328346000 \\
$\mathrm{O}$ & -1.010785000 & 2.499286000 & -1.046871000 \\
$\mathrm{~K}$ & 1.328338000 & 2.486548000 & -0.526291000 \\
$\mathrm{H}$ & -1.813375000 & 2.684536000 & -1.543177000 \\
$\mathrm{H}$ & -1.434026000 & 0.950762000 & 1.467465000
\end{tabular}

\begin{tabular}{lccc} 
A3 $_{\text {Kон }}$ & & & \\
& & & \\
& Coordinates (Angstroms) & \\
\hline $\mathrm{C}$ & -4.652660000 & -0.624679000 & -0.618885000 \\
$\mathrm{C}$ & -3.459686000 & -1.250520000 & -0.269275000 \\
$\mathrm{C}$ & -2.340456000 & -0.501628000 & 0.119171000 \\
$\mathrm{C}$ & -2.446130000 & 0.897932000 & 0.180505000 \\
$\mathrm{C}$ & -3.644728000 & 1.510107000 & -0.169907000 \\
$\mathrm{C}$ & -4.747592000 & 0.761490000 & -0.578287000 \\
$\mathrm{H}$ & -5.509840000 & -1.225283000 & -0.909849000 \\
$\mathrm{H}$ & -3.427045000 & -2.337804000 & -0.255711000 \\
$\mathrm{H}$ & -1.597955000 & 1.519284000 & 0.527322000 \\
$\mathrm{H}$ & -3.715695000 & 2.593446000 & -0.117420000 \\
$\mathrm{H}$ & -5.676816000 & 1.254289000 & -0.849933000 \\
$\mathrm{C}$ & -1.070261000 & -1.196650000 & 0.459136000 \\
$\mathrm{C}$ & -0.105039000 & -0.696654000 & 1.242487000 \\
$\mathrm{H}$ & -0.193990000 & 0.329794000 & 1.614210000 \\
$\mathrm{O}$ & -0.918534000 & -2.466797000 & -0.056945000 \\
$\mathrm{C}$ & 1.181735000 & -1.434928000 & 1.531600000 \\
$\mathrm{H}$ & 1.046114000 & -2.511008000 & 1.390867000
\end{tabular}




$\begin{array}{lrrr}\text { C } & 2.283872000 & -0.936911000 & 0.618669000 \\ \mathrm{C} & 2.307486000 & -1.334122000 & -0.725554000 \\ \mathrm{C} & 3.225514000 & -0.000108000 & 1.052422000 \\ \mathrm{C} & 3.239150000 & -0.801319000 & -1.612756000 \\ \mathrm{H} & 1.571322000 & -2.057113000 & -1.067560000 \\ \mathrm{C} & 4.159066000 & 0.539625000 & 0.166023000 \\ \mathrm{H} & 3.210595000 & 0.329476000 & 2.087618000 \\ \mathrm{C} & 4.167054000 & 0.145149000 & -1.170706000 \\ \mathrm{H} & 3.248065000 & -1.128453000 & -2.648996000 \\ \mathrm{H} & 4.880141000 & 1.269615000 & 0.522481000 \\ \mathrm{H} & 4.898601000 & 0.557197000 & -1.859654000 \\ \mathrm{O} & -0.030509000 & 2.448966000 & 1.192395000 \\ \mathrm{H} & 1.470256000 & -1.270125000 & 2.575284000 \\ \mathrm{H} & -1.516192000 & -2.561590000 & -0.807813000 \\ \mathrm{H} & -0.447754000 & 2.852826000 & 1.957890000 \\ \mathrm{~K} & 1.093085000 & 1.687758000 & -0.662642000 \\ \text {-_--_-_- }\end{array}$

\begin{tabular}{|c|c|c|c|}
\hline \multicolumn{4}{|c|}{$\underline{\mathbf{A}} \mathbf{1}_{\mathbf{N a O H}}$} \\
\hline & $\mathrm{X}$ & $\mathrm{Y}$ & Z \\
\hline $\mathrm{C}$ & -2.509188000 & -2.777457000 & -0.742272000 \\
\hline $\mathrm{C}$ & -1.577896000 & -1.749936000 & -0.621252000 \\
\hline $\mathrm{C}$ & -1.917750000 & -0.566976000 & 0.040385000 \\
\hline $\mathrm{C}$ & -3.194040000 & -0.418256000 & 0.593236000 \\
\hline $\mathrm{C}$ & -4.119344000 & -1.452213000 & 0.471037000 \\
\hline $\mathrm{C}$ & -3.781869000 & -2.628236000 & -0.195748000 \\
\hline $\mathrm{H}$ & -2.240243000 & -3.696720000 & -1.254977000 \\
\hline $\mathrm{H}$ & -0.577220000 & -1.871878000 & -1.032542000 \\
\hline $\mathrm{H}$ & -3.419987000 & 0.527354000 & 1.104828000 \\
\hline $\mathrm{H}$ & -5.109036000 & -1.340898000 & 0.905809000 \\
\hline $\mathrm{H}$ & -4.508052000 & -3.431557000 & -0.284664000 \\
\hline $\mathrm{C}$ & -0.947751000 & 0.598142000 & 0.111902000 \\
\hline $\mathrm{C}$ & 0.468143000 & 0.174249000 & 0.370126000 \\
\hline $\mathrm{H}$ & 0.586798000 & -0.486503000 & 1.227635000 \\
\hline $\mathrm{O}$ & -1.052288000 & 1.383701000 & -1.105857000 \\
\hline $\mathrm{C}$ & 1.529262000 & 0.558288000 & -0.343398000 \\
\hline $\mathrm{H}$ & 1.374026000 & 1.265935000 & -1.156432000 \\
\hline $\mathrm{C}$ & 2.925725000 & 0.154821000 & -0.102581000 \\
\hline $\mathrm{C}$ & 3.962906000 & 0.954280000 & -0.597343000 \\
\hline $\mathrm{C}$ & 3.264792000 & -1.007496000 & 0.602254000 \\
\hline $\mathrm{C}$ & 5.294532000 & 0.622650000 & -0.373034000 \\
\hline $\mathrm{H}$ & 3.718065000 & 1.856010000 & -1.153669000 \\
\hline $\mathrm{C}$ & 4.594716000 & -1.340719000 & 0.827767000 \\
\hline
\end{tabular}




$\begin{array}{lrrr}\mathrm{H} & 2.480291000 & -1.666751000 & 0.963036000 \\ \mathrm{C} & 5.616072000 & -0.525698000 & 0.344353000 \\ \mathrm{H} & 6.082035000 & 1.263588000 & -0.758790000 \\ \mathrm{H} & 4.835588000 & -2.247299000 & 1.375449000 \\ \mathrm{H} & 6.655111000 & -0.788034000 & 0.519503000 \\ \mathrm{H} & -1.308759000 & 1.285230000 & 0.897116000 \\ \mathrm{H} & -0.916683000 & 0.775329000 & -1.844616000 \\ \mathrm{O} & -2.950312000 & 2.495902000 & 1.390812000 \\ \mathrm{H} & -3.133549000 & 2.865370000 & 2.256406000 \\ \mathrm{Na} & -2.740677000 & 2.825576000 & -0.577849000\end{array}$

TSA1-2 $\mathrm{NaOH}$

\begin{tabular}{|c|c|c|c|}
\hline \multicolumn{4}{|c|}{ Coordinates (Angstroms) } \\
\hline & $X$ & $\mathrm{Y}$ & Z \\
\hline$\overline{\mathrm{C}}$ & -3.513369000 & -2.438752000 & 0.145364000 \\
\hline $\mathrm{C}$ & -2.340566000 & -1.688560000 & 0.100997000 \\
\hline $\mathrm{C}$ & -2.379376000 & -0.301529000 & -0.086214000 \\
\hline $\mathrm{C}$ & -3.633565000 & 0.305807000 & -0.251882000 \\
\hline $\mathrm{C}$ & -4.800967000 & -0.445213000 & -0.227430000 \\
\hline $\mathrm{C}$ & -4.748850000 & -1.823401000 & -0.021450000 \\
\hline $\mathrm{H}$ & -3.455119000 & -3.513527000 & 0.294762000 \\
\hline $\mathrm{H}$ & -1.384664000 & -2.195478000 & 0.198176000 \\
\hline $\mathrm{H}$ & -3.676985000 & 1.381665000 & -0.392113000 \\
\hline $\mathrm{H}$ & -5.760182000 & 0.048214000 & -0.358696000 \\
\hline $\mathrm{H}$ & -5.663032000 & -2.409295000 & 0.004543000 \\
\hline $\mathrm{C}$ & -1.160816000 & 0.556656000 & -0.089135000 \\
\hline $\mathrm{C}$ & 0.149668000 & -0.052131000 & 0.099677000 \\
\hline $\mathrm{H}$ & 0.210934000 & -0.742050000 & 0.941685000 \\
\hline $\mathrm{O}$ & -1.163742000 & 1.509424000 & -1.180793000 \\
\hline $\mathrm{C}$ & 1.288683000 & 0.260641000 & -0.563884000 \\
\hline $\mathrm{H}$ & 1.208137000 & 0.864051000 & -1.470995000 \\
\hline $\mathrm{C}$ & 2.640717000 & -0.219701000 & -0.247854000 \\
\hline $\mathrm{C}$ & 3.648540000 & -0.132080000 & -1.219707000 \\
\hline $\mathrm{C}$ & 2.985149000 & -0.762112000 & 1.000173000 \\
\hline $\mathrm{C}$ & 4.938461000 & -0.584766000 & -0.967532000 \\
\hline $\mathrm{H}$ & 3.408248000 & 0.286453000 & -2.194934000 \\
\hline $\mathrm{C}$ & 4.272110000 & -1.221832000 & 1.250699000 \\
\hline $\mathrm{H}$ & 2.239864000 & -0.814285000 & 1.788989000 \\
\hline $\mathrm{C}$ & 5.257438000 & -1.137590000 & 0.269459000 \\
\hline $\mathrm{H}$ & 5.696193000 & -0.509511000 & -1.742379000 \\
\hline $\mathrm{H}$ & 4.509254000 & -1.641759000 & 2.224209000 \\
\hline $\mathrm{H}$ & 6.263419000 & -1.493894000 & 0.469234000 \\
\hline $\mathrm{H}$ & -1.253430000 & 1.465625000 & 0.936684000 \\
\hline $\mathrm{H}$ & -1.156068000 & 1.018823000 & -2.014934000 \\
\hline
\end{tabular}




\begin{tabular}{lrrr}
$\mathrm{O}$ & -1.149890000 & 2.543662000 & 1.586199000 \\
$\mathrm{H}$ & -1.102542000 & 2.290150000 & 2.513153000 \\
$\mathrm{Na}$ & 0.255043000 & 2.877046000 & 0.037426000 \\
\hline
\end{tabular}

\begin{tabular}{|c|c|c|c|}
\hline \multicolumn{4}{|c|}{ A2 2 aㅇ } \\
\hline & $X$ & $\mathrm{Y}$ & Z \\
\hline $\mathrm{C}$ & 3.679957000 & 2.040047000 & 0.586886000 \\
\hline $\mathrm{C}$ & 2.456415000 & 1.560477000 & 0.155797000 \\
\hline $\mathrm{C}$ & 2.342730000 & 0.309217000 & -0.506155000 \\
\hline $\mathrm{C}$ & 3.547312000 & -0.419672000 & -0.686085000 \\
\hline $\mathrm{C}$ & 4.770946000 & 0.075582000 & -0.237238000 \\
\hline $\mathrm{C}$ & 4.855570000 & 1.303821000 & 0.405204000 \\
\hline $\mathrm{H}$ & 3.721193000 & 3.008107000 & 1.079499000 \\
\hline $\mathrm{H}$ & 1.570476000 & 2.165543000 & 0.322125000 \\
\hline $\mathrm{H}$ & 3.514495000 & -1.356831000 & -1.233142000 \\
\hline $\mathrm{H}$ & 5.670897000 & -0.509447000 & -0.410818000 \\
\hline $\mathrm{H}$ & 5.809292000 & 1.689072000 & 0.751005000 \\
\hline $\mathrm{C}$ & 1.077427000 & -0.264127000 & -0.888903000 \\
\hline $\mathrm{C}$ & -0.190887000 & 0.199536000 & -0.572781000 \\
\hline $\mathrm{H}$ & -0.237310000 & 1.230009000 & -0.227750000 \\
\hline $\mathrm{O}$ & 1.189208000 & -1.626817000 & -1.289682000 \\
\hline $\mathrm{C}$ & -1.384476000 & -0.517318000 & -0.606098000 \\
\hline $\mathrm{H}$ & -1.376816000 & -1.534860000 & -0.999179000 \\
\hline $\mathrm{C}$ & -2.712102000 & 0.029634000 & -0.347956000 \\
\hline $\mathrm{C}$ & -3.828209000 & -0.830920000 & -0.362907000 \\
\hline $\mathrm{C}$ & -2.964982000 & 1.384428000 & -0.055750000 \\
\hline $\mathrm{C}$ & -5.110614000 & -0.373059000 & -0.094416000 \\
\hline $\mathrm{H}$ & -3.673034000 & -1.884493000 & -0.587661000 \\
\hline $\mathrm{C}$ & -4.248831000 & 1.840164000 & 0.216790000 \\
\hline $\mathrm{H}$ & -2.149319000 & 2.102318000 & -0.055695000 \\
\hline $\mathrm{C}$ & -5.335824000 & 0.969330000 & 0.203014000 \\
\hline $\mathrm{H}$ & -5.942245000 & -1.072804000 & -0.117470000 \\
\hline $\mathrm{H}$ & -4.402384000 & 2.894461000 & 0.433131000 \\
\hline $\mathrm{H}$ & -6.338006000 & 1.331108000 & 0.411726000 \\
\hline $\mathrm{H}$ & 0.533055000 & -1.787821000 & -1.978394000 \\
\hline $\mathrm{O}$ & -0.443304000 & -1.777348000 & 1.896836000 \\
\hline $\mathrm{H}$ & -0.811225000 & -1.375612000 & 2.691293000 \\
\hline $\mathrm{H}$ & -0.903768000 & -1.320342000 & 1.139539000 \\
\hline $\mathrm{Na}$ & 1.574625000 & -1.835266000 & 0.969831000 \\
\hline
\end{tabular}

TSA2-3 aOㅂ $_{-}$ 


\begin{tabular}{|c|c|c|c|}
\hline & $\mathrm{X}$ & Y & Z \\
\hline $\mathrm{C}$ & -3.691639000 & -2.229544000 & 0.212070000 \\
\hline $\mathrm{C}$ & -2.479697000 & -1.665458000 & -0.153659000 \\
\hline $\mathrm{C}$ & -2.382829000 & -0.294770000 & -0.458420000 \\
\hline $\mathrm{C}$ & -3.561834000 & 0.471656000 & -0.410952000 \\
\hline $\mathrm{C}$ & -4.776684000 & -0.103672000 & -0.041840000 \\
\hline $\mathrm{C}$ & -4.850048000 & -1.453028000 & 0.279184000 \\
\hline $\mathrm{H}$ & -3.737402000 & -3.291081000 & 0.437873000 \\
\hline $\mathrm{H}$ & -1.600265000 & -2.298097000 & -0.219781000 \\
\hline $\mathrm{H}$ & -3.527736000 & 1.516802000 & -0.702138000 \\
\hline $\mathrm{H}$ & -5.672436000 & 0.511031000 & -0.020808000 \\
\hline $\mathrm{H}$ & -5.796822000 & -1.903048000 & 0.560778000 \\
\hline $\mathrm{C}$ & -1.099501000 & 0.350732000 & -0.752174000 \\
\hline $\mathrm{C}$ & 0.140884000 & -0.150961000 & -0.545104000 \\
\hline $\mathrm{H}$ & 0.206206000 & -1.176050000 & -0.185274000 \\
\hline $\mathrm{O}$ & -1.255626000 & 1.703809000 & -1.085424000 \\
\hline $\mathrm{C}$ & 1.369402000 & 0.627947000 & -0.654745000 \\
\hline $\mathrm{C}$ & 2.647137000 & -0.044187000 & -0.334968000 \\
\hline $\mathrm{C}$ & 2.761197000 & -0.946124000 & 0.736106000 \\
\hline $\mathrm{C}$ & 3.819448000 & 0.249360000 & -1.049519000 \\
\hline $\mathrm{C}$ & 3.975597000 & -1.543041000 & 1.057115000 \\
\hline $\mathrm{H}$ & 1.883884000 & -1.173942000 & 1.338293000 \\
\hline $\mathrm{C}$ & 5.036871000 & -0.334656000 & -0.721272000 \\
\hline $\mathrm{H}$ & 3.767053000 & 0.947362000 & -1.882363000 \\
\hline $\mathrm{C}$ & 5.125211000 & -1.242151000 & 0.331929000 \\
\hline $\mathrm{H}$ & 4.024715000 & -2.242821000 & 1.887682000 \\
\hline $\mathrm{H}$ & 5.923305000 & -0.085455000 & -1.299103000 \\
\hline $\mathrm{H}$ & -0.369302000 & 2.078528000 & -1.201419000 \\
\hline $\mathrm{H}$ & 1.161689000 & 1.622882000 & 0.358279000 \\
\hline $\mathrm{O}$ & 0.781236000 & 2.412378000 & 1.184959000 \\
\hline $\mathrm{H}$ & 1.548384000 & 2.616391000 & 1.728180000 \\
\hline $\mathrm{H}$ & 1.439994000 & 1.202230000 & -1.588247000 \\
\hline $\mathrm{H}$ & 6.074732000 & -1.704584000 & 0.584410000 \\
\hline $\mathrm{Na}$ & -1.248361000 & 1.845250000 & 1.313169000 \\
\hline
\end{tabular}

$\mathbf{A 3}_{\mathrm{NaOH}}$

$\begin{array}{lrrr} & & & \text { Coordinates (Angstroms) } \\ \text { C } & -4.619450000 & -0.587651000 & -0.500403000 \\ \mathrm{C} & -3.405981000 & -1.183126000 & -0.171700000 \\ \mathrm{C} & -2.276166000 & -0.401877000 & 0.107628000 \\ \mathrm{C} & -2.383019000 & 0.996834000 & 0.073851000 \\ \mathrm{C} & -3.603395000 & 1.578401000 & -0.254955000\end{array}$




\begin{tabular}{|c|c|c|c|}
\hline $\mathrm{C}$ & -4.720020000 & 0.798502000 & -0.550204000 \\
\hline $\mathrm{H}$ & -5.486576000 & -1.208704000 & -0.705968000 \\
\hline $\mathrm{H}$ & -3.360175000 & -2.266890000 & -0.086358000 \\
\hline $\mathrm{H}$ & -1.515229000 & 1.637599000 & 0.315994000 \\
\hline $\mathrm{H}$ & -3.679468000 & 2.662131000 & -0.282310000 \\
\hline $\mathrm{H}$ & -5.665104000 & 1.268369000 & -0.807327000 \\
\hline $\mathrm{C}$ & -0.989992000 & -1.069126000 & 0.437605000 \\
\hline $\mathrm{C}$ & -0.034432000 & -0.553966000 & 1.224246000 \\
\hline $\mathrm{H}$ & -0.159761000 & 0.458324000 & 1.609406000 \\
\hline $\mathrm{O}$ & -0.813846000 & -2.332394000 & -0.079792000 \\
\hline $\mathrm{C}$ & 1.262548000 & -1.272612000 & 1.521966000 \\
\hline $\mathrm{H}$ & 1.135715000 & -2.351810000 & 1.397210000 \\
\hline $\mathrm{C}$ & 2.354134000 & -0.775253000 & 0.596385000 \\
\hline $\mathrm{C}$ & 2.369186000 & -1.185224000 & -0.745580000 \\
\hline $\mathrm{C}$ & 3.276822000 & 0.190570000 & 1.008346000 \\
\hline $\mathrm{C}$ & 3.269978000 & -0.630277000 & -1.651936000 \\
\hline $\mathrm{H}$ & 1.647365000 & -1.929805000 & -1.071028000 \\
\hline $\mathrm{C}$ & 4.179668000 & 0.749933000 & 0.102354000 \\
\hline $\mathrm{H}$ & 3.265816000 & 0.532036000 & 2.039479000 \\
\hline $\mathrm{C}$ & 4.175658000 & 0.347160000 & -1.231485000 \\
\hline $\mathrm{H}$ & 3.270710000 & -0.963462000 & -2.686010000 \\
\hline $\mathrm{H}$ & 4.881489000 & 1.506284000 & 0.440424000 \\
\hline $\mathrm{H}$ & 4.879306000 & 0.779587000 & -1.936286000 \\
\hline $\mathrm{O}$ & 0.120867000 & 2.564404000 & 0.743978000 \\
\hline $\mathrm{H}$ & 1.552086000 & -1.089842000 & 2.562051000 \\
\hline $\mathrm{H}$ & -1.451015000 & -2.457990000 & -0.793133000 \\
\hline $\mathrm{H}$ & -0.084133000 & 3.470124000 & 0.986267000 \\
\hline $\mathrm{Na}$ & 1.198142000 & 1.462203000 & -0.553873000 \\
\hline
\end{tabular}

\begin{tabular}{|c|c|c|c|}
\hline \multicolumn{4}{|c|}{ Coordinates (Angstroms) } \\
\hline & $\mathrm{X}$ & Y & Z \\
\hline $\mathrm{C}$ & 2.727822000 & -2.529401000 & 0.604302000 \\
\hline $\mathrm{C}$ & 1.759211000 & -1.539119000 & 0.463436000 \\
\hline $\mathrm{C}$ & 2.103715000 & -0.289384000 & -0.056451000 \\
\hline $\mathrm{C}$ & 3.425183000 & -0.032085000 & -0.437810000 \\
\hline $\mathrm{C}$ & 4.387747000 & -1.026845000 & -0.294573000 \\
\hline $\mathrm{C}$ & 4.042690000 & -2.273356000 & 0.225019000 \\
\hline $\mathrm{H}$ & 2.454958000 & -3.500807000 & 1.006411000 \\
\hline $\mathrm{H}$ & 0.728713000 & -1.740452000 & 0.747694000 \\
\hline $\mathrm{H}$ & 3.665465000 & 0.961664000 & -0.827802000 \\
\hline $\mathrm{H}$ & 5.413520000 & -0.829090000 & -0.592385000 \\
\hline
\end{tabular}




$\begin{array}{lccc}\mathrm{H} & 4.798163000 & -3.046803000 & 0.331078000 \\ \mathrm{C} & 1.087493000 & 0.832905000 & -0.144369000 \\ \mathrm{C} & -0.310857000 & 0.362970000 & -0.418860000 \\ \mathrm{H} & -0.407097000 & -0.224024000 & -1.330724000 \\ \mathrm{O} & 1.151542000 & 1.626254000 & 1.075446000 \\ \mathrm{C} & -1.374302000 & 0.622174000 & 0.344352000 \\ \mathrm{H} & -1.235223000 & 1.254041000 & 1.219838000 \\ \mathrm{C} & -2.754767000 & 0.168312000 & 0.100679000 \\ \mathrm{C} & -3.806425000 & 0.808317000 & 0.766874000 \\ \mathrm{C} & -3.065762000 & -0.886777000 & -0.767132000 \\ \mathrm{C} & -5.127219000 & 0.426982000 & 0.557643000 \\ \mathrm{H} & -3.582421000 & 1.622576000 & 1.451869000 \\ \mathrm{C} & -4.384448000 & -1.268734000 & -0.978546000 \\ \mathrm{H} & -2.268242000 & -1.424395000 & -1.272066000 \\ \mathrm{C} & -5.421832000 & -0.612229000 & -0.319369000 \\ \mathrm{H} & -5.927399000 & 0.942298000 & 1.080895000 \\ \mathrm{H} & -4.604251000 & -2.088252000 & -1.656592000 \\ \mathrm{H} & -6.451862000 & -0.913519000 & -0.484425000 \\ \mathrm{H} & 1.419801000 & 1.535535000 & -0.920155000 \\ \mathrm{H} & 1.200086000 & 1.009313000 & 1.817432000 \\ \mathrm{O} & 3.120543000 & 3.027248000 & -0.918706000 \\ \mathrm{H} & 3.552584000 & 3.614803000 & -1.538170000 \\ \mathrm{Li} & 2.445680000 & 3.005569000 & 0.614006000 \\ -------1 .-1\end{array}$

TSA1-2

\begin{tabular}{lrrr} 
& \multicolumn{3}{c}{ Coordinates (Angstroms) } \\
& $\mathrm{X}$ & $\mathrm{Y}$ & $\mathrm{Z}$ \\
$\mathrm{C}$ & -3.605868000 & -2.213341000 & 0.132531000 \\
$\mathrm{C}$ & -2.410159000 & -1.501092000 & 0.128018000 \\
$\mathrm{C}$ & -2.397214000 & -0.114283000 & -0.074239000 \\
$\mathrm{C}$ & -3.624989000 & 0.528000000 & -0.296996000 \\
$\mathrm{C}$ & -4.814999000 & -0.187768000 & -0.310946000 \\
$\mathrm{C}$ & -4.815352000 & -1.563631000 & -0.089389000 \\
$\mathrm{H}$ & -3.585994000 & -3.287861000 & 0.293213000 \\
$\mathrm{H}$ & -1.477065000 & -2.039016000 & 0.267567000 \\
$\mathrm{H}$ & -3.633355000 & 1.601996000 & -0.455200000 \\
$\mathrm{H}$ & -5.751907000 & 0.333758000 & -0.486844000 \\
$\mathrm{H}$ & -5.747160000 & -2.121259000 & -0.094972000 \\
$\mathrm{C}$ & -1.153812000 & 0.694119000 & -0.022855000 \\
$\mathrm{C}$ & 0.153125000 & 0.072986000 & 0.081913000 \\
$\mathrm{H}$ & 0.226716000 & -0.718945000 & 0.826777000 \\
$\mathrm{O}$ & -1.142821000 & 1.784409000 & -0.984850000 \\
$\mathrm{C}$ & 1.294186000 & 0.492631000 & -0.522856000 \\
$\mathrm{H}$ & 1.220249000 & 1.196668000 & -1.355908000
\end{tabular}




\begin{tabular}{lrrr}
$\mathrm{C}$ & 2.648521000 & -0.009413000 & -0.252724000 \\
$\mathrm{C}$ & 3.657054000 & 0.171927000 & -1.210273000 \\
$\mathrm{C}$ & 2.991384000 & -0.666524000 & 0.938792000 \\
$\mathrm{C}$ & 4.946757000 & -0.301565000 & -0.999515000 \\
$\mathrm{H}$ & 3.418081000 & 0.680310000 & -2.142064000 \\
$\mathrm{C}$ & 4.278301000 & -1.147315000 & 1.147079000 \\
$\mathrm{H}$ & 2.245423000 & -0.790650000 & 1.719150000 \\
$\mathrm{C}$ & 5.264490000 & -0.969804000 & 0.179544000 \\
$\mathrm{H}$ & 5.705670000 & -0.151892000 & -1.762325000 \\
$\mathrm{H}$ & 4.515047000 & -1.654505000 & 2.078173000 \\
$\mathrm{H}$ & 6.270465000 & -1.342613000 & 0.346259000 \\
$\mathrm{H}$ & -1.124616000 & 1.571286000 & 1.110553000 \\
$\mathrm{H}$ & -1.069481000 & 1.415381000 & -1.877277000 \\
$\mathrm{O}$ & -0.819643000 & 2.600007000 & 1.677823000 \\
$\mathrm{H}$ & -0.487119000 & 2.352321000 & 2.547072000 \\
$\mathrm{Li}$ & 0.168739000 & 2.723380000 & 0.157845000 \\
\hline
\end{tabular}

A2

\begin{tabular}{|c|c|c|c|}
\hline \multicolumn{4}{|c|}{ Coordinates (Angstroms) } \\
\hline & $\mathrm{X}$ & Y & Z \\
\hline $\mathrm{C}$ & 3.797163000 & 1.974690000 & 0.262635000 \\
\hline $\mathrm{C}$ & 2.573487000 & 1.448450000 & -0.107347000 \\
\hline $\mathrm{C}$ & 2.434102000 & 0.078482000 & -0.447024000 \\
\hline $\mathrm{C}$ & 3.606107000 & -0.715832000 & -0.385735000 \\
\hline $\mathrm{C}$ & 4.831236000 & -0.168767000 & -0.002959000 \\
\hline $\mathrm{C}$ & 4.942681000 & 1.173732000 & 0.328079000 \\
\hline $\mathrm{H}$ & 3.864511000 & 3.032293000 & 0.503641000 \\
\hline $\mathrm{H}$ & 1.711749000 & 2.107372000 & -0.152277000 \\
\hline $\mathrm{H}$ & 3.559692000 & -1.753751000 & -0.699623000 \\
\hline $\mathrm{H}$ & 5.708732000 & -0.809939000 & 0.019136000 \\
\hline $\mathrm{H}$ & 5.897261000 & 1.598232000 & 0.621296000 \\
\hline $\mathrm{C}$ & 1.156964000 & -0.526882000 & -0.745539000 \\
\hline $\mathrm{C}$ & -0.108309000 & 0.000842000 & -0.526738000 \\
\hline $\mathrm{H}$ & -0.136133000 & 1.076310000 & -0.368422000 \\
\hline $\mathrm{O}$ & 1.257039000 & -1.949950000 & -0.860637000 \\
\hline $\mathrm{C}$ & -1.311847000 & -0.691819000 & -0.428208000 \\
\hline $\mathrm{H}$ & -1.337544000 & -1.752237000 & -0.685550000 \\
\hline $\mathrm{C}$ & -2.630048000 & -0.083759000 & -0.256689000 \\
\hline $\mathrm{C}$ & -3.775892000 & -0.900679000 & -0.295872000 \\
\hline $\mathrm{C}$ & -2.838947000 & 1.288758000 & -0.024829000 \\
\hline $\mathrm{C}$ & -5.050843000 & -0.381847000 & -0.114463000 \\
\hline $\mathrm{H}$ & -3.653332000 & -1.967194000 & -0.473949000 \\
\hline $\mathrm{C}$ & -4.115104000 & 1.807142000 & 0.154912000 \\
\hline $\mathrm{H}$ & -1.993597000 & 1.970754000 & 0.012740000 \\
\hline
\end{tabular}




$\begin{array}{lrrr}\mathrm{C} & -5.234798000 & 0.979628000 & 0.113764000 \\ \mathrm{H} & -5.908465000 & -1.048505000 & -0.153684000 \\ \mathrm{H} & -4.235883000 & 2.873445000 & 0.327802000 \\ \mathrm{H} & -6.230295000 & 1.389291000 & 0.254539000 \\ \mathrm{H} & 0.533166000 & -2.261359000 & -1.416931000 \\ \mathrm{O} & -0.115805000 & -1.650170000 & 2.075012000 \\ \mathrm{H} & -0.280919000 & -1.101149000 & 2.850561000 \\ \mathrm{H} & -0.710796000 & -1.285337000 & 1.355708000 \\ \mathrm{Li} & 1.475426000 & -1.653363000 & 1.048697000\end{array}$

TSA2-3

\begin{tabular}{|c|c|c|c|}
\hline \multicolumn{4}{|c|}{ Coordinates (Angstroms) } \\
\hline & $\mathrm{X}$ & $\mathrm{Y}$ & $\mathrm{Z}$ \\
\hline $\mathrm{C}$ & -3.847303000 & -1.939022000 & 0.482231000 \\
\hline $\mathrm{C}$ & -2.612066000 & -1.316326000 & 0.374360000 \\
\hline $\mathrm{C}$ & -2.488942000 & -0.059014000 & -0.242528000 \\
\hline $\mathrm{C}$ & -3.656028000 & 0.557472000 & -0.719565000 \\
\hline $\mathrm{C}$ & -4.892814000 & -0.068014000 & -0.599515000 \\
\hline $\mathrm{C}$ & -4.998124000 & -1.319890000 & -0.002978000 \\
\hline $\mathrm{H}$ & -3.913929000 & -2.911463000 & 0.961453000 \\
\hline $\mathrm{H}$ & -1.734589000 & -1.806511000 & 0.785584000 \\
\hline $\mathrm{H}$ & -3.583713000 & 1.528097000 & -1.197544000 \\
\hline $\mathrm{H}$ & -5.780027000 & 0.427958000 & -0.982928000 \\
\hline $\mathrm{H}$ & -5.964075000 & -1.806362000 & 0.090326000 \\
\hline $\mathrm{C}$ & -1.190613000 & 0.613013000 & -0.360202000 \\
\hline $\mathrm{C}$ & 0.054496000 & 0.090917000 & -0.210883000 \\
\hline $\mathrm{H}$ & 0.099888000 & -0.981047000 & -0.033445000 \\
\hline $\mathrm{O}$ & -1.316794000 & 2.015952000 & -0.571478000 \\
\hline $\mathrm{C}$ & 1.301671000 & 0.823066000 & -0.187419000 \\
\hline $\mathrm{C}$ & 2.578355000 & 0.075867000 & -0.207121000 \\
\hline $\mathrm{C}$ & 2.761813000 & -1.109152000 & 0.523420000 \\
\hline $\mathrm{C}$ & 3.683739000 & 0.567870000 & -0.917368000 \\
\hline $\mathrm{C}$ & 3.979640000 & -1.779135000 & 0.521592000 \\
\hline $\mathrm{H}$ & 1.943653000 & -1.503038000 & 1.122053000 \\
\hline $\mathrm{C}$ & 4.906862000 & -0.092733000 & -0.908485000 \\
\hline $\mathrm{H}$ & 3.575770000 & 1.486130000 & -1.491309000 \\
\hline $\mathrm{C}$ & 5.063716000 & -1.277208000 & -0.194091000 \\
\hline $\mathrm{H}$ & 4.085364000 & -2.696961000 & 1.094058000 \\
\hline $\mathrm{H}$ & 5.741166000 & 0.317583000 & -1.471450000 \\
\hline $\mathrm{H}$ & -0.595083000 & 2.301287000 & -1.148932000 \\
\hline $\mathrm{H}$ & 1.145137000 & 1.498673000 & 1.108012000 \\
\hline $\mathrm{O}$ & 0.719425000 & 2.075475000 & 2.042457000 \\
\hline $\mathrm{H}$ & 1.343298000 & 2.776893000 & 2.251683000 \\
\hline $\mathrm{H}$ & 1.332257000 & 1.701393000 & -0.845903000 \\
\hline
\end{tabular}




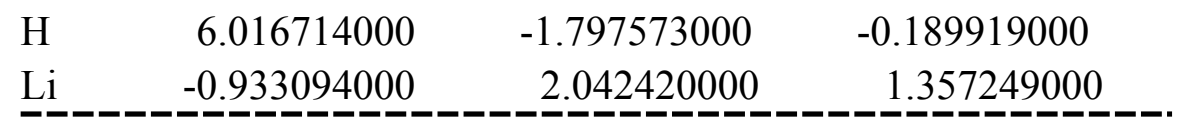

\begin{tabular}{|c|c|c|c|}
\hline \multicolumn{4}{|c|}{ Coordinates (Angstroms) } \\
\hline & $X$ & Y & $\mathrm{Z}$ \\
\hline $\mathrm{C}$ & 4.579963000 & -0.444282000 & 0.322140000 \\
\hline $\mathrm{C}$ & 3.351377000 & -1.077972000 & 0.178241000 \\
\hline $\mathrm{C}$ & 2.189237000 & -0.329052000 & -0.053798000 \\
\hline $\mathrm{C}$ & 2.264367000 & 1.066616000 & -0.133832000 \\
\hline $\mathrm{C}$ & 3.502318000 & 1.687762000 & 0.010693000 \\
\hline $\mathrm{C}$ & 4.657049000 & 0.943499000 & 0.237730000 \\
\hline $\mathrm{H}$ & 5.476766000 & -1.033417000 & 0.489933000 \\
\hline $\mathrm{H}$ & 3.301962000 & -2.164490000 & 0.208665000 \\
\hline $\mathrm{H}$ & 1.355306000 & 1.670928000 & -0.262355000 \\
\hline $\mathrm{H}$ & 3.558301000 & 2.771149000 & -0.044960000 \\
\hline $\mathrm{H}$ & 5.615485000 & 1.441932000 & 0.351411000 \\
\hline $\mathrm{C}$ & 0.897410000 & -1.042286000 & -0.210983000 \\
\hline $\mathrm{C}$ & -0.024560000 & -0.764063000 & -1.144857000 \\
\hline $\mathrm{H}$ & 0.172738000 & 0.059209000 & -1.824619000 \\
\hline $\mathrm{O}$ & 0.689779000 & -2.103665000 & 0.631433000 \\
\hline $\mathrm{C}$ & -1.325081000 & -1.523389000 & -1.288744000 \\
\hline $\mathrm{H}$ & -1.211162000 & -2.539649000 & -0.901024000 \\
\hline $\mathrm{C}$ & -2.417348000 & -0.798677000 & -0.529968000 \\
\hline $\mathrm{C}$ & -2.560049000 & -0.998153000 & 0.851199000 \\
\hline $\mathrm{C}$ & -3.173872000 & 0.205975000 & -1.142560000 \\
\hline $\mathrm{C}$ & -3.425313000 & -0.201997000 & 1.599088000 \\
\hline $\mathrm{H}$ & -1.961298000 & -1.764118000 & 1.336116000 \\
\hline $\mathrm{C}$ & -4.039584000 & 1.003812000 & -0.394478000 \\
\hline $\mathrm{H}$ & -3.049335000 & 0.393799000 & -2.205357000 \\
\hline $\mathrm{C}$ & -4.163829000 & 0.806231000 & 0.978210000 \\
\hline $\mathrm{H}$ & -3.518734000 & -0.363995000 & 2.668674000 \\
\hline $\mathrm{H}$ & -4.597149000 & 1.795923000 & -0.883663000 \\
\hline $\mathrm{H}$ & -4.824778000 & 1.436970000 & 1.563761000 \\
\hline $\mathrm{O}$ & -0.413168000 & 2.570928000 & -0.133004000 \\
\hline $\mathrm{H}$ & -1.591980000 & -1.600351000 & -2.347781000 \\
\hline $\mathrm{H}$ & 1.349767000 & -2.061799000 & 1.334814000 \\
\hline $\mathrm{H}$ & -0.443670000 & 3.499143000 & 0.099529000 \\
\hline $\mathrm{Li}$ & -1.239206000 & 1.172167000 & 0.253649000 \\
\hline
\end{tabular}

\footnotetext{
$\mathbf{A 1}_{\text {tBuOK }}$
} 


\begin{tabular}{|c|c|c|c|}
\hline \multicolumn{4}{|c|}{ Coordinates (Angstroms) } \\
\hline & $X$ & $\mathrm{Y}$ & Z \\
\hline $\mathrm{C}$ & -0.817777000 & 4.032262000 & -0.713354000 \\
\hline $\mathrm{C}$ & -0.175040000 & 2.890891000 & -0.243285000 \\
\hline $\mathrm{C}$ & -0.869604000 & 1.683589000 & -0.134020000 \\
\hline $\mathrm{C}$ & -2.213671000 & 1.623439000 & -0.516091000 \\
\hline $\mathrm{C}$ & -2.851725000 & 2.767534000 & -0.989708000 \\
\hline $\mathrm{C}$ & -2.159258000 & 3.972202000 & -1.085242000 \\
\hline $\mathrm{H}$ & -0.270486000 & 4.966628000 & -0.800321000 \\
\hline $\mathrm{H}$ & 0.879595000 & 2.932118000 & 0.024065000 \\
\hline $\mathrm{H}$ & -2.723866000 & 0.656827000 & -0.452070000 \\
\hline $\mathrm{H}$ & -3.892744000 & 2.713828000 & -1.297100000 \\
\hline $\mathrm{H}$ & -2.658289000 & 4.861055000 & -1.460801000 \\
\hline $\mathrm{C}$ & -0.209182000 & 0.450088000 & 0.457836000 \\
\hline $\mathrm{C}$ & 1.178206000 & 0.217978000 & -0.064928000 \\
\hline $\mathrm{H}$ & 1.255492000 & 0.253979000 & -1.150321000 \\
\hline $\mathrm{O}$ & -0.239967000 & 0.534354000 & 1.900391000 \\
\hline $\mathrm{C}$ & 2.250106000 & -0.024697000 & 0.693383000 \\
\hline $\mathrm{H}$ & 2.114661000 & -0.050278000 & 1.773797000 \\
\hline $\mathrm{C}$ & 3.625147000 & -0.273335000 & 0.226903000 \\
\hline $\mathrm{C}$ & 4.664324000 & -0.282048000 & 1.164887000 \\
\hline $\mathrm{C}$ & 3.947306000 & -0.510240000 & -1.116373000 \\
\hline $\mathrm{C}$ & 5.981960000 & -0.504748000 & 0.779463000 \\
\hline $\mathrm{H}$ & 4.433606000 & -0.109216000 & 2.213557000 \\
\hline $\mathrm{C}$ & 5.262359000 & -0.732460000 & -1.503973000 \\
\hline $\mathrm{H}$ & 3.162492000 & -0.536288000 & -1.866266000 \\
\hline $\mathrm{C}$ & 6.286731000 & -0.729171000 & -0.559068000 \\
\hline $\mathrm{H}$ & 6.769845000 & -0.505264000 & 1.526957000 \\
\hline $\mathrm{H}$ & 5.488091000 & -0.916887000 & -2.550251000 \\
\hline $\mathrm{H}$ & 7.313528000 & -0.906725000 & -0.864506000 \\
\hline $\mathrm{H}$ & -0.855540000 & -0.415500000 & 0.227477000 \\
\hline $\mathrm{H}$ & 0.226592000 & 1.345543000 & 2.143610000 \\
\hline $\mathrm{C}$ & -4.087617000 & -3.009571000 & -0.861407000 \\
\hline $\mathrm{C}$ & -2.711056000 & -2.314002000 & -0.787335000 \\
\hline $\mathrm{H}$ & -4.297983000 & -3.509163000 & 0.092903000 \\
\hline $\mathrm{H}$ & -4.867046000 & -2.256263000 & -1.028294000 \\
\hline $\mathrm{H}$ & -4.148769000 & -3.758134000 & -1.662645000 \\
\hline $\mathrm{C}$ & -2.428540000 & -1.647445000 & -2.153048000 \\
\hline $\mathrm{C}$ & -1.627083000 & -3.385788000 & -0.536377000 \\
\hline $\mathrm{H}$ & -1.456108000 & -1.139148000 & -2.127971000 \\
\hline $\mathrm{H}$ & -2.416437000 & -2.368691000 & -2.980842000 \\
\hline $\mathrm{H}$ & -3.194704000 & -0.892143000 & -2.366114000 \\
\hline $\mathrm{H}$ & -1.602220000 & -4.161906000 & -1.312781000 \\
\hline $\mathrm{H}$ & -0.638979000 & -2.910436000 & -0.492724000 \\
\hline
\end{tabular}




\begin{tabular}{lrrr}
$\mathrm{H}$ & -1.808337000 & -3.872848000 & 0.430362000 \\
$\mathrm{O}$ & -2.701363000 & -1.382160000 & 0.220924000 \\
$\mathrm{~K}$ & $\mathbf{- 2 . 7 1 3 4 3 5 0 0 0}$ & -0.420290000 & 2.350537000 \\
\hline
\end{tabular}

TSA1-2 ${ }_{t \mathrm{BuOK}}$

\begin{tabular}{|c|c|c|c|}
\hline & & nates (Angstrom & \\
\hline & $X$ & $\mathrm{Y}$ & $\mathrm{Z}$ \\
\hline $\mathrm{C}$ & 3.538750000 & -2.933105000 & 0.299917000 \\
\hline $\mathrm{C}$ & 2.527696000 & -2.131191000 & 0.831948000 \\
\hline $\mathrm{C}$ & 1.654262000 & -1.412095000 & 0.000498000 \\
\hline $\mathrm{C}$ & 1.845572000 & -1.520496000 & -1.389198000 \\
\hline $\mathrm{C}$ & 2.842975000 & -2.327600000 & -1.915871000 \\
\hline $\mathrm{C}$ & 3.702782000 & -3.038717000 & -1.075877000 \\
\hline $\mathrm{H}$ & 4.188798000 & -3.490614000 & 0.969955000 \\
\hline $\mathrm{H}$ & 2.390503000 & -2.081571000 & 1.909687000 \\
\hline $\mathrm{H}$ & 1.213663000 & -0.937191000 & -2.054247000 \\
\hline $\mathrm{H}$ & 2.964012000 & -2.394262000 & -2.993712000 \\
\hline $\mathrm{H}$ & 4.485912000 & -3.665093000 & -1.492026000 \\
\hline $\mathrm{C}$ & 0.637731000 & -0.463282000 & 0.538423000 \\
\hline $\mathrm{C}$ & -0.744560000 & -0.692297000 & 0.122893000 \\
\hline $\mathrm{H}$ & -0.865209000 & -1.136999000 & -0.864536000 \\
\hline $\mathrm{O}$ & 0.769725000 & -0.265811000 & 1.956712000 \\
\hline $\mathrm{C}$ & -1.839982000 & -0.360099000 & 0.833936000 \\
\hline $\mathrm{H}$ & -1.703364000 & 0.148147000 & 1.787544000 \\
\hline $\mathrm{C}$ & -3.232825000 & -0.561783000 & 0.415172000 \\
\hline $\mathrm{C}$ & -4.251803000 & 0.161703000 & 1.051504000 \\
\hline $\mathrm{C}$ & -3.606910000 & -1.459657000 & -0.596163000 \\
\hline $\mathrm{C}$ & -5.583952000 & 0.011806000 & 0.684133000 \\
\hline $\mathrm{H}$ & -3.986439000 & 0.861240000 & 1.840923000 \\
\hline $\mathrm{C}$ & -4.937189000 & -1.606667000 & -0.968626000 \\
\hline $\mathrm{H}$ & -2.849347000 & -2.065975000 & -1.084934000 \\
\hline $\mathrm{C}$ & -5.935389000 & -0.871944000 & -0.332227000 \\
\hline $\mathrm{H}$ & -6.350712000 & 0.589160000 & 1.193510000 \\
\hline $\mathrm{H}$ & -5.198643000 & -2.311023000 & -1.753623000 \\
\hline $\mathrm{H}$ & -6.975559000 & -0.994789000 & -0.619094000 \\
\hline $\mathrm{H}$ & 1.022426000 & 0.773088000 & 0.031057000 \\
\hline $\mathrm{H}$ & 0.251033000 & -0.951189000 & 2.406470000 \\
\hline $\mathrm{C}$ & 1.513978000 & 4.025756000 & -1.159120000 \\
\hline $\mathrm{C}$ & 0.706187000 & 2.773824000 & -0.795762000 \\
\hline $\mathrm{H}$ & 1.984357000 & 4.443657000 & -0.260393000 \\
\hline $\mathrm{H}$ & 2.304542000 & 3.766257000 & -1.871949000 \\
\hline $\mathrm{H}$ & 0.882520000 & 4.802444000 & -1.607054000 \\
\hline $\mathrm{C}$ & 0.061557000 & 2.190331000 & -2.064343000 \\
\hline
\end{tabular}




$\begin{array}{lrrr}\mathrm{C} & -0.389531000 & 3.136445000 & 0.219728000 \\ \mathrm{H} & -0.550192000 & 1.317012000 & -1.811258000 \\ \mathrm{H} & -0.585537000 & 2.919745000 & -2.566085000 \\ \mathrm{H} & 0.843292000 & 1.876179000 & -2.765039000 \\ \mathrm{H} & -1.091620000 & 3.878034000 & -0.180595000 \\ \mathrm{H} & -0.957344000 & 2.240967000 & 0.495754000 \\ \mathrm{H} & 0.065335000 & 3.549500000 & 1.128686000 \\ \mathrm{O} & 1.589696000 & 1.841251000 & -0.237727000 \\ \mathrm{~K} & 3.083479000 & 0.986009000 & 1.484384000\end{array}$

\begin{tabular}{|c|c|c|c|}
\hline & & inates (Angstrom & \\
\hline & $X$ & $\mathrm{Y}$ & Z \\
\hline $\mathrm{C}$ & -5.025768000 & -0.633686000 & 0.163486000 \\
\hline $\mathrm{C}$ & -3.871426000 & -1.054886000 & -0.491078000 \\
\hline $\mathrm{C}$ & -2.613564000 & -1.078995000 & 0.165387000 \\
\hline $\mathrm{C}$ & -2.602293000 & -0.641883000 & 1.517518000 \\
\hline $\mathrm{C}$ & -3.761725000 & -0.235816000 & 2.156942000 \\
\hline $\mathrm{C}$ & -4.989955000 & -0.216833000 & 1.490542000 \\
\hline $\mathrm{H}$ & -5.970460000 & -0.645149000 & -0.375402000 \\
\hline $\mathrm{H}$ & -3.930769000 & -1.411146000 & -1.514697000 \\
\hline $\mathrm{H}$ & -1.667574000 & -0.622817000 & 2.070169000 \\
\hline $\mathrm{H}$ & -3.705227000 & 0.080626000 & 3.195647000 \\
\hline $\mathrm{H}$ & -5.892642000 & 0.108007000 & 1.997923000 \\
\hline $\mathrm{C}$ & -1.415508000 & -1.412503000 & -0.557269000 \\
\hline $\mathrm{C}$ & -0.116002000 & -1.393341000 & -0.075688000 \\
\hline $\mathrm{H}$ & -0.022575000 & -1.354193000 & 1.007589000 \\
\hline $\mathrm{O}$ & -1.592236000 & -1.466677000 & -1.964045000 \\
\hline $\mathrm{C}$ & 1.061377000 & -1.378521000 & -0.816879000 \\
\hline $\mathrm{H}$ & 0.998089000 & -1.394362000 & -1.904888000 \\
\hline $\mathrm{C}$ & 2.403459000 & -1.385538000 & -0.261618000 \\
\hline $\mathrm{C}$ & 3.509853000 & -1.137941000 & -1.102802000 \\
\hline $\mathrm{C}$ & 2.686799000 & -1.575330000 & 1.107892000 \\
\hline $\mathrm{C}$ & 4.802928000 & -1.051922000 & -0.606793000 \\
\hline $\mathrm{H}$ & 3.334275000 & -0.992887000 & -2.166961000 \\
\hline $\mathrm{C}$ & 3.981549000 & -1.477404000 & 1.602931000 \\
\hline $\mathrm{H}$ & 1.882799000 & -1.820611000 & 1.796709000 \\
\hline $\mathrm{C}$ & 5.054112000 & -1.209675000 & 0.755722000 \\
\hline $\mathrm{H}$ & 5.623574000 & -0.852718000 & -1.291503000 \\
\hline $\mathrm{H}$ & 4.154537000 & -1.626085000 & 2.665883000 \\
\hline $\mathrm{H}$ & 6.064232000 & -1.136007000 & 1.146666000 \\
\hline $\mathrm{H}$ & 0.962598000 & 0.672724000 & -0.778341000 \\
\hline $\mathrm{H}$ & -1.086582000 & -2.220978000 & -2.292089000 \\
\hline
\end{tabular}




$\begin{array}{lrrr}\mathrm{C} & 0.855388000 & 1.672489000 & 1.572407000 \\ \mathrm{C} & 1.229312000 & 2.345820000 & 0.249283000 \\ \mathrm{H} & -0.231749000 & 1.552214000 & 1.652571000 \\ \mathrm{H} & 1.322858000 & 0.684632000 & 1.642103000 \\ \mathrm{H} & 1.200882000 & 2.268819000 & 2.423841000 \\ \mathrm{C} & 2.748741000 & 2.404408000 & 0.084322000 \\ \mathrm{C} & 0.615183000 & 3.740234000 & 0.158068000 \\ \mathrm{H} & 3.006216000 & 2.869928000 & -0.872570000 \\ \mathrm{H} & 3.205594000 & 2.987808000 & 0.891258000 \\ \mathrm{H} & 3.179324000 & 1.397457000 & 0.105816000 \\ \mathrm{H} & 1.021407000 & 4.397500000 & 0.933022000 \\ \mathrm{H} & 0.826238000 & 4.187142000 & -0.819262000 \\ \mathrm{H} & -0.472871000 & 3.693918000 & 0.297080000 \\ \mathrm{O} & 0.661355000 & 1.610048000 & -0.842028000 \\ \mathrm{~K} & -1.772100000 & 1.176692000 & -1.580853000 \\ - \text {-_-_-_-_. }\end{array}$

TSA2-3 $_{t \mathrm{BuOK}}$

\begin{tabular}{lrcc} 
& \multicolumn{3}{c}{ Coordinates (Angstroms) } \\
& $\mathrm{X}$ & $\mathrm{Y}$ & $\mathrm{Z}$ \\
$\mathrm{C}$ & -5.170051000 & -1.115604000 & -0.062554000 \\
$\mathrm{C}$ & -3.953288000 & -1.173044000 & -0.734538000 \\
$\mathrm{C}$ & -2.741129000 & -0.987075000 & -0.049151000 \\
$\mathrm{C}$ & -2.801119000 & -0.709588000 & 1.329484000 \\
$\mathrm{C}$ & -4.018163000 & -0.666689000 & 1.997066000 \\
$\mathrm{C}$ & -5.211973000 & -0.868017000 & 1.306455000 \\
$\mathrm{H}$ & -6.093028000 & -1.273756000 & -0.613490000 \\
$\mathrm{H}$ & -3.926948000 & -1.378418000 & -1.799239000 \\
$\mathrm{H}$ & -1.884676000 & -0.516686000 & 1.880273000 \\
$\mathrm{H}$ & -4.034560000 & -0.460969000 & 3.063673000 \\
$\mathrm{H}$ & -6.162637000 & -0.825752000 & 1.829012000 \\
$\mathrm{C}$ & -1.465154000 & -1.004845000 & -0.774820000 \\
$\mathrm{C}$ & -0.229278000 & -1.091970000 & -0.237914000 \\
$\mathrm{H}$ & -0.190538000 & -1.303637000 & 0.826868000 \\
$\mathrm{O}$ & -1.631087000 & -0.731453000 & -2.142784000 \\
$\mathrm{C}$ & 1.025051000 & -0.760638000 & -0.898431000 \\
$\mathrm{H}$ & 0.989573000 & -0.794137000 & -1.994724000 \\
$\mathrm{C}$ & 2.310903000 & -1.306490000 & -0.397078000 \\
$\mathrm{C}$ & 3.476702000 & -1.085012000 & -1.150582000 \\
$\mathrm{C}$ & 2.462635000 & -1.966628000 & 0.830701000 \\
$\mathrm{C}$ & 4.725156000 & -1.492043000 & -0.700488000 \\
$\mathrm{H}$ & 3.391551000 & -0.567989000 & -2.104128000 \\
$\mathrm{C}$ & 3.715022000 & -2.369731000 & 1.287473000 \\
$\mathrm{H}$ & 1.593081000 & -2.186664000 & 1.443356000
\end{tabular}




\begin{tabular}{lrcc}
$\mathrm{C}$ & 4.856287000 & -2.136231000 & 0.528733000 \\
$\mathrm{H}$ & 5.603778000 & -1.300704000 & -1.310996000 \\
$\mathrm{H}$ & 3.794073000 & -2.878075000 & 2.244900000 \\
$\mathrm{H}$ & 5.831592000 & -2.453452000 & 0.885582000 \\
$\mathrm{H}$ & 1.036602000 & 0.574493000 & -0.694325000 \\
$\mathrm{H}$ & -0.888643000 & -1.138807000 & -2.608951000 \\
$\mathrm{C}$ & 1.198987000 & 1.552305000 & 1.799053000 \\
$\mathrm{C}$ & 1.473901000 & 2.386764000 & 0.532805000 \\
$\mathrm{H}$ & 0.115850000 & 1.457878000 & 1.958065000 \\
$\mathrm{H}$ & 1.619827000 & 0.546440000 & 1.693301000 \\
$\mathrm{H}$ & 1.637888000 & 2.008669000 & 2.694473000 \\
$\mathrm{C}$ & 2.987216000 & 2.435325000 & 0.270424000 \\
$\mathrm{C}$ & 0.946033000 & 3.814663000 & 0.747138000 \\
$\mathrm{H}$ & 3.185558000 & 3.016424000 & -0.636999000 \\
$\mathrm{H}$ & 3.533708000 & 2.891443000 & 1.105317000 \\
$\mathrm{H}$ & 3.376061000 & 1.422695000 & 0.117718000 \\
$\mathrm{H}$ & 1.446069000 & 4.321277000 & 1.581383000 \\
$\mathrm{H}$ & 1.099731000 & 4.408467000 & -0.161296000 \\
$\mathrm{H}$ & -0.130914000 & 3.793896000 & 0.971084000 \\
$\mathrm{O}$ & 0.808021000 & 1.839345000 & -0.567410000 \\
$\mathrm{~K}$ & -1.572889000 & 1.844290000 & -1.180630000 \\
\hline---- - &
\end{tabular}

\begin{tabular}{lrrr} 
A3 $_{\text {tBuoK }}$ & \multicolumn{3}{c}{ Coordinates (Angstroms) } \\
& $\mathrm{X}$ & $\mathrm{Y}$ & $\mathrm{Z}$ \\
$\mathrm{C}$ & -4.695110000 & 1.427444000 & 0.381823000 \\
$\mathrm{C}$ & -3.526423000 & 1.922370000 & -0.190048000 \\
$\mathrm{C}$ & -2.358022000 & 1.149805000 & -0.217197000 \\
$\mathrm{C}$ & -2.389695000 & -0.146517000 & 0.321635000 \\
$\mathrm{C}$ & -3.563014000 & -0.632057000 & 0.887841000 \\
$\mathrm{C}$ & -4.716131000 & 0.149929000 & 0.930216000 \\
$\mathrm{H}$ & -5.592828000 & 2.038968000 & 0.383999000 \\
$\mathrm{H}$ & -3.550532000 & 2.902134000 & -0.661891000 \\
$\mathrm{H}$ & -1.506947000 & -0.802311000 & 0.280727000 \\
$\mathrm{H}$ & -3.569457000 & -1.639915000 & 1.294322000 \\
$\mathrm{H}$ & -5.627052000 & -0.239186000 & 1.375590000 \\
$\mathrm{C}$ & -1.113503000 & 1.703829000 & -0.813470000 \\
$\mathrm{C}$ & -0.121514000 & 0.961831000 & -1.323622000 \\
$\mathrm{H}$ & -0.183026000 & -0.122993000 & -1.247810000 \\
$\mathrm{O}$ & -1.017273000 & 3.075750000 & -0.863344000 \\
$\mathrm{C}$ & 1.154406000 & 1.537548000 & -1.887362000 \\
$\mathrm{H}$ & 1.020666000 & 2.591103000 & -2.146104000 \\
$\mathrm{C}$ & 2.264537000 & 1.389825000 & -0.867508000
\end{tabular}




$\begin{array}{lccc}\mathrm{C} & 2.466845000 & 2.379043000 & 0.102773000 \\ \mathrm{C} & 3.021110000 & 0.216223000 & -0.794493000 \\ \mathrm{C} & 3.399703000 & 2.198588000 & 1.123165000 \\ \mathrm{H} & 1.875771000 & 3.290453000 & 0.056280000 \\ \mathrm{C} & 3.953771000 & 0.030852000 & 0.224859000 \\ \mathrm{H} & 2.852072000 & -0.572378000 & -1.521618000 \\ \mathrm{C} & 4.144479000 & 1.019378000 & 1.190282000 \\ \mathrm{H} & 3.552637000 & 2.981482000 & 1.861120000 \\ \mathrm{H} & 4.524653000 & -0.891941000 & 0.268291000 \\ \mathrm{H} & 4.877647000 & 0.879558000 & 1.979280000 \\ \mathrm{C} & 0.278676000 & -2.941562000 & -1.881377000 \\ \mathrm{C} & 0.127403000 & -3.074783000 & -0.348792000 \\ \mathrm{H} & -0.520795000 & -2.303459000 & -2.277814000 \\ \mathrm{H} & 1.239289000 & -2.463404000 & -2.113116000 \\ \mathrm{H} & 0.239439000 & -3.906689000 & -2.403967000 \\ \mathrm{C} & 1.251546000 & -3.996871000 & 0.174005000 \\ \mathrm{C} & -1.234945000 & -3.741259000 & -0.050497000 \\ \mathrm{H} & 1.147524000 & -4.122168000 & 1.259262000 \\ \mathrm{H} & 1.246460000 & -4.992114000 & -0.290402000 \\ \mathrm{H} & 2.225084000 & -3.528703000 & -0.021474000 \\ \mathrm{H} & -1.330131000 & -4.741919000 & -0.493034000 \\ \mathrm{H} & -1.368417000 & -3.828549000 & 1.035174000 \\ \mathrm{H} & -2.046175000 & -3.115604000 & -0.443037000 \\ \mathrm{O} & 0.204849000 & -1.844283000 & 0.248278000 \\ \mathrm{H} & 1.422549000 & 1.000047000 & -2.803599000 \\ \mathrm{~K} & 0.902805000 & -0.054483000 & 1.579626000 \\ \mathrm{H} & -1.647063000 & 3.449348000 & -0.235940000 \\ -------0 & \end{array}$

\begin{tabular}{|c|c|c|c|}
\hline \multicolumn{4}{|c|}{ Coordinates (Angstroms) } \\
\hline & $\mathrm{X}$ & Y & Z \\
\hline$\overline{\mathrm{C}}$ & -0.594590000 & 4.052459000 & -0.410546000 \\
\hline $\mathrm{C}$ & -0.014326000 & 2.829075000 & -0.087400000 \\
\hline $\mathrm{C}$ & -0.800100000 & 1.676780000 & -0.008355000 \\
\hline $\mathrm{C}$ & -2.173146000 & 1.753195000 & -0.263529000 \\
\hline $\mathrm{C}$ & -2.747503000 & 2.979011000 & -0.588543000 \\
\hline $\mathrm{C}$ & -1.962739000 & 4.128333000 & -0.659915000 \\
\hline $\mathrm{H}$ & 0.021558000 & 4.944783000 & -0.474417000 \\
\hline $\mathrm{H}$ & 1.057248000 & 2.763491000 & 0.090843000 \\
\hline $\mathrm{H}$ & -2.761510000 & 0.832383000 & -0.205769000 \\
\hline $\mathrm{H}$ & -3.812734000 & 3.036352000 & -0.794432000 \\
\hline $\mathrm{H}$ & -2.415408000 & 5.081866000 & -0.916578000 \\
\hline
\end{tabular}




\begin{tabular}{lrrr}
$\mathrm{C}$ & -0.205612000 & 0.351636000 & 0.430377000 \\
$\mathrm{C}$ & 1.177864000 & 0.112217000 & -0.099828000 \\
$\mathrm{H}$ & 1.268136000 & 0.243511000 & -1.176681000 \\
$\mathrm{O}$ & -0.245353000 & 0.271003000 & 1.879633000 \\
$\mathrm{C}$ & 2.235240000 & -0.227559000 & 0.640785000 \\
$\mathrm{H}$ & 2.094348000 & -0.334998000 & 1.715222000 \\
$\mathrm{C}$ & 3.604702000 & -0.475041000 & 0.156731000 \\
$\mathrm{C}$ & 4.652637000 & -0.510503000 & 1.084064000 \\
$\mathrm{C}$ & 3.910228000 & -0.684311000 & -1.194614000 \\
$\mathrm{C}$ & 5.965109000 & -0.728510000 & 0.679089000 \\
$\mathrm{H}$ & 4.433680000 & -0.359796000 & 2.138582000 \\
$\mathrm{C}$ & 5.220296000 & -0.902427000 & -1.601527000 \\
$\mathrm{H}$ & 3.115735000 & -0.694564000 & -1.934833000 \\
$\mathrm{C}$ & 6.254353000 & -0.923420000 & -0.667598000 \\
$\mathrm{H}$ & 6.761053000 & -0.748520000 & 1.417649000 \\
$\mathrm{H}$ & 5.434243000 & -1.066535000 & -2.653575000 \\
$\mathrm{H}$ & 7.277027000 & -1.097965000 & -0.988090000 \\
$\mathrm{H}$ & -0.889935000 & -0.448736000 & 0.108281000 \\
$\mathrm{H}$ & 0.188267000 & 1.066346000 & 2.216949000 \\
$\mathrm{C}$ & -4.933426000 & -2.283755000 & -0.288114000 \\
$\mathrm{C}$ & -3.413983000 & -2.108423000 & -0.483679000 \\
$\mathrm{H}$ & -5.133618000 & -2.655065000 & 0.725054000 \\
$\mathrm{H}$ & -5.430775000 & -1.312896000 & -0.397707000 \\
$\mathrm{H}$ & -5.377069000 & -2.985923000 & -1.006226000 \\
$\mathrm{C}$ & -3.153733000 & -1.607162000 & -1.920340000 \\
$\mathrm{C}$ & -2.725364000 & -3.477140000 & -0.306543000 \\
$\mathrm{H}$ & -2.077105000 & -1.462310000 & -2.073251000 \\
$\mathrm{H}$ & -3.518617000 & -2.306014000 & -2.684074000 \\
$\mathrm{H}$ & -3.647596000 & -0.640365000 & -2.074676000 \\
$\mathrm{H}$ & -3.079364000 & -4.230793000 & -1.022130000 \\
$\mathrm{H}$ & -1.642268000 & -3.360038000 & -0.433594000 \\
$\mathrm{H}$ & -2.909030000 & -3.854247000 & 0.707933000 \\
$\mathrm{H}$ & -2.916676000 & -1.205991000 & 0.426165000 \\
-2.312316000 & -0.605163000 & 2.255096000 \\
\hline & & &
\end{tabular}

TSA1-2 ${ }_{t \mathrm{BuONa}}$

\begin{tabular}{llll} 
& & \multicolumn{3}{c}{ Coordinates (Angstroms) } & Z \\
\hline $\mathrm{C}$ & -3.857069000 & -2.638687000 & -0.587946000 \\
$\mathrm{C}$ & -2.775839000 & -1.864050000 & -1.014875000 \\
$\mathrm{C}$ & -1.832174000 & -1.359853000 & -0.103782000 \\
$\mathrm{C}$ & -2.023569000 & -1.663260000 & 1.257553000 \\
$\mathrm{C}$ & -3.090268000 & -2.441870000 & 1.676176000
\end{tabular}




\begin{tabular}{lrcc}
$\mathrm{C}$ & -4.022718000 & -2.932784000 & 0.758029000 \\
$\mathrm{H}$ & -4.561296000 & -3.023481000 & -1.321388000 \\
$\mathrm{H}$ & -2.636525000 & -1.684014000 & -2.078944000 \\
$\mathrm{H}$ & -1.335483000 & -1.254728000 & 1.993025000 \\
$\mathrm{H}$ & -3.210903000 & -2.656147000 & 2.734516000 \\
$\mathrm{H}$ & -4.861718000 & -3.534209000 & 1.093746000 \\
$\mathrm{C}$ & -0.757367000 & -0.419505000 & -0.518323000 \\
$\mathrm{C}$ & 0.610062000 & -0.704922000 & -0.100345000 \\
$\mathrm{H}$ & 0.703799000 & -1.221577000 & 0.853953000 \\
$\mathrm{O}$ & -0.858136000 & -0.060552000 & -1.911923000 \\
$\mathrm{C}$ & 1.724116000 & -0.337674000 & -0.763638000 \\
$\mathrm{H}$ & 1.612908000 & 0.227529000 & -1.687829000 \\
$\mathrm{C}$ & 3.107368000 & -0.585485000 & -0.338247000 \\
$\mathrm{C}$ & 4.147554000 & 0.129666000 & -0.949196000 \\
$\mathrm{C}$ & 3.453381000 & -1.517704000 & 0.651985000 \\
$\mathrm{C}$ & 5.473543000 & -0.061916000 & -0.578874000 \\
$\mathrm{H}$ & 3.904326000 & 0.855259000 & -1.722013000 \\
$\mathrm{C}$ & 4.777426000 & -1.706684000 & 1.027262000 \\
$\mathrm{H}$ & 2.678796000 & -2.116230000 & 1.123360000 \\
$\mathrm{C}$ & 5.797081000 & -0.980311000 & 0.415588000 \\
$\mathrm{H}$ & 6.257179000 & 0.509429000 & -1.068874000 \\
$\mathrm{H}$ & 5.016915000 & -2.436325000 & 1.795929000 \\
$\mathrm{H}$ & 6.832088000 & -1.135287000 & 0.705354000 \\
$\mathrm{H}$ & -1.166537000 & 0.794770000 & 0.067083000 \\
$\mathrm{H}$ & -0.419509000 & -0.742757000 & -2.443184000 \\
$\mathrm{C}$ & -1.768268000 & 4.100426000 & 0.969483000 \\
$\mathrm{C}$ & -0.910350000 & 2.883780000 & 0.611070000 \\
$\mathrm{H}$ & -2.403014000 & 4.380724000 & 0.119317000 \\
$\mathrm{H}$ & -2.419231000 & 3.862626000 & 1.817295000 \\
$\mathrm{H}$ & -1.780857000 & 1.822736000 & 0.298655000 \\
$\mathrm{H}$ & -1.150508000 & 4.966546000 & 1.234757000 \\
$\mathrm{C}$ & -0.033479000 & 2.488795000 & 1.808601000 \\
$\mathrm{H}$ & -0.018128000 & 3.204123000 & -0.597883000 \\
$\mathrm{H}$ & 0.602478000 & 1.635636000 & 1.546599000 \\
$\mathrm{H}$ & 0.617696000 & 3.313335000 & 2.121443000 \\
$\mathrm{H}$ & 0.667828000 & 2.203295000 & 2.654498000 \\
$\mathrm{H}$ & 0.560381000 & 2.318000000000 \\
$\mathrm{H}$ & -1.051343000 & -1.422417000 \\
\hline
\end{tabular}

\section{A2 t $_{\text {BuONa }}$}




\begin{tabular}{|c|c|c|c|}
\hline & $X$ & Y & Z \\
\hline $\mathrm{C}$ & -4.963357000 & -0.376183000 & -0.048433000 \\
\hline $\mathrm{C}$ & -3.809004000 & -0.826391000 & -0.688217000 \\
\hline $\mathrm{C}$ & -2.606186000 & -1.059263000 & 0.029783000 \\
\hline $\mathrm{C}$ & -2.650949000 & -0.810610000 & 1.428562000 \\
\hline $\mathrm{C}$ & -3.807497000 & -0.372583000 & 2.047239000 \\
\hline $\mathrm{C}$ & -4.979721000 & -0.138830000 & 1.319921000 \\
\hline $\mathrm{H}$ & -5.865542000 & -0.224676000 & -0.636194000 \\
\hline $\mathrm{H}$ & -3.837203000 & -1.055129000 & -1.749241000 \\
\hline $\mathrm{H}$ & -1.763939000 & -0.971473000 & 2.033431000 \\
\hline $\mathrm{H}$ & -3.796422000 & -0.203635000 & 3.121063000 \\
\hline $\mathrm{H}$ & -5.880149000 & 0.209229000 & 1.815369000 \\
\hline $\mathrm{C}$ & -1.395759000 & -1.410956000 & -0.665173000 \\
\hline $\mathrm{C}$ & -0.108412000 & -1.410337000 & -0.146641000 \\
\hline $\mathrm{H}$ & -0.050181000 & -1.423586000 & 0.939110000 \\
\hline $\mathrm{O}$ & -1.525004000 & -1.317168000 & -2.081464000 \\
\hline $\mathrm{C}$ & 1.089623000 & -1.327809000 & -0.845263000 \\
\hline $\mathrm{H}$ & 1.071775000 & -1.326875000 & -1.935362000 \\
\hline $\mathrm{C}$ & 2.411729000 & -1.276449000 & -0.240772000 \\
\hline $\mathrm{C}$ & 3.532256000 & -0.972707000 & -1.042342000 \\
\hline $\mathrm{C}$ & 2.654313000 & -1.455602000 & 1.136895000 \\
\hline $\mathrm{C}$ & 4.802612000 & -0.829884000 & -0.501627000 \\
\hline $\mathrm{H}$ & 3.386788000 & -0.831532000 & -2.111502000 \\
\hline $\mathrm{C}$ & 3.925970000 & -1.304830000 & 1.676424000 \\
\hline $\mathrm{H}$ & 1.838629000 & -1.732747000 & 1.799308000 \\
\hline $\mathrm{C}$ & 5.013739000 & -0.985872000 & 0.867384000 \\
\hline $\mathrm{H}$ & 5.636234000 & -0.588870000 & -1.156429000 \\
\hline $\mathrm{H}$ & 4.069100000 & -1.449448000 & 2.744244000 \\
\hline $\mathrm{H}$ & 6.005536000 & -0.869580000 & 1.293170000 \\
\hline $\mathrm{H}$ & 0.797108000 & 0.675725000 & -0.922550000 \\
\hline $\mathrm{H}$ & -0.964549000 & -1.995195000 & -2.476519000 \\
\hline $\mathrm{C}$ & 0.492401000 & 1.692400000 & 1.396088000 \\
\hline $\mathrm{C}$ & 0.845971000 & 2.388204000 & 0.079615000 \\
\hline $\mathrm{H}$ & -0.577632000 & 1.453318000 & 1.436870000 \\
\hline $\mathrm{H}$ & 1.062165000 & 0.763581000 & 1.503290000 \\
\hline $\mathrm{H}$ & 0.734290000 & 2.336320000 & 2.248359000 \\
\hline $\mathrm{C}$ & 2.356263000 & 2.595992000 & -0.034367000 \\
\hline $\mathrm{C}$ & 0.093489000 & 3.707514000 & -0.059289000 \\
\hline $\mathrm{H}$ & 2.601257000 & 3.077260000 & -0.986481000 \\
\hline $\mathrm{H}$ & 2.723998000 & 3.227294000 & 0.781578000 \\
\hline $\mathrm{H}$ & 2.880463000 & 1.635018000 & 0.014008000 \\
\hline $\mathrm{H}$ & 0.402798000 & 4.413820000 & 0.716998000 \\
\hline $\mathrm{H}$ & 0.290349000 & 4.160005000 & -1.036776000 \\
\hline $\mathrm{H}$ & -0.988391000 & 3.551498000 & 0.045757000 \\
\hline
\end{tabular}




$\begin{array}{lccc}\mathrm{O} & 0.406237000 & 1.579302000 & -1.025530000 \\ \mathrm{Na} & -1.655566000 & 0.919797000 & -1.534934000\end{array}$

TSA2-3 ${ }_{t \text { BuONa }}$

\begin{tabular}{|c|c|c|c|}
\hline \multicolumn{4}{|c|}{ Coordinates (Angstroms) } \\
\hline & $X$ & $\mathrm{Y}$ & Z \\
\hline$\overline{\mathrm{C}}$ & -5.245247000 & 0.312289000 & 0.022144000 \\
\hline $\mathrm{C}$ & -3.984354000 & 0.447310000 & -0.557164000 \\
\hline $\mathrm{C}$ & -3.002971000 & -0.548845000 & -0.395915000 \\
\hline $\mathrm{C}$ & -3.352523000 & -1.699166000 & 0.336590000 \\
\hline $\mathrm{C}$ & -4.607867000 & -1.824622000 & 0.909847000 \\
\hline $\mathrm{C}$ & -5.563621000 & -0.816856000 & 0.765870000 \\
\hline $\mathrm{H}$ & -5.985614000 & 1.093791000 & -0.125502000 \\
\hline $\mathrm{H}$ & -3.765550000 & 1.312690000 & -1.175309000 \\
\hline $\mathrm{H}$ & -2.638061000 & -2.509336000 & 0.442053000 \\
\hline $\mathrm{H}$ & -4.849600000 & -2.725206000 & 1.467078000 \\
\hline $\mathrm{H}$ & -6.546675000 & -0.924847000 & 1.213163000 \\
\hline $\mathrm{C}$ & -1.651648000 & -0.341260000 & -0.924276000 \\
\hline $\mathrm{C}$ & -0.527466000 & -1.018506000 & -0.585082000 \\
\hline $\mathrm{H}$ & -0.643011000 & -1.848473000 & 0.109155000 \\
\hline $\mathrm{O}$ & -1.559184000 & 0.826469000 & -1.693866000 \\
\hline $\mathrm{C}$ & 0.814719000 & -0.625473000 & -0.983728000 \\
\hline $\mathrm{H}$ & 0.911286000 & -0.397802000 & -2.052913000 \\
\hline $\mathrm{C}$ & 1.968156000 & -1.389089000 & -0.475950000 \\
\hline $\mathrm{C}$ & 3.135557000 & -1.522757000 & -1.246806000 \\
\hline $\mathrm{C}$ & 1.997131000 & -1.927684000 & 0.822362000 \\
\hline $\mathrm{C}$ & 4.266765000 & -2.160313000 & -0.751895000 \\
\hline $\mathrm{H}$ & 3.146683000 & -1.117460000 & -2.256573000 \\
\hline $\mathrm{C}$ & 3.123364000 & -2.579254000 & 1.313118000 \\
\hline $\mathrm{H}$ & 1.130491000 & -1.812908000 & 1.469786000 \\
\hline $\mathrm{C}$ & 4.269845000 & -2.699775000 & 0.532243000 \\
\hline $\mathrm{H}$ & 5.151286000 & -2.242701000 & -1.378211000 \\
\hline $\mathrm{H}$ & 3.107841000 & -2.986452000 & 2.320953000 \\
\hline $\mathrm{H}$ & 5.151914000 & -3.200320000 & 0.919933000 \\
\hline $\mathrm{H}$ & 0.899861000 & 0.679465000 & -0.361631000 \\
\hline $\mathrm{H}$ & -0.618237000 & 0.986461000 & -1.868910000 \\
\hline $\mathrm{C}$ & 2.661461000 & 1.719546000 & 1.558003000 \\
\hline $\mathrm{C}$ & 1.960721000 & 2.408008000 & 0.380468000 \\
\hline $\mathrm{H}$ & 2.020353000 & 1.751407000 & 2.447463000 \\
\hline $\mathrm{H}$ & 2.864657000 & 0.669879000 & 1.321517000 \\
\hline $\mathrm{H}$ & 3.611619000 & 2.209283000 & 1.800871000 \\
\hline $\mathrm{C}$ & 2.855001000 & 2.354530000 & -0.866132000 \\
\hline $\mathrm{C}$ & 1.640606000 & 3.863555000 & 0.732050000 \\
\hline
\end{tabular}




$\begin{array}{lrrr}\mathrm{H} & 2.334035000 & 2.796846000 & -1.722796000 \\ \mathrm{H} & 3.791603000 & 2.900984000 & -0.708422000 \\ \mathrm{H} & 3.105685000 & 1.316149000 & -1.108679000 \\ \mathrm{H} & 2.549092000 & 4.434253000 & 0.955539000 \\ \mathrm{H} & 1.124786000 & 4.349438000 & -0.104434000 \\ \mathrm{H} & 0.990827000 & 3.905770000 & 1.616331000 \\ \mathrm{O} & 0.736421000 & 1.760138000 & 0.109445000 \\ \mathrm{Na} & -1.346268000 & 1.723310000 & 0.540040000\end{array}$

\begin{tabular}{lrcc} 
A3 $\mathbf{B}_{\text {B BuONa }}$ & & \\
& \multicolumn{3}{c}{ Coordinates (Angstroms) } \\
& $\mathrm{X}$ & $\mathrm{Y}$ & $\mathrm{Z}$ \\
$\mathrm{C}$ & -4.752216000 & -1.157087000 & -0.357858000 \\
$\mathrm{C}$ & -3.586974000 & -1.753021000 & 0.113034000 \\
$\mathrm{C}$ & -2.381248000 & -1.039250000 & 0.138668000 \\
$\mathrm{C}$ & -2.360339000 & 0.290718000 & -0.304355000 \\
$\mathrm{C}$ & -3.532391000 & 0.875673000 & -0.773022000 \\
$\mathrm{C}$ & -4.726673000 & 0.159610000 & -0.807459000 \\
$\mathrm{H}$ & -5.681498000 & -1.719218000 & -0.361990000 \\
$\mathrm{H}$ & -3.633577000 & -2.766731000 & 0.505805000 \\
$\mathrm{H}$ & -1.434997000 & 0.885819000 & -0.291336000 \\
$\mathrm{H}$ & -3.501751000 & 1.904958000 & -1.119451000 \\
$\mathrm{H}$ & -5.634539000 & 0.625798000 & -1.178996000 \\
$\mathrm{C}$ & -1.147811000 & -1.702859000 & 0.633148000 \\
$\mathrm{C}$ & -0.159409000 & -1.082233000 & 1.293344000 \\
$\mathrm{H}$ & -0.242639000 & -0.011243000 & 1.451976000 \\
$\mathrm{O}$ & -1.058613000 & -3.057031000 & 0.421585000 \\
$\mathrm{C}$ & 1.101740000 & -1.771108000 & 1.761353000 \\
$\mathrm{H}$ & 0.917026000 & -2.837573000 & 1.917772000 \\
$\mathrm{C}$ & 2.196805000 & -1.581256000 & 0.731424000 \\
$\mathrm{C}$ & 2.249562000 & -2.414323000 & -0.395584000 \\
$\mathrm{C}$ & 3.076203000 & -0.496329000 & 0.802062000 \\
$\mathrm{C}$ & 3.146186000 & -2.155973000 & -1.431492000 \\
$\mathrm{H}$ & 1.562540000 & -3.254000000 & -0.459925000 \\
$\mathrm{C}$ & 3.973344000 & -0.234398000 & -0.233511000 \\
$\mathrm{H}$ & 3.032418000 & 0.172151000 & 1.657359000 \\
$\mathrm{C}$ & 4.007560000 & -1.059048000 & -1.356728000 \\
$\mathrm{H}$ & 3.177850000 & -2.814791000 & -2.294797000 \\
$\mathrm{H}$ & 4.635127000 & 0.623502000 & -0.167122000 \\
$\mathrm{H}$ & 4.706011000 & -0.855557000 & -2.162519000 \\
$\mathrm{C}$ & 0.190368000 & 2.855218000 & 1.710184000 \\
$\mathrm{C}$ & 0.407716000 & 3.047797000 & 0.192891000 \\
$\mathrm{H}$ & -0.803746000 & 2.426245000 & 1.888466000
\end{tabular}




\begin{tabular}{lrcc}
$\mathrm{H}$ & 0.938747000 & 2.151774000 & 2.099593000 \\
$\mathrm{H}$ & 0.268900000 & 3.790916000 & 2.279116000 \\
$\mathrm{C}$ & 1.813165000 & 3.645903000 & -0.029087000 \\
$\mathrm{C}$ & -0.646895000 & 4.044149000 & -0.334332000 \\
$\mathrm{H}$ & 1.983963000 & 3.792026000 & -1.102652000 \\
$\mathrm{H}$ & 1.958278000 & 4.608421000 & 0.479320000 \\
$\mathrm{H}$ & 2.569208000 & 2.940788000 & 0.341729000 \\
$\mathrm{H}$ & -0.587449000 & 5.026270000 & 0.153074000 \\
$\mathrm{H}$ & -0.513011000 & 4.181869000 & -1.413475000 \\
$\mathrm{H}$ & -1.651754000 & 3.636950000 & -0.166916000 \\
$\mathrm{O}$ & 0.291239000 & 1.845783000 & -0.454015000 \\
$\mathrm{H}$ & 1.417356000 & -1.344801000 & 2.719121000 \\
$\mathrm{H}$ & -1.733699000 & -3.309336000 & -0.220187000 \\
$\mathrm{Na}$ & 1.016188000 & 0.091149000 & -1.128236000 \\
\hline
\end{tabular}

\begin{tabular}{|c|c|c|c|}
\hline \multicolumn{4}{|c|}{$\mathrm{B} 1_{t \mathrm{BuONa}}$} \\
\hline & \multicolumn{3}{|c|}{ Coordinates (Angstroms) } \\
\hline & $X$ & $\mathrm{Y}$ & Z \\
\hline$\overline{\mathrm{C}}$ & -1.216304000 & 4.078956000 & 0.192148000 \\
\hline $\mathrm{C}$ & -0.658606000 & 2.874527000 & 0.600601000 \\
\hline $\mathrm{C}$ & -1.017170000 & 1.672684000 & -0.019885000 \\
\hline $\mathrm{C}$ & -1.977445000 & 1.697526000 & -1.036840000 \\
\hline $\mathrm{C}$ & -2.555474000 & 2.907942000 & -1.433771000 \\
\hline $\mathrm{C}$ & -2.171962000 & 4.100795000 & -0.829102000 \\
\hline $\mathrm{H}$ & -0.917776000 & 5.009030000 & 0.672584000 \\
\hline $\mathrm{H}$ & 0.053408000 & 2.820650000 & 1.419857000 \\
\hline $\mathrm{H}$ & -2.294189000 & 0.746064000 & -1.467285000 \\
\hline $\mathrm{H}$ & -3.309940000 & 2.913231000 & -2.218019000 \\
\hline $\mathrm{H}$ & -2.617408000 & 5.042866000 & -1.141723000 \\
\hline $\mathrm{C}$ & -0.437912000 & 0.339414000 & 0.518152000 \\
\hline $\mathrm{C}$ & 0.939288000 & 0.163683000 & -0.106721000 \\
\hline $\mathrm{H}$ & 0.998089000 & 0.210131000 & -1.196614000 \\
\hline $\mathrm{O}$ & -0.447325000 & 0.293888000 & 1.874750000 \\
\hline $\mathrm{C}$ & 2.018532000 & -0.045534000 & 0.653337000 \\
\hline $\mathrm{H}$ & 1.828594000 & -0.046779000 & 1.726885000 \\
\hline $\mathrm{C}$ & 3.400100000 & -0.244665000 & 0.201011000 \\
\hline $\mathrm{C}$ & 4.445189000 & -0.192953000 & 1.136654000 \\
\hline $\mathrm{C}$ & 3.749336000 & -0.495868000 & -1.136692000 \\
\hline $\mathrm{C}$ & 5.772588000 & -0.366346000 & 0.759721000 \\
\hline $\mathrm{H}$ & 4.199685000 & -0.008429000 & 2.179869000 \\
\hline $\mathrm{C}$ & 5.074309000 & -0.667634000 & -1.517054000 \\
\hline $\mathrm{H}$ & 2.965206000 & -0.568302000 & -1.884752000 \\
\hline $\mathrm{C}$ & 6.098227000 & -0.602930000 & -0.572982000 \\
\hline
\end{tabular}




\begin{tabular}{lrrr}
\hline $\mathrm{H}$ & 6.556431000 & -0.317750000 & 1.512111000 \\
$\mathrm{H}$ & 5.309594000 & -0.861376000 & -2.561094000 \\
$\mathrm{H}$ & 7.133986000 & -0.742277000 & -0.871841000 \\
$\mathrm{H}$ & -1.088850000 & -0.431950000 & 0.016610000 \\
$\mathrm{C}$ & -3.935520000 & -3.433432000 & -0.623707000 \\
$\mathrm{C}$ & -2.712341000 & -2.552323000 & -0.264647000 \\
$\mathrm{H}$ & -4.806509000 & -3.098605000 & -0.044270000 \\
$\mathrm{H}$ & -4.172641000 & -3.305853000 & -1.686747000 \\
$\mathrm{H}$ & -3.775055000 & -4.504186000 & -0.425319000 \\
$\mathrm{C}$ & -1.498156000 & -3.049479000 & -1.089084000 \\
$\mathrm{C}$ & -2.386182000 & -2.766756000 & 1.239366000 \\
$\mathrm{H}$ & -0.600685000 & -2.488975000 & -0.804033000 \\
$\mathrm{H}$ & -1.295163000 & -4.121801000 & -0.950908000 \\
$\mathrm{H}$ & -1.690642000 & -2.863613000 & -2.152500000 \\
$\mathrm{H}$ & -2.116654000 & -3.804082000 & 1.485870000 \\
$\mathrm{H}$ & -1.561000000 & -2.108746000 & 1.545938000 \\
$\mathrm{H}$ & -3.276652000 & -2.514670000 & 1.842575000 \\
$\mathrm{O}$ & -2.965537000 & -1.238967000 & -0.542860000 \\
$\mathrm{Na}$ & -3.072902000 & 0.190948000 & 1.302243000 \\
\hline
\end{tabular}

TSB1-2 ${ }_{t \mathrm{BuONa}}$

$\begin{array}{lccc} & & \text { Coordinates (Angstroms) } \\ & \mathrm{X} & \mathrm{Y} & \mathrm{Z} \\ \mathrm{C} & -4.335288000 & -2.099370000 & -0.466322000 \\ \mathrm{C} & -3.162620000 & -1.531741000 & -0.962284000 \\ \mathrm{C} & -2.068783000 & -1.237998000 & -0.123245000 \\ \mathrm{C} & -2.223213000 & -1.543186000 & 1.247290000 \\ \mathrm{C} & -3.388352000 & -2.110071000 & 1.736640000 \\ \mathrm{C} & -4.465348000 & -2.392474000 & 0.887076000 \\ \mathrm{H} & -5.151047000 & -2.327467000 & -1.151842000 \\ \mathrm{H} & -3.025099000 & -1.331462000 & -2.022188000 \\ \mathrm{H} & -1.422932000 & -1.295935000 & 1.940922000 \\ \mathrm{H} & -3.471792000 & -2.322916000 & 2.801080000 \\ \mathrm{H} & -5.379928000 & -2.830366000 & 1.279531000 \\ \mathrm{C} & -0.905953000 & -0.502137000 & -0.699900000 \\ \mathrm{C} & 0.402723000 & -0.857900000 & -0.206400000 \\ \mathrm{H} & 0.448591000 & -1.272678000 & 0.801631000 \\ \mathrm{O} & -1.033903000 & -0.109809000 & -1.995472000 \\ \mathrm{C} & 1.564674000 & -0.626884000 & -0.886577000 \\ \mathrm{H} & 1.459509000 & -0.275148000 & -1.910789000 \\ \mathrm{C} & 2.907936000 & -0.822649000 & -0.386156000 \\ \mathrm{C} & 4.009231000 & -0.719790000 & -1.266579000 \\ \mathrm{C} & 3.220209000 & -1.105386000 & 0.964213000\end{array}$




\begin{tabular}{lrrr}
\hline $\mathrm{C}$ & 5.316925000 & -0.899595000 & -0.838713000 \\
$\mathrm{H}$ & 3.811814000 & -0.499087000 & -2.313680000 \\
$\mathrm{C}$ & 4.528389000 & -1.288805000 & 1.388961000 \\
$\mathrm{H}$ & 2.416549000 & -1.166961000 & 1.693165000 \\
$\mathrm{C}$ & 5.596600000 & -1.190907000 & 0.496331000 \\
$\mathrm{H}$ & 6.129671000 & -0.813264000 & -1.558029000 \\
$\mathrm{H}$ & 4.719313000 & -1.502431000 & 2.439241000 \\
$\mathrm{H}$ & 6.620009000 & -1.332245000 & 0.834334000 \\
$\mathrm{H}$ & -1.037952000 & 0.681359000 & 0.092091000 \\
$\mathrm{C}$ & -0.956417000 & 4.061781000 & 1.104607000 \\
$\mathrm{C}$ & -0.386271000 & 2.740172000 & 0.570254000 \\
$\mathrm{H}$ & -1.737329000 & 4.434012000 & 0.427980000 \\
$\mathrm{H}$ & -1.410549000 & 3.901916000 & 2.088891000 \\
$\mathrm{H}$ & -0.182496000 & 4.835802000 & 1.195520000 \\
$\mathrm{C}$ & 0.682874000 & 2.212621000 & 1.542590000 \\
$\mathrm{C}$ & 0.253848000 & 2.968447000 & -0.811919000 \\
$\mathrm{H}$ & 1.149789000 & 1.311808000 & 1.132969000 \\
$\mathrm{H}$ & 1.470208000 & 2.956626000 & 1.721846000 \\
$\mathrm{H}$ & 0.212066000 & 1.961096000 & 2.499735000 \\
$\mathrm{H}$ & 1.148291000 & 3.602195000 & -0.757595000 \\
$\mathrm{H}$ & 0.522979000 & 2.003815000 & -1.256808000 \\
$\mathrm{H}$ & -0.464061000 & 3.468201000 & -1.482855000 \\
$\mathrm{O}$ & -1.445024000 & 1.828790000 & 0.474121000 \\
$\mathrm{Na}$ & -2.412834000 & 1.430592000 & -1.460323000 \\
- & & &
\end{tabular}

\begin{tabular}{|c|c|c|c|}
\hline \multicolumn{4}{|c|}{$\mathrm{B} 2_{t \mathrm{BuONa}}$} \\
\hline & $X$ & $\mathrm{Y}$ & Z \\
\hline$\overline{\mathrm{C}}$ & -0.887235000 & 4.184335000 & -0.295851000 \\
\hline $\mathrm{C}$ & -0.322894000 & 3.002531000 & 0.170529000 \\
\hline $\mathrm{C}$ & -1.019270000 & 1.793035000 & 0.087461000 \\
\hline $\mathrm{C}$ & -2.305172000 & 1.796638000 & -0.457572000 \\
\hline $\mathrm{C}$ & -2.876279000 & 2.981263000 & -0.923380000 \\
\hline $\mathrm{C}$ & -2.169376000 & 4.178292000 & -0.848744000 \\
\hline $\mathrm{H}$ & -0.329909000 & 5.117212000 & -0.231112000 \\
\hline $\mathrm{H}$ & 0.671611000 & 2.997974000 & 0.610743000 \\
\hline $\mathrm{H}$ & -2.846831000 & 0.851449000 & -0.489616000 \\
\hline $\mathrm{H}$ & -3.877647000 & 2.967408000 & -1.349915000 \\
\hline $\mathrm{H}$ & -2.611417000 & 5.101583000 & -1.217727000 \\
\hline $\mathrm{C}$ & -0.425977000 & 0.496424000 & 0.660262000 \\
\hline $\mathrm{C}$ & 0.903586000 & 0.224609000 & -0.016665000 \\
\hline $\mathrm{H}$ & 0.897104000 & 0.197807000 & -1.109212000 \\
\hline $\mathrm{O}$ & -0.352610000 & 0.549208000 & 2.029645000 \\
\hline
\end{tabular}




\begin{tabular}{lrrr}
\hline $\mathrm{C}$ & 2.022236000 & 0.013933000 & 0.685475000 \\
$\mathrm{H}$ & 1.904418000 & 0.088410000 & 1.766547000 \\
$\mathrm{C}$ & 3.362119000 & -0.278233000 & 0.163737000 \\
$\mathrm{C}$ & 4.454202000 & -0.297860000 & 1.045722000 \\
$\mathrm{C}$ & 3.628340000 & -0.548027000 & -1.189536000 \\
$\mathrm{C}$ & 5.746460000 & -0.560746000 & 0.603987000 \\
$\mathrm{H}$ & 4.273616000 & -0.097755000 & 2.099334000 \\
$\mathrm{C}$ & 4.917901000 & -0.809299000 & -1.634512000 \\
$\mathrm{H}$ & 2.807353000 & -0.558909000 & -1.900591000 \\
$\mathrm{C}$ & 5.989535000 & -0.817205000 & -0.742404000 \\
$\mathrm{H}$ & 6.567688000 & -0.565309000 & 1.317123000 \\
$\mathrm{H}$ & 5.088690000 & -1.013161000 & -2.689077000 \\
$\mathrm{H}$ & 6.997530000 & -1.023322000 & -1.092970000 \\
$\mathrm{H}$ & -1.123477000 & -0.313513000 & 0.290893000 \\
$\mathrm{C}$ & -4.160863000 & -3.239073000 & -0.094128000 \\
$\mathrm{C}$ & -2.902162000 & -2.405219000 & -0.438961000 \\
$\mathrm{H}$ & -4.041773000 & -3.679973000 & 0.904671000 \\
$\mathrm{H}$ & -5.036117000 & -2.578379000 & -0.069461000 \\
$\mathrm{H}$ & -4.354228000 & -4.051231000 & -0.810912000 \\
$\mathrm{C}$ & -3.078469000 & -1.821486000 & -1.861934000 \\
$\mathrm{C}$ & -1.676474000 & -3.350986000 & -0.450403000 \\
$\mathrm{H}$ & -2.196618000 & -1.222624000 & -2.118268000 \\
$\mathrm{H}$ & -3.215822000 & -2.593913000 & -2.633044000 \\
$\mathrm{H}$ & -3.949457000 & -1.154675000 & -1.876890000 \\
$\mathrm{H}$ & -1.760646000 & -4.166635000 & -1.184027000 \\
$\mathrm{H}$ & -0.773628000 & -2.770140000 & -0.672010000 \\
$\mathrm{H}$ & -1.550243000 & -3.795576000 & 0.546133000 \\
$\mathrm{O}$ & -2.719100000 & -1.400612000 & 0.461392000 \\
$\mathrm{Na}$ & -2.015251000 & -0.743914000 & 2.291145000 \\
\hline---2 & & \\
& & &
\end{tabular}

TSB2-3 ${ }_{t \mathrm{BuONa}}$

\begin{tabular}{|c|c|c|c|}
\hline & \multicolumn{3}{|c|}{ Coordinates (Angstroms) } \\
\hline & $\mathrm{X}$ & Y & Z \\
\hline $\mathrm{C}$ & -5.470271000 & 0.328672000 & 0.105197000 \\
\hline $\mathrm{C}$ & -4.242447000 & 0.463023000 & -0.532881000 \\
\hline $\mathrm{C}$ & -3.155926000 & -0.369311000 & -0.220848000 \\
\hline $\mathrm{C}$ & -3.374323000 & -1.375703000 & 0.735260000 \\
\hline $\mathrm{C}$ & -4.599972000 & -1.509830000 & 1.377258000 \\
\hline $\mathrm{C}$ & -5.659648000 & -0.655409000 & 1.073522000 \\
\hline $\mathrm{H}$ & -6.289293000 & 0.996022000 & -0.158141000 \\
\hline $\mathrm{H}$ & -4.080815000 & 1.209119000 & -1.304431000 \\
\hline $\mathrm{H}$ & -2.578171000 & -2.078709000 & 0.962678000 \\
\hline $\mathrm{H}$ & -4.733863000 & -2.299553000 & 2.114215000 \\
\hline
\end{tabular}




\begin{tabular}{|c|c|c|c|}
\hline $\mathrm{H}$ & -6.620140000 & -0.767554000 & 1.571161000 \\
\hline $\mathrm{C}$ & -1.863375000 & -0.140850000 & -0.944972000 \\
\hline $\mathrm{C}$ & -0.701238000 & -0.661920000 & -0.426274000 \\
\hline $\mathrm{H}$ & -0.773104000 & -1.195429000 & 0.518091000 \\
\hline $\mathrm{O}$ & -1.937417000 & 0.625648000 & -2.016296000 \\
\hline $\mathrm{C}$ & 0.637229000 & -0.532503000 & -1.034321000 \\
\hline $\mathrm{H}$ & 0.596510000 & -0.430900000 & -2.126580000 \\
\hline $\mathrm{C}$ & 1.753369000 & -1.343874000 & -0.592627000 \\
\hline $\mathrm{C}$ & 2.913039000 & -1.466985000 & -1.404745000 \\
\hline $\mathrm{C}$ & 1.840890000 & -1.961173000 & 0.681447000 \\
\hline $\mathrm{C}$ & 4.056758000 & -2.115794000 & -0.975092000 \\
\hline $\mathrm{H}$ & 2.893744000 & -1.012483000 & -2.393901000 \\
\hline $\mathrm{C}$ & 2.993648000 & -2.612696000 & 1.107632000 \\
\hline $\mathrm{H}$ & 0.985952000 & -1.925098000 & 1.351444000 \\
\hline $\mathrm{C}$ & 4.121651000 & -2.698974000 & 0.295181000 \\
\hline $\mathrm{H}$ & 4.917273000 & -2.170513000 & -1.640650000 \\
\hline $\mathrm{H}$ & 3.006699000 & -3.064820000 & 2.098575000 \\
\hline $\mathrm{H}$ & 5.021053000 & -3.205717000 & 0.634830000 \\
\hline $\mathrm{H}$ & 1.326885000 & 0.842093000 & -0.656169000 \\
\hline $\mathrm{C}$ & 1.264266000 & 1.573161000 & 1.847395000 \\
\hline $\mathrm{C}$ & 2.188769000 & 2.137085000 & 0.758263000 \\
\hline $\mathrm{H}$ & 0.270583000 & 2.034809000 & 1.781837000 \\
\hline $\mathrm{H}$ & 1.143555000 & 0.493669000 & 1.718056000 \\
\hline $\mathrm{H}$ & 1.667143000 & 1.761049000 & 2.850602000 \\
\hline $\mathrm{C}$ & 3.565878000 & 1.464364000 & 0.843140000 \\
\hline $\mathrm{C}$ & 2.335427000 & 3.652444000 & 0.928802000 \\
\hline $\mathrm{H}$ & 4.215742000 & 1.847256000 & 0.048552000 \\
\hline $\mathrm{H}$ & 4.044569000 & 1.658596000 & 1.811719000 \\
\hline $\mathrm{H}$ & 3.471432000 & 0.382059000 & 0.713680000 \\
\hline $\mathrm{H}$ & 2.781456000 & 3.905841000 & 1.898751000 \\
\hline $\mathrm{H}$ & 2.972095000 & 4.059018000 & 0.135157000 \\
\hline $\mathrm{H}$ & 1.353851000 & 4.138862000 & 0.863961000 \\
\hline $\mathrm{O}$ & 1.625887000 & 1.919725000 & -0.517082000 \\
\hline $\mathrm{Na}$ & -0.429387000 & 1.947002000 & -1.295857000 \\
\hline
\end{tabular}

\begin{tabular}{|c|c|c|c|}
\hline \multicolumn{4}{|c|}{$\mathrm{B3}_{t \mathrm{BuONa}}$} \\
\hline & \multicolumn{3}{|c|}{ Coordinates (Angstroms) } \\
\hline & $\mathrm{X}$ & Y & $\mathrm{Z}$ \\
\hline$\overline{\mathrm{C}}$ & -4.572601000 & 2.417390000 & -0.364317000 \\
\hline $\mathrm{C}$ & -3.505235000 & 1.597380000 & -0.715021000 \\
\hline $\mathrm{C}$ & -3.361695000 & 0.320891000 & -0.161596000 \\
\hline $\mathrm{C}$ & -4.305938000 & -0.093983000 & 0.784895000 \\
\hline $\mathrm{C}$ & -5.372983000 & 0.724522000 & 1.142248000 \\
\hline
\end{tabular}




\begin{tabular}{|c|c|c|c|}
\hline $\mathrm{C}$ & -5.516500000 & 1.984095000 & 0.564452000 \\
\hline $\mathrm{H}$ & -4.667297000 & 3.402750000 & -0.815330000 \\
\hline $\mathrm{H}$ & -2.749084000 & 1.923630000 & -1.421854000 \\
\hline $\mathrm{H}$ & -4.187501000 & -1.061633000 & 1.263900000 \\
\hline $\mathrm{H}$ & -6.090726000 & 0.381853000 & 1.884457000 \\
\hline $\mathrm{H}$ & -6.347606000 & 2.626575000 & 0.845673000 \\
\hline $\mathrm{C}$ & -2.187636000 & -0.523500000 & -0.598724000 \\
\hline $\mathrm{C}$ & -2.251155000 & -1.883726000 & -0.457901000 \\
\hline $\mathrm{H}$ & -3.151973000 & -2.371451000 & -0.101248000 \\
\hline $\mathrm{O}$ & -1.191859000 & 0.112744000 & -1.112395000 \\
\hline $\mathrm{C}$ & -1.105162000 & -2.738718000 & -0.918117000 \\
\hline $\mathrm{H}$ & -0.927146000 & -2.606596000 & -1.997729000 \\
\hline $\mathrm{C}$ & 0.223632000 & -2.484877000 & -0.218568000 \\
\hline $\mathrm{C}$ & 1.433731000 & -2.641028000 & -0.902054000 \\
\hline $\mathrm{C}$ & 0.275056000 & -2.078254000 & 1.118415000 \\
\hline $\mathrm{C}$ & 2.655348000 & -2.398719000 & -0.277507000 \\
\hline $\mathrm{H}$ & 1.413354000 & -2.928270000 & -1.951735000 \\
\hline $\mathrm{C}$ & 1.493066000 & -1.840388000 & 1.749726000 \\
\hline $\mathrm{H}$ & -0.664989000 & -1.910120000 & 1.637242000 \\
\hline $\mathrm{C}$ & 2.690208000 & -2.000018000 & 1.055048000 \\
\hline $\mathrm{H}$ & 3.580156000 & -2.488920000 & -0.840015000 \\
\hline $\mathrm{H}$ & 1.508278000 & -1.501752000 & 2.782617000 \\
\hline $\mathrm{H}$ & 3.638606000 & -1.779631000 & 1.535328000 \\
\hline $\mathrm{C}$ & 5.004371000 & 0.926297000 & -0.340567000 \\
\hline $\mathrm{C}$ & 3.797497000 & 1.833622000 & 0.018967000 \\
\hline $\mathrm{H}$ & 4.760487000 & -0.112484000 & -0.085606000 \\
\hline $\mathrm{H}$ & 5.179360000 & 0.971860000 & -1.422507000 \\
\hline $\mathrm{H}$ & 5.934721000 & 1.206141000 & 0.177029000 \\
\hline $\mathrm{C}$ & 4.169250000 & 3.294680000 & -0.348346000 \\
\hline $\mathrm{C}$ & 3.588858000 & 1.767330000 & 1.555607000 \\
\hline $\mathrm{H}$ & 3.330381000 & 3.953047000 & -0.091762000 \\
\hline $\mathrm{H}$ & 5.071880000 & 3.659194000 & 0.165660000 \\
\hline $\mathrm{H}$ & 4.331046000 & 3.365701000 & -1.430813000 \\
\hline $\mathrm{H}$ & 4.467438000 & 2.087516000 & 2.136606000 \\
\hline $\mathrm{H}$ & 2.738752000 & 2.405753000 & 1.828189000 \\
\hline $\mathrm{H}$ & 3.338369000 & 0.737562000 & 1.839438000 \\
\hline $\mathrm{O}$ & 2.684755000 & 1.439981000 & -0.640721000 \\
\hline $\mathrm{H}$ & -1.356859000 & -3.800546000 & -0.779778000 \\
\hline $\mathrm{Na}$ & 0.908592000 & 0.392732000 & -0.659335000 \\
\hline
\end{tabular}

$\mathrm{C1}_{\text {tBuONa }}$

Coordinates (Angstroms)

X $\quad \mathrm{Y}$ Z 


\begin{tabular}{|c|c|c|c|}
\hline $\mathrm{C}$ & 2.773065000 & -1.047599000 & 1.954562000 \\
\hline $\mathrm{C}$ & 1.713938000 & -1.055139000 & 1.049913000 \\
\hline $\mathrm{C}$ & 1.872062000 & -1.610133000 & -0.223652000 \\
\hline $\mathrm{C}$ & 3.107340000 & -2.172223000 & -0.565616000 \\
\hline $\mathrm{C}$ & 4.171496000 & -2.162905000 & 0.336543000 \\
\hline $\mathrm{C}$ & 4.006088000 & -1.593098000 & 1.599067000 \\
\hline $\mathrm{H}$ & 2.637492000 & -0.602296000 & 2.935628000 \\
\hline $\mathrm{H}$ & 0.769044000 & -0.589110000 & 1.307597000 \\
\hline $\mathrm{H}$ & 3.236940000 & -2.610674000 & -1.553017000 \\
\hline $\mathrm{H}$ & 5.122015000 & -2.610342000 & 0.060107000 \\
\hline $\mathrm{H}$ & 4.829851000 & -1.586115000 & 2.306649000 \\
\hline $\mathrm{C}$ & 0.802774000 & -1.417386000 & -1.299452000 \\
\hline $\mathrm{C}$ & -0.590482000 & -1.556621000 & -0.738283000 \\
\hline $\mathrm{H}$ & -0.772290000 & -2.468974000 & -0.170384000 \\
\hline $\mathrm{O}$ & 1.036055000 & -0.201836000 & -1.972020000 \\
\hline $\mathrm{C}$ & -1.548827000 & -0.642338000 & -0.889166000 \\
\hline $\mathrm{H}$ & -1.305748000 & 0.262964000 & -1.440701000 \\
\hline $\mathrm{C}$ & -2.912887000 & -0.713030000 & -0.337586000 \\
\hline $\mathrm{C}$ & -3.621543000 & 0.477011000 & -0.132570000 \\
\hline $\mathrm{C}$ & -3.533404000 & -1.922592000 & -0.001627000 \\
\hline $\mathrm{C}$ & -4.899477000 & 0.462892000 & 0.415294000 \\
\hline $\mathrm{H}$ & -3.154247000 & 1.423551000 & -0.392700000 \\
\hline $\mathrm{C}$ & -4.811600000 & -1.938544000 & 0.543095000 \\
\hline $\mathrm{H}$ & -3.018198000 & -2.861082000 & -0.186316000 \\
\hline $\mathrm{C}$ & -5.498789000 & -0.745581000 & 0.757514000 \\
\hline $\mathrm{H}$ & -5.427138000 & 1.398832000 & 0.573925000 \\
\hline $\mathrm{H}$ & -5.278432000 & -2.887121000 & 0.792497000 \\
\hline $\mathrm{H}$ & -6.498007000 & -0.759999000 & 1.182650000 \\
\hline $\mathrm{H}$ & 0.944164000 & -2.210417000 & -2.048460000 \\
\hline $\mathrm{C}$ & -0.473196000 & 3.278359000 & -0.892389000 \\
\hline $\mathrm{C}$ & 0.513105000 & 2.721707000 & 0.151435000 \\
\hline $\mathrm{H}$ & 0.080955000 & 3.689988000 & -1.743307000 \\
\hline $\mathrm{H}$ & -1.114817000 & 2.474925000 & -1.272624000 \\
\hline $\mathrm{H}$ & -1.118197000 & 4.065274000 & -0.481401000 \\
\hline $\mathrm{C}$ & -0.276989000 & 2.129123000 & 1.334541000 \\
\hline $\mathrm{C}$ & 1.392725000 & 3.875323000 & 0.666657000 \\
\hline $\mathrm{H}$ & 0.422315000 & 1.759175000 & 2.094833000 \\
\hline $\mathrm{H}$ & -0.941020000 & 2.863436000 & 1.807934000 \\
\hline $\mathrm{H}$ & -0.886934000 & 1.284251000 & 0.994514000 \\
\hline $\mathrm{H}$ & 0.808236000 & 4.673200000 & 1.141993000 \\
\hline $\mathrm{H}$ & 2.110738000 & 3.490489000 & 1.402068000 \\
\hline $\mathrm{H}$ & 1.956025000 & 4.310404000 & -0.167789000 \\
\hline $\mathrm{O}$ & 1.315507000 & 1.752345000 & -0.417116000 \\
\hline $\mathrm{H}$ & 0.886685000 & 0.597927000 & -1.333230000 \\
\hline
\end{tabular}




Na-- 3.071425000

TSC1-2 ${ }_{t \mathrm{BuONa}}$

\begin{tabular}{|c|c|c|c|}
\hline \multicolumn{4}{|c|}{ Coordinates (Angstroms) } \\
\hline & $\mathrm{X}$ & Y & $\mathrm{Z}$ \\
\hline $\mathrm{C}$ & 2.864417000 & -1.112297000 & 1.953218000 \\
\hline $\mathrm{C}$ & 1.784814000 & -1.072938000 & 1.074396000 \\
\hline $\mathrm{C}$ & 1.905590000 & -1.569647000 & -0.227544000 \\
\hline $\mathrm{C}$ & 3.128474000 & -2.127513000 & -0.619635000 \\
\hline $\mathrm{C}$ & 4.214250000 & -2.165867000 & 0.256382000 \\
\hline $\mathrm{C}$ & 4.084937000 & -1.649476000 & 1.545254000 \\
\hline $\mathrm{H}$ & 2.754671000 & -0.713322000 & 2.957464000 \\
\hline $\mathrm{H}$ & 0.846100000 & -0.623032000 & 1.381039000 \\
\hline $\mathrm{H}$ & 3.229791000 & -2.526545000 & -1.627301000 \\
\hline $\mathrm{H}$ & 5.153563000 & -2.609381000 & -0.062300000 \\
\hline $\mathrm{H}$ & 4.925275000 & -1.678711000 & 2.232564000 \\
\hline $\mathrm{C}$ & 0.822075000 & -1.300787000 & -1.277229000 \\
\hline $\mathrm{C}$ & -0.565184000 & -1.503801000 & -0.711024000 \\
\hline $\mathrm{H}$ & -0.723881000 & -2.430145000 & -0.158031000 \\
\hline $\mathrm{O}$ & 1.033501000 & -0.041322000 & -1.837764000 \\
\hline $\mathrm{C}$ & -1.550724000 & -0.620941000 & -0.872217000 \\
\hline $\mathrm{H}$ & -1.313904000 & 0.287539000 & -1.421416000 \\
\hline $\mathrm{C}$ & -2.920605000 & -0.728329000 & -0.342894000 \\
\hline $\mathrm{C}$ & -3.662706000 & 0.442138000 & -0.141742000 \\
\hline $\mathrm{C}$ & -3.516940000 & -1.954566000 & -0.023294000 \\
\hline $\mathrm{C}$ & -4.948650000 & 0.393367000 & 0.385106000 \\
\hline $\mathrm{H}$ & -3.214827000 & 1.401383000 & -0.389976000 \\
\hline $\mathrm{C}$ & -4.803118000 & -2.005598000 & 0.500325000 \\
\hline $\mathrm{H}$ & -2.974977000 & -2.878468000 & -0.204888000 \\
\hline $\mathrm{C}$ & -5.523625000 & -0.831578000 & 0.710438000 \\
\hline $\mathrm{H}$ & -5.501975000 & 1.315084000 & 0.540500000 \\
\hline $\mathrm{H}$ & -5.250401000 & -2.967001000 & 0.736321000 \\
\hline $\mathrm{H}$ & -6.529061000 & -0.873649000 & 1.118894000 \\
\hline $\mathrm{H}$ & 0.956422000 & -2.058086000 & -2.070251000 \\
\hline $\mathrm{C}$ & -0.503390000 & 3.251070000 & -0.875283000 \\
\hline $\mathrm{C}$ & 0.456620000 & 2.724350000 & 0.202555000 \\
\hline $\mathrm{H}$ & 0.066396000 & 3.702583000 & -1.694662000 \\
\hline $\mathrm{H}$ & -1.096606000 & 2.431868000 & -1.295183000 \\
\hline $\mathrm{H}$ & -1.194108000 & 4.002596000 & -0.474246000 \\
\hline $\mathrm{C}$ & -0.340537000 & 2.070427000 & 1.342957000 \\
\hline $\mathrm{C}$ & 1.282459000 & 3.890748000 & 0.760516000 \\
\hline $\mathrm{H}$ & 0.347796000 & 1.722015000 & 2.122102000 \\
\hline
\end{tabular}




\begin{tabular}{lrrr}
\hline $\mathrm{H}$ & -1.052322000 & 2.768684000 & 1.799760000 \\
$\mathrm{H}$ & -0.898609000 & 1.205241000 & 0.969775000 \\
$\mathrm{H}$ & 0.649974000 & 4.652585000 & 1.231467000 \\
$\mathrm{H}$ & 1.991636000 & 3.519481000 & 1.509880000 \\
$\mathrm{H}$ & 1.851903000 & 4.365179000 & -0.047247000 \\
$\mathrm{O}$ & 1.340876000 & 1.798147000 & -0.352126000 \\
$\mathrm{H}$ & 0.941953000 & 0.867366000 & -1.060259000 \\
$\mathrm{Na}$ & 3.071931000 & 0.753597000 & -0.946963000
\end{tabular}

C2 $2_{\text {tBuONa }}$

\begin{tabular}{lccc} 
& & Coordinates (Angstroms) & \\
& $\mathrm{X}$ & $\mathrm{Y}$ & $\mathrm{Z}$ \\
\hline $\mathrm{C}$ & 2.914637000 & -1.007857000 & 1.937775000 \\
$\mathrm{C}$ & 1.840920000 & -1.035976000 & 1.051574000 \\
$\mathrm{C}$ & 1.979456000 & -1.583294000 & -0.230007000 \\
$\mathrm{C}$ & 3.219912000 & -2.123919000 & -0.588712000 \\
$\mathrm{C}$ & 4.300399000 & -2.096747000 & 0.294471000 \\
$\mathrm{C}$ & 4.152298000 & -1.529622000 & 1.559429000 \\
$\mathrm{H}$ & 2.787098000 & -0.575474000 & 2.926423000 \\
$\mathrm{H}$ & 0.884142000 & -0.607020000 & 1.335840000 \\
$\mathrm{H}$ & 3.338164000 & -2.558865000 & -1.579526000 \\
$\mathrm{H}$ & 5.253698000 & -2.526781000 & -0.001031000 \\
$\mathrm{H}$ & 4.988721000 & -1.509620000 & 2.251869000 \\
$\mathrm{C}$ & 0.903283000 & -1.346614000 & -1.302797000 \\
$\mathrm{C}$ & -0.479793000 & -1.593040000 & -0.730451000 \\
$\mathrm{H}$ & -0.618809000 & -2.532377000 & -0.193361000 \\
$\mathrm{O}$ & 1.088099000 & -0.092446000 & -1.830033000 \\
$\mathrm{C}$ & -1.479429000 & -0.722288000 & -0.874908000 \\
$\mathrm{H}$ & -1.248477000 & 0.187274000 & -1.427525000 \\
$\mathrm{C}$ & -2.847368000 & -0.841458000 & -0.343883000 \\
$\mathrm{C}$ & -3.654114000 & 0.302091000 & -0.284846000 \\
$\mathrm{C}$ & -3.384748000 & -2.049060000 & 0.122368000 \\
$\mathrm{C}$ & -4.941906000 & 0.250292000 & 0.237800000 \\
$\mathrm{H}$ & -3.257618000 & 1.246662000 & -0.650123000 \\
$\mathrm{C}$ & -4.670648000 & -2.104067000 & 0.644543000 \\
$\mathrm{H}$ & -2.794074000 & -2.958757000 & 0.063382000 \\
$\mathrm{C}$ & -5.455559000 & -0.954053000 & 0.708199000 \\
$\mathrm{H}$ & -5.544912000 & 1.153198000 & 0.275929000 \\
$\mathrm{H}$ & -5.066962000 & -3.051455000 & 0.999237000 \\
$\mathrm{H}$ & -6.460839000 & -1.000188000 & 1.116584000 \\
$\mathrm{H}$ & 1.054855000 & -2.137159000 & -2.071002000 \\
$\mathrm{C}$ & -0.722960000 & 3.329445000 & -0.822550000 \\
$\mathrm{C}$ & 0.282965000 & 2.844109000 & 0.225476000
\end{tabular}




\begin{tabular}{lrrr}
\hline $\mathrm{H}$ & -0.207265000 & 3.882122000 & -1.614468000 \\
$\mathrm{H}$ & -1.234970000 & 2.478636000 & -1.284642000 \\
$\mathrm{H}$ & -1.479342000 & 3.982776000 & -0.373650000 \\
$\mathrm{C}$ & -0.424401000 & 2.053942000 & 1.331284000 \\
$\mathrm{C}$ & 1.045299000 & 4.025538000 & 0.821278000 \\
$\mathrm{H}$ & 0.305855000 & 1.715278000 & 2.074514000 \\
$\mathrm{H}$ & -1.174948000 & 2.669045000 & 1.839744000 \\
$\mathrm{H}$ & -0.927335000 & 1.173834000 & 0.917280000 \\
$\mathrm{H}$ & 0.364955000 & 4.712130000 & 1.335776000 \\
$\mathrm{H}$ & 1.787924000 & 3.670028000 & 1.544221000 \\
$\mathrm{H}$ & 1.566055000 & 4.578575000 & 0.032177000 \\
$\mathrm{O}$ & 1.255460000 & 2.023201000 & -0.403450000 \\
$\mathrm{H}$ & 0.849793000 & 1.227429000 & -0.884096000 \\
$\mathrm{Na}$ & 2.965125000 & 0.766376000 & -1.027450000 \\
\hline
\end{tabular}

\begin{tabular}{|c|c|c|c|}
\hline & $\mathrm{Coc}$ & lates (Angstrom & \\
\hline & $\mathrm{X}$ & Y & Z \\
\hline $\mathrm{C}$ & -2.969282000 & -1.645162000 & -1.118142000 \\
\hline $\mathrm{C}$ & -1.908124000 & -0.956144000 & -0.530237000 \\
\hline $\mathrm{C}$ & -2.111232000 & -0.127352000 & 0.582917000 \\
\hline $\mathrm{C}$ & -3.406944000 & -0.036915000 & 1.106739000 \\
\hline $\mathrm{C}$ & -4.471049000 & -0.724441000 & 0.524749000 \\
\hline $\mathrm{C}$ & -4.258365000 & -1.523851000 & -0.598435000 \\
\hline $\mathrm{H}$ & -2.787899000 & -2.287282000 & -1.976344000 \\
\hline $\mathrm{H}$ & -0.903460000 & -1.043170000 & -0.938895000 \\
\hline $\mathrm{H}$ & -3.578405000 & 0.602298000 & 1.970317000 \\
\hline $\mathrm{H}$ & -5.467607000 & -0.639018000 & 0.949909000 \\
\hline $\mathrm{H}$ & -5.085425000 & -2.062113000 & -1.052049000 \\
\hline $\mathrm{C}$ & -1.034596000 & 0.889893000 & 1.021957000 \\
\hline $\mathrm{C}$ & 0.338037000 & 0.241736000 & 0.967011000 \\
\hline $\mathrm{H}$ & 0.489173000 & -0.638659000 & 1.594577000 \\
\hline $\mathrm{O}$ & -1.167371000 & 2.002623000 & 0.247317000 \\
\hline $\mathrm{C}$ & 1.316379000 & 0.728769000 & 0.202285000 \\
\hline $\mathrm{H}$ & 1.080082000 & 1.641127000 & -0.344217000 \\
\hline $\mathrm{C}$ & 2.679545000 & 0.190175000 & 0.064108000 \\
\hline $\mathrm{C}$ & 3.694982000 & 1.012556000 & -0.440432000 \\
\hline $\mathrm{C}$ & 3.013448000 & -1.127643000 & 0.406801000 \\
\hline $\mathrm{C}$ & 4.998646000 & 0.547527000 & -0.577598000 \\
\hline $\mathrm{H}$ & 3.454634000 & 2.036515000 & -0.716325000 \\
\hline $\mathrm{C}$ & 4.314720000 & -1.595381000 & 0.271317000 \\
\hline $\mathrm{H}$ & 2.239768000 & -1.797962000 & 0.771014000 \\
\hline $\mathrm{C}$ & 5.315605000 & -0.759617000 & -0.220194000 \\
\hline
\end{tabular}




\begin{tabular}{lrrr}
\hline $\mathrm{H}$ & 5.768786000 & 1.209124000 & -0.964585000 \\
$\mathrm{H}$ & 4.547628000 & -2.621657000 & 0.541854000 \\
$\mathrm{H}$ & 6.331477000 & -1.128014000 & -0.330050000 \\
$\mathrm{H}$ & -1.230349000 & 1.092202000 & 2.101557000 \\
$\mathrm{Na}$ & -2.599096000 & 1.720975000 & -1.232944000 \\
\hline
\end{tabular}

\section{$\mathrm{C4}_{\text {tBuONa }}$}

\begin{tabular}{lrcc} 
& Coordinates (Angstroms) & \\
& & $\mathrm{X}$ & $\mathrm{Z}$ \\
\hline $\mathrm{C}$ & -4.639987000 & -1.673668000 & -0.002864000 \\
$\mathrm{C}$ & -3.518678000 & -1.059958000 & -0.565099000 \\
$\mathrm{C}$ & -2.454998000 & -0.625881000 & 0.231885000 \\
$\mathrm{C}$ & -2.564688000 & -0.782837000 & 1.619938000 \\
$\mathrm{C}$ & -3.682011000 & -1.387128000 & 2.182738000 \\
$\mathrm{C}$ & -4.721114000 & -1.848006000 & 1.372863000 \\
$\mathrm{H}$ & -5.458413000 & -1.993274000 & -0.642811000 \\
$\mathrm{H}$ & -3.465841000 & -0.851382000 & -1.631397000 \\
$\mathrm{H}$ & -1.779978000 & -0.407853000 & 2.271809000 \\
$\mathrm{H}$ & -3.748222000 & -1.496477000 & 3.261526000 \\
$\mathrm{H}$ & -5.592578000 & -2.319299000 & 1.817345000 \\
$\mathrm{C}$ & -1.226639000 & 0.018309000 & -0.437268000 \\
$\mathrm{C}$ & 0.049707000 & -0.599276000 & 0.122613000 \\
$\mathrm{H}$ & 0.111820000 & -0.738400000 & 1.200720000 \\
$\mathrm{O}$ & -1.282123000 & -0.050002000 & -1.820351000 \\
$\mathrm{C}$ & 1.109384000 & -0.908153000 & -0.638807000 \\
$\mathrm{H}$ & 1.024171000 & -0.723172000 & -1.711826000 \\
$\mathrm{C}$ & 2.406469000 & -1.411737000 & -0.149562000 \\
$\mathrm{C}$ & 3.209419000 & -2.200777000 & -0.981563000 \\
$\mathrm{C}$ & 2.877908000 & -1.106275000 & 1.133408000 \\
$\mathrm{C}$ & 4.428426000 & -2.701247000 & -0.537007000 \\
$\mathrm{H}$ & 2.872903000 & -2.421914000 & -1.992994000 \\
$\mathrm{C}$ & 4.095717000 & -1.605340000 & 1.579501000 \\
$\mathrm{H}$ & 2.298134000 & -0.446379000 & 1.772262000 \\
$\mathrm{C}$ & 4.873119000 & -2.409731000 & 0.749496000 \\
$\mathrm{H}$ & 5.034410000 & -3.315308000 & -1.196934000 \\
$\mathrm{H}$ & 4.447086000 & -1.352606000 & 2.575563000 \\
$\mathrm{H}$ & 5.826099000 & -2.795541000 & 1.098899000 \\
$\mathrm{C}$ & -1.172872000 & 1.075224000 & -0.081610000 \\
$\mathrm{H}$ & 0.645634000 & 4.969761000 & 0.491282000 \\
$\mathrm{H}$ & 0.632782000 & 3.431453000 & 0.349713000 \\
$\mathrm{H}$ & 0.799492000 & 5.424189000 & -0.495175000 \\
$\mathrm{H}$ & 1.431400000 & 5.332732000 & 1.168337000
\end{tabular}




\begin{tabular}{lrrr}
\hline $\mathrm{C}$ & 0.417914000 & 2.812831000 & 1.752450000 \\
$\mathrm{C}$ & 2.017767000 & 2.972233000 & -0.166447000 \\
$\mathrm{H}$ & 0.467862000 & 1.717959000 & 1.682169000 \\
$\mathrm{H}$ & 1.166270000 & 3.142289000 & 2.486130000 \\
$\mathrm{H}$ & -0.578075000 & 3.083118000 & 2.121776000 \\
$\mathrm{H}$ & 2.847763000 & 3.251141000 & 0.497414000 \\
$\mathrm{H}$ & 2.018015000 & 1.880018000 & -0.279860000 \\
$\mathrm{H}$ & 2.204466000 & 3.419561000 & -1.152483000 \\
$\mathrm{O}$ & -0.357516000 & 3.035183000 & -0.503909000 \\
$\mathrm{Na}$ & -1.171762000 & -2.214065000 & -1.731909000 \\
$\mathrm{Na}$ & -0.741733000 & 2.035564000 & -2.239743000 \\
\hline
\end{tabular}

TSC4-5 ${ }_{t \mathrm{BuONa}}$

$\begin{array}{lccc} & & \text { Coordinates (Angstroms) } & \\ & \mathrm{X} & \mathrm{Y} & \mathrm{Z} \\ \mathrm{C} & 4.043658000 & -2.447882000 & -0.054184000 \\ \mathrm{C} & 3.010248000 & -1.744062000 & 0.568713000 \\ \mathrm{C} & 2.025717000 & -1.074965000 & -0.178291000 \\ \mathrm{C} & 2.144866000 & -1.114739000 & -1.582176000 \\ \mathrm{C} & 3.172720000 & -1.811686000 & -2.197018000 \\ \mathrm{C} & 4.128959000 & -2.494446000 & -1.438718000 \\ \mathrm{H} & 4.792734000 & -2.949576000 & 0.554216000 \\ \mathrm{H} & 2.969937000 & -1.655553000 & 1.651241000 \\ \mathrm{H} & 1.436383000 & -0.565424000 & -2.196903000 \\ \mathrm{H} & 3.239681000 & -1.817895000 & -3.281985000 \\ \mathrm{H} & 4.934907000 & -3.034262000 & -1.926876000 \\ \mathrm{C} & 0.930938000 & -0.334604000 & 0.529755000 \\ \mathrm{C} & -0.396946000 & -0.540662000 & -0.043482000 \\ \mathrm{H} & -0.454807000 & -0.669222000 & -1.123681000 \\ \mathrm{O} & 0.979318000 & -0.443650000 & 1.921470000 \\ \mathrm{C} & -1.552753000 & -0.558246000 & 0.677672000 \\ \mathrm{H} & -1.468557000 & -0.407008000 & 1.754376000 \\ \mathrm{C} & -2.911762000 & -0.665045000 & 0.132855000 \\ \mathrm{C} & -3.979188000 & -0.990206000 & 0.984936000 \\ \mathrm{C} & -3.211890000 & -0.440664000 & -1.220740000 \\ \mathrm{C} & -5.278915000 & -1.111229000 & 0.507642000 \\ \mathrm{H} & -3.778350000 & -1.152366000 & 2.042352000 \\ \mathrm{C} & -4.509515000 & -0.566873000 & -1.700601000 \\ \mathrm{H} & -2.421835000 & -0.141649000 & -1.903872000 \\ \mathrm{C} & -5.552867000 & -0.906513000 & -0.842013000 \\ \mathrm{H} & -6.081599000 & -1.367066000 & 1.194132000 \\ \mathrm{H} & -4.709452000 & -0.385570000 & -2.753179000 \\ \mathrm{H} & -6.566738000 & -1.001191000 & -1.219290000 \\ & & & \\ \mathrm{H} & & \end{array}$




\begin{tabular}{lrrr}
\hline $\mathrm{H}$ & 1.184361000 & 0.994527000 & 0.248751000 \\
$\mathrm{C}$ & 1.398777000 & 4.440159000 & -0.429317000 \\
$\mathrm{C}$ & 0.710501000 & 3.070808000 & -0.380971000 \\
$\mathrm{H}$ & 1.530036000 & 4.832960000 & 0.586557000 \\
$\mathrm{H}$ & 2.388968000 & 4.343046000 & -0.886404000 \\
$\mathrm{H}$ & 0.815377000 & 5.168298000 & -1.006196000 \\
$\mathrm{C}$ & 0.560632000 & 2.525787000 & -1.811617000 \\
$\mathrm{C}$ & -0.678534000 & 3.208243000 & 0.265713000 \\
$\mathrm{H}$ & 0.015296000 & 1.575831000 & -1.804140000 \\
$\mathrm{H}$ & 0.011750000 & 3.223814000 & -2.454727000 \\
$\mathrm{H}$ & 1.550527000 & 2.349865000 & -2.245441000 \\
$\mathrm{H}$ & -1.346936000 & 3.853896000 & -0.316524000 \\
$\mathrm{H}$ & -1.148690000 & 2.222625000 & 0.363001000 \\
$\mathrm{H}$ & -0.581278000 & 3.647517000 & 1.269205000 \\
$\mathrm{O}$ & 1.514420000 & 2.210516000 & 0.379570000 \\
$\mathrm{Na}$ & 0.287665000 & -2.474110000 & 1.596355000 \\
$\mathrm{Na}$ & 1.476926000 & 1.577784000 & 2.432564000 \\
\hline
\end{tabular}

$\mathrm{C5}_{t \mathrm{BuONa}}$

\begin{tabular}{lccc} 
& \multicolumn{3}{c}{ Coordinates (Angstroms) } \\
& $\mathrm{X}$ & $\mathrm{Y}$ & $\mathrm{Z}$ \\
\hline $\mathrm{C}$ & -5.271483000 & -0.059382000 & 0.175961000 \\
$\mathrm{C}$ & -4.093894000 & -0.508214000 & -0.412976000 \\
$\mathrm{C}$ & -2.858837000 & -0.397335000 & 0.248918000 \\
$\mathrm{C}$ & -2.856469000 & 0.209464000 & 1.519940000 \\
$\mathrm{C}$ & -4.034266000 & 0.653341000 & 2.105460000 \\
$\mathrm{C}$ & -5.253584000 & 0.522018000 & 1.440609000 \\
$\mathrm{H}$ & -6.212422000 & -0.166459000 & -0.358212000 \\
$\mathrm{H}$ & -4.098363000 & -0.958924000 & -1.399821000 \\
$\mathrm{H}$ & -1.919821000 & 0.350470000 & 2.052164000 \\
$\mathrm{H}$ & -3.998767000 & 1.117939000 & 3.087484000 \\
$\mathrm{H}$ & -6.171646000 & 0.876499000 & 1.899855000 \\
$\mathrm{C}$ & -1.642654000 & -0.879594000 & -0.440133000 \\
$\mathrm{C}$ & -0.414513000 & -0.973969000 & 0.180578000 \\
$\mathrm{H}$ & -0.369296000 & -0.762954000 & 1.245225000 \\
$\mathrm{O}$ & -1.763925000 & -1.192848000 & -1.756612000 \\
$\mathrm{C}$ & 0.820189000 & -1.336949000 & -0.471243000 \\
$\mathrm{H}$ & 0.830547000 & -1.361054000 & -1.566061000 \\
$\mathrm{C}$ & 2.118336000 & -1.342794000 & 0.161493000 \\
$\mathrm{C}$ & 3.297839000 & -1.424942000 & -0.622800000 \\
$\mathrm{C}$ & 2.316319000 & -1.207141000 & 1.557847000 \\
$\mathrm{C}$ & 4.562583000 & -1.354777000 & -0.062549000 \\
$\mathrm{H}$ & 3.196785000 & -1.530354000 & -1.702231000
\end{tabular}




\begin{tabular}{lrrr}
\hline $\mathrm{C}$ & 3.589112000 & -1.131965000 & 2.111446000 \\
$\mathrm{H}$ & 1.457255000 & -1.167145000 & 2.222371000 \\
$\mathrm{C}$ & 4.729797000 & -1.198690000 & 1.315180000 \\
$\mathrm{H}$ & 5.433540000 & -1.413373000 & -0.711330000 \\
$\mathrm{H}$ & 3.688766000 & -1.020008000 & 3.188707000 \\
$\mathrm{H}$ & 5.720517000 & -1.136928000 & 1.754156000 \\
$\mathrm{H}$ & 1.326230000 & 0.622681000 & -0.908614000 \\
$\mathrm{C}$ & 0.788451000 & 2.240369000 & 0.990343000 \\
$\mathrm{C}$ & 1.540070000 & 2.496924000 & -0.317454000 \\
$\mathrm{H}$ & -0.294059000 & 2.207904000 & 0.818335000 \\
$\mathrm{H}$ & 1.098075000 & 1.283593000 & 1.424051000 \\
$\mathrm{H}$ & 1.000965000 & 3.029121000 & 1.719868000 \\
$\mathrm{C}$ & 3.050334000 & 2.399041000 & -0.099341000 \\
$\mathrm{C}$ & 1.150823000 & 3.841843000 & -0.920303000 \\
$\mathrm{H}$ & 3.579829000 & 2.551110000 & -1.045067000 \\
$\mathrm{H}$ & 3.387415000 & 3.155896000 & 0.617197000 \\
$\mathrm{H}$ & 3.319414000 & 1.412258000 & 0.293783000 \\
$\mathrm{H}$ & 1.442859000 & 4.660799000 & -0.255806000 \\
$\mathrm{H}$ & 1.645679000 & 3.985460000 & -1.886367000 \\
$\mathrm{H}$ & 0.065475000 & 3.900689000 & -1.070324000 \\
$\mathrm{O}$ & 1.151062000 & 1.512306000 & -1.292202000 \\
$\mathrm{Na}$ & -0.833319000 & -3.103028000 & -1.216940000 \\
$\mathrm{Na}$ & -0.844408000 & 0.786995000 & -2.037125000 \\
\hline$-------~$
\end{tabular}

TSC5-6 ${ }_{t \mathrm{BuONa}}$

\begin{tabular}{lccc} 
& & \\
& Coordinates (Angstroms) & \\
\hline $\mathrm{C}$ & -5.312452000 & -0.602616000 & 0.005759000 \\
$\mathrm{C}$ & -4.069605000 & -0.669149000 & -0.615301000 \\
$\mathrm{C}$ & -2.888414000 & -0.453048000 & 0.106569000 \\
$\mathrm{C}$ & -2.997441000 & -0.132349000 & 1.468127000 \\
$\mathrm{C}$ & -4.239371000 & -0.067181000 & 2.088398000 \\
$\mathrm{C}$ & -5.404897000 & -0.306779000 & 1.362890000 \\
$\mathrm{H}$ & -6.214647000 & -0.779795000 & -0.573829000 \\
$\mathrm{H}$ & -3.985671000 & -0.880837000 & -1.676106000 \\
$\mathrm{H}$ & -2.104674000 & 0.088933000 & 2.045917000 \\
$\mathrm{H}$ & -4.298624000 & 0.183409000 & 3.144030000 \\
$\mathrm{H}$ & -6.374423000 & -0.249325000 & 1.848914000 \\
$\mathrm{C}$ & -1.584762000 & -0.543385000 & -0.614495000 \\
$\mathrm{C}$ & -0.387571000 & -0.658629000 & 0.042223000 \\
$\mathrm{H}$ & -0.396138000 & -0.694117000 & 1.127033000 \\
$\mathrm{O}$ & -1.640589000 & -0.503071000 & -1.957285000 \\
$\mathrm{C}$ & 0.937217000 & -0.544039000 & -0.608511000
\end{tabular}




\begin{tabular}{|c|c|c|c|}
\hline $\mathrm{H}$ & 0.918587000 & -0.609731000 & -1.707932000 \\
\hline $\mathrm{C}$ & 2.113282000 & -1.239216000 & -0.047730000 \\
\hline $\mathrm{C}$ & 3.342461000 & -1.183804000 & -0.737450000 \\
\hline $\mathrm{C}$ & 2.107727000 & -1.922374000 & 1.181077000 \\
\hline $\mathrm{C}$ & 4.492690000 & -1.768427000 & -0.228695000 \\
\hline $\mathrm{H}$ & 3.385504000 & -0.644486000 & -1.682228000 \\
\hline $\mathrm{C}$ & 3.266059000 & -2.502693000 & 1.694644000 \\
\hline $\mathrm{H}$ & 1.184040000 & -2.005084000 & 1.746905000 \\
\hline $\mathrm{C}$ & 4.466759000 & -2.434243000 & 0.997728000 \\
\hline $\mathrm{H}$ & 5.422170000 & -1.697223000 & -0.787949000 \\
\hline $\mathrm{H}$ & 3.222142000 & -3.017426000 & 2.651291000 \\
\hline $\mathrm{H}$ & 5.367596000 & -2.886988000 & 1.400700000 \\
\hline $\mathrm{H}$ & 1.158893000 & 0.835139000 & -0.624939000 \\
\hline $\mathrm{C}$ & 1.027191000 & 2.243759000 & 1.556328000 \\
\hline $\mathrm{C}$ & 1.604726000 & 2.776151000 & 0.233409000 \\
\hline $\mathrm{H}$ & -0.069307000 & 2.265921000 & 1.522502000 \\
\hline $\mathrm{H}$ & 1.341847000 & 1.207156000 & 1.721947000 \\
\hline $\mathrm{H}$ & 1.360703000 & 2.841974000 & 2.412448000 \\
\hline $\mathrm{C}$ & 3.134688000 & 2.642150000 & 0.248422000 \\
\hline $\mathrm{C}$ & 1.207123000 & 4.243846000 & 0.047188000 \\
\hline $\mathrm{H}$ & 3.552431000 & 3.021739000 & -0.690484000 \\
\hline $\mathrm{H}$ & 3.584112000 & 3.198082000 & 1.080266000 \\
\hline $\mathrm{H}$ & 3.417141000 & 1.587688000 & 0.346011000 \\
\hline $\mathrm{H}$ & 1.605634000 & 4.878699000 & 0.847470000 \\
\hline $\mathrm{H}$ & 1.586402000 & 4.616408000 & -0.911025000 \\
\hline $\mathrm{H}$ & 0.113783000 & 4.342735000 & 0.048240000 \\
\hline $\mathrm{O}$ & 1.084417000 & 2.043534000 & -0.846314000 \\
\hline $\mathrm{Na}$ & -0.781697000 & 1.564281000 & -1.811800000 \\
\hline $\mathrm{Na}$ & -0.511082000 & -2.400137000 & -1.819461000 \\
\hline
\end{tabular}

\begin{tabular}{llcc} 
C6 $_{t \text { BuONa }}$ & & & \\
& & & \\
& Coordinates (Angstroms) & \\
\hline $\mathrm{C}$ & -4.434495000 & 0.853670000 & -1.248931000 \\
$\mathrm{C}$ & -3.692835000 & -0.125253000 & -0.596479000 \\
$\mathrm{C}$ & -2.408441000 & 0.149855000 & -0.113686000 \\
$\mathrm{C}$ & -1.870023000 & 1.431434000 & -0.309840000 \\
$\mathrm{C}$ & -2.616171000 & 2.404392000 & -0.971195000 \\
$\mathrm{C}$ & -3.898360000 & 2.125694000 & -1.436811000 \\
$\mathrm{H}$ & -5.430114000 & 0.622263000 & -1.618287000 \\
$\mathrm{H}$ & -4.084793000 & -1.127938000 & -0.460107000 \\
$\mathrm{H}$ & -0.854861000 & 1.689981000 & 0.011480000 \\
$\mathrm{H}$ & -2.179753000 & 3.387940000 & -1.122240000
\end{tabular}




\begin{tabular}{lrrr}
$-\mathrm{H}$ & -4.473348000 & 2.891169000 & -1.950800000 \\
$\mathrm{C}$ & -1.657733000 & -0.975038000 & 0.565021000 \\
$\mathrm{C}$ & -0.475739000 & -0.720403000 & 1.240211000 \\
$\mathrm{H}$ & -0.037667000 & 0.274935000 & 1.233876000 \\
$\mathrm{O}$ & -2.218463000 & -2.147066000 & 0.533554000 \\
$\mathrm{C}$ & 0.495015000 & -1.838033000 & 1.593637000 \\
$\mathrm{H}$ & -0.017309000 & -2.769111000 & 1.879567000 \\
$\mathrm{C}$ & 1.356061000 & -2.135420000 & 0.380444000 \\
$\mathrm{C}$ & 0.801947000 & -2.831476000 & -0.705206000 \\
$\mathrm{C}$ & 2.631815000 & -1.583009000 & 0.243337000 \\
$\mathrm{C}$ & 1.503529000 & -2.950101000 & -1.902016000 \\
$\mathrm{H}$ & -0.204354000 & -3.231498000 & -0.609168000 \\
$\mathrm{C}$ & 3.335393000 & -1.700931000 & -0.956079000 \\
$\mathrm{H}$ & 3.058548000 & -1.012429000 & 1.063093000 \\
$\mathrm{C}$ & 2.772464000 & -2.379202000 & -2.034384000 \\
$\mathrm{H}$ & 1.060273000 & -3.488216000 & -2.735304000 \\
$\mathrm{H}$ & 4.315455000 & -1.243205000 & -1.050226000 \\
$\mathrm{H}$ & 3.317087000 & -2.469106000 & -2.969516000 \\
$\mathrm{C}$ & 2.122023000 & 2.203990000 & 2.009583000 \\
$\mathrm{C}$ & 2.084464000 & 2.559700000 & 0.505449000 \\
$\mathrm{H}$ & 1.119865000 & 2.325736000 & 2.439147000 \\
$\mathrm{H}$ & 2.411873000 & 1.151468000 & 2.128731000 \\
$\mathrm{H}$ & 2.823016000 & 2.824026000 & 2.584568000 \\
$\mathrm{C}$ & 3.507443000 & 2.394305000 & -0.072580000 \\
$\mathrm{C}$ & 1.659820000 & 4.037431000 & 0.359915000 \\
$\mathrm{H}$ & 3.495970000 & 2.635780000 & -1.142630000 \\
$\mathrm{H}$ & 4.252090000 & 3.033964000 & 0.420322000 \\
$\mathrm{H}$ & 3.824915000 & 1.348725000 & 0.034576000 \\
$\mathrm{H}$ & 2.341688000 & 4.732997000 & 0.867797000 \\
$\mathrm{H}$ & 1.620368000 & 4.301297000 & -0.703465000 \\
$\mathrm{H}$ & 0.654283000 & 4.170659000 & 0.777167000 \\
$\mathrm{O}$ & 1.201306000 & 1.745284000 & -0.146531000 \\
$\mathrm{H}$ & 1.135789000 & -1.541321000 & 2.431674000 \\
$\mathrm{Na}$ & -2.297740000 & -1.973941000 & 2.684509000 \\
$\mathrm{Na}$ & 0.724210000 & 0.080896000 & -1.221671000 \\
\hline------1000
\end{tabular}

A1 $1_{\text {tBuOLi }}$

Coordinates (Angstroms)

\begin{tabular}{|c|c|c|c|}
\hline & $X$ & Y & $\mathrm{Z}$ \\
\hline $\mathrm{C}$ & -0.535678000 & 4.010471000 & -0.206201000 \\
\hline $\mathrm{C}$ & 0.019667000 & 2.749903000 & -0.002477000 \\
\hline $\mathrm{C}$ & -0.800809000 & 1.620898000 & 0.041289000 \\
\hline
\end{tabular}




\begin{tabular}{|c|c|c|c|}
\hline $\mathrm{C}$ & -2.183498000 & 1.759655000 & -0.118440000 \\
\hline $\mathrm{C}$ & -2.732829000 & 3.021083000 & -0.322807000 \\
\hline $\mathrm{C}$ & -1.911673000 & 4.146582000 & -0.367207000 \\
\hline $\mathrm{H}$ & 0.107387000 & 4.884970000 & -0.243204000 \\
\hline $\mathrm{H}$ & 1.095333000 & 2.640070000 & 0.114081000 \\
\hline $\mathrm{H}$ & -2.806342000 & 0.864549000 & -0.068284000 \\
\hline $\mathrm{H}$ & -3.805832000 & 3.126037000 & -0.453684000 \\
\hline $\mathrm{H}$ & -2.344707000 & 5.129275000 & -0.530104000 \\
\hline $\mathrm{C}$ & -0.236344000 & 0.246808000 & 0.343018000 \\
\hline $\mathrm{C}$ & 1.167838000 & 0.039934000 & -0.144283000 \\
\hline $\mathrm{H}$ & 1.302090000 & 0.246092000 & -1.204681000 \\
\hline $\mathrm{O}$ & -0.348642000 & -0.004363000 & 1.772344000 \\
\hline $\mathrm{C}$ & 2.192992000 & -0.354878000 & 0.613184000 \\
\hline $\mathrm{H}$ & 2.011049000 & -0.531538000 & 1.671927000 \\
\hline $\mathrm{C}$ & 3.579396000 & -0.573937000 & 0.164589000 \\
\hline $\mathrm{C}$ & 4.597552000 & -0.637310000 & 1.122954000 \\
\hline $\mathrm{C}$ & 3.927202000 & -0.728979000 & -1.183723000 \\
\hline $\mathrm{C}$ & 5.923797000 & -0.827975000 & 0.750538000 \\
\hline $\mathrm{H}$ & 4.344783000 & -0.528980000 & 2.175015000 \\
\hline $\mathrm{C}$ & 5.251164000 & -0.920324000 & -1.557875000 \\
\hline $\mathrm{H}$ & 3.154895000 & -0.719921000 & -1.947092000 \\
\hline $\mathrm{C}$ & 6.255683000 & -0.968080000 & -0.593307000 \\
\hline $\mathrm{H}$ & 6.697341000 & -0.869911000 & 1.511483000 \\
\hline $\mathrm{H}$ & 5.498905000 & -1.044019000 & -2.607981000 \\
\hline $\mathrm{H}$ & 7.289343000 & -1.122408000 & -0.887733000 \\
\hline $\mathrm{H}$ & -0.906818000 & -0.504487000 & -0.094716000 \\
\hline $\mathrm{H}$ & -0.033286000 & 0.786584000 & 2.229435000 \\
\hline $\mathrm{C}$ & -5.225558000 & -2.225576000 & 0.428345000 \\
\hline $\mathrm{C}$ & -3.835138000 & -2.023828000 & -0.201819000 \\
\hline $\mathrm{H}$ & -5.121507000 & -2.724896000 & 1.399244000 \\
\hline $\mathrm{H}$ & -5.698499000 & -1.251177000 & 0.596514000 \\
\hline $\mathrm{H}$ & -5.888716000 & -2.830834000 & -0.202845000 \\
\hline $\mathrm{C}$ & -4.000112000 & -1.331859000 & -1.569249000 \\
\hline $\mathrm{C}$ & -3.171945000 & -3.397607000 & -0.414389000 \\
\hline $\mathrm{H}$ & -3.015168000 & -1.158657000 & -2.019619000 \\
\hline $\mathrm{H}$ & -4.600173000 & -1.927858000 & -2.268035000 \\
\hline $\mathrm{H}$ & -4.489400000 & -0.358852000 & -1.440887000 \\
\hline $\mathrm{H}$ & -3.761333000 & -4.053527000 & -1.067670000 \\
\hline $\mathrm{H}$ & -2.179653000 & -3.263315000 & -0.861556000 \\
\hline $\mathrm{H}$ & -3.043177000 & -3.898577000 & 0.552835000 \\
\hline $\mathrm{O}$ & -3.051378000 & -1.248803000 & 0.623656000 \\
\hline $\mathrm{Li}$ & -2.098866000 & -0.831058000 & 1.928908000 \\
\hline
\end{tabular}

TSA1-2 ${ }_{\text {tBuOLi }}$

Coordinates (Angstroms) 


\begin{tabular}{|c|c|c|c|}
\hline & $\mathrm{X}$ & Y & Z \\
\hline $\mathrm{C}$ & -4.155479000 & -2.310726000 & -0.760466000 \\
\hline $\mathrm{C}$ & -3.033023000 & -1.559982000 & -1.113921000 \\
\hline $\mathrm{C}$ & -2.014103000 & -1.283045000 & -0.186099000 \\
\hline $\mathrm{C}$ & -2.176763000 & -1.794796000 & 1.116225000 \\
\hline $\mathrm{C}$ & -3.287429000 & -2.547705000 & 1.459919000 \\
\hline $\mathrm{C}$ & -4.292770000 & -2.810671000 & 0.525957000 \\
\hline $\mathrm{H}$ & -4.917613000 & -2.513049000 & -1.508547000 \\
\hline $\mathrm{H}$ & -2.931745000 & -1.225634000 & -2.144484000 \\
\hline $\mathrm{H}$ & -1.429153000 & -1.574881000 & 1.873372000 \\
\hline $\mathrm{H}$ & -3.379726000 & -2.927924000 & 2.473676000 \\
\hline $\mathrm{H}$ & -5.164821000 & -3.394146000 & 0.803864000 \\
\hline $\mathrm{C}$ & -0.886282000 & -0.383195000 & -0.513877000 \\
\hline $\mathrm{C}$ & 0.466149000 & -0.703541000 & -0.093291000 \\
\hline $\mathrm{H}$ & 0.532926000 & -1.264950000 & 0.836996000 \\
\hline $\mathrm{O}$ & -0.952659000 & 0.088503000 & -1.885820000 \\
\hline $\mathrm{C}$ & 1.599803000 & -0.329367000 & -0.720726000 \\
\hline $\mathrm{H}$ & 1.511662000 & 0.227171000 & -1.652450000 \\
\hline $\mathrm{C}$ & 2.974378000 & -0.597653000 & -0.282284000 \\
\hline $\mathrm{C}$ & 4.036802000 & -0.224398000 & -1.118732000 \\
\hline $\mathrm{C}$ & 3.298241000 & -1.209597000 & 0.939161000 \\
\hline $\mathrm{C}$ & 5.360213000 & -0.455550000 & -0.761549000 \\
\hline $\mathrm{H}$ & 3.815050000 & 0.255751000 & -2.069234000 \\
\hline $\mathrm{C}$ & 4.619633000 & -1.442557000 & 1.297440000 \\
\hline $\mathrm{H}$ & 2.509758000 & -1.504605000 & 1.625619000 \\
\hline $\mathrm{C}$ & 5.661251000 & -1.069099000 & 0.450387000 \\
\hline $\mathrm{H}$ & 6.158407000 & -0.154491000 & -1.434469000 \\
\hline $\mathrm{H}$ & 4.838075000 & -1.918213000 & 2.249644000 \\
\hline $\mathrm{H}$ & 6.693488000 & -1.252153000 & 0.733344000 \\
\hline $\mathrm{H}$ & -1.315272000 & 0.893562000 & 0.088949000 \\
\hline $\mathrm{H}$ & -0.671088000 & -0.614589000 & -2.490231000 \\
\hline $\mathrm{C}$ & -1.902865000 & 4.194988000 & 0.768346000 \\
\hline $\mathrm{C}$ & -1.024865000 & 2.987749000 & 0.440137000 \\
\hline $\mathrm{H}$ & -2.533373000 & 4.455098000 & -0.090659000 \\
\hline $\mathrm{H}$ & -2.557610000 & 3.964109000 & 1.614359000 \\
\hline $\mathrm{H}$ & -1.294976000 & 5.070484000 & 1.023390000 \\
\hline $\mathrm{C}$ & -0.162026000 & 2.607612000 & 1.648348000 \\
\hline $\mathrm{C}$ & -0.128679000 & 3.285762000 & -0.768623000 \\
\hline $\mathrm{H}$ & 0.482446000 & 1.755912000 & 1.403311000 \\
\hline $\mathrm{H}$ & 0.478706000 & 3.442115000 & 1.954016000 \\
\hline $\mathrm{H}$ & -0.802597000 & 2.330685000 & 2.491864000 \\
\hline $\mathrm{H}$ & 0.558622000 & 4.114978000 & -0.567668000 \\
\hline $\mathrm{H}$ & 0.464344000 & 2.401229000 & -1.025454000 \\
\hline \multirow[t]{2}{*}{$\mathrm{H}$} & -0.740106000 & 3.562298000 & -1.639478000 \\
\hline & & S117 & \\
\hline
\end{tabular}




\begin{tabular}{|c|c|c|c|}
\hline $\mathrm{O}$ & -1.894577000 & 1.907010000 & 0.133733000 \\
\hline $\mathrm{Li}$ & -2.413548000 & 1.260583000 & -1.497051000 \\
\hline
\end{tabular}

\begin{tabular}{|c|c|c|c|}
\hline \multicolumn{4}{|c|}{ Coordinates (Angstroms) } \\
\hline & $\mathrm{X}$ & Y & Z \\
\hline$\overline{\mathrm{C}}$ & -4.870458000 & -0.226936000 & -0.207457000 \\
\hline $\mathrm{C}$ & -3.708433000 & -0.629764000 & -0.867725000 \\
\hline $\mathrm{C}$ & -2.570359000 & -1.077515000 & -0.148162000 \\
\hline $\mathrm{C}$ & -2.685894000 & -1.110003000 & 1.266709000 \\
\hline $\mathrm{C}$ & -3.847061000 & -0.712379000 & 1.901631000 \\
\hline $\mathrm{C}$ & -4.954020000 & -0.255165000 & 1.176678000 \\
\hline $\mathrm{H}$ & -5.723232000 & 0.098495000 & -0.797836000 \\
\hline $\mathrm{H}$ & -3.695141000 & -0.661128000 & -1.952876000 \\
\hline $\mathrm{H}$ & -1.855419000 & -1.467167000 & 1.867795000 \\
\hline $\mathrm{H}$ & -3.896507000 & -0.761508000 & 2.986277000 \\
\hline $\mathrm{H}$ & -5.859911000 & 0.055119000 & 1.686905000 \\
\hline $\mathrm{C}$ & -1.338061000 & -1.365725000 & -0.839193000 \\
\hline $\mathrm{C}$ & -0.060932000 & -1.394196000 & -0.289236000 \\
\hline $\mathrm{H}$ & -0.036371000 & -1.499966000 & 0.792291000 \\
\hline $\mathrm{O}$ & -1.436245000 & -1.074736000 & -2.237660000 \\
\hline $\mathrm{C}$ & 1.151806000 & -1.210262000 & -0.936792000 \\
\hline $\mathrm{H}$ & 1.181449000 & -1.153402000 & -2.025699000 \\
\hline $\mathrm{C}$ & 2.452695000 & -1.132736000 & -0.284244000 \\
\hline $\mathrm{C}$ & 3.584743000 & -0.771566000 & -1.042504000 \\
\hline $\mathrm{C}$ & 2.656606000 & -1.342287000 & 1.094189000 \\
\hline $\mathrm{C}$ & 4.835415000 & -0.614590000 & -0.461064000 \\
\hline $\mathrm{H}$ & 3.466754000 & -0.604074000 & -2.111238000 \\
\hline $\mathrm{C}$ & 3.908991000 & -1.181121000 & 1.674473000 \\
\hline $\mathrm{H}$ & 1.827834000 & -1.650496000 & 1.725973000 \\
\hline $\mathrm{C}$ & 5.011050000 & -0.812944000 & 0.907009000 \\
\hline $\mathrm{H}$ & 5.681078000 & -0.332618000 & -1.083032000 \\
\hline $\mathrm{H}$ & 4.025238000 & -1.352921000 & 2.741416000 \\
\hline $\mathrm{H}$ & 5.987486000 & -0.688317000 & 1.364582000 \\
\hline $\mathrm{H}$ & 0.671038000 & 0.739553000 & -1.009725000 \\
\hline $\mathrm{H}$ & -0.762244000 & -1.583433000 & -2.702345000 \\
\hline $\mathrm{C}$ & 0.153746000 & 1.642691000 & 1.333577000 \\
\hline $\mathrm{C}$ & 0.425618000 & 2.412561000 & 0.039549000 \\
\hline $\mathrm{H}$ & -0.866534000 & 1.239662000 & 1.346308000 \\
\hline $\mathrm{H}$ & 0.859113000 & 0.811947000 & 1.442239000 \\
\hline $\mathrm{H}$ & 0.272777000 & 2.301841000 & 2.199831000 \\
\hline $\mathrm{C}$ & 1.892592000 & 2.829132000 & -0.047223000 \\
\hline $\mathrm{C}$ & -0.509523000 & 3.608147000 & -0.096013000 \\
\hline $\mathrm{H}$ & 2.081010000 & 3.364348000 & -0.983006000 \\
\hline
\end{tabular}




$\begin{array}{cccc} & & & \\ \mathrm{H} & 2.159870000 & 3.481955000 & 0.790238000 \\ \mathrm{H} & 2.543014000 & 1.947043000 & -0.013485000 \\ \mathrm{H} & -0.323979000 & 4.334488000 & 0.701020000 \\ \mathrm{H} & -0.358980000 & 4.104551000 & -1.059934000 \\ \mathrm{H} & -1.558392000 & 3.293048000 & -0.022368000 \\ \mathrm{O} & 0.130417000 & 1.567304000 & -1.094626000 \\ \mathrm{Li} & -1.514822000 & 0.703464000 & -1.423415000\end{array}$

TSA2-3 ${ }_{\text {tBuOLi }}$

\begin{tabular}{|c|c|c|c|}
\hline \multicolumn{4}{|c|}{ Coordinates (Angstroms) } \\
\hline & $\mathrm{X}$ & Y & $\mathrm{Z}$ \\
\hline $\mathrm{C}$ & -5.458784000 & -0.128002000 & -0.392857000 \\
\hline $\mathrm{C}$ & -4.188164000 & -0.015327000 & -0.948789000 \\
\hline $\mathrm{C}$ & -3.052361000 & -0.427347000 & -0.235173000 \\
\hline $\mathrm{C}$ & -3.237390000 & -0.941318000 & 1.060051000 \\
\hline $\mathrm{C}$ & -4.507643000 & -1.059880000 & 1.604824000 \\
\hline $\mathrm{C}$ & -5.629071000 & -0.653547000 & 0.883071000 \\
\hline $\mathrm{H}$ & -6.322157000 & 0.193361000 & -0.968620000 \\
\hline $\mathrm{H}$ & -4.068749000 & 0.383232000 & -1.950148000 \\
\hline $\mathrm{H}$ & -2.379065000 & -1.244857000 & 1.652057000 \\
\hline $\mathrm{H}$ & -4.622750000 & -1.466220000 & 2.605554000 \\
\hline $\mathrm{H}$ & -6.621038000 & -0.743506000 & 1.314793000 \\
\hline $\mathrm{C}$ & -1.714962000 & -0.294856000 & -0.823868000 \\
\hline $\mathrm{C}$ & -0.544125000 & -0.840417000 & -0.407838000 \\
\hline $\mathrm{H}$ & -0.609530000 & -1.494090000 & 0.458542000 \\
\hline $\mathrm{O}$ & -1.689709000 & 0.594223000 & -1.934469000 \\
\hline $\mathrm{C}$ & 0.766338000 & -0.579708000 & -0.964014000 \\
\hline $\mathrm{H}$ & 0.791370000 & -0.467097000 & -2.055869000 \\
\hline $\mathrm{C}$ & 1.939216000 & -1.323217000 & -0.464072000 \\
\hline $\mathrm{C}$ & 3.047991000 & -1.534328000 & -1.300953000 \\
\hline $\mathrm{C}$ & 2.051593000 & -1.765893000 & 0.864791000 \\
\hline $\mathrm{C}$ & 4.202214000 & -2.154875000 & -0.839585000 \\
\hline $\mathrm{H}$ & 2.996264000 & -1.200951000 & -2.335304000 \\
\hline $\mathrm{C}$ & 3.203643000 & -2.394862000 & 1.325514000 \\
\hline $\mathrm{H}$ & 1.231648000 & -1.604902000 & 1.560927000 \\
\hline $\mathrm{C}$ & 4.290001000 & -2.594675000 & 0.479188000 \\
\hline $\mathrm{H}$ & 5.037741000 & -2.301000000 & -1.519111000 \\
\hline $\mathrm{H}$ & 3.253225000 & -2.724330000 & 2.360102000 \\
\hline $\mathrm{H}$ & 5.190553000 & -3.080604000 & 0.841872000 \\
\hline $\mathrm{H}$ & 0.954589000 & 0.820700000 & -0.628016000 \\
\hline $\mathrm{H}$ & -1.038605000 & 0.270649000 & -2.572590000 \\
\hline $\mathrm{C}$ & 1.653468000 & 1.839872000 & 1.800722000 \\
\hline $\mathrm{C}$ & 1.795568000 & 2.518643000 & 0.433392000 \\
\hline $\mathrm{H}$ & 0.639552000 & 1.984684000 & 2.194889000 \\
\hline $\mathrm{H}$ & 1.843128000 & 0.765256000 & 1.712121000 \\
\hline
\end{tabular}




\begin{tabular}{cccc}
\hline $\mathrm{H}$ & 2.364120000 & 2.251295000 & 2.526359000 \\
$\mathrm{C}$ & 3.195308000 & 2.268891000 & -0.138360000 \\
$\mathrm{C}$ & 1.528142000 & 4.019529000 & 0.555080000 \\
$\mathrm{H}$ & 3.288780000 & 2.740302000 & -1.122257000 \\
$\mathrm{H}$ & 3.969718000 & 2.678434000 & 0.519923000 \\
$\mathrm{H}$ & 3.375746000 & 1.194297000 & -0.252084000 \\
$\mathrm{H}$ & 2.250947000 & 4.499234000 & 1.224406000 \\
$\mathrm{H}$ & 1.595396000 & 4.497697000 & -0.427817000 \\
$\mathrm{H}$ & 0.523787000 & 4.197989000 & 0.960631000 \\
$\mathrm{O}$ & 0.831167000 & 1.989766000 & -0.460466000 \\
$\mathrm{Li}$ & -0.940742000 & 1.848805000 & -0.629209000
\end{tabular}

$\underline{\mathbf{A}}_{\text {B }} \underline{\text { unOLi }}_{-}$

Coordinates (Angstroms)

\begin{tabular}{|c|c|c|c|}
\hline & $X$ & Y & Z \\
\hline $\mathrm{C}$ & -4.828013000 & -0.667332000 & -0.269772000 \\
\hline $\mathrm{C}$ & -3.713624000 & -1.437130000 & 0.044354000 \\
\hline $\mathrm{C}$ & -2.437167000 & -0.859809000 & 0.065004000 \\
\hline $\mathrm{C}$ & -2.285142000 & 0.497379000 & -0.240663000 \\
\hline $\mathrm{C}$ & -3.407735000 & 1.257788000 & -0.554832000 \\
\hline $\mathrm{C}$ & -4.676246000 & 0.683755000 & -0.570244000 \\
\hline $\mathrm{H}$ & -5.814402000 & -1.121510000 & -0.270828000 \\
\hline $\mathrm{H}$ & -3.843541000 & -2.483962000 & 0.310526000 \\
\hline $\mathrm{H}$ & -1.289576000 & 0.954098000 & -0.266948000 \\
\hline $\mathrm{H}$ & -3.281357000 & 2.308171000 & -0.800970000 \\
\hline $\mathrm{H}$ & -5.545469000 & 1.285868000 & -0.818641000 \\
\hline $\mathrm{C}$ & -1.264049000 & -1.702051000 & 0.404755000 \\
\hline $\mathrm{C}$ & -0.261555000 & -1.322981000 & 1.210600000 \\
\hline $\mathrm{H}$ & -0.301745000 & -0.325920000 & 1.638514000 \\
\hline $\mathrm{O}$ & -1.259345000 & -2.969877000 & -0.116261000 \\
\hline $\mathrm{C}$ & 0.935877000 & -2.190449000 & 1.526331000 \\
\hline $\mathrm{H}$ & 0.678836000 & -3.247202000 & 1.412188000 \\
\hline $\mathrm{C}$ & 2.069788000 & -1.828235000 & 0.589547000 \\
\hline $\mathrm{C}$ & 2.087570000 & -2.343182000 & -0.715932000 \\
\hline $\mathrm{C}$ & 3.007588000 & -0.849639000 & 0.934409000 \\
\hline $\mathrm{C}$ & 3.008239000 & -1.875690000 & -1.652323000 \\
\hline $\mathrm{H}$ & 1.351129000 & -3.091438000 & -0.995629000 \\
\hline $\mathrm{C}$ & 3.929455000 & -0.381064000 & -0.002230000 \\
\hline $\mathrm{H}$ & 2.989959000 & -0.422050000 & 1.933061000 \\
\hline $\mathrm{C}$ & 3.928435000 & -0.887189000 & -1.299244000 \\
\hline $\mathrm{H}$ & 3.004012000 & -2.280433000 & -2.659960000 \\
\hline $\mathrm{H}$ & 4.632610000 & 0.396292000 & 0.278178000 \\
\hline $\mathrm{H}$ & 4.634854000 & -0.512144000 & -2.032452000 \\
\hline $\mathrm{C}$ & 0.563644000 & 2.678946000 & 1.623192000 \\
\hline
\end{tabular}




$\begin{array}{cccc}\text { C } & 0.804715000 & 2.850705000 & 0.108989000 \\ \mathrm{H} & -0.448614000 & 2.292420000 & 1.795284000 \\ \mathrm{H} & 1.279947000 & 1.951758000 & 2.028772000 \\ \mathrm{H} & 0.672960000 & 3.617526000 & 2.181508000 \\ \mathrm{C} & 2.234559000 & 3.380364000 & -0.112644000 \\ \mathrm{C} & -0.201409000 & 3.879734000 & -0.442365000 \\ \mathrm{H} & 2.420473000 & 3.498246000 & -1.186339000 \\ \mathrm{H} & 2.411173000 & 4.345398000 & 0.380050000 \\ \mathrm{H} & 2.958920000 & 2.654342000 & 0.278972000 \\ \mathrm{H} & -0.090677000 & 4.866087000 & 0.026089000 \\ \mathrm{H} & -0.062115000 & 3.987950000 & -1.523468000 \\ \mathrm{H} & -1.225749000 & 3.530475000 & -0.265181000 \\ \mathrm{O} & 0.642440000 & 1.641856000 & -0.527843000 \\ \mathrm{H} & 1.243949000 & -2.033639000 & 2.565071000 \\ \mathrm{H} & -1.944553000 & -3.020171000 & -0.794513000 \\ \mathrm{Li} & 1.178214000 & 0.056825000 & -0.647346000\end{array}$

\begin{tabular}{|c|c|c|c|}
\hline \multicolumn{4}{|c|}{$\mathbf{A 1}_{\text {TBD }}$} \\
\hline & \multicolumn{3}{|c|}{ Coordinates (Angstroms) } \\
\hline & $\mathrm{X}$ & Y & Z \\
\hline $\mathrm{C}$ & 2.907551000 & -0.661748000 & 2.423328000 \\
\hline $\mathrm{C}$ & 3.790940000 & -1.906286000 & 2.442490000 \\
\hline $\mathrm{C}$ & 4.890073000 & -1.738056000 & 1.403969000 \\
\hline $\mathrm{N}$ & 2.353653000 & -0.409425000 & 1.110591000 \\
\hline $\mathrm{H}$ & 3.497443000 & 0.203638000 & 2.770254000 \\
\hline $\mathrm{H}$ & 2.080632000 & -0.774495000 & 3.133319000 \\
\hline $\mathrm{H}$ & 3.180306000 & -2.782137000 & 2.193788000 \\
\hline $\mathrm{H}$ & 4.236923000 & -2.073596000 & 3.428480000 \\
\hline $\mathrm{H}$ & 5.657344000 & -1.038314000 & 1.768773000 \\
\hline $\mathrm{H}$ & 5.391086000 & -2.698004000 & 1.220726000 \\
\hline $\mathrm{N}$ & 4.351688000 & -1.259191000 & 0.139304000 \\
\hline $\mathrm{C}$ & 5.143364000 & -1.602103000 & -1.030753000 \\
\hline $\mathrm{C}$ & 4.682750000 & -0.858170000 & -2.274634000 \\
\hline $\mathrm{C}$ & 3.167840000 & -0.957769000 & -2.362460000 \\
\hline $\mathrm{N}$ & 2.614074000 & -0.372408000 & -1.164102000 \\
\hline $\mathrm{C}$ & 3.090962000 & -0.696468000 & 0.086408000 \\
\hline $\mathrm{H}$ & 5.089359000 & -2.689269000 & -1.207908000 \\
\hline $\mathrm{H}$ & 6.194161000 & -1.367509000 & -0.814904000 \\
\hline $\mathrm{H}$ & 4.965968000 & 0.197621000 & -2.210183000 \\
\hline $\mathrm{H}$ & 5.161727000 & -1.291844000 & -3.157849000 \\
\hline $\mathrm{H}$ & 2.787763000 & -0.398706000 & -3.222453000 \\
\hline $\mathrm{H}$ & 2.871261000 & -2.012157000 & -2.497706000 \\
\hline $\mathrm{H}$ & 1.618080000 & -0.165879000 & -1.173852000 \\
\hline
\end{tabular}




\begin{tabular}{lrrr}
$\mathrm{C}$ & -1.240410000 & 4.299453000 & -0.300630000 \\
$\mathrm{C}$ & -1.477332000 & 2.927647000 & -0.319766000 \\
$\mathrm{C}$ & -0.484006000 & 2.032024000 & 0.083251000 \\
$\mathrm{C}$ & 0.753303000 & 2.524814000 & 0.505241000 \\
$\mathrm{C}$ & 0.986365000 & 3.896092000 & 0.528599000 \\
$\mathrm{C}$ & -0.007574000 & 4.785574000 & 0.125524000 \\
$\mathrm{H}$ & -2.020136000 & 4.988582000 & -0.612310000 \\
$\mathrm{H}$ & -2.445195000 & 2.546797000 & -0.639004000 \\
$\mathrm{H}$ & 1.520060000 & 1.815138000 & 0.810502000 \\
$\mathrm{H}$ & 1.948365000 & 4.273761000 & 0.863723000 \\
$\mathrm{H}$ & 0.177034000 & 5.855948000 & 0.146814000 \\
$\mathrm{C}$ & -0.701042000 & 0.530740000 & -0.013797000 \\
$\mathrm{C}$ & -2.107539000 & 0.125766000 & 0.340830000 \\
$\mathrm{H}$ & -2.455318000 & 0.500478000 & 1.302575000 \\
$\mathrm{O}$ & -0.320854000 & 0.066655000 & -1.315733000 \\
$\mathrm{C}$ & -2.892805000 & -0.624563000 & -0.434082000 \\
$\mathrm{H}$ & -2.481704000 & -0.964308000 & -1.383229000 \\
$\mathrm{C}$ & -4.272572000 & -1.054101000 & -0.147947000 \\
$\mathrm{C}$ & -5.002009000 & -1.690552000 & -1.159477000 \\
$\mathrm{C}$ & -4.897597000 & -0.860476000 & 1.091636000 \\
$\mathrm{C}$ & -6.311356000 & -2.109940000 & -0.950322000 \\
$\mathrm{H}$ & -4.531858000 & -1.855596000 & -2.125958000 \\
$\mathrm{C}$ & -6.205024000 & -1.278285000 & 1.303440000 \\
$\mathrm{H}$ & -4.355443000 & -0.387045000 & 1.904861000 \\
$\mathrm{C}$ & -6.919757000 & -1.903914000 & 0.283447000 \\
$\mathrm{H}$ & -6.855208000 & -2.599712000 & -1.752963000 \\
$\mathrm{H}$ & -6.668796000 & -1.119555000 & 2.272783000 \\
$\mathrm{H}$ & -7.941372000 & -2.230885000 & 0.452639000 \\
$\mathrm{H}$ & 0.007698000 & 0.037247000 & 0.662898000 \\
$\mathrm{H}$ & -0.664005000 & 0.705654000 & -1.954295000 \\
\hline---- & & \\
& & &
\end{tabular}

TSA1-2

\begin{tabular}{lccc} 
& & Coordinates (Angstroms) & Z \\
\hline $\mathrm{C}$ & 0.427570000 & -0.782033000 & 2.414746000 \\
$\mathrm{C}$ & -0.795074000 & 0.118632000 & 2.537761000 \\
$\mathrm{C}$ & -2.047319000 & -0.708125000 & 2.293704000 \\
$\mathrm{~N}$ & 0.450720000 & -1.451104000 & 1.126329000 \\
$\mathrm{H}$ & 0.439364000 & -1.527062000 & 3.221263000 \\
$\mathrm{H}$ & 1.353855000 & -0.204074000 & 2.492192000 \\
$\mathrm{H}$ & -0.737803000 & 0.913807000 & 1.788953000 \\
$\mathrm{H}$ & -0.852471000 & 0.582677000 & 3.526789000 \\
$\mathrm{H}$ & -2.293610000 & -1.318083000 & 3.173472000
\end{tabular}




$\begin{array}{lrrr}\mathrm{H} & -2.897020000 & -0.044617000 & 2.096328000 \\ \mathrm{~N} & -1.902284000 & -1.587559000 & 1.132337000 \\ \mathrm{C} & -3.155213000 & -1.915023000 & 0.457895000 \\ \mathrm{C} & -2.958827000 & -2.981932000 & -0.606905000 \\ \mathrm{C} & -1.787610000 & -2.582601000 & -1.489717000 \\ \mathrm{~N} & -0.620248000 & -2.417010000 & -0.648477000 \\ \mathrm{C} & -0.694379000 & -1.787140000 & 0.553651000 \\ \mathrm{H} & -3.564314000 & -0.998681000 & 0.008874000 \\ \mathrm{H} & -3.864245000 & -2.258301000 & 1.220008000 \\ \mathrm{H} & -2.749890000 & -3.950394000 & -0.140290000 \\ \mathrm{H} & -3.874439000 & -3.077847000 & -1.197022000 \\ \mathrm{H} & -1.566410000 & -3.359166000 & -2.226282000 \\ \mathrm{H} & -2.025948000 & -1.657137000 & -2.034726000 \\ \mathrm{H} & 0.268209000 & -2.257648000 & -1.124098000 \\ \mathrm{C} & 4.899592000 & 1.424745000 & 0.926848000 \\ \mathrm{C} & 3.573740000 & 1.176217000 & 0.598882000 \\ \mathrm{C} & 3.227702000 & 0.244181000 & -0.397141000 \\ \mathrm{C} & 4.278676000 & -0.438901000 & -1.028058000 \\ \mathrm{C} & 5.606224000 & -0.189157000 & -0.695804000 \\ \mathrm{H} & -2.742725000 & 4.184387000 & 1.081901000 \\ \mathrm{H} & 5.931492000 & 0.746381000 & 0.280697000 \\ \mathrm{H} & 5.129424000 & 2.156522000 & 1.697137000 \\ \mathrm{H} & 1.610402000 & 3.541116000 & 0.044348000 \\ \mathrm{H} & 2.795886000 & 1.716216000 & 1.133692000 \\ \mathrm{H} & 4.038379000 & -1.180056000 & -1.782432000 \\ \mathrm{H} & 6.394573000 & -0.734383000 & -1.208844000 \\ \mathrm{H} & 6.968181000 & 0.941336000 & 0.538143000 \\ \mathrm{C} & 1.821416000 & -0.067193000 & -0.729840000 \\ \mathrm{C} & 0.790046000 & 0.933547000 & -0.656638000 \\ \mathrm{H} & 0.969362000 & 1.725804000 & 0.068820000 \\ \mathrm{O} & 1.664242000 & -1.005004000 & -1.794519000 \\ \mathrm{C} & -0.428578000 & 0.864708000 & -1.251558000 \\ \mathrm{H} & -0.595358000 & 0.073337000 & -1.979119000 \\ \mathrm{C} & -1.604631000 & 1.665341000 & -0.923987000 \\ \mathrm{H} & -2.839969000 & 1.339765000 & -1.514305000 \\ \mathrm{H} & -1.606839000 & 2.718655000 & 0.009242000 \\ \mathrm{H} & -4.013601000 & 2.001149000 & -1.173125000 \\ \mathrm{H} & -2.871033000 & 0.548070000 & -2.260551000 \\ \mathrm{H} & -3.67555454000 & 3.379142000 & 0.353026000 \\ \mathrm{H} & & -0.9137900000 & 0.474507000 \\ \mathrm{H} & -0.521666000 & -2.633931000\end{array}$




\begin{tabular}{|c|c|c|c|}
\hline \multicolumn{4}{|c|}{$\mathrm{A} 2_{\text {TBD }}$} \\
\hline & $X$ & Y & Z \\
\hline $\mathrm{C}$ & -2.459959000 & 1.167910000 & -1.716186000 \\
\hline $\mathrm{C}$ & -3.037426000 & 1.685860000 & -0.410066000 \\
\hline $\mathrm{C}$ & -2.247704000 & 2.911642000 & 0.021751000 \\
\hline $\mathrm{N}$ & -1.027134000 & 0.951243000 & -1.566808000 \\
\hline $\mathrm{H}$ & -2.644030000 & 1.879077000 & -2.531920000 \\
\hline $\mathrm{H}$ & -2.904734000 & 0.207991000 & -1.981704000 \\
\hline $\mathrm{H}$ & -2.973041000 & 0.901818000 & 0.351972000 \\
\hline $\mathrm{H}$ & -4.090257000 & 1.950630000 & -0.536528000 \\
\hline $\mathrm{H}$ & -2.453053000 & 3.766759000 & -0.636592000 \\
\hline $\mathrm{H}$ & -2.522437000 & 3.203537000 & 1.041021000 \\
\hline $\mathrm{N}$ & -0.810190000 & 2.637189000 & 0.026202000 \\
\hline $\mathrm{C}$ & -0.006913000 & 3.440102000 & 0.946631000 \\
\hline $\mathrm{C}$ & 1.483386000 & 3.298026000 & 0.670357000 \\
\hline $\mathrm{C}$ & 1.826922000 & 1.823390000 & 0.537268000 \\
\hline $\mathrm{N}$ & 0.994358000 & 1.257046000 & -0.510923000 \\
\hline $\mathrm{C}$ & -0.285116000 & 1.605764000 & -0.665400000 \\
\hline $\mathrm{H}$ & -0.236098000 & 3.134140000 & 1.976682000 \\
\hline $\mathrm{H}$ & -0.321777000 & 4.483613000 & 0.834085000 \\
\hline $\mathrm{H}$ & 1.744786000 & 3.818116000 & -0.257320000 \\
\hline $\mathrm{H}$ & 2.047698000 & 3.756808000 & 1.486421000 \\
\hline $\mathrm{H}$ & 2.868887000 & 1.665874000 & 0.251148000 \\
\hline $\mathrm{H}$ & 1.666733000 & 1.295032000 & 1.486529000 \\
\hline $\mathrm{H}$ & 1.246966000 & 0.291571000 & -0.806538000 \\
\hline $\mathrm{C}$ & -3.249364000 & -1.333409000 & 2.233880000 \\
\hline $\mathrm{C}$ & -2.050718000 & -1.486678000 & 1.552930000 \\
\hline $\mathrm{C}$ & -2.019304000 & -1.823994000 & 0.179085000 \\
\hline $\mathrm{C}$ & -3.268685000 & -1.988678000 & -0.459653000 \\
\hline $\mathrm{C}$ & -4.464154000 & -1.816981000 & 0.227194000 \\
\hline $\mathrm{C}$ & -4.473091000 & -1.485943000 & 1.580393000 \\
\hline $\mathrm{H}$ & -3.227816000 & -1.079214000 & 3.291055000 \\
\hline $\mathrm{H}$ & -1.119721000 & -1.328499000 & 2.089629000 \\
\hline $\mathrm{H}$ & -3.281471000 & -2.258614000 & -1.510413000 \\
\hline $\mathrm{H}$ & -5.404141000 & -1.953360000 & -0.302343000 \\
\hline $\mathrm{H}$ & -5.408994000 & -1.355184000 & 2.114805000 \\
\hline $\mathrm{C}$ & -0.791006000 & -1.919855000 & -0.573591000 \\
\hline $\mathrm{C}$ & 0.493213000 & -1.819240000 & -0.099103000 \\
\hline $\mathrm{H}$ & 0.598529000 & -1.851540000 & 0.983006000 \\
\hline $\mathrm{O}$ & -0.971976000 & -1.843969000 & -1.975884000 \\
\hline $\mathrm{C}$ & 1.659271000 & -1.605965000 & -0.852621000 \\
\hline
\end{tabular}




\begin{tabular}{lrrr}
$\mathrm{H}$ & 1.598019000 & -1.605780000 & -1.940755000 \\
$\mathrm{C}$ & 2.969928000 & -1.354568000 & -0.295435000 \\
$\mathrm{C}$ & 4.015548000 & -0.903413000 & -1.136014000 \\
$\mathrm{C}$ & 3.274669000 & -1.435913000 & 1.083797000 \\
$\mathrm{C}$ & 5.254712000 & -0.532251000 & -0.635519000 \\
$\mathrm{H}$ & 3.828488000 & -0.831425000 & -2.205932000 \\
$\mathrm{C}$ & 4.516473000 & -1.060320000 & 1.579708000 \\
$\mathrm{H}$ & 2.528619000 & -1.809360000 & 1.780023000 \\
$\mathrm{C}$ & 5.520819000 & -0.595310000 & 0.732724000 \\
$\mathrm{H}$ & 6.023418000 & -0.187062000 & -1.323098000 \\
$\mathrm{H}$ & 4.704415000 & -1.141847000 & 2.647961000 \\
$\mathrm{H}$ & 6.490149000 & -0.304784000 & 1.126116000 \\
$\mathrm{H}$ & -0.685272000 & 0.043655000 & -1.893253000 \\
$\mathrm{H}$ & -0.366310000 & -2.479589000 & -2.379075000 \\
\hline
\end{tabular}

TSA2-3 ${ }_{\text {TBD }}$

\begin{tabular}{|c|c|c|c|}
\hline & & inates (Angstrom & \\
\hline & $X$ & $\mathrm{Y}$ & $\mathrm{Z}$ \\
\hline $\bar{C}$ & -2.456417000 & 1.479458000 & -1.427967000 \\
\hline $\mathrm{C}$ & -2.847032000 & 1.929799000 & -0.030300000 \\
\hline $\mathrm{C}$ & -1.990468000 & 3.126685000 & 0.353466000 \\
\hline $\mathrm{N}$ & -1.017805000 & 1.275908000 & -1.502203000 \\
\hline $\mathrm{H}$ & -2.767544000 & 2.227544000 & -2.169394000 \\
\hline $\mathrm{H}$ & -2.937689000 & 0.531187000 & -1.677101000 \\
\hline $\mathrm{H}$ & -2.688959000 & 1.106902000 & 0.675829000 \\
\hline $\mathrm{H}$ & -3.905029000 & 2.204025000 & 0.002017000 \\
\hline $\mathrm{H}$ & -2.264222000 & 4.009008000 & -0.243431000 \\
\hline $\mathrm{H}$ & -2.143790000 & 3.383105000 & 1.407994000 \\
\hline $\mathrm{N}$ & -0.572977000 & 2.828308000 & 0.180393000 \\
\hline $\mathrm{C}$ & 0.354906000 & 3.601276000 & 0.998058000 \\
\hline $\mathrm{C}$ & 1.786593000 & 3.438175000 & 0.508756000 \\
\hline $\mathrm{C}$ & 2.066951000 & 1.958210000 & 0.294209000 \\
\hline $\mathrm{N}$ & 1.110751000 & 1.409663000 & -0.652587000 \\
\hline $\mathrm{C}$ & -0.140747000 & 1.832370000 & -0.636698000 \\
\hline $\mathrm{H}$ & 0.268723000 & 3.279895000 & 2.046082000 \\
\hline $\mathrm{H}$ & 0.047662000 & 4.653249000 & 0.951337000 \\
\hline $\mathrm{H}$ & 1.921773000 & 3.973811000 & -0.437342000 \\
\hline $\mathrm{H}$ & 2.475394000 & 3.867115000 & 1.242207000 \\
\hline $\mathrm{H}$ & 3.068373000 & 1.792751000 & -0.110625000 \\
\hline $\mathrm{H}$ & 2.019439000 & 1.418274000 & 1.250928000 \\
\hline $\mathrm{H}$ & 1.295678000 & 0.238198000 & -0.873497000 \\
\hline $\mathrm{C}$ & -3.481751000 & -1.643009000 & 2.107090000 \\
\hline $\mathrm{C}$ & -2.263962000 & -1.597932000 & 1.442634000 \\
\hline
\end{tabular}




\begin{tabular}{lrrr}
$\mathrm{C}$ & -2.183995000 & -1.810302000 & 0.053170000 \\
$\mathrm{C}$ & -3.385251000 & -2.048716000 & -0.636043000 \\
$\mathrm{C}$ & -4.604301000 & -2.079235000 & 0.032416000 \\
$\mathrm{C}$ & -4.664830000 & -1.879266000 & 1.408177000 \\
$\mathrm{H}$ & -3.509175000 & -1.478960000 & 3.181122000 \\
$\mathrm{H}$ & -1.362393000 & -1.378373000 & 2.006895000 \\
$\mathrm{H}$ & -3.347705000 & -2.212024000 & -1.707522000 \\
$\mathrm{H}$ & -5.515819000 & -2.267307000 & -0.528755000 \\
$\mathrm{H}$ & -5.617135000 & -1.904959000 & 1.929075000 \\
$\mathrm{C}$ & -0.920514000 & -1.720295000 & -0.675030000 \\
$\mathrm{C}$ & 0.325807000 & -1.641055000 & -0.155154000 \\
$\mathrm{H}$ & 0.411037000 & -1.799910000 & 0.916194000 \\
$\mathrm{O}$ & -1.092323000 & -1.542086000 & -2.057617000 \\
$\mathrm{C}$ & 1.518761000 & -1.259794000 & -0.876416000 \\
$\mathrm{H}$ & 1.524617000 & -1.456212000 & -1.952793000 \\
$\mathrm{C}$ & 2.853249000 & -1.358582000 & -0.273021000 \\
$\mathrm{C}$ & 3.997650000 & -1.345288000 & -1.094522000 \\
$\mathrm{C}$ & 3.079311000 & -1.355045000 & 1.117162000 \\
$\mathrm{C}$ & 5.280849000 & -1.325008000 & -0.564913000 \\
$\mathrm{H}$ & 3.864811000 & -1.347935000 & -2.174628000 \\
$\mathrm{C}$ & 4.364607000 & -1.339581000 & 1.647157000 \\
$\mathrm{H}$ & 2.236566000 & -1.355731000 & 1.803854000 \\
$\mathrm{C}$ & 5.480248000 & -1.321545000 & 0.814525000 \\
$\mathrm{H}$ & 6.134845000 & -1.317150000 & -1.237891000 \\
$\mathrm{H}$ & 4.494282000 & -1.341048000 & 2.726841000 \\
$\mathrm{H}$ & 6.482805000 & -1.308520000 & 1.231267000 \\
$\mathrm{H}$ & -0.728295000 & 0.392935000 & -1.917588000 \\
$\mathrm{H}$ & -0.377428000 & -2.018269000 & -2.499898000 \\
\hline-----0. & \\
& & & \\
$\mathrm{H}$ & & & \\
$\mathrm{H}$ & & &
\end{tabular}

\begin{tabular}{|c|c|c|c|}
\hline \multicolumn{4}{|c|}{$\mathbf{A} \mathbf{3}_{\text {TBD }}$} \\
\hline & \multicolumn{3}{|c|}{ Coordinates (Angstroms) } \\
\hline & $\mathrm{X}$ & Y & $\mathrm{Z}$ \\
\hline $\mathrm{C}$ & 0.195700000 & 2.553481000 & 0.616195000 \\
\hline $\mathrm{C}$ & 0.962562000 & 2.193280000 & 1.880865000 \\
\hline $\mathrm{C}$ & 2.449248000 & 2.076690000 & 1.564992000 \\
\hline $\mathrm{N}$ & 0.482431000 & 1.549456000 & -0.375988000 \\
\hline $\mathrm{H}$ & 0.470864000 & 3.571079000 & 0.286901000 \\
\hline $\mathrm{H}$ & -0.882296000 & 2.549322000 & 0.806148000 \\
\hline $\mathrm{H}$ & 0.584452000 & 1.238887000 & 2.256473000 \\
\hline $\mathrm{H}$ & 0.816704000 & 2.948675000 & 2.659854000 \\
\hline $\mathrm{H}$ & 2.909768000 & 3.078316000 & 1.568190000 \\
\hline $\mathrm{H}$ & 2.947144000 & 1.496174000 & 2.354064000 \\
\hline $\mathrm{N}$ & 2.717438000 & 1.437406000 & 0.289119000 \\
\hline
\end{tabular}




\begin{tabular}{|c|c|c|c|}
\hline $\mathrm{C}$ & 4.092121000 & 1.019519000 & 0.080645000 \\
\hline $\mathrm{C}$ & 4.402742000 & 0.925484000 & -1.406025000 \\
\hline $\mathrm{C}$ & 3.311173000 & 0.102770000 & -2.087930000 \\
\hline $\mathrm{N}$ & 1.979767000 & 0.597460000 & -1.815660000 \\
\hline $\mathrm{C}$ & 1.773473000 & 1.176711000 & -0.681457000 \\
\hline $\mathrm{H}$ & 4.263507000 & 0.050114000 & 0.573140000 \\
\hline $\mathrm{H}$ & 4.748990000 & 1.755191000 & 0.563426000 \\
\hline $\mathrm{H}$ & 4.423398000 & 1.933367000 & -1.837836000 \\
\hline $\mathrm{H}$ & 5.391253000 & 0.476453000 & -1.551677000 \\
\hline $\mathrm{H}$ & 3.465926000 & 0.105427000 & -3.173660000 \\
\hline $\mathrm{H}$ & 3.399578000 & -0.945944000 & -1.762112000 \\
\hline $\mathrm{C}$ & -5.830730000 & -0.954587000 & 0.468585000 \\
\hline $\mathrm{C}$ & -4.576642000 & -1.227124000 & -0.064448000 \\
\hline $\mathrm{C}$ & -3.695715000 & -0.186943000 & -0.379925000 \\
\hline $\mathrm{C}$ & -4.112695000 & 1.133572000 & -0.170531000 \\
\hline $\mathrm{C}$ & -5.370223000 & 1.405267000 & 0.357806000 \\
\hline $\mathrm{C}$ & -6.232727000 & 0.362246000 & 0.681378000 \\
\hline $\mathrm{H}$ & -6.503407000 & -1.773158000 & 0.706848000 \\
\hline $\mathrm{H}$ & -4.278627000 & -2.253332000 & -0.257108000 \\
\hline $\mathrm{H}$ & -3.434650000 & 1.951517000 & -0.399501000 \\
\hline $\mathrm{H}$ & -5.672814000 & 2.434994000 & 0.523790000 \\
\hline $\mathrm{H}$ & -7.214667000 & 0.572763000 & 1.094031000 \\
\hline $\mathrm{C}$ & -2.346329000 & -0.458129000 & -0.929513000 \\
\hline $\mathrm{C}$ & -1.577032000 & -1.486327000 & -0.558356000 \\
\hline $\mathrm{H}$ & -1.968239000 & -2.138233000 & 0.219179000 \\
\hline $\mathrm{O}$ & -1.871608000 & 0.477799000 & -1.822632000 \\
\hline $\mathrm{C}$ & -0.208841000 & -1.796111000 & -1.087665000 \\
\hline $\mathrm{H}$ & 0.133488000 & -1.005055000 & -1.765664000 \\
\hline $\mathrm{C}$ & 0.849249000 & -1.965619000 & -0.014483000 \\
\hline $\mathrm{C}$ & 2.009772000 & -2.694361000 & -0.287486000 \\
\hline $\mathrm{C}$ & 0.727190000 & -1.366551000 & 1.238922000 \\
\hline $\mathrm{C}$ & 3.028287000 & -2.803732000 & 0.653542000 \\
\hline $\mathrm{H}$ & 2.120404000 & -3.172631000 & -1.258125000 \\
\hline $\mathrm{C}$ & 1.742781000 & -1.471654000 & 2.185442000 \\
\hline $\mathrm{H}$ & -0.169301000 & -0.796260000 & 1.462912000 \\
\hline $\mathrm{C}$ & 2.900450000 & -2.187728000 & 1.896683000 \\
\hline $\mathrm{H}$ & 3.923811000 & -3.371500000 & 0.416137000 \\
\hline $\mathrm{H}$ & 1.626601000 & -0.992357000 & 3.154382000 \\
\hline $\mathrm{H}$ & 3.691602000 & -2.273433000 & 2.636116000 \\
\hline $\mathrm{H}$ & -0.165647000 & 1.413218000 & -1.140743000 \\
\hline $\mathrm{H}$ & -2.619462000 & 0.855489000 & -2.301804000 \\
\hline $\mathrm{H}$ & -0.255854000 & -2.722666000 & -1.678017000 \\
\hline
\end{tabular}

b1 $_{\text {TBD }}$ 


\begin{tabular}{|c|c|c|c|}
\hline \multicolumn{4}{|c|}{ Coordinates (Angstroms) } \\
\hline & $\mathrm{X}$ & Y & Z \\
\hline $\mathrm{C}$ & -0.080413000 & 3.094080000 & -1.558083000 \\
\hline $\mathrm{C}$ & -1.043625000 & 2.062894000 & -2.140863000 \\
\hline $\mathrm{C}$ & -2.272083000 & 1.971331000 & -1.248025000 \\
\hline $\mathrm{N}$ & 0.260401000 & 2.817955000 & -0.182549000 \\
\hline $\mathrm{H}$ & -0.529477000 & 4.098943000 & -1.654543000 \\
\hline $\mathrm{H}$ & 0.846096000 & 3.113161000 & -2.145143000 \\
\hline $\mathrm{H}$ & -0.544391000 & 1.088682000 & -2.170527000 \\
\hline $\mathrm{H}$ & -1.344306000 & 2.318734000 & -3.163758000 \\
\hline $\mathrm{H}$ & -2.919139000 & 2.850343000 & -1.400938000 \\
\hline $\mathrm{H}$ & -2.859058000 & 1.081728000 & -1.506629000 \\
\hline $\mathrm{N}$ & -1.900074000 & 1.871471000 & 0.155148000 \\
\hline $\mathrm{C}$ & -2.795915000 & 1.065575000 & 0.973200000 \\
\hline $\mathrm{C}$ & -2.491885000 & 1.208455000 & 2.455893000 \\
\hline $\mathrm{C}$ & -0.998663000 & 1.026767000 & 2.676326000 \\
\hline $\mathrm{N}$ & -0.312849000 & 2.036711000 & 1.887080000 \\
\hline $\mathrm{C}$ & -0.630302000 & 2.240819000 & 0.553142000 \\
\hline $\mathrm{H}$ & -2.722417000 & 0.009654000 & 0.678584000 \\
\hline $\mathrm{H}$ & -3.824446000 & 1.391190000 & 0.765364000 \\
\hline $\mathrm{H}$ & -2.792170000 & 2.202600000 & 2.808541000 \\
\hline $\mathrm{H}$ & -3.060223000 & 0.457308000 & 3.014068000 \\
\hline $\mathrm{H}$ & -0.741021000 & 1.191075000 & 3.727470000 \\
\hline $\mathrm{H}$ & -0.689025000 & 0.007180000 & 2.408386000 \\
\hline $\mathrm{H}$ & 0.695001000 & 2.036632000 & 1.989457000 \\
\hline $\mathrm{C}$ & 5.205382000 & -1.343822000 & -0.564003000 \\
\hline $\mathrm{C}$ & 4.107613000 & -1.534528000 & 0.271645000 \\
\hline $\mathrm{C}$ & 3.111277000 & -0.567769000 & 0.368308000 \\
\hline $\mathrm{C}$ & 3.236684000 & 0.610612000 & -0.373098000 \\
\hline $\mathrm{C}$ & 4.334153000 & 0.806871000 & -1.208042000 \\
\hline $\mathrm{C}$ & 5.322985000 & -0.171988000 & -1.310549000 \\
\hline $\mathrm{H}$ & 5.978589000 & -2.107904000 & -0.631630000 \\
\hline $\mathrm{H}$ & 3.971580000 & -2.417119000 & 0.892260000 \\
\hline $\mathrm{H}$ & 2.471293000 & 1.384529000 & -0.286780000 \\
\hline $\mathrm{H}$ & 4.423113000 & 1.730935000 & -1.777010000 \\
\hline $\mathrm{H}$ & 6.182033000 & -0.017572000 & -1.960837000 \\
\hline $\mathrm{C}$ & 1.908103000 & -0.856334000 & 1.317959000 \\
\hline $\mathrm{C}$ & 0.675447000 & -0.919564000 & 0.384637000 \\
\hline $\mathrm{H}$ & 0.513975000 & -0.109144000 & -0.330276000 \\
\hline $\mathrm{O}$ & 2.046649000 & -1.881951000 & 2.119372000 \\
\hline $\mathrm{C}$ & -0.187000000 & -1.932716000 & 0.509674000 \\
\hline $\mathrm{H}$ & 0.063421000 & -2.639917000 & 1.303571000 \\
\hline $\mathrm{C}$ & -1.434381000 & -2.092838000 & -0.241688000 \\
\hline $\mathrm{C}$ & -2.537727000 & -2.732331000 & 0.347009000 \\
\hline
\end{tabular}




\begin{tabular}{llcc}
\hline C & -1.600030000 & -1.594174000 & -1.544177000 \\
C & -3.760975000 & -2.821249000 & -0.308753000 \\
H & -2.425968000 & -3.143336000 & 1.347632000 \\
C & -2.822452000 & -1.676949000 & -2.200747000 \\
H & -0.745035000 & -1.144947000 & -2.040490000 \\
$\mathrm{C}$ & -3.916461000 & -2.283125000 & -1.584486000 \\
H & -4.600902000 & -3.309013000 & 0.180741000 \\
H & -2.919870000 & -1.271371000 & -3.205410000 \\
H & -4.872326000 & -2.348633000 & -2.097446000 \\
H & 1.760737000 & 0.158816000 & 1.830933000 \\
\hline
\end{tabular}

TSb1-2

\begin{tabular}{lccc} 
& Coordinates (Angstroms) & \\
\hline $\mathrm{C}$ & -0.526085000 & -1.563922000 & 1.796629000 \\
$\mathrm{C}$ & -0.862172000 & -2.515322000 & 0.658059000 \\
$\mathrm{C}$ & 0.299265000 & -3.476296000 & 0.475703000 \\
$\mathrm{~N}$ & 0.726304000 & -0.859445000 & 1.548857000 \\
$\mathrm{H}$ & -0.454234000 & -2.107710000 & 2.750409000 \\
$\mathrm{H}$ & -1.307673000 & -0.805661000 & 1.899777000 \\
$\mathrm{H}$ & -0.988048000 & -1.921417000 & -0.250419000 \\
$\mathrm{H}$ & -1.787310000 & -3.066430000 & 0.857534000 \\
$\mathrm{H}$ & 0.351941000 & -4.187827000 & 1.314263000 \\
$\mathrm{H}$ & 0.162295000 & -4.062097000 & -0.439376000 \\
$\mathrm{~N}$ & 1.579325000 & -2.771546000 & 0.368169000 \\
$\mathrm{C}$ & 2.349006000 & -3.090369000 & -0.831594000 \\
$\mathrm{C}$ & 3.751284000 & -2.503750000 & -0.756315000 \\
$\mathrm{C}$ & 3.641901000 & -1.012877000 & -0.481257000 \\
$\mathrm{~N}$ & 2.871814000 & -0.832700000 & 0.740187000 \\
$\mathrm{C}$ & 1.656262000 & -1.478920000 & 0.832974000 \\
$\mathrm{H}$ & 1.822994000 & -2.683219000 & -1.707874000 \\
$\mathrm{H}$ & 2.398816000 & -4.181995000 & -0.919489000 \\
$\mathrm{H}$ & 4.317203000 & -2.995353000 & 0.044207000 \\
$\mathrm{H}$ & 4.270212000 & -2.678963000 & -1.705179000 \\
$\mathrm{H}$ & 4.627705000 & -0.559848000 & -0.337015000 \\
$\mathrm{H}$ & 3.116692000 & -0.517108000 & -1.306774000 \\
$\mathrm{H}$ & 2.747573000 & 0.145292000 & 0.981614000 \\
$\mathrm{C}$ & 3.336222000 & 3.142035000 & -0.923745000 \\
$\mathrm{C}$ & 2.388525000 & 2.146263000 & -1.127309000 \\
$\mathrm{C}$ & 1.320288000 & 1.947504000 & -0.232067000 \\
$\mathrm{C}$ & 1.264403000 & 2.801035000 & 0.888772000 \\
$\mathrm{C}$ & 2.210992000 & 3.797480000 & 1.091296000 \\
$\mathrm{C}$ & 3.260656000 & 3.979901000 & 0.188268000
\end{tabular}




\begin{tabular}{cccc}
\hline$H$ & 4.141512000 & 3.271177000 & -1.645529000 \\
$\mathrm{H}$ & 2.428042000 & 1.493174000 & -1.993276000 \\
$\mathrm{H}$ & 0.476765000 & 2.657395000 & 1.625889000 \\
$\mathrm{H}$ & 2.139208000 & 4.430972000 & 1.973729000 \\
$\mathrm{H}$ & 4.006262000 & 4.753651000 & 0.354496000 \\
$\mathrm{C}$ & 0.380405000 & 0.809140000 & -0.483402000 \\
$\mathrm{C}$ & -1.016424000 & 1.071272000 & -0.274297000 \\
$\mathrm{H}$ & -1.265900000 & 1.893976000 & 0.397693000 \\
$\mathrm{O}$ & 0.793535000 & -0.161206000 & -1.271899000 \\
$\mathrm{C}$ & -2.031764000 & 0.339671000 & -0.852007000 \\
$\mathrm{H}$ & -1.730166000 & -0.362733000 & -1.626989000 \\
$\mathrm{C}$ & -3.428469000 & 0.405161000 & -0.531510000 \\
$\mathrm{C}$ & -4.372898000 & -0.320722000 & -1.302971000 \\
$\mathrm{C}$ & -3.966161000 & 1.146036000 & 0.553415000 \\
$\mathrm{C}$ & -5.730613000 & -0.295626000 & -1.027730000 \\
$\mathrm{H}$ & -4.005334000 & -0.910417000 & -2.140981000 \\
$\mathrm{C}$ & -5.327164000 & 1.172139000 & 0.820097000 \\
$\mathrm{H}$ & -3.291968000 & 1.702100000 & 1.200106000 \\
$\mathrm{C}$ & -6.233932000 & 0.454890000 & 0.037515000 \\
$\mathrm{H}$ & -6.410486000 & -0.871644000 & -1.654125000 \\
$\mathrm{H}$ & -5.688972000 & 1.757979000 & 1.664065000 \\
$\mathrm{H}$ & -7.299021000 & 0.476170000 & 0.253386000 \\
$\mathrm{H}$ & 0.450351000 & 0.137674000 & 0.873411000 \\
\hline & & &
\end{tabular}

b2

$\begin{array}{lrrr} & & \\ & & & \\ \text { Coordinates (Angstroms) } & \\ \text { C } & 1.985178000 & -2.971791000 & -1.292584000 \\ \mathrm{C} & 3.105259000 & -1.976378000 & -1.012272000 \\ \mathrm{C} & 3.154706000 & -1.676600000 & 0.479018000 \\ \mathrm{~N} & 0.705289000 & -2.472247000 & -0.837476000 \\ \mathrm{H} & 2.216874000 & -3.937493000 & -0.808115000 \\ \mathrm{H} & 1.916339000 & -3.168836000 & -2.368576000 \\ \mathrm{H} & 2.881386000 & -1.057247000 & -1.557365000 \\ \mathrm{H} & 4.082036000 & -2.346734000 & -1.345675000 \\ \mathrm{H} & 3.756658000 & -2.431216000 & 1.009206000 \\ \mathrm{H} & 3.633465000 & -0.700210000 & 0.636602000 \\ \mathrm{~N} & 1.826500000 & -1.637049000 & 1.086490000 \\ \mathrm{C} & 1.781419000 & -0.830773000 & 2.298213000 \\ \mathrm{C} & 0.423880000 & -0.904520000 & 2.977575000 \\ \mathrm{C} & -0.640892000 & -0.608785000 & 1.935568000 \\ \mathrm{~N} & -0.523957000 & -1.594732000 & 0.881594000 \\ \mathrm{C} & 0.703400000 & -1.892282000 & 0.324409000\end{array}$




\begin{tabular}{|c|c|c|c|}
\hline $\mathrm{H}$ & 2.014150000 & 0.217040000 & 2.050444000 \\
\hline $\mathrm{H}$ & 2.570742000 & -1.193444000 & 2.971689000 \\
\hline $\mathrm{H}$ & 0.260564000 & -1.902820000 & 3.400241000 \\
\hline $\mathrm{H}$ & 0.385655000 & -0.173433000 & 3.792092000 \\
\hline $\mathrm{H}$ & -1.649795000 & -0.678121000 & 2.352739000 \\
\hline $\mathrm{H}$ & -0.506557000 & 0.412991000 & 1.553916000 \\
\hline $\mathrm{H}$ & -1.224190000 & -1.493062000 & 0.149445000 \\
\hline $\mathrm{C}$ & 2.569005000 & 3.003007000 & 0.962918000 \\
\hline $\mathrm{C}$ & 1.432098000 & 2.422323000 & 0.422636000 \\
\hline $\mathrm{C}$ & 1.479937000 & 1.630307000 & -0.757387000 \\
\hline $\mathrm{C}$ & 2.756963000 & 1.520750000 & -1.366730000 \\
\hline $\mathrm{C}$ & 3.889511000 & 2.099941000 & -0.813994000 \\
\hline $\mathrm{C}$ & 3.821699000 & 2.842655000 & 0.365725000 \\
\hline $\mathrm{H}$ & 2.476621000 & 3.585263000 & 1.878503000 \\
\hline $\mathrm{H}$ & 0.485644000 & 2.564644000 & 0.937162000 \\
\hline $\mathrm{H}$ & 2.826836000 & 0.973172000 & -2.299780000 \\
\hline $\mathrm{H}$ & 4.845786000 & 1.975223000 & -1.319951000 \\
\hline $\mathrm{H}$ & 4.710641000 & 3.292042000 & 0.799845000 \\
\hline $\mathrm{C}$ & 0.346061000 & 0.940594000 & -1.315867000 \\
\hline $\mathrm{C}$ & -0.955485000 & 1.059519000 & -0.863923000 \\
\hline $\mathrm{H}$ & -1.115557000 & 1.794046000 & -0.075916000 \\
\hline $\mathrm{O}$ & 0.616815000 & 0.050225000 & -2.357667000 \\
\hline $\mathrm{C}$ & -2.063793000 & 0.317883000 & -1.272623000 \\
\hline $\mathrm{H}$ & -1.942252000 & -0.343685000 & -2.127847000 \\
\hline $\mathrm{C}$ & -3.366218000 & 0.372821000 & -0.677236000 \\
\hline $\mathrm{C}$ & -4.444793000 & -0.355414000 & -1.248979000 \\
\hline $\mathrm{C}$ & -3.683883000 & 1.096771000 & 0.504788000 \\
\hline $\mathrm{C}$ & -5.714678000 & -0.357247000 & -0.696998000 \\
\hline $\mathrm{H}$ & -4.253154000 & -0.927503000 & -2.155008000 \\
\hline $\mathrm{C}$ & -4.959541000 & 1.092159000 & 1.049313000 \\
\hline $\mathrm{H}$ & -2.904597000 & 1.662857000 & 1.009178000 \\
\hline $\mathrm{C}$ & -5.998774000 & 0.368312000 & 0.462955000 \\
\hline $\mathrm{H}$ & -6.502012000 & -0.933824000 & -1.180738000 \\
\hline $\mathrm{H}$ & -5.145540000 & 1.661814000 & 1.958979000 \\
\hline $\mathrm{H}$ & -6.994473000 & 0.364873000 & 0.898199000 \\
\hline $\mathrm{H}$ & 0.459021000 & -0.849458000 & -1.999335000 \\
\hline
\end{tabular}

TSb2-3 ${ }_{\text {TBD }}$

\begin{tabular}{|c|c|c|c|}
\hline & \multicolumn{3}{|c|}{ Coordinates (Angstroms) } \\
\hline & $\mathrm{X}$ & $\mathrm{Y}$ & Z \\
\hline $\mathrm{C}$ & -2.034283000 & 2.417887000 & -1.638570000 \\
\hline $\mathrm{C}$ & -2.746485000 & 2.440664000 & -0.288513000 \\
\hline $\mathrm{C}$ & -1.919177000 & 3.298613000 & 0.655246000 \\
\hline
\end{tabular}




\begin{tabular}{|c|c|c|c|}
\hline $\mathrm{N}$ & -0.714808000 & 1.845169000 & -1.530681000 \\
\hline $\mathrm{H}$ & -2.002313000 & 3.449050000 & -2.040233000 \\
\hline $\mathrm{H}$ & -2.608065000 & 1.819276000 & -2.356547000 \\
\hline $\mathrm{H}$ & -2.810389000 & 1.413435000 & 0.088903000 \\
\hline $\mathrm{H}$ & -3.764734000 & 2.843397000 & -0.357848000 \\
\hline $\mathrm{H}$ & -2.115757000 & 4.368123000 & 0.462631000 \\
\hline $\mathrm{H}$ & -2.211183000 & 3.110710000 & 1.701751000 \\
\hline $\mathrm{N}$ & -0.500751000 & 3.032654000 & 0.513684000 \\
\hline $\mathrm{C}$ & 0.297687000 & 3.531469000 & 1.612004000 \\
\hline $\mathrm{C}$ & 1.780838000 & 3.282351000 & 1.390637000 \\
\hline $\mathrm{C}$ & 1.939396000 & 1.851112000 & 0.895572000 \\
\hline $\mathrm{N}$ & 1.249990000 & 1.682898000 & -0.361977000 \\
\hline $\mathrm{C}$ & 0.001577000 & 2.170348000 & -0.473960000 \\
\hline $\mathrm{H}$ & -0.016137000 & 3.042555000 & 2.552383000 \\
\hline $\mathrm{H}$ & 0.095242000 & 4.608022000 & 1.739870000 \\
\hline $\mathrm{H}$ & 2.171138000 & 3.966184000 & 0.627910000 \\
\hline $\mathrm{H}$ & 2.325867000 & 3.461575000 & 2.325509000 \\
\hline $\mathrm{H}$ & 2.994894000 & 1.601441000 & 0.741790000 \\
\hline $\mathrm{H}$ & 1.568556000 & 1.159762000 & 1.677473000 \\
\hline $\mathrm{C}$ & -3.322783000 & -3.381419000 & 1.485124000 \\
\hline $\mathrm{C}$ & -2.177094000 & -2.931478000 & 0.841677000 \\
\hline $\mathrm{C}$ & -2.236774000 & -1.919847000 & -0.133809000 \\
\hline $\mathrm{C}$ & -3.507641000 & -1.417863000 & -0.463149000 \\
\hline $\mathrm{C}$ & -4.653594000 & -1.872842000 & 0.178331000 \\
\hline $\mathrm{C}$ & -4.573117000 & -2.854409000 & 1.163693000 \\
\hline $\mathrm{H}$ & -3.240603000 & -4.163506000 & 2.237014000 \\
\hline $\mathrm{H}$ & -1.219983000 & -3.385182000 & 1.082176000 \\
\hline $\mathrm{H}$ & -3.566728000 & -0.659991000 & -1.236800000 \\
\hline $\mathrm{H}$ & -5.620377000 & -1.454542000 & -0.094084000 \\
\hline $\mathrm{H}$ & -5.469302000 & -3.213793000 & 1.663195000 \\
\hline $\mathrm{C}$ & -1.047319000 & -1.377051000 & -0.811568000 \\
\hline $\mathrm{C}$ & 0.211606000 & -1.496603000 & -0.326535000 \\
\hline $\mathrm{H}$ & 0.327782000 & -1.986014000 & 0.638693000 \\
\hline $\mathrm{O}$ & -1.311810000 & -0.698194000 & -1.966341000 \\
\hline $\mathrm{C}$ & 1.400116000 & -0.936443000 & -0.966477000 \\
\hline $\mathrm{H}$ & 1.339358000 & 0.426550000 & -0.738206000 \\
\hline $\mathrm{C}$ & 2.733217000 & -1.306796000 & -0.481559000 \\
\hline $\mathrm{C}$ & 3.842231000 & -1.290686000 & -1.353987000 \\
\hline $\mathrm{C}$ & 3.014711000 & -1.595183000 & 0.871106000 \\
\hline $\mathrm{C}$ & 5.133147000 & -1.545300000 & -0.912117000 \\
\hline $\mathrm{H}$ & 3.668141000 & -1.069083000 & -2.404795000 \\
\hline $\mathrm{C}$ & 4.306531000 & -1.855601000 & 1.312498000 \\
\hline $\mathrm{H}$ & 2.204239000 & -1.591071000 & 1.595561000 \\
\hline $\mathrm{C}$ & 5.384127000 & -1.835487000 & 0.428979000 \\
\hline
\end{tabular}




\begin{tabular}{lrrr} 
H & 5.954761000 & -1.522234000 & -1.625902000 \\
H & 4.474557000 & -2.069730000 & 2.366598000 \\
H & 6.394187000 & -2.036166000 & 0.776953000 \\
H & -0.904610000 & 0.227123000 & -1.889339000 \\
H & 1.323421000 & -0.938005000 & -2.058844000 \\
\hline
\end{tabular}

b3 $_{\text {TBD }}$

\begin{tabular}{|c|c|c|c|}
\hline \multicolumn{4}{|c|}{ Coordinates (Angstroms) } \\
\hline & $X$ & $\mathrm{Y}$ & $\mathrm{Z}$ \\
\hline $\mathrm{C}$ & 0.366648000 & -1.879014000 & 1.060003000 \\
\hline $\mathrm{C}$ & -0.076442000 & -0.666404000 & 1.865115000 \\
\hline $\mathrm{C}$ & -1.585407000 & -0.746846000 & 2.070105000 \\
\hline $\mathrm{N}$ & -0.326298000 & -1.903755000 & -0.204540000 \\
\hline $\mathrm{H}$ & 0.170207000 & -2.803518000 & 1.627742000 \\
\hline $\mathrm{H}$ & 1.435101000 & -1.836234000 & 0.838431000 \\
\hline $\mathrm{H}$ & 0.195500000 & 0.222350000 & 1.288437000 \\
\hline $\mathrm{H}$ & 0.426654000 & -0.609927000 & 2.837772000 \\
\hline $\mathrm{H}$ & -1.813281000 & -1.309649000 & 2.990328000 \\
\hline $\mathrm{H}$ & -2.002305000 & 0.264467000 & 2.199625000 \\
\hline $\mathrm{N}$ & -2.275593000 & -1.409644000 & 0.966994000 \\
\hline $\mathrm{C}$ & -3.713625000 & -1.256286000 & 1.028097000 \\
\hline $\mathrm{C}$ & -4.379106000 & -1.962359000 & -0.141674000 \\
\hline $\mathrm{C}$ & -3.700174000 & -1.487392000 & -1.426254000 \\
\hline $\mathrm{N}$ & -2.273172000 & -1.706220000 & -1.413695000 \\
\hline $\mathrm{C}$ & -1.666520000 & -1.640521000 & -0.270705000 \\
\hline $\mathrm{H}$ & -3.981937000 & -0.186116000 & 1.015936000 \\
\hline $\mathrm{H}$ & -4.064904000 & -1.665018000 & 1.988640000 \\
\hline $\mathrm{H}$ & -4.249238000 & -3.047155000 & -0.041472000 \\
\hline $\mathrm{H}$ & -5.455303000 & -1.747972000 & -0.143481000 \\
\hline $\mathrm{H}$ & -4.123981000 & -2.019993000 & -2.289161000 \\
\hline $\mathrm{H}$ & -3.949893000 & -0.422578000 & -1.574603000 \\
\hline $\mathrm{C}$ & 5.531540000 & 1.059044000 & 0.587516000 \\
\hline $\mathrm{C}$ & 4.277800000 & 1.201750000 & 0.000749000 \\
\hline $\mathrm{C}$ & 3.556563000 & 0.089572000 & -0.452583000 \\
\hline $\mathrm{C}$ & 4.155416000 & -1.168022000 & -0.317111000 \\
\hline $\mathrm{C}$ & 5.406036000 & -1.317788000 & 0.273310000 \\
\hline $\mathrm{C}$ & 6.103516000 & -0.203378000 & 0.733637000 \\
\hline $\mathrm{H}$ & 6.068592000 & 1.941909000 & 0.928803000 \\
\hline $\mathrm{H}$ & 3.862513000 & 2.198141000 & -0.117368000 \\
\hline $\mathrm{H}$ & 3.599039000 & -2.014987000 & -0.706315000 \\
\hline $\mathrm{H}$ & 5.842923000 & -2.309692000 & 0.371329000 \\
\hline $\mathrm{H}$ & 7.082895000 & -0.314737000 & 1.193480000 \\
\hline $\mathrm{C}$ & 2.176351000 & 0.136976000 & -1.093766000 \\
\hline
\end{tabular}




\begin{tabular}{|c|c|c|c|}
\hline $\mathrm{C}$ & 1.447921000 & 1.298677000 & -0.991954000 \\
\hline $\mathrm{H}$ & 1.880800000 & 2.182566000 & -0.532388000 \\
\hline $\mathrm{O}$ & 1.797790000 & -0.953634000 & -1.651078000 \\
\hline $\mathrm{C}$ & 0.092109000 & 1.422331000 & -1.614261000 \\
\hline $\mathrm{H}$ & -0.186620000 & 0.443249000 & -2.021895000 \\
\hline $\mathrm{C}$ & -1.041889000 & 1.908978000 & -0.726206000 \\
\hline $\mathrm{C}$ & -2.363153000 & 1.755772000 & -1.160307000 \\
\hline $\mathrm{C}$ & -0.834090000 & 2.536540000 & 0.503870000 \\
\hline $\mathrm{C}$ & -3.436073000 & 2.208314000 & -0.400948000 \\
\hline $\mathrm{H}$ & -2.540485000 & 1.249249000 & -2.105566000 \\
\hline $\mathrm{C}$ & -1.904187000 & 2.994106000 & 1.272230000 \\
\hline $\mathrm{H}$ & 0.181925000 & 2.645700000 & 0.871560000 \\
\hline $\mathrm{C}$ & -3.212123000 & 2.833307000 & 0.825180000 \\
\hline $\mathrm{H}$ & -4.451752000 & 2.061500000 & -0.762049000 \\
\hline $\mathrm{H}$ & -1.712361000 & 3.474543000 & 2.229139000 \\
\hline $\mathrm{H}$ & -4.047744000 & 3.184593000 & 1.425565000 \\
\hline $\mathrm{H}$ & 0.261343000 & -1.624896000 & -1.005517000 \\
\hline $\mathrm{H}$ & 0.108763000 & 2.106459000 & -2.483931000 \\
\hline
\end{tabular}

\begin{tabular}{|c|c|c|c|}
\hline \multicolumn{4}{|c|}{ Coordinates (Angstroms) } \\
\hline & $\mathrm{X}$ & Y & $\mathrm{Z}$ \\
\hline $\mathrm{C}$ & -0.758369000 & -0.830012000 & -1.957810000 \\
\hline $\mathrm{C}$ & -1.750374000 & -0.252488000 & -2.961454000 \\
\hline $\mathrm{C}$ & -2.759179000 & 0.587351000 & -2.196384000 \\
\hline $\mathrm{N}$ & -1.401504000 & -1.545865000 & -0.877296000 \\
\hline $\mathrm{H}$ & -0.153119000 & -0.002340000 & -1.555691000 \\
\hline $\mathrm{H}$ & -0.061935000 & -1.514094000 & -2.456212000 \\
\hline $\mathrm{H}$ & -2.268722000 & -1.067080000 & -3.481046000 \\
\hline $\mathrm{H}$ & -1.245254000 & 0.362223000 & -3.713762000 \\
\hline $\mathrm{H}$ & -2.284622000 & 1.501033000 & -1.805538000 \\
\hline $\mathrm{H}$ & -3.581691000 & 0.897778000 & -2.853351000 \\
\hline $\mathrm{N}$ & -3.331804000 & -0.176675000 & -1.100040000 \\
\hline $\mathrm{C}$ & -4.605554000 & 0.327814000 & -0.620119000 \\
\hline $\mathrm{C}$ & -5.006281000 & -0.281495000 & 0.713658000 \\
\hline $\mathrm{C}$ & -4.603150000 & -1.745818000 & 0.729387000 \\
\hline $\mathrm{N}$ & -3.164625000 & -1.826284000 & 0.574051000 \\
\hline $\mathrm{C}$ & -2.580885000 & -1.176237000 & -0.510510000 \\
\hline $\mathrm{H}$ & -5.378511000 & 0.114163000 & -1.375061000 \\
\hline $\mathrm{H}$ & -4.539009000 & 1.421345000 & -0.532149000 \\
\hline $\mathrm{H}$ & -4.491936000 & 0.223648000 & 1.538083000 \\
\hline $\mathrm{H}$ & -6.083821000 & -0.164787000 & 0.864140000 \\
\hline
\end{tabular}




\begin{tabular}{lrrr}
$-\mathrm{H}$ & -4.865812000 & -2.203593000 & 1.687468000 \\
$\mathrm{H}$ & -5.140102000 & -2.302511000 & -0.059059000 \\
$\mathrm{C}$ & -0.403609000 & 3.412631000 & 0.042597000 \\
$\mathrm{C}$ & 0.404703000 & 2.300667000 & 0.276271000 \\
$\mathrm{C}$ & -0.106401000 & 1.169714000 & 0.912735000 \\
$\mathrm{C}$ & -1.440143000 & 1.178657000 & 1.332270000 \\
$\mathrm{C}$ & -2.244076000 & 2.289223000 & 1.111601000 \\
$\mathrm{C}$ & -1.730160000 & 3.409909000 & 0.459473000 \\
$\mathrm{H}$ & 0.009355000 & 4.283678000 & -0.457927000 \\
$\mathrm{H}$ & 1.444541000 & 2.316896000 & -0.039060000 \\
$\mathrm{H}$ & -1.845196000 & 0.296624000 & 1.821045000 \\
$\mathrm{H}$ & -3.277375000 & 2.284572000 & 1.448538000 \\
$\mathrm{H}$ & -2.360351000 & 4.276939000 & 0.283252000 \\
$\mathrm{C}$ & 0.742300000 & -0.056911000 & 1.229650000 \\
$\mathrm{C}$ & 2.038029000 & -0.095521000 & 0.461418000 \\
$\mathrm{H}$ & 1.936267000 & -0.021209000 & -0.620312000 \\
$\mathrm{O}$ & 0.925162000 & -0.176776000 & 2.632827000 \\
$\mathrm{C}$ & 3.239793000 & -0.252538000 & 1.018147000 \\
$\mathrm{H}$ & 3.288152000 & -0.397304000 & 2.096166000 \\
$\mathrm{C}$ & 4.526059000 & -0.300467000 & 0.301035000 \\
$\mathrm{C}$ & 5.631002000 & -0.898552000 & 0.918175000 \\
$\mathrm{C}$ & 4.697437000 & 0.241134000 & -0.979831000 \\
$\mathrm{C}$ & 6.859301000 & -0.977844000 & 0.271284000 \\
$\mathrm{H}$ & 5.517635000 & -1.317513000 & 1.915060000 \\
$\mathrm{C}$ & 5.923550000 & 0.163342000 & -1.628371000 \\
$\mathrm{H}$ & 3.865593000 & 0.742673000 & -1.466778000 \\
$\mathrm{C}$ & 7.010085000 & -0.448634000 & -1.006991000 \\
$\mathrm{H}$ & 7.701022000 & -1.452729000 & 0.767040000 \\
$\mathrm{H}$ & 6.035594000 & 0.592694000 & -2.619920000 \\
$\mathrm{H}$ & 7.969050000 & -0.505472000 & -1.513299000 \\
$\mathrm{H}$ & 0.154964000 & -0.936636000 & 0.935903000 \\
$\mathrm{H}$ & 1.286243000 & 0.665214000 & 2.940852000 \\
$\mathrm{H}$ & -2.527916000 & -3.052981000 & 1.016226000 \\
$\mathrm{H}$ & -2.644325000 & -3.870509000 & 0.290107000 \\
-1.461688000 & -2.883278000 & 1.159175000 \\
$\mathrm{H}$ & -2.977676000 & -3.350111000 & 1.967984000 \\
\hline
\end{tabular}

TSA1-2

\begin{tabular}{cccc} 
& & Coordinates (Angstroms) & \\
\hline $\mathrm{C}$ & $\mathrm{X}$ & $\mathrm{Y}$ & $\mathrm{Z}$ \\
$\mathrm{C}$ & 0.1732682000 & -1.357663000 & 1.865293000 \\
$\mathrm{C}$ & -1.100692000 & -1.498954000 & 2.946833000 \\
$\mathrm{~N}$ & 0.719094000 & -1.637026000 & 0.527064000
\end{tabular}




\begin{tabular}{|c|c|c|c|}
\hline $\mathrm{H}$ & 2.075262000 & -2.030205000 & 2.055059000 \\
\hline $\mathrm{H}$ & 1.638769000 & -0.338971000 & 1.873918000 \\
\hline $\mathrm{H}$ & 0.519885000 & -1.040919000 & 3.877653000 \\
\hline $\mathrm{H}$ & -0.036998000 & -2.555325000 & 3.149017000 \\
\hline $\mathrm{H}$ & -1.908061000 & -0.937397000 & 3.191342000 \\
\hline $\mathrm{H}$ & -0.944427000 & 0.248076000 & 2.297521000 \\
\hline $\mathrm{N}$ & -1.536543000 & -1.470245000 & 1.229035000 \\
\hline $\mathrm{C}$ & -2.947185000 & -1.301465000 & 0.893102000 \\
\hline $\mathrm{C}$ & -3.327667000 & -2.150560000 & -0.305931000 \\
\hline $\mathrm{C}$ & -2.315527000 & -1.937315000 & -1.417567000 \\
\hline $\mathrm{N}$ & -0.972166000 & -2.201213000 & -0.926454000 \\
\hline $\mathrm{C}$ & -0.586174000 & -1.741381000 & 0.288582000 \\
\hline $\mathrm{H}$ & -3.151654000 & -0.241881000 & 0.692557000 \\
\hline $\mathrm{H}$ & -3.534288000 & -1.595286000 & 1.770178000 \\
\hline $\mathrm{H}$ & -3.345702000 & -3.210259000 & -0.029245000 \\
\hline $\mathrm{H}$ & -4.327622000 & -1.868492000 & -0.647423000 \\
\hline $\mathrm{H}$ & -2.506658000 & -2.627643000 & -2.243662000 \\
\hline $\mathrm{H}$ & -2.378612000 & -0.914189000 & -1.810286000 \\
\hline $\mathrm{C}$ & 4.639778000 & 1.545007000 & 1.202949000 \\
\hline $\mathrm{C}$ & 3.354457000 & 1.397491000 & 0.696084000 \\
\hline $\mathrm{C}$ & 3.103231000 & 0.641354000 & -0.464627000 \\
\hline $\mathrm{C}$ & 4.216572000 & 0.061821000 & -1.098893000 \\
\hline $\mathrm{C}$ & 5.501759000 & 0.218228000 & -0.594074000 \\
\hline $\mathrm{C}$ & 5.728212000 & 0.955440000 & 0.565149000 \\
\hline $\mathrm{H}$ & 4.792039000 & 2.138445000 & 2.101036000 \\
\hline $\mathrm{H}$ & 2.537422000 & 1.896687000 & 1.210188000 \\
\hline $\mathrm{H}$ & 4.052844000 & -0.520958000 & -1.999000000 \\
\hline $\mathrm{H}$ & 6.337017000 & -0.246058000 & -1.112577000 \\
\hline $\mathrm{H}$ & 6.732279000 & 1.075084000 & 0.960952000 \\
\hline $\mathrm{C}$ & 1.747537000 & 0.381691000 & -0.994640000 \\
\hline $\mathrm{C}$ & 0.605471000 & 1.139984000 & -0.572078000 \\
\hline $\mathrm{H}$ & 0.668040000 & 1.541148000 & 0.438913000 \\
\hline $\mathrm{O}$ & 1.722352000 & -0.030034000 & -2.350612000 \\
\hline $\mathrm{C}$ & -0.583122000 & 1.238441000 & -1.223098000 \\
\hline $\mathrm{H}$ & -0.649545000 & 0.827389000 & -2.229068000 \\
\hline $\mathrm{C}$ & -1.823254000 & 1.772275000 & -0.668559000 \\
\hline $\mathrm{C}$ & -3.007414000 & 1.695311000 & -1.426270000 \\
\hline $\mathrm{C}$ & -1.935404000 & 2.322289000 & 0.623021000 \\
\hline $\mathrm{C}$ & -4.236106000 & 2.087483000 & -0.909890000 \\
\hline $\mathrm{H}$ & -2.953220000 & 1.306287000 & -2.441225000 \\
\hline $\mathrm{C}$ & -3.162695000 & 2.717044000 & 1.139791000 \\
\hline $\mathrm{H}$ & -1.046723000 & 2.445465000 & 1.235991000 \\
\hline $\mathrm{C}$ & -4.328616000 & 2.590810000 & 0.385852000 \\
\hline $\mathrm{H}$ & -5.128134000 & 2.001728000 & -1.524939000 \\
\hline
\end{tabular}




\begin{tabular}{lrcc}
\hline $\mathrm{H}$ & -3.209549000 & 3.132368000 & 2.143188000 \\
$\mathrm{H}$ & -5.286660000 & 2.896331000 & 0.794955000 \\
$\mathrm{H}$ & 1.259527000 & -0.891881000 & -0.231855000 \\
$\mathrm{H}$ & 1.854447000 & 0.757001000 & -2.899295000 \\
$\mathrm{C}$ & 0.026598000 & -2.580026000 & -1.916084000 \\
$\mathrm{H}$ & 0.391499000 & -1.715470000 & -2.481219000 \\
$\mathrm{H}$ & 0.876465000 & -3.038961000 & -1.410041000 \\
$\mathrm{H}$ & -0.427528000 & -3.309835000 & -2.592632000 \\
\hline
\end{tabular}

A2

\begin{tabular}{|c|c|c|c|}
\hline \multicolumn{4}{|c|}{ Coordinates (Angstroms) } \\
\hline & $X$ & $\mathrm{Y}$ & Z \\
\hline$\overline{\mathrm{C}}$ & -2.450122000 & 0.655531000 & -2.091539000 \\
\hline $\mathrm{C}$ & -3.289639000 & 1.115253000 & -0.911676000 \\
\hline $\mathrm{C}$ & -2.725899000 & 2.416055000 & -0.366967000 \\
\hline $\mathrm{N}$ & -1.024217000 & 0.934012000 & -1.898230000 \\
\hline $\mathrm{H}$ & -2.760115000 & 1.159270000 & -3.016187000 \\
\hline $\mathrm{H}$ & -2.560558000 & -0.425243000 & -2.232790000 \\
\hline $\mathrm{H}$ & -3.275177000 & 0.364684000 & -0.120058000 \\
\hline $\mathrm{H}$ & -4.326655000 & 1.250016000 & -1.230379000 \\
\hline $\mathrm{H}$ & -2.798194000 & 3.227277000 & -1.104547000 \\
\hline $\mathrm{H}$ & -3.268610000 & 2.720683000 & 0.530138000 \\
\hline $\mathrm{N}$ & -1.330921000 & 2.217936000 & 0.011922000 \\
\hline $\mathrm{C}$ & -0.861369000 & 2.722961000 & 1.299189000 \\
\hline $\mathrm{C}$ & 0.619505000 & 3.061363000 & 1.216840000 \\
\hline $\mathrm{C}$ & 1.378358000 & 1.839617000 & 0.732395000 \\
\hline $\mathrm{N}$ & 0.772844000 & 1.323997000 & -0.490990000 \\
\hline $\mathrm{C}$ & -0.532015000 & 1.467390000 & -0.768789000 \\
\hline $\mathrm{H}$ & -1.057942000 & 1.977050000 & 2.080655000 \\
\hline $\mathrm{H}$ & -1.445317000 & 3.617611000 & 1.530689000 \\
\hline $\mathrm{H}$ & 0.764898000 & 3.897719000 & 0.524189000 \\
\hline $\mathrm{H}$ & 0.990934000 & 3.366694000 & 2.198636000 \\
\hline $\mathrm{H}$ & 2.425986000 & 2.063149000 & 0.519021000 \\
\hline $\mathrm{H}$ & 1.368658000 & 1.054842000 & 1.498529000 \\
\hline $\mathrm{H}$ & 1.191820000 & 0.421804000 & -0.779040000 \\
\hline $\mathrm{C}$ & -2.953410000 & -0.705774000 & 2.377221000 \\
\hline $\mathrm{C}$ & -1.762096000 & -0.934838000 & 1.704688000 \\
\hline $\mathrm{C}$ & -1.707660000 & -1.746304000 & 0.542619000 \\
\hline $\mathrm{C}$ & -2.936532000 & -2.289665000 & 0.098481000 \\
\hline $\mathrm{C}$ & -4.125497000 & -2.042490000 & 0.771355000 \\
\hline $\mathrm{C}$ & -4.155325000 & -1.246490000 & 1.916826000 \\
\hline $\mathrm{H}$ & -2.945748000 & -0.086385000 & 3.271939000 \\
\hline $\mathrm{H}$ & -0.851834000 & -0.477258000 & 2.084338000 \\
\hline $\mathrm{H}$ & -2.932134000 & -2.914930000 & -0.787641000 \\
\hline & & S137 & \\
\hline
\end{tabular}




$\begin{array}{lrrr}-\mathrm{H} & -5.046249000 & -2.482254000 & 0.395173000 \\ \mathrm{H} & -5.087644000 & -1.057435000 & 2.439767000 \\ \mathrm{C} & -0.494128000 & -1.966468000 & -0.199525000 \\ \mathrm{C} & 0.770147000 & -1.583654000 & 0.186661000 \\ \mathrm{H} & 0.854923000 & -1.245856000 & 1.218633000 \\ \mathrm{O} & -0.670841000 & -2.524296000 & -1.478566000 \\ \mathrm{C} & 1.929007000 & -1.503803000 & -0.594662000 \\ \mathrm{H} & 1.874502000 & -1.771774000 & -1.648895000 \\ \mathrm{C} & 3.215320000 & -1.054520000 & -0.110231000 \\ \mathrm{C} & 4.229803000 & -0.691443000 & -1.027316000 \\ \mathrm{C} & 3.527366000 & -0.887521000 & 1.259731000 \\ \mathrm{C} & 5.443775000 & -0.167288000 & -0.610790000 \\ \mathrm{H} & 4.038266000 & -0.818986000 & -2.091325000 \\ \mathrm{C} & 4.740488000 & -0.349533000 & 1.671175000 \\ \mathrm{H} & 2.813647000 & -1.207562000 & 2.014538000 \\ \mathrm{C} & 5.712027000 & 0.024738000 & 0.745341000 \\ \mathrm{H} & 6.190604000 & 0.101620000 & -1.354158000 \\ \mathrm{H} & 4.935370000 & -0.236554000 & 2.735375000 \\ \mathrm{H} & 6.661193000 & 0.438780000 & 1.071678000 \\ \mathrm{H} & 0.002722000 & -3.210838000 & -1.576517000 \\ \mathrm{C} & -0.151922000 & 0.308788000 & -2.885307000 \\ \mathrm{H} & 0.825758000 & 0.792638000 & -2.884410000 \\ \mathrm{H} & -0.051117000 & -0.761985000 & -2.683713000 \\ \mathrm{H} & -0.610296000 & 0.454550000 & -3.868340000 \\ ----1 & \end{array}$

TSA2-3 ${ }_{\text {MTBD }}$

\begin{tabular}{lrrr} 
& \multicolumn{3}{c}{ Coordinates (Angstroms) } \\
& $\mathrm{X}$ & $\mathrm{Y}$ & $\mathrm{Z}$ \\
$\mathrm{C}$ & 2.355874000 & 1.348307000 & 1.812680000 \\
$\mathrm{C}$ & 3.168295000 & 1.379510000 & 0.526085000 \\
$\mathrm{C}$ & 2.597460000 & 2.434704000 & -0.405693000 \\
$\mathrm{~N}$ & 0.937088000 & 1.620417000 & 1.586840000 \\
$\mathrm{H}$ & 2.725639000 & 2.102839000 & 2.520409000 \\
$\mathrm{H}$ & 2.441105000 & 0.364998000 & 2.289533000 \\
$\mathrm{H}$ & 3.127642000 & 0.410980000 & 0.024416000 \\
$\mathrm{H}$ & 4.215257000 & 1.594185000 & 0.757976000 \\
$\mathrm{H}$ & 2.702192000 & 3.441782000 & 0.024130000 \\
$\mathrm{H}$ & 3.120693000 & 2.420149000 & -1.364379000 \\
$\mathrm{~N}$ & 1.195254000 & 2.140353000 & -0.662762000 \\
$\mathrm{C}$ & 0.663677000 & 2.252578000 & -2.014744000 \\
$\mathrm{C}$ & -0.777502000 & 2.736247000 & -1.960386000 \\
$\mathrm{C}$ & -1.575637000 & 1.797710000 & -1.070384000 \\
$\mathrm{~N}$ & -0.921730000 & 1.577943000 & 0.214376000 \\
$\mathrm{C}$ & 0.386238000 & 1.757531000 & 0.355960000
\end{tabular}




\begin{tabular}{|c|c|c|c|}
\hline $\mathrm{H}$ & 0.731885000 & 1.284446000 & -2.529992000 \\
\hline $\mathrm{H}$ & 1.290098000 & 2.963994000 & -2.559814000 \\
\hline $\mathrm{H}$ & -0.800561000 & 3.754293000 & -1.555023000 \\
\hline $\mathrm{H}$ & -1.208379000 & 2.763495000 & -2.965448000 \\
\hline $\mathrm{H}$ & -2.577491000 & 2.191337000 & -0.876890000 \\
\hline $\mathrm{H}$ & -1.712058000 & 0.829538000 & -1.571263000 \\
\hline $\mathrm{H}$ & -1.333513000 & 0.569295000 & 0.671998000 \\
\hline $\mathrm{C}$ & 3.122014000 & -1.566135000 & -2.121479000 \\
\hline $\mathrm{C}$ & 1.929932000 & -1.462206000 & -1.418581000 \\
\hline $\mathrm{C}$ & 1.845060000 & -1.834505000 & -0.062288000 \\
\hline $\mathrm{C}$ & 3.019781000 & -2.298757000 & 0.554622000 \\
\hline $\mathrm{C}$ & 4.215458000 & -2.385791000 & -0.149538000 \\
\hline $\mathrm{C}$ & 4.279979000 & -2.021451000 & -1.491905000 \\
\hline $\mathrm{H}$ & 3.151707000 & -1.273796000 & -3.168126000 \\
\hline $\mathrm{H}$ & 1.053572000 & -1.064408000 & -1.922008000 \\
\hline $\mathrm{H}$ & 2.980993000 & -2.582707000 & 1.600163000 \\
\hline $\mathrm{H}$ & 5.106906000 & -2.746098000 & 0.357116000 \\
\hline $\mathrm{H}$ & 5.215780000 & -2.088654000 & -2.038433000 \\
\hline $\mathrm{C}$ & 0.618692000 & -1.670599000 & 0.711444000 \\
\hline $\mathrm{C}$ & -0.612432000 & -1.414905000 & 0.206472000 \\
\hline $\mathrm{H}$ & -0.702442000 & -1.469846000 & -0.876258000 \\
\hline $\mathrm{O}$ & 0.822454000 & -1.714365000 & 2.091291000 \\
\hline $\mathrm{C}$ & -1.753635000 & -0.904284000 & 0.917277000 \\
\hline $\mathrm{H}$ & -1.715147000 & -0.914181000 & 2.009250000 \\
\hline $\mathrm{C}$ & -3.109764000 & -0.950514000 & 0.364513000 \\
\hline $\mathrm{C}$ & -4.165799000 & -0.329209000 & 1.062742000 \\
\hline $\mathrm{C}$ & -3.428384000 & -1.513836000 & -0.886045000 \\
\hline $\mathrm{C}$ & -5.448136000 & -0.256309000 & 0.538321000 \\
\hline $\mathrm{H}$ & -3.957910000 & 0.114694000 & 2.034745000 \\
\hline $\mathrm{C}$ & -4.711779000 & -1.428891000 & -1.416483000 \\
\hline $\mathrm{H}$ & -2.666039000 & -2.049524000 & -1.445077000 \\
\hline $\mathrm{C}$ & -5.734588000 & -0.798302000 & -0.714779000 \\
\hline $\mathrm{H}$ & -6.232978000 & 0.232713000 & 1.110386000 \\
\hline $\mathrm{H}$ & -4.915757000 & -1.878843000 & -2.385201000 \\
\hline $\mathrm{H}$ & -6.736308000 & -0.737804000 & -1.129499000 \\
\hline $\mathrm{H}$ & 0.014270000 & -2.076110000 & 2.478874000 \\
\hline $\mathrm{C}$ & 0.102929000 & 1.451796000 & 2.763910000 \\
\hline $\mathrm{H}$ & -0.889893000 & 1.858978000 & 2.574272000 \\
\hline $\mathrm{H}$ & 0.024977000 & 0.394397000 & 3.030017000 \\
\hline $\mathrm{H}$ & 0.565452000 & 1.997659000 & 3.594304000 \\
\hline
\end{tabular}

A3 $3_{\text {MTBD }}$

Coordinates (Angstroms)

$\mathrm{X}$

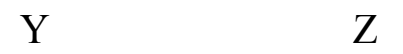




\begin{tabular}{|c|c|c|c|}
\hline $\mathrm{C}$ & -1.027776000 & -2.881098000 & 1.279422000 \\
\hline $\mathrm{C}$ & -1.874390000 & -2.106376000 & 2.273785000 \\
\hline $\mathrm{C}$ & -3.152060000 & -1.647710000 & 1.590253000 \\
\hline $\mathrm{N}$ & -0.709890000 & -2.013457000 & 0.165081000 \\
\hline $\mathrm{H}$ & -1.551665000 & -3.798644000 & 0.956106000 \\
\hline $\mathrm{H}$ & -0.086608000 & -3.192654000 & 1.742844000 \\
\hline $\mathrm{H}$ & -1.294880000 & -1.244578000 & 2.618827000 \\
\hline $\mathrm{H}$ & -2.122188000 & -2.719888000 & 3.145816000 \\
\hline $\mathrm{H}$ & -3.898829000 & -2.457420000 & 1.613724000 \\
\hline $\mathrm{H}$ & -3.586053000 & -0.797545000 & 2.134394000 \\
\hline $\mathrm{N}$ & -2.936845000 & -1.251987000 & 0.210394000 \\
\hline $\mathrm{C}$ & -4.037745000 & -0.505287000 & -0.375845000 \\
\hline $\mathrm{C}$ & -3.998081000 & -0.595066000 & -1.892133000 \\
\hline $\mathrm{C}$ & -2.600415000 & -0.199615000 & -2.357084000 \\
\hline $\mathrm{N}$ & -1.552684000 & -0.944917000 & -1.695238000 \\
\hline $\mathrm{C}$ & -1.754916000 & -1.372845000 & -0.496807000 \\
\hline $\mathrm{H}$ & -3.990762000 & 0.545233000 & -0.050443000 \\
\hline $\mathrm{H}$ & -4.972672000 & -0.929924000 & 0.011907000 \\
\hline $\mathrm{H}$ & -4.213387000 & -1.624321000 & -2.203005000 \\
\hline $\mathrm{H}$ & -4.763511000 & 0.057395000 & -2.325584000 \\
\hline $\mathrm{H}$ & -2.500737000 & -0.356659000 & -3.437543000 \\
\hline $\mathrm{H}$ & -2.459605000 & 0.879875000 & -2.184961000 \\
\hline $\mathrm{C}$ & 5.690279000 & 0.941093000 & 1.088824000 \\
\hline $\mathrm{C}$ & 4.496957000 & 1.275854000 & 0.460589000 \\
\hline $\mathrm{C}$ & 3.798461000 & 0.326451000 & -0.293195000 \\
\hline $\mathrm{C}$ & 4.335996000 & -0.960999000 & -0.417949000 \\
\hline $\mathrm{C}$ & 5.534587000 & -1.292417000 & 0.205367000 \\
\hline $\mathrm{C}$ & 6.215521000 & -0.343032000 & 0.961486000 \\
\hline $\mathrm{H}$ & 6.221439000 & 1.690487000 & 1.668269000 \\
\hline $\mathrm{H}$ & 4.104637000 & 2.285454000 & 0.536795000 \\
\hline $\mathrm{H}$ & 3.793879000 & -1.717174000 & -0.980505000 \\
\hline $\mathrm{H}$ & 5.934155000 & -2.297291000 & 0.104303000 \\
\hline $\mathrm{H}$ & 7.152360000 & -0.600051000 & 1.446485000 \\
\hline $\mathrm{C}$ & 2.515412000 & 0.660506000 & -0.954356000 \\
\hline $\mathrm{C}$ & 1.577791000 & 1.446424000 & -0.415049000 \\
\hline $\mathrm{H}$ & 1.773520000 & 1.860426000 & 0.569861000 \\
\hline $\mathrm{O}$ & 2.276607000 & 0.052505000 & -2.160235000 \\
\hline $\mathrm{C}$ & 0.260073000 & 1.763450000 & -1.066232000 \\
\hline $\mathrm{H}$ & -0.042827000 & 0.912639000 & -1.690656000 \\
\hline $\mathrm{C}$ & -0.849716000 & 2.046514000 & -0.077665000 \\
\hline $\mathrm{C}$ & -1.830240000 & 3.001708000 & -0.358572000 \\
\hline $\mathrm{C}$ & -0.939148000 & 1.345330000 & 1.126935000 \\
\hline $\mathrm{C}$ & -2.867673000 & 3.253909000 & 0.535080000 \\
\hline $\mathrm{H}$ & -1.776064000 & 3.558818000 & -1.291338000 \\
\hline
\end{tabular}




\begin{tabular}{lrrr}
\hline $\mathrm{C}$ & -1.967045000 & 1.601263000 & 2.028661000 \\
$\mathrm{H}$ & -0.201739000 & 0.577788000 & 1.345541000 \\
$\mathrm{C}$ & -2.937367000 & 2.556895000 & 1.738325000 \\
$\mathrm{H}$ & -3.615999000 & 4.003889000 & 0.294816000 \\
$\mathrm{H}$ & -2.009879000 & 1.053149000 & 2.966606000 \\
$\mathrm{H}$ & -3.736589000 & 2.759972000 & 2.445332000 \\
$\mathrm{H}$ & 3.118602000 & -0.226026000 & -2.539493000 \\
$\mathrm{H}$ & 0.369532000 & 2.625655000 & -1.739105000 \\
$\mathrm{C}$ & 0.454096000 & -2.378996000 & -0.616830000 \\
$\mathrm{H}$ & 0.302004000 & -3.316927000 & -1.173757000 \\
$\mathrm{H}$ & 0.678251000 & -1.589546000 & -1.331412000 \\
$\mathrm{H}$ & 1.303086000 & -2.505375000 & 0.064338000 \\
\hline
\end{tabular}

A1

\begin{tabular}{|c|c|c|c|}
\hline \multicolumn{4}{|c|}{ Coordinates (Angstroms) } \\
\hline & $X$ & $\mathrm{Y}$ & Z \\
\hline$\overline{\mathrm{C}}$ & -1.456929000 & 4.072389000 & -1.064775000 \\
\hline $\mathrm{C}$ & -1.724968000 & 2.718349000 & -0.879878000 \\
\hline $\mathrm{C}$ & -0.715475000 & 1.843363000 & -0.473415000 \\
\hline $\mathrm{C}$ & 0.569933000 & 2.345328000 & -0.255696000 \\
\hline $\mathrm{C}$ & 0.837887000 & 3.697848000 & -0.436380000 \\
\hline $\mathrm{C}$ & -0.174540000 & 4.565249000 & -0.842345000 \\
\hline $\mathrm{H}$ & -2.251551000 & 4.743676000 & -1.377906000 \\
\hline $\mathrm{H}$ & -2.730258000 & 2.335464000 & -1.041349000 \\
\hline $\mathrm{H}$ & 1.348287000 & 1.655353000 & 0.060533000 \\
\hline $\mathrm{H}$ & 1.840061000 & 4.079300000 & -0.259918000 \\
\hline $\mathrm{H}$ & 0.034491000 & 5.621919000 & -0.982730000 \\
\hline $\mathrm{C}$ & -0.972270000 & 0.348320000 & -0.347993000 \\
\hline $\mathrm{C}$ & -2.354726000 & 0.051016000 & 0.173330000 \\
\hline $\mathrm{H}$ & -2.607825000 & 0.555654000 & 1.105149000 \\
\hline $\mathrm{O}$ & -0.705056000 & -0.301703000 & -1.588387000 \\
\hline $\mathrm{C}$ & -3.232462000 & -0.751363000 & -0.431527000 \\
\hline $\mathrm{H}$ & -2.929076000 & -1.217232000 & -1.367611000 \\
\hline $\mathrm{C}$ & -4.594282000 & -1.068523000 & 0.033081000 \\
\hline $\mathrm{C}$ & -5.513761000 & -1.607456000 & -0.874753000 \\
\hline $\mathrm{C}$ & -5.018029000 & -0.854613000 & 1.351553000 \\
\hline $\mathrm{C}$ & -6.817785000 & -1.901331000 & -0.490712000 \\
\hline $\mathrm{H}$ & -5.199942000 & -1.790761000 & -1.899633000 \\
\hline $\mathrm{C}$ & -6.319779000 & -1.147664000 & 1.737993000 \\
\hline $\mathrm{H}$ & -4.316859000 & -0.471413000 & 2.087209000 \\
\hline $\mathrm{C}$ & -7.227708000 & -1.668859000 & 0.818477000 \\
\hline $\mathrm{H}$ & -7.514313000 & -2.312577000 & -1.215764000 \\
\hline $\mathrm{H}$ & -6.625429000 & -0.976053000 & 2.766175000 \\
\hline
\end{tabular}




\begin{tabular}{lrrr}
$\mathrm{H}$ & -8.244559000 & -1.898233000 & 1.123017000 \\
$\mathrm{H}$ & -0.229459000 & -0.066060000 & 0.346584000 \\
$\mathrm{H}$ & -1.127322000 & 0.224270000 & -2.280219000 \\
$\mathrm{C}$ & 5.396130000 & -1.343194000 & 0.125471000 \\
$\mathrm{C}$ & 5.103726000 & -2.602506000 & -0.684304000 \\
$\mathrm{C}$ & 2.482458000 & -1.435488000 & -0.832348000 \\
$\mathrm{C}$ & 4.521789000 & -2.318085000 & -2.071162000 \\
$\mathrm{C}$ & 3.426039000 & -1.250570000 & -2.022330000 \\
$\mathrm{H}$ & 5.913991000 & -0.605658000 & -0.509847000 \\
$\mathrm{H}$ & 2.292748000 & -2.504051000 & -0.664802000 \\
$\mathrm{H}$ & 4.433361000 & -3.245116000 & -0.102416000 \\
$\mathrm{H}$ & 4.114178000 & -3.249067000 & -2.483771000 \\
$\mathrm{H}$ & 6.102575000 & -1.609239000 & 0.922559000 \\
$\mathrm{H}$ & 6.043157000 & -3.156822000 & -0.789535000 \\
$\mathrm{H}$ & 1.508618000 & -0.993453000 & -1.050695000 \\
$\mathrm{H}$ & 5.322039000 & -1.998090000 & -2.750009000 \\
$\mathrm{H}$ & 2.845253000 & -1.276880000 & -2.950108000 \\
$\mathrm{H}$ & 3.876264000 & -0.250873000 & -1.974081000 \\
$\mathrm{C}$ & 2.923646000 & -0.800903000 & 0.476905000 \\
$\mathrm{~N}$ & 1.964815000 & -0.352428000 & 1.213673000 \\
$\mathrm{~N}$ & 4.263114000 & -0.711099000 & 0.798279000 \\
$\mathrm{C}$ & 4.694948000 & 0.134187000 & 1.909012000 \\
$\mathrm{H}$ & 5.025075000 & -0.503100000 & 2.743697000 \\
$\mathrm{H}$ & 5.571004000 & 0.709970000 & 1.580773000 \\
$\mathrm{C}$ & 3.592569000 & 1.072136000 & 2.370259000 \\
$\mathrm{H}$ & 3.872280000 & 1.532244000 & 3.323284000 \\
$\mathrm{H}$ & 3.455231000 & 1.877476000 & 1.638442000 \\
$\mathrm{C}$ & 2.299489000 & 0.272351000 & 2.476973000 \\
$\mathrm{H}$ & 1.466221000 & 0.920076000 & 2.770724000 \\
$\mathrm{H}$ & 2.401325000 & -0.492320000 & 3.263070000 \\
\hline---1.200 \\
\end{tabular}

TSA1-2

\begin{tabular}{lccc} 
& & & Coordinates (Angstroms) \\
\hline $\mathrm{C}$ & 1.678840000 & 1.978469000 & 2.290499000 \\
$\mathrm{C}$ & 0.647036000 & 1.501731000 & 1.492382000 \\
$\mathrm{C}$ & 0.434633000 & 1.988010000 & 0.182398000 \\
$\mathrm{C}$ & 1.337535000 & 2.969849000 & -0.278648000 \\
$\mathrm{C}$ & 2.368596000 & 3.441378000 & 0.523952000 \\
$\mathrm{C}$ & 2.557359000 & 2.951644000 & 1.815903000 \\
$\mathrm{H}$ & 1.799054000 & 1.582333000 & 3.296498000 \\
$\mathrm{H}$ & -0.019632000 & 0.741889000 & 1.894511000 \\
$\mathrm{H}$ & 1.209423000 & 3.352279000 & -1.286116000
\end{tabular}




\begin{tabular}{|c|c|c|c|}
\hline $\mathrm{H}$ & 3.036066000 & 4.206798000 & 0.134308000 \\
\hline $\mathrm{H}$ & 3.364887000 & 3.322695000 & 2.439736000 \\
\hline $\mathrm{C}$ & -0.575160000 & 1.409307000 & -0.698370000 \\
\hline $\mathrm{C}$ & -1.850876000 & 0.988360000 & -0.205490000 \\
\hline $\mathrm{H}$ & -1.921649000 & 0.893140000 & 0.878383000 \\
\hline $\mathrm{O}$ & -0.540232000 & 1.847535000 & -2.039595000 \\
\hline $\mathrm{C}$ & -2.938567000 & 0.656978000 & -0.953584000 \\
\hline $\mathrm{H}$ & -2.849528000 & 0.705127000 & -2.037305000 \\
\hline $\mathrm{C}$ & -4.208118000 & 0.156843000 & -0.436387000 \\
\hline $\mathrm{C}$ & -5.174208000 & -0.334464000 & -1.333609000 \\
\hline $\mathrm{C}$ & -4.534551000 & 0.116215000 & 0.932832000 \\
\hline $\mathrm{C}$ & -6.386360000 & -0.849962000 & -0.892360000 \\
\hline $\mathrm{H}$ & -4.957872000 & -0.311126000 & -2.399480000 \\
\hline $\mathrm{C}$ & -5.743813000 & -0.404712000 & 1.374421000 \\
\hline $\mathrm{H}$ & -3.837790000 & 0.513295000 & 1.666229000 \\
\hline $\mathrm{C}$ & -6.681595000 & -0.895350000 & 0.467936000 \\
\hline $\mathrm{H}$ & -7.106358000 & -1.220340000 & -1.617756000 \\
\hline $\mathrm{H}$ & -5.960096000 & -0.419042000 & 2.439871000 \\
\hline $\mathrm{H}$ & -7.627991000 & -1.298390000 & 0.816250000 \\
\hline $\mathrm{H}$ & 0.035841000 & -0.140103000 & -0.777920000 \\
\hline $\mathrm{H}$ & -1.229611000 & 2.522006000 & -2.143845000 \\
\hline $\mathrm{C}$ & 3.909047000 & -2.279834000 & 0.295026000 \\
\hline $\mathrm{C}$ & 4.729474000 & -1.895510000 & -0.930452000 \\
\hline $\mathrm{C}$ & 2.492984000 & -0.168957000 & -1.407068000 \\
\hline $\mathrm{C}$ & 5.000755000 & -0.393370000 & -1.050032000 \\
\hline $\mathrm{C}$ & 3.749066000 & 0.450349000 & -0.794101000 \\
\hline $\mathrm{H}$ & 4.287794000 & -1.756770000 & 1.184536000 \\
\hline $\mathrm{H}$ & 2.713612000 & -0.642613000 & -2.372287000 \\
\hline $\mathrm{H}$ & 4.229053000 & -2.279717000 & -1.826656000 \\
\hline $\mathrm{H}$ & 5.390761000 & -0.189355000 & -2.054214000 \\
\hline $\mathrm{H}$ & 4.040268000 & -3.352120000 & 0.477252000 \\
\hline $\mathrm{H}$ & 5.681297000 & -2.433753000 & -0.864326000 \\
\hline $\mathrm{H}$ & 1.753620000 & 0.604825000 & -1.623282000 \\
\hline $\mathrm{H}$ & 5.788198000 & -0.103894000 & -0.344410000 \\
\hline $\mathrm{H}$ & 3.880659000 & 1.453419000 & -1.207366000 \\
\hline $\mathrm{H}$ & 3.582568000 & 0.601917000 & 0.278669000 \\
\hline $\mathrm{C}$ & 1.794590000 & -1.185588000 & -0.535856000 \\
\hline $\mathrm{N}$ & 0.481704000 & -1.148230000 & -0.580305000 \\
\hline $\mathrm{N}$ & 2.453356000 & -2.080895000 & 0.220843000 \\
\hline $\mathrm{C}$ & 1.733499000 & -2.915332000 & 1.194299000 \\
\hline $\mathrm{H}$ & 1.656970000 & -3.934462000 & 0.792662000 \\
\hline $\mathrm{H}$ & 2.347668000 & -2.961964000 & 2.099717000 \\
\hline $\mathrm{C}$ & 0.359419000 & -2.357547000 & 1.520262000 \\
\hline $\mathrm{H}$ & -0.203007000 & -3.085776000 & 2.110359000 \\
\hline
\end{tabular}




\begin{tabular}{lrrr}
$\mathrm{H}$ & 0.464573000 & -1.443881000 & 2.113857000 \\
$\mathrm{C}$ & -0.351567000 & -2.033503000 & 0.216262000 \\
$\mathrm{H}$ & -1.304577000 & -1.524369000 & 0.376380000 \\
$\mathrm{H}$ & -0.555867000 & -2.948471000 & -0.352555000 \\
\hline
\end{tabular}

\begin{tabular}{|c|c|c|c|}
\hline \multicolumn{4}{|c|}{$\mathrm{A} 2_{\text {DBU }}$} \\
\hline & $X$ & $\mathrm{Y}$ & Z \\
\hline$\overline{\mathrm{C}}$ & 2.695434000 & 2.163175000 & 1.854822000 \\
\hline $\mathrm{C}$ & 1.526062000 & 1.978055000 & 1.132186000 \\
\hline $\mathrm{C}$ & 1.529743000 & 1.882772000 & -0.280284000 \\
\hline $\mathrm{C}$ & 2.785172000 & 2.041753000 & -0.912082000 \\
\hline $\mathrm{C}$ & 3.952970000 & 2.215105000 & -0.180614000 \\
\hline $\mathrm{C}$ & 3.930425000 & 2.261902000 & 1.212600000 \\
\hline $\mathrm{H}$ & 2.639813000 & 2.238303000 & 2.938839000 \\
\hline $\mathrm{H}$ & 0.587838000 & 1.922267000 & 1.675213000 \\
\hline $\mathrm{H}$ & 2.823269000 & 2.011959000 & -1.995511000 \\
\hline $\mathrm{H}$ & 4.896947000 & 2.322554000 & -0.709553000 \\
\hline $\mathrm{H}$ & 4.845140000 & 2.399752000 & 1.780675000 \\
\hline $\mathrm{C}$ & 0.353534000 & 1.566104000 & -1.058069000 \\
\hline $\mathrm{C}$ & -0.920314000 & 1.364246000 & -0.576118000 \\
\hline $\mathrm{H}$ & -1.068975000 & 1.609955000 & 0.472515000 \\
\hline $\mathrm{O}$ & 0.586247000 & 1.350372000 & -2.423026000 \\
\hline $\mathrm{C}$ & -2.015087000 & 0.817915000 & -1.257258000 \\
\hline $\mathrm{H}$ & -1.904640000 & 0.551804000 & -2.307934000 \\
\hline $\mathrm{C}$ & -3.327313000 & 0.617495000 & -0.680030000 \\
\hline $\mathrm{C}$ & -4.301084000 & -0.136326000 & -1.376725000 \\
\hline $\mathrm{C}$ & -3.708788000 & 1.090597000 & 0.598715000 \\
\hline $\mathrm{C}$ & -5.544093000 & -0.417372000 & -0.830373000 \\
\hline $\mathrm{H}$ & -4.056098000 & -0.512085000 & -2.368519000 \\
\hline $\mathrm{C}$ & -4.952491000 & 0.797696000 & 1.145091000 \\
\hline $\mathrm{H}$ & -3.027851000 & 1.719719000 & 1.166221000 \\
\hline $\mathrm{C}$ & -5.885296000 & 0.036386000 & 0.444018000 \\
\hline $\mathrm{H}$ & -6.256344000 & -1.003631000 & -1.406152000 \\
\hline $\mathrm{H}$ & -5.200997000 & 1.186582000 & 2.130043000 \\
\hline $\mathrm{H}$ & -6.857860000 & -0.184242000 & 0.872780000 \\
\hline $\mathrm{H}$ & -1.461824000 & -1.107157000 & -0.538283000 \\
\hline $\mathrm{H}$ & -0.119474000 & 1.806353000 & -2.900179000 \\
\hline $\mathrm{C}$ & 2.732011000 & -1.633161000 & 0.855091000 \\
\hline $\mathrm{C}$ & 3.171291000 & -2.733780000 & -0.110524000 \\
\hline $\mathrm{C}$ & 0.787433000 & -1.638838000 & -1.497885000 \\
\hline $\mathrm{C}$ & 3.273339000 & -2.271040000 & -1.568555000 \\
\hline $\mathrm{C}$ & 2.197075000 & -1.251245000 & -1.929835000 \\
\hline
\end{tabular}




$\begin{array}{lrrr}\mathrm{H} & 3.173856000 & -0.658643000 & 0.614663000 \\ \mathrm{H} & 0.550562000 & -2.666507000 & -1.806496000 \\ \mathrm{H} & 2.464017000 & -3.567554000 & -0.017446000 \\ \mathrm{H} & 3.213824000 & -3.145828000 & -2.227549000 \\ \mathrm{H} & 3.069060000 & -1.893714000 & 1.861975000 \\ \mathrm{H} & 4.139736000 & -3.119256000 & 0.225260000 \\ \mathrm{H} & 0.079569000 & -0.983522000 & -2.021450000 \\ \mathrm{H} & 4.254925000 & -1.814883000 & -1.741161000 \\ \mathrm{H} & 2.176645000 & -1.095183000 & -3.012165000 \\ \mathrm{H} & 2.441224000 & -0.277768000 & -1.503069000 \\ \mathrm{C} & 0.412423000 & -1.535289000 & -0.039860000 \\ \mathrm{~N} & -0.902726000 & -1.594949000 & 0.179463000 \\ \mathrm{~N} & 1.269287000 & -1.486675000 & 0.974558000 \\ \mathrm{C} & 0.826481000 & -1.222984000 & 2.353992000 \\ \mathrm{H} & 0.963547000 & -2.147477000 & 2.930011000 \\ \mathrm{H} & 1.501159000 & -0.463424000 & 2.758902000 \\ \mathrm{C} & -0.612713000 & -0.746163000 & 2.437935000 \\ \mathrm{H} & -0.956414000 & -0.818103000 & 3.473389000 \\ \mathrm{H} & -0.682225000 & 0.299372000 & 2.132645000 \\ \mathrm{C} & -1.480388000 & -1.587159000 & 1.516117000 \\ \mathrm{H} & -2.490105000 & -1.180970000 & 1.434270000 \\ \mathrm{H} & -1.549205000 & -2.619343000 & 1.880004000 \\ - & & & \end{array}$

TSA2-3

\begin{tabular}{|c|c|c|c|}
\hline & \multicolumn{3}{|c|}{ Coordinates (Angstroms) } \\
\hline & $\mathrm{X}$ & Y & $\mathrm{Z}$ \\
\hline $\mathrm{C}$ & -2.879449000 & -1.726225000 & 2.089431000 \\
\hline $\mathrm{C}$ & -1.711620000 & -1.529603000 & 1.364198000 \\
\hline $\mathrm{C}$ & -1.581206000 & -2.007211000 & 0.046644000 \\
\hline $\mathrm{C}$ & -2.685454000 & -2.669546000 & -0.515320000 \\
\hline $\mathrm{C}$ & -3.857348000 & -2.852348000 & 0.208640000 \\
\hline $\mathrm{C}$ & -3.966962000 & -2.382811000 & 1.515289000 \\
\hline $\mathrm{H}$ & -2.947391000 & -1.349268000 & 3.107062000 \\
\hline $\mathrm{H}$ & -0.891438000 & -0.982590000 & 1.819420000 \\
\hline $\mathrm{H}$ & -2.610892000 & -3.028093000 & -1.535883000 \\
\hline $\mathrm{H}$ & -4.695534000 & -3.366392000 & -0.254143000 \\
\hline $\mathrm{H}$ & -4.885721000 & -2.523434000 & 2.076650000 \\
\hline $\mathrm{C}$ & -0.387396000 & -1.747194000 & -0.757016000 \\
\hline $\mathrm{C}$ & 0.828020000 & -1.378197000 & -0.282335000 \\
\hline $\mathrm{H}$ & 0.944431000 & -1.431714000 & 0.797035000 \\
\hline $\mathrm{O}$ & -0.615981000 & -1.825914000 & -2.126944000 \\
\hline $\mathrm{C}$ & 1.908657000 & -0.796653000 & -1.031182000 \\
\hline $\mathrm{H}$ & 1.844553000 & -0.842118000 & -2.120988000 \\
\hline
\end{tabular}




\begin{tabular}{lrrr}
$\mathrm{C}$ & 3.277365000 & -0.715968000 & -0.519683000 \\
$\mathrm{C}$ & 4.294499000 & -0.177306000 & -1.335388000 \\
$\mathrm{C}$ & 3.647807000 & -1.067596000 & 0.794515000 \\
$\mathrm{C}$ & 5.585010000 & 0.017751000 & -0.865743000 \\
$\mathrm{H}$ & 4.049929000 & 0.099384000 & -2.359140000 \\
$\mathrm{C}$ & 4.939677000 & -0.860582000 & 1.266611000 \\
$\mathrm{H}$ & 2.919424000 & -1.525385000 & 1.458285000 \\
$\mathrm{C}$ & 5.922155000 & -0.313089000 & 0.446533000 \\
$\mathrm{H}$ & 6.337037000 & 0.434381000 & -1.531400000 \\
$\mathrm{H}$ & 5.182125000 & -1.144479000 & 2.287995000 \\
$\mathrm{H}$ & 6.930986000 & -0.158291000 & 0.816907000 \\
$\mathrm{H}$ & 1.408203000 & 0.716120000 & -0.812038000 \\
$\mathrm{H}$ & 0.216792000 & -2.098355000 & -2.534292000 \\
$\mathrm{C}$ & -2.584667000 & 2.200786000 & 0.709941000 \\
$\mathrm{C}$ & -3.133973000 & 2.890081000 & -0.537317000 \\
$\mathrm{C}$ & -0.961665000 & 1.356889000 & -1.737881000 \\
$\mathrm{C}$ & -3.450501000 & 1.921700000 & -1.681074000 \\
$\mathrm{C}$ & -2.391734000 & 0.829106000 & -1.812114000 \\
$\mathrm{H}$ & -3.109700000 & 1.257927000 & 0.912861000 \\
$\mathrm{H}$ & -0.845848000 & 2.263363000 & -2.347621000 \\
$\mathrm{H}$ & -2.408898000 & 3.647548000 & -0.859075000 \\
$\mathrm{H}$ & -3.532973000 & 2.488328000 & -2.616576000 \\
$\mathrm{H}$ & -2.756284000 & 2.853373000 & 1.572376000 \\
$\mathrm{H}$ & -4.039571000 & 3.436771000 & -0.253304000 \\
$\mathrm{H}$ & -0.285307000 & 0.613226000 & -2.173273000 \\
$\mathrm{H}$ & -4.427077000 & 1.453852000 & -1.510343000 \\
$\mathrm{H}$ & -2.510689000 & 0.299180000 & -2.761098000 \\
$\mathrm{H}$ & -2.533962000 & 0.070220000 & -1.038385000 \\
$\mathrm{C}$ & -0.386205000 & 1.660331000 & -0.374800000 \\
$\mathrm{H}$ & 0.934712000 & 1.654522000 & -0.330875000 \\
$\mathrm{H}$ & -1.134667000 & 1.954133000 & 0.703502000 \\
$\mathrm{C}$ & -0.555995000 & 1.989152000 & 2.053442000 \\
$\mathrm{H}$ & -0.603317000 & 3.024381000 & 2.417577000 \\
$\mathrm{H}$ & -1.203204000 & 1.383829000 & 2.698701000 \\
$\mathrm{H}$ & 0.873881000 & 1.477045000 & 2.101211000 \\
$\mathrm{H}$ & 1.339868000 & 1.786548000 & 3.040870000 \\
$\mathrm{H}$ & 0.896194000 & 0.384256000 & 2.063408000 \\
$\mathrm{H}$ & 1.625535000 & 2.023054000 & 0.896149000 \\
$\mathrm{H}$ & 1.69784361000 & 1.618114000 & 0.837334000 \\
0 & 3.116663000 & 0.951745000 \\
\hline
\end{tabular}

A3

Coordinates (Angstroms) 


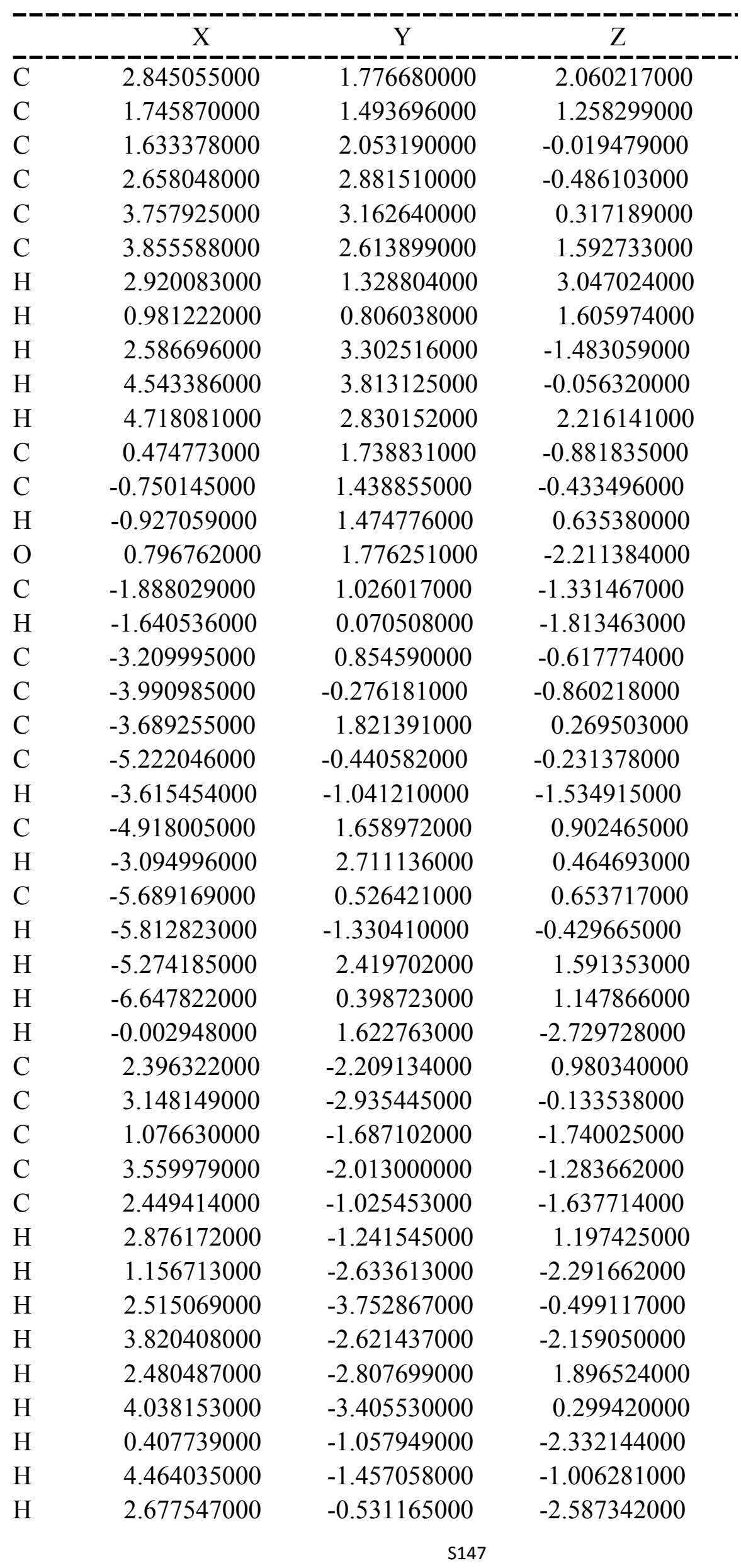




\begin{tabular}{lrrr}
$\mathrm{H}$ & 2.414582000 & -0.227018000 & -0.891665000 \\
$\mathrm{C}$ & 0.305454000 & -1.961795000 & -0.454081000 \\
$\mathrm{~N}$ & -0.965334000 & -2.110711000 & -0.604195000 \\
$\mathrm{~N}$ & 0.963319000 & -2.029116000 & 0.760149000 \\
$\mathrm{C}$ & 0.213861000 & -1.960468000 & 2.011734000 \\
$\mathrm{H}$ & 0.305612000 & -2.924691000 & 2.535330000 \\
$\mathrm{H}$ & 0.684050000 & -1.205606000 & 2.659378000 \\
$\mathrm{C}$ & -1.252495000 & -1.637257000 & 1.781970000 \\
$\mathrm{H}$ & -1.825479000 & -1.860936000 & 2.687651000 \\
$\mathrm{H}$ & -1.387674000 & -0.574080000 & 1.556459000 \\
$\mathrm{C}$ & -1.725210000 & -2.451991000 & 0.583834000 \\
$\mathrm{H}$ & -2.786130000 & -2.272615000 & 0.391439000 \\
$\mathrm{H}$ & -1.613022000 & -3.525925000 & 0.806613000 \\
$\mathrm{H}$ & -2.032563000 & 1.772189000 & -2.132248000 \\
\hline-
\end{tabular}

\section{(2) $\mathbf{R}_{1}=\mathrm{OMe}, \mathrm{R}_{2}=\mathrm{H}$}

\begin{tabular}{lccc} 
A1 & \multicolumn{3}{c}{ Coordinates (Angstroms) } \\
& $\mathrm{X}$ & $\mathrm{Y}$ & $\mathrm{Z}$ \\
$\mathrm{C}$ & -2.150347000 & -2.269156000 & -0.759056000 \\
$\mathrm{C}$ & -1.092839000 & -1.381989000 & -0.639415000 \\
$\mathrm{C}$ & -1.254049000 & -0.157419000 & 0.019402000 \\
$\mathrm{C}$ & -2.498642000 & 0.158417000 & 0.564835000 \\
$\mathrm{C}$ & -3.567855000 & -0.729755000 & 0.453095000 \\
$\mathrm{C}$ & -3.396204000 & -1.946631000 & -0.209654000 \\
$\mathrm{H}$ & -2.034533000 & -3.225402000 & -1.259011000 \\
$\mathrm{H}$ & -0.121055000 & -1.651487000 & -1.049397000 \\
$\mathrm{H}$ & -2.590672000 & 1.122682000 & 1.085373000 \\
$\mathrm{H}$ & -4.521859000 & -0.464660000 & 0.895941000 \\
$\mathrm{C}$ & -0.125460000 & 0.851770000 & 0.109063000 \\
$\mathrm{C}$ & 1.201703000 & 0.211730000 & 0.394909000 \\
$\mathrm{H}$ & 1.192638000 & -0.461095000 & 1.251262000 \\
$\mathrm{O}$ & -0.081327000 & 1.652677000 & -1.097140000 \\
$\mathrm{C}$ & 2.320096000 & 0.403044000 & -0.309394000 \\
$\mathrm{H}$ & 2.276402000 & 1.077860000 & -1.163014000 \\
$\mathrm{C}$ & 3.634653000 & -0.213913000 & -0.059728000 \\
$\mathrm{C}$ & 4.625111000 & -0.120449000 & -1.044973000 \\
$\mathrm{C}$ & 3.948239000 & -0.893983000 & 1.125163000 \\
$\mathrm{C}$ & 5.877900000 & -0.697525000 & -0.865927000 \\
$\mathrm{H}$ & 4.404670000 & 0.409253000 & -1.968961000 \\
$\mathrm{C}$ & 5.198176000 & -1.471615000 & 1.306463000 \\
$\mathrm{H}$ & 3.211797000 & -0.961158000 & 1.920359000
\end{tabular}




\begin{tabular}{lrrr}
$\mathrm{C}$ & 6.169481000 & -1.378690000 & 0.311454000 \\
$\mathrm{H}$ & 6.627612000 & -0.613696000 & -1.647464000 \\
$\mathrm{H}$ & 5.418476000 & -1.991379000 & 2.234510000 \\
$\mathrm{H}$ & 7.146483000 & -1.829806000 & 0.456830000 \\
$\mathrm{H}$ & -0.400599000 & 1.576480000 & 0.901503000 \\
$\mathrm{H}$ & -0.034597000 & 1.033395000 & -1.837719000 \\
$\mathrm{O}$ & -1.757331000 & 2.912552000 & 1.673865000 \\
$\mathrm{H}$ & -1.760187000 & 2.668094000 & 2.602885000 \\
$\mathrm{~K}$ & -1.621607000 & 3.747034000 & -0.458289000 \\
$\mathrm{O}$ & -4.368300000 & -2.884261000 & -0.369017000 \\
$\mathrm{C}$ & -5.630359000 & -2.628758000 & 0.201885000 \\
$\mathrm{H}$ & -6.099199000 & -1.737278000 & -0.235128000 \\
$\mathrm{H}$ & -6.244380000 & -3.502589000 & -0.020870000 \\
$\mathrm{H}$ & -5.562436000 & -2.501069000 & 1.289994000 \\
\hline
\end{tabular}

TSA1-2

\begin{tabular}{|c|c|c|c|}
\hline \multicolumn{4}{|c|}{ Coordinates (Angstroms) } \\
\hline & $X$ & $\mathrm{Y}$ & Z \\
\hline $\bar{C}$ & -3.121990000 & -1.936990000 & -0.158644000 \\
\hline $\mathrm{C}$ & -1.865486000 & -1.351994000 & -0.222038000 \\
\hline $\mathrm{C}$ & -1.701377000 & 0.036753000 & -0.135686000 \\
\hline $\mathrm{C}$ & -2.853867000 & 0.815860000 & 0.000919000 \\
\hline $\mathrm{C}$ & -4.123564000 & 0.248512000 & 0.047245000 \\
\hline $\mathrm{C}$ & -4.260843000 & -1.139672000 & -0.028957000 \\
\hline $\mathrm{H}$ & -3.247002000 & -3.013313000 & -0.222720000 \\
\hline $\mathrm{H}$ & -0.991417000 & -1.986969000 & -0.344827000 \\
\hline $\mathrm{H}$ & -2.739771000 & 1.893199000 & 0.089749000 \\
\hline $\mathrm{H}$ & -4.989337000 & 0.892784000 & 0.154633000 \\
\hline $\mathrm{C}$ & -0.370129000 & 0.716288000 & -0.191613000 \\
\hline $\mathrm{C}$ & 0.818249000 & -0.080886000 & 0.071236000 \\
\hline $\mathrm{H}$ & 0.747120000 & -0.704965000 & 0.963263000 \\
\hline $\mathrm{O}$ & -0.236728000 & 1.541037000 & -1.374597000 \\
\hline $\mathrm{C}$ & 1.998326000 & -0.023758000 & -0.592754000 \\
\hline $\mathrm{H}$ & 2.031108000 & 0.523471000 & -1.536836000 \\
\hline $\mathrm{C}$ & 3.245430000 & -0.696657000 & -0.210252000 \\
\hline $\mathrm{C}$ & 4.276189000 & -0.830961000 & -1.153483000 \\
\hline $\mathrm{C}$ & 3.474546000 & -1.210726000 & 1.076654000 \\
\hline $\mathrm{C}$ & 5.471235000 & -1.466416000 & -0.837005000 \\
\hline $\mathrm{H}$ & 4.125800000 & -0.439595000 & -2.157722000 \\
\hline $\mathrm{C}$ & 4.665755000 & -1.852343000 & 1.391940000 \\
\hline $\mathrm{H}$ & 2.713848000 & -1.097881000 & 1.844247000 \\
\hline $\mathrm{C}$ & 5.672938000 & -1.986368000 & 0.438494000 \\
\hline $\mathrm{H}$ & 6.246660000 & -1.559507000 & -1.592520000 \\
\hline
\end{tabular}




\begin{tabular}{lrrr}
$\mathrm{H}$ & 4.812235000 & -2.244855000 & 2.394590000 \\
$\mathrm{H}$ & 6.604803000 & -2.484449000 & 0.688668000 \\
$\mathrm{H}$ & -0.420032000 & 1.675085000 & 0.792778000 \\
$\mathrm{H}$ & -0.314978000 & 0.963876000 & -2.148098000 \\
$\mathrm{O}$ & -0.375647000 & 2.717525000 & 1.485568000 \\
$\mathrm{H}$ & -1.096292000 & 2.641646000 & 2.116848000 \\
$\mathrm{~K}$ & 1.454718000 & 3.089126000 & -0.026734000 \\
$\mathrm{O}$ & -5.449141000 & -1.803659000 & 0.013304000 \\
$\mathrm{C}$ & -6.624184000 & -1.042558000 & 0.153840000 \\
$\mathrm{H}$ & -7.447800000 & -1.758065000 & 0.161159000 \\
$\mathrm{H}$ & -6.630320000 & -0.476381000 & 1.094894000 \\
$\mathrm{H}$ & -6.759515000 & -0.345211000 & -0.683719000 \\
\hline
\end{tabular}

\begin{tabular}{|c|c|c|c|}
\hline \multicolumn{4}{|c|}{ Coordinates (Angstroms) } \\
\hline & $X$ & Y & $\mathrm{Z}$ \\
\hline$\overline{\mathrm{C}}$ & -3.183689000 & -1.414933000 & 0.684774000 \\
\hline $\mathrm{C}$ & -1.896927000 & -1.125694000 & 0.261235000 \\
\hline $\mathrm{C}$ & -1.629260000 & -0.053529000 & -0.628877000 \\
\hline $\mathrm{C}$ & -2.746953000 & 0.715346000 & -1.038665000 \\
\hline $\mathrm{C}$ & -4.035605000 & 0.425698000 & -0.599020000 \\
\hline $\mathrm{C}$ & -4.264380000 & -0.636964000 & 0.266629000 \\
\hline $\mathrm{H}$ & -3.364671000 & -2.240450000 & 1.367821000 \\
\hline $\mathrm{H}$ & -1.081748000 & -1.743551000 & 0.625876000 \\
\hline $\mathrm{H}$ & -2.589824000 & 1.530253000 & -1.737642000 \\
\hline $\mathrm{H}$ & -4.876846000 & 1.030334000 & -0.928244000 \\
\hline $\mathrm{C}$ & -0.294134000 & 0.332431000 & -1.017370000 \\
\hline $\mathrm{C}$ & 0.900719000 & -0.243202000 & -0.622808000 \\
\hline $\mathrm{H}$ & 0.815927000 & -1.216748000 & -0.144642000 \\
\hline $\mathrm{O}$ & -0.233950000 & 1.610578000 & -1.630284000 \\
\hline $\mathrm{C}$ & 2.179639000 & 0.301608000 & -0.752049000 \\
\hline $\mathrm{H}$ & 2.290675000 & 1.250518000 & -1.277126000 \\
\hline $\mathrm{C}$ & 3.428468000 & -0.361862000 & -0.408148000 \\
\hline $\mathrm{C}$ & 4.652197000 & 0.304350000 & -0.631851000 \\
\hline $\mathrm{C}$ & 3.510462000 & -1.639852000 & 0.183740000 \\
\hline $\mathrm{C}$ & 5.871124000 & -0.262682000 & -0.287874000 \\
\hline $\mathrm{H}$ & 4.632562000 & 1.290703000 & -1.091863000 \\
\hline $\mathrm{C}$ & 4.731619000 & -2.204111000 & 0.528458000 \\
\hline $\mathrm{H}$ & 2.603684000 & -2.208297000 & 0.372453000 \\
\hline $\mathrm{C}$ & 5.926638000 & -1.525011000 & 0.299989000 \\
\hline $\mathrm{H}$ & 6.789259000 & 0.285963000 & -0.483533000 \\
\hline $\mathrm{H}$ & 4.749172000 & -3.192925000 & 0.980342000 \\
\hline $\mathrm{H}$ & 6.878973000 & -1.972252000 & 0.567786000 \\
\hline
\end{tabular}




$\begin{array}{lrcc}\mathrm{H} & 0.392369000 & 1.552087000 & -2.362895000 \\ \mathrm{O} & 1.754580000 & 1.773614000 & 1.770115000 \\ \mathrm{H} & 2.466530000 & 1.648443000 & 2.405660000 \\ \mathrm{~K} & -0.602724000 & 2.306199000 & 0.941184000 \\ \mathrm{H} & 2.011409000 & 1.219984000 & 0.986594000 \\ \mathrm{O} & -5.532932000 & -0.903196000 & 0.735809000 \\ \mathrm{C} & -6.272238000 & -1.765591000 & -0.106468000 \\ \mathrm{H} & -7.245754000 & -1.913326000 & 0.366464000 \\ \mathrm{H} & -6.415292000 & -1.325333000 & -1.103020000 \\ \mathrm{H} & -5.772334000 & -2.737177000 & -0.221835000\end{array}$

TSA2-3

\begin{tabular}{|c|c|c|c|}
\hline \multicolumn{4}{|c|}{ Coordinates (Angstroms) } \\
\hline & $X$ & $\mathrm{Y}$ & Z \\
\hline$\overline{\mathrm{C}}$ & 3.198186000 & -1.169341000 & -1.174685000 \\
\hline $\mathrm{C}$ & 1.914671000 & -0.813281000 & -0.803042000 \\
\hline $\mathrm{C}$ & 1.654943000 & -0.186852000 & 0.431733000 \\
\hline $\mathrm{C}$ & 2.754804000 & 0.100308000 & 1.247625000 \\
\hline $\mathrm{C}$ & 4.054286000 & -0.243355000 & 0.877732000 \\
\hline $\mathrm{C}$ & 4.283139000 & -0.889695000 & -0.335889000 \\
\hline $\mathrm{H}$ & 3.388960000 & -1.659069000 & -2.124169000 \\
\hline $\mathrm{H}$ & 1.096184000 & -1.022847000 & -1.486003000 \\
\hline $\mathrm{H}$ & 2.588240000 & 0.591147000 & 2.200278000 \\
\hline $\mathrm{H}$ & 4.870267000 & -0.006168000 & 1.551151000 \\
\hline $\mathrm{C}$ & 0.297158000 & 0.208190000 & 0.831481000 \\
\hline $\mathrm{C}$ & -0.868826000 & -0.224232000 & 0.306765000 \\
\hline $\mathrm{H}$ & -0.800369000 & -1.033709000 & -0.416199000 \\
\hline $\mathrm{O}$ & 0.308601000 & 1.258957000 & 1.762741000 \\
\hline $\mathrm{C}$ & -2.179651000 & 0.394106000 & 0.487542000 \\
\hline $\mathrm{C}$ & -3.405513000 & -0.420088000 & 0.257167000 \\
\hline $\mathrm{C}$ & -3.404906000 & -1.801418000 & 0.024999000 \\
\hline $\mathrm{C}$ & -4.649108000 & 0.233304000 & 0.238475000 \\
\hline $\mathrm{C}$ & -4.586714000 & -2.493519000 & -0.230687000 \\
\hline $\mathrm{H}$ & -2.470234000 & -2.354279000 & 0.065468000 \\
\hline $\mathrm{C}$ & -5.830269000 & -0.455364000 & -0.000941000 \\
\hline $\mathrm{H}$ & -4.673610000 & 1.308428000 & 0.405927000 \\
\hline $\mathrm{C}$ & -5.807432000 & -1.828316000 & -0.244339000 \\
\hline $\mathrm{H}$ & -4.549656000 & -3.565773000 & -0.406011000 \\
\hline $\mathrm{H}$ & -6.775284000 & 0.081893000 & -0.006278000 \\
\hline $\mathrm{H}$ & -6.729503000 & -2.368882000 & -0.437206000 \\
\hline $\mathrm{H}$ & -0.524928000 & 1.213466000 & 2.251177000 \\
\hline $\mathrm{H}$ & -2.163021000 & 1.398899000 & -0.411892000 \\
\hline $\mathrm{O}$ & -1.985802000 & 2.421982000 & -1.177740000 \\
\hline
\end{tabular}




$\begin{array}{lrrr}\mathrm{K} & 0.324568000 & 2.707830000 & -0.608398000 \\ \mathrm{H} & -2.796805000 & 2.484971000 & -1.690561000 \\ \mathrm{H} & -2.283804000 & 0.974929000 & 1.415100000 \\ \mathrm{O} & 5.502438000 & -1.274125000 & -0.796241000 \\ \mathrm{C} & 6.620838000 & -1.040628000 & 0.027633000 \\ \mathrm{H} & 7.480800000 & -1.444592000 & -0.507862000 \\ \mathrm{H} & 6.776759000 & 0.032083000 & 0.203444000 \\ \mathrm{H} & 6.523679000 & -1.552703000 & 0.993705000\end{array}$

\begin{tabular}{|c|c|c|c|}
\hline \multicolumn{4}{|c|}{$\mathbf{A 3}_{\text {KOH }}$} \\
\hline & $X$ & Y & Z \\
\hline$\overline{\mathrm{C}}$ & -3.940996000 & 0.991856000 & 0.286466000 \\
\hline $\mathrm{C}$ & -2.682191000 & 1.540633000 & 0.040842000 \\
\hline $\mathrm{C}$ & -1.591757000 & 0.748179000 & -0.322597000 \\
\hline $\mathrm{C}$ & -1.794402000 & -0.638186000 & -0.472403000 \\
\hline $\mathrm{C}$ & -3.041774000 & -1.184497000 & -0.230701000 \\
\hline $\mathrm{C}$ & -4.122464000 & -0.382974000 & 0.158492000 \\
\hline $\mathrm{H}$ & -4.758647000 & 1.649148000 & 0.559609000 \\
\hline $\mathrm{H}$ & -2.583004000 & 2.622489000 & 0.097647000 \\
\hline $\mathrm{H}$ & -0.973260000 & -1.302753000 & -0.806530000 \\
\hline $\mathrm{H}$ & -3.205501000 & -2.251878000 & -0.344546000 \\
\hline $\mathrm{C}$ & -0.259679000 & 1.365465000 & -0.544709000 \\
\hline $\mathrm{C}$ & 0.725216000 & 0.836040000 & -1.283476000 \\
\hline $\mathrm{H}$ & 0.590747000 & -0.159130000 & -1.720678000 \\
\hline $\mathrm{O}$ & -0.059447000 & 2.596223000 & 0.046790000 \\
\hline $\mathrm{C}$ & 2.080158000 & 1.489253000 & -1.433285000 \\
\hline $\mathrm{H}$ & 2.008523000 & 2.565242000 & -1.250077000 \\
\hline $\mathrm{C}$ & 3.067240000 & 0.871998000 & -0.462811000 \\
\hline $\mathrm{C}$ & 2.983390000 & 1.175360000 & 0.903533000 \\
\hline $\mathrm{C}$ & 3.998175000 & -0.084829000 & -0.875192000 \\
\hline $\mathrm{C}$ & 3.798118000 & 0.531207000 & 1.830842000 \\
\hline $\mathrm{H}$ & 2.254589000 & 1.913611000 & 1.228211000 \\
\hline $\mathrm{C}$ & 4.814724000 & -0.736131000 & 0.051078000 \\
\hline $\mathrm{H}$ & 4.068123000 & -0.339690000 & -1.929106000 \\
\hline $\mathrm{C}$ & 4.714522000 & -0.435269000 & 1.408066000 \\
\hline $\mathrm{H}$ & 3.722685000 & 0.784152000 & 2.884965000 \\
\hline $\mathrm{H}$ & 5.529153000 & -1.479686000 & -0.290415000 \\
\hline $\mathrm{H}$ & 5.354189000 & -0.936292000 & 2.128732000 \\
\hline $\mathrm{O}$ & 0.550424000 & -2.312897000 & -1.429192000 \\
\hline $\mathrm{H}$ & 2.443244000 & 1.350926000 & -2.457410000 \\
\hline $\mathrm{H}$ & -0.694008000 & 2.689333000 & 0.767026000 \\
\hline $\mathrm{H}$ & 0.152058000 & -2.636948000 & -2.241285000 \\
\hline
\end{tabular}




$\begin{array}{lrrr}\text { K } & 1.614858000 & -1.746448000 & 0.525852000 \\ \mathrm{O} & -5.295241000 & -1.031790000 & 0.378425000 \\ \mathrm{C} & -6.414359000 & -0.264224000 & 0.754439000 \\ \mathrm{H} & -7.237083000 & -0.970285000 & 0.874745000 \\ \mathrm{H} & -6.244884000 & 0.258155000 & 1.705411000 \\ \mathrm{H} & -6.678646000 & 0.470565000 & -0.017169000\end{array}$

\begin{tabular}{|c|c|c|c|}
\hline \multicolumn{4}{|c|}{$\mathbf{A 1}_{\mathrm{NaOH}}$} \\
\hline & \multicolumn{3}{|c|}{ Coordinates (Angstroms) } \\
\hline & $\mathrm{X}$ & $\mathrm{Y}$ & $\mathrm{Z}$ \\
\hline $\mathrm{C}$ & -2.333648000 & -1.978791000 & -0.691828000 \\
\hline $\mathrm{C}$ & -1.231973000 & -1.146394000 & -0.581010000 \\
\hline $\mathrm{C}$ & -1.339759000 & 0.104398000 & 0.038996000 \\
\hline $\mathrm{C}$ & -2.572893000 & 0.503273000 & 0.555813000 \\
\hline $\mathrm{C}$ & -3.685863000 & -0.330240000 & 0.451831000 \\
\hline $\mathrm{C}$ & -3.569056000 & -1.573088000 & -0.173962000 \\
\hline $\mathrm{H}$ & -2.262590000 & -2.953018000 & -1.164423000 \\
\hline $\mathrm{H}$ & -0.270183000 & -1.478427000 & -0.967966000 \\
\hline $\mathrm{H}$ & -2.629840000 & 1.489499000 & 1.037535000 \\
\hline $\mathrm{H}$ & -4.630673000 & 0.000207000 & 0.869280000 \\
\hline $\mathrm{C}$ & -0.165749000 & 1.058903000 & 0.103504000 \\
\hline $\mathrm{C}$ & 1.137884000 & 0.375024000 & 0.396865000 \\
\hline $\mathrm{H}$ & 1.107876000 & -0.269403000 & 1.274004000 \\
\hline $\mathrm{O}$ & -0.093951000 & 1.829137000 & -1.128947000 \\
\hline $\mathrm{C}$ & 2.256684000 & 0.505123000 & -0.319740000 \\
\hline $\mathrm{H}$ & 2.230866000 & 1.156545000 & -1.192013000 \\
\hline $\mathrm{C}$ & 3.552124000 & -0.146919000 & -0.059076000 \\
\hline $\mathrm{C}$ & 4.546431000 & -0.091670000 & -1.042980000 \\
\hline $\mathrm{C}$ & 3.843866000 & -0.819922000 & 1.135209000 \\
\hline $\mathrm{C}$ & 5.783952000 & -0.697161000 & -0.852431000 \\
\hline $\mathrm{H}$ & 4.342427000 & 0.432322000 & -1.973868000 \\
\hline $\mathrm{C}$ & 5.078563000 & -1.425757000 & 1.327990000 \\
\hline $\mathrm{H}$ & 3.103887000 & -0.859386000 & 1.928882000 \\
\hline $\mathrm{C}$ & 6.054716000 & -1.369309000 & 0.334981000 \\
\hline $\mathrm{H}$ & 6.537929000 & -0.641811000 & -1.632205000 \\
\hline $\mathrm{H}$ & 5.283175000 & -1.938975000 & 2.263147000 \\
\hline $\mathrm{H}$ & 7.020219000 & -1.841480000 & 0.489718000 \\
\hline $\mathrm{H}$ & -0.395348000 & 1.816181000 & 0.872560000 \\
\hline $\mathrm{H}$ & -0.119025000 & 1.192599000 & -1.855648000 \\
\hline $\mathrm{O}$ & -1.818359000 & 3.334082000 & 1.314791000 \\
\hline $\mathrm{H}$ & -1.946998000 & 3.738083000 & 2.174784000 \\
\hline $\mathrm{O}$ & -4.586720000 & -2.460278000 & -0.326736000 \\
\hline $\mathrm{C}$ & -5.845066000 & -2.120991000 & 0.208874000 \\
\hline
\end{tabular}




$\begin{array}{llll}\mathrm{H} & -6.252986000 & -1.214965000 & -0.257890000 \\ \mathrm{H} & -6.502662000 & -2.963484000 & -0.009710000 \\ \mathrm{H} & -5.793826000 & -1.973163000 & 1.295204000 \\ \mathrm{Na} & -1.444664000 & 3.600570000 & -0.638407000\end{array}$

TSA1-2 $\mathrm{NaOH}$

\begin{tabular}{|c|c|c|c|}
\hline \multicolumn{4}{|c|}{ Coordinates (Angstroms) } \\
\hline & $X$ & Y & Z \\
\hline $\mathrm{C}$ & -3.028891000 & -1.815587000 & -0.208429000 \\
\hline $\mathrm{C}$ & -1.784621000 & -1.204129000 & -0.257531000 \\
\hline $\mathrm{C}$ & -1.646251000 & 0.182812000 & -0.111276000 \\
\hline $\mathrm{C}$ & -2.811368000 & 0.933713000 & 0.068068000 \\
\hline $\mathrm{C}$ & -4.069264000 & 0.340209000 & 0.098550000 \\
\hline $\mathrm{C}$ & -4.181169000 & -1.046131000 & -0.035099000 \\
\hline $\mathrm{H}$ & -3.134453000 & -2.890239000 & -0.317872000 \\
\hline $\mathrm{H}$ & -0.899906000 & -1.815765000 & -0.417590000 \\
\hline $\mathrm{H}$ & -2.719401000 & 2.008047000 & 0.202050000 \\
\hline $\mathrm{H}$ & -4.945639000 & 0.962590000 & 0.241446000 \\
\hline $\mathrm{C}$ & -0.327125000 & 0.879266000 & -0.148570000 \\
\hline $\mathrm{C}$ & 0.880602000 & 0.108878000 & 0.080007000 \\
\hline $\mathrm{H}$ & 0.826821000 & -0.559992000 & 0.940310000 \\
\hline $\mathrm{O}$ & -0.202970000 & 1.765405000 & -1.294020000 \\
\hline $\mathrm{C}$ & 2.066453000 & 0.235091000 & -0.570324000 \\
\hline $\mathrm{H}$ & 2.087271000 & 0.801936000 & -1.504325000 \\
\hline $\mathrm{C}$ & 3.329103000 & -0.418541000 & -0.204495000 \\
\hline $\mathrm{C}$ & 4.360464000 & -0.511806000 & -1.151634000 \\
\hline $\mathrm{C}$ & 3.567816000 & -0.957918000 & 1.069886000 \\
\hline $\mathrm{C}$ & 5.567428000 & -1.131809000 & -0.850391000 \\
\hline $\mathrm{H}$ & 4.202320000 & -0.100446000 & -2.146605000 \\
\hline $\mathrm{C}$ & 4.770990000 & -1.584775000 & 1.369413000 \\
\hline $\mathrm{H}$ & 2.806252000 & -0.875998000 & 1.840482000 \\
\hline $\mathrm{C}$ & 5.779182000 & -1.677830000 & 0.412487000 \\
\hline $\mathrm{H}$ & 6.343864000 & -1.192583000 & -1.607904000 \\
\hline $\mathrm{H}$ & 4.925752000 & -1.997511000 & 2.362524000 \\
\hline $\mathrm{H}$ & 6.720297000 & -2.164154000 & 0.650540000 \\
\hline $\mathrm{H}$ & -0.315125000 & 1.875663000 & 0.846474000 \\
\hline $\mathrm{H}$ & -0.229246000 & 1.227474000 & -2.098453000 \\
\hline $\mathrm{O}$ & -0.126773000 & 2.942208000 & 1.419791000 \\
\hline $\mathrm{H}$ & -0.109058000 & 2.734905000 & 2.359682000 \\
\hline $\mathrm{O}$ & -5.355705000 & -1.733598000 & -0.010602000 \\
\hline $\mathrm{C}$ & -6.544289000 & -1.001527000 & 0.169327000 \\
\hline $\mathrm{H}$ & -7.353746000 & -1.732774000 & 0.154299000 \\
\hline $\mathrm{H}$ & -6.553165000 & -0.473666000 & 1.132168000 \\
\hline
\end{tabular}




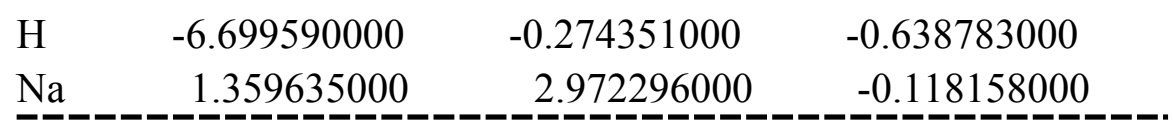

$\mathrm{A} 2_{\mathrm{NaOH}}$

\begin{tabular}{|c|c|c|c|}
\hline \multicolumn{4}{|c|}{ Coordinates (Angstroms) } \\
\hline & $X$ & $\mathrm{Y}$ & $\mathrm{Z}$ \\
\hline$\overline{\mathrm{C}}$ & 3.217683000 & 1.421011000 & 0.381113000 \\
\hline $\mathrm{C}$ & 1.935336000 & 1.072356000 & -0.002003000 \\
\hline $\mathrm{C}$ & 1.647025000 & -0.199575000 & -0.560697000 \\
\hline $\mathrm{C}$ & 2.739079000 & -1.091772000 & -0.692900000 \\
\hline $\mathrm{C}$ & 4.028390000 & -0.733163000 & -0.301510000 \\
\hline $\mathrm{C}$ & 4.276452000 & 0.518374000 & 0.241819000 \\
\hline $\mathrm{H}$ & 3.410566000 & 2.399050000 & 0.813625000 \\
\hline $\mathrm{H}$ & 1.140091000 & 1.800052000 & 0.127417000 \\
\hline $\mathrm{H}$ & 2.572461000 & -2.059305000 & -1.155194000 \\
\hline $\mathrm{H}$ & 4.852857000 & -1.430987000 & -0.417997000 \\
\hline $\mathrm{C}$ & 0.306130000 & -0.629767000 & -0.886527000 \\
\hline $\mathrm{C}$ & -0.883987000 & 0.014081000 & -0.596256000 \\
\hline $\mathrm{H}$ & -0.789653000 & 1.062824000 & -0.322972000 \\
\hline $\mathrm{O}$ & 0.237455000 & -2.021309000 & -1.185413000 \\
\hline $\mathrm{C}$ & -2.165876000 & -0.539432000 & -0.584207000 \\
\hline $\mathrm{H}$ & -2.304256000 & -1.550772000 & -0.968657000 \\
\hline $\mathrm{C}$ & -3.402136000 & 0.195420000 & -0.342128000 \\
\hline $\mathrm{C}$ & -4.641108000 & -0.447668000 & -0.536548000 \\
\hline $\mathrm{C}$ & -3.449959000 & 1.526488000 & 0.119347000 \\
\hline $\mathrm{C}$ & -5.846996000 & 0.193700000 & -0.290199000 \\
\hline $\mathrm{H}$ & -4.646306000 & -1.475822000 & -0.893631000 \\
\hline $\mathrm{C}$ & -4.657848000 & 2.167786000 & 0.362037000 \\
\hline $\mathrm{H}$ & -2.526855000 & 2.073545000 & 0.293126000 \\
\hline $\mathrm{C}$ & -5.870080000 & 1.511293000 & 0.162265000 \\
\hline $\mathrm{H}$ & -6.779522000 & -0.339568000 & -0.456972000 \\
\hline $\mathrm{H}$ & -4.650408000 & 3.196486000 & 0.714190000 \\
\hline $\mathrm{H}$ & -6.812302000 & 2.015693000 & 0.353225000 \\
\hline $\mathrm{H}$ & -0.445308000 & -2.147505000 & -1.855104000 \\
\hline $\mathrm{O}$ & -1.462713000 & -1.752706000 & 1.992067000 \\
\hline $\mathrm{H}$ & -1.803629000 & -1.262079000 & 2.747625000 \\
\hline $\mathrm{H}$ & -1.849060000 & -1.297382000 & 1.190424000 \\
\hline $\mathrm{O}$ & 5.543732000 & 0.848019000 & 0.667118000 \\
\hline $\mathrm{C}$ & 6.251822000 & 1.670942000 & -0.239238000 \\
\hline $\mathrm{H}$ & 7.231467000 & 1.858599000 & 0.205527000 \\
\hline $\mathrm{H}$ & 6.380297000 & 1.173670000 & -1.210348000 \\
\hline $\mathrm{H}$ & 5.739577000 & 2.629157000 & -0.401427000 \\
\hline $\mathrm{Na}$ & 0.529878000 & -2.103466000 & 1.094615000 \\
\hline
\end{tabular}


TSA2-3 ${ }_{\mathrm{NaOH}}$

\begin{tabular}{|c|c|c|c|}
\hline \multicolumn{4}{|c|}{ Coordinates (Angstroms) } \\
\hline & $\mathrm{X}$ & $\mathrm{Y}$ & $\mathrm{Z}$ \\
\hline $\mathrm{C}$ & -3.158422000 & -1.635676000 & 0.375319000 \\
\hline $\mathrm{C}$ & -1.883909000 & -1.162223000 & 0.136407000 \\
\hline $\mathrm{C}$ & -1.668063000 & 0.099639000 & -0.453845000 \\
\hline $\mathrm{C}$ & -2.799149000 & 0.859827000 & -0.771996000 \\
\hline $\mathrm{C}$ & -4.091674000 & 0.392979000 & -0.527066000 \\
\hline $\mathrm{C}$ & -4.279357000 & -0.861414000 & 0.046650000 \\
\hline $\mathrm{H}$ & -3.316213000 & -2.610124000 & 0.825926000 \\
\hline $\mathrm{H}$ & -1.038652000 & -1.785025000 & 0.413052000 \\
\hline $\mathrm{H}$ & -2.669829000 & 1.828695000 & -1.241507000 \\
\hline $\mathrm{H}$ & -4.934745000 & 1.017378000 & -0.801002000 \\
\hline $\mathrm{C}$ & -0.318596000 & 0.635418000 & -0.677502000 \\
\hline $\mathrm{C}$ & 0.870698000 & 0.012775000 & -0.519798000 \\
\hline $\mathrm{H}$ & 0.841247000 & -1.049137000 & -0.283730000 \\
\hline $\mathrm{O}$ & -0.345784000 & 2.019355000 & -0.934241000 \\
\hline $\mathrm{C}$ & 2.174204000 & 0.673069000 & -0.574564000 \\
\hline $\mathrm{C}$ & 3.373383000 & -0.162165000 & -0.353955000 \\
\hline $\mathrm{C}$ & 3.401888000 & -1.167266000 & 0.628291000 \\
\hline $\mathrm{C}$ & 4.563446000 & 0.067761000 & -1.063176000 \\
\hline $\mathrm{C}$ & 4.548279000 & -1.916242000 & 0.870321000 \\
\hline $\mathrm{H}$ & 2.511304000 & -1.355507000 & 1.224752000 \\
\hline $\mathrm{C}$ & 5.714289000 & -0.669555000 & -0.812757000 \\
\hline $\mathrm{H}$ & 4.577937000 & 0.840031000 & -1.829199000 \\
\hline $\mathrm{C}$ & 5.716485000 & -1.673524000 & 0.153096000 \\
\hline $\mathrm{H}$ & 4.530844000 & -2.689033000 & 1.634735000 \\
\hline $\mathrm{H}$ & 6.616549000 & -0.463415000 & -1.383034000 \\
\hline $\mathrm{H}$ & 6.614709000 & -2.252427000 & 0.346249000 \\
\hline $\mathrm{H}$ & 0.545106000 & 2.279372000 & -1.208662000 \\
\hline $\mathrm{H}$ & 2.121193000 & 1.582877000 & 0.546642000 \\
\hline $\mathrm{O}$ & 1.899264000 & 2.302431000 & 1.476137000 \\
\hline $\mathrm{H}$ & 2.638305000 & 2.173335000 & 2.078716000 \\
\hline $\mathrm{H}$ & 2.301320000 & 1.330076000 & -1.446457000 \\
\hline $\mathrm{O}$ & -5.485292000 & -1.417782000 & 0.328937000 \\
\hline $\mathrm{C}$ & -6.642147000 & -0.683730000 & 0.000300000 \\
\hline $\mathrm{H}$ & -7.488027000 & -1.302937000 & 0.301360000 \\
\hline $\mathrm{H}$ & -6.682678000 & 0.270568000 & 0.542017000 \\
\hline $\mathrm{H}$ & -6.703128000 & -0.488290000 & -1.078153000 \\
\hline $\mathrm{Na}$ & -0.194444000 & 2.053044000 & 1.423390000 \\
\hline
\end{tabular}

$\mathrm{A3}_{\mathrm{NaOH}}$ 


\begin{tabular}{lrcc} 
& \multicolumn{3}{c}{ Coordinates (Angstroms) } \\
& $\mathrm{X}$ & $\mathrm{Y}$ & $\mathrm{Z}$ \\
\hline $\mathrm{C}$ & 3.859060000 & -0.947860000 & 0.215733000 \\
$\mathrm{C}$ & 2.586587000 & -1.468981000 & -0.014610000 \\
$\mathrm{C}$ & 1.496335000 & -0.643826000 & -0.297779000 \\
$\mathrm{C}$ & 1.705909000 & 0.746291000 & -0.374325000 \\
$\mathrm{C}$ & 2.968414000 & 1.264648000 & -0.148932000 \\
$\mathrm{C}$ & 4.051774000 & 0.430754000 & 0.154365000 \\
$\mathrm{H}$ & 4.677862000 & -1.626638000 & 0.425210000 \\
$\mathrm{H}$ & 2.473085000 & -2.551126000 & -0.012924000 \\
$\mathrm{H}$ & 0.874366000 & 1.433086000 & -0.620365000 \\
$\mathrm{H}$ & 3.141875000 & 2.335035000 & -0.202989000 \\
$\mathrm{C}$ & 0.154513000 & -1.237628000 & -0.517561000 \\
$\mathrm{C}$ & -0.816168000 & -0.701204000 & -1.271713000 \\
$\mathrm{H}$ & -0.649176000 & 0.274480000 & -1.728681000 \\
$\mathrm{O}$ & -0.070904000 & -2.457340000 & 0.081195000 \\
$\mathrm{C}$ & -2.179384000 & -1.335860000 & -1.435034000 \\
$\mathrm{H}$ & -2.120636000 & -2.414216000 & -1.261540000 \\
$\mathrm{C}$ & -3.158196000 & -0.711842000 & -0.460777000 \\
$\mathrm{C}$ & -3.086381000 & -1.037297000 & 0.902425000 \\
$\mathrm{C}$ & -4.046934000 & 0.290347000 & -0.859892000 \\
$\mathrm{C}$ & -3.866533000 & -0.364048000 & 1.839707000 \\
$\mathrm{H}$ & -2.389340000 & -1.809586000 & 1.217082000 \\
$\mathrm{C}$ & -4.829139000 & 0.968179000 & 0.076909000 \\
$\mathrm{H}$ & -4.102740000 & 0.565425000 & -1.909252000 \\
$\mathrm{C}$ & -4.736921000 & 0.649424000 & 1.429996000 \\
$\mathrm{H}$ & -3.798936000 & -0.630430000 & 2.890783000 \\
$\mathrm{H}$ & -5.504990000 & 1.751343000 & -0.253026000 \\
$\mathrm{H}$ & -5.345043000 & 1.176522000 & 2.158835000 \\
$\mathrm{O}$ & -0.714852000 & 2.446240000 & -0.998964000 \\
$\mathrm{H}$ & -2.535693000 & -1.183315000 & -2.459150000 \\
$\mathrm{H}$ & 0.587758000 & -2.575849000 & 0.775993000 \\
$\mathrm{H}$ & -0.456907000 & 3.320824000 & -1.298564000 \\
$\mathrm{C}$ & 5.239852000 & 1.053301000 & 0.364665000 \\
$\mathrm{H}$ & 6.357899000 & 0.256137000 & 0.677640000 \\
$\mathrm{H}$ & 7.192536000 & 0.946150000 & 0.808170000 \\
$\mathrm{H}$ & 6.204255000 & -0.305539000 & 1.608609000 \\
$\mathrm{H}$ & -1.749072000 & -0.446851000 & -0.131908000 \\
$\mathrm{H}$ & 1.496335000 & 0.447421000 \\
\hline
\end{tabular}

A1 $1_{\text {LiOH }}$

Coordinates (Angstroms)

X

Y Z 


\begin{tabular}{lrrr}
$\mathrm{C}$ & -2.416841000 & -1.804384000 & -0.481120000 \\
$\mathrm{C}$ & -1.296972000 & -0.995464000 & -0.378722000 \\
$\mathrm{C}$ & -1.407882000 & 0.321904000 & 0.081203000 \\
$\mathrm{C}$ & -2.664340000 & 0.814657000 & 0.437759000 \\
$\mathrm{C}$ & -3.795478000 & 0.007772000 & 0.338230000 \\
$\mathrm{C}$ & -3.674212000 & -1.305434000 & -0.122971000 \\
$\mathrm{H}$ & -2.344549000 & -2.828758000 & -0.831537000 \\
$\mathrm{H}$ & -0.322033000 & -1.395135000 & -0.648774000 \\
$\mathrm{H}$ & -2.737241000 & 1.853154000 & 0.777719000 \\
$\mathrm{H}$ & -4.758332000 & 0.415293000 & 0.624948000 \\
$\mathrm{C}$ & -0.213429000 & 1.249195000 & 0.126488000 \\
$\mathrm{C}$ & 1.083749000 & 0.554615000 & 0.424680000 \\
$\mathrm{H}$ & 1.065039000 & -0.028076000 & 1.344072000 \\
$\mathrm{O}$ & -0.136107000 & 2.000980000 & -1.121143000 \\
$\mathrm{C}$ & 2.179916000 & 0.613558000 & -0.333792000 \\
$\mathrm{H}$ & 2.137364000 & 1.211102000 & -1.242877000 \\
$\mathrm{C}$ & 3.471248000 & -0.048134000 & -0.077817000 \\
$\mathrm{C}$ & 4.460457000 & 0.003580000 & -1.066841000 \\
$\mathrm{C}$ & 3.763512000 & -0.727177000 & 1.112835000 \\
$\mathrm{C}$ & 5.696324000 & -0.607205000 & -0.882646000 \\
$\mathrm{H}$ & 4.254755000 & 0.530273000 & -1.995722000 \\
$\mathrm{C}$ & 4.996585000 & -1.337890000 & 1.299342000 \\
$\mathrm{H}$ & 3.025052000 & -0.771711000 & 1.907651000 \\
$\mathrm{C}$ & 5.969070000 & -1.282179000 & 0.302550000 \\
$\mathrm{H}$ & 6.447113000 & -0.553688000 & -1.665477000 \\
$\mathrm{H}$ & 5.202354000 & -1.856013000 & 2.231442000 \\
$\mathrm{H}$ & 6.933127000 & -1.758778000 & 0.452299000 \\
$\mathrm{H}$ & -0.411044000 & 2.025186000 & 0.877560000 \\
$\mathrm{H}$ & -0.317737000 & 1.378328000 & -1.837415000 \\
$\mathrm{O}$ & -1.931569000 & 3.802406000 & 0.768194000 \\
$\mathrm{H}$ & -2.244734000 & 4.494325000 & 1.350224000 \\
$\mathrm{O}$ & -4.708035000 & -2.173579000 & -0.258660000 \\
$\mathrm{C}$ & -5.999628000 & -1.726222000 & 0.086930000 \\
$\mathrm{H}$ & -6.307927000 & -0.870794000 & -0.527814000 \\
$\mathrm{H}$ & -6.669730000 & -2.565340000 & -0.104155000 \\
$\mathrm{H}$ & -6.060631000 & -1.449108000 & 1.147052000 \\
.199212000 & 3.581379000 & -0.720989000 \\
\hline
\end{tabular}

TSA1-2

\begin{tabular}{|c|c|c|c|}
\hline & \multicolumn{3}{|c|}{ Coordinates (Angstroms) } \\
\hline & $\mathrm{X}$ & $\mathrm{Y}$ & Z \\
\hline $\mathrm{C}$ & -3.022368000 & -1.688189000 & 0.063904000 \\
\hline $\mathrm{C}$ & -1.766345000 & -1.102152000 & 0.021319000 \\
\hline
\end{tabular}




\begin{tabular}{|c|c|c|c|}
\hline $\mathrm{C}$ & -1.610671000 & 0.289141000 & -0.063131000 \\
\hline $\mathrm{C}$ & -2.773912000 & 1.062004000 & -0.133438000 \\
\hline $\mathrm{C}$ & -4.040931000 & 0.487043000 & -0.118478000 \\
\hline $\mathrm{C}$ & -4.171343000 & -0.898549000 & -0.008087000 \\
\hline $\mathrm{H}$ & -3.136654000 & -2.765204000 & 0.133757000 \\
\hline $\mathrm{H}$ & -0.890766000 & -1.745318000 & 0.041120000 \\
\hline $\mathrm{H}$ & -2.681402000 & 2.141961000 & -0.201389000 \\
\hline $\mathrm{H}$ & -4.912049000 & 1.130016000 & -0.179164000 \\
\hline $\mathrm{C}$ & -0.285235000 & 0.959406000 & -0.055550000 \\
\hline $\mathrm{C}$ & 0.941000000 & 0.203394000 & 0.081122000 \\
\hline $\mathrm{H}$ & 0.926778000 & -0.550506000 & 0.868442000 \\
\hline $\mathrm{O}$ & -0.162158000 & 1.987451000 & -1.080739000 \\
\hline $\mathrm{C}$ & 2.119658000 & 0.458465000 & -0.548146000 \\
\hline $\mathrm{H}$ & 2.113277000 & 1.109912000 & -1.426340000 \\
\hline $\mathrm{C}$ & 3.413655000 & -0.167815000 & -0.248292000 \\
\hline $\mathrm{C}$ & 4.435380000 & -0.139027000 & -1.209137000 \\
\hline $\mathrm{C}$ & 3.688705000 & -0.797090000 & 0.975866000 \\
\hline $\mathrm{C}$ & 5.670587000 & -0.729340000 & -0.969554000 \\
\hline $\mathrm{H}$ & 4.248890000 & 0.344352000 & -2.165927000 \\
\hline $\mathrm{C}$ & 4.920523000 & -1.394589000 & 1.213086000 \\
\hline $\mathrm{H}$ & 2.934353000 & -0.807686000 & 1.757747000 \\
\hline $\mathrm{C}$ & 5.919630000 & -1.366068000 & 0.242996000 \\
\hline $\mathrm{H}$ & 6.440145000 & -0.695318000 & -1.735478000 \\
\hline $\mathrm{H}$ & 5.104721000 & -1.877142000 & 2.168863000 \\
\hline $\mathrm{H}$ & 6.882987000 & -1.829435000 & 0.432628000 \\
\hline $\mathrm{H}$ & -0.142610000 & 1.905597000 & 1.033643000 \\
\hline $\mathrm{H}$ & -0.137511000 & 1.560847000 & -1.950127000 \\
\hline $\mathrm{O}$ & 0.275720000 & 2.914116000 & 1.532257000 \\
\hline $\mathrm{H}$ & 0.587475000 & 2.678756000 & 2.412857000 \\
\hline $\mathrm{Li}$ & 1.273242000 & 2.804173000 & 0.006976000 \\
\hline $\mathrm{O}$ & -5.356894000 & -1.566789000 & 0.029635000 \\
\hline $\mathrm{C}$ & -6.541065000 & -0.810507000 & -0.051776000 \\
\hline $\mathrm{H}$ & -6.603591000 & -0.256608000 & -0.998062000 \\
\hline $\mathrm{H}$ & -7.361084000 & -1.528396000 & -0.002550000 \\
\hline $\mathrm{H}$ & -6.629647000 & -0.103338000 & 0.783613000 \\
\hline
\end{tabular}

A2 $2_{\text {LiOH }}$

\begin{tabular}{llcc} 
& & \multicolumn{3}{c}{ Coordinates (Angstroms) } & Z \\
\hline $\mathrm{C}$ & -3.232421000 & -1.392673000 & 0.213936000 \\
$\mathrm{C}$ & -1.954542000 & -0.994467000 & -0.132444000 \\
$\mathrm{C}$ & -1.660681000 & 0.356852000 & -0.440078000 \\
$\mathrm{C}$ & -2.735358000 & 1.273764000 & -0.373221000
\end{tabular}




\begin{tabular}{lrrr}
$\mathrm{C}$ & -4.020456000 & 0.864029000 & -0.018357000 \\
$\mathrm{C}$ & -4.276200000 & -0.464752000 & 0.282542000 \\
$\mathrm{H}$ & -3.436865000 & -2.433616000 & 0.448328000 \\
$\mathrm{H}$ & -1.170386000 & -1.743770000 & -0.176924000 \\
$\mathrm{H}$ & -2.567364000 & 2.309396000 & -0.649860000 \\
$\mathrm{H}$ & -4.833586000 & 1.582859000 & 0.024733000 \\
$\mathrm{C}$ & -0.316587000 & 0.821507000 & -0.716833000 \\
$\mathrm{C}$ & 0.880020000 & 0.154488000 & -0.511299000 \\
$\mathrm{H}$ & 0.786910000 & -0.918178000 & -0.357577000 \\
$\mathrm{O}$ & -0.258167000 & 2.248253000 & -0.817544000 \\
$\mathrm{C}$ & 2.159286000 & 0.705593000 & -0.421941000 \\
$\mathrm{H}$ & 2.310380000 & 1.745620000 & -0.716963000 \\
$\mathrm{C}$ & 3.394152000 & -0.059317000 & -0.259102000 \\
$\mathrm{C}$ & 4.634888000 & 0.580908000 & -0.437967000 \\
$\mathrm{C}$ & 3.430410000 & -1.418801000 & 0.105913000 \\
$\mathrm{C}$ & 5.837062000 & -0.092637000 & -0.268494000 \\
$\mathrm{H}$ & 4.647105000 & 1.632147000 & -0.719126000 \\
$\mathrm{C}$ & 4.633633000 & -2.093264000 & 0.269296000 \\
$\mathrm{H}$ & 2.503162000 & -1.961479000 & 0.271376000 \\
$\mathrm{C}$ & 5.849816000 & -1.439758000 & 0.085499000 \\
$\mathrm{H}$ & 6.773344000 & 0.438556000 & -0.418793000 \\
$\mathrm{H}$ & 4.619333000 & -3.144180000 & 0.547131000 \\
$\mathrm{H}$ & 6.788721000 & -1.968668000 & 0.217126000 \\
$\mathrm{H}$ & 0.492008000 & 2.482831000 & -1.376886000 \\
$\mathrm{O}$ & 1.179296000 & 1.798471000 & 2.087826000 \\
$\mathrm{H}$ & 1.315824000 & 1.243913000 & 2.864963000 \\
$\mathrm{H}$ & 1.708270000 & 1.367219000 & 1.349359000 \\
$\mathrm{O}$ & -5.536096000 & -0.861210000 & 0.667553000 \\
$\mathrm{C}$ & -6.317008000 & -1.375604000 & -0.393972000 \\
$\mathrm{H}$ & -7.280887000 & -1.660007000 & 0.033200000 \\
$\mathrm{H}$ & -6.472440000 & -0.619445000 & -1.175682000 \\
$\mathrm{H}$ & -5.846916000 & -2.258415000 & -0.848438000 \\
$\mathrm{Li}$ & -0.417656000 & 1.955297000 & 1.093497000 \\
\hline----- &
\end{tabular}

TSA2-3 ${ }_{\mathrm{LiOH}}$

$\begin{array}{lrrr} & & & \text { Coordinates (Angstroms) } \\ & & \mathrm{X} & \mathrm{Z} \\ \mathrm{C} & -3.193054000 & -1.475878000 & 0.497580000 \\ \mathrm{C} & -1.917500000 & -0.960484000 & 0.376489000 \\ \mathrm{C} & -1.687975000 & 0.297256000 & -0.213843000 \\ \mathrm{C} & -2.805178000 & 1.017699000 & -0.646836000 \\ \mathrm{C} & -4.098157000 & 0.510962000 & -0.519673000 \\ \mathrm{C} & -4.299832000 & -0.744289000 & 0.049428000\end{array}$




\begin{tabular}{|c|c|c|c|}
\hline $\mathrm{H}$ & -3.362104000 & -2.447110000 & 0.950879000 \\
\hline $\mathrm{H}$ & -1.081884000 & -1.543467000 & 0.752688000 \\
\hline $\mathrm{H}$ & -2.663665000 & 1.990316000 & -1.105091000 \\
\hline $\mathrm{H}$ & -4.931287000 & 1.106593000 & -0.875361000 \\
\hline $\mathrm{C}$ & -0.334548000 & 0.848455000 & -0.346194000 \\
\hline $\mathrm{C}$ & 0.860759000 & 0.211642000 & -0.246888000 \\
\hline $\mathrm{H}$ & 0.809652000 & -0.865174000 & -0.098717000 \\
\hline $\mathrm{O}$ & -0.334329000 & 2.263235000 & -0.506517000 \\
\hline $\mathrm{C}$ & 2.170057000 & 0.828206000 & -0.253127000 \\
\hline $\mathrm{H}$ & 2.279317000 & 1.680121000 & -0.936822000 \\
\hline $\mathrm{C}$ & 3.367250000 & -0.036303000 & -0.247375000 \\
\hline $\mathrm{C}$ & 4.526780000 & 0.335233000 & -0.946719000 \\
\hline $\mathrm{C}$ & 3.426421000 & -1.224486000 & 0.500821000 \\
\hline $\mathrm{C}$ & 5.678235000 & -0.442038000 & -0.912095000 \\
\hline $\mathrm{H}$ & 4.517079000 & 1.252162000 & -1.532333000 \\
\hline $\mathrm{C}$ & 4.573211000 & -2.010316000 & 0.525453000 \\
\hline $\mathrm{H}$ & 2.563585000 & -1.534062000 & 1.087185000 \\
\hline $\mathrm{C}$ & 5.710355000 & -1.626624000 & -0.180186000 \\
\hline $\mathrm{H}$ & 6.555740000 & -0.123210000 & -1.468715000 \\
\hline $\mathrm{H}$ & 4.580351000 & -2.926946000 & 1.109930000 \\
\hline $\mathrm{H}$ & 6.607773000 & -2.237566000 & -0.156631000 \\
\hline $\mathrm{H}$ & 0.414342000 & 2.505497000 & -1.069222000 \\
\hline $\mathrm{O}$ & 1.831003000 & 2.243458000 & 1.943517000 \\
\hline $\mathrm{H}$ & 2.269844000 & 1.873924000 & 2.717241000 \\
\hline $\mathrm{H}$ & 2.137567000 & 1.597421000 & 1.038941000 \\
\hline $\mathrm{O}$ & -5.510164000 & -1.336120000 & 0.221810000 \\
\hline $\mathrm{C}$ & -6.649857000 & -0.649473000 & -0.241238000 \\
\hline $\mathrm{H}$ & -7.501007000 & -1.295389000 & -0.022321000 \\
\hline $\mathrm{H}$ & -6.782417000 & 0.309903000 & 0.276095000 \\
\hline $\mathrm{H}$ & -6.599363000 & -0.470559000 & -1.323188000 \\
\hline $\mathrm{Li}$ & 0.110866000 & 2.161864000 & 1.403795000 \\
\hline
\end{tabular}

\begin{tabular}{lrrr} 
& & & \\
& & & \\
& Coordinates (Angstroms) & \\
\hline C & 3.779170000 & -0.887293000 & 0.190652000 \\
$\mathrm{C}$ & 2.492671000 & -1.411583000 & 0.093898000 \\
$\mathrm{C}$ & 1.388407000 & -0.589429000 & -0.141641000 \\
$\mathrm{C}$ & 1.585259000 & 0.795031000 & -0.275729000 \\
$\mathrm{C}$ & 2.863505000 & 1.317598000 & -0.182124000 \\
$\mathrm{C}$ & 3.967138000 & 0.488173000 & 0.048050000 \\
$\mathrm{H}$ & 4.613077000 & -1.558187000 & 0.362142000 \\
$\mathrm{H}$ & 2.363882000 & -2.489348000 & 0.168672000
\end{tabular}




\begin{tabular}{lrrr}
$\mathrm{H}$ & 0.730698000 & 1.474192000 & -0.406046000 \\
$\mathrm{H}$ & 3.030657000 & 2.385834000 & -0.274890000 \\
$\mathrm{C}$ & 0.042187000 & -1.197366000 & -0.256598000 \\
$\mathrm{C}$ & -0.881204000 & -0.858035000 & -1.169933000 \\
$\mathrm{H}$ & -0.633278000 & -0.069564000 & -1.873912000 \\
$\mathrm{O}$ & -0.229042000 & -2.227192000 & 0.607522000 \\
$\mathrm{C}$ & -2.246306000 & -1.504658000 & -1.262897000 \\
$\mathrm{H}$ & -2.206615000 & -2.524765000 & -0.870600000 \\
$\mathrm{C}$ & -3.249080000 & -0.686580000 & -0.475401000 \\
$\mathrm{C}$ & -3.363130000 & -0.864616000 & 0.911219000 \\
$\mathrm{C}$ & -3.941047000 & 0.372342000 & -1.072918000 \\
$\mathrm{C}$ & -4.140449000 & 0.001252000 & 1.677870000 \\
$\mathrm{H}$ & -2.813756000 & -1.673916000 & 1.384014000 \\
$\mathrm{C}$ & -4.718665000 & 1.239675000 & -0.306055000 \\
$\mathrm{H}$ & -3.834281000 & 0.543789000 & -2.140433000 \\
$\mathrm{C}$ & -4.817328000 & 1.059662000 & 1.070974000 \\
$\mathrm{H}$ & -4.214443000 & -0.147369000 & 2.750972000 \\
$\mathrm{H}$ & -5.227408000 & 2.070094000 & -0.784881000 \\
$\mathrm{H}$ & -5.412299000 & 1.741403000 & 1.670080000 \\
$\mathrm{O}$ & -0.958587000 & 2.506334000 & -0.240177000 \\
$\mathrm{H}$ & -2.554409000 & -1.564949000 & -2.311756000 \\
$\mathrm{H}$ & 0.440687000 & -2.215382000 & 1.302886000 \\
$\mathrm{H}$ & -0.925507000 & 3.440363000 & -0.032862000 \\
$\mathrm{O}$ & 5.170163000 & 1.110493000 & 0.120604000 \\
$\mathrm{C}$ & 6.312980000 & 0.321835000 & 0.359315000 \\
$\mathrm{H}$ & 7.156521000 & 1.012920000 & 0.377362000 \\
$\mathrm{H}$ & 6.249748000 & -0.197071000 & 1.324753000 \\
$\mathrm{H}$ & 6.467969000 & -0.417006000 & -0.437754000 \\
$\mathrm{Li}$ & -1.845390000 & 1.165281000 & 0.212187000 \\
\hline---2 & &
\end{tabular}

\begin{tabular}{|c|c|c|c|}
\hline \multicolumn{4}{|c|}{$\mathrm{A1}_{\text {tBuOK }}$} \\
\hline & \multicolumn{3}{|c|}{ Coordinates (Angstroms) } \\
\hline & $X$ & $\mathrm{Y}$ & $\mathrm{Z}$ \\
\hline$\overline{\mathrm{C}}$ & -1.626240000 & 3.248680000 & 0.016650000 \\
\hline $\mathrm{C}$ & -0.666619000 & 2.272371000 & 0.281580000 \\
\hline $\mathrm{C}$ & -0.974483000 & 0.916402000 & 0.209294000 \\
\hline $\mathrm{C}$ & -2.273347000 & 0.536559000 & -0.159266000 \\
\hline $\mathrm{C}$ & -3.234468000 & 1.498067000 & -0.426620000 \\
\hline $\mathrm{C}$ & -2.919895000 & 2.858945000 & -0.335710000 \\
\hline $\mathrm{H}$ & -1.351275000 & 4.295575000 & 0.077924000 \\
\hline $\mathrm{H}$ & 0.346262000 & 2.582204000 & 0.533690000 \\
\hline $\mathrm{H}$ & -2.489492000 & -0.532901000 & -0.252785000 \\
\hline $\mathrm{H}$ & -4.240850000 & 1.220188000 & -0.724738000 \\
\hline
\end{tabular}




\begin{tabular}{lrcc}
$\mathrm{C}$ & 0.039331000 & -0.146824000 & 0.586091000 \\
$\mathrm{C}$ & 1.400261000 & 0.110123000 & 0.008891000 \\
$\mathrm{H}$ & 1.393887000 & 0.294088000 & -1.064079000 \\
$\mathrm{O}$ & 0.077032000 & -0.297946000 & 2.024398000 \\
$\mathrm{C}$ & 2.540566000 & 0.113236000 & 0.703562000 \\
$\mathrm{H}$ & 2.484270000 & -0.094564000 & 1.771201000 \\
$\mathrm{C}$ & 3.897029000 & 0.356205000 & 0.183155000 \\
$\mathrm{C}$ & 4.969279000 & 0.373622000 & 1.083156000 \\
$\mathrm{C}$ & 4.173505000 & 0.575102000 & -1.173489000 \\
$\mathrm{C}$ & 6.271139000 & 0.604209000 & 0.651268000 \\
$\mathrm{H}$ & 4.776536000 & 0.201752000 & 2.139602000 \\
$\mathrm{C}$ & 5.472367000 & 0.805206000 & -1.607533000 \\
$\mathrm{H}$ & 3.368471000 & 0.560630000 & -1.901957000 \\
$\mathrm{C}$ & 6.528332000 & 0.822483000 & -0.697981000 \\
$\mathrm{H}$ & 7.084291000 & 0.611824000 & 1.371281000 \\
$\mathrm{H}$ & 5.662495000 & 0.970304000 & -2.664082000 \\
$\mathrm{H}$ & 7.542903000 & 1.001450000 & -1.041167000 \\
$\mathrm{H}$ & -0.350779000 & -1.115549000 & 0.227851000 \\
$\mathrm{H}$ & 0.284151000 & 0.572745000 & 2.390055000 \\
$\mathrm{C}$ & -2.675289000 & -4.360378000 & -1.270068000 \\
$\mathrm{C}$ & -1.591144000 & -3.281444000 & -1.059766000 \\
$\mathrm{H}$ & -2.688770000 & -5.042043000 & -0.410049000 \\
$\mathrm{H}$ & -3.658408000 & -3.879352000 & -1.337051000 \\
$\mathrm{H}$ & -2.516644000 & -4.955699000 & -2.179259000 \\
$\mathrm{C}$ & -1.570186000 & -2.362261000 & -2.302230000 \\
$\mathrm{C}$ & -0.216069000 & -3.976727000 & -0.944034000 \\
$\mathrm{H}$ & -0.809101000 & -1.580932000 & -2.178577000 \\
$\mathrm{H}$ & -1.351951000 & -2.907296000 & -3.230188000 \\
$\mathrm{H}$ & -2.541759000 & -1.866173000 & -2.415058000 \\
$\mathrm{H}$ & 0.036626000 & -4.580749000 & -1.825943000 \\
$\mathrm{H}$ & 0.571169000 & -3.224336000 & -0.807039000 \\
$\mathrm{H}$ & -0.210113000 & -4.634483000 & -0.065200000 \\
$\mathrm{H}$ & -1.852555000 & -2.556517000 & 0.076821000 \\
$\mathrm{H}$ & -2.007158000 & -1.978970000 & 2.333356000 \\
$\mathrm{H}$ & -3.933263000 & 3.719031000 & -0.615051000 \\
$\mathrm{H}$ & -3.661373000 & 5.100508000 & -0.570878000 \\
$\mathrm{H}$ & & 5.383283000 & -1.296441000 \\
$\mathrm{H}$ & 5.59997790000 & 5.419447000 & 0.432124000 \\
\hline
\end{tabular}

TSA1-2 tBuOK $_{\text {}}$

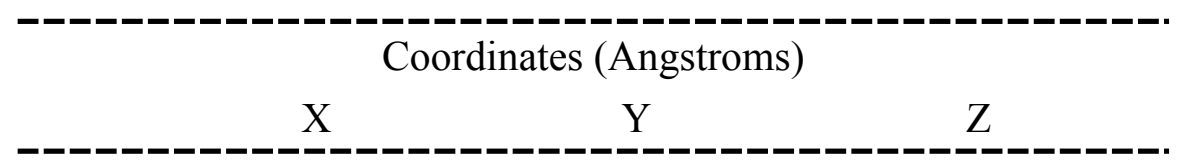




\begin{tabular}{|c|c|c|c|}
\hline $\mathrm{C}$ & -3.757960000 & -1.275292000 & -1.077309000 \\
\hline $\mathrm{C}$ & -2.489887000 & -0.787371000 & -1.399052000 \\
\hline $\mathrm{C}$ & -1.522946000 & -0.549179000 & -0.411677000 \\
\hline $\mathrm{C}$ & -1.888368000 & -0.807338000 & 0.922491000 \\
\hline $\mathrm{C}$ & -3.142852000 & -1.297769000 & 1.248294000 \\
\hline $\mathrm{C}$ & -4.088807000 & -1.531841000 & 0.246229000 \\
\hline $\mathrm{H}$ & -4.497524000 & -1.465828000 & -1.849726000 \\
\hline $\mathrm{H}$ & -2.238274000 & -0.610622000 & -2.442126000 \\
\hline $\mathrm{H}$ & -1.181769000 & -0.576836000 & 1.715549000 \\
\hline $\mathrm{H}$ & -3.408226000 & -1.468501000 & 2.287836000 \\
\hline $\mathrm{C}$ & -0.206592000 & 0.088305000 & -0.711002000 \\
\hline $\mathrm{C}$ & 0.985293000 & -0.644062000 & -0.292333000 \\
\hline $\mathrm{H}$ & 0.864821000 & -1.237717000 & 0.613260000 \\
\hline $\mathrm{O}$ & -0.129434000 & 0.567156000 & -2.063947000 \\
\hline $\mathrm{C}$ & 2.193216000 & -0.583358000 & -0.886853000 \\
\hline $\mathrm{H}$ & 2.310690000 & 0.067323000 & -1.752418000 \\
\hline $\mathrm{C}$ & 3.415535000 & -1.259559000 & -0.434839000 \\
\hline $\mathrm{C}$ & 4.653435000 & -0.845228000 & -0.947700000 \\
\hline $\mathrm{C}$ & 3.417371000 & -2.310162000 & 0.495706000 \\
\hline $\mathrm{C}$ & 5.842832000 & -1.436008000 & -0.536972000 \\
\hline $\mathrm{H}$ & 4.677769000 & -0.036605000 & -1.674649000 \\
\hline $\mathrm{C}$ & 4.605261000 & -2.898171000 & 0.911433000 \\
\hline $\mathrm{H}$ & 2.477037000 & -2.683393000 & 0.891644000 \\
\hline $\mathrm{C}$ & 5.826773000 & -2.465025000 & 0.399830000 \\
\hline $\mathrm{H}$ & 6.785940000 & -1.088104000 & -0.949509000 \\
\hline $\mathrm{H}$ & 4.576684000 & -3.707400000 & 1.636044000 \\
\hline $\mathrm{H}$ & 6.753443000 & -2.929116000 & 0.724161000 \\
\hline $\mathrm{H}$ & -0.227517000 & 1.266106000 & 0.030010000 \\
\hline $\mathrm{H}$ & 0.177884000 & -0.160847000 & -2.626578000 \\
\hline $\mathrm{C}$ & 0.287101000 & 4.299068000 & 1.695484000 \\
\hline $\mathrm{C}$ & 0.682406000 & 2.942285000 & 1.100260000 \\
\hline $\mathrm{H}$ & -0.091949000 & 4.961604000 & 0.907011000 \\
\hline $\mathrm{H}$ & -0.505463000 & 4.164871000 & 2.439870000 \\
\hline $\mathrm{H}$ & 1.137835000 & 4.795318000 & 2.177913000 \\
\hline $\mathrm{C}$ & 1.205790000 & 2.022181000 & 2.215725000 \\
\hline $\mathrm{C}$ & 1.779103000 & 3.138525000 & 0.040039000 \\
\hline $\mathrm{H}$ & 1.529486000 & 1.063387000 & 1.796025000 \\
\hline $\mathrm{H}$ & 2.060725000 & 2.465650000 & 2.740196000 \\
\hline $\mathrm{H}$ & 0.408951000 & 1.833412000 & 2.943806000 \\
\hline $\mathrm{H}$ & 2.687193000 & 3.581965000 & 0.465927000 \\
\hline $\mathrm{H}$ & 2.044714000 & 2.175080000 & -0.408437000 \\
\hline $\mathrm{H}$ & 1.416661000 & 3.801484000 & -0.756387000 \\
\hline $\mathrm{O}$ & -0.459888000 & 2.382637000 & 0.512753000 \\
\hline $\mathrm{K}$ & -1.993599000 & 2.389671000 & -1.396366000 \\
\hline
\end{tabular}




$\begin{array}{llll}\mathrm{O} & -5.362322000 & -1.956934000 & 0.541905000 \\ \mathrm{C} & -5.437182000 & -3.258985000 & 1.088953000 \\ \mathrm{H} & -6.495878000 & -3.464553000 & 1.258791000 \\ \mathrm{H} & -5.027126000 & -4.002819000 & 0.392789000 \\ \mathrm{H} & -4.901698000 & -3.336573000 & 2.044032000\end{array}$

\begin{tabular}{|c|c|c|c|}
\hline \multicolumn{4}{|c|}{$\mathbf{A} 2_{\text {tBuOK }}$} \\
\hline & $X$ & Y & Z \\
\hline $\mathrm{C}$ & 4.452566000 & -0.567605000 & 0.665808000 \\
\hline $\mathrm{C}$ & 3.229379000 & -1.065823000 & 1.106439000 \\
\hline $\mathrm{C}$ & 2.076736000 & -1.044678000 & 0.282443000 \\
\hline $\mathrm{C}$ & 2.238120000 & -0.474991000 & -1.008203000 \\
\hline $\mathrm{C}$ & 3.460002000 & 0.012363000 & -1.441456000 \\
\hline $\mathrm{C}$ & 4.578110000 & -0.021260000 & -0.605270000 \\
\hline $\mathrm{H}$ & 5.324573000 & -0.597503000 & 1.313798000 \\
\hline $\mathrm{H}$ & 3.155506000 & -1.510672000 & 2.093276000 \\
\hline $\mathrm{H}$ & 1.387266000 & -0.408323000 & -1.679849000 \\
\hline $\mathrm{H}$ & 3.553873000 & 0.451129000 & -2.431411000 \\
\hline $\mathrm{C}$ & 0.797030000 & -1.460209000 & 0.799665000 \\
\hline $\mathrm{C}$ & -0.425494000 & -1.400909000 & 0.152712000 \\
\hline $\mathrm{H}$ & -0.375467000 & -1.278876000 & -0.927520000 \\
\hline $\mathrm{O}$ & 0.784609000 & -1.625176000 & 2.208811000 \\
\hline $\mathrm{C}$ & -1.691140000 & -1.440302000 & 0.734389000 \\
\hline $\mathrm{H}$ & -1.770024000 & -1.531931000 & 1.817631000 \\
\hline $\mathrm{C}$ & -2.951515000 & -1.416357000 & 0.015158000 \\
\hline $\mathrm{C}$ & -4.156344000 & -1.223931000 & 0.726442000 \\
\hline $\mathrm{C}$ & -3.062259000 & -1.521178000 & -1.388343000 \\
\hline $\mathrm{C}$ & -5.379032000 & -1.110730000 & 0.081848000 \\
\hline $\mathrm{H}$ & -4.113578000 & -1.143785000 & 1.811021000 \\
\hline $\mathrm{C}$ & -4.287776000 & -1.395379000 & -2.031723000 \\
\hline $\mathrm{H}$ & -2.176377000 & -1.722108000 & -1.985134000 \\
\hline $\mathrm{C}$ & -5.459330000 & -1.183679000 & -1.308827000 \\
\hline $\mathrm{H}$ & -6.280432000 & -0.956556000 & 0.669713000 \\
\hline $\mathrm{H}$ & -4.328084000 & -1.478888000 & -3.115039000 \\
\hline $\mathrm{H}$ & -6.414924000 & -1.089180000 & -1.815062000 \\
\hline $\mathrm{H}$ & -1.691565000 & 0.626554000 & 0.777217000 \\
\hline $\mathrm{H}$ & 0.225840000 & -2.388247000 & 2.403800000 \\
\hline $\mathrm{C}$ & -1.211187000 & 1.740725000 & -1.481734000 \\
\hline $\mathrm{C}$ & -1.826020000 & 2.338321000 & -0.213053000 \\
\hline $\mathrm{H}$ & -0.122318000 & 1.657945000 & -1.380728000 \\
\hline $\mathrm{H}$ & -1.623751000 & 0.743701000 & -1.668788000 \\
\hline $\mathrm{H}$ & -1.431732000 & 2.365378000 & -2.354050000 \\
\hline
\end{tabular}




$\begin{array}{lrrr}\mathrm{C} & -3.352552000 & 2.347299000 & -0.307640000 \\ \mathrm{C} & -1.284771000 & 3.742458000 & 0.040559000 \\ \mathrm{H} & -3.785576000 & 2.752480000 & 0.612624000 \\ \mathrm{H} & -3.685691000 & 2.962642000 & -1.150645000 \\ \mathrm{H} & -3.738176000 & 1.332484000 & -0.453020000 \\ \mathrm{H} & -1.566738000 & 4.420596000 & -0.770720000 \\ \mathrm{H} & -1.684781000 & 4.141809000 & 0.978692000 \\ \mathrm{H} & -0.188883000 & 3.731060000 & 0.102401000 \\ \mathrm{O} & -1.424154000 & 1.564139000 & 0.924790000 \\ \mathrm{~K} & 0.856741000 & 1.054872000 & 2.021870000 \\ \mathrm{O} & 5.780704000 & 0.514093000 & -1.013727000 \\ \mathrm{C} & 6.536350000 & -0.356871000 & -1.832181000 \\ \mathrm{H} & 7.464056000 & 0.164550000 & -2.078328000 \\ \mathrm{H} & 6.771787000 & -1.292163000 & -1.306087000 \\ \mathrm{H} & 6.002603000 & -0.602696000 & -2.760524000 \\ - \text { - - - - } & \end{array}$

TSA2-3 ${ }_{\mathrm{tBuOK}}$

\begin{tabular}{lccc} 
& \multicolumn{3}{c}{ Coordinates (Angstroms) } \\
& $\mathrm{X}$ & $\mathrm{Y}$ & $\mathrm{Z}$ \\
$\mathrm{C}$ & 4.614601000 & -0.745635000 & 0.634549000 \\
$\mathrm{C}$ & 3.340513000 & -0.845675000 & 1.192151000 \\
$\mathrm{C}$ & 2.183510000 & -0.742463000 & 0.411366000 \\
$\mathrm{C}$ & 2.355082000 & -0.498963000 & -0.966222000 \\
$\mathrm{C}$ & 3.613860000 & -0.411134000 & -1.531420000 \\
$\mathrm{C}$ & 4.759156000 & -0.535710000 & -0.735956000 \\
$\mathrm{H}$ & 5.479110000 & -0.842549000 & 1.281875000 \\
$\mathrm{H}$ & 3.240104000 & -1.022396000 & 2.257653000 \\
$\mathrm{H}$ & 1.484524000 & -0.370585000 & -1.603715000 \\
$\mathrm{H}$ & 3.738436000 & -0.231870000 & -2.594481000 \\
$\mathrm{C}$ & 0.849979000 & -0.813785000 & 1.022722000 \\
$\mathrm{C}$ & -0.325839000 & -1.005318000 & 0.388639000 \\
$\mathrm{H}$ & -0.256744000 & -1.266979000 & -0.663701000 \\
$\mathrm{O}$ & 0.877935000 & -0.471330000 & 2.385713000 \\
$\mathrm{C}$ & -1.653136000 & -0.730677000 & 0.923849000 \\
$\mathrm{H}$ & -1.711392000 & -0.705461000 & 2.019468000 \\
$\mathrm{C}$ & -2.848724000 & -1.394603000 & 0.352087000 \\
$\mathrm{C}$ & -4.085493000 & -1.239958000 & 1.003183000 \\
$\mathrm{C}$ & -2.852562000 & -2.112116000 & -0.853426000 \\
$\mathrm{C}$ & -5.259294000 & -1.761947000 & 0.477481000 \\
$\mathrm{H}$ & -4.117804000 & -0.682289000 & 1.937072000 \\
$\mathrm{C}$ & -4.029956000 & -2.631146000 & -1.385850000 \\
$\mathrm{H}$ & -1.922490000 & -2.284157000 & -1.387574000 \\
$\mathrm{C}$ & -5.242992000 & -2.461841000 & -0.728012000
\end{tabular}




$\begin{array}{lrrr}\mathrm{H} & -6.195888000 & -1.618282000 & 1.010010000 \\ \mathrm{H} & -3.992196000 & -3.180048000 & -2.323365000 \\ \mathrm{H} & -6.159356000 & -2.869758000 & -1.143877000 \\ \mathrm{H} & -1.739534000 & 0.604248000 & 0.644863000 \\ \mathrm{H} & 0.115543000 & -0.897169000 & 2.800861000 \\ \mathrm{C} & -1.849330000 & 1.470748000 & -1.894635000 \\ \mathrm{C} & -2.252233000 & 2.313179000 & -0.669380000 \\ \mathrm{H} & -0.757546000 & 1.484536000 & -2.016745000 \\ \mathrm{H} & -2.167640000 & 0.430723000 & -1.765158000 \\ \mathrm{H} & -2.301472000 & 1.850564000 & -2.818781000 \\ \mathrm{C} & -3.773903000 & 2.232692000 & -0.470561000 \\ \mathrm{C} & -1.844739000 & 3.775776000 & -0.904988000 \\ \mathrm{H} & -4.062277000 & 2.816731000 & 0.410650000 \\ \mathrm{H} & -4.320597000 & 2.617904000 & -1.340190000 \\ \mathrm{H} & -4.078386000 & 1.193686000 & -0.305830000 \\ \mathrm{H} & -2.346222000 & 4.209761000 & -1.778248000 \\ \mathrm{H} & -2.097481000 & 4.379889000 & -0.025738000 \\ \mathrm{H} & -0.760633000 & 3.848058000 & -1.075612000 \\ \mathrm{O} & -1.588602000 & 1.857637000 & 0.474442000 \\ \mathrm{~K} & 0.722854000 & 2.043927000 & 1.276382000 \\ \mathrm{O} & 5.946225000 & -0.423292000 & -1.386257000 \\ \mathrm{C} & 7.128324000 & -0.563368000 & -0.632729000 \\ \mathrm{H} & 7.950559000 & -0.459553000 & -1.341687000 \\ \mathrm{H} & 7.212898000 & 0.216735000 & 0.135538000 \\ \mathrm{H} & 7.186855000 & -1.548803000 & -0.152796000 \\ -------0 .-1\end{array}$

\begin{tabular}{lccc} 
A3 & \multicolumn{3}{c}{ Coordinates (Angstroms) } \\
& $\mathrm{X}$ & $\mathrm{Y}$ & $\mathrm{Z}$ \\
$\mathrm{C}$ & -3.946005000 & -2.006898000 & 0.139233000 \\
$\mathrm{C}$ & -2.676758000 & -2.369211000 & 0.559275000 \\
$\mathrm{C}$ & -1.610615000 & -1.456991000 & 0.527163000 \\
$\mathrm{C}$ & -1.867114000 & -0.155164000 & 0.078981000 \\
$\mathrm{C}$ & -3.141056000 & 0.215326000 & -0.340849000 \\
$\mathrm{C}$ & -4.187885000 & -0.709045000 & -0.319741000 \\
$\mathrm{H}$ & -4.771288000 & -2.710340000 & 0.175024000 \\
$\mathrm{H}$ & -2.535475000 & -3.369216000 & 0.963925000 \\
$\mathrm{H}$ & -1.075265000 & 0.610500000 & 0.063896000 \\
$\mathrm{H}$ & -3.293450000 & 1.235850000 & -0.675500000 \\
$\mathrm{C}$ & -0.253912000 & -1.867537000 & 0.969337000 \\
$\mathrm{C}$ & 0.681307000 & -1.028897000 & 1.435877000 \\
$\mathrm{H}$ & 0.468421000 & 0.037594000 & 1.448444000 \\
$\mathrm{O}$ & 0.028616000 & -3.213561000 & 0.906085000
\end{tabular}




\begin{tabular}{|c|c|c|c|}
\hline $\mathrm{C}$ & 2.077728000 & -1.455156000 & 1.818659000 \\
\hline $\mathrm{H}$ & 2.101910000 & -2.520427000 & 2.063514000 \\
\hline $\mathrm{C}$ & 3.024526000 & -1.166896000 & 0.671711000 \\
\hline $\mathrm{C}$ & 3.217089000 & -2.118409000 & -0.337719000 \\
\hline $\mathrm{C}$ & 3.618773000 & 0.090768000 & 0.527922000 \\
\hline $\mathrm{C}$ & 3.979112000 & -1.818607000 & -1.466040000 \\
\hline $\mathrm{H}$ & 2.748303000 & -3.093860000 & -0.235754000 \\
\hline $\mathrm{C}$ & 4.380526000 & 0.395177000 & -0.599342000 \\
\hline $\mathrm{H}$ & 3.450644000 & 0.849891000 & 1.286224000 \\
\hline $\mathrm{C}$ & 4.560753000 & -0.556702000 & -1.602977000 \\
\hline $\mathrm{H}$ & 4.126993000 & -2.572544000 & -2.234583000 \\
\hline $\mathrm{H}$ & 4.826213000 & 1.380650000 & -0.696708000 \\
\hline $\mathrm{H}$ & 5.159476000 & -0.322061000 & -2.478287000 \\
\hline $\mathrm{C}$ & 0.526948000 & 2.883877000 & 2.033445000 \\
\hline $\mathrm{C}$ & 0.325059000 & 3.049849000 & 0.509879000 \\
\hline $\mathrm{H}$ & -0.219707000 & 2.184658000 & 2.430321000 \\
\hline $\mathrm{H}$ & 1.521362000 & 2.460229000 & 2.225462000 \\
\hline $\mathrm{H}$ & 0.441765000 & 3.829371000 & 2.585719000 \\
\hline $\mathrm{C}$ & 1.371040000 & 4.058473000 & -0.015423000 \\
\hline $\mathrm{C}$ & -1.086443000 & 3.633016000 & 0.267364000 \\
\hline $\mathrm{H}$ & 1.231501000 & 4.202360000 & -1.094312000 \\
\hline $\mathrm{H}$ & 1.311413000 & 5.039619000 & 0.474645000 \\
\hline $\mathrm{H}$ & 2.377914000 & 3.651529000 & 0.144530000 \\
\hline $\mathrm{H}$ & -1.234971000 & 4.613230000 & 0.740229000 \\
\hline $\mathrm{H}$ & -1.256025000 & 3.739420000 & -0.811491000 \\
\hline $\mathrm{H}$ & -1.842489000 & 2.943887000 & 0.664927000 \\
\hline $\mathrm{O}$ & 0.465282000 & 1.845473000 & -0.125361000 \\
\hline $\mathrm{H}$ & 2.396950000 & -0.901701000 & 2.708435000 \\
\hline K & 1.188783000 & 0.125661000 & -1.534142000 \\
\hline $\mathrm{H}$ & -0.616531000 & -3.633605000 & 0.325425000 \\
\hline $\mathrm{O}$ & -5.459515000 & -0.446639000 & -0.710975000 \\
\hline $\mathrm{C}$ & -5.760195000 & 0.862690000 & -1.141757000 \\
\hline $\mathrm{H}$ & -6.823438000 & 0.861925000 & -1.384864000 \\
\hline $\mathrm{H}$ & -5.569071000 & 1.600631000 & -0.352694000 \\
\hline $\mathrm{H}$ & -5.184353000 & 1.134707000 & -2.035812000 \\
\hline
\end{tabular}

\begin{tabular}{|c|c|c|c|}
\hline \multicolumn{4}{|c|}{ A1 $1_{\text {tBuONa }}$} \\
\hline & \multicolumn{3}{|c|}{ Coordinates (Angstroms) } \\
\hline & $\mathrm{X}$ & Y & Z \\
\hline $\mathrm{C}$ & 1.242324000 & -3.363540000 & 0.116128000 \\
\hline $\mathrm{C}$ & 0.356916000 & -2.314386000 & 0.297564000 \\
\hline $\mathrm{C}$ & 0.798536000 & -0.986885000 & 0.238560000 \\
\hline $\mathrm{C}$ & 2.146248000 & -0.732836000 & -0.016036000 \\
\hline
\end{tabular}




$\begin{array}{lrcc}\mathrm{C} & 3.044908000 & -1.782027000 & -0.202920000 \\ \mathrm{C} & 2.594443000 & -3.102148000 & -0.135268000 \\ \mathrm{H} & 0.912078000 & -4.396543000 & 0.154011000 \\ \mathrm{H} & -0.696103000 & -2.525903000 & 0.473045000 \\ \mathrm{H} & 2.476834000 & 0.309823000 & -0.068492000 \\ \mathrm{H} & 4.084984000 & -1.555110000 & -0.408929000 \\ \mathrm{C} & -0.138883000 & 0.168896000 & 0.518184000 \\ \mathrm{C} & -1.509187000 & -0.022806000 & -0.064210000 \\ \mathrm{H} & -1.503462000 & -0.265514000 & -1.125259000 \\ \mathrm{O} & -0.195167000 & 0.412664000 & 1.950247000 \\ \mathrm{C} & -2.654052000 & 0.082651000 & 0.614090000 \\ \mathrm{H} & -2.596510000 & 0.334212000 & 1.672048000 \\ \mathrm{C} & -4.017628000 & -0.100989000 & 0.087386000 \\ \mathrm{C} & -5.094181000 & -0.055990000 & 0.981041000 \\ \mathrm{C} & -4.295243000 & -0.322095000 & -1.268423000 \\ \mathrm{C} & -6.402622000 & -0.232125000 & 0.543567000 \\ \mathrm{H} & -4.899926000 & 0.119817000 & 2.036498000 \\ \mathrm{C} & -5.600695000 & -0.497997000 & -1.707922000 \\ \mathrm{H} & -3.486348000 & -0.350337000 & -1.992261000 \\ \mathrm{C} & -6.661208000 & -0.455731000 & -0.804521000 \\ \mathrm{H} & -7.219474000 & -0.193849000 & 1.258285000 \\ \mathrm{H} & 4.747812000 & -3.990111000 & -0.579944000 \\ \mathrm{H} & -5.792418000 & -0.666539000 & -2.763550000 \\ \mathrm{H} & -7.680757000 & -0.593173000 & -1.151977000 \\ \mathrm{H} & 0.325425000 & 1.083209000 & 0.118793000 \\ \mathrm{H} & -0.412698000 & -0.431494000 & 2.367965000 \\ \mathrm{C} & 3.753919000 & 3.901844000 & -0.473977000 \\ \mathrm{C} & 2.344296000 & 3.291376000 & -0.606729000 \\ \mathrm{H} & 3.857691000 & 4.379536000 & 0.508493000 \\ \mathrm{H} & 4.505572000 & 3.106871000 & -0.543610000 \\ \mathrm{H} & 3.968038000 & 4.652268000 & -1.246355000 \\ \mathrm{H} & 2.212833000 & 2.642595000 & -2.001189000 \\ \mathrm{H} & 1.297194000 & 4.417919000 & -0.485833000 \\ \mathrm{H} & 1.218296000 & 2.192308000 & -2.109437000 \\ \mathrm{H} & 2.355394000 & 3.362516000 & -2.817480000 \\ \mathrm{H} & 2.956775000 & 1.844549000 & -2.111784000 \\ \mathrm{H} & 1.406780000 & 5.192450000 & -1.256391000 \\ \mathrm{H} & 0.290335000 & 3.989895000 & -0.565680000 \\ \mathrm{H} & 1.385984000 & 4.898631000 & 0.497327000 \\ \mathrm{H} & 2.138301000 & 2.347454000 & 0.371054000 \\ \mathrm{H} & 3.380794000 & -4.195384000 & -0.302667000 \\ \mathrm{H} & & -4.983166000 & -0.681849000 \\ \mathrm{H} & & & -1.515552000\end{array}$


TSA1-2 ${ }_{\mathrm{tBuONa}}$

\begin{tabular}{|c|c|c|c|}
\hline \multicolumn{4}{|c|}{ Coordinates (Angstroms) } \\
\hline & $X$ & Y & Z \\
\hline $\mathrm{C}$ & -3.869900000 & -1.173723000 & -0.848307000 \\
\hline $\mathrm{C}$ & -2.589825000 & -0.720380000 & -1.199091000 \\
\hline $\mathrm{C}$ & -1.577455000 & -0.539366000 & -0.251785000 \\
\hline $\mathrm{C}$ & -1.906798000 & -0.824038000 & 1.090086000 \\
\hline $\mathrm{C}$ & -3.156623000 & -1.286592000 & 1.446320000 \\
\hline $\mathrm{C}$ & -4.156744000 & -1.465205000 & 0.478792000 \\
\hline $\mathrm{H}$ & -4.611242000 & -1.309159000 & -1.628493000 \\
\hline $\mathrm{H}$ & -2.373694000 & -0.545656000 & -2.251157000 \\
\hline $\mathrm{H}$ & -1.163883000 & -0.655957000 & 1.865290000 \\
\hline $\mathrm{H}$ & -3.396407000 & -1.504528000 & 2.482257000 \\
\hline $\mathrm{C}$ & -0.254349000 & 0.056030000 & -0.591808000 \\
\hline $\mathrm{C}$ & 0.946843000 & -0.648744000 & -0.167983000 \\
\hline $\mathrm{H}$ & 0.850828000 & -1.200346000 & 0.766587000 \\
\hline $\mathrm{O}$ & -0.199308000 & 0.488802000 & -1.967816000 \\
\hline $\mathrm{C}$ & 2.141282000 & -0.614909000 & -0.793126000 \\
\hline $\mathrm{H}$ & 2.236390000 & -0.012275000 & -1.695211000 \\
\hline $\mathrm{C}$ & 3.372910000 & -1.274117000 & -0.342357000 \\
\hline $\mathrm{C}$ & 4.600733000 & -0.875478000 & -0.891139000 \\
\hline $\mathrm{C}$ & 3.392952000 & -2.297989000 & 0.617337000 \\
\hline $\mathrm{C}$ & 5.797742000 & -1.455225000 & -0.487137000 \\
\hline $\mathrm{H}$ & 4.610976000 & -0.087884000 & -1.641104000 \\
\hline $\mathrm{C}$ & 4.588741000 & -2.874777000 & 1.026363000 \\
\hline $\mathrm{H}$ & 2.459960000 & -2.660776000 & 1.039583000 \\
\hline $\mathrm{C}$ & 5.800050000 & -2.457140000 & 0.478902000 \\
\hline $\mathrm{H}$ & 6.732680000 & -1.120247000 & -0.927969000 \\
\hline $\mathrm{H}$ & 4.573975000 & -3.664348000 & 1.772643000 \\
\hline $\mathrm{H}$ & 6.732777000 & -2.913174000 & 0.797178000 \\
\hline $\mathrm{H}$ & -0.287563000 & 1.325885000 & 0.062544000 \\
\hline $\mathrm{H}$ & 0.028743000 & -0.270946000 & -2.525684000 \\
\hline $\mathrm{C}$ & 0.139943000 & 4.614885000 & 1.058209000 \\
\hline $\mathrm{C}$ & 0.587361000 & 3.198347000 & 0.688659000 \\
\hline $\mathrm{H}$ & -0.350222000 & 5.092979000 & 0.200638000 \\
\hline $\mathrm{H}$ & -0.577437000 & 4.578425000 & 1.884622000 \\
\hline $\mathrm{H}$ & 0.988568000 & 5.240595000 & 1.358588000 \\
\hline $\mathrm{C}$ & 1.269755000 & 2.529907000 & 1.890875000 \\
\hline $\mathrm{C}$ & 1.561526000 & 3.244318000 & -0.498135000 \\
\hline $\mathrm{H}$ & 1.608786000 & 1.523059000 & 1.622301000 \\
\hline $\mathrm{H}$ & 2.141075000 & 3.102360000 & 2.229761000 \\
\hline
\end{tabular}




$\begin{array}{lrrr}\mathrm{H} & 0.560060000 & 2.447945000 & 2.720916000 \\ \mathrm{H} & 2.470106000 & 3.808932000 & -0.258113000 \\ \mathrm{H} & 1.852685000 & 2.228281000 & -0.785183000 \\ \mathrm{H} & 1.082592000 & 3.723295000 & -1.362909000 \\ \mathrm{O} & -0.563472000 & 2.464977000 & 0.337892000 \\ \mathrm{Na} & -1.736751000 & 2.103962000 & -1.432421000 \\ \mathrm{O} & -5.355439000 & -1.916541000 & 0.941992000 \\ \mathrm{C} & -6.377896000 & -2.132643000 & 0.000640000 \\ \mathrm{H} & -6.087293000 & -2.882870000 & -0.746921000 \\ \mathrm{H} & -7.237059000 & -2.500813000 & 0.562938000 \\ \mathrm{H} & -6.656873000 & -1.203232000 & -0.514893000\end{array}$

\begin{tabular}{|c|c|c|c|}
\hline \multicolumn{4}{|c|}{$\mathrm{A} \boldsymbol{2}_{\text {tBuONa }}$} \\
\hline & $\mathrm{X}$ & Y & Z \\
\hline$\overline{\mathrm{C}}$ & 4.340563000 & -0.254337000 & 0.835000000 \\
\hline $\mathrm{C}$ & 3.115610000 & -0.711612000 & 1.319860000 \\
\hline $\mathrm{C}$ & 2.024995000 & -0.977739000 & 0.454119000 \\
\hline $\mathrm{C}$ & 2.249347000 & -0.745705000 & -0.928930000 \\
\hline $\mathrm{C}$ & 3.469197000 & -0.296856000 & -1.400876000 \\
\hline $\mathrm{C}$ & 4.526253000 & -0.038961000 & -0.522258000 \\
\hline $\mathrm{H}$ & 5.164459000 & -0.063400000 & 1.517138000 \\
\hline $\mathrm{H}$ & 3.004138000 & -0.914737000 & 2.380144000 \\
\hline $\mathrm{H}$ & 1.451788000 & -0.921343000 & -1.644214000 \\
\hline $\mathrm{H}$ & 3.612237000 & -0.121904000 & -2.463798000 \\
\hline $\mathrm{C}$ & 0.737310000 & -1.347597000 & 0.990453000 \\
\hline $\mathrm{C}$ & -0.471910000 & -1.383730000 & 0.314379000 \\
\hline $\mathrm{H}$ & -0.392496000 & -1.418802000 & -0.769653000 \\
\hline $\mathrm{O}$ & 0.682953000 & -1.229754000 & 2.410191000 \\
\hline $\mathrm{C}$ & -1.751401000 & -1.319730000 & 0.857725000 \\
\hline $\mathrm{H}$ & -1.870270000 & -1.309191000 & 1.941347000 \\
\hline $\mathrm{C}$ & -2.990017000 & -1.313300000 & 0.096882000 \\
\hline $\mathrm{C}$ & -4.209997000 & -1.063798000 & 0.760921000 \\
\hline $\mathrm{C}$ & -3.059382000 & -1.480244000 & -1.302389000 \\
\hline $\mathrm{C}$ & -5.411369000 & -0.963139000 & 0.074512000 \\
\hline $\mathrm{H}$ & -4.198773000 & -0.932437000 & 1.841237000 \\
\hline $\mathrm{C}$ & -4.263488000 & -1.372298000 & -1.987770000 \\
\hline $\mathrm{H}$ & -2.158624000 & -1.711622000 & -1.864975000 \\
\hline $\mathrm{C}$ & -5.451980000 & -1.108545000 & -1.311638000 \\
\hline $\mathrm{H}$ & -6.326757000 & -0.765183000 & 0.626398000 \\
\hline $\mathrm{H}$ & -4.272270000 & -1.506037000 & -3.066676000 \\
\hline $\mathrm{H}$ & -6.390848000 & -1.026099000 & -1.850270000 \\
\hline $\mathrm{H}$ & -1.589214000 & 0.693918000 & 0.919762000 \\
\hline
\end{tabular}




\begin{tabular}{|c|c|c|c|}
\hline $\mathrm{H}$ & 0.079720000 & -1.906612000 & 2.739106000 \\
\hline $\mathrm{C}$ & -0.905382000 & 1.679041000 & -1.346125000 \\
\hline $\mathrm{C}$ & -1.522096000 & 2.377902000 & -0.131854000 \\
\hline $\mathrm{H}$ & 0.164187000 & 1.490645000 & -1.189027000 \\
\hline $\mathrm{H}$ & -1.404787000 & 0.722278000 & -1.530144000 \\
\hline $\mathrm{H}$ & -1.016474000 & 2.295460000 & -2.244425000 \\
\hline $\mathrm{C}$ & -3.036430000 & 2.507994000 & -0.294787000 \\
\hline $\mathrm{C}$ & -0.871355000 & 3.737076000 & 0.102931000 \\
\hline $\mathrm{H}$ & -3.472501000 & 2.988713000 & 0.586496000 \\
\hline $\mathrm{H}$ & -3.281087000 & 3.107953000 & -1.177933000 \\
\hline $\mathrm{H}$ & -3.497176000 & 1.521128000 & -0.414944000 \\
\hline $\mathrm{H}$ & -1.059205000 & 4.408346000 & -0.740465000 \\
\hline $\mathrm{H}$ & -1.271494000 & 4.200114000 & 1.010705000 \\
\hline $\mathrm{H}$ & 0.216533000 & 3.634218000 & 0.211271000 \\
\hline $\mathrm{O}$ & -1.246216000 & 1.613077000 & 1.055153000 \\
\hline $\mathrm{Na}$ & 0.750860000 & 0.998070000 & 1.825831000 \\
\hline $\mathrm{O}$ & 5.725440000 & 0.451336000 & -0.989324000 \\
\hline $\mathrm{C}$ & 6.571373000 & -0.545639000 & -1.528483000 \\
\hline $\mathrm{H}$ & 7.479738000 & -0.041656000 & -1.865701000 \\
\hline $\mathrm{H}$ & 6.830659000 & -1.298379000 & -0.771592000 \\
\hline $\mathrm{H}$ & 6.102603000 & -1.054119000 & -2.382118000 \\
\hline
\end{tabular}

TSA2-3 ${ }_{\text {tBuONa }}$

\begin{tabular}{lrcc} 
& \multicolumn{3}{c}{ Coordinates (Angstroms) } \\
& $\mathrm{X}$ & $\mathrm{Y}$ & $\mathrm{Z}$ \\
$\mathrm{C}$ & 4.672352000 & -0.518922000 & 0.720850000 \\
$\mathrm{C}$ & 3.393365000 & -0.600516000 & 1.268922000 \\
$\mathrm{C}$ & 2.247255000 & -0.577735000 & 0.467142000 \\
$\mathrm{C}$ & 2.431352000 & -0.440158000 & -0.922477000 \\
$\mathrm{C}$ & 3.695240000 & -0.371200000 & -1.477610000 \\
$\mathrm{C}$ & 4.831161000 & -0.410433000 & -0.660141000 \\
$\mathrm{H}$ & 5.529635000 & -0.549000000 & 1.383995000 \\
$\mathrm{H}$ & 3.282789000 & -0.698560000 & 2.343576000 \\
$\mathrm{H}$ & 1.566200000 & -0.377760000 & -1.576946000 \\
$\mathrm{H}$ & 3.831233000 & -0.268215000 & -2.549169000 \\
$\mathrm{C}$ & 0.904547000 & -0.638254000 & 1.058578000 \\
$\mathrm{C}$ & -0.255753000 & -0.955555000 & 0.442936000 \\
$\mathrm{H}$ & -0.154716000 & -1.337739000 & -0.569111000 \\
$\mathrm{O}$ & 0.883990000 & -0.133574000 & 2.379126000 \\
$\mathrm{C}$ & -1.596582000 & -0.721048000 & 0.957820000 \\
$\mathrm{H}$ & -1.680387000 & -0.751262000 & 2.052032000 \\
$\mathrm{C}$ & -2.767549000 & -1.368113000 & 0.325772000 \\
$\mathrm{C}$ & -3.977895000 & -1.437656000 & 1.037869000
\end{tabular}




\begin{tabular}{lrrr}
$\mathrm{C}$ & -2.783796000 & -1.839288000 & -0.996879000 \\
$\mathrm{C}$ & -5.136909000 & -1.942351000 & 0.464053000 \\
$\mathrm{H}$ & -4.003719000 & -1.072862000 & 2.062704000 \\
$\mathrm{C}$ & -3.944430000 & -2.346967000 & -1.573355000 \\
$\mathrm{H}$ & -1.878506000 & -1.813707000 & -1.597623000 \\
$\mathrm{C}$ & -5.131553000 & -2.402931000 & -0.851229000 \\
$\mathrm{H}$ & -6.052663000 & -1.976543000 & 1.048604000 \\
$\mathrm{H}$ & -3.915290000 & -2.703847000 & -2.599709000 \\
$\mathrm{H}$ & -6.035861000 & -2.799371000 & -1.302778000 \\
$\mathrm{H}$ & -1.734224000 & 0.662583000 & 0.755312000 \\
$\mathrm{H}$ & 0.141953000 & -0.550658000 & 2.838228000 \\
$\mathrm{C}$ & -1.686206000 & 1.727874000 & -1.722553000 \\
$\mathrm{C}$ & -2.259680000 & 2.407475000 & -0.468046000 \\
$\mathrm{H}$ & -0.596117000 & 1.863280000 & -1.764144000 \\
$\mathrm{H}$ & -1.900784000 & 0.654388000 & -1.705719000 \\
$\mathrm{H}$ & -2.114179000 & 2.144780000 & -2.641594000 \\
$\mathrm{C}$ & -3.774176000 & 2.169090000 & -0.396099000 \\
$\mathrm{C}$ & -1.969386000 & 3.911770000 & -0.519013000 \\
$\mathrm{H}$ & -4.181793000 & 2.640408000 & 0.504459000 \\
$\mathrm{H}$ & -4.288535000 & 2.583789000 & -1.271133000 \\
$\mathrm{H}$ & -3.988742000 & 1.096382000 & -0.346849000 \\
$\mathrm{H}$ & -2.443278000 & 4.389189000 & -1.384585000 \\
$\mathrm{H}$ & -2.338702000 & 4.395880000 & 0.391317000 \\
$\mathrm{H}$ & -0.886832000 & 4.089904000 & -0.591243000 \\
$\mathrm{O}$ & -1.640775000 & 1.892075000 & 0.686279000 \\
$\mathrm{Na}$ & 0.418999000 & 1.861092000 & 1.240396000 \\
$\mathrm{O}$ & 6.024162000 & -0.323826000 & -1.302003000 \\
$\mathrm{C}$ & 7.198654000 & -0.378092000 & -0.525200000 \\
$\mathrm{H}$ & 7.275473000 & -1.326217000 & 0.022537000 \\
$\mathrm{H}$ & 8.027990000 & -0.301609000 & -1.229292000 \\
$\mathrm{H}$ & 7.250981000 & 0.455415000 & 0.187510000 \\
\hline-----
\end{tabular}

\begin{tabular}{llcc} 
& & & \\
& & & \\
& Coordinates (Angstroms) & Z \\
\hline $\mathrm{C}$ & -3.953314000 & -1.973761000 & 0.029296000 \\
$\mathrm{C}$ & -2.671818000 & -2.414695000 & 0.308454000 \\
$\mathrm{C}$ & -1.583862000 & -1.526444000 & 0.306262000 \\
$\mathrm{C}$ & -1.819332000 & -0.178912000 & 0.019808000 \\
$\mathrm{C}$ & -3.106946000 & 0.268509000 & -0.262509000 \\
$\mathrm{C}$ & -4.180427000 & -0.623754000 & -0.260671000 \\
$\mathrm{H}$ & -4.798223000 & -2.654254000 & 0.042531000 \\
$\mathrm{H}$ & -2.525987000 & -3.460418000 & 0.571505000
\end{tabular}




\begin{tabular}{lrcc}
$\mathrm{H}$ & -1.003996000 & 0.559707000 & -0.007248000 \\
$\mathrm{H}$ & -3.244187000 & 1.320002000 & -0.489240000 \\
$\mathrm{C}$ & -0.222879000 & -2.029297000 & 0.608933000 \\
$\mathrm{C}$ & 0.709519000 & -1.359153000 & 1.303786000 \\
$\mathrm{H}$ & 0.462933000 & -0.364742000 & 1.663958000 \\
$\mathrm{O}$ & 0.065472000 & -3.297525000 & 0.168374000 \\
$\mathrm{C}$ & 2.097383000 & -1.892267000 & 1.578661000 \\
$\mathrm{H}$ & 2.096004000 & -2.984949000 & 1.537625000 \\
$\mathrm{C}$ & 3.073186000 & -1.338337000 & 0.561170000 \\
$\mathrm{C}$ & 3.246151000 & -1.981634000 & -0.672349000 \\
$\mathrm{C}$ & 3.701940000 & -0.105474000 & 0.765416000 \\
$\mathrm{C}$ & 4.011110000 & -1.395715000 & -1.680796000 \\
$\mathrm{H}$ & 2.756906000 & -2.937569000 & -0.840006000 \\
$\mathrm{C}$ & 4.464746000 & 0.484153000 & -0.241747000 \\
$\mathrm{H}$ & 3.563420000 & 0.412875000 & 1.710222000 \\
$\mathrm{C}$ & 4.616764000 & -0.155360000 & -1.471085000 \\
$\mathrm{H}$ & 4.138950000 & -1.909186000 & -2.629535000 \\
$\mathrm{H}$ & 4.928769000 & 1.450183000 & -0.069751000 \\
$\mathrm{H}$ & 5.208134000 & 0.304134000 & -2.256862000 \\
$\mathrm{C}$ & 0.629080000 & 2.768023000 & 1.808775000 \\
$\mathrm{C}$ & 0.382368000 & 3.030127000 & 0.306564000 \\
$\mathrm{H}$ & -0.136605000 & 2.081619000 & 2.191993000 \\
$\mathrm{H}$ & 1.609255000 & 2.289460000 & 1.937336000 \\
$\mathrm{H}$ & 0.608291000 & 3.681700000 & 2.417689000 \\
$\mathrm{C}$ & 1.464466000 & 4.001603000 & -0.210249000 \\
$\mathrm{C}$ & -1.002470000 & 3.695281000 & 0.143570000 \\
$\mathrm{H}$ & 1.305301000 & 4.191319000 & -1.278333000 \\
$\mathrm{H}$ & 1.468360000 & 4.964247000 & 0.318276000 \\
$\mathrm{H}$ & 2.453254000 & 3.538288000 & -0.092140000 \\
$\mathrm{H}$ & -1.078977000 & 4.659042000 & 0.664127000 \\
$\mathrm{H}$ & -1.205181000 & 3.856941000 & -0.921503000 \\
$\mathrm{H}$ & -1.778629000 & 3.029192000 & 0.541375000 \\
$\mathrm{O}$ & 0.426039000 & 1.851294000 & -0.389354000 \\
$\mathrm{H}$ & 2.408624000 & -1.600390000 & 2.587373000 \\
$\mathrm{H}$ & -0.625508000 & -3.566042000 & -0.449603000 \\
$\mathrm{H}$ & 1.460687000 & 0.244283000 & -1.038191000 \\
$\mathrm{H}$ & -5.466095000 & -0.285850000 & -0.523776000 \\
$\mathrm{H}$ & -5.748257000 & 1.069367000 & -0.799653000 \\
$\mathrm{H}$ & -5.473038000 & 1.716767000 & 0.042234000 \\
$\mathrm{H}$ & & 1.412663000 & -1.702519000 \\
\hline
\end{tabular}

$\mathrm{A1}_{\text {tBuOLi }}$ 


\begin{tabular}{|c|c|c|c|}
\hline \multicolumn{4}{|c|}{ Coordinates (Angstroms) } \\
\hline & $X$ & $\mathrm{Y}$ & $\mathrm{Z}$ \\
\hline $\mathrm{C}$ & -1.095043000 & -3.316712000 & -0.097489000 \\
\hline $\mathrm{C}$ & -0.252928000 & -2.223806000 & -0.218501000 \\
\hline $\mathrm{C}$ & -0.764671000 & -0.921325000 & -0.192697000 \\
\hline $\mathrm{C}$ & -2.140022000 & -0.737768000 & -0.046292000 \\
\hline $\mathrm{C}$ & -2.995464000 & -1.829541000 & 0.077033000 \\
\hline $\mathrm{C}$ & -2.473190000 & -3.125347000 & 0.053305000 \\
\hline $\mathrm{H}$ & -0.709664000 & -4.330907000 & -0.109852000 \\
\hline $\mathrm{H}$ & 0.817585000 & -2.382938000 & -0.325276000 \\
\hline $\mathrm{H}$ & -2.534957000 & 0.280362000 & -0.043763000 \\
\hline $\mathrm{H}$ & -4.058361000 & -1.654142000 & 0.197307000 \\
\hline $\mathrm{C}$ & 0.124172000 & 0.285381000 & -0.398195000 \\
\hline $\mathrm{C}$ & 1.519998000 & 0.105989000 & 0.124394000 \\
\hline $\mathrm{H}$ & 1.559108000 & -0.198335000 & 1.168705000 \\
\hline $\mathrm{O}$ & 0.123742000 & 0.651812000 & -1.808751000 \\
\hline $\mathrm{C}$ & 2.635724000 & 0.282911000 & -0.585714000 \\
\hline $\mathrm{H}$ & 2.534467000 & 0.585382000 & -1.626639000 \\
\hline $\mathrm{C}$ & 4.021089000 & 0.108092000 & -0.115545000 \\
\hline $\mathrm{C}$ & 5.059482000 & 0.172131000 & -1.051807000 \\
\hline $\mathrm{C}$ & 4.353825000 & -0.123340000 & 1.225966000 \\
\hline $\mathrm{C}$ & 6.385542000 & -0.000416000 & -0.669593000 \\
\hline $\mathrm{H}$ & 4.821838000 & 0.357725000 & -2.096574000 \\
\hline $\mathrm{C}$ & 5.677113000 & -0.295743000 & 1.610199000 \\
\hline $\mathrm{H}$ & 3.574895000 & -0.159997000 & 1.981609000 \\
\hline $\mathrm{C}$ & 6.699237000 & -0.237439000 & 0.664544000 \\
\hline $\mathrm{H}$ & 7.172953000 & 0.051353000 & -1.415591000 \\
\hline $\mathrm{H}$ & 5.912553000 & -0.472527000 & 2.655550000 \\
\hline $\mathrm{H}$ & 7.733007000 & -0.372261000 & 0.968074000 \\
\hline $\mathrm{H}$ & -0.351756000 & 1.150159000 & 0.081501000 \\
\hline $\mathrm{H}$ & 0.254187000 & -0.161542000 & -2.314393000 \\
\hline $\mathrm{C}$ & -4.110296000 & 3.922676000 & -0.230022000 \\
\hline $\mathrm{C}$ & -2.788609000 & 3.319054000 & 0.279038000 \\
\hline $\mathrm{H}$ & -3.941073000 & 4.425925000 & -1.189414000 \\
\hline $\mathrm{H}$ & -4.843172000 & 3.123864000 & -0.391369000 \\
\hline $\mathrm{H}$ & -4.536542000 & 4.650093000 & 0.472791000 \\
\hline $\mathrm{C}$ & -3.041860000 & 2.623379000 & 1.631147000 \\
\hline $\mathrm{C}$ & -1.761349000 & 4.448456000 & 0.484757000 \\
\hline $\mathrm{H}$ & -2.114303000 & 2.162833000 & 1.992652000 \\
\hline $\mathrm{H}$ & -3.402629000 & 3.320563000 & 2.397659000 \\
\hline $\mathrm{H}$ & -3.788871000 & 1.829459000 & 1.510887000 \\
\hline $\mathrm{H}$ & -2.098668000 & 5.200631000 & 1.209251000 \\
\hline $\mathrm{H}$ & -0.814735000 & 4.024471000 & 0.841590000 \\
\hline $\mathrm{H}$ & -1.570358000 & 4.951323000 & -0.471208000 \\
\hline
\end{tabular}




$\begin{array}{llrr}\mathrm{O} & -2.304137000 & 2.407232000 & -0.632128000 \\ \mathrm{O} & -3.212382000 & -4.255653000 & 0.168489000 \\ \mathrm{C} & -4.606329000 & -4.122595000 & 0.339343000 \\ \mathrm{H} & -4.998071000 & -5.137303000 & 0.418407000 \\ \mathrm{H} & -4.848215000 & -3.569609000 & 1.255703000 \\ \mathrm{H} & -5.071226000 & -3.621491000 & -0.519190000 \\ \mathrm{Li} & -1.411436000 & 1.838520000 & -1.924171000\end{array}$

TSA1-2 tBuOLi

\begin{tabular}{|c|c|c|c|}
\hline \multicolumn{4}{|c|}{ Coordinates (Angstroms) } \\
\hline & $\mathrm{X}$ & Y & Z \\
\hline$\overline{\mathrm{C}}$ & 3.297328000 & -1.873785000 & -0.549995000 \\
\hline $\mathrm{C}$ & 1.991501000 & -1.557223000 & -0.168477000 \\
\hline $\mathrm{C}$ & 1.705631000 & -0.438359000 & 0.613190000 \\
\hline $\mathrm{C}$ & 2.789472000 & 0.353643000 & 1.033458000 \\
\hline $\mathrm{C}$ & 4.089037000 & 0.039607000 & 0.685080000 \\
\hline $\mathrm{C}$ & 4.353945000 & -1.075437000 & -0.120126000 \\
\hline $\mathrm{H}$ & 3.468705000 & -2.752162000 & -1.162215000 \\
\hline $\mathrm{H}$ & 1.184552000 & -2.211803000 & -0.487260000 \\
\hline $\mathrm{H}$ & 2.590670000 & 1.234323000 & 1.637667000 \\
\hline $\mathrm{H}$ & 4.923010000 & 0.653497000 & 1.010122000 \\
\hline $\mathrm{C}$ & 0.326700000 & -0.027045000 & 0.968197000 \\
\hline $\mathrm{C}$ & -0.834488000 & -0.635887000 & 0.369839000 \\
\hline $\mathrm{H}$ & -0.757846000 & -0.798506000 & -0.705352000 \\
\hline $\mathrm{O}$ & 0.132988000 & 0.232781000 & 2.385776000 \\
\hline $\mathrm{C}$ & -2.040518000 & -0.840070000 & 0.968971000 \\
\hline $\mathrm{H}$ & -2.097098000 & -0.825611000 & 2.060773000 \\
\hline $\mathrm{C}$ & -3.283857000 & -1.205075000 & 0.279276000 \\
\hline $\mathrm{C}$ & -4.325911000 & -1.814799000 & 0.994034000 \\
\hline $\mathrm{C}$ & -3.491124000 & -0.952105000 & -1.086078000 \\
\hline $\mathrm{C}$ & -5.514696000 & -2.175580000 & 0.371032000 \\
\hline $\mathrm{H}$ & -4.190841000 & -2.018872000 & 2.054215000 \\
\hline $\mathrm{C}$ & -4.675618000 & -1.321873000 & -1.711295000 \\
\hline $\mathrm{H}$ & -2.722880000 & -0.441445000 & -1.660618000 \\
\hline $\mathrm{C}$ & -5.695490000 & -1.936675000 & -0.988536000 \\
\hline $\mathrm{H}$ & -6.302271000 & -2.650183000 & 0.949775000 \\
\hline $\mathrm{H}$ & -4.808985000 & -1.115724000 & -2.769624000 \\
\hline $\mathrm{H}$ & -6.623439000 & -2.217054000 & -1.477526000 \\
\hline $\mathrm{H}$ & 0.117297000 & 1.350016000 & 0.598493000 \\
\hline $\mathrm{H}$ & 0.129027000 & -0.610975000 & 2.861323000 \\
\hline $\mathrm{C}$ & 1.134962000 & 3.436474000 & -0.853732000 \\
\hline $\mathrm{C}$ & -0.328598000 & 3.168128000 & -0.482815000 \\
\hline $\mathrm{H}$ & 1.625519000 & 4.000391000 & -0.053782000 \\
\hline
\end{tabular}




\begin{tabular}{lrrr}
$\mathrm{H}$ & 1.672849000 & 2.489786000 & -0.979981000 \\
$\mathrm{H}$ & 1.216113000 & 4.006585000 & -1.786266000 \\
$\mathrm{C}$ & -1.017766000 & 2.366171000 & -1.596305000 \\
$\mathrm{C}$ & -1.069841000 & 4.484528000 & -0.245309000 \\
$\mathrm{H}$ & -2.047511000 & 2.122681000 & -1.308188000 \\
$\mathrm{H}$ & -1.045747000 & 2.928866000 & -2.536180000 \\
$\mathrm{H}$ & -0.482266000 & 1.427764000 & -1.780564000 \\
$\mathrm{H}$ & -1.055676000 & 5.118477000 & -1.139293000 \\
$\mathrm{H}$ & -2.116345000 & 4.289387000 & 0.018063000 \\
$\mathrm{H}$ & -0.602671000 & 5.033698000 & 0.578731000 \\
$\mathrm{O}$ & -0.359985000 & 2.438184000 & 0.724743000 \\
$\mathrm{Li}$ & -1.372997000 & 1.432677000 & 1.849182000 \\
$\mathrm{O}$ & 5.663517000 & -1.290118000 & -0.420708000 \\
$\mathrm{C}$ & 5.975669000 & -2.386252000 & -1.246755000 \\
$\mathrm{H}$ & 5.488893000 & -2.303787000 & -2.227619000 \\
$\mathrm{H}$ & 7.058178000 & -2.365943000 & -1.380687000 \\
$\mathrm{H}$ & 5.687556000 & -3.338522000 & -0.781972000 \\
\hline- - & &
\end{tabular}

\begin{tabular}{|c|c|c|c|}
\hline \multicolumn{4}{|c|}{$\mathrm{A} 2_{\mathrm{tBuOLi}}$} \\
\hline & $X$ & Y & Z \\
\hline$\overline{\mathrm{C}}$ & 4.235442000 & -0.000187000 & 0.889971000 \\
\hline $\mathrm{C}$ & 3.009116000 & -0.389198000 & 1.430308000 \\
\hline $\mathrm{C}$ & 1.969548000 & -0.896555000 & 0.614106000 \\
\hline $\mathrm{C}$ & 2.240655000 & -0.991571000 & -0.774929000 \\
\hline $\mathrm{C}$ & 3.459089000 & -0.605390000 & -1.300188000 \\
\hline $\mathrm{C}$ & 4.466865000 & -0.098485000 & -0.472815000 \\
\hline $\mathrm{H}$ & 5.022749000 & 0.381041000 & 1.533953000 \\
\hline $\mathrm{H}$ & 2.867863000 & -0.350193000 & 2.505594000 \\
\hline $\mathrm{H}$ & 1.484162000 & -1.380376000 & -1.449383000 \\
\hline $\mathrm{H}$ & 3.642849000 & -0.682819000 & -2.368218000 \\
\hline $\mathrm{C}$ & 0.670923000 & -1.190606000 & 1.178445000 \\
\hline $\mathrm{C}$ & -0.530910000 & -1.318874000 & 0.496386000 \\
\hline $\mathrm{H}$ & -0.426660000 & -1.484881000 & -0.572845000 \\
\hline $\mathrm{O}$ & 0.598681000 & -0.820093000 & 2.560018000 \\
\hline $\mathrm{C}$ & -1.820030000 & -1.173401000 & 0.994914000 \\
\hline $\mathrm{H}$ & -1.977096000 & -1.084654000 & 2.070572000 \\
\hline $\mathrm{C}$ & -3.038230000 & -1.202991000 & 0.196757000 \\
\hline $\mathrm{C}$ & -4.278788000 & -0.953258000 & 0.818216000 \\
\hline $\mathrm{C}$ & -3.061565000 & -1.404114000 & -1.198499000 \\
\hline $\mathrm{C}$ & -5.461777000 & -0.896100000 & 0.094773000 \\
\hline $\mathrm{H}$ & -4.302607000 & -0.793288000 & 1.894347000 \\
\hline $\mathrm{C}$ & -4.247258000 & -1.344338000 & -1.920698000 \\
\hline
\end{tabular}




$\begin{array}{lccc}\mathrm{H} & -2.139814000 & -1.620499000 & -1.732140000 \\ \mathrm{C} & -5.459464000 & -1.087182000 & -1.285769000 \\ \mathrm{H} & -6.396025000 & -0.699518000 & 0.614295000 \\ \mathrm{H} & -4.221054000 & -1.505649000 & -2.995371000 \\ \mathrm{H} & -6.383940000 & -1.040848000 & -1.852878000 \\ \mathrm{H} & -1.513012000 & 0.799308000 & 0.998238000 \\ \mathrm{H} & -0.094530000 & -1.343038000 & 2.978916000 \\ \mathrm{C} & -0.626607000 & 1.650939000 & -1.292633000 \\ \mathrm{C} & -1.226868000 & 2.425769000 & -0.117588000 \\ \mathrm{H} & 0.425710000 & 1.400778000 & -1.106872000 \\ \mathrm{H} & -1.180926000 & 0.722148000 & -1.461885000 \\ \mathrm{H} & -0.677115000 & 2.246472000 & -2.210134000 \\ \mathrm{C} & -2.724124000 & 2.648324000 & -0.321901000 \\ \mathrm{C} & -0.489609000 & 3.739840000 & 0.110133000 \\ \mathrm{H} & -3.149846000 & 3.181378000 & 0.533657000 \\ \mathrm{H} & -2.905266000 & 3.236550000 & -1.227437000 \\ \mathrm{H} & -3.244636000 & 1.689618000 & -0.429563000 \\ \mathrm{H} & -0.596574000 & 4.396956000 & -0.758174000 \\ \mathrm{H} & -0.889823000 & 4.254617000 & 0.988959000 \\ \mathrm{H} & 0.582366000 & 3.563642000 & 0.265847000 \\ \mathrm{O} & -1.042287000 & 1.668640000 & 1.099801000 \\ \mathrm{O} & 5.664859000 & 0.318774000 & -1.006087000 \\ \mathrm{C} & 6.582761000 & -0.734571000 & -1.227184000 \\ \mathrm{H} & 7.484604000 & -0.284617000 & -1.647586000 \\ \mathrm{H} & 6.833118000 & -1.246569000 & -0.288055000 \\ \mathrm{H} & 6.184713000 & -1.474984000 & -1.934728000 \\ \mathrm{Li} & 0.595250000 & 0.904184000 & 1.650069000 \\ - & & & \end{array}$

TSA2-3 tBuOLi $_{\text {in }}$

\begin{tabular}{llcc} 
& & \\
& & & \\
& Coordinates (Angstroms) & Y & \\
\hline $\mathrm{C}$ & 4.685700000 & 0.045692000 & 1.179874000 \\
$\mathrm{C}$ & 3.372268000 & 0.089011000 & 1.614816000 \\
$\mathrm{C}$ & 2.307942000 & -0.219042000 & 0.750028000 \\
$\mathrm{C}$ & 2.625272000 & -0.550422000 & -0.572426000 \\
$\mathrm{C}$ & 3.942484000 & -0.611345000 & -1.016420000 \\
$\mathrm{C}$ & 4.985026000 & -0.311126000 & -0.138028000 \\
$\mathrm{H}$ & 5.504479000 & 0.279827000 & 1.852436000 \\
$\mathrm{H}$ & 3.159366000 & 0.354765000 & 2.644646000 \\
$\mathrm{H}$ & 1.829689000 & -0.759818000 & -1.282156000 \\
$\mathrm{H}$ & 4.136461000 & -0.874965000 & -2.049835000 \\
$\mathrm{C}$ & 0.916174000 & -0.165862000 & 1.214740000 \\
$\mathrm{C}$ & -0.177009000 & -0.764212000 & 0.681369000
\end{tabular}




\begin{tabular}{lccc}
$\mathrm{H}$ & 0.023156000 & -1.423714000 & -0.159775000 \\
$\mathrm{O}$ & 0.743241000 & 0.728084000 & 2.308815000 \\
$\mathrm{C}$ & -1.555158000 & -0.546971000 & 1.071299000 \\
$\mathrm{H}$ & -1.719012000 & -0.408612000 & 2.147696000 \\
$\mathrm{C}$ & -2.634485000 & -1.335702000 & 0.449817000 \\
$\mathrm{C}$ & -3.864059000 & -1.488654000 & 1.115007000 \\
$\mathrm{C}$ & -2.541777000 & -1.882221000 & -0.842073000 \\
$\mathrm{C}$ & -4.935534000 & -2.148561000 & 0.527203000 \\
$\mathrm{H}$ & -3.975293000 & -1.072154000 & 2.114054000 \\
$\mathrm{C}$ & -3.611866000 & -2.550448000 & -1.428167000 \\
$\mathrm{H}$ & -1.620925000 & -1.778143000 & -1.410606000 \\
$\mathrm{C}$ & -4.819098000 & -2.690131000 & -0.751425000 \\
$\mathrm{H}$ & -5.869662000 & -2.244637000 & 1.074494000 \\
$\mathrm{H}$ & -3.498105000 & -2.962709000 & -2.427498000 \\
$\mathrm{H}$ & -5.655653000 & -3.206896000 & -1.211678000 \\
$\mathrm{H}$ & -1.742883000 & 0.854865000 & 0.670175000 \\
$\mathrm{H}$ & 0.027024000 & 0.393144000 & 2.866379000 \\
$\mathrm{C}$ & -1.961337000 & 1.670613000 & -1.889601000 \\
$\mathrm{C}$ & -2.395123000 & 2.440675000 & -0.636078000 \\
$\mathrm{H}$ & -0.888445000 & 1.814721000 & -2.072880000 \\
$\mathrm{H}$ & -2.153201000 & 0.600162000 & -1.763626000 \\
$\mathrm{H}$ & -2.506684000 & 2.012302000 & -2.776449000 \\
$\mathrm{C}$ & -3.877594000 & 2.185173000 & -0.346465000 \\
$\mathrm{C}$ & -2.132343000 & 3.936478000 & -0.812826000 \\
$\mathrm{H}$ & -4.177443000 & 2.714707000 & 0.563834000 \\
$\mathrm{H}$ & -4.508108000 & 2.528806000 & -1.174215000 \\
$\mathrm{H}$ & -4.058493000 & 1.114927000 & -0.198207000 \\
$\mathrm{H}$ & -2.707008000 & 4.345572000 & -1.651114000 \\
$\mathrm{H}$ & -2.409194000 & 4.479426000 & 0.096928000 \\
$\mathrm{H}$ & -1.068218000 & 4.117727000 & -1.013882000 \\
$\mathrm{O}$ & -1.627206000 & 2.008016000 & 0.475919000 \\
$\mathrm{O}$ & 6.302643000 & -0.327147000 & -0.465925000 \\
$\mathrm{H}$ & 6.650890000 & -0.694308000 & -1.780974000 \\
$\mathrm{H}$ & 7.739737000 & -0.652279000 & -1.827974000 \\
$\mathrm{H}$ & 6.318153000 & -1.713575000 & -2.016435000 \\
$\mathrm{H}$ & 6.232161000 & 0.002227000 & -2.519264000 \\
0.118812000 & 1.920293000 & 0.878819000 \\
\hline-------- &
\end{tabular}

A3 ${ }_{\text {tBuOLi }}$

\begin{tabular}{|c|c|c|c|}
\hline \multicolumn{4}{|c|}{ Coordinates (Angstroms) } \\
\hline & $\mathrm{X}$ & $\mathrm{Y}$ & $\mathrm{Z}$ \\
\hline $\mathrm{C}$ & -4.002660000 & -1.761925000 & 0.031516000 \\
\hline $\mathrm{C}$ & -2.740794000 & -2.280495000 & 0.259736000 \\
\hline
\end{tabular}




\begin{tabular}{|c|c|c|c|}
\hline $\mathrm{C}$ & -1.607423000 & -1.451652000 & 0.242885000 \\
\hline $\mathrm{C}$ & -1.772569000 & -0.089585000 & -0.015493000 \\
\hline $\mathrm{C}$ & -3.040366000 & 0.436879000 & -0.247133000 \\
\hline $\mathrm{C}$ & -4.161232000 & -0.394948000 & -0.224737000 \\
\hline $\mathrm{H}$ & -4.884863000 & -2.393052000 & 0.054911000 \\
\hline $\mathrm{H}$ & -2.639081000 & -3.339359000 & 0.487740000 \\
\hline $\mathrm{H}$ & -0.904971000 & 0.577807000 & -0.072239000 \\
\hline $\mathrm{H}$ & -3.129460000 & 1.496488000 & -0.458651000 \\
\hline $\mathrm{C}$ & -0.267816000 & -2.033309000 & 0.487207000 \\
\hline $\mathrm{C}$ & 0.686381000 & -1.474030000 & 1.247958000 \\
\hline $\mathrm{H}$ & 0.464568000 & -0.525156000 & 1.727143000 \\
\hline $\mathrm{O}$ & -0.029474000 & -3.256380000 & -0.084338000 \\
\hline $\mathrm{C}$ & 2.061665000 & -2.072419000 & 1.443949000 \\
\hline $\mathrm{H}$ & 2.024819000 & -3.156020000 & 1.301349000 \\
\hline $\mathrm{C}$ & 3.019156000 & -1.448668000 & 0.449227000 \\
\hline $\mathrm{C}$ & 3.056213000 & -1.918758000 & -0.872438000 \\
\hline $\mathrm{C}$ & 3.738467000 & -0.291472000 & 0.766442000 \\
\hline $\mathrm{C}$ & 3.778915000 & -1.237563000 & -1.850252000 \\
\hline $\mathrm{H}$ & 2.488583000 & -2.808765000 & -1.129848000 \\
\hline $\mathrm{C}$ & 4.462253000 & 0.390791000 & -0.211828000 \\
\hline $\mathrm{H}$ & 3.699600000 & 0.100274000 & 1.779227000 \\
\hline $\mathrm{C}$ & 4.479582000 & -0.075665000 & -1.523360000 \\
\hline $\mathrm{H}$ & 3.791759000 & -1.612155000 & -2.869463000 \\
\hline $\mathrm{H}$ & 4.992101000 & 1.301216000 & 0.048990000 \\
\hline $\mathrm{H}$ & 5.028633000 & 0.464397000 & -2.287728000 \\
\hline $\mathrm{C}$ & 0.737157000 & 2.609462000 & 1.768575000 \\
\hline $\mathrm{C}$ & 0.764469000 & 2.864423000 & 0.247114000 \\
\hline $\mathrm{H}$ & -0.143936000 & 2.008601000 & 2.026981000 \\
\hline $\mathrm{H}$ & 1.632884000 & 2.046527000 & 2.063480000 \\
\hline $\mathrm{H}$ & 0.705538000 & 3.536151000 & 2.356125000 \\
\hline $\mathrm{C}$ & 2.014239000 & 3.696192000 & -0.099229000 \\
\hline $\mathrm{C}$ & -0.494102000 & 3.664431000 & -0.144175000 \\
\hline $\mathrm{H}$ & 2.051179000 & 3.871975000 & -1.180325000 \\
\hline $\mathrm{H}$ & 2.034586000 & 4.666295000 & 0.414248000 \\
\hline $\mathrm{H}$ & 2.915478000 & 3.136808000 & 0.182998000 \\
\hline $\mathrm{H}$ & -0.539806000 & 4.643684000 & 0.349254000 \\
\hline $\mathrm{H}$ & -0.510339000 & 3.817060000 & -1.228868000 \\
\hline $\mathrm{H}$ & -1.393661000 & 3.101185000 & 0.133719000 \\
\hline $\mathrm{O}$ & 0.791965000 & 1.665673000 & -0.427049000 \\
\hline $\mathrm{H}$ & 2.407316000 & -1.883301000 & 2.465369000 \\
\hline $\mathrm{H}$ & -0.726057000 & -3.426009000 & -0.731024000 \\
\hline $\mathrm{O}$ & -5.433134000 & 0.018233000 & -0.441895000 \\
\hline $\mathrm{C}$ & -5.647350000 & 1.389064000 & -0.700554000 \\
\hline $\mathrm{H}$ & -6.723062000 & 1.504615000 & -0.837193000 \\
\hline
\end{tabular}




\begin{tabular}{llll}
$\mathrm{H}$ & -5.319099000 & 2.012870000 & 0.140243000 \\
$\mathrm{H}$ & -5.129636000 & 1.711464000 & -1.612608000 \\
$\mathrm{Li}$ & 1.595658000 & 0.200560000 & -0.610011000 \\
\hline
\end{tabular}

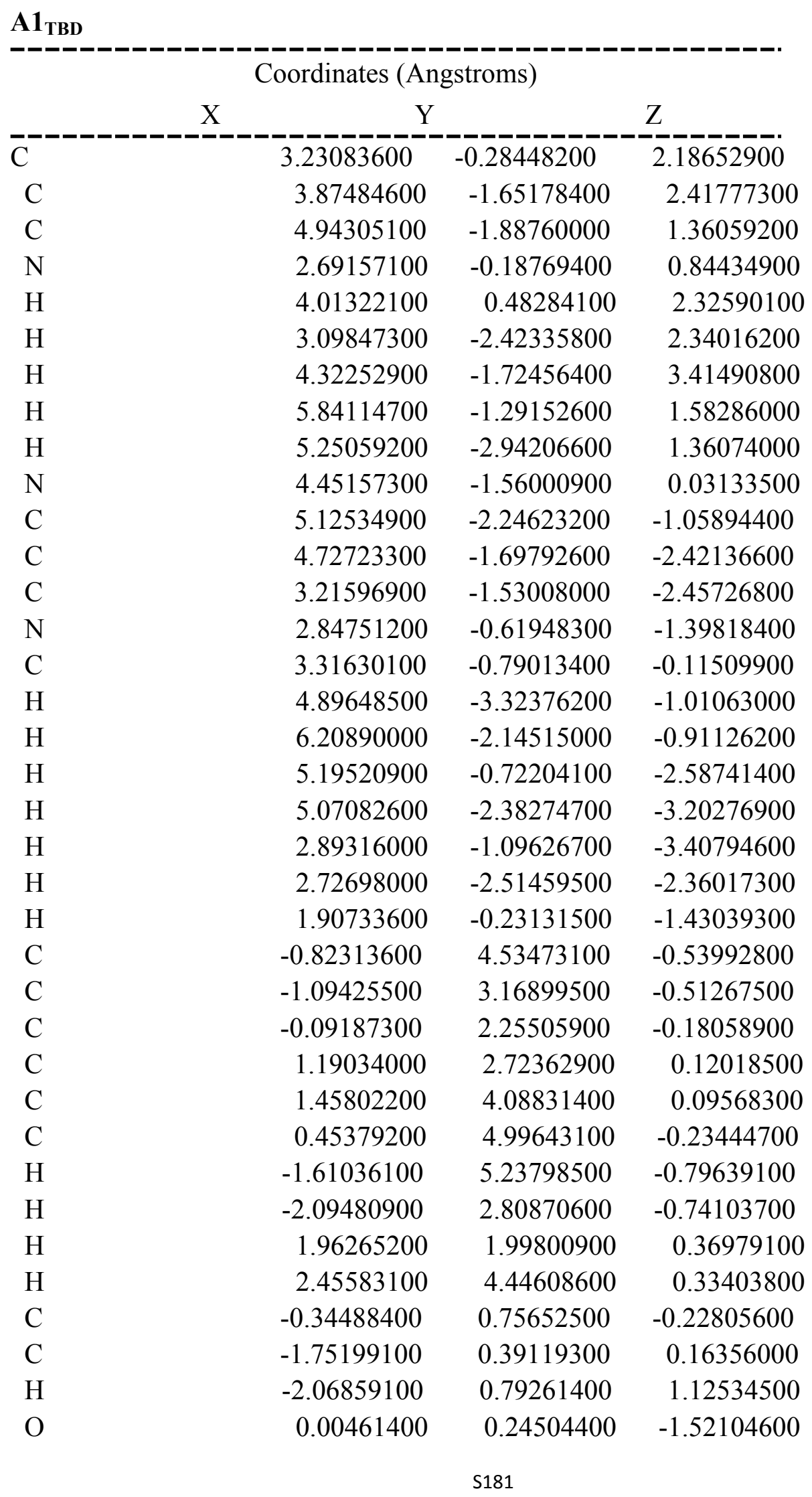




\begin{tabular}{|c|c|c|c|}
\hline $\mathrm{C}$ & -2.57437100 & -0.35294500 & -0.57822200 \\
\hline $\mathrm{H}$ & -2.19677700 & -0.72067800 & -1.53089000 \\
\hline $\mathrm{C}$ & -3.95827900 & -0.73630900 & -0.25256300 \\
\hline $\mathrm{C}$ & -4.74179900 & -1.34831300 & -1.23521600 \\
\hline $\mathrm{C}$ & -4.54613100 & -0.51856200 & 1.00185700 \\
\hline $\mathrm{C}$ & -6.06058900 & -1.71450100 & -0.98571400 \\
\hline $\mathrm{H}$ & -4.31063900 & -1.53593300 & -2.21576800 \\
\hline $\mathrm{C}$ & -5.86018600 & -0.88489600 & 1.24788800 \\
\hline $\mathrm{H}$ & -3.96777400 & -0.06439800 & 1.80118300 \\
\hline $\mathrm{C}$ & -6.64510600 & -1.48849000 & 0.25878300 \\
\hline $\mathrm{H}$ & -6.64264400 & -2.18534000 & -1.77441000 \\
\hline $\mathrm{H}$ & -6.28942300 & -0.70437800 & 2.23096400 \\
\hline $\mathrm{H}$ & 0.36531700 & 0.27008600 & 0.45186200 \\
\hline $\mathrm{H}$ & -0.32137400 & 0.87853700 & -2.17363300 \\
\hline $\mathrm{C}$ & 2.11441000 & -0.00156800 & 3.18665200 \\
\hline $\mathrm{H}$ & 2.49031500 & -0.00469100 & 4.21635400 \\
\hline $\mathrm{H}$ & 1.66385900 & 0.97472000 & 2.98126400 \\
\hline $\mathrm{H}$ & 1.32942500 & -0.76143700 & 3.09801100 \\
\hline $\mathrm{H}$ & -7.66002162 & -1.76705513 & 0.45177979 \\
\hline $\mathrm{O}$ & 0.73222198 & 6.39886482 & -0.25802913 \\
\hline $\mathrm{C}$ & 2.07829813 & 6.62551605 & 0.16812604 \\
\hline $\mathrm{H}$ & 2.37019018 & 7.62271558 & -0.08739720 \\
\hline $\mathrm{H}$ & 2.14351780 & 6.49534750 & 1.22817441 \\
\hline $\mathrm{H}$ & 2.72838982 & 5.92807713 & -0.31752790 \\
\hline
\end{tabular}

TSA1-2

\begin{tabular}{lccc} 
& \multicolumn{3}{c}{ Coordinates (Angstroms) } \\
\hline $\mathrm{C}$ & -0.085972000 & -0.797007000 & 2.382695000 \\
$\mathrm{C}$ & -1.210882000 & 0.215780000 & 2.554315000 \\
$\mathrm{C}$ & -2.546198000 & -0.499327000 & 2.425564000 \\
$\mathrm{~N}$ & -0.215653000 & -1.508716000 & 1.124159000 \\
$\mathrm{H}$ & -0.081939000 & -1.512264000 & 3.215762000 \\
$\mathrm{H}$ & 0.890836000 & -0.303299000 & 2.371963000 \\
$\mathrm{H}$ & -1.135613000 & 0.979682000 & 1.775035000 \\
$\mathrm{H}$ & -1.155023000 & 0.711972000 & 3.527754000 \\
$\mathrm{H}$ & -2.779682000 & -1.062827000 & 3.339095000 \\
$\mathrm{H}$ & -3.345792000 & 0.233693000 & 2.269574000 \\
$\mathrm{~N}$ & -2.564996000 & -1.415113000 & 1.283886000 \\
$\mathrm{C}$ & -3.885056000 & -1.635196000 & 0.699800000 \\
$\mathrm{C}$ & -3.862130000 & -2.755453000 & -0.327248000 \\
$\mathrm{C}$ & -2.720547000 & -2.504190000 & -1.298950000 \\
$\mathrm{~N}$ & -1.488666000 & -2.423994000 & -0.540546000
\end{tabular}




\begin{tabular}{|c|c|c|c|}
\hline $\mathrm{C}$ & -1.423737000 & -1.749890000 & 0.637237000 \\
\hline $\mathrm{H}$ & -4.227578000 & -0.698767000 & 0.236430000 \\
\hline $\mathrm{H}$ & -4.574957000 & -1.876628000 & 1.516519000 \\
\hline $\mathrm{H}$ & -3.713827000 & -3.721262000 & 0.167317000 \\
\hline $\mathrm{H}$ & -4.819816000 & -2.784891000 & -0.854201000 \\
\hline $\mathrm{H}$ & -2.624621000 & -3.323641000 & -2.015772000 \\
\hline $\mathrm{H}$ & -2.905599000 & -1.578723000 & -1.863694000 \\
\hline $\mathrm{H}$ & -0.622289000 & -2.366983000 & -1.076764000 \\
\hline $\mathrm{C}$ & 4.463561000 & 0.950818000 & 0.525282000 \\
\hline $\mathrm{C}$ & 3.101112000 & 0.797620000 & 0.278369000 \\
\hline $\mathrm{C}$ & 2.608628000 & -0.138622000 & -0.639977000 \\
\hline $\mathrm{C}$ & 3.560786000 & -0.941775000 & -1.289740000 \\
\hline $\mathrm{C}$ & 4.918956000 & -0.801298000 & -1.052015000 \\
\hline $\mathrm{C}$ & 5.386719000 & 0.149266000 & -0.144530000 \\
\hline $\mathrm{H}$ & 4.784776000 & 1.697226000 & 1.243712000 \\
\hline $\mathrm{H}$ & 2.414001000 & 1.433488000 & 0.831692000 \\
\hline $\mathrm{H}$ & 3.217506000 & -1.694866000 & -1.990566000 \\
\hline $\mathrm{H}$ & 5.641589000 & -1.427620000 & -1.566269000 \\
\hline $\mathrm{C}$ & 1.160264000 & -0.337614000 & -0.877294000 \\
\hline $\mathrm{C}$ & 0.226436000 & 0.749007000 & -0.794681000 \\
\hline $\mathrm{H}$ & 0.518099000 & 1.551251000 & -0.117916000 \\
\hline $\mathrm{O}$ & 0.860523000 & -1.307783000 & -1.881003000 \\
\hline $\mathrm{C}$ & -1.027958000 & 0.767808000 & -1.319139000 \\
\hline $\mathrm{H}$ & -1.302487000 & -0.028694000 & -2.007205000 \\
\hline $\mathrm{C}$ & -2.107791000 & 1.684289000 & -0.971317000 \\
\hline $\mathrm{C}$ & -3.385287000 & 1.481507000 & -1.527436000 \\
\hline $\mathrm{C}$ & -1.979518000 & 2.743360000 & -0.052511000 \\
\hline $\mathrm{C}$ & -4.474966000 & 2.266637000 & -1.170968000 \\
\hline $\mathrm{H}$ & -3.515983000 & 0.688618000 & -2.261778000 \\
\hline $\mathrm{C}$ & -3.068933000 & 3.527224000 & 0.306559000 \\
\hline $\mathrm{H}$ & -1.010557000 & 2.960709000 & 0.388788000 \\
\hline $\mathrm{C}$ & -4.328692000 & 3.293877000 & -0.241632000 \\
\hline $\mathrm{H}$ & -5.443954000 & 2.077777000 & -1.625868000 \\
\hline $\mathrm{H}$ & -2.931052000 & 4.332926000 & 1.022985000 \\
\hline $\mathrm{H}$ & -5.177352000 & 3.908730000 & 0.042116000 \\
\hline $\mathrm{H}$ & 0.532770000 & -1.081253000 & 0.300841000 \\
\hline $\mathrm{H}$ & 0.878124000 & -0.868277000 & -2.744798000 \\
\hline $\mathrm{O}$ & 6.741595000 & 0.212496000 & 0.019200000 \\
\hline $\mathrm{C}$ & 7.243063000 & 1.157356000 & 0.929871000 \\
\hline $\mathrm{H}$ & 6.873127000 & 0.977261000 & 1.948808000 \\
\hline $\mathrm{H}$ & 8.328516000 & 1.044203000 & 0.919239000 \\
\hline $\mathrm{H}$ & 6.985915000 & 2.184473000 & 0.635586000 \\
\hline
\end{tabular}

A2 ${ }_{\text {TBD }}$ 


\begin{tabular}{|c|c|c|c|}
\hline \multicolumn{4}{|c|}{ Coordinates (Angstroms) } \\
\hline & $X$ & $\mathrm{Y}$ & Z \\
\hline $\bar{C}$ & 1.564038000 & 1.793768000 & 1.963404000 \\
\hline $\mathrm{C}$ & 2.256049000 & 2.207944000 & 0.676474000 \\
\hline $\mathrm{C}$ & 1.400753000 & 3.249639000 & -0.027383000 \\
\hline $\mathrm{N}$ & 0.198512000 & 1.375630000 & 1.674797000 \\
\hline $\mathrm{H}$ & 1.552808000 & 2.621293000 & 2.684430000 \\
\hline $\mathrm{H}$ & 2.070767000 & 0.942099000 & 2.419479000 \\
\hline $\mathrm{H}$ & 2.387184000 & 1.329680000 & 0.036104000 \\
\hline $\mathrm{H}$ & 3.243858000 & 2.624895000 & 0.887807000 \\
\hline $\mathrm{H}$ & 1.411677000 & 4.204500000 & 0.515505000 \\
\hline $\mathrm{H}$ & 1.783903000 & 3.436271000 & -1.036080000 \\
\hline $\mathrm{N}$ & 0.018505000 & 2.789114000 & -0.168857000 \\
\hline $\mathrm{C}$ & -0.729016000 & 3.340644000 & -1.297503000 \\
\hline $\mathrm{C}$ & -2.219894000 & 3.059229000 & -1.178229000 \\
\hline $\mathrm{C}$ & -2.425017000 & 1.587945000 & -0.857790000 \\
\hline $\mathrm{N}$ & -1.682307000 & 1.276998000 & 0.352802000 \\
\hline $\mathrm{C}$ & -0.480533000 & 1.802650000 & 0.602665000 \\
\hline $\mathrm{H}$ & -0.330531000 & 2.914229000 & -2.228424000 \\
\hline $\mathrm{H}$ & -0.540820000 & 4.419764000 & -1.321494000 \\
\hline $\mathrm{H}$ & -2.654506000 & 3.670866000 & -0.380410000 \\
\hline $\mathrm{H}$ & -2.715427000 & 3.324160000 & -2.115834000 \\
\hline $\mathrm{H}$ & -3.473861000 & 1.348839000 & -0.670365000 \\
\hline $\mathrm{H}$ & -2.090348000 & 0.953225000 & -1.688964000 \\
\hline $\mathrm{H}$ & -1.866337000 & 0.331938000 & 0.749819000 \\
\hline $\mathrm{C}$ & 3.131634000 & -0.987124000 & -1.507470000 \\
\hline $\mathrm{C}$ & 1.880759000 & -1.228437000 & -0.958830000 \\
\hline $\mathrm{C}$ & 1.705213000 & -1.431525000 & 0.429651000 \\
\hline $\mathrm{C}$ & 2.868253000 & -1.368321000 & 1.228049000 \\
\hline $\mathrm{C}$ & 4.118390000 & -1.110056000 & 0.679279000 \\
\hline $\mathrm{C}$ & 4.259633000 & -0.913133000 & -0.690557000 \\
\hline $\mathrm{H}$ & 3.243746000 & -0.824204000 & -2.576175000 \\
\hline $\mathrm{H}$ & 1.018488000 & -1.237679000 & -1.619259000 \\
\hline $\mathrm{H}$ & 2.772412000 & -1.524180000 & 2.297085000 \\
\hline $\mathrm{H}$ & 4.999782000 & -1.055492000 & 1.312779000 \\
\hline $\mathrm{C}$ & 0.404930000 & -1.604482000 & 1.036503000 \\
\hline $\mathrm{C}$ & -0.806320000 & -1.729249000 & 0.404895000 \\
\hline $\mathrm{H}$ & -0.760489000 & -1.900872000 & -0.668417000 \\
\hline $\mathrm{O}$ & 0.389484000 & -1.345265000 & 2.428410000 \\
\hline $\mathrm{C}$ & -2.079312000 & -1.584730000 & 0.982793000 \\
\hline $\mathrm{H}$ & -2.164039000 & -1.459322000 & 2.061958000 \\
\hline $\mathrm{C}$ & -3.326796000 & -1.575482000 & 0.252419000 \\
\hline $\mathrm{C}$ & -4.523033000 & -1.207242000 & 0.913599000 \\
\hline $\mathrm{C}$ & -3.438321000 & -1.823565000 & -1.136398000 \\
\hline
\end{tabular}




$\begin{array}{lrrr}\mathrm{C} & -5.725961000 & -1.068517000 & 0.238314000 \\ \mathrm{H} & -4.485924000 & -1.015061000 & 1.984420000 \\ \mathrm{C} & -4.645588000 & -1.680435000 & -1.808186000 \\ \mathrm{H} & -2.564823000 & -2.142669000 & -1.698862000 \\ \mathrm{C} & -5.803828000 & -1.293564000 & -1.136760000 \\ \mathrm{H} & -6.616493000 & -0.779241000 & 0.791691000 \\ \mathrm{H} & -4.682616000 & -1.881374000 & -2.876664000 \\ \mathrm{H} & -6.745407000 & -1.184213000 & -1.666032000 \\ \mathrm{H} & -0.079985000 & 0.478870000 & 2.081765000 \\ \mathrm{H} & -0.194012000 & -1.998750000 & 2.835741000 \\ \mathrm{O} & 5.493897000 & -0.613708000 & -1.228955000 \\ \mathrm{C} & 6.196253000 & -1.750892000 & -1.688368000 \\ \mathrm{H} & 7.149372000 & -1.395310000 & -2.087031000 \\ \mathrm{H} & 6.384910000 & -2.461884000 & -0.872191000 \\ \mathrm{H} & 5.642980000 & -2.271169000 & -2.482641000 \\ -- \text { - - - - } & \end{array}$

TSA2-3 ${ }_{\text {TBD }}$

\begin{tabular}{|c|c|c|c|}
\hline \multicolumn{4}{|c|}{ Coordinates (Angstroms) } \\
\hline & $\mathrm{X}$ & $\mathrm{Y}$ & Z \\
\hline $\mathrm{C}$ & 1.592263000 & 1.898422000 & 1.689514000 \\
\hline $\mathrm{C}$ & 2.099567000 & 2.327919000 & 0.323035000 \\
\hline $\mathrm{C}$ & 1.160474000 & 3.385224000 & -0.236729000 \\
\hline $\mathrm{N}$ & 0.189886000 & 1.520803000 & 1.607581000 \\
\hline $\mathrm{H}$ & 1.717841000 & 2.712840000 & 2.415850000 \\
\hline $\mathrm{H}$ & 2.145251000 & 1.029215000 & 2.051917000 \\
\hline $\mathrm{H}$ & 2.134036000 & 1.458599000 & -0.343024000 \\
\hline $\mathrm{H}$ & 3.110872000 & 2.736290000 & 0.401543000 \\
\hline $\mathrm{H}$ & 1.253014000 & 4.325675000 & 0.325801000 \\
\hline $\mathrm{H}$ & 1.409898000 & 3.602135000 & -1.281726000 \\
\hline $\mathrm{N}$ & -0.223301000 & 2.924245000 & -0.208416000 \\
\hline $\mathrm{C}$ & -1.124393000 & 3.529237000 & -1.182706000 \\
\hline $\mathrm{C}$ & -2.578479000 & 3.231177000 & -0.848280000 \\
\hline $\mathrm{C}$ & -2.720519000 & 1.744286000 & -0.560048000 \\
\hline $\mathrm{N}$ & -1.829136000 & 1.368381000 & 0.524164000 \\
\hline $\mathrm{C}$ & -0.636027000 & 1.928230000 & 0.618004000 \\
\hline $\mathrm{H}$ & -0.878633000 & 3.152700000 & -2.186086000 \\
\hline $\mathrm{H}$ & -0.940610000 & 4.610479000 & -1.183740000 \\
\hline $\mathrm{H}$ & -2.884001000 & 3.803608000 & 0.034593000 \\
\hline $\mathrm{H}$ & -3.215557000 & 3.533427000 & -1.684436000 \\
\hline $\mathrm{H}$ & -3.738977000 & 1.487824000 & -0.258464000 \\
\hline $\mathrm{H}$ & -2.501095000 & 1.157054000 & -1.463679000 \\
\hline $\mathrm{H}$ & -1.924323000 & 0.212350000 & 0.818857000 \\
\hline $\mathrm{C}$ & 3.242372000 & -1.128345000 & -1.645092000 \\
\hline
\end{tabular}




$\begin{array}{lrrr}\mathrm{C} & 1.985198000 & -1.238429000 & -1.080430000 \\ \mathrm{C} & 1.812099000 & -1.436355000 & 0.303979000 \\ \mathrm{C} & 2.972334000 & -1.498280000 & 1.084254000 \\ \mathrm{C} & 4.245280000 & -1.372848000 & 0.528037000 \\ \mathrm{C} & 4.388944000 & -1.189093000 & -0.844824000 \\ \mathrm{H} & 3.364323000 & -0.977910000 & -2.713148000 \\ \mathrm{H} & 1.116844000 & -1.151789000 & -1.726754000 \\ \mathrm{H} & 2.873689000 & -1.646141000 & 2.154171000 \\ \mathrm{H} & 5.109358000 & -1.427127000 & 1.181309000 \\ \mathrm{C} & 0.489353000 & -1.493052000 & 0.924518000 \\ \mathrm{C} & -0.709652000 & -1.586042000 & 0.307293000 \\ \mathrm{H} & -0.684223000 & -1.790466000 & -0.759635000 \\ \mathrm{O} & 0.526129000 & -1.244747000 & 2.307379000 \\ \mathrm{C} & -1.994155000 & -1.311653000 & 0.912348000 \\ \mathrm{H} & -2.066694000 & -1.442464000 & 1.995944000 \\ \mathrm{C} & -3.258790000 & -1.581924000 & 0.223017000 \\ \mathrm{C} & -4.463887000 & -1.590249000 & 0.954928000 \\ \mathrm{C} & -3.374696000 & -1.729265000 & -1.173655000 \\ \mathrm{C} & -5.698497000 & -1.728527000 & 0.336830000 \\ \mathrm{H} & -4.416956000 & -1.478371000 & 2.036469000 \\ \mathrm{C} & -4.612185000 & -1.871450000 & -1.791697000 \\ \mathrm{H} & -2.482453000 & -1.729827000 & -1.794594000 \\ \mathrm{C} & -5.788577000 & -1.869818000 & -1.047341000 \\ \mathrm{H} & -6.601499000 & -1.730415000 & 0.942733000 \\ \mathrm{H} & -4.655544000 & -1.987798000 & -2.872128000 \\ \mathrm{H} & -6.753253000 & -1.982192000 & -1.532794000 \\ \mathrm{H} & -0.040669000 & 0.627026000 & 2.036559000 \\ \mathrm{H} & -0.171829000 & -1.777132000 & 2.711040000 \\ \mathrm{O} & 5.577066000 & -1.047992000 & -1.496212000 \\ \mathrm{C} & 6.755418000 & -1.126749000 & -0.731335000 \\ \mathrm{H} & 7.580753000 & -1.002299000 & -1.433946000 \\ \mathrm{H} & 6.802173000 & -0.331625000 & 0.025170000 \\ \mathrm{H} & 6.849879000 & -2.100244000 & -0.232157000 \\ ------ & \end{array}$

\begin{tabular}{lrrr}
$\mathbf{A 3}_{\text {TBD }}$ & & & \\
& & & \\
& Coordinates $($ Angstroms) & Y & \\
\hline $\mathrm{C}$ & -0.649798000 & -2.506332000 & 0.409591000 \\
$\mathrm{C}$ & -1.369438000 & -2.274526000 & 1.731012000 \\
$\mathrm{C}$ & -2.875949000 & -2.236754000 & 1.497978000 \\
$\mathrm{~N}$ & -1.052024000 & -1.465261000 & -0.500342000 \\
$\mathrm{H}$ & -0.876395000 & -3.517863000 & 0.028743000 \\
$\mathrm{H}$ & 0.434138000 & -2.440149000 & 0.546514000
\end{tabular}




$\begin{array}{lrrr}\mathrm{H} & -1.032083000 & -1.320995000 & 2.144562000 \\ \mathrm{H} & -1.134426000 & -3.062259000 & 2.454055000 \\ \mathrm{H} & -3.275692000 & -3.263867000 & 1.480236000 \\ \mathrm{H} & -3.362956000 & -1.720296000 & 2.337323000 \\ \mathrm{~N} & -3.253249000 & -1.565936000 & 0.266304000 \\ \mathrm{C} & -4.663824000 & -1.245736000 & 0.143696000 \\ \mathrm{C} & -5.048069000 & -1.083354000 & -1.319965000 \\ \mathrm{C} & -4.058193000 & -0.127823000 & -1.983328000 \\ \mathrm{~N} & -2.681201000 & -0.532207000 & -1.803731000 \\ \mathrm{C} & -2.378247000 & -1.168162000 & -0.722721000 \\ \mathrm{H} & -4.885854000 & -0.325014000 & 0.704985000 \\ \mathrm{H} & -5.238733000 & -2.060124000 & 0.604411000 \\ \mathrm{H} & -5.003576000 & -2.059455000 & -1.817816000 \\ \mathrm{H} & -6.075707000 & -0.711760000 & -1.396709000 \\ \mathrm{H} & -4.262186000 & -0.064246000 & -3.058987000 \\ \mathrm{H} & -4.213331000 & 0.883697000 & -1.575209000 \\ \mathrm{C} & 5.176128000 & 1.392495000 & 0.130159000 \\ \mathrm{C} & 3.876366000 & 1.599177000 & -0.291997000 \\ \mathrm{C} & 3.042956000 & 0.521536000 & -0.626709000 \\ \mathrm{H} & -4.746041000 & 3.061355000 & 0.766187000 \\ \mathrm{C} & -2.114246000 & 0.773758000 & 3.270102000 \\ \mathrm{C} & -4.308710000 & 1.882869000 & 2.912081000 \\ \mathrm{C} & -0.449817000 & -1.231484000 & -1.278910000 \\ \mathrm{H} & & & \end{array}$




$\begin{array}{lrrr}\mathrm{H} & 1.899082000 & -0.468349000 & -2.535493000 \\ \mathrm{H} & -0.622747000 & 2.902681000 & -1.533598000 \\ \mathrm{O} & 6.978497000 & -0.001858000 & 0.636360000 \\ \mathrm{C} & 7.547999000 & -1.287434000 & 0.740556000 \\ \mathrm{H} & 8.567646000 & -1.136652000 & 1.096446000 \\ \mathrm{H} & 7.002834000 & -1.912103000 & 1.459691000 \\ \mathrm{H} & 7.575687000 & -1.795401000 & -0.232028000\end{array}$

\begin{tabular}{|c|c|c|c|}
\hline \multicolumn{4}{|c|}{$\mathbf{A} 1_{\text {DBU }}$} \\
\hline & $X$ & $\mathrm{Y}$ & $\mathrm{Z}$ \\
\hline$\overline{\mathrm{C}}$ & -1.307296000 & 3.719377000 & -0.772992000 \\
\hline $\mathrm{C}$ & -1.650946000 & 2.381434000 & -0.659337000 \\
\hline $\mathrm{C}$ & -0.702160000 & 1.420433000 & -0.292533000 \\
\hline $\mathrm{C}$ & 0.602719000 & 1.840955000 & -0.047903000 \\
\hline $\mathrm{C}$ & 0.964905000 & 3.181883000 & -0.154241000 \\
\hline $\mathrm{C}$ & 0.005403000 & 4.128316000 & -0.519105000 \\
\hline $\mathrm{H}$ & -2.040014000 & 4.469311000 & -1.052483000 \\
\hline $\mathrm{H}$ & -2.677963000 & 2.077238000 & -0.848247000 \\
\hline $\mathrm{H}$ & 1.342673000 & 1.096237000 & 0.234186000 \\
\hline $\mathrm{H}$ & 1.990388000 & 3.470674000 & 0.048404000 \\
\hline $\mathrm{C}$ & -1.045589000 & -0.059515000 & -0.241488000 \\
\hline $\mathrm{C}$ & -2.450755000 & -0.297043000 & 0.251269000 \\
\hline $\mathrm{H}$ & -2.677949000 & 0.167395000 & 1.209967000 \\
\hline $\mathrm{O}$ & -0.802754000 & -0.674103000 & -1.505984000 \\
\hline $\mathrm{C}$ & -3.364325000 & -1.013357000 & -0.406413000 \\
\hline $\mathrm{H}$ & -3.055740000 & -1.472132000 & -1.344295000 \\
\hline $\mathrm{C}$ & -4.754165000 & -1.278775000 & 0.002884000 \\
\hline $\mathrm{C}$ & -5.531522000 & -2.143830000 & -0.776771000 \\
\hline $\mathrm{C}$ & -5.348513000 & -0.699753000 & 1.132852000 \\
\hline $\mathrm{C}$ & -6.851501000 & -2.427852000 & -0.443044000 \\
\hline $\mathrm{H}$ & -5.088881000 & -2.601215000 & -1.658365000 \\
\hline $\mathrm{C}$ & -6.665839000 & -0.982625000 & 1.469466000 \\
\hline $\mathrm{H}$ & -4.777630000 & -0.016490000 & 1.754691000 \\
\hline $\mathrm{C}$ & -7.425248000 & -1.847757000 & 0.683652000 \\
\hline $\mathrm{H}$ & -7.432813000 & -3.101752000 & -1.065790000 \\
\hline $\mathrm{H}$ & -7.105368000 & -0.521925000 & 2.349606000 \\
\hline $\mathrm{H}$ & -8.456619000 & -2.063348000 & 0.946485000 \\
\hline $\mathrm{H}$ & -0.338389000 & -0.547782000 & 0.441642000 \\
\hline $\mathrm{H}$ & -1.167217000 & -0.085232000 & -2.179619000 \\
\hline $\mathrm{C}$ & 5.240349000 & -2.088647000 & 0.043522000 \\
\hline $\mathrm{C}$ & 4.865501000 & -3.316341000 & -0.780555000 \\
\hline $\mathrm{C}$ & 2.291349000 & -2.037791000 & -0.801627000 \\
\hline
\end{tabular}




\begin{tabular}{lrrr}
$\mathrm{C}$ & 4.244694000 & -2.976782000 & -2.137859000 \\
$\mathrm{C}$ & 3.194407000 & -1.869052000 & -2.025320000 \\
$\mathrm{H}$ & 5.767142000 & -1.360784000 & -0.595801000 \\
$\mathrm{H}$ & 2.066950000 & -3.101065000 & -0.643029000 \\
$\mathrm{H}$ & 4.190640000 & -3.943976000 & -0.187622000 \\
$\mathrm{H}$ & 3.786550000 & -3.881776000 & -2.555057000 \\
$\mathrm{H}$ & 5.962770000 & -2.401931000 & 0.808543000 \\
$\mathrm{H}$ & 5.776467000 & -3.906026000 & -0.932957000 \\
$\mathrm{H}$ & 1.328652000 & -1.553803000 & -0.976047000 \\
$\mathrm{H}$ & 5.031678000 & -2.672285000 & -2.838965000 \\
$\mathrm{H}$ & 2.575820000 & -1.855145000 & -2.928677000 \\
$\mathrm{H}$ & 3.684810000 & -0.888332000 & -1.978239000 \\
$\mathrm{C}$ & 2.808598000 & -1.442877000 & 0.497721000 \\
$\mathrm{~N}$ & 1.899970000 & -0.959568000 & 1.275083000 \\
$\mathrm{~N}$ & 4.161976000 & -1.421785000 & 0.770151000 \\
$\mathrm{C}$ & 4.674106000 & -0.612981000 & 1.873585000 \\
$\mathrm{H}$ & 5.002795000 & -1.276053000 & 2.688444000 \\
$\mathrm{H}$ & 5.564806000 & -0.076179000 & 1.519843000 \\
$\mathrm{C}$ & 3.636276000 & 0.370961000 & 2.385179000 \\
$\mathrm{H}$ & 3.973526000 & 0.806201000 & 3.331323000 \\
$\mathrm{H}$ & 3.511715000 & 1.190229000 & 1.666420000 \\
$\mathrm{C}$ & 2.310466000 & -0.366685000 & 2.531749000 \\
$\mathrm{H}$ & 1.521075000 & 0.317420000 & 2.861787000 \\
$\mathrm{H}$ & 2.403335000 & -1.143906000 & 3.306429000 \\
$\mathrm{O}$ & 0.244000000 & 5.459792000 & -0.651052000 \\
$\mathrm{C}$ & 1.546769000 & 5.926786000 & -0.390207000 \\
$\mathrm{H}$ & 1.515248000 & 7.006674000 & -0.541299000 \\
$\mathrm{H}$ & 1.851905000 & 5.715212000 & 0.643014000 \\
$\mathrm{H}$ & 2.279948000 & 5.487614000 & -1.079539000 \\
\hline---- & & \\
& & &
\end{tabular}

TSA1-2

\begin{tabular}{lccc} 
& \multicolumn{3}{c}{ Coordinates (Angstroms) } \\
C & 1.863673000 & -1.778943000 & -1.612551000 \\
$\mathrm{C}$ & 0.699369000 & -1.370613000 & -0.982552000 \\
$\mathrm{C}$ & 0.439129000 & -1.658840000 & 0.377151000 \\
$\mathrm{C}$ & 1.446683000 & -2.362533000 & 1.059718000 \\
$\mathrm{C}$ & 2.624388000 & -2.771676000 & 0.436213000 \\
$\mathrm{C}$ & 2.845011000 & -2.481909000 & -0.909111000 \\
$\mathrm{H}$ & 2.035269000 & -1.560348000 & -2.662727000 \\
$\mathrm{H}$ & -0.037802000 & -0.822069000 & -1.564810000 \\
$\mathrm{H}$ & 1.293700000 & -2.586261000 & 2.110285000 \\
$\mathrm{H}$ & 3.357366000 & -3.321283000 & 1.018100000
\end{tabular}




\begin{tabular}{|c|c|c|c|}
\hline $\mathrm{C}$ & -0.739171000 & -1.144107000 & 1.068337000 \\
\hline $\mathrm{C}$ & -2.005160000 & -1.019936000 & 0.436115000 \\
\hline $\mathrm{H}$ & -1.993261000 & -1.133001000 & -0.648693000 \\
\hline $\mathrm{O}$ & -0.743651000 & -1.311620000 & 2.468647000 \\
\hline $\mathrm{C}$ & -3.195254000 & -0.732216000 & 1.043526000 \\
\hline $\mathrm{H}$ & -3.193960000 & -0.568640000 & 2.119671000 \\
\hline $\mathrm{C}$ & -4.470068000 & -0.535154000 & 0.369915000 \\
\hline $\mathrm{C}$ & -5.580644000 & -0.081121000 & 1.108583000 \\
\hline $\mathrm{C}$ & -4.676602000 & -0.755879000 & -1.007128000 \\
\hline $\mathrm{C}$ & -6.813458000 & 0.148449000 & 0.512724000 \\
\hline $\mathrm{H}$ & -5.459379000 & 0.095189000 & 2.175238000 \\
\hline $\mathrm{C}$ & -5.908642000 & -0.520538000 & -1.603268000 \\
\hline $\mathrm{H}$ & -3.864614000 & -1.135979000 & -1.621535000 \\
\hline $\mathrm{C}$ & -6.990774000 & -0.064705000 & -0.852642000 \\
\hline $\mathrm{H}$ & -7.644185000 & 0.498182000 & 1.120798000 \\
\hline $\mathrm{H}$ & -6.028698000 & -0.705323000 & -2.668122000 \\
\hline $\mathrm{H}$ & -7.954340000 & 0.111744000 & -1.321314000 \\
\hline $\mathrm{H}$ & -0.439300000 & 0.557523000 & 0.864797000 \\
\hline $\mathrm{H}$ & -1.370246000 & -2.024100000 & 2.670920000 \\
\hline $\mathrm{C}$ & 3.020216000 & 3.134137000 & -0.531848000 \\
\hline $\mathrm{C}$ & 3.831095000 & 3.147142000 & 0.758889000 \\
\hline $\mathrm{C}$ & 1.935594000 & 1.130291000 & 1.505432000 \\
\hline $\mathrm{C}$ & 4.372023000 & 1.773480000 & 1.166121000 \\
\hline $\mathrm{C}$ & 3.317439000 & 0.671592000 & 1.038556000 \\
\hline $\mathrm{H}$ & 3.533739000 & 2.536765000 & -1.298214000 \\
\hline $\mathrm{H}$ & 2.010844000 & 1.800246000 & 2.371694000 \\
\hline $\mathrm{H}$ & 3.218311000 & 3.581944000 & 1.556981000 \\
\hline $\mathrm{H}$ & 4.729218000 & 1.832423000 & 2.201109000 \\
\hline $\mathrm{H}$ & 2.960211000 & 4.158691000 & -0.914713000 \\
\hline $\mathrm{H}$ & 4.665387000 & 3.841164000 & 0.610289000 \\
\hline $\mathrm{H}$ & 1.345613000 & 0.275827000 & 1.842948000 \\
\hline $\mathrm{H}$ & 5.242380000 & 1.521172000 & 0.548983000 \\
\hline $\mathrm{H}$ & 3.610549000 & -0.204200000 & 1.622954000 \\
\hline $\mathrm{H}$ & 3.240712000 & 0.313873000 & 0.005315000 \\
\hline $\mathrm{C}$ & 1.107165000 & 1.823505000 & 0.451076000 \\
\hline $\mathrm{N}$ & -0.179192000 & 1.548969000 & 0.480480000 \\
\hline $\mathrm{N}$ & 1.622986000 & 2.679775000 & -0.444928000 \\
\hline $\mathrm{C}$ & 0.808855000 & 3.182852000 & -1.561691000 \\
\hline $\mathrm{H}$ & 0.537119000 & 4.225783000 & -1.352069000 \\
\hline $\mathrm{H}$ & 1.445113000 & 3.180941000 & -2.452908000 \\
\hline $\mathrm{C}$ & -0.430454000 & 2.337589000 & -1.799803000 \\
\hline $\mathrm{H}$ & -1.088590000 & 2.845452000 & -2.509449000 \\
\hline $\mathrm{H}$ & -0.143941000 & 1.373455000 & -2.231975000 \\
\hline $\mathrm{C}$ & -1.127692000 & 2.109898000 & -0.468531000 \\
\hline
\end{tabular}




$\begin{array}{lrrr}\mathrm{H} & -1.959508000 & 1.407100000 & -0.547425000 \\ \mathrm{H} & -1.523549000 & 3.050906000 & -0.068574000 \\ \mathrm{O} & 3.967716000 & -2.821172000 & -1.616810000 \\ \mathrm{C} & 4.947184000 & -3.574136000 & -0.949316000 \\ \mathrm{H} & 4.547567000 & -4.530900000 & -0.585845000 \\ \mathrm{H} & 5.733748000 & -3.769475000 & -1.680451000 \\ \mathrm{H} & \mathbf{5 . 3 7 6 0 1 7 0 0 0} & -\mathbf{- 3 . 0 2 5 4 4 8 0 0 0} & -0.098314000\end{array}$

A2

\begin{tabular}{lrcc} 
& \multicolumn{3}{c}{ Coordinates (Angstroms) } \\
& $\mathrm{X}$ & $\mathrm{Y}$ & $\mathrm{Z}$ \\
\hline $\mathrm{C}$ & -2.762216000 & -1.873414000 & 0.841187000 \\
$\mathrm{C}$ & -1.508444000 & -1.713644000 & 0.269604000 \\
$\mathrm{C}$ & -1.339254000 & -1.163017000 & -1.022370000 \\
$\mathrm{C}$ & -2.524927000 & -0.837911000 & -1.720882000 \\
$\mathrm{C}$ & -3.779598000 & -0.986304000 & -1.143740000 \\
$\mathrm{C}$ & -3.909107000 & -1.486853000 & 0.149103000 \\
$\mathrm{H}$ & -2.865424000 & -2.296854000 & 1.837325000 \\
$\mathrm{H}$ & -0.642265000 & -2.034853000 & 0.838950000 \\
$\mathrm{H}$ & -2.437289000 & -0.442436000 & -2.726727000 \\
$\mathrm{H}$ & -4.675011000 & -0.707218000 & -1.693112000 \\
$\mathrm{C}$ & -0.046282000 & -0.855101000 & -1.593677000 \\
$\mathrm{C}$ & 1.183478000 & -1.070964000 & -1.015450000 \\
$\mathrm{H}$ & 1.178811000 & -1.670755000 & -0.107991000 \\
$\mathrm{O}$ & -0.092962000 & -0.163068000 & -2.811236000 \\
$\mathrm{C}$ & 2.419388000 & -0.549490000 & -1.421650000 \\
$\mathrm{H}$ & 2.463622000 & 0.066201000 & -2.319408000 \\
$\mathrm{C}$ & 3.680472000 & -0.804444000 & -0.760120000 \\
$\mathrm{C}$ & 4.829451000 & -0.057540000 & -1.113492000 \\
$\mathrm{C}$ & 3.848754000 & -1.742821000 & 0.287024000 \\
$\mathrm{C}$ & 6.040738000 & -0.215606000 & -0.458136000 \\
$\mathrm{H}$ & 4.748778000 & 0.669479000 & -1.919603000 \\
$\mathrm{C}$ & 5.063330000 & -1.890220000 & 0.946220000 \\
$\mathrm{H}$ & 3.020191000 & -2.386147000 & 0.572356000 \\
$\mathrm{C}$ & 6.173689000 & -1.128111000 & 0.589160000 \\
$\mathrm{H}$ & 6.894149000 & 0.383874000 & -0.766312000 \\
$\mathrm{H}$ & 5.146231000 & -2.627567000 & 1.741638000 \\
$\mathrm{H}$ & 7.122448000 & -1.252421000 & 1.101870000 \\
$\mathrm{H}$ & 2.137464000 & 1.087376000 & -0.081164000 \\
$\mathrm{H}$ & 0.584316000 & -0.555045000 & -3.378095000 \\
$\mathrm{C}$ & -2.042127000 & 1.952929000 & 1.175046000 \\
$\mathrm{C}$ & -2.163715000 & 3.378008000 & 0.634398000 \\
$\mathrm{C}$ & 0.145958000 & 2.337322000 & -0.916796000 \\
& & &
\end{tabular}




\begin{tabular}{lrrr}
$\mathrm{C}$ & -2.160060000 & 3.462492000 & -0.896808000 \\
$\mathrm{C}$ & -1.245250000 & 2.419990000 & -1.533281000 \\
$\mathrm{H}$ & -2.607521000 & 1.227155000 & 0.578503000 \\
$\mathrm{H}$ & 0.600520000 & 3.334736000 & -0.839610000 \\
$\mathrm{H}$ & -1.342966000 & 3.971274000 & 1.056733000 \\
$\mathrm{H}$ & -1.862152000 & 4.474060000 & -1.198642000 \\
$\mathrm{H}$ & -2.456517000 & 1.922240000 & 2.185881000 \\
$\mathrm{H}$ & -3.087265000 & 3.815920000 & 1.027136000 \\
$\mathrm{H}$ & 0.785248000 & 1.754786000 & -1.592888000 \\
$\mathrm{H}$ & -3.176710000 & 3.309371000 & -1.276489000 \\
$\mathrm{H}$ & -1.118308000 & 2.631582000 & -2.598834000 \\
$\mathrm{H}$ & -1.710843000 & 1.434768000 & -1.489032000 \\
$\mathrm{C}$ & 0.316902000 & 1.688289000 & 0.434484000 \\
$\mathrm{~N}$ & 1.587044000 & 1.405836000 & 0.731560000 \\
$\mathrm{~N}$ & -0.651490000 & 1.483561000 & 1.320794000 \\
$\mathrm{C}$ & -0.425456000 & 0.705641000 & 2.550168000 \\
$\mathrm{H}$ & -0.459999000 & 1.402133000 & 3.398096000 \\
$\mathrm{H}$ & -1.266040000 & 0.012200000 & 2.642216000 \\
$\mathrm{C}$ & 0.885928000 & -0.060121000 & 2.536615000 \\
$\mathrm{H}$ & 1.110038000 & -0.409157000 & 3.548173000 \\
$\mathrm{H}$ & 0.803938000 & -0.934409000 & 1.888745000 \\
$\mathrm{C}$ & 1.991967000 & 0.839256000 & 2.010072000 \\
$\mathrm{H}$ & 2.918144000 & 0.285745000 & 1.844999000 \\
$\mathrm{H}$ & 2.196847000 & 1.655608000 & 2.712995000 \\
$\mathrm{O}$ & -5.149352000 & -1.591884000 & 0.743631000 \\
$\mathrm{C}$ & -5.790474000 & -2.826007000 & 0.489393000 \\
$\mathrm{H}$ & -6.756332000 & -2.792914000 & 0.998897000 \\
$\mathrm{H}$ & -5.952221000 & -2.979408000 & -0.586337000 \\
$\mathrm{H}$ & -5.203102000 & -3.669635000 & 0.877675000 \\
\hline---- & &
\end{tabular}

TSA2-3 ${ }_{\text {DBU }}$

\begin{tabular}{lccc} 
& \multicolumn{3}{c}{ Coordinates (Angstroms) } \\
\hline $\mathrm{C}$ & $\mathbf{X}$ & $\mathrm{Y}$ & $\mathrm{Z}$ \\
$\mathrm{C}$ & 1.536950000 & -1.322988000 & -1.441519000 \\
$\mathrm{C}$ & 1.421462000 & -1.484219000 & 0.538285000 \\
$\mathrm{C}$ & 2.566464000 & -1.820540000 & 1.269782000 \\
$\mathrm{C}$ & 3.822786000 & -1.910143000 & 0.673721000 \\
$\mathrm{C}$ & 3.966860000 & -1.664641000 & -0.690732000 \\
$\mathrm{H}$ & 2.959445000 & -1.129142000 & -2.502944000 \\
$\mathrm{H}$ & 0.745789000 & -0.924016000 & -1.436805000 \\
$\mathrm{H}$ & 2.467789000 & -2.004322000 & 2.333867000 \\
$\mathrm{H}$ & 4.676385000 & -2.174555000 & 1.288355000
\end{tabular}




\begin{tabular}{|c|c|c|c|}
\hline $\mathrm{C}$ & 0.126857000 & -1.321018000 & 1.200079000 \\
\hline $\mathrm{C}$ & -1.082825000 & -1.284562000 & 0.589412000 \\
\hline $\mathrm{H}$ & -1.080697000 & -1.547094000 & -0.465707000 \\
\hline $\mathrm{O}$ & 0.232782000 & -1.108328000 & 2.571430000 \\
\hline $\mathrm{C}$ & -2.318204000 & -0.798794000 & 1.139994000 \\
\hline $\mathrm{H}$ & -2.357846000 & -0.618385000 & 2.216640000 \\
\hline $\mathrm{C}$ & -3.618480000 & -1.052848000 & 0.526887000 \\
\hline $\mathrm{C}$ & -4.787081000 & -0.536810000 & 1.128689000 \\
\hline $\mathrm{C}$ & -3.792060000 & -1.719534000 & -0.704762000 \\
\hline $\mathrm{C}$ & -6.033484000 & -0.655695000 & 0.532826000 \\
\hline $\mathrm{H}$ & -4.695726000 & -0.020941000 & 2.082673000 \\
\hline $\mathrm{C}$ & -5.042154000 & -1.827264000 & -1.304938000 \\
\hline $\mathrm{H}$ & -2.938221000 & -2.179368000 & -1.194875000 \\
\hline $\mathrm{C}$ & -6.176702000 & -1.294410000 & -0.699302000 \\
\hline $\mathrm{H}$ & -6.905324000 & -0.240596000 & 1.032823000 \\
\hline $\mathrm{H}$ & -5.130472000 & -2.348718000 & -2.255257000 \\
\hline $\mathrm{H}$ & -7.151661000 & -1.385996000 & -1.168150000 \\
\hline $\mathrm{H}$ & -2.065720000 & 0.745327000 & 0.637576000 \\
\hline $\mathrm{H}$ & -0.581108000 & -1.446381000 & 2.967950000 \\
\hline $\mathrm{C}$ & 1.750284000 & 2.618426000 & -0.877627000 \\
\hline $\mathrm{C}$ & 2.026147000 & 3.631763000 & 0.231340000 \\
\hline $\mathrm{C}$ & 0.049008000 & 1.951655000 & 1.572130000 \\
\hline $\mathrm{C}$ & 2.381749000 & 2.986032000 & 1.574168000 \\
\hline $\mathrm{C}$ & 1.534549000 & 1.746611000 & 1.854675000 \\
\hline $\mathrm{H}$ & 2.451921000 & 1.774888000 & -0.838263000 \\
\hline $\mathrm{H}$ & -0.297313000 & 2.911971000 & 1.978494000 \\
\hline $\mathrm{H}$ & 1.146437000 & 4.279233000 & 0.331248000 \\
\hline $\mathrm{H}$ & 2.251352000 & 3.727793000 & 2.371697000 \\
\hline $\mathrm{H}$ & 1.898744000 & 3.110420000 & -1.844383000 \\
\hline $\mathrm{H}$ & 2.845059000 & 4.281347000 & -0.095789000 \\
\hline $\mathrm{H}$ & -0.522465000 & 1.179947000 & 2.099729000 \\
\hline $\mathrm{H}$ & 3.441133000 & 2.703782000 & 1.578727000 \\
\hline $\mathrm{H}$ & 1.643125000 & 1.442768000 & 2.899510000 \\
\hline $\mathrm{H}$ & 1.897938000 & 0.898150000 & 1.269245000 \\
\hline $\mathrm{C}$ & -0.421197000 & 1.880733000 & 0.138667000 \\
\hline $\mathrm{N}$ & -1.711885000 & 1.623209000 & 0.005373000 \\
\hline $\mathrm{N}$ & 0.373226000 & 2.102894000 & -0.922558000 \\
\hline $\mathrm{C}$ & -0.065122000 & 1.789358000 & -2.289825000 \\
\hline $\mathrm{H}$ & -0.166326000 & 2.735413000 & -2.838607000 \\
\hline $\mathrm{H}$ & 0.740323000 & 1.217447000 & -2.764799000 \\
\hline $\mathrm{C}$ & -1.370427000 & 1.013047000 & -2.331726000 \\
\hline $\mathrm{H}$ & -1.784350000 & 1.053248000 & -3.343189000 \\
\hline $\mathrm{H}$ & -1.206339000 & -0.038985000 & -2.082044000 \\
\hline $\mathrm{C}$ & -2.327040000 & 1.616475000 & -1.314004000 \\
\hline
\end{tabular}




$\begin{array}{lrrr}\mathrm{H} & -3.252755000 & 1.043034000 & -1.242325000 \\ \mathrm{H} & -2.583019000 & 2.646842000 & -1.591524000 \\ \mathrm{O} & 5.143045000 & -1.718677000 & -1.376367000 \\ \mathrm{C} & 6.299255000 & -2.085979000 & -0.662823000 \\ \mathrm{H} & 7.113017000 & -2.094478000 & -1.389406000 \\ \mathrm{H} & 6.532164000 & -1.363745000 & 0.131329000 \\ \mathrm{H} & \mathbf{6 . 2 0 0 3 1 4 0 0 0} & -\mathbf{- 3 . 0 8 5 4 8 8 0 0 0} & -0.219263000\end{array}$

\begin{tabular}{|c|c|c|c|}
\hline \multicolumn{4}{|c|}{$\mathbf{A} \mathbf{3}_{\text {DBU }}$} \\
\hline & \multicolumn{3}{|c|}{ Coordinates (Angstroms) } \\
\hline & $X$ & Y & Z \\
\hline$\overline{\mathrm{C}}$ & 2.693151000 & -1.370672000 & -1.456565000 \\
\hline $\mathrm{C}$ & 1.492382000 & -1.184876000 & -0.796819000 \\
\hline $\mathrm{C}$ & 1.360529000 & -1.481826000 & 0.567346000 \\
\hline $\mathrm{C}$ & 2.484215000 & -1.946689000 & 1.248555000 \\
\hline $\mathrm{C}$ & 3.702075000 & -2.134393000 & 0.598779000 \\
\hline $\mathrm{C}$ & 3.810514000 & -1.849236000 & -0.762433000 \\
\hline $\mathrm{H}$ & 2.798086000 & -1.137650000 & -2.511267000 \\
\hline $\mathrm{H}$ & 0.646675000 & -0.774199000 & -1.338473000 \\
\hline $\mathrm{H}$ & 2.408662000 & -2.158501000 & 2.309611000 \\
\hline $\mathrm{H}$ & 4.551348000 & -2.499661000 & 1.164763000 \\
\hline $\mathrm{C}$ & 0.086968000 & -1.247594000 & 1.276501000 \\
\hline $\mathrm{C}$ & -1.122009000 & -1.253144000 & 0.701862000 \\
\hline $\mathrm{H}$ & -1.190032000 & -1.516923000 & -0.347289000 \\
\hline $\mathrm{O}$ & 0.278917000 & -0.986939000 & 2.607189000 \\
\hline $\mathrm{C}$ & -2.393134000 & -0.852219000 & 1.406142000 \\
\hline $\mathrm{H}$ & -2.351886000 & 0.223173000 & 1.629055000 \\
\hline $\mathrm{C}$ & -3.641718000 & -1.120490000 & 0.595898000 \\
\hline $\mathrm{C}$ & -4.615445000 & -0.131017000 & 0.453257000 \\
\hline $\mathrm{C}$ & -3.853991000 & -2.360771000 & -0.011184000 \\
\hline $\mathrm{C}$ & -5.774516000 & -0.372241000 & -0.280118000 \\
\hline $\mathrm{H}$ & -4.452295000 & 0.840162000 & 0.913970000 \\
\hline $\mathrm{C}$ & -5.009956000 & -2.605337000 & -0.745436000 \\
\hline $\mathrm{H}$ & -3.102842000 & -3.140469000 & 0.092037000 \\
\hline $\mathrm{C}$ & -5.975029000 & -1.610539000 & -0.882721000 \\
\hline $\mathrm{H}$ & -6.520125000 & 0.411135000 & -0.382645000 \\
\hline $\mathrm{H}$ & -5.157967000 & -3.575195000 & -1.211958000 \\
\hline $\mathrm{H}$ & -6.878028000 & -1.800162000 & -1.455663000 \\
\hline $\mathrm{H}$ & -0.583370000 & -0.895536000 & 3.031045000 \\
\hline $\mathrm{C}$ & 1.810651000 & 2.577790000 & -0.983265000 \\
\hline $\mathrm{C}$ & 2.310173000 & 3.575527000 & 0.059530000 \\
\hline $\mathrm{C}$ & 0.180716000 & 2.346651000 & 1.600212000 \\
\hline $\mathrm{C}$ & 2.653896000 & 2.925623000 & 1.401906000 \\
\hline
\end{tabular}




\begin{tabular}{lrrr}
$\mathrm{C}$ & 1.619962000 & 1.874413000 & 1.802586000 \\
$\mathrm{H}$ & 2.424718000 & 1.663239000 & -0.964153000 \\
$\mathrm{H}$ & 0.074037000 & 3.385061000 & 1.942689000 \\
$\mathrm{H}$ & 1.546393000 & 4.352237000 & 0.187625000 \\
$\mathrm{H}$ & 2.721877000 & 3.703980000 & 2.172381000 \\
$\mathrm{H}$ & 1.953706000 & 3.025501000 & -1.974977000 \\
$\mathrm{H}$ & 3.196077000 & 4.078732000 & -0.343665000 \\
$\mathrm{H}$ & -0.492812000 & 1.758261000 & 2.227315000 \\
$\mathrm{H}$ & 3.642875000 & 2.454828000 & 1.341048000 \\
$\mathrm{H}$ & 1.759652000 & 1.598172000 & 2.852559000 \\
$\mathrm{H}$ & 1.785863000 & 0.955600000 & 1.233499000 \\
$\mathrm{C}$ & -0.422133000 & 2.245417000 & 0.204176000 \\
$\mathrm{~N}$ & -1.708437000 & 2.172757000 & 0.174164000 \\
$\mathrm{~N}$ & 0.392004000 & 2.234849000 & -0.912014000 \\
$\mathrm{C}$ & -0.146164000 & 1.850363000 & -2.213489000 \\
$\mathrm{H}$ & -0.158951000 & 2.731899000 & -2.873360000 \\
$\mathrm{H}$ & 0.542341000 & 1.124608000 & -2.670025000 \\
$\mathrm{C}$ & -1.543221000 & 1.266468000 & -2.100238000 \\
$\mathrm{H}$ & -2.007859000 & 1.220400000 & -3.090319000 \\
$\mathrm{H}$ & -1.502997000 & 0.246994000 & -1.702287000 \\
$\mathrm{C}$ & -2.340409000 & 2.130718000 & -1.130758000 \\
$\mathrm{H}$ & -3.355475000 & 1.739252000 & -1.017618000 \\
$\mathrm{H}$ & -2.432128000 & 3.150101000 & -1.540077000 \\
$\mathrm{H}$ & -2.494005000 & -1.386944000 & 2.366574000 \\
$\mathrm{O}$ & 4.943986000 & -1.989207000 & -1.495101000 \\
$\mathrm{C}$ & 6.101537000 & -2.454716000 & -0.839397000 \\
$\mathrm{H}$ & 5.951319000 & -3.457361000 & -0.418928000 \\
$\mathrm{H}$ & 6.881771000 & -2.497047000 & -1.600116000 \\
$\mathrm{H}$ & 6.412905000 & -1.771016000 & -0.039058000 \\
\hline----1 &
\end{tabular}

A1 $1_{\text {MTBd }}$

\begin{tabular}{lrrr} 
& \multicolumn{3}{c}{ Coordinates (Angstroms) } \\
\hline $\mathrm{C}$ & -1.231112000 & -2.589828000 & -1.363502000 \\
$\mathrm{C}$ & -0.906194000 & -1.317559000 & -2.138937000 \\
$\mathrm{C}$ & 0.590488000 & -1.281930000 & -2.412246000 \\
$\mathrm{~N}$ & -0.484322000 & -2.671988000 & -0.128027000 \\
$\mathrm{H}$ & -1.024602000 & -3.466331000 & -2.000962000 \\
$\mathrm{H}$ & -2.300737000 & -2.620008000 & -1.127548000 \\
$\mathrm{H}$ & -1.211993000 & -0.454833000 & -1.536877000 \\
$\mathrm{H}$ & -1.451681000 & -1.266209000 & -3.087085000 \\
$\mathrm{H}$ & 0.833285000 & -1.904159000 & -3.286902000 \\
$\mathrm{H}$ & 0.909997000 & -0.257055000 & -2.653652000
\end{tabular}




\begin{tabular}{|c|c|c|c|}
\hline $\mathrm{N}$ & 1.374136000 & -1.741404000 & -1.272510000 \\
\hline $\mathrm{C}$ & 2.765747000 & -1.323414000 & -1.323308000 \\
\hline $\mathrm{C}$ & 3.593847000 & -1.972224000 & -0.231040000 \\
\hline $\mathrm{C}$ & 2.839302000 & -1.860064000 & 1.081296000 \\
\hline $\mathrm{N}$ & 1.547508000 & -2.500941000 & 0.948881000 \\
\hline $\mathrm{C}$ & 0.755161000 & -2.303178000 & -0.169811000 \\
\hline $\mathrm{H}$ & 2.827142000 & -0.229028000 & -1.230617000 \\
\hline $\mathrm{H}$ & 3.164865000 & -1.584722000 & -2.312917000 \\
\hline $\mathrm{H}$ & 3.767010000 & -3.030063000 & -0.456672000 \\
\hline $\mathrm{H}$ & 4.563031000 & -1.468778000 & -0.163783000 \\
\hline $\mathrm{H}$ & 3.384214000 & -2.374571000 & 1.879877000 \\
\hline $\mathrm{H}$ & 2.739536000 & -0.804079000 & 1.376451000 \\
\hline $\mathrm{C}$ & -3.694973000 & 2.147531000 & 0.000773000 \\
\hline $\mathrm{C}$ & -2.450845000 & 1.874261000 & 0.544047000 \\
\hline $\mathrm{C}$ & -2.112109000 & 0.580731000 & 0.957087000 \\
\hline $\mathrm{C}$ & -3.051098000 & -0.432647000 & 0.794907000 \\
\hline $\mathrm{C}$ & -4.304956000 & -0.178462000 & 0.242106000 \\
\hline $\mathrm{C}$ & -4.632175000 & 1.119856000 & -0.152239000 \\
\hline $\mathrm{H}$ & -3.964397000 & 3.147776000 & -0.322556000 \\
\hline $\mathrm{H}$ & -1.720542000 & 2.676303000 & 0.636009000 \\
\hline $\mathrm{H}$ & -2.786947000 & -1.446829000 & 1.083339000 \\
\hline $\mathrm{H}$ & -5.008368000 & -0.995008000 & 0.125824000 \\
\hline $\mathrm{C}$ & -0.762564000 & 0.282705000 & 1.580745000 \\
\hline $\mathrm{C}$ & 0.363637000 & 0.852798000 & 0.758626000 \\
\hline $\mathrm{H}$ & 0.307565000 & 0.642277000 & -0.307711000 \\
\hline $\mathrm{O}$ & -0.692480000 & 0.671159000 & 2.944666000 \\
\hline $\mathrm{C}$ & 1.413248000 & 1.515569000 & 1.246510000 \\
\hline $\mathrm{H}$ & 1.493959000 & 1.650528000 & 2.323766000 \\
\hline $\mathrm{C}$ & 2.538083000 & 1.983041000 & 0.415798000 \\
\hline $\mathrm{C}$ & 3.841869000 & 1.934900000 & 0.922809000 \\
\hline $\mathrm{C}$ & 2.351994000 & 2.427610000 & -0.899435000 \\
\hline $\mathrm{C}$ & 4.930614000 & 2.279356000 & 0.128633000 \\
\hline $\mathrm{H}$ & 4.001066000 & 1.603318000 & 1.946081000 \\
\hline $\mathrm{C}$ & 3.439151000 & 2.775316000 & -1.693737000 \\
\hline $\mathrm{H}$ & 1.343815000 & 2.507755000 & -1.297192000 \\
\hline $\mathrm{C}$ & 4.733636000 & 2.695468000 & -1.185457000 \\
\hline $\mathrm{H}$ & 5.935092000 & 2.222523000 & 0.538044000 \\
\hline $\mathrm{H}$ & 3.274488000 & 3.117863000 & -2.711292000 \\
\hline $\mathrm{H}$ & 5.582106000 & 2.967971000 & -1.805964000 \\
\hline $\mathrm{H}$ & -0.647099000 & -0.805273000 & 1.583157000 \\
\hline $\mathrm{H}$ & -0.970971000 & 1.595023000 & 2.993635000 \\
\hline $\mathrm{C}$ & 0.932296000 & -2.972664000 & 2.170967000 \\
\hline $\mathrm{H}$ & 0.651593000 & -2.144146000 & 2.838736000 \\
\hline $\mathrm{H}$ & 0.035837000 & -3.536315000 & 1.918495000 \\
\hline
\end{tabular}




$\begin{array}{lrrr}\mathrm{H} & 1.639465000 & -3.619026000 & 2.703293000 \\ \mathrm{O} & -5.820657000 & 1.483809000 & -0.699024000 \\ \mathrm{C} & -6.797024000 & 0.483958000 & -0.880853000 \\ \mathrm{H} & -7.662093000 & 0.982985000 & -1.319204000 \\ \mathrm{H} & -6.447966000 & -0.300437000 & -1.564910000 \\ \mathrm{H} & -7.088962000 & 0.026364000 & 0.073148000\end{array}$

TSA1-2

\begin{tabular}{|c|c|c|c|}
\hline \multicolumn{4}{|c|}{ Coordinates (Angstroms) } \\
\hline & $\mathrm{X}$ & Y & Z \\
\hline$\overline{\mathrm{C}}$ & 0.653088000 & -1.349466000 & 1.848084000 \\
\hline $\mathrm{C}$ & -0.367826000 & -1.396480000 & 2.974547000 \\
\hline $\mathrm{C}$ & -1.629238000 & -0.689148000 & 2.510159000 \\
\hline $\mathrm{N}$ & 0.074359000 & -1.673228000 & 0.547165000 \\
\hline $\mathrm{H}$ & 1.476216000 & -2.045471000 & 2.038344000 \\
\hline $\mathrm{H}$ & 1.097171000 & -0.348167000 & 1.789520000 \\
\hline $\mathrm{H}$ & 0.036745000 & -0.916673000 & 3.870676000 \\
\hline $\mathrm{H}$ & -0.613867000 & -2.433361000 & 3.229796000 \\
\hline $\mathrm{H}$ & -2.411320000 & -0.736248000 & 3.273624000 \\
\hline $\mathrm{H}$ & -1.435450000 & 0.371969000 & 2.298906000 \\
\hline $\mathrm{N}$ & -2.140995000 & -1.358333000 & 1.321873000 \\
\hline $\mathrm{C}$ & -3.557780000 & -1.149628000 & 1.037716000 \\
\hline $\mathrm{C}$ & -4.021106000 & -2.032687000 & -0.106387000 \\
\hline $\mathrm{C}$ & -3.046673000 & -1.911104000 & -1.264183000 \\
\hline $\mathrm{N}$ & -1.698428000 & -2.218568000 & -0.813609000 \\
\hline $\mathrm{C}$ & -1.243744000 & -1.721954000 & 0.362153000 \\
\hline $\mathrm{H}$ & -3.731457000 & -0.092318000 & 0.800873000 \\
\hline $\mathrm{H}$ & -4.116505000 & -1.383933000 & 1.950519000 \\
\hline $\mathrm{H}$ & -4.072102000 & -3.077666000 & 0.217971000 \\
\hline $\mathrm{H}$ & -5.021457000 & -1.722169000 & -0.421003000 \\
\hline $\mathrm{H}$ & -3.301965000 & -2.623535000 & -2.053262000 \\
\hline $\mathrm{H}$ & -3.078007000 & -0.902251000 & -1.694910000 \\
\hline $\mathrm{C}$ & 4.177457000 & 1.363206000 & 0.895003000 \\
\hline $\mathrm{C}$ & 2.873298000 & 1.251379000 & 0.442877000 \\
\hline $\mathrm{C}$ & 2.532274000 & 0.432182000 & -0.651338000 \\
\hline $\mathrm{C}$ & 3.587975000 & -0.246688000 & -1.272050000 \\
\hline $\mathrm{C}$ & 4.906518000 & -0.135683000 & -0.833314000 \\
\hline $\mathrm{C}$ & 5.210776000 & 0.668248000 & 0.262509000 \\
\hline $\mathrm{H}$ & 4.422638000 & 2.004058000 & 1.736572000 \\
\hline $\mathrm{H}$ & 2.108432000 & 1.832507000 & 0.951068000 \\
\hline $\mathrm{H}$ & 3.363439000 & -0.882686000 & -2.121933000 \\
\hline $\mathrm{H}$ & 5.680389000 & -0.686195000 & -1.357793000 \\
\hline $\mathrm{C}$ & 1.143349000 & 0.214753000 & -1.118394000 \\
\hline
\end{tabular}




$\begin{array}{lrcc}\mathrm{C} & 0.056984000 & 1.042170000 & -0.692760000 \\ \mathrm{H} & 0.175012000 & 1.478624000 & 0.298870000 \\ \mathrm{O} & 1.051157000 & -0.262927000 & -2.449756000 \\ \mathrm{C} & -1.152248000 & 1.172593000 & -1.303544000 \\ \mathrm{H} & -1.276333000 & 0.728249000 & -2.289652000 \\ \mathrm{C} & -2.342124000 & 1.783407000 & -0.723399000 \\ \mathrm{C} & -3.560652000 & 1.731039000 & -1.428191000 \\ \mathrm{C} & -2.376388000 & 2.389671000 & 0.547968000 \\ \mathrm{C} & -4.747902000 & 2.198323000 & -0.878928000 \\ \mathrm{H} & -3.567420000 & 1.300592000 & -2.427722000 \\ \mathrm{C} & -3.562285000 & 2.859869000 & 1.097155000 \\ \mathrm{H} & -1.457406000 & 2.498359000 & 1.117530000 \\ \mathrm{C} & -4.763990000 & 2.756028000 & 0.397894000 \\ \mathrm{H} & -5.668298000 & 2.128143000 & -1.452922000 \\ \mathrm{H} & -3.547301000 & 3.316731000 & 2.083360000 \\ \mathrm{H} & -5.689517000 & 3.121503000 & 0.831933000 \\ \mathrm{H} & 0.614116000 & -1.001762000 & -0.265547000 \\ \mathrm{H} & 1.201352000 & 0.488492000 & -3.042247000 \\ \mathrm{C} & -0.760641000 & -2.691612000 & -1.822032000 \\ \mathrm{H} & -0.387290000 & -1.874827000 & -2.449492000 \\ \mathrm{H} & 0.091939000 & -3.158218000 & -1.327785000 \\ \mathrm{H} & -1.274034000 & -3.436893000 & -2.436538000 \\ \mathrm{O} & 6.461110000 & 0.847900000 & 0.782805000 \\ \mathrm{C} & 7.522261000 & 0.157706000 & 0.172685000 \\ \mathrm{H} & 7.648229000 & 0.449855000 & -0.878925000 \\ \mathrm{H} & 8.421936000 & 0.428418000 & 0.728177000 \\ \mathrm{H} & 7.381117000 & -0.930750000 & 0.224215000 \\ -------0 & \end{array}$

$\begin{array}{lrrr}\text { A2 } & & \\ \text { MTBD } & & \\ & \text { Coordinates (Angstroms) } & \text { Z } \\ \text { C } & 1.598330000 & 1.476444000 & 2.224094000 \\ \mathrm{C} & 2.564524000 & 1.715418000 & 1.075105000 \\ \mathrm{C} & 1.994815000 & 2.769377000 & 0.141813000 \\ \mathrm{~N} & 0.197761000 & 1.573529000 & 1.804334000 \\ \mathrm{H} & 1.750216000 & 2.212293000 & 3.024341000 \\ \mathrm{H} & 1.749317000 & 0.474703000 & 2.641860000 \\ \mathrm{H} & 2.720186000 & 0.793400000 & 0.513180000 \\ \mathrm{H} & 3.531355000 & 2.037546000 & 1.471183000 \\ \mathrm{H} & 1.909269000 & 3.745067000 & 0.640211000 \\ \mathrm{H} & 2.636871000 & 2.887843000 & -0.733326000 \\ \mathrm{~N} & 0.683324000 & 2.341586000 & -0.331490000 \\ \mathrm{C} & 0.354569000 & 2.484279000 & -1.747063000\end{array}$




\begin{tabular}{|c|c|c|c|}
\hline $\mathrm{C}$ & -1.142880000 & 2.691397000 & -1.919199000 \\
\hline $\mathrm{C}$ & -1.882994000 & 1.562714000 & -1.224987000 \\
\hline $\mathrm{N}$ & -1.416800000 & 1.424809000 & 0.150554000 \\
\hline $\mathrm{C}$ & -0.171257000 & 1.750776000 & 0.524455000 \\
\hline $\mathrm{H}$ & 0.701113000 & 1.599611000 & -2.296722000 \\
\hline $\mathrm{H}$ & 0.905039000 & 3.350538000 & -2.123357000 \\
\hline $\mathrm{H}$ & -1.429960000 & 3.655430000 & -1.484942000 \\
\hline $\mathrm{H}$ & -1.398433000 & 2.709735000 & -2.981988000 \\
\hline $\mathrm{H}$ & -2.961005000 & 1.735868000 & -1.195816000 \\
\hline $\mathrm{H}$ & -1.726145000 & 0.615338000 & -1.755416000 \\
\hline $\mathrm{H}$ & -1.811694000 & 0.576612000 & 0.606441000 \\
\hline $\mathrm{C}$ & 2.809452000 & -0.834820000 & -1.694731000 \\
\hline $\mathrm{C}$ & 1.568461000 & -1.014707000 & -1.111171000 \\
\hline $\mathrm{C}$ & 1.420413000 & -1.543348000 & 0.194164000 \\
\hline $\mathrm{C}$ & 2.612834000 & -1.863131000 & 0.868676000 \\
\hline $\mathrm{C}$ & 3.868087000 & -1.672682000 & 0.292027000 \\
\hline $\mathrm{C}$ & 3.979408000 & -1.152963000 & -0.996554000 \\
\hline $\mathrm{H}$ & 2.899869000 & -0.432612000 & -2.699981000 \\
\hline $\mathrm{H}$ & 0.685234000 & -0.731919000 & -1.678144000 \\
\hline $\mathrm{H}$ & 2.545614000 & -2.271726000 & 1.871002000 \\
\hline $\mathrm{H}$ & 4.750252000 & -1.940503000 & 0.864422000 \\
\hline $\mathrm{C}$ & 0.132653000 & -1.683860000 & 0.837558000 \\
\hline $\mathrm{C}$ & -1.090293000 & -1.539352000 & 0.235718000 \\
\hline $\mathrm{H}$ & -1.067271000 & -1.491202000 & -0.852363000 \\
\hline $\mathrm{O}$ & 0.194590000 & -1.872440000 & 2.229363000 \\
\hline $\mathrm{C}$ & -2.344620000 & -1.361914000 & 0.847193000 \\
\hline $\mathrm{H}$ & -2.404144000 & -1.348749000 & 1.934816000 \\
\hline $\mathrm{C}$ & -3.594193000 & -1.217146000 & 0.139055000 \\
\hline $\mathrm{C}$ & -4.749955000 & -0.772363000 & 0.826293000 \\
\hline $\mathrm{C}$ & -3.748495000 & -1.438237000 & -1.251027000 \\
\hline $\mathrm{C}$ & -5.949807000 & -0.536910000 & 0.174474000 \\
\hline $\mathrm{H}$ & -4.680750000 & -0.601604000 & 1.899386000 \\
\hline $\mathrm{C}$ & -4.950844000 & -1.188622000 & -1.901053000 \\
\hline $\mathrm{H}$ & -2.917143000 & -1.838490000 & -1.825932000 \\
\hline $\mathrm{C}$ & -6.066175000 & -0.729910000 & -1.203364000 \\
\hline $\mathrm{H}$ & -6.808142000 & -0.193489000 & 0.747641000 \\
\hline $\mathrm{H}$ & -5.022164000 & -1.374203000 & -2.970697000 \\
\hline $\mathrm{H}$ & -7.006376000 & -0.545230000 & -1.713931000 \\
\hline $\mathrm{H}$ & -0.475301000 & -2.535531000 & 2.445243000 \\
\hline $\mathrm{C}$ & -0.756332000 & 1.142992000 & 2.818825000 \\
\hline $\mathrm{H}$ & -1.759022000 & 1.490778000 & 2.567941000 \\
\hline $\mathrm{H}$ & -0.741493000 & 0.053610000 & 2.914483000 \\
\hline $\mathrm{H}$ & -0.457324000 & 1.599367000 & 3.767874000 \\
\hline $\mathrm{O}$ & 5.155303000 & -0.908428000 & -1.651509000 \\
\hline
\end{tabular}




\begin{tabular}{llll}
$\mathrm{C}$ & 6.346275000 & -1.262544000 & -0.995599000 \\
$\mathrm{H}$ & 7.158143000 & -1.004646000 & -1.677837000 \\
$\mathrm{H}$ & 6.476370000 & -0.707034000 & -0.055985000 \\
$\mathrm{H}$ & 6.386522000 & -2.338628000 & -0.778397000 \\
\hline
\end{tabular}

TSA2-3 ${ }_{\text {MTBD }}$

\begin{tabular}{lrcc} 
& Coordinates (Angstroms) & \\
& $\mathrm{X}$ & $\mathrm{Y}$ & $\mathrm{Z}$ \\
\hline $\mathrm{C}$ & 1.378535000 & 2.208520000 & 1.809095000 \\
$\mathrm{C}$ & 2.380648000 & 2.057824000 & 0.674710000 \\
$\mathrm{C}$ & 1.849748000 & 2.745718000 & -0.570213000 \\
$\mathrm{~N}$ & -0.006111000 & 2.162904000 & 1.344571000 \\
$\mathrm{H}$ & 1.522225000 & 3.167888000 & 2.325145000 \\
$\mathrm{H}$ & 1.513397000 & 1.403266000 & 2.540217000 \\
$\mathrm{H}$ & 2.546633000 & 1.002855000 & 0.449977000 \\
$\mathrm{H}$ & 3.338299000 & 2.494106000 & 0.972301000 \\
$\mathrm{H}$ & 1.773496000 & 3.833357000 & -0.423068000 \\
$\mathrm{H}$ & 2.519041000 & 2.563433000 & -1.414399000 \\
$\mathrm{~N}$ & 0.547414000 & 2.193764000 & -0.914082000 \\
$\mathrm{C}$ & 0.215319000 & 1.991791000 & -2.319209000 \\
$\mathrm{C}$ & -1.274489000 & 2.201149000 & -2.542198000 \\
$\mathrm{C}$ & -2.042589000 & 1.318675000 & -1.573419000 \\
$\mathrm{~N}$ & -1.601335000 & 1.527964000 & -0.199999000 \\
$\mathrm{C}$ & -0.361529000 & 1.932593000 & 0.057257000 \\
$\mathrm{H}$ & 0.520234000 & 0.985294000 & -2.636674000 \\
$\mathrm{H}$ & 0.796339000 & 2.712121000 & -2.902570000 \\
$\mathrm{H}$ & -1.523632000 & 3.254687000 & -2.371089000 \\
$\mathrm{H}$ & -1.537269000 & 1.955664000 & -3.575220000 \\
$\mathrm{H}$ & -3.116873000 & 1.516587000 & -1.615395000 \\
$\mathrm{H}$ & -1.907157000 & 0.261013000 & -1.839197000 \\
$\mathrm{H}$ & -1.956337000 & 0.619651000 & 0.442081000 \\
$\mathrm{C}$ & 2.995203000 & -1.244851000 & -1.433743000 \\
$\mathrm{C}$ & 1.741123000 & -1.194370000 & -0.853961000 \\
$\mathrm{C}$ & 1.557558000 & -1.364286000 & 0.532856000 \\
$\mathrm{C}$ & 2.708614000 & -1.567892000 & 1.302933000 \\
$\mathrm{C}$ & 3.980421000 & -1.606604000 & 0.732124000 \\
$\mathrm{C}$ & 4.132666000 & -1.446231000 & -0.643914000 \\
$\mathrm{H}$ & 3.124021000 & -1.112047000 & -2.503595000 \\
$\mathrm{H}$ & 0.880685000 & -0.992511000 & -1.485332000 \\
$\mathrm{H}$ & 2.602973000 & -1.688507000 & 2.375381000 \\
$\mathrm{C}$ & 4.838819000 & -1.765587000 & 1.375761000 \\
$\mathrm{H}$ & 0.243352000 & -1.236358000 & 1.157428000 \\
-0.948903000 & -1.303107000 & 0.518983000
\end{tabular}




$\begin{array}{lrrr}\mathrm{H} & -0.906881000 & -1.618166000 & -0.521576000 \\ \mathrm{O} & 0.308174000 & -0.938161000 & 2.519661000 \\ \mathrm{C} & -2.219347000 & -0.831767000 & 1.004994000 \\ \mathrm{H} & -2.294564000 & -0.599869000 & 2.069973000 \\ \mathrm{C} & -3.494565000 & -1.185982000 & 0.385277000 \\ \mathrm{C} & -4.694090000 & -0.678366000 & 0.928696000 \\ \mathrm{C} & -3.615522000 & -1.945946000 & -0.795585000 \\ \mathrm{C} & -5.924063000 & -0.896802000 & 0.326712000 \\ \mathrm{H} & -4.642323000 & -0.091204000 & 1.844084000 \\ \mathrm{C} & -4.849010000 & -2.155239000 & -1.403528000 \\ \mathrm{H} & -2.733055000 & -2.400093000 & -1.238176000 \\ \mathrm{C} & -6.016397000 & -1.632916000 & -0.854756000 \\ \mathrm{H} & -6.821909000 & -0.485804000 & 0.782207000 \\ \mathrm{H} & -4.896934000 & -2.748190000 & -2.314068000 \\ \mathrm{H} & -6.978132000 & -1.802816000 & -1.329309000 \\ \mathrm{H} & -0.484132000 & -1.318339000 & 2.922413000 \\ \mathrm{C} & -0.988779000 & 2.126651000 & 2.413411000 \\ \mathrm{H} & -1.985923000 & 2.292849000 & 2.007342000 \\ \mathrm{H} & -0.955910000 & 1.163866000 & 2.930090000 \\ \mathrm{H} & -0.751062000 & 2.925059000 & 3.125925000 \\ \mathrm{O} & 5.323461000 & -1.454417000 & -1.306803000 \\ \mathrm{C} & 6.489247000 & -1.683968000 & -0.553060000 \\ \mathrm{H} & 7.316930000 & -1.668809000 & -1.263690000 \\ \mathrm{H} & 6.646227000 & -0.900379000 & 0.200846000 \\ \mathrm{H}--------1\end{array}$

\begin{tabular}{|c|c|c|c|}
\hline \multicolumn{4}{|c|}{ A3 $3_{\text {MTBD }}$} \\
\hline & $\mathrm{X}$ & Y & Z \\
\hline $\mathrm{C}$ & 1.463921000 & -3.007686000 & -1.038789000 \\
\hline $\mathrm{C}$ & 2.218850000 & -2.331468000 & -2.169881000 \\
\hline $\mathrm{C}$ & 3.569112000 & -1.860323000 & -1.654818000 \\
\hline $\mathrm{N}$ & 1.275806000 & -2.049226000 & 0.029792000 \\
\hline $\mathrm{H}$ & 2.002624000 & -3.910924000 & -0.699586000 \\
\hline $\mathrm{H}$ & 0.475580000 & -3.330084000 & -1.380767000 \\
\hline $\mathrm{H}$ & 1.625078000 & -1.480668000 & -2.518815000 \\
\hline $\mathrm{H}$ & 2.363046000 & -3.014625000 & -3.012806000 \\
\hline $\mathrm{H}$ & 4.291264000 & -2.692015000 & -1.686373000 \\
\hline $\mathrm{H}$ & 3.965242000 & -1.069825000 & -2.307212000 \\
\hline $\mathrm{N}$ & 3.501490000 & -1.351901000 & -0.297620000 \\
\hline $\mathrm{C}$ & 4.673931000 & -0.600461000 & 0.119455000 \\
\hline $\mathrm{C}$ & 4.783260000 & -0.575707000 & 1.634836000 \\
\hline $\mathrm{C}$ & 3.447728000 & -0.108902000 & 2.205071000 \\
\hline $\mathrm{N}$ & 2.324812000 & -0.874316000 & 1.710609000 \\
\hline
\end{tabular}




\begin{tabular}{|c|c|c|c|}
\hline $\mathrm{C}$ & 2.396248000 & -1.391145000 & 0.532729000 \\
\hline $\mathrm{H}$ & 4.621496000 & 0.423324000 & -0.280759000 \\
\hline $\mathrm{H}$ & 5.555096000 & -1.083059000 & -0.322698000 \\
\hline $\mathrm{H}$ & 5.007559000 & -1.584250000 & 2.002265000 \\
\hline $\mathrm{H}$ & 5.601202000 & 0.085517000 & 1.940150000 \\
\hline $\mathrm{H}$ & 3.457150000 & -0.184106000 & 3.298919000 \\
\hline $\mathrm{H}$ & 3.308907000 & 0.957921000 & 1.966967000 \\
\hline $\mathrm{C}$ & -5.150391000 & 1.381226000 & -0.611491000 \\
\hline $\mathrm{C}$ & -3.890300000 & 1.620652000 & -0.095797000 \\
\hline $\mathrm{C}$ & -3.191213000 & 0.623928000 & 0.600223000 \\
\hline $\mathrm{C}$ & -3.813314000 & -0.613330000 & 0.773118000 \\
\hline $\mathrm{C}$ & -5.086300000 & -0.867301000 & 0.265781000 \\
\hline $\mathrm{C}$ & -5.760622000 & 0.133909000 & -0.432899000 \\
\hline $\mathrm{H}$ & -5.695403000 & 2.152545000 & -1.145592000 \\
\hline $\mathrm{H}$ & -3.438162000 & 2.600466000 & -0.215857000 \\
\hline $\mathrm{H}$ & -3.285894000 & -1.412635000 & 1.288164000 \\
\hline $\mathrm{H}$ & -5.529495000 & -1.845119000 & 0.414995000 \\
\hline $\mathrm{C}$ & -1.834295000 & 0.864557000 & 1.140913000 \\
\hline $\mathrm{C}$ & -0.893106000 & 1.581834000 & 0.518649000 \\
\hline $\mathrm{H}$ & -1.138161000 & 1.995938000 & -0.455244000 \\
\hline $\mathrm{O}$ & -1.539411000 & 0.248489000 & 2.331542000 \\
\hline $\mathrm{C}$ & 0.487791000 & 1.830938000 & 1.061015000 \\
\hline $\mathrm{H}$ & 0.794796000 & 0.978936000 & 1.681987000 \\
\hline $\mathrm{C}$ & 1.527875000 & 2.029525000 & -0.019296000 \\
\hline $\mathrm{C}$ & 2.538672000 & 2.982690000 & 0.125048000 \\
\hline $\mathrm{C}$ & 1.516849000 & 1.249185000 & -1.178131000 \\
\hline $\mathrm{C}$ & 3.511680000 & 3.155096000 & -0.856219000 \\
\hline $\mathrm{H}$ & 2.560956000 & 3.601561000 & 1.019390000 \\
\hline $\mathrm{C}$ & 2.480209000 & 1.423822000 & -2.166093000 \\
\hline $\mathrm{H}$ & 0.752058000 & 0.485053000 & -1.289814000 \\
\hline $\mathrm{C}$ & 3.483235000 & 2.377916000 & -2.010833000 \\
\hline $\mathrm{H}$ & 4.286137000 & 3.905344000 & -0.722760000 \\
\hline $\mathrm{H}$ & 2.446612000 & 0.813366000 & -3.065259000 \\
\hline $\mathrm{H}$ & 4.233629000 & 2.516225000 & -2.784015000 \\
\hline $\mathrm{H}$ & -2.365835000 & 0.051029000 & 2.788177000 \\
\hline $\mathrm{H}$ & 0.480923000 & 2.712126000 & 1.718307000 \\
\hline $\mathrm{C}$ & 0.200405000 & -2.328803000 & 0.960805000 \\
\hline $\mathrm{H}$ & 0.427753000 & -3.185478000 & 1.614365000 \\
\hline $\mathrm{H}$ & 0.021644000 & -1.458865000 & 1.589770000 \\
\hline $\mathrm{H}$ & -0.705088000 & -2.550896000 & 0.385586000 \\
\hline $\mathrm{O}$ & -7.000063000 & 0.002212000 & -0.968361000 \\
\hline $\mathrm{C}$ & -7.669272000 & -1.226075000 & -0.794600000 \\
\hline $\mathrm{H}$ & -8.637471000 & -1.112622000 & -1.283500000 \\
\hline $\mathrm{H}$ & -7.122656000 & -2.054264000 & -1.263914000 \\
\hline
\end{tabular}


(3) $\mathrm{R}_{1}=\mathrm{Me}, \mathrm{R}_{2}=\mathrm{H}$

\begin{tabular}{|c|c|c|c|}
\hline \multicolumn{4}{|c|}{ А1 кон } \\
\hline & \multicolumn{3}{|c|}{ Coordinates (Angstroms) } \\
\hline & $X$ & $\mathrm{Y}$ & Z \\
\hline $\mathrm{C}$ & -2.182771000 & -2.606113000 & -0.714810000 \\
\hline $\mathrm{C}$ & -1.212960000 & -1.615271000 & -0.610095000 \\
\hline $\mathrm{C}$ & -1.479710000 & -0.433052000 & 0.084519000 \\
\hline $\mathrm{C}$ & -2.728442000 & -0.263812000 & 0.689704000 \\
\hline $\mathrm{C}$ & -3.690550000 & -1.264571000 & 0.580275000 \\
\hline $\mathrm{C}$ & -3.437607000 & -2.446274000 & -0.120430000 \\
\hline $\mathrm{H}$ & -1.960981000 & -3.522258000 & -1.258018000 \\
\hline $\mathrm{H}$ & -0.234956000 & -1.767304000 & -1.064225000 \\
\hline $\mathrm{H}$ & -2.901334000 & 0.675371000 & 1.235551000 \\
\hline $\mathrm{H}$ & -4.659772000 & -1.126723000 & 1.055861000 \\
\hline $\mathrm{C}$ & -0.466152000 & 0.697863000 & 0.134245000 \\
\hline $\mathrm{C}$ & 0.926509000 & 0.211395000 & 0.408190000 \\
\hline $\mathrm{H}$ & 1.001330000 & -0.454521000 & 1.266488000 \\
\hline $\mathrm{O}$ & -0.534805000 & 1.461387000 & -1.093031000 \\
\hline $\mathrm{C}$ & 2.008515000 & 0.526191000 & -0.308846000 \\
\hline $\mathrm{H}$ & 1.880448000 & 1.193703000 & -1.159817000 \\
\hline $\mathrm{C}$ & 3.386532000 & 0.060447000 & -0.074300000 \\
\hline $\mathrm{C}$ & 4.358490000 & 0.297996000 & -1.053766000 \\
\hline $\mathrm{C}$ & 3.778504000 & -0.615601000 & 1.089426000 \\
\hline $\mathrm{C}$ & 5.670303000 & -0.133848000 & -0.888622000 \\
\hline $\mathrm{H}$ & 4.077279000 & 0.827626000 & -1.961159000 \\
\hline $\mathrm{C}$ & 5.087274000 & -1.048440000 & 1.256763000 \\
\hline $\mathrm{H}$ & 3.056015000 & -0.795750000 & 1.879801000 \\
\hline $\mathrm{C}$ & 6.040538000 & -0.812533000 & 0.267906000 \\
\hline $\mathrm{H}$ & 6.404441000 & 0.061902000 & -1.664947000 \\
\hline $\mathrm{H}$ & 5.367220000 & -1.568373000 & 2.168488000 \\
\hline $\mathrm{H}$ & 7.063886000 & -1.149985000 & 0.402482000 \\
\hline $\mathrm{H}$ & -0.806964000 & 1.411644000 & 0.912134000 \\
\hline $\mathrm{H}$ & -0.380735000 & 0.837280000 & -1.815197000 \\
\hline $\mathrm{O}$ & -2.283437000 & 2.592111000 & 1.688553000 \\
\hline $\mathrm{H}$ & -2.172618000 & 2.453733000 & 2.632633000 \\
\hline $\mathrm{K}$ & -2.549599000 & 3.151287000 & -0.520883000 \\
\hline $\mathrm{C}$ & -4.475884000 & -3.537336000 & -0.209064000 \\
\hline $\mathrm{H}$ & -4.433100000 & -4.052913000 & -1.173945000 \\
\hline $\mathrm{H}$ & -4.321089000 & -4.291630000 & 0.572059000 \\
\hline $\mathrm{H}$ & -5.486295000 & -3.136475000 & -0.082147000 \\
\hline
\end{tabular}


TSA1-2

\begin{tabular}{lccc} 
& Coordinates (Angstroms) & \\
& $\mathrm{X}$ & $\mathrm{Y}$ & $\mathrm{Z}$ \\
\hline $\mathrm{C}$ & -3.417370000 & -2.106158000 & 0.012535000 \\
$\mathrm{C}$ & -2.169301000 & -1.490120000 & -0.063793000 \\
$\mathrm{C}$ & -2.056551000 & -0.099964000 & -0.134903000 \\
$\mathrm{C}$ & -3.242273000 & 0.650773000 & -0.147161000 \\
$\mathrm{C}$ & -4.481748000 & 0.033179000 & -0.090569000 \\
$\mathrm{C}$ & -4.593338000 & -1.359746000 & -0.001504000 \\
$\mathrm{H}$ & -3.473945000 & -3.191097000 & 0.071130000 \\
$\mathrm{H}$ & -1.272558000 & -2.104497000 & -0.079042000 \\
$\mathrm{H}$ & -3.167520000 & 1.734210000 & -0.188696000 \\
$\mathrm{H}$ & -5.385242000 & 0.639928000 & -0.102934000 \\
$\mathrm{C}$ & -0.752289000 & 0.627686000 & -0.180276000 \\
$\mathrm{C}$ & 0.472413000 & -0.123868000 & 0.067914000 \\
$\mathrm{H}$ & 0.434685000 & -0.759356000 & 0.953572000 \\
$\mathrm{O}$ & -0.654494000 & 1.483895000 & -1.341269000 \\
$\mathrm{C}$ & 1.643580000 & -0.004746000 & -0.599883000 \\
$\mathrm{H}$ & 1.647610000 & 0.560437000 & -1.533907000 \\
$\mathrm{C}$ & 2.922430000 & -0.627317000 & -0.234725000 \\
$\mathrm{C}$ & 3.952103000 & -0.697968000 & -1.185556000 \\
$\mathrm{C}$ & 3.180801000 & -1.153187000 & 1.041604000 \\
$\mathrm{C}$ & 5.176224000 & -1.285136000 & -0.887005000 \\
$\mathrm{H}$ & 3.778935000 & -0.295074000 & -2.181480000 \\
$\mathrm{C}$ & 4.401328000 & -1.746551000 & 1.338925000 \\
$\mathrm{H}$ & 2.420261000 & -1.088026000 & 1.814684000 \\
$\mathrm{C}$ & 5.407568000 & -1.818536000 & 0.377837000 \\
$\mathrm{H}$ & 5.950729000 & -1.329943000 & -1.647650000 \\
$\mathrm{H}$ & 4.570754000 & -2.150472000 & 2.333310000 \\
$\mathrm{H}$ & 6.361588000 & -2.280159000 & 0.613874000 \\
$\mathrm{H}$ & -0.831679000 & 1.544373000 & 0.806672000 \\
$\mathrm{H}$ & -0.718274000 & 0.924216000 & -2.128430000 \\
$\mathrm{O}$ & -0.804669000 & 2.594094000 & 1.539549000 \\
$\mathrm{H}$ & -1.570776000 & 2.526179000 & 2.115673000 \\
$\mathrm{H}$ & 0.992307000 & 3.082058000 & 0.039641000 \\
$\mathrm{C}$ & -5.945798000 & -2.020713000 & 0.094348000 \\
$\mathrm{H}$ & -5.871567000 & -3.102274000 & -0.055199000 \\
$\mathrm{H}$ & -6.401010000 & -1.852915000 & 1.077995000 \\
-6.639195000 & -1.624307000 & -0.655712000 \\
\hline
\end{tabular}

A2 


\begin{tabular}{lrcc} 
& \multicolumn{3}{c}{ Coordinates (Angstroms) } \\
& $\mathrm{X}$ & $\mathrm{Y}$ & $\mathrm{Z}$ \\
\hline $\mathrm{C}$ & -3.395459000 & -1.706391000 & 0.678409000 \\
$\mathrm{C}$ & -2.126600000 & -1.326900000 & 0.276369000 \\
$\mathrm{C}$ & -1.926568000 & -0.276819000 & -0.657088000 \\
$\mathrm{C}$ & -3.095724000 & 0.374856000 & -1.116585000 \\
$\mathrm{C}$ & -4.362914000 & -0.016455000 & -0.691260000 \\
$\mathrm{C}$ & -4.548972000 & -1.062821000 & 0.209685000 \\
$\mathrm{H}$ & -3.495912000 & -2.523394000 & 1.390906000 \\
$\mathrm{H}$ & -1.270453000 & -1.850823000 & 0.691600000 \\
$\mathrm{H}$ & -2.995907000 & 1.173637000 & -1.844418000 \\
$\mathrm{H}$ & -5.232107000 & 0.507042000 & -1.086083000 \\
$\mathrm{C}$ & -0.619001000 & 0.198859000 & -1.035371000 \\
$\mathrm{C}$ & 0.608031000 & -0.292206000 & -0.626353000 \\
$\mathrm{H}$ & 0.587636000 & -1.270925000 & -0.151017000 \\
$\mathrm{O}$ & -0.640329000 & 1.470428000 & -1.663913000 \\
$\mathrm{C}$ & 1.845866000 & 0.344949000 & -0.730225000 \\
$\mathrm{H}$ & 1.891955000 & 1.321622000 & -1.212810000 \\
$\mathrm{C}$ & 3.133937000 & -0.241796000 & -0.392769000 \\
$\mathrm{C}$ & 4.293179000 & 0.562603000 & -0.422143000 \\
$\mathrm{C}$ & 3.311860000 & -1.584752000 & -0.000572000 \\
$\mathrm{C}$ & 5.540707000 & 0.065195000 & -0.073156000 \\
$\mathrm{H}$ & 4.197790000 & 1.603635000 & -0.726278000 \\
$\mathrm{C}$ & 4.560696000 & -2.077999000 & 0.354993000 \\
$\mathrm{H}$ & 2.462534000 & -2.262574000 & 0.007069000 \\
$\mathrm{C}$ & 5.689621000 & -1.261898000 & 0.326016000 \\
$\mathrm{H}$ & 6.406692000 & 0.721691000 & -0.112541000 \\
$\mathrm{H}$ & 4.654987000 & -3.121241000 & 0.646724000 \\
$\mathrm{H}$ & 6.664636000 & -1.654542000 & 0.598103000 \\
$\mathrm{H}$ & 0.013056000 & 1.451711000 & -2.375035000 \\
$\mathrm{O}$ & 1.242046000 & 1.751307000 & 1.790728000 \\
$\mathrm{H}$ & 1.944950000 & 1.677770000 & 2.444123000 \\
$\mathrm{H}$ & -1.118770000 & 2.155576000 & 0.890792000 \\
$\mathrm{H}$ & 1.555959000 & 1.213759000 & 1.016506000 \\
$\mathrm{H}$ & -5.922172000 & -1.505249000 & 0.647797000 \\
$\mathrm{H}$ & -6.202917000 & -2.458805000 & 0.183376000 \\
$\mathrm{H}$ & -6.683863000 & -1.647879000 & 1.733472000 \\
$\mathrm{H}$ & & & 0.370238000 \\
\hline
\end{tabular}

TSA2-3 ${ }_{\mathrm{KOH}}$

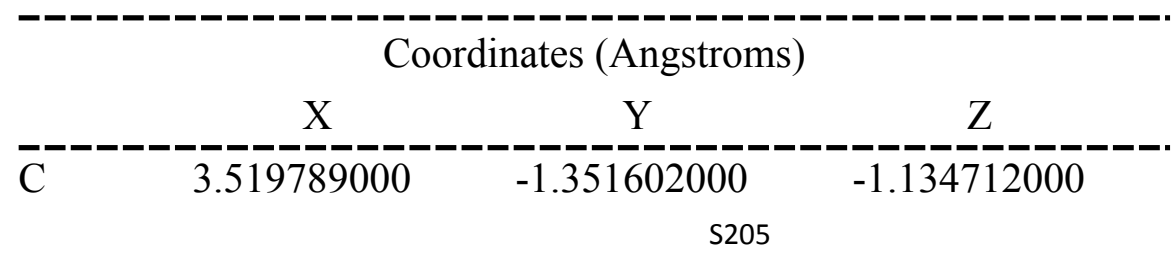




\begin{tabular}{lrcc}
$\mathrm{C}$ & 2.237965000 & -0.948018000 & -0.779908000 \\
$\mathrm{C}$ & 1.991978000 & -0.319273000 & 0.451108000 \\
$\mathrm{C}$ & 3.095616000 & -0.074850000 & 1.285220000 \\
$\mathrm{C}$ & 4.373637000 & -0.471228000 & 0.914959000 \\
$\mathrm{C}$ & 4.613151000 & -1.122792000 & -0.297830000 \\
$\mathrm{H}$ & 3.673010000 & -1.844334000 & -2.092068000 \\
$\mathrm{H}$ & 1.419359000 & -1.120339000 & -1.473315000 \\
$\mathrm{H}$ & 2.935551000 & 0.419016000 & 2.237428000 \\
$\mathrm{H}$ & 5.205006000 & -0.275904000 & 1.589139000 \\
$\mathrm{C}$ & 0.648573000 & 0.129783000 & 0.839763000 \\
$\mathrm{C}$ & -0.528821000 & -0.258899000 & 0.305257000 \\
$\mathrm{H}$ & -0.486428000 & -1.072003000 & -0.415641000 \\
$\mathrm{O}$ & 0.691743000 & 1.179366000 & 1.771038000 \\
$\mathrm{C}$ & -1.814679000 & 0.410907000 & 0.474385000 \\
$\mathrm{C}$ & -3.072215000 & -0.354447000 & 0.242189000 \\
$\mathrm{C}$ & -3.129569000 & -1.739188000 & 0.041693000 \\
$\mathrm{C}$ & -4.284078000 & 0.353918000 & 0.192620000 \\
$\mathrm{C}$ & -4.338579000 & -2.383910000 & -0.211176000 \\
$\mathrm{H}$ & -2.220203000 & -2.330950000 & 0.105118000 \\
$\mathrm{C}$ & -5.492320000 & -0.286939000 & -0.043999000 \\
$\mathrm{H}$ & -4.260526000 & 1.432667000 & 0.334517000 \\
$\mathrm{C}$ & -5.527944000 & -1.665060000 & -0.254005000 \\
$\mathrm{H}$ & -4.348809000 & -3.460480000 & -0.361813000 \\
$\mathrm{H}$ & -6.412481000 & 0.291139000 & -0.073210000 \\
$\mathrm{H}$ & -6.471060000 & -2.169161000 & -0.443699000 \\
$\mathrm{H}$ & -0.142665000 & 1.160738000 & 2.259691000 \\
$\mathrm{H}$ & -1.751111000 & 1.400361000 & -0.425596000 \\
$\mathrm{H}$ & -1.524488000 & 2.420369000 & -1.201814000 \\
$\mathrm{H}$ & 0.780985000 & 2.638837000 & -0.590490000 \\
$\mathrm{H}$ & -2.323559000 & 2.507010000 & -1.729654000 \\
$\mathrm{H}$ & -1.900532000 & 1.001856000 & 1.397353000 \\
$\mathrm{H}$ & 6.005237000 & -1.559678000 & -0.678652000 \\
$\mathrm{H}$ & 6.705056000 & -0.716274000 & -0.675459000 \\
$\mathrm{H}$ & 6.391345000 & -2.303684000 & 0.027743000 \\
6.024847000 & -2.007774000 & -1.676473000 \\
\hline$---0 .--$ &
\end{tabular}

\begin{tabular}{|c|c|c|c|}
\hline \multicolumn{4}{|c|}{$\mathbf{A} 3_{\mathrm{KOH}}$} \\
\hline & \multicolumn{3}{|c|}{ Coordinates (Angstroms) } \\
\hline & $\mathrm{X}$ & $\mathrm{Y}$ & Z \\
\hline$\overline{\mathrm{C}}$ & 4.264887000 & 0.973054000 & -0.467543000 \\
\hline $\mathrm{C}$ & 3.023476000 & 1.533681000 & -0.181493000 \\
\hline $\mathrm{C}$ & 1.945386000 & 0.734685000 & 0.216703000 \\
\hline $\mathrm{C}$ & 2.149600000 & -0.649052000 & 0.349304000 \\
\hline
\end{tabular}




\begin{tabular}{|c|c|c|c|}
\hline $\mathrm{C}$ & 3.395846000 & -1.189388000 & 0.061354000 \\
\hline $\mathrm{C}$ & 4.472770000 & -0.399709000 & -0.356053000 \\
\hline $\mathrm{H}$ & 5.084854000 & 1.620248000 & -0.770270000 \\
\hline $\mathrm{H}$ & 2.921802000 & 2.615760000 & -0.231748000 \\
\hline $\mathrm{H}$ & 1.338482000 & -1.314981000 & 0.701455000 \\
\hline $\mathrm{H}$ & 3.535275000 & -2.263476000 & 0.168205000 \\
\hline $\mathrm{C}$ & 0.620432000 & 1.351729000 & 0.485925000 \\
\hline $\mathrm{C}$ & -0.336171000 & 0.818339000 & 1.258152000 \\
\hline $\mathrm{H}$ & -0.189026000 & -0.181390000 & 1.680837000 \\
\hline $\mathrm{O}$ & 0.398675000 & 2.584448000 & -0.092049000 \\
\hline $\mathrm{C}$ & -1.682701000 & 1.473018000 & 1.465198000 \\
\hline $\mathrm{H}$ & -1.615287000 & 2.550615000 & 1.289991000 \\
\hline $\mathrm{C}$ & -2.706767000 & 0.867345000 & 0.526553000 \\
\hline $\mathrm{C}$ & -2.680580000 & 1.192564000 & -0.836976000 \\
\hline $\mathrm{C}$ & -3.618211000 & -0.097743000 & 0.962638000 \\
\hline $\mathrm{C}$ & -3.533980000 & 0.562604000 & -1.738942000 \\
\hline $\mathrm{H}$ & -1.966280000 & 1.936550000 & -1.180312000 \\
\hline $\mathrm{C}$ & -4.473204000 & -0.734848000 & 0.061641000 \\
\hline $\mathrm{H}$ & -3.641997000 & -0.370418000 & 2.014163000 \\
\hline $\mathrm{C}$ & -4.431559000 & -0.411100000 & -1.293226000 \\
\hline $\mathrm{H}$ & -3.504328000 & 0.833137000 & -2.790991000 \\
\hline $\mathrm{H}$ & -5.172217000 & -1.484625000 & 0.421038000 \\
\hline $\mathrm{H}$ & -5.101746000 & -0.900173000 & -1.994062000 \\
\hline $\mathrm{O}$ & -0.178907000 & -2.329726000 & 1.365359000 \\
\hline $\mathrm{H}$ & -2.006190000 & 1.324784000 & 2.501077000 \\
\hline $\mathrm{H}$ & 1.020311000 & 2.691656000 & -0.821454000 \\
\hline $\mathrm{H}$ & 0.244169000 & -2.662690000 & 2.161187000 \\
\hline K & -1.295560000 & -1.734748000 & -0.552913000 \\
\hline $\mathrm{C}$ & 5.821094000 & -1.014491000 & -0.634312000 \\
\hline $\mathrm{H}$ & 6.444691000 & -0.356790000 & -1.247775000 \\
\hline $\mathrm{H}$ & 6.362033000 & -1.207860000 & 0.299863000 \\
\hline $\mathrm{H}$ & 5.721050000 & -1.972035000 & -1.155875000 \\
\hline
\end{tabular}

\begin{tabular}{|c|c|c|c|}
\hline \multicolumn{4}{|c|}{$\mathrm{A1}_{\mathrm{NaOH}}$} \\
\hline & \multicolumn{3}{|c|}{ Coordinates (Angstroms) } \\
\hline & $\mathrm{X}$ & $\mathrm{Y}$ & Z \\
\hline$\overline{\mathrm{C}}$ & -2.470953000 & -2.309029000 & -0.612887000 \\
\hline $\mathrm{C}$ & -1.442072000 & -1.378806000 & -0.521579000 \\
\hline $\mathrm{C}$ & -1.663202000 & -0.136975000 & 0.079078000 \\
\hline $\mathrm{C}$ & -2.927112000 & 0.159714000 & 0.596621000 \\
\hline $\mathrm{C}$ & -3.947697000 & -0.782393000 & 0.501830000 \\
\hline $\mathrm{C}$ & -3.739905000 & -2.025944000 & -0.099477000 \\
\hline $\mathrm{H}$ & -2.286311000 & -3.272661000 & -1.082603000 \\
\hline
\end{tabular}




\begin{tabular}{lrrr}
$\mathrm{H}$ & -0.455146000 & -1.623655000 & -0.910447000 \\
$\mathrm{H}$ & -3.069525000 & 1.144541000 & 1.061424000 \\
$\mathrm{H}$ & -4.928196000 & -0.548354000 & 0.911236000 \\
$\mathrm{C}$ & -0.578613000 & 0.922339000 & 0.119809000 \\
$\mathrm{C}$ & 0.781197000 & 0.359260000 & 0.412710000 \\
$\mathrm{H}$ & 0.813266000 & -0.266389000 & 1.303144000 \\
$\mathrm{O}$ & -0.587762000 & 1.668016000 & -1.128214000 \\
$\mathrm{C}$ & 1.877642000 & 0.564601000 & -0.320754000 \\
$\mathrm{H}$ & 1.790213000 & 1.195026000 & -1.204301000 \\
$\mathrm{C}$ & 3.222711000 & 0.018225000 & -0.068438000 \\
$\mathrm{C}$ & 4.207181000 & 0.164955000 & -1.052924000 \\
$\mathrm{C}$ & 3.569910000 & -0.647394000 & 1.115189000 \\
$\mathrm{C}$ & 5.488561000 & -0.345374000 & -0.873206000 \\
$\mathrm{H}$ & 3.960543000 & 0.684720000 & -1.975860000 \\
$\mathrm{C}$ & 4.848268000 & -1.158204000 & 1.297131000 \\
$\mathrm{H}$ & 2.837083000 & -0.759056000 & 1.908718000 \\
$\mathrm{C}$ & 5.814342000 & -1.012202000 & 0.303255000 \\
$\mathrm{H}$ & 6.233586000 & -0.220674000 & -1.653736000 \\
$\mathrm{H}$ & 5.094237000 & -1.668802000 & 2.223735000 \\
$\mathrm{H}$ & 6.813234000 & -1.412465000 & 0.448638000 \\
$\mathrm{H}$ & -0.872553000 & 1.671253000 & 0.875845000 \\
$\mathrm{H}$ & -0.552166000 & 1.017127000 & -1.841713000 \\
$\mathrm{O}$ & -2.380656000 & 3.067805000 & 1.302605000 \\
$\mathrm{H}$ & -2.555114000 & 3.466702000 & 2.156856000 \\
$\mathrm{C}$ & -4.842699000 & -3.052718000 & -0.170766000 \\
$\mathrm{H}$ & -4.834711000 & -3.581945000 & -1.129378000 \\
$\mathrm{H}$ & -4.729741000 & -3.805610000 & 0.618686000 \\
$\mathrm{H}$ & -5.826501000 & -2.590281000 & -0.046286000 \\
$\mathrm{Na}$ & -2.079108000 & 3.331239000 & -0.662967000 \\
\hline---- &
\end{tabular}

TSA1-2 ${ }_{\mathrm{NaOH}}$

$\begin{array}{lrrr} & & & \text { Coordinates (Angstroms) } \\ & \mathrm{X} & \mathrm{Y} & \mathrm{Z} \\ \mathrm{C} & -3.367818000 & -1.960025000 & 0.074698000 \\ \mathrm{C} & -2.125215000 & -1.332568000 & 0.016730000 \\ \mathrm{C} & -2.024313000 & 0.056838000 & -0.098596000 \\ \mathrm{C} & -3.217432000 & 0.791037000 & -0.180366000 \\ \mathrm{C} & -4.450772000 & 0.159320000 & -0.144245000 \\ \mathrm{C} & -4.551386000 & -1.230093000 & -0.009300000 \\ \mathrm{H} & -3.413320000 & -3.042495000 & 0.174060000 \\ \mathrm{H} & -1.224430000 & -1.939629000 & 0.052901000 \\ \mathrm{H} & -3.158778000 & 1.872658000 & -0.259226000 \\ \mathrm{H} & -5.359113000 & 0.755397000 & -0.205338000\end{array}$




\begin{tabular}{lrrr}
$\mathrm{C}$ & -0.726710000 & 0.789413000 & -0.107792000 \\
$\mathrm{C}$ & 0.514624000 & 0.055087000 & 0.085913000 \\
$\mathrm{H}$ & 0.501632000 & -0.637059000 & 0.928561000 \\
$\mathrm{O}$ & -0.635582000 & 1.733588000 & -1.204513000 \\
$\mathrm{C}$ & 1.683703000 & 0.251250000 & -0.571863000 \\
$\mathrm{H}$ & 1.669102000 & 0.854272000 & -1.482966000 \\
$\mathrm{C}$ & 2.978638000 & -0.359124000 & -0.242956000 \\
$\mathrm{C}$ & 3.997881000 & -0.380583000 & -1.206706000 \\
$\mathrm{C}$ & 3.257995000 & -0.923656000 & 1.011686000 \\
$\mathrm{C}$ & 5.234165000 & -0.957792000 & -0.940681000 \\
$\mathrm{H}$ & 3.808130000 & 0.052669000 & -2.186665000 \\
$\mathrm{C}$ & 4.490575000 & -1.507679000 & 1.276008000 \\
$\mathrm{H}$ & 2.505323000 & -0.895126000 & 1.794621000 \\
$\mathrm{C}$ & 5.486915000 & -1.530424000 & 0.302623000 \\
$\mathrm{H}$ & 6.001327000 & -0.963942000 & -1.709788000 \\
$\mathrm{H}$ & 4.677173000 & -1.941685000 & 2.254369000 \\
$\mathrm{H}$ & 6.450598000 & -1.984151000 & 0.512967000 \\
$\mathrm{H}$ & -0.733052000 & 1.712647000 & 0.917654000 \\
$\mathrm{H}$ & -0.669622000 & 1.239531000 & -2.035942000 \\
$\mathrm{O}$ & -0.538036000 & 2.774381000 & 1.558954000 \\
$\mathrm{H}$ & -0.514862000 & 2.521558000 & 2.487101000 \\
$\mathrm{C}$ & -5.897807000 & -1.909195000 & 0.021800000 \\
$\mathrm{H}$ & -6.319555000 & -2.005025000 & -0.986361000 \\
$\mathrm{H}$ & -5.826865000 & -2.915389000 & 0.446525000 \\
$\mathrm{H}$ & -6.615992000 & -1.340155000 & 0.621780000 \\
$\mathrm{Na}$ & 0.901180000 & 2.956485000 & 0.010772000 \\
\hline$------1-2$ &
\end{tabular}

A2 $2_{\mathrm{NaOH}}$

\begin{tabular}{lrrr} 
& \multicolumn{3}{c}{ Coordinates (Angstroms) } \\
& $\mathrm{X}$ & $\mathrm{Y}$ & $\mathrm{Z}$ \\
\hline $\mathrm{C}$ & 3.460218000 & 1.734540000 & 0.284983000 \\
$\mathrm{C}$ & 2.195573000 & 1.305903000 & -0.070037000 \\
$\mathrm{C}$ & 1.977312000 & 0.014935000 & -0.617498000 \\
$\mathrm{C}$ & 3.125013000 & -0.801926000 & -0.758177000 \\
$\mathrm{C}$ & 4.391374000 & -0.351180000 & -0.384070000 \\
$\mathrm{C}$ & 4.593854000 & 0.918879000 & 0.144016000 \\
$\mathrm{H}$ & 3.577483000 & 2.736850000 & 0.692968000 \\
$\mathrm{H}$ & 1.359492000 & 1.985102000 & 0.066783000 \\
$\mathrm{H}$ & 3.020597000 & -1.780495000 & -1.216344000 \\
$\mathrm{H}$ & 5.243498000 & -1.013790000 & -0.524653000 \\
$\mathrm{C}$ & 0.663361000 & -0.500476000 & -0.919998000 \\
$\mathrm{C}$ & -0.559637000 & 0.072195000 & -0.613658000 \\
$\mathrm{H}$ & -0.525519000 & 1.128564000 & -0.356346000
\end{tabular}




$\begin{array}{lrrr}\mathrm{O} & 0.675036000 & -1.896191000 & -1.206867000 \\ \mathrm{C} & -1.801249000 & -0.561622000 & -0.559096000 \\ \mathrm{H} & -1.876024000 & -1.599736000 & -0.885816000 \\ \mathrm{C} & -3.081096000 & 0.094830000 & -0.318781000 \\ \mathrm{C} & -4.262648000 & -0.674182000 & -0.317085000 \\ \mathrm{C} & -3.226627000 & 1.471726000 & -0.056303000 \\ \mathrm{C} & -5.505031000 & -0.109241000 & -0.065780000 \\ \mathrm{H} & -4.191497000 & -1.741549000 & -0.517856000 \\ \mathrm{C} & -4.470899000 & 2.035049000 & 0.196980000 \\ \mathrm{H} & -2.355207000 & 2.121170000 & -0.060380000 \\ \mathrm{C} & -5.624254000 & 1.253871000 & 0.197057000 \\ \mathrm{H} & -6.390177000 & -0.740495000 & -0.076615000 \\ \mathrm{H} & -4.540426000 & 3.102688000 & 0.390366000 \\ \mathrm{H} & -6.595042000 & 1.699367000 & 0.391433000 \\ \mathrm{H} & -0.013733000 & -2.069430000 & -1.859848000 \\ \mathrm{O} & -0.910793000 & -1.675775000 & 2.014929000 \\ \mathrm{H} & -1.237475000 & -1.185366000 & 2.776915000 \\ \mathrm{H} & -1.355000000 & -1.253870000 & 1.225947000 \\ \mathrm{C} & 5.963610000 & 1.418741000 & 0.526554000 \\ \mathrm{H} & 6.318151000 & 2.187279000 & -0.171414000 \\ \mathrm{H} & 5.965035000 & 1.866477000 & 1.527066000 \\ \mathrm{H} & 6.698790000 & 0.607852000 & 0.522439000 \\ \mathrm{Na} & 1.068170000 & -1.946103000 & 1.054202000 \\ --------1 .--1\end{array}$

TSA2-3 ${ }_{\mathrm{NaOH}}$

\begin{tabular}{lrrr} 
& \multicolumn{3}{c}{ Coordinates (Angstroms) } \\
& $\mathrm{X}$ & $\mathrm{Y}$ & $\mathrm{Z}$ \\
\hline $\mathrm{C}$ & -3.483800000 & -1.838013000 & -0.007510000 \\
$\mathrm{C}$ & -2.226664000 & -1.353753000 & -0.325764000 \\
$\mathrm{C}$ & -2.013578000 & 0.019300000 & -0.548005000 \\
$\mathrm{C}$ & -3.130773000 & 0.866796000 & -0.461629000 \\
$\mathrm{C}$ & -4.390975000 & 0.365485000 & -0.138691000 \\
$\mathrm{C}$ & -4.594777000 & -0.990644000 & 0.098153000 \\
$\mathrm{H}$ & -3.612708000 & -2.905555000 & 0.155919000 \\
$\mathrm{H}$ & -1.401897000 & -2.053462000 & -0.417244000 \\
$\mathrm{H}$ & -3.015853000 & 1.923403000 & -0.682795000 \\
$\mathrm{H}$ & -5.233395000 & 1.051674000 & -0.085467000 \\
$\mathrm{C}$ & -0.679144000 & 0.577725000 & -0.790021000 \\
$\mathrm{C}$ & 0.515555000 & -0.028581000 & -0.597997000 \\
$\mathrm{H}$ & 0.496685000 & -1.075158000 & -0.299801000 \\
$\mathrm{O}$ & -0.725305000 & 1.955973000 & -1.044844000 \\
$\mathrm{C}$ & 1.802911000 & 0.657813000 & -0.647959000 \\
$\mathrm{C}$ & 3.012983000 & -0.129046000 & -0.328420000
\end{tabular}




\begin{tabular}{lrrr}
$\mathrm{C}$ & 3.025048000 & -1.070452000 & 0.714934000 \\
$\mathrm{C}$ & 4.222981000 & 0.085019000 & -1.007849000 \\
$\mathrm{C}$ & 4.176363000 & -1.778148000 & 1.042591000 \\
$\mathrm{H}$ & 2.117065000 & -1.240951000 & 1.289997000 \\
$\mathrm{C}$ & 5.378383000 & -0.610436000 & -0.672247000 \\
$\mathrm{H}$ & 4.249279000 & 0.810102000 & -1.818417000 \\
$\mathrm{C}$ & 5.364579000 & -1.553778000 & 0.352814000 \\
$\mathrm{H}$ & 4.146772000 & -2.504471000 & 1.850979000 \\
$\mathrm{H}$ & 6.296765000 & -0.419914000 & -1.221771000 \\
$\mathrm{H}$ & 6.266161000 & -2.101015000 & 0.611712000 \\
$\mathrm{H}$ & 0.189301000 & 2.264540000 & -1.134125000 \\
$\mathrm{H}$ & 1.654709000 & 1.612092000 & 0.422792000 \\
$\mathrm{O}$ & 1.329220000 & 2.364142000 & 1.299023000 \\
$\mathrm{H}$ & 2.095781000 & 2.440175000 & 1.875322000 \\
$\mathrm{H}$ & 1.941460000 & 1.264744000 & -1.552523000 \\
$\mathrm{C}$ & -5.961189000 & -1.543865000 & 0.412849000 \\
$\mathrm{H}$ & -5.914456000 & -2.296954000 & 1.206545000 \\
$\mathrm{H}$ & -6.647809000 & -0.755361000 & 0.735456000 \\
$\mathrm{H}$ & -6.402765000 & -2.026075000 & -0.467520000 \\
$\mathrm{Na}$ & -0.742167000 & 1.945340000 & 1.356910000 \\
\hline- - & &
\end{tabular}

\begin{tabular}{|c|c|c|c|}
\hline \multicolumn{4}{|c|}{$\mathrm{A} 3_{\mathrm{NaOH}}$} \\
\hline & $X$ & $\mathrm{Y}$ & Z \\
\hline$\overline{\mathrm{C}}$ & 4.204964000 & -0.928294000 & 0.375003000 \\
\hline $\mathrm{C}$ & 2.949553000 & -1.463945000 & 0.107219000 \\
\hline $\mathrm{C}$ & 1.865427000 & -0.633960000 & -0.202877000 \\
\hline $\mathrm{C}$ & 2.070376000 & 0.753107000 & -0.259584000 \\
\hline $\mathrm{C}$ & 3.332415000 & 1.268064000 & 0.008423000 \\
\hline $\mathrm{C}$ & 4.417901000 & 0.448525000 & 0.333619000 \\
\hline $\mathrm{H}$ & 5.031199000 & -1.595541000 & 0.609214000 \\
\hline $\mathrm{H}$ & 2.837235000 & -2.546339000 & 0.099704000 \\
\hline $\mathrm{H}$ & 1.244236000 & 1.439316000 & -0.521176000 \\
\hline $\mathrm{H}$ & 3.476391000 & 2.345804000 & -0.035159000 \\
\hline $\mathrm{C}$ & 0.529944000 & -1.229084000 & -0.463332000 \\
\hline $\mathrm{C}$ & -0.416115000 & -0.689068000 & -1.245352000 \\
\hline $\mathrm{H}$ & -0.237151000 & 0.291217000 & -1.687920000 \\
\hline $\mathrm{O}$ & 0.286984000 & -2.450617000 & 0.123073000 \\
\hline $\mathrm{C}$ & -1.771379000 & -1.325688000 & -1.458471000 \\
\hline $\mathrm{H}$ & -1.715479000 & -2.405077000 & -1.290374000 \\
\hline $\mathrm{C}$ & -2.783523000 & -0.710627000 & -0.513160000 \\
\hline $\mathrm{C}$ & -2.759692000 & -1.048877000 & 0.848517000 \\
\hline $\mathrm{C}$ & -3.658857000 & 0.294293000 & -0.934445000 \\
\hline
\end{tabular}




$\begin{array}{lrrr}\mathrm{C} & -3.574460000 & -0.385696000 & 1.763335000 \\ \mathrm{H} & -2.072927000 & -1.823065000 & 1.180691000 \\ \mathrm{C} & -4.475515000 & 0.961984000 & -0.020176000 \\ \mathrm{H} & -3.676638000 & 0.579672000 & -1.982393000 \\ \mathrm{C} & -4.431749000 & 0.630006000 & 1.332248000 \\ \mathrm{H} & -3.544839000 & -0.662418000 & 2.813511000 \\ \mathrm{H} & -5.140417000 & 1.747345000 & -0.366736000 \\ \mathrm{H} & -5.066991000 & 1.148881000 & 2.043625000 \\ \mathrm{O} & -0.343320000 & 2.454461000 & -0.947283000 \\ \mathrm{H} & -2.092859000 & -1.166549000 & -2.493013000 \\ \mathrm{H} & 0.936580000 & -2.582795000 & 0.823862000 \\ \mathrm{H} & -0.085548000 & 3.330896000 & -1.241446000 \\ \mathrm{C} & 5.782281000 & 1.036316000 & 0.591364000 \\ \mathrm{H} & 6.401535000 & 0.366728000 & 1.196429000 \\ \mathrm{H} & 6.313291000 & 1.216396000 & -0.351180000 \\ \mathrm{H} & 5.709243000 & 1.996107000 & 1.112709000 \\ \mathrm{Na} & -1.414146000 & 1.493303000 & 0.464485000 \\ -\end{array}$

\begin{tabular}{|c|c|c|c|}
\hline \multicolumn{4}{|c|}{$\mathrm{A1}_{\text {LiOH }}$} \\
\hline & $\mathrm{X}$ & $\mathrm{Y}$ & Z \\
\hline$\overline{\mathrm{C}}$ & -2.641346000 & -2.063254000 & -0.460424000 \\
\hline $\mathrm{C}$ & -1.575010000 & -1.174263000 & -0.367974000 \\
\hline $\mathrm{C}$ & -1.781319000 & 0.127882000 & 0.089775000 \\
\hline $\mathrm{C}$ & -3.070263000 & 0.533263000 & 0.452567000 \\
\hline $\mathrm{C}$ & -4.126591000 & -0.364920000 & 0.356131000 \\
\hline $\mathrm{C}$ & -3.931629000 & -1.673770000 & -0.096231000 \\
\hline $\mathrm{H}$ & -2.467426000 & -3.075766000 & -0.817041000 \\
\hline $\mathrm{H}$ & -0.574184000 & -1.498609000 & -0.644928000 \\
\hline $\mathrm{H}$ & -3.213140000 & 1.564517000 & 0.789443000 \\
\hline $\mathrm{H}$ & -5.126434000 & -0.043962000 & 0.640013000 \\
\hline $\mathrm{C}$ & -0.653551000 & 1.139038000 & 0.128851000 \\
\hline $\mathrm{C}$ & 0.686720000 & 0.534562000 & 0.430524000 \\
\hline $\mathrm{H}$ & 0.707908000 & -0.035235000 & 1.357852000 \\
\hline $\mathrm{O}$ & -0.632220000 & 1.880370000 & -1.125492000 \\
\hline $\mathrm{C}$ & 1.773904000 & 0.648254000 & -0.334612000 \\
\hline $\mathrm{H}$ & 1.692924000 & 1.231670000 & -1.250166000 \\
\hline $\mathrm{C}$ & 3.101454000 & 0.061518000 & -0.080543000 \\
\hline $\mathrm{C}$ & 4.091886000 & 0.190581000 & -1.061283000 \\
\hline $\mathrm{C}$ & 3.425679000 & -0.625656000 & 1.097210000 \\
\hline $\mathrm{C}$ & 5.359485000 & -0.352954000 & -0.881461000 \\
\hline $\mathrm{H}$ & 3.861714000 & 0.724005000 & -1.980554000 \\
\hline $\mathrm{C}$ & 4.690387000 & -1.169018000 & 1.279450000 \\
\hline
\end{tabular}




\begin{tabular}{lccc}
$\mathrm{H}$ & 2.685976000 & -0.732857000 & 1.884936000 \\
$\mathrm{C}$ & 5.663770000 & -1.037016000 & 0.290688000 \\
$\mathrm{H}$ & 6.110118000 & -0.240648000 & -1.658315000 \\
$\mathrm{H}$ & 4.919593000 & -1.695580000 & 2.201328000 \\
$\mathrm{H}$ & 6.651691000 & -1.463455000 & 0.436170000 \\
$\mathrm{H}$ & -0.907780000 & 1.905925000 & 0.872450000 \\
$\mathrm{H}$ & -0.760018000 & 1.239365000 & -1.837069000 \\
$\mathrm{O}$ & -2.471680000 & 3.574167000 & 0.780312000 \\
$\mathrm{H}$ & -2.844048000 & 4.230140000 & 1.368892000 \\
$\mathrm{C}$ & -5.081577000 & -2.647501000 & -0.158402000 \\
$\mathrm{H}$ & -4.907257000 & -3.427417000 & -0.906139000 \\
$\mathrm{H}$ & -5.224108000 & -3.143913000 & 0.809033000 \\
$\mathrm{H}$ & -6.019564000 & -2.141130000 & -0.406669000 \\
$\mathrm{Li}$ & -1.782251000 & 3.402061000 & -0.734468000 \\
\hline
\end{tabular}

TSA1-2

\begin{tabular}{|c|c|c|c|}
\hline & & inates (Angstrom & \\
\hline & $X$ & $\mathrm{Y}$ & $\mathrm{Z}$ \\
\hline $\bar{C}$ & -3.404194000 & -1.769912000 & 0.205010000 \\
\hline $\mathrm{C}$ & -2.149585000 & -1.170559000 & 0.194102000 \\
\hline $\mathrm{C}$ & -2.005338000 & 0.200735000 & -0.053359000 \\
\hline $\mathrm{C}$ & -3.170949000 & 0.936409000 & -0.309725000 \\
\hline $\mathrm{C}$ & -4.418244000 & 0.326504000 & -0.316444000 \\
\hline $\mathrm{C}$ & -4.562896000 & -1.037801000 & -0.056043000 \\
\hline $\mathrm{H}$ & -3.480488000 & -2.836977000 & 0.403499000 \\
\hline $\mathrm{H}$ & -1.274103000 & -1.788966000 & 0.370521000 \\
\hline $\mathrm{H}$ & -3.087959000 & 2.002335000 & -0.497856000 \\
\hline $\mathrm{H}$ & -5.302265000 & 0.927184000 & -0.520065000 \\
\hline $\mathrm{C}$ & -0.696054000 & 0.897963000 & -0.012586000 \\
\hline $\mathrm{C}$ & 0.553408000 & 0.169204000 & 0.093530000 \\
\hline $\mathrm{H}$ & 0.564892000 & -0.618448000 & 0.846342000 \\
\hline $\mathrm{O}$ & -0.595140000 & 1.971497000 & -0.988727000 \\
\hline $\mathrm{C}$ & 1.721285000 & 0.485438000 & -0.523943000 \\
\hline $\mathrm{H}$ & 1.696912000 & 1.183052000 & -1.365228000 \\
\hline $\mathrm{C}$ & 3.032496000 & -0.121570000 & -0.258202000 \\
\hline $\mathrm{C}$ & 4.043649000 & -0.033865000 & -1.226222000 \\
\hline $\mathrm{C}$ & 3.332660000 & -0.787647000 & 0.939925000 \\
\hline $\mathrm{C}$ & 5.294019000 & -0.604258000 & -1.018750000 \\
\hline $\mathrm{H}$ & 3.836909000 & 0.478564000 & -2.163486000 \\
\hline $\mathrm{C}$ & 4.579900000 & -1.364840000 & 1.145152000 \\
\hline $\mathrm{H}$ & 2.585727000 & -0.842188000 & 1.727184000 \\
\hline $\mathrm{C}$ & 5.568661000 & -1.278182000 & 0.167900000 \\
\hline $\mathrm{H}$ & 6.055637000 & -0.525458000 & -1.789344000 \\
\hline
\end{tabular}




$\begin{array}{lccc}\mathrm{H} & 4.784531000 & -1.875748000 & 2.081815000 \\ \mathrm{H} & 6.543938000 & -1.725878000 & 0.332608000 \\ \mathrm{H} & -0.586093000 & 1.786391000 & 1.111469000 \\ \mathrm{H} & -0.559883000 & 1.586269000 & -1.876736000 \\ \mathrm{O} & -0.192124000 & 2.789729000 & 1.663291000 \\ \mathrm{H} & 0.122352000 & 2.524476000 & 2.534101000 \\ \mathrm{Li} & 0.798606000 & 2.805250000 & 0.138415000 \\ \mathrm{C} & -5.915737000 & -1.703848000 & -0.088099000 \\ \mathrm{H} & -6.101501000 & -2.178549000 & -1.059541000 \\ \mathrm{H} & -5.995499000 & -2.483656000 & 0.676482000 \\ \mathrm{H} & -6.719309000 & -0.980600000 & 0.083005000\end{array}$

\begin{tabular}{|c|c|c|c|}
\hline \multicolumn{4}{|c|}{ A2 ${ }_{\mathrm{LiOH}}$} \\
\hline & $X$ & $\mathrm{Y}$ & Z \\
\hline$\overline{\mathrm{C}}$ & 3.526248000 & 1.645252000 & 0.011180000 \\
\hline $\mathrm{C}$ & 2.263179000 & 1.176249000 & -0.290751000 \\
\hline $\mathrm{C}$ & 2.025878000 & -0.201114000 & -0.527728000 \\
\hline $\mathrm{C}$ & 3.146652000 & -1.057146000 & -0.431878000 \\
\hline $\mathrm{C}$ & 4.413596000 & -0.560703000 & -0.118379000 \\
\hline $\mathrm{C}$ & 4.636152000 & 0.790592000 & 0.112175000 \\
\hline $\mathrm{H}$ & 3.663927000 & 2.712382000 & 0.173906000 \\
\hline $\mathrm{H}$ & 1.446171000 & 1.887681000 & -0.363754000 \\
\hline $\mathrm{H}$ & 3.032566000 & -2.111454000 & -0.662821000 \\
\hline $\mathrm{H}$ & 5.247938000 & -1.257414000 & -0.068553000 \\
\hline $\mathrm{C}$ & 0.703780000 & -0.741240000 & -0.758099000 \\
\hline $\mathrm{C}$ & -0.518384000 & -0.120741000 & -0.549908000 \\
\hline $\mathrm{H}$ & -0.472929000 & 0.962127000 & -0.459846000 \\
\hline $\mathrm{O}$ & 0.713439000 & -2.172298000 & -0.777944000 \\
\hline $\mathrm{C}$ & -1.762739000 & -0.727106000 & -0.382518000 \\
\hline $\mathrm{H}$ & -1.865178000 & -1.794000000 & -0.589449000 \\
\hline $\mathrm{C}$ & -3.034075000 & -0.023150000 & -0.227924000 \\
\hline $\mathrm{C}$ & -4.235674000 & -0.756451000 & -0.252578000 \\
\hline $\mathrm{C}$ & -3.144624000 & 1.365525000 & -0.023544000 \\
\hline $\mathrm{C}$ & -5.470402000 & -0.144446000 & -0.085276000 \\
\hline $\mathrm{H}$ & -4.189728000 & -1.832310000 & -0.409697000 \\
\hline $\mathrm{C}$ & -4.380898000 & 1.977370000 & 0.140333000 \\
\hline $\mathrm{H}$ & -2.251268000 & 1.983924000 & 0.008644000 \\
\hline $\mathrm{C}$ & -5.556997000 & 1.231517000 & 0.112803000 \\
\hline $\mathrm{H}$ & -6.374055000 & -0.747947000 & -0.113222000 \\
\hline $\mathrm{H}$ & -4.425197000 & 3.052817000 & 0.292675000 \\
\hline $\mathrm{H}$ & -6.521106000 & 1.713446000 & 0.242546000 \\
\hline $\mathrm{H}$ & -0.047120000 & -2.473412000 & -1.289074000 \\
\hline
\end{tabular}




$\begin{array}{lrcc}\mathrm{O} & -0.594204000 & -1.596954000 & 2.151378000 \\ \mathrm{H} & -0.715898000 & -0.985348000 & 2.887138000 \\ \mathrm{H} & -1.178326000 & -1.247880000 & 1.412783000 \\ \mathrm{C} & 6.002356000 & 1.333291000 & 0.445016000 \\ \mathrm{H} & 6.330174000 & 2.072425000 & -0.295365000 \\ \mathrm{H} & 6.010752000 & 1.829693000 & 1.422778000 \\ \mathrm{H} & 6.751209000 & 0.535835000 & 0.471021000 \\ \mathrm{Li} & 0.973296000 & -1.757583000 & 1.105094000\end{array}$

TSA2-3 ${ }_{\text {LiOH }}$

\begin{tabular}{lrcc} 
& & Coordinates (Angstroms) & \\
& $\mathrm{X}$ & $\mathrm{Y}$ & $\mathrm{Z}$ \\
\hline $\mathrm{C}$ & -3.567177000 & -1.584641000 & 0.377608000 \\
$\mathrm{C}$ & -2.292097000 & -1.054381000 & 0.269267000 \\
$\mathrm{C}$ & -2.084151000 & 0.229377000 & -0.265170000 \\
$\mathrm{C}$ & -3.214782000 & 0.958201000 & -0.657979000 \\
$\mathrm{C}$ & -4.491593000 & 0.418217000 & -0.535225000 \\
$\mathrm{C}$ & -4.696124000 & -0.860789000 & -0.022364000 \\
$\mathrm{H}$ & -3.692636000 & -2.580857000 & 0.795844000 \\
$\mathrm{H}$ & -1.446929000 & -1.643353000 & 0.613522000 \\
$\mathrm{H}$ & -3.088612000 & 1.951566000 & -1.074202000 \\
$\mathrm{H}$ & -5.347335000 & 1.009891000 & -0.852368000 \\
$\mathrm{C}$ & -0.740978000 & 0.807900000 & -0.375901000 \\
$\mathrm{C}$ & 0.464488000 & 0.191874000 & -0.266300000 \\
$\mathrm{H}$ & 0.433007000 & -0.886741000 & -0.127541000 \\
$\mathrm{O}$ & -0.764239000 & 2.223664000 & -0.519608000 \\
$\mathrm{C}$ & 1.761061000 & 0.833489000 & -0.245492000 \\
$\mathrm{H}$ & 1.864418000 & 1.693637000 & -0.919726000 \\
$\mathrm{C}$ & 2.975175000 & -0.009137000 & -0.230162000 \\
$\mathrm{C}$ & 4.136186000 & 0.389935000 & -0.910752000 \\
$\mathrm{C}$ & 3.046132000 & -1.200696000 & 0.510881000 \\
$\mathrm{C}$ & 5.302141000 & -0.365001000 & -0.864934000 \\
$\mathrm{H}$ & 4.116826000 & 1.310817000 & -1.489803000 \\
$\mathrm{C}$ & 4.207530000 & -1.964377000 & 0.546602000 \\
$\mathrm{H}$ & 2.181286000 & -1.530390000 & 1.083060000 \\
$\mathrm{C}$ & 5.346659000 & -1.553575000 & -0.140348000 \\
$\mathrm{H}$ & 6.181170000 & -0.025184000 & -1.406366000 \\
$\mathrm{H}$ & 4.224537000 & -2.884592000 & 1.125118000 \\
$\mathrm{H}$ & 6.255617000 & -2.146744000 & -0.107641000 \\
$\mathrm{H}$ & -0.014583000 & 2.487168000 & -1.071199000 \\
$\mathrm{O}$ & 1.358845000 & 2.224754000 & 1.949439000 \\
$\mathrm{H}$ & 1.797452000 & 1.864080000 & 2.727279000 \\
$\mathrm{H}$ & 1.691040000 & 1.581119000 & 1.036376000 \\
& & & \\
$\mathrm{H}$ & &
\end{tabular}




$\begin{array}{llll}\mathrm{C} & -6.076045000 & -1.457065000 & 0.090577000 \\ \mathrm{H} & -6.240619000 & -2.220079000 & -0.679789000 \\ \mathrm{H} & -6.223975000 & -1.939534000 & 1.062766000 \\ \mathrm{H} & -6.851269000 & -0.694152000 & -0.028550000 \\ \mathrm{Li} & -0.351601000 & 2.130001000 & 1.397579000\end{array}$

\begin{tabular}{|c|c|c|c|}
\hline \multicolumn{4}{|c|}{ Coordinates (Angstroms) } \\
\hline & $\mathrm{X}$ & Y & Z \\
\hline $\mathrm{C}$ & 4.146208000 & -0.848051000 & 0.282485000 \\
\hline $\mathrm{C}$ & 2.875291000 & -1.394960000 & 0.154693000 \\
\hline $\mathrm{C}$ & 1.770078000 & -0.574234000 & -0.102693000 \\
\hline $\mathrm{C}$ & 1.956849000 & 0.807873000 & -0.224267000 \\
\hline $\mathrm{C}$ & 3.236834000 & 1.335434000 & -0.095455000 \\
\hline $\mathrm{C}$ & 4.348556000 & 0.527337000 & 0.156332000 \\
\hline $\mathrm{H}$ & 4.993440000 & -1.501772000 & 0.475600000 \\
\hline $\mathrm{H}$ & 2.751108000 & -2.473775000 & 0.223476000 \\
\hline $\mathrm{H}$ & 1.101269000 & 1.481848000 & -0.368977000 \\
\hline $\mathrm{H}$ & 3.369576000 & 2.411497000 & -0.180614000 \\
\hline $\mathrm{C}$ & 0.427380000 & -1.189011000 & -0.237996000 \\
\hline $\mathrm{C}$ & -0.483163000 & -0.849907000 & -1.163751000 \\
\hline $\mathrm{H}$ & -0.229016000 & -0.055889000 & -1.859233000 \\
\hline $\mathrm{O}$ & 0.147853000 & -2.220762000 & 0.620102000 \\
\hline $\mathrm{C}$ & -1.843482000 & -1.502630000 & -1.279285000 \\
\hline $\mathrm{H}$ & -1.803726000 & -2.525089000 & -0.893029000 \\
\hline $\mathrm{C}$ & -2.859701000 & -0.693970000 & -0.499152000 \\
\hline $\mathrm{C}$ & -2.984068000 & -0.875769000 & 0.886194000 \\
\hline $\mathrm{C}$ & -3.554877000 & 0.360561000 & -1.100493000 \\
\hline $\mathrm{C}$ & -3.773776000 & -0.017001000 & 1.648059000 \\
\hline $\mathrm{H}$ & -2.432415000 & -1.681701000 & 1.362030000 \\
\hline $\mathrm{C}$ & -4.345249000 & 1.220670000 & -0.338355000 \\
\hline $\mathrm{H}$ & -3.440855000 & 0.534709000 & -2.166795000 \\
\hline $\mathrm{C}$ & -4.453420000 & 1.037663000 & 1.037530000 \\
\hline $\mathrm{H}$ & -3.855265000 & -0.168002000 & 2.720271000 \\
\hline $\mathrm{H}$ & -4.856487000 & 2.048004000 & -0.819867000 \\
\hline $\mathrm{H}$ & -5.057849000 & 1.714135000 & 1.633131000 \\
\hline $\mathrm{O}$ & -0.598553000 & 2.517614000 & -0.236522000 \\
\hline $\mathrm{H}$ & -2.137663000 & -1.557302000 & -2.332386000 \\
\hline $\mathrm{H}$ & 0.815619000 & -2.219218000 & 1.317461000 \\
\hline $\mathrm{H}$ & -0.597004000 & 3.456584000 & -0.049862000 \\
\hline $\mathrm{C}$ & 5.729003000 & 1.123134000 & 0.269499000 \\
\hline $\mathrm{H}$ & 6.393936000 & 0.485222000 & 0.859919000 \\
\hline $\mathrm{H}$ & 6.183562000 & 1.247720000 & -0.720795000 \\
\hline
\end{tabular}




\begin{tabular}{lrcc}
$\mathrm{H}$ & 5.697342000 & 2.110334000 & 0.740904000 \\
$\mathrm{Li}$ & $\mathbf{- 1 . 4 8} 1581000$ & 1.171682000 & 0.210020000 \\
\hline
\end{tabular}

\begin{tabular}{|c|c|c|c|}
\hline \multicolumn{4}{|c|}{ A1 $1_{\text {tBuOK }}$} \\
\hline & \multicolumn{3}{|c|}{ Coordinates (Angstroms) } \\
\hline & $\mathrm{X}$ & Y & Z \\
\hline$\overline{\mathrm{C}}$ & -1.051249000 & 3.704439000 & -0.198910000 \\
\hline $\mathrm{C}$ & -0.281780000 & 2.594660000 & 0.132951000 \\
\hline $\mathrm{C}$ & -0.853602000 & 1.321220000 & 0.165532000 \\
\hline $\mathrm{C}$ & -2.206716000 & 1.175005000 & -0.151149000 \\
\hline $\mathrm{C}$ & -2.967437000 & 2.291544000 & -0.485282000 \\
\hline $\mathrm{C}$ & -2.405886000 & 3.570253000 & -0.515277000 \\
\hline $\mathrm{H}$ & -0.590696000 & 4.689498000 & -0.224271000 \\
\hline $\mathrm{H}$ & 0.777430000 & 2.717593000 & 0.352398000 \\
\hline $\mathrm{H}$ & -2.627679000 & 0.164559000 & -0.150427000 \\
\hline $\mathrm{H}$ & -4.018003000 & 2.163963000 & -0.738093000 \\
\hline $\mathrm{C}$ & -0.052821000 & 0.107992000 & 0.601115000 \\
\hline $\mathrm{C}$ & 1.328784000 & 0.075534000 & 0.017249000 \\
\hline $\mathrm{H}$ & 1.353427000 & 0.192029000 & -1.065075000 \\
\hline $\mathrm{O}$ & -0.035761000 & 0.030335000 & 2.045570000 \\
\hline $\mathrm{C}$ & 2.455787000 & -0.081974000 & 0.715291000 \\
\hline $\mathrm{H}$ & 2.378529000 & -0.185278000 & 1.796762000 \\
\hline $\mathrm{C}$ & 3.823365000 & -0.130662000 & 0.169288000 \\
\hline $\mathrm{C}$ & 4.908275000 & 0.043588000 & 1.036710000 \\
\hline $\mathrm{C}$ & 4.092170000 & -0.350909000 & -1.188186000 \\
\hline $\mathrm{C}$ & 6.216964000 & 0.020207000 & 0.566410000 \\
\hline $\mathrm{H}$ & 4.719980000 & 0.205266000 & 2.095592000 \\
\hline $\mathrm{C}$ & 5.398287000 & -0.374148000 & -1.660368000 \\
\hline $\mathrm{H}$ & 3.273425000 & -0.524120000 & -1.880426000 \\
\hline $\mathrm{C}$ & 6.467316000 & -0.186093000 & -0.786400000 \\
\hline $\mathrm{H}$ & 7.041229000 & 0.160953000 & 1.259727000 \\
\hline $\mathrm{H}$ & 5.583552000 & -0.549493000 & -2.716100000 \\
\hline $\mathrm{H}$ & 7.487427000 & -0.209118000 & -1.158103000 \\
\hline $\mathrm{H}$ & -0.613287000 & -0.789818000 & 0.289853000 \\
\hline $\mathrm{H}$ & 0.308436000 & 0.874442000 & 2.366982000 \\
\hline $\mathrm{C}$ & -3.865665000 & -2.372831000 & -1.613276000 \\
\hline $\mathrm{C}$ & -2.464741000 & -2.601251000 & -1.004724000 \\
\hline $\mathrm{H}$ & -4.631105000 & -2.724430000 & -0.910377000 \\
\hline $\mathrm{H}$ & -4.027133000 & -1.300387000 & -1.779273000 \\
\hline $\mathrm{H}$ & -4.008071000 & -2.893340000 & -2.569805000 \\
\hline $\mathrm{C}$ & -1.406373000 & -2.081882000 & -2.006107000 \\
\hline $\mathrm{C}$ & -2.250405000 & -4.118836000 & -0.814166000 \\
\hline $\mathrm{H}$ & -0.399463000 & -2.257760000 & -1.605733000 \\
\hline
\end{tabular}




$\begin{array}{lrrr}\mathrm{H} & -1.474219000 & -2.571101000 & -2.986730000 \\ \mathrm{H} & -1.531030000 & -1.001664000 & -2.152818000 \\ \mathrm{H} & -2.313373000 & -4.684232000 & -1.753625000 \\ \mathrm{H} & -1.262393000 & -4.292385000 & -0.370088000 \\ \mathrm{H} & -3.007137000 & -4.512592000 & -0.123472000 \\ \mathrm{O} & -2.355189000 & -1.950619000 & 0.199093000 \\ \mathrm{~K} & -2.259405000 & -1.411900000 & 2.462548000 \\ \mathrm{C} & -3.225516000 & 4.770677000 & -0.918759000 \\ \mathrm{H} & -2.917074000 & 5.668757000 & -0.373895000 \\ \mathrm{H} & -3.107736000 & 4.980065000 & -1.988776000 \\ \mathrm{H} & -4.291277000 & 4.607788000 & -0.731584000\end{array}$

TSA1-2 ${ }_{\text {tBuOK }}$

\begin{tabular}{|c|c|c|c|}
\hline & & inates (Angstrom & \\
\hline & $\mathrm{X}$ & $\mathrm{Y}$ & Z \\
\hline$\overline{\mathrm{C}}$ & -3.699333000 & -2.117439000 & -0.721162000 \\
\hline $\mathrm{C}$ & -2.545291000 & -1.465970000 & -1.161887000 \\
\hline $\mathrm{C}$ & -1.593026000 & -0.975476000 & -0.257298000 \\
\hline $\mathrm{C}$ & -1.859404000 & -1.161729000 & 1.111740000 \\
\hline $\mathrm{C}$ & -2.998702000 & -1.822859000 & 1.540423000 \\
\hline $\mathrm{C}$ & -3.947782000 & -2.314107000 & 0.634088000 \\
\hline $\mathrm{H}$ & -4.410624000 & -2.492096000 & -1.455329000 \\
\hline $\mathrm{H}$ & -2.367515000 & -1.357933000 & -2.229573000 \\
\hline $\mathrm{H}$ & -1.167666000 & -0.754572000 & 1.845012000 \\
\hline $\mathrm{H}$ & -3.168543000 & -1.950227000 & 2.607632000 \\
\hline $\mathrm{C}$ & -0.413278000 & -0.170915000 & -0.686356000 \\
\hline $\mathrm{C}$ & 0.893754000 & -0.643086000 & -0.237079000 \\
\hline $\mathrm{H}$ & 0.893074000 & -1.163532000 & 0.720227000 \\
\hline $\mathrm{O}$ & -0.445921000 & 0.142741000 & -2.089467000 \\
\hline $\mathrm{C}$ & 2.063426000 & -0.442000000 & -0.875649000 \\
\hline $\mathrm{H}$ & 2.057046000 & 0.140503000 & -1.796066000 \\
\hline $\mathrm{C}$ & 3.384918000 & -0.882813000 & -0.411669000 \\
\hline $\mathrm{C}$ & 4.535098000 & -0.306743000 & -0.970299000 \\
\hline $\mathrm{C}$ & 3.563977000 & -1.868172000 & 0.571477000 \\
\hline $\mathrm{C}$ & 5.807608000 & -0.680080000 & -0.553587000 \\
\hline $\mathrm{H}$ & 4.422635000 & 0.455702000 & -1.737836000 \\
\hline $\mathrm{C}$ & 4.834646000 & -2.238334000 & 0.993483000 \\
\hline $\mathrm{H}$ & 2.697971000 & -2.363706000 & 1.001179000 \\
\hline $\mathrm{C}$ & 5.965826000 & -1.646903000 & 0.435156000 \\
\hline $\mathrm{H}$ & 6.679505000 & -0.212155000 & -1.002471000 \\
\hline $\mathrm{H}$ & 4.942757000 & -3.003623000 & 1.757334000 \\
\hline $\mathrm{H}$ & 6.958509000 & -1.941637000 & 0.762601000 \\
\hline $\mathrm{H}$ & -0.620704000 & 1.077594000 & -0.098231000 \\
\hline
\end{tabular}




\begin{tabular}{lrrr}
$\mathrm{H}$ & -0.033450000 & -0.590672000 & -2.572107000 \\
$\mathrm{C}$ & -0.725015000 & 4.219529000 & 1.426236000 \\
$\mathrm{C}$ & -0.081846000 & 2.899225000 & 0.984970000 \\
$\mathrm{H}$ & -1.012910000 & 4.808742000 & 0.546696000 \\
$\mathrm{H}$ & -1.627836000 & 4.016877000 & 2.013546000 \\
$\mathrm{H}$ & -0.042347000 & 4.824284000 & 2.035109000 \\
$\mathrm{C}$ & 0.311907000 & 2.078043000 & 2.224501000 \\
$\mathrm{C}$ & 1.167689000 & 3.185919000 & 0.136768000 \\
$\mathrm{H}$ & 0.810394000 & 1.150411000 & 1.921516000 \\
$\mathrm{H}$ & 0.996735000 & 2.630701000 & 2.878621000 \\
$\mathrm{H}$ & -0.586751000 & 1.819409000 & 2.795994000 \\
$\mathrm{H}$ & 1.921791000 & 3.751756000 & 0.697241000 \\
$\mathrm{H}$ & 1.620991000 & 2.245521000 & -0.196353000 \\
$\mathrm{H}$ & 0.890024000 & 3.766558000 & -0.751573000 \\
$\mathrm{O}$ & -1.027034000 & 2.196505000 & 0.227881000 \\
$\mathrm{~K}$ & -2.554007000 & 1.714755000 & -1.598816000 \\
$\mathrm{C}$ & -5.174192000 & -3.048353000 & 1.115354000 \\
$\mathrm{H}$ & -4.934737000 & -4.083080000 & 1.389847000 \\
$\mathrm{H}$ & -5.607174000 & -2.570056000 & 2.000526000 \\
$\mathrm{H}$ & -5.947280000 & -3.085502000 & 0.341392000 \\
\hline$-\boldsymbol{- 1}$ - & &
\end{tabular}

\begin{tabular}{|c|c|c|c|}
\hline \multicolumn{4}{|c|}{$\mathrm{A} 2_{\mathrm{tBuOK}}$} \\
\hline & \multicolumn{3}{|c|}{ Coordinates (Angstroms) } \\
\hline & $\mathrm{X}$ & Y & $\mathrm{Z}$ \\
\hline $\mathrm{C}$ & -4.741473000 & -0.700637000 & -0.236635000 \\
\hline $\mathrm{C}$ & -3.540576000 & -1.178881000 & -0.755945000 \\
\hline $\mathrm{C}$ & -2.336709000 & -1.120494000 & -0.013456000 \\
\hline $\mathrm{C}$ & -2.431648000 & -0.534576000 & 1.276389000 \\
\hline $\mathrm{C}$ & -3.637134000 & -0.075318000 & 1.777127000 \\
\hline $\mathrm{C}$ & -4.825401000 & -0.139868000 & 1.035944000 \\
\hline $\mathrm{H}$ & -5.641807000 & -0.778499000 & -0.844112000 \\
\hline $\mathrm{H}$ & -3.525577000 & -1.638011000 & -1.739225000 \\
\hline $\mathrm{H}$ & -1.542184000 & -0.437770000 & 1.892397000 \\
\hline $\mathrm{H}$ & -3.657425000 & 0.360281000 & 2.774673000 \\
\hline $\mathrm{C}$ & -1.084150000 & -1.517066000 & -0.604569000 \\
\hline $\mathrm{C}$ & 0.175137000 & -1.420033000 & -0.038081000 \\
\hline $\mathrm{H}$ & 0.190146000 & -1.270668000 & 1.039605000 \\
\hline $\mathrm{O}$ & -1.157928000 & -1.717489000 & -2.007400000 \\
\hline $\mathrm{C}$ & 1.403372000 & -1.452627000 & -0.695661000 \\
\hline $\mathrm{H}$ & 1.417403000 & -1.576128000 & -1.778619000 \\
\hline $\mathrm{C}$ & 2.703608000 & -1.383063000 & -0.054728000 \\
\hline $\mathrm{C}$ & 3.861844000 & -1.196826000 & -0.841264000 \\
\hline $\mathrm{C}$ & 2.897357000 & -1.436144000 & 1.342671000 \\
\hline
\end{tabular}




\begin{tabular}{lrrr}
$\mathrm{C}$ & 5.117354000 & -1.040899000 & -0.272536000 \\
$\mathrm{H}$ & 3.755467000 & -1.156705000 & -1.923663000 \\
$\mathrm{C}$ & 4.155071000 & -1.267661000 & 1.909365000 \\
$\mathrm{H}$ & 2.051911000 & -1.629614000 & 1.997720000 \\
$\mathrm{C}$ & 5.279064000 & -1.062771000 & 1.112619000 \\
$\mathrm{H}$ & 5.979925000 & -0.893705000 & -0.917770000 \\
$\mathrm{H}$ & 4.259084000 & -1.311686000 & 2.990740000 \\
$\mathrm{H}$ & 6.260047000 & -0.935242000 & 1.559658000 \\
$\mathrm{H}$ & 1.338412000 & 0.602157000 & -0.824694000 \\
$\mathrm{H}$ & -0.601739000 & -2.477899000 & -2.219096000 \\
$\mathrm{C}$ & 0.984542000 & 1.789811000 & 1.419214000 \\
$\mathrm{C}$ & 1.500956000 & 2.352154000 & 0.091742000 \\
$\mathrm{H}$ & -0.106362000 & 1.679191000 & 1.394444000 \\
$\mathrm{H}$ & 1.432237000 & 0.809877000 & 1.615347000 \\
$\mathrm{H}$ & 1.246804000 & 2.452565000 & 2.250958000 \\
$\mathrm{C}$ & 3.029777000 & 2.396505000 & 0.084275000 \\
$\mathrm{C}$ & 0.911956000 & 3.734802000 & -0.174329000 \\
$\mathrm{H}$ & 3.392060000 & 2.774185000 & -0.877427000 \\
$\mathrm{H}$ & 3.402995000 & 3.051608000 & 0.879131000 \\
$\mathrm{H}$ & 3.447420000 & 1.396355000 & 0.242155000 \\
$\mathrm{H}$ & 1.236198000 & 4.449523000 & 0.588244000 \\
$\mathrm{H}$ & 1.233971000 & 4.104861000 & -1.153592000 \\
$\mathrm{H}$ & -0.185138000 & 3.699385000 & -0.154800000 \\
$\mathrm{O}$ & 1.043071000 & 1.529118000 & -0.988936000 \\
$\mathrm{~K}$ & -1.305223000 & 0.955844000 & -1.890979000 \\
$\mathrm{C}$ & -6.129266000 & 0.356067000 & 1.607955000 \\
$\mathrm{H}$ & -6.894913000 & 0.450206000 & 0.831082000 \\
$\mathrm{H}$ & -6.519062000 & -0.329622000 & 2.370797000 \\
$\mathrm{H}$ & -6.013861000 & 1.336436000 & 2.084738000 \\
\hline---- & &
\end{tabular}

TSA2-3 ${ }_{\text {tBuOK }}$

\begin{tabular}{llcc} 
& \multicolumn{3}{c}{ Coordinates (Angstroms) } \\
& & $\mathrm{X}$ & $\mathrm{Y}$ \\
$\mathrm{C}$ & -4.875942000 & -0.897856000 & -0.518999000 \\
$\mathrm{C}$ & -3.621044000 & -0.985877000 & -1.109521000 \\
$\mathrm{C}$ & -2.450088000 & -0.870808000 & -0.342022000 \\
$\mathrm{C}$ & -2.601259000 & -0.633131000 & 1.035243000 \\
$\mathrm{C}$ & -3.860549000 & -0.562066000 & 1.616389000 \\
$\mathrm{C}$ & -5.024141000 & -0.692943000 & 0.854393000 \\
$\mathrm{H}$ & -5.762556000 & -0.997130000 & -1.141689000 \\
$\mathrm{H}$ & -3.534572000 & -1.157137000 & -2.177097000 \\
$\mathrm{H}$ & -1.723006000 & -0.490184000 & 1.658858000 \\
$\mathrm{H}$ & -3.940335000 & -0.384427000 & 2.686427000
\end{tabular}




\begin{tabular}{|c|c|c|c|}
\hline $\mathrm{C}$ & -1.128584000 & -0.912413000 & -0.979670000 \\
\hline $\mathrm{C}$ & 0.064867000 & -1.051426000 & -0.363817000 \\
\hline $\mathrm{H}$ & 0.024343000 & -1.294573000 & 0.694354000 \\
\hline $\mathrm{O}$ & -1.192605000 & -0.592972000 & -2.346532000 \\
\hline $\mathrm{C}$ & 1.370171000 & -0.732969000 & -0.924448000 \\
\hline $\mathrm{H}$ & 1.411185000 & -0.718753000 & -2.020884000 \\
\hline $\mathrm{C}$ & 2.602131000 & -1.337281000 & -0.360783000 \\
\hline $\mathrm{C}$ & 3.828345000 & -1.096849000 & -1.005047000 \\
\hline $\mathrm{C}$ & 2.643819000 & -2.080994000 & 0.827421000 \\
\hline $\mathrm{C}$ & 5.028888000 & -1.565437000 & -0.489283000 \\
\hline $\mathrm{H}$ & 3.829586000 & -0.515889000 & -1.925195000 \\
\hline $\mathrm{C}$ & 3.847994000 & -2.545409000 & 1.350815000 \\
\hline $\mathrm{H}$ & 1.723959000 & -2.319581000 & 1.353497000 \\
\hline $\mathrm{C}$ & 5.049928000 & -2.293326000 & 0.699520000 \\
\hline $\mathrm{H}$ & 5.956499000 & -1.358167000 & -1.016491000 \\
\hline $\mathrm{H}$ & 3.840337000 & -3.118014000 & 2.274776000 \\
\hline $\mathrm{H}$ & 5.987119000 & -2.659841000 & 1.107489000 \\
\hline $\mathrm{H}$ & 1.399594000 & 0.598506000 & -0.664673000 \\
\hline $\mathrm{H}$ & -0.425452000 & -1.000249000 & -2.770999000 \\
\hline $\mathrm{C}$ & 1.478923000 & 1.477669000 & 1.861981000 \\
\hline $\mathrm{C}$ & 1.813094000 & 2.354813000 & 0.639934000 \\
\hline $\mathrm{H}$ & 0.389588000 & 1.392129000 & 1.976307000 \\
\hline $\mathrm{H}$ & 1.891200000 & 0.470672000 & 1.735419000 \\
\hline $\mathrm{H}$ & 1.888908000 & 1.895941000 & 2.789254000 \\
\hline $\mathrm{C}$ & 3.336240000 & 2.397815000 & 0.441898000 \\
\hline $\mathrm{C}$ & 1.288704000 & 3.779007000 & 0.884478000 \\
\hline $\mathrm{H}$ & 3.577643000 & 3.010187000 & -0.434131000 \\
\hline $\mathrm{H}$ & 3.851352000 & 2.817275000 & 1.315035000 \\
\hline $\mathrm{H}$ & 3.721810000 & 1.387277000 & 0.268451000 \\
\hline $\mathrm{H}$ & 1.757570000 & 4.249543000 & 1.756990000 \\
\hline $\mathrm{H}$ & 1.486034000 & 4.405273000 & 0.006577000 \\
\hline $\mathrm{H}$ & 0.203334000 & 3.760369000 & 1.062613000 \\
\hline $\mathrm{O}$ & 1.189076000 & 1.855207000 & -0.506868000 \\
\hline $\mathrm{K}$ & -1.141112000 & 1.948897000 & -1.280042000 \\
\hline $\mathrm{C}$ & -6.390070000 & -0.626857000 & 1.489177000 \\
\hline $\mathrm{H}$ & -7.092648000 & -0.061651000 & 0.867819000 \\
\hline $\mathrm{H}$ & -6.810542000 & -1.630456000 & 1.626092000 \\
\hline $\mathrm{H}$ & -6.350162000 & -0.150012000 & 2.473248000 \\
\hline
\end{tabular}

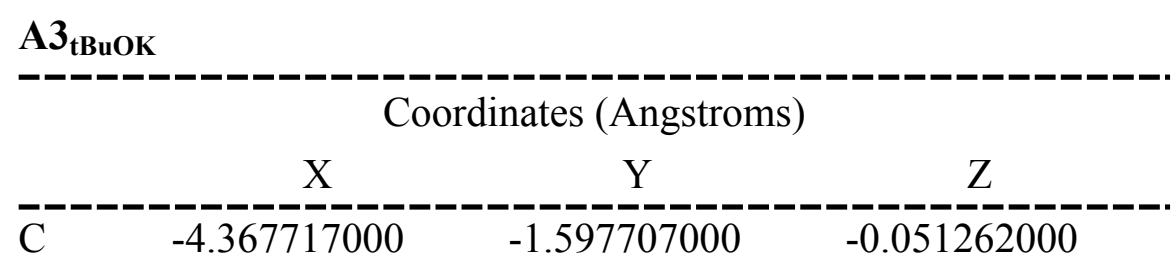




\begin{tabular}{|c|c|c|c|}
\hline $\mathrm{C}$ & -3.150771000 & -2.061021000 & 0.441839000 \\
\hline $\mathrm{C}$ & -2.010052000 & -1.251928000 & 0.416390000 \\
\hline $\mathrm{C}$ & -2.126541000 & 0.050071000 & -0.098257000 \\
\hline $\mathrm{C}$ & -3.347065000 & 0.497295000 & -0.583640000 \\
\hline $\mathrm{C}$ & -4.487940000 & -0.313413000 & -0.577215000 \\
\hline $\mathrm{H}$ & -5.239353000 & -2.246703000 & -0.011668000 \\
\hline $\mathrm{H}$ & -3.117480000 & -3.050220000 & 0.893387000 \\
\hline $\mathrm{H}$ & -1.269083000 & 0.739766000 & -0.104312000 \\
\hline $\mathrm{H}$ & -3.411286000 & 1.511157000 & -0.974076000 \\
\hline $\mathrm{C}$ & -0.712484000 & -1.766500000 & 0.926176000 \\
\hline $\mathrm{C}$ & 0.282688000 & -0.994630000 & 1.383453000 \\
\hline $\mathrm{H}$ & 0.174343000 & 0.087344000 & 1.332262000 \\
\hline $\mathrm{O}$ & -0.561884000 & -3.134158000 & 0.946117000 \\
\hline $\mathrm{C}$ & 1.613565000 & -1.528509000 & 1.852754000 \\
\hline $\mathrm{H}$ & 1.536032000 & -2.589921000 & 2.102439000 \\
\hline $\mathrm{C}$ & 2.646921000 & -1.324750000 & 0.763958000 \\
\hline $\mathrm{C}$ & 2.836360000 & -2.302107000 & -0.220733000 \\
\hline $\mathrm{C}$ & 3.332836000 & -0.112040000 & 0.643838000 \\
\hline $\mathrm{C}$ & 3.686977000 & -2.071910000 & -1.301291000 \\
\hline $\mathrm{H}$ & 2.299875000 & -3.244065000 & -0.137396000 \\
\hline $\mathrm{C}$ & 4.183551000 & 0.122689000 & -0.435134000 \\
\hline $\mathrm{H}$ & 3.168879000 & 0.668138000 & 1.381138000 \\
\hline $\mathrm{C}$ & 4.361053000 & -0.854470000 & -1.414632000 \\
\hline $\mathrm{H}$ & 3.831242000 & -2.845675000 & -2.050559000 \\
\hline $\mathrm{H}$ & 4.698546000 & 1.075333000 & -0.515277000 \\
\hline $\mathrm{H}$ & 5.029913000 & -0.675037000 & -2.251332000 \\
\hline $\mathrm{C}$ & 0.514385000 & 2.913898000 & 1.944788000 \\
\hline $\mathrm{C}$ & 0.323721000 & 3.061428000 & 0.417849000 \\
\hline $\mathrm{H}$ & -0.264519000 & 2.257640000 & 2.352513000 \\
\hline $\mathrm{H}$ & 1.488007000 & 2.449176000 & 2.148071000 \\
\hline $\mathrm{H}$ & 0.471732000 & 3.872351000 & 2.479331000 \\
\hline $\mathrm{C}$ & 1.419271000 & 4.007914000 & -0.121811000 \\
\hline $\mathrm{C}$ & -1.056669000 & 3.708270000 & 0.160844000 \\
\hline $\mathrm{H}$ & 1.287048000 & 4.142835000 & -1.202797000 \\
\hline $\mathrm{H}$ & 1.409495000 & 4.997929000 & 0.353625000 \\
\hline $\mathrm{H}$ & 2.404586000 & 3.553352000 & 0.044701000 \\
\hline $\mathrm{H}$ & -1.158039000 & 4.702243000 & 0.617026000 \\
\hline $\mathrm{H}$ & -1.218133000 & 3.805088000 & -0.920154000 \\
\hline $\mathrm{H}$ & -1.847015000 & 3.064037000 & 0.565973000 \\
\hline $\mathrm{O}$ & 0.406191000 & 1.839671000 & -0.195202000 \\
\hline $\mathrm{H}$ & 1.922061000 & -0.993973000 & 2.757998000 \\
\hline $\mathrm{K}$ & 1.046791000 & 0.063388000 & -1.571260000 \\
\hline $\mathrm{H}$ & -1.222411000 & -3.521985000 & 0.360260000 \\
\hline $\mathrm{C}$ & -5.804159000 & 0.207441000 & -1.095722000 \\
\hline
\end{tabular}




$\begin{array}{lrrr}\mathrm{H} & -6.550038000 & -0.589083000 & -1.174067000 \\ \mathrm{H} & -6.207482000 & 0.978332000 & -0.428762000 \\ \mathrm{H} & -5.687988000 & 0.663346000 & -2.084860000\end{array}$

\begin{tabular}{|c|c|c|c|}
\hline \multicolumn{4}{|c|}{ A1 $1_{\text {tBuONa }}$} \\
\hline & \multicolumn{3}{|c|}{ Coordinates (Angstroms) } \\
\hline & $\mathrm{X}$ & Y & Z \\
\hline$\overline{\mathrm{C}}$ & -0.943868000 & 3.723830000 & -0.111823000 \\
\hline $\mathrm{C}$ & -0.209288000 & 2.566761000 & 0.129630000 \\
\hline $\mathrm{C}$ & -0.839268000 & 1.321813000 & 0.145846000 \\
\hline $\mathrm{C}$ & -2.215874000 & 1.248796000 & -0.089785000 \\
\hline $\mathrm{C}$ & -2.939135000 & 2.411093000 & -0.331767000 \\
\hline $\mathrm{C}$ & -2.318859000 & 3.663990000 & -0.347737000 \\
\hline $\mathrm{H}$ & -0.439899000 & 4.687407000 & -0.123394000 \\
\hline $\mathrm{H}$ & 0.864286000 & 2.632669000 & 0.295875000 \\
\hline $\mathrm{H}$ & -2.691955000 & 0.264104000 & -0.082834000 \\
\hline $\mathrm{H}$ & -4.008512000 & 2.343152000 & -0.519833000 \\
\hline $\mathrm{C}$ & -0.081578000 & 0.056655000 & 0.495707000 \\
\hline $\mathrm{C}$ & 1.311328000 & 0.014799000 & -0.061174000 \\
\hline $\mathrm{H}$ & 1.365044000 & 0.203479000 & -1.132111000 \\
\hline $\mathrm{O}$ & -0.087683000 & -0.118133000 & 1.938144000 \\
\hline $\mathrm{C}$ & 2.416503000 & -0.227706000 & 0.646984000 \\
\hline $\mathrm{H}$ & 2.312075000 & -0.398593000 & 1.717238000 \\
\hline $\mathrm{C}$ & 3.794766000 & -0.289103000 & 0.129619000 \\
\hline $\mathrm{C}$ & 4.860265000 & -0.237531000 & 1.035906000 \\
\hline $\mathrm{C}$ & 4.092389000 & -0.405115000 & -1.234644000 \\
\hline $\mathrm{C}$ & 6.179760000 & -0.281397000 & 0.598612000 \\
\hline $\mathrm{H}$ & 4.648981000 & -0.156807000 & 2.099624000 \\
\hline $\mathrm{C}$ & 5.409384000 & -0.449307000 & -1.673874000 \\
\hline $\mathrm{H}$ & 3.287724000 & -0.479732000 & -1.960182000 \\
\hline $\mathrm{C}$ & 6.459562000 & -0.385391000 & -0.760097000 \\
\hline $\mathrm{H}$ & 6.988965000 & -0.237723000 & 1.321670000 \\
\hline $\mathrm{H}$ & 5.617258000 & -0.545110000 & -2.735548000 \\
\hline $\mathrm{H}$ & 7.488024000 & -0.425325000 & -1.106324000 \\
\hline $\mathrm{H}$ & -0.669119000 & -0.799698000 & 0.128843000 \\
\hline $\mathrm{H}$ & 0.236428000 & 0.707950000 & 2.321157000 \\
\hline $\mathrm{C}$ & -4.314662000 & -3.381334000 & -0.163247000 \\
\hline $\mathrm{C}$ & -2.978327000 & -2.719729000 & -0.556844000 \\
\hline $\mathrm{H}$ & -4.194705000 & -3.912164000 & 0.790021000 \\
\hline $\mathrm{H}$ & -5.079680000 & -2.607698000 & -0.026854000 \\
\hline $\mathrm{H}$ & -4.674225000 & -4.098067000 & -0.913267000 \\
\hline $\mathrm{C}$ & -3.162511000 & -1.995470000 & -1.907313000 \\
\hline $\mathrm{C}$ & -1.909344000 & -3.818807000 & -0.728617000 \\
\hline
\end{tabular}




$\begin{array}{lrrr}\mathrm{H} & -2.225243000 & -1.502182000 & -2.192603000 \\ \mathrm{H} & -3.455745000 & -2.677138000 & -2.716069000 \\ \mathrm{H} & -3.933658000 & -1.221273000 & -1.812690000 \\ \mathrm{H} & -2.178229000 & -4.561511000 & -1.491002000 \\ \mathrm{H} & -0.953325000 & -3.361919000 & -1.012033000 \\ \mathrm{H} & -1.763617000 & -4.341387000 & 0.225451000 \\ \mathrm{O} & -2.584246000 & -1.824572000 & 0.408514000 \\ \mathrm{Na} & -2.018410000 & -1.278186000 & 2.265526000 \\ \mathrm{C} & -3.111296000 & 4.912216000 & -0.645603000 \\ \mathrm{H} & -3.241497000 & 5.044494000 & -1.726242000 \\ \mathrm{H} & -4.110096000 & 4.863983000 & -0.200158000 \\ \mathrm{H} & -2.611026000 & 5.806403000 & -0.261448000\end{array}$

TSA1-2 ${ }_{\mathrm{tBuONa}}$

\begin{tabular}{|c|c|c|c|}
\hline \multicolumn{4}{|c|}{ Coordinates (Angstroms) } \\
\hline & $\mathrm{X}$ & $\mathrm{Y}$ & Z \\
\hline $\mathrm{C}$ & 3.032014000 & -2.244683000 & -1.183608000 \\
\hline $\mathrm{C}$ & 1.810882000 & -1.745790000 & -0.743776000 \\
\hline $\mathrm{C}$ & 1.722027000 & -0.973716000 & 0.422576000 \\
\hline $\mathrm{C}$ & 2.907046000 & -0.747949000 & 1.138019000 \\
\hline $\mathrm{C}$ & 4.120067000 & -1.264167000 & 0.700300000 \\
\hline $\mathrm{C}$ & 4.210923000 & -2.014145000 & -0.474284000 \\
\hline $\mathrm{H}$ & 3.063499000 & -2.840659000 & -2.093397000 \\
\hline $\mathrm{H}$ & 0.915604000 & -1.980091000 & -1.312091000 \\
\hline $\mathrm{H}$ & 2.866748000 & -0.152682000 & 2.045043000 \\
\hline $\mathrm{H}$ & 5.019980000 & -1.071091000 & 1.280519000 \\
\hline $\mathrm{C}$ & 0.462410000 & -0.330204000 & 0.872958000 \\
\hline $\mathrm{C}$ & -0.805696000 & -0.642506000 & 0.237723000 \\
\hline $\mathrm{H}$ & -0.780153000 & -0.643819000 & -0.851819000 \\
\hline $\mathrm{O}$ & 0.349829000 & -0.217065000 & 2.307088000 \\
\hline $\mathrm{C}$ & -2.011711000 & -0.757745000 & 0.847886000 \\
\hline $\mathrm{H}$ & -2.040134000 & -0.872622000 & 1.933957000 \\
\hline $\mathrm{C}$ & -3.308480000 & -0.842906000 & 0.162205000 \\
\hline $\mathrm{C}$ & -4.401739000 & -1.441805000 & 0.805281000 \\
\hline $\mathrm{C}$ & -3.511292000 & -0.326590000 & -1.127433000 \\
\hline $\mathrm{C}$ & -5.638664000 & -1.545202000 & 0.179630000 \\
\hline $\mathrm{H}$ & -4.269016000 & -1.849410000 & 1.805287000 \\
\hline $\mathrm{C}$ & -4.745669000 & -0.436559000 & -1.756274000 \\
\hline $\mathrm{H}$ & -2.695639000 & 0.180678000 & -1.636391000 \\
\hline $\mathrm{C}$ & -5.816977000 & -1.046892000 & -1.108184000 \\
\hline $\mathrm{H}$ & -6.466255000 & -2.021186000 & 0.698189000 \\
\hline $\mathrm{H}$ & -4.875419000 & -0.029086000 & -2.755053000 \\
\hline $\mathrm{H}$ & -6.782161000 & -1.126807000 & -1.598952000 \\
\hline
\end{tabular}




$\begin{array}{lrrr}\mathrm{H} & 0.591775000 & 1.011674000 & 0.571920000 \\ \mathrm{H} & 0.264547000 & -1.105106000 & 2.682827000 \\ \mathrm{C} & 2.582186000 & 2.861489000 & -0.364548000 \\ \mathrm{C} & 1.058454000 & 2.828476000 & -0.549338000 \\ \mathrm{H} & 2.835708000 & 3.436743000 & 0.532689000 \\ \mathrm{H} & 2.967989000 & 1.843684000 & -0.236373000 \\ \mathrm{H} & 3.086371000 & 3.316819000 & -1.225446000 \\ \mathrm{C} & 0.706777000 & 2.010962000 & -1.804518000 \\ \mathrm{C} & 0.511661000 & 4.252689000 & -0.690783000 \\ \mathrm{H} & -0.382380000 & 1.922328000 & -1.900081000 \\ \mathrm{H} & 1.096949000 & 2.481583000 & -2.714240000 \\ \mathrm{H} & 1.133545000 & 1.002905000 & -1.739201000 \\ \mathrm{H} & 0.952084000 & 4.773042000 & -1.549404000 \\ \mathrm{H} & -0.576334000 & 4.224805000 & -0.825438000 \\ \mathrm{H} & 0.733170000 & 4.829331000 & 0.214413000 \\ \mathrm{O} & 0.463993000 & 2.247749000 & 0.580178000 \\ \mathrm{Na} & -1.031058000 & 1.645452000 & 1.941017000 \\ \mathrm{C} & 5.535060000 & -2.536625000 & -0.972733000 \\ \mathrm{H} & 5.978212000 & -1.852978000 & -1.707481000 \\ \mathrm{H} & 6.254442000 & -2.649502000 & -0.155387000 \\ \mathrm{H} & 5.422246000 & -3.510774000 & -1.459996000 \\ ----- \text { - } & \end{array}$

\section{A2 ${ }_{\text {tBuONa }}$}

$\begin{array}{lrcc} & & \\ & & & \text { Coordinates }(\text { Angstroms }) \\ \text { C } & -4.643622000 & -0.457123000 & -0.461120000 \\ \mathrm{C} & -3.438911000 & -0.924435000 & -0.989037000 \\ \mathrm{C} & -2.297660000 & -1.117104000 & -0.172698000 \\ \mathrm{C} & -2.460099000 & -0.808566000 & 1.204294000 \\ \mathrm{C} & -3.664927000 & -0.356148000 & 1.705852000 \\ \mathrm{C} & -4.790004000 & -0.159541000 & 0.888743000 \\ \mathrm{H} & -5.494264000 & -0.338659000 & -1.130028000 \\ \mathrm{H} & -3.386463000 & -1.196864000 & -2.038719000 \\ \mathrm{H} & -1.627968000 & -0.936478000 & 1.890098000 \\ \mathrm{H} & -3.741402000 & -0.144250000 & 2.770775000 \\ \mathrm{C} & -1.032096000 & -1.494329000 & -0.751300000 \\ \mathrm{C} & 0.212241000 & -1.436862000 & -0.143243000 \\ \mathrm{H} & 0.191481000 & -1.377807000 & 0.942405000 \\ \mathrm{O} & -1.058445000 & -1.490858000 & -2.176366000 \\ \mathrm{C} & 1.457147000 & -1.375292000 & -0.761637000 \\ \mathrm{H} & 1.516667000 & -1.449819000 & -1.847682000 \\ \mathrm{C} & 2.734692000 & -1.263032000 & -0.077176000 \\ \mathrm{C} & 3.901693000 & -1.001504000 & -0.826499000\end{array}$




\begin{tabular}{|c|c|c|c|}
\hline $\mathrm{C}$ & 2.890764000 & -1.337851000 & 1.322836000 \\
\hline $\mathrm{C}$ & 5.133790000 & -0.804272000 & -0.219992000 \\
\hline $\mathrm{H}$ & 3.822272000 & -0.937956000 & -1.910035000 \\
\hline $\mathrm{C}$ & 4.125232000 & -1.133541000 & 1.927694000 \\
\hline $\mathrm{H}$ & 2.036498000 & -1.576406000 & 1.951069000 \\
\hline $\mathrm{C}$ & 5.259643000 & -0.860067000 & 1.167482000 \\
\hline $\mathrm{H}$ & 6.005397000 & -0.599835000 & -0.836683000 \\
\hline $\mathrm{H}$ & 4.202391000 & -1.200218000 & 3.010063000 \\
\hline $\mathrm{H}$ & 6.222197000 & -0.703264000 & 1.644224000 \\
\hline $\mathrm{H}$ & 1.198543000 & 0.612436000 & -0.967190000 \\
\hline $\mathrm{H}$ & -0.446150000 & -2.169814000 & -2.483312000 \\
\hline $\mathrm{C}$ & 0.619940000 & 1.755671000 & 1.250746000 \\
\hline $\mathrm{C}$ & 1.131592000 & 2.374948000 & -0.052514000 \\
\hline $\mathrm{H}$ & -0.446748000 & 1.509232000 & 1.175573000 \\
\hline $\mathrm{H}$ & 1.173591000 & 0.839869000 & 1.482598000 \\
\hline $\mathrm{H}$ & 0.753756000 & 2.451589000 & 2.085745000 \\
\hline $\mathrm{C}$ & 2.646077000 & 2.575166000 & 0.003301000 \\
\hline $\mathrm{C}$ & 0.410071000 & 3.685022000 & -0.351782000 \\
\hline $\mathrm{H}$ & 3.007222000 & 3.003616000 & -0.937001000 \\
\hline $\mathrm{H}$ & 2.917891000 & 3.250356000 & 0.821739000 \\
\hline $\mathrm{H}$ & 3.154395000 & 1.618308000 & 0.165880000 \\
\hline $\mathrm{H}$ & 0.619871000 & 4.429486000 & 0.422157000 \\
\hline $\mathrm{H}$ & 0.733726000 & 4.088059000 & -1.316945000 \\
\hline $\mathrm{H}$ & -0.677103000 & 3.532619000 & -0.381082000 \\
\hline $\mathrm{O}$ & 0.817282000 & 1.507220000 & -1.155585000 \\
\hline $\mathrm{Na}$ & -1.193596000 & 0.777538000 & -1.772280000 \\
\hline $\mathrm{C}$ & -6.090549000 & 0.339052000 & 1.465393000 \\
\hline $\mathrm{H}$ & -6.483332000 & -0.350775000 & 2.222081000 \\
\hline $\mathrm{H}$ & -5.969721000 & 1.315261000 & 1.950521000 \\
\hline $\mathrm{H}$ & -6.853981000 & 0.446342000 & 0.688405000 \\
\hline
\end{tabular}

TSA2-3

Coordinates (Angstroms)

$\begin{array}{llcc} & & & \\ \text { C } & 4.988043000 & -0.599589000 & \\ \text { C } & 3.721370000 & -0.661773000 & 1.215449000 \\ \text { C } & 2.569735000 & -0.657500000 & 0.414736000 \\ \text { C } & 2.745518000 & -0.563666000 & -0.976825000 \\ \text { C } & 4.014260000 & -0.515229000 & -1.535157000 \\ \text { C } & 5.162558000 & -0.527449000 & -0.737107000 \\ \text { H } & 5.861391000 & -0.614771000 & 1.293305000 \\ \text { H } & 3.615251000 & -0.732727000 & 2.292627000 \\ \text { H } & 1.877528000 & -0.520140000 & -1.629123000\end{array}$




\begin{tabular}{|c|c|c|c|}
\hline $\mathrm{H}$ & 4.116130000 & -0.449638000 & -2.616011000 \\
\hline $\mathrm{C}$ & 1.232459000 & -0.689706000 & 1.018595000 \\
\hline $\mathrm{C}$ & 0.059100000 & -0.984274000 & 0.415201000 \\
\hline $\mathrm{H}$ & 0.138893000 & -1.368633000 & -0.597807000 \\
\hline $\mathrm{O}$ & 1.233840000 & -0.182721000 & 2.337766000 \\
\hline $\mathrm{C}$ & -1.268576000 & -0.720645000 & 0.947011000 \\
\hline $\mathrm{H}$ & -1.338127000 & -0.747966000 & 2.042247000 \\
\hline $\mathrm{C}$ & -2.463732000 & -1.342003000 & 0.332544000 \\
\hline $\mathrm{C}$ & -3.663003000 & -1.384187000 & 1.064540000 \\
\hline $\mathrm{C}$ & -2.510757000 & -1.812841000 & -0.988987000 \\
\hline $\mathrm{C}$ & -4.841960000 & -1.863992000 & 0.510138000 \\
\hline $\mathrm{H}$ & -3.663859000 & -1.018333000 & 2.089283000 \\
\hline $\mathrm{C}$ & -3.691350000 & -2.295798000 & -1.546126000 \\
\hline $\mathrm{H}$ & -1.614834000 & -1.806021000 & -1.604215000 \\
\hline $\mathrm{C}$ & -4.867428000 & -2.325433000 & -0.804464000 \\
\hline $\mathrm{H}$ & -5.748663000 & -1.877990000 & 1.109402000 \\
\hline $\mathrm{H}$ & -3.687140000 & -2.653619000 & -2.572522000 \\
\hline $\mathrm{H}$ & -5.787090000 & -2.702648000 & -1.241224000 \\
\hline $\mathrm{H}$ & -1.372780000 & 0.655993000 & 0.748837000 \\
\hline $\mathrm{H}$ & 0.490909000 & -0.586543000 & 2.806925000 \\
\hline $\mathrm{C}$ & -1.362770000 & 1.739040000 & -1.725803000 \\
\hline $\mathrm{C}$ & -1.885369000 & 2.428136000 & -0.454306000 \\
\hline $\mathrm{H}$ & -0.271208000 & 1.847349000 & -1.795794000 \\
\hline $\mathrm{H}$ & -1.603344000 & 0.671031000 & -1.706193000 \\
\hline $\mathrm{H}$ & -1.804667000 & 2.169286000 & -2.632039000 \\
\hline $\mathrm{C}$ & -3.403144000 & 2.227471000 & -0.343507000 \\
\hline $\mathrm{C}$ & -1.559709000 & 3.925233000 & -0.509024000 \\
\hline $\mathrm{H}$ & -3.775503000 & 2.706008000 & 0.568460000 \\
\hline $\mathrm{H}$ & -3.929795000 & 2.657206000 & -1.203883000 \\
\hline $\mathrm{H}$ & -3.642752000 & 1.160122000 & -0.291375000 \\
\hline $\mathrm{H}$ & -2.044167000 & 4.417075000 & -1.360640000 \\
\hline $\mathrm{H}$ & -1.893158000 & 4.415327000 & 0.411950000 \\
\hline $\mathrm{H}$ & -0.475375000 & 4.077174000 & -0.609164000 \\
\hline $\mathrm{O}$ & -1.249919000 & 1.892854000 & 0.680882000 \\
\hline $\mathrm{Na}$ & 0.811457000 & 1.828603000 & 1.213577000 \\
\hline $\mathrm{C}$ & 6.535585000 & -0.426901000 & -1.351679000 \\
\hline $\mathrm{H}$ & 6.790950000 & 0.615810000 & -1.577260000 \\
\hline $\mathrm{H}$ & 7.303792000 & -0.814903000 & -0.675926000 \\
\hline $\mathrm{H}$ & 6.595238000 & -0.987517000 & -2.290083000 \\
\hline
\end{tabular}

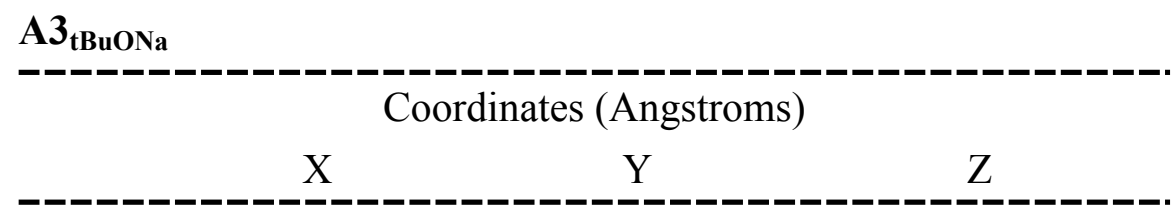




\begin{tabular}{|c|c|c|c|}
\hline $\mathrm{C}$ & -4.359971000 & -1.511647000 & -0.095923000 \\
\hline $\mathrm{C}$ & -3.137067000 & -2.031087000 & 0.313648000 \\
\hline $\mathrm{C}$ & -1.984314000 & -1.236377000 & 0.306575000 \\
\hline $\mathrm{C}$ & -2.086210000 & 0.097967000 & -0.110686000 \\
\hline $\mathrm{C}$ & -3.316334000 & 0.600375000 & -0.516085000 \\
\hline $\mathrm{C}$ & -4.471309000 & -0.186981000 & -0.519493000 \\
\hline $\mathrm{H}$ & -5.243095000 & -2.145903000 & -0.075656000 \\
\hline $\mathrm{H}$ & -3.098582000 & -3.054664000 & 0.681289000 \\
\hline $\mathrm{H}$ & -1.210143000 & 0.762927000 & -0.130381000 \\
\hline $\mathrm{H}$ & -3.371817000 & 1.636139000 & -0.844180000 \\
\hline $\mathrm{C}$ & -0.685171000 & -1.814433000 & 0.732272000 \\
\hline $\mathrm{C}$ & 0.290830000 & -1.132047000 & 1.349309000 \\
\hline $\mathrm{H}$ & 0.141234000 & -0.071620000 & 1.528916000 \\
\hline $\mathrm{O}$ & -0.510341000 & -3.156513000 & 0.497238000 \\
\hline $\mathrm{C}$ & 1.622263000 & -1.733795000 & 1.735948000 \\
\hline $\mathrm{H}$ & 1.521496000 & -2.811573000 & 1.891161000 \\
\hline $\mathrm{C}$ & 2.637572000 & -1.460145000 & 0.644882000 \\
\hline $\mathrm{C}$ & 2.679757000 & -2.278518000 & -0.493248000 \\
\hline $\mathrm{C}$ & 3.437432000 & -0.313481000 & 0.672707000 \\
\hline $\mathrm{C}$ & 3.486253000 & -1.946150000 & -1.580780000 \\
\hline $\mathrm{H}$ & 2.053898000 & -3.166580000 & -0.524566000 \\
\hline $\mathrm{C}$ & 4.244429000 & 0.022385000 & -0.414379000 \\
\hline $\mathrm{H}$ & 3.397999000 & 0.344107000 & 1.536574000 \\
\hline $\mathrm{C}$ & 4.266995000 & -0.788552000 & -1.547765000 \\
\hline $\mathrm{H}$ & 3.510486000 & -2.593865000 & -2.452715000 \\
\hline $\mathrm{H}$ & 4.842394000 & 0.927738000 & -0.379685000 \\
\hline $\mathrm{H}$ & 4.893525000 & -0.526528000 & -2.394794000 \\
\hline $\mathrm{C}$ & 0.516771000 & 2.850834000 & 1.786573000 \\
\hline $\mathrm{C}$ & 0.483278000 & 3.059560000 & 0.256259000 \\
\hline $\mathrm{H}$ & -0.383373000 & 2.309944000 & 2.104808000 \\
\hline $\mathrm{H}$ & 1.391562000 & 2.241997000 & 2.051934000 \\
\hline $\mathrm{H}$ & 0.568272000 & 3.792280000 & 2.349268000 \\
\hline $\mathrm{C}$ & 1.748917000 & 3.837889000 & -0.161128000 \\
\hline $\mathrm{C}$ & -0.760344000 & 3.903439000 & -0.098201000 \\
\hline $\mathrm{H}$ & 1.741889000 & 3.993318000 & -1.246771000 \\
\hline $\mathrm{H}$ & 1.834824000 & 4.816596000 & 0.329555000 \\
\hline $\mathrm{H}$ & 2.639162000 & 3.246132000 & 0.090971000 \\
\hline $\mathrm{H}$ & -0.757906000 & 4.890270000 & 0.383290000 \\
\hline $\mathrm{H}$ & -0.809899000 & 4.045324000 & -1.184049000 \\
\hline $\mathrm{H}$ & -1.667110000 & 3.371385000 & 0.215809000 \\
\hline $\mathrm{O}$ & 0.432545000 & 1.848638000 & -0.381585000 \\
\hline $\mathrm{H}$ & 1.964148000 & -1.293135000 & 2.678049000 \\
\hline $\mathrm{H}$ & -1.196452000 & -3.448743000 & -0.115184000 \\
\hline $\mathrm{Na}$ & 1.220933000 & 0.145368000 & -1.112549000 \\
\hline
\end{tabular}




\begin{tabular}{lrrr}
$\mathrm{C}$ & -5.800459000 & 0.388519000 & -0.937121000 \\
$\mathrm{H}$ & -6.481979000 & -0.389631000 & -1.294421000 \\
$\mathrm{H}$ & -6.287745000 & 0.891757000 & -0.093264000 \\
$\mathrm{H}$ & $-\mathbf{- 5 . 6 8 0 5 8 8 0 0 0}$ & 1.129752000 & -1.733319000 \\
\hline
\end{tabular}

\begin{tabular}{|c|c|c|c|}
\hline \multicolumn{4}{|c|}{$\mathbf{A 1}_{\text {tBuOLi }}$} \\
\hline & $X$ & Y & $\mathrm{Z}$ \\
\hline $\mathrm{C}$ & -0.800608000 & 3.653713000 & -0.052809000 \\
\hline $\mathrm{C}$ & -0.118761000 & 2.451951000 & 0.113721000 \\
\hline $\mathrm{C}$ & -0.817346000 & 1.245013000 & 0.143407000 \\
\hline $\mathrm{C}$ & -2.209635000 & 1.257076000 & 0.008819000 \\
\hline $\mathrm{C}$ & -2.879879000 & 2.462205000 & -0.157913000 \\
\hline $\mathrm{C}$ & -2.189041000 & 3.677751000 & -0.195351000 \\
\hline $\mathrm{H}$ & -0.243960000 & 4.587494000 & -0.076352000 \\
\hline $\mathrm{H}$ & 0.964223000 & 2.453398000 & 0.214495000 \\
\hline $\mathrm{H}$ & -2.746798000 & 0.307919000 & 0.047844000 \\
\hline $\mathrm{H}$ & -3.961958000 & 2.459368000 & -0.267159000 \\
\hline $\mathrm{C}$ & -0.116430000 & -0.072450000 & 0.402004000 \\
\hline $\mathrm{C}$ & 1.294550000 & -0.122821000 & -0.106431000 \\
\hline $\mathrm{H}$ & 1.388945000 & 0.109876000 & -1.165769000 \\
\hline $\mathrm{O}$ & -0.185001000 & -0.375779000 & 1.824441000 \\
\hline $\mathrm{C}$ & 2.369972000 & -0.410248000 & 0.629166000 \\
\hline $\mathrm{H}$ & 2.227973000 & -0.618173000 & 1.688279000 \\
\hline $\mathrm{C}$ & 3.763820000 & -0.464399000 & 0.153769000 \\
\hline $\mathrm{C}$ & 4.800657000 & -0.421105000 & 1.092863000 \\
\hline $\mathrm{C}$ & 4.101526000 & -0.559915000 & -1.202600000 \\
\hline $\mathrm{C}$ & 6.132708000 & -0.450026000 & 0.694175000 \\
\hline $\mathrm{H}$ & 4.557341000 & -0.357115000 & 2.150780000 \\
\hline $\mathrm{C}$ & 5.431281000 & -0.589687000 & -1.603056000 \\
\hline $\mathrm{H}$ & 3.318784000 & -0.630589000 & -1.952127000 \\
\hline $\mathrm{C}$ & 6.452966000 & -0.531685000 & -0.657270000 \\
\hline $\mathrm{H}$ & 6.920152000 & -0.411619000 & 1.440951000 \\
\hline $\mathrm{H}$ & 5.671372000 & -0.669272000 & -2.659225000 \\
\hline $\mathrm{H}$ & 7.491602000 & -0.559462000 & -0.972804000 \\
\hline $\mathrm{H}$ & -0.712017000 & -0.875157000 & -0.052126000 \\
\hline $\mathrm{H}$ & 0.044393000 & 0.432872000 & 2.301239000 \\
\hline $\mathrm{C}$ & -4.699636000 & -3.246318000 & 0.389209000 \\
\hline $\mathrm{C}$ & -3.422823000 & -2.663580000 & -0.243807000 \\
\hline $\mathrm{H}$ & -4.435166000 & -3.841782000 & 1.271479000 \\
\hline $\mathrm{H}$ & -5.354806000 & -2.430194000 & 0.714987000 \\
\hline $\mathrm{H}$ & -5.259640000 & -3.885877000 & -0.304916000 \\
\hline $\mathrm{C}$ & -3.810550000 & -1.840306000 & -1.488139000 \\
\hline
\end{tabular}




$\begin{array}{lrrr}\mathrm{C} & -2.501004000 & -3.819352000 & -0.676780000 \\ \mathrm{H} & -2.912465000 & -1.398519000 & -1.936815000 \\ \mathrm{H} & -4.314974000 & -2.447244000 & -2.250264000 \\ \mathrm{H} & -4.482642000 & -1.022904000 & -1.200280000 \\ \mathrm{H} & -2.976890000 & -4.489748000 & -1.403721000 \\ \mathrm{H} & -1.586691000 & -3.414098000 & -1.127093000 \\ \mathrm{H} & -2.212805000 & -4.409881000 & 0.201426000 \\ \mathrm{O} & -2.769167000 & -1.860480000 & 0.663349000 \\ \mathrm{C} & -2.924552000 & 4.975763000 & -0.413634000 \\ \mathrm{H} & -3.105564000 & 5.145498000 & -1.481677000 \\ \mathrm{H} & -3.898454000 & 4.970053000 & 0.085756000 \\ \mathrm{H} & -2.353275000 & 5.829136000 & -0.036065000 \\ \mathrm{Li} & -1.845541000 & -1.377793000 & 1.963594000\end{array}$

TSA1-2 tBuOLi $_{\text {i }}$

\begin{tabular}{lccc} 
& \multicolumn{3}{c}{ Coordinates (Angstroms) } \\
& $\mathrm{X}$ & $\mathrm{Y}$ & $\mathrm{Z}$ \\
\hline $\mathrm{C}$ & 3.276867000 & -2.198147000 & -1.054005000 \\
$\mathrm{C}$ & 2.029261000 & -1.780957000 & -0.597625000 \\
$\mathrm{C}$ & 1.915361000 & -0.822082000 & 0.414527000 \\
$\mathrm{C}$ & 3.102766000 & -0.315963000 & 0.966648000 \\
$\mathrm{C}$ & 4.341448000 & -0.752093000 & 0.521065000 \\
$\mathrm{C}$ & 4.455048000 & -1.698912000 & -0.502571000 \\
$\mathrm{H}$ & 3.329886000 & -2.942731000 & -1.845229000 \\
$\mathrm{H}$ & 1.135873000 & -2.221522000 & -1.031296000 \\
$\mathrm{H}$ & 3.039209000 & 0.435672000 & 1.747973000 \\
$\mathrm{H}$ & 5.243535000 & -0.339296000 & 0.967898000 \\
$\mathrm{C}$ & 0.613967000 & -0.280592000 & 0.869077000 \\
$\mathrm{C}$ & -0.640317000 & -0.693824000 & 0.285137000 \\
$\mathrm{H}$ & -0.630902000 & -0.777124000 & -0.801165000 \\
$\mathrm{O}$ & 0.510067000 & -0.106190000 & 2.306162000 \\
$\mathrm{C}$ & -1.835877000 & -0.798481000 & 0.925527000 \\
$\mathrm{H}$ & -1.847496000 & -0.851982000 & 2.017477000 \\
$\mathrm{C}$ & -3.140849000 & -0.973216000 & 0.274800000 \\
$\mathrm{C}$ & -4.221551000 & -1.485993000 & 1.007560000 \\
$\mathrm{C}$ & -3.367024000 & -0.633263000 & -1.068381000 \\
$\mathrm{C}$ & -5.468142000 & -1.672371000 & 0.421550000 \\
$\mathrm{H}$ & -4.073122000 & -1.753899000 & 2.051611000 \\
$\mathrm{C}$ & -4.610345000 & -0.828304000 & -1.657047000 \\
$\mathrm{H}$ & -2.564380000 & -0.195402000 & -1.655670000 \\
$\mathrm{C}$ & -5.669412000 & -1.350097000 & -0.917643000 \\
$\mathrm{H}$ & -6.284971000 & -2.075988000 & 1.013326000 \\
$\mathrm{H}$ & -4.756509000 & -0.559819000 & -2.699551000 \\
& & &
\end{tabular}




$\begin{array}{lccc}\mathrm{H} & -6.641676000 & -1.495566000 & -1.378374000 \\ \mathrm{H} & 0.575379000 & 1.121519000 & 0.605637000 \\ \mathrm{H} & 0.418201000 & -0.975895000 & 2.722056000 \\ \mathrm{C} & 1.805361000 & 3.309544000 & -0.646188000 \\ \mathrm{C} & 0.324923000 & 3.057909000 & -0.336912000 \\ \mathrm{H} & 2.281283000 & 3.820449000 & 0.197040000 \\ \mathrm{H} & 2.323061000 & 2.355966000 & -0.800986000 \\ \mathrm{H} & 1.931386000 & 3.923040000 & -1.545672000 \\ \mathrm{C} & -0.345217000 & 2.338533000 & -1.516951000 \\ \mathrm{C} & -0.395023000 & 4.375691000 & -0.046642000 \\ \mathrm{H} & -1.387841000 & 2.100685000 & -1.273734000 \\ \mathrm{H} & -0.333634000 & 2.958203000 & -2.420616000 \\ \mathrm{H} & 0.178911000 & 1.402632000 & -1.743248000 \\ \mathrm{H} & -0.336956000 & 5.061330000 & -0.899675000 \\ \mathrm{H} & -1.453997000 & 4.189990000 & 0.170190000 \\ \mathrm{H} & 0.055667000 & 4.865164000 & 0.823182000 \\ \mathrm{O} & 0.238156000 & 2.258493000 & 0.822236000 \\ \mathrm{C} & 5.806586000 & -2.179757000 & -0.968380000 \\ \mathrm{H} & 6.522720000 & -1.353992000 & -1.037702000 \\ \mathrm{H} & 6.225987000 & -2.916479000 & -0.272083000 \\ \mathrm{H} & 5.743395000 & -2.655009000 & -1.952091000 \\ \mathrm{Li} & -0.848592000 & 1.318626000 & 1.924078000 \\ - & & & \end{array}$

\begin{tabular}{|c|c|c|c|}
\hline \multicolumn{4}{|c|}{ A2 tBuOLi } \\
\hline & \multicolumn{3}{|c|}{ Coordinates (Angstroms) } \\
\hline & $\mathrm{X}$ & $\mathrm{Y}$ & Z \\
\hline$\overline{\mathrm{C}}$ & 4.520060000 & -0.304204000 & 0.602405000 \\
\hline $\mathrm{C}$ & 3.311239000 & -0.706432000 & 1.175946000 \\
\hline $\mathrm{C}$ & 2.219096000 & -1.122229000 & 0.376828000 \\
\hline $\mathrm{C}$ & 2.432350000 & -1.119053000 & -1.026520000 \\
\hline $\mathrm{C}$ & 3.638005000 & -0.722816000 & -1.568912000 \\
\hline $\mathrm{C}$ & 4.714136000 & -0.295839000 & -0.772380000 \\
\hline $\mathrm{H}$ & 5.332910000 & -0.002705000 & 1.260119000 \\
\hline $\mathrm{H}$ & 3.228553000 & -0.759016000 & 2.257213000 \\
\hline $\mathrm{H}$ & 1.641459000 & -1.446166000 & -1.694585000 \\
\hline $\mathrm{H}$ & 3.757413000 & -0.744921000 & -2.650471000 \\
\hline $\mathrm{C}$ & 0.937841000 & -1.410679000 & 0.975948000 \\
\hline $\mathrm{C}$ & -0.298541000 & -1.408869000 & 0.341904000 \\
\hline $\mathrm{H}$ & -0.251157000 & -1.497376000 & -0.740595000 \\
\hline $\mathrm{O}$ & 0.947137000 & -1.149594000 & 2.383823000 \\
\hline $\mathrm{C}$ & -1.550340000 & -1.214357000 & 0.909989000 \\
\hline $\mathrm{H}$ & -1.653145000 & -1.175460000 & 1.995204000 \\
\hline $\mathrm{C}$ & -2.803775000 & -1.113723000 & 0.173990000 \\
\hline
\end{tabular}




\begin{tabular}{lccc}
$\mathrm{C}$ & -3.978482000 & -0.745375000 & 0.860885000 \\
$\mathrm{C}$ & -2.920740000 & -1.308364000 & -1.216976000 \\
$\mathrm{C}$ & -5.187289000 & -0.569409000 & 0.202216000 \\
$\mathrm{H}$ & -3.927716000 & -0.588152000 & 1.936541000 \\
$\mathrm{C}$ & -4.131797000 & -1.128324000 & -1.874667000 \\
$\mathrm{H}$ & -2.055863000 & -1.620948000 & -1.796253000 \\
$\mathrm{C}$ & -5.277005000 & -0.754751000 & -1.176172000 \\
$\mathrm{H}$ & -6.068513000 & -0.283707000 & 0.770855000 \\
$\mathrm{H}$ & -4.181169000 & -1.290385000 & -2.948412000 \\
$\mathrm{H}$ & -6.221481000 & -0.616667000 & -1.693163000 \\
$\mathrm{H}$ & -1.056220000 & 0.722259000 & 1.046532000 \\
$\mathrm{H}$ & 0.229120000 & -1.649046000 & 2.788952000 \\
$\mathrm{C}$ & -0.368014000 & 1.649748000 & -1.234412000 \\
$\mathrm{C}$ & -0.697693000 & 2.410626000 & 0.051563000 \\
$\mathrm{H}$ & 0.638667000 & 1.216261000 & -1.187636000 \\
$\mathrm{H}$ & -1.089431000 & 0.842331000 & -1.399382000 \\
$\mathrm{H}$ & -0.411541000 & 2.323888000 & -2.096250000 \\
$\mathrm{C}$ & -2.150884000 & 2.880618000 & 0.048827000 \\
$\mathrm{C}$ & 0.270631000 & 3.567368000 & 0.268732000 \\
$\mathrm{H}$ & -2.381488000 & 3.407788000 & 0.979711000 \\
$\mathrm{H}$ & -2.338116000 & 3.556206000 & -0.792353000 \\
$\mathrm{H}$ & -2.828900000 & 2.024055000 & -0.043439000 \\
$\mathrm{H}$ & 0.170152000 & 4.309760000 & -0.528798000 \\
$\mathrm{H}$ & 0.073406000 & 4.058270000 & 1.227064000 \\
$\mathrm{H}$ & 1.309066000 & 3.211189000 & 0.263562000 \\
$\mathrm{O}$ & -0.509244000 & 1.539014000 & 1.188113000 \\
$\mathrm{C}$ & 6.019943000 & 0.125334000 & -1.396187000 \\
$\mathrm{H}$ & 6.508350000 & -0.713823000 & -1.906355000 \\
$\mathrm{H}$ & 5.873848000 & 0.915800000 & -2.141786000 \\
$\mathrm{H}$ & 6.717022000 & 0.503315000 & -0.641872000 \\
$\mathrm{Li}$ & 1.096396000 & 0.642089000 & 1.607356000 \\
\hline----- & & \\
& & &
\end{tabular}

TSA2-3 ${ }_{\text {tBuOLi }}$

\begin{tabular}{llcc} 
& \multicolumn{3}{c}{ Coordinates (Angstroms) } \\
C & S.079909000 & 0.086389000 & 0.716952000 \\
$\mathrm{C}$ & 3.784553000 & 0.176864000 & 1.217873000 \\
$\mathrm{C}$ & 2.690928000 & -0.285235000 & 0.473503000 \\
$\mathrm{C}$ & 2.951425000 & -0.825494000 & -0.797744000 \\
$\mathrm{C}$ & 4.246204000 & -0.919463000 & -1.281455000 \\
$\mathrm{C}$ & 5.339488000 & -0.463778000 & -0.536538000 \\
$\mathrm{H}$ & 5.906914000 & 0.447107000 & 1.324226000 \\
$\mathrm{H}$ & 3.617941000 & 0.596218000 & 2.204024000
\end{tabular}




\begin{tabular}{lrcc}
$\mathrm{H}$ & 2.131965000 & -1.172343000 & -1.420446000 \\
$\mathrm{H}$ & 4.413023000 & -1.350268000 & -2.266098000 \\
$\mathrm{C}$ & 1.324474000 & -0.179574000 & 0.997499000 \\
$\mathrm{C}$ & 0.196123000 & -0.786685000 & 0.551385000 \\
$\mathrm{H}$ & 0.334273000 & -1.482472000 & -0.272569000 \\
$\mathrm{O}$ & 1.216396000 & 0.765454000 & 2.055449000 \\
$\mathrm{C}$ & -1.153834000 & -0.546193000 & 1.015870000 \\
$\mathrm{H}$ & -1.253482000 & -0.389095000 & 2.097650000 \\
$\mathrm{C}$ & -2.267312000 & -1.345358000 & 0.470762000 \\
$\mathrm{C}$ & -3.427396000 & -1.557389000 & 1.235381000 \\
$\mathrm{C}$ & -2.272018000 & -1.846518000 & -0.842616000 \\
$\mathrm{C}$ & -4.526445000 & -2.234328000 & 0.721268000 \\
$\mathrm{H}$ & -3.461431000 & -1.178121000 & 2.254630000 \\
$\mathrm{C}$ & -3.367833000 & -2.533206000 & -1.354549000 \\
$\mathrm{H}$ & -1.410948000 & -1.685059000 & -1.486926000 \\
$\mathrm{C}$ & -4.505705000 & -2.733587000 & -0.579089000 \\
$\mathrm{H}$ & -5.405068000 & -2.378093000 & 1.344623000 \\
$\mathrm{H}$ & -3.331633000 & -2.908851000 & -2.373905000 \\
$\mathrm{H}$ & -5.363096000 & -3.263890000 & -0.981956000 \\
$\mathrm{H}$ & -1.358503000 & 0.841432000 & 0.600948000 \\
$\mathrm{H}$ & 0.527642000 & 0.465947000 & 2.664975000 \\
$\mathrm{C}$ & -2.060191000 & 1.742995000 & -1.875398000 \\
$\mathrm{C}$ & -2.219798000 & 2.470919000 & -0.535825000 \\
$\mathrm{H} \mathrm{H}$ & -1.047951000 & 1.892280000 & -2.271775000 \\
$\mathrm{H}$ & -2.229283000 & 0.668852000 & -1.746884000 \\
$\mathrm{H}$ & -2.776356000 & 2.113474000 & -2.617464000 \\
$\mathrm{C}$ & -3.616791000 & 2.217595000 & 0.040998000 \\
$\mathrm{C}$ & -1.978690000 & 3.970579000 & -0.713181000 \\
$\mathrm{H}$ & -3.719825000 & 2.717872000 & 1.009589000 \\
$\mathrm{H}$ & -4.395552000 & 2.594463000 & -0.631469000 \\
$\mathrm{H}$ & -3.781065000 & 1.144256000 & 0.187109000 \\
$\mathrm{H}$ & -2.707402000 & 4.410893000 & -1.402857000 \\
$\mathrm{H}$ & -2.059086000 & 4.483771000 & 0.250951000 \\
$\mathrm{H}$ & -0.976060000 & 4.151575000 & -1.121580000 \\
$\mathrm{H}$ & -1.249639000 & 1.994537000 & 0.381158000 \\
$\mathrm{H}$ & 6.739239000 & -0.546964000 & -1.090004000 \\
$\mathrm{H}$ & 6.921344000 & -1.516253000 & -1.566044000 \\
$\mathrm{H}$ & 7.911498000 & 0.226021000 & -1.848917000 \\
$\mathrm{H}$ & -517240000 & 1.910745000 & 0.623641000 \\
\hline
\end{tabular}

A3 ${ }_{\text {tBuOLi }}$ 


\begin{tabular}{|c|c|c|c|}
\hline & X & $\mathrm{Y}$ & Z \\
\hline $\mathrm{C}$ & -4.419065000 & -1.255659000 & -0.086466000 \\
\hline $\mathrm{C}$ & -3.212936000 & -1.893639000 & 0.176621000 \\
\hline $\mathrm{C}$ & -2.012320000 & -1.174283000 & 0.165442000 \\
\hline $\mathrm{C}$ & -2.039297000 & 0.194427000 & -0.125839000 \\
\hline $\mathrm{C}$ & -3.254121000 & 0.816208000 & -0.387634000 \\
\hline $\mathrm{C}$ & -4.460350000 & 0.110878000 & -0.369704000 \\
\hline $\mathrm{H}$ & -5.343694000 & -1.827277000 & -0.063341000 \\
\hline $\mathrm{H}$ & -3.213693000 & -2.952760000 & 0.426132000 \\
\hline $\mathrm{H}$ & -1.108698000 & 0.770097000 & -0.183780000 \\
\hline $\mathrm{H}$ & -3.258585000 & 1.877746000 & -0.624730000 \\
\hline $\mathrm{C}$ & -0.737841000 & -1.874951000 & 0.453598000 \\
\hline $\mathrm{C}$ & 0.237558000 & -1.392375000 & 1.238478000 \\
\hline $\mathrm{H}$ & 0.090573000 & -0.415331000 & 1.688872000 \\
\hline $\mathrm{O}$ & -0.598132000 & -3.124111000 & -0.092272000 \\
\hline $\mathrm{C}$ & 1.538097000 & -2.117686000 & 1.504030000 \\
\hline $\mathrm{H}$ & 1.398479000 & -3.196266000 & 1.389608000 \\
\hline $\mathrm{C}$ & 2.590653000 & -1.624033000 & 0.532635000 \\
\hline $\mathrm{C}$ & 2.635768000 & -2.142272000 & -0.770580000 \\
\hline $\mathrm{C}$ & 3.408192000 & -0.533475000 & 0.846999000 \\
\hline $\mathrm{C}$ & 3.467593000 & -1.573495000 & -1.733590000 \\
\hline $\mathrm{H}$ & 1.990205000 & -2.978082000 & -1.026351000 \\
\hline $\mathrm{C}$ & 4.240476000 & 0.036405000 & -0.116573000 \\
\hline $\mathrm{H}$ & 3.363997000 & -0.102845000 & 1.843590000 \\
\hline $\mathrm{C}$ & 4.268568000 & -0.477464000 & -1.409937000 \\
\hline $\mathrm{H}$ & 3.487517000 & -1.984423000 & -2.738541000 \\
\hline $\mathrm{H}$ & 4.848401000 & 0.897801000 & 0.140386000 \\
\hline $\mathrm{H}$ & 4.905102000 & -0.025032000 & -2.163260000 \\
\hline $\mathrm{C}$ & 0.533242000 & 2.657937000 & 1.662259000 \\
\hline $\mathrm{C}$ & 0.786118000 & 2.867534000 & 0.154563000 \\
\hline $\mathrm{H}$ & -0.450635000 & 2.196588000 & 1.813207000 \\
\hline $\mathrm{H}$ & 1.293862000 & 1.979507000 & 2.071618000 \\
\hline $\mathrm{H}$ & 0.565185000 & 3.594325000 & 2.233998000 \\
\hline $\mathrm{C}$ & 2.176219000 & 3.505189000 & -0.034080000 \\
\hline $\mathrm{C}$ & -0.285035000 & 3.825697000 & -0.401973000 \\
\hline $\mathrm{H}$ & 2.372680000 & 3.647945000 & -1.102857000 \\
\hline $\mathrm{H}$ & 2.271559000 & 4.475066000 & 0.471298000 \\
\hline $\mathrm{H}$ & 2.944740000 & 2.830391000 & 0.364502000 \\
\hline $\mathrm{H}$ & -0.264347000 & 4.808268000 & 0.086528000 \\
\hline $\mathrm{H}$ & -0.130544000 & 3.965541000 & -1.477383000 \\
\hline $\mathrm{H}$ & -1.281708000 & 3.391237000 & -0.257177000 \\
\hline $\mathrm{O}$ & 0.725942000 & 1.659453000 & -0.500900000 \\
\hline $\mathrm{H}$ & 1.861982000 & -1.931870000 & 2.533110000 \\
\hline $\mathrm{H}$ & -1.297189000 & -3.246102000 & -0.746966000 \\
\hline
\end{tabular}




$\begin{array}{llll}\mathrm{C} & -5.770072000 & 0.811364000 & -0.627631000 \\ \mathrm{H} & -6.540197000 & 0.110007000 & -0.962827000 \\ \mathrm{H} & -6.138586000 & 1.296448000 & 0.284124000 \\ \mathrm{H} & -5.661631000 & 1.588822000 & -1.390330000 \\ \mathrm{Li} & 1.374684000 & 0.116539000 & -0.629257000\end{array}$

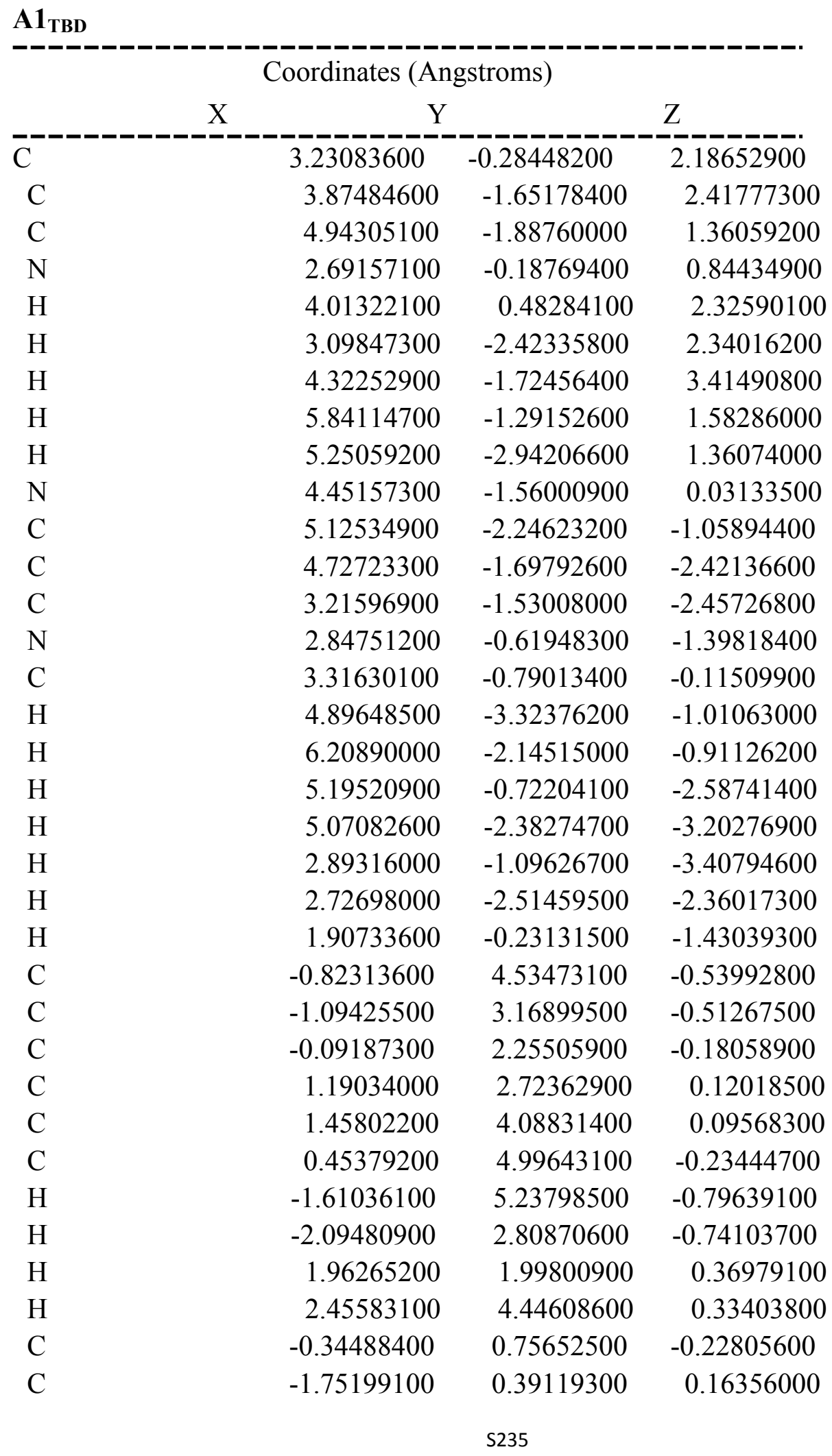




\begin{tabular}{|c|c|c|c|}
\hline $\mathrm{H}$ & -2.06859100 & 0.79261400 & 1.12534500 \\
\hline $\mathrm{O}$ & 0.00461400 & 0.24504400 & -1.52104600 \\
\hline $\mathrm{C}$ & -2.57437100 & -0.35294500 & -0.57822200 \\
\hline $\mathrm{H}$ & -2.19677700 & -0.72067800 & -1.53089000 \\
\hline $\mathrm{C}$ & -3.95827900 & -0.73630900 & -0.25256300 \\
\hline $\mathrm{C}$ & -4.74179900 & -1.34831300 & -1.23521600 \\
\hline $\mathrm{C}$ & -4.54613100 & -0.51856200 & 1.00185700 \\
\hline $\mathrm{C}$ & -6.06058900 & -1.71450100 & -0.98571400 \\
\hline $\mathrm{H}$ & -4.31063900 & -1.53593300 & -2.21576800 \\
\hline $\mathrm{C}$ & -5.86018600 & -0.88489600 & 1.24788800 \\
\hline $\mathrm{H}$ & -3.96777400 & -0.06439800 & 1.80118300 \\
\hline $\mathrm{C}$ & -6.64510600 & -1.48849000 & 0.25878300 \\
\hline $\mathrm{H}$ & -6.64264400 & -2.18534000 & -1.77441000 \\
\hline $\mathrm{H}$ & -6.28942300 & -0.70437800 & 2.23096400 \\
\hline $\mathrm{H}$ & 0.36531700 & 0.27008600 & 0.45186200 \\
\hline $\mathrm{H}$ & -0.32137400 & 0.87853700 & -2.17363300 \\
\hline $\mathrm{C}$ & 2.11441000 & -0.00156800 & 3.18665200 \\
\hline $\mathrm{H}$ & 2.49031500 & -0.00469100 & 4.21635400 \\
\hline $\mathrm{H}$ & 1.66385900 & 0.97472000 & 2.98126400 \\
\hline $\mathrm{H}$ & 1.32942500 & -0.76143700 & 3.09801100 \\
\hline $\mathrm{H}$ & -7.66002162 & -1.76705513 & 0.45177979 \\
\hline $\mathrm{C}$ & 0.75363967 & 6.50674435 & -0.25984314 \\
\hline $\mathrm{H}$ & 0.19413680 & 6.96827338 & -1.04651174 \\
\hline $\mathrm{H}$ & 0.47600847 & 6.94130523 & 0.67769500 \\
\hline $\mathrm{H}$ & 1.79910941 & 6.66002755 & -0.42835805 \\
\hline
\end{tabular}

TSA1-2

\begin{tabular}{lrcc} 
& \multicolumn{3}{c}{ Coordinates (Angstroms) } \\
C & X & Y & Z \\
$\mathrm{C}$ & -1.0157950000 & -0.859065000 & 2.387339000 \\
$\mathrm{C}$ & -2.312207000 & -0.657488000 & 2.347278000 \\
$\mathrm{~N}$ & 0.104498000 & -1.517680000 & 1.094711000 \\
$\mathrm{H}$ & 0.157399000 & -1.610150000 & 3.188253000 \\
$\mathrm{H}$ & 1.114633000 & -0.329711000 & 2.438099000 \\
$\mathrm{H}$ & -0.938146000 & 0.899223000 & 1.811145000 \\
$\mathrm{H}$ & -1.012884000 & 0.560181000 & 3.549745000 \\
$\mathrm{H}$ & -2.559245000 & -1.264652000 & 3.228761000 \\
$\mathrm{H}$ & -3.132822000 & 0.050945000 & 2.186516000 \\
$\mathrm{~N}$ & -2.251271000 & -1.528759000 & 1.172430000 \\
$\mathrm{C}$ & -3.539627000 & -1.779688000 & 0.532698000 \\
$\mathrm{C}$ & -3.432765000 & -2.851260000 & -0.539860000 \\
$\mathrm{C}$ & -2.269602000 & -2.510134000 & -1.457180000
\end{tabular}




\begin{tabular}{|c|c|c|c|}
\hline $\mathrm{N}$ & -1.069589000 & -2.412639000 & -0.651562000 \\
\hline $\mathrm{C}$ & -1.074261000 & -1.787726000 & 0.555229000 \\
\hline $\mathrm{H}$ & -3.907917000 & -0.839545000 & 0.097472000 \\
\hline $\mathrm{H}$ & -4.245360000 & -2.086028000 & 1.313251000 \\
\hline $\mathrm{H}$ & -3.260958000 & -3.831111000 & -0.081841000 \\
\hline $\mathrm{H}$ & -4.369469000 & -2.896557000 & -1.102321000 \\
\hline $\mathrm{H}$ & -2.113604000 & -3.292807000 & -2.204074000 \\
\hline $\mathrm{H}$ & -2.474373000 & -1.569392000 & -1.989017000 \\
\hline $\mathrm{H}$ & -0.188419000 & -2.297052000 & -1.152931000 \\
\hline $\mathrm{C}$ & 4.657364000 & 1.183098000 & 0.783320000 \\
\hline $\mathrm{C}$ & 3.317055000 & 0.984392000 & 0.487702000 \\
\hline $\mathrm{C}$ & 2.909917000 & 0.066169000 & -0.497249000 \\
\hline $\mathrm{C}$ & 3.923205000 & -0.654287000 & -1.143808000 \\
\hline $\mathrm{C}$ & 5.265513000 & -0.450436000 & -0.839471000 \\
\hline $\mathrm{C}$ & 5.665498000 & 0.474765000 & 0.122660000 \\
\hline $\mathrm{H}$ & 4.928781000 & 1.906647000 & 1.549820000 \\
\hline $\mathrm{H}$ & 2.575565000 & 1.553323000 & 1.043993000 \\
\hline $\mathrm{H}$ & 3.645522000 & -1.392276000 & -1.888559000 \\
\hline $\mathrm{H}$ & 6.020059000 & -1.030252000 & -1.367917000 \\
\hline $\mathrm{C}$ & 1.485248000 & -0.190637000 & -0.797670000 \\
\hline $\mathrm{C}$ & 0.498886000 & 0.850570000 & -0.700604000 \\
\hline $\mathrm{H}$ & 0.729377000 & 1.631654000 & 0.022393000 \\
\hline $\mathrm{O}$ & 1.265829000 & -1.123191000 & -1.855737000 \\
\hline $\mathrm{C}$ & -0.734027000 & 0.839515000 & -1.270685000 \\
\hline $\mathrm{H}$ & -0.948718000 & 0.064166000 & -2.002871000 \\
\hline $\mathrm{C}$ & -1.864770000 & 1.692115000 & -0.917633000 \\
\hline $\mathrm{C}$ & -3.118464000 & 1.451041000 & -1.510331000 \\
\hline $\mathrm{C}$ & -1.806016000 & 2.720090000 & 0.042154000 \\
\hline $\mathrm{C}$ & -4.251808000 & 2.169721000 & -1.149040000 \\
\hline $\mathrm{H}$ & -3.196102000 & 0.680342000 & -2.275082000 \\
\hline $\mathrm{C}$ & -2.938602000 & 3.437703000 & 0.405678000 \\
\hline $\mathrm{H}$ & -0.857827000 & 2.965569000 & 0.512707000 \\
\hline $\mathrm{C}$ & -4.174019000 & 3.166201000 & -0.179168000 \\
\hline $\mathrm{H}$ & -5.201362000 & 1.952687000 & -1.631471000 \\
\hline $\mathrm{H}$ & -2.854267000 & 4.221524000 & 1.153822000 \\
\hline $\mathrm{H}$ & -5.057268000 & 3.728394000 & 0.108151000 \\
\hline $\mathrm{H}$ & 0.863088000 & -1.014558000 & 0.315493000 \\
\hline $\mathrm{H}$ & 1.307101000 & -0.644615000 & -2.697515000 \\
\hline $\mathrm{C}$ & 7.121298000 & 0.722042000 & 0.429330000 \\
\hline $\mathrm{H}$ & 7.492063000 & 1.618750000 & -0.083173000 \\
\hline $\mathrm{H}$ & 7.285230000 & 0.871530000 & 1.502222000 \\
\hline $\mathrm{H}$ & 7.744847000 & -0.119053000 & 0.109395000 \\
\hline
\end{tabular}

A2 ${ }_{\text {TBD }}$ 


\begin{tabular}{|c|c|c|c|}
\hline \multicolumn{4}{|c|}{ Coordinates (Angstroms) } \\
\hline & $X$ & $\mathrm{Y}$ & $\mathrm{Z}$ \\
\hline $\mathrm{C}$ & 2.043531000 & 1.374163000 & 1.949590000 \\
\hline $\mathrm{C}$ & 2.721898000 & 1.832209000 & 0.670085000 \\
\hline $\mathrm{C}$ & 1.945420000 & 3.007581000 & 0.098203000 \\
\hline $\mathrm{N}$ & 0.634507000 & 1.108977000 & 1.691256000 \\
\hline $\mathrm{H}$ & 2.138741000 & 2.135548000 & 2.734679000 \\
\hline $\mathrm{H}$ & 2.484298000 & 0.443994000 & 2.310962000 \\
\hline $\mathrm{H}$ & 2.741299000 & 1.005255000 & -0.047457000 \\
\hline $\mathrm{H}$ & 3.753385000 & 2.133463000 & 0.869970000 \\
\hline $\mathrm{H}$ & 2.071201000 & 3.905919000 & 0.717710000 \\
\hline $\mathrm{H}$ & 2.303196000 & 3.246059000 & -0.909062000 \\
\hline $\mathrm{N}$ & 0.520507000 & 2.692906000 & -0.014100000 \\
\hline $\mathrm{C}$ & -0.212905000 & 3.410868000 & -1.055377000 \\
\hline $\mathrm{C}$ & -1.718668000 & 3.243697000 & -0.906328000 \\
\hline $\mathrm{C}$ & -2.038676000 & 1.771661000 & -0.704912000 \\
\hline $\mathrm{N}$ & -1.293856000 & 1.299155000 & 0.450238000 \\
\hline $\mathrm{C}$ & -0.040225000 & 1.689679000 & 0.690985000 \\
\hline $\mathrm{H}$ & 0.118552000 & 3.044595000 & -2.036826000 \\
\hline $\mathrm{H}$ & 0.065886000 & 4.468262000 & -0.985273000 \\
\hline $\mathrm{H}$ & -2.076524000 & 3.815071000 & -0.043266000 \\
\hline $\mathrm{H}$ & -2.215884000 & 3.631995000 & -1.798977000 \\
\hline $\mathrm{H}$ & -3.098445000 & 1.602583000 & -0.503804000 \\
\hline $\mathrm{H}$ & -1.778873000 & 1.186828000 & -1.597617000 \\
\hline $\mathrm{H}$ & -1.553821000 & 0.345577000 & 0.780790000 \\
\hline $\mathrm{C}$ & 3.173134000 & -1.207414000 & -1.852577000 \\
\hline $\mathrm{C}$ & 1.934546000 & -1.381229000 & -1.254478000 \\
\hline $\mathrm{C}$ & 1.813046000 & -1.689207000 & 0.119782000 \\
\hline $\mathrm{C}$ & 3.019588000 & -1.798432000 & 0.842517000 \\
\hline $\mathrm{C}$ & 4.253132000 & -1.604076000 & 0.233364000 \\
\hline $\mathrm{C}$ & 4.364897000 & -1.305969000 & -1.125659000 \\
\hline $\mathrm{H}$ & 3.216738000 & -0.972706000 & -2.914982000 \\
\hline $\mathrm{H}$ & 1.041242000 & -1.255781000 & -1.859899000 \\
\hline $\mathrm{H}$ & 2.971862000 & -2.036989000 & 1.899859000 \\
\hline $\mathrm{H}$ & 5.157108000 & -1.693494000 & 0.833590000 \\
\hline $\mathrm{C}$ & 0.536877000 & -1.800249000 & 0.787612000 \\
\hline $\mathrm{C}$ & -0.711930000 & -1.760476000 & 0.221951000 \\
\hline $\mathrm{H}$ & -0.738951000 & -1.841761000 & -0.862555000 \\
\hline $\mathrm{O}$ & 0.617565000 & -1.669539000 & 2.195221000 \\
\hline $\mathrm{C}$ & -1.934035000 & -1.552153000 & 0.885190000 \\
\hline $\mathrm{H}$ & -1.947915000 & -1.507555000 & 1.974169000 \\
\hline $\mathrm{C}$ & -3.214610000 & -1.389006000 & 0.234273000 \\
\hline $\mathrm{C}$ & -4.337768000 & -0.969874000 & 0.987021000 \\
\hline $\mathrm{C}$ & -3.422877000 & -1.538812000 & -1.157309000 \\
\hline
\end{tabular}




\begin{tabular}{lrcc}
$\mathrm{C}$ & -5.562362000 & -0.699693000 & 0.395184000 \\
$\mathrm{H}$ & -4.224722000 & -0.846004000 & 2.062535000 \\
$\mathrm{C}$ & -4.650975000 & -1.263951000 & -1.744933000 \\
$\mathrm{H}$ & -2.612041000 & -1.890012000 & -1.789797000 \\
$\mathrm{C}$ & -5.735806000 & -0.834553000 & -0.982767000 \\
$\mathrm{H}$ & -6.393940000 & -0.378025000 & 1.018095000 \\
$\mathrm{H}$ & -4.764646000 & -1.397667000 & -2.818410000 \\
$\mathrm{H}$ & -6.694595000 & -0.625323000 & -1.447084000 \\
$\mathrm{H}$ & 0.292083000 & 0.205415000 & 2.028540000 \\
$\mathrm{H}$ & -0.007412000 & -2.299619000 & 2.577311000 \\
$\mathrm{C}$ & 5.706701000 & -1.124718000 & -1.789255000 \\
$\mathrm{H}$ & 6.054342000 & -2.054338000 & -2.257651000 \\
$\mathrm{H}$ & 5.666725000 & -0.362590000 & -2.575575000 \\
$\mathrm{H}$ & 6.470889000 & -0.819911000 & -1.066487000 \\
\hline
\end{tabular}

TSA2-3 ${ }_{\text {TBD }}$

\begin{tabular}{|c|c|c|c|}
\hline & $\mathrm{Co}$ & nates (Angstrom & \\
\hline & $\mathrm{X}$ & $Y$ & Z \\
\hline$\overline{\mathrm{C}}$ & 1.994085000 & 1.744896000 & 1.635935000 \\
\hline $\mathrm{C}$ & 2.488507000 & 2.142766000 & 0.255313000 \\
\hline $\mathrm{C}$ & 1.598676000 & 3.254598000 & -0.278764000 \\
\hline $\mathrm{N}$ & 0.572147000 & 1.442371000 & 1.589272000 \\
\hline $\mathrm{H}$ & 2.180945000 & 2.553958000 & 2.355039000 \\
\hline $\mathrm{H}$ & 2.509453000 & 0.849200000 & 1.988824000 \\
\hline $\mathrm{H}$ & 2.452690000 & 1.272459000 & -0.409555000 \\
\hline $\mathrm{H}$ & 3.524429000 & 2.489331000 & 0.305172000 \\
\hline $\mathrm{H}$ & 1.764364000 & 4.187861000 & 0.278909000 \\
\hline $\mathrm{H}$ & 1.829360000 & 3.456522000 & -1.331187000 \\
\hline $\mathrm{N}$ & 0.190937000 & 2.877825000 & -0.208511000 \\
\hline $\mathrm{C}$ & -0.702045000 & 3.546972000 & -1.147277000 \\
\hline $\mathrm{C}$ & -2.160395000 & 3.322952000 & -0.775660000 \\
\hline $\mathrm{C}$ & -2.374707000 & 1.842167000 & -0.500442000 \\
\hline $\mathrm{N}$ & -1.478770000 & 1.405971000 & 0.557029000 \\
\hline $\mathrm{C}$ & -0.255508000 & 1.900504000 & 0.623412000 \\
\hline $\mathrm{H}$ & -0.505447000 & 3.173763000 & -2.162735000 \\
\hline $\mathrm{H}$ & -0.459642000 & 4.616724000 & -1.138022000 \\
\hline $\mathrm{H}$ & -2.410546000 & 3.898980000 & 0.122215000 \\
\hline $\mathrm{H}$ & -2.802985000 & 3.670005000 & -1.589889000 \\
\hline $\mathrm{H}$ & -3.397848000 & 1.636515000 & -0.176594000 \\
\hline $\mathrm{H}$ & -2.209310000 & 1.256382000 & -1.416472000 \\
\hline $\mathrm{H}$ & -1.625110000 & 0.242417000 & 0.833898000 \\
\hline $\mathrm{C}$ & 3.428433000 & -1.389346000 & -1.733092000 \\
\hline $\mathrm{C}$ & 2.169467000 & -1.431743000 & -1.155142000 \\
\hline
\end{tabular}




\begin{tabular}{lrrr}
$\mathrm{C}$ & 2.008378000 & -1.597654000 & 0.233558000 \\
$\mathrm{C}$ & 3.176942000 & -1.702636000 & 1.002596000 \\
$\mathrm{C}$ & 4.436368000 & -1.643801000 & 0.413838000 \\
$\mathrm{C}$ & 4.592442000 & -1.487317000 & -0.961886000 \\
$\mathrm{H}$ & 3.513024000 & -1.261408000 & -2.810515000 \\
$\mathrm{H}$ & 1.296774000 & -1.316834000 & -1.791316000 \\
$\mathrm{H}$ & 3.086166000 & -1.831204000 & 2.075714000 \\
$\mathrm{H}$ & 5.318938000 & -1.726722000 & 1.044774000 \\
$\mathrm{C}$ & 0.696090000 & -1.581805000 & 0.875542000 \\
$\mathrm{C}$ & -0.516356000 & -1.601864000 & 0.276516000 \\
$\mathrm{H}$ & -0.520790000 & -1.796233000 & -0.792442000 \\
$\mathrm{O}$ & 0.766206000 & -1.346670000 & 2.258758000 \\
$\mathrm{C}$ & -1.772929000 & -1.264495000 & 0.905600000 \\
$\mathrm{H}$ & -1.833755000 & -1.404485000 & 1.988810000 \\
$\mathrm{C}$ & -3.062484000 & -1.466251000 & 0.235641000 \\
$\mathrm{C}$ & -4.252725000 & -1.429573000 & 0.989467000 \\
$\mathrm{C}$ & -3.208462000 & -1.589951000 & -1.159903000 \\
$\mathrm{C}$ & -5.503037000 & -1.506512000 & 0.392043000 \\
$\mathrm{H}$ & -4.181675000 & -1.332811000 & 2.071069000 \\
$\mathrm{C}$ & -4.461404000 & -1.670819000 & -1.757477000 \\
$\mathrm{H}$ & -2.328052000 & -1.619933000 & -1.796761000 \\
$\mathrm{C}$ & -5.623018000 & -1.627667000 & -0.991515000 \\
$\mathrm{H}$ & -6.394467000 & -1.476424000 & 1.013997000 \\
$\mathrm{H}$ & -4.529107000 & -1.771639000 & -2.838119000 \\
$\mathrm{H}$ & -6.599883000 & -1.692672000 & -1.460955000 \\
$\mathrm{H}$ & 0.303990000 & 0.559563000 & 2.018900000 \\
$\mathrm{H}$ & 0.051754000 & -1.849299000 & 2.671429000 \\
$\mathrm{C}$ & 5.954544000 & -1.414813000 & -1.604615000 \\
$\mathrm{H}$ & 6.079507000 & -2.194682000 & -2.364772000 \\
$\mathrm{H}$ & 6.111885000 & -0.450155000 & -2.102049000 \\
$\mathrm{H}$ & 6.750340000 & -1.540636000 & -0.864084000 \\
\hline-----
\end{tabular}

\begin{tabular}{lccc} 
& & & \\
& & & \\
A3 & & Y & Y \\
\hline $\mathrm{C}$ & -0.474748000 & -2.535288000 & 0.571386000 \\
$\mathrm{C}$ & -1.224036000 & -2.206766000 & 1.855099000 \\
$\mathrm{C}$ & -2.718850000 & -2.118523000 & 1.569821000 \\
$\mathrm{~N}$ & -0.803191000 & -1.526907000 & -0.403042000 \\
$\mathrm{H}$ & -0.733692000 & -3.554977000 & 0.235466000 \\
$\mathrm{H}$ & 0.606444000 & -2.508642000 & 0.739961000 \\
$\mathrm{H}$ & -0.859494000 & -1.248603000 & 2.234206000 \\
$\mathrm{H}$ & -1.046577000 & -2.967609000 & 2.622142000
\end{tabular}




\begin{tabular}{|c|c|c|c|}
\hline $\mathrm{H}$ & -3.157869000 & -3.129820000 & 1.569190000 \\
\hline $\mathrm{H}$ & -3.213046000 & -1.558543000 & 2.375976000 \\
\hline $\mathrm{N}$ & -3.025908000 & -1.469393000 & 0.307759000 \\
\hline $\mathrm{C}$ & -4.413395000 & -1.080401000 & 0.131073000 \\
\hline $\mathrm{C}$ & -4.753936000 & -0.972807000 & -1.348162000 \\
\hline $\mathrm{C}$ & -3.694559000 & -0.114399000 & -2.036741000 \\
\hline $\mathrm{N}$ & -2.347666000 & -0.583914000 & -1.798298000 \\
\hline $\mathrm{C}$ & -2.106621000 & -1.175198000 & -0.677019000 \\
\hline $\mathrm{H}$ & -4.597274000 & -0.121974000 & 0.640193000 \\
\hline $\mathrm{H}$ & -5.044049000 & -1.837530000 & 0.615678000 \\
\hline $\mathrm{H}$ & -4.758455000 & -1.974541000 & -1.794416000 \\
\hline $\mathrm{H}$ & -5.755200000 & -0.545257000 & -1.469532000 \\
\hline $\mathrm{H}$ & -3.869991000 & -0.102053000 & -3.119263000 \\
\hline $\mathrm{H}$ & -3.800315000 & 0.926365000 & -1.691078000 \\
\hline $\mathrm{C}$ & 5.525828000 & 1.091295000 & 0.218892000 \\
\hline $\mathrm{C}$ & 4.240304000 & 1.339780000 & -0.241397000 \\
\hline $\mathrm{C}$ & 3.369779000 & 0.284936000 & -0.535091000 \\
\hline $\mathrm{C}$ & 3.838780000 & -1.023213000 & -0.371887000 \\
\hline $\mathrm{C}$ & 5.129725000 & -1.265001000 & 0.083777000 \\
\hline $\mathrm{C}$ & 5.996588000 & -0.214263000 & 0.386309000 \\
\hline $\mathrm{H}$ & 6.183856000 & 1.928029000 & 0.440977000 \\
\hline $\mathrm{H}$ & 3.907627000 & 2.362448000 & -0.392310000 \\
\hline $\mathrm{H}$ & 3.176439000 & -1.860422000 & -0.576754000 \\
\hline $\mathrm{H}$ & 5.466288000 & -2.290716000 & 0.214222000 \\
\hline $\mathrm{C}$ & 1.989474000 & 0.530157000 & -1.013026000 \\
\hline $\mathrm{C}$ & 1.214907000 & 1.537974000 & -0.598892000 \\
\hline $\mathrm{H}$ & 1.623312000 & 2.189176000 & 0.170350000 \\
\hline $\mathrm{O}$ & 1.495511000 & -0.405563000 & -1.896473000 \\
\hline $\mathrm{C}$ & -0.174793000 & 1.831293000 & -1.079093000 \\
\hline $\mathrm{H}$ & -0.522950000 & 1.047163000 & -1.762075000 \\
\hline $\mathrm{C}$ & -1.206517000 & 1.963753000 & 0.024169000 \\
\hline $\mathrm{C}$ & -2.394003000 & 2.660685000 & -0.214121000 \\
\hline $\mathrm{C}$ & -1.035971000 & 1.360333000 & 1.269639000 \\
\hline $\mathrm{C}$ & -3.391752000 & 2.733987000 & 0.751927000 \\
\hline $\mathrm{H}$ & -2.541867000 & 3.141665000 & -1.178463000 \\
\hline $\mathrm{C}$ & -2.030552000 & 1.430040000 & 2.241702000 \\
\hline $\mathrm{H}$ & -0.118779000 & 0.813413000 & 1.467021000 \\
\hline $\mathrm{C}$ & -3.215549000 & 2.113365000 & 1.986946000 \\
\hline $\mathrm{H}$ & -4.309167000 & 3.276549000 & 0.540693000 \\
\hline $\mathrm{H}$ & -1.877198000 & 0.947692000 & 3.203970000 \\
\hline $\mathrm{H}$ & -3.990764000 & 2.170341000 & 2.745697000 \\
\hline $\mathrm{H}$ & -0.171594000 & -1.364356000 & -1.176505000 \\
\hline $\mathrm{H}$ & 2.229087000 & -0.759473000 & -2.414315000 \\
\hline $\mathrm{H}$ & -0.161163000 & 2.768758000 & -1.653988000 \\
\hline
\end{tabular}




\begin{tabular}{llll}
$\mathrm{C}$ & 7.407652000 & -0.474466000 & 0.848611000 \\
$\mathrm{H}$ & 7.500728000 & -1.460505000 & 1.313647000 \\
$\mathrm{H}$ & 8.109241000 & -0.439720000 & 0.006422000 \\
$\mathrm{H}$ & 7.733158000 & 0.275508000 & 1.576220000 \\
\hline
\end{tabular}

\begin{tabular}{|c|c|c|c|}
\hline \multicolumn{4}{|c|}{$\mathbf{A} 1_{\text {DBU }}$} \\
\hline & \multicolumn{3}{|c|}{ Coordinates (Angstroms) } \\
\hline & $X$ & Y & Z \\
\hline $\bar{C}$ & -1.428262000 & 3.893581000 & -0.789990000 \\
\hline $\mathrm{C}$ & -1.712652000 & 2.535781000 & -0.676294000 \\
\hline $\mathrm{C}$ & -0.715235000 & 1.626393000 & -0.322539000 \\
\hline $\mathrm{C}$ & 0.574847000 & 2.107900000 & -0.090252000 \\
\hline $\mathrm{C}$ & 0.853310000 & 3.464303000 & -0.201546000 \\
\hline $\mathrm{C}$ & -0.142769000 & 4.380904000 & -0.550381000 \\
\hline $\mathrm{H}$ & -2.219782000 & 4.586448000 & -1.066447000 \\
\hline $\mathrm{H}$ & -2.724157000 & 2.178935000 & -0.857554000 \\
\hline $\mathrm{H}$ & 1.352520000 & 1.397826000 & 0.179661000 \\
\hline $\mathrm{H}$ & 1.864018000 & 3.821856000 & -0.015178000 \\
\hline $\mathrm{C}$ & -0.983889000 & 0.129988000 & -0.274244000 \\
\hline $\mathrm{C}$ & -2.373412000 & -0.183845000 & 0.218588000 \\
\hline $\mathrm{H}$ & -2.635489000 & 0.283377000 & 1.167217000 \\
\hline $\mathrm{O}$ & -0.709616000 & -0.462857000 & -1.542067000 \\
\hline $\mathrm{C}$ & -3.245197000 & -0.958865000 & -0.429003000 \\
\hline $\mathrm{H}$ & -2.929243000 & -1.388006000 & -1.378332000 \\
\hline $\mathrm{C}$ & -4.613728000 & -1.292430000 & 0.002892000 \\
\hline $\mathrm{C}$ & -5.509092000 & -1.827361000 & -0.931157000 \\
\hline $\mathrm{C}$ & -5.068668000 & -1.098063000 & 1.314138000 \\
\hline $\mathrm{C}$ & -6.818646000 & -2.136760000 & -0.579437000 \\
\hline $\mathrm{H}$ & -5.170963000 & -1.995729000 & -1.950881000 \\
\hline $\mathrm{C}$ & -6.375942000 & -1.406344000 & 1.668296000 \\
\hline $\mathrm{H}$ & -4.387959000 & -0.716280000 & 2.069487000 \\
\hline $\mathrm{C}$ & -7.259374000 & -1.923888000 & 0.723005000 \\
\hline $\mathrm{H}$ & -7.495620000 & -2.544932000 & -1.324485000 \\
\hline $\mathrm{H}$ & -6.706085000 & -1.248740000 & 2.691170000 \\
\hline $\mathrm{H}$ & -8.280904000 & -2.164669000 & 1.001971000 \\
\hline $\mathrm{H}$ & -0.250566000 & -0.323429000 & 0.405812000 \\
\hline $\mathrm{H}$ & -1.115644000 & 0.103357000 & -2.211433000 \\
\hline $\mathrm{C}$ & 5.377212000 & -1.640224000 & 0.047001000 \\
\hline $\mathrm{C}$ & 5.057763000 & -2.871632000 & -0.794964000 \\
\hline $\mathrm{C}$ & 2.443603000 & -1.683253000 & -0.850455000 \\
\hline $\mathrm{C}$ & 4.450088000 & -2.539379000 & -2.159993000 \\
\hline $\mathrm{C}$ & 3.363037000 & -1.467177000 & -2.054279000 \\
\hline $\mathrm{H}$ & 5.889415000 & -0.888284000 & -0.575984000 \\
\hline
\end{tabular}




\begin{tabular}{|c|c|c|c|}
\hline $\mathrm{H}$ & 2.252357000 & -2.755457000 & -0.709859000 \\
\hline $\mathrm{H}$ & 4.393502000 & -3.526328000 & -0.219658000 \\
\hline $\mathrm{H}$ & 4.027897000 & -3.454292000 & -2.593286000 \\
\hline $\mathrm{H}$ & 6.096579000 & -1.936577000 & 0.821540000 \\
\hline $\mathrm{H}$ & 5.990226000 & -3.429642000 & -0.936295000 \\
\hline $\mathrm{H}$ & 1.468153000 & -1.229813000 & -1.035622000 \\
\hline $\mathrm{H}$ & 5.238712000 & -2.203076000 & -2.844571000 \\
\hline $\mathrm{H}$ & 2.762205000 & -1.460372000 & -2.969621000 \\
\hline $\mathrm{H}$ & 3.821119000 & -0.472339000 & -1.983998000 \\
\hline $\mathrm{C}$ & 2.916812000 & -1.088267000 & 0.465930000 \\
\hline $\mathrm{N}$ & 1.977572000 & -0.651055000 & 1.234005000 \\
\hline $\mathrm{N}$ & 4.263781000 & -1.018387000 & 0.760728000 \\
\hline $\mathrm{C}$ & 4.725607000 & -0.208184000 & 1.885191000 \\
\hline $\mathrm{H}$ & 5.062617000 & -0.870991000 & 2.696928000 \\
\hline $\mathrm{H}$ & 5.602671000 & 0.365720000 & 1.556367000 \\
\hline $\mathrm{C}$ & 3.643112000 & 0.730326000 & 2.389943000 \\
\hline $\mathrm{H}$ & 3.946959000 & 1.165548000 & 3.347286000 \\
\hline $\mathrm{H}$ & 3.500399000 & 1.553399000 & 1.679195000 \\
\hline $\mathrm{C}$ & 2.343670000 & -0.058187000 & 2.504118000 \\
\hline $\mathrm{H}$ & 1.523397000 & 0.592045000 & 2.827480000 \\
\hline $\mathrm{H}$ & 2.451834000 & -0.840474000 & 3.271796000 \\
\hline $\mathrm{C}$ & 0.156892000 & 5.856736000 & -0.634215000 \\
\hline $\mathrm{H}$ & -0.508890000 & 6.360803000 & -1.341679000 \\
\hline $\mathrm{H}$ & 0.024825000 & 6.339547000 & 0.341757000 \\
\hline$\underline{\mathrm{H}}$ & 1.188848000 & 6.037203000 & -0.951496000 \\
\hline
\end{tabular}

TSA1-2

\begin{tabular}{lrcr} 
& \multicolumn{3}{c}{ Coordinates (Angstroms) } \\
& $\mathrm{X}$ & $\mathrm{Y}$ & $\mathrm{Z}$ \\
$\mathrm{C}$ & 1.808171000 & 2.025662000 & 1.758064000 \\
$\mathrm{C}$ & 0.703179000 & 1.507935000 & 1.091806000 \\
$\mathrm{C}$ & 0.431367000 & 1.828267000 & -0.255468000 \\
$\mathrm{C}$ & 1.359764000 & 2.682229000 & -0.889007000 \\
$\mathrm{C}$ & 2.462396000 & 3.186510000 & -0.215334000 \\
$\mathrm{C}$ & 2.719801000 & 2.870365000 & 1.122907000 \\
$\mathrm{H}$ & 1.965995000 & 1.761998000 & 2.802931000 \\
$\mathrm{H}$ & 0.025417000 & 0.853621000 & 1.636152000 \\
$\mathrm{H}$ & 1.192942000 & 2.938666000 & -1.930014000 \\
$\mathrm{H}$ & 3.143849000 & 3.851308000 & -0.744463000 \\
$\mathrm{C}$ & -0.667630000 & 1.216868000 & -0.995566000 \\
$\mathrm{C}$ & -1.928658000 & 0.948093000 & -0.382265000 \\
$\mathrm{H}$ & -1.933683000 & 0.991554000 & 0.707556000 \\
$\mathrm{O}$ & -0.692570000 & 1.479973000 & -2.381692000 \\
& & &
\end{tabular}




\begin{tabular}{|c|c|c|c|}
\hline $\mathrm{C}$ & -3.084082000 & 0.608080000 & -1.020178000 \\
\hline $\mathrm{H}$ & -3.060393000 & 0.504739000 & -2.103497000 \\
\hline $\mathrm{C}$ & -4.348655000 & 0.282098000 & -0.371550000 \\
\hline $\mathrm{C}$ & -5.389468000 & -0.286673000 & -1.129454000 \\
\hline $\mathrm{C}$ & -4.602730000 & 0.492025000 & 0.997841000 \\
\hline $\mathrm{C}$ & -6.603549000 & -0.640478000 & -0.555134000 \\
\hline $\mathrm{H}$ & -5.229220000 & -0.455641000 & -2.192072000 \\
\hline $\mathrm{C}$ & -5.814161000 & 0.131409000 & 1.573303000 \\
\hline $\mathrm{H}$ & -3.847667000 & 0.964863000 & 1.620143000 \\
\hline $\mathrm{C}$ & -6.826714000 & -0.440081000 & 0.805072000 \\
\hline $\mathrm{H}$ & -7.381984000 & -1.077382000 & -1.175649000 \\
\hline $\mathrm{H}$ & -5.974571000 & 0.310663000 & 2.633612000 \\
\hline $\mathrm{H}$ & -7.775505000 & -0.713630000 & 1.257069000 \\
\hline $\mathrm{H}$ & -0.155172000 & -0.398367000 & -0.915642000 \\
\hline $\mathrm{H}$ & -1.357707000 & 2.169858000 & -2.533336000 \\
\hline $\mathrm{C}$ & 3.507968000 & -2.681527000 & 0.512920000 \\
\hline $\mathrm{C}$ & 4.382627000 & -2.519653000 & -0.725101000 \\
\hline $\mathrm{C}$ & 2.315502000 & -0.672206000 & -1.457221000 \\
\hline $\mathrm{C}$ & 4.786988000 & -1.070789000 & -1.011029000 \\
\hline $\mathrm{C}$ & 3.610504000 & -0.100768000 & -0.880030000 \\
\hline $\mathrm{H}$ & 3.910587000 & -2.084675000 & 1.343492000 \\
\hline $\mathrm{H}$ & 2.510208000 & -1.263228000 & -2.361598000 \\
\hline $\mathrm{H}$ & 3.867101000 & -2.963705000 & -1.584364000 \\
\hline $\mathrm{H}$ & 5.206893000 & -1.017136000 & -2.022449000 \\
\hline $\mathrm{H}$ & 3.544917000 & -3.730078000 & 0.828413000 \\
\hline $\mathrm{H}$ & 5.281388000 & -3.125858000 & -0.568285000 \\
\hline $\mathrm{H}$ & 1.651025000 & 0.135244000 & -1.771580000 \\
\hline $\mathrm{H}$ & 5.587124000 & -0.769822000 & -0.324604000 \\
\hline $\mathrm{H}$ & 3.834518000 & 0.839903000 & -1.389057000 \\
\hline $\mathrm{H}$ & 3.438183000 & 0.173543000 & 0.167016000 \\
\hline $\mathrm{C}$ & 1.513431000 & -1.526109000 & -0.505135000 \\
\hline $\mathrm{N}$ & 0.208196000 & -1.399219000 & -0.603241000 \\
\hline $\mathrm{N}$ & 2.075664000 & -2.377129000 & 0.370098000 \\
\hline $\mathrm{C}$ & 1.269213000 & -3.032460000 & 1.410700000 \\
\hline $\mathrm{H}$ & 1.140972000 & -4.088723000 & 1.139668000 \\
\hline $\mathrm{H}$ & 1.848317000 & -2.998940000 & 2.339643000 \\
\hline $\mathrm{C}$ & -0.077924000 & -2.359165000 & 1.606106000 \\
\hline $\mathrm{H}$ & -0.709736000 & -2.982000000 & 2.244730000 \\
\hline $\mathrm{H}$ & 0.057643000 & -1.391854000 & 2.100386000 \\
\hline $\mathrm{C}$ & -0.712304000 & -2.143074000 & 0.241813000 \\
\hline $\mathrm{H}$ & -1.637704000 & -1.566565000 & 0.298562000 \\
\hline $\mathrm{H}$ & -0.943639000 & -3.102697000 & -0.235880000 \\
\hline $\mathrm{C}$ & 3.942536000 & 3.396621000 & 1.831767000 \\
\hline $\mathrm{H}$ & 3.864820000 & 3.256343000 & 2.914990000 \\
\hline
\end{tabular}




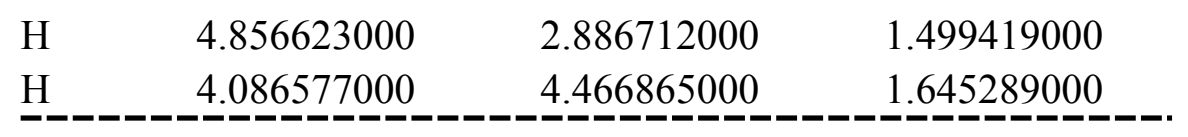

\begin{tabular}{|c|c|c|c|}
\hline \multicolumn{4}{|c|}{$\mathbf{A} 2_{\mathrm{DBU}}$} \\
\hline & \multicolumn{3}{|c|}{ Coordinates (Angstroms) } \\
\hline & $X$ & $\mathrm{Y}$ & Z \\
\hline$\overline{\mathrm{C}}$ & -2.765500000 & -2.216581000 & 1.041936000 \\
\hline $\mathrm{C}$ & -1.535516000 & -1.983911000 & 0.444522000 \\
\hline $\mathrm{C}$ & -1.429049000 & -1.510351000 & -0.883003000 \\
\hline $\mathrm{C}$ & -2.649271000 & -1.350886000 & -1.577720000 \\
\hline $\mathrm{C}$ & -3.874181000 & -1.576526000 & -0.964433000 \\
\hline $\mathrm{C}$ & -3.969381000 & -1.999564000 & 0.364008000 \\
\hline $\mathrm{H}$ & -2.790912000 & -2.583204000 & 2.067535000 \\
\hline $\mathrm{H}$ & -0.638000000 & -2.186442000 & 1.020889000 \\
\hline $\mathrm{H}$ & -2.615053000 & -1.024585000 & -2.611479000 \\
\hline $\mathrm{H}$ & -4.786214000 & -1.421485000 & -1.538602000 \\
\hline $\mathrm{C}$ & -0.178592000 & -1.120679000 & -1.496123000 \\
\hline $\mathrm{C}$ & 1.070877000 & -1.165898000 & -0.921452000 \\
\hline $\mathrm{H}$ & 1.133107000 & -1.687755000 & 0.030708000 \\
\hline $\mathrm{O}$ & -0.303741000 & -0.533181000 & -2.762267000 \\
\hline $\mathrm{C}$ & 2.243766000 & -0.556971000 & -1.388693000 \\
\hline $\mathrm{H}$ & 2.216026000 & -0.018829000 & -2.335465000 \\
\hline $\mathrm{C}$ & 3.530540000 & -0.620206000 & -0.731757000 \\
\hline $\mathrm{C}$ & 4.594354000 & 0.197265000 & -1.183033000 \\
\hline $\mathrm{C}$ & 3.805763000 & -1.424502000 & 0.400986000 \\
\hline $\mathrm{C}$ & 5.822065000 & 0.229741000 & -0.540810000 \\
\hline $\mathrm{H}$ & 4.431533000 & 0.824095000 & -2.057903000 \\
\hline $\mathrm{C}$ & 5.036379000 & -1.381132000 & 1.045673000 \\
\hline $\mathrm{H}$ & 3.049724000 & -2.114200000 & 0.767977000 \\
\hline $\mathrm{C}$ & 6.059113000 & -0.551509000 & 0.590862000 \\
\hline $\mathrm{H}$ & 6.605715000 & 0.878292000 & -0.925450000 \\
\hline $\mathrm{H}$ & 5.202389000 & -2.019687000 & 1.910526000 \\
\hline $\mathrm{H}$ & 7.020255000 & -0.524668000 & 1.094727000 \\
\hline $\mathrm{H}$ & 1.810959000 & 1.134671000 & -0.158539000 \\
\hline $\mathrm{H}$ & 0.402771000 & -0.901166000 & -3.309131000 \\
\hline $\mathrm{C}$ & -2.429167000 & 1.689860000 & 1.075799000 \\
\hline $\mathrm{C}$ & -2.686713000 & 3.049820000 & 0.426897000 \\
\hline $\mathrm{C}$ & -0.296614000 & 2.119355000 & -1.066038000 \\
\hline $\mathrm{C}$ & -2.700247000 & 3.012668000 & -1.105925000 \\
\hline $\mathrm{C}$ & -1.691815000 & 2.016349000 & -1.670854000 \\
\hline $\mathrm{H}$ & -2.927638000 & 0.867218000 & 0.548756000 \\
\hline $\mathrm{H}$ & 0.057411000 & 3.159629000 & -1.070636000 \\
\hline $\mathrm{H}$ & -1.922891000 & 3.748600000 & 0.790550000 \\
\hline
\end{tabular}




$\begin{array}{lrrr}\mathrm{H} & -2.504045000 & 4.020924000 & -1.490539000 \\ \mathrm{H} & -2.830247000 & 1.704243000 & 2.092697000 \\ \mathrm{H} & -3.644681000 & 3.430934000 & 0.796436000 \\ \mathrm{H} & 0.393826000 & 1.552069000 & -1.703918000 \\ \mathrm{H} & -3.699757000 & 2.732964000 & -1.458388000 \\ \mathrm{H} & -1.591022000 & 2.155626000 & -2.750927000 \\ \mathrm{H} & -2.058599000 & 0.997213000 & -1.543870000 \\ \mathrm{C} & -0.058766000 & 1.594402000 & 0.328274000 \\ \mathrm{~N} & 1.232927000 & 1.457461000 & 0.633228000 \\ \mathrm{~N} & -0.999835000 & 1.365535000 & 1.238363000 \\ \mathrm{C} & -0.700060000 & 0.696925000 & 2.515239000 \\ \mathrm{H} & -0.810148000 & 1.441016000 & 3.315037000 \\ \mathrm{H} & -1.465850000 & -0.071630000 & 2.653617000 \\ \mathrm{C} & 0.683062000 & 0.071125000 & 2.550837000 \\ \mathrm{H} & 0.940783000 & -0.172428000 & 3.585123000 \\ \mathrm{H} & 0.691371000 & -0.855630000 & 1.974398000 \\ \mathrm{C} & 1.691859000 & 1.035614000 & 1.948880000 \\ \mathrm{H} & 2.669573000 & 0.567724000 & 1.820993000 \\ \mathrm{H} & 1.810783000 & 1.921387000 & 2.584354000 \\ \mathrm{C} & -5.302814000 & -2.262304000 & 1.017079000 \\ \mathrm{H} & -6.073560000 & -1.580261000 & 0.641730000 \\ \mathrm{H} & -5.652975000 & -3.284528000 & 0.824753000 \\ \mathrm{H} & -5.246902000 & -2.138245000 & 2.104127000 \\ --------1 .-1\end{array}$

TSA2-3 ${ }_{\text {DBU }}$

\begin{tabular}{|c|c|c|c|}
\hline \multicolumn{4}{|c|}{ Coordinates (Angstroms) } \\
\hline & $X$ & Y & Z \\
\hline $\mathrm{C}$ & -2.923580000 & -1.618847000 & 1.574063000 \\
\hline $\mathrm{C}$ & -1.701763000 & -1.431774000 & 0.941462000 \\
\hline $\mathrm{C}$ & -1.532000000 & -1.724636000 & -0.422871000 \\
\hline $\mathrm{C}$ & -2.658708000 & -2.188115000 & -1.121401000 \\
\hline $\mathrm{C}$ & -3.880243000 & -2.358284000 & -0.483905000 \\
\hline $\mathrm{C}$ & -4.042745000 & -2.078016000 & 0.874900000 \\
\hline $\mathrm{H}$ & -3.013683000 & -1.392239000 & 2.635003000 \\
\hline $\mathrm{H}$ & -0.867146000 & -1.037091000 & 1.513423000 \\
\hline $\mathrm{H}$ & -2.561334000 & -2.405236000 & -2.179341000 \\
\hline $\mathrm{H}$ & -4.730651000 & -2.722262000 & -1.056966000 \\
\hline $\mathrm{C}$ & -0.271185000 & -1.477855000 & -1.120939000 \\
\hline $\mathrm{C}$ & 0.941094000 & -1.310736000 & -0.537268000 \\
\hline $\mathrm{H}$ & 0.980179000 & -1.528127000 & 0.527095000 \\
\hline $\mathrm{O}$ & -0.419152000 & -1.332566000 & -2.496711000 \\
\hline $\mathrm{C}$ & 2.120102000 & -0.742141000 & -1.129995000 \\
\hline $\mathrm{H}$ & 2.130338000 & -0.610368000 & -2.214446000 \\
\hline
\end{tabular}




\begin{tabular}{|c|c|c|c|}
\hline $\mathrm{C}$ & 3.446682000 & -0.865383000 & -0.527808000 \\
\hline $\mathrm{C}$ & 4.557072000 & -0.263256000 & -1.157669000 \\
\hline $\mathrm{C}$ & 3.690771000 & -1.484074000 & 0.715811000 \\
\hline $\mathrm{C}$ & 5.815471000 & -0.255340000 & -0.574811000 \\
\hline $\mathrm{H}$ & 4.410023000 & 0.219361000 & -2.122001000 \\
\hline $\mathrm{C}$ & 4.951599000 & -1.464949000 & 1.302977000 \\
\hline $\mathrm{H}$ & 2.886297000 & -2.005986000 & 1.226642000 \\
\hline $\mathrm{C}$ & 6.026940000 & -0.848099000 & 0.670197000 \\
\hline $\mathrm{H}$ & 6.642106000 & 0.222249000 & -1.095299000 \\
\hline $\mathrm{H}$ & 5.096087000 & -1.953699000 & 2.263656000 \\
\hline $\mathrm{H}$ & 7.010653000 & -0.839451000 & 1.129538000 \\
\hline $\mathrm{H}$ & 1.746239000 & 0.775182000 & -0.703660000 \\
\hline $\mathrm{H}$ & 0.411890000 & -1.619068000 & -2.898053000 \\
\hline $\mathrm{C}$ & -2.173161000 & 2.403788000 & 0.847492000 \\
\hline $\mathrm{C}$ & -2.577158000 & 3.326600000 & -0.300387000 \\
\hline $\mathrm{C}$ & -0.492759000 & 1.771384000 & -1.625830000 \\
\hline $\mathrm{C}$ & -2.913594000 & 2.580738000 & -1.595297000 \\
\hline $\mathrm{C}$ & -1.960282000 & 1.413829000 & -1.844269000 \\
\hline $\mathrm{H}$ & -2.796636000 & 1.500182000 & 0.875943000 \\
\hline $\mathrm{H}$ & -0.254553000 & 2.740761000 & -2.084789000 \\
\hline $\mathrm{H}$ & -1.766440000 & 4.047069000 & -0.464797000 \\
\hline $\mathrm{H}$ & -2.881974000 & 3.288434000 & -2.432626000 \\
\hline $\mathrm{H}$ & -2.333999000 & 2.931713000 & 1.793199000 \\
\hline $\mathrm{H}$ & -3.443001000 & 3.913852000 & 0.024136000 \\
\hline $\mathrm{H}$ & 0.133154000 & 1.033970000 & -2.140193000 \\
\hline $\mathrm{H}$ & -3.939724000 & 2.198141000 & -1.545214000 \\
\hline $\mathrm{H}$ & -2.072794000 & 1.042377000 & -2.866579000 \\
\hline $\mathrm{H}$ & -2.222889000 & 0.569561000 & -1.201707000 \\
\hline $\mathrm{C}$ & 0.025535000 & 1.809958000 & -0.207757000 \\
\hline $\mathrm{N}$ & 1.335935000 & 1.666470000 & -0.105455000 \\
\hline $\mathrm{N}$ & -0.755238000 & 2.013155000 & 0.868261000 \\
\hline $\mathrm{C}$ & -0.255272000 & 1.793248000 & 2.232505000 \\
\hline $\mathrm{H}$ & -0.224561000 & 2.764818000 & 2.743883000 \\
\hline $\mathrm{H}$ & -0.993502000 & 1.171055000 & 2.751607000 \\
\hline $\mathrm{C}$ & 1.114403000 & 1.136570000 & 2.265147000 \\
\hline $\mathrm{H}$ & 1.551110000 & 1.255255000 & 3.260766000 \\
\hline $\mathrm{H}$ & 1.036221000 & 0.064849000 & 2.062053000 \\
\hline $\mathrm{C}$ & 1.983977000 & 1.777076000 & 1.193456000 \\
\hline $\mathrm{H}$ & 2.956212000 & 1.286855000 & 1.118608000 \\
\hline $\mathrm{H}$ & 2.152309000 & 2.837942000 & 1.417921000 \\
\hline $\mathrm{C}$ & -5.381403000 & -2.237332000 & 1.550914000 \\
\hline $\mathrm{H}$ & -6.012427000 & -1.351468000 & 1.404808000 \\
\hline $\mathrm{H}$ & -5.929395000 & -3.095971000 & 1.149484000 \\
\hline $\mathrm{H}$ & -5.268518000 & -2.385637000 & 2.629655000 \\
\hline
\end{tabular}




\begin{tabular}{|c|c|c|c|}
\hline \multicolumn{4}{|c|}{$\mathrm{A} 3_{\mathrm{DBU}}$} \\
\hline & $\mathrm{X}$ & Y & Z \\
\hline $\mathrm{C}$ & 2.809871000 & -1.642779000 & -1.621844000 \\
\hline $\mathrm{C}$ & 1.635893000 & -1.399499000 & -0.921629000 \\
\hline $\mathrm{C}$ & 1.523582000 & -1.745775000 & 0.428762000 \\
\hline $\mathrm{C}$ & 2.629551000 & -2.321842000 & 1.059513000 \\
\hline $\mathrm{C}$ & 3.802663000 & -2.558620000 & 0.353444000 \\
\hline $\mathrm{C}$ & 3.916296000 & -2.223912000 & -0.997142000 \\
\hline $\mathrm{H}$ & 2.872548000 & -1.364471000 & -2.671476000 \\
\hline $\mathrm{H}$ & 0.803979000 & -0.912419000 & -1.420108000 \\
\hline $\mathrm{H}$ & 2.564831000 & -2.579748000 & 2.110999000 \\
\hline $\mathrm{H}$ & 4.648948000 & -3.013174000 & 0.863062000 \\
\hline $\mathrm{C}$ & 0.287705000 & -1.462886000 & 1.187109000 \\
\hline $\mathrm{C}$ & -0.933066000 & -1.351201000 & 0.649771000 \\
\hline $\mathrm{H}$ & -1.049964000 & -1.542529000 & -0.410513000 \\
\hline $\mathrm{O}$ & 0.529690000 & -1.298696000 & 2.524529000 \\
\hline $\mathrm{C}$ & -2.154947000 & -0.934689000 & 1.429245000 \\
\hline $\mathrm{H}$ & -2.014591000 & 0.088500000 & 1.803558000 \\
\hline $\mathrm{C}$ & -3.429671000 & -0.966914000 & 0.616767000 \\
\hline $\mathrm{C}$ & -4.274607000 & 0.142738000 & 0.584427000 \\
\hline $\mathrm{C}$ & -3.795298000 & -2.109451000 & -0.099665000 \\
\hline $\mathrm{C}$ & -5.458776000 & 0.113274000 & -0.148176000 \\
\hline $\mathrm{H}$ & -3.985607000 & 1.041524000 & 1.123175000 \\
\hline $\mathrm{C}$ & -4.976454000 & -2.141907000 & -0.833851000 \\
\hline $\mathrm{H}$ & -3.145648000 & -2.981742000 & -0.080774000 \\
\hline $\mathrm{C}$ & -5.813433000 & -1.028772000 & -0.859832000 \\
\hline $\mathrm{H}$ & -6.103093000 & 0.987873000 & -0.164960000 \\
\hline $\mathrm{H}$ & -5.245133000 & -3.039060000 & -1.384456000 \\
\hline $\mathrm{H}$ & -6.736518000 & -1.052240000 & -1.431718000 \\
\hline $\mathrm{H}$ & -0.312581000 & -1.174930000 & 2.979225000 \\
\hline $\mathrm{C}$ & 2.134586000 & 2.338128000 & -0.961058000 \\
\hline $\mathrm{C}$ & 2.715430000 & 3.267993000 & 0.102743000 \\
\hline $\mathrm{C}$ & 0.579191000 & 2.070361000 & 1.667735000 \\
\hline $\mathrm{C}$ & 3.068030000 & 2.545814000 & 1.405569000 \\
\hline $\mathrm{C}$ & 2.000238000 & 1.522942000 & 1.789597000 \\
\hline $\mathrm{H}$ & 2.695609000 & 1.390734000 & -0.993019000 \\
\hline $\mathrm{H}$ & 0.530374000 & 3.084165000 & 2.087789000 \\
\hline $\mathrm{H}$ & 1.992484000 & 4.071795000 & 0.287328000 \\
\hline $\mathrm{H}$ & 3.190941000 & 3.286196000 & 2.206187000 \\
\hline $\mathrm{H}$ & 2.273911000 & 2.812920000 & -1.940737000 \\
\hline $\mathrm{H}$ & 3.611043000 & 3.747326000 & -0.307979000 \\
\hline
\end{tabular}




\begin{tabular}{|c|c|c|c|}
\hline $\mathrm{H}$ & -0.099700000 & 1.468080000 & 2.276138000 \\
\hline $\mathrm{H}$ & 4.033572000 & 2.036916000 & 1.295693000 \\
\hline $\mathrm{H}$ & 2.163126000 & 1.182844000 & 2.817206000 \\
\hline $\mathrm{H}$ & 2.102093000 & 0.630653000 & 1.166062000 \\
\hline $\mathrm{C}$ & -0.072834000 & 2.103103000 & 0.290278000 \\
\hline $\mathrm{N}$ & -1.360353000 & 2.151081000 & 0.299346000 \\
\hline $\mathrm{N}$ & 0.701408000 & 2.075996000 & -0.854489000 \\
\hline $\mathrm{C}$ & 0.094666000 & 1.786169000 & -2.150392000 \\
\hline $\mathrm{H}$ & 0.158105000 & 2.684202000 & -2.784578000 \\
\hline $\mathrm{H}$ & 0.691397000 & 1.007303000 & -2.647336000 \\
\hline $\mathrm{C}$ & -1.355284000 & 1.353301000 & -2.017236000 \\
\hline $\mathrm{H}$ & -1.847567000 & 1.404295000 & -2.993664000 \\
\hline $\mathrm{H}$ & -1.423614000 & 0.319852000 & -1.661183000 \\
\hline $\mathrm{C}$ & -2.018111000 & 2.262056000 & -0.988807000 \\
\hline $\mathrm{H}$ & -3.073865000 & 2.003980000 & -0.870838000 \\
\hline $\mathrm{H}$ & -1.977883000 & 3.304693000 & -1.345232000 \\
\hline $\mathrm{H}$ & -2.295490000 & -1.590576000 & 2.305729000 \\
\hline $\mathrm{C}$ & 5.204358000 & -2.449328000 & -1.747621000 \\
\hline $\mathrm{H}$ & 5.865707000 & -1.578207000 & -1.664558000 \\
\hline $\mathrm{H}$ & 5.748675000 & -3.312619000 & -1.352709000 \\
\hline $\mathrm{H}$ & 5.021524000 & -2.621923000 & -2.812765000 \\
\hline
\end{tabular}

\begin{tabular}{lrrr} 
A1 & \multicolumn{3}{c}{ Coordinates (Angstroms) } \\
& X & Y & \\
\hline $\mathrm{C}$ & -1.364263000 & -2.651570000 & -1.482327000 \\
$\mathrm{C}$ & -1.020200000 & -1.375208000 & -2.242205000 \\
$\mathrm{C}$ & 0.487930000 & -1.315188000 & -2.436691000 \\
$\mathrm{~N}$ & -0.681579000 & -2.719504000 & -0.210022000 \\
$\mathrm{H}$ & -1.110345000 & -3.524981000 & -2.106850000 \\
$\mathrm{H}$ & -2.443867000 & -2.700507000 & -1.301679000 \\
$\mathrm{H}$ & -1.371279000 & -0.516478000 & -1.659242000 \\
$\mathrm{H}$ & -1.516856000 & -1.333656000 & -3.217224000 \\
$\mathrm{H}$ & 0.785774000 & -1.934263000 & -3.296278000 \\
$\mathrm{H}$ & 0.802204000 & -0.285318000 & -2.662821000 \\
$\mathrm{~N}$ & 1.219149000 & -1.759963000 & -1.256482000 \\
$\mathrm{C}$ & 2.596457000 & -1.295773000 & -1.222765000 \\
$\mathrm{C}$ & 3.378671000 & -1.932835000 & -0.091015000 \\
$\mathrm{C}$ & 2.553881000 & -1.844048000 & 1.179922000 \\
$\mathrm{~N}$ & 1.281031000 & -2.503959000 & 0.978832000 \\
$\mathrm{C}$ & 0.551246000 & -2.326849000 & -0.185406000 \\
$\mathrm{H}$ & 2.617179000 & -0.200967000 & -1.115821000 \\
$\mathrm{H}$ & 3.059745000 & -1.533060000 & -2.189979000
\end{tabular}




\begin{tabular}{|c|c|c|c|}
\hline $\mathrm{H}$ & 3.585618000 & -2.985381000 & -0.313220000 \\
\hline $\mathrm{H}$ & 4.332236000 & -1.410191000 & 0.031020000 \\
\hline $\mathrm{H}$ & 3.065764000 & -2.354277000 & 2.002992000 \\
\hline $\mathrm{H}$ & 2.421561000 & -0.791828000 & 1.476060000 \\
\hline $\mathrm{C}$ & -4.002687000 & 2.211777000 & -0.075632000 \\
\hline $\mathrm{C}$ & -2.759293000 & 1.901082000 & 0.465046000 \\
\hline $\mathrm{C}$ & -2.437421000 & 0.584581000 & 0.796096000 \\
\hline $\mathrm{C}$ & -3.381491000 & -0.415361000 & 0.560988000 \\
\hline $\mathrm{C}$ & -4.619734000 & -0.102679000 & 0.012681000 \\
\hline $\mathrm{C}$ & -4.951530000 & 1.215288000 & -0.312582000 \\
\hline $\mathrm{H}$ & -4.237310000 & 3.244401000 & -0.323514000 \\
\hline $\mathrm{H}$ & -2.025151000 & 2.689886000 & 0.620705000 \\
\hline $\mathrm{H}$ & -3.128714000 & -1.445820000 & 0.798412000 \\
\hline $\mathrm{H}$ & -5.343313000 & -0.894816000 & -0.165490000 \\
\hline $\mathrm{C}$ & -1.100064000 & 0.235407000 & 1.422888000 \\
\hline $\mathrm{C}$ & 0.046136000 & 0.853351000 & 0.666072000 \\
\hline $\mathrm{H}$ & 0.015519000 & 0.710381000 & -0.412774000 \\
\hline $\mathrm{O}$ & -1.059791000 & 0.528491000 & 2.811084000 \\
\hline $\mathrm{C}$ & 1.084841000 & 1.479378000 & 1.221410000 \\
\hline $\mathrm{H}$ & 1.135587000 & 1.545919000 & 2.306971000 \\
\hline $\mathrm{C}$ & 2.235221000 & 1.992417000 & 0.455548000 \\
\hline $\mathrm{C}$ & 3.519838000 & 1.927327000 & 1.007812000 \\
\hline $\mathrm{C}$ & 2.094806000 & 2.496521000 & -0.843851000 \\
\hline $\mathrm{C}$ & 4.634845000 & 2.312938000 & 0.270964000 \\
\hline $\mathrm{H}$ & 3.643914000 & 1.548274000 & 2.019455000 \\
\hline $\mathrm{C}$ & 3.208090000 & 2.885168000 & -1.580663000 \\
\hline $\mathrm{H}$ & 1.101749000 & 2.591171000 & -1.274777000 \\
\hline $\mathrm{C}$ & 4.483543000 & 2.787989000 & -1.029118000 \\
\hline $\mathrm{H}$ & 5.624017000 & 2.241918000 & 0.713927000 \\
\hline $\mathrm{H}$ & 3.078435000 & 3.274692000 & -2.586339000 \\
\hline $\mathrm{H}$ & 5.352724000 & 3.091868000 & -1.604721000 \\
\hline $\mathrm{H}$ & -0.990559000 & -0.851082000 & 1.351671000 \\
\hline $\mathrm{H}$ & -1.328778000 & 1.450409000 & 2.918064000 \\
\hline $\mathrm{C}$ & 0.592284000 & -2.943888000 & 2.174550000 \\
\hline $\mathrm{H}$ & 0.253064000 & -2.096721000 & 2.789696000 \\
\hline $\mathrm{H}$ & -0.274710000 & -3.534993000 & 1.885105000 \\
\hline $\mathrm{H}$ & 1.274017000 & -3.556502000 & 2.775291000 \\
\hline $\mathrm{C}$ & -6.285573000 & 1.543061000 & -0.935345000 \\
\hline $\mathrm{H}$ & -6.260773000 & 1.384343000 & -2.020250000 \\
\hline $\mathrm{H}$ & -7.080529000 & 0.908922000 & -0.530373000 \\
\hline $\mathrm{H}$ & -6.561997000 & 2.587393000 & -0.761249000 \\
\hline
\end{tabular}

TSA1-2 


\begin{tabular}{|c|c|c|c|}
\hline \multicolumn{4}{|c|}{ Coordinates (Angstroms) } \\
\hline & $\mathrm{X}$ & Y & Z \\
\hline $\mathrm{C}$ & 0.950073000 & -1.354734000 & 1.852326000 \\
\hline $\mathrm{C}$ & -0.072754000 & -1.395208000 & 2.977569000 \\
\hline $\mathrm{C}$ & -1.331930000 & -0.686893000 & 2.508521000 \\
\hline $\mathrm{N}$ & 0.372335000 & -1.675260000 & 0.549998000 \\
\hline $\mathrm{H}$ & 1.768772000 & -2.055514000 & 2.044395000 \\
\hline $\mathrm{H}$ & 1.400427000 & -0.356441000 & 1.793742000 \\
\hline $\mathrm{H}$ & 0.331611000 & -0.913134000 & 3.872614000 \\
\hline $\mathrm{H}$ & -0.322032000 & -2.430425000 & 3.236368000 \\
\hline $\mathrm{H}$ & -2.114983000 & -0.728073000 & 3.271302000 \\
\hline $\mathrm{H}$ & -1.134929000 & 0.372743000 & 2.292152000 \\
\hline $\mathrm{N}$ & -1.843566000 & -1.361044000 & 1.323162000 \\
\hline $\mathrm{C}$ & -3.260239000 & -1.154221000 & 1.037944000 \\
\hline $\mathrm{C}$ & -3.722054000 & -2.040381000 & -0.104366000 \\
\hline $\mathrm{C}$ & -2.747682000 & -1.919218000 & -1.262325000 \\
\hline $\mathrm{N}$ & -1.399019000 & -2.223546000 & -0.811204000 \\
\hline $\mathrm{C}$ & -0.945021000 & -1.725915000 & 0.364514000 \\
\hline $\mathrm{H}$ & -3.435036000 & -0.097602000 & 0.798387000 \\
\hline $\mathrm{H}$ & -3.819359000 & -1.386804000 & 1.950963000 \\
\hline $\mathrm{H}$ & -3.770913000 & -3.084777000 & 0.222169000 \\
\hline $\mathrm{H}$ & -4.723064000 & -1.732574000 & -0.419577000 \\
\hline $\mathrm{H}$ & -3.001754000 & -2.633368000 & -2.050233000 \\
\hline $\mathrm{H}$ & -2.781336000 & -0.911196000 & -1.695005000 \\
\hline $\mathrm{C}$ & 4.464296000 & 1.356333000 & 0.903147000 \\
\hline $\mathrm{C}$ & 3.152511000 & 1.245381000 & 0.455992000 \\
\hline $\mathrm{C}$ & 2.816476000 & 0.439829000 & -0.645401000 \\
\hline $\mathrm{C}$ & 3.878061000 & -0.228972000 & -1.280337000 \\
\hline $\mathrm{C}$ & 5.185722000 & -0.105470000 & -0.832602000 \\
\hline $\mathrm{C}$ & 5.511401000 & 0.685773000 & 0.271859000 \\
\hline $\mathrm{H}$ & 4.678052000 & 1.993614000 & 1.759262000 \\
\hline $\mathrm{H}$ & 2.384389000 & 1.813002000 & 0.974839000 \\
\hline $\mathrm{H}$ & 3.653748000 & -0.858247000 & -2.135324000 \\
\hline $\mathrm{H}$ & 5.976433000 & -0.642577000 & -1.353924000 \\
\hline $\mathrm{C}$ & 1.430933000 & 0.216031000 & -1.111729000 \\
\hline $\mathrm{C}$ & 0.341486000 & 1.043968000 & -0.686134000 \\
\hline $\mathrm{H}$ & 0.459422000 & 1.483448000 & 0.303944000 \\
\hline $\mathrm{O}$ & 1.336456000 & -0.261083000 & -2.443143000 \\
\hline $\mathrm{C}$ & -0.865367000 & 1.171111000 & -1.298878000 \\
\hline $\mathrm{H}$ & -0.987401000 & 0.722088000 & -2.283117000 \\
\hline $\mathrm{C}$ & -2.057715000 & 1.784380000 & -0.723854000 \\
\hline $\mathrm{C}$ & -3.274163000 & 1.726201000 & -1.430758000 \\
\hline $\mathrm{C}$ & -2.094167000 & 2.396689000 & 0.544035000 \\
\hline $\mathrm{C}$ & -4.463036000 & 2.195484000 & -0.886336000 \\
\hline
\end{tabular}




$\begin{array}{lrcc}\mathrm{H} & -3.278506000 & 1.289828000 & -2.427686000 \\ \mathrm{C} & -3.281476000 & 2.868814000 & 1.088537000 \\ \mathrm{H} & -1.176288000 & 2.508660000 & 1.114652000 \\ \mathrm{C} & -4.481483000 & 2.760293000 & 0.387150000 \\ \mathrm{H} & -5.382399000 & 2.121179000 & -1.461366000 \\ \mathrm{H} & -3.269059000 & 3.330653000 & 2.072401000 \\ \mathrm{H} & -5.408123000 & 3.127270000 & 0.817547000 \\ \mathrm{H} & 0.914188000 & -0.997341000 & -0.263802000 \\ \mathrm{H} & 1.485924000 & 0.490092000 & -3.035901000 \\ \mathrm{C} & -0.460733000 & -2.700777000 & -1.817218000 \\ \mathrm{H} & -0.086961000 & -1.886504000 & -2.447608000 \\ \mathrm{H} & 0.391589000 & -3.165201000 & -1.320527000 \\ \mathrm{H} & -0.973721000 & -3.448509000 & -2.429098000 \\ \mathrm{C} & 6.938238000 & 0.822477000 & 0.741305000 \\ \mathrm{H} & 7.535411000 & 1.422679000 & 0.043634000 \\ \mathrm{H} & 6.990653000 & 1.309402000 & 1.720408000 \\ \mathrm{H} & 7.427063000 & -0.154610000 & 0.828059000 \\ \text {--_-_-_. }\end{array}$

\begin{tabular}{lrrr} 
A2 & \multicolumn{3}{c}{ Coordinates (Angstroms) } \\
& X & Y & Z \\
\hline $\mathrm{C}$ & 2.016626000 & 1.124379000 & 2.211784000 \\
$\mathrm{C}$ & 2.935910000 & 1.433532000 & 1.041420000 \\
$\mathrm{C}$ & 2.379250000 & 2.610718000 & 0.259433000 \\
$\mathrm{~N}$ & 0.604425000 & 1.337623000 & 1.879892000 \\
$\mathrm{H}$ & 2.249047000 & 1.762723000 & 3.073812000 \\
$\mathrm{H}$ & 2.131808000 & 0.077746000 & 2.514444000 \\
$\mathrm{H}$ & 3.007483000 & 0.571553000 & 0.376302000 \\
$\mathrm{H}$ & 3.939234000 & 1.657334000 & 1.413532000 \\
$\mathrm{H}$ & 2.363120000 & 3.526874000 & 0.866030000 \\
$\mathrm{H}$ & 2.984826000 & 2.801241000 & -0.628836000 \\
$\mathrm{~N}$ & 1.028102000 & 2.294822000 & -0.191189000 \\
$\mathrm{C}$ & 0.654629000 & 2.550790000 & -1.579363000 \\
$\mathrm{C}$ & -0.836212000 & 2.840927000 & -1.672282000 \\
$\mathrm{C}$ & -1.605073000 & 1.696992000 & -1.036531000 \\
$\mathrm{~N}$ & -1.086218000 & 1.417978000 & 0.298494000 \\
$\mathrm{C}$ & 0.187969000 & 1.654915000 & 0.642984000 \\
$\mathrm{H}$ & 0.933889000 & 1.689943000 & -2.201322000 \\
$\mathrm{H}$ & 1.233417000 & 3.413974000 & -1.918118000 \\
$\mathrm{H}$ & -1.056963000 & 3.781448000 & -1.155530000 \\
$\mathrm{H}$ & -1.131615000 & 2.953586000 & -2.718762000 \\
$\mathrm{H}$ & -2.669879000 & 1.920634000 & -0.940733000 \\
$\mathrm{H}$ & -1.522426000 & 0.791697000 & -1.650324000 \\
& & &
\end{tabular}




\begin{tabular}{lrrr}
$\mathrm{H}$ & -1.502157000 & 0.555753000 & 0.698813000 \\
$\mathrm{C}$ & 2.943479000 & -0.910357000 & -1.902869000 \\
$\mathrm{C}$ & 1.706866000 & -1.074158000 & -1.298337000 \\
$\mathrm{C}$ & 1.572985000 & -1.686482000 & -0.027907000 \\
$\mathrm{C}$ & 2.774077000 & -2.101150000 & 0.588586000 \\
$\mathrm{C}$ & 4.006985000 & -1.917436000 & -0.023839000 \\
$\mathrm{C}$ & 4.128940000 & -1.319717000 & -1.280909000 \\
$\mathrm{H}$ & 2.993268000 & -0.437670000 & -2.883153000 \\
$\mathrm{H}$ & 0.821965000 & -0.711025000 & -1.815265000 \\
$\mathrm{H}$ & 2.717844000 & -2.570899000 & 1.564524000 \\
$\mathrm{H}$ & 4.905163000 & -2.252027000 & 0.492650000 \\
$\mathrm{C}$ & 0.306379000 & -1.822719000 & 0.646426000 \\
$\mathrm{C}$ & -0.928919000 & -1.560840000 & 0.103728000 \\
$\mathrm{H}$ & -0.935646000 & -1.409242000 & -0.974884000 \\
$\mathrm{O}$ & 0.394984000 & -2.145121000 & 2.012275000 \\
$\mathrm{C}$ & -2.151397000 & -1.389015000 & 0.768518000 \\
$\mathrm{H}$ & -2.179712000 & -1.474923000 & 1.854017000 \\
$\mathrm{C}$ & -3.410339000 & -1.109934000 & 0.115060000 \\
$\mathrm{C}$ & -4.513417000 & -0.646767000 & 0.871221000 \\
$\mathrm{C}$ & -3.616373000 & -1.216613000 & -1.280919000 \\
$\mathrm{C}$ & -5.713708000 & -0.288011000 & 0.278057000 \\
$\mathrm{H}$ & -4.402176000 & -0.559441000 & 1.950663000 \\
$\mathrm{C}$ & -4.817398000 & -0.843607000 & -1.871554000 \\
$\mathrm{H}$ & -2.829236000 & -1.624244000 & -1.910121000 \\
$\mathrm{C}$ & -5.880210000 & -0.369983000 & -1.105325000 \\
$\mathrm{H}$ & -6.531158000 & 0.064898000 & 0.902741000 \\
$\mathrm{H}$ & -4.931372000 & -0.945788000 & -2.948536000 \\
$\mathrm{H}$ & -6.820025000 & -0.088947000 & -1.570690000 \\
$\mathrm{H}$ & -0.276304000 & -2.820287000 & 2.180428000 \\
$\mathrm{C}$ & -0.327287000 & 0.854167000 & 2.891955000 \\
$\mathrm{H}$ & -1.311217000 & 1.300760000 & 2.742054000 \\
$\mathrm{H}$ & -0.391411000 & -0.237558000 & 2.862119000 \\
$\mathrm{H}$ & 0.053565000 & 1.171743000 & 3.867594000 \\
$\mathrm{H}$ & 5.468729000 & -1.143865000 & -1.950033000 \\
$\mathrm{H}$ & 5.650861000 & -1.921985000 & -2.702417000 \\
$\mathrm{H}$ & 5.541281000 & -0.177551000 & -2.462804000 \\
\hline--285541000 & -1.196917000 & -1.222627000 \\
\hline
\end{tabular}

TSA2-3

\begin{tabular}{|c|c|c|c|}
\hline \multicolumn{4}{|c|}{ Coordinates (Angstroms) } \\
\hline & $\mathrm{X}$ & $\mathrm{Y}$ & $\mathrm{Z}$ \\
\hline $\mathrm{C}$ & 1.835973000 & 1.853019000 & 1.832914000 \\
\hline $\mathrm{C}$ & 2.769084000 & 1.784978000 & 0.631630000 \\
\hline
\end{tabular}




\begin{tabular}{|c|c|c|c|}
\hline $\mathrm{C}$ & 2.213185000 & 2.649164000 & -0.487055000 \\
\hline $\mathrm{N}$ & 0.430871000 & 1.984804000 & 1.447326000 \\
\hline $\mathrm{H}$ & 2.083749000 & 2.718145000 & 2.462916000 \\
\hline $\mathrm{H}$ & 1.936335000 & 0.947741000 & 2.442480000 \\
\hline $\mathrm{H}$ & 2.857572000 & 0.758301000 & 0.270760000 \\
\hline $\mathrm{H}$ & 3.766574000 & 2.125976000 & 0.923344000 \\
\hline $\mathrm{H}$ & 2.192557000 & 3.709977000 & -0.196942000 \\
\hline $\mathrm{H}$ & 2.829186000 & 2.556261000 & -1.384277000 \\
\hline $\mathrm{N}$ & 0.871663000 & 2.194746000 & -0.822509000 \\
\hline $\mathrm{C}$ & 0.474137000 & 2.062261000 & -2.217781000 \\
\hline $\mathrm{C}$ & -0.998183000 & 2.415076000 & -2.368413000 \\
\hline $\mathrm{C}$ & -1.809120000 & 1.549707000 & -1.417677000 \\
\hline $\mathrm{N}$ & -1.275834000 & 1.584568000 & -0.060996000 \\
\hline $\mathrm{C}$ & -0.005089000 & 1.893678000 & 0.166787000 \\
\hline $\mathrm{H}$ & 0.668652000 & 1.040531000 & -2.572010000 \\
\hline $\mathrm{H}$ & 1.096517000 & 2.741801000 & -2.806429000 \\
\hline $\mathrm{H}$ & -1.141521000 & 3.476002000 & -2.134071000 \\
\hline $\mathrm{H}$ & -1.322868000 & 2.251126000 & -3.400101000 \\
\hline $\mathrm{H}$ & -2.853328000 & 1.872292000 & -1.378142000 \\
\hline $\mathrm{H}$ & -1.816651000 & 0.509664000 & -1.771375000 \\
\hline $\mathrm{H}$ & -1.661115000 & 0.633849000 & 0.515839000 \\
\hline $\mathrm{C}$ & 3.159111000 & -1.498853000 & -1.580791000 \\
\hline $\mathrm{C}$ & 1.910017000 & -1.384136000 & -0.989595000 \\
\hline $\mathrm{C}$ & 1.732208000 & -1.579492000 & 0.392887000 \\
\hline $\mathrm{C}$ & 2.879273000 & -1.874167000 & 1.146760000 \\
\hline $\mathrm{C}$ & 4.129436000 & -1.972306000 & 0.546780000 \\
\hline $\mathrm{C}$ & 4.300517000 & -1.787578000 & -0.825683000 \\
\hline $\mathrm{H}$ & 3.254501000 & -1.347100000 & -2.654357000 \\
\hline $\mathrm{H}$ & 1.056835000 & -1.122227000 & -1.609174000 \\
\hline $\mathrm{H}$ & 2.776213000 & -2.024684000 & 2.215571000 \\
\hline $\mathrm{H}$ & 4.994892000 & -2.205569000 & 1.163912000 \\
\hline $\mathrm{C}$ & 0.436473000 & -1.407641000 & 1.041964000 \\
\hline $\mathrm{C}$ & -0.763332000 & -1.333836000 & 0.417475000 \\
\hline $\mathrm{H}$ & -0.757036000 & -1.555125000 & -0.647682000 \\
\hline $\mathrm{O}$ & 0.528733000 & -1.234983000 & 2.423434000 \\
\hline $\mathrm{C}$ & -1.992311000 & -0.823875000 & 0.965195000 \\
\hline $\mathrm{H}$ & -2.032971000 & -0.669572000 & 2.046188000 \\
\hline $\mathrm{C}$ & -3.299320000 & -1.065507000 & 0.352595000 \\
\hline $\mathrm{C}$ & -4.455280000 & -0.518467000 & 0.947810000 \\
\hline $\mathrm{C}$ & -3.485784000 & -1.753036000 & -0.862865000 \\
\hline $\mathrm{C}$ & -5.707334000 & -0.635616000 & 0.362711000 \\
\hline $\mathrm{H}$ & -4.351730000 & 0.015089000 & 1.891031000 \\
\hline $\mathrm{C}$ & -4.740368000 & -1.859797000 & -1.454215000 \\
\hline $\mathrm{H}$ & -2.639318000 & -2.234041000 & -1.345615000 \\
\hline
\end{tabular}




$\begin{array}{lrrr}\mathrm{C} & -5.864464000 & -1.301647000 & -0.852803000 \\ \mathrm{H} & -6.571555000 & -0.199376000 & 0.857910000 \\ \mathrm{H} & -4.840327000 & -2.399767000 & -2.392883000 \\ \mathrm{H} & -6.843384000 & -1.393138000 & -1.313578000 \\ \mathrm{H} & -0.285062000 & -1.594115000 & 2.801848000 \\ \mathrm{C} & -0.497810000 & 1.928992000 & 2.562547000 \\ \mathrm{H} & -1.499390000 & 2.193159000 & 2.224871000 \\ \mathrm{H} & -0.508754000 & 0.928681000 & 3.003507000 \\ \mathrm{H} & -0.169855000 & 2.650930000 & 3.319585000 \\ \mathrm{C} & 5.661772000 & -1.870909000 & -1.469211000 \\ \mathrm{H} & 5.606758000 & -2.334271000 & -2.459987000 \\ \mathrm{H} & 6.108593000 & -0.876803000 & -1.598704000 \\ \mathrm{H} & 6.352437000 & -2.462784000 & -0.860432000\end{array}$

\begin{tabular}{|c|c|c|c|}
\hline \multicolumn{4}{|c|}{ Coordinates (Angstroms) } \\
\hline & $X$ & Y & $\mathrm{Z}$ \\
\hline$\overline{\mathrm{C}}$ & 1.159485000 & -2.951441000 & -1.104761000 \\
\hline $\mathrm{C}$ & 1.952564000 & -2.265046000 & -2.202827000 \\
\hline $\mathrm{C}$ & 3.291436000 & -1.814111000 & -1.641995000 \\
\hline $\mathrm{N}$ & 0.952331000 & -2.011584000 & -0.023612000 \\
\hline $\mathrm{H}$ & 1.677717000 & -3.867003000 & -0.766779000 \\
\hline $\mathrm{H}$ & 0.177055000 & -3.255780000 & -1.478964000 \\
\hline $\mathrm{H}$ & 1.376615000 & -1.403815000 & -2.555838000 \\
\hline $\mathrm{H}$ & 2.115118000 & -2.936350000 & -3.051888000 \\
\hline $\mathrm{H}$ & 4.007536000 & -2.651165000 & -1.667378000 \\
\hline $\mathrm{H}$ & 3.712238000 & -1.016128000 & -2.269336000 \\
\hline $\mathrm{N}$ & 3.190450000 & -1.327272000 & -0.278660000 \\
\hline $\mathrm{C}$ & 4.355059000 & -0.587624000 & 0.179643000 \\
\hline $\mathrm{C}$ & 4.424862000 & -0.584980000 & 1.697521000 \\
\hline $\mathrm{C}$ & 3.077778000 & -0.117059000 & 2.238452000 \\
\hline $\mathrm{N}$ & 1.963580000 & -0.867227000 & 1.702935000 \\
\hline $\mathrm{C}$ & 2.062669000 & -1.369600000 & 0.520889000 \\
\hline $\mathrm{H}$ & 4.317560000 & 0.442208000 & -0.206986000 \\
\hline $\mathrm{H}$ & 5.245216000 & -1.068114000 & -0.246384000 \\
\hline $\mathrm{H}$ & 4.632988000 & -1.600108000 & 2.056136000 \\
\hline $\mathrm{H}$ & 5.238699000 & 0.066417000 & 2.033421000 \\
\hline $\mathrm{H}$ & 3.056603000 & -0.207736000 & 3.330959000 \\
\hline $\mathrm{H}$ & 2.952590000 & 0.954102000 & 2.011756000 \\
\hline $\mathrm{C}$ & -5.406737000 & 1.069150000 & -0.788599000 \\
\hline $\mathrm{C}$ & -4.180826000 & 1.400119000 & -0.228127000 \\
\hline $\mathrm{C}$ & -3.462217000 & 0.463593000 & 0.521891000 \\
\hline $\mathrm{C}$ & -4.021320000 & -0.806004000 & 0.705626000 \\
\hline
\end{tabular}




$\begin{array}{lrrr}\mathrm{C} & -5.252764000 & -1.128548000 & 0.146441000 \\ \mathrm{C} & -5.968165000 & -0.198243000 & -0.608449000 \\ \mathrm{H} & -5.946887000 & 1.814220000 & -1.367966000 \\ \mathrm{H} & -3.775742000 & 2.399160000 & -0.358876000 \\ \mathrm{H} & -3.471130000 & -1.561214000 & 1.261868000 \\ \mathrm{H} & -5.661371000 & -2.125507000 & 0.293217000 \\ \mathrm{C} & -2.141814000 & 0.789949000 & 1.107707000 \\ \mathrm{C} & -1.213507000 & 1.531214000 & 0.494285000 \\ \mathrm{H} & -1.447019000 & 1.908907000 & -0.496986000 \\ \mathrm{O} & -1.861708000 & 0.225183000 & 2.325936000 \\ \mathrm{C} & 0.141001000 & 1.845573000 & 1.066982000 \\ \mathrm{H} & 0.458808000 & 1.017454000 & 1.714173000 \\ \mathrm{C} & 1.204441000 & 2.058194000 & 0.011890000 \\ \mathrm{C} & 2.208109000 & 3.013737000 & 0.190178000 \\ \mathrm{C} & 1.226123000 & 1.288292000 & -1.153349000 \\ \mathrm{C} & 3.204594000 & 3.197937000 & -0.764673000 \\ \mathrm{H} & 2.206063000 & 3.624265000 & 1.090558000 \\ \mathrm{C} & 2.213001000 & 1.475380000 & -2.115571000 \\ \mathrm{H} & 0.469586000 & 0.520356000 & -1.290834000 \\ \mathrm{C} & 3.208015000 & 2.431350000 & -1.926862000 \\ \mathrm{H} & 3.972901000 & 3.949348000 & -0.604846000 \\ \mathrm{H} & 2.204066000 & 0.872896000 & -3.020656000 \\ \mathrm{H} & 3.976493000 & 2.579894000 & -2.680116000 \\ \mathrm{H} & -2.691683000 & -0.026482000 & 2.748059000 \\ \mathrm{H} & 0.083969000 & 2.739785000 & 1.703791000 \\ \mathrm{C} & -0.155896000 & -2.299581000 & 0.864478000 \\ \mathrm{H} & 0.011951000 & -3.215872000 & 1.452266000 \\ \mathrm{H} & -0.296704000 & -1.470697000 & 1.555133000 \\ \mathrm{H} & -1.061689000 & -2.427247000 & 0.261085000 \\ \mathrm{C} & -7.319280000 & -0.534638000 & -1.186797000 \\ \mathrm{H} & -7.437243000 & -1.613330000 & -1.327334000 \\ \mathrm{H} & -8.124098000 & -0.198943000 & -0.521715000 \\ \mathrm{H} & -7.470747000 & -0.048097000 & -2.155507000 \\ -----. & \\ & & & \\ \mathrm{H} & ----0 & \end{array}$

\section{(4) $\mathrm{R}_{1}=\mathrm{CF}_{3}, \mathrm{R}_{2}=\mathrm{H}$}

\begin{tabular}{|c|c|c|c|}
\hline \multicolumn{4}{|c|}{$\mathbf{A 1}_{\text {КОН }}$} \\
\hline & $\mathrm{X}$ & Y & $\mathrm{Z}$ \\
\hline$\overline{\mathrm{C}}$ & -2.082021000 & -1.620163000 & -0.602864000 \\
\hline $\mathrm{C}$ & -0.872240000 & -0.941822000 & -0.528821000 \\
\hline $\mathrm{C}$ & -0.798882000 & 0.306936000 & 0.093636000 \\
\hline $\mathrm{C}$ & -1.947375000 & 0.871092000 & 0.656056000 \\
\hline
\end{tabular}




\begin{tabular}{|c|c|c|c|}
\hline $\mathrm{C}$ & -3.157161000 & 0.187575000 & 0.587712000 \\
\hline $\mathrm{C}$ & -3.226032000 & -1.052018000 & -0.044307000 \\
\hline $\mathrm{H}$ & -2.139439000 & -2.588535000 & -1.089475000 \\
\hline $\mathrm{H}$ & 0.025608000 & -1.391544000 & -0.947710000 \\
\hline $\mathrm{H}$ & -1.838506000 & 1.852074000 & 1.141928000 \\
\hline $\mathrm{H}$ & -4.050381000 & 0.619882000 & 1.026640000 \\
\hline $\mathrm{C}$ & 0.495800000 & 1.103346000 & 0.116928000 \\
\hline $\mathrm{C}$ & 1.699355000 & 0.251215000 & 0.388801000 \\
\hline $\mathrm{H}$ & 1.605658000 & -0.378050000 & 1.272704000 \\
\hline $\mathrm{O}$ & 0.623207000 & 1.846094000 & -1.115490000 \\
\hline $\mathrm{C}$ & 2.812968000 & 0.227790000 & -0.348252000 \\
\hline $\mathrm{H}$ & 2.859201000 & 0.867577000 & -1.228589000 \\
\hline $\mathrm{C}$ & 4.015128000 & -0.585711000 & -0.094439000 \\
\hline $\mathrm{C}$ & 4.975096000 & -0.714867000 & -1.105376000 \\
\hline $\mathrm{C}$ & 4.249891000 & -1.237940000 & 1.123594000 \\
\hline $\mathrm{C}$ & 6.121937000 & -1.478997000 & -0.917345000 \\
\hline $\mathrm{H}$ & 4.815535000 & -0.210159000 & -2.055517000 \\
\hline $\mathrm{C}$ & 5.393668000 & -2.002165000 & 1.313710000 \\
\hline $\mathrm{H}$ & 3.538830000 & -1.135589000 & 1.937885000 \\
\hline $\mathrm{C}$ & 6.335261000 & -2.128507000 & 0.294248000 \\
\hline $\mathrm{H}$ & 6.851155000 & -1.564027000 & -1.717820000 \\
\hline $\mathrm{H}$ & 5.555769000 & -2.496016000 & 2.267401000 \\
\hline $\mathrm{H}$ & 7.231583000 & -2.721954000 & 0.447411000 \\
\hline $\mathrm{H}$ & 0.374990000 & 1.889605000 & 0.891689000 \\
\hline $\mathrm{H}$ & 0.580756000 & 1.205616000 & -1.838205000 \\
\hline $\mathrm{O}$ & -0.655816000 & 3.440208000 & 1.633587000 \\
\hline $\mathrm{H}$ & -0.684999000 & 3.256202000 & 2.576132000 \\
\hline K & -0.422306000 & 4.247013000 & -0.506574000 \\
\hline $\mathrm{C}$ & -4.515445000 & -1.823750000 & -0.076558000 \\
\hline $\mathrm{F}$ & -4.561360000 & -2.756470000 & 0.893335000 \\
\hline $\mathrm{F}$ & -5.587753000 & -1.031270000 & 0.090388000 \\
\hline $\mathrm{F}$ & -4.677640000 & -2.474575000 & -1.244241000 \\
\hline
\end{tabular}

TSA1-2 кон

\begin{tabular}{|c|c|c|c|}
\hline & \multicolumn{3}{|c|}{ Coordinates (Angstroms) } \\
\hline & $\mathrm{X}$ & Y & Z \\
\hline$\overline{\mathrm{C}}$ & -2.789816000 & -1.380823000 & 0.207270000 \\
\hline $\mathrm{C}$ & -1.465927000 & -0.960336000 & 0.154429000 \\
\hline $\mathrm{C}$ & -1.140568000 & 0.364349000 & -0.160867000 \\
\hline $\mathrm{C}$ & -2.192736000 & 1.250736000 & -0.441093000 \\
\hline $\mathrm{C}$ & -3.513112000 & 0.833112000 & -0.402633000 \\
\hline $\mathrm{C}$ & -3.818619000 & -0.487720000 & -0.073516000 \\
\hline $\mathrm{H}$ & -3.019084000 & -2.414124000 & 0.446029000 \\
\hline
\end{tabular}




$\begin{array}{lrrr}\mathrm{H} & -0.679462000 & -1.684118000 & 0.343484000 \\ \mathrm{H} & -1.954918000 & 2.281256000 & -0.684674000 \\ \mathrm{H} & -4.311274000 & 1.533545000 & -0.626986000 \\ \mathrm{C} & 0.253804000 & 0.894783000 & -0.167830000 \\ \mathrm{C} & 1.362726000 & -0.039181000 & 0.064727000 \\ \mathrm{H} & 1.251767000 & -0.662763000 & 0.951520000 \\ \mathrm{O} & 0.491975000 & 1.763915000 & -1.292651000 \\ \mathrm{C} & 2.526261000 & -0.079053000 & -0.616682000 \\ \mathrm{H} & 2.602543000 & 0.497446000 & -1.540353000 \\ \mathrm{C} & 3.715249000 & -0.872153000 & -0.270079000 \\ \mathrm{C} & 4.706827000 & -1.085252000 & -1.238466000 \\ \mathrm{C} & 3.921238000 & -1.416796000 & 1.006742000 \\ \mathrm{C} & 5.847035000 & -1.827929000 & -0.953514000 \\ \mathrm{H} & 4.570527000 & -0.670020000 & -2.234932000 \\ \mathrm{C} & 5.057134000 & -2.164565000 & 1.290893000 \\ \mathrm{H} & 3.190046000 & -1.242361000 & 1.791255000 \\ \mathrm{C} & 6.027223000 & -2.375618000 & 0.313318000 \\ \mathrm{H} & 6.596326000 & -1.981284000 & -1.725101000 \\ \mathrm{H} & 5.190804000 & -2.578315000 & 2.286467000 \\ \mathrm{H} & 6.917395000 & -2.954672000 & 0.539733000 \\ \mathrm{H} & 0.323828000 & 1.740577000 & 0.820848000 \\ \mathrm{H} & 0.360607000 & 1.250003000 & -2.102260000 \\ \mathrm{O} & 0.598366000 & 2.763925000 & 1.640715000 \\ \mathrm{H} & -0.175279000 & 2.864276000 & 2.201735000 \\ \mathrm{~K} & 2.405574000 & 3.064624000 & 0.129319000 \\ \mathrm{C} & -5.250149000 & -0.923343000 & 0.029230000 \\ \mathrm{~F} & -6.037116000 & -0.279520000 & -0.852984000 \\ \mathrm{~F} & -5.395412000 & -2.242300000 & -0.193885000 \\ \mathrm{~F} & -5.764347000 & -0.679432000 & 1.251323000 \\ \mathrm{H} & & & \end{array}$

\section{A2 кон}

\begin{tabular}{lrrr} 
& \multicolumn{3}{c}{ Coordinates (Angstroms) } \\
& $\mathrm{X}$ & $\mathrm{Y}$ & $\mathrm{Z}$ \\
\hline $\mathrm{C}$ & -2.634288000 & -1.338470000 & 0.283942000 \\
$\mathrm{C}$ & -1.327339000 & -1.054625000 & -0.052453000 \\
$\mathrm{C}$ & -0.984087000 & 0.103648000 & -0.809134000 \\
$\mathrm{C}$ & -2.069728000 & 0.943302000 & -1.189175000 \\
$\mathrm{C}$ & -3.378800000 & 0.648289000 & -0.838468000 \\
$\mathrm{C}$ & -3.681945000 & -0.486507000 & -0.089750000 \\
$\mathrm{H}$ & -2.851221000 & -2.232189000 & 0.861725000 \\
$\mathrm{H}$ & -0.550084000 & -1.737790000 & 0.274926000 \\
$\mathrm{H}$ & -1.862879000 & 1.815876000 & -1.799926000 \\
$\mathrm{H}$ & -4.176239000 & 1.313792000 & -1.155550000
\end{tabular}




\begin{tabular}{|c|c|c|c|}
\hline $\mathrm{C}$ & 0.361909000 & 0.490085000 & -1.083810000 \\
\hline $\mathrm{C}$ & 1.529836000 & -0.165313000 & -0.692290000 \\
\hline $\mathrm{H}$ & 1.396347000 & -1.172882000 & -0.304143000 \\
\hline $\mathrm{O}$ & 0.497361000 & 1.810801000 & -1.580486000 \\
\hline $\mathrm{C}$ & 2.815983000 & 0.345072000 & -0.723474000 \\
\hline $\mathrm{H}$ & 2.968053000 & 1.348356000 & -1.120821000 \\
\hline $\mathrm{C}$ & 4.033689000 & -0.374553000 & -0.364781000 \\
\hline $\mathrm{C}$ & 5.256673000 & 0.322788000 & -0.314593000 \\
\hline $\mathrm{C}$ & 4.070652000 & -1.743564000 & -0.035743000 \\
\hline $\mathrm{C}$ & 6.441202000 & -0.302943000 & 0.049964000 \\
\hline $\mathrm{H}$ & 5.267136000 & 1.381043000 & -0.569443000 \\
\hline $\mathrm{C}$ & 5.255446000 & -2.366656000 & 0.334329000 \\
\hline $\mathrm{H}$ & 3.161910000 & -2.337012000 & -0.086410000 \\
\hline $\mathrm{C}$ & 6.452510000 & -1.655541000 & 0.383359000 \\
\hline $\mathrm{H}$ & 7.364478000 & 0.270431000 & 0.072699000 \\
\hline $\mathrm{H}$ & 5.244370000 & -3.426329000 & 0.576511000 \\
\hline $\mathrm{H}$ & 7.377424000 & -2.148284000 & 0.666968000 \\
\hline $\mathrm{H}$ & 1.086472000 & 1.785094000 & -2.345158000 \\
\hline $\mathrm{O}$ & 2.152005000 & 1.672866000 & 1.874051000 \\
\hline $\mathrm{H}$ & 2.816889000 & 1.528585000 & 2.554962000 \\
\hline K & -0.139760000 & 2.372492000 & 0.958433000 \\
\hline $\mathrm{H}$ & 2.462340000 & 1.145393000 & 1.098487000 \\
\hline $\mathrm{C}$ & -5.092581000 & -0.830943000 & 0.253142000 \\
\hline $\mathrm{F}$ & -5.890599000 & 0.256152000 & 0.272493000 \\
\hline $\mathrm{F}$ & -5.647737000 & -1.695431000 & -0.622413000 \\
\hline $\mathrm{F}$ & -5.188250000 & -1.414631000 & 1.466591000 \\
\hline
\end{tabular}

TSA2-3 кон

\begin{tabular}{lrcc} 
& \multicolumn{3}{c}{ Coordinates (Angstroms) } \\
& $\mathrm{X}$ & $\mathrm{Y}$ & $\mathrm{Z}$ \\
$\mathrm{C}$ & 2.752105000 & -0.968619000 & -0.833566000 \\
$\mathrm{C}$ & 1.427412000 & -0.681763000 & -0.546418000 \\
$\mathrm{C}$ & 1.076685000 & 0.074156000 & 0.587647000 \\
$\mathrm{C}$ & 2.117070000 & 0.557651000 & 1.399492000 \\
$\mathrm{C}$ & 3.444220000 & 0.278218000 & 1.104918000 \\
$\mathrm{C}$ & 3.770366000 & -0.486248000 & -0.011910000 \\
$\mathrm{H}$ & 2.998514000 & -1.552262000 & -1.714396000 \\
$\mathrm{H}$ & 0.655787000 & -1.039636000 & -1.220746000 \\
$\mathrm{H}$ & 1.870449000 & 1.147362000 & 2.274798000 \\
$\mathrm{H}$ & 4.230324000 & 0.659169000 & 1.748806000 \\
$\mathrm{C}$ & -0.315509000 & 0.411676000 & 0.894754000 \\
$\mathrm{C}$ & -1.426767000 & -0.136843000 & 0.355238000 \\
$\mathrm{H}$ & -1.279316000 & -0.983035000 & -0.311542000
\end{tabular}




$\begin{array}{lrrr}\mathrm{O} & -0.418572000 & 1.512635000 & 1.758169000 \\ \mathrm{C} & -2.781495000 & 0.390577000 & 0.457207000 \\ \mathrm{C} & -3.931215000 & -0.534117000 & 0.219258000 \\ \mathrm{C} & -3.826810000 & -1.929388000 & 0.216087000 \\ \mathrm{C} & -5.194277000 & 0.023510000 & -0.029894000 \\ \mathrm{C} & -4.934567000 & -2.734884000 & -0.040282000 \\ \mathrm{H} & -2.871523000 & -2.398995000 & 0.436485000 \\ \mathrm{C} & -6.304084000 & -0.775977000 & -0.270345000 \\ \mathrm{H} & -5.290490000 & 1.107178000 & -0.044809000 \\ \mathrm{C} & -6.179343000 & -2.164430000 & -0.282153000 \\ \mathrm{H} & -4.822710000 & -3.815991000 & -0.035908000 \\ \mathrm{H} & -7.270067000 & -0.314727000 & -0.458008000 \\ \mathrm{H} & -7.044056000 & -2.792446000 & -0.475821000 \\ \mathrm{H} & -1.251330000 & 1.423197000 & 2.241726000 \\ \mathrm{H} & -2.803792000 & 1.327498000 & -0.463632000 \\ \mathrm{O} & -2.684583000 & 2.362521000 & -1.293433000 \\ \mathrm{~K} & -0.456187000 & 2.916260000 & -0.631103000 \\ \mathrm{H} & -3.445926000 & 2.298731000 & -1.877206000 \\ \mathrm{H} & -2.965656000 & 0.996866000 & 1.355720000 \\ \mathrm{C} & 5.202920000 & -0.826888000 & -0.301535000 \\ \mathrm{~F} & 6.039630000 & 0.148053000 & 0.098660000 \\ \mathrm{~F} & 5.415729000 & -1.024191000 & -1.615185000 \\ \mathrm{~F} & 5.589343000 & -1.951961000 & 0.328370000 \\ \mathrm{H}--------\end{array}$

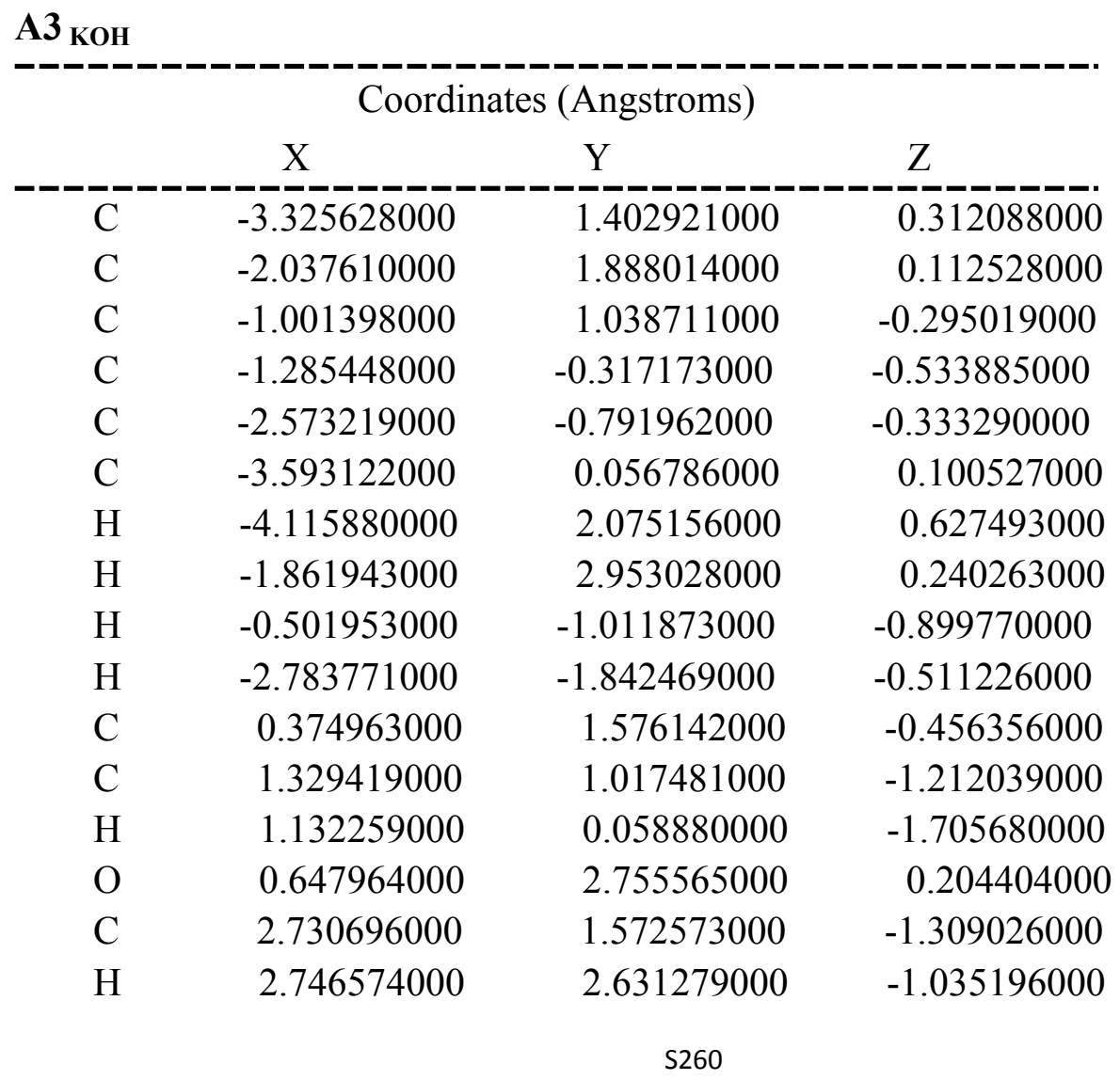




$\begin{array}{cccc}\mathrm{C} & 3.655731000 & 0.793988000 & -0.394873000 \\ \mathrm{C} & 3.581216000 & 0.981197000 & 0.992580000 \\ \mathrm{C} & 4.520168000 & -0.186473000 & -0.888105000 \\ \mathrm{C} & 4.342198000 & 0.203020000 & 1.861003000 \\ \mathrm{H} & 2.905880000 & 1.739624000 & 1.380542000 \\ \mathrm{C} & 5.282913000 & -0.971584000 & -0.021551000 \\ \mathrm{H} & 4.581012000 & -0.352563000 & -1.960088000 \\ \mathrm{C} & 5.193913000 & -0.783337000 & 1.356591000 \\ \mathrm{H} & 4.277516000 & 0.369206000 & 2.932870000 \\ \mathrm{H} & 5.948105000 & -1.729279000 & -0.425685000 \\ \mathrm{H} & 5.793766000 & -1.387109000 & 2.031176000 \\ \mathrm{O} & 0.935206000 & -2.061275000 & -1.585402000 \\ \mathrm{H} & 3.086916000 & 1.490696000 & -2.341526000 \\ \mathrm{H} & 0.031756000 & 2.844229000 & 0.940897000 \\ \mathrm{H} & 0.524298000 & -2.294488000 & -2.422209000 \\ \mathrm{~K} & 2.065348000 & -1.830761000 & 0.406646000 \\ \mathrm{C} & -4.980974000 & -0.489178000 & 0.279255000 \\ \mathrm{~F} & -5.760808000 & 0.329457000 & 1.007542000 \\ \mathrm{~F} & -5.599751000 & -0.674625000 & -0.901455000 \\ \mathrm{~F} & -4.967708000 & -1.683823000 & 0.899166000 \\ - \text { - - - - } & & & \end{array}$

\begin{tabular}{|c|c|c|c|}
\hline \multicolumn{4}{|c|}{$\mathrm{A1}_{\mathrm{NaOH}}$} \\
\hline & $\mathrm{X}$ & $\mathrm{Y}$ & Z \\
\hline $\mathrm{C}$ & -2.108528000 & -1.392223000 & -0.558057000 \\
\hline $\mathrm{C}$ & -0.893658000 & -0.722689000 & -0.493893000 \\
\hline $\mathrm{C}$ & -0.816679000 & 0.545500000 & 0.088072000 \\
\hline $\mathrm{C}$ & -1.964884000 & 1.139495000 & 0.618925000 \\
\hline $\mathrm{C}$ & -3.179996000 & 0.463947000 & 0.559998000 \\
\hline $\mathrm{C}$ & -3.252681000 & -0.795665000 & -0.029868000 \\
\hline $\mathrm{H}$ & -2.170118000 & -2.374927000 & -1.014465000 \\
\hline $\mathrm{H}$ & 0.004401000 & -1.192831000 & -0.888903000 \\
\hline $\mathrm{H}$ & -1.859719000 & 2.138535000 & 1.063830000 \\
\hline $\mathrm{H}$ & -4.073736000 & 0.918771000 & 0.973808000 \\
\hline $\mathrm{C}$ & 0.485013000 & 1.327901000 & 0.098815000 \\
\hline $\mathrm{C}$ & 1.683440000 & 0.473579000 & 0.387289000 \\
\hline $\mathrm{H}$ & 1.588616000 & -0.121882000 & 1.293819000 \\
\hline $\mathrm{O}$ & 0.621137000 & 2.044713000 & -1.154787000 \\
\hline $\mathrm{C}$ & 2.789049000 & 0.411442000 & -0.358673000 \\
\hline $\mathrm{H}$ & 2.835486000 & 1.020151000 & -1.260603000 \\
\hline $\mathrm{C}$ & 3.983726000 & -0.408380000 & -0.089107000 \\
\hline $\mathrm{C}$ & 4.947488000 & -0.554103000 & -1.093897000 \\
\hline $\mathrm{C}$ & 4.206368000 & -1.050697000 & 1.136354000 \\
\hline
\end{tabular}




\begin{tabular}{lrrr}
$\mathrm{C}$ & 6.087941000 & -1.324336000 & -0.892008000 \\
$\mathrm{H}$ & 4.796933000 & -0.056999000 & -2.049422000 \\
$\mathrm{C}$ & 5.343683000 & -1.820733000 & 1.340268000 \\
$\mathrm{H}$ & 3.491225000 & -0.937682000 & 1.945593000 \\
$\mathrm{C}$ & 6.289862000 & -1.963160000 & 0.327087000 \\
$\mathrm{H}$ & 6.820912000 & -1.422265000 & -1.687474000 \\
$\mathrm{H}$ & 5.496955000 & -2.307162000 & 2.299143000 \\
$\mathrm{H}$ & 7.180963000 & -2.561534000 & 0.490961000 \\
$\mathrm{H}$ & 0.377518000 & 2.131467000 & 0.850228000 \\
$\mathrm{H}$ & 0.526226000 & 1.399060000 & -1.867351000 \\
$\mathrm{O}$ & -0.711665000 & 3.805373000 & 1.275981000 \\
$\mathrm{H}$ & -0.792294000 & 4.217658000 & 2.138109000 \\
$\mathrm{C}$ & -4.548265000 & -1.558396000 & -0.055513000 \\
$\mathrm{~F}$ & -4.593524000 & -2.490921000 & 0.914223000 \\
$\mathrm{~F}$ & -5.614076000 & -0.759491000 & 0.116103000 \\
$\mathrm{~F}$ & -4.718532000 & -2.207976000 & -1.222613000 \\
$\mathrm{Na}$ & -0.315930000 & 4.076868000 & -0.676453000 \\
\hline-- - - & & &
\end{tabular}

TSA1-2 $\mathrm{NaOH}$

\begin{tabular}{lrrr} 
& \multicolumn{3}{c}{ Coordinates (Angstroms) } \\
\hline $\mathrm{C}$ & -2.660129000 & -1.260413000 & 0.356205000 \\
$\mathrm{C}$ & -1.347517000 & -0.809465000 & 0.311332000 \\
$\mathrm{C}$ & -1.045339000 & 0.503638000 & -0.075627000 \\
$\mathrm{C}$ & -2.112739000 & 1.342650000 & -0.432870000 \\
$\mathrm{C}$ & -3.422896000 & 0.891405000 & -0.401921000 \\
$\mathrm{C}$ & -3.705147000 & -0.413368000 & -0.000744000 \\
$\mathrm{H}$ & -2.869681000 & -2.280495000 & 0.661335000 \\
$\mathrm{H}$ & -0.552747000 & -1.501254000 & 0.570956000 \\
$\mathrm{H}$ & -1.899588000 & 2.365854000 & -0.723183000 \\
$\mathrm{H}$ & -4.231789000 & 1.560772000 & -0.676193000 \\
$\mathrm{C}$ & 0.334557000 & 1.058078000 & -0.067464000 \\
$\mathrm{C}$ & 1.478097000 & 0.158736000 & 0.097381000 \\
$\mathrm{H}$ & 1.395969000 & -0.541241000 & 0.928342000 \\
$\mathrm{O}$ & 0.550494000 & 2.011347000 & -1.132117000 \\
$\mathrm{C}$ & 2.649055000 & 0.219903000 & -0.574243000 \\
$\mathrm{H}$ & 2.706864000 & 0.851841000 & -1.463260000 \\
$\mathrm{C}$ & 3.860982000 & -0.558391000 & -0.275949000 \\
$\mathrm{C}$ & 4.843559000 & -0.712845000 & -1.264096000 \\
$\mathrm{C}$ & 4.091712000 & -1.151167000 & 0.974519000 \\
$\mathrm{C}$ & 6.000471000 & -1.445150000 & -1.023139000 \\
$\mathrm{H}$ & 4.687882000 & -0.260493000 & -2.241305000 \\
$\mathrm{C}$ & 5.244214000 & -1.889059000 & 1.214310000
\end{tabular}




\begin{tabular}{lccc}
$\mathrm{H}$ & 3.367822000 & -1.021354000 & 1.774323000 \\
$\mathrm{C}$ & 6.205506000 & -2.041568000 & 0.217700000 \\
$\mathrm{H}$ & 6.743234000 & -1.552045000 & -1.808557000 \\
$\mathrm{H}$ & 5.397786000 & -2.339996000 & 2.190550000 \\
$\mathrm{H}$ & 7.109136000 & -2.611943000 & 0.409759000 \\
$\mathrm{H}$ & 0.450448000 & 1.908780000 & 0.966196000 \\
$\mathrm{H}$ & 0.465294000 & 1.552108000 & -1.979784000 \\
$\mathrm{O}$ & 0.824619000 & 2.934240000 & 1.662314000 \\
$\mathrm{H}$ & 0.818860000 & 2.658076000 & 2.583598000 \\
$\mathrm{C}$ & -5.115518000 & -0.922677000 & -0.017810000 \\
$\mathrm{~F}$ & -5.320670000 & -1.870326000 & 0.915541000 \\
$\mathrm{~F}$ & -6.009563000 & 0.057833000 & 0.203644000 \\
$\mathrm{~F}$ & -5.437193000 & -1.476586000 & -1.204715000 \\
$\mathrm{Na}$ & 2.229384000 & 3.038779000 & 0.100902000 \\
\hline-
\end{tabular}

\begin{tabular}{|c|c|c|c|}
\hline \multicolumn{4}{|c|}{$\mathrm{A2}_{\mathrm{NaOH}}$} \\
\hline & $\mathrm{X}$ & $\mathrm{Y}$ & Z \\
\hline $\mathrm{C}$ & 2.627669000 & 1.311533000 & -0.119110000 \\
\hline $\mathrm{C}$ & 1.325595000 & 0.965846000 & -0.402867000 \\
\hline $\mathrm{C}$ & 0.970932000 & -0.364860000 & -0.772939000 \\
\hline $\mathrm{C}$ & 2.041811000 & -1.301778000 & -0.840884000 \\
\hline $\mathrm{C}$ & 3.349854000 & -0.938713000 & -0.541773000 \\
\hline $\mathrm{C}$ & 3.661061000 & 0.363508000 & -0.171839000 \\
\hline $\mathrm{H}$ & 2.857526000 & 2.338896000 & 0.148441000 \\
\hline $\mathrm{H}$ & 0.561721000 & 1.734865000 & -0.353878000 \\
\hline $\mathrm{H}$ & 1.835806000 & -2.307720000 & -1.192663000 \\
\hline $\mathrm{H}$ & 4.137466000 & -1.682003000 & -0.615368000 \\
\hline $\mathrm{C}$ & -0.377844000 & -0.790402000 & -0.967359000 \\
\hline $\mathrm{C}$ & -1.542786000 & -0.071931000 & -0.689045000 \\
\hline $\mathrm{H}$ & -1.407569000 & 0.998336000 & -0.551296000 \\
\hline $\mathrm{O}$ & -0.508155000 & -2.202705000 & -1.088670000 \\
\hline $\mathrm{C}$ & -2.819370000 & -0.582888000 & -0.540883000 \\
\hline $\mathrm{H}$ & -2.985301000 & -1.643450000 & -0.731558000 \\
\hline $\mathrm{C}$ & -4.031780000 & 0.192968000 & -0.288363000 \\
\hline $\mathrm{C}$ & -5.266912000 & -0.474797000 & -0.191589000 \\
\hline $\mathrm{C}$ & -4.048816000 & 1.589046000 & -0.108021000 \\
\hline $\mathrm{C}$ & -6.447629000 & 0.206581000 & 0.072041000 \\
\hline $\mathrm{H}$ & -5.292378000 & -1.553959000 & -0.329626000 \\
\hline $\mathrm{C}$ & -5.230285000 & 2.269620000 & 0.156620000 \\
\hline $\mathrm{H}$ & -3.126095000 & 2.158185000 & -0.181963000 \\
\hline $\mathrm{C}$ & -6.441279000 & 1.587773000 & 0.251215000 \\
\hline $\mathrm{H}$ & -7.381385000 & -0.345816000 & 0.136605000 \\
\hline
\end{tabular}




$\begin{array}{lrrr}\mathrm{H} & -5.203427000 & 3.348293000 & 0.287833000 \\ \mathrm{H} & -7.363320000 & 2.122967000 & 0.456237000 \\ \mathrm{H} & -1.173209000 & -2.392465000 & -1.760887000 \\ \mathrm{O} & -1.783626000 & -1.526548000 & 2.116708000 \\ \mathrm{H} & -2.011420000 & -0.943871000 & 2.849174000 \\ \mathrm{H} & -2.246844000 & -1.143349000 & 1.328402000 \\ \mathrm{C} & 5.047965000 & 0.755260000 & 0.216615000 \\ \mathrm{~F} & 5.962771000 & -0.156246000 & -0.161058000 \\ \mathrm{~F} & 5.411158000 & 1.935797000 & -0.322541000 \\ \mathrm{~F} & 5.179822000 & 0.901685000 & 1.555679000 \\ \mathrm{Na} & 0.112709000 & -2.116965000 & 1.115146000\end{array}$

TSA2-3 $\mathrm{NaOH}$

\begin{tabular}{|c|c|c|c|}
\hline & $\mathrm{Co}$ & nates (Angstrom & \\
\hline & $\mathrm{X}$ & $\mathrm{Y}$ & Z \\
\hline$\overline{\mathrm{C}}$ & -2.665241000 & -1.333252000 & -0.271536000 \\
\hline $\mathrm{C}$ & -1.360078000 & -0.951944000 & -0.519633000 \\
\hline $\mathrm{C}$ & -1.015588000 & 0.407235000 & -0.666629000 \\
\hline $\mathrm{C}$ & -2.054818000 & 1.354651000 & -0.598170000 \\
\hline $\mathrm{C}$ & -3.367287000 & 0.966197000 & -0.348366000 \\
\hline $\mathrm{C}$ & -3.678763000 & -0.375586000 & -0.172577000 \\
\hline $\mathrm{H}$ & -2.905304000 & -2.385986000 & -0.162275000 \\
\hline $\mathrm{H}$ & -0.598787000 & -1.718659000 & -0.615321000 \\
\hline $\mathrm{H}$ & -1.834040000 & 2.401207000 & -0.779085000 \\
\hline $\mathrm{H}$ & -4.150738000 & 1.715312000 & -0.305513000 \\
\hline $\mathrm{C}$ & 0.368385000 & 0.851995000 & -0.817457000 \\
\hline $\mathrm{C}$ & 1.489547000 & 0.115410000 & -0.617470000 \\
\hline $\mathrm{H}$ & 1.353133000 & -0.938138000 & -0.381196000 \\
\hline $\mathrm{O}$ & 0.460383000 & 2.239172000 & -0.987260000 \\
\hline $\mathrm{C}$ & 2.834662000 & 0.666078000 & -0.570369000 \\
\hline $\mathrm{H}$ & 3.063709000 & 1.330090000 & -1.414052000 \\
\hline $\mathrm{C}$ & 3.953247000 & -0.258560000 & -0.277821000 \\
\hline $\mathrm{C}$ & 5.191853000 & -0.125932000 & -0.923165000 \\
\hline $\mathrm{C}$ & 3.842667000 & -1.260159000 & 0.700012000 \\
\hline $\mathrm{C}$ & 6.262570000 & -0.957937000 & -0.617121000 \\
\hline $\mathrm{H}$ & 5.309324000 & 0.641897000 & -1.684806000 \\
\hline $\mathrm{C}$ & 4.907990000 & -2.102809000 & 0.998072000 \\
\hline $\mathrm{H}$ & 2.908845000 & -1.371684000 & 1.247528000 \\
\hline $\mathrm{C}$ & 6.127862000 & -1.958004000 & 0.342989000 \\
\hline $\mathrm{H}$ & 7.207611000 & -0.828750000 & -1.138170000 \\
\hline $\mathrm{H}$ & 4.787328000 & -2.871320000 & 1.757337000 \\
\hline $\mathrm{H}$ & 6.961763000 & -2.611888000 & 0.580149000 \\
\hline $\mathrm{H}$ & 1.397923000 & 2.483090000 & -0.934935000 \\
\hline
\end{tabular}




$\begin{array}{lrcc}\mathrm{O} & 2.369905000 & 2.360494000 & 1.376460000 \\ \mathrm{H} & 3.143267000 & 2.520853000 & 1.925156000 \\ \mathrm{H} & 2.709909000 & 1.565926000 & 0.521259000 \\ \mathrm{C} & -5.076538000 & -0.801091000 & 0.167992000 \\ \mathrm{~F} & -5.988107000 & 0.116011000 & -0.196563000 \\ \mathrm{~F} & -5.406142000 & -1.959844000 & -0.428482000 \\ \mathrm{~F} & -5.225867000 & -0.994411000 & 1.494542000 \\ \mathrm{Na} & 0.274724000 & 2.127015000 & 1.417485000\end{array}$

\begin{tabular}{|c|c|c|c|}
\hline \multicolumn{4}{|c|}{$\mathrm{A3}{ }_{\mathrm{NaOH}}$} \\
\hline & $X$ & Y & Z \\
\hline$\overline{\mathrm{C}}$ & -3.234777000 & 1.361293000 & 0.230311000 \\
\hline $\mathrm{C}$ & -1.933408000 & 1.813221000 & 0.046240000 \\
\hline $\mathrm{C}$ & -0.899557000 & 0.921357000 & -0.265962000 \\
\hline $\mathrm{C}$ & -1.191861000 & -0.443630000 & -0.415175000 \\
\hline $\mathrm{C}$ & -2.494929000 & -0.885197000 & -0.231785000 \\
\hline $\mathrm{C}$ & -3.515335000 & 0.006606000 & 0.099483000 \\
\hline $\mathrm{H}$ & -4.025435000 & 2.063307000 & 0.471379000 \\
\hline $\mathrm{H}$ & -1.742403000 & 2.881667000 & 0.108795000 \\
\hline $\mathrm{H}$ & -0.399580000 & -1.168146000 & -0.683166000 \\
\hline $\mathrm{H}$ & -2.716711000 & -1.942521000 & -0.339619000 \\
\hline $\mathrm{C}$ & 0.485600000 & 1.432666000 & -0.431246000 \\
\hline $\mathrm{C}$ & 1.425400000 & 0.858185000 & -1.194102000 \\
\hline $\mathrm{H}$ & 1.201605000 & -0.091696000 & -1.683125000 \\
\hline $\mathrm{O}$ & 0.780411000 & 2.609747000 & 0.219026000 \\
\hline $\mathrm{C}$ & 2.829100000 & 1.403326000 & -1.322669000 \\
\hline $\mathrm{H}$ & 2.852442000 & 2.466992000 & -1.069174000 \\
\hline $\mathrm{C}$ & 3.761023000 & 0.635985000 & -0.406772000 \\
\hline $\mathrm{C}$ & 3.694702000 & 0.839851000 & 0.980064000 \\
\hline $\mathrm{C}$ & 4.603764000 & -0.367723000 & -0.892472000 \\
\hline $\mathrm{C}$ & 4.435120000 & 0.047731000 & 1.854662000 \\
\hline $\mathrm{H}$ & 3.036563000 & 1.615841000 & 1.362288000 \\
\hline $\mathrm{C}$ & 5.346447000 & -1.164948000 & -0.019048000 \\
\hline $\mathrm{H}$ & 4.656197000 & -0.547504000 & -1.962450000 \\
\hline $\mathrm{C}$ & 5.259571000 & -0.965494000 & 1.357460000 \\
\hline $\mathrm{H}$ & 4.373881000 & 0.222513000 & 2.925078000 \\
\hline $\mathrm{H}$ & 5.988394000 & -1.945240000 & -0.416621000 \\
\hline $\mathrm{H}$ & 5.839058000 & -1.582810000 & 2.037035000 \\
\hline $\mathrm{O}$ & 1.103634000 & -2.235176000 & -1.151509000 \\
\hline $\mathrm{H}$ & 3.169381000 & 1.300739000 & -2.358466000 \\
\hline $\mathrm{H}$ & 0.135086000 & 2.739959000 & 0.923974000 \\
\hline $\mathrm{H}$ & 0.784969000 & -3.035774000 & -1.574660000 \\
\hline
\end{tabular}




$\begin{array}{llcc}\mathrm{C} & -4.922274000 & -0.498340000 & 0.252099000 \\ \mathrm{~F} & -5.688563000 & 0.344459000 & 0.967677000 \\ \mathrm{~F} & -5.525442000 & -0.659367000 & -0.940495000 \\ \mathrm{~F} & -4.958933000 & -1.693205000 & 0.868525000 \\ \mathrm{Na} & 2.312646000 & -1.624566000 & 0.346427000\end{array}$

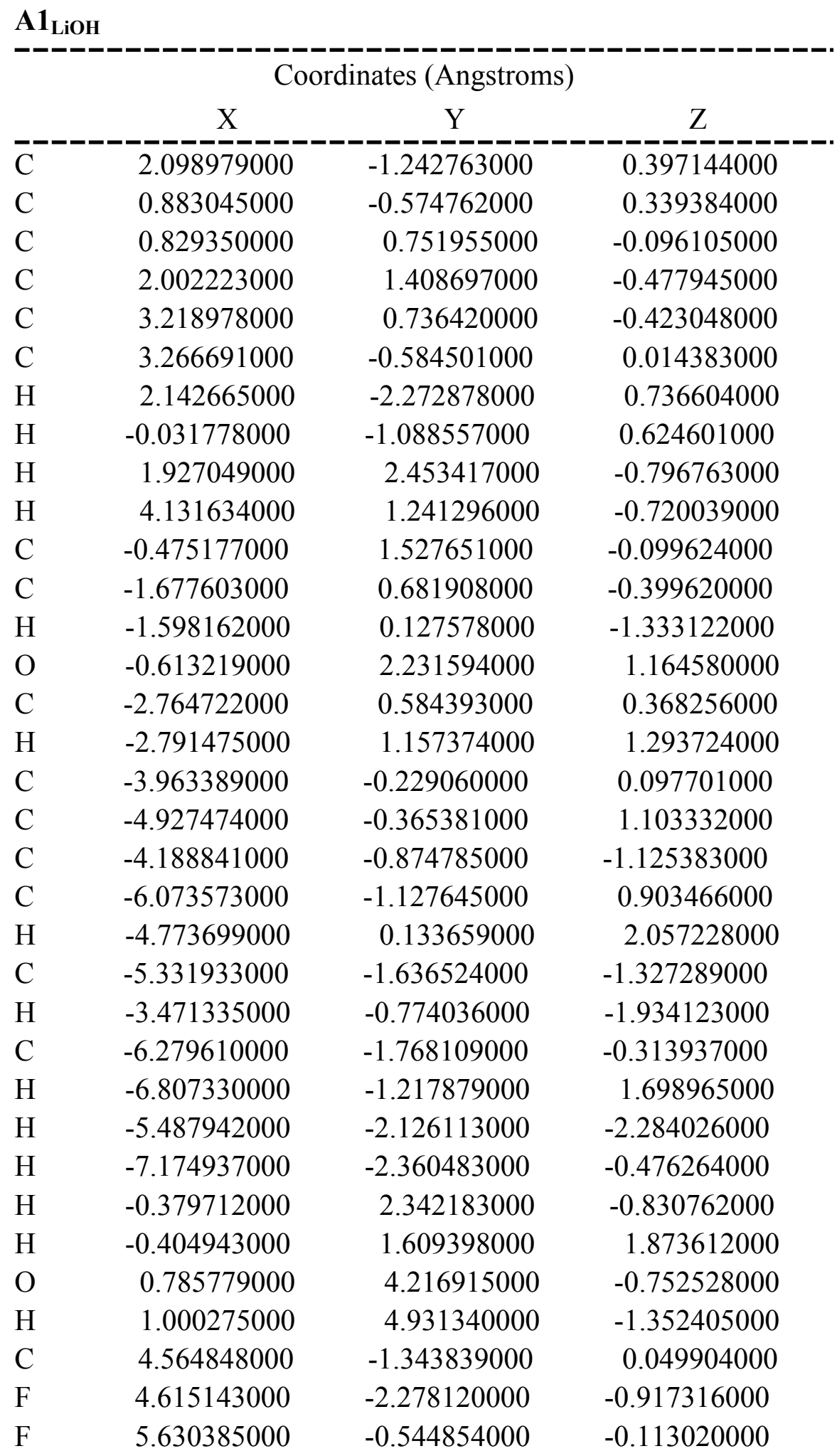




$\begin{array}{llrl}\mathrm{F} & 4.723391000 & -1.991625000 & 1.219441000 \\ \mathrm{Li} & 0.176066000 & 3.975032000 & 0.793565000\end{array}$

TSA1-2 ${ }_{\text {LiOH }}$

\begin{tabular}{|c|c|c|c|}
\hline \multicolumn{4}{|c|}{ Coordinates (Angstroms) } \\
\hline & $\mathrm{X}$ & $\mathrm{Y}$ & $\mathrm{Z}$ \\
\hline $\bar{C}$ & -2.595179000 & -1.112467000 & 0.512194000 \\
\hline $\mathrm{C}$ & -1.288069000 & -0.649054000 & 0.509122000 \\
\hline $\mathrm{C}$ & -0.967743000 & 0.619310000 & -0.000455000 \\
\hline $\mathrm{C}$ & -2.017302000 & 1.401616000 & -0.506918000 \\
\hline $\mathrm{C}$ & -3.323636000 & 0.935749000 & -0.512385000 \\
\hline $\mathrm{C}$ & -3.622534000 & -0.324581000 & -0.001164000 \\
\hline $\mathrm{H}$ & -2.815665000 & -2.096286000 & 0.913277000 \\
\hline $\mathrm{H}$ & -0.511153000 & -1.295182000 & 0.905021000 \\
\hline $\mathrm{H}$ & -1.798455000 & 2.392062000 & -0.890220000 \\
\hline $\mathrm{H}$ & -4.117111000 & 1.562191000 & -0.907137000 \\
\hline $\mathrm{C}$ & 0.406199000 & 1.167316000 & 0.039994000 \\
\hline $\mathrm{C}$ & 1.571282000 & 0.293984000 & 0.113499000 \\
\hline $\mathrm{H}$ & 1.502627000 & -0.518906000 & 0.834586000 \\
\hline $\mathrm{O}$ & 0.621560000 & 2.236056000 & -0.916951000 \\
\hline $\mathrm{C}$ & 2.758011000 & 0.494928000 & -0.507061000 \\
\hline $\mathrm{H}$ & 2.815887000 & 1.234612000 & -1.309394000 \\
\hline $\mathrm{C}$ & 3.992568000 & -0.271267000 & -0.279627000 \\
\hline $\mathrm{C}$ & 4.984335000 & -0.293219000 & -1.270084000 \\
\hline $\mathrm{C}$ & 4.235133000 & -0.980132000 & 0.906060000 \\
\hline $\mathrm{C}$ & 6.163407000 & -1.007982000 & -1.094273000 \\
\hline $\mathrm{H}$ & 4.819129000 & 0.248956000 & -2.198727000 \\
\hline $\mathrm{C}$ & 5.410637000 & -1.700356000 & 1.080327000 \\
\hline $\mathrm{H}$ & 3.503633000 & -0.953592000 & 1.709072000 \\
\hline $\mathrm{C}$ & 6.381763000 & -1.719240000 & 0.082150000 \\
\hline $\mathrm{H}$ & 6.913241000 & -1.010984000 & -1.879990000 \\
\hline $\mathrm{H}$ & 5.575006000 & -2.239118000 & 2.009105000 \\
\hline $\mathrm{H}$ & 7.303554000 & -2.274946000 & 0.224076000 \\
\hline $\mathrm{H}$ & 0.607357000 & 1.995115000 & 1.168079000 \\
\hline $\mathrm{H}$ & 0.646270000 & 1.861038000 & -1.810024000 \\
\hline $\mathrm{O}$ & 1.111712000 & 2.952284000 & 1.748249000 \\
\hline $\mathrm{H}$ & 1.399433000 & 2.647341000 & 2.614865000 \\
\hline $\mathrm{Li}$ & 2.071109000 & 2.962393000 & 0.218211000 \\
\hline $\mathrm{C}$ & -5.022916000 & -0.854778000 & -0.070457000 \\
\hline $\mathrm{F}$ & -5.939101000 & 0.125616000 & 0.030325000 \\
\hline $\mathrm{F}$ & -5.264174000 & -1.487119000 & -1.237129000 \\
\hline $\mathrm{F}$ & -5.276614000 & -1.743515000 & 0.907566000 \\
\hline
\end{tabular}




\begin{tabular}{|c|c|c|c|}
\hline \multicolumn{4}{|c|}{ A2 $2_{\mathrm{LiOH}}$} \\
\hline & $X$ & Y & Z \\
\hline $\mathrm{C}$ & -2.615138000 & -1.211716000 & -0.188937000 \\
\hline $\mathrm{C}$ & -1.315570000 & -0.848729000 & -0.461299000 \\
\hline $\mathrm{C}$ & -0.959450000 & 0.509767000 & -0.700594000 \\
\hline $\mathrm{C}$ & -2.017005000 & 1.460063000 & -0.645847000 \\
\hline $\mathrm{C}$ & -3.323651000 & 1.076331000 & -0.360658000 \\
\hline $\mathrm{C}$ & -3.638931000 & -0.254167000 & -0.124411000 \\
\hline $\mathrm{H}$ & -2.850372000 & -2.258753000 & -0.022728000 \\
\hline $\mathrm{H}$ & -0.556900000 & -1.622967000 & -0.507830000 \\
\hline $\mathrm{H}$ & -1.812024000 & 2.495511000 & -0.896882000 \\
\hline $\mathrm{H}$ & -4.104962000 & 1.829164000 & -0.339753000 \\
\hline $\mathrm{C}$ & 0.392427000 & 0.941940000 & -0.878677000 \\
\hline $\mathrm{C}$ & 1.563426000 & 0.215065000 & -0.638162000 \\
\hline $\mathrm{H}$ & 1.441041000 & -0.865298000 & -0.607753000 \\
\hline $\mathrm{O}$ & 0.506426000 & 2.367687000 & -0.859973000 \\
\hline $\mathrm{C}$ & 2.822434000 & 0.724554000 & -0.384927000 \\
\hline $\mathrm{H}$ & 2.979697000 & 1.803365000 & -0.430058000 \\
\hline $\mathrm{C}$ & 4.044613000 & -0.058669000 & -0.197793000 \\
\hline $\mathrm{C}$ & 5.216754000 & 0.593359000 & 0.224880000 \\
\hline $\mathrm{C}$ & 4.124724000 & -1.446379000 & -0.409363000 \\
\hline $\mathrm{C}$ & 6.399883000 & -0.100725000 & 0.439214000 \\
\hline $\mathrm{H}$ & 5.189444000 & 1.668499000 & 0.391310000 \\
\hline $\mathrm{C}$ & 5.307341000 & -2.141596000 & -0.188344000 \\
\hline $\mathrm{H}$ & 3.257665000 & -1.991621000 & -0.771957000 \\
\hline $\mathrm{C}$ & 6.454747000 & -1.477964000 & 0.238934000 \\
\hline $\mathrm{H}$ & 7.285533000 & 0.437742000 & 0.765537000 \\
\hline $\mathrm{H}$ & 5.335149000 & -3.213649000 & -0.364392000 \\
\hline $\mathrm{H}$ & 7.378936000 & -2.023011000 & 0.404398000 \\
\hline $\mathrm{H}$ & 1.283537000 & 2.622667000 & -1.371282000 \\
\hline $\mathrm{O}$ & 1.426618000 & 1.537963000 & 2.148921000 \\
\hline $\mathrm{H}$ & 1.368725000 & 0.876028000 & 2.848154000 \\
\hline $\mathrm{H}$ & 2.043532000 & 1.156746000 & 1.469434000 \\
\hline $\mathrm{C}$ & -5.024813000 & -0.669188000 & 0.249583000 \\
\hline $\mathrm{F}$ & -5.942461000 & 0.254353000 & -0.088485000 \\
\hline $\mathrm{F}$ & -5.381218000 & -1.826313000 & -0.341233000 \\
\hline $\mathrm{F}$ & -5.153295000 & -0.871803000 & 1.579950000 \\
\hline $\mathrm{Li}$ & -0.000448000 & 1.987527000 & 0.97617600 \\
\hline
\end{tabular}

TSA2-3 ${ }_{\mathrm{LiOH}}$ 


\begin{tabular}{|c|c|c|c|}
\hline & $X$ & Y & Z \\
\hline $\mathrm{C}$ & -2.682186000 & -1.132274000 & 0.275204000 \\
\hline $\mathrm{C}$ & -1.363581000 & -0.719181000 & 0.198097000 \\
\hline $\mathrm{C}$ & -1.031515000 & 0.560544000 & -0.283584000 \\
\hline $\mathrm{C}$ & -2.081781000 & 1.409316000 & -0.668253000 \\
\hline $\mathrm{C}$ & -3.404582000 & 0.995091000 & -0.584105000 \\
\hline $\mathrm{C}$ & -3.713110000 & -0.276956000 & -0.114838000 \\
\hline $\mathrm{H}$ & -2.914028000 & -2.126267000 & 0.643520000 \\
\hline $\mathrm{H}$ & -0.583688000 & -1.401020000 & 0.521454000 \\
\hline $\mathrm{H}$ & -1.852832000 & 2.396667000 & -1.051911000 \\
\hline $\mathrm{H}$ & -4.198357000 & 1.665126000 & -0.897096000 \\
\hline $\mathrm{C}$ & 0.355942000 & 1.018138000 & -0.361702000 \\
\hline $\mathrm{C}$ & 1.499263000 & 0.294080000 & -0.234785000 \\
\hline $\mathrm{H}$ & 1.371492000 & -0.774652000 & -0.077505000 \\
\hline $\mathrm{O}$ & 0.464199000 & 2.426397000 & -0.531478000 \\
\hline $\mathrm{C}$ & 2.846496000 & 0.815837000 & -0.220844000 \\
\hline $\mathrm{H}$ & 3.021425000 & 1.653434000 & -0.908674000 \\
\hline $\mathrm{C}$ & 3.981037000 & -0.136634000 & -0.208162000 \\
\hline $\mathrm{C}$ & 5.152454000 & 0.135508000 & -0.929378000 \\
\hline $\mathrm{C}$ & 3.958907000 & -1.307428000 & 0.565903000 \\
\hline $\mathrm{C}$ & 6.243190000 & -0.725200000 & -0.892864000 \\
\hline $\mathrm{H}$ & 5.202420000 & 1.038387000 & -1.534093000 \\
\hline $\mathrm{C}$ & 5.043751000 & -2.176911000 & 0.593194000 \\
\hline $\mathrm{H}$ & 3.082983000 & -1.537257000 & 1.169378000 \\
\hline $\mathrm{C}$ & 6.195315000 & -1.892887000 & -0.135806000 \\
\hline $\mathrm{H}$ & 7.134743000 & -0.485051000 & -1.466024000 \\
\hline $\mathrm{H}$ & 4.992091000 & -3.078560000 & 1.197865000 \\
\hline $\mathrm{H}$ & 7.043773000 & -2.569989000 & -0.110843000 \\
\hline $\mathrm{H}$ & 1.205194000 & 2.612065000 & -1.125251000 \\
\hline $\mathrm{O}$ & 2.596894000 & 2.312493000 & 1.912364000 \\
\hline $\mathrm{H}$ & 3.013405000 & 1.961236000 & 2.705836000 \\
\hline $\mathrm{H}$ & 2.852458000 & 1.575838000 & 1.006911000 \\
\hline $\mathrm{C}$ & -5.142108000 & -0.709113000 & 0.036606000 \\
\hline $\mathrm{F}$ & -5.956158000 & -0.069132000 & -0.820178000 \\
\hline $\mathrm{F}$ & -5.289907000 & -2.028911000 & -0.174908000 \\
\hline $\mathrm{F}$ & -5.609679000 & -0.461983000 & 1.276381000 \\
\hline $\mathrm{Li}$ & 0.898973000 & 2.429195000 & 1.379867000 \\
\hline
\end{tabular}

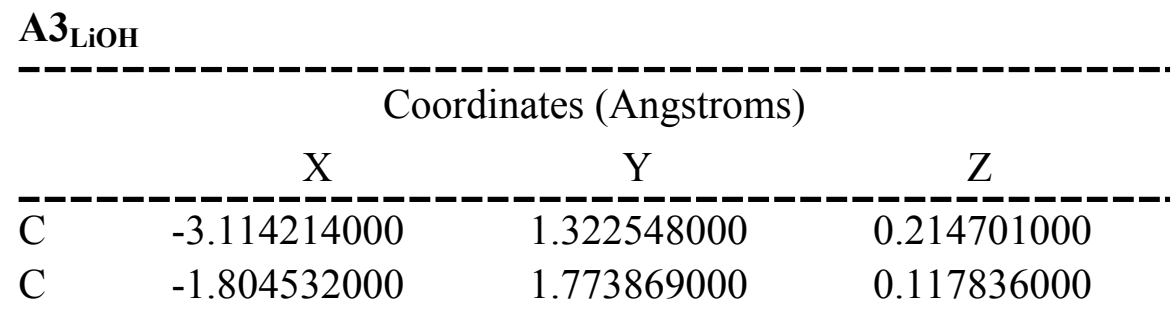




\begin{tabular}{lrrr}
$\mathrm{C}$ & -0.758320000 & 0.876915000 & -0.132478000 \\
$\mathrm{C}$ & -1.032903000 & -0.488703000 & -0.279444000 \\
$\mathrm{C}$ & -2.346870000 & -0.930789000 & -0.182893000 \\
$\mathrm{C}$ & -3.384778000 & -0.034048000 & 0.064507000 \\
$\mathrm{H}$ & -3.920158000 & 2.023372000 & 0.402522000 \\
$\mathrm{H}$ & -1.604320000 & 2.839099000 & 0.204035000 \\
$\mathrm{H}$ & -0.218041000 & -1.214206000 & -0.428328000 \\
$\mathrm{H}$ & -2.560310000 & -1.989934000 & -0.285392000 \\
$\mathrm{C}$ & 0.628569000 & 1.394268000 & -0.235718000 \\
$\mathrm{C}$ & 1.531962000 & 0.964491000 & -1.126482000 \\
$\mathrm{H}$ & 1.246134000 & 0.159987000 & -1.796924000 \\
$\mathrm{O}$ & 0.955955000 & 2.426126000 & 0.607279000 \\
$\mathrm{C}$ & 2.943112000 & 1.498086000 & -1.210851000 \\
$\mathrm{H}$ & 2.991908000 & 2.508535000 & -0.795797000 \\
$\mathrm{C}$ & 3.864490000 & 0.576361000 & -0.440023000 \\
$\mathrm{C}$ & 3.931284000 & 0.668520000 & 0.958697000 \\
$\mathrm{C}$ & 4.524334000 & -0.482364000 & -1.072671000 \\
$\mathrm{C}$ & 4.623537000 & -0.284984000 & 1.702553000 \\
$\mathrm{H}$ & 3.405342000 & 1.478361000 & 1.456531000 \\
$\mathrm{C}$ & 5.217746000 & -1.438949000 & -0.329227000 \\
$\mathrm{H}$ & 4.454394000 & -0.585938000 & -2.151667000 \\
$\mathrm{C}$ & 5.263703000 & -1.347400000 & 1.060201000 \\
$\mathrm{H}$ & 4.658070000 & -0.203851000 & 2.784765000 \\
$\mathrm{H}$ & 5.699929000 & -2.268292000 & -0.836460000 \\
$\mathrm{H}$ & 5.790170000 & -2.098539000 & 1.640034000 \\
$\mathrm{O}$ & 1.350539000 & -2.374937000 & -0.408033000 \\
$\mathrm{H}$ & 3.259230000 & 1.552767000 & -2.257654000 \\
$\mathrm{H}$ & 0.293332000 & 2.468964000 & 1.307764000 \\
$\mathrm{H} \mathrm{F}$ & 1.157278000 & -3.312121000 & -0.366837000 \\
$\mathrm{C}$ & -4.802286000 & -0.532969000 & 0.117425000 \\
$\mathrm{~F}$ & -5.606677000 & 0.302037000 & 0.799041000 \\
$\mathrm{~F}$ & -5.327724000 & -0.664306000 & -1.114769000 \\
-4.885200000 & -1.738621000 & 0.705555000 \\
& 2.520829000 & -1.356275000 & 0.221400000 \\
\hline--- & &
\end{tabular}

\begin{tabular}{|c|c|c|c|}
\hline \multicolumn{4}{|c|}{$\mathrm{A1}_{\text {tBuOK }}$} \\
\hline & $\mathrm{X}$ & $\mathrm{Y}$ & Z \\
\hline$\overline{\mathrm{C}}$ & 1.961207000 & -2.529338000 & 0.243574000 \\
\hline $\mathrm{C}$ & 0.769665000 & -1.840708000 & 0.439007000 \\
\hline $\mathrm{C}$ & 0.728945000 & -0.449729000 & 0.325017000 \\
\hline $\mathrm{C}$ & 1.892616000 & 0.251520000 & -0.006392000 \\
\hline $\mathrm{C}$ & 3.083266000 & -0.436968000 & -0.206454000 \\
\hline
\end{tabular}




\begin{tabular}{|c|c|c|c|}
\hline $\mathrm{C}$ & 3.119596000 & -1.824289000 & -0.074265000 \\
\hline $\mathrm{H}$ & 1.991157000 & -3.609615000 & 0.337274000 \\
\hline $\mathrm{H}$ & -0.140489000 & -2.390587000 & 0.669698000 \\
\hline $\mathrm{H}$ & 1.822858000 & 1.339072000 & -0.118292000 \\
\hline $\mathrm{H}$ & 3.987231000 & 0.104481000 & -0.467586000 \\
\hline $\mathrm{C}$ & -0.541581000 & 0.327994000 & 0.626147000 \\
\hline $\mathrm{C}$ & -1.768581000 & -0.297139000 & 0.031946000 \\
\hline $\mathrm{H}$ & -1.673864000 & -0.539690000 & -1.025269000 \\
\hline $\mathrm{O}$ & -0.656822000 & 0.517005000 & 2.052795000 \\
\hline $\mathrm{C}$ & -2.902542000 & -0.540868000 & 0.693342000 \\
\hline $\mathrm{H}$ & -2.945776000 & -0.280044000 & 1.750035000 \\
\hline $\mathrm{C}$ & -4.132491000 & -1.135895000 & 0.142557000 \\
\hline $\mathrm{C}$ & -5.129820000 & -1.565555000 & 1.025958000 \\
\hline $\mathrm{C}$ & -4.355789000 & -1.291670000 & -1.232050000 \\
\hline $\mathrm{C}$ & -6.304114000 & -2.146255000 & 0.558903000 \\
\hline $\mathrm{H}$ & -4.978696000 & -1.442940000 & 2.095933000 \\
\hline $\mathrm{C}$ & -5.527413000 & -1.871460000 & -1.700894000 \\
\hline $\mathrm{H}$ & -3.614636000 & -0.942473000 & -1.944794000 \\
\hline $\mathrm{C}$ & -6.506213000 & -2.304553000 & -0.808213000 \\
\hline $\mathrm{H}$ & -7.061594000 & -2.473809000 & 1.264910000 \\
\hline $\mathrm{H}$ & -5.681173000 & -1.981270000 & -2.770345000 \\
\hline $\mathrm{H}$ & -7.422087000 & -2.755087000 & -1.178610000 \\
\hline $\mathrm{H}$ & -0.399639000 & 1.350787000 & 0.232620000 \\
\hline $\mathrm{H}$ & -0.693211000 & -0.361686000 & 2.454580000 \\
\hline $\mathrm{C}$ & 1.128646000 & 5.047954000 & -1.241475000 \\
\hline $\mathrm{C}$ & 0.366072000 & 3.711973000 & -1.115591000 \\
\hline $\mathrm{H}$ & 0.836367000 & 5.718841000 & -0.423343000 \\
\hline $\mathrm{H}$ & 2.205881000 & 4.860931000 & -1.157149000 \\
\hline $\mathrm{H}$ & 0.938709000 & 5.563263000 & -2.192369000 \\
\hline $\mathrm{C}$ & 0.756877000 & 2.811890000 & -2.309223000 \\
\hline $\mathrm{C}$ & -1.149980000 & 3.996554000 & -1.196218000 \\
\hline $\mathrm{H}$ & 0.234232000 & 1.849216000 & -2.239763000 \\
\hline $\mathrm{H}$ & 0.513283000 & 3.265113000 & -3.278899000 \\
\hline $\mathrm{H}$ & 1.834704000 & 2.609428000 & -2.286432000 \\
\hline $\mathrm{H}$ & -1.443480000 & 4.498749000 & -2.127553000 \\
\hline $\mathrm{H}$ & -1.709336000 & 3.055219000 & -1.122759000 \\
\hline $\mathrm{H}$ & -1.450585000 & 4.633185000 & -0.354148000 \\
\hline $\mathrm{O}$ & 0.671040000 & 3.099688000 & 0.076815000 \\
\hline K & 0.786134000 & 2.764490000 & 2.389732000 \\
\hline $\mathrm{C}$ & 4.403654000 & -2.559857000 & -0.340802000 \\
\hline $\mathrm{F}$ & 4.436359000 & -3.757405000 & 0.269904000 \\
\hline $\mathrm{F}$ & 5.472953000 & -1.863846000 & 0.085944000 \\
\hline $\mathrm{F}$ & 4.584882000 & -2.784478000 & -1.654602000 \\
\hline
\end{tabular}


TSA1-2 ${ }_{\text {tBuOK }}$

\begin{tabular}{|c|c|c|c|}
\hline \multicolumn{4}{|c|}{ Coordinates (Angstroms) } \\
\hline & $\mathrm{X}$ & Y & $\mathrm{Z}$ \\
\hline & -3.318174000 & -0.817003000 & -1.196295000 \\
\hline $\mathrm{C}$ & -2.010652000 & -0.460505000 & -1.517185000 \\
\hline $\mathrm{C}$ & -1.018045000 & -0.352514000 & -0.530126000 \\
\hline $\mathrm{C}$ & -1.395340000 & -0.618781000 & 0.801010000 \\
\hline $\mathrm{C}$ & -2.690803000 & -0.980092000 & 1.120797000 \\
\hline $\mathrm{C}$ & -3.667396000 & -1.073029000 & 0.123909000 \\
\hline $\mathrm{H}$ & -4.064562000 & -0.900355000 & -1.979860000 \\
\hline $\mathrm{H}$ & -1.744284000 & -0.286811000 & -2.556488000 \\
\hline $\mathrm{H}$ & -0.661346000 & -0.501270000 & 1.593228000 \\
\hline $\mathrm{H}$ & -2.958071000 & -1.168578000 & 2.156039000 \\
\hline $\mathrm{C}$ & 0.340755000 & 0.168214000 & -0.822502000 \\
\hline $\mathrm{C}$ & 1.473943000 & -0.641019000 & -0.364625000 \\
\hline $\mathrm{H}$ & 1.284442000 & -1.272176000 & 0.503181000 \\
\hline $\mathrm{O}$ & 0.479965000 & 0.609725000 & -2.182987000 \\
\hline $\mathrm{C}$ & 2.709492000 & -0.613759000 & -0.896781000 \\
\hline $\mathrm{H}$ & 2.910771000 & 0.082305000 & -1.710089000 \\
\hline $\mathrm{C}$ & 3.859303000 & -1.393891000 & -0.418206000 \\
\hline $\mathrm{C}$ & 5.157758000 & -0.923157000 & -0.657377000 \\
\hline $\mathrm{C}$ & 3.717922000 & -2.603432000 & 0.276755000 \\
\hline $\mathrm{C}$ & 6.271338000 & -1.616589000 & -0.197811000 \\
\hline $\mathrm{H}$ & 5.289228000 & 0.011602000 & -1.197479000 \\
\hline $\mathrm{C}$ & 4.830211000 & -3.296315000 & 0.739900000 \\
\hline $\mathrm{H}$ & 2.726095000 & -3.017054000 & 0.437612000 \\
\hline $\mathrm{C}$ & 6.113288000 & -2.806426000 & 0.507642000 \\
\hline $\mathrm{H}$ & 7.266181000 & -1.223750000 & -0.388236000 \\
\hline $\mathrm{H}$ & 4.694407000 & -4.232860000 & 1.273624000 \\
\hline $\mathrm{H}$ & 6.981608000 & -3.351107000 & 0.866254000 \\
\hline $\mathrm{H}$ & 0.384077000 & 1.341481000 & -0.133605000 \\
\hline $\mathrm{H}$ & 0.775875000 & -0.144010000 & -2.716259000 \\
\hline $\mathrm{C}$ & 0.844083000 & 4.270519000 & 1.779318000 \\
\hline $\mathrm{C}$ & 1.218453000 & 2.893888000 & 1.215074000 \\
\hline $\mathrm{H}$ & 0.788685000 & 5.006805000 & 0.967911000 \\
\hline $\mathrm{H}$ & -0.136665000 & 4.220080000 & 2.265384000 \\
\hline $\mathrm{H}$ & 1.577855000 & 4.625499000 & 2.512901000 \\
\hline $\mathrm{C}$ & 1.285653000 & 1.870263000 & 2.361643000 \\
\hline $\mathrm{C}$ & 2.586342000 & 2.972636000 & 0.517253000 \\
\hline $\mathrm{H}$ & 1.597208000 & 0.891731000 & 1.978230000 \\
\hline $\mathrm{H}$ & 2.002412000 & 2.170571000 & 3.135063000 \\
\hline $\mathrm{H}$ & 0.296964000 & 1.765255000 & 2.822716000 \\
\hline $\mathrm{H}$ & 3.383265000 & 3.270915000 & 1.209171000 \\
\hline
\end{tabular}




\begin{tabular}{lrrr}
$\mathrm{H}$ & 2.847235000 & 1.996606000 & 0.092744000 \\
$\mathrm{H}$ & 2.547428000 & 3.704372000 & -0.299049000 \\
$\mathrm{O}$ & 0.230746000 & 2.522534000 & 0.297041000 \\
$\mathrm{~K}$ & -1.139550000 & 2.681731000 & -1.694104000 \\
$\mathrm{C}$ & -5.061621000 & -1.486894000 & 0.485160000 \\
$\mathrm{~F}$ & -5.950854000 & -1.160365000 & -0.471494000 \\
$\mathrm{~F}$ & -5.474706000 & -0.906577000 & 1.628353000 \\
$\mathrm{~F}$ & $-\mathbf{- 5 . 1 6 3 5 6 3 0 0 0}$ & -2.816712000 & 0.673941000 \\
\hline
\end{tabular}

\begin{tabular}{|c|c|c|c|}
\hline \multicolumn{4}{|c|}{$\mathrm{A} 2_{\mathrm{tBuOK}}$} \\
\hline & $\mathrm{X}$ & Y & Z \\
\hline$\overline{\mathrm{C}}$ & 3.825979000 & -0.886146000 & 0.799687000 \\
\hline $\mathrm{C}$ & 2.576917000 & -1.362724000 & 1.166616000 \\
\hline $\mathrm{C}$ & 1.455448000 & -1.278024000 & 0.291308000 \\
\hline $\mathrm{C}$ & 1.703373000 & -0.688030000 & -0.982955000 \\
\hline $\mathrm{C}$ & 2.953929000 & -0.225793000 & -1.337088000 \\
\hline $\mathrm{C}$ & 4.033398000 & -0.300307000 & -0.448062000 \\
\hline $\mathrm{H}$ & 4.653415000 & -0.972732000 & 1.498367000 \\
\hline $\mathrm{H}$ & 2.449281000 & -1.843169000 & 2.131066000 \\
\hline $\mathrm{H}$ & 0.895258000 & -0.589061000 & -1.700685000 \\
\hline $\mathrm{H}$ & 3.096562000 & 0.220524000 & -2.316519000 \\
\hline $\mathrm{C}$ & 0.154130000 & -1.649385000 & 0.739907000 \\
\hline $\mathrm{C}$ & -1.045400000 & -1.491219000 & 0.044177000 \\
\hline $\mathrm{H}$ & -0.940132000 & -1.284294000 & -1.018486000 \\
\hline $\mathrm{O}$ & 0.067101000 & -1.911171000 & 2.130465000 \\
\hline $\mathrm{C}$ & -2.325358000 & -1.530009000 & 0.562741000 \\
\hline $\mathrm{H}$ & -2.455406000 & -1.720401000 & 1.627599000 \\
\hline $\mathrm{C}$ & -3.551725000 & -1.325579000 & -0.195692000 \\
\hline $\mathrm{C}$ & -4.769381000 & -1.146445000 & 0.491217000 \\
\hline $\mathrm{C}$ & -3.599306000 & -1.234357000 & -1.601711000 \\
\hline $\mathrm{C}$ & -5.951895000 & -0.863834000 & -0.178190000 \\
\hline $\mathrm{H}$ & -4.772899000 & -1.218790000 & 1.576961000 \\
\hline $\mathrm{C}$ & -4.782213000 & -0.940873000 & -2.269121000 \\
\hline $\mathrm{H}$ & -2.699366000 & -1.409336000 & -2.185157000 \\
\hline $\mathrm{C}$ & -5.969443000 & -0.746670000 & -1.567121000 \\
\hline $\mathrm{H}$ & -6.868704000 & -0.727560000 & 0.389388000 \\
\hline $\mathrm{H}$ & -4.776450000 & -0.872393000 & -3.353923000 \\
\hline $\mathrm{H}$ & -6.892519000 & -0.520672000 & -2.091770000 \\
\hline $\mathrm{H}$ & -2.137108000 & 0.538562000 & 0.861786000 \\
\hline $\mathrm{H}$ & -0.436740000 & -2.725752000 & 2.250830000 \\
\hline $\mathrm{C}$ & -1.465794000 & 1.859525000 & -1.231879000 \\
\hline $\mathrm{C}$ & -2.102356000 & 2.351880000 & 0.070608000 \\
\hline
\end{tabular}




\begin{tabular}{lrrr}
$\mathrm{H}$ & -0.392992000 & 1.677603000 & -1.094991000 \\
$\mathrm{H}$ & -1.940358000 & 0.928337000 & -1.558453000 \\
$\mathrm{H}$ & -1.591349000 & 2.598989000 & -2.029978000 \\
$\mathrm{C}$ & -3.618385000 & 2.480820000 & -0.083655000 \\
$\mathrm{C}$ & -1.483026000 & 3.675046000 & 0.511805000 \\
$\mathrm{H}$ & -4.067846000 & 2.805765000 & 0.860123000 \\
$\mathrm{H}$ & -3.868613000 & 3.212475000 & -0.859424000 \\
$\mathrm{H}$ & -4.063396000 & 1.520376000 & -0.365503000 \\
$\mathrm{H}$ & -1.677036000 & 4.459809000 & -0.225531000 \\
$\mathrm{H}$ & -1.902841000 & 3.991364000 & 1.472473000 \\
$\mathrm{H}$ & -0.394552000 & 3.578666000 & 0.617157000 \\
$\mathrm{O}$ & -1.809254000 & 1.425958000 & 1.127360000 \\
$\mathrm{~K}$ & 0.420906000 & 0.739477000 & 2.234892000 \\
$\mathrm{C}$ & 5.390755000 & 0.170796000 & -0.849715000 \\
$\mathrm{~F}$ & 6.089179000 & 0.650878000 & 0.201461000 \\
$\mathrm{~F}$ & 6.148747000 & -0.808337000 & -1.387335000 \\
$\mathrm{~F}$ & 5.333843000 & 1.154271000 & -1.770587000 \\
\hline
\end{tabular}

TSA2-3 ${ }_{\mathrm{tBuOK}}$

\begin{tabular}{lrcc} 
& \multicolumn{3}{c}{ Coordinates (Angstroms) } \\
\hline $\mathrm{C}$ & -3.918242000 & 0.801351000 & -0.660441000 \\
$\mathrm{C}$ & -2.579508000 & 0.885135000 & -1.026481000 \\
$\mathrm{C}$ & -1.705063000 & -0.210156000 & -0.866268000 \\
$\mathrm{C}$ & -2.258891000 & -1.411093000 & -0.373005000 \\
$\mathrm{C}$ & -3.589120000 & -1.488390000 & -0.004430000 \\
$\mathrm{C}$ & -4.429406000 & -0.378805000 & -0.132681000 \\
$\mathrm{H}$ & -4.569517000 & 1.657971000 & -0.802059000 \\
$\mathrm{H}$ & -2.203487000 & 1.795322000 & -1.481573000 \\
$\mathrm{H}$ & -1.643156000 & -2.300706000 & -0.295760000 \\
$\mathrm{H}$ & -3.988141000 & -2.424227000 & 0.373764000 \\
$\mathrm{C}$ & -0.280752000 & -0.054095000 & -1.147339000 \\
$\mathrm{C}$ & 0.699011000 & -0.928944000 & -0.788833000 \\
$\mathrm{H}$ & 0.392621000 & -1.815481000 & -0.234996000 \\
$\mathrm{O}$ & 0.029567000 & 1.181562000 & -1.706259000 \\
$\mathrm{C}$ & 2.111855000 & -0.694817000 & -0.987014000 \\
$\mathrm{H}$ & 2.363683000 & -0.353664000 & -1.997293000 \\
$\mathrm{C}$ & 3.100812000 & -1.616966000 & -0.410997000 \\
$\mathrm{C}$ & 4.336049000 & -1.836738000 & -1.045485000 \\
$\mathrm{C}$ & 2.906085000 & -2.237690000 & 0.835879000 \\
$\mathrm{C}$ & 5.317936000 & -2.634523000 & -0.470686000 \\
$\mathrm{H}$ & 4.516904000 & -1.375576000 & -2.014278000 \\
$\mathrm{C}$ & 3.882019000 & -3.048368000 & 1.404498000
\end{tabular}




\begin{tabular}{lrrr}
$\mathrm{H}$ & 1.977360000 & -2.068060000 & 1.377284000 \\
$\mathrm{C}$ & 5.098746000 & -3.252395000 & 0.758621000 \\
$\mathrm{H}$ & 6.260558000 & -2.781730000 & -0.991508000 \\
$\mathrm{H}$ & 3.694812000 & -3.516053000 & 2.367947000 \\
$\mathrm{H}$ & 5.863703000 & -3.879588000 & 1.206301000 \\
$\mathrm{H}$ & 2.247857000 & 0.564066000 & -0.295598000 \\
$\mathrm{H}$ & 0.954063000 & 1.399611000 & -1.472511000 \\
$\mathrm{C}$ & 4.136993000 & 1.580995000 & 1.418245000 \\
$\mathrm{C}$ & 3.385361000 & 2.276015000 & 0.276018000 \\
$\mathrm{H}$ & 3.565973000 & 1.660219000 & 2.351497000 \\
$\mathrm{H}$ & 4.274884000 & 0.518059000 & 1.192251000 \\
$\mathrm{H}$ & 5.123202000 & 2.030688000 & 1.581998000 \\
$\mathrm{C}$ & 4.192398000 & 2.163493000 & -1.024582000 \\
$\mathrm{C}$ & 3.144390000 & 3.750132000 & 0.613820000 \\
$\mathrm{H}$ & 3.630078000 & 2.598053000 & -1.858837000 \\
$\mathrm{H}$ & 5.151717000 & 2.686856000 & -0.942941000 \\
$\mathrm{H}$ & 4.398986000 & 1.113092000 & -1.254626000 \\
$\mathrm{H}$ & 4.087004000 & 4.292889000 & 0.745634000 \\
$\mathrm{H}$ & 2.577322000 & 4.234536000 & -0.189589000 \\
$\mathrm{H}$ & 2.575647000 & 3.840577000 & 1.549339000 \\
$\mathrm{O}$ & 2.117870000 & 1.668947000 & 0.111398000 \\
$\mathrm{~K}$ & -0.207851000 & 1.842135000 & 1.039958000 \\
$\mathrm{C}$ & -5.848849000 & -0.454552000 & 0.342155000 \\
$\mathrm{~F}$ & -5.943493000 & -0.207684000 & 1.667045000 \\
$\mathrm{~F}$ & -6.383066000 & -1.671576000 & 0.141480000 \\
$\mathrm{~F}$ & -6.639368000 & 0.441591000 & -0.273050000 \\
\hline------1 &
\end{tabular}

\section{$\mathrm{A}_{\text {tBuOK }}$}

Coordinates (Angstroms)

$\mathrm{X}$ Y Z

\begin{tabular}{lrrr}
\hline $\mathrm{C}$ & -3.597316000 & -1.733117000 & 0.361732000 \\
$\mathrm{C}$ & -2.340079000 & -2.168742000 & 0.763395000 \\
$\mathrm{C}$ & -1.219920000 & -1.335367000 & 0.651906000 \\
$\mathrm{C}$ & -1.391247000 & -0.034752000 & 0.150112000 \\
$\mathrm{C}$ & -2.649587000 & 0.395974000 & -0.245340000 \\
$\mathrm{C}$ & -3.753254000 & -0.451535000 & -0.154099000 \\
$\mathrm{H}$ & -4.454365000 & -2.391640000 & 0.454896000 \\
$\mathrm{H}$ & -2.251137000 & -3.156125000 & 1.208850000 \\
$\mathrm{H}$ & -0.545612000 & 0.668294000 & 0.082516000 \\
$\mathrm{H}$ & -2.766309000 & 1.403182000 & -0.633543000 \\
$\mathrm{C}$ & 0.122257000 & -1.831036000 & 1.055967000 \\
$\mathrm{C}$ & 1.145939000 & -1.035512000 & 1.392864000 \\
$\mathrm{H}$ & 1.024944000 & 0.045239000 & 1.313439000
\end{tabular}




\begin{tabular}{|c|c|c|c|}
\hline $\mathrm{O}$ & 0.280008000 & -3.196342000 & 1.119775000 \\
\hline $\mathrm{C}$ & 2.518994000 & -1.537442000 & 1.760647000 \\
\hline $\mathrm{H}$ & 2.493286000 & -2.606694000 & 1.985533000 \\
\hline $\mathrm{C}$ & 3.468955000 & -1.267674000 & 0.611669000 \\
\hline $\mathrm{C}$ & 3.638475000 & -2.219462000 & -0.400840000 \\
\hline $\mathrm{C}$ & 4.094293000 & -0.024411000 & 0.473803000 \\
\hline $\mathrm{C}$ & 4.411664000 & -1.936038000 & -1.526237000 \\
\hline $\mathrm{H}$ & 3.150108000 & -3.185949000 & -0.302347000 \\
\hline $\mathrm{C}$ & 4.868232000 & 0.263428000 & -0.649220000 \\
\hline $\mathrm{H}$ & 3.942061000 & 0.736430000 & 1.233346000 \\
\hline $\mathrm{C}$ & 5.027034000 & -0.689534000 & -1.655790000 \\
\hline $\mathrm{H}$ & 4.543024000 & -2.691194000 & -2.296461000 \\
\hline $\mathrm{H}$ & 5.339313000 & 1.237461000 & -0.741474000 \\
\hline $\mathrm{H}$ & 5.635709000 & -0.467943000 & -2.527602000 \\
\hline $\mathrm{C}$ & 1.229205000 & 2.800118000 & 2.070867000 \\
\hline $\mathrm{C}$ & 1.025592000 & 2.980846000 & 0.549931000 \\
\hline $\mathrm{H}$ & 0.448055000 & 2.143385000 & 2.473347000 \\
\hline $\mathrm{H}$ & 2.200192000 & 2.322879000 & 2.257542000 \\
\hline $\mathrm{H}$ & 1.200388000 & 3.748435000 & 2.623524000 \\
\hline $\mathrm{C}$ & 2.131443000 & 3.917162000 & 0.015521000 \\
\hline $\mathrm{C}$ & -0.346146000 & 3.652958000 & 0.318498000 \\
\hline $\mathrm{H}$ & 1.989907000 & 4.075651000 & -1.061187000 \\
\hline $\mathrm{H}$ & 2.144606000 & 4.897798000 & 0.509378000 \\
\hline $\mathrm{H}$ & 3.110656000 & 3.442870000 & 0.162464000 \\
\hline $\mathrm{H}$ & -0.425683000 & 4.641085000 & 0.790564000 \\
\hline $\mathrm{H}$ & -0.519431000 & 3.769046000 & -0.758608000 \\
\hline $\mathrm{H}$ & -1.142766000 & 3.017182000 & 0.724865000 \\
\hline $\mathrm{O}$ & 1.082232000 & 1.767798000 & -0.088481000 \\
\hline $\mathrm{H}$ & 2.869428000 & -1.015071000 & 2.657926000 \\
\hline $\mathrm{K}$ & 1.702353000 & 0.109491000 & -1.624653000 \\
\hline $\mathrm{H}$ & -0.380053000 & -3.611560000 & 0.552450000 \\
\hline $\mathrm{C}$ & -5.118002000 & 0.042409000 & -0.544260000 \\
\hline $\mathrm{F}$ & -5.912986000 & -0.959334000 & -0.962900000 \\
\hline $\mathrm{F}$ & -5.057212000 & 0.947633000 & -1.535842000 \\
\hline $\mathrm{F}$ & -5.746262000 & 0.633438000 & 0.487839000 \\
\hline
\end{tabular}

\begin{tabular}{|c|c|c|c|}
\hline \multicolumn{4}{|c|}{$\mathbf{A 1}_{\text {tBuONa }}$} \\
\hline & \multicolumn{3}{|c|}{ Coordinates (Angstroms) } \\
\hline & $X$ & $\mathrm{Y}$ & Z \\
\hline$\overline{\mathrm{C}}$ & 1.708448000 & -2.581082000 & 0.181895000 \\
\hline $\mathrm{C}$ & 0.568161000 & -1.800539000 & 0.335573000 \\
\hline $\mathrm{C}$ & 0.656952000 & -0.407752000 & 0.303184000 \\
\hline $\mathrm{C}$ & 1.898753000 & 0.205234000 & 0.109230000 \\
\hline
\end{tabular}




\begin{tabular}{|c|c|c|c|}
\hline $\mathrm{C}$ & 3.037694000 & -0.575518000 & -0.046761000 \\
\hline $\mathrm{C}$ & 2.943108000 & -1.966005000 & -0.007422000 \\
\hline $\mathrm{H}$ & 1.637933000 & -3.663172000 & 0.209298000 \\
\hline $\mathrm{H}$ & -0.399213000 & -2.278812000 & 0.470705000 \\
\hline $\mathrm{H}$ & 1.936696000 & 1.298883000 & 0.079743000 \\
\hline $\mathrm{H}$ & 4.002555000 & -0.103099000 & -0.200372000 \\
\hline $\mathrm{C}$ & -0.561032000 & 0.464470000 & 0.549843000 \\
\hline $\mathrm{C}$ & -1.824293000 & -0.098759000 & -0.031271000 \\
\hline $\mathrm{H}$ & -1.744717000 & -0.358033000 & -1.085649000 \\
\hline $\mathrm{O}$ & -0.683026000 & 0.715788000 & 1.972256000 \\
\hline $\mathrm{C}$ & -2.964738000 & -0.285577000 & 0.636687000 \\
\hline $\mathrm{H}$ & -2.991723000 & -0.017709000 & 1.692025000 \\
\hline $\mathrm{C}$ & -4.220637000 & -0.834469000 & 0.095777000 \\
\hline $\mathrm{C}$ & -5.227419000 & -1.221898000 & 0.987735000 \\
\hline $\mathrm{C}$ & -4.456885000 & -0.989952000 & -1.276588000 \\
\hline $\mathrm{C}$ & -6.424114000 & -1.763457000 & 0.530576000 \\
\hline $\mathrm{H}$ & -5.066161000 & -1.098453000 & 2.056073000 \\
\hline $\mathrm{C}$ & -5.650997000 & -1.530500000 & -1.735436000 \\
\hline $\mathrm{H}$ & -3.708104000 & -0.671803000 & -1.995819000 \\
\hline $\mathrm{C}$ & -6.639101000 & -1.923114000 & -0.834387000 \\
\hline $\mathrm{H}$ & -7.188615000 & -2.060055000 & 1.242521000 \\
\hline $\mathrm{H}$ & -5.814502000 & -1.641664000 & -2.803247000 \\
\hline $\mathrm{H}$ & -7.571977000 & -2.344217000 & -1.196804000 \\
\hline $\mathrm{H}$ & -0.347508000 & 1.459894000 & 0.129909000 \\
\hline $\mathrm{H}$ & -0.699672000 & -0.143029000 & 2.415244000 \\
\hline $\mathrm{C}$ & 2.111803000 & 5.109529000 & -0.409490000 \\
\hline $\mathrm{C}$ & 1.025220000 & 4.046233000 & -0.664010000 \\
\hline $\mathrm{H}$ & 1.886814000 & 5.651343000 & 0.518200000 \\
\hline $\mathrm{H}$ & 3.084034000 & 4.618219000 & -0.286517000 \\
\hline $\mathrm{H}$ & 2.191328000 & 5.841068000 & -1.224044000 \\
\hline $\mathrm{C}$ & 1.350674000 & 3.309118000 & -1.980343000 \\
\hline $\mathrm{C}$ & -0.338453000 & 4.750107000 & -0.816681000 \\
\hline $\mathrm{H}$ & 0.595888000 & 2.535572000 & -2.169267000 \\
\hline $\mathrm{H}$ & 1.376770000 & 3.983268000 & -2.845903000 \\
\hline $\mathrm{H}$ & 2.326264000 & 2.814825000 & -1.900803000 \\
\hline $\mathrm{H}$ & -0.356040000 & 5.476494000 & -1.639439000 \\
\hline $\mathrm{H}$ & -1.120767000 & 4.002629000 & -0.997310000 \\
\hline $\mathrm{H}$ & -0.584545000 & 5.280191000 & 0.112808000 \\
\hline $\mathrm{O}$ & 0.979475000 & 3.148667000 & 0.378578000 \\
\hline $\mathrm{Na}$ & 0.504037000 & 2.637399000 & 2.277253000 \\
\hline $\mathrm{C}$ & 4.172094000 & -2.802378000 & -0.237409000 \\
\hline $\mathrm{F}$ & 4.071869000 & -4.012902000 & 0.339805000 \\
\hline $\mathrm{F}$ & 5.275602000 & -2.213493000 & 0.254820000 \\
\hline $\mathrm{F}$ & 4.391177000 & -3.010442000 & -1.548183000 \\
\hline
\end{tabular}


TSA1-2 ${ }_{\text {tBuONa }}$

\begin{tabular}{|c|c|c|c|}
\hline \multicolumn{4}{|c|}{ Coordinates (Angstroms) } \\
\hline & $X$ & Y & Z \\
\hline $\mathrm{C}$ & 2.695974000 & -1.287400000 & -0.919281000 \\
\hline $\mathrm{C}$ & 1.376783000 & -1.011115000 & -0.592744000 \\
\hline $\mathrm{C}$ & 1.043801000 & -0.399427000 & 0.627105000 \\
\hline $\mathrm{C}$ & 2.091476000 & -0.092005000 & 1.508684000 \\
\hline $\mathrm{C}$ & 3.410470000 & -0.378778000 & 1.188175000 \\
\hline $\mathrm{C}$ & 3.722562000 & -0.973389000 & -0.031351000 \\
\hline $\mathrm{H}$ & 2.927471000 & -1.754174000 & -1.871390000 \\
\hline $\mathrm{H}$ & 0.601692000 & -1.281556000 & -1.302329000 \\
\hline $\mathrm{H}$ & 1.860059000 & 0.397247000 & 2.448288000 \\
\hline $\mathrm{H}$ & 4.201902000 & -0.123991000 & 1.885117000 \\
\hline $\mathrm{C}$ & -0.335716000 & 0.029087000 & 0.955476000 \\
\hline $\mathrm{C}$ & -1.488372000 & -0.567839000 & 0.286883000 \\
\hline $\mathrm{H}$ & -1.394978000 & -0.672786000 & -0.792698000 \\
\hline $\mathrm{O}$ & -0.562048000 & 0.215912000 & 2.365314000 \\
\hline $\mathrm{C}$ & -2.685121000 & -0.844441000 & 0.853275000 \\
\hline $\mathrm{H}$ & -2.765792000 & -0.832850000 & 1.942485000 \\
\hline $\mathrm{C}$ & -3.903374000 & -1.248488000 & 0.135728000 \\
\hline $\mathrm{C}$ & -4.919800000 & -1.925885000 & 0.824054000 \\
\hline $\mathrm{C}$ & -4.107365000 & -0.967298000 & -1.223586000 \\
\hline $\mathrm{C}$ & -6.083520000 & -2.327645000 & 0.178681000 \\
\hline $\mathrm{H}$ & -4.784210000 & -2.152156000 & 1.879594000 \\
\hline $\mathrm{C}$ & -5.267005000 & -1.375444000 & -1.871243000 \\
\hline $\mathrm{H}$ & -3.357932000 & -0.406719000 & -1.776176000 \\
\hline $\mathrm{C}$ & -6.261741000 & -2.058894000 & -1.175401000 \\
\hline $\mathrm{H}$ & -6.852270000 & -2.856446000 & 0.735009000 \\
\hline $\mathrm{H}$ & -5.400565000 & -1.148075000 & -2.925002000 \\
\hline $\mathrm{H}$ & -7.169796000 & -2.371082000 & -1.682018000 \\
\hline $\mathrm{H}$ & -0.412883000 & 1.319650000 & 0.561716000 \\
\hline $\mathrm{H}$ & -0.555321000 & -0.649701000 & 2.799324000 \\
\hline $\mathrm{C}$ & 1.327988000 & 3.289170000 & -0.471393000 \\
\hline $\mathrm{C}$ & -0.188842000 & 3.142331000 & -0.662757000 \\
\hline $\mathrm{H}$ & 1.533702000 & 3.890486000 & 0.420649000 \\
\hline $\mathrm{H}$ & 1.790478000 & 2.305369000 & -0.331092000 \\
\hline $\mathrm{H}$ & 1.801915000 & 3.771056000 & -1.334778000 \\
\hline $\mathrm{C}$ & -0.472474000 & 2.274946000 & -1.901450000 \\
\hline $\mathrm{C}$ & -0.832603000 & 4.521670000 & -0.839396000 \\
\hline $\mathrm{H}$ & -1.551636000 & 2.106806000 & -2.001203000 \\
\hline $\mathrm{H}$ & -0.107635000 & 2.747221000 & -2.820770000 \\
\hline $\mathrm{H}$ & 0.024063000 & 1.301412000 & -1.807023000 \\
\hline
\end{tabular}




$\begin{array}{lrrr}\mathrm{H} & -0.425033000 & 5.052150000 & -1.707853000 \\ \mathrm{H} & -1.915328000 & 4.415151000 & -0.977288000 \\ \mathrm{H} & -0.656416000 & 5.131775000 & 0.053499000 \\ \mathrm{O} & -0.749352000 & 2.543688000 & 0.472892000 \\ \mathrm{Na} & -2.185363000 & 1.832059000 & 1.823857000 \\ \mathrm{C} & 5.138788000 & -1.328282000 & -0.371450000 \\ \mathrm{~F} & 5.419893000 & -2.615678000 & -0.083674000 \\ \mathrm{~F} & 6.026185000 & -0.578021000 & 0.305528000 \\ \mathrm{~F} & 5.394728000 & -1.166688000 & -1.683208000\end{array}$

\begin{tabular}{|c|c|c|c|}
\hline \multicolumn{4}{|c|}{$\mathrm{A} 2_{\mathrm{tBuONa}}$} \\
\hline & \multicolumn{3}{|c|}{ Coordinates (Angstroms) } \\
\hline & $\mathrm{X}$ & $\mathrm{Y}$ & $\mathrm{Z}$ \\
\hline$\overline{\mathrm{C}}$ & -3.702565000 & -0.810825000 & -0.930260000 \\
\hline $\mathrm{C}$ & -2.448348000 & -1.292481000 & -1.287283000 \\
\hline $\mathrm{C}$ & -1.374148000 & -1.347900000 & -0.352590000 \\
\hline $\mathrm{C}$ & -1.675160000 & -0.913095000 & 0.972862000 \\
\hline $\mathrm{C}$ & -2.925014000 & -0.446525000 & 1.311850000 \\
\hline $\mathrm{C}$ & -3.957157000 & -0.373160000 & 0.363539000 \\
\hline $\mathrm{H}$ & -4.494803000 & -0.792619000 & -1.672030000 \\
\hline $\mathrm{H}$ & -2.293980000 & -1.688461000 & -2.285954000 \\
\hline $\mathrm{H}$ & -0.910420000 & -0.953067000 & 1.741512000 \\
\hline $\mathrm{H}$ & -3.114069000 & -0.129303000 & 2.333375000 \\
\hline $\mathrm{C}$ & -0.060914000 & -1.709033000 & -0.777137000 \\
\hline $\mathrm{C}$ & 1.125592000 & -1.522782000 & -0.063625000 \\
\hline $\mathrm{H}$ & 0.998321000 & -1.351802000 & 1.002304000 \\
\hline $\mathrm{O}$ & 0.053855000 & -1.849934000 & -2.188940000 \\
\hline $\mathrm{C}$ & 2.409187000 & -1.468398000 & -0.568921000 \\
\hline $\mathrm{H}$ & 2.570189000 & -1.651709000 & -1.631120000 \\
\hline $\mathrm{C}$ & 3.610359000 & -1.172921000 & 0.203247000 \\
\hline $\mathrm{C}$ & 4.825433000 & -0.943079000 & -0.471563000 \\
\hline $\mathrm{C}$ & 3.628533000 & -1.037141000 & 1.605561000 \\
\hline $\mathrm{C}$ & 5.980167000 & -0.577365000 & 0.206984000 \\
\hline $\mathrm{H}$ & 4.851126000 & -1.045678000 & -1.554547000 \\
\hline $\mathrm{C}$ & 4.783424000 & -0.664202000 & 2.282111000 \\
\hline $\mathrm{H}$ & 2.728876000 & -1.237132000 & 2.181313000 \\
\hline $\mathrm{C}$ & 5.969399000 & -0.425619000 & 1.592188000 \\
\hline $\mathrm{H}$ & 6.896870000 & -0.405697000 & -0.350931000 \\
\hline $\mathrm{H}$ & 4.755857000 & -0.565183000 & 3.364112000 \\
\hline $\mathrm{H}$ & 6.870273000 & -0.135710000 & 2.123953000 \\
\hline $\mathrm{H}$ & 2.002303000 & 0.497938000 & -1.062267000 \\
\hline $\mathrm{H}$ & 0.659663000 & -2.576756000 & -2.374201000 \\
\hline $\mathrm{C}$ & 1.151652000 & 1.815138000 & 0.952700000 \\
\hline
\end{tabular}




\begin{tabular}{|c|c|c|c|}
\hline $\mathrm{C}$ & 1.721415000 & 2.334440000 & -0.369353000 \\
\hline $\mathrm{H}$ & 0.119937000 & 1.464260000 & 0.825924000 \\
\hline $\mathrm{H}$ & 1.758356000 & 0.985486000 & 1.329935000 \\
\hline $\mathrm{H}$ & 1.155100000 & 2.605664000 & 1.710174000 \\
\hline $\mathrm{C}$ & 3.204898000 & 2.671996000 & -0.222934000 \\
\hline $\mathrm{C}$ & 0.925707000 & 3.533714000 & -0.872860000 \\
\hline $\mathrm{H}$ & 3.609718000 & 3.031634000 & -1.174129000 \\
\hline $\mathrm{H}$ & 3.352024000 & 3.448351000 & 0.535407000 \\
\hline $\mathrm{H}$ & 3.771974000 & 1.785579000 & 0.083173000 \\
\hline $\mathrm{H}$ & 1.006008000 & 4.371097000 & -0.173390000 \\
\hline $\mathrm{H}$ & 1.299908000 & 3.860272000 & -1.848672000 \\
\hline $\mathrm{H}$ & -0.139559000 & 3.284770000 & -0.968809000 \\
\hline $\mathrm{O}$ & 1.574861000 & 1.324881000 & -1.386632000 \\
\hline $\mathrm{Na}$ & -0.327916000 & 0.423994000 & -2.110126000 \\
\hline $\mathrm{C}$ & -5.273275000 & 0.217064000 & 0.745209000 \\
\hline $\mathrm{F}$ & -5.210766000 & 1.563872000 & 0.866459000 \\
\hline $\mathrm{F}$ & -5.710266000 & -0.241401000 & 1.934910000 \\
\hline $\mathrm{F}$ & -6.233570000 & -0.041627000 & -0.161092000 \\
\hline
\end{tabular}

TSA2-3 ${ }_{\text {tBuONa }}$

\begin{tabular}{lrcc} 
& \multicolumn{3}{c}{ Coordinates (Angstroms) } \\
& $\mathrm{X}$ & $\mathrm{Y}$ & $\mathrm{Z}$ \\
$\mathrm{C}$ & -3.933466000 & 0.808504000 & -0.672184000 \\
$\mathrm{C}$ & -2.611338000 & 0.870689000 & -1.097691000 \\
$\mathrm{C}$ & -1.726655000 & -0.206203000 & -0.886771000 \\
$\mathrm{C}$ & -2.246340000 & -1.365976000 & -0.277600000 \\
$\mathrm{C}$ & -3.561063000 & -1.421664000 & 0.147804000 \\
$\mathrm{C}$ & -4.413353000 & -0.330146000 & -0.035567000 \\
$\mathrm{H}$ & -4.596820000 & 1.648328000 & -0.853129000 \\
$\mathrm{H}$ & -2.263435000 & 1.746411000 & -1.635636000 \\
$\mathrm{H}$ & -1.617228000 & -2.240402000 & -0.150647000 \\
$\mathrm{H}$ & -3.936386000 & -2.326021000 & 0.614911000 \\
$\mathrm{C}$ & -0.315040000 & -0.072410000 & -1.238174000 \\
$\mathrm{C}$ & 0.701939000 & -0.873895000 & -0.827620000 \\
$\mathrm{H}$ & 0.435822000 & -1.729861000 & -0.210161000 \\
$\mathrm{O}$ & -0.043888000 & 1.130608000 & -1.899892000 \\
$\mathrm{C}$ & 2.105516000 & -0.583838000 & -1.043552000 \\
$\mathrm{H}$ & 2.344916000 & -0.299057000 & -2.075281000 \\
$\mathrm{C}$ & 3.124098000 & -1.469147000 & -0.449009000 \\
$\mathrm{C}$ & 4.350454000 & -1.691489000 & -1.096397000 \\
$\mathrm{C}$ & 2.955693000 & -2.049046000 & 0.819870000 \\
$\mathrm{C}$ & 5.351757000 & -2.458575000 & -0.512563000 \\
$\mathrm{H}$ & 4.511857000 & -1.255194000 & -2.079908000
\end{tabular}




$\begin{array}{lrrr}\mathrm{C} & 3.951157000 & -2.828859000 & 1.398581000 \\ \mathrm{H} & 2.036271000 & -1.868979000 & 1.373072000 \\ \mathrm{C} & 5.158777000 & -3.039548000 & 0.738480000 \\ \mathrm{H} & 6.288323000 & -2.609829000 & -1.042757000 \\ \mathrm{H} & 3.785705000 & -3.266225000 & 2.379777000 \\ \mathrm{H} & 5.938859000 & -3.642110000 & 1.193710000 \\ \mathrm{H} & 2.201262000 & 0.675263000 & -0.365565000 \\ \mathrm{H} & 0.917440000 & 1.272748000 & -1.880044000 \\ \mathrm{C} & 3.703798000 & 1.568387000 & 1.826664000 \\ \mathrm{C} & 3.259513000 & 2.317919000 & 0.564986000 \\ \mathrm{H} & 2.932817000 & 1.648872000 & 2.603099000 \\ \mathrm{H} & 3.857122000 & 0.506659000 & 1.605714000 \\ \mathrm{H} & 4.638504000 & 1.975460000 & 2.229506000 \\ \mathrm{C} & 4.331044000 & 2.191091000 & -0.526465000 \\ \mathrm{C} & 3.005191000 & 3.793506000 & 0.883700000 \\ \mathrm{H} & 3.983359000 & 2.666827000 & -1.450285000 \\ \mathrm{H} & 5.268596000 & 2.668832000 & -0.221144000 \\ \mathrm{H} & 4.539757000 & 1.136158000 & -0.735135000 \\ \mathrm{H} & 3.910461000 & 4.290049000 & 1.250409000 \\ \mathrm{H} & 2.661154000 & 4.319942000 & -0.013860000 \\ \mathrm{H} & 2.234407000 & 3.887145000 & 1.660735000 \\ \mathrm{O} & 2.043745000 & 1.771411000 & 0.095663000 \\ \mathrm{Na} & -0.065516000 & 1.874892000 & 0.417264000 \\ \mathrm{C} & -5.816004000 & -0.360417000 & 0.495616000 \\ \mathrm{~F} & -6.659397000 & 0.352412000 & -0.271235000 \\ \mathrm{~F} & -6.299921000 & -1.610813000 & 0.576548000 \\ \mathrm{~F} & -5.885682000 & 0.165258000 & 1.736231000 \\ ------\end{array}$

\begin{tabular}{|c|c|c|c|}
\hline \multicolumn{4}{|c|}{$\mathrm{A}_{\mathrm{tBuONa}}$} \\
\hline & \multicolumn{3}{|c|}{ Coordinates (Angstroms) } \\
\hline & $\mathrm{X}$ & Y & Z \\
\hline$\overline{\mathrm{C}}$ & -3.508800000 & -1.778635000 & 0.263300000 \\
\hline $\mathrm{C}$ & -2.235182000 & -2.228951000 & 0.586968000 \\
\hline $\mathrm{C}$ & -1.127457000 & -1.377635000 & 0.485056000 \\
\hline $\mathrm{C}$ & -1.316701000 & -0.054723000 & 0.061943000 \\
\hline $\mathrm{C}$ & -2.592756000 & 0.389228000 & -0.258622000 \\
\hline $\mathrm{C}$ & -3.687940000 & -0.468159000 & -0.167446000 \\
\hline $\mathrm{H}$ & -4.359284000 & -2.446228000 & 0.349936000 \\
\hline $\mathrm{H}$ & -2.119016000 & -3.242013000 & 0.964399000 \\
\hline $\mathrm{H}$ & -0.472340000 & 0.647529000 & -0.017847000 \\
\hline $\mathrm{H}$ & -2.727973000 & 1.413211000 & -0.592851000 \\
\hline $\mathrm{C}$ & 0.225627000 & -1.888136000 & 0.823914000 \\
\hline $\mathrm{C}$ & 1.211637000 & -1.140222000 & 1.338491000 \\
\hline
\end{tabular}




\begin{tabular}{lrrr}
$\mathrm{H}$ & 1.040263000 & -0.075242000 & 1.470672000 \\
$\mathrm{O}$ & 0.435590000 & -3.232564000 & 0.636546000 \\
$\mathrm{C}$ & 2.586345000 & -1.676037000 & 1.661790000 \\
$\mathrm{H}$ & 2.546609000 & -2.757961000 & 1.814887000 \\
$\mathrm{C}$ & 3.535454000 & -1.346715000 & 0.528043000 \\
$\mathrm{C}$ & 3.532965000 & -2.131264000 & -0.634497000 \\
$\mathrm{C}$ & 4.321374000 & -0.190615000 & 0.551687000 \\
$\mathrm{C}$ & 4.283067000 & -1.758207000 & -1.748622000 \\
$\mathrm{H}$ & 2.919863000 & -3.028547000 & -0.660442000 \\
$\mathrm{C}$ & 5.072120000 & 0.186976000 & -0.562433000 \\
$\mathrm{H}$ & 4.318014000 & 0.438517000 & 1.437341000 \\
$\mathrm{C}$ & 5.050784000 & -0.591179000 & -1.718870000 \\
$\mathrm{H}$ & 4.275282000 & -2.381116000 & -2.638668000 \\
$\mathrm{H}$ & 5.663772000 & 1.096589000 & -0.528449000 \\
$\mathrm{H}$ & 5.635191000 & -0.298857000 & -2.585834000 \\
$\mathrm{C}$ & 1.255445000 & 2.889568000 & 1.881683000 \\
$\mathrm{C}$ & 1.027533000 & 3.031592000 & 0.361456000 \\
$\mathrm{H}$ & 0.480953000 & 2.242343000 & 2.311759000 \\
$\mathrm{H}$ & 2.230519000 & 2.417823000 & 2.061206000 \\
$\mathrm{H}$ & 1.233007000 & 3.850688000 & 2.411542000 \\
$\mathrm{C}$ & 2.116042000 & 3.958777000 & -0.217309000 \\
$\mathrm{C}$ & -0.354097000 & 3.678877000 & 0.127690000 \\
$\mathrm{H}$ & 1.962097000 & 4.077529000 & -1.296854000 \\
$\mathrm{H}$ & 2.121407000 & 4.955341000 & 0.243261000 \\
$\mathrm{H}$ & 3.102492000 & 3.501612000 & -0.061841000 \\
$\mathrm{H}$ & -0.437129000 & 4.678318000 & 0.573897000 \\
$\mathrm{H}$ & -0.542593000 & 3.762035000 & -0.949181000 \\
$\mathrm{H}$ & -1.137442000 & 3.046269000 & 0.563354000 \\
$\mathrm{O}$ & 1.082677000 & 1.796819000 & -0.235812000 \\
$\mathrm{H}$ & 2.948505000 & -1.221581000 & 2.589795000 \\
$\mathrm{H}$ & -0.255176000 & -3.574976000 & 0.056550000 \\
$\mathrm{~F}$ & 2.067107000 & 0.302843000 & -1.170005000 \\
$\mathrm{C}$ & -5.069458000 & 0.043113000 & -0.468728000 \\
$\mathrm{~F}$ & -5.891468000 & -0.941433000 & -0.875329000 \\
--5.057174000 & 0.979457000 & -1.431673000 \\
\hline-5.636621000 & 0.602806000 & 0.615288000 \\
\hline
\end{tabular}

\begin{tabular}{|c|c|c|c|}
\hline \multicolumn{4}{|c|}{$\mathrm{A1}_{\mathrm{tBuOLi}}$} \\
\hline & \multicolumn{3}{|c|}{ Coordinates (Angstroms) } \\
\hline & $\mathrm{X}$ & $\mathrm{Y}$ & $\mathrm{Z}$ \\
\hline $\mathrm{C}$ & -1.565648000 & -2.561636000 & -0.142899000 \\
\hline $\mathrm{C}$ & -0.457363000 & -1.729088000 & -0.254620000 \\
\hline $\mathrm{C}$ & -0.615614000 & -0.342740000 & -0.250970000 \\
\hline
\end{tabular}




\begin{tabular}{|c|c|c|c|}
\hline $\mathrm{C}$ & -1.895151000 & 0.210132000 & -0.137993000 \\
\hline $\mathrm{C}$ & -3.001928000 & -0.621460000 & -0.024751000 \\
\hline $\mathrm{C}$ & -2.836568000 & -2.005723000 & -0.027000000 \\
\hline $\mathrm{H}$ & -1.441560000 & -3.639164000 & -0.147735000 \\
\hline $\mathrm{H}$ & 0.535927000 & -2.162446000 & -0.338962000 \\
\hline $\mathrm{H}$ & -1.997094000 & 1.297071000 & -0.152648000 \\
\hline $\mathrm{H}$ & -3.994471000 & -0.192763000 & 0.065431000 \\
\hline $\mathrm{C}$ & 0.565581000 & 0.590884000 & -0.441036000 \\
\hline $\mathrm{C}$ & 1.861427000 & 0.045657000 & 0.083191000 \\
\hline $\mathrm{H}$ & 1.812493000 & -0.278799000 & 1.121152000 \\
\hline $\mathrm{O}$ & 0.659245000 & 0.955164000 & -1.843787000 \\
\hline $\mathrm{C}$ & 2.994267000 & -0.054454000 & -0.614876000 \\
\hline $\mathrm{H}$ & 2.988918000 & 0.274733000 & -1.652808000 \\
\hline $\mathrm{C}$ & 4.281109000 & -0.581677000 & -0.127689000 \\
\hline $\mathrm{C}$ & 5.281861000 & -0.882412000 & -1.058694000 \\
\hline $\mathrm{C}$ & 4.551666000 & -0.798111000 & 1.229852000 \\
\hline $\mathrm{C}$ & 6.507195000 & -1.400637000 & -0.653406000 \\
\hline $\mathrm{H}$ & 5.093584000 & -0.710899000 & -2.115836000 \\
\hline $\mathrm{C}$ & 5.774501000 & -1.315412000 & 1.636797000 \\
\hline $\mathrm{H}$ & 3.807231000 & -0.545474000 & 1.978996000 \\
\hline $\mathrm{C}$ & 6.756794000 & -1.622008000 & 0.696984000 \\
\hline $\mathrm{H}$ & 7.267428000 & -1.630043000 & -1.394046000 \\
\hline $\mathrm{H}$ & 5.964965000 & -1.473999000 & 2.694051000 \\
\hline $\mathrm{H}$ & 7.712470000 & -2.024956000 & 1.018236000 \\
\hline $\mathrm{H}$ & 0.325483000 & 1.545540000 & 0.044626000 \\
\hline $\mathrm{H}$ & 0.622011000 & 0.141885000 & -2.364177000 \\
\hline $\mathrm{C}$ & -2.428960000 & 5.227045000 & -0.255414000 \\
\hline $\mathrm{C}$ & -1.401609000 & 4.228766000 & 0.307891000 \\
\hline $\mathrm{H}$ & -2.023451000 & 5.706665000 & -1.154360000 \\
\hline $\mathrm{H}$ & -3.344074000 & 4.694320000 & -0.538125000 \\
\hline $\mathrm{H}$ & -2.691301000 & 6.010364000 & 0.466775000 \\
\hline $\mathrm{C}$ & -1.978592000 & 3.578676000 & 1.580642000 \\
\hline $\mathrm{C}$ & -0.110514000 & 4.983894000 & 0.674782000 \\
\hline $\mathrm{H}$ & -1.269094000 & 2.842025000 & 1.977544000 \\
\hline $\mathrm{H}$ & -2.186839000 & 4.315093000 & 2.366413000 \\
\hline $\mathrm{H}$ & -2.913885000 & 3.056229000 & 1.346164000 \\
\hline $\mathrm{H}$ & -0.276835000 & 5.765080000 & 1.427241000 \\
\hline $\mathrm{H}$ & 0.631281000 & 4.277630000 & 1.067400000 \\
\hline $\mathrm{H}$ & 0.310715000 & 5.453463000 & -0.222570000 \\
\hline $\mathrm{O}$ & -1.123258000 & 3.256947000 & -0.629675000 \\
\hline $\mathrm{C}$ & -4.030835000 & -2.903260000 & 0.155994000 \\
\hline $\mathrm{F}$ & -3.847783000 & -4.103688000 & -0.421121000 \\
\hline $\mathrm{F}$ & -5.143845000 & -2.368152000 & -0.372346000 \\
\hline $\mathrm{F}$ & -4.282457000 & -3.128185000 & 1.457924000 \\
\hline
\end{tabular}


TSA1-2 tBuOLi $_{\text {i }}$

\begin{tabular}{|c|c|c|c|}
\hline \multicolumn{4}{|c|}{ Coordinates (Angstroms) } \\
\hline & $\mathrm{X}$ & Y & $\mathrm{Z}$ \\
\hline $\mathrm{C}$ & 2.824273000 & -0.826599000 & -1.064599000 \\
\hline $\mathrm{C}$ & 1.504748000 & -0.589945000 & -0.708378000 \\
\hline $\mathrm{C}$ & 1.152864000 & -0.280145000 & 0.615427000 \\
\hline $\mathrm{C}$ & 2.184488000 & -0.200563000 & 1.563494000 \\
\hline $\mathrm{C}$ & 3.503299000 & -0.438628000 & 1.208122000 \\
\hline $\mathrm{C}$ & 3.832882000 & -0.755845000 & -0.107944000 \\
\hline $\mathrm{H}$ & 3.069131000 & -1.064833000 & -2.093860000 \\
\hline $\mathrm{H}$ & 0.742339000 & -0.643557000 & -1.479650000 \\
\hline $\mathrm{H}$ & 1.943657000 & 0.064238000 & 2.586841000 \\
\hline $\mathrm{H}$ & 4.284010000 & -0.371977000 & 1.959950000 \\
\hline $\mathrm{C}$ & -0.238253000 & 0.047773000 & 0.984619000 \\
\hline $\mathrm{C}$ & -1.373807000 & -0.599337000 & 0.346957000 \\
\hline $\mathrm{H}$ & -1.278836000 & -0.774218000 & -0.722725000 \\
\hline $\mathrm{O}$ & -0.457815000 & 0.200428000 & 2.406468000 \\
\hline $\mathrm{C}$ & -2.569540000 & -0.861978000 & 0.926880000 \\
\hline $\mathrm{H}$ & -2.662090000 & -0.806164000 & 2.014551000 \\
\hline $\mathrm{C}$ & -3.774772000 & -1.324344000 & 0.223111000 \\
\hline $\mathrm{C}$ & -4.765025000 & -2.023719000 & 0.926654000 \\
\hline $\mathrm{C}$ & -3.988358000 & -1.078331000 & -1.141162000 \\
\hline $\mathrm{C}$ & -5.912488000 & -2.482414000 & 0.290492000 \\
\hline $\mathrm{H}$ & -4.622021000 & -2.222049000 & 1.986780000 \\
\hline $\mathrm{C}$ & -5.131356000 & -1.544076000 & -1.779742000 \\
\hline $\mathrm{H}$ & -3.262144000 & -0.497391000 & -1.703477000 \\
\hline $\mathrm{C}$ & -6.099396000 & -2.249905000 & -1.069188000 \\
\hline $\mathrm{H}$ & -6.661060000 & -3.027530000 & 0.858328000 \\
\hline $\mathrm{H}$ & -5.273843000 & -1.342595000 & -2.837560000 \\
\hline $\mathrm{H}$ & -6.994478000 & -2.607715000 & -1.568664000 \\
\hline $\mathrm{H}$ & -0.483021000 & 1.396343000 & 0.678513000 \\
\hline $\mathrm{H}$ & -0.515371000 & -0.677720000 & 2.812919000 \\
\hline $\mathrm{C}$ & 0.413202000 & 3.555882000 & -0.755720000 \\
\hline $\mathrm{C}$ & -1.033579000 & 3.242104000 & -0.355722000 \\
\hline $\mathrm{H}$ & 0.911213000 & 4.110369000 & 0.045828000 \\
\hline $\mathrm{H}$ & 0.970341000 & 2.626165000 & -0.921111000 \\
\hline $\mathrm{H}$ & 0.456584000 & 4.151085000 & -1.674975000 \\
\hline $\mathrm{C}$ & -1.727334000 & 2.445502000 & -1.470672000 \\
\hline $\mathrm{C}$ & -1.803465000 & 4.533475000 & -0.074393000 \\
\hline $\mathrm{H}$ & -2.738200000 & 2.155626000 & -1.159943000 \\
\hline $\mathrm{H}$ & -1.802227000 & 3.029962000 & -2.394412000 \\
\hline
\end{tabular}




$\begin{array}{lrrr}\mathrm{H} & -1.164245000 & 1.532059000 & -1.696268000 \\ \mathrm{H} & -1.822954000 & 5.187895000 & -0.953300000 \\ \mathrm{H} & -2.839407000 & 4.306003000 & 0.204762000 \\ \mathrm{H} & -1.333434000 & 5.075795000 & 0.752540000 \\ \mathrm{O} & -1.018887000 & 2.485222000 & 0.834315000 \\ \mathrm{C} & 5.262446000 & -1.040415000 & -0.456704000 \\ \mathrm{~F} & 5.696385000 & -2.186919000 & 0.104939000 \\ \mathrm{~F} & 6.086777000 & -0.069823000 & -0.016918000 \\ \mathrm{~F} & 5.453471000 & -1.155735000 & -1.782355000 \\ \mathrm{Li} & -1.935002000 & 1.485452000 & 2.006164000\end{array}$

\begin{tabular}{|c|c|c|c|}
\hline \multicolumn{4}{|c|}{$\mathrm{A} 2_{\mathrm{tBuOLi}}$} \\
\hline & $X$ & $\mathrm{Y}$ & Z \\
\hline$\overline{\mathrm{C}}$ & 3.556410000 & -0.601996000 & 1.097282000 \\
\hline $\mathrm{C}$ & 2.306538000 & -1.041495000 & 1.522621000 \\
\hline $\mathrm{C}$ & 1.266986000 & -1.333815000 & 0.594366000 \\
\hline $\mathrm{C}$ & 1.595074000 & -1.194124000 & -0.785646000 \\
\hline $\mathrm{C}$ & 2.838362000 & -0.762915000 & -1.188321000 \\
\hline $\mathrm{C}$ & 3.835663000 & -0.445668000 & -0.253159000 \\
\hline $\mathrm{H}$ & 4.324515000 & -0.393544000 & 1.835365000 \\
\hline $\mathrm{H}$ & 2.139115000 & -1.222221000 & 2.579448000 \\
\hline $\mathrm{H}$ & 0.858584000 & -1.441379000 & -1.542989000 \\
\hline $\mathrm{H}$ & 3.050778000 & -0.670194000 & -2.249138000 \\
\hline $\mathrm{C}$ & -0.053750000 & -1.627914000 & 1.054792000 \\
\hline $\mathrm{C}$ & -1.236281000 & -1.507563000 & 0.315117000 \\
\hline $\mathrm{H}$ & -1.097083000 & -1.494579000 & -0.762862000 \\
\hline $\mathrm{O}$ & -0.166043000 & -1.501749000 & 2.475014000 \\
\hline $\mathrm{C}$ & -2.514674000 & -1.306787000 & 0.790267000 \\
\hline $\mathrm{H}$ & -2.702839000 & -1.324424000 & 1.864337000 \\
\hline $\mathrm{C}$ & -3.689579000 & -1.052081000 & -0.040188000 \\
\hline $\mathrm{C}$ & -4.862500000 & -0.555992000 & 0.559712000 \\
\hline $\mathrm{C}$ & -3.710977000 & -1.223484000 & -1.437042000 \\
\hline $\mathrm{C}$ & -5.984557000 & -0.231346000 & -0.191675000 \\
\hline $\mathrm{H}$ & -4.882497000 & -0.415749000 & 1.638703000 \\
\hline $\mathrm{C}$ & -4.832794000 & -0.893289000 & -2.187870000 \\
\hline $\mathrm{H}$ & -2.845645000 & -1.639761000 & -1.945394000 \\
\hline $\mathrm{C}$ & -5.978317000 & -0.391267000 & -1.575576000 \\
\hline $\mathrm{H}$ & -6.870148000 & 0.152394000 & 0.307597000 \\
\hline $\mathrm{H}$ & -4.813016000 & -1.040618000 & -3.264411000 \\
\hline $\mathrm{H}$ & -6.853637000 & -0.136594000 & -2.165029000 \\
\hline $\mathrm{H}$ & -1.908210000 & 0.635585000 & 1.133355000 \\
\hline $\mathrm{H}$ & -0.900519000 & -2.047606000 & 2.777446000 \\
\hline
\end{tabular}




\begin{tabular}{lrrr}
$\mathrm{C}$ & -0.974087000 & 1.666434000 & -0.987673000 \\
$\mathrm{C}$ & -1.312460000 & 2.357701000 & 0.333915000 \\
$\mathrm{H}$ & -0.015945000 & 1.137388000 & -0.919748000 \\
$\mathrm{H}$ & -1.754290000 & 0.947074000 & -1.259729000 \\
$\mathrm{H}$ & -0.900941000 & 2.405412000 & -1.792096000 \\
$\mathrm{C}$ & -2.696322000 & 3.000347000 & 0.275050000 \\
$\mathrm{C}$ & -0.235571000 & 3.364227000 & 0.719497000 \\
$\mathrm{H}$ & -2.935930000 & 3.477436000 & 1.230237000 \\
$\mathrm{H}$ & -2.737480000 & 3.757318000 & -0.514745000 \\
$\mathrm{H}$ & -3.459329000 & 2.242495000 & 0.061984000 \\
$\mathrm{H}$ & -0.178920000 & 4.168004000 & -0.020378000 \\
$\mathrm{H}$ & -0.453072000 & 3.807314000 & 1.696629000 \\
$\mathrm{H}$ & 0.750228000 & 2.882896000 & 0.761058000 \\
$\mathrm{O}$ & -1.317253000 & 1.379624000 & 1.400565000 \\
$\mathrm{C}$ & 5.143765000 & 0.107382000 & -0.715507000 \\
$\mathrm{~F}$ & 5.067523000 & 1.429697000 & -0.988454000 \\
$\mathrm{~F}$ & 5.572219000 & -0.484376000 & -1.847108000 \\
$\mathrm{~F}$ & 6.113118000 & -0.037240000 & 0.206425000 \\
$\mathrm{Li}$ & 0.170451000 & 0.353165000 & 1.936060000 \\
\hline
\end{tabular}

TSA2-3 tBuOLi $_{\text {in }}$

\begin{tabular}{lccc} 
& \multicolumn{3}{c}{ Coordinates (Angstroms) } \\
C & X & Y & Z \\
$\mathrm{C}$ & 2.089799000 & 0.442187000 & 1.106128000 \\
$\mathrm{C}$ & 1.750711000 & -0.104223000 & 0.731515000 \\
$\mathrm{C}$ & 2.135166000 & -0.787433000 & -0.437223000 \\
$\mathrm{C}$ & 3.464080000 & -0.862941000 & -0.815067000 \\
$\mathrm{C}$ & 4.452008000 & -0.247373000 & -0.045598000 \\
$\mathrm{H}$ & 4.851224000 & 0.918676000 & 1.714445000 \\
$\mathrm{H}$ & 2.490090000 & 1.036988000 & 2.401409000 \\
$\mathrm{H}$ & 1.387029000 & -1.261642000 & -1.064088000 \\
$\mathrm{H}$ & 3.737118000 & -1.401150000 & -1.716859000 \\
$\mathrm{C}$ & 0.348237000 & -0.007762000 & 1.138460000 \\
$\mathrm{C}$ & -0.721013000 & -0.692681000 & 0.656911000 \\
$\mathrm{H}$ & -0.501993000 & -1.429417000 & -0.111975000 \\
$\mathrm{O}$ & 0.127294000 & 0.996101000 & 2.121070000 \\
$\mathrm{C}$ & -2.105496000 & -0.486464000 & 1.016282000 \\
$\mathrm{H}$ & -2.279659000 & -0.266614000 & 2.077505000 \\
$\mathrm{C}$ & -3.141525000 & -1.382171000 & 0.459386000 \\
$\mathrm{C}$ & -4.314095000 & -1.647657000 & 1.184243000 \\
$\mathrm{C}$ & -3.048005000 & -1.930415000 & -0.830284000 \\
$\mathrm{C}$ & -5.334450000 & -2.429164000 & 0.655059000 \\
& & &
\end{tabular}




\begin{tabular}{lrrr}
$\mathrm{H}$ & -4.421704000 & -1.231138000 & 2.183557000 \\
$\mathrm{C}$ & -4.063027000 & -2.722461000 & -1.356240000 \\
$\mathrm{H}$ & -2.174982000 & -1.722344000 & -1.444562000 \\
$\mathrm{C}$ & -5.214721000 & -2.979594000 & -0.618647000 \\
$\mathrm{H}$ & -6.227463000 & -2.615231000 & 1.245641000 \\
$\mathrm{H}$ & -3.953729000 & -3.135626000 & -2.355421000 \\
$\mathrm{H}$ & -6.008685000 & -3.594532000 & -1.031114000 \\
$\mathrm{H}$ & -2.339503000 & 0.831770000 & 0.513527000 \\
$\mathrm{H}$ & -0.559572000 & 0.689283000 & 2.729309000 \\
$\mathrm{C}$ & -3.140713000 & 1.643235000 & -1.982412000 \\
$\mathrm{C}$ & -3.282301000 & 2.418756000 & -0.667766000 \\
$\mathrm{H}$ & -2.141221000 & 1.795866000 & -2.407547000 \\
$\mathrm{H}$ & -3.285724000 & 0.571286000 & -1.811026000 \\
$\mathrm{H}$ & -3.881227000 & 1.972725000 & -2.719777000 \\
$\mathrm{C}$ & -4.657770000 & 2.156024000 & -0.044709000 \\
$\mathrm{C}$ & -3.082318000 & 3.915978000 & -0.908939000 \\
$\mathrm{H}$ & -4.745381000 & 2.684689000 & 0.910309000 \\
$\mathrm{H}$ & -5.462875000 & 2.494459000 & -0.706393000 \\
$\mathrm{H}$ & -4.794557000 & 1.084432000 & 0.138837000 \\
$\mathrm{H}$ & -3.837709000 & 4.313559000 & -1.595759000 \\
$\mathrm{H}$ & -3.151849000 & 4.463928000 & 0.037072000 \\
$\mathrm{H}$ & -2.094632000 & 4.102777000 & -1.349449000 \\
$\mathrm{O}$ & -2.279236000 & 2.000263000 & 0.238577000 \\
$\mathrm{C}$ & 5.881657000 & -0.277834000 & -0.501320000 \\
$\mathrm{~F}$ & 6.140723000 & 0.696116000 & -1.396149000 \\
$\mathrm{~F}$ & 6.190062000 & -1.442086000 & -1.099805000 \\
$\mathrm{~F}$ & 6.740498000 & -0.108213000 & 0.518231000 \\
$\mathrm{Li}$ & -0.556305000 & 2.076667000 & 0.632714000 \\
\hline & & &
\end{tabular}

\begin{tabular}{|c|c|c|c|}
\hline \multicolumn{4}{|c|}{$\mathbf{A 3}_{\text {tBuOLi }}$} \\
\hline & \multicolumn{3}{|c|}{ Coordinates (Angstroms) } \\
\hline & $X$ & $\mathrm{Y}$ & $\mathrm{Z}$ \\
\hline$\overline{\mathrm{C}}$ & 3.475446000 & -1.726595000 & -0.148415000 \\
\hline $\mathrm{C}$ & 2.212230000 & -2.262377000 & -0.362491000 \\
\hline $\mathrm{C}$ & 1.074148000 & -1.449197000 & -0.296605000 \\
\hline $\mathrm{C}$ & 1.212192000 & -0.088099000 & -0.000290000 \\
\hline $\mathrm{C}$ & 2.478546000 & 0.441483000 & 0.212383000 \\
\hline $\mathrm{C}$ & 3.608069000 & -0.371734000 & 0.140736000 \\
\hline $\mathrm{H}$ & 4.353561000 & -2.360263000 & -0.207632000 \\
\hline $\mathrm{H}$ & 2.117179000 & -3.315357000 & -0.615944000 \\
\hline $\mathrm{H}$ & 0.328907000 & 0.555130000 & 0.093252000 \\
\hline $\mathrm{H}$ & 2.583687000 & 1.494050000 & 0.455172000 \\
\hline $\mathrm{C}$ & -0.264116000 & -2.045406000 & -0.528102000 \\
\hline
\end{tabular}




\begin{tabular}{lrrr}
$\mathrm{C}$ & -1.239217000 & -1.457157000 & -1.233087000 \\
$\mathrm{H}$ & -1.049745000 & -0.470117000 & -1.643730000 \\
$\mathrm{O}$ & -0.464244000 & -3.303536000 & -0.019998000 \\
$\mathrm{C}$ & -2.609615000 & -2.060114000 & -1.431746000 \\
$\mathrm{H}$ & -2.568242000 & -3.144599000 & -1.298685000 \\
$\mathrm{C}$ & -3.562067000 & -1.447925000 & -0.426133000 \\
$\mathrm{C}$ & -3.557823000 & -1.901844000 & 0.901867000 \\
$\mathrm{C}$ & -4.327000000 & -0.320679000 & -0.744204000 \\
$\mathrm{C}$ & -4.282812000 & -1.232459000 & 1.886371000 \\
$\mathrm{H}$ & -2.953044000 & -2.767187000 & 1.158908000 \\
$\mathrm{C}$ & -5.051877000 & 0.352903000 & 0.240790000 \\
$\mathrm{H}$ & -4.321226000 & 0.058942000 & -1.762125000 \\
$\mathrm{C}$ & -5.026076000 & -0.095607000 & 1.559602000 \\
$\mathrm{H}$ & -4.261618000 & -1.592623000 & 2.910502000 \\
$\mathrm{H}$ & -5.614326000 & 1.243408000 & -0.020283000 \\
$\mathrm{H}$ & -5.575907000 & 0.437041000 & 2.328562000 \\
$\mathrm{C}$ & -0.921416000 & 2.654329000 & -1.748497000 \\
$\mathrm{C}$ & -1.243779000 & 2.870694000 & -0.256114000 \\
$\mathrm{H}$ & 0.014187000 & 2.091416000 & -1.851899000 \\
$\mathrm{H}$ & -1.724242000 & 2.070642000 & -2.217706000 \\
$\mathrm{H}$ & -0.815418000 & 3.597632000 & -2.298943000 \\
$\mathrm{C}$ & -2.564380000 & 3.655569000 & -0.138811000 \\
$\mathrm{C}$ & -0.112799000 & 3.693519000 & 0.388752000 \\
$\mathrm{H}$ & -2.810108000 & 3.804384000 & 0.919361000 \\
$\mathrm{H}$ & -2.519655000 & 4.637762000 & -0.626683000 \\
$\mathrm{H}$ & -3.376983000 & 3.079443000 & -0.601054000 \\
$\mathrm{H}$ & 0.004787000 & 4.680816000 & -0.075580000 \\
$\mathrm{H}$ & -0.317373000 & 3.830538000 & 1.456147000 \\
$\mathrm{~F}$ & 0.839170000 & 3.157807000 & 0.294098000 \\
$\mathrm{O}$ & -1.359584000 & 1.651550000 & 0.375241000 \\
$\mathrm{H}$ & -2.962704000 & -1.862404000 & -2.449117000 \\
$\mathrm{H}$ & 0.232836000 & -3.488391000 & 0.621545000 \\
$\mathrm{C}$ & 4.972620000 & 0.232987000 & 0.326757000 \\
$\mathrm{~F}$ & 5.879498000 & -0.680934000 & 0.715194000 \\
$\mathrm{H}$ & 4.963360000 & 1.208206000 & 1.250443000 \\
$\mathrm{H}$ & 5.430176000 & 0.779300000 & -0.814396000 \\
0.364019000 & 0.354975000 & 0.724046000 \\
\hline
\end{tabular}

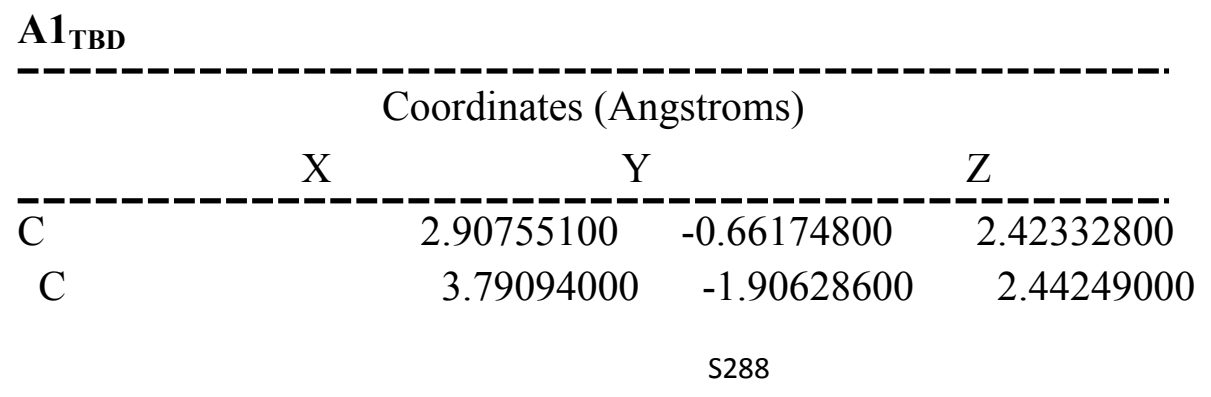


C

$\mathrm{N}$

$\mathrm{H}$

$\mathrm{H}$

$\mathrm{H}$

$\mathrm{H}$

$\mathrm{H}$

$\mathrm{H}$

$\mathrm{N}$

C

C

C

$\mathrm{N}$

C

$\mathrm{H}$

$\mathrm{H}$

$\mathrm{H}$

$\mathrm{H}$

$\mathrm{H}$

$\mathrm{H}$

$\mathrm{H}$

C

C

C

C

C

C

$\mathrm{H}$

$\mathrm{H}$

$\mathrm{H}$

$\mathrm{H}$

C

C

$\mathrm{H}$

$\mathrm{O}$

C

$\mathrm{H}$

C

C

C

C

$\mathrm{H}$

C

$\mathrm{H}$
4.89007300

$-1.73805600$

1.40396900

2.35365300

$-0.40942500$

1.11059100

$3.49744300 \quad 0.20363800$

2.77025400

2.08063200

$-0.77449500$

3.13331900

3.18030600

$-2.78213700$

2.19378800

4.23692300

$-2.07359600$

3.42848000

5.65734400

$-1.03831400$

1.76877300

5.39108600

$-2.69800400$

1.22072600

4.35168800

$-1.25919100$

0.13930400

5.14336400

$-1.60210300$

$-1.03075300$

4.68275000

3.16784000

$-0.85817000$

$-0.95776900$

$-2.27463400$

2.61407400

$-0.37240800$

$-0.69646800$

$-2.68926900$

$-1.36750900$

0.19762100

$-1.29184400$

$-2.36246000$

$-1.16410200$

0.08640800

$-1.20790800$

$-0.81490400$

$-2.21018300$

$-3.15784900$

5.16172700

$-0.39870600$

$-3.22245300$

$-2.01215700$

$-2.49770600$

2.87126100

$-0.16587900$

$-1.17385200$

4.29945300

$-0.30063000$

2.92764700

$-0.31976600$

$-1.47733200$

2.03202400

0.08325100

0.75330300

2.52481400

0.50524100

0.98636500

3.89609200

0.52859900

$-0.00757400$

4.78557400

0.12552400

$-2.02013600$

4.98858200

$-0.61231000$

$-2.44519500$

2.54679700

$-0.63900400$

1.52006000

1.81513800

0.81050200

1.94836500

4.27376100

0.86372300

$-0.70104200$

0.53074000

$-0.01379700$

$-2.10753900$

0.12576600

0.34083000

0.50047800

1.30257500

$-2.45531800$

0.06665500

$-1.31573300$

$-2.89280500$

$-0.62456300$

$-0.43408200$

$-2.48170400$

$-0.96430800$

$-1.38322900$

$-4.27257200$

$-1.05410100$

$-0.14794700$

$-5.00200900$

$-1.69055200$

$-1.15947700$

$-4.89759700$

$-0.86047600$

1.09163600

$-6.31135600$

$-2.10994000$

$-0.95032200$

$-4.53185800$

$-1.85559600$

$-2.12595800$

$-6.20502400$

$-1.27828500$

1.30344000

$-4.35544300$

$-0.38704500$

1.90486100 


$\begin{array}{lccc}\mathrm{C} & -6.91975700 & -1.90391400 & 0.28344700 \\ \mathrm{H} & -6.85520800 & -2.59971200 & -1.75296300 \\ \mathrm{H} & -6.66879600 & -1.11955500 & 2.27278300 \\ \mathrm{H} & -7.94137200 & -2.23088500 & 0.45263900 \\ \mathrm{H} & 0.00769800 & 0.03724700 & 0.66289800 \\ \mathrm{H} & -0.66400500 & 0.70565400 & -1.95429500 \\ \mathrm{C} & 0.25411604 & 6.30287671 & 0.15570352 \\ \mathrm{~F} & -0.43438584 & 6.89818599 & -0.84132808 \\ \mathrm{~F} & 1.57390095 & 6.53523259 & -0.00763217 \\ \mathrm{~F} & -0.14776350 & 6.80531402 & 1.34252683\end{array}$

TSA1-2 TBD

\begin{tabular}{|c|c|c|c|}
\hline \multicolumn{4}{|c|}{ Coordinates (Angstroms) } \\
\hline & $\mathrm{X}$ & Y & $\mathrm{Z}$ \\
\hline $\mathrm{C}$ & -0.624739000 & -0.946514000 & 2.345334000 \\
\hline $\mathrm{C}$ & -1.747574000 & 0.058489000 & 2.571523000 \\
\hline $\mathrm{C}$ & -3.083770000 & -0.646611000 & 2.403464000 \\
\hline $\mathrm{N}$ & -0.752824000 & -1.586104000 & 1.048373000 \\
\hline $\mathrm{H}$ & -0.624563000 & -1.706190000 & 3.138054000 \\
\hline $\mathrm{H}$ & 0.353419000 & -0.455483000 & 2.367280000 \\
\hline $\mathrm{H}$ & -1.670829000 & 0.863232000 & 1.834056000 \\
\hline $\mathrm{H}$ & -1.690695000 & 0.502344000 & 3.569768000 \\
\hline $\mathrm{H}$ & -3.316040000 & -1.263316000 & 3.282242000 \\
\hline $\mathrm{H}$ & -3.883840000 & 0.094067000 & 2.292920000 \\
\hline $\mathrm{N}$ & -3.103366000 & -1.492243000 & 1.209035000 \\
\hline $\mathrm{C}$ & -4.422972000 & -1.675888000 & 0.611814000 \\
\hline $\mathrm{C}$ & -4.403034000 & -2.738095000 & -0.475048000 \\
\hline $\mathrm{C}$ & -3.258077000 & -2.439838000 & -1.429394000 \\
\hline $\mathrm{N}$ & -2.028078000 & -2.403166000 & -0.664899000 \\
\hline $\mathrm{C}$ & -1.960076000 & -1.797165000 & 0.549922000 \\
\hline $\mathrm{H}$ & -4.762714000 & -0.714484000 & 0.199881000 \\
\hline $\mathrm{H}$ & -5.115209000 & -1.959322000 & 1.412751000 \\
\hline $\mathrm{H}$ & -4.259972000 & -3.729767000 & -0.033003000 \\
\hline $\mathrm{H}$ & -5.359669000 & -2.734377000 & -1.004601000 \\
\hline $\mathrm{H}$ & -3.162873000 & -3.221177000 & -2.187660000 \\
\hline $\mathrm{H}$ & -3.439160000 & -1.485729000 & -1.946187000 \\
\hline $\mathrm{H}$ & -1.159577000 & -2.330565000 & -1.193429000 \\
\hline $\mathrm{C}$ & 3.888151000 & 0.919179000 & 0.623774000 \\
\hline $\mathrm{C}$ & 2.535761000 & 0.789709000 & 0.361151000 \\
\hline $\mathrm{C}$ & 2.056994000 & -0.114872000 & -0.607734000 \\
\hline $\mathrm{C}$ & 3.011643000 & -0.896956000 & -1.278350000 \\
\hline $\mathrm{C}$ & 4.368379000 & -0.768308000 & -1.015900000 \\
\hline $\mathrm{C}$ & 4.820167000 & 0.141480000 & -0.064847000 \\
\hline
\end{tabular}




$\begin{array}{lrrr}\mathrm{H} & 4.225258000 & 1.626858000 & 1.374887000 \\ \mathrm{H} & 1.840837000 & 1.401638000 & 0.929533000 \\ \mathrm{H} & 2.671665000 & -1.621069000 & -2.009699000 \\ \mathrm{H} & 5.080098000 & -1.385830000 & -1.554356000 \\ \mathrm{C} & 0.618942000 & -0.302836000 & -0.865367000 \\ \mathrm{C} & -0.315446000 & 0.792192000 & -0.729726000 \\ \mathrm{H} & -0.031547000 & 1.553676000 & -0.005511000 \\ \mathrm{O} & 0.327324000 & -1.200290000 & -1.934847000 \\ \mathrm{C} & -1.556042000 & 0.840966000 & -1.269529000 \\ \mathrm{H} & -1.823618000 & 0.077112000 & -1.996333000 \\ \mathrm{C} & -2.640604000 & 1.747667000 & -0.894762000 \\ \mathrm{C} & -3.909284000 & 1.568560000 & -1.473525000 \\ \mathrm{C} & -2.517228000 & 2.763683000 & 0.069640000 \\ \mathrm{C} & -5.001495000 & 2.341742000 & -1.097380000 \\ \mathrm{H} & -4.033229000 & 0.805316000 & -2.239496000 \\ \mathrm{C} & -3.608044000 & 3.536765000 & 0.447513000 \\ \mathrm{H} & -1.553120000 & 2.957047000 & 0.531922000 \\ \mathrm{C} & -4.860846000 & 3.329786000 & -0.126419000 \\ \mathrm{H} & -5.966277000 & 2.174399000 & -1.568747000 \\ \mathrm{H} & -3.477258000 & 4.312093000 & 1.197616000 \\ \mathrm{H} & -5.710782000 & 3.935737000 & 0.172161000 \\ \mathrm{H} & 0.014109000 & -1.100772000 & 0.236375000 \\ \mathrm{H} & 0.364564000 & -0.705958000 & -2.767612000 \\ \mathrm{C} & 6.282699000 & 0.336311000 & 0.184077000 \\ \mathrm{~F} & 7.010078000 & -0.737856000 & -0.174297000 \\ \mathrm{~F} & 6.544652000 & 0.579548000 & 1.484044000 \\ \mathrm{~F} & 6.780966000 & 1.385723000 & -0.503412000 \\ \mathrm{H} & & & \end{array}$

\begin{tabular}{|c|c|c|c|}
\hline \multicolumn{4}{|c|}{ A2 } \\
\hline & \multicolumn{3}{|c|}{ Coordinates (Angstroms) } \\
\hline & $X$ & $\mathrm{Y}$ & $\mathrm{Z}$ \\
\hline$\overline{\mathrm{C}}$ & 1.072839000 & 1.707824000 & 2.196306000 \\
\hline $\mathrm{C}$ & 1.869839000 & 2.119472000 & 0.970380000 \\
\hline $\mathrm{C}$ & 1.096161000 & 3.190086000 & 0.218346000 \\
\hline $\mathrm{N}$ & -0.276235000 & 1.325178000 & 1.798873000 \\
\hline $\mathrm{H}$ & 1.025206000 & 2.527430000 & 2.924553000 \\
\hline $\mathrm{H}$ & 1.523860000 & 0.839619000 & 2.678439000 \\
\hline $\mathrm{H}$ & 2.028542000 & 1.246979000 & 0.328727000 \\
\hline $\mathrm{H}$ & 2.848476000 & 2.508642000 & 1.262145000 \\
\hline $\mathrm{H}$ & 1.087016000 & 4.138112000 & 0.772654000 \\
\hline $\mathrm{H}$ & 1.558273000 & 3.378223000 & -0.756165000 \\
\hline $\mathrm{N}$ & -0.282292000 & 2.764455000 & -0.033150000 \\
\hline $\mathrm{C}$ & -0.926039000 & 3.342562000 & -1.212187000 \\
\hline
\end{tabular}




\begin{tabular}{|c|c|c|c|}
\hline $\mathrm{C}$ & -2.428816000 & 3.101067000 & -1.212217000 \\
\hline $\mathrm{C}$ & -2.700453000 & 1.634934000 & -0.917827000 \\
\hline $\mathrm{N}$ & -2.058687000 & 1.299184000 & 0.342911000 \\
\hline $\mathrm{C}$ & -0.862148000 & 1.785608000 & 0.687023000 \\
\hline $\mathrm{H}$ & -0.466963000 & 2.911494000 & -2.112206000 \\
\hline $\mathrm{H}$ & -0.709026000 & 4.416304000 & -1.211934000 \\
\hline $\mathrm{H}$ & -2.908683000 & 3.720963000 & -0.447491000 \\
\hline $\mathrm{H}$ & -2.841868000 & 3.383495000 & -2.183941000 \\
\hline $\mathrm{H}$ & -3.767031000 & 1.428430000 & -0.807640000 \\
\hline $\mathrm{H}$ & -2.323432000 & 0.993548000 & -1.725388000 \\
\hline $\mathrm{H}$ & -2.305460000 & 0.371773000 & 0.728810000 \\
\hline $\mathrm{C}$ & 2.679340000 & -1.043342000 & -1.277559000 \\
\hline $\mathrm{C}$ & 1.410009000 & -1.295028000 & -0.796403000 \\
\hline $\mathrm{C}$ & 1.164394000 & -1.497947000 & 0.587811000 \\
\hline $\mathrm{C}$ & 2.295972000 & -1.444319000 & 1.440061000 \\
\hline $\mathrm{C}$ & 3.564877000 & -1.182840000 & 0.951530000 \\
\hline $\mathrm{C}$ & 3.775084000 & -0.965311000 & -0.410159000 \\
\hline $\mathrm{H}$ & 2.825061000 & -0.881163000 & -2.341783000 \\
\hline $\mathrm{H}$ & 0.584205000 & -1.311266000 & -1.500882000 \\
\hline $\mathrm{H}$ & 2.151989000 & -1.611472000 & 2.501658000 \\
\hline $\mathrm{H}$ & 4.404155000 & -1.143503000 & 1.639115000 \\
\hline $\mathrm{C}$ & -0.150061000 & -1.658611000 & 1.130703000 \\
\hline $\mathrm{C}$ & -1.339677000 & -1.738449000 & 0.432420000 \\
\hline $\mathrm{H}$ & -1.244101000 & -1.884885000 & -0.641186000 \\
\hline $\mathrm{O}$ & -0.234517000 & -1.426035000 & 2.523507000 \\
\hline $\mathrm{C}$ & -2.626460000 & -1.581198000 & 0.944976000 \\
\hline $\mathrm{H}$ & -2.760735000 & -1.446655000 & 2.017853000 \\
\hline $\mathrm{C}$ & -3.835976000 & -1.517005000 & 0.145965000 \\
\hline $\mathrm{C}$ & -5.037573000 & -1.064334000 & 0.734996000 \\
\hline $\mathrm{C}$ & -3.884997000 & -1.799776000 & -1.237266000 \\
\hline $\mathrm{C}$ & -6.195579000 & -0.880057000 & -0.006958000 \\
\hline $\mathrm{H}$ & -5.044818000 & -0.844040000 & 1.800884000 \\
\hline $\mathrm{C}$ & -5.045120000 & -1.609843000 & -1.977205000 \\
\hline $\mathrm{H}$ & -3.003582000 & -2.188736000 & -1.739764000 \\
\hline $\mathrm{C}$ & -6.211966000 & -1.141055000 & -1.376843000 \\
\hline $\mathrm{H}$ & -7.095963000 & -0.525978000 & 0.489083000 \\
\hline $\mathrm{H}$ & -5.038570000 & -1.840097000 & -3.040016000 \\
\hline $\mathrm{H}$ & -7.117373000 & -0.996847000 & -1.958256000 \\
\hline $\mathrm{H}$ & -0.612126000 & 0.436696000 & 2.177752000 \\
\hline $\mathrm{H}$ & -0.818003000 & -2.098894000 & 2.898028000 \\
\hline $\mathrm{C}$ & 5.137548000 & -0.701271000 & -0.956448000 \\
\hline $\mathrm{F}$ & 5.996212000 & -0.277972000 & -0.007943000 \\
\hline $\mathrm{F}$ & 5.117338000 & 0.246787000 & -1.920919000 \\
\hline $\mathrm{F}$ & 5.696495000 & -1.791993000 & -1.522051000 \\
\hline
\end{tabular}


TSA2-3 TBD

\begin{tabular}{|c|c|c|c|}
\hline & & nates (Angstr & \\
\hline & $\mathrm{X}$ & $\mathrm{Y}$ & $\mathrm{Z}$ \\
\hline $\bar{C}$ & 0.987573000 & 2.058990000 & 1.879738000 \\
\hline $\mathrm{C}$ & 1.629153000 & 2.373578000 & 0.538657000 \\
\hline $\mathrm{C}$ & 0.754339000 & 3.380884000 & -0.191495000 \\
\hline $\mathrm{N}$ & -0.397366000 & 1.658320000 & 1.688177000 \\
\hline $\mathrm{H}$ & 1.035946000 & 2.936149000 & 2.539149000 \\
\hline $\mathrm{H}$ & 1.503645000 & 1.233471000 & 2.374725000 \\
\hline $\mathrm{H}$ & 1.719982000 & 1.453370000 & -0.048952000 \\
\hline $\mathrm{H}$ & 2.631716000 & 2.786610000 & 0.678825000 \\
\hline $\mathrm{H}$ & 0.799441000 & 4.362696000 & 0.301632000 \\
\hline $\mathrm{H}$ & 1.104965000 & 3.514741000 & -1.221180000 \\
\hline $\mathrm{N}$ & -0.629483000 & 2.922063000 & -0.257958000 \\
\hline $\mathrm{C}$ & -1.431489000 & 3.452061000 & -1.354440000 \\
\hline $\mathrm{C}$ & -2.911707000 & 3.178293000 & -1.134682000 \\
\hline $\mathrm{C}$ & -3.088322000 & 1.716169000 & -0.752724000 \\
\hline $\mathrm{N}$ & -2.303743000 & 1.414532000 & 0.432526000 \\
\hline $\mathrm{C}$ & -1.126213000 & 1.988140000 & 0.595759000 \\
\hline $\mathrm{H}$ & -1.094981000 & 3.002194000 & -2.299696000 \\
\hline $\mathrm{H}$ & -1.242171000 & 4.530350000 & -1.419504000 \\
\hline $\mathrm{H}$ & -3.293554000 & 3.814263000 & -0.328493000 \\
\hline $\mathrm{H}$ & -3.466725000 & 3.417794000 & -2.046329000 \\
\hline $\mathrm{H}$ & -4.132831000 & 1.483479000 & -0.530408000 \\
\hline $\mathrm{H}$ & -2.791962000 & 1.066791000 & -1.589891000 \\
\hline $\mathrm{H}$ & -2.421744000 & 0.233037000 & 0.780679000 \\
\hline $\mathrm{C}$ & 2.897073000 & -1.143941000 & -1.206588000 \\
\hline $\mathrm{C}$ & 1.603900000 & -1.277323000 & -0.732787000 \\
\hline $\mathrm{C}$ & 1.333727000 & -1.348589000 & 0.648739000 \\
\hline $\mathrm{C}$ & 2.431134000 & -1.283027000 & 1.525969000 \\
\hline $\mathrm{C}$ & 3.726477000 & -1.135195000 & 1.050625000 \\
\hline $\mathrm{C}$ & 3.970272000 & -1.061584000 & -0.317678000 \\
\hline $\mathrm{H}$ & 3.076169000 & -1.090938000 & -2.275845000 \\
\hline $\mathrm{H}$ & 0.788756000 & -1.304937000 & -1.448371000 \\
\hline $\mathrm{H}$ & 2.251296000 & -1.347238000 & 2.592884000 \\
\hline $\mathrm{H}$ & 4.553773000 & -1.084553000 & 1.751173000 \\
\hline $\mathrm{C}$ & -0.023223000 & -1.400671000 & 1.175102000 \\
\hline $\mathrm{C}$ & -1.169337000 & -1.530082000 & 0.464943000 \\
\hline $\mathrm{H}$ & -1.058481000 & -1.776162000 & -0.587050000 \\
\hline $\mathrm{O}$ & -0.097734000 & -1.091838000 & 2.541011000 \\
\hline $\mathrm{C}$ & -2.495757000 & -1.230031000 & 0.945539000 \\
\hline $\mathrm{H}$ & -2.651472000 & -1.305146000 & 2.025548000 \\
\hline
\end{tabular}




\begin{tabular}{lrrr}
$\mathrm{C}$ & -3.700539000 & -1.576268000 & 0.171798000 \\
$\mathrm{C}$ & -4.955225000 & -1.587259000 & 0.808073000 \\
$\mathrm{C}$ & -3.695316000 & -1.799761000 & -1.216997000 \\
$\mathrm{C}$ & -6.131891000 & -1.804576000 & 0.103412000 \\
$\mathrm{H}$ & -4.999637000 & -1.416645000 & 1.881804000 \\
$\mathrm{C}$ & -4.873019000 & -2.020970000 & -1.922664000 \\
$\mathrm{H}$ & -2.756441000 & -1.798548000 & -1.764623000 \\
$\mathrm{C}$ & -6.103515000 & -2.023672000 & -1.272453000 \\
$\mathrm{H}$ & -7.080308000 & -1.806855000 & 0.634554000 \\
$\mathrm{H}$ & -4.824848000 & -2.193749000 & -2.994956000 \\
$\mathrm{H}$ & -7.022440000 & -2.196488000 & -1.824315000 \\
$\mathrm{H}$ & -0.670326000 & 0.797613000 & 2.154200000 \\
$\mathrm{H}$ & -0.801467000 & -1.632686000 & 2.922566000 \\
$\mathrm{C}$ & 5.352914000 & -0.816492000 & -0.837901000 \\
$\mathrm{~F}$ & 5.587032000 & 0.499563000 & -1.037044000 \\
$\mathrm{~F}$ & 6.301328000 & -1.250907000 & 0.010194000 \\
$\mathrm{~F}$ & 5.562047000 & -1.422029000 & -2.021272000 \\
\hline
\end{tabular}

\begin{tabular}{|c|c|c|c|}
\hline \multicolumn{4}{|c|}{$\begin{array}{l}\mathbf{A} 3_{\mathrm{TBD}} \\
-\end{array}$} \\
\hline & $\mathrm{X}$ & $\mathrm{Y}$ & $\mathrm{Z}$ \\
\hline $\bar{C}$ & -1.180122000 & -2.504121000 & 0.419845000 \\
\hline $\mathrm{C}$ & -1.859768000 & -2.252946000 & 1.758592000 \\
\hline $\mathrm{C}$ & -3.371914000 & -2.213978000 & 1.568900000 \\
\hline $\mathrm{N}$ & -1.602918000 & -1.469449000 & -0.489946000 \\
\hline $\mathrm{H}$ & -1.423982000 & -3.518352000 & 0.057813000 \\
\hline $\mathrm{H}$ & -0.092219000 & -2.443982000 & 0.525461000 \\
\hline $\mathrm{H}$ & -1.508153000 & -1.295396000 & 2.151384000 \\
\hline $\mathrm{H}$ & -1.605451000 & -3.032583000 & 2.483593000 \\
\hline $\mathrm{H}$ & -3.773053000 & -3.240651000 & 1.565144000 \\
\hline $\mathrm{H}$ & -3.834822000 & -1.694885000 & 2.419723000 \\
\hline $\mathrm{N}$ & -3.781665000 & -1.544146000 & 0.347025000 \\
\hline $\mathrm{C}$ & -5.193707000 & -1.216819000 & 0.261776000 \\
\hline $\mathrm{C}$ & -5.623976000 & -1.089305000 & -1.192374000 \\
\hline $\mathrm{C}$ & -4.651217000 & -0.157762000 & -1.912870000 \\
\hline $\mathrm{N}$ & -3.269999000 & -0.560560000 & -1.762181000 \\
\hline $\mathrm{C}$ & -2.937243000 & -1.174296000 & -0.677379000 \\
\hline $\mathrm{H}$ & -5.391242000 & -0.280834000 & 0.806172000 \\
\hline $\mathrm{H}$ & -5.758354000 & -2.015250000 & 0.761012000 \\
\hline $\mathrm{H}$ & -5.601930000 & -2.078068000 & -1.666038000 \\
\hline $\mathrm{H}$ & -6.651145000 & -0.712644000 & -1.245763000 \\
\hline $\mathrm{H}$ & -4.886527000 & -0.125911000 & -2.983493000 \\
\hline $\mathrm{H}$ & -4.791039000 & 0.866260000 & -1.531301000 \\
\hline
\end{tabular}




\begin{tabular}{|c|c|c|c|}
\hline $\mathrm{C}$ & 4.599064000 & 1.300631000 & -0.009715000 \\
\hline $\mathrm{C}$ & 3.294693000 & 1.532060000 & -0.421159000 \\
\hline $\mathrm{C}$ & 2.454271000 & 0.467925000 & -0.767310000 \\
\hline $\mathrm{C}$ & 2.964769000 & -0.834938000 & -0.712388000 \\
\hline $\mathrm{C}$ & 4.271508000 & -1.069888000 & -0.306744000 \\
\hline $\mathrm{C}$ & 5.090639000 & -0.001936000 & 0.048173000 \\
\hline $\mathrm{H}$ & 5.239441000 & 2.133908000 & 0.259114000 \\
\hline $\mathrm{H}$ & 2.924524000 & 2.549729000 & -0.493603000 \\
\hline $\mathrm{H}$ & 2.322490000 & -1.673777000 & -0.964155000 \\
\hline $\mathrm{H}$ & 4.650956000 & -2.084700000 & -0.252940000 \\
\hline $\mathrm{C}$ & 1.052916000 & 0.696542000 & -1.189175000 \\
\hline $\mathrm{C}$ & 0.267016000 & 1.654967000 & -0.688355000 \\
\hline $\mathrm{H}$ & 0.688584000 & 2.279588000 & 0.095760000 \\
\hline $\mathrm{O}$ & 0.550583000 & -0.212489000 & -2.094084000 \\
\hline $\mathrm{C}$ & -1.154298000 & 1.918924000 & -1.083350000 \\
\hline $\mathrm{H}$ & -1.512611000 & 1.149815000 & -1.778131000 \\
\hline $\mathrm{C}$ & -2.127598000 & 1.972489000 & 0.078138000 \\
\hline $\mathrm{C}$ & -3.356056000 & 2.619774000 & -0.078155000 \\
\hline $\mathrm{C}$ & -1.864742000 & 1.342068000 & 1.293568000 \\
\hline $\mathrm{C}$ & -4.304595000 & 2.616829000 & 0.938515000 \\
\hline $\mathrm{H}$ & -3.575391000 & 3.120040000 & -1.018697000 \\
\hline $\mathrm{C}$ & -2.810245000 & 1.334912000 & 2.315885000 \\
\hline $\mathrm{H}$ & -0.915705000 & 0.832056000 & 1.429001000 \\
\hline $\mathrm{C}$ & -4.036780000 & 1.968277000 & 2.142446000 \\
\hline $\mathrm{H}$ & -5.255363000 & 3.121476000 & 0.790992000 \\
\hline $\mathrm{H}$ & -2.585894000 & 0.831838000 & 3.253166000 \\
\hline $\mathrm{H}$ & -4.774793000 & 1.965271000 & 2.939344000 \\
\hline $\mathrm{H}$ & -1.039281000 & -1.279615000 & -1.308101000 \\
\hline $\mathrm{H}$ & 1.254532000 & -0.475816000 & -2.699853000 \\
\hline $\mathrm{H}$ & -1.199257000 & 2.878329000 & -1.618711000 \\
\hline $\mathrm{C}$ & 6.524021000 & -0.251000000 & 0.428295000 \\
\hline $\mathrm{F}$ & 6.683663000 & -1.449988000 & 1.013915000 \\
\hline $\mathrm{F}$ & 7.331595000 & -0.230734000 & -0.647918000 \\
\hline $\mathrm{F}$ & 6.983996000 & 0.679910000 & 1.280728000 \\
\hline
\end{tabular}

\begin{tabular}{|c|c|c|c|}
\hline \multicolumn{4}{|c|}{$\mathbf{A} 1_{\text {DBU }}$} \\
\hline & \multicolumn{3}{|c|}{ Coordinates (Angstroms) } \\
\hline & $\mathrm{X}$ & Y & Z \\
\hline$\overline{\mathrm{C}}$ & -1.346709000 & 3.328177000 & -0.525206000 \\
\hline $\mathrm{C}$ & -1.680017000 & 1.978592000 & -0.481086000 \\
\hline $\mathrm{C}$ & -0.705765000 & 1.012424000 & -0.225679000 \\
\hline $\mathrm{C}$ & 0.615002000 & 1.419146000 & -0.021362000 \\
\hline $\mathrm{C}$ & 0.953708000 & 2.764568000 & -0.060259000 \\
\hline
\end{tabular}




\begin{tabular}{|c|c|c|c|}
\hline $\mathrm{C}$ & -0.028428000 & 3.721049000 & -0.313178000 \\
\hline $\mathrm{H}$ & -2.109729000 & 4.072782000 & -0.725919000 \\
\hline $\mathrm{H}$ & -2.711349000 & 1.674456000 & -0.640852000 \\
\hline $\mathrm{H}$ & 1.366973000 & 0.659272000 & 0.173548000 \\
\hline $\mathrm{H}$ & 1.981431000 & 3.073410000 & 0.100977000 \\
\hline $\mathrm{C}$ & -1.032519000 & -0.475285000 & -0.247316000 \\
\hline $\mathrm{C}$ & -2.432279000 & -0.761435000 & 0.232001000 \\
\hline $\mathrm{H}$ & -2.684470000 & -0.316355000 & 1.193734000 \\
\hline $\mathrm{O}$ & -0.772356000 & -1.010215000 & -1.540741000 \\
\hline $\mathrm{C}$ & -3.321079000 & -1.494964000 & -0.440421000 \\
\hline $\mathrm{H}$ & -3.011505000 & -1.908782000 & -1.398758000 \\
\hline $\mathrm{C}$ & -4.700334000 & -1.806103000 & -0.026488000 \\
\hline $\mathrm{C}$ & -5.592010000 & -2.321111000 & -0.974934000 \\
\hline $\mathrm{C}$ & -5.168744000 & -1.608620000 & 1.279476000 \\
\hline $\mathrm{C}$ & -6.911033000 & -2.609892000 & -0.641601000 \\
\hline $\mathrm{H}$ & -5.243546000 & -2.490982000 & -1.990915000 \\
\hline $\mathrm{C}$ & -6.485373000 & -1.896446000 & 1.615152000 \\
\hline $\mathrm{H}$ & -4.492886000 & -1.240053000 & 2.045620000 \\
\hline $\mathrm{C}$ & -7.364701000 & -2.395268000 & 0.655996000 \\
\hline $\mathrm{H}$ & -7.585047000 & -3.003385000 & -1.397001000 \\
\hline $\mathrm{H}$ & -6.826677000 & -1.737011000 & 2.633970000 \\
\hline $\mathrm{H}$ & -8.393671000 & -2.619779000 & 0.920446000 \\
\hline $\mathrm{H}$ & -0.313283000 & -0.984947000 & 0.407531000 \\
\hline $\mathrm{H}$ & -1.226371000 & -0.446332000 & -2.181171000 \\
\hline $\mathrm{C}$ & 5.254857000 & -2.505232000 & 0.037921000 \\
\hline $\mathrm{C}$ & 4.898968000 & -3.724941000 & -0.806199000 \\
\hline $\mathrm{C}$ & 2.326006000 & -2.448411000 & -0.870127000 \\
\hline $\mathrm{C}$ & 4.307902000 & -3.371361000 & -2.173181000 \\
\hline $\mathrm{C}$ & 3.255564000 & -2.264581000 & -2.071689000 \\
\hline $\mathrm{H}$ & 5.791973000 & -1.769083000 & -0.582613000 \\
\hline $\mathrm{H}$ & 2.099638000 & -3.513551000 & -0.728284000 \\
\hline $\mathrm{H}$ & 4.212141000 & -4.359060000 & -0.234334000 \\
\hline $\mathrm{H}$ & 3.858758000 & -4.271733000 & -2.609647000 \\
\hline $\mathrm{H}$ & 5.962189000 & -2.824534000 & 0.814217000 \\
\hline $\mathrm{H}$ & 5.813433000 & -4.312434000 & -0.944882000 \\
\hline $\mathrm{H}$ & 1.366703000 & -1.962931000 & -1.058367000 \\
\hline $\mathrm{H}$ & 5.109698000 & -3.059539000 & -2.853842000 \\
\hline $\mathrm{H}$ & 2.658010000 & -2.239896000 & -2.988813000 \\
\hline $\mathrm{H}$ & 3.744842000 & -1.284697000 & -2.001773000 \\
\hline $\mathrm{C}$ & 2.814337000 & -1.867817000 & 0.447177000 \\
\hline $\mathrm{N}$ & 1.887053000 & -1.393785000 & 1.209354000 \\
\hline $\mathrm{N}$ & 4.159754000 & -1.848848000 & 0.749955000 \\
\hline $\mathrm{C}$ & 4.645994000 & -1.054687000 & 1.876288000 \\
\hline $\mathrm{H}$ & 4.954865000 & -1.729129000 & 2.689302000 \\
\hline
\end{tabular}




$\begin{array}{lrrr}\mathrm{H} & 5.544584000 & -0.513805000 & 1.550554000 \\ \mathrm{C} & 3.596859000 & -0.076742000 & 2.375927000 \\ \mathrm{H} & 3.911574000 & 0.346406000 & 3.335015000 \\ \mathrm{H} & 3.489849000 & 0.752070000 & 1.665455000 \\ \mathrm{C} & 2.268136000 & -0.815713000 & 2.482477000 \\ \mathrm{H} & 1.471277000 & -0.135110000 & 2.802142000 \\ \mathrm{H} & 2.342274000 & -1.601901000 & 3.249852000 \\ \mathrm{C} & 0.322535000 & 5.182486000 & -0.294112000 \\ \mathrm{~F} & 0.183948000 & 5.709716000 & 0.937286000 \\ \mathrm{~F} & -0.465618000 & 5.899465000 & -1.114069000 \\ \mathrm{~F} & 1.595476000 & 5.399684000 & -0.668982000 \\ -\end{array}$

TSA1-2 DBU

\begin{tabular}{|c|c|c|c|}
\hline & & inates (Angstr & \\
\hline & $X$ & Y & $\mathrm{Z}$ \\
\hline $\mathrm{C}$ & 1.530819000 & -1.784648000 & -1.134233000 \\
\hline $\mathrm{C}$ & 0.313558000 & -1.315210000 & -0.673924000 \\
\hline $\mathrm{C}$ & 0.002749000 & -1.282861000 & 0.705707000 \\
\hline $\mathrm{C}$ & 0.978376000 & -1.796937000 & 1.586817000 \\
\hline $\mathrm{C}$ & 2.194830000 & -2.270206000 & 1.124996000 \\
\hline $\mathrm{C}$ & 2.493334000 & -2.254079000 & -0.237896000 \\
\hline $\mathrm{H}$ & 1.741720000 & -1.783112000 & -2.199798000 \\
\hline $\mathrm{H}$ & -0.412474000 & -0.948944000 & -1.395672000 \\
\hline $\mathrm{H}$ & 0.765678000 & -1.788595000 & 2.650286000 \\
\hline $\mathrm{H}$ & 2.927890000 & -2.648398000 & 1.831325000 \\
\hline $\mathrm{C}$ & -1.180914000 & -0.594812000 & 1.205910000 \\
\hline $\mathrm{C}$ & -2.446713000 & -0.706534000 & 0.516258000 \\
\hline $\mathrm{H}$ & -2.395425000 & -1.065949000 & -0.511688000 \\
\hline $\mathrm{O}$ & -1.268146000 & -0.491084000 & 2.611679000 \\
\hline $\mathrm{C}$ & -3.654610000 & -0.380541000 & 1.033544000 \\
\hline $\mathrm{H}$ & -3.684607000 & 0.025035000 & 2.043269000 \\
\hline $\mathrm{C}$ & -4.941836000 & -0.465304000 & 0.342299000 \\
\hline $\mathrm{C}$ & -6.074768000 & 0.120597000 & 0.930851000 \\
\hline $\mathrm{C}$ & -5.120235000 & -1.105489000 & -0.896521000 \\
\hline $\mathrm{C}$ & -7.318037000 & 0.083823000 & 0.311464000 \\
\hline $\mathrm{H}$ & -5.968308000 & 0.616541000 & 1.892977000 \\
\hline $\mathrm{C}$ & -6.361125000 & -1.137608000 & -1.519452000 \\
\hline $\mathrm{H}$ & -4.280008000 & -1.602970000 & -1.373233000 \\
\hline $\mathrm{C}$ & -7.470731000 & -0.542277000 & -0.922545000 \\
\hline $\mathrm{H}$ & -8.173159000 & 0.548058000 & 0.795559000 \\
\hline $\mathrm{H}$ & -6.465934000 & -1.642094000 & -2.476414000 \\
\hline $\mathrm{H}$ & -8.441234000 & -0.575095000 & -1.408561000 \\
\hline $\mathrm{H}$ & -0.760761000 & 0.856787000 & 0.751868000 \\
\hline
\end{tabular}




\begin{tabular}{|c|c|c|c|}
\hline $\mathrm{H}$ & -1.829857000 & -1.217719000 & 2.924820000 \\
\hline $\mathrm{C}$ & 2.934010000 & 3.183638000 & -0.709212000 \\
\hline $\mathrm{C}$ & 3.593689000 & 3.447844000 & 0.639410000 \\
\hline $\mathrm{C}$ & 1.570925000 & 1.627971000 & 1.537212000 \\
\hline $\mathrm{C}$ & 4.047117000 & 2.180274000 & 1.368085000 \\
\hline $\mathrm{C}$ & 2.984690000 & 1.079516000 & 1.332546000 \\
\hline $\mathrm{H}$ & 3.520152000 & 2.450321000 & -1.281038000 \\
\hline $\mathrm{H}$ & 1.571689000 & 2.458945000 & 2.254071000 \\
\hline $\mathrm{H}$ & 2.909136000 & 4.038005000 & 1.259584000 \\
\hline $\mathrm{H}$ & 4.283302000 & 2.438646000 & 2.407166000 \\
\hline $\mathrm{H}$ & 2.942563000 & 4.115702000 & -1.285044000 \\
\hline $\mathrm{H}$ & 4.459856000 & 4.092215000 & 0.453521000 \\
\hline $\mathrm{H}$ & 0.922556000 & 0.867338000 & 1.976348000 \\
\hline $\mathrm{H}$ & 4.973690000 & 1.804874000 & 0.918845000 \\
\hline $\mathrm{H}$ & 3.189648000 & 0.336493000 & 2.107549000 \\
\hline $\mathrm{H}$ & 3.028209000 & 0.529372000 & 0.386099000 \\
\hline $\mathrm{C}$ & 0.879533000 & 2.094428000 & 0.277732000 \\
\hline $\mathrm{N}$ & -0.402516000 & 1.822770000 & 0.209511000 \\
\hline $\mathrm{N}$ & 1.525975000 & 2.754823000 & -0.701818000 \\
\hline $\mathrm{C}$ & 0.865234000 & 3.023094000 & -1.986465000 \\
\hline $\mathrm{H}$ & 0.587258000 & 4.084929000 & -2.024204000 \\
\hline $\mathrm{H}$ & 1.604433000 & 2.848726000 & -2.775377000 \\
\hline $\mathrm{C}$ & -0.349016000 & 2.137051000 & -2.201126000 \\
\hline $\mathrm{H}$ & -0.905247000 & 2.481109000 & -3.077099000 \\
\hline $\mathrm{H}$ & -0.024275000 & 1.107870000 & -2.385120000 \\
\hline $\mathrm{C}$ & -1.206610000 & 2.179117000 & -0.946050000 \\
\hline $\mathrm{H}$ & -2.038106000 & 1.470819000 & -0.991235000 \\
\hline $\mathrm{H}$ & -1.633532000 & 3.178890000 & -0.799706000 \\
\hline $\mathrm{C}$ & 3.862344000 & -2.594749000 & -0.719610000 \\
\hline $\mathrm{F}$ & 3.858445000 & -3.126425000 & -1.956868000 \\
\hline $\mathrm{F}$ & 4.657451000 & -1.494362000 & -0.789306000 \\
\hline $\mathrm{F}$ & 4.495930000 & -3.462419000 & 0.089378000 \\
\hline
\end{tabular}

A2

\begin{tabular}{llll} 
& & \multicolumn{2}{c}{ Coordinates (Angstroms) } \\
& $\mathrm{X}$ & $\mathrm{Y}$ & $\mathrm{Z}$ \\
\hline $\mathrm{C}$ & 2.226239000 & -2.097332000 & 0.326521000 \\
$\mathrm{C}$ & 0.983225000 & -1.868246000 & 0.877539000 \\
$\mathrm{C}$ & 0.733986000 & -0.745545000 & 1.718925000 \\
$\mathrm{C}$ & 1.888405000 & -0.034102000 & 2.150085000 \\
$\mathrm{C}$ & 3.131648000 & -0.267010000 & 1.592660000 \\
$\mathrm{C}$ & 3.310664000 & -1.264076000 & 0.628203000 \\
$\mathrm{H}$ & 2.367309000 & -2.939367000 & -0.346212000
\end{tabular}




\begin{tabular}{|c|c|c|c|}
\hline $\mathrm{H}$ & 0.180341000 & -2.565070000 & 0.656665000 \\
\hline $\mathrm{H}$ & 1.767302000 & 0.738514000 & 2.900882000 \\
\hline $\mathrm{H}$ & 3.978674000 & 0.338317000 & 1.902732000 \\
\hline $\mathrm{C}$ & -0.573362000 & -0.294173000 & 2.057573000 \\
\hline $\mathrm{C}$ & -1.748306000 & -0.715317000 & 1.442798000 \\
\hline $\mathrm{H}$ & -1.652526000 & -1.586100000 & 0.797077000 \\
\hline $\mathrm{O}$ & -0.635252000 & 0.838070000 & 2.883707000 \\
\hline $\mathrm{C}$ & -2.985711000 & -0.094122000 & 1.482871000 \\
\hline $\mathrm{H}$ & -3.106500000 & 0.794783000 & 2.099736000 \\
\hline $\mathrm{C}$ & -4.152395000 & -0.510158000 & 0.725112000 \\
\hline $\mathrm{C}$ & -5.268087000 & 0.350501000 & 0.627958000 \\
\hline $\mathrm{C}$ & -4.240935000 & -1.732062000 & 0.019616000 \\
\hline $\mathrm{C}$ & -6.380682000 & 0.026233000 & -0.134588000 \\
\hline $\mathrm{H}$ & -5.243711000 & 1.297103000 & 1.164117000 \\
\hline $\mathrm{C}$ & -5.354482000 & -2.049424000 & -0.749528000 \\
\hline $\mathrm{H}$ & -3.437520000 & -2.460202000 & 0.098742000 \\
\hline $\mathrm{C}$ & -6.434870000 & -1.174481000 & -0.841371000 \\
\hline $\mathrm{H}$ & -7.215198000 & 0.721503000 & -0.180281000 \\
\hline $\mathrm{H}$ & -5.384310000 & -3.003336000 & -1.270662000 \\
\hline $\mathrm{H}$ & -7.305332000 & -1.428772000 & -1.438171000 \\
\hline $\mathrm{H}$ & -2.367219000 & 0.803682000 & -0.524357000 \\
\hline $\mathrm{H}$ & -1.264722000 & 0.641104000 & 3.588874000 \\
\hline $\mathrm{C}$ & 1.922195000 & 1.770421000 & -1.351129000 \\
\hline $\mathrm{C}$ & 1.769139000 & 3.289419000 & -1.325880000 \\
\hline $\mathrm{C}$ & -0.661035000 & 2.465058000 & 0.140004000 \\
\hline $\mathrm{C}$ & 1.485767000 & 3.857437000 & 0.069290000 \\
\hline $\mathrm{C}$ & 0.575650000 & 2.947173000 & 0.889498000 \\
\hline $\mathrm{H}$ & 2.470724000 & 1.386653000 & -0.484334000 \\
\hline $\mathrm{H}$ & -1.167331000 & 3.300423000 & -0.363094000 \\
\hline $\mathrm{H}$ & 0.971179000 & 3.564759000 & -2.027010000 \\
\hline $\mathrm{H}$ & 1.039352000 & 4.853988000 & -0.033591000 \\
\hline $\mathrm{H}$ & 2.495858000 & 1.483268000 & -2.235834000 \\
\hline $\mathrm{H}$ & 2.687587000 & 3.731166000 & -1.726076000 \\
\hline $\mathrm{H}$ & -1.371311000 & 2.061339000 & 0.872905000 \\
\hline $\mathrm{H}$ & 2.429541000 & 3.991259000 & 0.610191000 \\
\hline $\mathrm{H}$ & 0.234404000 & 3.464392000 & 1.790479000 \\
\hline $\mathrm{H}$ & 1.127889000 & 2.076258000 & 1.247226000 \\
\hline $\mathrm{C}$ & -0.494157000 & 1.373142000 & -0.888636000 \\
\hline $\mathrm{N}$ & -1.634823000 & 0.780888000 & -1.236939000 \\
\hline $\mathrm{N}$ & 0.647519000 & 1.035524000 & -1.473433000 \\
\hline $\mathrm{C}$ & 0.757993000 & -0.161111000 & -2.325728000 \\
\hline $\mathrm{H}$ & 0.871732000 & 0.177049000 & -3.363695000 \\
\hline $\mathrm{H}$ & 1.680373000 & -0.669774000 & -2.029736000 \\
\hline $\mathrm{C}$ & -0.430453000 & -1.093691000 & -2.183594000 \\
\hline
\end{tabular}




$\begin{array}{lrrr}\mathrm{H} & -0.407453000 & -1.833317000 & -2.988174000 \\ \mathrm{H} & -0.360121000 & -1.620913000 & -1.230475000 \\ \mathrm{C} & -1.719034000 & -0.287741000 & -2.221048000 \\ \mathrm{H} & -2.591344000 & -0.894545000 & -1.969145000 \\ \mathrm{H} & -1.880688000 & 0.150115000 & -3.213378000 \\ \mathrm{C} & 4.571091000 & -1.375616000 & -0.147782000 \\ \mathrm{~F} & 4.477499000 & -0.730866000 & -1.350644000 \\ \mathrm{~F} & 4.891952000 & -2.648678000 & -0.449155000 \\ \mathrm{~F} & 5.627349000 & -0.829877000 & 0.479948000\end{array}$

TSA2-3 DBU

\begin{tabular}{|c|c|c|c|}
\hline \multicolumn{4}{|c|}{ Coordinates (Angstroms) } \\
\hline & $X$ & Y & $\mathrm{Z}$ \\
\hline $\mathrm{C}$ & 2.540267000 & -1.309297000 & -0.965103000 \\
\hline $\mathrm{C}$ & 1.252851000 & -1.255173000 & -0.461111000 \\
\hline $\mathrm{C}$ & 1.003121000 & -1.310936000 & 0.924616000 \\
\hline $\mathrm{C}$ & 2.115605000 & -1.426199000 & 1.777294000 \\
\hline $\mathrm{C}$ & 3.407236000 & -1.465340000 & 1.273629000 \\
\hline $\mathrm{C}$ & 3.631459000 & -1.400944000 & -0.099693000 \\
\hline $\mathrm{H}$ & 2.703984000 & -1.264531000 & -2.037569000 \\
\hline $\mathrm{H}$ & 0.427201000 & -1.143040000 & -1.155555000 \\
\hline $\mathrm{H}$ & 1.949142000 & -1.462426000 & 2.847448000 \\
\hline $\mathrm{H}$ & 4.248285000 & -1.545245000 & 1.954778000 \\
\hline $\mathrm{C}$ & -0.334523000 & -1.139779000 & 1.477482000 \\
\hline $\mathrm{C}$ & -1.495186000 & -1.209207000 & 0.777230000 \\
\hline $\mathrm{H}$ & -1.411768000 & -1.595736000 & -0.235247000 \\
\hline $\mathrm{O}$ & -0.334815000 & -0.760895000 & 2.814482000 \\
\hline $\mathrm{C}$ & -2.769195000 & -0.668261000 & 1.160489000 \\
\hline $\mathrm{H}$ & -2.893947000 & -0.368286000 & 2.203710000 \\
\hline $\mathrm{C}$ & -4.023406000 & -1.055020000 & 0.499923000 \\
\hline $\mathrm{C}$ & -5.225521000 & -0.430184000 & 0.886973000 \\
\hline $\mathrm{C}$ & -4.097945000 & -1.961475000 & -0.574030000 \\
\hline $\mathrm{C}$ & -6.422326000 & -0.678248000 & 0.230668000 \\
\hline $\mathrm{H}$ & -5.203659000 & 0.276561000 & 1.714102000 \\
\hline $\mathrm{C}$ & -5.297041000 & -2.200715000 & -1.238751000 \\
\hline $\mathrm{H}$ & -3.211057000 & -2.506758000 & -0.884612000 \\
\hline $\mathrm{C}$ & -6.469435000 & -1.561212000 & -0.847866000 \\
\hline $\mathrm{H}$ & -7.327574000 & -0.175023000 & 0.560675000 \\
\hline $\mathrm{H}$ & -5.314838000 & -2.910466000 & -2.062128000 \\
\hline $\mathrm{H}$ & -7.404953000 & -1.754447000 & -1.363894000 \\
\hline $\mathrm{H}$ & -2.507216000 & 0.720199000 & 0.520750000 \\
\hline $\mathrm{H}$ & -1.160998000 & -1.081830000 & 3.198832000 \\
\hline $\mathrm{C}$ & 1.335284000 & 2.658064000 & -0.969427000 \\
\hline
\end{tabular}




\begin{tabular}{|c|c|c|c|}
\hline $\mathrm{C}$ & 1.496222000 & 3.787070000 & 0.046089000 \\
\hline $\mathrm{C}$ & -0.504211000 & 2.181212000 & 1.427403000 \\
\hline $\mathrm{C}$ & 1.789309000 & 3.295645000 & 1.466606000 \\
\hline $\mathrm{C}$ & 0.965581000 & 2.059604000 & 1.820578000 \\
\hline $\mathrm{H}$ & 2.079247000 & 1.866256000 & -0.808377000 \\
\hline $\mathrm{H}$ & -0.899868000 & 3.170231000 & 1.695444000 \\
\hline $\mathrm{H}$ & 0.586811000 & 4.400287000 & 0.026035000 \\
\hline $\mathrm{H}$ & 1.582934000 & 4.106766000 & 2.175394000 \\
\hline $\mathrm{H}$ & 1.515716000 & 3.063659000 & -1.970466000 \\
\hline $\mathrm{H}$ & 2.308295000 & 4.437587000 & -0.295462000 \\
\hline $\mathrm{H}$ & -1.089692000 & 1.458681000 & 2.005583000 \\
\hline $\mathrm{H}$ & 2.854758000 & 3.057259000 & 1.563812000 \\
\hline $\mathrm{H}$ & 1.018855000 & 1.863614000 & 2.895145000 \\
\hline $\mathrm{H}$ & 1.393286000 & 1.173966000 & 1.343514000 \\
\hline $\mathrm{C}$ & -0.873779000 & 1.927038000 & -0.015991000 \\
\hline $\mathrm{N}$ & -2.135097000 & 1.587281000 & -0.200182000 \\
\hline $\mathrm{N}$ & -0.007327000 & 2.060099000 & -1.038577000 \\
\hline $\mathrm{C}$ & -0.325527000 & 1.557046000 & -2.381609000 \\
\hline $\mathrm{H}$ & -0.418174000 & 2.418553000 & -3.056577000 \\
\hline $\mathrm{H}$ & 0.534680000 & 0.967258000 & -2.719329000 \\
\hline $\mathrm{C}$ & -1.594793000 & 0.722414000 & -2.417262000 \\
\hline $\mathrm{H}$ & -1.931969000 & 0.616998000 & -3.452279000 \\
\hline $\mathrm{H}$ & -1.411389000 & -0.282133000 & -2.025544000 \\
\hline $\mathrm{C}$ & -2.645645000 & 1.400795000 & -1.550248000 \\
\hline $\mathrm{H}$ & -3.555868000 & 0.802060000 & -1.479597000 \\
\hline $\mathrm{H}$ & -2.914485000 & 2.378416000 & -1.970229000 \\
\hline $\mathrm{C}$ & 5.022651000 & -1.336552000 & -0.648714000 \\
\hline $\mathrm{F}$ & 5.428198000 & -0.060207000 & -0.834410000 \\
\hline $\mathrm{F}$ & 5.922352000 & -1.910683000 & 0.167547000 \\
\hline $\mathrm{F}$ & 5.120943000 & -1.941888000 & -1.847353000 \\
\hline
\end{tabular}

\begin{tabular}{lrrr} 
A3 & & & \\
& & & \\
Cobu & & Y & \\
\hline $\mathrm{C}$ & 2.498351000 & -1.106119000 & -1.014718000 \\
$\mathrm{C}$ & 1.233994000 & -1.020249000 & -0.451695000 \\
$\mathrm{C}$ & 1.039941000 & -1.256050000 & 0.913923000 \\
$\mathrm{C}$ & 2.149355000 & -1.566484000 & 1.706557000 \\
$\mathrm{C}$ & 3.417164000 & -1.649476000 & 1.146715000 \\
$\mathrm{C}$ & 3.595332000 & -1.418906000 & -0.214561000 \\
$\mathrm{H}$ & 2.634919000 & -0.918812000 & -2.074540000 \\
$\mathrm{H}$ & 0.392649000 & -0.733794000 & -1.072710000 \\
$\mathrm{H}$ & 2.012017000 & -1.733389000 & 2.768631000
\end{tabular}




\begin{tabular}{|c|c|c|c|}
\hline $\mathrm{H}$ & 4.269947000 & -1.895287000 & 1.770717000 \\
\hline $\mathrm{C}$ & -0.297736000 & -1.115338000 & 1.525387000 \\
\hline $\mathrm{C}$ & -1.455513000 & -1.246395000 & 0.867539000 \\
\hline $\mathrm{H}$ & -1.427679000 & -1.539307000 & -0.175323000 \\
\hline $\mathrm{O}$ & -0.221586000 & -0.800771000 & 2.854841000 \\
\hline $\mathrm{C}$ & -2.803386000 & -0.963508000 & 1.479526000 \\
\hline $\mathrm{H}$ & -2.859291000 & 0.102060000 & 1.739534000 \\
\hline $\mathrm{C}$ & -3.961076000 & -1.284150000 & 0.561645000 \\
\hline $\mathrm{C}$ & -4.920530000 & -0.313477000 & 0.273247000 \\
\hline $\mathrm{C}$ & -4.097564000 & -2.553762000 & -0.005107000 \\
\hline $\mathrm{C}$ & -5.995419000 & -0.604075000 & -0.563375000 \\
\hline $\mathrm{H}$ & -4.804525000 & 0.682513000 & 0.692646000 \\
\hline $\mathrm{C}$ & -5.168504000 & -2.846501000 & -0.843395000 \\
\hline $\mathrm{H}$ & -3.355570000 & -3.318758000 & 0.212979000 \\
\hline $\mathrm{C}$ & -6.122413000 & -1.871043000 & -1.124489000 \\
\hline $\mathrm{H}$ & -6.731805000 & 0.164374000 & -0.780669000 \\
\hline $\mathrm{H}$ & -5.260128000 & -3.837964000 & -1.277666000 \\
\hline $\mathrm{H}$ & -6.958339000 & -2.098518000 & -1.779506000 \\
\hline $\mathrm{H}$ & -1.114353000 & -0.770309000 & 3.220388000 \\
\hline $\mathrm{C}$ & 1.253746000 & 2.770737000 & -0.910432000 \\
\hline $\mathrm{C}$ & 1.507337000 & 3.867541000 & 0.121662000 \\
\hline $\mathrm{C}$ & -0.581078000 & 2.427082000 & 1.522623000 \\
\hline $\mathrm{C}$ & 1.799939000 & 3.323889000 & 1.522188000 \\
\hline $\mathrm{C}$ & 0.880422000 & 2.156361000 & 1.876359000 \\
\hline $\mathrm{H}$ & 1.985377000 & 1.955721000 & -0.789433000 \\
\hline $\mathrm{H}$ & -0.849694000 & 3.454582000 & 1.803370000 \\
\hline $\mathrm{H}$ & 0.637828000 & 4.535725000 & 0.136453000 \\
\hline $\mathrm{H}$ & 1.684457000 & 4.132072000 & 2.255242000 \\
\hline $\mathrm{H}$ & 1.428421000 & 3.196011000 & -1.906939000 \\
\hline $\mathrm{H}$ & 2.353239000 & 4.473237000 & -0.221911000 \\
\hline $\mathrm{H}$ & -1.232600000 & 1.780180000 & 2.114418000 \\
\hline $\mathrm{H}$ & 2.844310000 & 2.993514000 & 1.579868000 \\
\hline $\mathrm{H}$ & 0.955589000 & 1.933416000 & 2.945350000 \\
\hline $\mathrm{H}$ & 1.218891000 & 1.252841000 & 1.361673000 \\
\hline $\mathrm{C}$ & -1.025826000 & 2.201271000 & 0.082692000 \\
\hline $\mathrm{N}$ & -2.285784000 & 1.979884000 & -0.069149000 \\
\hline $\mathrm{N}$ & -0.104606000 & 2.231996000 & -0.947532000 \\
\hline $\mathrm{C}$ & -0.455804000 & 1.718601000 & -2.268108000 \\
\hline $\mathrm{H}$ & -0.479258000 & 2.553806000 & -2.984796000 \\
\hline $\mathrm{H}$ & 0.345446000 & 1.042249000 & -2.600920000 \\
\hline $\mathrm{C}$ & -1.796581000 & 1.004646000 & -2.265408000 \\
\hline $\mathrm{H}$ & -2.146536000 & 0.870241000 & -3.293845000 \\
\hline $\mathrm{H}$ & -1.708079000 & 0.010972000 & -1.812570000 \\
\hline $\mathrm{C}$ & -2.767534000 & 1.829922000 & -1.429137000 \\
\hline
\end{tabular}




$\begin{array}{lrrr}\mathrm{H} & -3.751567000 & 1.353972000 & -1.404567000 \\ \mathrm{H} & -2.898390000 & 2.820272000 & -1.895174000 \\ \mathrm{H} & -2.933957000 & -1.538936000 & 2.411799000 \\ \mathrm{C} & 4.977879000 & -1.425866000 & -0.802779000 \\ \mathrm{~F} & 4.967050000 & -1.741657000 & -2.109124000 \\ \mathrm{~F} & 5.562888000 & -0.216763000 & -0.698653000 \\ \mathrm{~F} & \mathbf{5 . 7 8 4 1 8 8 0 0 0} & -2.302867000 & -0.181735000\end{array}$

\begin{tabular}{|c|c|c|c|}
\hline \multicolumn{4}{|c|}{$\mathbf{A 1}_{\text {MTBD }}$} \\
\hline & $X$ & $\mathrm{Y}$ & $\mathrm{Z}$ \\
\hline$\overline{\mathrm{C}}$ & -0.841935000 & -2.886227000 & -0.491742000 \\
\hline $\mathrm{C}$ & -0.795762000 & -1.827554000 & -1.588667000 \\
\hline $\mathrm{C}$ & 0.587298000 & -1.854997000 & -2.220421000 \\
\hline $\mathrm{N}$ & 0.172750000 & -2.660681000 & 0.514590000 \\
\hline $\mathrm{H}$ & -0.722426000 & -3.883560000 & -0.947686000 \\
\hline $\mathrm{H}$ & -1.823316000 & -2.881882000 & -0.006343000 \\
\hline $\mathrm{H}$ & -0.995373000 & -0.846030000 & -1.144079000 \\
\hline $\mathrm{H}$ & -1.555479000 & -2.001093000 & -2.357727000 \\
\hline $\mathrm{H}$ & 0.661692000 & -2.686933000 & -2.937418000 \\
\hline $\mathrm{H}$ & 0.769536000 & -0.930359000 & -2.787349000 \\
\hline $\mathrm{N}$ & 1.641276000 & -1.986570000 & -1.223159000 \\
\hline $\mathrm{C}$ & 2.962474000 & -1.649870000 & -1.726452000 \\
\hline $\mathrm{C}$ & 4.056925000 & -2.006703000 & -0.738736000 \\
\hline $\mathrm{C}$ & 3.626218000 & -1.550173000 & 0.643212000 \\
\hline $\mathrm{N}$ & 2.385256000 & -2.208299000 & 0.996023000 \\
\hline $\mathrm{C}$ & 1.339942000 & -2.296411000 & 0.092919000 \\
\hline $\mathrm{H}$ & 3.006244000 & -0.572074000 & -1.945683000 \\
\hline $\mathrm{H}$ & 3.114751000 & -2.178514000 & -2.677807000 \\
\hline $\mathrm{H}$ & 4.221894000 & -3.089386000 & -0.723059000 \\
\hline $\mathrm{H}$ & 4.990155000 & -1.519209000 & -1.035929000 \\
\hline $\mathrm{H}$ & 4.375196000 & -1.835616000 & 1.388879000 \\
\hline $\mathrm{H}$ & 3.532660000 & -0.453894000 & 0.676779000 \\
\hline $\mathrm{C}$ & -3.230074000 & 1.642759000 & -0.135733000 \\
\hline $\mathrm{C}$ & -1.974080000 & 1.562676000 & 0.449765000 \\
\hline $\mathrm{C}$ & -1.619463000 & 0.458582000 & 1.229283000 \\
\hline $\mathrm{C}$ & -2.548265000 & -0.563484000 & 1.416127000 \\
\hline $\mathrm{C}$ & -3.805607000 & -0.495005000 & 0.827058000 \\
\hline $\mathrm{C}$ & -4.145883000 & 0.608925000 & 0.050768000 \\
\hline $\mathrm{H}$ & -3.498585000 & 2.503011000 & -0.740751000 \\
\hline $\mathrm{H}$ & -1.250073000 & 2.357187000 & 0.284102000 \\
\hline $\mathrm{H}$ & -2.270541000 & -1.429941000 & 2.009035000 \\
\hline $\mathrm{H}$ & -4.520161000 & -1.298038000 & 0.970319000 \\
\hline
\end{tabular}




\begin{tabular}{lrrr}
$\mathrm{C}$ & -0.249978000 & 0.378994000 & 1.886294000 \\
$\mathrm{C}$ & 0.829746000 & 0.804341000 & 0.926834000 \\
$\mathrm{H}$ & 0.811486000 & 0.294029000 & -0.034268000 \\
$\mathrm{O}$ & -0.218513000 & 1.089958000 & 3.112041000 \\
$\mathrm{C}$ & 1.773269000 & 1.710687000 & 1.187407000 \\
$\mathrm{H}$ & 1.812126000 & 2.153579000 & 2.181265000 \\
$\mathrm{C}$ & 2.825696000 & 2.115713000 & 0.237919000 \\
$\mathrm{C}$ & 4.047335000 & 2.599660000 & 0.720082000 \\
$\mathrm{C}$ & 2.654025000 & 2.015377000 & -1.149241000 \\
$\mathrm{C}$ & 5.076732000 & 2.940360000 & -0.151378000 \\
$\mathrm{H}$ & 4.193429000 & 2.696526000 & 1.792960000 \\
$\mathrm{C}$ & 3.681972000 & 2.353031000 & -2.021526000 \\
$\mathrm{H}$ & 1.699502000 & 1.682716000 & -1.548388000 \\
$\mathrm{C}$ & 4.900442000 & 2.812570000 & -1.526069000 \\
$\mathrm{H}$ & 6.018375000 & 3.308251000 & 0.245512000 \\
$\mathrm{H}$ & 3.527009000 & 2.267600000 & -3.093289000 \\
$\mathrm{H}$ & 5.700920000 & 3.081797000 & -2.208496000 \\
$\mathrm{H}$ & -0.075581000 & -0.669548000 & 2.144916000 \\
$\mathrm{H}$ & -0.514092000 & 1.992158000 & 2.931059000 \\
$\mathrm{C}$ & 2.113686000 & -2.354251000 & 2.409489000 \\
$\mathrm{H}$ & 1.987844000 & -1.378578000 & 2.902483000 \\
$\mathrm{H}$ & 1.201089000 & -2.934624000 & 2.533946000 \\
$\mathrm{H}$ & 2.951245000 & -2.875406000 & 2.887312000 \\
$\mathrm{C}$ & -5.477663000 & 0.681922000 & -0.642819000 \\
$\mathrm{~F}$ & -6.362739000 & -0.193679000 & -0.140900000 \\
$\mathrm{~F}$ & -5.361852000 & 0.418082000 & -1.958338000 \\
$\mathrm{~F}$ & -6.021929000 & 1.907984000 & -0.542432000 \\
\hline$-----0 .-$ &
\end{tabular}

TSA1-2 MTBD 


\begin{tabular}{|c|c|c|c|}
\hline $\mathrm{C}$ & -4.611065000 & -1.900565000 & 0.118619000 \\
\hline $\mathrm{C}$ & -3.685315000 & -1.900145000 & -1.085038000 \\
\hline $\mathrm{N}$ & -2.333707000 & -2.244166000 & -0.671999000 \\
\hline $\mathrm{C}$ & -1.804535000 & -1.708670000 & 0.454901000 \\
\hline $\mathrm{H}$ & -4.187499000 & 0.080085000 & 0.872430000 \\
\hline $\mathrm{H}$ & -4.586709000 & -1.106233000 & 2.125918000 \\
\hline $\mathrm{H}$ & -4.692357000 & -2.917808000 & 0.516315000 \\
\hline $\mathrm{H}$ & -5.610481000 & -1.565743000 & -0.173244000 \\
\hline $\mathrm{H}$ & -4.007926000 & -2.646305000 & -1.815961000 \\
\hline $\mathrm{H}$ & -3.691694000 & -0.919524000 & -1.578458000 \\
\hline $\mathrm{C}$ & 3.779521000 & 1.071473000 & 0.601227000 \\
\hline $\mathrm{C}$ & 2.447335000 & 1.005725000 & 0.227252000 \\
\hline $\mathrm{C}$ & 2.016807000 & 0.174810000 & -0.826985000 \\
\hline $\mathrm{C}$ & 3.007848000 & -0.569699000 & -1.494200000 \\
\hline $\mathrm{C}$ & 4.341820000 & -0.500395000 & -1.124502000 \\
\hline $\mathrm{C}$ & 4.740972000 & 0.315230000 & -0.067616000 \\
\hline $\mathrm{H}$ & 4.077015000 & 1.722993000 & 1.417153000 \\
\hline $\mathrm{H}$ & 1.735268000 & 1.627275000 & 0.761954000 \\
\hline $\mathrm{H}$ & 2.705579000 & -1.217025000 & -2.309796000 \\
\hline $\mathrm{H}$ & 5.078767000 & -1.093104000 & -1.657236000 \\
\hline $\mathrm{C}$ & 0.604100000 & 0.002213000 & -1.206906000 \\
\hline $\mathrm{C}$ & -0.414704000 & 0.930480000 & -0.783660000 \\
\hline $\mathrm{H}$ & -0.231063000 & 1.415237000 & 0.174298000 \\
\hline $\mathrm{O}$ & 0.416410000 & -0.532969000 & -2.505258000 \\
\hline $\mathrm{C}$ & -1.628973000 & 1.096334000 & -1.361113000 \\
\hline $\mathrm{H}$ & -1.814427000 & 0.595564000 & -2.309807000 \\
\hline $\mathrm{C}$ & -2.765136000 & 1.814600000 & -0.785418000 \\
\hline $\mathrm{C}$ & -4.006012000 & 1.778743000 & -1.446243000 \\
\hline $\mathrm{C}$ & -2.717867000 & 2.502365000 & 0.441074000 \\
\hline $\mathrm{C}$ & -5.147114000 & 2.346595000 & -0.892399000 \\
\hline $\mathrm{H}$ & -4.070821000 & 1.282720000 & -2.412571000 \\
\hline $\mathrm{C}$ & -3.856665000 & 3.072564000 & 0.995450000 \\
\hline $\mathrm{H}$ & -1.774491000 & 2.595648000 & 0.971930000 \\
\hline $\mathrm{C}$ & -5.085256000 & 2.988154000 & 0.342241000 \\
\hline $\mathrm{H}$ & -6.090356000 & 2.288419000 & -1.428853000 \\
\hline $\mathrm{H}$ & -3.783428000 & 3.589819000 & 1.948377000 \\
\hline $\mathrm{H}$ & -5.974464000 & 3.430550000 & 0.780522000 \\
\hline $\mathrm{H}$ & 0.076584000 & -1.113588000 & -0.289498000 \\
\hline $\mathrm{H}$ & 0.567852000 & 0.179883000 & -3.143174000 \\
\hline $\mathrm{C}$ & -1.467195000 & -2.843268000 & -1.676890000 \\
\hline $\mathrm{H}$ & -1.073011000 & -2.096272000 & -2.374230000 \\
\hline $\mathrm{H}$ & -0.623893000 & -3.324661000 & -1.181014000 \\
\hline $\mathrm{H}$ & -2.046427000 & -3.596990000 & -2.218125000 \\
\hline $\mathrm{C}$ & 6.185190000 & 0.440099000 & 0.302349000 \\
\hline
\end{tabular}




$\begin{array}{lrrr}\text { F } & 6.891618000 & -0.658667000 & -0.023864000 \\ F & 6.351466000 & 0.642613000 & 1.624965000 \\ \text { F } & 6.784503000 & 1.478973000 & -0.317165000\end{array}$

A2

Coordinates (Angstroms)

$\begin{array}{lrrr} & \mathrm{X} & \mathrm{Y} & \mathrm{Z} \\ \mathrm{C} & 1.099263000 & 1.506979000 & 2.485303000 \\ \mathrm{C} & 2.153941000 & 1.755494000 & 1.422566000 \\ \mathrm{C} & 1.657959000 & 2.809641000 & 0.449381000 \\ \mathrm{~N} & -0.256074000 & 1.518061000 & 1.928831000 \\ \mathrm{H} & 1.141552000 & 2.271018000 & 3.272163000 \\ \mathrm{H} & 1.256476000 & 0.526288000 & 2.947342000 \\ \mathrm{H} & 2.360891000 & 0.836237000 & 0.873567000 \\ \mathrm{H} & 3.083963000 & 2.079431000 & 1.896713000 \\ \mathrm{H} & 1.528734000 & 3.784344000 & 0.939299000 \\ \mathrm{H} & 2.370049000 & 2.930687000 & -0.369611000 \\ \mathrm{~N} & 0.389681000 & 2.379917000 & -0.132238000 \\ \mathrm{C} & 0.189515000 & 2.522470000 & -1.572235000 \\ \mathrm{C} & -1.290359000 & 2.686879000 & -1.882390000 \\ \mathrm{C} & -2.056728000 & 1.525384000 & -1.276529000 \\ \mathrm{~N} & -1.705027000 & 1.375839000 & 0.130656000 \\ \mathrm{C} & -0.509754000 & 1.732180000 & 0.629333000 \\ \mathrm{H} & 0.612523000 & 1.650272000 & -2.087552000 \\ \mathrm{H} & 0.746881000 & 3.406877000 & -1.891156000 \\ \mathrm{H} & -1.648986000 & 3.634334000 & -1.465376000 \\ \mathrm{H} & -1.445998000 & 2.713652000 & -2.963986000 \\ \mathrm{H} & -3.137588000 & 1.670739000 & -1.338291000 \\ \mathrm{H} & -1.826229000 & 0.594519000 & -1.809639000 \\ \mathrm{H} & -2.139853000 & 0.550896000 & 0.563447000 \\ \mathrm{C} & 2.432312000 & -0.748609000 & -1.311281000 \\ \mathrm{C} & 1.157830000 & -1.003885000 & -0.845939000 \\ \mathrm{C} & 0.924235000 & -1.563295000 & 0.443648000 \\ \mathrm{C} & 2.083312000 & -1.833657000 & 1.223118000 \\ \mathrm{C} & 3.354483000 & -1.566489000 & 0.752469000 \\ \mathrm{C} & 3.553420000 & -1.005835000 & -0.514926000 \\ \mathrm{H} & 2.564535000 & -0.319980000 & -2.300908000 \\ \mathrm{H} & 0.316632000 & -0.760073000 & -1.488798000 \\ \mathrm{H} & 1.950825000 & -2.263378000 & 2.209340000 \\ \mathrm{H} & 4.212849000 & -1.783626000 & 1.381891000 \\ \mathrm{H} & -0.380474000 & -1.781924000 & 0.970230000 \\ \mathrm{H} & -1.567574000 & -1.602269000 & 0.274036000 \\ \mathrm{H} & -456149000 & -1.494535000 & -0.803851000\end{array}$




$\begin{array}{lrrr}\mathrm{O} & -0.446584000 & -2.052897000 & 2.347561000 \\ \mathrm{C} & -2.852781000 & -1.474513000 & 0.780939000 \\ \mathrm{H} & -3.002917000 & -1.506671000 & 1.858915000 \\ \mathrm{C} & -4.029461000 & -1.218057000 & -0.029518000 \\ \mathrm{C} & -5.200842000 & -0.706634000 & 0.570235000 \\ \mathrm{C} & -4.067572000 & -1.396877000 & -1.430069000 \\ \mathrm{C} & -6.317774000 & -0.364788000 & -0.178228000 \\ \mathrm{H} & -5.215573000 & -0.566259000 & 1.649479000 \\ \mathrm{C} & -5.183626000 & -1.042003000 & -2.177910000 \\ \mathrm{H} & -3.217121000 & -1.846089000 & -1.936342000 \\ \mathrm{C} & -6.318975000 & -0.516581000 & -1.564844000 \\ \mathrm{H} & -7.197068000 & 0.030395000 & 0.324469000 \\ \mathrm{H} & -5.171955000 & -1.197009000 & -3.254245000 \\ \mathrm{H} & -7.192401000 & -0.247909000 & -2.151212000 \\ \mathrm{H} & -1.050409000 & -2.799484000 & 2.458691000 \\ \mathrm{C} & -1.282393000 & 1.018723000 & 2.837371000 \\ \mathrm{H} & -2.267989000 & 1.368687000 & 2.526410000 \\ \mathrm{H} & -1.259793000 & -0.075102000 & 2.884314000 \\ \mathrm{H} & -1.071530000 & 1.430859000 & 3.828665000 \\ \mathrm{C} & 4.927287000 & -0.732233000 & -1.019749000 \\ \mathrm{~F} & 4.940807000 & 0.259332000 & -1.939137000 \\ \mathrm{~F} & 5.766933000 & -0.359559000 & -0.029573000 \\ \mathrm{~F} & 5.500793000 & -1.801247000 & -1.612960000 \\ \mathrm{H}--------1 .-1\end{array}$

TSA2-3 MTBD

\begin{tabular}{lrrr} 
& \multicolumn{3}{c}{ Coordinates (Angstroms) } \\
& $\mathrm{X}$ & $\mathrm{Y}$ & $\mathrm{Z}$ \\
$\mathrm{C}$ & 0.855312000 & 2.420672000 & 1.703311000 \\
$\mathrm{C}$ & 1.888825000 & 2.240793000 & 0.600391000 \\
$\mathrm{~N}$ & 1.352381000 & 2.825269000 & -0.694737000 \\
$\mathrm{H}$ & -0.517453000 & 2.318562000 & 1.211041000 \\
$\mathrm{H}$ & 0.963437000 & 3.408082000 & 2.172433000 \\
$\mathrm{H}$ & 0.994697000 & 1.660491000 & 2.481135000 \\
$\mathrm{H}$ & 2.101952000 & 1.182365000 & 0.442208000 \\
$\mathrm{H}$ & 2.823168000 & 2.730892000 & 0.887409000 \\
$\mathrm{H}$ & 1.210937000 & 3.912753000 & -0.610561000 \\
$\mathrm{~N}$ & 2.050705000 & 2.636867000 & -1.513281000 \\
$\mathrm{C}$ & -0.091751000 & 2.177396000 & -1.026738000 \\
$\mathrm{C}$ & -1.668566000 & 1.9796000 & -2.405530000 \\
$\mathrm{C}$ & -2.467741000 & 1.189801000 & -1.671036000 \\
$\mathrm{~N}$ & -2.052329000 & 1.497742000 & -0.308293000 \\
$\mathrm{C}$ & -0.837861000 & 1.968610000 & -0.060517000
\end{tabular}




\begin{tabular}{|c|c|c|c|}
\hline $\mathrm{H}$ & 0.124685000 & 0.756944000 & -2.581043000 \\
\hline $\mathrm{H}$ & 0.416440000 & 2.436732000 & -3.054827000 \\
\hline $\mathrm{H}$ & -1.922742000 & 3.037025000 & -2.648637000 \\
\hline $\mathrm{H}$ & -1.898023000 & 1.618821000 & -3.710150000 \\
\hline $\mathrm{H}$ & -3.537552000 & 1.401044000 & -1.754930000 \\
\hline $\mathrm{H}$ & -2.348975000 & 0.111330000 & -1.847282000 \\
\hline $\mathrm{H}$ & -2.402646000 & 0.586109000 & 0.408684000 \\
\hline $\mathrm{C}$ & 2.703134000 & -1.241860000 & -0.972249000 \\
\hline $\mathrm{C}$ & 1.408010000 & -1.234671000 & -0.486650000 \\
\hline $\mathrm{C}$ & 1.141894000 & -1.212479000 & 0.898211000 \\
\hline $\mathrm{C}$ & 2.248139000 & -1.205158000 & 1.768061000 \\
\hline $\mathrm{C}$ & 3.546749000 & -1.196808000 & 1.280470000 \\
\hline $\mathrm{C}$ & 3.786309000 & -1.209163000 & -0.091908000 \\
\hline $\mathrm{H}$ & 2.878126000 & -1.255888000 & -2.043569000 \\
\hline $\mathrm{H}$ & 0.586054000 & -1.215201000 & -1.195096000 \\
\hline $\mathrm{H}$ & 2.070670000 & -1.189680000 & 2.836965000 \\
\hline $\mathrm{H}$ & 4.381677000 & -1.181173000 & 1.973756000 \\
\hline $\mathrm{C}$ & -0.209067000 & -1.098598000 & 1.423625000 \\
\hline $\mathrm{C}$ & -1.350085000 & -1.241522000 & 0.703971000 \\
\hline $\mathrm{H}$ & -1.231268000 & -1.626413000 & -0.306493000 \\
\hline $\mathrm{O}$ & -0.253821000 & -0.701458000 & 2.758400000 \\
\hline $\mathrm{C}$ & -2.656012000 & -0.754520000 & 1.051020000 \\
\hline $\mathrm{H}$ & -2.814888000 & -0.439532000 & 2.085171000 \\
\hline $\mathrm{C}$ & -3.876969000 & -1.219504000 & 0.376820000 \\
\hline $\mathrm{C}$ & -5.106122000 & -0.603376000 & 0.681779000 \\
\hline $\mathrm{C}$ & -3.892425000 & -2.201027000 & -0.629755000 \\
\hline $\mathrm{C}$ & -6.275843000 & -0.936532000 & 0.013959000 \\
\hline $\mathrm{H}$ & -5.128370000 & 0.160658000 & 1.456571000 \\
\hline $\mathrm{C}$ & -5.063331000 & -2.525979000 & -1.308206000 \\
\hline $\mathrm{H}$ & -2.980189000 & -2.739532000 & -0.871783000 \\
\hline $\mathrm{C}$ & -6.264862000 & -1.897764000 & -0.996376000 \\
\hline $\mathrm{H}$ & -7.204942000 & -0.438516000 & 0.279587000 \\
\hline $\mathrm{H}$ & -5.036139000 & -3.292354000 & -2.078732000 \\
\hline $\mathrm{H}$ & -7.178771000 & -2.157805000 & -1.521923000 \\
\hline $\mathrm{H}$ & -1.057489000 & -1.081301000 & 3.137462000 \\
\hline $\mathrm{C}$ & -1.527210000 & 2.353872000 & 2.253956000 \\
\hline $\mathrm{H}$ & -2.514780000 & 2.467504000 & 1.807959000 \\
\hline $\mathrm{H}$ & -1.494588000 & 1.439478000 & 2.853239000 \\
\hline $\mathrm{H}$ & -1.322427000 & 3.213393000 & 2.902715000 \\
\hline $\mathrm{C}$ & 5.178819000 & -1.086134000 & -0.626029000 \\
\hline $\mathrm{F}$ & 6.097424000 & -1.580556000 & 0.221686000 \\
\hline $\mathrm{F}$ & 5.516296000 & 0.204584000 & -0.848732000 \\
\hline $\mathrm{F}$ & 5.329271000 & -1.724130000 & -1.801590000 \\
\hline
\end{tabular}




\begin{tabular}{|c|c|c|c|}
\hline \multicolumn{4}{|c|}{ A3 MTBD } \\
\hline & $X$ & $\mathrm{Y}$ & $\mathrm{Z}$ \\
\hline $\mathrm{C}$ & -1.426724000 & -2.971082000 & 0.812052000 \\
\hline $\mathrm{C}$ & -2.270820000 & -2.537978000 & 1.997417000 \\
\hline $\mathrm{C}$ & -3.690587000 & -2.280553000 & 1.522450000 \\
\hline $\mathrm{N}$ & -1.432513000 & -1.916595000 & -0.179625000 \\
\hline $\mathrm{H}$ & -1.799766000 & -3.927417000 & 0.402780000 \\
\hline $\mathrm{H}$ & -0.389538000 & -3.134769000 & 1.120846000 \\
\hline $\mathrm{H}$ & -1.836387000 & -1.626258000 & 2.420349000 \\
\hline $\mathrm{H}$ & -2.276046000 & -3.304296000 & 2.778509000 \\
\hline $\mathrm{H}$ & -4.238659000 & -3.234258000 & 1.455711000 \\
\hline $\mathrm{H}$ & -4.226158000 & -1.655979000 & 2.250763000 \\
\hline $\mathrm{N}$ & -3.732795000 & -1.619764000 & 0.231448000 \\
\hline $\mathrm{C}$ & -5.029963000 & -1.057936000 & -0.109561000 \\
\hline $\mathrm{C}$ & -5.160433000 & -0.874410000 & -1.612157000 \\
\hline $\mathrm{C}$ & -3.933574000 & -0.116837000 & -2.108020000 \\
\hline $\mathrm{N}$ & -2.691939000 & -0.746040000 & -1.715771000 \\
\hline $\mathrm{C}$ & -2.654344000 & -1.396731000 & -0.604730000 \\
\hline $\mathrm{H}$ & -5.165977000 & -0.095955000 & 0.408165000 \\
\hline $\mathrm{H}$ & -5.800236000 & -1.745820000 & 0.262682000 \\
\hline $\mathrm{H}$ & -5.208647000 & -1.855486000 & -2.099386000 \\
\hline $\mathrm{H}$ & -6.085910000 & -0.336455000 & -1.842956000 \\
\hline $\mathrm{H}$ & -3.949572000 & -0.041219000 & -3.201504000 \\
\hline $\mathrm{H}$ & -3.971700000 & 0.915010000 & -1.721963000 \\
\hline $\mathrm{C}$ & 4.431640000 & 1.218901000 & 0.663755000 \\
\hline $\mathrm{C}$ & 3.176663000 & 1.614309000 & 0.220649000 \\
\hline $\mathrm{C}$ & 2.489180000 & 0.865180000 & -0.739496000 \\
\hline $\mathrm{C}$ & 3.101284000 & -0.283268000 & -1.259838000 \\
\hline $\mathrm{C}$ & 4.356636000 & -0.679318000 & -0.821400000 \\
\hline $\mathrm{C}$ & 5.024579000 & 0.071667000 & 0.143175000 \\
\hline $\mathrm{H}$ & 4.954905000 & 1.808907000 & 1.408084000 \\
\hline $\mathrm{H}$ & 2.728558000 & 2.523159000 & 0.609409000 \\
\hline $\mathrm{H}$ & 2.572629000 & -0.890777000 & -1.988918000 \\
\hline $\mathrm{H}$ & 4.812859000 & -1.579349000 & -1.220723000 \\
\hline $\mathrm{C}$ & 1.140437000 & 1.255433000 & -1.208005000 \\
\hline $\mathrm{C}$ & 0.194001000 & 1.772122000 & -0.417786000 \\
\hline $\mathrm{H}$ & 0.434693000 & 1.924994000 & 0.630083000 \\
\hline $\mathrm{O}$ & 0.840886000 & 0.963899000 & -2.514128000 \\
\hline $\mathrm{C}$ & -1.194086000 & 2.109095000 & -0.887621000 \\
\hline $\mathrm{H}$ & -1.495686000 & 1.369647000 & -1.641571000 \\
\hline $\mathrm{C}$ & -2.231974000 & 2.108089000 & 0.211979000 \\
\hline $\mathrm{C}$ & -3.296534000 & 3.012542000 & 0.187625000 \\
\hline
\end{tabular}




\begin{tabular}{lrrr}
$\mathrm{C}$ & -2.182792000 & 1.174163000 & 1.248933000 \\
$\mathrm{C}$ & -4.285259000 & 2.985765000 & 1.167215000 \\
$\mathrm{H}$ & -3.350691000 & 3.748891000 & -0.611076000 \\
$\mathrm{C}$ & -3.162289000 & 1.149899000 & 2.236232000 \\
$\mathrm{H}$ & -1.381195000 & 0.440633000 & 1.261121000 \\
$\mathrm{C}$ & -4.220032000 & 2.054709000 & 2.200434000 \\
$\mathrm{H}$ & -5.102172000 & 3.700791000 & 1.128043000 \\
$\mathrm{H}$ & -3.100725000 & 0.417444000 & 3.037242000 \\
$\mathrm{H}$ & -4.984374000 & 2.037313000 & 2.971798000 \\
$\mathrm{H}$ & 1.658769000 & 0.924603000 & -3.024057000 \\
$\mathrm{H}$ & -1.193697000 & 3.087670000 & -1.387376000 \\
$\mathrm{C}$ & -0.348110000 & -1.949594000 & -1.139710000 \\
$\mathrm{H}$ & -0.395478000 & -2.840344000 & -1.786199000 \\
$\mathrm{H}$ & -0.396052000 & -1.065290000 & -1.772141000 \\
$\mathrm{H}$ & 0.602506000 & -1.959288000 & -0.594644000 \\
$\mathrm{C}$ & 6.408210000 & -0.336409000 & 0.566605000 \\
$\mathrm{~F}$ & 7.328619000 & 0.019764000 & -0.348478000 \\
$\mathrm{~F}$ & 6.768983000 & 0.228593000 & 1.730075000 \\
$\mathrm{~F}$ & 6.507592000 & -1.669142000 & 0.7168950000 \\
\hline- - - - - - &
\end{tabular}

(5) $\mathrm{R}_{1}=\mathrm{NO}_{2}, \mathrm{R}_{2}=\mathrm{H}$

\begin{tabular}{|c|c|c|c|}
\hline \multicolumn{4}{|c|}{ Coordinates (Angstroms) } \\
\hline & $X$ & Y & $\mathrm{Z}$ \\
\hline$\overline{\mathrm{C}}$ & -2.241374000 & -1.987702000 & -0.558355000 \\
\hline $\mathrm{C}$ & -1.092128000 & -1.211089000 & -0.484314000 \\
\hline $\mathrm{C}$ & -1.129767000 & 0.057794000 & 0.100758000 \\
\hline $\mathrm{C}$ & -2.328283000 & 0.551954000 & 0.628546000 \\
\hline $\mathrm{C}$ & -3.482857000 & -0.218616000 & 0.563024000 \\
\hline $\mathrm{C}$ & -3.420062000 & -1.473021000 & -0.033070000 \\
\hline $\mathrm{H}$ & -2.237502000 & -2.973906000 & -1.005663000 \\
\hline $\mathrm{H}$ & -0.154565000 & -1.601750000 & -0.873343000 \\
\hline $\mathrm{H}$ & -2.297184000 & 1.551325000 & 1.089882000 \\
\hline $\mathrm{H}$ & -4.423387000 & 0.134368000 & 0.968889000 \\
\hline $\mathrm{C}$ & 0.098406000 & 0.954320000 & 0.124135000 \\
\hline $\mathrm{C}$ & 1.367657000 & 0.205304000 & 0.400466000 \\
\hline $\mathrm{H}$ & 1.323882000 & -0.438178000 & 1.278129000 \\
\hline $\mathrm{O}$ & 0.164211000 & 1.698820000 & -1.110858000 \\
\hline $\mathrm{C}$ & 2.484647000 & 0.290381000 & -0.326835000 \\
\hline $\mathrm{H}$ & 2.478271000 & 0.943175000 & -1.198903000 \\
\hline $\mathrm{C}$ & 3.755807000 & -0.410796000 & -0.075871000 \\
\hline $\mathrm{C}$ & 4.730947000 & -0.425403000 & -1.080186000 \\
\hline
\end{tabular}




$\begin{array}{lrrr}\mathrm{C} & 4.042396000 & -1.062972000 & 1.131024000 \\ \mathrm{C} & 5.944814000 & -1.078817000 & -0.896632000 \\ \mathrm{H} & 4.530978000 & 0.081613000 & -2.021378000 \\ \mathrm{C} & 5.253516000 & -1.716722000 & 1.316697000 \\ \mathrm{H} & 3.318559000 & -1.046481000 & 1.940316000 \\ \mathrm{C} & 6.210404000 & -1.729767000 & 0.303773000 \\ \mathrm{H} & 6.684675000 & -1.077189000 & -1.691639000 \\ \mathrm{H} & 5.455145000 & -2.212675000 & 2.261668000 \\ \mathrm{H} & 7.158436000 & -2.237494000 & 0.453459000 \\ \mathrm{H} & -0.090449000 & 1.733946000 & 0.892521000 \\ \mathrm{H} & 0.207680000 & 1.055229000 & -1.830894000 \\ \mathrm{O} & -1.247816000 & 3.201972000 & 1.581802000 \\ \mathrm{H} & -1.289604000 & 3.037867000 & 2.527634000 \\ \mathrm{~K} & -0.994346000 & 4.055963000 & -0.541709000 \\ \mathrm{~N} & -4.638926000 & -2.288284000 & -0.106935000 \\ \mathrm{O} & -5.668432000 & -1.817194000 & 0.352365000 \\ \mathrm{O} & -4.556041000 & -3.391628000 & -0.626888000 \\ ---- \text { - } & & & \end{array}$

TSA1-2 кон

\begin{tabular}{lrcc} 
& \multicolumn{3}{c}{ Coordinates (Angstroms) } \\
\hline $\mathrm{C}$ & -3.172306000 & -1.144311000 & 0.868964000 \\
$\mathrm{C}$ & -1.884345000 & -0.639985000 & 0.830302000 \\
$\mathrm{C}$ & -1.505743000 & 0.327343000 & -0.116953000 \\
$\mathrm{C}$ & -2.481466000 & 0.788470000 & -1.011974000 \\
$\mathrm{C}$ & -3.775873000 & 0.290546000 & -0.987512000 \\
$\mathrm{C}$ & -4.107700000 & -0.676375000 & -0.048609000 \\
$\mathrm{H}$ & -3.464676000 & -1.890677000 & 1.597219000 \\
$\mathrm{H}$ & -1.166764000 & -0.997030000 & 1.561765000 \\
$\mathrm{H}$ & -2.222407000 & 1.561730000 & -1.725827000 \\
$\mathrm{H}$ & -4.528350000 & 0.642734000 & -1.682464000 \\
$\mathrm{C}$ & -0.132948000 & 0.915430000 & -0.116780000 \\
$\mathrm{C}$ & 0.993090000 & -0.027760000 & 0.073126000 \\
$\mathrm{H}$ & 0.878467000 & -0.735366000 & 0.891283000 \\
$\mathrm{O}$ & 0.098568000 & 1.793755000 & -1.225964000 \\
$\mathrm{C}$ & 2.156679000 & -0.010702000 & -0.602335000 \\
$\mathrm{H}$ & 2.253538000 & 0.649968000 & -1.465377000 \\
$\mathrm{C}$ & 3.334502000 & -0.849442000 & -0.323290000 \\
$\mathrm{C}$ & 4.301807000 & -1.032870000 & -1.320549000 \\
$\mathrm{C}$ & 3.547010000 & -1.464006000 & 0.919122000 \\
$\mathrm{C}$ & 5.427611000 & -1.817598000 & -1.096769000 \\
$\mathrm{H}$ & 4.158798000 & -0.562350000 & -2.291105000 \\
$\mathrm{C}$ & 4.668605000 & -2.252922000 & 1.142424000
\end{tabular}




$\begin{array}{lrrr}\mathrm{H} & 2.835222000 & -1.310578000 & 1.725423000 \\ \mathrm{C} & 5.614886000 & -2.435241000 & 0.136370000 \\ \mathrm{H} & 6.159843000 & -1.947907000 & -1.888482000 \\ \mathrm{H} & 4.810431000 & -2.720158000 & 2.112662000 \\ \mathrm{H} & 6.493954000 & -3.046778000 & 0.315406000 \\ \mathrm{H} & -0.062089000 & 1.686707000 & 0.876415000 \\ \mathrm{H} & 0.116227000 & 1.259843000 & -2.034384000 \\ \mathrm{O} & 0.296773000 & 2.657429000 & 1.832157000 \\ \mathrm{H} & -0.449977000 & 2.729366000 & 2.432453000 \\ \mathrm{~K} & 1.967697000 & 3.175810000 & 0.254246000 \\ \mathrm{~N} & -5.466921000 & -1.206903000 & -0.016440000 \\ \mathrm{O} & -6.269578000 & -0.775015000 & -0.832838000 \\ \mathrm{O} & -5.729874000 & -2.055549000 & 0.824919000\end{array}$

\begin{tabular}{|c|c|c|c|}
\hline \multicolumn{4}{|c|}{ А2 кон } \\
\hline & \multicolumn{3}{|c|}{ Coordinates (Angstroms) } \\
\hline & $\mathrm{X}$ & $\mathrm{Y}$ & Z \\
\hline$\overline{\mathrm{C}}$ & -2.891441000 & -1.553105000 & 0.285445000 \\
\hline $\mathrm{C}$ & -1.604691000 & -1.215037000 & -0.055847000 \\
\hline $\mathrm{C}$ & -1.307008000 & -0.021092000 & -0.790241000 \\
\hline $\mathrm{C}$ & -2.433779000 & 0.783538000 & -1.162430000 \\
\hline $\mathrm{C}$ & -3.723145000 & 0.441172000 & -0.809369000 \\
\hline $\mathrm{C}$ & -3.961219000 & -0.720335000 & -0.073636000 \\
\hline $\mathrm{H}$ & -3.097543000 & -2.459562000 & 0.842591000 \\
\hline $\mathrm{H}$ & -0.800460000 & -1.876520000 & 0.249007000 \\
\hline $\mathrm{H}$ & -2.264284000 & 1.663998000 & -1.773179000 \\
\hline $\mathrm{H}$ & -4.563561000 & 1.058709000 & -1.105629000 \\
\hline $\mathrm{C}$ & 0.007854000 & 0.426056000 & -1.048597000 \\
\hline $\mathrm{C}$ & 1.210512000 & -0.202833000 & -0.679255000 \\
\hline $\mathrm{H}$ & 1.117097000 & -1.223839000 & -0.316202000 \\
\hline $\mathrm{O}$ & 0.102268000 & 1.755452000 & -1.523218000 \\
\hline $\mathrm{C}$ & 2.465415000 & 0.357709000 & -0.715028000 \\
\hline $\mathrm{H}$ & 2.566712000 & 1.381295000 & -1.074926000 \\
\hline $\mathrm{C}$ & 3.719748000 & -0.305975000 & -0.363115000 \\
\hline $\mathrm{C}$ & 4.896220000 & 0.460090000 & -0.271150000 \\
\hline $\mathrm{C}$ & 3.828008000 & -1.681690000 & -0.090089000 \\
\hline $\mathrm{C}$ & 6.111613000 & -0.110767000 & 0.083542000 \\
\hline $\mathrm{H}$ & 4.846554000 & 1.526196000 & -0.484240000 \\
\hline $\mathrm{C}$ & 5.042718000 & -2.250763000 & 0.270114000 \\
\hline $\mathrm{H}$ & 2.955321000 & -2.322800000 & -0.177664000 \\
\hline $\mathrm{C}$ & 6.195007000 & -1.472691000 & 0.361851000 \\
\hline $\mathrm{H}$ & 7.000159000 & 0.512314000 & 0.141124000 \\
\hline $\mathrm{H}$ & 5.092470000 & -3.317771000 & 0.469898000 \\
\hline
\end{tabular}




$\begin{array}{lrrr}\mathrm{H} & 7.143584000 & -1.923336000 & 0.636958000 \\ \mathrm{H} & 0.654258000 & 1.760035000 & -2.315776000 \\ \mathrm{O} & 1.612752000 & 1.664685000 & 1.893227000 \\ \mathrm{H} & 2.244660000 & 1.537665000 & 2.608293000 \\ \mathrm{~K} & -0.702773000 & 2.319127000 & 0.975849000 \\ \mathrm{H} & 1.969157000 & 1.140274000 & 1.141401000 \\ \mathrm{~N} & -5.299903000 & -1.057985000 & 0.321340000 \\ \mathrm{O} & -5.475517000 & -2.101402000 & 0.947396000 \\ \mathrm{O} & -6.207878000 & -0.283713000 & 0.021927000\end{array}$

TSA2-3 кон

\begin{tabular}{lccc} 
& \multicolumn{3}{c}{ Coordinates (Angstroms) } \\
& $\mathrm{X}$ & $\mathrm{Y}$ & $\mathrm{Z}$ \\
\hline $\mathrm{C}$ & 3.071656000 & -1.183045000 & -0.875140000 \\
$\mathrm{C}$ & 1.764556000 & -0.834967000 & -0.586948000 \\
$\mathrm{C}$ & 1.453111000 & -0.018072000 & 0.519152000 \\
$\mathrm{C}$ & 2.517333000 & 0.460331000 & 1.306792000 \\
$\mathrm{C}$ & 3.830830000 & 0.123965000 & 1.020546000 \\
$\mathrm{C}$ & 4.096158000 & -0.696830000 & -0.068147000 \\
$\mathrm{H}$ & 3.312040000 & -1.813709000 & -1.722279000 \\
$\mathrm{H}$ & 0.974475000 & -1.194532000 & -1.237788000 \\
$\mathrm{H}$ & 2.297238000 & 1.089783000 & 2.160729000 \\
$\mathrm{H}$ & 4.649680000 & 0.483429000 & 1.631799000 \\
$\mathrm{C}$ & 0.080053000 & 0.373974000 & 0.830225000 \\
$\mathrm{C}$ & -1.055659000 & -0.154937000 & 0.318870000 \\
$\mathrm{H}$ & -0.946066000 & -1.012053000 & -0.341108000 \\
$\mathrm{O}$ & 0.016336000 & 1.484081000 & 1.685157000 \\
$\mathrm{C}$ & -2.395419000 & 0.399530000 & 0.455809000 \\
$\mathrm{C}$ & -3.565106000 & -0.510266000 & 0.240630000 \\
$\mathrm{C}$ & -3.493458000 & -1.903842000 & 0.333763000 \\
$\mathrm{C}$ & -4.805132000 & 0.061509000 & -0.076337000 \\
$\mathrm{C}$ & -4.615196000 & -2.698391000 & 0.106257000 \\
$\mathrm{H}$ & -2.553138000 & -2.377420000 & 0.605483000 \\
$\mathrm{C}$ & -5.929374000 & -0.726277000 & -0.289350000 \\
$\mathrm{H}$ & -4.870285000 & 1.143489000 & -0.167809000 \\
$\mathrm{C}$ & -5.838823000 & -2.114612000 & -0.203202000 \\
$\mathrm{H}$ & -4.531704000 & -3.778907000 & 0.186351000 \\
$\mathrm{H}$ & -6.878896000 & -0.256836000 & -0.531906000 \\
$\mathrm{H}$ & -6.714890000 & -2.733232000 & -0.374545000 \\
$\mathrm{H}$ & -0.790203000 & 1.400327000 & 2.212381000 \\
$\mathrm{H}$ & -2.437292000 & 1.328355000 & -0.442638000 \\
$\mathrm{O}$ & -2.357223000 & 2.395121000 & -1.281327000 \\
$\mathrm{~K}$ & -0.130878000 & 2.957814000 & -0.642798000
\end{tabular}




$\begin{array}{lrrr}\mathrm{H} & -3.101885000 & 2.294763000 & -1.881228000 \\ \mathrm{H} & -2.544319000 & 0.996863000 & 1.367149000 \\ \mathrm{~N} & 5.478763000 & -1.047507000 & -0.379353000 \\ \mathrm{O} & 5.679683000 & -1.777451000 & -1.340108000 \\ \mathrm{O} & \mathbf{6 . 3 5 8 3 8 3 0 0 0} & -0.589833000 & 0.337094000\end{array}$

$\mathbf{A 3}_{\text {кон }}$

\begin{tabular}{|c|c|c|c|}
\hline \multicolumn{4}{|c|}{ Coordinates (Angstroms) } \\
\hline & $\mathrm{X}$ & Y & Z \\
\hline $\mathrm{C}$ & -3.723274000 & 1.269423000 & 0.302639000 \\
\hline $\mathrm{C}$ & -2.451096000 & 1.781071000 & 0.087684000 \\
\hline $\mathrm{C}$ & -1.388231000 & 0.946125000 & -0.289564000 \\
\hline $\mathrm{C}$ & -1.627663000 & -0.426093000 & -0.482154000 \\
\hline $\mathrm{C}$ & -2.899220000 & -0.939121000 & -0.270434000 \\
\hline $\mathrm{C}$ & -3.926654000 & -0.091288000 & 0.129984000 \\
\hline $\mathrm{H}$ & -4.549633000 & 1.907235000 & 0.590572000 \\
\hline $\mathrm{H}$ & -2.305298000 & 2.853841000 & 0.177230000 \\
\hline $\mathrm{H}$ & -0.820157000 & -1.105971000 & -0.825283000 \\
\hline $\mathrm{H}$ & -3.099436000 & -1.994564000 & -0.411807000 \\
\hline $\mathrm{C}$ & -0.030660000 & 1.520558000 & -0.470685000 \\
\hline $\mathrm{C}$ & 0.938307000 & 0.961231000 & -1.207399000 \\
\hline $\mathrm{H}$ & 0.770418000 & -0.024281000 & -1.661044000 \\
\hline $\mathrm{O}$ & 0.203120000 & 2.736304000 & 0.137609000 \\
\hline $\mathrm{C}$ & 2.318338000 & 1.559329000 & -1.339112000 \\
\hline $\mathrm{H}$ & 2.301868000 & 2.625456000 & -1.095750000 \\
\hline $\mathrm{C}$ & 3.281325000 & 0.839328000 & -0.415945000 \\
\hline $\mathrm{C}$ & 3.198131000 & 1.042643000 & 0.968686000 \\
\hline $\mathrm{C}$ & 4.195639000 & -0.101394000 & -0.896516000 \\
\hline $\mathrm{C}$ & 3.999546000 & 0.318557000 & 1.847363000 \\
\hline $\mathrm{H}$ & 2.485798000 & 1.772013000 & 1.346158000 \\
\hline $\mathrm{C}$ & 4.999489000 & -0.832421000 & -0.019562000 \\
\hline $\mathrm{H}$ & 4.266256000 & -0.277823000 & -1.966279000 \\
\hline $\mathrm{C}$ & 4.901309000 & -0.629203000 & 1.355870000 \\
\hline $\mathrm{H}$ & 3.927275000 & 0.496775000 & 2.916748000 \\
\hline $\mathrm{H}$ & 5.703866000 & -1.559512000 & -0.413445000 \\
\hline $\mathrm{H}$ & 5.531941000 & -1.191257000 & 2.038334000 \\
\hline $\mathrm{O}$ & 0.636990000 & -2.101167000 & -1.509589000 \\
\hline $\mathrm{H}$ & 2.663228000 & 1.459072000 & -2.373791000 \\
\hline $\mathrm{H}$ & -0.392770000 & 2.825461000 & 0.890668000 \\
\hline $\mathrm{H}$ & 0.220796000 & -2.373411000 & -2.331990000 \\
\hline K & 1.854205000 & -1.873128000 & 0.436381000 \\
\hline $\mathrm{N}$ & -5.263052000 & -0.643012000 & 0.368724000 \\
\hline $\mathrm{O}$ & -5.419709000 & -1.845071000 & 0.215016000 \\
\hline
\end{tabular}




\begin{tabular}{|c|c|c|c|}
\hline \multicolumn{4}{|c|}{$\mathrm{A1}_{\mathrm{NaOH}}$} \\
\hline & \multicolumn{3}{|c|}{ Coordinates (Angstroms) } \\
\hline & $\mathrm{X}$ & Y & Z \\
\hline$\overline{\mathrm{C}}$ & -2.319962000 & -1.715478000 & -0.510656000 \\
\hline $\mathrm{C}$ & -1.156520000 & -0.959240000 & -0.452159000 \\
\hline $\mathrm{C}$ & -1.175692000 & 0.329889000 & 0.087777000 \\
\hline $\mathrm{C}$ & -2.368537000 & 0.866771000 & 0.585377000 \\
\hline $\mathrm{C}$ & -3.537160000 & 0.115913000 & 0.534930000 \\
\hline $\mathrm{C}$ & -3.492545000 & -1.159914000 & -0.014676000 \\
\hline $\mathrm{H}$ & -2.331503000 & -2.716290000 & -0.924049000 \\
\hline $\mathrm{H}$ & -0.223006000 & -1.380940000 & -0.817397000 \\
\hline $\mathrm{H}$ & -2.330508000 & 1.883863000 & 1.001212000 \\
\hline $\mathrm{H}$ & -4.474467000 & 0.501827000 & 0.917338000 \\
\hline $\mathrm{C}$ & 0.071878000 & 1.197718000 & 0.094242000 \\
\hline $\mathrm{C}$ & 1.324722000 & 0.428686000 & 0.391246000 \\
\hline $\mathrm{H}$ & 1.267823000 & -0.172583000 & 1.297236000 \\
\hline $\mathrm{O}$ & 0.159109000 & 1.911236000 & -1.163802000 \\
\hline $\mathrm{C}$ & 2.436658000 & 0.448210000 & -0.347650000 \\
\hline $\mathrm{H}$ & 2.443614000 & 1.060685000 & -1.248385000 \\
\hline $\mathrm{C}$ & 3.687278000 & -0.281632000 & -0.074477000 \\
\hline $\mathrm{C}$ & 4.660728000 & -0.355609000 & -1.077630000 \\
\hline $\mathrm{C}$ & 3.954114000 & -0.905328000 & 1.151737000 \\
\hline $\mathrm{C}$ & 5.854880000 & -1.038717000 & -0.873556000 \\
\hline $\mathrm{H}$ & 4.476109000 & 0.129405000 & -2.033379000 \\
\hline $\mathrm{C}$ & 5.145325000 & -1.588509000 & 1.357782000 \\
\hline $\mathrm{H}$ & 3.231526000 & -0.844648000 & 1.959972000 \\
\hline $\mathrm{C}$ & 6.101057000 & -1.660515000 & 0.346185000 \\
\hline $\mathrm{H}$ & 6.594437000 & -1.082437000 & -1.667573000 \\
\hline $\mathrm{H}$ & 5.332236000 & -2.062378000 & 2.316924000 \\
\hline $\mathrm{H}$ & 7.033916000 & -2.190831000 & 0.511807000 \\
\hline $\mathrm{H}$ & -0.094096000 & 1.999670000 & 0.836929000 \\
\hline $\mathrm{H}$ & 0.138153000 & 1.255470000 & -1.873197000 \\
\hline $\mathrm{O}$ & -1.290903000 & 3.594452000 & 1.214662000 \\
\hline $\mathrm{H}$ & -1.421561000 & 4.003856000 & 2.072166000 \\
\hline $\mathrm{N}$ & -4.726519000 & -1.955101000 & -0.070838000 \\
\hline $\mathrm{O}$ & -5.747138000 & -1.454498000 & 0.375875000 \\
\hline $\mathrm{O}$ & -4.661957000 & -3.071631000 & -0.564077000 \\
\hline $\mathrm{Na}$ & -0.849752000 & 3.921009000 & -0.722349000 \\
\hline
\end{tabular}

TSA1-2 ${ }_{\mathrm{NaOH}}$ 


\begin{tabular}{|c|c|c|c|}
\hline \multicolumn{4}{|c|}{ Coordinates (Angstroms) } \\
\hline & $X$ & $\mathrm{Y}$ & $\mathrm{Z}$ \\
\hline $\mathrm{C}$ & -2.999357000 & -1.343326000 & 0.578023000 \\
\hline $\mathrm{C}$ & -1.716005000 & -0.824275000 & 0.577326000 \\
\hline $\mathrm{C}$ & -1.422452000 & 0.398721000 & -0.051431000 \\
\hline $\mathrm{C}$ & -2.476288000 & 1.085189000 & -0.677540000 \\
\hline $\mathrm{C}$ & -3.763894000 & 0.573969000 & -0.689488000 \\
\hline $\mathrm{C}$ & -4.013898000 & -0.639501000 & -0.060957000 \\
\hline $\mathrm{H}$ & -3.223988000 & -2.288092000 & 1.057665000 \\
\hline $\mathrm{H}$ & -0.933733000 & -1.389841000 & 1.071910000 \\
\hline $\mathrm{H}$ & -2.275630000 & 2.040562000 & -1.148336000 \\
\hline $\mathrm{H}$ & -4.576298000 & 1.102272000 & -1.173488000 \\
\hline $\mathrm{C}$ & -0.069745000 & 1.010231000 & -0.005642000 \\
\hline $\mathrm{C}$ & 1.107530000 & 0.134846000 & 0.116860000 \\
\hline $\mathrm{H}$ & 1.046911000 & -0.624897000 & 0.893875000 \\
\hline $\mathrm{O}$ & 0.124063000 & 2.008323000 & -1.027939000 \\
\hline $\mathrm{C}$ & 2.272537000 & 0.273498000 & -0.547240000 \\
\hline $\mathrm{H}$ & 2.320134000 & 0.977476000 & -1.380595000 \\
\hline $\mathrm{C}$ & 3.502747000 & -0.498332000 & -0.307182000 \\
\hline $\mathrm{C}$ & 4.469823000 & -0.588354000 & -1.317386000 \\
\hline $\mathrm{C}$ & 3.762421000 & -1.144751000 & 0.909810000 \\
\hline $\mathrm{C}$ & 5.642328000 & -1.311599000 & -1.130060000 \\
\hline $\mathrm{H}$ & 4.290698000 & -0.093686000 & -2.269685000 \\
\hline $\mathrm{C}$ & 4.931064000 & -1.872649000 & 1.096376000 \\
\hline $\mathrm{H}$ & 3.050221000 & -1.063483000 & 1.726270000 \\
\hline $\mathrm{C}$ & 5.877410000 & -1.961293000 & 0.078171000 \\
\hline $\mathrm{H}$ & 6.373708000 & -1.369004000 & -1.930952000 \\
\hline $\mathrm{H}$ & 5.108915000 & -2.364557000 & 2.048278000 \\
\hline $\mathrm{H}$ & 6.793731000 & -2.523679000 & 0.228891000 \\
\hline $\mathrm{H}$ & 0.005517000 & 1.790617000 & 1.051387000 \\
\hline $\mathrm{H}$ & 0.117142000 & 1.571114000 & -1.892003000 \\
\hline $\mathrm{O}$ & 0.361751000 & 2.809486000 & 1.824950000 \\
\hline $\mathrm{H}$ & 0.365610000 & 2.492381000 & 2.732608000 \\
\hline $\mathrm{N}$ & -5.365888000 & -1.187109000 & -0.072614000 \\
\hline $\mathrm{O}$ & -6.237032000 & -0.548933000 & -0.648152000 \\
\hline $\mathrm{O}$ & -5.555463000 & -2.255606000 & 0.493151000 \\
\hline $\mathrm{Na}$ & 1.720582000 & 3.108381000 & 0.267144000 \\
\hline
\end{tabular}

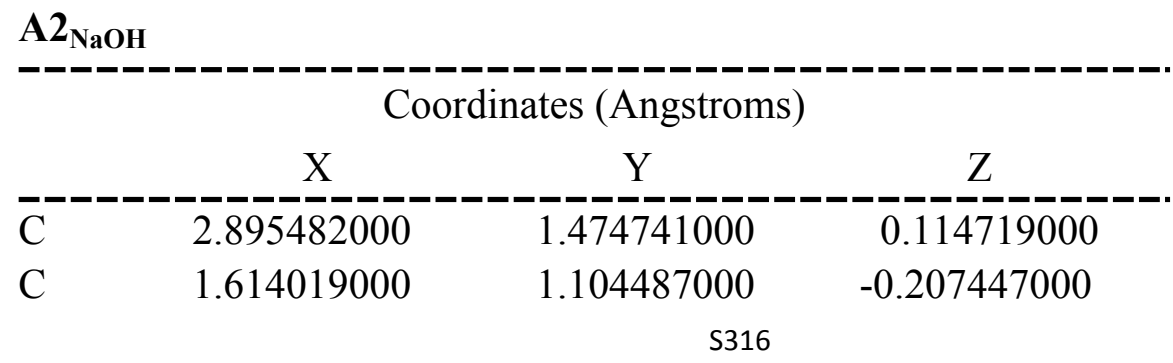




$\begin{array}{lrrr}\mathrm{C} & 1.314066000 & -0.194837000 & -0.733649000 \\ \mathrm{C} & 2.434107000 & -1.073124000 & -0.917412000 \\ \mathrm{C} & 3.720432000 & -0.693705000 & -0.581529000 \\ \mathrm{C} & 3.957850000 & 0.573069000 & -0.054552000 \\ \mathrm{H} & 3.103841000 & 2.460171000 & 0.514499000 \\ \mathrm{H} & 0.815203000 & 1.822745000 & -0.055957000 \\ \mathrm{H} & 2.271424000 & -2.036302000 & -1.390454000 \\ \mathrm{H} & 4.556013000 & -1.366887000 & -0.735937000 \\ \mathrm{C} & -0.001428000 & -0.656367000 & -0.957795000 \\ \mathrm{C} & -1.205013000 & 0.009238000 & -0.656775000 \\ \mathrm{H} & -1.120417000 & 1.079195000 & -0.480620000 \\ \mathrm{O} & -0.090801000 & -2.054990000 & -1.188777000 \\ \mathrm{C} & -2.447534000 & -0.564474000 & -0.540546000 \\ \mathrm{H} & -2.550713000 & -1.637108000 & -0.707009000 \\ \mathrm{C} & -3.700710000 & 0.144930000 & -0.277883000 \\ \mathrm{C} & -4.843320000 & -0.591427000 & 0.080165000 \\ \mathrm{C} & -3.834567000 & 1.541559000 & -0.360839000 \\ \mathrm{C} & -6.052658000 & 0.032792000 & 0.356540000 \\ \mathrm{H} & -4.771886000 & -1.675311000 & 0.145560000 \\ \mathrm{C} & -5.042837000 & 2.166247000 & -0.078015000 \\ \mathrm{H} & -2.990250000 & 2.149373000 & -0.674197000 \\ \mathrm{C} & -6.161387000 & 1.419310000 & 0.284434000 \\ \mathrm{H} & -6.915994000 & -0.567588000 & 0.630416000 \\ \mathrm{H} & -5.115373000 & 3.247544000 & -0.156698000 \\ \mathrm{H} & -7.106155000 & 1.910082000 & 0.496854000 \\ \mathrm{H} & -0.728187000 & -2.214424000 & -1.895454000 \\ \mathrm{O} & -1.182674000 & -1.626913000 & 2.055100000 \\ \mathrm{H} & -1.349878000 & -1.092538000 & 2.839137000 \\ \mathrm{H} & -1.689650000 & -1.189156000 & 1.332285000 \\ \mathrm{~N} & 5.292803000 & 0.955955000 & 0.318301000 \\ \mathrm{O} & 5.468441000 & 2.088594000 & 0.761050000 \\ \mathrm{O} & 6.193258000 & 0.129976000 & 0.182248000 \\ \mathrm{Na} & 0.687053000 & -2.184495000 & 0.965092000 \\ ------ & \end{array}$

TSA2-3 ${ }_{\mathrm{NaOH}}$

$\begin{array}{lrrr} & & & \text { Coordinates (Angstroms) } \\ & & \mathrm{X} & \mathrm{Z} \\ \mathrm{C} & -2.991749000 & -1.438309000 & 0.218178000 \\ \mathrm{C} & -1.695025000 & -1.006114000 & 0.017967000 \\ \mathrm{C} & -1.423993000 & 0.282803000 & -0.487416000 \\ \mathrm{C} & -2.518486000 & 1.118550000 & -0.783400000 \\ \mathrm{C} & -3.823226000 & 0.693176000 & -0.581173000 \\ \mathrm{C} & -4.048214000 & -0.580428000 & -0.078689000\end{array}$




\begin{tabular}{lrrr}
$\mathrm{H}$ & -3.199687000 & -2.427776000 & 0.606391000 \\
$\mathrm{H}$ & -0.882273000 & -1.682553000 & 0.258287000 \\
$\mathrm{H}$ & -2.336387000 & 2.102129000 & -1.199862000 \\
$\mathrm{H}$ & -4.665401000 & 1.333239000 & -0.814556000 \\
$\mathrm{C}$ & -0.062145000 & 0.778577000 & -0.661243000 \\
$\mathrm{C}$ & 1.097817000 & 0.111192000 & -0.449065000 \\
$\mathrm{H}$ & 1.018655000 & -0.936508000 & -0.169075000 \\
$\mathrm{O}$ & -0.029092000 & 2.151234000 & -0.968585000 \\
$\mathrm{C}$ & 2.430243000 & 0.698389000 & -0.484436000 \\
$\mathrm{H}$ & 2.596916000 & 1.370645000 & -1.338225000 \\
$\mathrm{C}$ & 3.573592000 & -0.233660000 & -0.297749000 \\
$\mathrm{C}$ & 4.711416000 & -0.175613000 & -1.112432000 \\
$\mathrm{C}$ & 3.571241000 & -1.170620000 & 0.746141000 \\
$\mathrm{C}$ & 5.791791000 & -1.026972000 & -0.905765000 \\
$\mathrm{H}$ & 4.743474000 & 0.545940000 & -1.925706000 \\
$\mathrm{C}$ & 4.643746000 & -2.032382000 & 0.946924000 \\
$\mathrm{H}$ & 2.720127000 & -1.212678000 & 1.423248000 \\
$\mathrm{C}$ & 5.762670000 & -1.966969000 & 0.120938000 \\
$\mathrm{H}$ & 6.658167000 & -0.961467000 & -1.558496000 \\
$\mathrm{H}$ & 4.609989000 & -2.752721000 & 1.759916000 \\
$\mathrm{H}$ & 6.602587000 & -2.636975000 & 0.278163000 \\
$\mathrm{H}$ & 0.845027000 & 2.354378000 & -1.329088000 \\
$\mathrm{O}$ & 2.212858000 & 2.384318000 & 1.514678000 \\
$\mathrm{H}$ & 2.936282000 & 2.269867000 & 2.137434000 \\
$\mathrm{H}$ & 2.417319000 & 1.558857000 & 0.570853000 \\
$\mathrm{~N}$ & -5.418764000 & -1.031213000 & 0.143131000 \\
$\mathrm{O}$ & -5.582745000 & -2.160530000 & 0.583348000 \\
$\mathrm{O}$ & -6.324806000 & -0.252871000 & -0.120562000 \\
$\mathrm{Na}$ & 0.139887000 & 2.455197000 & 1.361371000 \\
\hline---- & & \\
& & & \\
$\mathrm{H}$ & & &
\end{tabular}

\begin{tabular}{|c|c|c|c|}
\hline \multicolumn{4}{|c|}{$\mathrm{A} 3_{\mathrm{NaOH}}$} \\
\hline & $X$ & Y & Z \\
\hline$\overline{\mathrm{C}}$ & -3.650277000 & 1.226493000 & 0.218578000 \\
\hline $\mathrm{C}$ & -2.364373000 & 1.712385000 & 0.029932000 \\
\hline $\mathrm{C}$ & -1.298202000 & 0.844695000 & -0.251493000 \\
\hline $\mathrm{C}$ & -1.539730000 & -0.534334000 & -0.364123000 \\
\hline $\mathrm{C}$ & -2.826114000 & -1.021809000 & -0.178895000 \\
\hline $\mathrm{C}$ & -3.860068000 & -0.140913000 & 0.117975000 \\
\hline $\mathrm{H}$ & -4.481270000 & 1.887384000 & 0.430805000 \\
\hline $\mathrm{H}$ & -2.206932000 & 2.787062000 & 0.062238000 \\
\hline $\mathrm{H}$ & -0.719426000 & -1.236400000 & -0.608513000 \\
\hline $\mathrm{H}$ & -3.032837000 & -2.082306000 & -0.258482000 \\
\hline
\end{tabular}




$\begin{array}{lrrr}\mathrm{C} & 0.068046000 & 1.399431000 & -0.424430000 \\ \mathrm{C} & 1.027275000 & 0.834739000 & -1.169393000 \\ \mathrm{H} & 0.838269000 & -0.137703000 & -1.628932000 \\ \mathrm{O} & 0.318633000 & 2.606042000 & 0.190243000 \\ \mathrm{C} & 2.410913000 & 1.422498000 & -1.313622000 \\ \mathrm{H} & 2.403751000 & 2.486703000 & -1.061392000 \\ \mathrm{C} & 3.375969000 & 0.686547000 & -0.406478000 \\ \mathrm{C} & 3.291114000 & 0.861401000 & 0.983121000 \\ \mathrm{C} & 4.279542000 & -0.256206000 & -0.904714000 \\ \mathrm{C} & 4.074638000 & 0.101186000 & 1.848364000 \\ \mathrm{H} & 2.587055000 & 1.591338000 & 1.374377000 \\ \mathrm{C} & 5.065830000 & -1.022159000 & -0.040947000 \\ \mathrm{H} & 4.349730000 & -0.412389000 & -1.977418000 \\ \mathrm{C} & 4.961264000 & -0.851926000 & 1.338541000 \\ \mathrm{H} & 3.998496000 & 0.254154000 & 2.921080000 \\ \mathrm{H} & 5.756654000 & -1.754390000 & -0.448060000 \\ \mathrm{H} & 5.574316000 & -1.444436000 & 2.010691000 \\ \mathrm{O} & 0.801271000 & -2.248909000 & -1.095418000 \\ \mathrm{H} & 2.743916000 & 1.328942000 & -2.352703000 \\ \mathrm{H} & -0.310736000 & 2.722126000 & 0.911869000 \\ \mathrm{H} & 0.481898000 & -3.033668000 & -1.547118000 \\ \mathrm{~N} & -5.213553000 & -0.665213000 & 0.324736000 \\ \mathrm{O} & -5.378701000 & -1.871878000 & 0.228310000 \\ \mathrm{O} & -6.100958000 & 0.135449000 & 0.584027000 \\ \mathrm{Na} & 2.117735000 & -1.712520000 & 0.340601000 \\ ------ & & \\ & & & -1.000 \\ & & & \end{array}$

\begin{tabular}{|c|c|c|c|}
\hline \multicolumn{4}{|c|}{ A1 $1_{\text {LiOH }}$} \\
\hline & \multicolumn{3}{|c|}{ Coordinates (Angstroms) } \\
\hline & $X$ & $\mathrm{Y}$ & Z \\
\hline $\mathrm{C}$ & -2.349931000 & -1.526830000 & -0.352632000 \\
\hline $\mathrm{C}$ & -1.178214000 & -0.782301000 & -0.295692000 \\
\hline $\mathrm{C}$ & -1.210837000 & 0.558575000 & 0.095553000 \\
\hline $\mathrm{C}$ & -2.426794000 & 1.163427000 & 0.435093000 \\
\hline $\mathrm{C}$ & -3.603617000 & 0.427136000 & 0.380369000 \\
\hline $\mathrm{C}$ & -3.543465000 & -0.904778000 & -0.012375000 \\
\hline $\mathrm{H}$ & -2.350860000 & -2.568488000 & -0.648513000 \\
\hline $\mathrm{H}$ & -0.230734000 & -1.252111000 & -0.546100000 \\
\hline $\mathrm{H}$ & -2.411025000 & 2.220736000 & 0.723798000 \\
\hline $\mathrm{H}$ & -4.558835000 & 0.867844000 & 0.638204000 \\
\hline $\mathrm{C}$ & 0.048106000 & 1.408961000 & 0.101212000 \\
\hline $\mathrm{C}$ & 1.296655000 & 0.636094000 & 0.410034000 \\
\hline $\mathrm{H}$ & 1.246944000 & 0.078742000 & 1.343786000 \\
\hline $\mathrm{O}$ & 0.147870000 & 2.112425000 & -1.164677000 \\
\hline
\end{tabular}




$\begin{array}{lrrr}\mathrm{C} & 2.390606000 & 0.605904000 & -0.353800000 \\ \mathrm{H} & 2.385357000 & 1.183629000 & -1.276853000 \\ \mathrm{C} & 3.636851000 & -0.132836000 & -0.085862000 \\ \mathrm{C} & 4.616398000 & -0.182691000 & -1.084380000 \\ \mathrm{C} & 3.892893000 & -0.789778000 & 1.125162000 \\ \mathrm{C} & 5.809279000 & -0.870438000 & -0.888984000 \\ \mathrm{H} & 4.438369000 & 0.325609000 & -2.029104000 \\ \mathrm{C} & 5.083077000 & -1.476955000 & 1.322714000 \\ \mathrm{H} & 3.161874000 & -0.756739000 & 1.927371000 \\ \mathrm{C} & 6.046624000 & -1.521854000 & 0.316781000 \\ \mathrm{H} & 6.554196000 & -0.894593000 & -1.678643000 \\ \mathrm{H} & 5.262580000 & -1.976513000 & 2.270080000 \\ \mathrm{H} & 6.978229000 & -2.056301000 & 0.475770000 \\ \mathrm{H} & -0.101254000 & 2.221315000 & 0.825763000 \\ \mathrm{H} & 0.016615000 & 1.471566000 & -1.875427000 \\ \mathrm{O} & -1.379627000 & 4.004278000 & 0.706045000 \\ \mathrm{H} & -1.653508000 & 4.701321000 & 1.302459000 \\ \mathrm{~N} & -4.786539000 & -1.687473000 & -0.069449000 \\ \mathrm{O} & -5.826808000 & -1.123872000 & 0.232163000 \\ \mathrm{O} & -4.706238000 & -2.855964000 & -0.417703000 \\ \mathrm{Li} & -0.719847000 & 3.827180000 & -0.830745000 \\ ----- \text { - } & \end{array}$

TSA1-2

\begin{tabular}{lrrr} 
& \multicolumn{3}{c}{ Coordinates (Angstroms) } \\
& $\mathrm{X}$ & $\mathrm{Y}$ & $\mathrm{Z}$ \\
\hline $\mathrm{C}$ & -2.940964000 & -1.220990000 & 0.600142000 \\
$\mathrm{C}$ & -1.667426000 & -0.685337000 & 0.658780000 \\
$\mathrm{C}$ & -1.355521000 & 0.533786000 & 0.027137000 \\
$\mathrm{C}$ & -2.387361000 & 1.203624000 & -0.651866000 \\
$\mathrm{C}$ & -3.666713000 & 0.675334000 & -0.719682000 \\
$\mathrm{C}$ & -3.932541000 & -0.536658000 & -0.095727000 \\
$\mathrm{H}$ & -3.178272000 & -2.161497000 & 1.082004000 \\
$\mathrm{H}$ & -0.906268000 & -1.226942000 & 1.210907000 \\
$\mathrm{H}$ & -2.179435000 & 2.157395000 & -1.122578000 \\
$\mathrm{H}$ & -4.460877000 & 1.189507000 & -1.247391000 \\
$\mathrm{C}$ & -0.010360000 & 1.131867000 & 0.130456000 \\
$\mathrm{C}$ & 1.179555000 & 0.282067000 & 0.154402000 \\
$\mathrm{H}$ & 1.100155000 & -0.625702000 & 0.748781000 \\
$\mathrm{O}$ & 0.185702000 & 2.262696000 & -0.755186000 \\
$\mathrm{C}$ & 2.376294000 & 0.579071000 & -0.397808000 \\
$\mathrm{H}$ & 2.451703000 & 1.433752000 & -1.074631000 \\
$\mathrm{C}$ & 3.614078000 & -0.204347000 & -0.262513000 \\
$\mathrm{C}$ & 4.643483000 & -0.026305000 & -1.196726000
\end{tabular}




\begin{tabular}{|c|c|c|c|}
\hline $\mathrm{C}$ & 3.819794000 & -1.129019000 & 0.771558000 \\
\hline $\mathrm{C}$ & 5.824157000 & -0.755666000 & -1.116630000 \\
\hline $\mathrm{H}$ & 4.508621000 & 0.688605000 & -2.005737000 \\
\hline $\mathrm{C}$ & 4.996656000 & -1.863360000 & 0.849208000 \\
\hline $\mathrm{H}$ & 3.058189000 & -1.265402000 & 1.534384000 \\
\hline $\mathrm{C}$ & 6.005023000 & -1.683039000 & -0.094883000 \\
\hline $\mathrm{H}$ & 6.603062000 & -0.601905000 & -1.857858000 \\
\hline $\mathrm{H}$ & 5.130797000 & -2.575822000 & 1.658071000 \\
\hline $\mathrm{H}$ & 6.926167000 & -2.253799000 & -0.028822000 \\
\hline $\mathrm{H}$ & 0.124918000 & 1.882416000 & 1.308731000 \\
\hline $\mathrm{H}$ & 0.306666000 & 1.936789000 & -1.660112000 \\
\hline $\mathrm{O}$ & 0.554809000 & 2.830957000 & 1.968381000 \\
\hline $\mathrm{H}$ & 0.866388000 & 2.487552000 & 2.811789000 \\
\hline $\mathrm{Li}$ & 1.489354000 & 3.092130000 & 0.457188000 \\
\hline $\mathrm{N}$ & -5.274128000 & -1.101699000 & -0.169595000 \\
\hline $\mathrm{O}$ & -5.477012000 & -2.169716000 & 0.392836000 \\
\hline $\mathrm{O}$ & -6.124738000 & -0.478387000 & -0.790659000 \\
\hline
\end{tabular}

\begin{tabular}{lrrr} 
A2 & \multicolumn{3}{c}{ Coordinates $($ Angstroms) } \\
& X & Y & Z \\
\hline C & 2.937847000 & 1.365795000 & -0.081762000 \\
$\mathrm{C}$ & 1.658440000 & 0.969813000 & -0.381329000 \\
$\mathrm{C}$ & 1.343615000 & -0.397783000 & -0.661043000 \\
$\mathrm{C}$ & 2.435680000 & -1.323330000 & -0.620279000 \\
$\mathrm{C}$ & 3.721109000 & -0.914891000 & -0.306122000 \\
$\mathrm{C}$ & 3.975050000 & 0.422635000 & -0.028425000 \\
$\mathrm{H}$ & 3.163231000 & 2.405887000 & 0.121890000 \\
$\mathrm{H}$ & 0.877434000 & 1.721721000 & -0.415292000 \\
$\mathrm{H}$ & 2.263992000 & -2.355166000 & -0.908788000 \\
$\mathrm{H}$ & 4.539511000 & -1.625179000 & -0.286535000 \\
$\mathrm{C}$ & 0.019764000 & -0.863837000 & -0.856576000 \\
$\mathrm{C}$ & -1.181474000 & -0.165437000 & -0.626632000 \\
$\mathrm{H}$ & -1.091552000 & 0.917642000 & -0.590944000 \\
$\mathrm{O}$ & -0.064134000 & -2.288830000 & -0.869541000 \\
$\mathrm{C}$ & -2.420608000 & -0.714671000 & -0.403080000 \\
$\mathrm{H}$ & -2.537399000 & -1.798810000 & -0.438796000 \\
$\mathrm{C}$ & -3.669687000 & 0.025318000 & -0.206381000 \\
$\mathrm{C}$ & -4.814850000 & -0.669919000 & 0.217049000 \\
$\mathrm{C}$ & -3.794639000 & 1.409203000 & -0.414139000 \\
$\mathrm{C}$ & -6.020857000 & -0.017172000 & 0.436657000 \\
$\mathrm{H}$ & -4.749579000 & -1.743910000 & 0.379194000 \\
$\mathrm{C}$ & -4.999490000 & 2.063057000 & -0.188546000 \\
& & &
\end{tabular}




\begin{tabular}{lccc}
$\mathrm{H}$ & -2.946462000 & 1.983205000 & -0.776772000 \\
$\mathrm{C}$ & -6.121636000 & 1.357540000 & 0.239709000 \\
$\mathrm{H}$ & -6.887007000 & -0.585708000 & 0.763642000 \\
$\mathrm{H}$ & -5.065775000 & 3.133716000 & -0.360989000 \\
$\mathrm{H}$ & -7.063332000 & 1.870511000 & 0.408574000 \\
$\mathrm{H}$ & -0.800493000 & -2.558885000 & -1.430797000 \\
$\mathrm{O}$ & -0.926639000 & -1.550640000 & 2.143877000 \\
$\mathrm{H}$ & -0.862891000 & -0.912144000 & 2.864148000 \\
$\mathrm{H}$ & -1.558235000 & -1.158463000 & 1.493051000 \\
$\mathrm{~N}$ & 5.311815000 & 0.843860000 & 0.311603000 \\
$\mathrm{O}$ & 5.501865000 & 2.036065000 & 0.533552000 \\
$\mathrm{O}$ & 6.191503000 & -0.010862000 & 0.366527000 \\
$\mathrm{Li}$ & 0.501833000 & -1.988412000 & 0.962426000 \\
\hline
\end{tabular}

TSA2-3 ${ }_{\mathrm{LiOH}}$

\begin{tabular}{lccc} 
& \multicolumn{3}{c}{ Coordinates (Angstroms) } \\
& $\mathrm{X}$ & $\mathrm{Y}$ & $\mathrm{Z}$ \\
\hline $\mathrm{C}$ & -3.022263000 & -1.303309000 & 0.254836000 \\
$\mathrm{C}$ & -1.720411000 & -0.847945000 & 0.167720000 \\
$\mathrm{C}$ & -1.433285000 & 0.467030000 & -0.251706000 \\
$\mathrm{C}$ & -2.514659000 & 1.309418000 & -0.570613000 \\
$\mathrm{C}$ & -3.823529000 & 0.861859000 & -0.481391000 \\
$\mathrm{C}$ & -4.065991000 & -0.441043000 & -0.068926000 \\
$\mathrm{H}$ & -3.243075000 & -2.313770000 & 0.575955000 \\
$\mathrm{H}$ & -0.916685000 & -1.525130000 & 0.435896000 \\
$\mathrm{H}$ & -2.318603000 & 2.321141000 & -0.904715000 \\
$\mathrm{H}$ & -4.656497000 & 1.507315000 & -0.732251000 \\
$\mathrm{C}$ & -0.064742000 & 0.965764000 & -0.333192000 \\
$\mathrm{C}$ & 1.098923000 & 0.271211000 & -0.214908000 \\
$\mathrm{H}$ & 1.003955000 & -0.799788000 & -0.050977000 \\
$\mathrm{O}$ & 0.006083000 & 2.375766000 & -0.502129000 \\
$\mathrm{C}$ & 2.430388000 & 0.828481000 & -0.215348000 \\
$\mathrm{H}$ & 2.575803000 & 1.664998000 & -0.911284000 \\
$\mathrm{C}$ & 3.587424000 & -0.100625000 & -0.211787000 \\
$\mathrm{C}$ & 4.726060000 & 0.168319000 & -0.982818000 \\
$\mathrm{C}$ & 3.610911000 & -1.244122000 & 0.600466000 \\
$\mathrm{C}$ & 5.832518000 & -0.673110000 & -0.957550000 \\
$\mathrm{H}$ & 4.738338000 & 1.050805000 & -1.618695000 \\
$\mathrm{C}$ & 4.711143000 & -2.094501000 & 0.617572000 \\
$\mathrm{H}$ & 2.760701000 & -1.466136000 & 1.242543000 \\
$\mathrm{C}$ & 5.830686000 & -1.815274000 & -0.161429000 \\
$\mathrm{H}$ & 6.698868000 & -0.437919000 & -1.569821000 \\
$\mathrm{H}$ & 4.697519000 & -2.975401000 & 1.253892000 \\
& & &
\end{tabular}




$\begin{array}{lrrr}\mathrm{H} & 6.691430000 & -2.476918000 & -0.144292000 \\ \mathrm{H} & 0.714898000 & 2.581755000 & -1.127724000 \\ \mathrm{O} & 2.175465000 & 2.344014000 & 1.898627000 \\ \mathrm{H} & 2.619305000 & 2.024966000 & 2.690422000 \\ \mathrm{H} & 2.430982000 & 1.582225000 & 0.983805000 \\ \mathrm{~N} & -5.442653000 & -0.915479000 & 0.031753000 \\ \mathrm{O} & -5.622698000 & -2.070662000 & 0.391318000 \\ \mathrm{O} & -6.338125000 & -0.130370000 & -0.247411000 \\ \mathrm{Li} & 0.478931000 & 2.469098000 & 1.395392000\end{array}$

\begin{tabular}{|c|c|c|c|}
\hline \multicolumn{4}{|c|}{ A3 $3_{\text {LiOH }}$} \\
\hline & $X$ & $\mathrm{Y}$ & Z \\
\hline$\overline{\mathrm{C}}$ & -3.583959000 & 1.144923000 & 0.167074000 \\
\hline $\mathrm{C}$ & -2.314311000 & 1.692978000 & 0.054895000 \\
\hline $\mathrm{C}$ & -1.195220000 & 0.875446000 & -0.159453000 \\
\hline $\mathrm{C}$ & -1.360236000 & -0.513729000 & -0.269091000 \\
\hline $\mathrm{C}$ & -2.629658000 & -1.064910000 & -0.161718000 \\
\hline $\mathrm{C}$ & -3.720203000 & -0.232038000 & 0.060650000 \\
\hline $\mathrm{H}$ & -4.458488000 & 1.763434000 & 0.325076000 \\
\hline $\mathrm{H}$ & -2.202340000 & 2.772862000 & 0.099060000 \\
\hline $\mathrm{H}$ & -0.492314000 & -1.169558000 & -0.431676000 \\
\hline $\mathrm{H}$ & -2.780646000 & -2.134763000 & -0.238805000 \\
\hline $\mathrm{C}$ & 0.152633000 & 1.485949000 & -0.253209000 \\
\hline $\mathrm{C}$ & 1.147353000 & 0.987988000 & -0.996013000 \\
\hline $\mathrm{H}$ & 0.977984000 & 0.058795000 & -1.534926000 \\
\hline $\mathrm{O}$ & 0.344530000 & 2.654999000 & 0.443893000 \\
\hline $\mathrm{C}$ & 2.539808000 & 1.563398000 & -1.021280000 \\
\hline $\mathrm{H}$ & 2.553803000 & 2.545820000 & -0.541456000 \\
\hline $\mathrm{C}$ & 3.468278000 & 0.615270000 & -0.291328000 \\
\hline $\mathrm{C}$ & 3.362367000 & 0.480125000 & 1.101632000 \\
\hline $\mathrm{C}$ & 4.338693000 & -0.236163000 & -0.978361000 \\
\hline $\mathrm{C}$ & 4.094530000 & -0.488627000 & 1.785071000 \\
\hline $\mathrm{H}$ & 2.673464000 & 1.126025000 & 1.639027000 \\
\hline $\mathrm{C}$ & 5.072588000 & -1.211897000 & -0.298383000 \\
\hline $\mathrm{H}$ & 4.415470000 & -0.160767000 & -2.059014000 \\
\hline $\mathrm{C}$ & 4.947726000 & -1.346656000 & 1.083772000 \\
\hline $\mathrm{H}$ & 3.991221000 & -0.585216000 & 2.861385000 \\
\hline $\mathrm{H}$ & 5.726536000 & -1.876558000 & -0.853839000 \\
\hline $\mathrm{H}$ & 5.508689000 & -2.110862000 & 1.612034000 \\
\hline $\mathrm{O}$ & 1.144042000 & -2.220112000 & -0.817367000 \\
\hline $\mathrm{H}$ & 2.882856000 & 1.691784000 & -2.053922000 \\
\hline $\mathrm{H}$ & -0.305043000 & 2.698693000 & 1.156084000 \\
\hline
\end{tabular}




$\begin{array}{lrrr}\mathrm{H} & 0.853856000 & -3.069017000 & -1.154079000 \\ \mathrm{~N} & -5.057403000 & -0.821190000 & 0.187861000 \\ \mathrm{O} & -5.157970000 & -2.034117000 & 0.083275000 \\ \mathrm{O} & -5.995636000 & -0.064230000 & 0.392582000 \\ \mathrm{Li} & 2.484906000 & -1.669002000 & 0.031804000\end{array}$

\begin{tabular}{|c|c|c|c|}
\hline \multicolumn{4}{|c|}{ Coordinates (Angstroms) } \\
\hline & $\mathrm{X}$ & Y & Z \\
\hline$\overline{\mathrm{C}}$ & 1.695477000 & -3.021978000 & 0.105250000 \\
\hline $\mathrm{C}$ & 0.648367000 & -2.133232000 & 0.311967000 \\
\hline $\mathrm{C}$ & 0.870194000 & -0.753801000 & 0.280875000 \\
\hline $\mathrm{C}$ & 2.153566000 & -0.257940000 & 0.025736000 \\
\hline $\mathrm{C}$ & 3.208254000 & -1.137431000 & -0.185593000 \\
\hline $\mathrm{C}$ & 2.961669000 & -2.504068000 & -0.136374000 \\
\hline $\mathrm{H}$ & 1.546875000 & -4.094557000 & 0.122140000 \\
\hline $\mathrm{H}$ & -0.354650000 & -2.516656000 & 0.483902000 \\
\hline $\mathrm{H}$ & 2.282437000 & 0.829154000 & -0.029161000 \\
\hline $\mathrm{H}$ & 4.209684000 & -0.780662000 & -0.394022000 \\
\hline $\mathrm{C}$ & -0.247218000 & 0.231217000 & 0.584408000 \\
\hline $\mathrm{C}$ & -1.564234000 & -0.168896000 & -0.011434000 \\
\hline $\mathrm{H}$ & -1.517034000 & -0.400729000 & -1.074224000 \\
\hline $\mathrm{O}$ & -0.325891000 & 0.430186000 & 2.011517000 \\
\hline $\mathrm{C}$ & -2.717488000 & -0.237500000 & 0.658036000 \\
\hline $\mathrm{H}$ & -2.706348000 & 0.008895000 & 1.719095000 \\
\hline $\mathrm{C}$ & -4.034739000 & -0.617408000 & 0.119358000 \\
\hline $\mathrm{C}$ & -5.089630000 & -0.833963000 & 1.013695000 \\
\hline $\mathrm{C}$ & -4.286616000 & -0.774785000 & -1.250143000 \\
\hline $\mathrm{C}$ & -6.350599000 & -1.208869000 & 0.562581000 \\
\hline $\mathrm{H}$ & -4.915684000 & -0.707911000 & 2.079812000 \\
\hline $\mathrm{C}$ & -5.544846000 & -1.149326000 & -1.703075000 \\
\hline $\mathrm{H}$ & -3.497172000 & -0.591827000 & -1.973042000 \\
\hline $\mathrm{C}$ & -6.582445000 & -1.370627000 & -0.799245000 \\
\hline $\mathrm{H}$ & -7.151840000 & -1.373102000 & 1.277141000 \\
\hline $\mathrm{H}$ & -5.718632000 & -1.265084000 & -2.768773000 \\
\hline $\mathrm{H}$ & -7.565550000 & -1.661379000 & -1.157077000 \\
\hline $\mathrm{H}$ & 0.075718000 & 1.214216000 & 0.196473000 \\
\hline $\mathrm{H}$ & -0.505803000 & -0.431005000 & 2.412360000 \\
\hline $\mathrm{C}$ & 2.410928000 & 4.436604000 & -1.308918000 \\
\hline $\mathrm{C}$ & 1.316379000 & 3.362137000 & -1.140167000 \\
\hline $\mathrm{H}$ & 2.316195000 & 5.189378000 & -0.515650000 \\
\hline $\mathrm{H}$ & 3.397922000 & 3.968665000 & -1.214411000 \\
\hline $\mathrm{H}$ & 2.360174000 & 4.950642000 & -2.277739000 \\
\hline
\end{tabular}




\begin{tabular}{lrcc}
$\mathrm{C}$ & 1.450423000 & 2.343901000 & -2.294846000 \\
$\mathrm{C}$ & -0.067159000 & 4.039944000 & -1.249089000 \\
$\mathrm{H}$ & 0.686996000 & 1.560971000 & -2.197697000 \\
$\mathrm{H}$ & 1.335859000 & 2.809493000 & -3.282218000 \\
$\mathrm{H}$ & 2.433284000 & 1.859375000 & -2.256853000 \\
$\mathrm{H}$ & -0.219003000 & 4.554968000 & -2.206733000 \\
$\mathrm{H}$ & -0.858355000 & 3.287938000 & -1.134447000 \\
$\mathrm{H}$ & -0.181954000 & 4.777477000 & -0.443744000 \\
$\mathrm{O}$ & 1.443430000 & 2.738147000 & 0.079514000 \\
$\mathrm{~K}$ & 1.241557000 & 2.570567000 & 2.411186000 \\
$\mathrm{~N}$ & 4.075223000 & -3.437197000 & -0.352365000 \\
$\mathrm{O}$ & 5.185137000 & -2.963556000 & -0.541634000 \\
$\mathrm{O}$ & 3.828452000 & -4.633845000 & -0.325554000 \\
\hline$-\boldsymbol{C}$ & & &
\end{tabular}

TSA1-2 tBuOK $_{\text {}}$

\begin{tabular}{|c|c|c|c|}
\hline \multicolumn{4}{|c|}{ Coordinates (Angstroms) } \\
\hline & $\mathrm{X}$ & Y & Z \\
\hline $\mathrm{C}$ & -3.562235000 & -1.161012000 & -1.083627000 \\
\hline $\mathrm{C}$ & -2.294479000 & -0.712541000 & -1.429424000 \\
\hline $\mathrm{C}$ & -1.284822000 & -0.548072000 & -0.463359000 \\
\hline $\mathrm{C}$ & -1.603088000 & -0.851314000 & 0.876472000 \\
\hline $\mathrm{C}$ & -2.858409000 & -1.304280000 & 1.232520000 \\
\hline $\mathrm{C}$ & -3.834255000 & -1.449828000 & 0.246801000 \\
\hline $\mathrm{H}$ & -4.336241000 & -1.301961000 & -1.828978000 \\
\hline $\mathrm{H}$ & -2.064480000 & -0.514813000 & -2.472424000 \\
\hline $\mathrm{H}$ & -0.856635000 & -0.693317000 & 1.649102000 \\
\hline $\mathrm{H}$ & -3.103730000 & -1.530294000 & 2.263102000 \\
\hline $\mathrm{C}$ & 0.024305000 & 0.061348000 & -0.790266000 \\
\hline $\mathrm{C}$ & 1.224766000 & -0.658706000 & -0.341584000 \\
\hline $\mathrm{H}$ & 1.097644000 & -1.297450000 & 0.531453000 \\
\hline $\mathrm{O}$ & 0.107939000 & 0.488768000 & -2.159122000 \\
\hline $\mathrm{C}$ & 2.443660000 & -0.539175000 & -0.895513000 \\
\hline $\mathrm{H}$ & 2.575895000 & 0.165676000 & -1.715542000 \\
\hline $\mathrm{C}$ & 3.658012000 & -1.228870000 & -0.436371000 \\
\hline $\mathrm{C}$ & 4.912483000 & -0.667701000 & -0.709901000 \\
\hline $\mathrm{C}$ & 3.618959000 & -2.436173000 & 0.274987000 \\
\hline $\mathrm{C}$ & 6.083032000 & -1.272733000 & -0.267193000 \\
\hline $\mathrm{H}$ & 4.964437000 & 0.266573000 & -1.264151000 \\
\hline $\mathrm{C}$ & 4.788020000 & -3.040645000 & 0.720995000 \\
\hline $\mathrm{H}$ & 2.663137000 & -2.917578000 & 0.462719000 \\
\hline $\mathrm{C}$ & 6.026611000 & -2.461465000 & 0.455203000 \\
\hline $\mathrm{H}$ & 7.042622000 & -0.812008000 & -0.483897000 \\
\hline $\mathrm{H}$ & 4.732042000 & -3.977172000 & 1.268715000 \\
\hline
\end{tabular}




\begin{tabular}{|c|c|c|c|}
\hline $\mathrm{H}$ & 6.939349000 & -2.937275000 & 0.801039000 \\
\hline $\mathrm{H}$ & -0.005628000 & 1.221758000 & -0.133927000 \\
\hline $\mathrm{H}$ & 0.461564000 & -0.245986000 & -2.683182000 \\
\hline $\mathrm{C}$ & 0.227353000 & 4.201250000 & 1.797107000 \\
\hline $\mathrm{C}$ & 0.706399000 & 2.856736000 & 1.232916000 \\
\hline $\mathrm{H}$ & 0.144453000 & 4.939485000 & 0.989725000 \\
\hline $\mathrm{H}$ & -0.759947000 & 4.082158000 & 2.257297000 \\
\hline $\mathrm{H}$ & 0.917257000 & 4.597480000 & 2.551939000 \\
\hline $\mathrm{C}$ & 0.809159000 & 1.830918000 & 2.375524000 \\
\hline $\mathrm{C}$ & 2.086534000 & 3.031329000 & 0.577394000 \\
\hline $\mathrm{H}$ & 1.198802000 & 0.878709000 & 1.996576000 \\
\hline $\mathrm{H}$ & 1.480882000 & 2.172413000 & 3.171953000 \\
\hline $\mathrm{H}$ & -0.183261000 & 1.656213000 & 2.806355000 \\
\hline $\mathrm{H}$ & 2.841823000 & 3.376719000 & 1.293755000 \\
\hline $\mathrm{H}$ & 2.425000000 & 2.078134000 & 0.155287000 \\
\hline $\mathrm{H}$ & 2.023421000 & 3.765116000 & -0.235735000 \\
\hline $\mathrm{O}$ & -0.225915000 & 2.428814000 & 0.284017000 \\
\hline $\mathrm{K}$ & -1.528863000 & 2.550652000 & -1.745425000 \\
\hline $\mathrm{N}$ & -5.167145000 & -1.905494000 & 0.618264000 \\
\hline $\mathrm{O}$ & -6.013447000 & -1.979528000 & -0.264086000 \\
\hline$\underline{\mathrm{O}}$ & -5.370078000 & -2.186919000 & 1.791436000 \\
\hline
\end{tabular}

\begin{tabular}{lccc} 
A2 & \multicolumn{3}{c}{ Coordinates (Angstroms) } \\
& X & Y & Z \\
\hline $\mathrm{C}$ & -4.109789000 & -0.908131000 & -0.570764000 \\
$\mathrm{C}$ & -2.882513000 & -1.402411000 & -0.962807000 \\
$\mathrm{C}$ & -1.722918000 & -1.301105000 & -0.125006000 \\
$\mathrm{C}$ & -1.927960000 & -0.694716000 & 1.158243000 \\
$\mathrm{C}$ & -3.153946000 & -0.210663000 & 1.543408000 \\
$\mathrm{C}$ & -4.252681000 & -0.295581000 & 0.675422000 \\
$\mathrm{H}$ & -4.976514000 & -0.995307000 & -1.216269000 \\
$\mathrm{H}$ & -2.791505000 & -1.914140000 & -1.915132000 \\
$\mathrm{H}$ & -1.098391000 & -0.601905000 & 1.851282000 \\
$\mathrm{H}$ & -3.287515000 & 0.251582000 & 2.514440000 \\
$\mathrm{C}$ & -0.450672000 & -1.668302000 & -0.613599000 \\
$\mathrm{C}$ & 0.780598000 & -1.500596000 & 0.044954000 \\
$\mathrm{H}$ & 0.713330000 & -1.277017000 & 1.106727000 \\
$\mathrm{O}$ & -0.402675000 & -1.958120000 & -1.997204000 \\
$\mathrm{C}$ & 2.030469000 & -1.548582000 & -0.520983000 \\
$\mathrm{H}$ & 2.116143000 & -1.751454000 & -1.587926000 \\
$\mathrm{C}$ & 3.289489000 & -1.300106000 & 0.175366000 \\
$\mathrm{C}$ & 4.462235000 & -1.102245000 & -0.577068000 \\
& & 5 & \\
& & 5 &
\end{tabular}




\begin{tabular}{lrrr}
$\mathrm{C}$ & 3.399823000 & -1.184536000 & 1.574062000 \\
$\mathrm{C}$ & 5.669992000 & -0.780118000 & 0.027476000 \\
$\mathrm{H}$ & 4.411696000 & -1.192353000 & -1.660102000 \\
$\mathrm{C}$ & 4.606952000 & -0.853521000 & 2.177139000 \\
$\mathrm{H}$ & 2.533276000 & -1.369480000 & 2.203107000 \\
$\mathrm{C}$ & 5.751625000 & -0.642829000 & 1.411523000 \\
$\mathrm{H}$ & 6.553570000 & -0.629933000 & -0.586812000 \\
$\mathrm{H}$ & 4.655619000 & -0.767433000 & 3.259268000 \\
$\mathrm{H}$ & 6.693533000 & -0.387505000 & 1.886825000 \\
$\mathrm{H}$ & 1.721729000 & 0.527249000 & -0.883703000 \\
$\mathrm{H}$ & 0.070325000 & -2.790979000 & -2.118961000 \\
$\mathrm{C}$ & 1.131995000 & 1.880771000 & 1.203462000 \\
$\mathrm{C}$ & 1.684257000 & 2.356347000 & -0.142709000 \\
$\mathrm{H}$ & 0.058671000 & 1.669506000 & 1.129566000 \\
$\mathrm{H}$ & 1.647441000 & 0.969791000 & 1.525788000 \\
$\mathrm{H}$ & 1.281715000 & 2.642040000 & 1.976298000 \\
$\mathrm{C}$ & 3.202743000 & 2.523585000 & -0.076152000 \\
$\mathrm{C}$ & 1.007673000 & 3.651480000 & -0.581313000 \\
$\mathrm{H}$ & 3.593136000 & 2.839544000 & -1.048834000 \\
$\mathrm{H}$ & 3.477555000 & 3.275478000 & 0.671157000 \\
$\mathrm{H}$ & 3.684928000 & 1.579183000 & 0.199546000 \\
$\mathrm{H}$ & 1.228759000 & 4.460987000 & 0.120561000 \\
$\mathrm{H}$ & 1.360104000 & 3.949520000 & -1.574506000 \\
$\mathrm{H}$ & -0.082691000 & 3.529386000 & -0.614682000 \\
$\mathrm{O}$ & 1.358177000 & 1.394241000 & -1.159372000 \\
$\mathrm{~K}$ & -0.900182000 & 0.683596000 & -2.203634000 \\
$\mathrm{~N}$ & -5.521510000 & 0.251515000 & 1.064109000 \\
$\mathrm{O}$ & -6.454257000 & 0.182413000 & 0.264612000 \\
$\mathrm{O}$ & -5.615631000 & 0.771701000 & 2.174303000 \\
\hline---1 & & \\
& & &
\end{tabular}

TSA2-3 ${ }_{\text {tBuOK }}$

\begin{tabular}{llcc} 
& \multicolumn{3}{c}{ Coordinates (Angstroms) } \\
& & $\mathrm{X}$ & $\mathrm{Y}$ \\
$\mathrm{C}$ & 4.352073000 & -0.632082000 & 0.975778000 \\
$\mathrm{C}$ & 3.063730000 & -0.692335000 & 1.483540000 \\
$\mathrm{C}$ & 1.943474000 & -0.706905000 & 0.629899000 \\
$\mathrm{C}$ & 2.170503000 & -0.632256000 & -0.760435000 \\
$\mathrm{C}$ & 3.452978000 & -0.582638000 & -1.275716000 \\
$\mathrm{C}$ & 4.535180000 & -0.579890000 & -0.400201000 \\
$\mathrm{H}$ & 5.214832000 & -0.630910000 & 1.630814000 \\
$\mathrm{H}$ & 2.909006000 & -0.747943000 & 2.554658000 \\
$\mathrm{H}$ & 1.331423000 & -0.597371000 & -1.447652000 \\
$\mathrm{H}$ & 3.628336000 & -0.526990000 & -2.343121000
\end{tabular}




\begin{tabular}{lrrr}
$\mathrm{C}$ & 0.594735000 & -0.727086000 & 1.188609000 \\
$\mathrm{C}$ & -0.555598000 & -0.954245000 & 0.513208000 \\
$\mathrm{H}$ & -0.452237000 & -1.258727000 & -0.524586000 \\
$\mathrm{O}$ & 0.562353000 & -0.330224000 & 2.534712000 \\
$\mathrm{C}$ & -1.899339000 & -0.659746000 & 0.975634000 \\
$\mathrm{H}$ & -2.005982000 & -0.573670000 & 2.064396000 \\
$\mathrm{C}$ & -3.066828000 & -1.369858000 & 0.380293000 \\
$\mathrm{C}$ & -4.355672000 & -0.982141000 & 0.779118000 \\
$\mathrm{C}$ & -2.965630000 & -2.368509000 & -0.595052000 \\
$\mathrm{C}$ & -5.490525000 & -1.559580000 & 0.226085000 \\
$\mathrm{H}$ & -4.457729000 & -0.199576000 & 1.527879000 \\
$\mathrm{C}$ & -4.103080000 & -2.941704000 & -1.160200000 \\
$\mathrm{H}$ & -1.989239000 & -2.725742000 & -0.910523000 \\
$\mathrm{C}$ & -5.371367000 & -2.542905000 & -0.754811000 \\
$\mathrm{H}$ & -6.473674000 & -1.235305000 & 0.556303000 \\
$\mathrm{H}$ & -3.991874000 & -3.714752000 & -1.915765000 \\
$\mathrm{H}$ & -6.256751000 & -2.993250000 & -1.193242000 \\
$\mathrm{H}$ & -1.970630000 & 0.600611000 & 0.620470000 \\
$\mathrm{H}$ & -0.173860000 & -0.792365000 & 2.957862000 \\
$\mathrm{C}$ & -1.825741000 & 1.272857000 & -1.962123000 \\
$\mathrm{C}$ & -2.277055000 & 2.281944000 & -0.887650000 \\
$\mathrm{H}$ & -0.733452000 & 1.158529000 & -1.929036000 \\
$\mathrm{H}$ & -2.279318000 & 0.291115000 & -1.784892000 \\
$\mathrm{H}$ & -2.105482000 & 1.595178000 & -2.972195000 \\
$\mathrm{C}$ & -3.811611000 & 2.363092000 & -0.875610000 \\
$\mathrm{C}$ & -1.696568000 & 3.665572000 & -1.227258000 \\
$\mathrm{H}$ & -4.137783000 & 3.072179000 & -0.106642000 \\
$\mathrm{H}$ & -4.216068000 & 2.686440000 & -1.842891000 \\
$\mathrm{H}$ & -4.235755000 & 1.381546000 & -0.638118000 \\
$\mathrm{H}$ & -2.061685000 & 4.047315000 & -2.188348000 \\
$\mathrm{H}$ & -1.968900000 & 4.386034000 & -0.446804000 \\
$\mathrm{H}$ & -0.599399000 & 3.613710000 & -1.289062000 \\
$\mathrm{O}$ & -1.801797000 & 1.904794000 & 0.369371000 \\
$\mathrm{~N}$ & 0.360717000 & 2.197562000 & 1.465799000 \\
$\mathrm{O}$ & 5.889750000 & -0.503952000 & -0.939674000 \\
$\mathrm{H}$ & 6.819584000 & -0.482732000 & -0.145044000 \\
---.017661000 & -0.463285000 & -2.155364000 \\
\hline
\end{tabular}

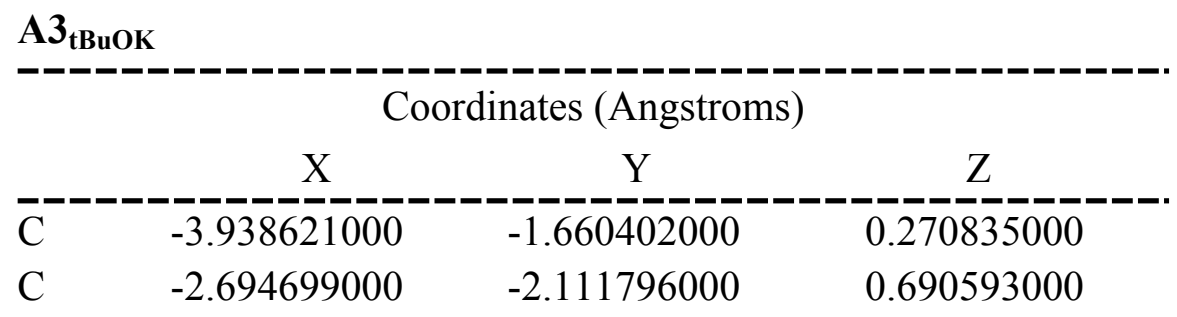




\begin{tabular}{|c|c|c|c|}
\hline $\mathrm{C}$ & -1.556090000 & -1.300000000 & 0.582358000 \\
\hline $\mathrm{C}$ & -1.692540000 & 0.001810000 & 0.068963000 \\
\hline $\mathrm{C}$ & -2.933646000 & 0.458567000 & -0.348792000 \\
\hline $\mathrm{C}$ & -4.037763000 & -0.381677000 & -0.256323000 \\
\hline $\mathrm{H}$ & -4.823816000 & -2.278465000 & 0.355171000 \\
\hline $\mathrm{H}$ & -2.629333000 & -3.094550000 & 1.148605000 \\
\hline $\mathrm{H}$ & -0.832062000 & 0.689027000 & 0.013892000 \\
\hline $\mathrm{H}$ & -3.048490000 & 1.461224000 & -0.744136000 \\
\hline $\mathrm{C}$ & -0.231215000 & -1.822984000 & 1.005147000 \\
\hline $\mathrm{C}$ & 0.807779000 & -1.046502000 & 1.340431000 \\
\hline $\mathrm{H}$ & 0.714669000 & 0.036681000 & 1.245037000 \\
\hline $\mathrm{O}$ & -0.110672000 & -3.190274000 & 1.099509000 \\
\hline $\mathrm{C}$ & 2.161015000 & -1.574813000 & 1.740519000 \\
\hline $\mathrm{H}$ & 2.111697000 & -2.645393000 & 1.954381000 \\
\hline $\mathrm{C}$ & 3.147571000 & -1.309362000 & 0.621685000 \\
\hline $\mathrm{C}$ & 3.316454000 & -2.245929000 & -0.405060000 \\
\hline $\mathrm{C}$ & 3.815077000 & -0.084163000 & 0.526896000 \\
\hline $\mathrm{C}$ & 4.131633000 & -1.965941000 & -1.501323000 \\
\hline $\mathrm{H}$ & 2.797140000 & -3.199038000 & -0.339965000 \\
\hline $\mathrm{C}$ & 4.632116000 & 0.199946000 & -0.566541000 \\
\hline $\mathrm{H}$ & 3.665714000 & 0.665864000 & 1.297868000 \\
\hline $\mathrm{C}$ & 4.790825000 & -0.738170000 & -1.587067000 \\
\hline $\mathrm{H}$ & 4.262475000 & -2.710309000 & -2.281997000 \\
\hline $\mathrm{H}$ & 5.137837000 & 1.159194000 & -0.624065000 \\
\hline $\mathrm{H}$ & 5.434136000 & -0.520371000 & -2.434586000 \\
\hline $\mathrm{C}$ & 0.941957000 & 2.747569000 & 2.104452000 \\
\hline $\mathrm{C}$ & 0.742876000 & 2.963632000 & 0.587914000 \\
\hline $\mathrm{H}$ & 0.158085000 & 2.084367000 & 2.490695000 \\
\hline $\mathrm{H}$ & 1.911309000 & 2.263838000 & 2.283001000 \\
\hline $\mathrm{H}$ & 0.914279000 & 3.683481000 & 2.677707000 \\
\hline $\mathrm{C}$ & 1.853135000 & 3.907561000 & 0.077562000 \\
\hline $\mathrm{C}$ & -0.625914000 & 3.643627000 & 0.366692000 \\
\hline $\mathrm{H}$ & 1.716754000 & 4.087797000 & -0.996409000 \\
\hline $\mathrm{H}$ & 1.865438000 & 4.878242000 & 0.590469000 \\
\hline $\mathrm{H}$ & 2.831017000 & 3.429067000 & 0.220430000 \\
\hline $\mathrm{H}$ & -0.702516000 & 4.622757000 & 0.857167000 \\
\hline $\mathrm{H}$ & -0.797211000 & 3.780922000 & -0.708116000 \\
\hline $\mathrm{H}$ & -1.426005000 & 3.003883000 & 0.759518000 \\
\hline $\mathrm{O}$ & 0.799426000 & 1.763803000 & -0.078462000 \\
\hline $\mathrm{H}$ & 2.494186000 & -1.065974000 & 2.652101000 \\
\hline $\mathrm{K}$ & 1.512417000 & 0.183746000 & -1.660024000 \\
\hline $\mathrm{H}$ & -0.749064000 & -3.602357000 & 0.505601000 \\
\hline $\mathrm{N}$ & -5.344673000 & 0.094675000 & -0.719696000 \\
\hline $\mathrm{O}$ & -6.299302000 & -0.660137000 & -0.601616000 \\
\hline
\end{tabular}




\begin{tabular}{|c|c|c|c|}
\hline \multicolumn{4}{|c|}{ A1 $1_{\text {tBuONa }}$} \\
\hline & \multicolumn{3}{|c|}{ Coordinates (Angstroms) } \\
\hline & $X$ & $\mathrm{Y}$ & Z \\
\hline$\overline{\mathrm{C}}$ & 1.560190000 & -3.019916000 & 0.089114000 \\
\hline $\mathrm{C}$ & 0.549353000 & -2.083392000 & 0.263046000 \\
\hline $\mathrm{C}$ & 0.839019000 & -0.716420000 & 0.251026000 \\
\hline $\mathrm{C}$ & 2.153913000 & -0.280239000 & 0.053513000 \\
\hline $\mathrm{C}$ & 3.172436000 & -1.208464000 & -0.123830000 \\
\hline $\mathrm{C}$ & 2.857690000 & -2.561730000 & -0.099418000 \\
\hline $\mathrm{H}$ & 1.360338000 & -4.084213000 & 0.092664000 \\
\hline $\mathrm{H}$ & -0.476155000 & -2.418873000 & 0.397104000 \\
\hline $\mathrm{H}$ & 2.343157000 & 0.798254000 & 0.038314000 \\
\hline $\mathrm{H}$ & 4.197950000 & -0.899400000 & -0.285547000 \\
\hline $\mathrm{C}$ & -0.240706000 & 0.318767000 & 0.516049000 \\
\hline $\mathrm{C}$ & -1.575657000 & -0.048609000 & -0.061405000 \\
\hline $\mathrm{H}$ & -1.542197000 & -0.312403000 & -1.117024000 \\
\hline $\mathrm{O}$ & -0.312925000 & 0.565401000 & 1.941330000 \\
\hline $\mathrm{C}$ & -2.726680000 & -0.058539000 & 0.614854000 \\
\hline $\mathrm{H}$ & -2.702017000 & 0.217431000 & 1.668206000 \\
\hline $\mathrm{C}$ & -4.059393000 & -0.407380000 & 0.093243000 \\
\hline $\mathrm{C}$ & -5.120357000 & -0.536162000 & 0.997046000 \\
\hline $\mathrm{C}$ & -4.319891000 & -0.619202000 & -1.267223000 \\
\hline $\mathrm{C}$ & -6.397428000 & -0.876412000 & 0.564143000 \\
\hline $\mathrm{H}$ & -4.939150000 & -0.368212000 & 2.056081000 \\
\hline $\mathrm{C}$ & -5.594268000 & -0.958935000 & -1.701954000 \\
\hline $\mathrm{H}$ & -3.524638000 & -0.507589000 & -1.998283000 \\
\hline $\mathrm{C}$ & -6.638701000 & -1.091310000 & -0.788617000 \\
\hline $\mathrm{H}$ & -7.203545000 & -0.971599000 & 1.285550000 \\
\hline $\mathrm{H}$ & -5.775112000 & -1.116989000 & -2.760983000 \\
\hline $\mathrm{H}$ & -7.634476000 & -1.354547000 & -1.132309000 \\
\hline $\mathrm{H}$ & 0.116982000 & 1.278218000 & 0.109355000 \\
\hline $\mathrm{H}$ & -0.487440000 & -0.280613000 & 2.374789000 \\
\hline $\mathrm{C}$ & 2.868442000 & 4.678277000 & -0.327810000 \\
\hline $\mathrm{C}$ & 1.767095000 & 3.651394000 & -0.655281000 \\
\hline $\mathrm{H}$ & 2.609390000 & 5.216023000 & 0.593612000 \\
\hline $\mathrm{H}$ & 3.818504000 & 4.157844000 & -0.160401000 \\
\hline $\mathrm{H}$ & 3.013022000 & 5.417440000 & -1.126433000 \\
\hline $\mathrm{C}$ & 2.140053000 & 2.921331000 & -1.962271000 \\
\hline $\mathrm{C}$ & 0.434039000 & 4.397062000 & -0.867750000 \\
\hline $\mathrm{H}$ & 1.376278000 & 2.170335000 & -2.199361000 \\
\hline $\mathrm{H}$ & 2.228254000 & 3.604415000 & -2.816509000 \\
\hline
\end{tabular}




$\begin{array}{lrcc}\mathrm{H} & 3.097455000 & 2.401175000 & -1.838650000 \\ \mathrm{H} & 0.481808000 & 5.138088000 & -1.676010000 \\ \mathrm{H} & -0.359035000 & 3.677617000 & -1.105966000 \\ \mathrm{H} & 0.152010000 & 4.917954000 & 0.056761000 \\ \mathrm{O} & 1.639087000 & 2.740909000 & 0.370923000 \\ \mathrm{Na} & 1.101274000 & 2.321807000 & 2.281540000 \\ \mathrm{~N} & 3.933063000 & -3.546805000 & -0.281531000 \\ \mathrm{O} & 3.628299000 & -4.730048000 & -0.266658000 \\ \mathrm{O} & 5.069071000 & -3.125428000 & -0.433098000\end{array}$

TSA1-2 ${ }_{\text {tBuONa }}$

\begin{tabular}{|c|c|c|c|}
\hline \multicolumn{4}{|c|}{ Coordinates (Angstroms) } \\
\hline & $X$ & $\mathrm{Y}$ & Z \\
\hline $\mathrm{C}$ & 3.792453000 & -0.745227000 & 1.188367000 \\
\hline $\mathrm{C}$ & 2.496684000 & -0.364142000 & 1.499185000 \\
\hline $\mathrm{C}$ & 1.487624000 & -0.328534000 & 0.521538000 \\
\hline $\mathrm{C}$ & 1.841782000 & -0.680075000 & -0.797069000 \\
\hline $\mathrm{C}$ & 3.129275000 & -1.063890000 & -1.119816000 \\
\hline $\mathrm{C}$ & 4.099306000 & -1.094491000 & -0.120615000 \\
\hline $\mathrm{H}$ & 4.566412000 & -0.781359000 & 1.945491000 \\
\hline $\mathrm{H}$ & 2.254345000 & -0.094554000 & 2.521201000 \\
\hline $\mathrm{H}$ & 1.098577000 & -0.627117000 & -1.586595000 \\
\hline $\mathrm{H}$ & 3.399689000 & -1.330840000 & -2.134230000 \\
\hline $\mathrm{C}$ & 0.125246000 & 0.151048000 & 0.823178000 \\
\hline $\mathrm{C}$ & -1.008365000 & -0.621967000 & 0.315448000 \\
\hline $\mathrm{H}$ & -0.826722000 & -1.167921000 & -0.608590000 \\
\hline $\mathrm{O}$ & -0.047106000 & 0.509140000 & 2.215586000 \\
\hline $\mathrm{C}$ & -2.238555000 & -0.657202000 & 0.861972000 \\
\hline $\mathrm{H}$ & -2.429983000 & -0.060681000 & 1.753419000 \\
\hline $\mathrm{C}$ & -3.400650000 & -1.387215000 & 0.337745000 \\
\hline $\mathrm{C}$ & -4.680188000 & -1.058718000 & 0.806511000 \\
\hline $\mathrm{C}$ & -3.297671000 & -2.408705000 & -0.618023000 \\
\hline $\mathrm{C}$ & -5.814003000 & -1.705301000 & 0.328165000 \\
\hline $\mathrm{H}$ & -4.783837000 & -0.275064000 & 1.553952000 \\
\hline $\mathrm{C}$ & -4.429789000 & -3.052280000 & -1.101232000 \\
\hline $\mathrm{H}$ & -2.319795000 & -2.716256000 & -0.977804000 \\
\hline $\mathrm{C}$ & -5.695226000 & -2.704397000 & -0.633189000 \\
\hline $\mathrm{H}$ & -6.793063000 & -1.426772000 & 0.707784000 \\
\hline $\mathrm{H}$ & -4.322826000 & -3.839004000 & -1.842704000 \\
\hline $\mathrm{H}$ & -6.578062000 & -3.211970000 & -1.009860000 \\
\hline $\mathrm{H}$ & 0.029568000 & 1.420968000 & 0.247477000 \\
\hline $\mathrm{H}$ & -0.294367000 & -0.286445000 & 2.710817000 \\
\hline $\mathrm{C}$ & -0.479781000 & 4.445625000 & -1.391896000 \\
\hline
\end{tabular}




$\begin{array}{lrrr}\mathrm{C} & -0.797811000 & 2.984662000 & -1.061398000 \\ \mathrm{H} & -0.749691000 & 5.092469000 & -0.547662000 \\ \mathrm{H} & 0.591666000 & 4.563295000 & -1.583817000 \\ \mathrm{H} & -1.032745000 & 4.789293000 & -2.273770000 \\ \mathrm{C} & -0.397219000 & 2.086134000 & -2.240885000 \\ \mathrm{C} & -2.296207000 & 2.822404000 & -0.768974000 \\ \mathrm{H} & -0.677040000 & 1.045692000 & -2.040547000 \\ \mathrm{H} & -0.894734000 & 2.394359000 & -3.167143000 \\ \mathrm{H} & 0.686418000 & 2.130146000 & -2.391674000 \\ \mathrm{H} & -2.913528000 & 3.073333000 & -1.639400000 \\ \mathrm{H} & -2.513762000 & 1.788587000 & -0.478013000 \\ \mathrm{H} & -2.592353000 & 3.485724000 & 0.055237000 \\ \mathrm{O} & -0.048313000 & 2.631251000 & 0.078105000 \\ \mathrm{Na} & -0.082017000 & 2.729281000 & 2.184184000 \\ \mathrm{~N} & 5.459161000 & -1.491801000 & -0.454605000 \\ \mathrm{O} & 6.293688000 & -1.493364000 & 0.441826000 \\ \mathrm{O} & 5.697039000 & -1.803494000 & -1.614445000 \\ \text { - - - - } & & \end{array}$

\begin{tabular}{|c|c|c|c|}
\hline \multicolumn{4}{|c|}{$\mathrm{A} 2_{\mathrm{tBuONa}}$} \\
\hline & $X$ & Y & $\mathrm{Z}$ \\
\hline$\overline{\mathrm{C}}$ & -4.026044000 & -0.649438000 & -0.831141000 \\
\hline $\mathrm{C}$ & -2.791621000 & -1.091627000 & -1.269412000 \\
\hline $\mathrm{C}$ & -1.682275000 & -1.246112000 & -0.373211000 \\
\hline $\mathrm{C}$ & -1.943733000 & -0.953993000 & 1.005431000 \\
\hline $\mathrm{C}$ & -3.174955000 & -0.523700000 & 1.432629000 \\
\hline $\mathrm{C}$ & -4.222316000 & -0.351201000 & 0.514735000 \\
\hline $\mathrm{H}$ & -4.854768000 & -0.544252000 & -1.522094000 \\
\hline $\mathrm{H}$ & -2.669975000 & -1.383112000 & -2.307703000 \\
\hline $\mathrm{H}$ & -1.157261000 & -1.076427000 & 1.742387000 \\
\hline $\mathrm{H}$ & -3.354476000 & -0.307652000 & 2.479237000 \\
\hline $\mathrm{C}$ & -0.397057000 & -1.558815000 & -0.871615000 \\
\hline $\mathrm{C}$ & 0.818670000 & -1.480020000 & -0.167982000 \\
\hline $\mathrm{H}$ & 0.725265000 & -1.417835000 & 0.913092000 \\
\hline $\mathrm{O}$ & -0.311399000 & -1.553622000 & -2.289364000 \\
\hline $\mathrm{C}$ & 2.078541000 & -1.400800000 & -0.706510000 \\
\hline $\mathrm{H}$ & 2.197155000 & -1.460008000 & -1.788128000 \\
\hline $\mathrm{C}$ & 3.314645000 & -1.200159000 & 0.046543000 \\
\hline $\mathrm{C}$ & 4.489513000 & -0.855932000 & -0.646758000 \\
\hline $\mathrm{C}$ & 3.397099000 & -1.276578000 & 1.449126000 \\
\hline $\mathrm{C}$ & 5.675811000 & -0.583375000 & 0.021909000 \\
\hline $\mathrm{H}$ & 4.459118000 & -0.790700000 & -1.732418000 \\
\hline $\mathrm{C}$ & 4.583542000 & -0.999444000 & 2.117268000 \\
\hline
\end{tabular}




$\begin{array}{lrrr}\mathrm{H} & 2.526846000 & -1.572677000 & 2.028668000 \\ \mathrm{C} & 5.732053000 & -0.647091000 & 1.412290000 \\ \mathrm{H} & 6.562568000 & -0.317170000 & -0.546875000 \\ \mathrm{H} & 4.612780000 & -1.070026000 & 3.201183000 \\ \mathrm{H} & 6.658058000 & -0.434063000 & 1.937122000 \\ \mathrm{H} & 1.621672000 & 0.622922000 & -0.999802000 \\ \mathrm{H} & 0.219941000 & -2.306190000 & -2.574199000 \\ \mathrm{C} & 0.890243000 & 1.745499000 & 1.161735000 \\ \mathrm{C} & 1.385043000 & 2.387855000 & -0.135799000 \\ \mathrm{H} & -0.145669000 & 1.399955000 & 1.058462000 \\ \mathrm{H} & 1.518160000 & 0.888729000 & 1.430121000 \\ \mathrm{H} & 0.932422000 & 2.464661000 & 1.986092000 \\ \mathrm{C} & 2.873085000 & 2.723291000 & -0.043064000 \\ \mathrm{C} & 0.557891000 & 3.619487000 & -0.486939000 \\ \mathrm{H} & 3.223446000 & 3.164951000 & -0.981151000 \\ \mathrm{H} & 3.060156000 & 3.434102000 & 0.768640000 \\ \mathrm{H} & 3.458661000 & 1.818285000 & 0.155036000 \\ \mathrm{H} & 0.676235000 & 4.394824000 & 0.275621000 \\ \mathrm{H} & 0.873889000 & 4.031854000 & -1.450751000 \\ \mathrm{H} & -0.510495000 & 3.371292000 & -0.540692000 \\ \mathrm{O} & 1.186426000 & 1.472463000 & -1.232059000 \\ \mathrm{Na} & -0.746564000 & 0.699957000 & -2.020179000 \\ \mathrm{~N} & -5.501384000 & 0.128399000 & 0.964886000 \\ \mathrm{O} & -5.645387000 & 0.357581000 & 2.163257000 \\ \mathrm{O} & -6.387221000 & 0.291469000 & 0.128230000 \\ - & & & \end{array}$

TSA2-3

\begin{tabular}{|c|c|c|c|}
\hline \multicolumn{4}{|c|}{ Coordinates (Angstroms) } \\
\hline & $\mathrm{X}$ & $\mathrm{Y}$ & $\mathrm{Z}$ \\
\hline$\overline{\mathrm{C}}$ & -4.274942000 & 0.809901000 & -0.447194000 \\
\hline $\mathrm{C}$ & -2.958140000 & 0.906310000 & -0.874144000 \\
\hline $\mathrm{C}$ & -2.069765000 & -0.187461000 & -0.765928000 \\
\hline $\mathrm{C}$ & -2.583836000 & -1.402110000 & -0.258947000 \\
\hline $\mathrm{C}$ & -3.892482000 & -1.503108000 & 0.167959000 \\
\hline $\mathrm{C}$ & -4.728085000 & -0.389579000 & 0.083458000 \\
\hline $\mathrm{H}$ & -4.957013000 & 1.647815000 & -0.529361000 \\
\hline $\mathrm{H}$ & -2.613471000 & 1.825556000 & -1.335743000 \\
\hline $\mathrm{H}$ & -1.953189000 & -2.283230000 & -0.218747000 \\
\hline $\mathrm{H}$ & -4.284286000 & -2.433884000 & 0.559572000 \\
\hline $\mathrm{C}$ & -0.667435000 & -0.022260000 & -1.119083000 \\
\hline $\mathrm{C}$ & 0.351510000 & -0.857853000 & -0.772751000 \\
\hline $\mathrm{H}$ & 0.088233000 & -1.740377000 & -0.192496000 \\
\hline $\mathrm{O}$ & -0.403569000 & 1.214807000 & -1.713302000 \\
\hline
\end{tabular}




$\begin{array}{lrrr}\mathrm{C} & 1.749828000 & -0.573269000 & -0.995866000 \\ \mathrm{H} & 1.976807000 & -0.226417000 & -2.010669000 \\ \mathrm{C} & 2.773267000 & -1.484917000 & -0.449007000 \\ \mathrm{C} & 3.981439000 & -1.703935000 & -1.128856000 \\ \mathrm{C} & 2.623206000 & -2.093198000 & 0.808157000 \\ \mathrm{C} & 4.984154000 & -2.499945000 & -0.586897000 \\ \mathrm{H} & 4.127299000 & -1.245059000 & -2.104455000 \\ \mathrm{C} & 3.619237000 & -2.901200000 & 1.344804000 \\ \mathrm{H} & 1.718140000 & -1.914669000 & 1.385075000 \\ \mathrm{C} & 4.808886000 & -3.110505000 & 0.652460000 \\ \mathrm{H} & 5.907175000 & -2.650151000 & -1.140377000 \\ \mathrm{H} & 3.469275000 & -3.361169000 & 2.317995000 \\ \mathrm{H} & 5.589542000 & -3.736155000 & 1.074272000 \\ \mathrm{H} & 1.854153000 & 0.647449000 & -0.282455000 \\ \mathrm{H} & 0.550006000 & 1.395890000 & -1.631987000 \\ \mathrm{C} & 3.588073000 & 1.620660000 & 1.677243000 \\ \mathrm{C} & 2.933967000 & 2.355721000 & 0.501813000 \\ \mathrm{H} & 2.933163000 & 1.656613000 & 2.556244000 \\ \mathrm{H} & 3.759343000 & 0.569133000 & 1.422577000 \\ \mathrm{H} & 4.549623000 & 2.072210000 & 1.946460000 \\ \mathrm{C} & 3.849085000 & 2.297057000 & -0.728995000 \\ \mathrm{C} & 2.648056000 & 3.812669000 & 0.874215000 \\ \mathrm{H} & 3.356205000 & 2.764464000 & -1.589082000 \\ \mathrm{H} & 4.795349000 & 2.818002000 & -0.546699000 \\ \mathrm{H} & 4.079452000 & 1.256505000 & -0.982604000 \\ \mathrm{H} & 3.567698000 & 4.355165000 & 1.119826000 \\ \mathrm{H} & 2.157415000 & 4.326338000 & 0.039201000 \\ \mathrm{H} & 1.988002000 & 3.858313000 & 1.750894000 \\ \mathrm{O} & 1.695282000 & 1.746837000 & 0.194335000 \\ \mathrm{Na} & -0.391797000 & 1.837602000 & 0.657614000 \\ \mathrm{~N} & -6.107631000 & -0.492351000 & 0.543066000 \\ \mathrm{O} & -6.809857000 & 0.507237000 & 0.467896000 \\ \mathrm{O} & -6.483340000 & -1.569803000 & 0.983910000 \\ ------ & \end{array}$

\begin{tabular}{|c|c|c|c|}
\hline \multirow{2}{*}{\multicolumn{4}{|c|}{$\mathrm{A3}_{\mathrm{tBuONa}}$}} \\
\hline & & & \\
\hline & $X$ & Y & Z \\
\hline$\overline{\mathrm{C}}$ & -3.878595000 & -1.678990000 & 0.190468000 \\
\hline $\mathrm{C}$ & -2.620293000 & -2.152980000 & 0.533096000 \\
\hline $\mathrm{C}$ & -1.490780000 & -1.325985000 & 0.444925000 \\
\hline $\mathrm{C}$ & -1.640878000 & 0.003637000 & 0.022124000 \\
\hline $\mathrm{C}$ & -2.898203000 & 0.482978000 & -0.318466000 \\
\hline $\mathrm{C}$ & -3.996288000 & -0.365229000 & -0.239625000 \\
\hline
\end{tabular}




\begin{tabular}{|c|c|c|c|}
\hline $\mathrm{H}$ & -4.759422000 & -2.304857000 & 0.260462000 \\
\hline $\mathrm{H}$ & -2.532795000 & -3.166304000 & 0.915919000 \\
\hline $\mathrm{H}$ & -0.777207000 & 0.684937000 & -0.036549000 \\
\hline $\mathrm{H}$ & -3.028165000 & 1.507109000 & -0.648397000 \\
\hline $\mathrm{C}$ & -0.155636000 & -1.870387000 & 0.800054000 \\
\hline $\mathrm{C}$ & 0.851359000 & -1.138178000 & 1.295885000 \\
\hline $\mathrm{H}$ & 0.719614000 & -0.061939000 & 1.385533000 \\
\hline $\mathrm{O}$ & 0.009937000 & -3.226805000 & 0.656322000 \\
\hline $\mathrm{C}$ & 2.205334000 & -1.707962000 & 1.643981000 \\
\hline $\mathrm{H}$ & 2.137291000 & -2.788439000 & 1.796228000 \\
\hline $\mathrm{C}$ & 3.182281000 & -1.401245000 & 0.527590000 \\
\hline $\mathrm{C}$ & 3.162069000 & -2.168319000 & -0.646364000 \\
\hline $\mathrm{C}$ & 4.019011000 & -0.282422000 & 0.580018000 \\
\hline $\mathrm{C}$ & 3.945622000 & -1.814817000 & -1.743815000 \\
\hline $\mathrm{H}$ & 2.509555000 & -3.036535000 & -0.693856000 \\
\hline $\mathrm{C}$ & 4.803806000 & 0.075731000 & -0.517180000 \\
\hline $\mathrm{H}$ & 4.031184000 & 0.332725000 & 1.475282000 \\
\hline $\mathrm{C}$ & 4.765220000 & -0.684451000 & -1.685275000 \\
\hline $\mathrm{H}$ & 3.923887000 & -2.424336000 & -2.642811000 \\
\hline $\mathrm{H}$ & 5.437821000 & 0.955223000 & -0.460640000 \\
\hline $\mathrm{H}$ & 5.377134000 & -0.407687000 & -2.538277000 \\
\hline $\mathrm{C}$ & 1.105926000 & 2.930404000 & 1.900112000 \\
\hline $\mathrm{C}$ & 0.825377000 & 3.013815000 & 0.385185000 \\
\hline $\mathrm{H}$ & 0.330923000 & 2.325198000 & 2.386105000 \\
\hline $\mathrm{H}$ & 2.073552000 & 2.438639000 & 2.065067000 \\
\hline $\mathrm{H}$ & 1.130400000 & 3.913781000 & 2.387056000 \\
\hline $\mathrm{C}$ & 1.920029000 & 3.878235000 & -0.273364000 \\
\hline $\mathrm{C}$ & -0.543690000 & 3.690858000 & 0.168712000 \\
\hline $\mathrm{H}$ & 1.730515000 & 3.955777000 & -1.351339000 \\
\hline $\mathrm{H}$ & 1.974216000 & 4.893400000 & 0.140932000 \\
\hline $\mathrm{H}$ & 2.897646000 & 3.397560000 & -0.130894000 \\
\hline $\mathrm{H}$ & -0.581563000 & 4.711551000 & 0.570260000 \\
\hline $\mathrm{H}$ & -0.767536000 & 3.730910000 & -0.903960000 \\
\hline $\mathrm{H}$ & -1.329548000 & 3.103475000 & 0.659476000 \\
\hline $\mathrm{O}$ & 0.822662000 & 1.750392000 & -0.157341000 \\
\hline $\mathrm{H}$ & 2.561924000 & -1.261288000 & 2.577992000 \\
\hline $\mathrm{H}$ & -0.665957000 & -3.561286000 & 0.054738000 \\
\hline $\mathrm{Na}$ & 1.835925000 & 0.334463000 & -1.194735000 \\
\hline $\mathrm{N}$ & -5.321913000 & 0.138614000 & -0.616282000 \\
\hline $\mathrm{O}$ & -6.267063000 & -0.632683000 & -0.533879000 \\
\hline $\mathrm{O}$ & -5.403696000 & 1.297202000 & -0.992682000 \\
\hline
\end{tabular}

\section{A1 $1_{\text {tBuOLi }}$}




\begin{tabular}{|c|c|c|c|}
\hline \multicolumn{4}{|c|}{ Coordinates (Angstroms) } \\
\hline & $X$ & $\mathrm{Y}$ & Z \\
\hline $\bar{C}$ & 1.416458000 & -2.985048000 & 0.058064000 \\
\hline $\mathrm{C}$ & 0.442593000 & -2.003092000 & 0.193101000 \\
\hline $\mathrm{C}$ & 0.799255000 & -0.653012000 & 0.225293000 \\
\hline $\mathrm{C}$ & 2.144903000 & -0.281770000 & 0.125280000 \\
\hline $\mathrm{C}$ & 3.126581000 & -1.254244000 & -0.010803000 \\
\hline $\mathrm{C}$ & 2.743194000 & -2.589717000 & -0.042237000 \\
\hline $\mathrm{H}$ & 1.165551000 & -4.038016000 & 0.025856000 \\
\hline $\mathrm{H}$ & -0.603328000 & -2.289485000 & 0.264046000 \\
\hline $\mathrm{H}$ & 2.395465000 & 0.780409000 & 0.161957000 \\
\hline $\mathrm{H}$ & 4.174342000 & -0.994035000 & -0.098597000 \\
\hline $\mathrm{C}$ & -0.239453000 & 0.435389000 & 0.431060000 \\
\hline $\mathrm{C}$ & -1.600918000 & 0.085704000 & -0.093469000 \\
\hline $\mathrm{H}$ & -1.602985000 & -0.239851000 & -1.132214000 \\
\hline $\mathrm{O}$ & -0.274174000 & 0.792376000 & 1.836697000 \\
\hline $\mathrm{C}$ & -2.734694000 & 0.158799000 & 0.606728000 \\
\hline $\mathrm{H}$ & -2.675722000 & 0.487635000 & 1.643181000 \\
\hline $\mathrm{C}$ & -4.089560000 & -0.165772000 & 0.128078000 \\
\hline $\mathrm{C}$ & -5.126758000 & -0.262212000 & 1.062693000 \\
\hline $\mathrm{C}$ & -4.391386000 & -0.383710000 & -1.222713000 \\
\hline $\mathrm{C}$ & -6.421971000 & -0.580537000 & 0.668596000 \\
\hline $\mathrm{H}$ & -4.913144000 & -0.087093000 & 2.114427000 \\
\hline $\mathrm{C}$ & -5.683991000 & -0.701469000 & -1.618505000 \\
\hline $\mathrm{H}$ & -3.614901000 & -0.290680000 & -1.976234000 \\
\hline $\mathrm{C}$ & -6.704480000 & -0.804111000 & -0.674858000 \\
\hline $\mathrm{H}$ & -7.209947000 & -0.651409000 & 1.412287000 \\
\hline $\mathrm{H}$ & -5.897723000 & -0.864244000 & -2.670640000 \\
\hline $\mathrm{H}$ & -7.714870000 & -1.049258000 & -0.987740000 \\
\hline $\mathrm{H}$ & 0.136240000 & 1.351835000 & -0.042765000 \\
\hline $\mathrm{H}$ & -0.382831000 & -0.018145000 & 2.351269000 \\
\hline $\mathrm{C}$ & 3.191769000 & 4.722967000 & 0.320368000 \\
\hline $\mathrm{C}$ & 2.181272000 & 3.747498000 & -0.308847000 \\
\hline $\mathrm{H}$ & 2.723438000 & 5.248494000 & 1.161587000 \\
\hline $\mathrm{H}$ & 4.053102000 & 4.165299000 & 0.705332000 \\
\hline $\mathrm{H}$ & 3.553747000 & 5.471141000 & -0.395960000 \\
\hline $\mathrm{C}$ & 2.849817000 & 3.029742000 & -1.497241000 \\
\hline $\mathrm{C}$ & 0.965073000 & 4.540912000 & -0.821321000 \\
\hline $\mathrm{H}$ & 2.147568000 & 2.316117000 & -1.945787000 \\
\hline $\mathrm{H}$ & 3.174730000 & 3.729467000 & -2.276651000 \\
\hline $\mathrm{H}$ & 3.727731000 & 2.470995000 & -1.151121000 \\
\hline $\mathrm{H}$ & 1.238619000 & 5.292123000 & -1.572642000 \\
\hline $\mathrm{H}$ & 0.235229000 & 3.855159000 & -1.268912000 \\
\hline $\mathrm{H}$ & 0.478363000 & 5.053064000 & 0.017768000 \\
\hline
\end{tabular}




$\begin{array}{llll}\mathrm{O} & 1.770358000 & 2.820266000 & 0.627009000 \\ \mathrm{~N} & 3.777914000 & -3.623955000 & -0.188704000 \\ \mathrm{O} & 3.413733000 & -4.789639000 & -0.218816000 \\ \mathrm{O} & 4.939111000 & -3.256246000 & -0.269067000 \\ \mathrm{Li} & 0.989159000 & 2.265400000 & 2.000102000\end{array}$

TSA1-2 tBuOLi $_{\text {i }}$

\begin{tabular}{lrcc} 
& \multicolumn{3}{c}{ Coordinates (Angstroms) } \\
& $\mathrm{X}$ & $\mathrm{Y}$ & $\mathrm{Z}$ \\
\hline $\mathrm{C}$ & 3.077805000 & -1.005178000 & -1.161601000 \\
$\mathrm{C}$ & 1.782609000 & -0.711316000 & -0.775977000 \\
$\mathrm{C}$ & 1.465599000 & -0.429464000 & 0.567383000 \\
$\mathrm{C}$ & 2.510231000 & -0.440848000 & 1.506504000 \\
$\mathrm{C}$ & 3.810673000 & -0.738294000 & 1.131838000 \\
$\mathrm{C}$ & 4.083817000 & -1.021188000 & -0.200398000 \\
$\mathrm{H}$ & 3.321076000 & -1.222068000 & -2.194475000 \\
$\mathrm{H}$ & 1.008517000 & -0.693542000 & -1.536515000 \\
$\mathrm{H}$ & 2.294717000 & -0.200693000 & 2.541058000 \\
$\mathrm{H}$ & 4.615658000 & -0.752169000 & 1.856523000 \\
$\mathrm{C}$ & 0.102867000 & -0.035154000 & 0.959635000 \\
$\mathrm{C}$ & -1.074667000 & -0.620048000 & 0.328156000 \\
$\mathrm{H}$ & -0.998570000 & -0.799221000 & -0.742039000 \\
$\mathrm{O}$ & -0.088899000 & 0.113045000 & 2.384848000 \\
$\mathrm{C}$ & -2.272739000 & -0.822787000 & 0.920844000 \\
$\mathrm{H}$ & -2.353850000 & -0.748446000 & 2.008124000 \\
$\mathrm{C}$ & -3.508473000 & -1.225239000 & 0.232154000 \\
$\mathrm{C}$ & -4.525061000 & -1.869397000 & 0.950033000 \\
$\mathrm{C}$ & -3.723494000 & -0.972234000 & -1.130229000 \\
$\mathrm{C}$ & -5.702444000 & -2.268577000 & 0.328376000 \\
$\mathrm{H}$ & -4.380832000 & -2.071090000 & 2.009329000 \\
$\mathrm{C}$ & -4.896899000 & -1.377982000 & -1.754173000 \\
$\mathrm{H}$ & -2.973816000 & -0.432386000 & -1.702534000 \\
$\mathrm{C}$ & -5.892214000 & -2.029723000 & -1.029728000 \\
$\mathrm{H}$ & -6.472654000 & -2.771398000 & 0.906102000 \\
$\mathrm{H}$ & -5.041061000 & -1.172202000 & -2.810833000 \\
$\mathrm{H}$ & -6.810936000 & -2.340092000 & -1.517807000 \\
$\mathrm{H}$ & -0.066655000 & 1.311303000 & 0.669653000 \\
$\mathrm{H}$ & -0.201250000 & -0.764059000 & 2.781703000 \\
$\mathrm{C}$ & 0.912380000 & 3.485231000 & -0.742974000 \\
$\mathrm{C}$ & -0.540652000 & 3.206151000 & -0.340151000 \\
$\mathrm{H}$ & 1.427281000 & 4.021299000 & 0.060463000 \\
$\mathrm{H}$ & 1.444540000 & 2.542490000 & -0.916884000 \\
& 0.968531000 & 4.085133000 & -1.658376000 \\
& & &
\end{tabular}




$\begin{array}{lccc}\mathrm{C} & -1.258131000 & 2.435661000 & -1.458531000 \\ \mathrm{C} & -1.276208000 & 4.515514000 & -0.049481000 \\ \mathrm{H} & -2.276712000 & 2.173722000 & -1.148474000 \\ \mathrm{H} & -1.316169000 & 3.026841000 & -2.379101000 \\ \mathrm{H} & -0.721659000 & 1.507578000 & -1.689459000 \\ \mathrm{H} & -1.281764000 & 5.175157000 & -0.924597000 \\ \mathrm{H} & -2.316857000 & 4.312804000 & 0.231305000 \\ \mathrm{H} & -0.790137000 & 5.041790000 & 0.778704000 \\ \mathrm{O} & -0.542529000 & 2.440240000 & 0.843742000 \\ \mathrm{Li} & -1.467139000 & 1.522754000 & 2.061588000 \\ \mathrm{~N} & 5.449710000 & -1.334806000 & -0.600403000 \\ \mathrm{O} & 6.312477000 & -1.345125000 & 0.267493000 \\ \mathrm{O} & 5.659706000 & -1.571429000 & -1.782821000 \\ \text {-_-_-_-_._-_. }\end{array}$

\begin{tabular}{|c|c|c|c|}
\hline \multicolumn{4}{|c|}{$\mathrm{A} \boldsymbol{2}_{\mathrm{tBuOLi}}$} \\
\hline & \multicolumn{3}{|c|}{ Coordinates (Angstroms) } \\
\hline & $X$ & Y & Z \\
\hline$\overline{\mathrm{C}}$ & 3.893882000 & -0.484873000 & 0.973597000 \\
\hline $\mathrm{C}$ & 2.661919000 & -0.893852000 & 1.455837000 \\
\hline $\mathrm{C}$ & 1.589775000 & -1.244531000 & 0.572818000 \\
\hline $\mathrm{C}$ & 1.880297000 & -1.196483000 & -0.828072000 \\
\hline $\mathrm{C}$ & 3.107104000 & -0.797097000 & -1.295400000 \\
\hline $\mathrm{C}$ & 4.116794000 & -0.420457000 & -0.396800000 \\
\hline $\mathrm{H}$ & 4.696016000 & -0.223618000 & 1.654040000 \\
\hline $\mathrm{H}$ & 2.524074000 & -1.010612000 & 2.525762000 \\
\hline $\mathrm{H}$ & 1.122429000 & -1.489790000 & -1.546432000 \\
\hline $\mathrm{H}$ & 3.311790000 & -0.763568000 & -2.358968000 \\
\hline $\mathrm{C}$ & 0.294403000 & -1.499023000 & 1.087044000 \\
\hline $\mathrm{C}$ & -0.915649000 & -1.459834000 & 0.367856000 \\
\hline $\mathrm{H}$ & -0.808231000 & -1.537542000 & -0.710788000 \\
\hline $\mathrm{O}$ & 0.210216000 & -1.276069000 & 2.494067000 \\
\hline $\mathrm{C}$ & -2.173811000 & -1.242592000 & 0.869273000 \\
\hline $\mathrm{H}$ & -2.319341000 & -1.164607000 & 1.947255000 \\
\hline $\mathrm{C}$ & -3.385533000 & -1.061170000 & 0.069373000 \\
\hline $\mathrm{C}$ & -4.537248000 & -0.537836000 & 0.683587000 \\
\hline $\mathrm{C}$ & -3.458114000 & -1.332657000 & -1.308529000 \\
\hline $\mathrm{C}$ & -5.694695000 & -0.281744000 & -0.040432000 \\
\hline $\mathrm{H}$ & -4.514141000 & -0.323629000 & 1.750204000 \\
\hline $\mathrm{C}$ & -4.615269000 & -1.072332000 & -2.032495000 \\
\hline $\mathrm{H}$ & -2.606078000 & -1.770291000 & -1.821297000 \\
\hline $\mathrm{C}$ & -5.741523000 & -0.542209000 & -1.407770000 \\
\hline $\mathrm{H}$ & -6.565087000 & 0.125661000 & 0.466270000 \\
\hline $\mathrm{H}$ & -4.639184000 & -1.294042000 & -3.095892000 \\
\hline
\end{tabular}




$\begin{array}{lrcc}\mathrm{H} & -6.644586000 & -0.342319000 & -1.975957000 \\ \mathrm{H} & -1.535440000 & 0.752373000 & 1.076322000 \\ \mathrm{H} & -0.480574000 & -1.836947000 & 2.864435000 \\ \mathrm{C} & -0.684548000 & 1.635748000 & -1.135554000 \\ \mathrm{C} & -0.964806000 & 2.414699000 & 0.149891000 \\ \mathrm{H} & 0.272397000 & 1.104403000 & -1.071608000 \\ \mathrm{H} & -1.479060000 & 0.905876000 & -1.328121000 \\ \mathrm{H} & -0.640497000 & 2.319408000 & -1.989302000 \\ \mathrm{C} & -2.344626000 & 3.066609000 & 0.106823000 \\ \mathrm{C} & 0.134277000 & 3.432287000 & 0.428514000 \\ \mathrm{H} & -2.543896000 & 3.598778000 & 1.041911000 \\ \mathrm{H} & -2.409997000 & 3.778389000 & -0.722272000 \\ \mathrm{H} & -3.121543000 & 2.305998000 & -0.034039000 \\ \mathrm{H} & 0.162187000 & 4.192192000 & -0.357526000 \\ \mathrm{H} & -0.037468000 & 3.932458000 & 1.387112000 \\ \mathrm{H} & 1.118387000 & 2.946381000 & 0.453430000 \\ \mathrm{O} & -0.935120000 & 1.505081000 & 1.277272000 \\ \mathrm{~N} & 5.393895000 & 0.023997000 & -0.896818000 \\ \mathrm{O} & 5.559839000 & 0.050366000 & -2.112884000 \\ \mathrm{O} & 6.249777000 & 0.358953000 & -0.082485000 \\ \mathrm{Li} & 0.575699000 & 0.541500000 & 1.866025000 \\ - & \end{array}$

TSA2-3 ${ }_{\text {tBuOLi }}$

$\begin{array}{lrrr} & & \\ & \mathrm{X} & \mathrm{Y} & \mathrm{Z} \\ \mathrm{C} & -4.467974000 & 0.454694000 & -0.945564000 \\ \mathrm{C} & -3.145414000 & 0.541097000 & -1.351946000 \\ \mathrm{C} & -2.121546000 & -0.113030000 & -0.642135000 \\ \mathrm{C} & -2.479022000 & -0.853655000 & 0.503009000 \\ \mathrm{C} & -3.795600000 & -0.949507000 & 0.911614000 \\ \mathrm{C} & -4.781591000 & -0.290651000 & 0.182651000 \\ \mathrm{H} & -5.258492000 & 0.952795000 & -1.493518000 \\ \mathrm{H} & -2.896131000 & 1.109204000 & -2.240176000 \\ \mathrm{H} & -1.717334000 & -1.355896000 & 1.089508000 \\ \mathrm{H} & -4.071044000 & -1.517955000 & 1.791432000 \\ \mathrm{C} & -0.732690000 & 0.007675000 & -1.073322000 \\ \mathrm{C} & 0.351244000 & -0.680097000 & -0.626745000 \\ \mathrm{H} & 0.156109000 & -1.432990000 & 0.132694000 \\ \mathrm{O} & -0.536774000 & 1.032376000 & -2.038694000 \\ \mathrm{C} & 1.725212000 & -0.455134000 & -1.008464000 \\ \mathrm{H} & 1.875382000 & -0.208598000 & -2.067403000 \\ \mathrm{C} & 2.775052000 & -1.365810000 & -0.496459000 \\ \mathrm{C} & 3.916062000 & -1.638332000 & -1.265491000 \\ & & & \\ & & 5339 & \end{array}$




$\begin{array}{lrrr}\mathrm{C} & 2.718041000 & -1.923085000 & 0.790457000 \\ \mathrm{C} & 4.943258000 & -2.439598000 & -0.779950000 \\ \mathrm{H} & 3.993963000 & -1.213858000 & -2.264098000 \\ \mathrm{C} & 3.739282000 & -2.734228000 & 1.273266000 \\ \mathrm{H} & 1.870140000 & -1.707155000 & 1.436594000 \\ \mathrm{C} & 4.859793000 & -3.000277000 & 0.491901000 \\ \mathrm{H} & 5.811619000 & -2.633165000 & -1.403739000 \\ \mathrm{H} & 3.659977000 & -3.155169000 & 2.271845000 \\ \mathrm{H} & 5.658406000 & -3.631054000 & 0.869938000 \\ \mathrm{H} & 1.957647000 & 0.828864000 & -0.487968000 \\ \mathrm{H} & 0.116371000 & 0.731312000 & -2.685827000 \\ \mathrm{C} & 2.822387000 & 1.631903000 & 2.005040000 \\ \mathrm{C} & 2.928640000 & 2.425315000 & 0.697532000 \\ \mathrm{H} & 1.831529000 & 1.771735000 & 2.453939000 \\ \mathrm{H} & 2.971173000 & 0.563303000 & 1.816609000 \\ \mathrm{H} & 3.576766000 & 1.956865000 & 2.730231000 \\ \mathrm{C} & 4.289945000 & 2.175778000 & 0.038583000 \\ \mathrm{C} & 2.730750000 & 3.918734000 & 0.964916000 \\ \mathrm{H} & 4.352654000 & 2.716702000 & -0.911562000 \\ \mathrm{H} & 5.110048000 & 2.508547000 & 0.684523000 \\ \mathrm{H} & 4.425691000 & 1.107012000 & -0.161778000 \\ \mathrm{H} & 3.500128000 & 4.309545000 & 1.639965000 \\ \mathrm{H} & 2.777428000 & 4.480366000 & 0.025409000 \\ \mathrm{H} & 1.752697000 & 4.095928000 & 1.430259000 \\ \mathrm{O} & 1.906079000 & 2.014808000 & -0.188314000 \\ \mathrm{~N} & -6.172249000 & -0.381228000 & 0.617134000 \\ \mathrm{O} & -6.416056000 & -1.050043000 & 1.611812000 \\ \mathrm{O} & -7.014429000 & 0.217658000 & -0.037215000 \\ \mathrm{Li} & 0.195166000 & 2.134377000 & -0.590296000 \\ - & & & \\ & & & \end{array}$

\begin{tabular}{|c|c|c|c|}
\hline \multicolumn{4}{|c|}{ Coordinates (Angstroms) } \\
\hline & $X$ & $\mathrm{Y}$ & Z \\
\hline$\overline{\mathrm{C}}$ & -3.852529000 & -1.582469000 & 0.103319000 \\
\hline $\mathrm{C}$ & -2.607724000 & -2.152234000 & 0.325260000 \\
\hline $\mathrm{C}$ & -1.443866000 & -1.372360000 & 0.264379000 \\
\hline $\mathrm{C}$ & -1.532867000 & -0.006544000 & -0.034458000 \\
\hline $\mathrm{C}$ & -2.777941000 & 0.568689000 & -0.254907000 \\
\hline $\mathrm{C}$ & -3.915363000 & -0.225421000 & -0.184093000 \\
\hline $\mathrm{H}$ & -4.764047000 & -2.164357000 & 0.157949000 \\
\hline $\mathrm{H}$ & -2.546025000 & -3.205973000 & 0.583999000 \\
\hline $\mathrm{H}$ & -0.626584000 & 0.606637000 & -0.123626000 \\
\hline $\mathrm{H}$ & -2.870834000 & 1.621505000 & -0.493515000 \\
\hline
\end{tabular}




\begin{tabular}{lrrr}
$\mathrm{C}$ & -0.127443000 & -2.009857000 & 0.508387000 \\
$\mathrm{C}$ & 0.861687000 & -1.438928000 & 1.206784000 \\
$\mathrm{H}$ & 0.703392000 & -0.436866000 & 1.592803000 \\
$\mathrm{O}$ & 0.031497000 & -3.284692000 & 0.027303000 \\
$\mathrm{C}$ & 2.211694000 & -2.077683000 & 1.424663000 \\
$\mathrm{H}$ & 2.143243000 & -3.161149000 & 1.295271000 \\
$\mathrm{C}$ & 3.193417000 & -1.495770000 & 0.429379000 \\
$\mathrm{C}$ & 3.185980000 & -1.946346000 & -0.899755000 \\
$\mathrm{C}$ & 4.001624000 & -0.403052000 & 0.759878000 \\
$\mathrm{C}$ & 3.952600000 & -1.308113000 & -1.873611000 \\
$\mathrm{H}$ & 2.547602000 & -2.784267000 & -1.166226000 \\
$\mathrm{C}$ & 4.768616000 & 0.239722000 & -0.214068000 \\
$\mathrm{H}$ & 4.000110000 & -0.026001000 & 1.778716000 \\
$\mathrm{C}$ & 4.740711000 & -0.205095000 & -1.534505000 \\
$\mathrm{H}$ & 3.929483000 & -1.665559000 & -2.898597000 \\
$\mathrm{H}$ & 5.367686000 & 1.103066000 & 0.056743000 \\
$\mathrm{H}$ & 5.324780000 & 0.303390000 & -2.294511000 \\
$\mathrm{C}$ & 0.757724000 & 2.614514000 & 1.760671000 \\
$\mathrm{C}$ & 1.043482000 & 2.842936000 & 0.262947000 \\
$\mathrm{H}$ & -0.202722000 & 2.099348000 & 1.883855000 \\
$\mathrm{H}$ & 1.542649000 & 1.980073000 & 2.193293000 \\
$\mathrm{H}$ & 0.719091000 & 3.550570000 & 2.331765000 \\
$\mathrm{C}$ & 2.399276000 & 3.560206000 & 0.116713000 \\
$\mathrm{C}$ & -0.062512000 & 3.734617000 & -0.330264000 \\
$\mathrm{H}$ & 2.618813000 & 3.717387000 & -0.946159000 \\
$\mathrm{H}$ & 2.424154000 & 4.533257000 & 0.623509000 \\
$\mathrm{H}$ & 3.194616000 & 2.932886000 & 0.541903000 \\
$\mathrm{H}$ & -0.115517000 & 4.716944000 & 0.155496000 \\
$\mathrm{H}$ & 0.116232000 & 3.882504000 & -1.400809000 \\
$\mathrm{H}$ & -1.037252000 & 3.245754000 & -0.215515000 \\
$\mathrm{O}$ & 1.076889000 & 1.629759000 & -0.393604000 \\
$\mathrm{H}$ & 2.557444000 & -1.885074000 & 2.445558000 \\
$\mathrm{H}$ & -0.652431000 & -3.453318000 & -0.632427000 \\
$\mathrm{O}$ & -5.229705000 & 0.383909000 & -0.423523000 \\
$\mathrm{H}$ & -6.210227000 & -0.344433000 & -0.373368000 \\
$\mathrm{H}$ & -5.266453000 & 1.581129000 & -0.659087000 \\
-1336680000 & 0.375636000 & -0.778364000 \\
\hline
\end{tabular}

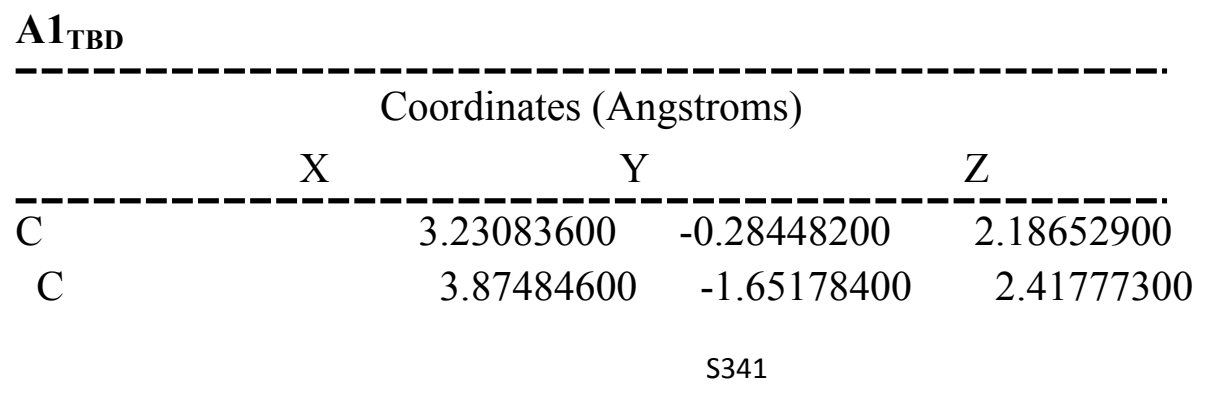


C

$\mathrm{N}$

$\mathrm{H}$

$\mathrm{H}$

$\mathrm{H}$

$\mathrm{H}$

$\mathrm{H}$

$\mathrm{N}$

C

C

C

$\mathrm{N}$

C

$\mathrm{H}$

$\mathrm{H}$

$\mathrm{H}$

$\mathrm{H}$

$\mathrm{H}$

$\mathrm{H}$

$\mathrm{H}$

C

C

C

C

C

C

$\mathrm{H}$

$\mathrm{H}$

$\mathrm{H}$

$\mathrm{H}$

C

C

$\mathrm{H}$

$\mathrm{O}$

C

$\mathrm{H}$

C

C

C

C

$\mathrm{H}$

C

$\mathrm{H}$

C

$\begin{array}{rrc}4.94305100 & -1.88760000 & 1.36059200 \\ 2.69157100 & -0.18769400 & 0.84434900 \\ 4.01322100 & 0.48284100 & 2.32590100 \\ 3.09847300 & -2.42335800 & 2.34016200 \\ 4.32252900 & -1.72456400 & 3.41490800 \\ 5.84114700 & -1.29152600 & 1.58286000 \\ 5.25059200 & -2.94206600 & 1.36074000 \\ 4.45157300 & -1.56000900 & 0.03133500 \\ 5.12534900 & -2.24623200 & -1.05894400 \\ 4.72723300 & -1.69792600 & -2.42136600 \\ 3.21596900 & -1.53008000 & -2.45726800 \\ 2.84751200 & -0.61948300 & -1.39818400 \\ 3.31630100 & -0.79013400 & -0.11509900 \\ 4.89648500 & -3.32376200 & -1.01063000 \\ 6.20890000 & -2.14515000 & -0.91126200 \\ 5.19520900 & -0.72204100 & -2.58741400 \\ 5.07082600 & -2.38274700 & -3.20276900 \\ 2.89316000 & -1.09626700 & -3.40794600 \\ 2.72698000 & -2.51459500 & -2.36017300 \\ 1.90733600 & -0.23131500 & -1.43039300 \\ -0.82313600 & 4.53473100 & -0.53992800 \\ -1.09425500 & 3.16899500 & -0.51267500 \\ -0.09187300 & 2.25505900 & -0.18058900 \\ 1.19034000 & 2.72362900 & 0.12018500 \\ 1.45802200 & 4.08831400 & 0.09568300 \\ 0.45379200 & 4.99643100 & -0.23444700 \\ -1.61036100 & 5.23798500 & -0.79639100 \\ -2.09480900 & 2.80870600 & -0.74103700 \\ 1.96265200 & 1.99800900 & 0.36979100 \\ 2.45583100 & 4.44608600 & 0.33403800 \\ -0.34488400 & 0.75652500 & -0.22805600 \\ -1.75199100 & 0.39119300 & 0.16356000 \\ -2.06859100 & 0.79261400 & 1.12534500 \\ 0.00461400 & 0.24504400 & -1.52104600 \\ -2.57437100 & -0.35294500 & -0.57822200 \\ -2.19677700 & -0.72067800 & -1.53089000 \\ -3.95827900 & -0.73630900 & -0.25256300 \\ -4.74179900 & -1.34831300 & -1.23521600 \\ -4.54613100 & -0.51856200 & 1.00185700 \\ -6.06058900 & -1.71450100 & -0.98571400 \\ -4.31063900 & -1.53593300 & -2.21576800 \\ -5.86018600 & -0.88489600 & 1.24788800 \\ -3.96777400 & -0.06439800 & 1.80118300 \\ -6.64510600 & -1.48849000 & 0.25878300 \\ & & \end{array}$




$\begin{array}{lrcc}\mathrm{H} & -6.64264400 & -2.18534000 & -1.77441000 \\ \mathrm{H} & -6.28942300 & -0.70437800 & 2.23096400 \\ \mathrm{H} & 0.36531700 & 0.27008600 & 0.45186200 \\ \mathrm{H} & -0.32137400 & 0.87853700 & -2.17363300 \\ \mathrm{C} & 2.11441000 & -0.00156800 & 3.18665200 \\ \mathrm{H} & 2.49031500 & -0.00469100 & 4.21635400 \\ \mathrm{H} & 1.66385900 & 0.97472000 & 2.98126400 \\ \mathrm{H} & 1.32942500 & -0.76143700 & 3.09801100 \\ \mathrm{H} & -7.66002162 & -1.76705513 & 0.45177979 \\ \mathrm{~N} & 0.74001023 & 6.43809374 & -0.25868877 \\ \mathrm{O} & -0.24313928 & 7.32124987 & -0.57970635 \\ \mathrm{O} & 1.87564478 & 6.84816439 & 0.01302797\end{array}$

TSA1-2 TBD

\begin{tabular}{|c|c|c|c|}
\hline \multicolumn{4}{|c|}{ Coordinates (Angstroms) } \\
\hline & $\mathrm{X}$ & $\mathrm{Y}$ & Z \\
\hline $\mathrm{C}$ & 1.300928000 & 2.029181000 & 1.632465000 \\
\hline $\mathrm{C}$ & 1.715123000 & 2.882991000 & 0.441079000 \\
\hline $\mathrm{C}$ & 0.575102000 & 3.830653000 & 0.097576000 \\
\hline $\mathrm{N}$ & 0.062663000 & 1.327852000 & 1.348854000 \\
\hline $\mathrm{H}$ & 1.185927000 & 2.653201000 & 2.529443000 \\
\hline $\mathrm{H}$ & 2.059703000 & 1.273837000 & 1.855184000 \\
\hline $\mathrm{H}$ & 1.932510000 & 2.225584000 & -0.408106000 \\
\hline $\mathrm{H}$ & 2.618431000 & 3.459501000 & 0.659424000 \\
\hline $\mathrm{H}$ & 0.507755000 & 4.639022000 & 0.839062000 \\
\hline $\mathrm{H}$ & 0.743632000 & 4.296526000 & -0.880558000 \\
\hline $\mathrm{N}$ & -0.704350000 & 3.127266000 & 0.037399000 \\
\hline $\mathrm{C}$ & -1.745513000 & 3.779834000 & -0.750790000 \\
\hline $\mathrm{C}$ & -3.115970000 & 3.151164000 & -0.535232000 \\
\hline $\mathrm{C}$ & -2.986473000 & 1.637023000 & -0.614374000 \\
\hline $\mathrm{N}$ & -2.010537000 & 1.228918000 & 0.373694000 \\
\hline $\mathrm{C}$ & -0.853710000 & 1.895845000 & 0.592727000 \\
\hline $\mathrm{H}$ & -1.471290000 & 3.728254000 & -1.814818000 \\
\hline $\mathrm{H}$ & -1.764226000 & 4.840277000 & -0.472132000 \\
\hline $\mathrm{H}$ & -3.506844000 & 3.425578000 & 0.449990000 \\
\hline $\mathrm{H}$ & -3.809079000 & 3.526507000 & -1.293199000 \\
\hline $\mathrm{H}$ & -3.928298000 & 1.134511000 & -0.381596000 \\
\hline $\mathrm{H}$ & -2.689621000 & 1.325891000 & -1.626615000 \\
\hline $\mathrm{H}$ & -2.050157000 & 0.283389000 & 0.739578000 \\
\hline $\mathrm{C}$ & 3.142977000 & -0.570985000 & -1.295664000 \\
\hline $\mathrm{C}$ & 1.889496000 & -0.731589000 & -0.736755000 \\
\hline $\mathrm{C}$ & 1.718825000 & -1.296005000 & 0.548167000 \\
\hline $\mathrm{C}$ & 2.880302000 & -1.677575000 & 1.249488000 \\
\hline
\end{tabular}




$\begin{array}{lrrr}\mathrm{C} & 4.139640000 & -1.521148000 & 0.698964000 \\ \mathrm{C} & 4.266138000 & -0.966086000 & -0.571977000 \\ \mathrm{H} & 3.270701000 & -0.137371000 & -2.280350000 \\ \mathrm{H} & 1.024010000 & -0.393736000 & -1.299658000 \\ \mathrm{H} & 2.770310000 & -2.087893000 & 2.246524000 \\ \mathrm{H} & 5.030282000 & -1.820545000 & 1.238485000 \\ \mathrm{C} & 0.401509000 & -1.340467000 & 1.193800000 \\ \mathrm{C} & -0.780605000 & -1.641738000 & 0.410189000 \\ \mathrm{H} & -0.696627000 & -1.453996000 & -0.659514000 \\ \mathrm{O} & 0.385666000 & -1.834971000 & 2.513563000 \\ \mathrm{C} & -1.998092000 & -1.966504000 & 0.906290000 \\ \mathrm{H} & -2.106658000 & -2.087267000 & 1.982426000 \\ \mathrm{C} & -3.231519000 & -2.003489000 & 0.109898000 \\ \mathrm{C} & -4.466727000 & -1.805688000 & 0.747838000 \\ \mathrm{C} & -3.238613000 & -2.152433000 & -1.286974000 \\ \mathrm{C} & -5.651332000 & -1.730098000 & 0.023478000 \\ \mathrm{H} & -4.489265000 & -1.694804000 & 1.829544000 \\ \mathrm{C} & -4.421542000 & -2.071801000 & -2.011595000 \\ \mathrm{H} & -2.307691000 & -2.352245000 & -1.810241000 \\ \mathrm{C} & -5.635618000 & -1.854486000 & -1.363654000 \\ \mathrm{H} & -6.591400000 & -1.572793000 & 0.544968000 \\ \mathrm{H} & -4.396359000 & -2.194250000 & -3.090975000 \\ \mathrm{H} & -6.559738000 & -1.797788000 & -1.930864000 \\ \mathrm{H} & 0.171255000 & 0.073323000 & 1.328279000 \\ \mathrm{H} & 0.260432000 & -2.794509000 & 2.467443000 \\ \mathrm{~N} & 5.585566000 & -0.784833000 & -1.149355000 \\ \mathrm{O} & 5.662106000 & -0.280448000 & -2.264498000 \\ \mathrm{O} & 6.556347000 & -1.141109000 & -0.492255000 \\ -----0 .- & \end{array}$

A2 TBD

\begin{tabular}{llcc} 
& \multicolumn{3}{c}{ Coordinates (Angstroms) } \\
C & X & Y & Z \\
C & 1.527042000 & 1.544351000 & 2.151942000 \\
$\mathrm{C}$ & 2.295462000 & 1.969997000 & 0.912988000 \\
$\mathrm{~N}$ & 1.542445000 & 3.099509000 & 0.230125000 \\
$\mathrm{H}$ & 1.536206000 & 2.336966000 & 2.910434000 \\
$\mathrm{H}$ & 1.959248000 & 0.641653000 & 2.585199000 \\
$\mathrm{H}$ & 2.389677000 & 1.117623000 & 0.232797000 \\
$\mathrm{H}$ & 3.301377000 & 2.303101000 & 1.179720000 \\
$\mathrm{H}$ & 1.588354000 & 4.024542000 & 0.819876000 \\
$\mathrm{H}$ & 1.979130000 & 3.307183000 & -0.751817000 \\
$\mathrm{~N}$ & 0.139885000 & 2.736602000 & 0.010576000
\end{tabular}




\begin{tabular}{|c|c|c|c|}
\hline $\mathrm{C}$ & -0.518080000 & 3.381771000 & -1.126101000 \\
\hline $\mathrm{C}$ & -2.027947000 & 3.192607000 & -1.090287000 \\
\hline $\mathrm{C}$ & -2.342538000 & 1.727662000 & -0.836639000 \\
\hline $\mathrm{N}$ & -1.681346000 & 1.331638000 & 0.396540000 \\
\hline $\mathrm{C}$ & -0.452436000 & 1.756413000 & 0.715464000 \\
\hline $\mathrm{H}$ & -0.099536000 & 2.969848000 & -2.054144000 \\
\hline $\mathrm{H}$ & -0.263181000 & 4.446410000 & -1.091379000 \\
\hline $\mathrm{H}$ & -2.464388000 & 3.801809000 & -0.291815000 \\
\hline $\mathrm{H}$ & -2.457031000 & 3.522415000 & -2.039799000 \\
\hline $\mathrm{H}$ & -3.412480000 & 1.553587000 & -0.705161000 \\
\hline $\mathrm{H}$ & -2.005751000 & 1.101803000 & -1.673779000 \\
\hline $\mathrm{H}$ & -1.953220000 & 0.409777000 & 0.762709000 \\
\hline $\mathrm{C}$ & 2.821714000 & -1.169959000 & -1.507294000 \\
\hline $\mathrm{C}$ & 1.570453000 & -1.401277000 & -0.985849000 \\
\hline $\mathrm{C}$ & 1.364889000 & -1.615626000 & 0.410867000 \\
\hline $\mathrm{C}$ & 2.534484000 & -1.614345000 & 1.228431000 \\
\hline $\mathrm{C}$ & 3.786700000 & -1.377871000 & 0.706925000 \\
\hline $\mathrm{C}$ & 3.938383000 & -1.137057000 & -0.662178000 \\
\hline $\mathrm{H}$ & 2.961485000 & -0.995066000 & -2.567648000 \\
\hline $\mathrm{H}$ & 0.722433000 & -1.396263000 & -1.663242000 \\
\hline $\mathrm{H}$ & 2.421776000 & -1.808446000 & 2.288878000 \\
\hline $\mathrm{H}$ & 4.664563000 & -1.375148000 & 1.342600000 \\
\hline $\mathrm{C}$ & 0.080354000 & -1.742564000 & 0.994565000 \\
\hline $\mathrm{C}$ & -1.145896000 & -1.751365000 & 0.333324000 \\
\hline $\mathrm{H}$ & -1.096144000 & -1.856372000 & -0.748032000 \\
\hline $\mathrm{O}$ & 0.046364000 & -1.564336000 & 2.395059000 \\
\hline $\mathrm{C}$ & -2.394103000 & -1.574382000 & 0.903182000 \\
\hline $\mathrm{H}$ & -2.474765000 & -1.463834000 & 1.984187000 \\
\hline $\mathrm{C}$ & -3.639210000 & -1.427182000 & 0.163467000 \\
\hline $\mathrm{C}$ & -4.778280000 & -0.919534000 & 0.821560000 \\
\hline $\mathrm{C}$ & -3.769293000 & -1.690112000 & -1.215811000 \\
\hline $\mathrm{C}$ & -5.963650000 & -0.668585000 & 0.143753000 \\
\hline $\mathrm{H}$ & -4.716674000 & -0.712185000 & 1.888093000 \\
\hline $\mathrm{C}$ & -4.955120000 & -1.434097000 & -1.892364000 \\
\hline $\mathrm{H}$ & -2.934965000 & -2.118038000 & -1.764657000 \\
\hline $\mathrm{C}$ & -6.062659000 & -0.915113000 & -1.224510000 \\
\hline $\mathrm{H}$ & -6.818102000 & -0.275065000 & 0.688222000 \\
\hline $\mathrm{H}$ & -5.018145000 & -1.651985000 & -2.955506000 \\
\hline $\mathrm{H}$ & -6.988996000 & -0.720554000 & -1.756078000 \\
\hline $\mathrm{H}$ & -0.215302000 & 0.347607000 & 2.150649000 \\
\hline $\mathrm{H}$ & -0.524161000 & -2.248110000 & 2.770108000 \\
\hline $\mathrm{N}$ & 5.234571000 & -0.838162000 & -1.200974000 \\
\hline $\mathrm{O}$ & 5.329319000 & -0.627219000 & -2.409872000 \\
\hline $\mathrm{O}$ & 6.191560000 & -0.793798000 & -0.428902000 \\
\hline
\end{tabular}


TSA2-3 TBD

\begin{tabular}{|c|c|c|c|}
\hline \multicolumn{4}{|c|}{ Coordinates (Angstroms) } \\
\hline & $\mathrm{X}$ & Y & Z \\
\hline $\bar{C}$ & 1.332890000 & 2.029787000 & 1.766777000 \\
\hline $\mathrm{C}$ & 1.902223000 & 2.394951000 & 0.405862000 \\
\hline $\mathrm{C}$ & 0.987967000 & 3.423442000 & -0.242159000 \\
\hline $\mathrm{N}$ & -0.061880000 & 1.640156000 & 1.634925000 \\
\hline $\mathrm{H}$ & 1.421476000 & 2.880766000 & 2.455438000 \\
\hline $\mathrm{H}$ & 1.872636000 & 1.185704000 & 2.201847000 \\
\hline $\mathrm{H}$ & 1.964097000 & 1.496912000 & -0.219296000 \\
\hline $\mathrm{H}$ & 2.909463000 & 2.807230000 & 0.509844000 \\
\hline $\mathrm{H}$ & 1.051005000 & 4.385567000 & 0.286530000 \\
\hline $\mathrm{H}$ & 1.287869000 & 3.600029000 & -1.281288000 \\
\hline $\mathrm{N}$ & -0.393410000 & 2.954694000 & -0.261956000 \\
\hline $\mathrm{C}$ & -1.251425000 & 3.515263000 & -1.299472000 \\
\hline $\mathrm{C}$ & -2.717627000 & 3.229037000 & -1.011812000 \\
\hline $\mathrm{C}$ & -2.870356000 & 1.755034000 & -0.665392000 \\
\hline $\mathrm{N}$ & -2.025786000 & 1.418817000 & 0.468123000 \\
\hline $\mathrm{C}$ & -0.846333000 & 1.996045000 & 0.589169000 \\
\hline $\mathrm{H}$ & -0.963757000 & 3.095711000 & -2.274260000 \\
\hline $\mathrm{H}$ & -1.068103000 & 4.595687000 & -1.340781000 \\
\hline $\mathrm{H}$ & -3.058650000 & 3.838401000 & -0.167614000 \\
\hline $\mathrm{H}$ & -3.320276000 & 3.494025000 & -1.885447000 \\
\hline $\mathrm{H}$ & -3.902237000 & 1.512324000 & -0.398690000 \\
\hline $\mathrm{H}$ & -2.615584000 & 1.134018000 & -1.537378000 \\
\hline $\mathrm{H}$ & -2.114838000 & 0.202141000 & 0.789789000 \\
\hline $\mathrm{C}$ & 3.151600000 & -1.213232000 & -1.373318000 \\
\hline $\mathrm{C}$ & 1.873350000 & -1.319323000 & -0.860900000 \\
\hline $\mathrm{C}$ & 1.640711000 & -1.390752000 & 0.530489000 \\
\hline $\mathrm{C}$ & 2.764005000 & -1.350081000 & 1.382251000 \\
\hline $\mathrm{C}$ & 4.047937000 & -1.233182000 & 0.878239000 \\
\hline $\mathrm{C}$ & 4.234488000 & -1.160942000 & -0.497750000 \\
\hline $\mathrm{H}$ & 3.326084000 & -1.152150000 & -2.440601000 \\
\hline $\mathrm{H}$ & 1.038510000 & -1.324067000 & -1.553337000 \\
\hline $\mathrm{H}$ & 2.608693000 & -1.410341000 & 2.452885000 \\
\hline $\mathrm{H}$ & 4.907694000 & -1.199384000 & 1.536415000 \\
\hline $\mathrm{C}$ & 0.303218000 & -1.422680000 & 1.093282000 \\
\hline $\mathrm{C}$ & -0.864250000 & -1.535613000 & 0.411096000 \\
\hline $\mathrm{H}$ & -0.785914000 & -1.774192000 & -0.645441000 \\
\hline $\mathrm{O}$ & 0.268546000 & -1.128635000 & 2.462071000 \\
\hline $\mathrm{C}$ & -2.172265000 & -1.233498000 & 0.933469000 \\
\hline $\mathrm{H}$ & -2.293253000 & -1.322667000 & 2.016907000 \\
\hline
\end{tabular}




\begin{tabular}{lrrr}
$\mathrm{C}$ & -3.403816000 & -1.581796000 & 0.196678000 \\
$\mathrm{C}$ & -4.628498000 & -1.637170000 & 0.883704000 \\
$\mathrm{C}$ & -3.448139000 & -1.763348000 & -1.196289000 \\
$\mathrm{C}$ & -5.828508000 & -1.860762000 & 0.221032000 \\
$\mathrm{H}$ & -4.631664000 & -1.497165000 & 1.962673000 \\
$\mathrm{C}$ & -4.648432000 & -1.992006000 & -1.860334000 \\
$\mathrm{H}$ & -2.532459000 & -1.721250000 & -1.780543000 \\
$\mathrm{C}$ & -5.850293000 & -2.041271000 & -1.160125000 \\
$\mathrm{H}$ & -6.754563000 & -1.898561000 & 0.788737000 \\
$\mathrm{H}$ & -4.641740000 & -2.133256000 & -2.938122000 \\
$\mathrm{H}$ & -6.786874000 & -2.218914000 & -1.679699000 \\
$\mathrm{H}$ & -0.322220000 & 0.779668000 & 2.106700000 \\
$\mathrm{H}$ & -0.419314000 & -1.676171000 & 2.862744000 \\
$\mathrm{~N}$ & 5.578787000 & -1.015448000 & -1.033596000 \\
$\mathrm{O}$ & 6.510343000 & -0.961822000 & -0.240417000 \\
$\mathrm{O}$ & 5.707269000 & -0.948920000 & -2.250088000 \\
\hline- - - & &
\end{tabular}

\begin{tabular}{lccc} 
A3 & \multicolumn{3}{c}{ Coordinates (Angstroms) } \\
& $\mathrm{X}$ & $\mathrm{Y}$ & $\mathrm{Z}$ \\
$\mathrm{C}$ & -0.967170000 & -2.567962000 & 0.542498000 \\
$\mathrm{C}$ & -1.668356000 & -2.240161000 & 1.853030000 \\
$\mathrm{C}$ & -3.170653000 & -2.135103000 & 1.619292000 \\
$\mathrm{~N}$ & -1.312281000 & -1.540841000 & -0.409461000 \\
$\mathrm{H}$ & -1.254705000 & -3.578895000 & 0.204239000 \\
$\mathrm{H}$ & 0.119224000 & -2.561851000 & 0.675755000 \\
$\mathrm{H}$ & -1.281060000 & -1.288168000 & 2.225899000 \\
$\mathrm{H}$ & -1.472353000 & -3.007619000 & 2.608538000 \\
$\mathrm{H}$ & -3.618233000 & -3.142545000 & 1.619427000 \\
$\mathrm{H}$ & -3.633566000 & -1.581820000 & 2.447778000 \\
$\mathrm{~N}$ & -3.511899000 & -1.465462000 & 0.376502000 \\
$\mathrm{C}$ & -4.904567000 & -1.075785000 & 0.239921000 \\
$\mathrm{C}$ & -5.295820000 & -0.995735000 & -1.228702000 \\
$\mathrm{C}$ & -4.259173000 & -0.154924000 & -1.971225000 \\
$\mathrm{~N}$ & -2.904587000 & -0.619397000 & -1.765874000 \\
$\mathrm{C}$ & -2.628466000 & -1.194060000 & -0.644527000 \\
$\mathrm{H}$ & -5.068331000 & -0.108099000 & 0.737385000 \\
$\mathrm{H}$ & -5.519259000 & -1.822016000 & 0.759877000 \\
$\mathrm{H}$ & -5.319453000 & -2.005984000 & -1.654646000 \\
$\mathrm{H}$ & -6.299032000 & -0.566677000 & -1.324305000 \\
$\mathrm{H}$ & -4.468837000 & -0.168045000 & -3.047474000 \\
$\mathrm{H}$ & -4.352965000 & 0.893901000 & -1.647319000 \\
$\mathrm{C}$ & 4.959608000 & 1.248778000 & 0.028091000
\end{tabular}




$\begin{array}{lrrr}\mathrm{C} & 3.655404000 & 1.451970000 & -0.393864000 \\ \mathrm{C} & 2.826584000 & 0.368734000 & -0.716313000 \\ \mathrm{C} & 3.347794000 & -0.929861000 & -0.630487000 \\ \mathrm{C} & 4.653605000 & -1.148304000 & -0.216031000 \\ \mathrm{C} & 5.440783000 & -0.052329000 & 0.109499000 \\ \mathrm{H} & 5.610468000 & 2.077497000 & 0.277805000 \\ \mathrm{H} & 3.277336000 & 2.463621000 & -0.497160000 \\ \mathrm{H} & 2.713962000 & -1.778273000 & -0.868682000 \\ \mathrm{H} & 5.065066000 & -2.146806000 & -0.134879000 \\ \mathrm{C} & 1.427854000 & 0.575757000 & -1.154657000 \\ \mathrm{C} & 0.643628000 & 1.560376000 & -0.702849000 \\ \mathrm{H} & 1.061479000 & 2.219805000 & 0.054438000 \\ \mathrm{O} & 0.925456000 & -0.377065000 & -2.012637000 \\ \mathrm{C} & -0.770342000 & 1.817388000 & -1.125051000 \\ \mathrm{H} & -1.131396000 & 1.016041000 & -1.780797000 \\ \mathrm{C} & -1.752600000 & 1.950423000 & 0.021611000 \\ \mathrm{C} & -2.949203000 & 2.646403000 & -0.167300000 \\ \mathrm{C} & -1.529186000 & 1.345778000 & 1.258088000 \\ \mathrm{C} & -3.905764000 & 2.717930000 & 0.839589000 \\ \mathrm{H} & -3.136796000 & 3.128871000 & -1.123867000 \\ \mathrm{C} & -2.482541000 & 1.413915000 & 2.270556000 \\ \mathrm{H} & -0.605330000 & 0.798030000 & 1.418530000 \\ \mathrm{C} & -3.677499000 & 2.096556000 & 2.065569000 \\ \mathrm{H} & -4.831587000 & 3.259625000 & 0.667247000 \\ \mathrm{H} & -2.289474000 & 0.930632000 & 3.224992000 \\ \mathrm{H} & -4.421342000 & 2.151561000 & 2.855073000 \\ \mathrm{H} & -0.730133000 & -1.424321000 & -1.228246000 \\ \mathrm{H} & 1.611091000 & -0.630041000 & -2.643519000 \\ \mathrm{H} & -0.793943000 & 2.746272000 & -1.713256000 \\ \mathrm{~N} & 6.823898000 & -0.274094000 & 0.543908000 \\ \mathrm{O} & 7.493563000 & 0.707596000 & 0.826529000 \\ \mathrm{O} & 7.223450000 & -1.427384000 & 0.597509000 \\ ------- & \end{array}$

\begin{tabular}{|c|c|c|c|}
\hline \multicolumn{4}{|c|}{ A1 $1_{\text {DBU }}$} \\
\hline & \multicolumn{3}{|c|}{ Coordinates (Angstroms) } \\
\hline & $X$ & $\mathrm{Y}$ & Z \\
\hline$\overline{\mathrm{C}}$ & -1.395927000 & 3.579580000 & -0.533540000 \\
\hline $\mathrm{C}$ & -1.706093000 & 2.225409000 & -0.485665000 \\
\hline $\mathrm{C}$ & -0.714111000 & 1.272552000 & -0.241697000 \\
\hline $\mathrm{C}$ & 0.605730000 & 1.692775000 & -0.049501000 \\
\hline $\mathrm{C}$ & 0.932842000 & 3.039801000 & -0.090409000 \\
\hline $\mathrm{C}$ & -0.077366000 & 3.963468000 & -0.332859000 \\
\hline $\mathrm{H}$ & -2.153130000 & 4.331483000 & -0.718206000 \\
\hline
\end{tabular}




\begin{tabular}{|c|c|c|c|}
\hline $\mathrm{H}$ & -2.734800000 & 1.906986000 & -0.631712000 \\
\hline $\mathrm{H}$ & 1.365780000 & 0.940285000 & 0.142978000 \\
\hline $\mathrm{H}$ & 1.948023000 & 3.385175000 & 0.062698000 \\
\hline $\mathrm{C}$ & -1.019350000 & -0.220586000 & -0.260828000 \\
\hline $\mathrm{C}$ & -2.412189000 & -0.529682000 & 0.223465000 \\
\hline $\mathrm{H}$ & -2.672715000 & -0.082076000 & 1.181800000 \\
\hline $\mathrm{O}$ & -0.752418000 & -0.746405000 & -1.555382000 \\
\hline $\mathrm{C}$ & -3.286031000 & -1.289091000 & -0.439908000 \\
\hline $\mathrm{H}$ & -2.970724000 & -1.705986000 & -1.395088000 \\
\hline $\mathrm{C}$ & -4.656926000 & -1.625825000 & -0.018266000 \\
\hline $\mathrm{C}$ & -5.545561000 & -2.153392000 & -0.962513000 \\
\hline $\mathrm{C}$ & -5.119041000 & -1.440009000 & 1.291507000 \\
\hline $\mathrm{C}$ & -6.857428000 & -2.464463000 & -0.621160000 \\
\hline $\mathrm{H}$ & -5.201176000 & -2.315121000 & -1.981199000 \\
\hline $\mathrm{C}$ & -6.428514000 & -1.750266000 & 1.635107000 \\
\hline $\mathrm{H}$ & -4.443619000 & -1.063844000 & 2.054386000 \\
\hline $\mathrm{C}$ & -7.305709000 & -2.260495000 & 0.680041000 \\
\hline $\mathrm{H}$ & -7.529983000 & -2.866962000 & -1.373026000 \\
\hline $\mathrm{H}$ & -6.765542000 & -1.599606000 & 2.656619000 \\
\hline $\mathrm{H}$ & -8.329103000 & -2.502401000 & 0.950632000 \\
\hline $\mathrm{H}$ & -0.287542000 & -0.717153000 & 0.390322000 \\
\hline $\mathrm{H}$ & -1.248381000 & -0.214007000 & -2.191880000 \\
\hline $\mathrm{C}$ & 5.303872000 & -2.182931000 & 0.056316000 \\
\hline $\mathrm{C}$ & 4.955983000 & -3.401926000 & -0.792070000 \\
\hline $\mathrm{C}$ & 2.387933000 & -2.120587000 & -0.889208000 \\
\hline $\mathrm{C}$ & 4.384008000 & -3.046885000 & -2.166741000 \\
\hline $\mathrm{C}$ & 3.332911000 & -1.937760000 & -2.078842000 \\
\hline $\mathrm{H}$ & 5.849764000 & -1.447615000 & -0.557272000 \\
\hline $\mathrm{H}$ & 2.159134000 & -3.185478000 & -0.749869000 \\
\hline $\mathrm{H}$ & 4.260428000 & -4.034551000 & -0.229186000 \\
\hline $\mathrm{H}$ & 3.938591000 & -3.946083000 & -2.609319000 \\
\hline $\mathrm{H}$ & 6.000630000 & -2.503117000 & 0.841646000 \\
\hline $\mathrm{H}$ & 5.870754000 & -3.991568000 & -0.918709000 \\
\hline $\mathrm{H}$ & 1.430653000 & -1.635532000 & -1.088609000 \\
\hline $\mathrm{H}$ & 5.195393000 & -2.736627000 & -2.836571000 \\
\hline $\mathrm{H}$ & 2.747580000 & -1.911757000 & -3.003747000 \\
\hline $\mathrm{H}$ & 3.823341000 & -0.958960000 & -2.002328000 \\
\hline $\mathrm{C}$ & 2.860468000 & -1.540448000 & 0.434059000 \\
\hline $\mathrm{N}$ & 1.923812000 & -1.063906000 & 1.184127000 \\
\hline $\mathrm{N}$ & 4.200840000 & -1.524315000 & 0.754553000 \\
\hline $\mathrm{C}$ & 4.674863000 & -0.729113000 & 1.885860000 \\
\hline $\mathrm{H}$ & 4.975048000 & -1.403400000 & 2.702080000 \\
\hline $\mathrm{H}$ & 5.576643000 & -0.188301000 & 1.569316000 \\
\hline $\mathrm{C}$ & 3.620326000 & 0.248863000 & 2.373865000 \\
\hline
\end{tabular}




$\begin{array}{lrrr}\mathrm{H} & 3.923616000 & 0.670868000 & 3.337019000 \\ \mathrm{H} & 3.522210000 & 1.078612000 & 1.663233000 \\ \mathrm{C} & 2.290527000 & -0.490269000 & 2.463689000 \\ \mathrm{H} & 1.490020000 & 0.188972000 & 2.777335000 \\ \mathrm{H} & 2.356328000 & -1.279122000 & 3.228966000 \\ \mathrm{~N} & 0.260573000 & 5.390878000 & -0.374837000 \\ \mathrm{O} & 1.426775000 & 5.704460000 & -0.187602000 \\ \mathrm{O} & -0.642495000 & 6.183941000 & -0.594943000\end{array}$

TSA1-2 DBU

$\begin{array}{lrcc} & & \text { Coordinates (Angstroms) } & \\ & \mathrm{X} & \mathrm{Y} & \mathrm{Z} \\ \mathrm{C} & 1.436720000 & 2.287472000 & 1.118277000 \\ \mathrm{C} & 0.297472000 & 1.644576000 & 0.679613000 \\ \mathrm{C} & 0.096651000 & 1.330862000 & -0.688651000 \\ \mathrm{C} & 1.107206000 & 1.734101000 & -1.592106000 \\ \mathrm{C} & 2.249348000 & 2.381695000 & -1.162772000 \\ \mathrm{C} & 2.421664000 & 2.644165000 & 0.195815000 \\ \mathrm{H} & 1.586013000 & 2.514368000 & 2.167413000 \\ \mathrm{H} & -0.451989000 & 1.358867000 & 1.412689000 \\ \mathrm{H} & 0.975099000 & 1.510271000 & -2.644620000 \\ \mathrm{H} & 3.017809000 & 2.685870000 & -1.863624000 \\ \mathrm{C} & -0.996994000 & 0.475442000 & -1.118934000 \\ \mathrm{C} & -2.314044000 & 0.587384000 & -0.511632000 \\ \mathrm{H} & -2.363244000 & 1.119026000 & 0.438352000 \\ \mathrm{O} & -1.001236000 & 0.147032000 & -2.493425000 \\ \mathrm{C} & -3.447114000 & 0.059018000 & -1.020347000 \\ \mathrm{H} & -3.374732000 & -0.521389000 & -1.938456000 \\ \mathrm{C} & -4.780907000 & 0.143170000 & -0.417731000 \\ \mathrm{C} & -5.784409000 & -0.737085000 & -0.850669000 \\ \mathrm{C} & -5.117622000 & 1.068725000 & 0.583105000 \\ \mathrm{C} & -7.058420000 & -0.712698000 & -0.295845000 \\ \mathrm{H} & -5.551038000 & -1.457852000 & -1.630915000 \\ \mathrm{C} & -6.389064000 & 1.090755000 & 1.142418000 \\ \mathrm{H} & -4.381770000 & 1.796713000 & 0.913378000 \\ \mathrm{C} & -7.368272000 & 0.199225000 & 0.709218000 \\ \mathrm{H} & -7.812173000 & -1.410720000 & -0.649751000 \\ \mathrm{H} & -6.622127000 & 1.820143000 & 1.913361000 \\ \mathrm{H} & -8.362992000 & 0.222978000 & 1.144115000 \\ \mathrm{H} & -0.488613000 & -0.797323000 & -0.489670000 \\ \mathrm{H} & -1.586312000 & 0.774092000 & -2.946369000 \\ \mathrm{C} & 3.478009000 & -2.780219000 & 0.827543000 \\ \mathrm{C} & 3.951037000 & -3.286796000 & -0.529867000 \\ & & & \end{array}$




$\begin{array}{lrrr}\mathrm{C} & 1.745388000 & -1.695678000 & -1.445222000 \\ \mathrm{C} & 4.242390000 & -2.174037000 & -1.540052000 \\ \mathrm{C} & 3.151046000 & -1.100613000 & -1.554927000 \\ \mathrm{H} & 4.104706000 & -1.940244000 & 1.159492000 \\ \mathrm{H} & 1.680978000 & -2.644054000 & -1.993753000 \\ \mathrm{H} & 3.209716000 & -3.993028000 & -0.921567000 \\ \mathrm{H} & 4.339227000 & -2.622105000 & -2.536001000 \\ \mathrm{H} & 3.611574000 & -3.583034000 & 1.560918000 \\ \mathrm{H} & 4.861991000 & -3.870352000 & -0.358911000 \\ \mathrm{H} & 1.009986000 & -1.041189000 & -1.918145000 \\ \mathrm{H} & 5.208374000 & -1.709545000 & -1.311175000 \\ \mathrm{H} & 3.216094000 & -0.516291000 & -2.477127000 \\ \mathrm{H} & 3.301236000 & -0.381141000 & -0.741482000 \\ \mathrm{C} & 1.256373000 & -1.937987000 & -0.034929000 \\ \mathrm{~N} & -0.014711000 & -1.686436000 & 0.162043000 \\ \mathrm{~N} & 2.064575000 & -2.388881000 & 0.945194000 \\ \mathrm{C} & 1.596181000 & -2.432529000 & 2.337435000 \\ \mathrm{H} & 1.372722000 & -3.475333000 & 2.600039000 \\ \mathrm{H} & 2.425321000 & -2.102716000 & 2.971955000 \\ \mathrm{C} & 0.384786000 & -1.544525000 & 2.558015000 \\ \mathrm{H} & -0.030977000 & -1.727740000 & 3.552382000 \\ \mathrm{H} & 0.685071000 & -0.493163000 & 2.499824000 \\ \mathrm{C} & -0.632852000 & -1.837377000 & 1.466315000 \\ \mathrm{H} & -1.482663000 & -1.149055000 & 1.503054000 \\ \mathrm{H} & -1.032883000 & -2.853814000 & 1.568011000 \\ \mathrm{~N} & 3.636272000 & 3.278744000 & 0.657255000 \\ \mathrm{O} & 4.487931000 & 3.573423000 & -0.175411000 \\ \mathrm{O} & 3.761454000 & 3.482232000 & 1.861428000 \\ ----0 . & \end{array}$

\begin{tabular}{|c|c|c|c|}
\hline \multicolumn{4}{|c|}{ A2 DBU } \\
\hline & \multicolumn{3}{|c|}{ Coordinates (Angstroms) } \\
\hline & $\mathrm{X}$ & Y & Z \\
\hline$\overline{\mathrm{C}}$ & -2.528335000 & -2.026280000 & 0.231470000 \\
\hline $\mathrm{C}$ & -1.299322000 & -1.818450000 & -0.344351000 \\
\hline $\mathrm{C}$ & -1.122897000 & -0.938285000 & -1.461351000 \\
\hline $\mathrm{C}$ & -2.326363000 & -0.405477000 & -2.026220000 \\
\hline $\mathrm{C}$ & -3.556545000 & -0.612285000 & -1.447544000 \\
\hline $\mathrm{C}$ & -3.666032000 & -1.389924000 & -0.285833000 \\
\hline $\mathrm{H}$ & -2.640446000 & -2.688613000 & 1.082787000 \\
\hline $\mathrm{H}$ & -0.448267000 & -2.357334000 & 0.058356000 \\
\hline $\mathrm{H}$ & -2.246902000 & 0.197788000 & -2.923171000 \\
\hline $\mathrm{H}$ & -4.452608000 & -0.175600000 & -1.873435000 \\
\hline $\mathrm{C}$ & 0.139997000 & -0.545552000 & -1.953993000 \\
\hline
\end{tabular}




\begin{tabular}{|c|c|c|c|}
\hline $\mathrm{C}$ & 1.376918000 & -0.877188000 & -1.384022000 \\
\hline $\mathrm{H}$ & 1.349861000 & -1.635006000 & -0.605001000 \\
\hline $\mathrm{O}$ & 0.142227000 & 0.405940000 & -2.980694000 \\
\hline $\mathrm{C}$ & 2.595892000 & -0.297854000 & -1.646640000 \\
\hline $\mathrm{H}$ & 2.656111000 & 0.504532000 & -2.380208000 \\
\hline $\mathrm{C}$ & 3.807729000 & -0.590238000 & -0.896047000 \\
\hline $\mathrm{C}$ & 4.847599000 & 0.360885000 & -0.834190000 \\
\hline $\mathrm{C}$ & 3.987583000 & -1.775186000 & -0.149708000 \\
\hline $\mathrm{C}$ & 5.977885000 & 0.158072000 & -0.051999000 \\
\hline $\mathrm{H}$ & 4.751854000 & 1.279671000 & -1.410023000 \\
\hline $\mathrm{C}$ & 5.116468000 & -1.972377000 & 0.635653000 \\
\hline $\mathrm{H}$ & 3.242088000 & -2.563300000 & -0.210357000 \\
\hline $\mathrm{C}$ & 6.119556000 & -1.006049000 & 0.700454000 \\
\hline $\mathrm{H}$ & 6.754341000 & 0.918016000 & -0.028642000 \\
\hline $\mathrm{H}$ & 5.223108000 & -2.901612000 & 1.189563000 \\
\hline $\mathrm{H}$ & 7.003452000 & -1.166633000 & 1.309700000 \\
\hline $\mathrm{H}$ & 2.399694000 & 1.151548000 & 0.347927000 \\
\hline $\mathrm{H}$ & 0.712738000 & 0.069184000 & -3.683880000 \\
\hline $\mathrm{C}$ & -1.938816000 & 1.571034000 & 1.344571000 \\
\hline $\mathrm{C}$ & -2.010897000 & 3.087672000 & 1.174000000 \\
\hline $\mathrm{C}$ & 0.416467000 & 2.401804000 & -0.418764000 \\
\hline $\mathrm{C}$ & -1.874730000 & 3.552724000 & -0.281232000 \\
\hline $\mathrm{C}$ & -0.925074000 & 2.673796000 & -1.090048000 \\
\hline $\mathrm{H}$ & -2.451242000 & 1.031051000 & 0.541348000 \\
\hline $\mathrm{H}$ & 0.883812000 & 3.339302000 & -0.087181000 \\
\hline $\mathrm{H}$ & -1.231516000 & 3.538595000 & 1.801473000 \\
\hline $\mathrm{H}$ & -1.540243000 & 4.597097000 & -0.295507000 \\
\hline $\mathrm{H}$ & -2.432158000 & 1.293267000 & 2.278706000 \\
\hline $\mathrm{H}$ & -2.965519000 & 3.432443000 & 1.583970000 \\
\hline $\mathrm{H}$ & 1.086930000 & 1.959445000 & -1.167171000 \\
\hline $\mathrm{H}$ & -2.857072000 & 3.532036000 & -0.765856000 \\
\hline $\mathrm{H}$ & -0.714567000 & 3.140808000 & -2.056286000 \\
\hline $\mathrm{H}$ & -1.401557000 & 1.722176000 & -1.328746000 \\
\hline $\mathrm{C}$ & 0.473997000 & 1.457179000 & 0.759304000 \\
\hline $\mathrm{N}$ & 1.710931000 & 1.084929000 & 1.089693000 \\
\hline $\mathrm{N}$ & -0.561715000 & 1.050473000 & 1.475794000 \\
\hline $\mathrm{C}$ & -0.424859000 & 0.010803000 & 2.510546000 \\
\hline $\mathrm{H}$ & -0.503120000 & 0.499394000 & 3.490002000 \\
\hline $\mathrm{H}$ & -1.278072000 & -0.661653000 & 2.392457000 \\
\hline $\mathrm{C}$ & 0.872480000 & -0.769222000 & 2.394350000 \\
\hline $\mathrm{H}$ & 1.014966000 & -1.366746000 & 3.298204000 \\
\hline $\mathrm{H}$ & 0.819883000 & -1.450408000 & 1.542567000 \\
\hline $\mathrm{C}$ & 2.031544000 & 0.192058000 & 2.193722000 \\
\hline $\mathrm{H}$ & 2.952831000 & -0.334014000 & 1.935837000 \\
\hline
\end{tabular}




\begin{tabular}{lrrr}
$\mathrm{H}$ & 2.210860000 & 0.784783000 & 3.098537000 \\
$\mathrm{~N}$ & -4.929468000 & -1.547982000 & 0.363470000 \\
$\mathrm{O}$ & -5.915658000 & -0.999267000 & -0.129373000 \\
$\mathrm{O}$ & -4.973937000 & -2.213186000 & 1.401137000 \\
\hline
\end{tabular}

TSA2-3 DBU

\begin{tabular}{lccc} 
& Coordinates (Angstroms) & \\
& $\mathrm{X}$ & $\mathrm{Y}$ & $\mathrm{Z}$ \\
\hline $\mathrm{C}$ & 2.768291000 & -1.317741000 & -1.168362000 \\
$\mathrm{C}$ & 1.505042000 & -1.221024000 & -0.618036000 \\
$\mathrm{C}$ & 1.284447000 & -1.414818000 & 0.763480000 \\
$\mathrm{C}$ & 2.406163000 & -1.687806000 & 1.572257000 \\
$\mathrm{C}$ & 3.676791000 & -1.777966000 & 1.032540000 \\
$\mathrm{C}$ & 3.851640000 & -1.589979000 & -0.335421000 \\
$\mathrm{H}$ & 2.935971000 & -1.166771000 & -2.227971000 \\
$\mathrm{H}$ & 0.674304000 & -0.959293000 & -1.264458000 \\
$\mathrm{H}$ & 2.260081000 & -1.820514000 & 2.637376000 \\
$\mathrm{H}$ & 4.537410000 & -1.991116000 & 1.654914000 \\
$\mathrm{C}$ & -0.028052000 & -1.246042000 & 1.361823000 \\
$\mathrm{C}$ & -1.205485000 & -1.241680000 & 0.683353000 \\
$\mathrm{H}$ & -1.149267000 & -1.538729000 & -0.360706000 \\
$\mathrm{O}$ & 0.010045000 & -0.987731000 & 2.724589000 \\
$\mathrm{C}$ & -2.464125000 & -0.728873000 & 1.143003000 \\
$\mathrm{H}$ & -2.557176000 & -0.515332000 & 2.210763000 \\
$\mathrm{C}$ & -3.740045000 & -1.078709000 & 0.495191000 \\
$\mathrm{C}$ & -4.927990000 & -0.476388000 & 0.950803000 \\
$\mathrm{C}$ & -3.842418000 & -1.923844000 & -0.623791000 \\
$\mathrm{C}$ & -6.143243000 & -0.688093000 & 0.315039000 \\
$\mathrm{H}$ & -4.881879000 & 0.182725000 & 1.815386000 \\
$\mathrm{C}$ & -5.059453000 & -2.126044000 & -1.267983000 \\
$\mathrm{H}$ & -2.964380000 & -2.449754000 & -0.987991000 \\
$\mathrm{C}$ & -6.219322000 & -1.509587000 & -0.809143000 \\
$\mathrm{H}$ & -7.038786000 & -0.205463000 & 0.697549000 \\
$\mathrm{H}$ & -5.100951000 & -2.787940000 & -2.129350000 \\
$\mathrm{H}$ & -7.168597000 & -1.674323000 & -1.309615000 \\
$\mathrm{H}$ & -2.228691000 & 0.671979000 & 0.607099000 \\
$\mathrm{H}$ & -0.812478000 & -1.325098000 & 3.103118000 \\
$\mathrm{C}$ & 1.542474000 & 2.761540000 & -0.901935000 \\
$\mathrm{C}$ & 1.753442000 & 3.794618000 & 0.202435000 \\
$\mathrm{C}$ & -0.183629000 & 2.075545000 & 1.526227000 \\
$\mathrm{C}$ & 2.116549000 & 3.173442000 & 1.554560000 \\
$\mathrm{H}$ & 1.302101000 & 1.912471000 & 1.836929000 \\
& 2.292994000 & 1.961990000 & -0.846639000
\end{tabular}




$\begin{array}{lrrr}\mathrm{H} & -0.554599000 & 3.041438000 & 1.895133000 \\ \mathrm{H} & 0.844074000 & 4.402395000 & 0.283895000 \\ \mathrm{H} & 1.953902000 & 3.916824000 & 2.344292000 \\ \mathrm{H} & 1.676784000 & 3.254212000 & -1.870554000 \\ \mathrm{H} & 2.547782000 & 4.477032000 & -0.117378000 \\ \mathrm{H} & -0.749801000 & 1.312971000 & 2.070742000 \\ \mathrm{H} & 3.183964000 & 2.924791000 & 1.572927000 \\ \mathrm{H} & 1.404366000 & 1.621700000 & 2.886173000 \\ \mathrm{H} & 1.699176000 & 1.070436000 & 1.263116000 \\ \mathrm{C} & -0.622878000 & 1.945166000 & 0.084810000 \\ \mathrm{~N} & -1.888758000 & 1.613325000 & -0.067960000 \\ \mathrm{~N} & 0.198606000 & 2.165789000 & -0.960682000 \\ \mathrm{C} & -0.186302000 & 1.790096000 & -2.327841000 \\ \mathrm{H} & -0.325414000 & 2.711416000 & -2.909218000 \\ \mathrm{H} & 0.659855000 & 1.249799000 & -2.768050000 \\ \mathrm{C} & -1.446847000 & 0.943597000 & -2.374625000 \\ \mathrm{H} & -1.833838000 & 0.923248000 & -3.397295000 \\ \mathrm{H} & -1.233663000 & -0.089546000 & -2.083231000 \\ \mathrm{C} & -2.460815000 & 1.531388000 & -1.403710000 \\ \mathrm{H} & -3.360330000 & 0.915639000 & -1.341828000 \\ \mathrm{H} & -2.761540000 & 2.536143000 & -1.727230000 \\ \mathrm{~N} & 5.187210000 & -1.660514000 & -0.905709000 \\ \mathrm{O} & 6.121202000 & -1.908230000 & -0.153811000 \\ \mathrm{O} & 5.307916000 & -1.463624000 & -2.109542000 \\ ------- & \end{array}$

\begin{tabular}{lccc} 
A3 & & & \\
& & & \\
DBU & & Y & Z \\
\hline $\mathrm{C}$ & 2.661904000 & -1.242106000 & -1.185398000 \\
$\mathrm{C}$ & 1.428075000 & -1.087260000 & -0.575249000 \\
$\mathrm{C}$ & 1.257472000 & -1.372747000 & 0.786291000 \\
$\mathrm{C}$ & 2.362340000 & -1.796706000 & 1.533962000 \\
$\mathrm{C}$ & 3.604849000 & -1.952914000 & 0.936602000 \\
$\mathrm{C}$ & 3.736559000 & -1.673825000 & -0.416932000 \\
$\mathrm{H}$ & 2.807787000 & -1.019764000 & -2.235296000 \\
$\mathrm{H}$ & 0.594197000 & -0.704929000 & -1.152659000 \\
$\mathrm{H}$ & 2.241477000 & -1.995676000 & 2.592203000 \\
$\mathrm{H}$ & 4.467183000 & -2.283116000 & 1.502406000 \\
$\mathrm{C}$ & -0.050674000 & -1.172081000 & 1.441486000 \\
$\mathrm{C}$ & -1.228180000 & -1.209303000 & 0.806769000 \\
$\mathrm{H}$ & -1.236318000 & -1.457230000 & -0.248006000 \\
$\mathrm{O}$ & 0.079670000 & -0.919498000 & 2.779065000 \\
$\mathrm{C}$ & -2.547788000 & -0.878779000 & 1.452664000
\end{tabular}




\begin{tabular}{|c|c|c|c|}
\hline $\mathrm{H}$ & -2.563566000 & 0.193718000 & 1.688991000 \\
\hline $\mathrm{C}$ & -3.739535000 & -1.186730000 & 0.574275000 \\
\hline $\mathrm{C}$ & -4.710601000 & -0.212749000 & 0.340013000 \\
\hline $\mathrm{C}$ & -3.895702000 & -2.446177000 & -0.009526000 \\
\hline $\mathrm{C}$ & -5.815327000 & -0.490035000 & -0.461394000 \\
\hline $\mathrm{H}$ & -4.584339000 & 0.774275000 & 0.777582000 \\
\hline $\mathrm{C}$ & -4.996823000 & -2.725786000 & -0.812371000 \\
\hline $\mathrm{H}$ & -3.145183000 & -3.213796000 & 0.166126000 \\
\hline $\mathrm{C}$ & -5.961242000 & -1.746982000 & -1.040342000 \\
\hline $\mathrm{H}$ & -6.560797000 & 0.280442000 & -0.636280000 \\
\hline $\mathrm{H}$ & -5.103403000 & -3.709444000 & -1.260595000 \\
\hline $\mathrm{H}$ & -6.821131000 & -1.963948000 & -1.667226000 \\
\hline $\mathrm{H}$ & -0.799479000 & -0.846755000 & 3.170898000 \\
\hline $\mathrm{C}$ & 1.603372000 & 2.692479000 & -0.917299000 \\
\hline $\mathrm{C}$ & 1.954250000 & 3.744778000 & 0.132641000 \\
\hline $\mathrm{C}$ & -0.163872000 & 2.381374000 & 1.568230000 \\
\hline $\mathrm{C}$ & 2.262296000 & 3.148655000 & 1.508300000 \\
\hline $\mathrm{C}$ & 1.291795000 & 2.024149000 & 1.865372000 \\
\hline $\mathrm{H}$ & 2.292229000 & 1.835507000 & -0.843717000 \\
\hline $\mathrm{H}$ & -0.366622000 & 3.413590000 & 1.884066000 \\
\hline $\mathrm{H}$ & 1.126196000 & 4.461083000 & 0.194475000 \\
\hline $\mathrm{H}$ & 2.216263000 & 3.941801000 & 2.264662000 \\
\hline $\mathrm{H}$ & 1.768054000 & 3.134819000 & -1.907772000 \\
\hline $\mathrm{H}$ & 2.821861000 & 4.309614000 & -0.225685000 \\
\hline $\mathrm{H}$ & -0.829520000 & 1.755337000 & 2.166866000 \\
\hline $\mathrm{H}$ & 3.287871000 & 2.759923000 & 1.521780000 \\
\hline $\mathrm{H}$ & 1.389476000 & 1.770536000 & 2.925676000 \\
\hline $\mathrm{H}$ & 1.563716000 & 1.116759000 & 1.318419000 \\
\hline $\mathrm{C}$ & -0.669439000 & 2.218741000 & 0.139752000 \\
\hline $\mathrm{N}$ & -1.943932000 & 2.066965000 & 0.029564000 \\
\hline $\mathrm{N}$ & 0.216856000 & 2.228954000 & -0.920821000 \\
\hline $\mathrm{C}$ & -0.215142000 & 1.796521000 & -2.246940000 \\
\hline $\mathrm{H}$ & -0.232734000 & 2.665642000 & -2.922313000 \\
\hline $\mathrm{H}$ & 0.539519000 & 1.103521000 & -2.647373000 \\
\hline $\mathrm{C}$ & -1.584608000 & 1.140370000 & -2.214812000 \\
\hline $\mathrm{H}$ & -1.980247000 & 1.058580000 & -3.232092000 \\
\hline $\mathrm{H}$ & -1.520432000 & 0.127129000 & -1.803038000 \\
\hline $\mathrm{C}$ & -2.487448000 & 1.972831000 & -1.312282000 \\
\hline $\mathrm{H}$ & -3.485569000 & 1.529348000 & -1.257182000 \\
\hline $\mathrm{H}$ & -2.605076000 & 2.980137000 & -1.743501000 \\
\hline $\mathrm{H}$ & -2.666153000 & -1.430996000 & 2.400361000 \\
\hline $\mathrm{N}$ & 5.048715000 & -1.823520000 & -1.052169000 \\
\hline $\mathrm{O}$ & 5.137294000 & -1.555510000 & -2.241435000 \\
\hline $\mathrm{O}$ & 5.977295000 & -2.205895000 & -0.356463000 \\
\hline
\end{tabular}




\begin{tabular}{|c|c|c|c|}
\hline \multicolumn{4}{|c|}{ A1 $1_{\text {MTBD }}$} \\
\hline & \multicolumn{3}{|c|}{ Coordinates (Angstroms) } \\
\hline & $X$ & $\mathrm{Y}$ & Z \\
\hline $\mathrm{C}$ & -1.201412000 & -2.756682000 & -0.761790000 \\
\hline $\mathrm{C}$ & -1.033448000 & -1.653892000 & -1.801456000 \\
\hline $\mathrm{C}$ & 0.392254000 & -1.704201000 & -2.327828000 \\
\hline $\mathrm{N}$ & -0.256116000 & -2.620413000 & 0.324887000 \\
\hline $\mathrm{H}$ & -1.088135000 & -3.737093000 & -1.253971000 \\
\hline $\mathrm{H}$ & -2.215206000 & -2.732942000 & -0.348285000 \\
\hline $\mathrm{H}$ & -1.232740000 & -0.685343000 & -1.328691000 \\
\hline $\mathrm{H}$ & -1.737444000 & -1.765221000 & -2.632475000 \\
\hline $\mathrm{H}$ & 0.493087000 & -2.504981000 & -3.076135000 \\
\hline $\mathrm{H}$ & 0.647295000 & -0.762311000 & -2.834795000 \\
\hline $\mathrm{N}$ & 1.362789000 & -1.920169000 & -1.262701000 \\
\hline $\mathrm{C}$ & 2.725539000 & -1.582493000 & -1.639072000 \\
\hline $\mathrm{C}$ & 3.731804000 & -2.063991000 & -0.612154000 \\
\hline $\mathrm{C}$ & 3.228526000 & -1.689720000 & 0.770025000 \\
\hline $\mathrm{N}$ & 1.929955000 & -2.292421000 & 0.988528000 \\
\hline $\mathrm{C}$ & 0.953068000 & -2.284500000 & 0.008553000 \\
\hline $\mathrm{H}$ & 2.815688000 & -0.492114000 & -1.760897000 \\
\hline $\mathrm{H}$ & 2.931092000 & -2.035105000 & -2.618788000 \\
\hline $\mathrm{H}$ & 3.848440000 & -3.151439000 & -0.671865000 \\
\hline $\mathrm{H}$ & 4.703652000 & -1.601143000 & -0.807618000 \\
\hline $\mathrm{H}$ & 3.906654000 & -2.075832000 & 1.538271000 \\
\hline $\mathrm{H}$ & 3.195094000 & -0.595335000 & 0.884156000 \\
\hline $\mathrm{C}$ & -3.476157000 & 1.818968000 & -0.219580000 \\
\hline $\mathrm{C}$ & -2.230676000 & 1.682358000 & 0.378484000 \\
\hline $\mathrm{C}$ & -1.905095000 & 0.530703000 & 1.100122000 \\
\hline $\mathrm{C}$ & -2.848359000 & -0.491482000 & 1.218272000 \\
\hline $\mathrm{C}$ & -4.097229000 & -0.375948000 & 0.623437000 \\
\hline $\mathrm{C}$ & -4.389539000 & 0.781402000 & -0.087147000 \\
\hline $\mathrm{H}$ & -3.744310000 & 2.701806000 & -0.786650000 \\
\hline $\mathrm{H}$ & -1.492205000 & 2.472518000 & 0.265889000 \\
\hline $\mathrm{H}$ & -2.583825000 & -1.396262000 & 1.756797000 \\
\hline $\mathrm{H}$ & -4.837556000 & -1.163018000 & 0.695798000 \\
\hline $\mathrm{C}$ & -0.548755000 & 0.391650000 & 1.774307000 \\
\hline $\mathrm{C}$ & 0.564329000 & 0.827169000 & 0.858318000 \\
\hline $\mathrm{H}$ & 0.536835000 & 0.391499000 & -0.138732000 \\
\hline $\mathrm{O}$ & -0.527198000 & 1.045905000 & 3.030482000 \\
\hline $\mathrm{C}$ & 1.559849000 & 1.644237000 & 1.205775000 \\
\hline $\mathrm{H}$ & 1.607421000 & 2.002606000 & 2.232919000 \\
\hline $\mathrm{C}$ & 2.661449000 & 2.040314000 & 0.309787000 \\
\hline
\end{tabular}




$\begin{array}{lrrr}\mathrm{C} & 3.924253000 & 2.317952000 & 0.845892000 \\ \mathrm{C} & 2.494654000 & 2.126005000 & -1.078378000 \\ \mathrm{C} & 4.997095000 & 2.636582000 & 0.020079000 \\ \mathrm{H} & 4.067004000 & 2.264816000 & 1.922349000 \\ \mathrm{C} & 3.565432000 & 2.446950000 & -1.905216000 \\ \mathrm{H} & 1.512389000 & 1.954793000 & -1.510935000 \\ \mathrm{C} & 4.822583000 & 2.697670000 & -1.359627000 \\ \mathrm{H} & 5.971032000 & 2.838017000 & 0.456161000 \\ \mathrm{H} & 3.414967000 & 2.513980000 & -2.978806000 \\ \mathrm{H} & 5.657037000 & 2.952496000 & -2.005949000 \\ \mathrm{H} & -0.407472000 & -0.670564000 & 1.992280000 \\ \mathrm{H} & -0.744268000 & 1.975678000 & 2.879300000 \\ \mathrm{C} & 1.554515000 & -2.532252000 & 2.364744000 \\ \mathrm{H} & 1.443532000 & -1.592374000 & 2.926230000 \\ \mathrm{H} & 0.607951000 & -3.069967000 & 2.381590000 \\ \mathrm{H} & 2.329621000 & -3.133991000 & 2.853218000 \\ \mathrm{~N} & -5.703609000 & 0.909682000 & -0.728730000 \\ \mathrm{O} & -6.487581000 & -0.019146000 & -0.608945000 \\ \mathrm{O} & -5.935823000 & 1.938150000 & -1.346230000 \\ - \text { - - - - } & & \end{array}$

TSA1-2 MTBD

\begin{tabular}{lrcc} 
& \multicolumn{3}{c}{ Coordinates (Angstroms) } \\
C & 0.483873000 & -1.278735000 & 1.926995000 \\
$\mathrm{C}$ & -0.514551000 & -1.154874000 & 3.068577000 \\
$\mathrm{~N}$ & -1.749558000 & -0.439791000 & 2.549245000 \\
$\mathrm{H}$ & -0.131504000 & -1.687299000 & 0.667483000 \\
$\mathrm{H}$ & 1.269783000 & -1.999329000 & 2.174652000 \\
$\mathrm{H}$ & 0.982581000 & -0.314033000 & 1.770770000 \\
$\mathrm{H}$ & -0.069134000 & -0.604260000 & 3.902474000 \\
$\mathrm{H}$ & -0.806287000 & -2.144259000 & 3.437810000 \\
$\mathrm{H}$ & -2.517291000 & -0.363063000 & 3.324554000 \\
$\mathrm{~N}$ & -1.508670000 & 0.581207000 & 2.219105000 \\
$\mathrm{C}$ & -2.314880000 & -1.208965000 & 1.449914000 \\
$\mathrm{C}$ & -3.732171000 & -0.989702000 & 1.181733000 \\
$\mathrm{C}$ & -4.249990000 & -1.976795000 & 0.152101000 \\
$\mathrm{~N}$ & -3.309150000 & -1.995900000 & -1.039701000 \\
$\mathrm{C}$ & -1.954899000 & -2.290846000 & -0.598479000 \\
$\mathrm{H}$ & -1.452338000 & -1.704666000 & 0.515954000 \\
$\mathrm{H}$ & -3.885549000 & 0.040584000 & 0.834683000 \\
$\mathrm{H}$ & -4.271097000 & -1.107115000 & 2.128120000 \\
$\mathrm{H}$ & -4.309315000 & -2.980492000 & 0.586225000 \\
& -5.254325000 & -1.680204000 & -0.163248000 \\
& & &
\end{tabular}




\begin{tabular}{lrcc}
$\mathrm{H}$ & -3.604018000 & -2.774746000 & -1.747766000 \\
$\mathrm{H}$ & -3.335279000 & -1.033808000 & -1.568170000 \\
$\mathrm{C}$ & 4.053516000 & 1.201296000 & 0.641119000 \\
$\mathrm{C}$ & 2.729024000 & 1.088476000 & 0.262744000 \\
$\mathrm{C}$ & 2.326384000 & 0.228737000 & -0.783758000 \\
$\mathrm{C}$ & 3.340183000 & -0.498898000 & -1.442953000 \\
$\mathrm{C}$ & 4.668609000 & -0.390870000 & -1.075622000 \\
$\mathrm{C}$ & 5.020449000 & 0.456069000 & -0.028358000 \\
$\mathrm{H}$ & 4.354609000 & 1.868429000 & 1.439821000 \\
$\mathrm{H}$ & 1.997135000 & 1.696954000 & 0.784523000 \\
$\mathrm{H}$ & 3.055671000 & -1.162775000 & -2.251015000 \\
$\mathrm{H}$ & 5.440422000 & -0.954779000 & -1.585711000 \\
$\mathrm{C}$ & 0.924517000 & 0.009507000 & -1.159484000 \\
$\mathrm{C}$ & -0.118373000 & 0.940036000 & -0.775781000 \\
$\mathrm{H}$ & 0.047093000 & 1.466096000 & 0.163059000 \\
$\mathrm{O}$ & 0.752381000 & -0.569004000 & -2.440515000 \\
$\mathrm{C}$ & -1.324469000 & 1.054686000 & -1.374203000 \\
$\mathrm{H}$ & -1.485092000 & 0.506906000 & -2.301182000 \\
$\mathrm{C}$ & -2.487019000 & 1.773667000 & -0.848923000 \\
$\mathrm{C}$ & -3.713229000 & 1.679772000 & -1.528541000 \\
$\mathrm{C}$ & -2.473828000 & 2.513159000 & 0.346551000 \\
$\mathrm{C}$ & -4.875828000 & 2.246734000 & -1.019459000 \\
$\mathrm{H}$ & -3.749962000 & 1.140944000 & -2.473120000 \\
$\mathrm{C}$ & -3.633709000 & 3.082536000 & 0.856295000 \\
$\mathrm{H}$ & -1.542023000 & 2.648086000 & 0.888728000 \\
$\mathrm{C}$ & -4.847843000 & 2.943518000 & 0.185711000 \\
$\mathrm{H}$ & -5.808423000 & 2.144568000 & -1.567528000 \\
$\mathrm{H}$ & -3.589126000 & 3.641657000 & 1.786946000 \\
$\mathrm{H}$ & -5.753455000 & 3.385388000 & 0.589517000 \\
$\mathrm{H}$ & 0.433118000 & -1.070512000 & -0.232321000 \\
$\mathrm{H}$ & 0.886055000 & 0.127526000 & -3.100007000 \\
$\mathrm{H}$ & -1.064111000 & -2.919891000 & -1.562890000 \\
$\mathrm{H}$ & -0.672154000 & -2.198404000 & -2.287444000 \\
$\mathrm{H}$ & -0.220633000 & -3.364525000 & -1.034316000 \\
$\mathrm{H}$ & 6.412568000 & 0.571370000 & 0.366890000 \\
$\mathrm{H}$ & 7.242966000000000000 \\
$\mathrm{H}$ & & -0.086715000 & -0.248808000 \\
\hline
\end{tabular}

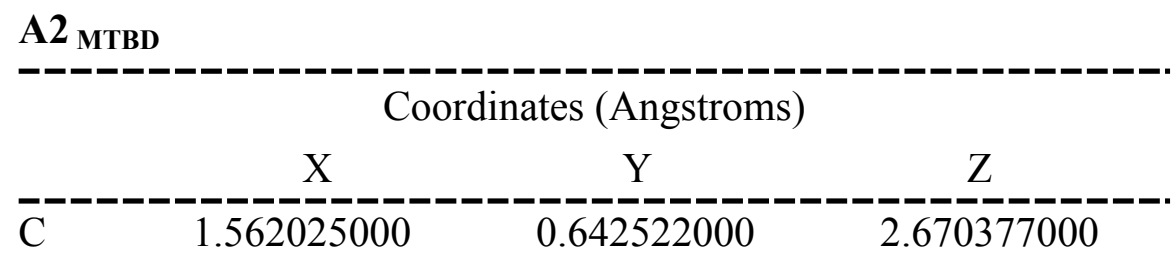




\begin{tabular}{|c|c|c|c|}
\hline $\mathrm{C}$ & 2.595316000 & 1.172056000 & 1.694097000 \\
\hline $\mathrm{C}$ & 2.116040000 & 2.486221000 & 1.105568000 \\
\hline $\mathrm{N}$ & 0.195347000 & 0.842104000 & 2.180393000 \\
\hline $\mathrm{H}$ & 1.643941000 & 1.140028000 & 3.645260000 \\
\hline $\mathrm{H}$ & 1.704847000 & -0.433264000 & 2.818357000 \\
\hline $\mathrm{H}$ & 2.747210000 & 0.456624000 & 0.885129000 \\
\hline $\mathrm{H}$ & 3.551440000 & 1.306472000 & 2.205995000 \\
\hline $\mathrm{H}$ & 2.035665000 & 3.268366000 & 1.872352000 \\
\hline $\mathrm{H}$ & 2.810366000 & 2.831193000 & 0.336808000 \\
\hline $\mathrm{N}$ & 0.817006000 & 2.288911000 & 0.467131000 \\
\hline $\mathrm{C}$ & 0.590055000 & 2.858222000 & -0.859435000 \\
\hline $\mathrm{C}$ & -0.889821000 & 3.146942000 & -1.059862000 \\
\hline $\mathrm{C}$ & -1.684053000 & 1.878358000 & -0.807094000 \\
\hline $\mathrm{N}$ & -1.298355000 & 1.297813000 & 0.473544000 \\
\hline $\mathrm{C}$ & -0.079802000 & 1.455898000 & 1.020400000 \\
\hline $\mathrm{H}$ & 0.970745000 & 2.166863000 & -1.622285000 \\
\hline $\mathrm{H}$ & 1.169780000 & 3.782363000 & -0.919628000 \\
\hline $\mathrm{H}$ & -1.206516000 & 3.934002000 & -0.367066000 \\
\hline $\mathrm{H}$ & -1.067373000 & 3.501606000 & -2.078302000 \\
\hline $\mathrm{H}$ & -2.759515000 & 2.067926000 & -0.778015000 \\
\hline $\mathrm{H}$ & -1.503308000 & 1.149329000 & -1.607108000 \\
\hline $\mathrm{H}$ & -1.759468000 & 0.406714000 & 0.670740000 \\
\hline $\mathrm{C}$ & 2.536504000 & -0.407586000 & -1.670362000 \\
\hline $\mathrm{C}$ & 1.279321000 & -0.775324000 & -1.259474000 \\
\hline $\mathrm{C}$ & 1.070519000 & -1.702369000 & -0.184106000 \\
\hline $\mathrm{C}$ & 2.254725000 & -2.223451000 & 0.431008000 \\
\hline $\mathrm{C}$ & 3.511656000 & -1.853763000 & 0.017151000 \\
\hline $\mathrm{C}$ & 3.669009000 & -0.926740000 & -1.025759000 \\
\hline $\mathrm{H}$ & 2.675452000 & 0.295202000 & -2.484467000 \\
\hline $\mathrm{H}$ & 0.425331000 & -0.345178000 & -1.774465000 \\
\hline $\mathrm{H}$ & 2.139519000 & -2.938659000 & 1.237112000 \\
\hline $\mathrm{H}$ & 4.396909000 & -2.260496000 & 0.492252000 \\
\hline $\mathrm{C}$ & -0.206008000 & -2.046815000 & 0.297442000 \\
\hline $\mathrm{C}$ & -1.421916000 & -1.610554000 & -0.248429000 \\
\hline $\mathrm{H}$ & -1.354496000 & -1.172973000 & -1.242742000 \\
\hline $\mathrm{O}$ & -0.247679000 & -2.746875000 & 1.511124000 \\
\hline $\mathrm{C}$ & -2.662229000 & -1.620970000 & 0.347060000 \\
\hline $\mathrm{H}$ & -2.755250000 & -1.992421000 & 1.366353000 \\
\hline $\mathrm{C}$ & -3.871483000 & -1.071635000 & -0.251637000 \\
\hline $\mathrm{C}$ & -4.964784000 & -0.735090000 & 0.570708000 \\
\hline $\mathrm{C}$ & -4.003340000 & -0.801653000 & -1.628707000 \\
\hline $\mathrm{C}$ & -6.108126000 & -0.139637000 & 0.056038000 \\
\hline $\mathrm{H}$ & -4.899245000 & -0.939399000 & 1.637462000 \\
\hline $\mathrm{C}$ & -5.144821000 & -0.196475000 & -2.140348000 \\
\hline
\end{tabular}




$\begin{array}{lrrr}\mathrm{H} & -3.212425000 & -1.096716000 & -2.313148000 \\ \mathrm{C} & -6.206081000 & 0.145415000 & -1.304834000 \\ \mathrm{H} & -6.928852000 & 0.108557000 & 0.723943000 \\ \mathrm{H} & -5.214435000 & -0.007847000 & -3.208600000 \\ \mathrm{H} & -7.099768000 & 0.610268000 & -1.709593000 \\ \mathrm{H} & -0.866581000 & -3.480733000 & 1.397879000 \\ \mathrm{C} & -0.827939000 & 0.119096000 & 2.927438000 \\ \mathrm{H} & -1.804453000 & 0.584405000 & 2.782642000 \\ \mathrm{H} & -0.858131000 & -0.936028000 & 2.635040000 \\ \mathrm{H} & -0.572630000 & 0.192479000 & 3.988484000 \\ \mathrm{~N} & 4.971304000 & -0.479668000 & -1.404023000 \\ \mathrm{O} & 5.062841000 & 0.380730000 & -2.284111000 \\ \mathrm{O} & 5.948028000 & -0.958707000 & -0.826156000\end{array}$

TSA2-3 MTBD

\begin{tabular}{|c|c|c|c|}
\hline \multicolumn{4}{|c|}{ Coordinates (Angstroms) } \\
\hline & $\mathrm{X}$ & Y & $\mathrm{Z}$ \\
\hline $\mathrm{C}$ & -1.154231000 & 2.385807000 & -1.683881000 \\
\hline $\mathrm{C}$ & -2.159057000 & 2.211129000 & -0.554051000 \\
\hline $\mathrm{C}$ & -1.599134000 & 2.825798000 & 0.717113000 \\
\hline $\mathrm{N}$ & 0.230050000 & 2.300757000 & -1.223089000 \\
\hline $\mathrm{H}$ & -1.283074000 & 3.366636000 & -2.161507000 \\
\hline $\mathrm{H}$ & -1.305931000 & 1.615637000 & -2.449510000 \\
\hline $\mathrm{H}$ & -2.352887000 & 1.152229000 & -0.372737000 \\
\hline $\mathrm{H}$ & -3.106419000 & 2.683248000 & -0.828200000 \\
\hline $\mathrm{H}$ & -1.469307000 & 3.912327000 & 0.607048000 \\
\hline $\mathrm{H}$ & -2.276019000 & 2.649320000 & 1.556006000 \\
\hline $\mathrm{N}$ & -0.325894000 & 2.194337000 & 1.031142000 \\
\hline $\mathrm{C}$ & -0.012912000 & 1.835080000 & 2.408586000 \\
\hline $\mathrm{C}$ & 1.473162000 & 2.032238000 & 2.667248000 \\
\hline $\mathrm{C}$ & 2.257860000 & 1.243528000 & 1.630606000 \\
\hline $\mathrm{N}$ & 1.807164000 & 1.520134000 & 0.272519000 \\
\hline $\mathrm{C}$ & 0.584798000 & 1.978243000 & 0.047886000 \\
\hline $\mathrm{H}$ & -0.307287000 & 0.794604000 & 2.605034000 \\
\hline $\mathrm{H}$ & -0.609136000 & 2.476810000 & 3.062874000 \\
\hline $\mathrm{H}$ & 1.713497000 & 3.098891000 & 2.592929000 \\
\hline $\mathrm{H}$ & 1.729866000 & 1.697542000 & 3.676562000 \\
\hline $\mathrm{H}$ & 3.326435000 & 1.470375000 & 1.686306000 \\
\hline $\mathrm{H}$ & 2.158186000 & 0.166554000 & 1.828703000 \\
\hline $\mathrm{H}$ & 2.130841000 & 0.564490000 & -0.442177000 \\
\hline $\mathrm{C}$ & -2.931501000 & -1.344210000 & 1.126305000 \\
\hline $\mathrm{C}$ & -1.654788000 & -1.291802000 & 0.604139000 \\
\hline $\mathrm{C}$ & -1.426346000 & -1.269064000 & -0.790796000 \\
\hline
\end{tabular}




$\begin{array}{lrrr}\mathrm{C} & -2.555547000 & -1.295428000 & -1.636441000 \\ \mathrm{C} & -3.839387000 & -1.336529000 & -1.122026000 \\ \mathrm{C} & -4.022021000 & -1.355445000 & 0.257659000 \\ \mathrm{H} & -3.102787000 & -1.356361000 & 2.195930000 \\ \mathrm{H} & -0.816251000 & -1.238040000 & 1.290495000 \\ \mathrm{H} & -2.403562000 & -1.272562000 & -2.708772000 \\ \mathrm{H} & -4.704743000 & -1.351903000 & -1.773606000 \\ \mathrm{C} & -0.097863000 & -1.127127000 & -1.351024000 \\ \mathrm{C} & 1.065257000 & -1.250632000 & -0.658897000 \\ \mathrm{H} & 0.980338000 & -1.626951000 & 0.357929000 \\ \mathrm{O} & -0.092903000 & -0.744564000 & -2.688757000 \\ \mathrm{C} & 2.353669000 & -0.756764000 & -1.050346000 \\ \mathrm{H} & 2.480452000 & -0.466030000 & -2.096130000 \\ \mathrm{C} & 3.600410000 & -1.210470000 & -0.406752000 \\ \mathrm{C} & 4.808877000 & -0.570310000 & -0.736824000 \\ \mathrm{C} & 3.653245000 & -2.200979000 & 0.587400000 \\ \mathrm{C} & 6.000447000 & -0.891781000 & -0.102079000 \\ \mathrm{H} & 4.798916000 & 0.201998000 & -1.503428000 \\ \mathrm{C} & 4.845627000 & -2.514654000 & 1.233355000 \\ \mathrm{H} & 2.754590000 & -2.754437000 & 0.846053000 \\ \mathrm{C} & 6.028246000 & -1.863637000 & 0.897260000 \\ \mathrm{H} & 6.914938000 & -0.376843000 & -0.384785000 \\ \mathrm{H} & 4.850043000 & -3.288247000 & 1.996766000 \\ \mathrm{H} & 6.958694000 & -2.113989000 & 1.397769000 \\ \mathrm{H} & 0.699664000 & -1.123826000 & -3.090989000 \\ \mathrm{C} & 1.215169000 & 2.334830000 & -2.288678000 \\ \mathrm{H} & 2.211436000 & 2.459620000 & -1.865873000 \\ \mathrm{H} & 1.177224000 & 1.415372000 & -2.880400000 \\ \mathrm{H} & 0.989503000 & 3.186173000 & -2.941293000 \\ \mathrm{~N} & -5.367919000 & -1.368110000 & 0.804240000 \\ \mathrm{O} & -6.307841000 & -1.376441000 & 0.018813000 \\ \mathrm{O} & -5.492368000 & -1.363921000 & 2.0237860000 \\ -----0 .-\end{array}$

\begin{tabular}{|c|c|c|c|}
\hline \multicolumn{4}{|c|}{ A3 } \\
\hline & \multicolumn{3}{|c|}{ Coordinates (Angstroms) } \\
\hline & $X$ & $\mathrm{Y}$ & Z \\
\hline$\overline{\mathrm{C}}$ & -1.601821000 & -2.948245000 & 1.149611000 \\
\hline $\mathrm{C}$ & -2.318771000 & -2.231000000 & 2.279539000 \\
\hline $\mathrm{C}$ & -3.688368000 & -1.784192000 & 1.795586000 \\
\hline $\mathrm{N}$ & -1.458485000 & -2.035304000 & 0.034126000 \\
\hline $\mathrm{H}$ & -2.146620000 & -3.867073000 & 0.867398000 \\
\hline $\mathrm{H}$ & -0.599322000 & -3.251647000 & 1.466922000 \\
\hline $\mathrm{H}$ & -1.717405000 & -1.365568000 & 2.576110000 \\
\hline
\end{tabular}




\begin{tabular}{|c|c|c|c|}
\hline $\mathrm{H}$ & -2.429564000 & -2.881688000 & 3.152444000 \\
\hline $\mathrm{H}$ & -4.404533000 & -2.617217000 & 1.879409000 \\
\hline $\mathrm{H}$ & -4.066464000 & -0.973589000 & 2.433516000 \\
\hline $\mathrm{N}$ & -3.669499000 & -1.319935000 & 0.420605000 \\
\hline $\mathrm{C}$ & -4.857427000 & -0.582869000 & 0.020515000 \\
\hline $\mathrm{C}$ & -5.027940000 & -0.620530000 & -1.488761000 \\
\hline $\mathrm{C}$ & -3.716672000 & -0.180731000 & -2.131228000 \\
\hline $\mathrm{N}$ & -2.574156000 & -0.925613000 & -1.649751000 \\
\hline $\mathrm{C}$ & -2.599533000 & -1.396929000 & -0.450623000 \\
\hline $\mathrm{H}$ & -4.787944000 & 0.456274000 & 0.376095000 \\
\hline $\mathrm{H}$ & -5.720393000 & -1.045001000 & 0.516607000 \\
\hline $\mathrm{H}$ & -5.267548000 & -1.643121000 & -1.803773000 \\
\hline $\mathrm{H}$ & -5.857163000 & 0.029035000 & -1.788285000 \\
\hline $\mathrm{H}$ & -3.769999000 & -0.301642000 & -3.219557000 \\
\hline $\mathrm{H}$ & -3.569131000 & 0.895206000 & -1.943594000 \\
\hline $\mathrm{C}$ & 4.913002000 & 1.254276000 & 0.430600000 \\
\hline $\mathrm{C}$ & 3.652773000 & 1.511637000 & -0.084859000 \\
\hline $\mathrm{C}$ & 2.946361000 & 0.522953000 & -0.782509000 \\
\hline $\mathrm{C}$ & 3.545488000 & -0.730245000 & -0.974172000 \\
\hline $\mathrm{C}$ & 4.809061000 & -1.000094000 & -0.468672000 \\
\hline $\mathrm{C}$ & 5.474456000 & -0.000847000 & 0.228595000 \\
\hline $\mathrm{H}$ & 5.470682000 & 2.009922000 & 0.969814000 \\
\hline $\mathrm{H}$ & 3.213292000 & 2.496022000 & 0.036709000 \\
\hline $\mathrm{H}$ & 3.001291000 & -1.511861000 & -1.496027000 \\
\hline $\mathrm{H}$ & 5.278121000 & -1.967538000 & -0.598723000 \\
\hline $\mathrm{C}$ & 1.590815000 & 0.782733000 & -1.315090000 \\
\hline $\mathrm{C}$ & 0.677885000 & 1.524097000 & -0.678126000 \\
\hline $\mathrm{H}$ & 0.956701000 & 1.954037000 & 0.279737000 \\
\hline $\mathrm{O}$ & 1.254032000 & 0.138047000 & -2.476973000 \\
\hline $\mathrm{C}$ & -0.719120000 & 1.767925000 & -1.175514000 \\
\hline $\mathrm{H}$ & -1.059858000 & 0.891448000 & -1.743301000 \\
\hline $\mathrm{C}$ & -1.711342000 & 2.033579000 & -0.065238000 \\
\hline $\mathrm{C}$ & -2.713616000 & 2.994569000 & -0.215211000 \\
\hline $\mathrm{C}$ & -1.665448000 & 1.305245000 & 1.125970000 \\
\hline $\mathrm{C}$ & -3.645373000 & 3.224161000 & 0.793774000 \\
\hline $\mathrm{H}$ & -2.763342000 & 3.572016000 & -1.135601000 \\
\hline $\mathrm{C}$ & -2.587657000 & 1.537313000 & 2.141037000 \\
\hline $\mathrm{H}$ & -0.909518000 & 0.533108000 & 1.243316000 \\
\hline $\mathrm{C}$ & -3.582695000 & 2.498588000 & 1.980271000 \\
\hline $\mathrm{H}$ & -4.414825000 & 3.978606000 & 0.656324000 \\
\hline $\mathrm{H}$ & -2.527961000 & 0.966356000 & 3.064234000 \\
\hline $\mathrm{H}$ & -4.301853000 & 2.682190000 & 2.773230000 \\
\hline $\mathrm{H}$ & 2.051452000 & -0.022068000 & -2.995813000 \\
\hline $\mathrm{H}$ & -0.719931000 & 2.615693000 & -1.874728000 \\
\hline
\end{tabular}




$\begin{array}{lrrr}\mathrm{C} & -0.428698000 & -2.372250000 & -0.928854000 \\ \mathrm{H} & -0.675805000 & -3.279825000 & -1.501614000 \\ \mathrm{H} & -0.298351000 & -1.551828000 & -1.631508000 \\ \mathrm{H} & 0.509340000 & -2.540495000 & -0.388178000 \\ \mathrm{~N} & 6.811352000 & -0.275929000 & 0.763956000 \\ \mathrm{O} & 7.284083000 & -1.385337000 & 0.567414000 \\ \mathrm{O} & \mathbf{7 . 3 7 3 1 3 7 0 0 0} & 0.620148000 & 1.375363000\end{array}$

(6) $\mathrm{R}_{1}=\mathrm{H}, \mathrm{R}_{2}=\mathrm{OMe}$

\begin{tabular}{|c|c|c|c|}
\hline \multicolumn{4}{|c|}{$\mathbf{A} 1_{\text {кон }}$} \\
\hline & \multicolumn{3}{|c|}{ Coordinates (Angstroms) } \\
\hline & $X$ & Y & Z \\
\hline$\overline{\mathrm{C}}$ & 2.847454000 & 3.134160000 & -0.842361000 \\
\hline $\mathrm{C}$ & 2.020609000 & 2.023352000 & -0.699141000 \\
\hline $\mathrm{C}$ & 2.459716000 & 0.903749000 & 0.012249000 \\
\hline $\mathrm{C}$ & 3.731764000 & 0.906776000 & 0.594253000 \\
\hline $\mathrm{C}$ & 4.552681000 & 2.023103000 & 0.450918000 \\
\hline $\mathrm{C}$ & 4.116515000 & 3.134366000 & -0.267748000 \\
\hline $\mathrm{H}$ & 2.497924000 & 4.003420000 & -1.392603000 \\
\hline $\mathrm{H}$ & 1.020293000 & 2.030801000 & -1.129029000 \\
\hline $\mathrm{H}$ & 4.030640000 & 0.009355000 & 1.155441000 \\
\hline $\mathrm{H}$ & 5.537514000 & 2.029260000 & 0.910866000 \\
\hline $\mathrm{H}$ & 4.759595000 & 4.003975000 & -0.372692000 \\
\hline $\mathrm{C}$ & 1.604959000 & -0.349105000 & 0.113297000 \\
\hline $\mathrm{C}$ & 0.159514000 & -0.048410000 & 0.383107000 \\
\hline $\mathrm{H}$ & -0.002367000 & 0.630158000 & 1.219303000 \\
\hline $\mathrm{O}$ & 1.773517000 & -1.144418000 & -1.083735000 \\
\hline $\mathrm{C}$ & -0.871912000 & -0.543922000 & -0.305919000 \\
\hline $\mathrm{H}$ & -0.652274000 & -1.220253000 & -1.131204000 \\
\hline $\mathrm{C}$ & -2.301350000 & -0.287478000 & -0.068534000 \\
\hline $\mathrm{C}$ & -3.242359000 & -0.763053000 & -0.982272000 \\
\hline $\mathrm{C}$ & -2.785980000 & 0.416155000 & 1.046971000 \\
\hline $\mathrm{C}$ & -4.610204000 & -0.551039000 & -0.818160000 \\
\hline $\mathrm{H}$ & -2.901452000 & -1.316747000 & -1.854104000 \\
\hline $\mathrm{C}$ & -4.137987000 & 0.636058000 & 1.227447000 \\
\hline $\mathrm{H}$ & -2.093020000 & 0.789917000 & 1.794778000 \\
\hline $\mathrm{C}$ & -5.064350000 & 0.154584000 & 0.294305000 \\
\hline $\mathrm{H}$ & -5.299818000 & -0.940113000 & -1.558547000 \\
\hline $\mathrm{H}$ & -4.508699000 & 1.175785000 & 2.092730000 \\
\hline $\mathrm{H}$ & 2.041112000 & -0.978634000 & 0.915271000 \\
\hline $\mathrm{H}$ & 1.538190000 & -0.575934000 & -1.829387000 \\
\hline $\mathrm{O}$ & 3.657066000 & -1.934116000 & 1.727586000 \\
\hline
\end{tabular}




$\begin{array}{lrcc}\mathrm{H} & 3.557799000 & -1.726980000 & 2.660344000 \\ \mathrm{~K} & 3.892674000 & -2.666885000 & -0.432163000 \\ \mathrm{O} & -6.368266000 & 0.421358000 & 0.565401000 \\ \mathrm{C} & -7.338808000 & -0.042489000 & -0.343905000 \\ \mathrm{H} & -8.302968000 & 0.269681000 & 0.059379000 \\ \mathrm{H} & -7.202612000 & 0.400442000 & -1.339305000 \\ \mathrm{H} & -\mathbf{- 7 . 3 2 0 7 7 5 0 0 0} & -1.136758000 & -0.431219000\end{array}$

TSA1-2 кон

\begin{tabular}{|c|c|c|c|}
\hline \multicolumn{4}{|c|}{ Coordinates (Angstroms) } \\
\hline & $X$ & $\mathrm{Y}$ & $\mathrm{Z}$ \\
\hline$\overline{\mathrm{C}}$ & 4.341414000 & 1.920415000 & -1.154408000 \\
\hline $\mathrm{C}$ & 3.412687000 & 0.878254000 & -1.175385000 \\
\hline $\mathrm{C}$ & 2.471951000 & 0.719137000 & -0.145902000 \\
\hline $\mathrm{C}$ & 2.507601000 & 1.645777000 & 0.911263000 \\
\hline $\mathrm{C}$ & 3.421296000 & 2.688791000 & 0.924161000 \\
\hline $\mathrm{C}$ & 4.351926000 & 2.833078000 & -0.106913000 \\
\hline $\mathrm{H}$ & 5.051063000 & 2.021739000 & -1.972136000 \\
\hline $\mathrm{H}$ & 3.396154000 & 0.188257000 & -2.015890000 \\
\hline $\mathrm{H}$ & 1.819788000 & 1.522425000 & 1.743678000 \\
\hline $\mathrm{H}$ & 3.420621000 & 3.390926000 & 1.753547000 \\
\hline $\mathrm{H}$ & 5.072470000 & 3.645083000 & -0.089726000 \\
\hline $\mathrm{C}$ & 1.544151000 & -0.451044000 & -0.080077000 \\
\hline $\mathrm{C}$ & 0.116492000 & -0.123262000 & 0.034074000 \\
\hline $\mathrm{H}$ & -0.107664000 & 0.797563000 & 0.572187000 \\
\hline $\mathrm{O}$ & 1.808664000 & -1.408234000 & -1.121240000 \\
\hline $\mathrm{C}$ & -0.898781000 & -0.873633000 & -0.428920000 \\
\hline $\mathrm{H}$ & -0.667722000 & -1.828875000 & -0.898724000 \\
\hline $\mathrm{C}$ & -2.327526000 & -0.553021000 & -0.302082000 \\
\hline $\mathrm{C}$ & -3.285307000 & -1.574037000 & -0.415093000 \\
\hline $\mathrm{C}$ & -2.804948000 & 0.740977000 & -0.074168000 \\
\hline $\mathrm{C}$ & -4.639816000 & -1.323252000 & -0.278878000 \\
\hline $\mathrm{H}$ & -2.952545000 & -2.592395000 & -0.602098000 \\
\hline $\mathrm{C}$ & -4.163336000 & 1.011681000 & 0.071563000 \\
\hline $\mathrm{H}$ & -2.104075000 & 1.569714000 & -0.024376000 \\
\hline $\mathrm{C}$ & -5.091222000 & -0.024974000 & -0.026972000 \\
\hline $\mathrm{H}$ & -5.371793000 & -2.120466000 & -0.360263000 \\
\hline $\mathrm{H}$ & -4.480389000 & 2.033934000 & 0.245976000 \\
\hline $\mathrm{H}$ & 1.915214000 & -1.120262000 & 1.033792000 \\
\hline $\mathrm{H}$ & 1.325913000 & -1.127300000 & -1.914269000 \\
\hline $\mathrm{O}$ & 2.505972000 & -1.828554000 & 1.924495000 \\
\hline $\mathrm{H}$ & 1.816792000 & -2.093419000 & 2.540075000 \\
\hline $\mathrm{K}$ & 4.104736000 & -2.010898000 & 0.149771000 \\
\hline
\end{tabular}




$\begin{array}{llll}\mathrm{O} & -6.439299000 & 0.124326000 & 0.095897000 \\ \mathrm{C} & -6.934997000 & 1.414660000 & 0.358399000 \\ \mathrm{H} & -8.018256000 & 1.312043000 & 0.438125000 \\ \mathrm{H} & -6.540595000 & 1.816347000 & 1.301524000 \\ \mathrm{H} & -6.697162000 & 2.114155000 & -0.454247000\end{array}$

\begin{tabular}{lrcc} 
A2 кон & \multicolumn{3}{c}{ Coordinates (Angstroms) } \\
- & $\mathrm{X}$ & $\mathrm{Y}$ & $\mathrm{Z}$ \\
\hline $\mathrm{C}$ & 4.212480000 & 2.249520000 & 0.749130000 \\
$\mathrm{C}$ & 3.023252000 & 1.718656000 & 0.278970000 \\
$\mathrm{C}$ & 3.001345000 & 0.572331000 & -0.560842000 \\
$\mathrm{C}$ & 4.264048000 & 0.003188000 & -0.875083000 \\
$\mathrm{C}$ & 5.449800000 & 0.545285000 & -0.386668000 \\
$\mathrm{C}$ & 5.444195000 & 1.669239000 & 0.432788000 \\
$\mathrm{H}$ & 4.179300000 & 3.134034000 & 1.380756000 \\
$\mathrm{H}$ & 2.090991000 & 2.200751000 & 0.557993000 \\
$\mathrm{H}$ & 4.297836000 & -0.849766000 & -1.545464000 \\
$\mathrm{H}$ & 6.393546000 & 0.081306000 & -0.664625000 \\
$\mathrm{H}$ & 6.370265000 & 2.091524000 & 0.809517000 \\
$\mathrm{C}$ & 1.785469000 & -0.065117000 & -0.987370000 \\
$\mathrm{C}$ & 0.483838000 & 0.284013000 & -0.649012000 \\
$\mathrm{H}$ & 0.368188000 & 1.262943000 & -0.187997000 \\
$\mathrm{O}$ & 1.981836000 & -1.347551000 & -1.564045000 \\
$\mathrm{C}$ & -0.662320000 & -0.485675000 & -0.814284000 \\
$\mathrm{H}$ & -0.576560000 & -1.439063000 & -1.335558000 \\
$\mathrm{C}$ & -2.028540000 & -0.065507000 & -0.514257000 \\
$\mathrm{C}$ & -3.104356000 & -0.913058000 & -0.821146000 \\
$\mathrm{C}$ & -2.363664000 & 1.151411000 & 0.117326000 \\
$\mathrm{C}$ & -4.427411000 & -0.590242000 & -0.526305000 \\
$\mathrm{H}$ & -2.900491000 & -1.862508000 & -1.312313000 \\
$\mathrm{C}$ & -3.673125000 & 1.485740000 & 0.415549000 \\
$\mathrm{H}$ & -1.583616000 & 1.860208000 & 0.381557000 \\
$\mathrm{C}$ & -4.722266000 & 0.618678000 & 0.099751000 \\
$\mathrm{H}$ & -5.210566000 & -1.290825000 & -0.795848000 \\
$\mathrm{H}$ & -3.908810000 & 2.429045000 & 0.899000000 \\
$\mathrm{H}$ & 1.433917000 & -1.401127000 & -2.356950000 \\
$\mathrm{O}$ & -0.004430000 & -1.821936000 & 1.745586000 \\
$\mathrm{H}$ & -0.736494000 & -1.793137000 & 2.369886000 \\
$\mathrm{~K}$ & 2.441356000 & -1.908736000 & 1.010159000 \\
$\mathrm{H}$ & -0.336534000 & -1.341686000 & 0.942719000 \\
$\mathrm{O}$ & -5.976135000 & 1.042070000 & 0.441663000 \\
$\mathrm{C}$ & -7.052848000 & 0.196371000 & 0.128008000
\end{tabular}




$\begin{array}{lrrr}\mathrm{H} & -7.952548000 & 0.703168000 & 0.481267000 \\ \mathrm{H} & -7.136854000 & 0.027618000 & -0.954530000 \\ \mathrm{H} & \mathbf{- 6 . 9 6 6 8 6 4 0 0 0} & -0.776160000 & 0.632610000\end{array}$

TSA2-3 кон

\begin{tabular}{lccc} 
& Coordinates (Angstroms) & \\
& $\mathrm{X}$ & $\mathrm{Y}$ & $\mathrm{Z}$ \\
\hline $\mathrm{C}$ & -3.963035000 & -2.666442000 & 0.175553000 \\
$\mathrm{C}$ & -2.851339000 & -1.953799000 & -0.247073000 \\
$\mathrm{C}$ & -2.944332000 & -0.586088000 & -0.577848000 \\
$\mathrm{C}$ & -4.221825000 & 0.008073000 & -0.512874000 \\
$\mathrm{C}$ & -5.334872000 & -0.717442000 & -0.092396000 \\
$\mathrm{C}$ & -5.214671000 & -2.054814000 & 0.268105000 \\
$\mathrm{H}$ & -3.855262000 & -3.719818000 & 0.419355000 \\
$\mathrm{H}$ & -1.900368000 & -2.467322000 & -0.346828000 \\
$\mathrm{H}$ & -4.337694000 & 1.037874000 & -0.836816000 \\
$\mathrm{H}$ & -6.306377000 & -0.230731000 & -0.061586000 \\
$\mathrm{H}$ & -6.083151000 & -2.621684000 & 0.588944000 \\
$\mathrm{C}$ & -1.773690000 & 0.225965000 & -0.913409000 \\
$\mathrm{C}$ & -0.477827000 & -0.114880000 & -0.666349000 \\
$\mathrm{H}$ & -0.314208000 & -1.078839000 & -0.183988000 \\
$\mathrm{O}$ & -2.108093000 & 1.501342000 & -1.360909000 \\
$\mathrm{C}$ & 0.671155000 & 0.734685000 & -0.883651000 \\
$\mathrm{C}$ & 2.004205000 & 0.249204000 & -0.485204000 \\
$\mathrm{C}$ & 2.213877000 & -0.494930000 & 0.691761000 \\
$\mathrm{C}$ & 3.148546000 & 0.553753000 & -1.232274000 \\
$\mathrm{C}$ & 3.473571000 & -0.921654000 & 1.080368000 \\
$\mathrm{H}$ & 1.365354000 & -0.737354000 & 1.329061000 \\
$\mathrm{C}$ & 4.425721000 & 0.146165000 & -0.849272000 \\
$\mathrm{H}$ & 3.038617000 & 1.121249000 & -2.153914000 \\
$\mathrm{C}$ & 4.595665000 & -0.602749000 & 0.313325000 \\
$\mathrm{H}$ & 3.614320000 & -1.496268000 & 1.991094000 \\
$\mathrm{H}$ & 5.270958000 & 0.412375000 & -1.475122000 \\
$\mathrm{H}$ & -1.374750000 & 2.103775000 & -1.128751000 \\
$\mathrm{H}$ & 0.260581000 & 1.938634000 & -0.044176000 \\
$\mathrm{O}$ & -0.328605000 & 2.793287000 & 0.419624000 \\
$\mathrm{H}$ & -2.266061000 & 1.635914000 & 1.510881000 \\
$\mathrm{H}$ & 0.229936000 & 3.570685000 & 0.307319000 \\
$\mathrm{H}$ & 0.665733000 & 1.237641000 & -1.857295000 \\
$\mathrm{H}$ & 5.795836000 & -1.060468000 & 0.784443000 \\
$\mathrm{H}$ & 6.939400000 & -0.782923000 & 0.017824000 \\
$\mathrm{H}$ & 7.115368000 & 0.297689000 & -0.077411000 \\
$\mathrm{H}$ & & -1.236021000 & 0.546504000 \\
$\mathrm{H}$ & &
\end{tabular}




\begin{tabular}{|c|c|c|c|}
\hline \multicolumn{4}{|c|}{ А3 кон } \\
\hline & $X$ & $\mathrm{Y}$ & Z \\
\hline$\overline{\mathrm{C}}$ & 5.203721000 & 0.175945000 & -1.016953000 \\
\hline $\mathrm{C}$ & 4.112831000 & 0.958074000 & -0.648013000 \\
\hline $\mathrm{C}$ & 2.981436000 & 0.381697000 & -0.055075000 \\
\hline $\mathrm{C}$ & 2.972725000 & -1.001452000 & 0.191821000 \\
\hline $\mathrm{C}$ & 4.069691000 & -1.770830000 & -0.181114000 \\
\hline $\mathrm{C}$ & 5.182629000 & -1.195662000 & -0.792419000 \\
\hline $\mathrm{H}$ & 6.073376000 & 0.644708000 & -1.468525000 \\
\hline $\mathrm{H}$ & 4.175203000 & 2.035287000 & -0.785783000 \\
\hline $\mathrm{H}$ & 2.116909000 & -1.485614000 & 0.700402000 \\
\hline $\mathrm{H}$ & 4.050342000 & -2.840077000 & 0.013307000 \\
\hline $\mathrm{H}$ & 6.031075000 & -1.810134000 & -1.080259000 \\
\hline $\mathrm{C}$ & 1.817468000 & 1.236531000 & 0.300543000 \\
\hline $\mathrm{C}$ & 0.900705000 & 0.941610000 & 1.231890000 \\
\hline $\mathrm{H}$ & 0.944490000 & -0.028926000 & 1.736570000 \\
\hline $\mathrm{O}$ & 1.720301000 & 2.436751000 & -0.373019000 \\
\hline $\mathrm{C}$ & -0.285797000 & 1.828502000 & 1.529428000 \\
\hline $\mathrm{H}$ & -0.081799000 & 2.858817000 & 1.224158000 \\
\hline $\mathrm{C}$ & -1.518280000 & 1.316685000 & 0.811088000 \\
\hline $\mathrm{C}$ & -1.664802000 & 1.518994000 & -0.570693000 \\
\hline $\mathrm{C}$ & -2.478018000 & 0.541787000 & 1.456162000 \\
\hline $\mathrm{C}$ & -2.720715000 & 0.959254000 & -1.274718000 \\
\hline $\mathrm{H}$ & -0.920334000 & 2.113825000 & -1.093999000 \\
\hline $\mathrm{C}$ & -3.548805000 & -0.034929000 & 0.764261000 \\
\hline $\mathrm{H}$ & -2.380196000 & 0.351635000 & 2.521419000 \\
\hline $\mathrm{C}$ & -3.675747000 & 0.175402000 & -0.609748000 \\
\hline $\mathrm{H}$ & -2.839718000 & 1.126002000 & -2.340926000 \\
\hline $\mathrm{H}$ & -4.267749000 & -0.634322000 & 1.311241000 \\
\hline $\mathrm{O}$ & 0.553916000 & -2.176723000 & 1.627638000 \\
\hline $\mathrm{H}$ & -0.473072000 & 1.836811000 & 2.608806000 \\
\hline $\mathrm{H}$ & 2.233697000 & 2.369279000 & -1.186592000 \\
\hline $\mathrm{H}$ & 1.012860000 & -2.515242000 & 2.400904000 \\
\hline $\mathrm{K}$ & -0.670791000 & -1.534478000 & -0.209750000 \\
\hline $\mathrm{O}$ & -4.666486000 & -0.335157000 & -1.385331000 \\
\hline $\mathrm{C}$ & -5.674152000 & -1.094599000 & -0.754180000 \\
\hline $\mathrm{H}$ & -6.372550000 & -1.381619000 & -1.540603000 \\
\hline $\mathrm{H}$ & -5.263878000 & -2.000071000 & -0.287660000 \\
\hline $\mathrm{H}$ & -6.204828000 & -0.506299000 & 0.004815000 \\
\hline
\end{tabular}




\begin{tabular}{|c|c|c|c|}
\hline \multicolumn{4}{|c|}{$\mathbf{A 1}_{\mathrm{NaOH}}$} \\
\hline & $X$ & $\mathrm{Y}$ & Z \\
\hline $\mathrm{C}$ & 3.277722000 & 2.799062000 & -0.789029000 \\
\hline $\mathrm{C}$ & 2.365403000 & 1.757375000 & -0.645904000 \\
\hline $\mathrm{C}$ & 2.729442000 & 0.592333000 & 0.034482000 \\
\hline $\mathrm{C}$ & 4.010192000 & 0.476576000 & 0.585010000 \\
\hline $\mathrm{C}$ & 4.916190000 & 1.524701000 & 0.440754000 \\
\hline $\mathrm{C}$ & 4.555227000 & 2.682179000 & -0.245812000 \\
\hline $\mathrm{H}$ & 2.990034000 & 3.704189000 & -1.316566000 \\
\hline $\mathrm{H}$ & 1.360555000 & 1.853835000 & -1.053633000 \\
\hline $\mathrm{H}$ & 4.256773000 & -0.455353000 & 1.112643000 \\
\hline $\mathrm{H}$ & 5.909359000 & 1.438813000 & 0.873401000 \\
\hline $\mathrm{H}$ & 5.266721000 & 3.496457000 & -0.352698000 \\
\hline $\mathrm{C}$ & 1.783359000 & -0.591314000 & 0.125210000 \\
\hline $\mathrm{C}$ & 0.357863000 & -0.197065000 & 0.381906000 \\
\hline $\mathrm{H}$ & 0.230966000 & 0.468004000 & 1.234521000 \\
\hline $\mathrm{O}$ & 1.905409000 & -1.390804000 & -1.082208000 \\
\hline $\mathrm{C}$ & -0.693695000 & -0.610675000 & -0.329772000 \\
\hline $\mathrm{H}$ & -0.511202000 & -1.306809000 & -1.147388000 \\
\hline $\mathrm{C}$ & -2.105454000 & -0.260222000 & -0.109698000 \\
\hline $\mathrm{C}$ & -3.096254000 & -0.950138000 & -0.809007000 \\
\hline $\mathrm{C}$ & -2.524538000 & 0.745161000 & 0.777537000 \\
\hline $\mathrm{C}$ & -4.452138000 & -0.679646000 & -0.635676000 \\
\hline $\mathrm{H}$ & -2.805307000 & -1.730819000 & -1.507913000 \\
\hline $\mathrm{C}$ & -3.864294000 & 1.027337000 & 0.963547000 \\
\hline $\mathrm{H}$ & -1.789705000 & 1.324214000 & 1.328955000 \\
\hline $\mathrm{C}$ & -4.842786000 & 0.314404000 & 0.259692000 \\
\hline $\mathrm{H}$ & -5.182111000 & -1.248463000 & -1.200204000 \\
\hline $\mathrm{H}$ & -4.184290000 & 1.803922000 & 1.650479000 \\
\hline $\mathrm{H}$ & 2.159700000 & -1.259113000 & 0.918839000 \\
\hline $\mathrm{H}$ & 1.755807000 & -0.794735000 & -1.828259000 \\
\hline $\mathrm{O}$ & 3.857739000 & -2.426654000 & 1.432019000 \\
\hline $\mathrm{H}$ & 4.053300000 & -2.783610000 & 2.300122000 \\
\hline $\mathrm{O}$ & -6.128376000 & 0.667405000 & 0.514367000 \\
\hline $\mathrm{C}$ & -7.149336000 & -0.017309000 & -0.174207000 \\
\hline $\mathrm{H}$ & -8.088786000 & 0.413403000 & 0.174189000 \\
\hline $\mathrm{H}$ & -7.066649000 & 0.123602000 & -1.259808000 \\
\hline $\mathrm{H}$ & -7.138017000 & -1.091575000 & 0.051652000 \\
\hline $\mathrm{Na}$ & 3.645752000 & -2.766554000 & -0.533269000 \\
\hline
\end{tabular}

TSA1-2 ${ }_{\mathrm{NaOH}}$ 


\begin{tabular}{lrcc} 
& \multicolumn{3}{c}{ Coordinates (Angstroms) } \\
& $\mathrm{X}$ & $\mathrm{Y}$ & $\mathrm{Z}$ \\
\hline $\mathrm{C}$ & 4.737160000 & 1.445343000 & -1.156923000 \\
$\mathrm{C}$ & 3.727270000 & 0.480800000 & -1.120465000 \\
$\mathrm{C}$ & 2.740446000 & 0.490367000 & -0.120090000 \\
$\mathrm{C}$ & 2.813948000 & 1.512168000 & 0.845550000 \\
$\mathrm{C}$ & 3.808955000 & 2.475203000 & 0.799561000 \\
$\mathrm{C}$ & 4.786155000 & 2.448129000 & -0.199198000 \\
$\mathrm{H}$ & 5.477486000 & 1.414826000 & -1.952423000 \\
$\mathrm{H}$ & 3.679305000 & -0.267093000 & -1.909254000 \\
$\mathrm{H}$ & 2.089692000 & 1.526389000 & 1.655516000 \\
$\mathrm{H}$ & 3.836367000 & 3.249593000 & 1.561172000 \\
$\mathrm{H}$ & 5.568662000 & 3.199980000 & -0.223995000 \\
$\mathrm{C}$ & 1.737302000 & -0.603605000 & 0.016944000 \\
$\mathrm{C}$ & 0.329258000 & -0.212187000 & 0.107774000 \\
$\mathrm{H}$ & 0.144920000 & 0.740672000 & 0.602846000 \\
$\mathrm{O}$ & 1.957260000 & -1.651863000 & -0.954666000 \\
$\mathrm{C}$ & -0.714878000 & -0.936234000 & -0.334835000 \\
$\mathrm{H}$ & -0.519042000 & -1.915253000 & -0.770302000 \\
$\mathrm{C}$ & -2.131900000 & -0.559373000 & -0.242917000 \\
$\mathrm{C}$ & -3.122388000 & -1.541544000 & -0.407754000 \\
$\mathrm{C}$ & -2.569557000 & 0.746800000 & -0.004092000 \\
$\mathrm{C}$ & -4.470847000 & -1.243506000 & -0.314627000 \\
$\mathrm{H}$ & -2.821551000 & -2.568085000 & -0.604079000 \\
$\mathrm{C}$ & -3.921554000 & 1.064854000 & 0.098843000 \\
$\mathrm{H}$ & -1.842715000 & 1.548738000 & 0.090877000 \\
$\mathrm{C}$ & -4.883103000 & 0.065831000 & -0.053632000 \\
$\mathrm{H}$ & -5.227436000 & -2.011856000 & -0.437798000 \\
$\mathrm{H}$ & -4.205896000 & 2.094681000 & 0.284332000 \\
$\mathrm{H}$ & 2.151327000 & -1.273067000 & 1.179930000 \\
$\mathrm{H}$ & 1.543286000 & -1.386983000 & -1.790927000 \\
$\mathrm{O}$ & 2.800555000 & -1.976808000 & 1.952454000 \\
$\mathrm{H}$ & 2.169434000 & -2.611484000 & 2.308706000 \\
$\mathrm{C}$ & -6.228208000 & 0.262621000 & 0.023893000 \\
$\mathrm{H}$ & -6.686959000 & 1.565265000 & 0.293039000 \\
$\mathrm{H}$ & -7.775506000 & 1.500876000 & 0.329192000 \\
$\mathrm{H}$ & -6.315189000 & 1.934723000 & 1.258262000 \\
$\mathrm{H}$ & 3.920650000 & -2.155310000 & 0.136133000 \\
\hline
\end{tabular}

A2 ${ }_{\mathrm{NaOH}}$

Coordinates (Angstroms)

X

Y Z 


\begin{tabular}{lrrr}
$\mathrm{C}$ & 4.329576000 & 2.178463000 & 0.570556000 \\
$\mathrm{C}$ & 3.145125000 & 1.618423000 & 0.128405000 \\
$\mathrm{C}$ & 3.116310000 & 0.345712000 & -0.504471000 \\
$\mathrm{C}$ & 4.366475000 & -0.316550000 & -0.638194000 \\
$\mathrm{C}$ & 5.549288000 & 0.260753000 & -0.178240000 \\
$\mathrm{C}$ & 5.550058000 & 1.508042000 & 0.432447000 \\
$\mathrm{H}$ & 4.303189000 & 3.158830000 & 1.039517000 \\
$\mathrm{H}$ & 2.221812000 & 2.173875000 & 0.262231000 \\
$\mathrm{H}$ & 4.401628000 & -1.267472000 & -1.160817000 \\
$\mathrm{H}$ & 6.484886000 & -0.275697000 & -0.317571000 \\
$\mathrm{H}$ & 6.472302000 & 1.956198000 & 0.787452000 \\
$\mathrm{C}$ & 1.897204000 & -0.308410000 & -0.895351000 \\
$\mathrm{C}$ & 0.594619000 & 0.094362000 & -0.621015000 \\
$\mathrm{H}$ & 0.482495000 & 1.136923000 & -0.330646000 \\
$\mathrm{O}$ & 2.089706000 & -1.680925000 & -1.230664000 \\
$\mathrm{C}$ & -0.552104000 & -0.689529000 & -0.641429000 \\
$\mathrm{H}$ & -0.476491000 & -1.723099000 & -0.981734000 \\
$\mathrm{C}$ & -1.918310000 & -0.209456000 & -0.434797000 \\
$\mathrm{C}$ & -2.984717000 & -1.120126000 & -0.431997000 \\
$\mathrm{C}$ & -2.255757000 & 1.140882000 & -0.209920000 \\
$\mathrm{C}$ & -4.304672000 & -0.732832000 & -0.211430000 \\
$\mathrm{H}$ & -2.776441000 & -2.174003000 & -0.605647000 \\
$\mathrm{C}$ & -3.561657000 & 1.540428000 & 0.016006000 \\
$\mathrm{H}$ & -1.482920000 & 1.904297000 & -0.224095000 \\
$\mathrm{C}$ & -4.602464000 & 0.608382000 & 0.020230000 \\
$\mathrm{H}$ & -5.082599000 & -1.488480000 & -0.223934000 \\
$\mathrm{H}$ & -3.801510000 & 2.585947000 & 0.183958000 \\
$\mathrm{H}$ & 1.469917000 & -1.903661000 & -1.935559000 \\
$\mathrm{O}$ & 0.446457000 & -1.750016000 & 1.943517000 \\
$\mathrm{H}$ & 0.080164000 & -1.279967000 & 2.700651000 \\
$\mathrm{H}$ & -0.025273000 & -1.366545000 & 1.153421000 \\
$\mathrm{O}$ & -5.853102000 & 1.106045000 & 0.252261000 \\
$\mathrm{H}$ & -6.924959000 & 0.197623000 & 0.240517000 \\
$\mathrm{H}$ & -7.824132000 & 0.783040000 & 0.440116000 \\
$\mathrm{H}$ & -7.027678000 & -0.297594000 & -0.734870000 \\
$\mathrm{H}$ & 2.478986000 & -1.768200000 & 1.019193000 \\
$\mathrm{H}$ & & & 1.032684000 \\
\hline
\end{tabular}

TSA2-3 ${ }_{\mathrm{NaOH}}$

\begin{tabular}{|c|c|c|c|}
\hline & \multicolumn{3}{|c|}{ Coordinates (Angstroms) } \\
\hline & $X$ & $\mathrm{Y}$ & Z \\
\hline $\mathrm{C}$ & -4.171584000 & -2.492433000 & 0.180973000 \\
\hline $\mathrm{C}$ & -3.039731000 & -1.790350000 & -0.201147000 \\
\hline
\end{tabular}




\begin{tabular}{lrrr}
$\mathrm{C}$ & -3.101664000 & -0.410502000 & -0.474668000 \\
$\mathrm{C}$ & -4.357286000 & 0.218779000 & -0.380601000 \\
$\mathrm{C}$ & -5.490685000 & -0.495560000 & 0.005160000 \\
$\mathrm{C}$ & -5.406338000 & -1.850964000 & 0.296775000 \\
$\mathrm{H}$ & -4.092498000 & -3.557026000 & 0.381823000 \\
$\mathrm{H}$ & -2.097926000 & -2.319148000 & -0.307063000 \\
$\mathrm{H}$ & -4.448687000 & 1.266562000 & -0.649945000 \\
$\mathrm{H}$ & -6.447661000 & 0.015685000 & 0.063467000 \\
$\mathrm{H}$ & -6.290088000 & -2.408027000 & 0.591614000 \\
$\mathrm{C}$ & -1.905375000 & 0.376786000 & -0.779363000 \\
$\mathrm{C}$ & -0.612788000 & 0.021554000 & -0.579191000 \\
$\mathrm{H}$ & -0.426242000 & -0.990100000 & -0.222816000 \\
$\mathrm{O}$ & -2.214388000 & 1.710893000 & -1.090406000 \\
$\mathrm{C}$ & 0.516313000 & 0.936760000 & -0.669377000 \\
$\mathrm{C}$ & 1.861881000 & 0.385532000 & -0.378440000 \\
$\mathrm{C}$ & 2.094594000 & -0.412387000 & 0.756146000 \\
$\mathrm{C}$ & 2.972318000 & 0.676235000 & -1.174748000 \\
$\mathrm{C}$ & 3.352115000 & -0.909636000 & 1.057251000 \\
$\mathrm{H}$ & 1.267863000 & -0.636953000 & 1.427501000 \\
$\mathrm{C}$ & 4.249143000 & 0.198459000 & -0.879280000 \\
$\mathrm{H}$ & 2.839257000 & 1.289805000 & -2.062936000 \\
$\mathrm{C}$ & 4.444515000 & -0.606513000 & 0.241031000 \\
$\mathrm{H}$ & 3.516569000 & -1.526606000 & 1.935420000 \\
$\mathrm{H}$ & 5.071637000 & 0.455364000 & -1.538078000 \\
$\mathrm{H}$ & -1.374768000 & 2.177222000 & -1.218699000 \\
$\mathrm{H}$ & 0.200905000 & 1.857333000 & 0.400864000 \\
$\mathrm{O}$ & -0.243706000 & 2.533826000 & 1.281428000 \\
$\mathrm{H}$ & 0.499078000 & 2.733475000 & 1.859196000 \\
$\mathrm{H}$ & 0.519339000 & 1.545333000 & -1.583836000 \\
$\mathrm{O}$ & 5.643620000 & -1.134639000 & 0.626663000 \\
$\mathrm{H}$ & 6.763592000 & -0.858881000 & -0.176360000 \\
$\mathrm{H}$ & 6.968568000 & 0.218993000 & -0.233053000 \\
$\mathrm{H}$ & 7.609717000 & -1.358844000 & 0.298158000 \\
$\mathrm{H}$ & 6.638673000 & -1.251160000 & -1.195001000 \\
-2.222916000 & 1.789652000 & 1.313360000 \\
\hline
\end{tabular}

\begin{tabular}{|c|c|c|c|}
\hline \multicolumn{4}{|c|}{ A3 ${ }_{\mathrm{NaOH}}$} \\
\hline & \multicolumn{3}{|c|}{ Coordinates (Angstroms) } \\
\hline & $\mathrm{X}$ & Y & Z \\
\hline$\overline{\mathrm{C}}$ & -5.199653000 & -0.036576000 & -0.901202000 \\
\hline $\mathrm{C}$ & -4.101287000 & -0.832849000 & -0.592453000 \\
\hline $\mathrm{C}$ & -2.934926000 & -0.269123000 & -0.057423000 \\
\hline $\mathrm{C}$ & -2.889430000 & 1.111854000 & 0.187879000 \\
\hline
\end{tabular}




$\begin{array}{lrrr}\mathrm{C} & -3.995830000 & 1.895409000 & -0.125236000 \\ \mathrm{C} & -5.147014000 & 1.334502000 & -0.674442000 \\ \mathrm{H} & -6.097773000 & -0.491750000 & -1.308555000 \\ \mathrm{H} & -4.179973000 & -1.909198000 & -0.730428000 \\ \mathrm{H} & -1.992651000 & 1.582144000 & 0.632207000 \\ \mathrm{H} & -3.951632000 & 2.964810000 & 0.062885000 \\ \mathrm{H} & -6.000965000 & 1.960725000 & -0.917099000 \\ \mathrm{C} & -1.771122000 & -1.143705000 & 0.242354000 \\ \mathrm{C} & -0.861677000 & -0.917093000 & 1.201083000 \\ \mathrm{H} & -0.928096000 & 0.005206000 & 1.777907000 \\ \mathrm{O} & -1.669988000 & -2.292875000 & -0.508618000 \\ \mathrm{C} & 0.318477000 & -1.827746000 & 1.453835000 \\ \mathrm{H} & 0.105440000 & -2.836809000 & 1.089647000 \\ \mathrm{C} & 1.553108000 & -1.282567000 & 0.764363000 \\ \mathrm{C} & 1.713331000 & -1.440051000 & -0.622665000 \\ \mathrm{C} & 2.482252000 & -0.493136000 & 1.437739000 \\ \mathrm{C} & 2.749891000 & -0.818220000 & -1.304515000 \\ \mathrm{H} & 0.993432000 & -2.044307000 & -1.168694000 \\ \mathrm{C} & 3.533878000 & 0.141461000 & 0.768657000 \\ \mathrm{H} & 2.368961000 & -0.331538000 & 2.505849000 \\ \mathrm{C} & 3.672931000 & -0.020022000 & -0.611138000 \\ \mathrm{H} & 2.878791000 & -0.948394000 & -2.374410000 \\ \mathrm{H} & 4.223255000 & 0.755977000 & 1.335770000 \\ \mathrm{O} & -0.321079000 & 2.221997000 & 1.348550000 \\ \mathrm{H} & 0.502363000 & -1.898567000 & 2.531146000 \\ \mathrm{H} & -2.227561000 & -2.192991000 & -1.289511000 \\ \mathrm{H} & -0.446881000 & 3.101205000 & 1.712145000 \\ \mathrm{O} & 4.647295000 & 0.545380000 & -1.364242000 \\ \mathrm{C} & 5.600120000 & 1.358723000 & -0.713049000 \\ \mathrm{H} & 6.285631000 & 1.698241000 & -1.489677000 \\ \mathrm{H} & 5.128471000 & 2.229684000 & -0.240175000 \\ \mathrm{H} & 6.160259000 & 0.793560000 & 0.042232000 \\ \mathrm{Na} & 0.690074000 & 1.200076000 & -0.063590000 \\ ------ & \end{array}$

\begin{tabular}{|c|c|c|c|}
\hline \multicolumn{4}{|c|}{$\mathbf{A 1}_{\text {LiOH }}$} \\
\hline & \multicolumn{3}{|c|}{ Coordinates (Angstroms) } \\
\hline & $\mathrm{X}$ & $\mathrm{Y}$ & Z \\
\hline$\overline{\mathrm{C}}$ & 3.491611000 & -2.586077000 & 0.622734000 \\
\hline $\mathrm{C}$ & 2.554423000 & -1.568087000 & 0.466786000 \\
\hline $\mathrm{C}$ & 2.941350000 & -0.332706000 & -0.057397000 \\
\hline $\mathrm{C}$ & 4.273428000 & -0.117658000 & -0.428048000 \\
\hline $\mathrm{C}$ & 5.204377000 & -1.139716000 & -0.269296000 \\
\hline $\mathrm{C}$ & 4.817070000 & -2.371916000 & 0.254811000 \\
\hline
\end{tabular}




\begin{tabular}{lrrr}
$\mathrm{H}$ & 3.185514000 & -3.546270000 & 1.027944000 \\
$\mathrm{H}$ & 1.515600000 & -1.735995000 & 0.742188000 \\
$\mathrm{H}$ & 4.548882000 & 0.865631000 & -0.822289000 \\
$\mathrm{H}$ & 6.238417000 & -0.974241000 & -0.558092000 \\
$\mathrm{H}$ & 5.548389000 & -3.166543000 & 0.373402000 \\
$\mathrm{C}$ & 1.961239000 & 0.820125000 & -0.159448000 \\
$\mathrm{C}$ & 0.546074000 & 0.396065000 & -0.426142000 \\
$\mathrm{H}$ & 0.431782000 & -0.217053000 & -1.318435000 \\
$\mathrm{O}$ & 2.054349000 & 1.626867000 & 1.050046000 \\
$\mathrm{C}$ & -0.507591000 & 0.724913000 & 0.324455000 \\
$\mathrm{H}$ & -0.336596000 & 1.373143000 & 1.182587000 \\
$\mathrm{C}$ & -1.907496000 & 0.330997000 & 0.103746000 \\
$\mathrm{C}$ & -2.915648000 & 0.957169000 & 0.837371000 \\
$\mathrm{C}$ & -2.295985000 & -0.659411000 & -0.813538000 \\
$\mathrm{C}$ & -4.261487000 & 0.638925000 & 0.668648000 \\
$\mathrm{H}$ & -2.647181000 & 1.723975000 & 1.560055000 \\
$\mathrm{C}$ & -3.625450000 & -0.988577000 & -0.994999000 \\
$\mathrm{H}$ & -1.544477000 & -1.191266000 & -1.389713000 \\
$\mathrm{C}$ & -4.622757000 & -0.339861000 & -0.255865000 \\
$\mathrm{H}$ & -5.006834000 & 1.158478000 & 1.259845000 \\
$\mathrm{H}$ & -3.922988000 & -1.754724000 & -1.703289000 \\
$\mathrm{H}$ & 2.315206000 & 1.501780000 & -0.943928000 \\
$\mathrm{H}$ & 2.069826000 & 1.018062000 & 1.800151000 \\
$\mathrm{O}$ & 4.111510000 & 2.945326000 & -0.938401000 \\
$\mathrm{H}$ & 4.572205000 & 3.514724000 & -1.554016000 \\
$\mathrm{O}$ & -5.895117000 & -0.737481000 & -0.506477000 \\
$\mathrm{C}$ & -6.934901000 & -0.123100000 & 0.220148000 \\
$\mathrm{H}$ & -7.859228000 & -0.585515000 & -0.127750000 \\
$\mathrm{H}$ & -6.827289000 & -0.294913000 & 1.298940000 \\
$\mathrm{H}$ & -6.977001000 & 0.957024000 & 0.029190000 \\
$\mathrm{Li}$ & 3.406760000 & 2.943166000 & 0.579348000 \\
\hline---- &
\end{tabular}

TSA1-2 ${ }_{\mathrm{LiOH}}$

\begin{tabular}{|c|c|c|c|}
\hline \multicolumn{4}{|c|}{ Coordinates (Angstroms) } \\
\hline & $\mathrm{X}$ & Y & $\mathrm{Z}$ \\
\hline$\overline{\mathrm{C}}$ & 4.203742000 & 2.422479000 & -0.017244000 \\
\hline $\mathrm{C}$ & 3.076744000 & 1.610196000 & 0.053702000 \\
\hline $\mathrm{C}$ & 3.181425000 & 0.216287000 & -0.061590000 \\
\hline $\mathrm{C}$ & 4.458116000 & -0.327262000 & -0.271752000 \\
\hline $\mathrm{C}$ & 5.579953000 & 0.488097000 & -0.357633000 \\
\hline $\mathrm{C}$ & 5.463337000 & 1.869476000 & -0.225677000 \\
\hline $\mathrm{H}$ & 4.090249000 & 3.498978000 & 0.077937000 \\
\hline $\mathrm{H}$ & 2.105277000 & 2.076349000 & 0.188858000 \\
\hline
\end{tabular}




\begin{tabular}{lrcc}
$\mathrm{H}$ & 4.562138000 & -1.403749000 & -0.361047000 \\
$\mathrm{H}$ & 6.555232000 & 0.037638000 & -0.521422000 \\
$\mathrm{H}$ & 6.341038000 & 2.505845000 & -0.287784000 \\
$\mathrm{C}$ & 2.016945000 & -0.690053000 & 0.083822000 \\
$\mathrm{C}$ & 0.650704000 & -0.197836000 & 0.027417000 \\
$\mathrm{H}$ & 0.458661000 & 0.704355000 & 0.607221000 \\
$\mathrm{O}$ & 2.155350000 & -1.925017000 & -0.669473000 \\
$\mathrm{C}$ & -0.410706000 & -0.837498000 & -0.526366000 \\
$\mathrm{H}$ & -0.223577000 & -1.671490000 & -1.208320000 \\
$\mathrm{C}$ & -1.822832000 & -0.450161000 & -0.396092000 \\
$\mathrm{C}$ & -2.765589000 & -0.926037000 & -1.322586000 \\
$\mathrm{C}$ & -2.298423000 & 0.376813000 & 0.625753000 \\
$\mathrm{C}$ & -4.103099000 & -0.580019000 & -1.245392000 \\
$\mathrm{H}$ & -2.434964000 & -1.575430000 & -2.130423000 \\
$\mathrm{C}$ & -3.639336000 & 0.742723000 & 0.713613000 \\
$\mathrm{H}$ & -1.613686000 & 0.740600000 & 1.387082000 \\
$\mathrm{C}$ & -4.552464000 & 0.265390000 & -0.226926000 \\
$\mathrm{H}$ & -4.822444000 & -0.948554000 & -1.969478000 \\
$\mathrm{H}$ & -3.955757000 & 1.388139000 & 1.525246000 \\
$\mathrm{H}$ & 2.015141000 & -1.357097000 & 1.360530000 \\
$\mathrm{H}$ & 2.082032000 & -1.726521000 & -1.614871000 \\
$\mathrm{O}$ & 1.776448000 & -2.289202000 & 2.090809000 \\
$\mathrm{H}$ & 1.385149000 & -1.925447000 & 2.892029000 \\
$\mathrm{Li}$ & 0.877200000 & -2.768918000 & 0.585108000 \\
$\mathrm{O}$ & -5.881381000 & 0.551828000 & -0.233650000 \\
$\mathrm{C}$ & -6.373780000 & 1.410511000 & 0.768275000 \\
$\mathrm{H}$ & -7.441513000 & 1.521000000 & 0.574878000 \\
$\mathrm{H}$ & -6.231075000 & 0.984990000 & 1.770423000 \\
$\mathrm{H}-----8.895256000$ & 2.397628000 & 0.724394000 \\
\hline
\end{tabular}

\section{A2}

\begin{tabular}{lrrr} 
& & \\
& & & \\
Coordinates (Angstroms) & Y & \\
\hline $\mathrm{C}$ & 4.470692000 & 2.129652000 & 0.177272000 \\
$\mathrm{C}$ & 3.295581000 & 1.508825000 & -0.200557000 \\
$\mathrm{C}$ & 3.248460000 & 0.113329000 & -0.458979000 \\
$\mathrm{C}$ & 4.464885000 & -0.601498000 & -0.308475000 \\
$\mathrm{C}$ & 5.640203000 & 0.043065000 & 0.080314000 \\
$\mathrm{C}$ & 5.659489000 & 1.406831000 & 0.332234000 \\
$\mathrm{H}$ & 4.464882000 & 3.201954000 & 0.354957000 \\
$\mathrm{H}$ & 2.398683000 & 2.109129000 & -0.318183000 \\
$\mathrm{H}$ & 4.494125000 & -1.656546000 & -0.562587000 \\
$\mathrm{H}$ & 6.553462000 & -0.539542000 & 0.170687000
\end{tabular}




$\begin{array}{lrcc}\mathrm{H} & 6.575621000 & 1.905800000 & 0.630644000 \\ \mathrm{C} & 2.022674000 & -0.585049000 & -0.754391000 \\ \mathrm{C} & 0.720546000 & -0.126503000 & -0.573579000 \\ \mathrm{H} & 0.624235000 & 0.952852000 & -0.481194000 \\ \mathrm{O} & 2.211738000 & -2.004477000 & -0.776527000 \\ \mathrm{C} & -0.436343000 & -0.882807000 & -0.439703000 \\ \mathrm{H} & -0.396387000 & -1.955802000 & -0.635480000 \\ \mathrm{C} & -1.792506000 & -0.345138000 & -0.302040000 \\ \mathrm{C} & -2.894286000 & -1.208141000 & -0.363284000 \\ \mathrm{C} & -2.080103000 & 1.015422000 & -0.075354000 \\ \mathrm{C} & -4.206240000 & -0.763802000 & -0.211327000 \\ \mathrm{H} & -2.723583000 & -2.268517000 & -0.537124000 \\ \mathrm{C} & -3.377392000 & 1.473230000 & 0.073693000 \\ \mathrm{H} & -1.273582000 & 1.740993000 & -0.011568000 \\ \mathrm{C} & -4.457272000 & 0.588385000 & 0.009336000 \\ \mathrm{H} & -5.014304000 & -1.484755000 & -0.270672000 \\ \mathrm{H} & -3.579596000 & 2.525885000 & 0.245697000 \\ \mathrm{H} & 1.520593000 & -2.394565000 & -1.324130000 \\ \mathrm{O} & 0.823420000 & -1.585907000 & 2.131368000 \\ \mathrm{H} & 0.634216000 & -0.973233000 & 2.852018000 \\ \mathrm{H} & 0.213309000 & -1.314745000 & 1.384760000 \\ \mathrm{O} & -5.694170000 & 1.140020000 & 0.174536000 \\ \mathrm{C} & -6.803518000 & 0.278326000 & 0.118215000 \\ \mathrm{H} & -7.683379000 & 0.903727000 & 0.277654000 \\ \mathrm{H} & -6.885771000 & -0.215222000 & -0.859759000 \\ \mathrm{H} & -6.761547000 & -0.489742000 & 0.902545000 \\ \mathrm{Li} & 2.420633000 & -1.569840000 & 1.108822000 \\ - & & & \end{array}$

TSA2-3 ${ }_{\text {LiOH }}$

\begin{tabular}{lrrr} 
& \multicolumn{3}{c}{ Coordinates (Angstroms) } \\
& $\mathrm{X}$ & $\mathrm{Y}$ & $\mathrm{Z}$ \\
\hline $\mathrm{C}$ & -4.434172000 & -2.213184000 & 0.431835000 \\
$\mathrm{C}$ & -3.264684000 & -1.475080000 & 0.321242000 \\
$\mathrm{C}$ & -3.271016000 & -0.194314000 & -0.259978000 \\
$\mathrm{C}$ & -4.500042000 & 0.319358000 & -0.703250000 \\
$\mathrm{C}$ & -5.670330000 & -0.423451000 & -0.582207000 \\
$\mathrm{C}$ & -5.647457000 & -1.693809000 & -0.017547000 \\
$\mathrm{H}$ & -4.398568000 & -3.200958000 & 0.882371000 \\
$\mathrm{H}$ & -2.337441000 & -1.898001000 & 0.696428000 \\
$\mathrm{H}$ & -4.529011000 & 1.303130000 & -1.158164000 \\
$\mathrm{H}$ & -6.605992000 & -0.002867000 & -0.939837000 \\
$\mathrm{H}$ & -6.561598000 & -2.271875000 & 0.075637000 \\
$\mathrm{C}$ & -2.047434000 & 0.603590000 & -0.378206000
\end{tabular}




\begin{tabular}{|c|c|c|c|}
\hline $\mathrm{C}$ & -0.752899000 & 0.221910000 & -0.207326000 \\
\hline $\mathrm{H}$ & -0.592817000 & -0.830719000 & 0.018136000 \\
\hline $\mathrm{O}$ & -2.309039000 & 1.980677000 & -0.633059000 \\
\hline $\mathrm{C}$ & 0.407490000 & 1.080991000 & -0.222811000 \\
\hline $\mathrm{H}$ & 0.370488000 & 1.901237000 & -0.951271000 \\
\hline $\mathrm{C}$ & 1.750713000 & 0.460369000 & -0.146518000 \\
\hline $\mathrm{C}$ & 2.816311000 & 0.922131000 & -0.922918000 \\
\hline $\mathrm{C}$ & 2.034495000 & -0.582375000 & 0.753079000 \\
\hline $\mathrm{C}$ & 4.098228000 & 0.381153000 & -0.828029000 \\
\hline $\mathrm{H}$ & 2.643679000 & 1.727866000 & -1.633036000 \\
\hline $\mathrm{C}$ & 3.297494000 & -1.142506000 & 0.850555000 \\
\hline $\mathrm{H}$ & 1.248414000 & -0.955645000 & 1.406620000 \\
\hline $\mathrm{C}$ & 4.344902000 & -0.663636000 & 0.059808000 \\
\hline $\mathrm{H}$ & 4.884601000 & 0.779229000 & -1.460003000 \\
\hline $\mathrm{H}$ & 3.501444000 & -1.947546000 & 1.549767000 \\
\hline $\mathrm{H}$ & -1.621816000 & 2.320034000 & -1.223051000 \\
\hline $\mathrm{O}$ & -0.267366000 & 2.529319000 & 1.868521000 \\
\hline $\mathrm{H}$ & 0.195690000 & 2.284995000 & 2.676937000 \\
\hline $\mathrm{H}$ & 0.183536000 & 1.891450000 & 1.007959000 \\
\hline $\mathrm{O}$ & 5.553888000 & -1.272439000 & 0.234549000 \\
\hline $\mathrm{C}$ & 6.632650000 & -0.809787000 & -0.539344000 \\
\hline $\mathrm{H}$ & 7.496176000 & -1.409831000 & -0.247485000 \\
\hline $\mathrm{H}$ & 6.448331000 & -0.943577000 & -1.614035000 \\
\hline $\mathrm{H}$ & 6.847619000 & 0.249669000 & -0.343888000 \\
\hline $\mathrm{Li}$ & -1.919361000 & 2.118261000 & 1.282169000 \\
\hline
\end{tabular}

\begin{tabular}{|c|c|c|c|}
\hline \multicolumn{4}{|c|}{ Coordinates (Angstroms) } \\
\hline & $X$ & $\mathrm{Y}$ & $\mathrm{Z}$ \\
\hline$\overline{\mathrm{C}}$ & -5.205686000 & 0.216301000 & -0.543326000 \\
\hline $\mathrm{C}$ & -4.116993000 & -0.643318000 & -0.451691000 \\
\hline $\mathrm{C}$ & -2.866606000 & -0.163268000 & -0.038104000 \\
\hline $\mathrm{C}$ & -2.710615000 & 1.191886000 & 0.276594000 \\
\hline $\mathrm{C}$ & -3.810055000 & 2.041271000 & 0.181391000 \\
\hline $\mathrm{C}$ & -5.052762000 & 1.563372000 & -0.226315000 \\
\hline $\mathrm{H}$ & -6.172950000 & -0.168375000 & -0.853237000 \\
\hline $\mathrm{H}$ & -4.250630000 & -1.701775000 & -0.665066000 \\
\hline $\mathrm{H}$ & -1.725885000 & 1.594748000 & 0.553299000 \\
\hline $\mathrm{H}$ & -3.684546000 & 3.094031000 & 0.417691000 \\
\hline $\mathrm{H}$ & -5.900626000 & 2.238729000 & -0.298129000 \\
\hline $\mathrm{C}$ & -1.728235000 & -1.111023000 & 0.054083000 \\
\hline $\mathrm{C}$ & -0.844159000 & -1.149317000 & 1.061923000 \\
\hline $\mathrm{H}$ & -0.955333000 & -0.422890000 & 1.861789000 \\
\hline
\end{tabular}




\begin{tabular}{|c|c|c|c|}
\hline $\mathrm{O}$ & -1.637651000 & -2.043280000 & -0.947018000 \\
\hline $\mathrm{C}$ & 0.312551000 & -2.121575000 & 1.129549000 \\
\hline $\mathrm{H}$ & 0.081463000 & -3.023147000 & 0.555063000 \\
\hline $\mathrm{C}$ & 1.559040000 & -1.457354000 & 0.582083000 \\
\hline $\mathrm{C}$ & 1.773671000 & -1.390102000 & -0.805308000 \\
\hline $\mathrm{C}$ & 2.412825000 & -0.726636000 & 1.407365000 \\
\hline $\mathrm{C}$ & 2.786338000 & -0.605734000 & -1.338470000 \\
\hline $\mathrm{H}$ & 1.108696000 & -1.936165000 & -1.468581000 \\
\hline $\mathrm{C}$ & 3.440129000 & 0.064262000 & 0.887365000 \\
\hline $\mathrm{H}$ & 2.250470000 & -0.731822000 & 2.481762000 \\
\hline $\mathrm{C}$ & 3.627979000 & 0.134034000 & -0.494083000 \\
\hline $\mathrm{H}$ & 2.944106000 & -0.542226000 & -2.410081000 \\
\hline $\mathrm{H}$ & 4.058674000 & 0.637269000 & 1.567256000 \\
\hline $\mathrm{O}$ & 0.174160000 & 2.230091000 & 0.686255000 \\
\hline $\mathrm{H}$ & 0.478329000 & -2.426581000 & 2.168100000 \\
\hline $\mathrm{H}$ & -2.223379000 & -1.770697000 & -1.664488000 \\
\hline $\mathrm{H}$ & 0.236312000 & 3.155221000 & 0.446516000 \\
\hline $\mathrm{O}$ & 4.573222000 & 0.879234000 & -1.108996000 \\
\hline $\mathrm{C}$ & 5.383073000 & 1.714543000 & -0.306809000 \\
\hline $\mathrm{H}$ & 6.030617000 & 2.255606000 & -0.996787000 \\
\hline $\mathrm{H}$ & 4.776326000 & 2.430468000 & 0.260841000 \\
\hline $\mathrm{H}$ & 6.000940000 & 1.127256000 & 0.384067000 \\
\hline $\mathrm{Li}$ & 0.768867000 & 0.772649000 & 0.120190000 \\
\hline
\end{tabular}

\begin{tabular}{|c|c|c|c|}
\hline \multicolumn{4}{|c|}{ Coordinates (Angstroms) } \\
\hline & $\mathrm{X}$ & Y & Z \\
\hline$\overline{\mathrm{C}}$ & -1.726774000 & 4.004185000 & -0.565895000 \\
\hline $\mathrm{C}$ & -1.017827000 & 2.890277000 & -0.124754000 \\
\hline $\mathrm{C}$ & -1.648343000 & 1.648280000 & -0.020743000 \\
\hline $\mathrm{C}$ & -2.995511000 & 1.526420000 & -0.376122000 \\
\hline $\mathrm{C}$ & -3.700013000 & 2.642509000 & -0.820210000 \\
\hline $\mathrm{C}$ & -3.070393000 & 3.881786000 & -0.912716000 \\
\hline $\mathrm{H}$ & -1.228175000 & 4.965643000 & -0.650391000 \\
\hline $\mathrm{H}$ & 0.038093000 & 2.981010000 & 0.123752000 \\
\hline $\mathrm{H}$ & -3.452482000 & 0.533077000 & -0.317650000 \\
\hline $\mathrm{H}$ & -4.743356000 & 2.540082000 & -1.106581000 \\
\hline $\mathrm{H}$ & -3.620980000 & 4.749408000 & -1.265305000 \\
\hline $\mathrm{C}$ & -0.915880000 & 0.439522000 & 0.536671000 \\
\hline $\mathrm{C}$ & 0.490687000 & 0.318937000 & 0.026907000 \\
\hline $\mathrm{H}$ & 0.570414000 & 0.351857000 & -1.058411000 \\
\hline $\mathrm{O}$ & -0.971914000 & 0.468709000 & 1.981471000 \\
\hline $\mathrm{C}$ & 1.572763000 & 0.161868000 & 0.793573000 \\
\hline
\end{tabular}




$\begin{array}{lrrr}\mathrm{H} & 1.426014000 & 0.091650000 & 1.870769000 \\ \mathrm{C} & 2.966971000 & 0.032380000 & 0.342316000 \\ \mathrm{C} & 3.951618000 & -0.328107000 & 1.262428000 \\ \mathrm{C} & 3.373576000 & 0.256382000 & -0.983762000 \\ \mathrm{C} & 5.288202000 & -0.480111000 & 0.898036000 \\ \mathrm{H} & 3.671107000 & -0.504244000 & 2.298306000 \\ \mathrm{C} & 4.693702000 & 0.109842000 & -1.364430000 \\ \mathrm{H} & 2.645619000 & 0.558040000 & -1.730831000 \\ \mathrm{C} & 5.664306000 & -0.263186000 & -0.426358000 \\ \mathrm{H} & 6.013297000 & -0.766809000 & 1.650894000 \\ \mathrm{H} & 5.004603000 & 0.283187000 & -2.389442000 \\ \mathrm{H} & -1.496675000 & -0.457299000 & 0.263734000 \\ \mathrm{H} & -0.583991000 & 1.309400000 & 2.259685000 \\ \mathrm{C} & -4.782792000 & -2.054980000 & -1.630913000 \\ \mathrm{C} & -3.396076000 & -2.270149000 & -0.986189000 \\ \mathrm{H} & -5.565413000 & -2.363052000 & -0.926442000 \\ \mathrm{H} & -4.927133000 & -0.989707000 & -1.850429000 \\ \mathrm{H} & -4.914876000 & -2.619005000 & -2.563907000 \\ \mathrm{C} & -2.311973000 & -1.826482000 & -1.996250000 \\ \mathrm{C} & -3.209363000 & -3.777055000 & -0.704924000 \\ \mathrm{H} & -1.315188000 & -1.997975000 & -1.569749000 \\ \mathrm{H} & -2.372769000 & -2.367989000 & -2.949471000 \\ \mathrm{H} & -2.413172000 & -0.754182000 & -2.204792000 \\ \mathrm{H} & -3.265666000 & -4.394717000 & -1.611390000 \\ \mathrm{H} & -2.231448000 & -3.938985000 & -0.235056000 \\ \mathrm{H} & -3.982953000 & -4.119562000 & -0.005739000 \\ \mathrm{O} & -3.296748000 & -1.552392000 & 0.180135000 \\ \mathrm{~K} & -3.276722000 & -0.836769000 & 2.394419000 \\ \mathrm{O} & 6.930258000 & -0.380675000 & -0.902298000 \\ \mathrm{C} & 7.945151000 & -0.741152000 & 0.005747000 \\ \mathrm{H} & 7.757757000 & -1.726719000 & 0.451486000 \\ \mathrm{H} & 8.869106000 & -0.778940000 & -0.572668000 \\ \mathrm{H} & 8.051017000 & 0.002303000 & 0.806724000 \\ -----.--\end{array}$

TSA1-2 ${ }_{\text {tBuOK }}$

\begin{tabular}{llcc} 
& & & Coordinates (Angstroms) \\
& & Y & Z \\
\hline C & -4.103556000 & -3.051121000 & -0.284346000 \\
$\mathrm{C}$ & -3.126526000 & -2.209670000 & -0.819106000 \\
$\mathrm{C}$ & -2.277313000 & -1.457648000 & 0.009196000 \\
$\mathrm{C}$ & -2.459056000 & -1.576731000 & 1.399859000 \\
$\mathrm{C}$ & -3.422226000 & -2.422571000 & 1.929021000 \\
$\mathrm{C}$ & -4.258001000 & -3.165242000 & 1.091813000
\end{tabular}




\begin{tabular}{|c|c|c|c|}
\hline $\mathrm{H}$ & -4.734286000 & -3.632305000 & -0.952834000 \\
\hline $\mathrm{H}$ & -2.995528000 & -2.154716000 & -1.897426000 \\
\hline $\mathrm{H}$ & -1.847300000 & -0.971389000 & 2.064036000 \\
\hline $\mathrm{H}$ & -3.535744000 & -2.495703000 & 3.007302000 \\
\hline $\mathrm{H}$ & -5.014279000 & -3.822404000 & 1.510129000 \\
\hline $\mathrm{C}$ & -1.303664000 & -0.467942000 & -0.531549000 \\
\hline $\mathrm{C}$ & 0.089899000 & -0.633333000 & -0.117053000 \\
\hline $\mathrm{H}$ & 0.226025000 & -1.063685000 & 0.874355000 \\
\hline $\mathrm{O}$ & -1.446697000 & -0.280569000 & -1.950534000 \\
\hline $\mathrm{C}$ & 1.167419000 & -0.267367000 & -0.837697000 \\
\hline $\mathrm{H}$ & 0.999840000 & 0.194149000 & -1.810189000 \\
\hline $\mathrm{C}$ & 2.575790000 & -0.383932000 & -0.439687000 \\
\hline $\mathrm{C}$ & 3.569079000 & 0.115751000 & -1.285350000 \\
\hline $\mathrm{C}$ & 3.004419000 & -0.976397000 & 0.761710000 \\
\hline $\mathrm{C}$ & 4.924943000 & 0.044353000 & -0.969678000 \\
\hline $\mathrm{H}$ & 3.277383000 & 0.582155000 & -2.223622000 \\
\hline $\mathrm{C}$ & 4.344471000 & -1.054288000 & 1.092930000 \\
\hline $\mathrm{H}$ & 2.276362000 & -1.387717000 & 1.454852000 \\
\hline $\mathrm{C}$ & 5.319897000 & -0.543769000 & 0.229537000 \\
\hline $\mathrm{H}$ & 5.651255000 & 0.449155000 & -1.665619000 \\
\hline $\mathrm{H}$ & 4.665847000 & -1.512591000 & 2.022822000 \\
\hline $\mathrm{H}$ & -1.740825000 & 0.760520000 & -0.021711000 \\
\hline $\mathrm{H}$ & -0.912726000 & -0.954995000 & -2.399387000 \\
\hline $\mathrm{C}$ & -2.335443000 & 3.993322000 & 1.150217000 \\
\hline $\mathrm{C}$ & -1.484697000 & 2.774129000 & 0.774520000 \\
\hline $\mathrm{H}$ & -2.845891000 & 4.384028000 & 0.261148000 \\
\hline $\mathrm{H}$ & -3.095859000 & 3.706233000 & 1.884833000 \\
\hline $\mathrm{H}$ & -1.726142000 & 4.799459000 & 1.576269000 \\
\hline $\mathrm{C}$ & -0.780124000 & 2.230482000 & 2.028688000 \\
\hline $\mathrm{C}$ & -0.434818000 & 3.173967000 & -0.274599000 \\
\hline $\mathrm{H}$ & -0.135142000 & 1.385097000 & 1.764082000 \\
\hline $\mathrm{H}$ & -0.154740000 & 2.993366000 & 2.507735000 \\
\hline $\mathrm{H}$ & -1.527773000 & 1.886278000 & 2.752013000 \\
\hline $\mathrm{H}$ & 0.249293000 & 3.944147000 & 0.102024000 \\
\hline $\mathrm{H}$ & 0.159249000 & 2.299862000 & -0.563585000 \\
\hline $\mathrm{H}$ & -0.931559000 & 3.564823000 & -1.171495000 \\
\hline $\mathrm{O}$ & -2.342816000 & 1.799177000 & 0.248792000 \\
\hline $\mathrm{K}$ & -3.808258000 & 0.871491000 & -1.464533000 \\
\hline $\mathrm{O}$ & 6.609471000 & -0.671197000 & 0.648385000 \\
\hline $\mathrm{C}$ & 7.620396000 & -0.156983000 & -0.183956000 \\
\hline $\mathrm{H}$ & 7.503956000 & 0.923710000 & -0.340835000 \\
\hline $\mathrm{H}$ & 8.563605000 & -0.344813000 & 0.331252000 \\
\hline $\mathrm{H}$ & 7.636885000 & -0.660340000 & -1.159986000 \\
\hline
\end{tabular}




\begin{tabular}{|c|c|c|c|}
\hline \multicolumn{4}{|c|}{$\mathrm{A} 2_{\text {tBuOK }}$} \\
\hline & $\mathrm{X}$ & Y & Z \\
\hline$\overline{\mathrm{C}}$ & -5.527081000 & -1.057661000 & 0.331696000 \\
\hline $\mathrm{C}$ & -4.375925000 & -1.334715000 & -0.400511000 \\
\hline $\mathrm{C}$ & -3.086924000 & -1.262075000 & 0.188593000 \\
\hline $\mathrm{C}$ & -3.042229000 & -0.881777000 & 1.557278000 \\
\hline $\mathrm{C}$ & -4.198175000 & -0.619464000 & 2.273748000 \\
\hline $\mathrm{C}$ & -5.457751000 & -0.695453000 & 1.673360000 \\
\hline $\mathrm{H}$ & -6.494612000 & -1.140420000 & -0.158108000 \\
\hline $\mathrm{H}$ & -4.458361000 & -1.649747000 & -1.435809000 \\
\hline $\mathrm{H}$ & -2.084308000 & -0.792013000 & 2.061127000 \\
\hline $\mathrm{H}$ & -4.114521000 & -0.343251000 & 3.322040000 \\
\hline $\mathrm{H}$ & -6.358207000 & -0.483543000 & 2.240978000 \\
\hline $\mathrm{C}$ & -1.905275000 & -1.447151000 & -0.609786000 \\
\hline $\mathrm{C}$ & -0.587441000 & -1.304748000 & -0.201356000 \\
\hline $\mathrm{H}$ & -0.433212000 & -1.296443000 & 0.875782000 \\
\hline $\mathrm{O}$ & -2.155180000 & -1.466133000 & -2.006745000 \\
\hline $\mathrm{C}$ & 0.533160000 & -1.136322000 & -1.007296000 \\
\hline $\mathrm{H}$ & 0.404640000 & -1.116860000 & -2.089320000 \\
\hline $\mathrm{C}$ & 1.902831000 & -1.018971000 & -0.533867000 \\
\hline $\mathrm{C}$ & 2.918420000 & -0.619423000 & -1.428802000 \\
\hline $\mathrm{C}$ & 2.294293000 & -1.223313000 & 0.805750000 \\
\hline $\mathrm{C}$ & 4.225261000 & -0.407589000 & -1.015038000 \\
\hline $\mathrm{H}$ & 2.659937000 & -0.449931000 & -2.471990000 \\
\hline $\mathrm{C}$ & 3.600799000 & -1.005110000 & 1.226802000 \\
\hline $\mathrm{H}$ & 1.568037000 & -1.573255000 & 1.534236000 \\
\hline $\mathrm{C}$ & 4.572356000 & -0.589199000 & 0.322604000 \\
\hline $\mathrm{H}$ & 4.983370000 & -0.080845000 & -1.721628000 \\
\hline $\mathrm{H}$ & 3.880257000 & -1.159197000 & 2.265194000 \\
\hline $\mathrm{H}$ & 0.264804000 & 0.905978000 & -0.834144000 \\
\hline $\mathrm{H}$ & -1.598745000 & -2.154564000 & -2.392312000 \\
\hline $\mathrm{C}$ & 0.128939000 & 1.764741000 & 1.580126000 \\
\hline $\mathrm{C}$ & 0.415874000 & 2.532804000 & 0.287141000 \\
\hline $\mathrm{H}$ & -0.945102000 & 1.569230000 & 1.685486000 \\
\hline $\mathrm{H}$ & 0.660734000 & 0.807453000 & 1.578440000 \\
\hline $\mathrm{H}$ & 0.463771000 & 2.333675000 & 2.454155000 \\
\hline $\mathrm{C}$ & 1.921493000 & 2.717058000 & 0.092152000 \\
\hline $\mathrm{C}$ & -0.304861000 & 3.878142000 & 0.284501000 \\
\hline $\mathrm{H}$ & 2.118979000 & 3.235109000 & -0.851739000 \\
\hline $\mathrm{H}$ & 2.351128000 & 3.304429000 & 0.910827000 \\
\hline $\mathrm{H}$ & 2.430117000 & 1.747574000 & 0.066584000 \\
\hline $\mathrm{H}$ & 0.068622000 & 4.522427000 & 1.086328000 \\
\hline
\end{tabular}




$\begin{array}{lrrr}\mathrm{H} & -0.150488000 & 4.390182000 & -0.671164000 \\ \mathrm{H} & -1.382844000 & 3.741016000 & 0.440004000 \\ \mathrm{O} & -0.124522000 & 1.811690000 & -0.828589000 \\ \mathrm{~K} & -2.529780000 & 1.134182000 & -1.492436000 \\ \mathrm{O} & 5.857893000 & -0.345726000 & 0.757765000 \\ \mathrm{C} & 6.744917000 & -1.419370000 & 0.520740000 \\ \mathrm{H} & 6.848504000 & -1.628927000 & -0.553051000 \\ \mathrm{H} & 7.716210000 & -1.123267000 & 0.923944000 \\ \mathrm{H} & 6.405474000 & -2.335350000 & 1.023974000\end{array}$

TSA2-3 ${ }_{\text {tBuOK }}$

\begin{tabular}{|c|c|c|c|}
\hline \multicolumn{4}{|c|}{ Coordinates (Angstroms) } \\
\hline & $X$ & Y & $\mathrm{Z}$ \\
\hline $\mathrm{C}$ & -5.555220000 & -1.767848000 & -0.054373000 \\
\hline $\mathrm{C}$ & -4.355847000 & -1.603953000 & -0.739381000 \\
\hline $\mathrm{C}$ & -3.174125000 & -1.267480000 & -0.057127000 \\
\hline $\mathrm{C}$ & -3.252966000 & -1.070352000 & 1.334684000 \\
\hline $\mathrm{C}$ & -4.451159000 & -1.247794000 & 2.014396000 \\
\hline $\mathrm{C}$ & -5.611861000 & -1.596262000 & 1.325751000 \\
\hline $\mathrm{H}$ & -6.451828000 & -2.039146000 & -0.604974000 \\
\hline $\mathrm{H}$ & -4.315696000 & -1.751897000 & -1.813112000 \\
\hline $\mathrm{H}$ & -2.367578000 & -0.767071000 & 1.886799000 \\
\hline $\mathrm{H}$ & -4.481321000 & -1.097957000 & 3.090024000 \\
\hline $\mathrm{H}$ & -6.548973000 & -1.726716000 & 1.858132000 \\
\hline $\mathrm{C}$ & -1.926263000 & -1.052854000 & -0.797151000 \\
\hline $\mathrm{C}$ & -0.677410000 & -0.973577000 & -0.286139000 \\
\hline $\mathrm{H}$ & -0.577577000 & -1.228834000 & 0.765336000 \\
\hline $\mathrm{O}$ & -2.160025000 & -0.743937000 & -2.148520000 \\
\hline $\mathrm{C}$ & 0.490705000 & -0.426621000 & -0.955903000 \\
\hline $\mathrm{H}$ & 0.426819000 & -0.418767000 & -2.051484000 \\
\hline $\mathrm{C}$ & 1.861341000 & -0.795496000 & -0.515492000 \\
\hline $\mathrm{C}$ & 2.954987000 & -0.413331000 & -1.300728000 \\
\hline $\mathrm{C}$ & 2.159938000 & -1.446340000 & 0.692265000 \\
\hline $\mathrm{C}$ & 4.273342000 & -0.652928000 & -0.920989000 \\
\hline $\mathrm{H}$ & 2.770754000 & 0.101168000 & -2.241526000 \\
\hline $\mathrm{C}$ & 3.466830000 & -1.687727000 & 1.090600000 \\
\hline $\mathrm{H}$ & 1.359588000 & -1.787686000 & 1.342711000 \\
\hline $\mathrm{C}$ & 4.537813000 & -1.293817000 & 0.288556000 \\
\hline $\mathrm{H}$ & 5.075784000 & -0.332303000 & -1.576378000 \\
\hline $\mathrm{H}$ & 3.678338000 & -2.193841000 & 2.027412000 \\
\hline $\mathrm{H}$ & 0.293837000 & 0.895121000 & -0.693188000 \\
\hline $\mathrm{H}$ & -1.374748000 & -1.013702000 & -2.643690000 \\
\hline $\mathrm{C}$ & 0.455449000 & 1.825270000 & 1.819951000 \\
\hline
\end{tabular}




\begin{tabular}{|c|c|c|c|}
\hline $\mathrm{C}$ & 0.537496000 & 2.707747000 & 0.559689000 \\
\hline $\mathrm{H}$ & -0.594882000 & 1.598792000 & 2.049395000 \\
\hline $\mathrm{H}$ & 0.983546000 & 0.879166000 & 1.660000000 \\
\hline $\mathrm{H}$ & 0.899104000 & 2.316194000 & 2.694439000 \\
\hline $\mathrm{C}$ & 2.011920000 & 2.961357000 & 0.207723000 \\
\hline $\mathrm{C}$ & -0.159731000 & 4.048972000 & 0.836219000 \\
\hline $\mathrm{H}$ & 2.074902000 & 3.576715000 & -0.696751000 \\
\hline $\mathrm{H}$ & 2.544010000 & 3.474126000 & 1.018721000 \\
\hline $\mathrm{H}$ & 2.519913000 & 2.011287000 & 0.010120000 \\
\hline $\mathrm{H}$ & 0.315515000 & 4.600740000 & 1.656126000 \\
\hline $\mathrm{H}$ & -0.135345000 & 4.675640000 & -0.062949000 \\
\hline $\mathrm{H}$ & -1.210678000 & 3.881789000 & 1.114069000 \\
\hline $\mathrm{O}$ & -0.117932000 & 2.091380000 & -0.510692000 \\
\hline $\mathrm{K}$ & -2.490394000 & 1.755861000 & -1.054652000 \\
\hline $\mathrm{O}$ & 5.784749000 & -1.580749000 & 0.764936000 \\
\hline $\mathrm{C}$ & 6.883855000 & -1.211010000 & -0.029122000 \\
\hline $\mathrm{H}$ & 6.928792000 & -0.123964000 & -0.182260000 \\
\hline $\mathrm{H}$ & 7.774286000 & -1.532026000 & 0.514054000 \\
\hline $\mathrm{H}$ & 6.862015000 & -1.707477000 & -1.009010000 \\
\hline
\end{tabular}

\begin{tabular}{lrrr} 
A3 & & \\
& & \\
Coordinates $($ Angstroms) & \\
\hline $\mathrm{C}$ & -5.281915000 & -0.920414000 & -0.805713000 \\
$\mathrm{C}$ & -4.242663000 & -1.584361000 & -0.160298000 \\
$\mathrm{C}$ & -2.993747000 & -0.972287000 & 0.007087000 \\
$\mathrm{C}$ & -2.811921000 & 0.337265000 & -0.466977000 \\
$\mathrm{C}$ & -3.857246000 & 0.991513000 & -1.109423000 \\
$\mathrm{C}$ & -5.090809000 & 0.368501000 & -1.290792000 \\
$\mathrm{H}$ & -6.245090000 & -1.409853000 & -0.917017000 \\
$\mathrm{H}$ & -4.430108000 & -2.570953000 & 0.257185000 \\
$\mathrm{H}$ & -1.861135000 & 0.870112000 & -0.317467000 \\
$\mathrm{H}$ & -3.699928000 & 2.004711000 & -1.469647000 \\
$\mathrm{H}$ & -5.900464000 & 0.888344000 & -1.794662000 \\
$\mathrm{C}$ & -1.889445000 & -1.712138000 & 0.675043000 \\
$\mathrm{C}$ & -0.866314000 & -1.129695000 & 1.313752000 \\
$\mathrm{H}$ & -0.803305000 & -0.042719000 & 1.314451000 \\
$\mathrm{O}$ & -1.973589000 & -3.085175000 & 0.648247000 \\
$\mathrm{C}$ & 0.280917000 & -1.874172000 & 1.948842000 \\
$\mathrm{H}$ & 0.081074000 & -2.948668000 & 1.965940000 \\
$\mathrm{C}$ & 1.556675000 & -1.588305000 & 1.181850000 \\
$\mathrm{C}$ & 2.046685000 & -2.488660000 & 0.227037000 \\
$\mathrm{C}$ & 2.201637000 & -0.358181000 & 1.307219000
\end{tabular}




\begin{tabular}{lrcc}
$\mathrm{C}$ & 3.133487000 & -2.168370000 & -0.579024000 \\
$\mathrm{H}$ & 1.560860000 & -3.454317000 & 0.107200000 \\
$\mathrm{C}$ & 3.295220000 & -0.018779000 & 0.511045000 \\
$\mathrm{H}$ & 1.816700000 & 0.380183000 & 2.003469000 \\
$\mathrm{C}$ & 3.766849000 & -0.926011000 & -0.441263000 \\
$\mathrm{H}$ & 3.518957000 & -2.871687000 & -1.310800000 \\
$\mathrm{H}$ & 3.745744000 & 0.959287000 & 0.631962000 \\
$\mathrm{C}$ & -0.733139000 & 2.790618000 & 1.955684000 \\
$\mathrm{C}$ & -0.094622000 & 2.923261000 & 0.554051000 \\
$\mathrm{H}$ & -1.735203000 & 2.353857000 & 1.863992000 \\
$\mathrm{H}$ & -0.127733000 & 2.116475000 & 2.575740000 \\
$\mathrm{H}$ & -0.821824000 & 3.751090000 & 2.480763000 \\
$\mathrm{C}$ & 1.321145000 & 3.522091000 & 0.717175000 \\
$\mathrm{C}$ & -0.949158000 & 3.904509000 & -0.280019000 \\
$\mathrm{H}$ & 1.791383000 & 3.626554000 & -0.269144000 \\
$\mathrm{H}$ & 1.321059000 & 4.505330000 & 1.206554000 \\
$\mathrm{H}$ & 1.939935000 & 2.841492000 & 1.316465000 \\
$\mathrm{H}$ & -1.026556000 & 4.902560000 & 0.172011000 \\
$\mathrm{H}$ & -0.512989000 & 4.010121000 & -1.281241000 \\
$\mathrm{H}$ & -1.963216000 & 3.501012000 & -0.392886000 \\
$\mathrm{O}$ & -0.028582000 & 1.701837000 & -0.060511000 \\
$\mathrm{H}$ & 0.397655000 & -1.546410000 & 2.989104000 \\
$\mathrm{~K}$ & 0.551286000 & -0.123991000 & -1.398042000 \\
$\mathrm{H}$ & -2.561842000 & -3.339887000 & -0.072388000 \\
$\mathrm{O}$ & 4.807718000 & -0.693378000 & -1.281398000 \\
$\mathrm{C}$ & 5.476222000 & 0.545888000 & -1.174996000 \\
$\mathrm{H}$ & 6.266228000 & 0.525562000 & -1.926017000 \\
$\mathrm{H}$ & 4.800768000 & 1.386370000 & -1.381399000 \\
$\mathrm{H}-----2.923054000$ & 0.677366000 & -0.181763000 \\
\hline & & & \\
& & &
\end{tabular}

\begin{tabular}{|c|c|c|c|}
\hline \multicolumn{4}{|c|}{$\mathrm{A1}_{\text {tBuONa }}$} \\
\hline & $X$ & $\mathrm{Y}$ & $\mathrm{Z}$ \\
\hline$\overline{\mathrm{C}}$ & -1.627667000 & 4.058057000 & -0.292203000 \\
\hline $\mathrm{C}$ & -0.956644000 & 2.876242000 & 0.009449000 \\
\hline $\mathrm{C}$ & -1.647978000 & 1.662882000 & 0.041442000 \\
\hline $\mathrm{C}$ & -3.018141000 & 1.637488000 & -0.238422000 \\
\hline $\mathrm{C}$ & -3.683546000 & 2.822194000 & -0.541561000 \\
\hline $\mathrm{C}$ & -2.992812000 & 4.031895000 & -0.567254000 \\
\hline $\mathrm{H}$ & -1.084215000 & 4.998200000 & -0.319957000 \\
\hline $\mathrm{H}$ & 0.113281000 & 2.892713000 & 0.207528000 \\
\hline $\mathrm{H}$ & -3.533062000 & 0.672135000 & -0.216495000 \\
\hline $\mathrm{H}$ & -4.746255000 & 2.798659000 & -0.766620000 \\
\hline
\end{tabular}




\begin{tabular}{lrcc}
$\mathrm{H}$ & -3.515027000 & 4.953573000 & -0.807998000 \\
$\mathrm{C}$ & -0.956194000 & 0.376304000 & 0.453554000 \\
$\mathrm{C}$ & 0.454377000 & 0.267230000 & -0.049163000 \\
$\mathrm{H}$ & 0.552501000 & 0.420990000 & -1.122256000 \\
$\mathrm{O}$ & -1.020977000 & 0.248865000 & 1.899157000 \\
$\mathrm{C}$ & 1.519931000 & 0.001550000 & 0.710541000 \\
$\mathrm{H}$ & 1.354903000 & -0.169665000 & 1.773435000 \\
$\mathrm{C}$ & 2.919720000 & -0.112985000 & 0.273397000 \\
$\mathrm{C}$ & 3.891643000 & -0.489870000 & 1.200536000 \\
$\mathrm{C}$ & 3.345529000 & 0.141344000 & -1.041235000 \\
$\mathrm{C}$ & 5.235286000 & -0.621684000 & 0.855669000 \\
$\mathrm{H}$ & 3.595594000 & -0.692344000 & 2.227188000 \\
$\mathrm{C}$ & 4.672847000 & 0.015045000 & -1.402614000 \\
$\mathrm{H}$ & 2.627777000 & 0.447041000 & -1.796429000 \\
$\mathrm{C}$ & 5.631616000 & -0.369559000 & -0.456628000 \\
$\mathrm{H}$ & 5.950128000 & -0.920404000 & 1.613566000 \\
$\mathrm{H}$ & 4.999034000 & 0.210949000 & -2.418745000 \\
$\mathrm{H}$ & -1.566005000 & -0.466086000 & 0.092582000 \\
$\mathrm{H}$ & -0.651372000 & 1.062033000 & 2.268665000 \\
$\mathrm{C}$ & -5.547773000 & -2.478117000 & -0.417586000 \\
$\mathrm{C}$ & -4.029403000 & -2.269624000 & -0.588986000 \\
$\mathrm{H}$ & -5.753362000 & -2.879052000 & 0.583159000 \\
$\mathrm{H}$ & -6.061613000 & -1.513916000 & -0.508943000 \\
$\mathrm{H}$ & -5.969522000 & -3.169725000 & -1.158812000 \\
$\mathrm{C}$ & -3.761183000 & -1.726619000 & -2.008915000 \\
$\mathrm{C}$ & -3.317341000 & -3.629350000 & -0.438153000 \\
$\mathrm{H}$ & -2.685513000 & -1.559887000 & -2.144568000 \\
$\mathrm{H}$ & -4.104970000 & -2.411723000 & -2.794608000 \\
$\mathrm{H}$ & -4.269884000 & -0.764763000 & -2.144847000 \\
$\mathrm{H}$ & -3.649909000 & -4.370992000 & -1.176232000 \\
$\mathrm{H}$ & -2.235453000 & -3.489267000 & -0.550272000 \\
$\mathrm{H}$ & -3.504779000 & -4.035224000 & 0.564444000 \\
$\mathrm{H}$ & -3.561739000 & -1.381615000 & 0.349943000 \\
$\mathrm{H}$ & -3.024069000 & -0.785019000 & 2.198966000 \\
$\mathrm{H}$ & 7.905401000 & -0.464343000 & -0.913531000 \\
$\mathrm{H}$ & 7.9439392000 & -0.830423000 & 0.004032000 \\
$\mathrm{H}$ & & -0.843183000 & -0.559585000 \\
$\mathrm{H}$ & -0.102461000 & 0.821721000 \\
$\mathrm{H}$ & -1.827642000 & 0.425499000 \\
\hline
\end{tabular}

TSA1-2 ${ }_{\mathrm{tBuONa}}$

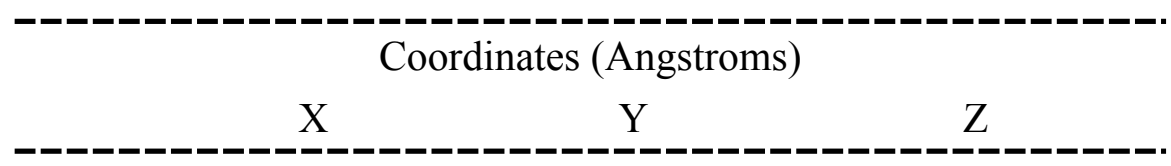




\begin{tabular}{|c|c|c|c|}
\hline $\mathrm{C}$ & -4.187737000 & -3.012912000 & -0.532355000 \\
\hline $\mathrm{C}$ & -3.256181000 & -2.076671000 & -0.987904000 \\
\hline $\mathrm{C}$ & -2.307757000 & -1.506548000 & -0.120820000 \\
\hline $\mathrm{C}$ & -2.337691000 & -1.917684000 & 1.225868000 \\
\hline $\mathrm{C}$ & -3.254175000 & -2.856124000 & 1.671139000 \\
\hline $\mathrm{C}$ & -4.194860000 & -3.409000000 & 0.797471000 \\
\hline $\mathrm{H}$ & -4.898655000 & -3.442754000 & -1.233764000 \\
\hline $\mathrm{H}$ & -3.234471000 & -1.819532000 & -2.045071000 \\
\hline $\mathrm{H}$ & -1.645759000 & -1.466808000 & 1.932249000 \\
\hline $\mathrm{H}$ & -3.250005000 & -3.150303000 & 2.717176000 \\
\hline $\mathrm{H}$ & -4.917529000 & -4.136218000 & 1.154086000 \\
\hline $\mathrm{C}$ & -1.404684000 & -0.406180000 & -0.547367000 \\
\hline $\mathrm{C}$ & 0.018508000 & -0.529961000 & -0.250325000 \\
\hline $\mathrm{H}$ & 0.254763000 & -1.088338000 & 0.654912000 \\
\hline $\mathrm{O}$ & -1.662886000 & 0.013909000 & -1.903570000 \\
\hline $\mathrm{C}$ & 1.022932000 & 0.018916000 & -0.960811000 \\
\hline $\mathrm{H}$ & 0.773917000 & 0.629031000 & -1.828225000 \\
\hline $\mathrm{C}$ & 2.454174000 & -0.087519000 & -0.646771000 \\
\hline $\mathrm{C}$ & 3.358749000 & 0.836622000 & -1.195103000 \\
\hline $\mathrm{C}$ & 2.986276000 & -1.080535000 & 0.180761000 \\
\hline $\mathrm{C}$ & 4.713149000 & 0.787182000 & -0.913952000 \\
\hline $\mathrm{H}$ & 2.983074000 & 1.618660000 & -1.850882000 \\
\hline $\mathrm{C}$ & 4.344919000 & -1.141940000 & 0.480368000 \\
\hline $\mathrm{H}$ & 2.332105000 & -1.843237000 & 0.594072000 \\
\hline $\mathrm{C}$ & 5.218707000 & -0.201653000 & -0.065711000 \\
\hline $\mathrm{H}$ & 5.403108000 & 1.510059000 & -1.337461000 \\
\hline $\mathrm{H}$ & 4.704567000 & -1.933734000 & 1.128033000 \\
\hline $\mathrm{H}$ & -1.923576000 & 0.712426000 & 0.153947000 \\
\hline $\mathrm{H}$ & -1.175407000 & -0.566635000 & -2.508305000 \\
\hline $\mathrm{C}$ & -2.913157000 & 3.854486000 & 1.254515000 \\
\hline $\mathrm{C}$ & -1.924262000 & 2.805099000 & 0.739307000 \\
\hline $\mathrm{H}$ & -3.697201000 & 4.034702000 & 0.508044000 \\
\hline $\mathrm{H}$ & -3.391631000 & 3.498483000 & 2.172625000 \\
\hline $\mathrm{H}$ & -2.416772000 & 4.809082000 & 1.465081000 \\
\hline $\mathrm{C}$ & -0.837891000 & 2.544655000 & 1.792808000 \\
\hline $\mathrm{C}$ & -1.270853000 & 3.289292000 & -0.564160000 \\
\hline $\mathrm{H}$ & -0.114555000 & 1.811286000 & 1.418596000 \\
\hline $\mathrm{H}$ & -0.293684000 & 3.462394000 & 2.044882000 \\
\hline $\mathrm{H}$ & -1.294398000 & 2.147036000 & 2.705368000 \\
\hline $\mathrm{H}$ & -0.701958000 & 4.214778000 & -0.417191000 \\
\hline $\mathrm{H}$ & -0.591621000 & 2.522141000 & -0.951512000 \\
\hline $\mathrm{H}$ & -2.040344000 & 3.484628000 & -1.324208000 \\
\hline $\mathrm{O}$ & -2.646798000 & 1.619574000 & 0.504199000 \\
\hline $\mathrm{Na}$ & -3.701269000 & 0.804424000 & -1.191953000 \\
\hline
\end{tabular}




$\begin{array}{llll}\mathrm{O} & 6.561953000 & -0.169165000 & 0.154650000 \\ \mathrm{C} & 7.108250000 & -1.142563000 & 1.011722000 \\ \mathrm{H} & 6.960983000 & -2.157043000 & 0.617570000 \\ \mathrm{H} & 8.177337000 & -0.932436000 & 1.068143000 \\ \mathrm{H} & 6.675041000 & -1.082657000 & 2.019005000\end{array}$

\begin{tabular}{|c|c|c|c|}
\hline \multicolumn{4}{|c|}{$\mathrm{A} 2_{\text {tBuONa }}$} \\
\hline & $X$ & Y & Z \\
\hline$\overline{\mathrm{C}}$ & -5.520638000 & -0.719208000 & 0.182401000 \\
\hline $\mathrm{C}$ & -4.384498000 & -1.040168000 & -0.559874000 \\
\hline $\mathrm{C}$ & -3.119034000 & -1.215118000 & 0.060364000 \\
\hline $\mathrm{C}$ & -3.082768000 & -1.049548000 & 1.471680000 \\
\hline $\mathrm{C}$ & -4.221522000 & -0.738960000 & 2.192139000 \\
\hline $\mathrm{C}$ & -5.456958000 & -0.559295000 & 1.560597000 \\
\hline $\mathrm{H}$ & -6.471295000 & -0.607371000 & -0.333485000 \\
\hline $\mathrm{H}$ & -4.472808000 & -1.211600000 & -1.628239000 \\
\hline $\mathrm{H}$ & -2.145408000 & -1.174039000 & 2.004974000 \\
\hline $\mathrm{H}$ & -4.145493000 & -0.628182000 & 3.270831000 \\
\hline $\mathrm{H}$ & -6.343665000 & -0.311936000 & 2.135167000 \\
\hline $\mathrm{C}$ & -1.937925000 & -1.428340000 & -0.733912000 \\
\hline $\mathrm{C}$ & -0.619688000 & -1.338187000 & -0.305334000 \\
\hline $\mathrm{H}$ & -0.481447000 & -1.405646000 & 0.771148000 \\
\hline $\mathrm{O}$ & -2.175841000 & -1.267240000 & -2.130412000 \\
\hline $\mathrm{C}$ & 0.512116000 & -1.111753000 & -1.076915000 \\
\hline $\mathrm{H}$ & 0.415115000 & -1.055652000 & -2.161121000 \\
\hline $\mathrm{C}$ & 1.865856000 & -0.962586000 & -0.561679000 \\
\hline $\mathrm{C}$ & 2.891785000 & -0.522337000 & -1.423654000 \\
\hline $\mathrm{C}$ & 2.223507000 & -1.164400000 & 0.786860000 \\
\hline $\mathrm{C}$ & 4.179100000 & -0.273042000 & -0.970263000 \\
\hline $\mathrm{H}$ & 2.658241000 & -0.351707000 & -2.472474000 \\
\hline $\mathrm{C}$ & 3.510639000 & -0.913119000 & 1.246968000 \\
\hline $\mathrm{H}$ & 1.486395000 & -1.534265000 & 1.494427000 \\
\hline $\mathrm{C}$ & 4.493928000 & -0.458953000 & 0.374270000 \\
\hline $\mathrm{H}$ & 4.946263000 & 0.084471000 & -1.651674000 \\
\hline $\mathrm{H}$ & 3.764137000 & -1.066051000 & 2.292086000 \\
\hline $\mathrm{H}$ & 0.066516000 & 0.868342000 & -0.989084000 \\
\hline $\mathrm{H}$ & -1.596155000 & -1.875984000 & -2.602825000 \\
\hline $\mathrm{C}$ & -0.227408000 & 1.712540000 & 1.410263000 \\
\hline $\mathrm{C}$ & 0.025681000 & 2.509612000 & 0.128489000 \\
\hline $\mathrm{H}$ & -1.274485000 & 1.390747000 & 1.473015000 \\
\hline $\mathrm{H}$ & 0.412671000 & 0.824456000 & 1.440140000 \\
\hline $\mathrm{H}$ & -0.000421000 & 2.319692000 & 2.293022000 \\
\hline
\end{tabular}




$\begin{array}{lrrr}\mathrm{C} & 1.512362000 & 2.830723000 & -0.024210000 \\ \mathrm{C} & -0.820811000 & 3.777964000 & 0.098915000 \\ \mathrm{H} & 1.688117000 & 3.377057000 & -0.956190000 \\ \mathrm{H} & 1.865600000 & 3.442413000 & 0.812670000 \\ \mathrm{H} & 2.104405000 & 1.909092000 & -0.045972000 \\ \mathrm{H} & -0.530483000 & 4.456373000 & 0.906768000 \\ \mathrm{H} & -0.691837000 & 4.299826000 & -0.854914000 \\ \mathrm{H} & -1.884884000 & 3.540784000 & 0.230082000 \\ \mathrm{O} & -0.401679000 & 1.739611000 & -1.009554000 \\ \mathrm{Na} & -2.425106000 & 0.920790000 & -1.439895000 \\ \mathrm{O} & 5.757036000 & -0.176722000 & 0.847994000 \\ \mathrm{C} & 6.684759000 & -1.221746000 & 0.638690000 \\ \mathrm{H} & 6.820355000 & -1.433129000 & -0.431075000 \\ \mathrm{H} & 7.635421000 & -0.890370000 & 1.062813000 \\ \mathrm{H} & 6.364288000 & -2.145664000 & 1.139759000 \\ - \text {-_-_-_-_- }\end{array}$

TSA2-3 ${ }_{\text {tBuONa }}$

\begin{tabular}{|c|c|c|c|}
\hline \multicolumn{4}{|c|}{ Coordinates (Angstroms) } \\
\hline & $\mathrm{X}$ & $\mathrm{Y}$ & Z \\
\hline$\overline{\mathrm{C}}$ & 5.904666000 & -1.131175000 & 0.355372000 \\
\hline $\mathrm{C}$ & 4.698225000 & -0.699085000 & 0.898580000 \\
\hline $\mathrm{C}$ & 3.478205000 & -0.977614000 & 0.259856000 \\
\hline $\mathrm{C}$ & 3.520563000 & -1.690147000 & -0.953252000 \\
\hline $\mathrm{C}$ & 4.725769000 & -2.126326000 & -1.483703000 \\
\hline $\mathrm{C}$ & 5.928932000 & -1.849044000 & -0.835406000 \\
\hline $\mathrm{H}$ & 6.832610000 & -0.909381000 & 0.875188000 \\
\hline $\mathrm{H}$ & 4.689224000 & -0.156534000 & 1.837501000 \\
\hline $\mathrm{H}$ & 2.600292000 & -1.900996000 & -1.489764000 \\
\hline $\mathrm{H}$ & 4.726728000 & -2.678847000 & -2.419013000 \\
\hline $\mathrm{H}$ & 6.870374000 & -2.187583000 & -1.256775000 \\
\hline $\mathrm{C}$ & 2.217013000 & -0.485419000 & 0.822425000 \\
\hline $\mathrm{C}$ & 0.952858000 & -0.792528000 & 0.442763000 \\
\hline $\mathrm{H}$ & 0.851965000 & -1.577456000 & -0.303077000 \\
\hline $\mathrm{O}$ & 2.421053000 & 0.537631000 & 1.776739000 \\
\hline $\mathrm{C}$ & -0.259459000 & -0.118595000 & 0.872172000 \\
\hline $\mathrm{H}$ & -0.250193000 & 0.224789000 & 1.915781000 \\
\hline $\mathrm{C}$ & -1.569081000 & -0.709028000 & 0.509425000 \\
\hline $\mathrm{C}$ & -2.652555000 & -0.677147000 & 1.390675000 \\
\hline $\mathrm{C}$ & -1.815444000 & -1.240174000 & -0.769523000 \\
\hline $\mathrm{C}$ & -3.916680000 & -1.153094000 & 1.039761000 \\
\hline $\mathrm{H}$ & -2.509258000 & -0.270684000 & 2.389797000 \\
\hline $\mathrm{C}$ & -3.058542000 & -1.732078000 & -1.129950000 \\
\hline $\mathrm{H}$ & -1.019560000 & -1.246577000 & -1.510476000 \\
\hline
\end{tabular}




$\begin{array}{lrcc}\mathrm{C} & -4.123988000 & -1.694046000 & -0.226532000 \\ \mathrm{H} & -4.717630000 & -1.102953000 & 1.769209000 \\ \mathrm{H} & -3.232696000 & -2.141586000 & -2.120123000 \\ \mathrm{H} & -0.165950000 & 1.116199000 & 0.181829000 \\ \mathrm{H} & 1.656376000 & 0.542889000 & 2.367970000 \\ \mathrm{C} & -1.993172000 & 2.473098000 & -1.481146000 \\ \mathrm{C} & -0.986874000 & 3.029050000 & -0.464803000 \\ \mathrm{H} & -1.507793000 & 2.345880000 & -2.455085000 \\ \mathrm{H} & -2.364653000 & 1.495910000 & -1.154901000 \\ \mathrm{H} & -2.850932000 & 3.144850000 & -1.604846000 \\ \mathrm{C} & -1.657936000 & 3.174151000 & 0.909499000 \\ \mathrm{C} & -0.463413000 & 4.389884000 & -0.935488000 \\ \mathrm{H} & -0.923435000 & 3.509709000 & 1.651467000 \\ \mathrm{H} & -2.477991000 & 3.900785000 & 0.881232000 \\ \mathrm{H} & -2.067810000 & 2.212416000 & 1.237722000 \\ \mathrm{H} & -1.271735000 & 5.122163000 & -1.044110000 \\ \mathrm{H} & 0.259886000 & 4.786427000 & -0.211206000 \\ \mathrm{H} & 0.036373000 & 4.284113000 & -1.905596000 \\ \mathrm{O} & 0.116038000 & 2.161400000 & -0.364814000 \\ \mathrm{Na} & 2.185994000 & 1.895261000 & -0.110484000 \\ \mathrm{O} & -5.308983000 & -2.199324000 & -0.677920000 \\ \mathrm{C} & -6.410963000 & -2.146607000 & 0.192981000 \\ \mathrm{H} & -7.249050000 & -2.589404000 & -0.347697000 \\ \mathrm{H} & -6.231801000 & -2.721941000 & 1.111574000 \\ \mathrm{H} & -6.662897000 & -1.112161000 & 0.464452000 \\ -------0 .-1\end{array}$

\begin{tabular}{llcc} 
A3 & \multicolumn{3}{c}{ Coordinates (Angstroms) } \\
& $\mathrm{X}$ & $\mathrm{Y}$ & $\mathrm{Z}$ \\
$\mathrm{C}$ & -5.319969000 & -0.684619000 & -0.745947000 \\
$\mathrm{C}$ & -4.254770000 & -1.445985000 & -0.278321000 \\
$\mathrm{C}$ & -3.001025000 & -0.857766000 & -0.060122000 \\
$\mathrm{C}$ & -2.825113000 & 0.508312000 & -0.316143000 \\
$\mathrm{C}$ & -3.899365000 & 1.259062000 & -0.785735000 \\
$\mathrm{C}$ & -5.143154000 & 0.672201000 & -1.002997000 \\
$\mathrm{H}$ & -6.289095000 & -1.150124000 & -0.899261000 \\
$\mathrm{H}$ & -4.415101000 & -2.496175000 & -0.042625000 \\
$\mathrm{H}$ & -1.852444000 & 1.004215000 & -0.180689000 \\
$\mathrm{H}$ & -3.748580000 & 2.315217000 & -0.989932000 \\
$\mathrm{H}$ & -5.973049000 & 1.268504000 & -1.371020000 \\
$\mathrm{C}$ & -1.883226000 & -1.700554000 & 0.436535000 \\
$\mathrm{C}$ & -0.971424000 & -1.305928000 & 1.337141000 \\
$\mathrm{H}$ & -1.034600000 & -0.288324000 & 1.711340000
\end{tabular}




\begin{tabular}{|c|c|c|c|}
\hline $\mathrm{O}$ & -1.831935000 & -2.985828000 & -0.044263000 \\
\hline $\mathrm{C}$ & 0.164952000 & -2.175365000 & 1.825204000 \\
\hline $\mathrm{H}$ & -0.089887000 & -3.231738000 & 1.703042000 \\
\hline $\mathrm{C}$ & 1.429948000 & -1.855420000 & 1.055044000 \\
\hline $\mathrm{C}$ & 1.723780000 & -2.519225000 & -0.145901000 \\
\hline $\mathrm{C}$ & 2.248164000 & -0.788712000 & 1.424607000 \\
\hline $\mathrm{C}$ & 2.775119000 & -2.109302000 & -0.958080000 \\
\hline $\mathrm{H}$ & 1.099511000 & -3.353256000 & -0.456381000 \\
\hline $\mathrm{C}$ & 3.309523000 & -0.364388000 & 0.624549000 \\
\hline $\mathrm{H}$ & 2.035591000 & -0.244878000 & 2.340854000 \\
\hline $\mathrm{C}$ & 3.573674000 & -1.018979000 & -0.581339000 \\
\hline $\mathrm{H}$ & 3.003351000 & -2.623697000 & -1.886302000 \\
\hline $\mathrm{H}$ & 3.901476000 & 0.484769000 & 0.943943000 \\
\hline $\mathrm{C}$ & 0.035964000 & 2.690681000 & 1.866627000 \\
\hline $\mathrm{C}$ & 0.257974000 & 2.946022000 & 0.359099000 \\
\hline $\mathrm{H}$ & -1.011831000 & 2.416494000 & 2.041596000 \\
\hline $\mathrm{H}$ & 0.665013000 & 1.850134000 & 2.189740000 \\
\hline $\mathrm{H}$ & 0.273902000 & 3.559945000 & 2.494274000 \\
\hline $\mathrm{C}$ & 1.738116000 & 3.330936000 & 0.138345000 \\
\hline $\mathrm{C}$ & -0.631120000 & 4.130472000 & -0.077223000 \\
\hline $\mathrm{H}$ & 1.914714000 & 3.500554000 & -0.930795000 \\
\hline $\mathrm{H}$ & 2.037699000 & 4.233333000 & 0.688248000 \\
\hline $\mathrm{H}$ & 2.380843000 & 2.500595000 & 0.461825000 \\
\hline $\mathrm{H}$ & -0.406964000 & 5.058229000 & 0.466110000 \\
\hline $\mathrm{H}$ & -0.495535000 & 4.312369000 & -1.149418000 \\
\hline $\mathrm{H}$ & -1.685461000 & 3.879246000 & 0.091773000 \\
\hline $\mathrm{O}$ & -0.058337000 & 1.824301000 & -0.359424000 \\
\hline $\mathrm{H}$ & 0.326601000 & -1.997931000 & 2.893865000 \\
\hline $\mathrm{H}$ & -2.457901000 & -3.061060000 & -0.774963000 \\
\hline $\mathrm{Na}$ & 0.535806000 & -0.053293000 & -0.830623000 \\
\hline $\mathrm{O}$ & 4.558589000 & -0.677943000 & -1.443591000 \\
\hline $\mathrm{C}$ & 5.331085000 & 0.468720000 & -1.144460000 \\
\hline $\mathrm{H}$ & 6.036242000 & 0.576681000 & -1.968552000 \\
\hline $\mathrm{H}$ & 4.703221000 & 1.366155000 & -1.079434000 \\
\hline $\mathrm{H}$ & 5.887070000 & 0.341728000 & -0.207468000 \\
\hline
\end{tabular}

\begin{tabular}{|c|c|c|c|}
\hline \multicolumn{4}{|c|}{ A1 tBuOLi $_{\text {t }}$} \\
\hline & \multicolumn{3}{|c|}{ Coordinates (Angstroms) } \\
\hline & $X$ & $\mathrm{Y}$ & Z \\
\hline$\overline{\mathrm{C}}$ & -1.574716000 & 4.019873000 & -0.006919000 \\
\hline $\mathrm{C}$ & -0.932431000 & 2.793969000 & 0.144821000 \\
\hline $\mathrm{C}$ & -1.666418000 & 1.606869000 & 0.104566000 \\
\hline $\mathrm{C}$ & -3.051587000 & 1.652650000 & -0.085266000 \\
\hline
\end{tabular}




\begin{tabular}{|c|c|c|c|}
\hline $\mathrm{C}$ & -3.688267000 & 2.880230000 & -0.236810000 \\
\hline $\mathrm{C}$ & -2.952848000 & 4.063558000 & -0.199141000 \\
\hline $\mathrm{H}$ & -0.998676000 & 4.940242000 & 0.020666000 \\
\hline $\mathrm{H}$ & 0.145287000 & 2.756607000 & 0.286227000 \\
\hline $\mathrm{H}$ & -3.608508000 & 0.713596000 & -0.098820000 \\
\hline $\mathrm{H}$ & -4.762992000 & 2.912905000 & -0.390622000 \\
\hline $\mathrm{H}$ & -3.453001000 & 5.019766000 & -0.322715000 \\
\hline $\mathrm{C}$ & -1.004225000 & 0.263952000 & 0.345985000 \\
\hline $\mathrm{C}$ & 0.425579000 & 0.200888000 & -0.107825000 \\
\hline $\mathrm{H}$ & 0.570053000 & 0.468873000 & -1.152926000 \\
\hline $\mathrm{O}$ & -1.131533000 & -0.075618000 & 1.755455000 \\
\hline $\mathrm{C}$ & 1.458110000 & -0.151786000 & 0.661214000 \\
\hline $\mathrm{H}$ & 1.250649000 & -0.452236000 & 1.687161000 \\
\hline $\mathrm{C}$ & 2.873606000 & -0.206218000 & 0.265296000 \\
\hline $\mathrm{C}$ & 3.787649000 & -0.862116000 & 1.090402000 \\
\hline $\mathrm{C}$ & 3.368182000 & 0.384446000 & -0.909292000 \\
\hline $\mathrm{C}$ & 5.140137000 & -0.953866000 & 0.769815000 \\
\hline $\mathrm{H}$ & 3.435915000 & -1.324645000 & 2.009462000 \\
\hline $\mathrm{C}$ & 4.706199000 & 0.303965000 & -1.244025000 \\
\hline $\mathrm{H}$ & 2.697789000 & 0.931103000 & -1.566116000 \\
\hline $\mathrm{C}$ & 5.605496000 & -0.369423000 & -0.407478000 \\
\hline $\mathrm{H}$ & 5.809501000 & -1.479700000 & 1.440761000 \\
\hline $\mathrm{H}$ & 5.088326000 & 0.764015000 & -2.149290000 \\
\hline $\mathrm{H}$ & -1.601367000 & -0.510984000 & -0.151517000 \\
\hline $\mathrm{H}$ & -0.860071000 & 0.700278000 & 2.263769000 \\
\hline $\mathrm{C}$ & -5.917090000 & -2.415374000 & 0.277008000 \\
\hline $\mathrm{C}$ & -4.520657000 & -2.203595000 & -0.336821000 \\
\hline $\mathrm{H}$ & -5.821002000 & -2.919973000 & 1.246040000 \\
\hline $\mathrm{H}$ & -6.395648000 & -1.443819000 & 0.446110000 \\
\hline $\mathrm{H}$ & -6.571352000 & -3.019570000 & -0.364457000 \\
\hline $\mathrm{C}$ & -4.673637000 & -1.504491000 & -1.701984000 \\
\hline $\mathrm{C}$ & -3.848652000 & -3.573019000 & -0.549790000 \\
\hline $\mathrm{H}$ & -3.684292000 & -1.323008000 & -2.138886000 \\
\hline $\mathrm{H}$ & -5.261757000 & -2.099788000 & -2.411496000 \\
\hline $\mathrm{H}$ & -5.170330000 & -0.535161000 & -1.574049000 \\
\hline $\mathrm{H}$ & -4.428468000 & -4.228143000 & -1.212407000 \\
\hline $\mathrm{H}$ & -2.852649000 & -3.431431000 & -0.985959000 \\
\hline $\mathrm{H}$ & -3.727497000 & -4.078307000 & 0.416185000 \\
\hline $\mathrm{O}$ & -3.751274000 & -1.429216000 & 0.502045000 \\
\hline $\mathrm{O}$ & 6.895401000 & -0.388322000 & -0.824469000 \\
\hline $\mathrm{C}$ & 7.845750000 & -1.035957000 & -0.009352000 \\
\hline $\mathrm{H}$ & 8.803040000 & -0.930121000 & -0.520797000 \\
\hline $\mathrm{H}$ & 7.912115000 & -0.567331000 & 0.981062000 \\
\hline $\mathrm{H}$ & 7.615749000 & -2.102432000 & 0.110157000 \\
\hline
\end{tabular}


TSA1-2 tBuOLi $_{\text {i }}$

\begin{tabular}{|c|c|c|c|}
\hline & \multicolumn{3}{|c|}{ Coordinates (Angstroms) } \\
\hline & $X$ & Y & Z \\
\hline $\mathrm{C}$ & -4.836891000 & -2.558401000 & -0.519245000 \\
\hline $\mathrm{C}$ & -3.857899000 & -1.702425000 & -1.016245000 \\
\hline $\mathrm{C}$ & -2.817932000 & -1.238429000 & -0.198286000 \\
\hline $\mathrm{C}$ & -2.817187000 & -1.651403000 & 1.146403000 \\
\hline $\mathrm{C}$ & -3.790227000 & -2.510955000 & 1.636115000 \\
\hline $\mathrm{C}$ & -4.810130000 & -2.975712000 & 0.806737000 \\
\hline $\mathrm{H}$ & -5.626270000 & -2.903906000 & -1.182033000 \\
\hline $\mathrm{H}$ & -3.898257000 & -1.387916000 & -2.054149000 \\
\hline $\mathrm{H}$ & -2.051675000 & -1.277883000 & 1.822599000 \\
\hline $\mathrm{H}$ & -3.757863000 & -2.812903000 & 2.679758000 \\
\hline $\mathrm{H}$ & -5.573381000 & -3.645133000 & 1.191804000 \\
\hline $\mathrm{C}$ & -1.785060000 & -0.305707000 & -0.689322000 \\
\hline $\mathrm{C}$ & -0.393825000 & -0.543080000 & -0.384143000 \\
\hline $\mathrm{H}$ & -0.212056000 & -1.051613000 & 0.561898000 \\
\hline $\mathrm{O}$ & -1.964454000 & 0.067341000 & -2.088450000 \\
\hline $\mathrm{C}$ & 0.676256000 & -0.138093000 & -1.108791000 \\
\hline $\mathrm{H}$ & 0.512468000 & 0.410911000 & -2.037380000 \\
\hline $\mathrm{C}$ & 2.084953000 & -0.317728000 & -0.737421000 \\
\hline $\mathrm{C}$ & 3.068309000 & 0.498922000 & -1.321077000 \\
\hline $\mathrm{C}$ & 2.523603000 & -1.271712000 & 0.186151000 \\
\hline $\mathrm{C}$ & 4.406531000 & 0.387560000 & -0.986071000 \\
\hline $\mathrm{H}$ & 2.768638000 & 1.247354000 & -2.051836000 \\
\hline $\mathrm{C}$ & 3.865102000 & -1.393343000 & 0.540916000 \\
\hline $\mathrm{H}$ & 1.808269000 & -1.956814000 & 0.632767000 \\
\hline $\mathrm{C}$ & 4.817551000 & -0.558272000 & -0.043248000 \\
\hline $\mathrm{H}$ & 5.155594000 & 1.028634000 & -1.439854000 \\
\hline $\mathrm{H}$ & 4.150593000 & -2.151486000 & 1.261609000 \\
\hline $\mathrm{H}$ & -2.047905000 & 1.129032000 & -0.113310000 \\
\hline $\mathrm{H}$ & -1.495414000 & -0.571063000 & -2.649106000 \\
\hline $\mathrm{C}$ & -2.151675000 & 4.257183000 & 1.044878000 \\
\hline $\mathrm{C}$ & -1.378231000 & 2.994315000 & 0.669176000 \\
\hline $\mathrm{H}$ & -2.430704000 & 4.816353000 & 0.144195000 \\
\hline $\mathrm{H}$ & -3.069035000 & 3.991724000 & 1.578827000 \\
\hline $\mathrm{H}$ & -1.549298000 & 4.912606000 & 1.683702000 \\
\hline $\mathrm{C}$ & -1.029923000 & 2.182931000 & 1.919730000 \\
\hline $\mathrm{C}$ & -0.102582000 & 3.351088000 & -0.102749000 \\
\hline $\mathrm{H}$ & -0.435936000 & 1.302545000 & 1.653957000 \\
\hline $\mathrm{H}$ & -0.447207000 & 2.787303000 & 2.622924000 \\
\hline
\end{tabular}




$\begin{array}{lccc}\mathrm{H} & -1.945839000 & 1.850119000 & 2.418550000 \\ \mathrm{H} & 0.581267000 & 3.958575000 & 0.499556000 \\ \mathrm{H} & 0.426326000 & 2.437224000 & -0.399245000 \\ \mathrm{H} & -0.353019000 & 3.934581000 & -1.001725000 \\ \mathrm{O} & -2.231840000 & 2.223757000 & -0.176395000 \\ \mathrm{O} & 6.151825000 & -0.591498000 & 0.226424000 \\ \mathrm{C} & 6.605543000 & -1.527735000 & 1.174070000 \\ \mathrm{H} & 6.407147000 & -2.558977000 & 0.852623000 \\ \mathrm{H} & 7.683219000 & -1.379044000 & 1.255733000 \\ \mathrm{H} & 6.144156000 & -1.364817000 & 2.157274000 \\ \mathrm{Li} & -2.015264000 & 1.927924000 & -1.962409000\end{array}$

\begin{tabular}{|c|c|c|c|}
\hline \multicolumn{4}{|c|}{$\mathrm{A} 2_{\text {tBuOLi }}$} \\
\hline & $\mathrm{X}$ & $\mathrm{Y}$ & Z \\
\hline $\mathrm{C}$ & -5.467395000 & -0.550925000 & 0.065251000 \\
\hline $\mathrm{C}$ & -4.340779000 & -0.804823000 & -0.719245000 \\
\hline $\mathrm{C}$ & -3.113911000 & -1.215202000 & -0.135623000 \\
\hline $\mathrm{C}$ & -3.103943000 & -1.372814000 & 1.275887000 \\
\hline $\mathrm{C}$ & -4.231258000 & -1.121296000 & 2.034339000 \\
\hline $\mathrm{C}$ & -5.427404000 & -0.696206000 & 1.443993000 \\
\hline $\mathrm{H}$ & -6.389826000 & -0.248714000 & -0.424002000 \\
\hline $\mathrm{H}$ & -4.420156000 & -0.745841000 & -1.800474000 \\
\hline $\mathrm{H}$ & -2.200855000 & -1.711747000 & 1.773908000 \\
\hline $\mathrm{H}$ & -4.181068000 & -1.261304000 & 3.110980000 \\
\hline $\mathrm{H}$ & -6.305464000 & -0.500440000 & 2.050593000 \\
\hline $\mathrm{C}$ & -1.927549000 & -1.341248000 & -0.944288000 \\
\hline $\mathrm{C}$ & -0.609798000 & -1.295010000 & -0.498102000 \\
\hline $\mathrm{H}$ & -0.487948000 & -1.477780000 & 0.566498000 \\
\hline $\mathrm{O}$ & -2.167510000 & -0.948129000 & -2.300153000 \\
\hline $\mathrm{C}$ & 0.525937000 & -0.953457000 & -1.215555000 \\
\hline $\mathrm{H}$ & 0.462345000 & -0.809697000 & -2.294821000 \\
\hline $\mathrm{C}$ & 1.864307000 & -0.810263000 & -0.652354000 \\
\hline $\mathrm{C}$ & 2.901386000 & -0.291011000 & -1.452884000 \\
\hline $\mathrm{C}$ & 2.189412000 & -1.102588000 & 0.686729000 \\
\hline $\mathrm{C}$ & 4.175169000 & -0.064237000 & -0.950574000 \\
\hline $\mathrm{H}$ & 2.692027000 & -0.050540000 & -2.493108000 \\
\hline $\mathrm{C}$ & 3.462177000 & -0.875393000 & 1.195732000 \\
\hline $\mathrm{H}$ & 1.439323000 & -1.525353000 & 1.349494000 \\
\hline $\mathrm{C}$ & 4.460859000 & -0.349832000 & 0.382288000 \\
\hline $\mathrm{H}$ & 4.955464000 & 0.348946000 & -1.583666000 \\
\hline $\mathrm{H}$ & 3.691046000 & -1.098795000 & 2.233789000 \\
\hline $\mathrm{H}$ & -0.123574000 & 0.952635000 & -1.072140000 \\
\hline
\end{tabular}




\begin{tabular}{|c|c|c|c|}
\hline $\mathrm{H}$ & -1.497232000 & -1.357782000 & -2.858512000 \\
\hline $\mathrm{C}$ & -0.483345000 & 1.590425000 & 1.373790000 \\
\hline $\mathrm{C}$ & -0.432666000 & 2.498641000 & 0.143694000 \\
\hline $\mathrm{H}$ & -1.452512000 & 1.081762000 & 1.447749000 \\
\hline $\mathrm{H}$ & 0.306781000 & 0.833674000 & 1.326716000 \\
\hline $\mathrm{H}$ & -0.333819000 & 2.177116000 & 2.286082000 \\
\hline $\mathrm{C}$ & 0.960783000 & 3.098530000 & -0.032463000 \\
\hline $\mathrm{C}$ & -1.506775000 & 3.577501000 & 0.213741000 \\
\hline $\mathrm{H}$ & 0.992065000 & 3.735471000 & -0.921907000 \\
\hline $\mathrm{H}$ & 1.236200000 & 3.700387000 & 0.839844000 \\
\hline $\mathrm{H}$ & 1.707118000 & 2.303515000 & -0.145990000 \\
\hline $\mathrm{H}$ & -1.326624000 & 4.244744000 & 1.061908000 \\
\hline $\mathrm{H}$ & -1.510756000 & 4.175222000 & -0.703485000 \\
\hline $\mathrm{H}$ & -2.499944000 & 3.129200000 & 0.346681000 \\
\hline $\mathrm{O}$ & -0.742428000 & 1.726950000 & -1.038290000 \\
\hline $\mathrm{O}$ & 5.711081000 & -0.094690000 & 0.901306000 \\
\hline $\mathrm{C}$ & 6.631946000 & -1.146493000 & 0.692184000 \\
\hline $\mathrm{H}$ & 6.791120000 & -1.334682000 & -0.378598000 \\
\hline $\mathrm{H}$ & 7.575068000 & -0.836934000 & 1.147999000 \\
\hline $\mathrm{H}$ & 6.287573000 & -2.077412000 & 1.163487000 \\
\hline $\mathrm{Li}$ & -2.326134000 & 0.745178000 & -1.323726000 \\
\hline
\end{tabular}

TSA2-3

\begin{tabular}{lrcc} 
& \multicolumn{3}{c}{ Coordinates (Angstroms) } \\
& $\mathrm{X}$ & $\mathrm{Y}$ & $\mathrm{Z}$ \\
$\mathrm{C}$ & -5.854322000 & -1.210240000 & -0.295456000 \\
$\mathrm{C}$ & -4.677740000 & -0.739260000 & -0.870164000 \\
$\mathrm{C}$ & -3.441368000 & -0.923735000 & -0.232392000 \\
$\mathrm{C}$ & -3.432416000 & -1.585268000 & 1.008250000 \\
$\mathrm{C}$ & -4.607708000 & -2.060614000 & 1.571073000 \\
$\mathrm{C}$ & -5.829137000 & -1.876244000 & 0.924720000 \\
$\mathrm{H}$ & -6.797528000 & -1.058337000 & -0.812549000 \\
$\mathrm{H}$ & -4.709661000 & -0.236276000 & -1.830185000 \\
$\mathrm{H}$ & -2.497175000 & -1.724292000 & 1.542343000 \\
$\mathrm{H}$ & -4.570390000 & -2.571414000 & 2.528921000 \\
$\mathrm{H}$ & -6.747421000 & -2.245492000 & 1.370611000 \\
$\mathrm{C}$ & -2.207192000 & -0.411175000 & -0.836934000 \\
$\mathrm{C}$ & -0.919085000 & -0.678024000 & -0.500925000 \\
$\mathrm{H}$ & -0.781477000 & -1.400429000 & 0.300150000 \\
$\mathrm{O}$ & -2.454945000 & 0.550410000 & -1.856184000 \\
$\mathrm{C}$ & 0.258740000 & -0.047424000 & -1.054830000 \\
$\mathrm{H}$ & 0.208663000 & 0.160569000 & -2.131617000 \\
$\mathrm{C}$ & 1.600029000 & -0.512388000 & -0.648022000
\end{tabular}




\begin{tabular}{lrrr}
$\mathrm{C}$ & 2.686425000 & -0.397616000 & -1.530823000 \\
$\mathrm{C}$ & 1.878589000 & -0.994551000 & 0.642041000 \\
$\mathrm{C}$ & 3.975883000 & -0.754865000 & -1.157351000 \\
$\mathrm{H}$ & 2.512014000 & -0.016102000 & -2.534425000 \\
$\mathrm{C}$ & 3.164706000 & -1.363207000 & 1.021785000 \\
$\mathrm{H}$ & 1.080438000 & -1.068916000 & 1.376803000 \\
$\mathrm{C}$ & 4.219167000 & -1.243730000 & 0.122756000 \\
$\mathrm{H}$ & 4.804717000 & -0.656468000 & -1.852445000 \\
$\mathrm{H}$ & 3.363513000 & -1.728456000 & 2.025369000 \\
$\mathrm{H}$ & 0.095040000 & 1.324598000 & -0.590028000 \\
$\mathrm{H}$ & -1.765321000 & 0.468580000 & -2.529475000 \\
$\mathrm{C}$ & 0.706814000 & 2.377860000 & 1.866869000 \\
$\mathrm{C}$ & 0.546629000 & 3.124463000 & 0.537651000 \\
$\mathrm{H}$ & -0.271887000 & 2.228992000 & 2.340028000 \\
$\mathrm{H}$ & 1.166125000 & 1.397889000 & 1.699562000 \\
$\mathrm{H}$ & 1.342406000 & 2.936903000 & 2.562644000 \\
$\mathrm{C}$ & 1.908432000 & 3.280651000 & -0.147503000 \\
$\mathrm{C}$ & -0.095553000 & 4.493210000 & 0.768463000 \\
$\mathrm{H}$ & 1.786622000 & 3.780778000 & -1.113976000 \\
$\mathrm{H}$ & 2.594052000 & 3.873133000 & 0.468404000 \\
$\mathrm{H}$ & 2.364853000 & 2.299818000 & -0.320854000 \\
$\mathrm{H}$ & 0.529795000 & 5.123679000 & 1.410217000 \\
$\mathrm{H}$ & -0.241589000 & 5.007685000 & -0.187270000 \\
$\mathrm{H}$ & -1.072816000 & 4.380102000 & 1.255286000 \\
$\mathrm{O}$ & -0.319046000 & 2.395161000 & -0.314736000 \\
$\mathrm{O}$ & 5.500837000 & -1.578175000 & 0.502383000 \\
$\mathrm{C}$ & 5.804447000 & -2.943502000 & 0.300472000 \\
$\mathrm{H}$ & 5.706089000 & -3.221760000 & -0.758021000 \\
$\mathrm{H}$ & 6.839246000 & -3.089220000 & 0.618386000 \\
$\mathrm{H}$ & 5.147268000 & -3.593034000 & 0.895414000 \\
$\mathrm{Li}$ & -2.004128000 & 1.827419000 & -0.441026000 \\
\hline---- &
\end{tabular}

\begin{tabular}{|c|c|c|c|}
\hline \multicolumn{4}{|c|}{$\mathrm{A3}_{\mathrm{tBuOLi}}$} \\
\hline & \multicolumn{3}{|c|}{ Coordinates (Angstroms) } \\
\hline & $X$ & $\mathrm{Y}$ & Z \\
\hline $\mathrm{C}$ & -5.296595000 & -0.318573000 & -0.721639000 \\
\hline $\mathrm{C}$ & -4.288664000 & -1.185022000 & -0.314339000 \\
\hline $\mathrm{C}$ & -2.989800000 & -0.706961000 & -0.094971000 \\
\hline $\mathrm{C}$ & -2.707184000 & 0.648655000 & -0.297581000 \\
\hline $\mathrm{C}$ & -3.723932000 & 1.506525000 & -0.706819000 \\
\hline $\mathrm{C}$ & -5.015277000 & 1.030841000 & -0.918762000 \\
\hline $\mathrm{H}$ & -6.302580000 & -0.696974000 & -0.877273000 \\
\hline $\mathrm{H}$ & -4.520755000 & -2.231893000 & -0.131050000 \\
\hline
\end{tabular}




\begin{tabular}{|c|c|c|c|}
\hline $\mathrm{H}$ & -1.688377000 & 1.032256000 & -0.174734000 \\
\hline $\mathrm{H}$ & -3.495566000 & 2.555180000 & -0.873947000 \\
\hline $\mathrm{H}$ & -5.800510000 & 1.708762000 & -1.240330000 \\
\hline $\mathrm{C}$ & -1.932889000 & -1.654598000 & 0.337497000 \\
\hline $\mathrm{C}$ & -1.029214000 & -1.405253000 & 1.297023000 \\
\hline $\mathrm{H}$ & -1.068706000 & -0.438141000 & 1.789827000 \\
\hline $\mathrm{O}$ & -1.938542000 & -2.882380000 & -0.271605000 \\
\hline $\mathrm{C}$ & 0.065509000 & -2.369674000 & 1.697783000 \\
\hline $\mathrm{H}$ & -0.238351000 & -3.398168000 & 1.483684000 \\
\hline $\mathrm{C}$ & 1.327367000 & -2.025373000 & 0.932899000 \\
\hline $\mathrm{C}$ & 1.495075000 & -2.472622000 & -0.388939000 \\
\hline $\mathrm{C}$ & 2.245360000 & -1.098752000 & 1.426127000 \\
\hline $\mathrm{C}$ & 2.515130000 & -1.979860000 & -1.190979000 \\
\hline $\mathrm{H}$ & 0.785928000 & -3.186980000 & -0.797972000 \\
\hline $\mathrm{C}$ & 3.281528000 & -0.598673000 & 0.635121000 \\
\hline $\mathrm{H}$ & 2.128228000 & -0.716181000 & 2.436495000 \\
\hline $\mathrm{C}$ & 3.411507000 & -1.024288000 & -0.688001000 \\
\hline $\mathrm{H}$ & 2.634545000 & -2.311858000 & -2.217097000 \\
\hline $\mathrm{H}$ & 3.951715000 & 0.142924000 & 1.052195000 \\
\hline $\mathrm{C}$ & 0.136046000 & 2.676065000 & 1.855854000 \\
\hline $\mathrm{C}$ & 0.556688000 & 2.762613000 & 0.373908000 \\
\hline $\mathrm{H}$ & -0.930127000 & 2.427165000 & 1.923813000 \\
\hline $\mathrm{H}$ & 0.703493000 & 1.877984000 & 2.352819000 \\
\hline $\mathrm{H}$ & 0.305731000 & 3.612288000 & 2.403234000 \\
\hline $\mathrm{C}$ & 2.058745000 & 3.105809000 & 0.302263000 \\
\hline $\mathrm{C}$ & -0.245619000 & 3.887891000 & -0.308129000 \\
\hline $\mathrm{H}$ & 2.374544000 & 3.146107000 & -0.747247000 \\
\hline $\mathrm{H}$ & 2.298641000 & 4.066044000 & 0.777068000 \\
\hline $\mathrm{H}$ & 2.636499000 & 2.318741000 & 0.804053000 \\
\hline $\mathrm{H}$ & -0.067786000 & 4.869063000 & 0.150644000 \\
\hline $\mathrm{H}$ & 0.022697000 & 3.942220000 & -1.368816000 \\
\hline $\mathrm{H}$ & -1.318368000 & 3.669491000 & -0.241784000 \\
\hline $\mathrm{O}$ & 0.313355000 & 1.563027000 & -0.250551000 \\
\hline $\mathrm{H}$ & 0.247211000 & -2.295983000 & 2.774888000 \\
\hline $\mathrm{H}$ & -2.521897000 & -2.835816000 & -1.039510000 \\
\hline $\mathrm{O}$ & 4.345695000 & -0.575698000 & -1.553291000 \\
\hline $\mathrm{C}$ & 5.151568000 & 0.515288000 & -1.149205000 \\
\hline $\mathrm{H}$ & 5.759323000 & 0.773234000 & -2.016582000 \\
\hline $\mathrm{H}$ & 4.535900000 & 1.376798000 & -0.863501000 \\
\hline $\mathrm{H}$ & 5.810696000 & 0.238230000 & -0.317014000 \\
\hline $\mathrm{Li}$ & 0.633906000 & -0.082771000 & -0.304320000 \\
\hline
\end{tabular}

$\mathbf{A 1}_{\text {TBD }}$ 


\begin{tabular}{|c|c|c|c|}
\hline \multicolumn{4}{|c|}{ Coordinates (Angstroms) } \\
\hline & $X$ & Y & Z \\
\hline $\bar{C}$ & 3.632685000 & -0.540202000 & 2.402350000 \\
\hline $\mathrm{C}$ & 4.258488000 & -1.922232000 & 2.568910000 \\
\hline $\mathrm{C}$ & 5.318202000 & -2.109481000 & 1.493201000 \\
\hline $\mathrm{N}$ & 3.075138000 & -0.353218000 & 1.080503000 \\
\hline $\mathrm{H}$ & 4.394815000 & 0.228242000 & 2.616272000 \\
\hline $\mathrm{H}$ & 2.834642000 & -0.395533000 & 3.139157000 \\
\hline $\mathrm{H}$ & 3.478423000 & -2.683891000 & 2.454096000 \\
\hline $\mathrm{H}$ & 4.710534000 & -2.048304000 & 3.558308000 \\
\hline $\mathrm{H}$ & 6.225350000 & -1.539184000 & 1.745328000 \\
\hline $\mathrm{H}$ & 5.611591000 & -3.165903000 & 1.426656000 \\
\hline $\mathrm{N}$ & 4.824432000 & -1.694817000 & 0.188428000 \\
\hline $\mathrm{C}$ & 5.493294000 & -2.319173000 & -0.941376000 \\
\hline $\mathrm{C}$ & 5.106387000 & -1.683587000 & -2.268367000 \\
\hline $\mathrm{C}$ & 3.597150000 & -1.498926000 & -2.296795000 \\
\hline $\mathrm{N}$ & 3.234783000 & -0.651735000 & -1.184621000 \\
\hline $\mathrm{C}$ & 3.696240000 & -0.904066000 & 0.088056000 \\
\hline $\mathrm{H}$ & 5.252274000 & -3.395104000 & -0.961792000 \\
\hline $\mathrm{H}$ & 6.577552000 & -2.240160000 & -0.785070000 \\
\hline $\mathrm{H}$ & 5.584350000 & -0.704052000 & -2.373201000 \\
\hline $\mathrm{H}$ & 5.445683000 & -2.322383000 & -3.089714000 \\
\hline $\mathrm{H}$ & 3.280717000 & -1.004460000 & -3.219640000 \\
\hline $\mathrm{H}$ & 3.098571000 & -2.482857000 & -2.261852000 \\
\hline $\mathrm{H}$ & 2.298791000 & -0.252833000 & -1.200383000 \\
\hline $\mathrm{C}$ & -0.122320000 & 4.606480000 & -0.382181000 \\
\hline $\mathrm{C}$ & -0.487414000 & 3.262748000 & -0.350468000 \\
\hline $\mathrm{C}$ & 0.444931000 & 2.284456000 & 0.001231000 \\
\hline $\mathrm{C}$ & 1.751829000 & 2.664958000 & 0.318991000 \\
\hline $\mathrm{C}$ & 2.113812000 & 4.007672000 & 0.288529000 \\
\hline $\mathrm{C}$ & 1.179557000 & 4.980435000 & -0.062387000 \\
\hline $\mathrm{H}$ & -0.856136000 & 5.360193000 & -0.653017000 \\
\hline $\mathrm{H}$ & -1.507541000 & 2.970936000 & -0.589651000 \\
\hline $\mathrm{H}$ & 2.468221000 & 1.891256000 & 0.590093000 \\
\hline $\mathrm{H}$ & 3.130593000 & 4.296713000 & 0.539588000 \\
\hline $\mathrm{H}$ & 1.464806000 & 6.028550000 & -0.084063000 \\
\hline $\mathrm{C}$ & 0.090244000 & 0.806697000 & -0.045878000 \\
\hline $\mathrm{C}$ & -1.344168000 & 0.536853000 & 0.324978000 \\
\hline $\mathrm{H}$ & -1.645797000 & 0.951276000 & 1.286092000 \\
\hline $\mathrm{O}$ & 0.421225000 & 0.272304000 & -1.335316000 \\
\hline $\mathrm{C}$ & -2.206237000 & -0.141603000 & -0.434702000 \\
\hline $\mathrm{H}$ & -1.842794000 & -0.522876000 & -1.387586000 \\
\hline $\mathrm{C}$ & -3.614755000 & -0.437456000 & -0.128596000 \\
\hline $\mathrm{C}$ & -4.439520000 & -0.944696000 & -1.133272000 \\
\hline
\end{tabular}




$\begin{array}{lccc}\mathrm{C} & -4.190088000 & -0.237586000 & 1.137377000 \\ \mathrm{C} & -5.785320000 & -1.231707000 & -0.913653000 \\ \mathrm{H} & -4.022407000 & -1.118105000 & -2.122417000 \\ \mathrm{C} & -5.522236000 & -0.517865000 & 1.375145000 \\ \mathrm{H} & -3.580764000 & 0.132592000 & 1.956584000 \\ \mathrm{C} & -6.334326000 & -1.016439000 & 0.349133000 \\ \mathrm{H} & -6.384457000 & -1.620342000 & -1.729250000 \\ \mathrm{H} & -5.962660000 & -0.366015000 & 2.355176000 \\ \mathrm{H} & 0.757257000 & 0.272871000 & 0.642348000 \\ \mathrm{H} & 0.146901000 & 0.927265000 & -1.990621000 \\ \mathrm{O} & -7.627179000 & -1.261885000 & 0.684769000 \\ \mathrm{C} & -8.483253000 & -1.762255000 & -0.315860000 \\ \mathrm{H} & -9.457052000 & -1.893273000 & 0.157597000 \\ \mathrm{H} & -8.579274000 & -1.057543000 & -1.152265000 \\ \mathrm{H} & -8.134108000 & -2.730189000 & -0.698773000 \\ \text { - - - - } & \end{array}$

TSA1-2 TBD

\begin{tabular}{|c|c|c|c|}
\hline \multicolumn{4}{|c|}{ Coordinates (Angstroms) } \\
\hline & $\mathrm{X}$ & Y & $\mathrm{Z}$ \\
\hline $\mathrm{C}$ & -1.173791000 & 0.935679000 & 2.366627000 \\
\hline $\mathrm{C}$ & 0.241219000 & 0.405598000 & 2.560014000 \\
\hline $\mathrm{C}$ & 1.230366000 & 1.533084000 & 2.311589000 \\
\hline $\mathrm{N}$ & -1.330060000 & 1.532689000 & 1.052252000 \\
\hline $\mathrm{H}$ & -1.417225000 & 1.673729000 & 3.142332000 \\
\hline $\mathrm{H}$ & -1.911181000 & 0.130108000 & 2.441613000 \\
\hline $\mathrm{H}$ & 0.429872000 & -0.400843000 & 1.845148000 \\
\hline $\mathrm{H}$ & 0.384668000 & 0.009201000 & 3.569529000 \\
\hline $\mathrm{H}$ & 1.254912000 & 2.229697000 & 3.160423000 \\
\hline $\mathrm{H}$ & 2.238255000 & 1.122460000 & 2.183861000 \\
\hline $\mathrm{N}$ & 0.905224000 & 2.278130000 & 1.094302000 \\
\hline $\mathrm{C}$ & 2.050211000 & 2.893679000 & 0.430210000 \\
\hline $\mathrm{C}$ & 1.615852000 & 3.824827000 & -0.689685000 \\
\hline $\mathrm{C}$ & 0.616383000 & 3.097268000 & -1.574413000 \\
\hline $\mathrm{N}$ & -0.492979000 & 2.666923000 & -0.748508000 \\
\hline $\mathrm{C}$ & -0.294546000 & 2.130360000 & 0.484683000 \\
\hline $\mathrm{H}$ & 2.699950000 & 2.098713000 & 0.037134000 \\
\hline $\mathrm{H}$ & 2.618954000 & 3.444212000 & 1.188320000 \\
\hline $\mathrm{H}$ & 1.147214000 & 4.723552000 & -0.275070000 \\
\hline $\mathrm{H}$ & 2.492290000 & 4.132141000 & -1.267082000 \\
\hline $\mathrm{H}$ & 0.224014000 & 3.759471000 & -2.350405000 \\
\hline $\mathrm{H}$ & 1.102949000 & 2.244639000 & -2.070906000 \\
\hline $\mathrm{H}$ & -1.294913000 & 2.262634000 & -1.232475000 \\
\hline $\mathrm{C}$ & -4.881317000 & -2.389206000 & 0.939746000 \\
\hline
\end{tabular}




$\begin{array}{lrrr}\mathrm{C} & -3.655127000 & -1.836023000 & 0.596904000 \\ \mathrm{C} & -3.545914000 & -0.864940000 & -0.416141000 \\ \mathrm{C} & -4.732779000 & -0.466989000 & -1.051252000 \\ \mathrm{C} & -5.960147000 & -1.022079000 & -0.704160000 \\ \mathrm{C} & -6.048397000 & -1.990017000 & 0.290933000 \\ \mathrm{H} & -4.925094000 & -3.141488000 & 1.723197000 \\ \mathrm{H} & -2.768776000 & -2.166467000 & 1.133093000 \\ \mathrm{H} & -4.680532000 & 0.295757000 & -1.820484000 \\ \mathrm{H} & -6.858007000 & -0.690799000 & -1.219907000 \\ \mathrm{C} & -2.259446000 & -0.224992000 & -0.759732000 \\ \mathrm{C} & -1.007561000 & -0.923262000 & -0.630570000 \\ \mathrm{H} & -0.992476000 & -1.696780000 & 0.135687000 \\ \mathrm{O} & -2.325667000 & 0.676346000 & -1.864629000 \\ \mathrm{C} & 0.165171000 & -0.573708000 & -1.217539000 \\ \mathrm{H} & 0.140663000 & 0.199260000 & -1.982575000 \\ \mathrm{C} & 1.499358000 & -1.029802000 & -0.835055000 \\ \mathrm{C} & 2.625899000 & -0.413113000 & -1.409392000 \\ \mathrm{C} & 1.747172000 & -2.011940000 & 0.141273000 \\ \mathrm{C} & 3.920771000 & -0.726766000 & -1.016355000 \\ \mathrm{H} & 2.475935000 & 0.339977000 & -2.180580000 \\ \mathrm{C} & 3.038526000 & -2.335762000 & 0.537787000 \\ \mathrm{H} & 0.915384000 & -2.537408000 & 0.602235000 \\ \mathrm{C} & 4.131830000 & -1.687634000 & -0.030417000 \\ \mathrm{H} & 4.774331000 & -0.223836000 & -1.462761000 \\ \mathrm{H} & 3.214011000 & -3.090642000 & 1.298557000 \\ \mathrm{H} & -1.908896000 & 0.794920000 & 0.302819000 \\ \mathrm{H} & -2.234112000 & 0.164129000 & -2.682163000 \\ \mathrm{H} & -7.006614000 & -2.424042000 & 0.560076000 \\ \mathrm{O} & 5.402107000 & -1.983382000 & 0.408305000 \\ \mathrm{C} & 6.127505000 & -2.824597000 & -0.467157000 \\ \mathrm{H} & 6.260415000 & -2.362471000 & -1.454835000 \\ \mathrm{H} & 7.106602000 & -2.984057000 & -0.010565000 \\ \mathrm{H} & 5.623173000 & -3.791654000 & -0.597331000 \\ ------ & \end{array}$

\begin{tabular}{|c|c|c|c|}
\hline \multicolumn{4}{|c|}{$\mathrm{A} 2_{\mathrm{TBD}}$} \\
\hline & \multicolumn{3}{|c|}{ Coordinates (Angstroms) } \\
\hline & $\mathrm{X}$ & Y & Z \\
\hline$\overline{\mathrm{C}}$ & -3.195600000 & 1.124487000 & -1.573462000 \\
\hline $\mathrm{C}$ & -3.701513000 & 1.585398000 & -0.217217000 \\
\hline $\mathrm{C}$ & -2.937797000 & 2.835928000 & 0.190289000 \\
\hline $\mathrm{N}$ & -1.748665000 & 0.965258000 & -1.527744000 \\
\hline $\mathrm{H}$ & -3.464637000 & 1.847060000 & -2.354824000 \\
\hline $\mathrm{H}$ & -3.618918000 & 0.153863000 & -1.834718000 \\
\hline
\end{tabular}




\begin{tabular}{|c|c|c|c|}
\hline $\mathrm{H}$ & -3.549124000 & 0.787174000 & 0.517493000 \\
\hline $\mathrm{H}$ & -4.771220000 & 1.805090000 & -0.262618000 \\
\hline $\mathrm{H}$ & -3.231573000 & 3.697147000 & -0.425275000 \\
\hline $\mathrm{H}$ & -3.147679000 & 3.087371000 & 1.235453000 \\
\hline $\mathrm{N}$ & -1.492694000 & 2.632108000 & 0.079268000 \\
\hline $\mathrm{C}$ & -0.660828000 & 3.456081000 & 0.954789000 \\
\hline $\mathrm{C}$ & 0.808558000 & 3.393573000 & 0.562323000 \\
\hline $\mathrm{C}$ & 1.212248000 & 1.939966000 & 0.376329000 \\
\hline $\mathrm{N}$ & 0.328525000 & 1.346708000 & -0.614177000 \\
\hline $\mathrm{C}$ & -0.973238000 & 1.638386000 & -0.669369000 \\
\hline $\mathrm{H}$ & -0.795830000 & 3.118913000 & 1.991763000 \\
\hline $\mathrm{H}$ & -1.034598000 & 4.484158000 & 0.890524000 \\
\hline $\mathrm{H}$ & 0.971183000 & 3.938658000 & -0.373540000 \\
\hline $\mathrm{H}$ & 1.412648000 & 3.867868000 & 1.340023000 \\
\hline $\mathrm{H}$ & 2.234770000 & 1.840382000 & 0.006259000 \\
\hline $\mathrm{H}$ & 1.153666000 & 1.390928000 & 1.325395000 \\
\hline $\mathrm{H}$ & 0.599369000 & 0.396762000 & -0.942281000 \\
\hline $\mathrm{C}$ & -3.630016000 & -1.530701000 & 2.339060000 \\
\hline $\mathrm{C}$ & -2.469738000 & -1.611465000 & 1.583246000 \\
\hline $\mathrm{C}$ & -2.508290000 & -1.897804000 & 0.197861000 \\
\hline $\mathrm{C}$ & -3.787188000 & -2.091016000 & -0.371185000 \\
\hline $\mathrm{C}$ & -4.944595000 & -1.992339000 & 0.391295000 \\
\hline $\mathrm{C}$ & -4.884449000 & -1.709285000 & 1.753918000 \\
\hline $\mathrm{H}$ & -3.553537000 & -1.314170000 & 3.402010000 \\
\hline $\mathrm{H}$ & -1.515189000 & -1.438083000 & 2.071702000 \\
\hline $\mathrm{H}$ & -3.854702000 & -2.324761000 & -1.428403000 \\
\hline $\mathrm{H}$ & -5.908521000 & -2.147787000 & -0.087536000 \\
\hline $\mathrm{C}$ & -1.325911000 & -1.917425000 & -0.629927000 \\
\hline $\mathrm{C}$ & -0.020246000 & -1.762395000 & -0.233220000 \\
\hline $\mathrm{H}$ & 0.152013000 & -1.809513000 & 0.839746000 \\
\hline $\mathrm{O}$ & -1.595647000 & -1.816739000 & -2.016528000 \\
\hline $\mathrm{C}$ & 1.085567000 & -1.480024000 & -1.050597000 \\
\hline $\mathrm{H}$ & 0.960249000 & -1.467719000 & -2.132942000 \\
\hline $\mathrm{C}$ & 2.414823000 & -1.171627000 & -0.566059000 \\
\hline $\mathrm{C}$ & 3.385876000 & -0.654479000 & -1.455692000 \\
\hline $\mathrm{C}$ & 2.801847000 & -1.252874000 & 0.791493000 \\
\hline $\mathrm{C}$ & 4.631569000 & -0.223625000 & -1.023339000 \\
\hline $\mathrm{H}$ & 3.135257000 & -0.571829000 & -2.511475000 \\
\hline $\mathrm{C}$ & 4.048696000 & -0.822386000 & 1.227148000 \\
\hline $\mathrm{H}$ & 2.115003000 & -1.666911000 & 1.524430000 \\
\hline $\mathrm{C}$ & 4.969167000 & -0.294413000 & 0.327281000 \\
\hline $\mathrm{H}$ & 5.350703000 & 0.184278000 & -1.729250000 \\
\hline $\mathrm{H}$ & 4.320072000 & -0.890713000 & 2.277352000 \\
\hline $\mathrm{H}$ & -1.391282000 & 0.081064000 & -1.898933000 \\
\hline
\end{tabular}




$\begin{array}{lrcc}\mathrm{H} & -0.992908000 & -2.418833000 & -2.471985000 \\ \mathrm{H} & -5.790331000 & -1.636260000 & 2.347807000 \\ \mathrm{O} & 6.191878000 & 0.165324000 & 0.775730000 \\ \mathrm{C} & 7.230013000 & -0.781956000 & 0.634812000 \\ \mathrm{H} & 7.396131000 & -1.044154000 & -0.419504000 \\ \mathrm{H} & 8.136566000 & -0.323954000 & 1.038089000 \\ \mathrm{H} & 7.008677000 & -1.703268000 & 1.191526000\end{array}$

TSA2-3 TBD

\begin{tabular}{|c|c|c|c|}
\hline \multicolumn{4}{|c|}{ Coordinates (Angstroms) } \\
\hline & $\mathrm{X}$ & $\mathrm{Y}$ & $\mathrm{Z}$ \\
\hline$\overline{\mathrm{C}}$ & -3.285680000 & 1.328024000 & -1.282620000 \\
\hline $\mathrm{C}$ & -3.612152000 & 1.649307000 & 0.166018000 \\
\hline $\mathrm{C}$ & -2.842523000 & 2.896825000 & 0.571514000 \\
\hline $\mathrm{N}$ & -1.844013000 & 1.260036000 & -1.466232000 \\
\hline $\mathrm{H}$ & -3.710801000 & 2.091823000 & -1.947759000 \\
\hline $\mathrm{H}$ & -3.700245000 & 0.358797000 & -1.567201000 \\
\hline $\mathrm{H}$ & -3.327015000 & 0.802469000 & 0.800275000 \\
\hline $\mathrm{H}$ & -4.685144000 & 1.819129000 & 0.289506000 \\
\hline $\mathrm{H}$ & -3.242949000 & 3.784369000 & 0.060405000 \\
\hline $\mathrm{H}$ & -2.936512000 & 3.069388000 & 1.649811000 \\
\hline $\mathrm{N}$ & -1.421125000 & 2.752790000 & 0.275491000 \\
\hline $\mathrm{C}$ & -0.510715000 & 3.559050000 & 1.080493000 \\
\hline $\mathrm{C}$ & 0.888280000 & 3.574099000 & 0.482066000 \\
\hline $\mathrm{C}$ & 1.290585000 & 2.145945000 & 0.145070000 \\
\hline $\mathrm{N}$ & 0.322221000 & 1.566378000 & -0.770016000 \\
\hline $\mathrm{C}$ & -0.960442000 & 1.854476000 & -0.633438000 \\
\hline $\mathrm{H}$ & -0.482957000 & 3.159117000 & 2.104428000 \\
\hline $\mathrm{H}$ & -0.921566000 & 4.574429000 & 1.136169000 \\
\hline $\mathrm{H}$ & 0.899140000 & 4.180287000 & -0.430535000 \\
\hline $\mathrm{H}$ & 1.586299000 & 4.022371000 & 1.194982000 \\
\hline $\mathrm{H}$ & 2.270118000 & 2.105342000 & -0.337507000 \\
\hline $\mathrm{H}$ & 1.365232000 & 1.545102000 & 1.063336000 \\
\hline $\mathrm{H}$ & 0.600233000 & 0.439645000 & -1.079172000 \\
\hline $\mathrm{C}$ & -3.617949000 & -1.950942000 & 2.283551000 \\
\hline $\mathrm{C}$ & -2.481758000 & -1.806083000 & 1.499692000 \\
\hline $\mathrm{C}$ & -2.538922000 & -1.943825000 & 0.099009000 \\
\hline $\mathrm{C}$ & -3.793745000 & -2.219889000 & -0.471968000 \\
\hline $\mathrm{C}$ & -4.931713000 & -2.349365000 & 0.317194000 \\
\hline $\mathrm{C}$ & -4.856253000 & -2.217203000 & 1.700370000 \\
\hline $\mathrm{H}$ & -3.536988000 & -1.840512000 & 3.361723000 \\
\hline $\mathrm{H}$ & -1.538321000 & -1.566803000 & 1.981151000 \\
\hline $\mathrm{H}$ & -3.863734000 & -2.332741000 & -1.548298000 \\
\hline
\end{tabular}




$\begin{array}{lrrr}\mathrm{H} & -5.886622000 & -2.560466000 & -0.156965000 \\ \mathrm{C} & -1.372275000 & -1.734268000 & -0.752265000 \\ \mathrm{C} & -0.087512000 & -1.550679000 & -0.363704000 \\ \mathrm{H} & 0.123935000 & -1.722276000 & 0.688326000 \\ \mathrm{O} & -1.702136000 & -1.534062000 & -2.104064000 \\ \mathrm{C} & 0.976692000 & -1.031819000 & -1.185336000 \\ \mathrm{H} & 0.881841000 & -1.181403000 & -2.264619000 \\ \mathrm{C} & 2.376500000 & -1.011543000 & -0.730927000 \\ \mathrm{C} & 3.412170000 & -0.797366000 & -1.663388000 \\ \mathrm{C} & 2.767352000 & -1.078322000 & 0.613571000 \\ \mathrm{C} & 4.734353000 & -0.653911000 & -1.281945000 \\ \mathrm{H} & 3.161905000 & -0.736167000 & -2.720484000 \\ \mathrm{C} & 4.097177000 & -0.938717000 & 1.014995000 \\ \mathrm{H} & 2.022270000 & -1.237446000 & 1.388818000 \\ \mathrm{C} & 5.092850000 & -0.721508000 & 0.067181000 \\ \mathrm{H} & 5.514367000 & -0.491480000 & -2.019671000 \\ \mathrm{H} & 4.332620000 & -1.005740000 & 2.071949000 \\ \mathrm{H} & -1.508736000 & 0.423883000 & -1.940561000 \\ \mathrm{H} & -1.004873000 & -1.944080000 & -2.632442000 \\ \mathrm{H} & -5.745427000 & -2.319019000 & 2.315120000 \\ \mathrm{O} & 6.422829000 & -0.564178000 & 0.345055000 \\ \mathrm{C} & 6.814630000 & -0.630900000 & 1.691803000 \\ \mathrm{H} & 7.896008000 & -0.483005000 & 1.703563000 \\ \mathrm{H} & 6.338432000 & 0.155880000 & 2.294022000 \\ \mathrm{H} & 6.580683000 & -1.607712000 & 2.137387000 \\ --------1 .-1\end{array}$

\begin{tabular}{lrrr} 
A3 & \multicolumn{3}{c}{ Coordinates $($ Angstroms) } \\
& X & Y & $\mathrm{Z}$ \\
\hline $\mathrm{C}$ & 0.095529000 & -2.277198000 & 1.573200000 \\
$\mathrm{C}$ & -0.800490000 & -1.433017000 & 2.468462000 \\
$\mathrm{C}$ & -2.248670000 & -1.524099000 & 1.992698000 \\
$\mathrm{~N}$ & -0.082622000 & -1.815452000 & 0.219767000 \\
$\mathrm{H}$ & -0.144197000 & -3.348172000 & 1.693515000 \\
$\mathrm{H}$ & 1.146991000 & -2.141415000 & 1.843540000 \\
$\mathrm{H}$ & -0.455448000 & -0.398350000 & 2.414187000 \\
$\mathrm{H}$ & -0.740518000 & -1.759260000 & 3.511833000 \\
$\mathrm{H}$ & -2.741216000 & -2.388676000 & 2.465102000 \\
$\mathrm{H}$ & -2.797935000 & -0.626984000 & 2.313442000 \\
$\mathrm{~N}$ & -2.383099000 & -1.658850000 & 0.550470000 \\
$\mathrm{C}$ & -3.738909000 & -1.535674000 & 0.050715000 \\
$\mathrm{C}$ & -3.853643000 & -2.149845000 & -1.336582000 \\
$\mathrm{C}$ & -2.737940000 & -1.588472000 & -2.216338000
\end{tabular}




\begin{tabular}{|c|c|c|c|}
\hline $\mathrm{N}$ & -1.425329000 & -1.756359000 & -1.632086000 \\
\hline $\mathrm{C}$ & -1.336517000 & -1.744746000 & -0.344771000 \\
\hline $\mathrm{H}$ & -4.034109000 & -0.474571000 & 0.027103000 \\
\hline $\mathrm{H}$ & -4.409404000 & -2.045599000 & 0.755390000 \\
\hline $\mathrm{H}$ & -3.745432000 & -3.238423000 & -1.260640000 \\
\hline $\mathrm{H}$ & -4.842266000 & -1.938368000 & -1.758547000 \\
\hline $\mathrm{H}$ & -2.747417000 & -2.085207000 & -3.193933000 \\
\hline $\mathrm{H}$ & -2.938576000 & -0.521852000 & -2.406074000 \\
\hline $\mathrm{C}$ & 6.201877000 & 1.478647000 & 0.030968000 \\
\hline $\mathrm{C}$ & 4.960628000 & 1.320895000 & -0.573550000 \\
\hline $\mathrm{C}$ & 4.171549000 & 0.198908000 & -0.297723000 \\
\hline $\mathrm{C}$ & 4.667601000 & -0.772568000 & 0.580394000 \\
\hline $\mathrm{C}$ & 5.912665000 & -0.616170000 & 1.180759000 \\
\hline $\mathrm{C}$ & 6.682969000 & 0.510847000 & 0.909821000 \\
\hline $\mathrm{H}$ & 6.802335000 & 2.354719000 & -0.195625000 \\
\hline $\mathrm{H}$ & 4.601986000 & 2.061456000 & -1.281996000 \\
\hline $\mathrm{H}$ & 4.060113000 & -1.642950000 & 0.814180000 \\
\hline $\mathrm{H}$ & 6.277411000 & -1.373329000 & 1.868639000 \\
\hline $\mathrm{C}$ & 2.838413000 & 0.026512000 & -0.922594000 \\
\hline $\mathrm{C}$ & 1.985296000 & 1.029269000 & -1.155197000 \\
\hline $\mathrm{H}$ & 2.286933000 & 2.017590000 & -0.815918000 \\
\hline $\mathrm{O}$ & 2.482361000 & -1.274370000 & -1.204707000 \\
\hline $\mathrm{C}$ & 0.636515000 & 0.908324000 & -1.796926000 \\
\hline $\mathrm{H}$ & 0.422485000 & -0.134647000 & -2.058637000 \\
\hline $\mathrm{C}$ & -0.509555000 & 1.411960000 & -0.940317000 \\
\hline $\mathrm{C}$ & -1.734140000 & 1.720638000 & -1.525083000 \\
\hline $\mathrm{C}$ & -0.415357000 & 1.509194000 & 0.449568000 \\
\hline $\mathrm{C}$ & -2.848935000 & 2.073184000 & -0.765994000 \\
\hline $\mathrm{H}$ & -1.836352000 & 1.660215000 & -2.606111000 \\
\hline $\mathrm{C}$ & -1.507713000 & 1.870503000 & 1.223945000 \\
\hline $\mathrm{H}$ & 0.527884000 & 1.274527000 & 0.934519000 \\
\hline $\mathrm{C}$ & -2.739460000 & 2.133530000 & 0.623224000 \\
\hline $\mathrm{H}$ & -3.786087000 & 2.286360000 & -1.267938000 \\
\hline $\mathrm{H}$ & -1.432409000 & 1.940305000 & 2.305006000 \\
\hline $\mathrm{H}$ & 0.655429000 & -1.970845000 & -0.453541000 \\
\hline $\mathrm{H}$ & 3.280661000 & -1.773422000 & -1.417372000 \\
\hline $\mathrm{H}$ & 0.641887000 & 1.474154000 & -2.739805000 \\
\hline $\mathrm{H}$ & 7.655145000 & 0.633014000 & 1.377640000 \\
\hline $\mathrm{O}$ & -3.763652000 & 2.433759000 & 1.471638000 \\
\hline $\mathrm{C}$ & -5.031315000 & 2.667009000 & 0.907508000 \\
\hline $\mathrm{H}$ & -5.703293000 & 2.870671000 & 1.742585000 \\
\hline $\mathrm{H}$ & -5.023004000 & 3.533549000 & 0.233128000 \\
\hline $\mathrm{H}$ & -5.396314000 & 1.789234000 & 0.356725000 \\
\hline
\end{tabular}




\begin{tabular}{|c|c|c|c|}
\hline \multicolumn{4}{|c|}{ A1 $1_{\text {DBU }}$} \\
\hline & \multicolumn{3}{|c|}{ Coordinates (Angstroms) } \\
\hline & $X$ & $\mathrm{Y}$ & Z \\
\hline$\overline{\mathrm{C}}$ & -0.240056000 & 4.329797000 & -1.144392000 \\
\hline $\mathrm{C}$ & -0.677761000 & 3.023312000 & -0.938959000 \\
\hline $\mathrm{C}$ & 0.209978000 & 2.038110000 & -0.502694000 \\
\hline $\mathrm{C}$ & 1.545713000 & 2.380146000 & -0.277371000 \\
\hline $\mathrm{C}$ & 1.983255000 & 3.684485000 & -0.478925000 \\
\hline $\mathrm{C}$ & 1.091224000 & 4.662948000 & -0.913846000 \\
\hline $\mathrm{H}$ & -0.941551000 & 5.087880000 & -1.480988000 \\
\hline $\mathrm{H}$ & -1.720891000 & 2.767332000 & -1.109951000 \\
\hline $\mathrm{H}$ & 2.228840000 & 1.604884000 & 0.060102000 \\
\hline $\mathrm{H}$ & 3.023837000 & 3.939499000 & -0.296735000 \\
\hline $\mathrm{H}$ & 1.432947000 & 5.682187000 & -1.070459000 \\
\hline $\mathrm{C}$ & -0.227047000 & 0.586973000 & -0.356611000 \\
\hline $\mathrm{C}$ & -1.653212000 & 0.461513000 & 0.116422000 \\
\hline $\mathrm{H}$ & -1.877528000 & 1.007234000 & 1.032218000 \\
\hline $\mathrm{O}$ & 0.008227000 & -0.117213000 & -1.574256000 \\
\hline $\mathrm{C}$ & -2.587454000 & -0.258396000 & -0.507909000 \\
\hline $\mathrm{H}$ & -2.283485000 & -0.793320000 & -1.406469000 \\
\hline $\mathrm{C}$ & -3.994445000 & -0.433952000 & -0.116205000 \\
\hline $\mathrm{C}$ & -4.823012000 & -1.233447000 & -0.904248000 \\
\hline $\mathrm{C}$ & -4.567877000 & 0.164618000 & 1.018245000 \\
\hline $\mathrm{C}$ & -6.165945000 & -1.442713000 & -0.596092000 \\
\hline $\mathrm{H}$ & -4.409701000 & -1.711724000 & -1.789002000 \\
\hline $\mathrm{C}$ & -5.897035000 & -0.031784000 & 1.341297000 \\
\hline $\mathrm{H}$ & -3.963125000 & 0.796019000 & 1.662417000 \\
\hline $\mathrm{C}$ & -6.709797000 & -0.839196000 & 0.536078000 \\
\hline $\mathrm{H}$ & -6.766057000 & -2.073287000 & -1.242117000 \\
\hline $\mathrm{H}$ & -6.336151000 & 0.430936000 & 2.219148000 \\
\hline $\mathrm{H}$ & 0.438975000 & 0.104702000 & 0.370817000 \\
\hline $\mathrm{H}$ & -0.340159000 & 0.431123000 & -2.289585000 \\
\hline $\mathrm{C}$ & 5.906763000 & -1.770498000 & 0.166080000 \\
\hline $\mathrm{C}$ & 5.487318000 & -3.031953000 & -0.581515000 \\
\hline $\mathrm{C}$ & 2.971012000 & -1.649589000 & -0.716338000 \\
\hline $\mathrm{C}$ & 4.893577000 & -2.757031000 & -1.965324000 \\
\hline $\mathrm{C}$ & 3.889974000 & -1.601240000 & -1.938698000 \\
\hline $\mathrm{H}$ & 6.471081000 & -1.108450000 & -0.511730000 \\
\hline $\mathrm{H}$ & 2.699068000 & -2.689804000 & -0.492259000 \\
\hline $\mathrm{H}$ & 4.780439000 & -3.590667000 & 0.042392000 \\
\hline $\mathrm{H}$ & 4.401436000 & -3.667880000 & -2.327548000 \\
\hline $\mathrm{H}$ & 6.608222000 & -2.063875000 & 0.958223000 \\
\hline $\mathrm{H}$ & 6.372879000 & -3.669271000 & -0.683816000 \\
\hline
\end{tabular}




$\begin{array}{lrrr}\mathrm{H} & 2.032488000 & -1.136417000 & -0.933025000 \\ \mathrm{H} & 5.699064000 & -2.532844000 & -2.675723000 \\ \mathrm{H} & 3.279451000 & -1.622827000 & -2.847387000 \\ \mathrm{H} & 4.420323000 & -0.640528000 & -1.953177000 \\ \mathrm{C} & 3.501628000 & -0.993680000 & 0.547597000 \\ \mathrm{~N} & 2.608014000 & -0.422661000 & 1.281523000 \\ \mathrm{~N} & 4.851831000 & -1.013277000 & 0.835498000 \\ \mathrm{C} & 5.392121000 & -0.145954000 & 1.879880000 \\ \mathrm{H} & 5.702709000 & -0.762068000 & 2.737700000 \\ \mathrm{H} & 6.298259000 & 0.336662000 & 1.489054000 \\ \mathrm{C} & 4.388124000 & 0.903183000 & 2.325677000 \\ \mathrm{H} & 4.736491000 & 1.378874000 & 3.247986000 \\ \mathrm{H} & 4.296946000 & 1.685147000 & 1.562035000 \\ \mathrm{C} & 3.035133000 & 0.224522000 & 2.505429000 \\ \mathrm{H} & 2.270599000 & 0.954080000 & 2.793186000 \\ \mathrm{H} & 3.097041000 & -0.512397000 & 3.321611000 \\ \mathrm{O} & -7.999804000 & -0.968473000 & 0.941338000 \\ \mathrm{C} & -8.858589000 & -1.766662000 & 0.160696000 \\ \mathrm{H} & -9.829008000 & -1.741166000 & 0.658004000 \\ \mathrm{H} & -8.964081000 & -1.368678000 & -0.857151000 \\ \mathrm{H} & -8.507174000 & -2.805355000 & 0.106762000 \\ ------ \text { - } & \end{array}$

TSA1-2 DBU

\begin{tabular}{lccc} 
& \multicolumn{3}{c}{ Coordinates (Angstroms) } \\
& $\mathrm{X}$ & $\mathrm{Y}$ & $\mathrm{Z}$ \\
$\mathrm{C}$ & 2.306776000 & 1.913874000 & 2.343601000 \\
$\mathrm{C}$ & 1.289837000 & 1.479825000 & 1.502871000 \\
$\mathrm{C}$ & 1.135060000 & 1.996081000 & 0.195514000 \\
$\mathrm{C}$ & 2.080759000 & 2.960204000 & -0.216537000 \\
$\mathrm{C}$ & 3.095541000 & 3.388930000 & 0.628952000 \\
$\mathrm{C}$ & 3.227027000 & 2.870871000 & 1.917234000 \\
$\mathrm{H}$ & 2.382465000 & 1.496517000 & 3.345400000 \\
$\mathrm{H}$ & 0.588317000 & 0.732409000 & 1.867840000 \\
$\mathrm{H}$ & 1.996276000 & 3.364234000 & -1.220090000 \\
$\mathrm{H}$ & 3.796058000 & 4.142793000 & 0.276564000 \\
$\mathrm{H}$ & 4.021959000 & 3.208435000 & 2.575304000 \\
$\mathrm{C}$ & 0.139697000 & 1.468303000 & -0.728607000 \\
$\mathrm{C}$ & -1.163773000 & 1.074021000 & -0.294472000 \\
$\mathrm{H}$ & -1.282555000 & 0.967809000 & 0.784446000 \\
$\mathrm{O}$ & 0.240687000 & 1.922611000 & -2.061044000 \\
$\mathrm{C}$ & -2.228801000 & 0.788706000 & -1.093672000 \\
$\mathrm{H}$ & -2.089146000 & 0.843752000 & -2.171693000 \\
$\mathrm{C}$ & -3.541351000 & 0.340088000 & -0.640788000 \\
& & 5 & \\
& & 5404 &
\end{tabular}




\begin{tabular}{|c|c|c|c|}
\hline $\mathrm{C}$ & -4.478895000 & -0.120262000 & -1.583532000 \\
\hline $\mathrm{C}$ & -3.941531000 & 0.322910000 & 0.709044000 \\
\hline $\mathrm{C}$ & -5.734818000 & -0.579436000 & -1.208897000 \\
\hline $\mathrm{H}$ & -4.205817000 & -0.121375000 & -2.636380000 \\
\hline $\mathrm{C}$ & -5.192535000 & -0.141604000 & 1.092435000 \\
\hline $\mathrm{H}$ & -3.268300000 & 0.694214000 & 1.476791000 \\
\hline $\mathrm{C}$ & -6.094829000 & -0.597679000 & 0.135182000 \\
\hline $\mathrm{H}$ & -6.442454000 & -0.938380000 & -1.951070000 \\
\hline $\mathrm{H}$ & -5.483371000 & -0.153981000 & 2.139361000 \\
\hline $\mathrm{H}$ & 0.711850000 & -0.125264000 & -0.810265000 \\
\hline $\mathrm{H}$ & -0.432757000 & 2.609836000 & -2.185816000 \\
\hline $\mathrm{C}$ & 4.496315000 & -2.360626000 & 0.344310000 \\
\hline $\mathrm{C}$ & 5.356902000 & -1.979400000 & -0.854485000 \\
\hline $\mathrm{C}$ & 3.177550000 & -0.193717000 & -1.363176000 \\
\hline $\mathrm{C}$ & 5.668639000 & -0.483086000 & -0.944170000 \\
\hline $\mathrm{C}$ & 4.431394000 & 0.387482000 & -0.709447000 \\
\hline $\mathrm{H}$ & 4.862788000 & -1.857849000 & 1.250355000 \\
\hline $\mathrm{H}$ & 3.411839000 & -0.659831000 & -2.328772000 \\
\hline $\mathrm{H}$ & 4.870034000 & -2.337929000 & -1.768624000 \\
\hline $\mathrm{H}$ & 6.090918000 & -0.274903000 & -1.934321000 \\
\hline $\mathrm{H}$ & 4.597656000 & -3.437907000 & 0.515322000 \\
\hline $\mathrm{H}$ & 6.293101000 & -2.542362000 & -0.772774000 \\
\hline $\mathrm{H}$ & 2.462882000 & 0.600621000 & -1.587805000 \\
\hline $\mathrm{H}$ & 6.443512000 & -0.223771000 & -0.213407000 \\
\hline $\mathrm{H}$ & 4.596376000 & 1.392298000 & -1.105857000 \\
\hline $\mathrm{H}$ & 4.240862000 & 0.529594000 & 0.360655000 \\
\hline $\mathrm{C}$ & 2.432644000 & -1.204532000 & -0.525120000 \\
\hline $\mathrm{N}$ & 1.121957000 & -1.134646000 & -0.603305000 \\
\hline $\mathrm{N}$ & 3.047728000 & -2.126424000 & 0.234505000 \\
\hline $\mathrm{C}$ & 2.280348000 & -2.958670000 & 1.173250000 \\
\hline $\mathrm{H}$ & 2.186431000 & -3.967558000 & 0.750194000 \\
\hline $\mathrm{H}$ & 2.868989000 & -3.038918000 & 2.092955000 \\
\hline $\mathrm{C}$ & 0.914220000 & -2.368137000 & 1.474963000 \\
\hline $\mathrm{H}$ & 0.318177000 & -3.089483000 & 2.039916000 \\
\hline $\mathrm{H}$ & 1.030116000 & -1.467030000 & 2.085465000 \\
\hline $\mathrm{C}$ & 0.243148000 & -2.004681000 & 0.160382000 \\
\hline $\mathrm{H}$ & -0.696830000 & -1.467760000 & 0.306082000 \\
\hline $\mathrm{H}$ & 0.023536000 & -2.904442000 & -0.426581000 \\
\hline $\mathrm{O}$ & -7.330484000 & -1.076189000 & 0.518231000 \\
\hline $\mathrm{C}$ & -8.326730000 & -0.075496000 & 0.559675000 \\
\hline $\mathrm{H}$ & -8.467038000 & 0.391829000 & -0.425071000 \\
\hline $\mathrm{H}$ & -9.255748000 & -0.563701000 & 0.863434000 \\
\hline $\mathrm{H}$ & -8.075093000 & 0.710401000 & 1.285495000 \\
\hline
\end{tabular}




\begin{tabular}{|c|c|c|c|}
\hline \multicolumn{4}{|c|}{$\mathrm{A} 2$ DBU } \\
\hline & $X$ & $\mathrm{Y}$ & $\mathrm{Z}$ \\
\hline $\mathrm{C}$ & -3.195807000 & -2.159532000 & 1.939311000 \\
\hline $\mathrm{C}$ & -2.054196000 & -1.975039000 & 1.173204000 \\
\hline $\mathrm{C}$ & -2.109415000 & -1.895663000 & -0.239401000 \\
\hline $\mathrm{C}$ & -3.385947000 & -2.069934000 & -0.823879000 \\
\hline $\mathrm{C}$ & -4.525375000 & -2.242639000 & -0.049077000 \\
\hline $\mathrm{C}$ & -4.452400000 & -2.273691000 & 1.342938000 \\
\hline $\mathrm{H}$ & -3.100955000 & -2.221749000 & 3.021411000 \\
\hline $\mathrm{H}$ & -1.097592000 & -1.905693000 & 1.681455000 \\
\hline $\mathrm{H}$ & -3.463343000 & -2.051841000 & -1.905431000 \\
\hline $\mathrm{H}$ & -5.486747000 & -2.362007000 & -0.543008000 \\
\hline $\mathrm{H}$ & -5.344916000 & -2.410977000 & 1.945417000 \\
\hline $\mathrm{C}$ & -0.965805000 & -1.579975000 & -1.063304000 \\
\hline $\mathrm{C}$ & 0.325614000 & -1.363954000 & -0.634204000 \\
\hline $\mathrm{H}$ & 0.518124000 & -1.596366000 & 0.410711000 \\
\hline $\mathrm{O}$ & -1.250958000 & -1.377232000 & -2.420817000 \\
\hline $\mathrm{C}$ & 1.385184000 & -0.814973000 & -1.364499000 \\
\hline $\mathrm{H}$ & 1.227511000 & -0.554663000 & -2.410464000 \\
\hline $\mathrm{C}$ & 2.718137000 & -0.588745000 & -0.842387000 \\
\hline $\mathrm{C}$ & 3.632850000 & 0.212373000 & -1.565111000 \\
\hline $\mathrm{C}$ & 3.172367000 & -1.073378000 & 0.406600000 \\
\hline $\mathrm{C}$ & 4.888556000 & 0.531031000 & -1.071877000 \\
\hline $\mathrm{H}$ & 3.328419000 & 0.606210000 & -2.532845000 \\
\hline $\mathrm{C}$ & 4.428746000 & -0.749901000 & 0.906345000 \\
\hline $\mathrm{H}$ & 2.537932000 & -1.733485000 & 0.992093000 \\
\hline $\mathrm{C}$ & 5.292678000 & 0.060168000 & 0.176443000 \\
\hline $\mathrm{H}$ & 5.562313000 & 1.163434000 & -1.644146000 \\
\hline $\mathrm{H}$ & 4.756034000 & -1.132174000 & 1.869666000 \\
\hline $\mathrm{H}$ & 0.842175000 & 1.110011000 & -0.639597000 \\
\hline $\mathrm{H}$ & -0.565032000 & -1.839340000 & -2.920499000 \\
\hline $\mathrm{C}$ & -3.293716000 & 1.622237000 & 0.920105000 \\
\hline $\mathrm{C}$ & -3.778075000 & 2.711209000 & -0.037020000 \\
\hline $\mathrm{C}$ & -1.446061000 & 1.618004000 & -1.510532000 \\
\hline $\mathrm{C}$ & -3.937161000 & 2.234279000 & -1.485111000 \\
\hline $\mathrm{C}$ & -2.869474000 & 1.218917000 & -1.882947000 \\
\hline $\mathrm{H}$ & -3.739049000 & 0.642981000 & 0.706852000 \\
\hline $\mathrm{H}$ & -1.228055000 & 2.644437000 & -1.836712000 \\
\hline $\mathrm{H}$ & -3.071863000 & 3.549188000 & 0.018866000 \\
\hline $\mathrm{H}$ & -3.911358000 & 3.103665000 & -2.153471000 \\
\hline $\mathrm{H}$ & -3.590445000 & 1.890361000 & 1.937597000 \\
\hline $\mathrm{H}$ & -4.733943000 & 3.095396000 & 0.334515000 \\
\hline
\end{tabular}




\begin{tabular}{lrcc}
$\mathrm{H}$ & -0.755964000 & 0.962704000 & -2.057307000 \\
$\mathrm{H}$ & -4.921739000 & 1.769984000 & -1.613416000 \\
$\mathrm{H}$ & -2.892200000 & 1.056371000 & -2.964316000 \\
$\mathrm{H}$ & -3.089807000 & 0.246473000 & -1.440866000 \\
$\mathrm{C}$ & -1.011984000 & 1.529313000 & -0.067881000 \\
$\mathrm{~N}$ & 0.310632000 & 1.599409000 & 0.097079000 \\
$\mathrm{~N}$ & -1.826594000 & 1.485116000 & 0.980984000 \\
$\mathrm{C}$ & -1.325153000 & 1.242318000 & 2.344124000 \\
$\mathrm{H}$ & -1.442760000 & 2.173841000 & 2.913103000 \\
$\mathrm{H}$ & -1.978091000 & 0.485170000 & 2.787192000 \\
$\mathrm{C}$ & 0.118630000 & 0.773190000 & 2.374359000 \\
$\mathrm{H}$ & 0.504914000 & 0.859058000 & 3.393589000 \\
$\mathrm{H}$ & 0.180074000 & -0.275718000 & 2.078966000 \\
$\mathrm{C}$ & 0.943886000 & 1.606973000 & 1.408172000 \\
$\mathrm{H}$ & 1.950945000 & 1.203541000 & 1.288629000 \\
$\mathrm{H}$ & 1.024977000 & 2.642972000 & 1.758135000 \\
$\mathrm{O}$ & 6.525075000 & 0.400955000 & 0.696426000 \\
$\mathrm{C}$ & 7.577714000 & -0.412708000 & 0.222087000 \\
$\mathrm{H}$ & 7.695832000 & -0.325838000 & -0.867110000 \\
$\mathrm{H}$ & 8.492006000 & -0.066908000 & 0.710494000 \\
$\mathrm{H}$ & 7.408640000 & -1.469787000 & 0.470452000 \\
\hline----- -
\end{tabular}

TSA2-3 DBU

\begin{tabular}{lrrr} 
& & Coordinates (Angstroms) \\
& $\mathrm{X}$ & $\mathrm{Y}$ & $\mathrm{Z}$ \\
\hline $\mathrm{C}$ & 3.295113000 & 1.842358000 & 2.135610000 \\
$\mathrm{C}$ & 2.168185000 & 1.596109000 & 1.362341000 \\
$\mathrm{C}$ & 2.084257000 & 2.043186000 & 0.030063000 \\
$\mathrm{C}$ & 3.193968000 & 2.727267000 & -0.494447000 \\
$\mathrm{C}$ & 4.325506000 & 2.959318000 & 0.278028000 \\
$\mathrm{C}$ & 4.388593000 & 2.519772000 & 1.598155000 \\
$\mathrm{H}$ & 3.326254000 & 1.488390000 & 3.163297000 \\
$\mathrm{H}$ & 1.345170000 & 1.032699000 & 1.791504000 \\
$\mathrm{H}$ & 3.156755000 & 3.062275000 & -1.525007000 \\
$\mathrm{H}$ & 5.168827000 & 3.488560000 & -0.157470000 \\
$\mathrm{H}$ & 5.275541000 & 2.699529000 & 2.198173000 \\
$\mathrm{C}$ & 0.937657000 & 1.731820000 & -0.821405000 \\
$\mathrm{C}$ & -0.284037000 & 1.323399000 & -0.395746000 \\
$\mathrm{H}$ & -0.449911000 & 1.383998000 & 0.677079000 \\
$\mathrm{O}$ & 1.223513000 & 1.798769000 & -2.181524000 \\
$\mathrm{C}$ & -1.304292000 & 0.683989000 & -1.178028000 \\
$\mathrm{H}$ & -1.192895000 & 0.700550000 & -2.264636000 \\
$\mathrm{C}$ & -2.688807000 & 0.549831000 & -0.718793000
\end{tabular}




\begin{tabular}{|c|c|c|c|}
\hline $\mathrm{C}$ & -3.618675000 & -0.151643000 & -1.514615000 \\
\hline $\mathrm{C}$ & -3.150228000 & 1.000367000 & 0.534106000 \\
\hline $\mathrm{C}$ & -4.911871000 & -0.408179000 & -1.084400000 \\
\hline $\mathrm{H}$ & -3.299670000 & -0.519122000 & -2.487866000 \\
\hline $\mathrm{C}$ & -4.442363000 & 0.734210000 & 0.976175000 \\
\hline $\mathrm{H}$ & -2.495284000 & 1.584995000 & 1.173855000 \\
\hline $\mathrm{C}$ & -5.327907000 & 0.023978000 & 0.173513000 \\
\hline $\mathrm{H}$ & -5.606952000 & -0.961810000 & -1.709701000 \\
\hline $\mathrm{H}$ & -4.776709000 & 1.083275000 & 1.949685000 \\
\hline $\mathrm{H}$ & -0.751266000 & -0.803868000 & -0.899563000 \\
\hline $\mathrm{H}$ & 0.400148000 & 2.035014000 & -2.628622000 \\
\hline $\mathrm{C}$ & 3.215163000 & -2.106361000 & 0.834330000 \\
\hline $\mathrm{C}$ & 3.843295000 & -2.802720000 & -0.371586000 \\
\hline $\mathrm{C}$ & 1.679588000 & -1.370650000 & -1.700625000 \\
\hline $\mathrm{C}$ & 4.178440000 & -1.847944000 & -1.521965000 \\
\hline $\mathrm{C}$ & 3.092144000 & -0.792736000 & -1.718232000 \\
\hline $\mathrm{H}$ & 3.698601000 & -1.141904000 & 1.038813000 \\
\hline $\mathrm{H}$ & 1.627430000 & -2.293985000 & -2.293943000 \\
\hline $\mathrm{H}$ & 3.158615000 & -3.590867000 & -0.708024000 \\
\hline $\mathrm{H}$ & 4.316249000 & -2.429586000 & -2.441550000 \\
\hline $\mathrm{H}$ & 3.369377000 & -2.733977000 & 1.718214000 \\
\hline $\mathrm{H}$ & 4.752438000 & -3.312212000 & -0.035068000 \\
\hline $\mathrm{H}$ & 0.998847000 & -0.663584000 & -2.186498000 \\
\hline $\mathrm{H}$ & 5.132547000 & -1.346816000 & -1.321479000 \\
\hline $\mathrm{H}$ & 3.236188000 & -0.272487000 & -2.669109000 \\
\hline $\mathrm{H}$ & 3.173220000 & -0.019195000 & -0.950372000 \\
\hline $\mathrm{C}$ & 1.051623000 & -1.667350000 & -0.359577000 \\
\hline $\mathrm{N}$ & -0.269324000 & -1.711160000 & -0.376385000 \\
\hline $\mathrm{N}$ & 1.759337000 & -1.910698000 & 0.757583000 \\
\hline $\mathrm{C}$ & 1.119713000 & -1.942829000 & 2.079933000 \\
\hline $\mathrm{H}$ & 1.185906000 & -2.969287000 & 2.465366000 \\
\hline $\mathrm{H}$ & 1.715560000 & -1.304404000 & 2.742382000 \\
\hline $\mathrm{C}$ & -0.328101000 & -1.481899000 & 2.051815000 \\
\hline $\mathrm{H}$ & -0.825655000 & -1.790326000 & 2.975487000 \\
\hline $\mathrm{H}$ & -0.388076000 & -0.391601000 & 1.990655000 \\
\hline $\mathrm{C}$ & -1.003740000 & -2.078253000 & 0.825820000 \\
\hline $\mathrm{H}$ & -2.025084000 & -1.710512000 & 0.711697000 \\
\hline $\mathrm{H}$ & -1.041739000 & -3.172255000 & 0.901875000 \\
\hline $\mathrm{O}$ & -6.600683000 & -0.264225000 & 0.622335000 \\
\hline $\mathrm{C}$ & -7.544075000 & 0.740085000 & 0.312139000 \\
\hline $\mathrm{H}$ & -7.634178000 & 0.885366000 & -0.773513000 \\
\hline $\mathrm{H}$ & -8.504828000 & 0.407774000 & 0.712847000 \\
\hline $\mathrm{H}$ & -7.273029000 & 1.701267000 & 0.770953000 \\
\hline
\end{tabular}




\begin{tabular}{|c|c|c|c|}
\hline \multicolumn{4}{|c|}{$\mathbf{A 3}$ DBU } \\
\hline & $X$ & $\mathrm{Y}$ & $\mathrm{Z}$ \\
\hline $\mathrm{C}$ & 3.079559000 & 1.664746000 & 2.308339000 \\
\hline $\mathrm{C}$ & 2.046241000 & 1.479954000 & 1.398688000 \\
\hline $\mathrm{C}$ & 2.119013000 & 2.026595000 & 0.112565000 \\
\hline $\mathrm{C}$ & 3.263002000 & 2.745328000 & -0.248502000 \\
\hline $\mathrm{C}$ & 4.299429000 & 2.925263000 & 0.661868000 \\
\hline $\mathrm{C}$ & 4.212704000 & 2.388136000 & 1.942837000 \\
\hline $\mathrm{H}$ & 3.004985000 & 1.229037000 & 3.300668000 \\
\hline $\mathrm{H}$ & 1.184845000 & 0.882796000 & 1.677930000 \\
\hline $\mathrm{H}$ & 3.336925000 & 3.155911000 & -1.249532000 \\
\hline $\mathrm{H}$ & 5.180985000 & 3.486886000 & 0.366640000 \\
\hline $\mathrm{H}$ & 5.024491000 & 2.526026000 & 2.650858000 \\
\hline $\mathrm{C}$ & 1.034487000 & 1.800244000 & -0.866267000 \\
\hline $\mathrm{C}$ & -0.236180000 & 1.522135000 & -0.550700000 \\
\hline $\mathrm{H}$ & -0.522928000 & 1.528633000 & 0.494352000 \\
\hline $\mathrm{O}$ & 1.480167000 & 1.876153000 & -2.158676000 \\
\hline $\mathrm{C}$ & -1.293889000 & 1.157859000 & -1.562566000 \\
\hline $\mathrm{H}$ & -0.997848000 & 0.233718000 & -2.077409000 \\
\hline $\mathrm{C}$ & -2.654670000 & 0.934667000 & -0.945016000 \\
\hline $\mathrm{C}$ & -3.312195000 & -0.281272000 & -1.088669000 \\
\hline $\mathrm{C}$ & -3.288792000 & 1.943573000 & -0.209841000 \\
\hline $\mathrm{C}$ & -4.567954000 & -0.503886000 & -0.520762000 \\
\hline $\mathrm{H}$ & -2.822914000 & -1.083906000 & -1.634492000 \\
\hline $\mathrm{C}$ & -4.533162000 & 1.741284000 & 0.362541000 \\
\hline $\mathrm{H}$ & -2.794395000 & 2.904019000 & -0.082139000 \\
\hline $\mathrm{C}$ & -5.182733000 & 0.511415000 & 0.209636000 \\
\hline $\mathrm{H}$ & -5.042986000 & -1.469371000 & -0.653245000 \\
\hline $\mathrm{H}$ & -5.026564000 & 2.521883000 & 0.932527000 \\
\hline $\mathrm{H}$ & 0.724962000 & 1.784620000 & -2.752621000 \\
\hline $\mathrm{C}$ & 3.030243000 & -2.146714000 & 0.919922000 \\
\hline $\mathrm{C}$ & 3.783360000 & -2.853929000 & -0.205395000 \\
\hline $\mathrm{C}$ & 1.694723000 & -1.591414000 & -1.781300000 \\
\hline $\mathrm{C}$ & 4.182691000 & -1.914018000 & -1.345902000 \\
\hline $\mathrm{C}$ & 3.065840000 & -0.925642000 & -1.676574000 \\
\hline $\mathrm{H}$ & 3.498650000 & -1.175787000 & 1.145991000 \\
\hline $\mathrm{H}$ & 1.777646000 & -2.533307000 & -2.340650000 \\
\hline $\mathrm{H}$ & 3.155067000 & -3.671723000 & -0.578228000 \\
\hline $\mathrm{H}$ & 4.438372000 & -2.508505000 & -2.232243000 \\
\hline $\mathrm{H}$ & 3.125992000 & -2.754092000 & 1.828949000 \\
\hline $\mathrm{H}$ & 4.678940000 & -3.322114000 & 0.218021000 \\
\hline $\mathrm{H}$ & 1.021013000 & -0.960750000 & -2.365832000 \\
\hline
\end{tabular}




$\begin{array}{lrrr}\mathrm{H} & 5.086781000 & -1.358335000 & -1.067949000 \\ \mathrm{H} & 3.286477000 & -0.415647000 & -2.619581000 \\ \mathrm{H} & 3.032718000 & -0.139085000 & -0.917933000 \\ \mathrm{C} & 0.928105000 & -1.879226000 & -0.495876000 \\ \mathrm{~N} & -0.345572000 & -2.011951000 & -0.640260000 \\ \mathrm{~N} & 1.593607000 & -1.982005000 & 0.710943000 \\ \mathrm{C} & 0.855030000 & -1.932526000 & 1.969553000 \\ \mathrm{H} & 0.938305000 & -2.909034000 & 2.471256000 \\ \mathrm{H} & 1.340639000 & -1.197675000 & 2.627944000 \\ \mathrm{C} & -0.608245000 & -1.585507000 & 1.757682000 \\ \mathrm{H} & -1.177872000 & -1.818708000 & 2.663127000 \\ \mathrm{H} & -0.730980000 & -0.516566000 & 1.551716000 \\ \mathrm{C} & -1.101064000 & -2.367268000 & 0.545857000 \\ \mathrm{H} & -2.160943000 & -2.167860000 & 0.365665000 \\ \mathrm{H} & -1.003698000 & -3.447224000 & 0.745713000 \\ \mathrm{H} & -1.382985000 & 1.946080000 & -2.330006000 \\ \mathrm{O} & -6.398960000 & 0.407358000 & 0.809478000 \\ \mathrm{C} & -7.095020000 & -0.809286000 & 0.675495000 \\ \mathrm{H} & -6.539680000 & -1.644979000 & 1.121523000 \\ \mathrm{H} & -8.037395000 & -0.678994000 & 1.209284000 \\ \mathrm{H} & -7.305647000 & -1.039428000 & -0.377184000 \\ ------ \text { - } & \end{array}$

\begin{tabular}{lccc} 
A1 & \multicolumn{3}{c}{ Coordinates (Angstroms) } \\
& X & Y & Z \\
\hline $\mathrm{C}$ & 2.806140000 & 2.032849000 & -1.482636000 \\
$\mathrm{C}$ & 2.031945000 & 0.991099000 & -2.283330000 \\
$\mathrm{C}$ & 0.588078000 & 1.452873000 & -2.413453000 \\
$\mathrm{~N}$ & 2.218700000 & 2.260167000 & -0.181222000 \\
$\mathrm{H}$ & 2.850154000 & 2.973486000 & -2.057347000 \\
$\mathrm{H}$ & 3.841371000 & 1.702510000 & -1.344206000 \\
$\mathrm{H}$ & 2.086437000 & 0.031102000 & -1.758168000 \\
$\mathrm{H}$ & 2.461076000 & 0.843452000 & -3.279948000 \\
$\mathrm{H}$ & 0.498272000 & 2.199743000 & -3.216740000 \\
$\mathrm{H}$ & -0.059671000 & 0.608310000 & -2.690965000 \\
$\mathrm{~N}$ & 0.076402000 & 2.024633000 & -1.173989000 \\
$\mathrm{C}$ & -1.375126000 & 2.042245000 & -1.098394000 \\
$\mathrm{C}$ & -1.869293000 & 2.847324000 & 0.087595000 \\
$\mathrm{C}$ & -1.082700000 & 2.430931000 & 1.316889000 \\
$\mathrm{~N}$ & 0.329359000 & 2.648481000 & 1.082764000 \\
$\mathrm{C}$ & 0.927552000 & 2.292631000 & -0.115789000 \\
$\mathrm{H}$ & -1.756899000 & 1.012518000 & -1.029179000 \\
$\mathrm{H}$ & -1.760344000 & 2.465992000 & -2.035839000 \\
& & & \\
& & 5 &
\end{tabular}




\begin{tabular}{|c|c|c|c|}
\hline $\mathrm{H}$ & -1.721911000 & 3.918307000 & -0.089143000 \\
\hline $\mathrm{H}$ & -2.938116000 & 2.663352000 & 0.232808000 \\
\hline $\mathrm{H}$ & -1.373216000 & 3.040962000 & 2.179121000 \\
\hline $\mathrm{H}$ & -1.295329000 & 1.380361000 & 1.569288000 \\
\hline $\mathrm{C}$ & 3.756373000 & -3.205266000 & -0.797713000 \\
\hline $\mathrm{C}$ & 2.753180000 & -2.617530000 & -0.032579000 \\
\hline $\mathrm{C}$ & 2.941287000 & -1.356739000 & 0.537663000 \\
\hline $\mathrm{C}$ & 4.147013000 & -0.688075000 & 0.322740000 \\
\hline $\mathrm{C}$ & 5.149197000 & -1.270458000 & -0.446848000 \\
\hline $\mathrm{C}$ & 4.956715000 & -2.531351000 & -1.006845000 \\
\hline $\mathrm{H}$ & 3.598099000 & -4.185195000 & -1.238875000 \\
\hline $\mathrm{H}$ & 1.805867000 & -3.134098000 & 0.110669000 \\
\hline $\mathrm{H}$ & 4.282439000 & 0.302849000 & 0.747918000 \\
\hline $\mathrm{H}$ & 6.081847000 & -0.738831000 & -0.612138000 \\
\hline $\mathrm{H}$ & 5.738232000 & -2.985196000 & -1.609271000 \\
\hline $\mathrm{C}$ & 1.863368000 & -0.720901000 & 1.399432000 \\
\hline $\mathrm{C}$ & 0.505442000 & -0.842121000 & 0.759442000 \\
\hline $\mathrm{H}$ & 0.461207000 & -0.541288000 & -0.285715000 \\
\hline $\mathrm{O}$ & 1.888247000 & -1.211791000 & 2.731015000 \\
\hline $\mathrm{C}$ & -0.606928000 & -1.232578000 & 1.384023000 \\
\hline $\mathrm{H}$ & -0.556232000 & -1.453491000 & 2.449190000 \\
\hline $\mathrm{C}$ & -1.930146000 & -1.301669000 & 0.740160000 \\
\hline $\mathrm{C}$ & -3.084087000 & -1.016240000 & 1.470133000 \\
\hline $\mathrm{C}$ & -2.081522000 & -1.603229000 & -0.622566000 \\
\hline $\mathrm{C}$ & -4.343472000 & -0.985374000 & 0.874000000 \\
\hline $\mathrm{H}$ & -2.998500000 & -0.792232000 & 2.530698000 \\
\hline $\mathrm{C}$ & -3.323697000 & -1.578926000 & -1.231135000 \\
\hline $\mathrm{H}$ & -1.206572000 & -1.868572000 & -1.210131000 \\
\hline $\mathrm{C}$ & -4.466335000 & -1.259938000 & -0.488173000 \\
\hline $\mathrm{H}$ & -5.210198000 & -0.747725000 & 1.480216000 \\
\hline $\mathrm{H}$ & -3.440330000 & -1.812668000 & -2.284353000 \\
\hline $\mathrm{H}$ & 2.101891000 & 0.343855000 & 1.476893000 \\
\hline $\mathrm{H}$ & 1.834878000 & -2.175386000 & 2.680930000 \\
\hline $\mathrm{C}$ & 1.155877000 & 2.797695000 & 2.261242000 \\
\hline $\mathrm{H}$ & 1.182083000 & 1.876612000 & 2.863227000 \\
\hline $\mathrm{H}$ & 2.169757000 & 3.042746000 & 1.949520000 \\
\hline $\mathrm{H}$ & 0.753543000 & 3.604838000 & 2.884337000 \\
\hline $\mathrm{O}$ & -5.635492000 & -1.254802000 & -1.177619000 \\
\hline $\mathrm{C}$ & -6.819182000 & -0.962541000 & -0.470731000 \\
\hline $\mathrm{H}$ & -6.787991000 & 0.041953000 & -0.029026000 \\
\hline $\mathrm{H}$ & -7.625552000 & -1.007508000 & -1.203525000 \\
\hline $\mathrm{H}$ & -7.007630000 & -1.699249000 & 0.320953000 \\
\hline
\end{tabular}

TSA1-2 MTBD 


\begin{tabular}{|c|c|c|c|}
\hline \multicolumn{4}{|c|}{ Coordinates (Angstroms) } \\
\hline & $X$ & $\mathrm{Y}$ & Z \\
\hline $\mathrm{C}$ & -1.895028000 & 1.125093000 & 1.955865000 \\
\hline $\mathrm{C}$ & -0.848297000 & 1.363583000 & 3.032783000 \\
\hline $\mathrm{C}$ & 0.504965000 & 0.941070000 & 2.486993000 \\
\hline $\mathrm{N}$ & -1.465092000 & 1.590880000 & 0.639841000 \\
\hline $\mathrm{H}$ & -2.835426000 & 1.626808000 & 2.204350000 \\
\hline $\mathrm{H}$ & -2.120111000 & 0.053922000 & 1.886539000 \\
\hline $\mathrm{H}$ & -1.096230000 & 0.794552000 & 3.933796000 \\
\hline $\mathrm{H}$ & -0.806401000 & 2.424146000 & 3.305362000 \\
\hline $\mathrm{H}$ & 1.300530000 & 1.130747000 & 3.213577000 \\
\hline $\mathrm{H}$ & 0.519514000 & -0.132500000 & 2.251614000 \\
\hline $\mathrm{N}$ & 0.804972000 & 1.726946000 & 1.297980000 \\
\hline $\mathrm{C}$ & 2.216539000 & 1.824104000 & 0.938939000 \\
\hline $\mathrm{C}$ & 2.424188000 & 2.816494000 & -0.190481000 \\
\hline $\mathrm{C}$ & 1.437036000 & 2.524829000 & -1.306757000 \\
\hline $\mathrm{N}$ & 0.079544000 & 2.527782000 & -0.784000000 \\
\hline $\mathrm{C}$ & -0.197374000 & 1.917922000 & 0.393781000 \\
\hline $\mathrm{H}$ & 2.591316000 & 0.833599000 & 0.651641000 \\
\hline $\mathrm{H}$ & 2.764651000 & 2.142731000 & 1.832323000 \\
\hline $\mathrm{H}$ & 2.272885000 & 3.839122000 & 0.171428000 \\
\hline $\mathrm{H}$ & 3.448922000 & 2.731691000 & -0.563211000 \\
\hline $\mathrm{H}$ & 1.495340000 & 3.296759000 & -2.078778000 \\
\hline $\mathrm{H}$ & 1.655161000 & 1.557460000 & -1.776893000 \\
\hline $\mathrm{C}$ & -4.774729000 & -2.264590000 & 1.115086000 \\
\hline $\mathrm{C}$ & -3.549824000 & -1.871452000 & 0.589274000 \\
\hline $\mathrm{C}$ & -3.467383000 & -0.969790000 & -0.489676000 \\
\hline $\mathrm{C}$ & -4.683862000 & -0.506669000 & -1.024456000 \\
\hline $\mathrm{C}$ & -5.906537000 & -0.908500000 & -0.501469000 \\
\hline $\mathrm{C}$ & -5.967191000 & -1.786696000 & 0.577930000 \\
\hline $\mathrm{H}$ & -4.795254000 & -2.967198000 & 1.944623000 \\
\hline $\mathrm{H}$ & -2.644996000 & -2.293060000 & 1.018701000 \\
\hline $\mathrm{H}$ & -4.649381000 & 0.184727000 & -1.859909000 \\
\hline $\mathrm{H}$ & -6.824149000 & -0.526438000 & -0.942159000 \\
\hline $\mathrm{H}$ & -6.923090000 & -2.099243000 & 0.987464000 \\
\hline $\mathrm{C}$ & -2.194835000 & -0.452424000 & -1.029489000 \\
\hline $\mathrm{C}$ & -0.927309000 & -1.030993000 & -0.687600000 \\
\hline $\mathrm{H}$ & -0.887035000 & -1.491940000 & 0.299223000 \\
\hline $\mathrm{O}$ & -2.285947000 & 0.056726000 & -2.349586000 \\
\hline $\mathrm{C}$ & 0.239579000 & -0.893944000 & -1.368916000 \\
\hline $\mathrm{H}$ & 0.208047000 & -0.421750000 & -2.349569000 \\
\hline $\mathrm{C}$ & 1.568315000 & -1.243819000 & -0.869528000 \\
\hline $\mathrm{C}$ & 2.706151000 & -0.919397000 & -1.620712000 \\
\hline $\mathrm{C}$ & 1.804516000 & -1.848193000 & 0.382176000 \\
\hline
\end{tabular}




\begin{tabular}{|c|c|c|c|}
\hline $\mathrm{C}$ & 4.002936000 & -1.120816000 & -1.149779000 \\
\hline $\mathrm{H}$ & 2.574966000 & -0.479112000 & -2.607127000 \\
\hline $\mathrm{C}$ & 3.084035000 & -2.057433000 & 0.865308000 \\
\hline $\mathrm{H}$ & 0.965860000 & -2.163523000 & 0.996710000 \\
\hline $\mathrm{C}$ & 4.200641000 & -1.683134000 & 0.110024000 \\
\hline $\mathrm{H}$ & 4.841425000 & -0.839168000 & -1.777579000 \\
\hline $\mathrm{H}$ & 3.246226000 & -2.520392000 & 1.833819000 \\
\hline $\mathrm{H}$ & -1.897239000 & 0.844887000 & -0.163591000 \\
\hline $\mathrm{H}$ & -2.315155000 & -0.698873000 & -2.954674000 \\
\hline $\mathrm{C}$ & -0.989443000 & 2.799779000 & -1.735192000 \\
\hline $\mathrm{H}$ & -1.229652000 & 1.921585000 & -2.345125000 \\
\hline $\mathrm{H}$ & -1.888233000 & 3.093764000 & -1.192071000 \\
\hline $\mathrm{H}$ & -0.668985000 & 3.627385000 & -2.374398000 \\
\hline $\mathrm{O}$ & 5.415837000 & -1.916970000 & 0.681772000 \\
\hline $\mathrm{C}$ & 6.561491000 & -1.586727000 & -0.064451000 \\
\hline $\mathrm{H}$ & 6.606940000 & -0.510970000 & -0.284014000 \\
\hline $\mathrm{H}$ & 7.417040000 & -1.861208000 & 0.554542000 \\
\hline $\mathrm{H}$ & 6.605956000 & -2.145600000 & -1.008785000 \\
\hline
\end{tabular}

\section{A2}

\begin{tabular}{lrcc} 
& \multicolumn{3}{c}{ Coordinates (Angstroms) } \\
& $\mathrm{X}$ & $\mathrm{Y}$ & $\mathrm{Z}$ \\
$\mathrm{C}$ & 3.080011000 & 0.652723000 & 2.046837000 \\
$\mathrm{C}$ & 3.857390000 & 1.186425000 & 0.855516000 \\
$\mathrm{C}$ & 3.224842000 & 2.482075000 & 0.378741000 \\
$\mathrm{~N}$ & 1.638828000 & 0.890389000 & 1.919468000 \\
$\mathrm{H}$ & 3.409121000 & 1.130485000 & 2.978558000 \\
$\mathrm{H}$ & 3.230040000 & -0.428544000 & 2.139829000 \\
$\mathrm{H}$ & 3.841834000 & 0.463903000 & 0.038215000 \\
$\mathrm{H}$ & 4.899879000 & 1.350151000 & 1.141048000 \\
$\mathrm{H}$ & 3.291490000 & 3.269027000 & 1.142654000 \\
$\mathrm{H}$ & 3.722618000 & 2.840276000 & -0.524476000 \\
$\mathrm{~N}$ & 1.825785000 & 2.241839000 & 0.041561000 \\
$\mathrm{C}$ & 1.294822000 & 2.759183000 & -1.216634000 \\
$\mathrm{C}$ & -0.194855000 & 3.034120000 & -1.077955000 \\
$\mathrm{C}$ & -0.888172000 & 1.771824000 & -0.599347000 \\
$\mathrm{~N}$ & -0.222981000 & 1.250425000 & 0.589777000 \\
$\mathrm{C}$ & 1.085319000 & 1.436703000 & 0.825290000 \\
$\mathrm{H}$ & 1.496401000 & 2.041653000 & -2.022887000 \\
$\mathrm{H}$ & 1.834099000 & 3.682814000 & -1.442221000 \\
$\mathrm{H}$ & -0.350479000 & 3.847396000 & -0.360467000 \\
$\mathrm{H}$ & -0.611126000 & 3.346829000 & -2.039185000 \\
$\mathrm{H}$ & -1.935976000 & 1.950064000 & -0.347432000
\end{tabular}




\begin{tabular}{|c|c|c|c|}
\hline $\mathrm{H}$ & -0.873442000 & 1.007198000 & -1.385510000 \\
\hline $\mathrm{H}$ & -0.594069000 & 0.323374000 & 0.865125000 \\
\hline $\mathrm{C}$ & 3.450228000 & -0.536143000 & -2.467684000 \\
\hline $\mathrm{C}$ & 2.297052000 & -0.834647000 & -1.757119000 \\
\hline $\mathrm{C}$ & 2.318977000 & -1.685342000 & -0.620625000 \\
\hline $\mathrm{C}$ & 3.587515000 & -2.191664000 & -0.244196000 \\
\hline $\mathrm{C}$ & 4.736687000 & -1.874917000 & -0.955052000 \\
\hline $\mathrm{C}$ & 4.690616000 & -1.042253000 & -2.074171000 \\
\hline $\mathrm{H}$ & 3.381761000 & 0.111722000 & -3.339471000 \\
\hline $\mathrm{H}$ & 1.355570000 & -0.400806000 & -2.085206000 \\
\hline $\mathrm{H}$ & 3.644011000 & -2.845887000 & 0.619002000 \\
\hline $\mathrm{H}$ & 5.688201000 & -2.290016000 & -0.630440000 \\
\hline $\mathrm{H}$ & 5.592967000 & -0.799343000 & -2.626626000 \\
\hline $\mathrm{C}$ & 1.148310000 & -1.980005000 & 0.157572000 \\
\hline $\mathrm{C}$ & -0.147349000 & -1.632380000 & -0.165480000 \\
\hline $\mathrm{H}$ & -0.282445000 & -1.264260000 & -1.181995000 \\
\hline $\mathrm{O}$ & 1.392976000 & -2.571920000 & 1.411258000 \\
\hline $\mathrm{C}$ & -1.275775000 & -1.625991000 & 0.656237000 \\
\hline $\mathrm{H}$ & -1.175070000 & -1.938702000 & 1.694617000 \\
\hline $\mathrm{C}$ & -2.603529000 & -1.219203000 & 0.232010000 \\
\hline $\mathrm{C}$ & -3.601105000 & -0.935315000 & 1.183367000 \\
\hline $\mathrm{C}$ & -2.974849000 & -1.023126000 & -1.118947000 \\
\hline $\mathrm{C}$ & -4.862872000 & -0.460687000 & 0.835301000 \\
\hline $\mathrm{H}$ & -3.374703000 & -1.082860000 & 2.237636000 \\
\hline $\mathrm{C}$ & -4.220816000 & -0.534723000 & -1.476695000 \\
\hline $\mathrm{H}$ & -2.276343000 & -1.280245000 & -1.910823000 \\
\hline $\mathrm{C}$ & -5.179645000 & -0.241713000 & -0.504913000 \\
\hline $\mathrm{H}$ & -5.583300000 & -0.262497000 & 1.622058000 \\
\hline $\mathrm{H}$ & -4.481960000 & -0.392537000 & -2.521407000 \\
\hline $\mathrm{H}$ & 0.769000000 & -3.305392000 & 1.497790000 \\
\hline $\mathrm{C}$ & 0.828470000 & 0.198277000 & 2.914774000 \\
\hline $\mathrm{H}$ & -0.163512000 & 0.648510000 & 2.972909000 \\
\hline $\mathrm{H}$ & 0.754954000 & -0.867281000 & 2.676634000 \\
\hline $\mathrm{H}$ & 1.321623000 & 0.323076000 & 3.883740000 \\
\hline $\mathrm{O}$ & -6.378389000 & 0.237266000 & -0.962912000 \\
\hline $\mathrm{C}$ & -7.378729000 & 0.487101000 & -0.010374000 \\
\hline $\mathrm{H}$ & -7.073842000 & 1.259541000 & 0.709997000 \\
\hline $\mathrm{H}$ & -8.249267000 & 0.841960000 & -0.565209000 \\
\hline $\mathrm{H}$ & -7.651426000 & -0.422880000 & 0.542410000 \\
\hline
\end{tabular}

TSA2-3 MTBD

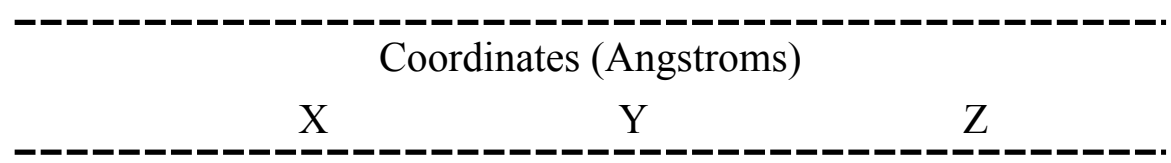




\begin{tabular}{|c|c|c|c|}
\hline $\mathrm{C}$ & 3.053174000 & 1.334263000 & 1.721536000 \\
\hline $\mathrm{C}$ & 3.822883000 & 1.307655000 & 0.408694000 \\
\hline $\mathrm{C}$ & 3.250359000 & 2.351947000 & -0.534112000 \\
\hline $\mathrm{N}$ & 1.637807000 & 1.653646000 & 1.537005000 \\
\hline $\mathrm{H}$ & 3.472269000 & 2.090554000 & 2.399082000 \\
\hline $\mathrm{H}$ & 3.118675000 & 0.359149000 & 2.217676000 \\
\hline $\mathrm{H}$ & 3.739009000 & 0.327655000 & -0.064468000 \\
\hline $\mathrm{H}$ & 4.882331000 & 1.499260000 & 0.600452000 \\
\hline $\mathrm{H}$ & 3.396009000 & 3.367716000 & -0.138013000 \\
\hline $\mathrm{H}$ & 3.740594000 & 2.294761000 & -1.508463000 \\
\hline $\mathrm{N}$ & 1.832638000 & 2.089896000 & -0.735838000 \\
\hline $\mathrm{C}$ & 1.258975000 & 2.179487000 & -2.072309000 \\
\hline $\mathrm{C}$ & -0.165033000 & 2.707112000 & -1.984590000 \\
\hline $\mathrm{C}$ & -0.958130000 & 1.819047000 & -1.040098000 \\
\hline $\mathrm{N}$ & -0.269353000 & 1.626448000 & 0.230546000 \\
\hline $\mathrm{C}$ & 1.048953000 & 1.766798000 & 0.321957000 \\
\hline $\mathrm{H}$ & 1.282398000 & 1.195866000 & -2.561061000 \\
\hline $\mathrm{H}$ & 1.886803000 & 2.856481000 & -2.658098000 \\
\hline $\mathrm{H}$ & -0.145437000 & 3.737132000 & -1.610477000 \\
\hline $\mathrm{H}$ & -0.628827000 & 2.716298000 & -2.975162000 \\
\hline $\mathrm{H}$ & -1.943491000 & 2.244190000 & -0.829695000 \\
\hline $\mathrm{H}$ & -1.132466000 & 0.838758000 & -1.504545000 \\
\hline $\mathrm{H}$ & -0.693078000 & 0.658380000 & 0.738730000 \\
\hline $\mathrm{C}$ & 3.547684000 & -1.690176000 & -2.174921000 \\
\hline $\mathrm{C}$ & 2.392263000 & -1.531549000 & -1.422936000 \\
\hline $\mathrm{C}$ & 2.357472000 & -1.863553000 & -0.053366000 \\
\hline $\mathrm{C}$ & 3.546783000 & -2.345363000 & 0.522875000 \\
\hline $\mathrm{C}$ & 4.705854000 & -2.487341000 & -0.231429000 \\
\hline $\mathrm{C}$ & 4.720171000 & -2.162289000 & -1.585581000 \\
\hline $\mathrm{H}$ & 3.537122000 & -1.428188000 & -3.230037000 \\
\hline $\mathrm{H}$ & 1.505452000 & -1.122347000 & -1.898385000 \\
\hline $\mathrm{H}$ & 3.547665000 & -2.598402000 & 1.577165000 \\
\hline $\mathrm{H}$ & 5.608578000 & -2.860319000 & 0.245401000 \\
\hline $\mathrm{H}$ & 5.627905000 & -2.272130000 & -2.171114000 \\
\hline $\mathrm{C}$ & 1.174188000 & -1.642648000 & 0.767385000 \\
\hline $\mathrm{C}$ & -0.074146000 & -1.367219000 & 0.311300000 \\
\hline $\mathrm{H}$ & -0.216057000 & -1.457533000 & -0.763817000 \\
\hline $\mathrm{O}$ & 1.436114000 & -1.649021000 & 2.139781000 \\
\hline $\mathrm{C}$ & -1.163145000 & -0.804231000 & 1.055504000 \\
\hline $\mathrm{H}$ & -1.072843000 & -0.785818000 & 2.144170000 \\
\hline $\mathrm{C}$ & -2.548724000 & -0.820225000 & 0.568482000 \\
\hline $\mathrm{C}$ & -3.551127000 & -0.151254000 & 1.288306000 \\
\hline $\mathrm{C}$ & -2.952077000 & -1.410864000 & -0.645628000 \\
\hline $\mathrm{C}$ & -4.865875000 & -0.057645000 & 0.839145000 \\
\hline
\end{tabular}




\begin{tabular}{lrcc}
$\mathrm{H}$ & -3.289462000 & 0.321038000 & 2.233385000 \\
$\mathrm{C}$ & -4.253705000 & -1.312943000 & -1.113892000 \\
$\mathrm{H}$ & -2.238259000 & -1.984191000 & -1.230646000 \\
$\mathrm{C}$ & -5.225831000 & -0.634860000 & -0.378103000 \\
$\mathrm{H}$ & -5.592381000 & 0.472076000 & 1.446416000 \\
$\mathrm{H}$ & -4.544721000 & -1.780851000 & -2.049671000 \\
$\mathrm{H}$ & 0.644648000 & -1.999622000 & 2.570115000 \\
$\mathrm{C}$ & 0.842793000 & 1.550078000 & 2.748337000 \\
$\mathrm{H}$ & -0.142436000 & 1.985464000 & 2.583715000 \\
$\mathrm{H}$ & 0.740363000 & 0.504635000 & 3.050723000 \\
$\mathrm{H}$ & 1.354196000 & 2.105655000 & 3.542974000 \\
$\mathrm{O}$ & -6.480626000 & -0.599438000 & -0.922393000 \\
$\mathrm{C}$ & -7.492056000 & 0.024675000 & -0.174630000 \\
$\mathrm{H}$ & -7.284120000 & 1.092157000 & -0.015280000 \\
$\mathrm{H}$ & -8.410027000 & -0.075750000 & -0.756545000 \\
$\mathrm{H}$ & -7.632177000 & -0.457725000 & 0.802821000 \\
\hline- - - - & &
\end{tabular}

\begin{tabular}{lrrr} 
A3 & & \\
& & \\
& Coordinates $($ Angstroms) & \\
\hline $\mathrm{C}$ & 0.137573000 & -2.776258000 & -1.777176000 \\
$\mathrm{C}$ & 1.226539000 & -2.024798000 & -2.522205000 \\
$\mathrm{C}$ & 2.503151000 & -2.052533000 & -1.699034000 \\
$\mathrm{~N}$ & -0.078075000 & -2.142861000 & -0.494367000 \\
$\mathrm{H}$ & 0.404840000 & -3.843456000 & -1.672533000 \\
$\mathrm{H}$ & -0.803997000 & -2.733293000 & -2.333824000 \\
$\mathrm{H}$ & 0.892591000 & -0.993731000 & -2.674540000 \\
$\mathrm{H}$ & 1.410942000 & -2.470642000 & -3.504606000 \\
$\mathrm{H}$ & 3.031736000 & -3.005096000 & -1.866136000 \\
$\mathrm{H}$ & 3.179761000 & -1.250509000 & -2.024154000 \\
$\mathrm{~N}$ & 2.252970000 & -1.896867000 & -0.277885000 \\
$\mathrm{C}$ & 3.431990000 & -1.566143000 & 0.504754000 \\
$\mathrm{C}$ & 3.236934000 & -1.953115000 & 1.960725000 \\
$\mathrm{C}$ & 1.916148000 & -1.361315000 & 2.442266000 \\
$\mathrm{~N}$ & 0.800225000 & -1.708278000 & 1.591787000 \\
$\mathrm{C}$ & 1.014349000 & -1.907010000 & 0.336832000 \\
$\mathrm{H}$ & 3.645172000 & -0.489717000 & 0.418017000 \\
$\mathrm{H}$ & 4.283919000 & -2.106354000 & 0.072277000 \\
$\mathrm{H}$ & 3.203508000 & -3.045768000 & 2.048132000 \\
$\mathrm{H}$ & 4.079843000 & -1.592195000 & 2.559866000 \\
$\mathrm{H}$ & 1.694812000 & -1.709408000 & 3.458174000 \\
$\mathrm{H}$ & 2.016341000 & -0.265264000 & 2.502804000 \\
$\mathrm{C}$ & -5.725919000 & 1.826806000 & -1.137717000
\end{tabular}




\begin{tabular}{lrrr}
$\mathrm{C}$ & -4.568633000 & 1.860722000 & -0.368578000 \\
$\mathrm{C}$ & -4.090576000 & 0.699940000 & 0.248597000 \\
$\mathrm{C}$ & -4.810659000 & -0.491316000 & 0.091946000 \\
$\mathrm{C}$ & -5.970965000 & -0.522671000 & -0.674520000 \\
$\mathrm{C}$ & -6.432179000 & 0.635886000 & -1.292397000 \\
$\mathrm{H}$ & -6.086838000 & 2.737447000 & -1.606634000 \\
$\mathrm{H}$ & -4.036001000 & 2.795984000 & -0.224666000 \\
$\mathrm{H}$ & -4.440102000 & -1.406077000 & 0.547839000 \\
$\mathrm{H}$ & -6.510282000 & -1.457592000 & -0.795370000 \\
$\mathrm{H}$ & -7.340041000 & 0.612971000 & -1.887726000 \\
$\mathrm{C}$ & -2.849182000 & 0.710092000 & 1.057106000 \\
$\mathrm{C}$ & -1.749575000 & 1.399232000 & 0.735293000 \\
$\mathrm{H}$ & -1.774561000 & 1.991073000 & -0.175324000 \\
$\mathrm{O}$ & -2.834558000 & -0.110360000 & 2.156158000 \\
$\mathrm{C}$ & -0.478120000 & 1.375740000 & 1.537331000 \\
$\mathrm{H}$ & -0.378178000 & 0.390695000 & 2.011879000 \\
$\mathrm{C}$ & 0.769731000 & 1.636511000 & 0.722507000 \\
$\mathrm{C}$ & 1.869524000 & 2.276827000 & 1.287210000 \\
$\mathrm{C}$ & 0.887403000 & 1.198319000 & -0.600978000 \\
$\mathrm{C}$ & 3.057249000 & 2.472160000 & 0.581002000 \\
$\mathrm{H}$ & 1.807719000 & 2.634172000 & 2.312843000 \\
$\mathrm{C}$ & 2.052198000 & 1.394298000 & -1.324439000 \\
$\mathrm{H}$ & 0.056250000 & 0.670241000 & -1.059905000 \\
$\mathrm{C}$ & 3.151167000 & 2.025179000 & -0.736301000 \\
$\mathrm{H}$ & 3.887079000 & 2.973177000 & 1.066763000 \\
$\mathrm{H}$ & 2.140452000 & 1.058019000 & -2.353145000 \\
$\mathrm{H}$ & -3.744508000 & -0.307161000 & 2.408147000 \\
$\mathrm{H}$ & -0.535015000 & 2.113227000 & 2.350763000 \\
$\mathrm{C}$ & -1.359730000 & -2.397752000 & 0.130139000 \\
$\mathrm{H}$ & -1.489226000 & -3.460916000 & 0.388330000 \\
$\mathrm{H}$ & -1.441429000 & -1.810305000 & 1.042301000 \\
$\mathrm{H}$ & -2.153365000 & -2.104639000 & -0.566591000 \\
$\mathrm{O}$ & 4.255884000 & 2.149232000 & -1.523835000 \\
$\mathrm{H}$ & 5.395451000 & 2.753191000 & -0.960471000 \\
$\mathrm{H}$ & 6.162427000 & 2.736441000 & -1.735999000 \\
$\mathrm{H}$ & 5.200602000 & 3.793925000 & -0.669595000 \\
---755890000 & 2.197876000 & -0.084055000 \\
\hline
\end{tabular}

\section{(7) $\mathbf{R}_{1}=\mathrm{H}, \mathrm{R}_{2}=\mathrm{Me}$}

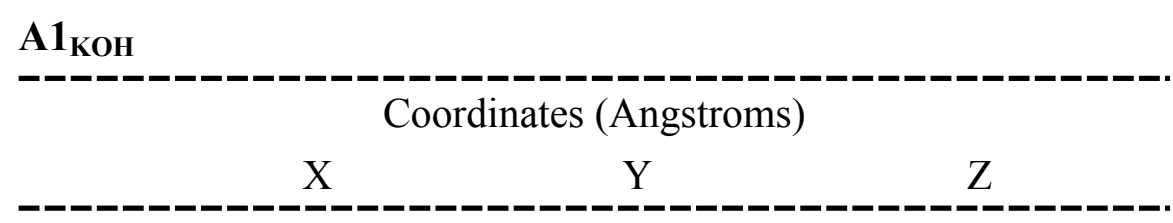




\begin{tabular}{|c|c|c|c|}
\hline $\mathrm{C}$ & 2.455260000 & 3.154194000 & -0.813585000 \\
\hline $\mathrm{C}$ & 1.633191000 & 2.037474000 & -0.689105000 \\
\hline $\mathrm{C}$ & 2.069479000 & 0.915935000 & 0.020845000 \\
\hline $\mathrm{C}$ & 3.333992000 & 0.922086000 & 0.618989000 \\
\hline $\mathrm{C}$ & 4.150069000 & 2.043984000 & 0.494206000 \\
\hline $\mathrm{C}$ & 3.716503000 & 3.157807000 & -0.222228000 \\
\hline $\mathrm{H}$ & 2.107889000 & 4.025113000 & -1.362538000 \\
\hline $\mathrm{H}$ & 0.638932000 & 2.041995000 & -1.132795000 \\
\hline $\mathrm{H}$ & 3.630131000 & 0.022887000 & 1.178233000 \\
\hline $\mathrm{H}$ & 5.129042000 & 2.052565000 & 0.966415000 \\
\hline $\mathrm{H}$ & 4.355652000 & 4.031945000 & -0.312529000 \\
\hline $\mathrm{C}$ & 1.221285000 & -0.342367000 & 0.104733000 \\
\hline $\mathrm{C}$ & -0.230334000 & -0.052347000 & 0.349493000 \\
\hline $\mathrm{H}$ & -0.412286000 & 0.623227000 & 1.183967000 \\
\hline $\mathrm{O}$ & 1.416468000 & -1.136496000 & -1.088582000 \\
\hline $\mathrm{C}$ & -1.245963000 & -0.553373000 & -0.358618000 \\
\hline $\mathrm{H}$ & -1.008470000 & -1.224991000 & -1.182600000 \\
\hline $\mathrm{C}$ & -2.681445000 & -0.305757000 & -0.143432000 \\
\hline $\mathrm{C}$ & -3.601844000 & -0.772600000 & -1.087309000 \\
\hline $\mathrm{C}$ & -3.187718000 & 0.378712000 & 0.970189000 \\
\hline $\mathrm{C}$ & -4.967456000 & -0.559306000 & -0.934312000 \\
\hline $\mathrm{H}$ & -3.239516000 & -1.308841000 & -1.961508000 \\
\hline $\mathrm{C}$ & -4.550056000 & 0.590058000 & 1.120285000 \\
\hline $\mathrm{H}$ & -2.510039000 & 0.745094000 & 1.735690000 \\
\hline $\mathrm{C}$ & -5.467631000 & 0.124582000 & 0.172768000 \\
\hline $\mathrm{H}$ & -5.655761000 & -0.932127000 & -1.689280000 \\
\hline $\mathrm{H}$ & -4.912829000 & 1.124057000 & 1.995676000 \\
\hline $\mathrm{H}$ & 1.646808000 & -0.969437000 & 0.915055000 \\
\hline $\mathrm{H}$ & 1.195366000 & -0.568910000 & -1.839223000 \\
\hline $\mathrm{O}$ & 3.240182000 & -1.921309000 & 1.750000000 \\
\hline $\mathrm{H}$ & 3.135142000 & -1.705416000 & 2.680153000 \\
\hline $\mathrm{K}$ & 3.498347000 & -2.688590000 & -0.396066000 \\
\hline $\mathrm{C}$ & -6.948605000 & 0.333169000 & 0.364181000 \\
\hline $\mathrm{H}$ & -7.491841000 & 0.225197000 & -0.579480000 \\
\hline $\mathrm{H}$ & -7.360503000 & -0.399053000 & 1.069357000 \\
\hline $\mathrm{H}$ & -7.161881000 & 1.328838000 & 0.766717000 \\
\hline
\end{tabular}

TSA1-2 кон

$\begin{array}{llrr} & & & \\ & & \text { Coordinates (Angstroms) } & \text { Y } \\ \text { C } & -3.715144000 & -2.789453000 & 0.165026000 \\ \text { C } & -2.593784000 & -1.966872000 & 0.078125000 \\ \text { C } & -2.727317000 & -0.585755000 & -0.102279000\end{array}$




$\begin{array}{lrrr}\mathrm{C} & -4.023041000 & -0.057350000 & -0.211334000 \\ \mathrm{C} & -5.140107000 & -0.878842000 & -0.143025000 \\ \mathrm{C} & -4.992464000 & -2.251835000 & 0.051651000 \\ \mathrm{H} & -3.584903000 & -3.858855000 & 0.307420000 \\ \mathrm{H} & -1.603761000 & -2.410358000 & 0.138620000 \\ \mathrm{H} & -4.136311000 & 1.015035000 & -0.342092000 \\ \mathrm{H} & -6.132953000 & -0.445903000 & -0.232508000 \\ \mathrm{H} & -5.865808000 & -2.895002000 & 0.110554000 \\ \mathrm{C} & -1.571168000 & 0.358593000 & -0.152179000 \\ \mathrm{C} & -0.221549000 & -0.169137000 & 0.041872000 \\ \mathrm{H} & -0.108732000 & -0.804005000 & 0.921090000 \\ \mathrm{O} & -1.658128000 & 1.251974000 & -1.284253000 \\ \mathrm{C} & 0.880478000 & 0.168352000 & -0.665261000 \\ \mathrm{H} & 0.743488000 & 0.736286000 & -1.587364000 \\ \mathrm{C} & 2.268876000 & -0.197175000 & -0.355215000 \\ \mathrm{C} & 3.261358000 & -0.042829000 & -1.331554000 \\ \mathrm{C} & 2.675350000 & -0.680984000 & 0.899096000 \\ \mathrm{C} & 4.588671000 & -0.371095000 & -1.077516000 \\ \mathrm{H} & 2.983895000 & 0.332660000 & -2.314627000 \\ \mathrm{C} & 3.999206000 & -1.012899000 & 1.146106000 \\ \mathrm{H} & 1.946335000 & -0.786224000 & 1.697991000 \\ \mathrm{C} & 4.984451000 & -0.866663000 & 0.164098000 \\ \mathrm{H} & 5.330278000 & -0.241256000 & -1.862683000 \\ \mathrm{H} & 4.278328000 & -1.385868000 & 2.129435000 \\ \mathrm{H} & -1.767902000 & 1.214148000 & 0.858207000 \\ \mathrm{H} & -1.657249000 & 0.714041000 & -2.088899000 \\ \mathrm{O} & -1.869062000 & 2.242234000 & 1.638363000 \\ \mathrm{H} & -2.636002000 & 2.068399000 & 2.190717000 \\ \mathrm{~K} & -0.237640000 & 3.067511000 & 0.101598000 \\ \mathrm{C} & 6.418531000 & -1.236129000 & 0.448100000 \\ \mathrm{H} & 7.078675000 & -0.932729000 & -0.370167000 \\ \mathrm{H} & 6.780022000 & -0.757693000 & 1.365249000 \\ \mathrm{H} & 6.531205000 & -2.318593000 & 0.581531000 \\ ------ & \end{array}$

\begin{tabular}{|c|c|c|c|}
\hline \multicolumn{4}{|c|}{ А2 ${ }_{\text {кон }}$} \\
\hline & \multicolumn{3}{|c|}{ Coordinates (Angstroms) } \\
\hline & $\mathrm{X}$ & Y & Z \\
\hline$\overline{\mathrm{C}}$ & -3.855168000 & -2.165698000 & 0.895187000 \\
\hline $\mathrm{C}$ & -2.658974000 & -1.659465000 & 0.413672000 \\
\hline $\mathrm{C}$ & -2.628325000 & -0.595608000 & -0.526956000 \\
\hline $\mathrm{C}$ & -3.886340000 & -0.070833000 & -0.921913000 \\
\hline $\mathrm{C}$ & -5.079004000 & -0.585152000 & -0.421332000 \\
\hline $\mathrm{C}$ & -5.083149000 & -1.634548000 & 0.492041000 \\
\hline
\end{tabular}




$\begin{array}{lrrr}\mathrm{H} & -3.829814000 & -2.986534000 & 1.607683000 \\ \mathrm{H} & -1.728796000 & -2.093947000 & 0.768058000 \\ \mathrm{H} & -3.909914000 & 0.723810000 & -1.660343000 \\ \mathrm{H} & -6.019579000 & -0.160193000 & -0.764394000 \\ \mathrm{H} & -6.014734000 & -2.035299000 & 0.878693000 \\ \mathrm{C} & -1.406754000 & 0.016061000 & -0.979374000 \\ \mathrm{C} & -0.109389000 & -0.319533000 & -0.622543000 \\ \mathrm{H} & 0.007649000 & -1.280319000 & -0.125055000 \\ \mathrm{O} & -1.601227000 & 1.263953000 & -1.626517000 \\ \mathrm{C} & 1.036838000 & 0.449494000 & -0.809734000 \\ \mathrm{H} & 0.944451000 & 1.403747000 & -1.328695000 \\ \mathrm{C} & 2.401606000 & 0.037300000 & -0.509664000 \\ \mathrm{C} & 3.456442000 & 0.959776000 & -0.671381000 \\ \mathrm{C} & 2.760971000 & -1.234876000 & -0.026465000 \\ \mathrm{C} & 4.768874000 & 0.636702000 & -0.364528000 \\ \mathrm{H} & 3.228375000 & 1.955283000 & -1.048559000 \\ \mathrm{C} & 4.078977000 & -1.549463000 & 0.283763000 \\ \mathrm{H} & 2.002179000 & -2.002784000 & 0.098054000 \\ \mathrm{C} & 5.113402000 & -0.627167000 & 0.124778000 \\ \mathrm{H} & 5.547323000 & 1.384306000 & -0.508029000 \\ \mathrm{H} & 4.307954000 & -2.547415000 & 0.653328000 \\ \mathrm{H} & -1.009295000 & 1.292344000 & -2.388975000 \\ \mathrm{O} & 0.378396000 & 1.878028000 & 1.695211000 \\ \mathrm{H} & 1.118297000 & 1.922376000 & 2.309227000 \\ \mathrm{~K} & -2.057623000 & 1.949350000 & 0.928382000 \\ \mathrm{H} & 0.717755000 & 1.352256000 & 0.924876000 \\ \mathrm{C} & 6.545738000 & -0.974378000 & 0.445556000 \\ \mathrm{H} & 7.167799000 & -0.991287000 & -0.458031000 \\ \mathrm{H} & 6.992700000 & -0.245017000 & 1.131684000 \\ \mathrm{H} & 6.620664000 & -1.960486000 & 0.915018000 \\ \mathrm{H} & & & \\ & & & \end{array}$

TSA2-3

$\begin{array}{lrrr} & & & \text { Coordinates (Angstroms) } \\ \text { C } & -3.674897000 & -2.599881000 & 0.134165000 \\ \mathrm{C} & -2.549090000 & -1.909748000 & -0.288514000 \\ \mathrm{C} & -2.605139000 & -0.531367000 & -0.578615000 \\ \mathrm{C} & -3.861164000 & 0.100138000 & -0.474250000 \\ \mathrm{C} & -4.989205000 & -0.602479000 & -0.054791000 \\ \mathrm{C} & -4.904693000 & -1.952733000 & 0.265630000 \\ \mathrm{H} & -3.595874000 & -3.662375000 & 0.347324000 \\ \mathrm{H} & -1.616070000 & -2.448568000 & -0.419061000 \\ \mathrm{H} & -3.950929000 & 1.142292000 & -0.765001000\end{array}$




$\begin{array}{lrcc}\mathrm{H} & -5.944518000 & -0.087610000 & 0.007487000 \\ \mathrm{H} & -5.784997000 & -2.501152000 & 0.586301000 \\ \mathrm{C} & -1.412486000 & 0.252843000 & -0.910624000 \\ \mathrm{C} & -0.127994000 & -0.138296000 & -0.686268000 \\ \mathrm{H} & 0.005590000 & -1.119478000 & -0.230619000 \\ \mathrm{O} & -1.712402000 & 1.546554000 & -1.324005000 \\ \mathrm{C} & 1.051388000 & 0.673138000 & -0.900543000 \\ \mathrm{C} & 2.365029000 & 0.142646000 & -0.507671000 \\ \mathrm{C} & 2.547729000 & -0.673162000 & 0.621223000 \\ \mathrm{C} & 3.527793000 & 0.478636000 & -1.225200000 \\ \mathrm{C} & 3.804713000 & -1.139939000 & 0.992552000 \\ \mathrm{H} & 1.688607000 & -0.943548000 & 1.232792000 \\ \mathrm{C} & 4.780853000 & 0.022680000 & -0.843691000 \\ \mathrm{H} & 3.435233000 & 1.103963000 & -2.110962000 \\ \mathrm{C} & 4.949662000 & -0.802276000 & 0.272935000 \\ \mathrm{H} & 3.895620000 & -1.776438000 & 1.871226000 \\ \mathrm{H} & 5.651309000 & 0.303990000 & -1.434284000 \\ \mathrm{H} & -0.955680000 & 2.121522000 & -1.094411000 \\ \mathrm{H} & 0.695677000 & 1.882563000 & -0.055131000 \\ \mathrm{O} & 0.137091000 & 2.755784000 & 0.419546000 \\ \mathrm{~K} & -1.792774000 & 1.628660000 & 1.553491000 \\ \mathrm{H} & 0.716127000 & 3.516946000 & 0.299793000 \\ \mathrm{H} & 1.060605000 & 1.185226000 & -1.869368000 \\ \mathrm{C} & 6.317500000 & -1.290490000 & 0.681472000 \\ \mathrm{H} & 6.251639000 & -2.032573000 & 1.483775000 \\ \mathrm{H} & 6.843628000 & -1.757080000 & -0.159596000 \\ \mathrm{H} & 6.949254000 & -0.469206000 & 1.042222000 \\ -------0 .-0\end{array}$

\begin{tabular}{|c|c|c|c|}
\hline \multicolumn{4}{|c|}{ A3 кон } \\
\hline & \multicolumn{3}{|c|}{ Coordinates (Angstroms) } \\
\hline & $\mathrm{X}$ & Y & $\mathrm{Z}$ \\
\hline$\overline{\mathrm{C}}$ & -4.892033000 & -0.430645000 & -0.941114000 \\
\hline $\mathrm{C}$ & -3.755529000 & -1.131331000 & -0.547545000 \\
\hline $\mathrm{C}$ & -2.651246000 & -0.463672000 & -0.000732000 \\
\hline $\mathrm{C}$ & -2.716464000 & 0.929104000 & 0.173485000 \\
\hline $\mathrm{C}$ & -3.859030000 & 1.616291000 & -0.221985000 \\
\hline $\mathrm{C}$ & -4.945195000 & 0.949767000 & -0.787115000 \\
\hline $\mathrm{H}$ & -5.739139000 & -0.969012000 & -1.356474000 \\
\hline $\mathrm{H}$ & -3.759875000 & -2.216091000 & -0.628754000 \\
\hline $\mathrm{H}$ & -1.881623000 & 1.485443000 & 0.642306000 \\
\hline $\mathrm{H}$ & -3.898475000 & 2.693358000 & -0.081266000 \\
\hline $\mathrm{H}$ & -5.830445000 & 1.500216000 & -1.092918000 \\
\hline $\mathrm{C}$ & -1.437782000 & -1.233934000 & 0.382429000 \\
\hline
\end{tabular}




\begin{tabular}{lrrr}
$\mathrm{C}$ & -0.523473000 & -0.834122000 & 1.276480000 \\
$\mathrm{H}$ & -0.610501000 & 0.162063000 & 1.722947000 \\
$\mathrm{O}$ & -1.286822000 & -2.465681000 & -0.220022000 \\
$\mathrm{C}$ & 0.715213000 & -1.636894000 & 1.601634000 \\
$\mathrm{H}$ & 0.558761000 & -2.694878000 & 1.372654000 \\
$\mathrm{C}$ & 1.897826000 & -1.111604000 & 0.812697000 \\
$\mathrm{C}$ & 2.017904000 & -1.402805000 & -0.551409000 \\
$\mathrm{C}$ & 2.827016000 & -0.234815000 & 1.378318000 \\
$\mathrm{C}$ & 3.026824000 & -0.829712000 & -1.321475000 \\
$\mathrm{H}$ & 1.294249000 & -2.073189000 & -1.008357000 \\
$\mathrm{C}$ & 3.833915000 & 0.342255000 & 0.605541000 \\
$\mathrm{H}$ & 2.742608000 & 0.023869000 & 2.430287000 \\
$\mathrm{C}$ & 3.954046000 & 0.055327000 & -0.757811000 \\
$\mathrm{H}$ & 3.101702000 & -1.078088000 & -2.378068000 \\
$\mathrm{H}$ & 4.537915000 & 1.027780000 & 1.072032000 \\
$\mathrm{O}$ & -0.342245000 & 2.304196000 & 1.488107000 \\
$\mathrm{H}$ & 0.929313000 & -1.557906000 & 2.672958000 \\
$\mathrm{H}$ & -1.816024000 & -2.474787000 & -1.026130000 \\
$\mathrm{H}$ & -0.795998000 & 2.665173000 & 2.254263000 \\
$\mathrm{~K}$ & 0.880156000 & 1.630751000 & -0.341182000 \\
$\mathrm{C}$ & 5.069814000 & 0.650791000 & -1.580589000 \\
$\mathrm{H}$ & 5.240053000 & 1.702468000 & -1.327291000 \\
$\mathrm{H}$ & 6.011218000 & 0.117991000 & -1.403415000 \\
$\mathrm{H}$ & 4.854993000 & 0.589208000 & -2.651838000 \\
\hline & & &
\end{tabular}

\begin{tabular}{|c|c|c|c|}
\hline \multicolumn{4}{|c|}{ Coordinates (Angstroms) } \\
\hline & $\mathrm{X}$ & Y & Z \\
\hline $\mathrm{C}$ & 2.860389000 & 2.814154000 & -0.747177000 \\
\hline $\mathrm{C}$ & 1.956798000 & 1.760983000 & -0.636383000 \\
\hline $\mathrm{C}$ & 2.315515000 & 0.595150000 & 0.045457000 \\
\hline $\mathrm{C}$ & 3.582459000 & 0.489727000 & 0.629067000 \\
\hline $\mathrm{C}$ & 4.480072000 & 1.548867000 & 0.516374000 \\
\hline $\mathrm{C}$ & 4.124213000 & 2.707489000 & -0.170886000 \\
\hline $\mathrm{H}$ & 2.576585000 & 3.719959000 & -1.275632000 \\
\hline $\mathrm{H}$ & 0.962667000 & 1.849480000 & -1.071361000 \\
\hline $\mathrm{H}$ & 3.824625000 & -0.442773000 & 1.157512000 \\
\hline $\mathrm{H}$ & 5.462488000 & 1.470740000 & 0.974223000 \\
\hline $\mathrm{H}$ & 4.828878000 & 3.530551000 & -0.252481000 \\
\hline $\mathrm{C}$ & 1.379970000 & -0.598705000 & 0.105993000 \\
\hline $\mathrm{C}$ & -0.054495000 & -0.218161000 & 0.327603000 \\
\hline $\mathrm{H}$ & -0.213010000 & 0.455536000 & 1.168372000 \\
\hline $\mathrm{O}$ & 1.538175000 & -1.392371000 & -1.100565000 \\
\hline
\end{tabular}




$\begin{array}{lrrr}\mathrm{C} & -1.087834000 & -0.654800000 & -0.396623000 \\ \mathrm{H} & -0.891847000 & -1.372714000 & -1.191755000 \\ \mathrm{C} & -2.501764000 & -0.298446000 & -0.189768000 \\ \mathrm{C} & -3.502875000 & -1.138551000 & -0.689557000 \\ \mathrm{C} & -2.901497000 & 0.860200000 & 0.486622000 \\ \mathrm{C} & -4.847870000 & -0.849540000 & -0.495843000 \\ \mathrm{H} & -3.220418000 & -2.038960000 & -1.230124000 \\ \mathrm{C} & -4.246684000 & 1.146134000 & 0.678459000 \\ \mathrm{H} & -2.152781000 & 1.558287000 & 0.850585000 \\ \mathrm{C} & -5.244859000 & 0.294937000 & 0.197911000 \\ \mathrm{H} & -5.602844000 & -1.525826000 & -0.890074000 \\ \mathrm{H} & -4.528166000 & 2.055605000 & 1.204117000 \\ \mathrm{H} & 1.742863000 & -1.266981000 & 0.905994000 \\ \mathrm{H} & 1.384592000 & -0.799372000 & -1.848285000 \\ \mathrm{O} & 3.424169000 & -2.419519000 & 1.453090000 \\ \mathrm{H} & 3.593817000 & -2.784030000 & 2.323519000 \\ \mathrm{C} & -6.703478000 & 0.592306000 & 0.437140000 \\ \mathrm{H} & -7.324297000 & 0.246641000 & -0.395538000 \\ \mathrm{H} & -7.062281000 & 0.090208000 & 1.343846000 \\ \mathrm{H} & -6.876401000 & 1.665112000 & 0.566689000 \\ \mathrm{Na} & 3.286219000 & -2.747892000 & -0.522740000 \\ ----------~\end{array}$

TSA1-2 ${ }_{\mathrm{NaOH}}$

\begin{tabular}{lrrr} 
& \multicolumn{3}{c}{ Coordinates (Angstroms) } \\
& $\mathrm{X}$ & $\mathrm{Y}$ & $\mathrm{Z}$ \\
$\mathrm{C}$ & -3.825003000 & -2.525501000 & 0.236794000 \\
$\mathrm{C}$ & -2.683375000 & -1.730980000 & 0.179187000 \\
$\mathrm{C}$ & -2.771696000 & -0.359193000 & -0.090837000 \\
$\mathrm{C}$ & -4.045797000 & 0.181220000 & -0.324149000 \\
$\mathrm{C}$ & -5.182900000 & -0.615310000 & -0.281462000 \\
$\mathrm{C}$ & -5.081283000 & -1.974959000 & 0.005997000 \\
$\mathrm{H}$ & -3.725768000 & -3.586758000 & 0.448095000 \\
$\mathrm{H}$ & -1.712587000 & -2.192789000 & 0.332716000 \\
$\mathrm{H}$ & -4.133181000 & 1.242452000 & -0.537707000 \\
$\mathrm{H}$ & -6.156829000 & -0.169687000 & -0.465570000 \\
$\mathrm{H}$ & -5.970439000 & -2.597385000 & 0.043829000 \\
$\mathrm{C}$ & -1.591371000 & 0.549063000 & -0.094346000 \\
$\mathrm{C}$ & -0.245245000 & 0.000101000 & 0.033333000 \\
$\mathrm{H}$ & -0.118374000 & -0.703951000 & 0.855221000 \\
$\mathrm{O}$ & -1.673160000 & 1.553653000 & -1.133810000 \\
$\mathrm{C}$ & 0.854395000 & 0.399547000 & -0.649284000 \\
$\mathrm{H}$ & 0.717501000 & 1.022334000 & -1.536474000 \\
$\mathrm{C}$ & 2.241781000 & 0.006757000 & -0.364677000
\end{tabular}




\begin{tabular}{lrrr}
$\mathrm{C}$ & 3.221844000 & 0.137535000 & -1.356092000 \\
$\mathrm{C}$ & 2.652451000 & -0.482856000 & 0.885393000 \\
$\mathrm{C}$ & 4.544389000 & -0.221488000 & -1.119479000 \\
$\mathrm{H}$ & 2.939246000 & 0.517380000 & -2.335928000 \\
$\mathrm{C}$ & 3.971433000 & -0.846422000 & 1.114180000 \\
$\mathrm{H}$ & 1.932643000 & -0.561085000 & 1.695701000 \\
$\mathrm{C}$ & 4.945055000 & -0.724973000 & 0.117634000 \\
$\mathrm{H}$ & 5.278367000 & -0.109711000 & -1.914336000 \\
$\mathrm{H}$ & 4.256381000 & -1.221118000 & 2.095013000 \\
$\mathrm{H}$ & -1.667168000 & 1.364005000 & 0.997170000 \\
$\mathrm{H}$ & -1.658726000 & 1.107014000 & -1.992494000 \\
$\mathrm{O}$ & -1.507429000 & 2.350806000 & 1.782258000 \\
$\mathrm{H}$ & -2.392769000 & 2.658131000 & 2.002841000 \\
$\mathrm{C}$ & 6.374413000 & -1.124949000 & 0.383265000 \\
$\mathrm{H}$ & 7.029441000 & -0.840923000 & -0.445913000 \\
$\mathrm{H}$ & 6.758979000 & -0.649330000 & 1.292379000 \\
$\mathrm{H}$ & 6.464438000 & -2.208831000 & 0.521799000 \\
$\mathrm{Na}$ & -0.242817000 & 2.880821000 & 0.154850000 \\
\hline- - & \multicolumn{1}{c}{ - } &
\end{tabular}

A2 ${ }_{\mathrm{NaOH}}$

\begin{tabular}{lrrr} 
& \multicolumn{3}{c}{ Coordinates (Angstroms) } \\
\hline $\mathrm{C}$ & 3.952439000 & 2.178115000 & 0.556075000 \\
$\mathrm{C}$ & 2.764315000 & 1.618900000 & 0.122089000 \\
$\mathrm{C}$ & 2.730579000 & 0.345999000 & -0.507927000 \\
$\mathrm{C}$ & 3.977337000 & -0.318541000 & -0.649810000 \\
$\mathrm{C}$ & 5.164169000 & 0.257054000 & -0.198246000 \\
$\mathrm{C}$ & 5.170356000 & 1.505148000 & 0.410773000 \\
$\mathrm{H}$ & 3.930872000 & 3.159112000 & 1.023799000 \\
$\mathrm{H}$ & 1.842475000 & 2.175475000 & 0.260999000 \\
$\mathrm{H}$ & 4.007126000 & -1.270874000 & -1.169977000 \\
$\mathrm{H}$ & 6.098016000 & -0.280979000 & -0.342514000 \\
$\mathrm{H}$ & 6.095662000 & 1.952211000 & 0.759187000 \\
$\mathrm{C}$ & 1.505963000 & -0.307203000 & -0.890729000 \\
$\mathrm{C}$ & 0.208016000 & 0.098374000 & -0.611713000 \\
$\mathrm{H}$ & 0.099119000 & 1.139576000 & -0.315817000 \\
$\mathrm{O}$ & 1.696170000 & -1.677942000 & -1.232235000 \\
$\mathrm{C}$ & -0.943046000 & -0.683354000 & -0.630001000 \\
$\mathrm{H}$ & -0.873035000 & -1.715843000 & -0.974817000 \\
$\mathrm{C}$ & -2.302960000 & -0.198813000 & -0.413078000 \\
$\mathrm{C}$ & -3.372950000 & -1.115752000 & -0.405417000 \\
$\mathrm{C}$ & -2.637001000 & 1.147333000 & -0.181882000 \\
$\mathrm{C}$ & -4.679678000 & -0.716438000 & -0.172797000
\end{tabular}




\begin{tabular}{|c|c|c|c|}
\hline $\mathrm{H}$ & -3.163121000 & -2.168606000 & -0.584974000 \\
\hline $\mathrm{C}$ & -3.949486000 & 1.540028000 & 0.055406000 \\
\hline $\mathrm{H}$ & -1.864572000 & 1.911379000 & -0.200538000 \\
\hline $\mathrm{C}$ & -5.000165000 & 0.623100000 & 0.068288000 \\
\hline $\mathrm{H}$ & -5.471850000 & -1.462895000 & -0.178063000 \\
\hline $\mathrm{H}$ & -4.160177000 & 2.593970000 & 0.226801000 \\
\hline $\mathrm{H}$ & 1.063009000 & -1.900852000 & -1.925222000 \\
\hline $\mathrm{O}$ & 0.037477000 & -1.763872000 & 1.945677000 \\
\hline $\mathrm{H}$ & -0.344951000 & -1.319710000 & 2.710267000 \\
\hline $\mathrm{H}$ & -0.435277000 & -1.373342000 & 1.159428000 \\
\hline $\mathrm{C}$ & -6.424227000 & 1.047506000 & 0.326157000 \\
\hline $\mathrm{H}$ & -7.085027000 & 0.760251000 & -0.500379000 \\
\hline $\mathrm{H}$ & -6.824556000 & 0.582443000 & 1.235329000 \\
\hline $\mathrm{H}$ & -6.500507000 & 2.132466000 & 0.449520000 \\
\hline $\mathrm{Na}$ & 2.066878000 & -1.773456000 & 1.036028000 \\
\hline
\end{tabular}

TSA2-3 $\mathrm{NaOH}$

\begin{tabular}{lrrr} 
& \multicolumn{3}{c}{ Coordinates (Angstroms) } \\
& $\mathrm{X}$ & $\mathrm{Y}$ & $\mathrm{Z}$ \\
\hline $\mathrm{C}$ & -3.869117000 & -2.422228000 & 0.170988000 \\
$\mathrm{C}$ & -2.717098000 & -1.745637000 & -0.196825000 \\
$\mathrm{C}$ & -2.742534000 & -0.362916000 & -0.460170000 \\
$\mathrm{C}$ & -3.981598000 & 0.297474000 & -0.368672000 \\
$\mathrm{C}$ & -5.135645000 & -0.390991000 & 0.002313000 \\
$\mathrm{C}$ & -5.087942000 & -1.750604000 & 0.282563000 \\
$\mathrm{H}$ & -3.818216000 & -3.489798000 & 0.365070000 \\
$\mathrm{H}$ & -1.788172000 & -2.297724000 & -0.297042000 \\
$\mathrm{H}$ & -4.042916000 & 1.350163000 & -0.626462000 \\
$\mathrm{H}$ & -6.079799000 & 0.143768000 & 0.058346000 \\
$\mathrm{H}$ & -5.987674000 & -2.287371000 & 0.566526000 \\
$\mathrm{C}$ & -1.524192000 & 0.397748000 & -0.751516000 \\
$\mathrm{C}$ & -0.242461000 & -0.003337000 & -0.577550000 \\
$\mathrm{H}$ & -0.085598000 & -1.032971000 & -0.261966000 \\
$\mathrm{O}$ & -1.798300000 & 1.746306000 & -1.027689000 \\
$\mathrm{C}$ & 0.917844000 & 0.875530000 & -0.659647000 \\
$\mathrm{H}$ & 0.928645000 & 1.506022000 & -1.559244000 \\
$\mathrm{C}$ & 2.247251000 & 0.277240000 & -0.402496000 \\
$\mathrm{C}$ & 3.373772000 & 0.641732000 & -1.154952000 \\
$\mathrm{C}$ & 2.456595000 & -0.638690000 & 0.639704000 \\
$\mathrm{C}$ & 4.629238000 & 0.109720000 & -0.891602000 \\
$\mathrm{H}$ & 3.255754000 & 1.349744000 & -1.972554000 \\
$\mathrm{C}$ & 3.712204000 & -1.180942000 & 0.891495000 \\
$\mathrm{H}$ & 1.620974000 & -0.928505000 & 1.274043000
\end{tabular}




\begin{tabular}{lrrr}
$\mathrm{C}$ & 4.826074000 & -0.818449000 & 0.133893000 \\
$\mathrm{H}$ & 5.474769000 & 0.412670000 & -1.506496000 \\
$\mathrm{H}$ & 3.828462000 & -1.898302000 & 1.701874000 \\
$\mathrm{H}$ & -0.948854000 & 2.192042000 & -1.163210000 \\
$\mathrm{O}$ & 0.224054000 & 2.498677000 & 1.290125000 \\
$\mathrm{H}$ & 0.978592000 & 2.704749000 & 1.849985000 \\
$\mathrm{H}$ & 0.648206000 & 1.791852000 & 0.412657000 \\
$\mathrm{C}$ & 6.194329000 & -1.384375000 & 0.423332000 \\
$\mathrm{H}$ & 6.757683000 & -1.555956000 & -0.500219000 \\
$\mathrm{H}$ & 6.788653000 & -0.703341000 & 1.045550000 \\
$\mathrm{H}$ & 6.126216000 & -2.338635000 & 0.955777000 \\
$\mathrm{Na}$ & -1.763916000 & 1.792190000 & 1.370248000 \\
\hline
\end{tabular}

\begin{tabular}{|c|c|c|c|}
\hline \multicolumn{4}{|c|}{$\mathrm{A3}$ NaOH } \\
\hline & \multicolumn{3}{|c|}{ Coordinates (Angstroms) } \\
\hline & $X$ & $\mathrm{Y}$ & $\mathrm{Z}$ \\
\hline$\overline{\mathrm{C}}$ & -4.898532000 & -0.323368000 & -0.818368000 \\
\hline $\mathrm{C}$ & -3.745497000 & -1.026898000 & -0.484881000 \\
\hline $\mathrm{C}$ & -2.606776000 & -0.356760000 & -0.017357000 \\
\hline $\mathrm{C}$ & -2.644583000 & 1.038099000 & 0.133013000 \\
\hline $\mathrm{C}$ & -3.805198000 & 1.728048000 & -0.202245000 \\
\hline $\mathrm{C}$ & -4.929657000 & 1.060349000 & -0.683557000 \\
\hline $\mathrm{H}$ & -5.774347000 & -0.860173000 & -1.171352000 \\
\hline $\mathrm{H}$ & -3.758739000 & -2.113020000 & -0.548491000 \\
\hline $\mathrm{H}$ & -1.770669000 & 1.591279000 & 0.523290000 \\
\hline $\mathrm{H}$ & -3.827201000 & 2.808123000 & -0.083933000 \\
\hline $\mathrm{H}$ & -5.827963000 & 1.613451000 & -0.943155000 \\
\hline $\mathrm{C}$ & -1.385551000 & -1.134852000 & 0.317777000 \\
\hline $\mathrm{C}$ & -0.472902000 & -0.777494000 & 1.232293000 \\
\hline $\mathrm{H}$ & -0.581741000 & 0.184033000 & 1.734428000 \\
\hline $\mathrm{O}$ & -1.230245000 & -2.332962000 & -0.341777000 \\
\hline $\mathrm{C}$ & 0.763698000 & -1.595733000 & 1.529372000 \\
\hline $\mathrm{H}$ & 0.603558000 & -2.642156000 & 1.254371000 \\
\hline $\mathrm{C}$ & 1.947517000 & -1.036871000 & 0.766536000 \\
\hline $\mathrm{C}$ & 2.068726000 & -1.267626000 & -0.610882000 \\
\hline $\mathrm{C}$ & 2.859700000 & -0.165469000 & 1.366612000 \\
\hline $\mathrm{C}$ & 3.055930000 & -0.631238000 & -1.359553000 \\
\hline $\mathrm{H}$ & 1.358784000 & -1.933567000 & -1.094727000 \\
\hline $\mathrm{C}$ & 3.846315000 & 0.472249000 & 0.614737000 \\
\hline $\mathrm{H}$ & 2.771821000 & 0.048915000 & 2.427910000 \\
\hline $\mathrm{C}$ & 3.962231000 & 0.254028000 & -0.760921000 \\
\hline $\mathrm{H}$ & 3.129879000 & -0.828432000 & -2.426720000 \\
\hline $\mathrm{H}$ & 4.531124000 & 1.157948000 & 1.107395000 \\
\hline
\end{tabular}




$\begin{array}{lrrr}\mathrm{O} & -0.133314000 & 2.370036000 & 1.180177000 \\ \mathrm{H} & 0.973598000 & -1.563896000 & 2.603763000 \\ \mathrm{H} & -1.805101000 & -2.328171000 & -1.116378000 \\ \mathrm{H} & -0.306919000 & 3.249190000 & 1.523734000 \\ \mathrm{C} & 5.051391000 & 0.919053000 & -1.564566000 \\ \mathrm{H} & 5.305036000 & 1.901743000 & -1.155701000 \\ \mathrm{H} & 5.965240000 & 0.313314000 & -1.554485000 \\ \mathrm{H} & 4.757199000 & 1.050334000 & -2.610390000 \\ \mathrm{Na} & 0.978549000 & 1.374672000 & -0.174983000\end{array}$

\begin{tabular}{|c|c|c|c|}
\hline \multicolumn{4}{|c|}{ A1 $1_{\text {LiOH }}$} \\
\hline & $X$ & $\mathrm{Y}$ & Z \\
\hline$\overline{\mathrm{C}}$ & 3.083545000 & -2.577995000 & 0.620501000 \\
\hline $\mathrm{C}$ & 2.147105000 & -1.556832000 & 0.481964000 \\
\hline $\mathrm{C}$ & 2.524827000 & -0.328308000 & -0.064771000 \\
\hline $\mathrm{C}$ & 3.846423000 & -0.123681000 & -0.476183000 \\
\hline $\mathrm{C}$ & 4.776491000 & -1.149255000 & -0.335691000 \\
\hline $\mathrm{C}$ & 4.398590000 & -2.374358000 & 0.211339000 \\
\hline $\mathrm{H}$ & 2.785007000 & -3.532662000 & 1.043915000 \\
\hline $\mathrm{H}$ & 1.116054000 & -1.717015000 & 0.789455000 \\
\hline $\mathrm{H}$ & 4.114236000 & 0.854942000 & -0.886121000 \\
\hline $\mathrm{H}$ & 5.802127000 & -0.992174000 & -0.657290000 \\
\hline $\mathrm{H}$ & 5.129133000 & -3.171614000 & 0.316027000 \\
\hline $\mathrm{C}$ & 1.547887000 & 0.828563000 & -0.148649000 \\
\hline $\mathrm{C}$ & 0.127700000 & 0.408592000 & -0.390188000 \\
\hline $\mathrm{H}$ & -0.007290000 & -0.187622000 & -1.291097000 \\
\hline $\mathrm{O}$ & 1.666104000 & 1.635614000 & 1.058276000 \\
\hline $\mathrm{C}$ & -0.910589000 & 0.721313000 & 0.387751000 \\
\hline $\mathrm{H}$ & -0.728009000 & 1.357510000 & 1.252300000 \\
\hline $\mathrm{C}$ & -2.313007000 & 0.325264000 & 0.176877000 \\
\hline $\mathrm{C}$ & -3.321928000 & 0.984239000 & 0.886239000 \\
\hline $\mathrm{C}$ & -2.696590000 & -0.696116000 & -0.702035000 \\
\hline $\mathrm{C}$ & -4.661007000 & 0.655523000 & 0.709034000 \\
\hline $\mathrm{H}$ & -3.052269000 & 1.772542000 & 1.585108000 \\
\hline $\mathrm{C}$ & -4.033690000 & -1.021161000 & -0.877246000 \\
\hline $\mathrm{H}$ & -1.941167000 & -1.256118000 & -1.245994000 \\
\hline $\mathrm{C}$ & -5.042608000 & -0.348975000 & -0.180014000 \\
\hline $\mathrm{H}$ & -5.421882000 & 1.189531000 & 1.273209000 \\
\hline $\mathrm{H}$ & -4.302338000 & -1.819541000 & -1.565074000 \\
\hline $\mathrm{H}$ & 1.889848000 & 1.508237000 & -0.940488000 \\
\hline $\mathrm{H}$ & 1.704603000 & 1.027561000 & 1.808117000 \\
\hline $\mathrm{O}$ & 3.654422000 & 2.938340000 & -0.994623000 \\
\hline
\end{tabular}




\begin{tabular}{lrrr}
$\mathrm{H}$ & 4.091787000 & 3.503579000 & -1.630839000 \\
$\mathrm{C}$ & -6.494181000 & -0.690319000 & -0.400946000 \\
$\mathrm{H}$ & -7.113413000 & -0.370104000 & 0.442443000 \\
$\mathrm{H}$ & -6.880422000 & -0.196118000 & -1.300545000 \\
$\mathrm{H}$ & -6.635169000 & -1.767629000 & -0.535267000 \\
$\mathrm{Li}$ & 3.003715000 & 2.955368000 & 0.548343000 \\
\hline
\end{tabular}

TSA1-2

\begin{tabular}{|c|c|c|c|}
\hline & & inates (Angstrom & \\
\hline & $\mathrm{X}$ & $\mathrm{Y}$ & Z \\
\hline$\overline{\mathrm{C}}$ & -3.919389000 & -2.327189000 & 0.104543000 \\
\hline $\mathrm{C}$ & -2.759495000 & -1.557949000 & 0.105696000 \\
\hline $\mathrm{C}$ & -2.815291000 & -0.167990000 & -0.066386000 \\
\hline $\mathrm{C}$ & -4.074931000 & 0.418343000 & -0.264321000 \\
\hline $\mathrm{C}$ & -5.229234000 & -0.353878000 & -0.283350000 \\
\hline $\mathrm{C}$ & -5.161170000 & -1.732515000 & -0.092451000 \\
\hline $\mathrm{H}$ & -3.845478000 & -3.402544000 & 0.241960000 \\
\hline $\mathrm{H}$ & -1.800707000 & -2.053533000 & 0.226688000 \\
\hline $\mathrm{H}$ & -4.137969000 & 1.493630000 & -0.398860000 \\
\hline $\mathrm{H}$ & -6.191472000 & 0.126192000 & -0.439506000 \\
\hline $\mathrm{H}$ & -6.064842000 & -2.334709000 & -0.102132000 \\
\hline $\mathrm{C}$ & -1.612291000 & 0.698010000 & -0.005671000 \\
\hline $\mathrm{C}$ & -0.273514000 & 0.140392000 & 0.055097000 \\
\hline $\mathrm{H}$ & -0.145439000 & -0.670188000 & 0.772115000 \\
\hline $\mathrm{O}$ & -1.671239000 & 1.821609000 & -0.926687000 \\
\hline $\mathrm{C}$ & 0.833286000 & 0.639389000 & -0.552940000 \\
\hline $\mathrm{H}$ & 0.706780000 & 1.365792000 & -1.360422000 \\
\hline $\mathrm{C}$ & 2.217779000 & 0.208323000 & -0.315532000 \\
\hline $\mathrm{C}$ & 3.204891000 & 0.473584000 & -1.273627000 \\
\hline $\mathrm{C}$ & 2.617234000 & -0.466049000 & 0.847777000 \\
\hline $\mathrm{C}$ & 4.521039000 & 0.068029000 & -1.088883000 \\
\hline $\mathrm{H}$ & 2.930596000 & 0.992760000 & -2.189732000 \\
\hline $\mathrm{C}$ & 3.931534000 & -0.876110000 & 1.024543000 \\
\hline $\mathrm{H}$ & 1.891991000 & -0.664912000 & 1.632307000 \\
\hline $\mathrm{C}$ & 4.911089000 & -0.617805000 & 0.062088000 \\
\hline $\mathrm{H}$ & 5.258696000 & 0.284816000 & -1.858175000 \\
\hline $\mathrm{H}$ & 4.205588000 & -1.400942000 & 1.937105000 \\
\hline $\mathrm{H}$ & -1.605851000 & 1.535046000 & 1.160964000 \\
\hline $\mathrm{H}$ & -1.594831000 & 1.489130000 & -1.833165000 \\
\hline $\mathrm{O}$ & -1.337502000 & 2.552963000 & 1.761551000 \\
\hline $\mathrm{H}$ & -0.979840000 & 2.287178000 & 2.615363000 \\
\hline $\mathrm{Li}$ & -0.378863000 & 2.771422000 & 0.231432000 \\
\hline $\mathrm{C}$ & 6.343824000 & -1.035121000 & 0.278337000 \\
\hline
\end{tabular}




$\begin{array}{llll}\mathrm{H} & 6.876475000 & -1.135802000 & -0.672362000 \\ \mathrm{H} & 6.885166000 & -0.297075000 & 0.882880000 \\ \mathrm{H} & 6.405475000 & -1.994468000 & 0.802246000\end{array}$

\begin{tabular}{|c|c|c|c|}
\hline \multicolumn{4}{|c|}{ A2 ${ }_{\text {LiOH }}$} \\
\hline & $\mathrm{X}$ & $\mathrm{Y}$ & Z \\
\hline$\overline{\mathrm{C}}$ & 4.088730000 & 2.118866000 & 0.203986000 \\
\hline $\mathrm{C}$ & 2.905295000 & 1.509913000 & -0.168807000 \\
\hline $\mathrm{C}$ & 2.848784000 & 0.120190000 & -0.449066000 \\
\hline $\mathrm{C}$ & 4.061419000 & -0.603806000 & -0.326996000 \\
\hline $\mathrm{C}$ & 5.245127000 & 0.027344000 & 0.057134000 \\
\hline $\mathrm{C}$ & 5.274737000 & 1.386939000 & 0.330852000 \\
\hline $\mathrm{H}$ & 4.091472000 & 3.188095000 & 0.399035000 \\
\hline $\mathrm{H}$ & 2.009792000 & 2.116234000 & -0.264217000 \\
\hline $\mathrm{H}$ & 4.080030000 & -1.655258000 & -0.596183000 \\
\hline $\mathrm{H}$ & 6.156229000 & -0.561410000 & 0.126386000 \\
\hline $\mathrm{H}$ & 6.197525000 & 1.876022000 & 0.625205000 \\
\hline $\mathrm{C}$ & 1.614324000 & -0.567148000 & -0.744415000 \\
\hline $\mathrm{C}$ & 0.317593000 & -0.105393000 & -0.555337000 \\
\hline $\mathrm{H}$ & 0.226789000 & 0.971613000 & -0.435331000 \\
\hline $\mathrm{O}$ & 1.796537000 & -1.986002000 & -0.801599000 \\
\hline $\mathrm{C}$ & -0.845720000 & -0.859692000 & -0.441836000 \\
\hline $\mathrm{H}$ & -0.811755000 & -1.927384000 & -0.665956000 \\
\hline $\mathrm{C}$ & -2.196252000 & -0.317345000 & -0.294747000 \\
\hline $\mathrm{C}$ & -3.301294000 & -1.187862000 & -0.344206000 \\
\hline $\mathrm{C}$ & -2.482133000 & 1.040330000 & -0.073625000 \\
\hline $\mathrm{C}$ & -4.600856000 & -0.731763000 & -0.184622000 \\
\hline $\mathrm{H}$ & -3.127755000 & -2.248671000 & -0.514194000 \\
\hline $\mathrm{C}$ & -3.787569000 & 1.491638000 & 0.082948000 \\
\hline $\mathrm{H}$ & -1.676617000 & 1.768265000 & -0.022803000 \\
\hline $\mathrm{C}$ & -4.876126000 & 0.621117000 & 0.033106000 \\
\hline $\mathrm{H}$ & -5.422461000 & -1.443990000 & -0.232471000 \\
\hline $\mathrm{H}$ & -3.961590000 & 2.552930000 & 0.248848000 \\
\hline $\mathrm{H}$ & 1.098495000 & -2.359763000 & -1.352256000 \\
\hline $\mathrm{O}$ & 0.380268000 & -1.648337000 & 2.108594000 \\
\hline $\mathrm{H}$ & 0.178166000 & -1.072126000 & 2.855170000 \\
\hline $\mathrm{H}$ & -0.225900000 & -1.349968000 & 1.368358000 \\
\hline $\mathrm{C}$ & -6.292860000 & 1.109225000 & 0.203864000 \\
\hline $\mathrm{H}$ & -6.904509000 & 0.877802000 & -0.676289000 \\
\hline $\mathrm{H}$ & -6.777921000 & 0.639100000 & 1.067729000 \\
\hline $\mathrm{H}$ & -6.325396000 & 2.192792000 & 0.354676000 \\
\hline $\mathrm{Li}$ & 1.980589000 & -1.601633000 & 1.096313000 \\
\hline
\end{tabular}


TSA2-3 ${ }_{\mathrm{LiOH}}$

\begin{tabular}{lccc} 
& Coordinates (Angstroms) & \\
& $\mathrm{X}$ & $\mathrm{Y}$ & $\mathrm{Z}$ \\
\hline $\mathrm{C}$ & -4.106519000 & -2.126938000 & 0.467111000 \\
$\mathrm{C}$ & -2.918526000 & -1.419760000 & 0.350043000 \\
$\mathrm{C}$ & -2.892820000 & -0.143989000 & -0.240498000 \\
$\mathrm{C}$ & -4.108078000 & 0.400295000 & -0.683844000 \\
$\mathrm{C}$ & -5.297181000 & -0.310460000 & -0.555578000 \\
$\mathrm{C}$ & -5.306097000 & -1.577926000 & 0.016598000 \\
$\mathrm{H}$ & -4.097040000 & -3.111490000 & 0.925822000 \\
$\mathrm{H}$ & -2.001421000 & -1.859930000 & 0.730607000 \\
$\mathrm{H}$ & -4.111306000 & 1.382658000 & -1.142895000 \\
$\mathrm{H}$ & -6.222660000 & 0.132099000 & -0.912978000 \\
$\mathrm{H}$ & -6.235375000 & -2.130131000 & 0.116656000 \\
$\mathrm{C}$ & -1.646742000 & 0.618952000 & -0.363381000 \\
$\mathrm{C}$ & -0.364977000 & 0.186390000 & -0.236721000 \\
$\mathrm{H}$ & -0.241496000 & -0.881076000 & -0.067306000 \\
$\mathrm{O}$ & -1.871608000 & 2.011414000 & -0.557974000 \\
$\mathrm{C}$ & 0.824484000 & 1.007853000 & -0.233344000 \\
$\mathrm{H}$ & 0.797473000 & 1.866575000 & -0.916681000 \\
$\mathrm{C}$ & 2.151853000 & 0.355207000 & -0.218203000 \\
$\mathrm{C}$ & 3.240319000 & 0.922678000 & -0.894348000 \\
$\mathrm{C}$ & 2.407047000 & -0.814751000 & 0.515506000 \\
$\mathrm{C}$ & 4.506178000 & 0.349759000 & -0.849216000 \\
$\mathrm{H}$ & 3.088164000 & 1.831749000 & -1.472350000 \\
$\mathrm{C}$ & 3.669553000 & -1.392544000 & 0.546098000 \\
$\mathrm{H}$ & 1.605167000 & -1.280622000 & 1.084733000 \\
$\mathrm{C}$ & 4.748724000 & -0.822547000 & -0.133321000 \\
$\mathrm{H}$ & 5.322580000 & 0.822442000 & -1.391620000 \\
$\mathrm{H}$ & 3.822202000 & -2.305513000 & 1.118698000 \\
$\mathrm{H}$ & -1.182179000 & 2.355348000 & -1.142947000 \\
$\mathrm{O}$ & 0.225310000 & 2.386359000 & 1.920766000 \\
$\mathrm{H}$ & 0.694031000 & 2.106014000 & 2.713825000 \\
$\mathrm{H}$ & 0.647914000 & 1.759397000 & 1.024994000 \\
$\mathrm{C}$ & 6.122314000 & -1.442686000 & -0.068708000 \\
$\mathrm{H}$ & 6.785053000 & -1.024590000 & -0.832987000 \\
$\mathrm{H}$ & 6.594470000 & -1.270468000 & 0.906468000 \\
$\mathrm{H}$ & 6.078583000 & -2.526859000 & -0.221026000 \\
$\mathrm{H}$ & -1.450326000 & 2.071668000 & 1.355826000 \\
\hline
\end{tabular}

A3 $3_{\text {LiOH }}$ 


\begin{tabular}{lccc} 
& \multicolumn{3}{c}{ Coordinates (Angstroms) } \\
& $\mathrm{X}$ & $\mathrm{Y}$ & $\mathrm{Z}$ \\
\hline $\mathrm{C}$ & -4.905869000 & -0.053379000 & -0.501407000 \\
$\mathrm{C}$ & -3.758941000 & -0.833717000 & -0.411056000 \\
$\mathrm{C}$ & -2.537636000 & -0.259593000 & -0.031956000 \\
$\mathrm{C}$ & -2.469915000 & 1.110250000 & 0.248500000 \\
$\mathrm{C}$ & -3.626903000 & 1.879725000 & 0.154148000 \\
$\mathrm{C}$ & -4.840783000 & 1.308428000 & -0.218425000 \\
$\mathrm{H}$ & -5.849863000 & -0.510778000 & -0.782879000 \\
$\mathrm{H}$ & -3.822753000 & -1.903476000 & -0.599001000 \\
$\mathrm{H}$ & -1.512641000 & 1.587379000 & 0.501636000 \\
$\mathrm{H}$ & -3.569471000 & 2.944047000 & 0.363673000 \\
$\mathrm{H}$ & -5.734978000 & 1.921235000 & -0.289628000 \\
$\mathrm{C}$ & -1.334709000 & -1.123814000 & 0.058145000 \\
$\mathrm{C}$ & -0.435849000 & -1.080879000 & 1.051764000 \\
$\mathrm{H}$ & -0.582752000 & -0.347875000 & 1.839486000 \\
$\mathrm{O}$ & -1.195037000 & -2.066361000 & -0.928231000 \\
$\mathrm{C}$ & 0.786584000 & -1.968640000 & 1.115510000 \\
$\mathrm{H}$ & 0.619644000 & -2.883678000 & 0.540600000 \\
$\mathrm{C}$ & 1.975100000 & -1.211705000 & 0.562690000 \\
$\mathrm{C}$ & 2.161706000 & -1.110306000 & -0.823697000 \\
$\mathrm{C}$ & 2.791295000 & -0.439399000 & 1.393355000 \\
$\mathrm{C}$ & 3.114703000 & -0.244005000 & -1.353066000 \\
$\mathrm{H}$ & 1.519902000 & -1.686653000 & -1.484298000 \\
$\mathrm{C}$ & 3.746170000 & 0.425431000 & 0.858891000 \\
$\mathrm{H}$ & 2.644266000 & -0.475498000 & 2.469345000 \\
$\mathrm{C}$ & 3.920353000 & 0.545247000 & -0.521615000 \\
$\mathrm{H}$ & 3.227352000 & -0.167485000 & -2.431649000 \\
$\mathrm{H}$ & 4.343576000 & 1.037954000 & 1.528269000 \\
$\mathrm{O}$ & 0.311598000 & 2.374007000 & 0.649788000 \\
$\mathrm{H}$ & 0.980065000 & -2.259967000 & 2.153290000 \\
$\mathrm{H}$ & -1.808837000 & -1.846650000 & -1.640305000 \\
$\mathrm{H}$ & 0.377388000 & 3.327726000 & 0.603444000 \\
$\mathrm{H}$ & 4.947190000 & 1.483429000 & -1.101608000 \\
$\mathrm{H}$ & 5.195665000 & 2.282724000 & -0.398049000 \\
$\mathrm{H}$ & 5.873929000 & 0.947874000 & -1.338996000 \\
$\mathrm{H}$ & 4.585812000 & 1.944428000 & -2.025716000 \\
$\mathrm{H}$ & 1.128744000 & 1.021701000 & 0.112425000 \\
\hline
\end{tabular}

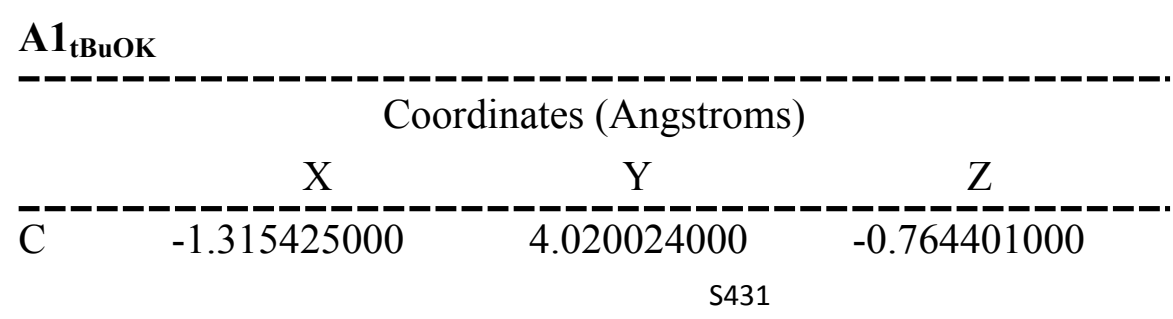




\begin{tabular}{|c|c|c|c|}
\hline $\mathrm{C}$ & -0.648063000 & 2.916305000 & -0.242165000 \\
\hline $\mathrm{C}$ & -1.289441000 & 1.677906000 & -0.157105000 \\
\hline $\mathrm{C}$ & -2.603747000 & 1.547946000 & -0.617229000 \\
\hline $\mathrm{C}$ & -3.266193000 & 2.655013000 & -1.143624000 \\
\hline $\mathrm{C}$ & -2.627764000 & 3.890690000 & -1.214718000 \\
\hline $\mathrm{H}$ & -0.808678000 & 4.978534000 & -0.832009000 \\
\hline $\mathrm{H}$ & 0.385803000 & 3.011771000 & 0.085786000 \\
\hline $\mathrm{H}$ & -3.071954000 & 0.558991000 & -0.567110000 \\
\hline $\mathrm{H}$ & -4.282732000 & 2.547311000 & -1.512672000 \\
\hline $\mathrm{H}$ & -3.145212000 & 4.750280000 & -1.631262000 \\
\hline $\mathrm{C}$ & -0.602008000 & 0.485362000 & 0.486527000 \\
\hline $\mathrm{C}$ & 0.811053000 & 0.300957000 & 0.016185000 \\
\hline $\mathrm{H}$ & 0.921618000 & 0.315560000 & -1.066803000 \\
\hline $\mathrm{O}$ & -0.689082000 & 0.596833000 & 1.924636000 \\
\hline $\mathrm{C}$ & 1.868046000 & 0.121807000 & 0.812189000 \\
\hline $\mathrm{H}$ & 1.701785000 & 0.115601000 & 1.888719000 \\
\hline $\mathrm{C}$ & 3.264538000 & -0.080917000 & 0.391023000 \\
\hline $\mathrm{C}$ & 4.281110000 & -0.020396000 & 1.350595000 \\
\hline $\mathrm{C}$ & 3.637884000 & -0.338257000 & -0.934042000 \\
\hline $\mathrm{C}$ & 5.614776000 & -0.196765000 & 1.002646000 \\
\hline $\mathrm{H}$ & 4.021580000 & 0.172961000 & 2.389020000 \\
\hline $\mathrm{C}$ & 4.970935000 & -0.514130000 & -1.278639000 \\
\hline $\mathrm{H}$ & 2.879413000 & -0.414793000 & -1.707381000 \\
\hline $\mathrm{C}$ & 5.984695000 & -0.448292000 & -0.319203000 \\
\hline $\mathrm{H}$ & 6.381183000 & -0.140026000 & 1.772079000 \\
\hline $\mathrm{H}$ & 5.229231000 & -0.713061000 & -2.316068000 \\
\hline $\mathrm{H}$ & -1.202940000 & -0.410505000 & 0.251623000 \\
\hline $\mathrm{H}$ & -0.256946000 & 1.426617000 & 2.168694000 \\
\hline $\mathrm{C}$ & -4.308980000 & -3.073107000 & -1.023561000 \\
\hline $\mathrm{C}$ & -2.929291000 & -2.423835000 & -0.782648000 \\
\hline $\mathrm{H}$ & -4.676413000 & -3.515634000 & -0.088921000 \\
\hline $\mathrm{H}$ & -5.024060000 & -2.302888000 & -1.336472000 \\
\hline $\mathrm{H}$ & -4.286026000 & -3.858429000 & -1.790944000 \\
\hline $\mathrm{C}$ & -2.424260000 & -1.839250000 & -2.121772000 \\
\hline $\mathrm{C}$ & -1.938251000 & -3.517162000 & -0.325459000 \\
\hline $\mathrm{H}$ & -1.442423000 & -1.369568000 & -1.979982000 \\
\hline $\mathrm{H}$ & -2.327354000 & -2.601378000 & -2.906317000 \\
\hline $\mathrm{H}$ & -3.116723000 & -1.066598000 & -2.476996000 \\
\hline $\mathrm{H}$ & -1.825760000 & -4.329343000 & -1.056256000 \\
\hline $\mathrm{H}$ & -0.951215000 & -3.069908000 & -0.152699000 \\
\hline $\mathrm{H}$ & -2.282933000 & -3.953250000 & 0.621126000 \\
\hline $\mathrm{O}$ & -3.024562000 & -1.442365000 & 0.172948000 \\
\hline $\mathrm{K}$ & -3.156476000 & -0.423461000 & 2.272021000 \\
\hline $\mathrm{C}$ & 7.427558000 & -0.678583000 & -0.691551000 \\
\hline
\end{tabular}




$\begin{array}{llll}\mathrm{H} & 8.099099000 & -0.064200000 & -0.083362000 \\ \mathrm{H} & 7.709446000 & -1.726877000 & -0.534882000 \\ \mathrm{H} & 7.611375000 & -0.443055000 & -1.744276000\end{array}$

TSA1-2 ${ }_{\text {tBuOK }}$

\begin{tabular}{lrcc} 
& Coordinates (Angstroms) & \\
& $\mathrm{X}$ & $\mathrm{Y}$ & $\mathrm{Z}$ \\
\hline $\mathrm{C}$ & -3.759171000 & -3.052045000 & -0.260004000 \\
$\mathrm{C}$ & -2.796629000 & -2.204670000 & -0.811219000 \\
$\mathrm{C}$ & -1.933066000 & -1.454251000 & 0.003165000 \\
$\mathrm{C}$ & -2.084101000 & -1.580234000 & 1.396647000 \\
$\mathrm{C}$ & -3.033203000 & -2.431925000 & 1.942006000 \\
$\mathrm{C}$ & -3.883786000 & -3.173173000 & 1.118697000 \\
$\mathrm{H}$ & -4.401944000 & -3.632290000 & -0.917690000 \\
$\mathrm{H}$ & -2.688341000 & -2.143165000 & -1.891632000 \\
$\mathrm{H}$ & -1.459823000 & -0.976282000 & 2.050395000 \\
$\mathrm{H}$ & -3.123966000 & -2.510652000 & 3.022042000 \\
$\mathrm{H}$ & -4.629061000 & -3.834744000 & 1.549661000 \\
$\mathrm{C}$ & -0.972788000 & -0.459219000 & -0.552963000 \\
$\mathrm{C}$ & 0.429518000 & -0.628684000 & -0.176266000 \\
$\mathrm{H}$ & 0.597456000 & -1.076163000 & 0.803074000 \\
$\mathrm{O}$ & -1.150381000 & -0.258760000 & -1.966315000 \\
$\mathrm{C}$ & 1.488326000 & -0.239729000 & -0.913262000 \\
$\mathrm{H}$ & 1.302049000 & 0.270182000 & -1.857651000 \\
$\mathrm{C}$ & 2.900202000 & -0.377763000 & -0.535432000 \\
$\mathrm{C}$ & 3.866867000 & 0.401485000 & -1.181883000 \\
$\mathrm{C}$ & 3.350224000 & -1.269337000 & 0.450767000 \\
$\mathrm{C}$ & 5.213977000 & 0.312239000 & -0.848296000 \\
$\mathrm{H}$ & 3.552658000 & 1.100094000 & -1.954084000 \\
$\mathrm{C}$ & 4.694053000 & -1.352210000 & 0.784144000 \\
$\mathrm{H}$ & 2.640317000 & -1.921621000 & 0.952083000 \\
$\mathrm{C}$ & 5.654608000 & -0.562976000 & 0.143257000 \\
$\mathrm{H}$ & 5.934950000 & 0.937808000 & -1.369989000 \\
$\mathrm{H}$ & 5.009173000 & -2.055884000 & 1.551979000 \\
$\mathrm{H}$ & -1.398020000 & 0.760444000 & -0.026441000 \\
$\mathrm{H}$ & -0.611943000 & -0.916267000 & -2.434269000 \\
$\mathrm{C}$ & -1.995932000 & 3.972558000 & 1.213652000 \\
$\mathrm{C}$ & -1.144881000 & 2.759273000 & 0.819398000 \\
$\mathrm{H}$ & -2.495337000 & 4.384762000 & 0.328088000 \\
$\mathrm{H}$ & -2.765101000 & 3.671465000 & 1.933529000 \\
$\mathrm{C}$ & -1.389044000 & 4.766914000 & 1.664590000 \\
-0.456915000 & 2.183567000 & 2.068503000 \\
& -0.081497000 & 3.181291000 & -0.207307000
\end{tabular}




$\begin{array}{lrrr}\mathrm{H} & 0.186733000 & 1.340925000 & 1.791966000 \\ \mathrm{H} & 0.167091000 & 2.931718000 & 2.571941000 \\ \mathrm{H} & -1.214093000 & 1.826816000 & 2.775745000 \\ \mathrm{H} & 0.597073000 & 3.943703000 & 0.194355000 \\ \mathrm{H} & 0.517081000 & 2.313796000 & -0.506839000 \\ \mathrm{H} & -0.567047000 & 3.590104000 & -1.102152000 \\ \mathrm{O} & -1.999624000 & 1.800206000 & 0.261136000 \\ \mathrm{~K} & -3.498164000 & 0.888257000 & -1.427606000 \\ \mathrm{C} & 7.112792000 & -0.670298000 & 0.512923000 \\ \mathrm{H} & 7.275176000 & -0.425833000 & 1.569137000 \\ \mathrm{H} & 7.490640000 & -1.687194000 & 0.354475000 \\ \mathrm{H} & 7.726904000 & 0.009509000 & -0.085528000\end{array}$

\begin{tabular}{|c|c|c|c|}
\hline \multicolumn{4}{|c|}{$\mathrm{A} 2_{\mathrm{tBuOK}}$} \\
\hline & \multicolumn{3}{|c|}{ Coordinates (Angstroms) } \\
\hline & $\mathrm{X}$ & Y & Z \\
\hline $\mathrm{C}$ & -5.298705000 & -0.748796000 & 0.322004000 \\
\hline $\mathrm{C}$ & -4.162272000 & -1.148791000 & -0.375664000 \\
\hline $\mathrm{C}$ & -2.877937000 & -1.135727000 & 0.228434000 \\
\hline $\mathrm{C}$ & -2.822166000 & -0.683308000 & 1.574877000 \\
\hline $\mathrm{C}$ & -3.964150000 & -0.298092000 & 2.257206000 \\
\hline $\mathrm{C}$ & -5.218896000 & -0.316341000 & 1.642136000 \\
\hline $\mathrm{H}$ & -6.263948000 & -0.789691000 & -0.177569000 \\
\hline $\mathrm{H}$ & -4.255764000 & -1.517778000 & -1.392050000 \\
\hline $\mathrm{H}$ & -1.866497000 & -0.635950000 & 2.088679000 \\
\hline $\mathrm{H}$ & -3.872781000 & 0.031372000 & 3.289398000 \\
\hline $\mathrm{H}$ & -6.107920000 & -0.008437000 & 2.183168000 \\
\hline $\mathrm{C}$ & -1.703615000 & -1.448348000 & -0.539693000 \\
\hline $\mathrm{C}$ & -0.383814000 & -1.371875000 & -0.118500000 \\
\hline $\mathrm{H}$ & -0.242840000 & -1.312717000 & 0.958787000 \\
\hline $\mathrm{O}$ & -1.938176000 & -1.525972000 & -1.937427000 \\
\hline $\mathrm{C}$ & 0.755670000 & -1.322763000 & -0.913423000 \\
\hline $\mathrm{H}$ & 0.642785000 & -1.356432000 & -1.996895000 \\
\hline $\mathrm{C}$ & 2.123222000 & -1.263710000 & -0.424405000 \\
\hline $\mathrm{C}$ & 3.175447000 & -0.978467000 & -1.318196000 \\
\hline $\mathrm{C}$ & 2.485913000 & -1.416005000 & 0.929953000 \\
\hline $\mathrm{C}$ & 4.484945000 & -0.824741000 & -0.886257000 \\
\hline $\mathrm{H}$ & 2.946485000 & -0.852721000 & -2.374662000 \\
\hline $\mathrm{C}$ & 3.797330000 & -1.248217000 & 1.355421000 \\
\hline $\mathrm{H}$ & 1.731524000 & -1.682381000 & 1.665504000 \\
\hline $\mathrm{C}$ & 4.828103000 & -0.946553000 & 0.462800000 \\
\hline $\mathrm{H}$ & 5.259741000 & -0.595101000 & -1.615339000 \\
\hline $\mathrm{H}$ & 4.026482000 & -1.368390000 & 2.412776000 \\
\hline
\end{tabular}




\begin{tabular}{lrcc}
$\mathrm{H}$ & 0.625194000 & 0.736602000 & -0.843530000 \\
$\mathrm{H}$ & -1.426410000 & -2.270838000 & -2.277151000 \\
$\mathrm{C}$ & 0.532840000 & 1.728238000 & 1.518441000 \\
$\mathrm{C}$ & 0.867674000 & 2.410909000 & 0.189676000 \\
$\mathrm{H}$ & -0.550012000 & 1.587998000 & 1.620574000 \\
$\mathrm{H}$ & 1.019511000 & 0.749012000 & 1.575330000 \\
$\mathrm{H}$ & 0.884882000 & 2.328618000 & 2.364315000 \\
$\mathrm{C}$ & 2.382377000 & 2.503195000 & -0.002518000 \\
$\mathrm{C}$ & 0.221633000 & 3.791580000 & 0.111798000 \\
$\mathrm{H}$ & 2.611934000 & 2.967928000 & -0.966805000 \\
$\mathrm{H}$ & 2.838752000 & 3.103396000 & 0.792342000 \\
$\mathrm{H}$ & 2.837758000 & 1.507279000 & 0.018138000 \\
$\mathrm{H}$ & 0.624218000 & 4.455219000 & 0.883237000 \\
$\mathrm{H}$ & 0.408624000 & 4.245838000 & -0.867004000 \\
$\mathrm{H}$ & -0.862977000 & 3.720134000 & 0.265286000 \\
$\mathrm{O}$ & 0.295093000 & 1.664378000 & -0.891901000 \\
$\mathrm{~K}$ & -2.138308000 & 1.119050000 & -1.569545000 \\
$\mathrm{C}$ & 6.253858000 & -0.801518000 & 0.932184000 \\
$\mathrm{H}$ & 6.751494000 & -1.776255000 & 1.014531000 \\
$\mathrm{H}$ & 6.842915000 & -0.192229000 & 0.238694000 \\
$\mathrm{H}$ & 6.304616000 & -0.327695000 & 1.918578000 \\
\hline---- - &
\end{tabular}

TSA2-3 ${ }_{\text {tBuOK }}$

\begin{tabular}{|c|c|c|c|}
\hline \multicolumn{4}{|c|}{ Coordinates (Angstroms) } \\
\hline & $\mathrm{X}$ & Y & $\mathrm{Z}$ \\
\hline$\overline{\mathrm{C}}$ & -5.334545000 & -1.554875000 & -0.022612000 \\
\hline $\mathrm{C}$ & -4.134504000 & -1.457170000 & -0.719284000 \\
\hline $\mathrm{C}$ & -2.931612000 & -1.176626000 & -0.049516000 \\
\hline $\mathrm{C}$ & -2.987316000 & -0.966740000 & 1.341295000 \\
\hline $\mathrm{C}$ & -4.186606000 & -1.078387000 & 2.032868000 \\
\hline $\mathrm{C}$ & -5.369591000 & -1.371569000 & 1.356673000 \\
\hline $\mathrm{H}$ & -6.248939000 & -1.783287000 & -0.563214000 \\
\hline $\mathrm{H}$ & -4.112597000 & -1.613737000 & -1.792283000 \\
\hline $\mathrm{H}$ & -2.082606000 & -0.705083000 & 1.883179000 \\
\hline $\mathrm{H}$ & -4.199096000 & -0.920438000 & 3.107660000 \\
\hline $\mathrm{H}$ & -6.307235000 & -1.450666000 & 1.898161000 \\
\hline $\mathrm{C}$ & -1.679626000 & -1.028879000 & -0.800632000 \\
\hline $\mathrm{C}$ & -0.426128000 & -1.008386000 & -0.297625000 \\
\hline $\mathrm{H}$ & -0.330649000 & -1.263628000 & 0.754127000 \\
\hline $\mathrm{O}$ & -1.908751000 & -0.714171000 & -2.150820000 \\
\hline $\mathrm{C}$ & 0.763917000 & -0.517665000 & -0.976066000 \\
\hline $\mathrm{H}$ & 0.694048000 & -0.503472000 & -2.071167000 \\
\hline $\mathrm{C}$ & 2.115973000 & -0.951163000 & -0.544193000 \\
\hline
\end{tabular}




\begin{tabular}{|c|c|c|c|}
\hline $\mathrm{C}$ & 3.223965000 & -0.594938000 & -1.332718000 \\
\hline $\mathrm{C}$ & 2.389705000 & -1.631892000 & 0.647736000 \\
\hline $\mathrm{C}$ & 4.521568000 & -0.896259000 & -0.950257000 \\
\hline $\mathrm{H}$ & 3.053267000 & -0.057110000 & -2.263142000 \\
\hline $\mathrm{C}$ & 3.696212000 & -1.927928000 & 1.032532000 \\
\hline $\mathrm{H}$ & 1.577815000 & -1.953323000 & 1.294263000 \\
\hline $\mathrm{C}$ & 4.787944000 & -1.569908000 & 0.246477000 \\
\hline $\mathrm{H}$ & 5.348562000 & -0.600131000 & -1.592941000 \\
\hline $\mathrm{H}$ & 3.862951000 & -2.457429000 & 1.968436000 \\
\hline $\mathrm{H}$ & 0.640384000 & 0.804290000 & -0.708714000 \\
\hline $\mathrm{H}$ & -1.138584000 & -1.017726000 & -2.650147000 \\
\hline $\mathrm{C}$ & 0.831999000 & 1.707865000 & 1.814114000 \\
\hline $\mathrm{C}$ & 0.955668000 & 2.602745000 & 0.565780000 \\
\hline $\mathrm{H}$ & -0.227430000 & 1.511585000 & 2.029403000 \\
\hline $\mathrm{H}$ & 1.332396000 & 0.747644000 & 1.648451000 \\
\hline $\mathrm{H}$ & 1.280682000 & 2.174381000 & 2.699401000 \\
\hline $\mathrm{C}$ & 2.441094000 & 2.805655000 & 0.227663000 \\
\hline $\mathrm{C}$ & 0.306516000 & 3.965466000 & 0.855969000 \\
\hline $\mathrm{H}$ & 2.533619000 & 3.429353000 & -0.668400000 \\
\hline $\mathrm{H}$ & 2.985693000 & 3.288473000 & 1.048730000 \\
\hline $\mathrm{H}$ & 2.915461000 & 1.840073000 & 0.021661000 \\
\hline $\mathrm{H}$ & 0.797056000 & 4.490386000 & 1.684541000 \\
\hline $\mathrm{H}$ & 0.357672000 & 4.601411000 & -0.035486000 \\
\hline $\mathrm{H}$ & -0.751104000 & 3.833040000 & 1.127723000 \\
\hline $\mathrm{O}$ & 0.285605000 & 2.026216000 & -0.516994000 \\
\hline K & -2.097808000 & 1.809101000 & -1.064027000 \\
\hline $\mathrm{C}$ & 6.203102000 & -1.899831000 & 0.650685000 \\
\hline $\mathrm{H}$ & 6.236999000 & -2.361653000 & 1.642372000 \\
\hline $\mathrm{H}$ & 6.668002000 & -2.597891000 & -0.056032000 \\
\hline $\mathrm{H}$ & 6.830732000 & -1.001414000 & 0.679113000 \\
\hline
\end{tabular}

\begin{tabular}{|c|c|c|c|}
\hline \multicolumn{4}{|c|}{$\mathrm{A3}_{\mathrm{tBuOK}}$} \\
\hline & \multicolumn{3}{|c|}{ Coordinates (Angstroms) } \\
\hline & $\mathrm{X}$ & Y & $\mathrm{Z}$ \\
\hline$\overline{\mathrm{C}}$ & -4.858899000 & -1.498777000 & -0.693377000 \\
\hline $\mathrm{C}$ & -3.742486000 & -1.957415000 & 0.000014000 \\
\hline $\mathrm{C}$ & -2.601960000 & -1.155075000 & 0.135676000 \\
\hline $\mathrm{C}$ & -2.610607000 & 0.135160000 & -0.420133000 \\
\hline $\mathrm{C}$ & -3.731639000 & 0.583158000 & -1.110183000 \\
\hline $\mathrm{C}$ & -4.855208000 & -0.228346000 & -1.258381000 \\
\hline $\mathrm{H}$ & -5.736327000 & -2.133319000 & -0.778269000 \\
\hline $\mathrm{H}$ & -3.789503000 & -2.933108000 & 0.478472000 \\
\hline $\mathrm{H}$ & -1.749491000 & 0.811184000 & -0.304352000 \\
\hline
\end{tabular}




\begin{tabular}{cccc}
$\mathrm{H}$ & -3.720786000 & 1.584050000 & -1.533451000 \\
$\mathrm{H}$ & -5.725480000 & 0.131917000 & -1.799100000 \\
$\mathrm{C}$ & -1.408280000 & -1.674315000 & 0.855167000 \\
$\mathrm{C}$ & -0.486922000 & -0.903538000 & 1.447554000 \\
$\mathrm{H}$ & -0.569105000 & 0.177211000 & 1.353332000 \\
$\mathrm{O}$ & -1.282849000 & -3.043636000 & 0.924544000 \\
$\mathrm{C}$ & 0.754657000 & -1.436528000 & 2.118131000 \\
$\mathrm{H}$ & 0.632659000 & -2.492479000 & 2.372927000 \\
$\mathrm{C}$ & 1.938236000 & -1.258871000 & 1.189091000 \\
$\mathrm{C}$ & 2.303458000 & -2.274391000 & 0.300760000 \\
$\mathrm{C}$ & 2.605210000 & -0.032724000 & 1.099240000 \\
$\mathrm{C}$ & 3.303115000 & -2.070475000 & -0.650435000 \\
$\mathrm{H}$ & 1.785714000 & -3.229392000 & 0.347725000 \\
$\mathrm{C}$ & 3.603486000 & 0.168830000 & 0.150473000 \\
$\mathrm{H}$ & 2.306458000 & 0.786943000 & 1.745622000 \\
$\mathrm{C}$ & 3.972526000 & -0.845357000 & -0.741006000 \\
$\mathrm{H}$ & 3.575183000 & -2.879564000 & -1.324949000 \\
$\mathrm{H}$ & 4.093772000 & 1.137674000 & 0.093423000 \\
$\mathrm{C}$ & -0.441307000 & 2.971521000 & 1.886082000 \\
$\mathrm{C}$ & -0.112433000 & 3.075147000 & 0.379124000 \\
$\mathrm{H}$ & -1.393521000 & 2.443501000 & 2.020521000 \\
$\mathrm{H}$ & 0.340421000 & 2.392456000 & 2.395332000 \\
$\mathrm{H}$ & -0.518533000 & 3.950210000 & 2.378299000 \\
$\mathrm{C}$ & 1.226787000 & 3.830274000 & 0.222835000 \\
$\mathrm{C}$ & -1.226773000 & 3.897839000 & -0.306119000 \\
$\mathrm{H}$ & 1.466311000 & 3.935845000 & -0.842931000 \\
$\mathrm{H}$ & 1.214952000 & 4.831094000 & 0.675103000 \\
$\mathrm{H}$ & 2.029870000 & 3.248618000 & 0.694087000 \\
$\mathrm{H}$ & -1.324561000 & 4.913593000 & 0.100495000 \\
$\mathrm{H}$ & -1.019067000 & 3.973080000 & -1.380681000 \\
$\mathrm{H}$ & -2.189729000 & 3.386582000 & -0.181547000 \\
$\mathrm{H}$ & -0.022452000 & 1.828574000 & -0.180127000 \\
$\mathrm{H}$ & 0.930973000 & -0.887444000 & 3.049822000 \\
$\mathrm{H}$ & 0.697344000 & -0.023537000 & -1.416918000 \\
$\mathrm{H}$ & -1.839156000 & -3.436729000 & 0.242013000 \\
$\mathrm{H}$ & 5.079684000 & -0.629971000 & -1.743289000 \\
$\mathrm{H}$ & 5.060802000 & -0.725703000 & -1.264074000 \\
$\mathrm{H}$ & & -1.365416000 & -2.552489000 \\
\hline
\end{tabular}

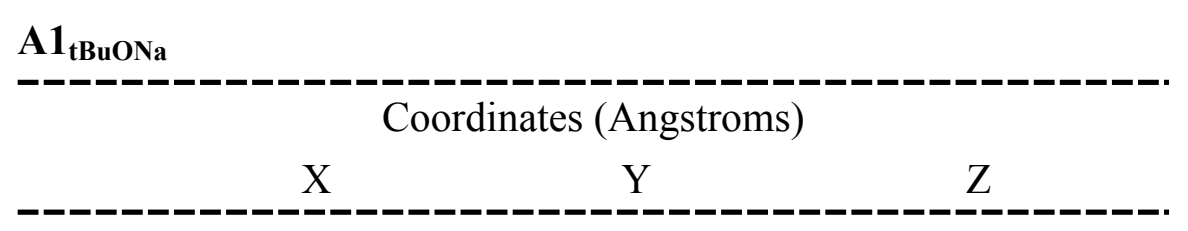




\begin{tabular}{|c|c|c|c|}
\hline $\mathrm{C}$ & -1.109875000 & 4.046628000 & -0.466385000 \\
\hline $\mathrm{C}$ & -0.488906000 & 2.854364000 & -0.104596000 \\
\hline $\mathrm{C}$ & -1.229307000 & 1.672894000 & -0.020966000 \\
\hline $\mathrm{C}$ & -2.597893000 & 1.689181000 & -0.309260000 \\
\hline $\mathrm{C}$ & -3.213001000 & 2.883884000 & -0.672705000 \\
\hline $\mathrm{C}$ & -2.473401000 & 4.062305000 & -0.749715000 \\
\hline $\mathrm{H}$ & -0.528915000 & 4.961967000 & -0.534132000 \\
\hline $\mathrm{H}$ & 0.579753000 & 2.836594000 & 0.100014000 \\
\hline $\mathrm{H}$ & -3.150296000 & 0.746741000 & -0.246467000 \\
\hline $\mathrm{H}$ & -4.274522000 & 2.893184000 & -0.904258000 \\
\hline $\mathrm{H}$ & -2.956997000 & 4.991570000 & -1.037283000 \\
\hline $\mathrm{C}$ & -0.593935000 & 0.380456000 & 0.456892000 \\
\hline $\mathrm{C}$ & 0.816008000 & 0.193216000 & -0.022795000 \\
\hline $\mathrm{H}$ & 0.935742000 & 0.303658000 & -1.099183000 \\
\hline $\mathrm{O}$ & -0.679076000 & 0.319230000 & 1.905502000 \\
\hline $\mathrm{C}$ & 1.863797000 & -0.077698000 & 0.758838000 \\
\hline $\mathrm{H}$ & 1.694137000 & -0.166247000 & 1.830903000 \\
\hline $\mathrm{C}$ & 3.257437000 & -0.272728000 & 0.324589000 \\
\hline $\mathrm{C}$ & 4.279876000 & -0.232561000 & 1.279039000 \\
\hline $\mathrm{C}$ & 3.620154000 & -0.501767000 & -1.008060000 \\
\hline $\mathrm{C}$ & 5.611024000 & -0.397118000 & 0.917372000 \\
\hline $\mathrm{H}$ & 4.027266000 & -0.061778000 & 2.323048000 \\
\hline $\mathrm{C}$ & 4.951348000 & -0.666988000 & -1.366209000 \\
\hline $\mathrm{H}$ & 2.854499000 & -0.566926000 & -1.775522000 \\
\hline $\mathrm{C}$ & 5.971521000 & -0.618648000 & -0.412759000 \\
\hline $\mathrm{H}$ & 6.383029000 & -0.355035000 & 1.682016000 \\
\hline $\mathrm{H}$ & 5.202635000 & -0.845259000 & -2.409010000 \\
\hline $\mathrm{H}$ & -1.232405000 & -0.452968000 & 0.124780000 \\
\hline $\mathrm{H}$ & -0.295038000 & 1.139057000 & 2.244007000 \\
\hline $\mathrm{C}$ & -5.238579000 & -2.389261000 & -0.344619000 \\
\hline $\mathrm{C}$ & -3.718029000 & -2.199842000 & -0.517950000 \\
\hline $\mathrm{H}$ & -5.450159000 & -2.760731000 & 0.666215000 \\
\hline $\mathrm{H}$ & -5.743139000 & -1.423167000 & -0.463301000 \\
\hline $\mathrm{H}$ & -5.665142000 & -3.096783000 & -1.067846000 \\
\hline $\mathrm{C}$ & -3.441176000 & -1.700568000 & -1.952224000 \\
\hline $\mathrm{C}$ & -3.019260000 & -3.561490000 & -0.326746000 \\
\hline $\mathrm{H}$ & -2.363859000 & -1.545888000 & -2.089457000 \\
\hline $\mathrm{H}$ & -3.788119000 & -2.405380000 & -2.718825000 \\
\hline $\mathrm{H}$ & -3.941856000 & -0.739078000 & -2.117452000 \\
\hline $\mathrm{H}$ & -3.356535000 & -4.320368000 & -1.044876000 \\
\hline $\mathrm{H}$ & -1.935713000 & -3.434683000 & -0.439203000 \\
\hline $\mathrm{H}$ & -3.213498000 & -3.937131000 & 0.686280000 \\
\hline $\mathrm{O}$ & -3.243744000 & -1.290005000 & 0.396650000 \\
\hline $\mathrm{Na}$ & -2.703237000 & -0.660739000 & 2.235384000 \\
\hline
\end{tabular}




$\begin{array}{llll}\mathrm{C} & 7.412696000 & -0.836060000 & -0.798893000 \\ \mathrm{H} & 8.083941000 & -0.214302000 & -0.197923000 \\ \mathrm{H} & 7.705879000 & -1.881216000 & -0.642499000 \\ \mathrm{H} & 7.584480000 & -0.600702000 & -1.853547000\end{array}$

TSA1-2 ${ }_{\text {tBuONa }}$

\begin{tabular}{lccc} 
& & Coordinates (Angstroms) & \\
& $\mathrm{X}$ & $\mathrm{Y}$ & $\mathrm{Z}$ \\
\hline $\mathrm{C}$ & 3.153727000 & -2.599246000 & -1.743901000 \\
$\mathrm{C}$ & 2.064761000 & -1.981094000 & -1.137500000 \\
$\mathrm{C}$ & 2.182375000 & -1.389232000 & 0.129018000 \\
$\mathrm{C}$ & 3.429157000 & -1.460671000 & 0.770357000 \\
$\mathrm{C}$ & 4.510614000 & -2.092036000 & 0.168178000 \\
$\mathrm{C}$ & 4.383399000 & -2.661769000 & -1.096562000 \\
$\mathrm{H}$ & 3.031913000 & -3.048923000 & -2.725578000 \\
$\mathrm{H}$ & 1.111032000 & -1.974943000 & -1.656302000 \\
$\mathrm{H}$ & 3.541748000 & -0.999750000 & 1.746431000 \\
$\mathrm{H}$ & 5.464018000 & -2.131468000 & 0.688165000 \\
$\mathrm{H}$ & 5.230795000 & -3.149629000 & -1.568729000 \\
$\mathrm{C}$ & 1.081257000 & -0.622739000 & 0.763238000 \\
$\mathrm{C}$ & -0.276729000 & -0.695196000 & 0.248637000 \\
$\mathrm{H}$ & -0.360897000 & -0.601805000 & -0.833474000 \\
$\mathrm{O}$ & 1.124378000 & -0.628787000 & 2.204814000 \\
$\mathrm{C}$ & -1.421555000 & -0.692254000 & 0.975017000 \\
$\mathrm{H}$ & -1.363061000 & -0.889694000 & 2.047976000 \\
$\mathrm{C}$ & -2.775784000 & -0.536715000 & 0.425205000 \\
$\mathrm{C}$ & -3.884436000 & -1.014462000 & 1.135904000 \\
$\mathrm{C}$ & -3.022207000 & 0.090593000 & -0.805726000 \\
$\mathrm{C}$ & -5.173725000 & -0.895993000 & 0.629749000 \\
$\mathrm{H}$ & -3.729609000 & -1.507501000 & 2.093394000 \\
$\mathrm{C}$ & -4.310499000 & 0.198972000 & -1.310591000 \\
$\mathrm{H}$ & -2.194197000 & 0.510959000 & -1.370755000 \\
$\mathrm{C}$ & -5.412714000 & -0.291438000 & -0.604860000 \\
$\mathrm{H}$ & -6.010172000 & -1.288778000 & 1.203448000 \\
$\mathrm{H}$ & -4.465379000 & 0.685379000 & -2.271198000 \\
$\mathrm{H}$ & 1.382618000 & 0.706278000 & 0.554394000 \\
$\mathrm{H}$ & 0.933156000 & -1.524654000 & 2.518110000 \\
$\mathrm{C}$ & 3.516616000 & 2.261322000 & -0.500848000 \\
$\mathrm{C}$ & 2.005312000 & 2.531135000 & -0.471871000 \\
$\mathrm{H}$ & 3.984514000 & 2.671785000 & 0.400488000 \\
$\mathrm{H}$ & 3.706727000 & 1.182313000 & -0.522503000 \\
& 3.993320000 & 2.713774000 & -1.378723000 \\
$\mathrm{H}$ & 1.349686000 & 1.941574000 & -1.732599000 \\
\hline
\end{tabular}




$\begin{array}{lrcc}\mathrm{C} & 1.738925000 & 4.038472000 & -0.407570000 \\ \mathrm{H} & 0.261377000 & 2.068511000 & -1.679312000 \\ \mathrm{H} & 1.712087000 & 2.431721000 & -2.643490000 \\ \mathrm{H} & 1.571672000 & 0.870886000 & -1.817267000 \\ \mathrm{H} & 2.161842000 & 4.562386000 & -1.272802000 \\ \mathrm{H} & 0.659000000 & 4.228119000 & -0.383506000 \\ \mathrm{H} & 2.182990000 & 4.458498000 & 0.501863000 \\ \mathrm{O} & 1.448168000 & 1.944397000 & 0.673327000 \\ \mathrm{Na} & 0.017340000 & 1.441364000 & 2.137748000 \\ \mathrm{C} & -6.810905000 & -0.139947000 & -1.148893000 \\ \mathrm{H} & -7.498429000 & -0.855714000 & -0.687650000 \\ \mathrm{H} & -7.205226000 & 0.865656000 & -0.957663000 \\ \mathrm{H} & -6.836604000 & -0.298955000 & -2.231968000 \\ \text {--_-_-_-_. }\end{array}$

\begin{tabular}{|c|c|c|c|}
\hline \multicolumn{4}{|c|}{ Coordinates (Angstroms) } \\
\hline & $\mathrm{X}$ & Y & $\mathrm{Z}$ \\
\hline$\overline{\mathrm{C}}$ & -5.254161000 & -0.496747000 & 0.144109000 \\
\hline $\mathrm{C}$ & -4.125821000 & -0.897498000 & -0.570796000 \\
\hline $\mathrm{C}$ & -2.878288000 & -1.112808000 & 0.073087000 \\
\hline $\mathrm{C}$ & -2.851521000 & -0.898191000 & 1.478327000 \\
\hline $\mathrm{C}$ & -3.982664000 & -0.508327000 & 2.171438000 \\
\hline $\mathrm{C}$ & -5.200224000 & -0.292298000 & 1.516823000 \\
\hline $\mathrm{H}$ & -6.192045000 & -0.357912000 & -0.388382000 \\
\hline $\mathrm{H}$ & -4.209040000 & -1.101501000 & -1.633901000 \\
\hline $\mathrm{H}$ & -1.927787000 & -1.048246000 & 2.028570000 \\
\hline $\mathrm{H}$ & -3.914744000 & -0.363770000 & 3.246707000 \\
\hline $\mathrm{H}$ & -6.081036000 & 0.016634000 & 2.070194000 \\
\hline $\mathrm{C}$ & -1.699553000 & -1.413791000 & -0.694928000 \\
\hline $\mathrm{C}$ & -0.384983000 & -1.382565000 & -0.247295000 \\
\hline $\mathrm{H}$ & -0.267017000 & -1.421431000 & 0.832923000 \\
\hline $\mathrm{O}$ & -1.908226000 & -1.289391000 & -2.099824000 \\
\hline $\mathrm{C}$ & 0.770501000 & -1.244502000 & -1.004379000 \\
\hline $\mathrm{H}$ & 0.696451000 & -1.217234000 & -2.091739000 \\
\hline $\mathrm{C}$ & 2.120281000 & -1.162790000 & -0.464830000 \\
\hline $\mathrm{C}$ & 3.191317000 & -0.805170000 & -1.306901000 \\
\hline $\mathrm{C}$ & 2.438501000 & -1.357280000 & 0.894494000 \\
\hline $\mathrm{C}$ & 4.479927000 & -0.629429000 & -0.821001000 \\
\hline $\mathrm{H}$ & 2.995852000 & -0.643404000 & -2.365160000 \\
\hline $\mathrm{C}$ & 3.728266000 & -1.171983000 & 1.373639000 \\
\hline $\mathrm{H}$ & 1.667079000 & -1.669007000 & 1.593679000 \\
\hline $\mathrm{C}$ & 4.779765000 & -0.802048000 & 0.531924000 \\
\hline $\mathrm{H}$ & 5.271458000 & -0.344350000 & -1.511382000 \\
\hline
\end{tabular}




$\begin{array}{lrcc}\mathrm{H} & 3.923796000 & -1.328294000 & 2.432930000 \\ \mathrm{H} & 0.419190000 & 0.752012000 & -0.995295000 \\ \mathrm{H} & -1.349212000 & -1.939467000 & -2.541194000 \\ \mathrm{C} & 0.177463000 & 1.692008000 & 1.368148000 \\ \mathrm{C} & 0.461875000 & 2.433528000 & 0.059846000 \\ \mathrm{H} & -0.883601000 & 1.423454000 & 1.443792000 \\ \mathrm{H} & 0.774718000 & 0.776079000 & 1.426092000 \\ \mathrm{H} & 0.436416000 & 2.317787000 & 2.228946000 \\ \mathrm{C} & 1.961086000 & 2.682698000 & -0.105824000 \\ \mathrm{C} & -0.327852000 & 3.736466000 & -0.010536000 \\ \mathrm{H} & 2.157448000 & 3.192943000 & -1.054079000 \\ \mathrm{H} & 2.344169000 & 3.302927000 & 0.711584000 \\ \mathrm{H} & 2.510046000 & 1.734440000 & -0.101932000 \\ \mathrm{H} & -0.007277000 & 4.427232000 & 0.775170000 \\ \mathrm{H} & -0.178392000 & 4.222311000 & -0.980406000 \\ \mathrm{H} & -1.400932000 & 3.549796000 & 0.128553000 \\ \mathrm{O} & -0.001628000 & 1.646206000 & -1.051162000 \\ \mathrm{Na} & -2.062086000 & 0.928785000 & -1.484702000 \\ \mathrm{C} & 6.181768000 & -0.632770000 & 1.060985000 \\ \mathrm{H} & 6.688653000 & -1.599789000 & 1.171167000 \\ \mathrm{H} & 6.790702000 & -0.018969000 & 0.389217000 \\ \mathrm{H} & 6.182782000 & -0.152425000 & 2.045585000 \\ --------1\end{array}$

TSA2-3 ${ }_{\text {tBuONa }}$

$\begin{array}{lccc} & & & \text { Coordinates (Angstroms) } \\ & & \mathrm{X} & \mathrm{Z} \\ \mathrm{C} & -5.552282000 & -0.413476000 & -0.018244000 \\ \mathrm{C} & -4.337375000 & -0.113932000 & -0.632656000 \\ \mathrm{C} & -3.178957000 & -0.862158000 & -0.351890000 \\ \mathrm{C} & -3.297675000 & -1.935674000 & 0.551995000 \\ \mathrm{C} & -4.509584000 & -2.226480000 & 1.157923000 \\ \mathrm{C} & -5.647215000 & -1.464414000 & 0.884645000 \\ \mathrm{H} & -6.431560000 & 0.176297000 & -0.262567000 \\ \mathrm{H} & -4.287635000 & 0.683700000 & -1.366485000 \\ \mathrm{H} & -2.435502000 & -2.555793000 & 0.775479000 \\ \mathrm{H} & -4.569603000 & -3.061004000 & 1.850713000 \\ \mathrm{H} & -6.593706000 & -1.699144000 & 1.361324000 \\ \mathrm{C} & -1.894785000 & -0.467476000 & -0.937695000 \\ \mathrm{C} & -0.661472000 & -0.929348000 & -0.620343000 \\ \mathrm{H} & -0.610912000 & -1.748418000 & 0.093516000 \\ \mathrm{O} & -2.022234000 & 0.662577000 & -1.762438000 \\ \mathrm{C} & 0.585377000 & -0.328929000 & -1.068076000 \\ \mathrm{H} & 0.603491000 & -0.084686000 & -2.138792000\end{array}$




$\begin{array}{lrrr}\mathrm{C} & 1.860246000 & -0.933152000 & -0.633032000 \\ \mathrm{C} & 2.991562000 & -0.910271000 & -1.465810000 \\ \mathrm{C} & 2.035975000 & -1.478576000 & 0.647413000 \\ \mathrm{C} & 4.216578000 & -1.409791000 & -1.046248000 \\ \mathrm{H} & 2.898896000 & -0.494650000 & -2.467201000 \\ \mathrm{C} & 3.262560000 & -1.990821000 & 1.059330000 \\ \mathrm{H} & 1.204433000 & -1.485543000 & 1.348895000 \\ \mathrm{C} & 4.379164000 & -1.967523000 & 0.224948000 \\ \mathrm{H} & 5.065122000 & -1.375427000 & -1.727110000 \\ \mathrm{H} & 3.353423000 & -2.410112000 & 2.059440000 \\ \mathrm{H} & 0.492338000 & 0.963121000 & -0.441121000 \\ \mathrm{H} & -1.134381000 & 0.900156000 & -2.067858000 \\ \mathrm{C} & 1.948722000 & 2.106737000 & 1.682202000 \\ \mathrm{C} & 1.294745000 & 2.770482000 & 0.463576000 \\ \mathrm{H} & 1.220598000 & 2.023578000 & 2.498641000 \\ \mathrm{H} & 2.294758000 & 1.099441000 & 1.427953000 \\ \mathrm{H} & 2.807082000 & 2.684705000 & 2.044172000 \\ \mathrm{C} & 2.307656000 & 2.870546000 & -0.685512000 \\ \mathrm{C} & 0.787151000 & 4.167213000 & 0.835486000 \\ \mathrm{H} & 1.826153000 & 3.294948000 & -1.573543000 \\ \mathrm{H} & 3.157781000 & 3.506653000 & -0.413350000 \\ \mathrm{H} & 2.694554000 & 1.877871000 & -0.940495000 \\ \mathrm{H} & 1.601006000 & 4.819522000 & 1.171869000 \\ \mathrm{H} & 0.304631000 & 4.635768000 & -0.030057000 \\ \mathrm{H} & 0.052862000 & 4.099384000 & 1.649498000 \\ \mathrm{O} & 0.180761000 & 2.013575000 & 0.048112000 \\ \mathrm{Na} & -1.892810000 & 1.712526000 & 0.379285000 \\ \mathrm{C} & 5.714783000 & -2.507550000 & 0.672346000 \\ \mathrm{H} & 6.102071000 & -3.251172000 & -0.033930000 \\ \mathrm{H} & 6.465146000 & -1.711319000 & 0.748059000 \\ \mathrm{H} & 5.640620000 & -2.987305000 & 1.653286000 \\ ----- & \end{array}$

\begin{tabular}{lrrr} 
& & & \\
& & & \\
& Coordinates (Angstroms) & Z \\
\hline $\mathrm{C}$ & -4.908178000 & -1.304780000 & -0.687391000 \\
$\mathrm{C}$ & -3.777488000 & -1.842694000 & -0.081624000 \\
$\mathrm{C}$ & -2.608996000 & -1.080449000 & 0.051545000 \\
$\mathrm{C}$ & -2.591573000 & 0.238749000 & -0.423378000 \\
$\mathrm{C}$ & -3.728924000 & 0.765439000 & -1.027192000 \\
$\mathrm{C}$ & -4.885251000 & 0.001456000 & -1.166749000 \\
$\mathrm{H}$ & -5.810059000 & -1.903748000 & -0.773362000 \\
$\mathrm{H}$ & -3.827225000 & -2.848311000 & 0.330994000
\end{tabular}




\begin{tabular}{lrcc}
$\mathrm{H}$ & -1.695799000 & 0.871154000 & -0.332071000 \\
$\mathrm{H}$ & -3.700208000 & 1.786856000 & -1.396254000 \\
$\mathrm{H}$ & -5.766535000 & 0.422920000 & -1.641420000 \\
$\mathrm{C}$ & -1.409160000 & -1.680930000 & 0.689204000 \\
$\mathrm{C}$ & -0.519270000 & -1.001917000 & 1.427094000 \\
$\mathrm{H}$ & -0.662454000 & 0.067601000 & 1.544681000 \\
$\mathrm{O}$ & -1.244850000 & -3.035218000 & 0.526209000 \\
$\mathrm{C}$ & 0.721288000 & -1.617813000 & 2.032377000 \\
$\mathrm{H}$ & 0.562565000 & -2.683099000 & 2.222878000 \\
$\mathrm{C}$ & 1.891951000 & -1.430351000 & 1.088554000 \\
$\mathrm{C}$ & 2.075158000 & -2.311169000 & 0.014976000 \\
$\mathrm{C}$ & 2.715264000 & -0.303027000 & 1.161578000 \\
$\mathrm{C}$ & 3.037464000 & -2.059161000 & -0.961356000 \\
$\mathrm{H}$ & 1.433797000 & -3.185028000 & -0.063797000 \\
$\mathrm{C}$ & 3.676398000 & -0.053052000 & 0.183627000 \\
$\mathrm{H}$ & 2.573475000 & 0.412893000 & 1.966384000 \\
$\mathrm{C}$ & 3.854414000 & -0.923472000 & -0.896259000 \\
$\mathrm{H}$ & 3.161986000 & -2.760864000 & -1.783110000 \\
$\mathrm{H}$ & 4.285300000 & 0.844466000 & 0.254642000 \\
$\mathrm{C}$ & -0.141010000 & 2.887301000 & 1.768598000 \\
$\mathrm{C}$ & 0.113119000 & 3.074704000 & 0.256083000 \\
$\mathrm{H}$ & -1.142170000 & 2.466068000 & 1.924747000 \\
$\mathrm{H}$ & 0.592617000 & 2.179240000 & 2.177573000 \\
$\mathrm{H}$ & -0.069368000 & 3.823572000 & 2.337530000 \\
$\mathrm{C}$ & 1.525560000 & 3.668539000 & 0.067253000 \\
$\mathrm{C}$ & -0.926034000 & 4.073200000 & -0.297200000 \\
$\mathrm{H}$ & 1.721763000 & 3.812901000 & -1.002181000 \\
$\mathrm{H}$ & 1.662220000 & 4.631007000 & 0.578157000 \\
$\mathrm{H}$ & 2.270339000 & 2.960940000 & 0.456397000 \\
$\mathrm{H}$ & -0.878030000 & 5.055029000 & 0.192204000 \\
$\mathrm{H}$ & -0.764824000 & 4.211454000 & -1.372520000 \\
$\mathrm{H}$ & -1.934888000 & 3.666127000 & -0.155847000 \\
$\mathrm{O}$ & 0.008620000 & 1.872338000 & -0.391936000 \\
$\mathrm{H}$ & 0.936700000 & -1.137117000 & 2.992075000 \\
$\mathrm{H}$ & -1.837876000 & -3.331480000 & -0.174949000 \\
$\mathrm{H}$ & 0.816081000 & 0.102720000 & -0.940900000 \\
$\mathrm{H}$ & 4.917704000 & -0.665214000 & -1.933779000 \\
$\mathrm{H}$ & 5.052477000 & 0.406365000 & -2.108989000 \\
$\mathrm{H}$ & 4.671732000 & -1.068437000 & -1.605290000 \\
-1.139544000 & -2.888537000 \\
\hline
\end{tabular}

A1 $1_{\text {tBuOLi }}$ 


\begin{tabular}{|c|c|c|c|}
\hline & $X$ & $\mathrm{Y}$ & Z \\
\hline $\mathrm{C}$ & -1.066758000 & 4.013526000 & -0.228805000 \\
\hline $\mathrm{C}$ & -0.466404000 & 2.777533000 & -0.003076000 \\
\hline $\mathrm{C}$ & -1.242390000 & 1.617402000 & 0.034237000 \\
\hline $\mathrm{C}$ & -2.626012000 & 1.699958000 & -0.154339000 \\
\hline $\mathrm{C}$ & -3.220419000 & 2.937039000 & -0.380053000 \\
\hline $\mathrm{C}$ & -2.443609000 & 4.093818000 & -0.417872000 \\
\hline $\mathrm{H}$ & -0.458498000 & 4.912787000 & -0.260830000 \\
\hline $\mathrm{H}$ & 0.610173000 & 2.710609000 & 0.135756000 \\
\hline $\mathrm{H}$ & -3.213960000 & 0.781234000 & -0.109166000 \\
\hline $\mathrm{H}$ & -4.294053000 & 2.998356000 & -0.532378000 \\
\hline $\mathrm{H}$ & -2.911332000 & 5.057583000 & -0.597137000 \\
\hline $\mathrm{C}$ & -0.630632000 & 0.269083000 & 0.360231000 \\
\hline $\mathrm{C}$ & 0.795287000 & 0.119148000 & -0.083316000 \\
\hline $\mathrm{H}$ & 0.951273000 & 0.318168000 & -1.142117000 \\
\hline $\mathrm{O}$ & -0.775064000 & 0.020874000 & 1.787234000 \\
\hline $\mathrm{C}$ & 1.814289000 & -0.218822000 & 0.709354000 \\
\hline $\mathrm{H}$ & 1.607605000 & -0.392563000 & 1.764168000 \\
\hline $\mathrm{C}$ & 3.221671000 & -0.380624000 & 0.307350000 \\
\hline $\mathrm{C}$ & 4.212129000 & -0.416287000 & 1.295419000 \\
\hline $\mathrm{C}$ & 3.627323000 & -0.504366000 & -1.026515000 \\
\hline $\mathrm{C}$ & 5.554342000 & -0.551328000 & 0.965252000 \\
\hline $\mathrm{H}$ & 3.924899000 & -0.327723000 & 2.340639000 \\
\hline $\mathrm{C}$ & 4.969944000 & -0.640773000 & -1.353143000 \\
\hline $\mathrm{H}$ & 2.886776000 & -0.510418000 & -1.820929000 \\
\hline $\mathrm{C}$ & 5.958326000 & -0.666880000 & -0.366302000 \\
\hline $\mathrm{H}$ & 6.301612000 & -0.569292000 & 1.754932000 \\
\hline $\mathrm{H}$ & 5.255544000 & -0.738389000 & -2.397715000 \\
\hline $\mathrm{H}$ & -1.255655000 & -0.511414000 & -0.093015000 \\
\hline $\mathrm{H}$ & -0.506840000 & 0.826849000 & 2.248055000 \\
\hline $\mathrm{C}$ & -5.573650000 & -2.326356000 & 0.384557000 \\
\hline $\mathrm{C}$ & -4.182381000 & -2.117262000 & -0.241511000 \\
\hline $\mathrm{H}$ & -5.469642000 & -2.822421000 & 1.357160000 \\
\hline $\mathrm{H}$ & -6.052981000 & -1.354317000 & 0.548505000 \\
\hline $\mathrm{H}$ & -6.231178000 & -2.937381000 & -0.247012000 \\
\hline $\mathrm{C}$ & -4.347601000 & -1.432434000 & -1.612544000 \\
\hline $\mathrm{C}$ & -3.508478000 & -3.486966000 & -0.445957000 \\
\hline $\mathrm{H}$ & -3.362227000 & -1.252658000 & -2.059199000 \\
\hline $\mathrm{H}$ & -4.939668000 & -2.036461000 & -2.311280000 \\
\hline $\mathrm{H}$ & -4.845862000 & -0.463137000 & -1.490549000 \\
\hline $\mathrm{H}$ & -4.090913000 & -4.149326000 & -1.098991000 \\
\hline $\mathrm{H}$ & -2.515524000 & -3.347256000 & -0.889764000 \\
\hline $\mathrm{H}$ & -3.379886000 & -3.983307000 & 0.523639000 \\
\hline $\mathrm{O}$ & -3.408050000 & -1.332688000 & 0.583613000 \\
\hline
\end{tabular}




$\begin{array}{llll}\mathrm{C} & 7.412587000 & -0.848680000 & -0.719867000 \\ \mathrm{H} & 8.055231000 & -0.214653000 & -0.100407000 \\ \mathrm{H} & 7.726847000 & -1.887209000 & -0.561082000 \\ \mathrm{H} & 7.602908000 & -0.603205000 & -1.768860000 \\ \mathrm{Li} & -2.491702000 & -0.876773000 & 1.900732000\end{array}$

TSA1-2 tBuOLi $_{\text {i }}$

\begin{tabular}{lccc} 
& \multicolumn{3}{c}{ Coordinates (Angstroms) } \\
& $\mathrm{X}$ & $\mathrm{Y}$ & $\mathrm{Z}$ \\
\hline $\mathrm{C}$ & 4.640266000 & -2.358260000 & 0.572571000 \\
$\mathrm{C}$ & 3.573807000 & -1.604702000 & 1.055412000 \\
$\mathrm{C}$ & 2.560809000 & -1.151127000 & 0.199092000 \\
$\mathrm{C}$ & 2.674606000 & -1.468011000 & -1.166352000 \\
$\mathrm{C}$ & 3.734839000 & -2.225854000 & -1.642848000 \\
$\mathrm{C}$ & 4.728857000 & -2.680334000 & -0.777004000 \\
$\mathrm{H}$ & 5.406519000 & -2.698847000 & 1.264337000 \\
$\mathrm{H}$ & 3.521480000 & -1.364385000 & 2.112509000 \\
$\mathrm{H}$ & 1.929623000 & -1.097593000 & -1.866838000 \\
$\mathrm{H}$ & 3.793177000 & -2.453747000 & -2.703940000 \\
$\mathrm{H}$ & 5.561402000 & -3.267574000 & -1.152371000 \\
$\mathrm{C}$ & 1.438633000 & -0.318265000 & 0.675658000 \\
$\mathrm{C}$ & 0.086594000 & -0.632108000 & 0.281023000 \\
$\mathrm{H}$ & -0.006981000 & -1.112818000 & -0.691823000 \\
$\mathrm{O}$ & 1.520977000 & -0.007300000 & 2.098526000 \\
$\mathrm{C}$ & -1.044624000 & -0.333620000 & 0.964150000 \\
$\mathrm{H}$ & -0.963181000 & 0.188097000 & 1.918407000 \\
$\mathrm{C}$ & -2.417446000 & -0.592626000 & 0.518714000 \\
$\mathrm{C}$ & -3.483698000 & 0.067046000 & 1.146873000 \\
$\mathrm{C}$ & -2.740742000 & -1.482658000 & -0.516317000 \\
$\mathrm{C}$ & -4.799529000 & -0.135558000 & 0.751284000 \\
$\mathrm{H}$ & -3.271895000 & 0.757807000 & 1.960820000 \\
$\mathrm{C}$ & -4.056876000 & -1.677384000 & -0.913220000 \\
$\mathrm{H}$ & -1.954225000 & -2.049691000 & -1.006646000 \\
$\mathrm{C}$ & -5.113742000 & -1.007139000 & -0.292605000 \\
$\mathrm{H}$ & -5.599369000 & 0.394910000 & 1.263888000 \\
$\mathrm{H}$ & -4.270031000 & -2.379751000 & -1.716236000 \\
$\mathrm{H}$ & 1.652048000 & 1.150196000 & 0.193296000 \\
$\mathrm{H}$ & 1.053262000 & -0.692369000 & 2.601746000 \\
$\mathrm{C}$ & 1.640393000 & 4.370352000 & -0.733815000 \\
$\mathrm{H}$ & 0.949179000 & 3.023334000 & -0.529180000 \\
$\mathrm{H}$ & 1.795151000 & 4.870966000 & 0.229307000 \\
$\mathrm{H}$ & 1.038090000 & 5.028921000 & -1.369337000
\end{tabular}




\begin{tabular}{lccc}
$\mathrm{C}$ & 0.772615000 & 2.299715000 & -1.866808000 \\
$\mathrm{C}$ & -0.409774000 & 3.213442000 & 0.153710000 \\
$\mathrm{H}$ & 0.254701000 & 1.345976000 & -1.721563000 \\
$\mathrm{H}$ & 0.180878000 & 2.906145000 & -2.560674000 \\
$\mathrm{H}$ & 1.749563000 & 2.101698000 & -2.319255000 \\
$\mathrm{H}$ & -1.087505000 & 3.820426000 & -0.456138000 \\
$\mathrm{H}$ & -0.885297000 & 2.241535000 & 0.329198000 \\
$\mathrm{H}$ & -0.282057000 & 3.730728000 & 1.116294000 \\
$\mathrm{O}$ & 1.796786000 & 2.248534000 & 0.317546000 \\
$\mathrm{Li}$ & 1.598543000 & 1.856304000 & 2.083072000 \\
$\mathrm{C}$ & -6.538455000 & -1.203952000 & -0.745807000 \\
$\mathrm{H}$ & -7.240404000 & -1.123145000 & 0.090669000 \\
$\mathrm{H}$ & -6.826580000 & -0.449030000 & -1.487908000 \\
$\mathrm{H}$ & -6.677883000 & -2.186265000 & -1.208423000 \\
\hline
\end{tabular}

\begin{tabular}{|c|c|c|c|}
\hline \multicolumn{4}{|c|}{$\mathrm{A} 2_{\mathrm{tBuOLi}}$} \\
\hline & \multicolumn{3}{|c|}{ Coordinates (Angstroms) } \\
\hline & $X$ & $\mathrm{Y}$ & Z \\
\hline$\overline{\mathrm{C}}$ & -5.181513000 & -0.299281000 & 0.009449000 \\
\hline $\mathrm{C}$ & -4.057643000 & -0.658468000 & -0.736954000 \\
\hline $\mathrm{C}$ & -2.870898000 & -1.116590000 & -0.107399000 \\
\hline $\mathrm{C}$ & -2.899057000 & -1.207596000 & 1.309865000 \\
\hline $\mathrm{C}$ & -4.023261000 & -0.852381000 & 2.030158000 \\
\hline $\mathrm{C}$ & -5.178556000 & -0.382468000 & 1.393856000 \\
\hline $\mathrm{H}$ & -6.073390000 & 0.036258000 & -0.513612000 \\
\hline $\mathrm{H}$ & -4.112167000 & -0.647754000 & -1.821342000 \\
\hline $\mathrm{H}$ & -2.029134000 & -1.578352000 & 1.842991000 \\
\hline $\mathrm{H}$ & -4.004113000 & -0.945876000 & 3.112882000 \\
\hline $\mathrm{H}$ & -6.054907000 & -0.105935000 & 1.970847000 \\
\hline $\mathrm{C}$ & -1.679396000 & -1.356214000 & -0.881623000 \\
\hline $\mathrm{C}$ & -0.370289000 & -1.365858000 & -0.407954000 \\
\hline $\mathrm{H}$ & -0.280713000 & -1.500912000 & 0.666878000 \\
\hline $\mathrm{O}$ & -1.867683000 & -1.018842000 & -2.260552000 \\
\hline $\mathrm{C}$ & 0.798029000 & -1.130534000 & -1.115375000 \\
\hline $\mathrm{H}$ & 0.765377000 & -1.040625000 & -2.202050000 \\
\hline $\mathrm{C}$ & 2.130341000 & -1.035060000 & -0.529251000 \\
\hline $\mathrm{C}$ & 3.214518000 & -0.622619000 & -1.325915000 \\
\hline $\mathrm{C}$ & 2.411720000 & -1.271736000 & 0.830377000 \\
\hline $\mathrm{C}$ & 4.486753000 & -0.445524000 & -0.797933000 \\
\hline $\mathrm{H}$ & 3.045621000 & -0.426438000 & -2.382905000 \\
\hline $\mathrm{C}$ & 3.685183000 & -1.088436000 & 1.351537000 \\
\hline $\mathrm{H}$ & 1.625930000 & -1.614804000 & 1.498178000 \\
\hline $\mathrm{C}$ & 4.753064000 & -0.670945000 & 0.553592000 \\
\hline
\end{tabular}




\begin{tabular}{|c|c|c|c|}
\hline $\mathrm{H}$ & 5.291495000 & -0.120868000 & -1.454530000 \\
\hline $\mathrm{H}$ & 3.854430000 & -1.280453000 & 2.409351000 \\
\hline $\mathrm{H}$ & 0.262681000 & 0.806036000 & -1.086925000 \\
\hline $\mathrm{H}$ & -1.211472000 & -1.494680000 & -2.781940000 \\
\hline $\mathrm{C}$ & -0.131838000 & 1.617040000 & 1.316001000 \\
\hline $\mathrm{C}$ & 0.043546000 & 2.435435000 & 0.035101000 \\
\hline $\mathrm{H}$ & -1.140916000 & 1.191064000 & 1.378023000 \\
\hline $\mathrm{H}$ & 0.595459000 & 0.799040000 & 1.352649000 \\
\hline $\mathrm{H}$ & 0.027953000 & 2.249238000 & 2.195706000 \\
\hline $\mathrm{C}$ & 1.493081000 & 2.890119000 & -0.124755000 \\
\hline $\mathrm{C}$ & -0.922841000 & 3.613304000 & -0.002303000 \\
\hline $\mathrm{H}$ & 1.613561000 & 3.456871000 & -1.053154000 \\
\hline $\mathrm{H}$ & 1.794222000 & 3.524679000 & 0.715298000 \\
\hline $\mathrm{H}$ & 2.164697000 & 2.024010000 & -0.156044000 \\
\hline $\mathrm{H}$ & -0.704705000 & 4.315543000 & 0.807886000 \\
\hline $\mathrm{H}$ & -0.839783000 & 4.145253000 & -0.955320000 \\
\hline $\mathrm{H}$ & -1.958442000 & 3.271409000 & 0.121382000 \\
\hline $\mathrm{O}$ & -0.301110000 & 1.622486000 & -1.108352000 \\
\hline $\mathrm{C}$ & 6.136998000 & -0.501201000 & 1.128003000 \\
\hline $\mathrm{H}$ & 6.641076000 & -1.468093000 & 1.249879000 \\
\hline $\mathrm{H}$ & 6.765317000 & 0.116622000 & 0.478400000 \\
\hline $\mathrm{H}$ & 6.105274000 & -0.025348000 & 2.114271000 \\
\hline $\mathrm{Li}$ & -1.940217000 & 0.726345000 & -1.375473000 \\
\hline
\end{tabular}

TSA2-3 ${ }_{\text {tBuOLi }}$

$\begin{array}{lccc} & & \\ & \mathrm{X} & \mathrm{Y} & \mathrm{Z} \\ \mathrm{C} & 5.690767000 & -0.683700000 & 0.302402000 \\ \mathrm{C} & 4.463250000 & -0.398012000 & 0.892050000 \\ \mathrm{C} & 3.261027000 & -0.699416000 & 0.233504000 \\ \mathrm{C} & 3.337717000 & -1.286339000 & -1.041973000 \\ \mathrm{C} & 4.564671000 & -1.577340000 & -1.619968000 \\ \mathrm{C} & 5.751896000 & -1.277025000 & -0.953546000 \\ \mathrm{H} & 6.606384000 & -0.444001000 & 0.835671000 \\ \mathrm{H} & 4.428085000 & 0.052021000 & 1.877916000 \\ \mathrm{H} & 2.428345000 & -1.509814000 & -1.591963000 \\ \mathrm{H} & 4.594612000 & -2.034489000 & -2.604794000 \\ \mathrm{H} & 6.710358000 & -1.498678000 & -1.412459000 \\ \mathrm{C} & 1.971998000 & -0.373602000 & 0.852714000 \\ \mathrm{C} & 0.726100000 & -0.772403000 & 0.489693000 \\ \mathrm{H} & 0.679781000 & -1.469500000 & -0.343370000 \\ \mathrm{O} & 2.099777000 & 0.563319000 & 1.916175000 \\ \mathrm{C} & -0.520504000 & -0.302284000 & 1.051869000\end{array}$




$\begin{array}{lrrr}\mathrm{H} & -0.503088000 & -0.123734000 & 2.135008000 \\ \mathrm{C} & -1.799031000 & -0.899151000 & 0.616731000 \\ \mathrm{C} & -2.912396000 & -0.885308000 & 1.473644000 \\ \mathrm{C} & -2.007057000 & -1.416255000 & -0.670708000 \\ \mathrm{C} & -4.150406000 & -1.365534000 & 1.070662000 \\ \mathrm{H} & -2.798126000 & -0.483042000 & 2.478198000 \\ \mathrm{C} & -3.248031000 & -1.905738000 & -1.067167000 \\ \mathrm{H} & -1.193329000 & -1.425798000 & -1.392074000 \\ \mathrm{C} & -4.345660000 & -1.893940000 & -0.208333000 \\ \mathrm{H} & -4.984246000 & -1.334933000 & 1.769385000 \\ \mathrm{H} & -3.363432000 & -2.300393000 & -2.074650000 \\ \mathrm{H} & -0.506851000 & 1.091206000 & 0.634842000 \\ \mathrm{H} & 1.416855000 & 0.374338000 & 2.574636000 \\ \mathrm{C} & -1.159638000 & 2.047237000 & -1.814547000 \\ \mathrm{C} & -1.113018000 & 2.838753000 & -0.502394000 \\ \mathrm{H} & -0.154184000 & 1.965622000 & -2.246349000 \\ \mathrm{H} & -1.545298000 & 1.037814000 & -1.637294000 \\ \mathrm{H} & -1.809430000 & 2.534653000 & -2.550109000 \\ \mathrm{C} & -2.504786000 & 2.883684000 & 0.137065000 \\ \mathrm{C} & -0.590771000 & 4.254596000 & -0.751072000 \\ \mathrm{H} & -2.463855000 & 3.425981000 & 1.087499000 \\ \mathrm{H} & -3.226397000 & 3.382717000 & -0.519452000 \\ \mathrm{H} & -2.865779000 & 1.868049000 & 0.333948000 \\ \mathrm{H} & -1.254267000 & 4.814464000 & -1.419420000 \\ \mathrm{H} & -0.512350000 & 4.799313000 & 0.195823000 \\ \mathrm{H} & 0.403301000 & 4.219499000 & -1.215527000 \\ \mathrm{O} & -0.213494000 & 2.213976000 & 0.396796000 \\ \mathrm{C} & -5.687250000 & -2.439742000 & -0.629242000 \\ \mathrm{H} & -5.883639000 & -3.415321000 & -0.167545000 \\ \mathrm{H} & -6.502058000 & -1.770123000 & -0.332445000 \\ \mathrm{H} & -5.741700000 & -2.572688000 & -1.714342000 \\ \mathrm{Li} & 1.525589000 & 1.835867000 & 0.542263000 \\ - & & & \\ & --2 & \end{array}$

\begin{tabular}{|c|c|c|c|}
\hline \multicolumn{4}{|c|}{ A3 tBuOLi $_{\text {. }}$} \\
\hline & \multicolumn{3}{|c|}{ Coordinates (Angstroms) } \\
\hline & $X$ & $\mathrm{Y}$ & Z \\
\hline$\overline{\mathrm{C}}$ & -5.049310000 & -0.598273000 & -0.575751000 \\
\hline $\mathrm{C}$ & -3.975709000 & -1.389697000 & -0.183456000 \\
\hline $\mathrm{C}$ & -2.700909000 & -0.828471000 & -0.029983000 \\
\hline $\mathrm{C}$ & -2.507746000 & 0.534161000 & -0.283556000 \\
\hline $\mathrm{C}$ & -3.589812000 & 1.316288000 & -0.677137000 \\
\hline $\mathrm{C}$ & -4.857225000 & 0.758479000 & -0.823238000 \\
\hline $\mathrm{H}$ & -6.035631000 & -1.040330000 & -0.681121000 \\
\hline
\end{tabular}




\begin{tabular}{|c|c|c|c|}
\hline $\mathrm{H}$ & -4.138072000 & -2.442155000 & 0.039120000 \\
\hline $\mathrm{H}$ & -1.509018000 & 0.979389000 & -0.213218000 \\
\hline $\mathrm{H}$ & -3.430720000 & 2.370668000 & -0.884019000 \\
\hline $\mathrm{H}$ & -5.693823000 & 1.378107000 & -1.133016000 \\
\hline $\mathrm{C}$ & -1.572251000 & -1.696508000 & 0.387402000 \\
\hline $\mathrm{C}$ & -0.650031000 & -1.362959000 & 1.302104000 \\
\hline $\mathrm{H}$ & -0.725976000 & -0.384600000 & 1.766836000 \\
\hline $\mathrm{O}$ & -1.525455000 & -2.941381000 & -0.184656000 \\
\hline $\mathrm{C}$ & 0.509914000 & -2.253319000 & 1.689388000 \\
\hline $\mathrm{H}$ & 0.254594000 & -3.303181000 & 1.520263000 \\
\hline $\mathrm{C}$ & 1.718424000 & -1.869718000 & 0.860871000 \\
\hline $\mathrm{C}$ & 1.844631000 & -2.338645000 & -0.453877000 \\
\hline $\mathrm{C}$ & 2.620103000 & -0.895609000 & 1.301398000 \\
\hline $\mathrm{C}$ & 2.820170000 & -1.822321000 & -1.305417000 \\
\hline $\mathrm{H}$ & 1.141267000 & -3.082633000 & -0.817651000 \\
\hline $\mathrm{C}$ & 3.595003000 & -0.384537000 & 0.447191000 \\
\hline $\mathrm{H}$ & 2.527388000 & -0.496983000 & 2.308008000 \\
\hline $\mathrm{C}$ & 3.706168000 & -0.828506000 & -0.873258000 \\
\hline $\mathrm{H}$ & 2.887420000 & -2.191090000 & -2.325837000 \\
\hline $\mathrm{H}$ & 4.261053000 & 0.395473000 & 0.805525000 \\
\hline $\mathrm{C}$ & 0.401610000 & 2.667300000 & 1.774749000 \\
\hline $\mathrm{C}$ & 0.625877000 & 2.839881000 & 0.257913000 \\
\hline $\mathrm{H}$ & -0.611565000 & 2.287329000 & 1.955849000 \\
\hline $\mathrm{H}$ & 1.116767000 & 1.933758000 & 2.170686000 \\
\hline $\mathrm{H}$ & 0.523618000 & 3.603632000 & 2.334391000 \\
\hline $\mathrm{C}$ & 2.059547000 & 3.352745000 & 0.019542000 \\
\hline $\mathrm{C}$ & -0.374958000 & 3.882470000 & -0.277826000 \\
\hline $\mathrm{H}$ & 2.232926000 & 3.472225000 & -1.056102000 \\
\hline $\mathrm{H}$ & 2.254738000 & 4.313660000 & 0.513404000 \\
\hline $\mathrm{H}$ & 2.779532000 & 2.615978000 & 0.399080000 \\
\hline $\mathrm{H}$ & -0.246053000 & 4.866972000 & 0.190060000 \\
\hline $\mathrm{H}$ & -0.249365000 & 3.990250000 & -1.360657000 \\
\hline $\mathrm{H}$ & -1.400870000 & 3.545370000 & -0.086189000 \\
\hline $\mathrm{O}$ & 0.440917000 & 1.635360000 & -0.380505000 \\
\hline $\mathrm{H}$ & 0.730669000 & -2.132117000 & 2.754750000 \\
\hline $\mathrm{H}$ & -2.144111000 & -2.956074000 & -0.925733000 \\
\hline $\mathrm{C}$ & 4.752152000 & -0.256090000 & -1.794093000 \\
\hline $\mathrm{H}$ & 4.853341000 & 0.823668000 & -1.649609000 \\
\hline $\mathrm{H}$ & 5.730973000 & -0.710940000 & -1.602130000 \\
\hline $\mathrm{H}$ & 4.502311000 & -0.436265000 & -2.843377000 \\
\hline $\mathrm{Li}$ & 0.937006000 & 0.029344000 & -0.411941000 \\
\hline
\end{tabular}

A1 $_{\text {TBD }}$ 


\begin{tabular}{|c|c|c|c|}
\hline \multicolumn{4}{|c|}{ Coordinates (Angstroms) } \\
\hline & $\mathrm{X}$ & Y & Z \\
\hline $\bar{C}$ & 3.183250000 & -0.814506000 & 2.427121000 \\
\hline $\mathrm{C}$ & 4.007194000 & -2.099359000 & 2.438040000 \\
\hline $\mathrm{C}$ & 5.128707000 & -1.966301000 & 1.418420000 \\
\hline $\mathrm{N}$ & 2.668311000 & -0.507919000 & 1.110285000 \\
\hline $\mathrm{H}$ & 3.805614000 & 0.014694000 & 2.804048000 \\
\hline $\mathrm{H}$ & 2.337677000 & -0.902460000 & 3.118489000 \\
\hline $\mathrm{H}$ & 3.360549000 & -2.941661000 & 2.165750000 \\
\hline $\mathrm{H}$ & 4.429505000 & -2.302469000 & 3.427680000 \\
\hline $\mathrm{H}$ & 5.918564000 & -1.303154000 & 1.803015000 \\
\hline $\mathrm{H}$ & 5.592542000 & -2.943959000 & 1.231592000 \\
\hline $\mathrm{N}$ & 4.629856000 & -1.452060000 & 0.151646000 \\
\hline $\mathrm{C}$ & 5.416667000 & -1.822603000 & -1.013276000 \\
\hline $\mathrm{C}$ & 5.016157000 & -1.035228000 & -2.251361000 \\
\hline $\mathrm{C}$ & 3.499451000 & -1.042451000 & -2.360285000 \\
\hline $\mathrm{N}$ & 2.964425000 & -0.445528000 & -1.159114000 \\
\hline $\mathrm{C}$ & 3.403766000 & -0.818199000 & 0.091815000 \\
\hline $\mathrm{H}$ & 5.303924000 & -2.902328000 & -1.207101000 \\
\hline $\mathrm{H}$ & 6.475860000 & -1.649864000 & -0.781127000 \\
\hline $\mathrm{H}$ & 5.361133000 & 0.000621000 & -2.167109000 \\
\hline $\mathrm{H}$ & 5.480505000 & -1.483419000 & -3.135164000 \\
\hline $\mathrm{H}$ & 3.166151000 & -0.446498000 & -3.215022000 \\
\hline $\mathrm{H}$ & 3.141114000 & -2.074266000 & -2.518201000 \\
\hline $\mathrm{H}$ & 1.982102000 & -0.181854000 & -1.178062000 \\
\hline $\mathrm{C}$ & -0.629503000 & 4.432637000 & -0.231739000 \\
\hline $\mathrm{C}$ & -0.945000000 & 3.077405000 & -0.281371000 \\
\hline $\mathrm{C}$ & -0.004014000 & 2.116788000 & 0.096552000 \\
\hline $\mathrm{C}$ & 1.261203000 & 2.528020000 & 0.523574000 \\
\hline $\mathrm{C}$ & 1.573237000 & 3.882796000 & 0.576445000 \\
\hline $\mathrm{C}$ & 0.630831000 & 4.837234000 & 0.198848000 \\
\hline $\mathrm{H}$ & -1.369925000 & 5.171794000 & -0.523829000 \\
\hline $\mathrm{H}$ & -1.934468000 & 2.760764000 & -0.604274000 \\
\hline $\mathrm{H}$ & 1.986634000 & 1.769553000 & 0.811865000 \\
\hline $\mathrm{H}$ & 2.556880000 & 4.196015000 & 0.915275000 \\
\hline $\mathrm{H}$ & 0.876345000 & 5.894553000 & 0.244267000 \\
\hline $\mathrm{C}$ & -0.308579000 & 0.632855000 & -0.032571000 \\
\hline $\mathrm{C}$ & -1.741734000 & 0.308139000 & 0.295947000 \\
\hline $\mathrm{H}$ & -2.077014000 & 0.682644000 & 1.262250000 \\
\hline $\mathrm{O}$ & 0.061722000 & 0.172004000 & -1.338755000 \\
\hline $\mathrm{C}$ & -2.566987000 & -0.368803000 & -0.504744000 \\
\hline $\mathrm{H}$ & -2.171399000 & -0.712245000 & -1.459242000 \\
\hline $\mathrm{C}$ & -3.975823000 & -0.705844000 & -0.240166000 \\
\hline $\mathrm{C}$ & -4.753042000 & -1.237440000 & -1.273970000 \\
\hline
\end{tabular}




\begin{tabular}{lrrr}
$\mathrm{C}$ & -4.593964000 & -0.517302000 & 1.003667000 \\
$\mathrm{C}$ & -6.093482000 & -1.554227000 & -1.082166000 \\
$\mathrm{H}$ & -4.299316000 & -1.398610000 & -2.249049000 \\
$\mathrm{C}$ & -5.930647000 & -0.834513000 & 1.192333000 \\
$\mathrm{H}$ & -4.023357000 & -0.123463000 & 1.839664000 \\
$\mathrm{C}$ & -6.707621000 & -1.358713000 & 0.153873000 \\
$\mathrm{H}$ & -6.670415000 & -1.960530000 & -1.909591000 \\
$\mathrm{H}$ & -6.383754000 & -0.675812000 & 2.168365000 \\
$\mathrm{H}$ & 0.360247000 & 0.084372000 & 0.642262000 \\
$\mathrm{H}$ & -0.237802000 & 0.840437000 & -1.969128000 \\
$\mathrm{C}$ & -8.153525000 & -1.721758000 & 0.379544000 \\
$\mathrm{H}$ & -8.689692000 & -1.828600000 & -0.568238000 \\
$\mathrm{H}$ & -8.241754000 & -2.673015000 & 0.918136000 \\
$\mathrm{H}$ & -8.667630000 & -0.960482000 & 0.975627000 \\
\hline-
\end{tabular}

TSA1-2 TBD

\begin{tabular}{|c|c|c|c|}
\hline & & nates (Angstrom & \\
\hline & $X$ & $\mathrm{Y}$ & $\mathrm{Z}$ \\
\hline $\bar{C}$ & -0.783723000 & 0.788744000 & 2.391008000 \\
\hline $\mathrm{C}$ & 0.573749000 & 0.112596000 & 2.535081000 \\
\hline $\mathrm{C}$ & 1.664846000 & 1.150974000 & 2.330543000 \\
\hline $\mathrm{N}$ & -0.892109000 & 1.472602000 & 1.114608000 \\
\hline $\mathrm{H}$ & -0.947200000 & 1.501355000 & 3.210297000 \\
\hline $\mathrm{H}$ & -1.595391000 & 0.055354000 & 2.430488000 \\
\hline $\mathrm{H}$ & 0.675970000 & -0.670504000 & 1.777294000 \\
\hline $\mathrm{H}$ & 0.686981000 & -0.347111000 & 3.521392000 \\
\hline $\mathrm{H}$ & 1.755333000 & 1.804052000 & 3.209137000 \\
\hline $\mathrm{H}$ & 2.629783000 & 0.654257000 & 2.180420000 \\
\hline $\mathrm{N}$ & 1.410200000 & 1.973918000 & 1.147290000 \\
\hline $\mathrm{C}$ & 2.606715000 & 2.479141000 & 0.480731000 \\
\hline $\mathrm{C}$ & 2.261089000 & 3.505636000 & -0.585660000 \\
\hline $\mathrm{C}$ & 1.174240000 & 2.933131000 & -1.481023000 \\
\hline $\mathrm{N}$ & 0.036800000 & 2.587889000 & -0.652689000 \\
\hline $\mathrm{C}$ & 0.193250000 & 1.981392000 & 0.553436000 \\
\hline $\mathrm{H}$ & 3.147387000 & 1.631810000 & 0.034905000 \\
\hline $\mathrm{H}$ & 3.252847000 & 2.922540000 & 1.246859000 \\
\hline $\mathrm{H}$ & 1.900206000 & 4.428904000 & -0.120231000 \\
\hline $\mathrm{H}$ & 3.156911000 & 3.742542000 & -1.166429000 \\
\hline $\mathrm{H}$ & 0.844275000 & 3.668144000 & -2.219467000 \\
\hline $\mathrm{H}$ & 1.557734000 & 2.055983000 & -2.022665000 \\
\hline $\mathrm{H}$ & -0.810949000 & 2.293363000 & -1.138572000 \\
\hline $\mathrm{C}$ & -4.877925000 & -1.968999000 & 0.931592000 \\
\hline $\mathrm{C}$ & -3.602549000 & -1.552983000 & 0.574778000 \\
\hline
\end{tabular}




\begin{tabular}{lrrr}
$\mathrm{C}$ & -3.401063000 & -0.564824000 & -0.407463000 \\
$\mathrm{C}$ & -4.546098000 & -0.007023000 & -0.997445000 \\
$\mathrm{C}$ & -5.822755000 & -0.425298000 & -0.636558000 \\
$\mathrm{C}$ & -6.003372000 & -1.410830000 & 0.328343000 \\
$\mathrm{H}$ & -4.993366000 & -2.737815000 & 1.691513000 \\
$\mathrm{H}$ & -2.749994000 & -2.005236000 & 1.075753000 \\
$\mathrm{H}$ & -4.421555000 & 0.771457000 & -1.742273000 \\
$\mathrm{H}$ & -6.686160000 & 0.028306000 & -1.116860000 \\
$\mathrm{C}$ & -2.055560000 & -0.067123000 & -0.758486000 \\
$\mathrm{C}$ & -0.891843000 & -0.910614000 & -0.693186000 \\
$\mathrm{H}$ & -0.952670000 & -1.718257000 & 0.034717000 \\
$\mathrm{O}$ & -2.037963000 & 0.890338000 & -1.817074000 \\
$\mathrm{C}$ & 0.303270000 & -0.671003000 & -1.290892000 \\
$\mathrm{H}$ & 0.356648000 & 0.136172000 & -2.018511000 \\
$\mathrm{C}$ & 1.580105000 & -1.300965000 & -0.966620000 \\
$\mathrm{C}$ & 2.760353000 & -0.809274000 & -1.547809000 \\
$\mathrm{C}$ & 1.731191000 & -2.349445000 & -0.039525000 \\
$\mathrm{C}$ & 4.012725000 & -1.308947000 & -1.206854000 \\
$\mathrm{H}$ & 2.690511000 & -0.015003000 & -2.288853000 \\
$\mathrm{C}$ & 2.982212000 & -2.844107000 & 0.298480000 \\
$\mathrm{H}$ & 0.853915000 & -2.791955000 & 0.424655000 \\
$\mathrm{C}$ & 4.153318000 & -2.331814000 & -0.269526000 \\
$\mathrm{H}$ & 4.898973000 & -0.893856000 & -1.682664000 \\
$\mathrm{H}$ & 3.052334000 & -3.652923000 & 1.023310000 \\
$\mathrm{H}$ & -1.564600000 & 0.849022000 & 0.344188000 \\
$\mathrm{H}$ & -2.010060000 & 0.412857000 & -2.659891000 \\
$\mathrm{H}$ & -7.000526000 & -1.736106000 & 0.609550000 \\
$\mathrm{C}$ & 5.505227000 & -2.875981000 & 0.118076000 \\
$\mathrm{H}$ & 5.699148000 & -2.740290000 & 1.188801000 \\
$\mathrm{H}$ & 5.579757000 & -3.949651000 & -0.089946000 \\
$\mathrm{H}$ & 6.308818000 & -2.375281000 & -0.430935000 \\
\hline-----
\end{tabular}

A2 TBD

\begin{tabular}{llcc} 
& \multicolumn{3}{c}{ Coordinates (Angstroms) } \\
C & X & Y & Z \\
C & -2.822043000 & 1.181992000 & -1.634259000 \\
$\mathrm{C}$ & -2.520619000 & 2.914366000 & 0.102441000 \\
$\mathrm{~N}$ & -1.384765000 & 0.959530000 & -1.556120000 \\
$\mathrm{H}$ & -3.043105000 & 1.899080000 & -2.435532000 \\
$\mathrm{H}$ & -3.282933000 & 0.225469000 & -1.883896000 \\
$\mathrm{H}$ & -3.234596000 & 0.903666000 & 0.454538000 \\
$\mathrm{H}$ & -4.390648000 & 1.961416000 & -0.371389000
\end{tabular}




\begin{tabular}{|c|c|c|c|}
\hline $\mathrm{H}$ & -2.759221000 & 3.775619000 & -0.536484000 \\
\hline $\mathrm{H}$ & -2.741681000 & 3.197048000 & 1.137245000 \\
\hline $\mathrm{N}$ & -1.084831000 & 2.639734000 & 0.029477000 \\
\hline $\mathrm{C}$ & -0.234524000 & 3.439353000 & 0.909517000 \\
\hline $\mathrm{C}$ & 1.239258000 & 3.297571000 & 0.555453000 \\
\hline $\mathrm{C}$ & 1.574755000 & 1.823127000 & 0.400536000 \\
\hline $\mathrm{N}$ & 0.688345000 & 1.259832000 & -0.604451000 \\
\hline $\mathrm{C}$ & -0.597027000 & 1.610347000 & -0.691993000 \\
\hline $\mathrm{H}$ & -0.410417000 & 3.130322000 & 1.949067000 \\
\hline $\mathrm{H}$ & -0.554339000 & 4.483354000 & 0.816737000 \\
\hline $\mathrm{H}$ & 1.451739000 & 3.819714000 & -0.383536000 \\
\hline $\mathrm{H}$ & 1.846333000 & 3.753926000 & 1.341637000 \\
\hline $\mathrm{H}$ & 2.600176000 & 1.666358000 & 0.059350000 \\
\hline $\mathrm{H}$ & 1.464173000 & 1.292225000 & 1.355341000 \\
\hline $\mathrm{H}$ & 0.921426000 & 0.292699000 & -0.910976000 \\
\hline $\mathrm{C}$ & -3.448058000 & -1.356153000 & 2.323917000 \\
\hline $\mathrm{C}$ & -2.279731000 & -1.507566000 & 1.591810000 \\
\hline $\mathrm{C}$ & -2.307144000 & -1.827664000 & 0.213413000 \\
\hline $\mathrm{C}$ & -3.583547000 & -1.979071000 & -0.373947000 \\
\hline $\mathrm{C}$ & -4.748207000 & -1.809583000 & 0.364440000 \\
\hline $\mathrm{C}$ & -4.699006000 & -1.494319000 & 1.720549000 \\
\hline $\mathrm{H}$ & -3.380415000 & -1.115547000 & 3.382320000 \\
\hline $\mathrm{H}$ & -1.326603000 & -1.362241000 & 2.092086000 \\
\hline $\mathrm{H}$ & -3.643097000 & -2.236823000 & -1.426100000 \\
\hline $\mathrm{H}$ & -5.709851000 & -1.935425000 & -0.127541000 \\
\hline $\mathrm{C}$ & -1.112702000 & -1.919280000 & -0.591174000 \\
\hline $\mathrm{C}$ & 0.191629000 & -1.819121000 & -0.172242000 \\
\hline $\mathrm{H}$ & 0.342475000 & -1.853992000 & 0.904450000 \\
\hline $\mathrm{O}$ & -1.352413000 & -1.834012000 & -1.984510000 \\
\hline $\mathrm{C}$ & 1.324069000 & -1.605014000 & -0.973453000 \\
\hline $\mathrm{H}$ & 1.218513000 & -1.606679000 & -2.058154000 \\
\hline $\mathrm{C}$ & 2.657177000 & -1.349182000 & -0.470544000 \\
\hline $\mathrm{C}$ & 3.666957000 & -0.893143000 & -1.348312000 \\
\hline $\mathrm{C}$ & 3.020305000 & -1.420383000 & 0.893609000 \\
\hline $\mathrm{C}$ & 4.920946000 & -0.510984000 & -0.895245000 \\
\hline $\mathrm{H}$ & 3.441479000 & -0.819261000 & -2.410698000 \\
\hline $\mathrm{C}$ & 4.277686000 & -1.032514000 & 1.336839000 \\
\hline $\mathrm{H}$ & 2.305516000 & -1.789229000 & 1.624412000 \\
\hline $\mathrm{C}$ & 5.258675000 & -0.562458000 & 0.460537000 \\
\hline $\mathrm{H}$ & 5.658197000 & -0.158607000 & -1.614998000 \\
\hline $\mathrm{H}$ & 4.504342000 & -1.104141000 & 2.399677000 \\
\hline $\mathrm{H}$ & -1.061282000 & 0.052523000 & -1.903122000 \\
\hline $\mathrm{H}$ & -0.766698000 & -2.469257000 & -2.416683000 \\
\hline $\mathrm{H}$ & -5.611044000 & -1.365424000 & 2.295179000 \\
\hline
\end{tabular}




$\begin{array}{llrl}\mathrm{C} & 6.630990000 & -0.169853000 & 0.947942000 \\ \mathrm{H} & 7.310460000 & -1.031281000 & 0.986421000 \\ \mathrm{H} & 7.090339000 & 0.574761000 & 0.288517000 \\ \mathrm{H} & 6.591547000 & 0.256510000 & 1.956533000\end{array}$

TSA2-3 TBD

\begin{tabular}{lrcc} 
& Coordinates (Angstroms) & \\
& $\mathrm{X}$ & $\mathrm{Y}$ & $\mathrm{Z}$ \\
\hline $\mathrm{C}$ & -2.901539000 & 1.368132000 & -1.390478000 \\
$\mathrm{C}$ & -3.285094000 & 1.743478000 & 0.031034000 \\
$\mathrm{C}$ & -2.494757000 & 2.977884000 & 0.437014000 \\
$\mathrm{~N}$ & -1.455782000 & 1.249153000 & -1.501617000 \\
$\mathrm{H}$ & -3.269935000 & 2.124159000 & -2.097042000 \\
$\mathrm{H}$ & -3.334307000 & 0.404178000 & -1.666075000 \\
$\mathrm{H}$ & -3.057774000 & 0.907659000 & 0.702278000 \\
$\mathrm{H}$ & -4.356726000 & 1.949815000 & 0.098172000 \\
$\mathrm{H}$ & -2.839819000 & 3.861507000 & -0.119537000 \\
$\mathrm{H}$ & -2.634668000 & 3.186744000 & 1.503887000 \\
$\mathrm{~N}$ & -1.066597000 & 2.778266000 & 0.216267000 \\
$\mathrm{C}$ & -0.169949000 & 3.588178000 & 1.033147000 \\
$\mathrm{C}$ & 1.257803000 & 3.529734000 & 0.509468000 \\
$\mathrm{C}$ & 1.622822000 & 2.076941000 & 0.242861000 \\
$\mathrm{~N}$ & 0.683023000 & 1.501245000 & -0.704121000 \\
$\mathrm{C}$ & -0.593084000 & 1.837671000 & -0.642426000 \\
$\mathrm{H}$ & -0.210792000 & 3.234153000 & 2.073384000 \\
$\mathrm{H}$ & -0.545448000 & 4.618811000 & 1.022874000 \\
$\mathrm{H}$ & 1.338651000 & 4.100639000 & -0.422079000 \\
$\mathrm{H}$ & 1.934850000 & 3.978699000 & 1.241855000 \\
$\mathrm{H}$ & 2.624092000 & 1.983914000 & -0.184734000 \\
$\mathrm{H}$ & 1.626182000 & 1.506236000 & 1.182970000 \\
$\mathrm{H}$ & 0.941325000 & 0.351135000 & -0.963874000 \\
$\mathrm{C}$ & -3.549142000 & -1.816936000 & 2.195349000 \\
$\mathrm{C}$ & -2.366344000 & -1.716168000 & 1.476244000 \\
$\mathrm{C}$ & -2.343617000 & -1.899747000 & 0.080221000 \\
$\mathrm{C}$ & -3.566903000 & -2.171312000 & -0.556401000 \\
$\mathrm{C}$ & -4.751572000 & -2.256829000 & 0.167225000 \\
$\mathrm{C}$ & -4.755056000 & -2.082267000 & 1.547728000 \\
$\mathrm{H}$ & -3.530736000 & -1.673052000 & 3.272480000 \\
$\mathrm{H}$ & -1.448457000 & -1.475119000 & 2.003982000 \\
$\mathrm{H}$ & -3.575120000 & -2.316238000 & -1.631127000 \\
$\mathrm{C}$ & -5.680989000 & -2.467441000 & -0.355295000 \\
& -1.121894000 & -1.742469000 & -0.704390000 \\
$\mathrm{H}$ & 0.140901000 & -1.591168000 & -0.241500000
\end{tabular}




\begin{tabular}{lrrr}
$\mathrm{H}$ & 0.284280000 & -1.747306000 & 0.823972000 \\
$\mathrm{O}$ & -1.366103000 & -1.565566000 & -2.076641000 \\
$\mathrm{C}$ & 1.270377000 & -1.124812000 & -1.010147000 \\
$\mathrm{H}$ & 1.235101000 & -1.297732000 & -2.089750000 \\
$\mathrm{C}$ & 2.638109000 & -1.136688000 & -0.474592000 \\
$\mathrm{C}$ & 3.732668000 & -0.979687000 & -1.347691000 \\
$\mathrm{C}$ & 2.944462000 & -1.182365000 & 0.896529000 \\
$\mathrm{C}$ & 5.033993000 & -0.869165000 & -0.882492000 \\
$\mathrm{H}$ & 3.546050000 & -0.939071000 & -2.419093000 \\
$\mathrm{C}$ & 4.253144000 & -1.074561000 & 1.357378000 \\
$\mathrm{H}$ & 2.150308000 & -1.299209000 & 1.629483000 \\
$\mathrm{C}$ & 5.327297000 & -0.912052000 & 0.484382000 \\
$\mathrm{H}$ & 5.844899000 & -0.751660000 & -1.599457000 \\
$\mathrm{H}$ & 4.439648000 & -1.120982000 & 2.428911000 \\
$\mathrm{H}$ & -1.125693000 & 0.391178000 & -1.938540000 \\
$\mathrm{H}$ & -0.646060000 & -1.998182000 & -2.553895000 \\
$\mathrm{H}$ & -5.680834000 & -2.150560000 & 2.110933000 \\
$\mathrm{C}$ & 6.745220000 & -0.782009000 & 0.982271000 \\
$\mathrm{H}$ & 6.804926000 & -0.951601000 & 2.062179000 \\
$\mathrm{H}$ & 7.409814000 & -1.505673000 & 0.495930000 \\
$\mathrm{H}$ & 7.154489000 & 0.216179000 & 0.782225000 \\
\hline-----------
\end{tabular}

\begin{tabular}{|c|c|c|c|}
\hline \multicolumn{4}{|c|}{ A3 ${ }_{\text {TBD }}$} \\
\hline & $\mathrm{X}$ & $\mathrm{Y}$ & Z \\
\hline $\mathrm{C}$ & 0.086419000 & -2.432331000 & 1.093747000 \\
\hline $\mathrm{C}$ & -0.747793000 & -1.819552000 & 2.209957000 \\
\hline $\mathrm{C}$ & -2.224815000 & -1.861794000 & 1.833958000 \\
\hline $\mathrm{N}$ & -0.206415000 & -1.713787000 & -0.119760000 \\
\hline $\mathrm{H}$ & -0.125736000 & -3.512942000 & 1.012197000 \\
\hline $\mathrm{H}$ & 1.154043000 & -2.320665000 & 1.307459000 \\
\hline $\mathrm{H}$ & -0.428696000 & -0.784003000 & 2.349654000 \\
\hline $\mathrm{H}$ & -0.599177000 & -2.352089000 & 3.154833000 \\
\hline $\mathrm{H}$ & -2.646138000 & -2.847733000 & 2.089342000 \\
\hline $\mathrm{H}$ & -2.775345000 & -1.118119000 & 2.427784000 \\
\hline $\mathrm{N}$ & -2.471586000 & -1.598026000 & 0.427247000 \\
\hline $\mathrm{C}$ & -3.855244000 & -1.329444000 & 0.082428000 \\
\hline $\mathrm{C}$ & -4.102917000 & -1.620993000 & -1.390591000 \\
\hline $\mathrm{C}$ & -3.031728000 & -0.913255000 & -2.218477000 \\
\hline $\mathrm{N}$ & -1.686919000 & -1.236477000 & -1.794528000 \\
\hline $\mathrm{C}$ & -1.500118000 & -1.511014000 & -0.548137000 \\
\hline $\mathrm{H}$ & -4.098943000 & -0.280678000 & 0.313648000 \\
\hline $\mathrm{H}$ & -4.492841000 & -1.963555000 & 0.712740000 \\
\hline
\end{tabular}




\begin{tabular}{|c|c|c|c|}
\hline $\mathrm{H}$ & -4.041429000 & -2.702569000 & -1.561371000 \\
\hline $\mathrm{H}$ & -5.109262000 & -1.291725000 & -1.671435000 \\
\hline $\mathrm{H}$ & -3.136687000 & -1.185582000 & -3.275594000 \\
\hline $\mathrm{H}$ & -3.196321000 & 0.174176000 & -2.157692000 \\
\hline $\mathrm{C}$ & 5.927478000 & 1.280037000 & 0.399423000 \\
\hline $\mathrm{C}$ & 4.696273000 & 1.344030000 & -0.241726000 \\
\hline $\mathrm{C}$ & 3.884015000 & 0.208613000 & -0.337405000 \\
\hline $\mathrm{C}$ & 4.347100000 & -0.996719000 & 0.204679000 \\
\hline $\mathrm{C}$ & 5.582125000 & -1.060808000 & 0.841312000 \\
\hline $\mathrm{C}$ & 6.375870000 & 0.077949000 & 0.942211000 \\
\hline $\mathrm{H}$ & 6.546760000 & 2.169940000 & 0.462676000 \\
\hline $\mathrm{H}$ & 4.363349000 & 2.274731000 & -0.691007000 \\
\hline $\mathrm{H}$ & 3.721721000 & -1.883956000 & 0.149138000 \\
\hline $\mathrm{H}$ & 5.921977000 & -2.001069000 & 1.265369000 \\
\hline $\mathrm{C}$ & 2.560059000 & 0.262740000 & -1.001213000 \\
\hline $\mathrm{C}$ & 1.724646000 & 1.303517000 & -0.927641000 \\
\hline $\mathrm{H}$ & 2.038360000 & 2.142264000 & -0.310550000 \\
\hline $\mathrm{O}$ & 2.183432000 & -0.885352000 & -1.664160000 \\
\hline $\mathrm{C}$ & 0.378466000 & 1.398901000 & -1.580521000 \\
\hline $\mathrm{H}$ & 0.118633000 & 0.452794000 & -2.070058000 \\
\hline $\mathrm{C}$ & -0.750067000 & 1.745642000 & -0.629264000 \\
\hline $\mathrm{C}$ & -1.938807000 & 2.288325000 & -1.119761000 \\
\hline $\mathrm{C}$ & -0.673327000 & 1.479242000 & 0.736709000 \\
\hline $\mathrm{C}$ & -3.021843000 & 2.527566000 & -0.281090000 \\
\hline $\mathrm{H}$ & -2.026560000 & 2.505988000 & -2.181915000 \\
\hline $\mathrm{C}$ & -1.755934000 & 1.718397000 & 1.576741000 \\
\hline $\mathrm{H}$ & 0.239833000 & 1.051594000 & 1.139679000 \\
\hline $\mathrm{C}$ & -2.952125000 & 2.240994000 & 1.083862000 \\
\hline $\mathrm{H}$ & -3.939124000 & 2.939876000 & -0.695881000 \\
\hline $\mathrm{H}$ & -1.669362000 & 1.490251000 & 2.637718000 \\
\hline $\mathrm{H}$ & 0.469505000 & -1.723168000 & -0.872242000 \\
\hline $\mathrm{H}$ & 2.975313000 & -1.320225000 & -2.003858000 \\
\hline $\mathrm{H}$ & 0.417393000 & 2.164520000 & -2.368777000 \\
\hline $\mathrm{H}$ & 7.341153000 & 0.028373000 & 1.436765000 \\
\hline $\mathrm{C}$ & -4.118201000 & 2.512473000 & 2.001887000 \\
\hline $\mathrm{H}$ & -4.188389000 & 1.755186000 & 2.790029000 \\
\hline $\mathrm{H}$ & -4.018451000 & 3.487867000 & 2.493483000 \\
\hline $\mathrm{H}$ & -5.065794000 & 2.516274000 & 1.453898000 \\
\hline
\end{tabular}

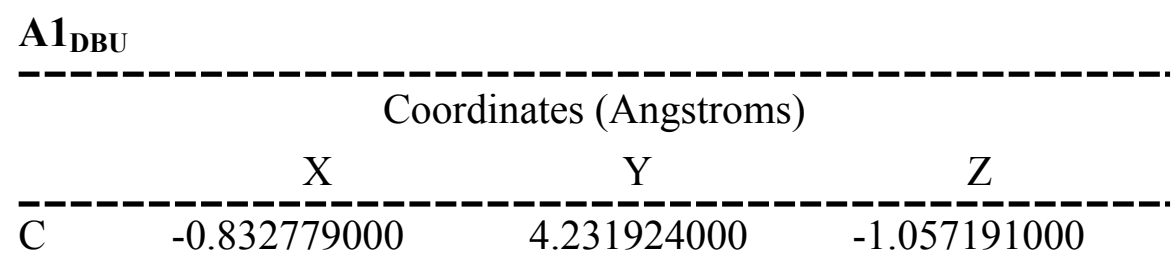




\begin{tabular}{|c|c|c|c|}
\hline $\mathrm{C}$ & -1.189768000 & 2.896176000 & -0.887842000 \\
\hline $\mathrm{C}$ & -0.245332000 & 1.956299000 & -0.470754000 \\
\hline $\mathrm{C}$ & 1.065612000 & 2.373540000 & -0.227313000 \\
\hline $\mathrm{C}$ & 1.422354000 & 3.707281000 & -0.392780000 \\
\hline $\mathrm{C}$ & 0.474366000 & 4.640045000 & -0.809162000 \\
\hline $\mathrm{H}$ & -1.577421000 & 4.954249000 & -1.379202000 \\
\hline $\mathrm{H}$ & -2.214316000 & 2.580579000 & -1.071333000 \\
\hline $\mathrm{H}$ & 1.793064000 & 1.633246000 & 0.095791000 \\
\hline $\mathrm{H}$ & 2.444030000 & 4.021695000 & -0.196900000 \\
\hline $\mathrm{H}$ & 0.752725000 & 5.682146000 & -0.938020000 \\
\hline $\mathrm{C}$ & -0.594139000 & 0.478287000 & -0.364909000 \\
\hline $\mathrm{C}$ & -2.009430000 & 0.255364000 & 0.103355000 \\
\hline $\mathrm{H}$ & -2.268751000 & 0.765048000 & 1.030721000 \\
\hline $\mathrm{O}$ & -0.319252000 & -0.178367000 & -1.600475000 \\
\hline $\mathrm{C}$ & -2.904924000 & -0.494160000 & -0.542066000 \\
\hline $\mathrm{H}$ & -2.588067000 & -0.968834000 & -1.469312000 \\
\hline $\mathrm{C}$ & -4.297417000 & -0.748433000 & -0.136299000 \\
\hline $\mathrm{C}$ & -5.197211000 & -1.269910000 & -1.073500000 \\
\hline $\mathrm{C}$ & -4.780239000 & -0.493561000 & 1.152621000 \\
\hline $\mathrm{C}$ & -6.525446000 & -1.507982000 & -0.744904000 \\
\hline $\mathrm{H}$ & -4.847813000 & -1.483153000 & -2.081084000 \\
\hline $\mathrm{C}$ & -6.108744000 & -0.733739000 & 1.478850000 \\
\hline $\mathrm{H}$ & -4.107125000 & -0.115245000 & 1.916682000 \\
\hline $\mathrm{C}$ & -7.006912000 & -1.243968000 & 0.538825000 \\
\hline $\mathrm{H}$ & -7.201267000 & -1.906054000 & -1.498543000 \\
\hline $\mathrm{H}$ & -6.454625000 & -0.527113000 & 2.489063000 \\
\hline $\mathrm{H}$ & 0.099094000 & 0.017491000 & 0.351254000 \\
\hline $\mathrm{H}$ & -0.687115000 & 0.374315000 & -2.302491000 \\
\hline $\mathrm{C}$ & 5.659074000 & -1.566418000 & 0.147108000 \\
\hline $\mathrm{C}$ & 5.302599000 & -2.819073000 & -0.647071000 \\
\hline $\mathrm{C}$ & 2.734449000 & -1.540252000 & -0.776232000 \\
\hline $\mathrm{C}$ & 4.718874000 & -2.522891000 & -2.030573000 \\
\hline $\mathrm{C}$ & 3.668715000 & -1.410488000 & -1.980809000 \\
\hline $\mathrm{H}$ & 6.204057000 & -0.859889000 & -0.500819000 \\
\hline $\mathrm{H}$ & 2.504061000 & -2.597944000 & -0.591176000 \\
\hline $\mathrm{H}$ & 4.611084000 & -3.426513000 & -0.052298000 \\
\hline $\mathrm{H}$ & 4.269512000 & -3.440079000 & -2.430424000 \\
\hline $\mathrm{H}$ & 6.360984000 & -1.856315000 & 0.940018000 \\
\hline $\mathrm{H}$ & 6.215697000 & -3.415131000 & -0.756066000 \\
\hline $\mathrm{H}$ & 1.777754000 & -1.060661000 & -0.990227000 \\
\hline $\mathrm{H}$ & 5.524835000 & -2.242321000 & -2.720026000 \\
\hline $\mathrm{H}$ & 3.073933000 & -1.424007000 & -2.899988000 \\
\hline $\mathrm{H}$ & 4.159973000 & -0.429562000 & -1.951829000 \\
\hline $\mathrm{C}$ & 3.218127000 & -0.905636000 & 0.517313000 \\
\hline
\end{tabular}




\begin{tabular}{|c|c|c|c|}
\hline $\mathrm{N}$ & 2.289798000 & -0.399156000 & 1.255734000 \\
\hline $\mathrm{N}$ & 4.563741000 & -0.875866000 & 0.824199000 \\
\hline $\mathrm{C}$ & 5.049125000 & -0.029128000 & 1.911205000 \\
\hline $\mathrm{H}$ & 5.363878000 & -0.664305000 & 2.753444000 \\
\hline $\mathrm{H}$ & 5.944648000 & 0.500569000 & 1.558899000 \\
\hline $\mathrm{C}$ & 3.996804000 & 0.965516000 & 2.370181000 \\
\hline $\mathrm{H}$ & 4.309811000 & 1.426973000 & 3.312173000 \\
\hline $\mathrm{H}$ & 3.887372000 & 1.765210000 & 1.627501000 \\
\hline $\mathrm{C}$ & 2.669327000 & 0.228598000 & 2.505196000 \\
\hline $\mathrm{H}$ & 1.871283000 & 0.919134000 & 2.798724000 \\
\hline $\mathrm{H}$ & 2.745847000 & -0.528099000 & 3.301765000 \\
\hline $\mathrm{C}$ & -8.441952000 & -1.531847000 & 0.901564000 \\
\hline $\mathrm{H}$ & -9.124538000 & -1.227367000 & 0.101240000 \\
\hline $\mathrm{H}$ & -8.597844000 & -2.603748000 & 1.073227000 \\
\hline $\mathrm{H}$ & -8.736303000 & -1.005483000 & 1.814621000 \\
\hline
\end{tabular}

TSA1-2 DBU

$\begin{array}{lrcc} & & \text { Coordinates (Angstroms) } \\ & \mathrm{X} & \mathrm{Y} & \mathrm{Z} \\ \mathrm{C} & 2.049145000 & 1.896918000 & 2.340873000 \\ \mathrm{C} & 1.012035000 & 1.483237000 & 1.514600000 \\ \mathrm{C} & 0.843063000 & 2.010573000 & 0.213195000 \\ \mathrm{C} & 1.796547000 & 2.963288000 & -0.208283000 \\ \mathrm{C} & 2.831374000 & 3.371328000 & 0.622831000 \\ \mathrm{C} & 2.976464000 & 2.842996000 & 1.905587000 \\ \mathrm{H} & 2.135110000 & 1.471974000 & 3.338683000 \\ \mathrm{H} & 0.305788000 & 0.743678000 & 1.886416000 \\ \mathrm{H} & 1.701288000 & 3.375411000 & -1.207547000 \\ \mathrm{H} & 3.536868000 & 4.117382000 & 0.263726000 \\ \mathrm{H} & 3.787143000 & 3.164472000 & 2.552429000 \\ \mathrm{C} & -0.173895000 & 1.503735000 & -0.697695000 \\ \mathrm{C} & -1.474013000 & 1.116851000 & -0.247986000 \\ \mathrm{H} & -1.580260000 & 1.007704000 & 0.831959000 \\ \mathrm{O} & -0.086008000 & 1.962745000 & -2.029319000 \\ \mathrm{C} & -2.550522000 & 0.839157000 & -1.034810000 \\ \mathrm{H} & -2.423795000 & 0.896625000 & -2.114404000 \\ \mathrm{C} & -3.857877000 & 0.391908000 & -0.567349000 \\ \mathrm{C} & -4.808800000 & -0.065085000 & -1.496702000 \\ \mathrm{C} & -4.244458000 & 0.367970000 & 0.785573000 \\ \mathrm{C} & -6.056551000 & -0.528124000 & -1.101094000 \\ \mathrm{H} & -4.552405000 & -0.062006000 & -2.554024000 \\ \mathrm{C} & -5.490919000 & -0.102049000 & 1.175954000 \\ \mathrm{H} & -3.564284000 & 0.736640000 & 1.548903000\end{array}$




\begin{tabular}{lrrr}
$\mathrm{C}$ & -6.426378000 & -0.559774000 & 0.244765000 \\
$\mathrm{H}$ & -6.759454000 & -0.875802000 & -1.855936000 \\
$\mathrm{H}$ & -5.747644000 & -0.104631000 & 2.233942000 \\
$\mathrm{H}$ & 0.372014000 & -0.101453000 & -0.795311000 \\
$\mathrm{H}$ & -0.759505000 & 2.651562000 & -2.144092000 \\
$\mathrm{C}$ & 4.131446000 & -2.409191000 & 0.293643000 \\
$\mathrm{C}$ & 4.982194000 & -2.033178000 & -0.913773000 \\
$\mathrm{C}$ & 2.828591000 & -0.205262000 & -1.378000000 \\
$\mathrm{C}$ & 5.319703000 & -0.541984000 & -0.994578000 \\
$\mathrm{C}$ & 4.101231000 & 0.348210000 & -0.736451000 \\
$\mathrm{H}$ & 4.519140000 & -1.920304000 & 1.198464000 \\
$\mathrm{H}$ & 3.041427000 & -0.666727000 & -2.350782000 \\
$\mathrm{H}$ & 4.476080000 & -2.374278000 & -1.824133000 \\
$\mathrm{H}$ & 5.733148000 & -0.332581000 & -1.988189000 \\
$\mathrm{H}$ & 4.216103000 & -3.489435000 & 0.454950000 \\
$\mathrm{H}$ & 5.908934000 & -2.613936000 & -0.850394000 \\
$\mathrm{H}$ & 2.125031000 & 0.603497000 & -1.585694000 \\
$\mathrm{H}$ & 6.108239000 & -0.303150000 & -0.271419000 \\
$\mathrm{H}$ & 4.278345000 & 1.353285000 & -1.126855000 \\
$\mathrm{H}$ & 3.926915000 & 0.485053000 & 0.337087000 \\
$\mathrm{C}$ & 2.077226000 & -1.210151000 & -0.538732000 \\
$\mathrm{~N}$ & 0.767033000 & -1.117853000 & -0.599857000 \\
$\mathrm{~N}$ & 2.685936000 & -2.148725000 & 0.205499000 \\
$\mathrm{C}$ & 1.916817000 & -2.974979000 & 1.147969000 \\
$\mathrm{H}$ & 1.801529000 & -3.979284000 & 0.719283000 \\
$\mathrm{H}$ & 2.515368000 & -3.071127000 & 2.059742000 \\
$\mathrm{C}$ & 0.564217000 & -2.364754000 & 1.470660000 \\
$\mathrm{H}$ & -0.036715000 & -3.080664000 & 2.037374000 \\
$\mathrm{H}$ & 0.701982000 & -1.470474000 & 2.086627000 \\
$\mathrm{C}$ & -0.116750000 & -1.979876000 & 0.167286000 \\
$\mathrm{H}$ & -1.046214000 & -1.429395000 & 0.328374000 \\
$\mathrm{H}$ & -0.357625000 & -2.871040000 & -0.424578000 \\
$\mathrm{C}$ & -7.792688000 & -1.033088000 & 0.673989000 \\
$\mathrm{H}$ & -7.761865000 & -1.497995000 & 1.665211000 \\
& -8.508270000 & -0.202513000 & 0.725477000 \\
---8.198350000 & -1.768463000 & -0.029070000 \\
\hline
\end{tabular}

\begin{tabular}{|c|c|c|c|}
\hline \multicolumn{4}{|c|}{ A2 } \\
\hline & \multicolumn{3}{|c|}{ Coordinates (Angstroms) } \\
\hline & $\mathrm{X}$ & $\mathrm{Y}$ & Z \\
\hline$\overline{\mathrm{C}}$ & -2.955921000 & -2.088109000 & 1.965255000 \\
\hline $\mathrm{C}$ & -1.808897000 & -1.940140000 & 1.199340000 \\
\hline $\mathrm{C}$ & -1.859356000 & -1.878367000 & -0.214436000 \\
\hline
\end{tabular}




\begin{tabular}{|c|c|c|c|}
\hline $\mathrm{C}$ & -3.138401000 & -2.032495000 & -0.799341000 \\
\hline $\mathrm{C}$ & -4.282951000 & -2.168820000 & -0.024839000 \\
\hline $\mathrm{C}$ & -4.213657000 & -2.181951000 & 1.367699000 \\
\hline $\mathrm{H}$ & -2.864552000 & -2.137490000 & 3.048355000 \\
\hline $\mathrm{H}$ & -0.851974000 & -1.886196000 & 1.708911000 \\
\hline $\mathrm{H}$ & -3.213084000 & -2.028423000 & -1.881235000 \\
\hline $\mathrm{H}$ & -5.245674000 & -2.274031000 & -0.519375000 \\
\hline $\mathrm{H}$ & -5.110276000 & -2.290512000 & 1.969972000 \\
\hline $\mathrm{C}$ & -0.707700000 & -1.598735000 & -1.039619000 \\
\hline $\mathrm{C}$ & 0.587849000 & -1.406649000 & -0.610656000 \\
\hline $\mathrm{H}$ & 0.773308000 & -1.631562000 & 0.437078000 \\
\hline $\mathrm{O}$ & -0.985508000 & -1.408314000 & -2.400531000 \\
\hline $\mathrm{C}$ & 1.661291000 & -0.892053000 & -1.345323000 \\
\hline $\mathrm{H}$ & 1.512742000 & -0.644966000 & -2.395949000 \\
\hline $\mathrm{C}$ & 2.997846000 & -0.689743000 & -0.823309000 \\
\hline $\mathrm{C}$ & 3.940373000 & 0.064037000 & -1.558387000 \\
\hline $\mathrm{C}$ & 3.435973000 & -1.152587000 & 0.438974000 \\
\hline $\mathrm{C}$ & 5.202991000 & 0.350686000 & -1.063103000 \\
\hline $\mathrm{H}$ & 3.655152000 & 0.442833000 & -2.538228000 \\
\hline $\mathrm{C}$ & 4.700305000 & -0.850799000 & 0.929909000 \\
\hline $\mathrm{H}$ & 2.781881000 & -1.777063000 & 1.042264000 \\
\hline $\mathrm{C}$ & 5.615746000 & -0.092421000 & 0.197098000 \\
\hline $\mathrm{H}$ & 5.886619000 & 0.941879000 & -1.670198000 \\
\hline $\mathrm{H}$ & 4.988741000 & -1.232249000 & 1.908358000 \\
\hline $\mathrm{H}$ & 1.159954000 & 1.054317000 & -0.650715000 \\
\hline $\mathrm{H}$ & -0.308401000 & -1.891562000 & -2.892184000 \\
\hline $\mathrm{C}$ & -2.967570000 & 1.676724000 & 0.890810000 \\
\hline $\mathrm{C}$ & -3.422900000 & 2.767414000 & -0.078728000 \\
\hline $\mathrm{C}$ & -1.115275000 & 1.592420000 & -1.535349000 \\
\hline $\mathrm{C}$ & -3.588865000 & 2.279820000 & -1.522676000 \\
\hline $\mathrm{C}$ & -2.549161000 & 1.229942000 & -1.904849000 \\
\hline $\mathrm{H}$ & -3.434569000 & 0.705914000 & 0.685883000 \\
\hline $\mathrm{H}$ & -0.864607000 & 2.604564000 & -1.881949000 \\
\hline $\mathrm{H}$ & -2.696617000 & 3.588383000 & -0.028889000 \\
\hline $\mathrm{H}$ & -3.535523000 & 3.140689000 & -2.200289000 \\
\hline $\mathrm{H}$ & -3.261318000 & 1.961651000 & 1.904597000 \\
\hline $\mathrm{H}$ & -4.370369000 & 3.178150000 & 0.285747000 \\
\hline $\mathrm{H}$ & -0.444976000 & 0.903486000 & -2.066036000 \\
\hline $\mathrm{H}$ & -4.585525000 & 1.841969000 & -1.650817000 \\
\hline $\mathrm{H}$ & -2.574480000 & 1.054625000 & -2.984193000 \\
\hline $\mathrm{H}$ & -2.798655000 & 0.269927000 & -1.451333000 \\
\hline $\mathrm{C}$ & -0.686975000 & 1.518915000 & -0.090193000 \\
\hline $\mathrm{N}$ & 0.636521000 & 1.565651000 & 0.076953000 \\
\hline $\mathrm{N}$ & -1.504233000 & 1.507210000 & 0.957613000 \\
\hline
\end{tabular}




$\begin{array}{lrrr}\mathrm{C} & -1.011288000 & 1.271431000 & 2.324952000 \\ \mathrm{H} & -1.111715000 & 2.212028000 & 2.882262000 \\ \mathrm{H} & -1.680276000 & 0.532757000 & 2.775327000 \\ \mathrm{C} & 0.422788000 & 0.774012000 & 2.364848000 \\ \mathrm{H} & 0.808447000 & 0.865566000 & 3.383869000 \\ \mathrm{H} & 0.463955000 & -0.279678000 & 2.083415000 \\ \mathrm{C} & 1.266399000 & 1.578296000 & 1.389525000 \\ \mathrm{H} & 2.265387000 & 1.152917000 & 1.277761000 \\ \mathrm{H} & 1.366602000 & 2.617272000 & 1.725733000 \\ \mathrm{C} & 7.000242000 & 0.194626000 & 0.721480000 \\ \mathrm{H} & 7.005931000 & 0.281326000 & 1.813698000 \\ \mathrm{H} & 7.707048000 & -0.602037000 & 0.455381000 \\ \mathrm{H} & 7.397550000 & 1.129279000 & 0.310584000 \\ \text {-_-_-_. }\end{array}$

TSA2-3 DBU

\begin{tabular}{|c|c|c|c|}
\hline \multicolumn{4}{|c|}{ Coordinates (Angstroms) } \\
\hline & $\mathrm{X}$ & $\mathrm{Y}$ & $\mathrm{Z}$ \\
\hline $\mathrm{C}$ & -3.073888000 & -1.790162000 & 2.133576000 \\
\hline $\mathrm{C}$ & -1.931757000 & -1.586782000 & 1.370501000 \\
\hline $\mathrm{C}$ & -1.865568000 & -2.004296000 & 0.027444000 \\
\hline $\mathrm{C}$ & -3.008080000 & -2.616361000 & -0.516077000 \\
\hline $\mathrm{C}$ & -4.154292000 & -2.804895000 & 0.246595000 \\
\hline $\mathrm{C}$ & -4.200336000 & -2.393730000 & 1.576464000 \\
\hline $\mathrm{H}$ & -3.089568000 & -1.461818000 & 3.170094000 \\
\hline $\mathrm{H}$ & -1.080662000 & -1.083528000 & 1.819060000 \\
\hline $\mathrm{H}$ & -2.984242000 & -2.928697000 & -1.554082000 \\
\hline $\mathrm{H}$ & -5.022185000 & -3.278803000 & -0.204155000 \\
\hline $\mathrm{H}$ & -5.098493000 & -2.539606000 & 2.168925000 \\
\hline $\mathrm{C}$ & -0.702157000 & -1.732176000 & -0.814299000 \\
\hline $\mathrm{C}$ & 0.533320000 & -1.377179000 & -0.379415000 \\
\hline $\mathrm{H}$ & 0.694235000 & -1.457581000 & 0.692689000 \\
\hline $\mathrm{O}$ & -0.981881000 & -1.774081000 & -2.177030000 \\
\hline $\mathrm{C}$ & 1.580384000 & -0.769819000 & -1.150948000 \\
\hline $\mathrm{H}$ & 1.473993000 & -0.774070000 & -2.238288000 \\
\hline $\mathrm{C}$ & 2.966415000 & -0.688507000 & -0.685106000 \\
\hline $\mathrm{C}$ & 3.930738000 & -0.035416000 & -1.480983000 \\
\hline $\mathrm{C}$ & 3.407929000 & -1.146108000 & 0.570157000 \\
\hline $\mathrm{C}$ & 5.230680000 & 0.161782000 & -1.044725000 \\
\hline $\mathrm{H}$ & 3.634233000 & 0.337234000 & -2.459690000 \\
\hline $\mathrm{C}$ & 4.713052000 & -0.933965000 & 1.005828000 \\
\hline $\mathrm{H}$ & 2.729458000 & -1.695927000 & 1.216730000 \\
\hline $\mathrm{C}$ & 5.654025000 & -0.277142000 & 0.214978000 \\
\hline $\mathrm{H}$ & 5.936856000 & 0.674761000 & -1.695508000 \\
\hline
\end{tabular}




\begin{tabular}{lrcc}
$\mathrm{H}$ & 5.008037000 & -1.307141000 & 1.985140000 \\
$\mathrm{H}$ & 1.078219000 & 0.734038000 & -0.868271000 \\
$\mathrm{H}$ & -0.166402000 & -2.042356000 & -2.620499000 \\
$\mathrm{C}$ & -2.872169000 & 2.149657000 & 0.810232000 \\
$\mathrm{C}$ & -3.456874000 & 2.882516000 & -0.395878000 \\
$\mathrm{C}$ & -1.316112000 & 1.397429000 & -1.707417000 \\
$\mathrm{C}$ & -3.801785000 & 1.956069000 & -1.566320000 \\
$\mathrm{C}$ & -2.746890000 & 0.868932000 & -1.759008000 \\
$\mathrm{H}$ & -3.387717000 & 1.197379000 & 0.992177000 \\
$\mathrm{H}$ & -1.219489000 & 2.324927000 & -2.288351000 \\
$\mathrm{H}$ & -2.742058000 & 3.653260000 & -0.709051000 \\
$\mathrm{H}$ & -3.904241000 & 2.555510000 & -2.479110000 \\
$\mathrm{H}$ & -3.023116000 & 2.768603000 & 1.700761000 \\
$\mathrm{H}$ & -4.355388000 & 3.415823000 & -0.067634000 \\
$\mathrm{H}$ & -0.651161000 & 0.671773000 & -2.187949000 \\
$\mathrm{H}$ & -4.774748000 & 1.482764000 & -1.390185000 \\
$\mathrm{H}$ & -2.890878000 & 0.366325000 & -2.719354000 \\
$\mathrm{H}$ & -2.868239000 & 0.088274000 & -1.003805000 \\
$\mathrm{C}$ & -0.703240000 & 1.655904000 & -0.351623000 \\
$\mathrm{~N}$ & 0.618752000 & 1.654566000 & -0.345745000 \\
$\mathrm{~N}$ & -1.421779000 & 1.910900000 & 0.756040000 \\
$\mathrm{C}$ & -0.805527000 & 1.903134000 & 2.089728000 \\
$\mathrm{H}$ & -0.846588000 & 2.925380000 & 2.489601000 \\
$\mathrm{H}$ & -1.432385000 & 1.273414000 & 2.731568000 \\
$\mathrm{C}$ & 0.627272000 & 1.396730000 & 2.080498000 \\
$\mathrm{H}$ & 1.118347000 & 1.678276000 & 3.016217000 \\
$\mathrm{H}$ & 0.652687000 & 0.306023000 & 2.007434000 \\
$\mathrm{C}$ & 1.343674000 & 1.983164000 & 0.872993000 \\
$\mathrm{H}$ & 2.353419000 & 1.580876000 & 0.771890000 \\
$\mathrm{H}$ & 1.417741000 & 3.074391000 & 0.962239000 \\
$\mathrm{C}$ & 7.076295000 & -0.073090000 & 0.673683000 \\
$\mathrm{H}$ & 7.182879000 & -0.272971000 & 1.744839000 \\
$\mathrm{H}$ & 7.768753000 & -0.739849000 & 0.144659000 \\
& 7.414290000 & 0.953560000 & 0.490815000 \\
\hline--- & &
\end{tabular}

$\mathrm{A3}_{\text {DBU }}$

\begin{tabular}{llll} 
& & \multicolumn{3}{c}{ Coordinates (Angstroms) } & Z \\
\hline $\mathrm{C}$ & 2.873378000 & 1.796502000 & 2.188129000 \\
$\mathrm{C}$ & 1.835527000 & 1.509454000 & 1.310175000 \\
$\mathrm{C}$ & 1.804341000 & 2.077628000 & 0.031635000 \\
$\mathrm{C}$ & 2.851535000 & 2.918160000 & -0.357399000 \\
$\mathrm{C}$ & 3.891604000 & 3.201881000 & 0.521518000
\end{tabular}




\begin{tabular}{|c|c|c|c|}
\hline $\mathrm{C}$ & 3.906507000 & 2.645401000 & 1.797247000 \\
\hline $\mathrm{H}$ & 2.882991000 & 1.342560000 & 3.175009000 \\
\hline $\mathrm{H}$ & 1.057015000 & 0.811998000 & 1.601629000 \\
\hline $\mathrm{H}$ & 2.845538000 & 3.345442000 & -1.354221000 \\
\hline $\mathrm{H}$ & 4.695094000 & 3.861246000 & 0.206482000 \\
\hline $\mathrm{H}$ & 4.721121000 & 2.865297000 & 2.480850000 \\
\hline $\mathrm{C}$ & 0.711052000 & 1.754600000 & -0.909270000 \\
\hline $\mathrm{C}$ & -0.528110000 & 1.404736000 & -0.543954000 \\
\hline $\mathrm{H}$ & -0.773003000 & 1.415441000 & 0.511791000 \\
\hline $\mathrm{O}$ & 1.113269000 & 1.831116000 & -2.215464000 \\
\hline $\mathrm{C}$ & -1.596894000 & 0.956752000 & -1.508155000 \\
\hline $\mathrm{H}$ & -1.291929000 & 0.008446000 & -1.970787000 \\
\hline $\mathrm{C}$ & -2.944621000 & 0.748211000 & -0.856045000 \\
\hline $\mathrm{C}$ & -3.633897000 & -0.453691000 & -1.021843000 \\
\hline $\mathrm{C}$ & -3.537908000 & 1.748653000 & -0.085797000 \\
\hline $\mathrm{C}$ & -4.878022000 & -0.648166000 & -0.432000000 \\
\hline $\mathrm{H}$ & -3.174688000 & -1.249285000 & -1.603140000 \\
\hline $\mathrm{C}$ & -4.782483000 & 1.551497000 & 0.504600000 \\
\hline $\mathrm{H}$ & -3.020576000 & 2.695215000 & 0.054514000 \\
\hline $\mathrm{C}$ & -5.473403000 & 0.349893000 & 0.343034000 \\
\hline $\mathrm{H}$ & -5.393402000 & -1.595862000 & -0.572622000 \\
\hline $\mathrm{H}$ & -5.224355000 & 2.347242000 & 1.099844000 \\
\hline $\mathrm{H}$ & 0.349664000 & 1.674562000 & -2.784770000 \\
\hline $\mathrm{C}$ & 2.797006000 & -2.109953000 & 0.981652000 \\
\hline $\mathrm{C}$ & 3.579981000 & -2.806172000 & -0.130162000 \\
\hline $\mathrm{C}$ & 1.468499000 & -1.633036000 & -1.739890000 \\
\hline $\mathrm{C}$ & 3.961146000 & -1.866700000 & -1.277245000 \\
\hline $\mathrm{C}$ & 2.815501000 & -0.920576000 & -1.632190000 \\
\hline $\mathrm{H}$ & 3.237496000 & -1.124518000 & 1.201332000 \\
\hline $\mathrm{H}$ & 1.586595000 & -2.576639000 & -2.289679000 \\
\hline $\mathrm{H}$ & 2.979911000 & -3.646443000 & -0.499552000 \\
\hline $\mathrm{H}$ & 4.246377000 & -2.463070000 & -2.153220000 \\
\hline $\mathrm{H}$ & 2.901626000 & -2.705577000 & 1.897498000 \\
\hline $\mathrm{H}$ & 4.485382000 & -3.243192000 & 0.305582000 \\
\hline $\mathrm{H}$ & 0.779684000 & -1.029651000 & -2.335702000 \\
\hline $\mathrm{H}$ & 4.843093000 & -1.278174000 & -0.995761000 \\
\hline $\mathrm{H}$ & 3.027299000 & -0.415863000 & -2.580096000 \\
\hline $\mathrm{H}$ & 2.748987000 & -0.125383000 & -0.884577000 \\
\hline $\mathrm{C}$ & 0.701543000 & -1.934236000 & -0.457419000 \\
\hline $\mathrm{N}$ & -0.563700000 & -2.121037000 & -0.612498000 \\
\hline $\mathrm{N}$ & 1.358480000 & -1.985802000 & 0.757976000 \\
\hline $\mathrm{C}$ & 0.603570000 & -1.957678000 & 2.007424000 \\
\hline $\mathrm{H}$ & 0.716900000 & -2.927597000 & 2.516169000 \\
\hline $\mathrm{H}$ & 1.052505000 & -1.201687000 & 2.668512000 \\
\hline
\end{tabular}




$\begin{array}{llrr}\mathrm{C} & -0.869034000 & -1.666822000 & 1.775989000 \\ \mathrm{H} & -1.439200000 & -1.909192000 & 2.678686000 \\ \mathrm{H} & -1.027680000 & -0.605388000 & 1.556182000 \\ \mathrm{C} & -1.320978000 & -2.482879000 & 0.570815000 \\ \mathrm{H} & -2.384316000 & -2.320763000 & 0.375393000 \\ \mathrm{H} & -1.191065000 & -3.555948000 & 0.787667000 \\ \mathrm{H} & -1.719507000 & 1.695535000 & -2.319065000 \\ \mathrm{C} & -6.815890000 & 0.122451000 & 0.992506000 \\ \mathrm{H} & -7.553854000 & -0.233034000 & 0.265073000 \\ \mathrm{H} & -6.749287000 & -0.633493000 & 1.783770000 \\ \mathrm{H} & -7.203727000 & 1.041262000 & 1.442446000\end{array}$

\begin{tabular}{|c|c|c|c|}
\hline \multicolumn{4}{|c|}{ A1 $1_{\text {MTBD }}$} \\
\hline & $\mathrm{X}$ & $\mathrm{Y}$ & Z \\
\hline $\mathrm{C}$ & 2.457896000 & 2.229044000 & -1.434583000 \\
\hline $\mathrm{C}$ & 1.781536000 & 1.138233000 & -2.258126000 \\
\hline $\mathrm{C}$ & 0.311482000 & 1.494184000 & -2.422943000 \\
\hline $\mathrm{N}$ & 1.824378000 & 2.404189000 & -0.147030000 \\
\hline $\mathrm{H}$ & 2.445911000 & 3.173557000 & -2.004481000 \\
\hline $\mathrm{H}$ & 3.511665000 & 1.976537000 & -1.272578000 \\
\hline $\mathrm{H}$ & 1.892231000 & 0.181973000 & -1.735137000 \\
\hline $\mathrm{H}$ & 2.244437000 & 1.026085000 & -3.244205000 \\
\hline $\mathrm{H}$ & 0.186147000 & 2.230951000 & -3.230557000 \\
\hline $\mathrm{H}$ & -0.265774000 & 0.603507000 & -2.712002000 \\
\hline $\mathrm{N}$ & -0.269929000 & 2.029705000 & -1.197610000 \\
\hline $\mathrm{C}$ & -1.718233000 & 1.918772000 & -1.147949000 \\
\hline $\mathrm{C}$ & -2.300917000 & 2.681739000 & 0.025200000 \\
\hline $\mathrm{C}$ & -1.515589000 & 2.323135000 & 1.273144000 \\
\hline $\mathrm{N}$ & -0.116307000 & 2.637762000 & 1.074671000 \\
\hline $\mathrm{C}$ & 0.532666000 & 2.342701000 & -0.113867000 \\
\hline $\mathrm{H}$ & -2.009313000 & 0.859109000 & -1.081037000 \\
\hline $\mathrm{H}$ & -2.121312000 & 2.305147000 & -2.093924000 \\
\hline $\mathrm{H}$ & -2.230148000 & 3.760875000 & -0.149354000 \\
\hline $\mathrm{H}$ & -3.355826000 & 2.417788000 & 0.145945000 \\
\hline $\mathrm{H}$ & -1.869584000 & 2.911355000 & 2.126698000 \\
\hline $\mathrm{H}$ & -1.661595000 & 1.260976000 & 1.522229000 \\
\hline $\mathrm{C}$ & 3.651884000 & -3.165015000 & -0.683050000 \\
\hline $\mathrm{C}$ & 2.609949000 & -2.564832000 & 0.018081000 \\
\hline $\mathrm{C}$ & 2.749143000 & -1.272455000 & 0.528145000 \\
\hline $\mathrm{C}$ & 3.944992000 & -0.584477000 & 0.316748000 \\
\hline $\mathrm{C}$ & 4.986086000 & -1.179831000 & -0.388359000 \\
\hline $\mathrm{C}$ & 4.842068000 & -2.472402000 & -0.887870000 \\
\hline
\end{tabular}




\begin{tabular}{lrcc}
$\mathrm{H}$ & 3.531222000 & -4.169717000 & -1.078069000 \\
$\mathrm{H}$ & 1.671537000 & -3.097590000 & 0.159670000 \\
$\mathrm{H}$ & 4.044804000 & 0.427974000 & 0.699574000 \\
$\mathrm{H}$ & 5.911307000 & -0.634546000 & -0.550629000 \\
$\mathrm{H}$ & 5.654676000 & -2.937000000 & -1.438746000 \\
$\mathrm{C}$ & 1.632939000 & -0.620663000 & 1.327081000 \\
$\mathrm{C}$ & 0.289184000 & -0.844376000 & 0.685175000 \\
$\mathrm{H}$ & 0.239111000 & -0.608918000 & -0.376694000 \\
$\mathrm{O}$ & 1.660685000 & -1.011501000 & 2.691375000 \\
$\mathrm{C}$ & -0.806921000 & -1.254427000 & 1.325718000 \\
$\mathrm{H}$ & -0.751449000 & -1.411617000 & 2.401780000 \\
$\mathrm{C}$ & -2.123599000 & -1.425920000 & 0.686695000 \\
$\mathrm{C}$ & -3.291667000 & -1.180349000 & 1.413644000 \\
$\mathrm{C}$ & -2.256687000 & -1.797031000 & -0.658202000 \\
$\mathrm{C}$ & -4.543055000 & -1.264733000 & 0.811613000 \\
$\mathrm{H}$ & -3.216500000 & -0.904545000 & 2.462852000 \\
$\mathrm{C}$ & -3.506757000 & -1.881693000 & -1.254872000 \\
$\mathrm{H}$ & -1.367491000 & -2.033778000 & -1.236741000 \\
$\mathrm{C}$ & -4.673960000 & -1.610411000 & -0.533295000 \\
$\mathrm{H}$ & -5.434113000 & -1.058810000 & 1.399824000 \\
$\mathrm{H}$ & -3.581940000 & -2.173847000 & -2.299962000 \\
$\mathrm{H}$ & 1.826724000 & 0.456756000 & 1.327609000 \\
$\mathrm{H}$ & 1.656185000 & -1.977700000 & 2.712565000 \\
$\mathrm{C}$ & 0.674331000 & 2.798785000 & 2.277290000 \\
$\mathrm{H}$ & 0.793741000 & 1.849485000 & 2.820943000 \\
$\mathrm{H}$ & 1.659651000 & 3.173460000 & 2.006327000 \\
$\mathrm{H}$ & 0.174797000 & 3.515831000 & 2.938307000 \\
$\mathrm{C}$ & -6.025571000 & -1.701109000 & -1.195570000 \\
$\mathrm{H}$ & -6.137730000 & -0.935353000 & -1.972022000 \\
$\mathrm{H}$ & -6.166888000 & -2.675126000 & -1.676726000 \\
$\mathrm{H}$ & -6.834176000 & -1.563619000 & -0.471779000 \\
\hline---- & & \\
& & & \\
$\mathrm{H}$ & --2 &
\end{tabular}

TSA1-2 MTBD

\begin{tabular}{lrcc} 
& \multicolumn{3}{c}{ Coordinates (Angstroms) } \\
& X & Y & Z \\
\hline $\mathrm{C}$ & -1.533880000 & 1.082836000 & 1.990329000 \\
$\mathrm{C}$ & -0.467700000 & 1.251822000 & 3.060908000 \\
$\mathrm{C}$ & 0.857796000 & 0.770778000 & 2.495384000 \\
$\mathrm{~N}$ & -1.097439000 & 1.564177000 & 0.682600000 \\
$\mathrm{H}$ & -2.450933000 & 1.615473000 & 2.260829000 \\
$\mathrm{H}$ & -1.803011000 & 0.023247000 & 1.899482000 \\
$\mathrm{H}$ & -0.735282000 & 0.681101000 & 3.955268000 \\
$\mathrm{H}$ & -0.371079000 & 2.304538000 & 3.349307000
\end{tabular}




$\begin{array}{lrrr}\mathrm{H} & 1.668319000 & 0.911081000 & 3.216907000 \\ \mathrm{H} & 0.817293000 & -0.298989000 & 2.245895000 \\ \mathrm{~N} & 1.185462000 & 1.557483000 & 1.314365000 \\ \mathrm{C} & 2.595045000 & 1.577012000 & 0.934126000 \\ \mathrm{C} & 2.846025000 & 2.586228000 & -0.171405000 \\ \mathrm{C} & 1.828837000 & 2.381973000 & -1.279989000 \\ \mathrm{~N} & 0.481002000 & 2.448525000 & -0.737121000 \\ \mathrm{C} & 0.183131000 & 1.825414000 & 0.429031000 \\ \mathrm{H} & 2.903189000 & 0.573347000 & 0.614722000 \\ \mathrm{H} & 3.174727000 & 1.837361000 & 1.826624000 \\ \mathrm{H} & 2.758095000 & 3.605295000 & 0.220391000 \\ \mathrm{H} & 3.859138000 & 2.453284000 & -0.561322000 \\ \mathrm{H} & 1.920882000 & 3.169353000 & -2.033055000 \\ \mathrm{H} & 1.986188000 & 1.416922000 & -1.778525000 \\ \mathrm{C} & -4.659765000 & -2.054399000 & 1.094760000 \\ \mathrm{C} & -3.416717000 & -1.737251000 & 0.560087000 \\ \mathrm{C} & -3.283518000 & -0.808454000 & -0.489583000 \\ \mathrm{C} & -4.468886000 & -0.238797000 & -0.988858000 \\ \mathrm{C} & -5.710637000 & -0.564917000 & -0.457954000 \\ \mathrm{C} & -5.820579000 & -1.471291000 & 0.593724000 \\ \mathrm{H} & -1.588402000 & 0.861629000 & -0.143498000 \\ \mathrm{H} & -4.720716000 & -2.780189000 & 1.901948000 \\ \mathrm{H} & -2.539420000 & -2.238118000 & 0.960390000 \\ \mathrm{H} & -4.395332000 & 0.475474000 & -1.802229000 \\ \mathrm{H} & -6.581724000 & 2.815547000 & -1.662413000 \\ \mathrm{H} & & & \end{array}$




$\begin{array}{lrrr}\mathrm{H} & -0.869698000 & 1.979582000 & -2.309316000 \\ \mathrm{H} & -1.459932000 & 3.125074000 & -1.094912000 \\ \mathrm{H} & -0.228013000 & 3.656207000 & -2.266346000 \\ \mathrm{C} & 5.735436000 & -2.289658000 & 0.533879000 \\ \mathrm{H} & 6.314852000 & -1.366541000 & 0.655856000 \\ \mathrm{H} & 5.706255000 & -2.793415000 & 1.504899000 \\ \mathrm{H} & \mathbf{6 . 2 9 3 0 2 4 0 0 0} & -2.932092000 & -0.157325000\end{array}$

A2

\begin{tabular}{|c|c|c|c|}
\hline \multicolumn{4}{|c|}{ Coordinates (Angstroms) } \\
\hline & $\mathrm{X}$ & Y & Z \\
\hline$\overline{\mathrm{C}}$ & 2.811225000 & 0.630169000 & 2.043298000 \\
\hline $\mathrm{C}$ & 3.588802000 & 1.141956000 & 0.842697000 \\
\hline $\mathrm{C}$ & 2.975421000 & 2.445101000 & 0.361338000 \\
\hline $\mathrm{N}$ & 1.372641000 & 0.883133000 & 1.920402000 \\
\hline $\mathrm{H}$ & 3.151394000 & 1.113419000 & 2.968263000 \\
\hline $\mathrm{H}$ & 2.949131000 & -0.451643000 & 2.147539000 \\
\hline $\mathrm{H}$ & 3.555788000 & 0.413817000 & 0.030799000 \\
\hline $\mathrm{H}$ & 4.635795000 & 1.290457000 & 1.119939000 \\
\hline $\mathrm{H}$ & 3.064415000 & 3.236775000 & 1.118148000 \\
\hline $\mathrm{H}$ & 3.471611000 & 2.786378000 & -0.549334000 \\
\hline $\mathrm{N}$ & 1.569015000 & 2.229058000 & 0.038698000 \\
\hline $\mathrm{C}$ & 1.035067000 & 2.759714000 & -1.212755000 \\
\hline $\mathrm{C}$ & -0.448637000 & 3.058680000 & -1.060411000 \\
\hline $\mathrm{C}$ & -1.158238000 & 1.806425000 & -0.579634000 \\
\hline $\mathrm{N}$ & -0.492016000 & 1.273780000 & 0.604028000 \\
\hline $\mathrm{C}$ & 0.820659000 & 1.438117000 & 0.829679000 \\
\hline $\mathrm{H}$ & 1.217394000 & 2.041861000 & -2.023388000 \\
\hline $\mathrm{H}$ & 1.587602000 & 3.675072000 & -1.440260000 \\
\hline $\mathrm{H}$ & -0.584788000 & 3.872143000 & -0.339164000 \\
\hline $\mathrm{H}$ & -0.867467000 & 3.381462000 & -2.017204000 \\
\hline $\mathrm{H}$ & -2.201330000 & 1.999168000 & -0.319184000 \\
\hline $\mathrm{H}$ & -1.161420000 & 1.042424000 & -1.366505000 \\
\hline $\mathrm{H}$ & -0.877997000 & 0.354335000 & 0.883852000 \\
\hline $\mathrm{C}$ & 3.143237000 & -0.568574000 & -2.483223000 \\
\hline $\mathrm{C}$ & 1.990603000 & -0.849631000 & -1.764581000 \\
\hline $\mathrm{C}$ & 2.007343000 & -1.704626000 & -0.632538000 \\
\hline $\mathrm{C}$ & 3.267675000 & -2.236001000 & -0.267631000 \\
\hline $\mathrm{C}$ & 4.417355000 & -1.936854000 & -0.985900000 \\
\hline $\mathrm{C}$ & 4.376854000 & -1.098881000 & -2.100862000 \\
\hline $\mathrm{H}$ & 3.080124000 & 0.084616000 & -3.351312000 \\
\hline $\mathrm{H}$ & 1.054456000 & -0.396501000 & -2.081350000 \\
\hline $\mathrm{H}$ & 3.318632000 & -2.894391000 & 0.592687000 \\
\hline
\end{tabular}




\begin{tabular}{|c|c|c|c|}
\hline $\mathrm{H}$ & 5.363871000 & -2.369973000 & -0.670752000 \\
\hline $\mathrm{H}$ & 5.279296000 & -0.869245000 & -2.658841000 \\
\hline $\mathrm{C}$ & 0.835828000 & -1.979281000 & 0.155795000 \\
\hline $\mathrm{C}$ & -0.453617000 & -1.612615000 & -0.159748000 \\
\hline $\mathrm{H}$ & -0.590199000 & -1.244180000 & -1.175645000 \\
\hline $\mathrm{O}$ & 1.083464000 & -2.572901000 & 1.406917000 \\
\hline $\mathrm{C}$ & -1.578278000 & -1.583937000 & 0.671520000 \\
\hline $\mathrm{H}$ & -1.474754000 & -1.887943000 & 1.712261000 \\
\hline $\mathrm{C}$ & -2.896588000 & -1.155529000 & 0.253844000 \\
\hline $\mathrm{C}$ & -3.883050000 & -0.846812000 & 1.218493000 \\
\hline $\mathrm{C}$ & -3.272772000 & -0.952168000 & -1.092644000 \\
\hline $\mathrm{C}$ & -5.125782000 & -0.345344000 & 0.866417000 \\
\hline $\mathrm{H}$ & -3.647587000 & -0.994717000 & 2.271048000 \\
\hline $\mathrm{C}$ & -4.516598000 & -0.435190000 & -1.434223000 \\
\hline $\mathrm{H}$ & -2.584768000 & -1.221662000 & -1.889908000 \\
\hline $\mathrm{C}$ & -5.473114000 & -0.115821000 & -0.469589000 \\
\hline $\mathrm{H}$ & -5.847194000 & -0.117838000 & 1.649762000 \\
\hline $\mathrm{H}$ & -4.755035000 & -0.292006000 & -2.487200000 \\
\hline $\mathrm{H}$ & 0.432570000 & -3.280110000 & 1.511192000 \\
\hline $\mathrm{C}$ & 0.558668000 & 0.206100000 & 2.923017000 \\
\hline $\mathrm{H}$ & -0.427677000 & 0.668031000 & 2.984056000 \\
\hline $\mathrm{H}$ & 0.471208000 & -0.859307000 & 2.689770000 \\
\hline $\mathrm{H}$ & 1.057820000 & 0.329112000 & 3.889127000 \\
\hline $\mathrm{C}$ & -6.836204000 & 0.407532000 & -0.847662000 \\
\hline $\mathrm{H}$ & -7.583816000 & -0.395480000 & -0.883959000 \\
\hline $\mathrm{H}$ & -7.195453000 & 1.149507000 & -0.125382000 \\
\hline $\mathrm{H}$ & -6.821970000 & 0.882649000 & -1.834467000 \\
\hline
\end{tabular}

TSA2-3 MTBD

$\begin{array}{llcc} & & & \\ & \mathrm{X} & \mathrm{Y} & \mathrm{Z} \\ \mathrm{C} & 2.759243000 & 1.335686000 & 1.721755000 \\ \mathrm{C} & 3.527926000 & 1.310711000 & 0.408286000 \\ \mathrm{C} & 2.953271000 & 2.354760000 & -0.533530000 \\ \mathrm{~N} & 1.342984000 & 1.650355000 & 1.538226000 \\ \mathrm{H} & 3.176995000 & 2.093628000 & 2.398364000 \\ \mathrm{H} & 2.828551000 & 0.361112000 & 2.218543000 \\ \mathrm{H} & 3.445179000 & 0.330750000 & -0.065262000 \\ \mathrm{H} & 4.587253000 & 1.503700000 & 0.599393000 \\ \mathrm{H} & 3.098433000 & 3.370469000 & -0.136987000 \\ \mathrm{H} & 3.442941000 & 2.298681000 & -1.508279000 \\ \mathrm{~N} & 1.535859000 & 2.090947000 & -0.734337000 \\ \mathrm{C} & 0.959914000 & 2.187934000 & -2.069262000\end{array}$




\begin{tabular}{|c|c|c|c|}
\hline $\mathrm{C}$ & -0.463981000 & 2.715173000 & -1.976616000 \\
\hline $\mathrm{C}$ & -1.255256000 & 1.821975000 & -1.035355000 \\
\hline $\mathrm{N}$ & -0.564909000 & 1.624267000 & 0.233569000 \\
\hline $\mathrm{C}$ & 0.752458000 & 1.766088000 & 0.323748000 \\
\hline $\mathrm{H}$ & 0.981971000 & 1.206909000 & -2.563447000 \\
\hline $\mathrm{H}$ & 1.586953000 & 2.867762000 & -2.652668000 \\
\hline $\mathrm{H}$ & -0.443810000 & 3.743153000 & -1.596960000 \\
\hline $\mathrm{H}$ & -0.929133000 & 2.729765000 & -2.966514000 \\
\hline $\mathrm{H}$ & -2.240863000 & 2.244918000 & -0.821671000 \\
\hline $\mathrm{H}$ & -1.429369000 & 0.843697000 & -1.504143000 \\
\hline $\mathrm{H}$ & -0.990563000 & 0.647778000 & 0.740600000 \\
\hline $\mathrm{C}$ & 3.260771000 & -1.688543000 & -2.178764000 \\
\hline $\mathrm{C}$ & 2.106007000 & -1.530821000 & -1.425471000 \\
\hline $\mathrm{C}$ & 2.072312000 & -1.867540000 & -0.057637000 \\
\hline $\mathrm{C}$ & 3.260315000 & -2.352489000 & 0.517089000 \\
\hline $\mathrm{C}$ & 4.419082000 & -2.493566000 & -0.238154000 \\
\hline $\mathrm{C}$ & 4.432577000 & -2.164253000 & -1.591133000 \\
\hline $\mathrm{H}$ & 3.250364000 & -1.422864000 & -3.232895000 \\
\hline $\mathrm{H}$ & 1.219560000 & -1.118097000 & -1.898392000 \\
\hline $\mathrm{H}$ & 3.261197000 & -2.608678000 & 1.570602000 \\
\hline $\mathrm{H}$ & 5.321670000 & -2.869011000 & 0.236854000 \\
\hline $\mathrm{H}$ & 5.339898000 & -2.273304000 & -2.177472000 \\
\hline $\mathrm{C}$ & 0.888279000 & -1.646755000 & 0.764844000 \\
\hline $\mathrm{C}$ & -0.358634000 & -1.372423000 & 0.308737000 \\
\hline $\mathrm{H}$ & -0.501338000 & -1.462389000 & -0.766013000 \\
\hline $\mathrm{O}$ & 1.153816000 & -1.652932000 & 2.135350000 \\
\hline $\mathrm{C}$ & -1.448540000 & -0.805591000 & 1.054101000 \\
\hline $\mathrm{H}$ & -1.357584000 & -0.784013000 & 2.142713000 \\
\hline $\mathrm{C}$ & -2.831505000 & -0.822288000 & 0.568896000 \\
\hline $\mathrm{C}$ & -3.835222000 & -0.158365000 & 1.302676000 \\
\hline $\mathrm{C}$ & -3.235063000 & -1.400352000 & -0.647727000 \\
\hline $\mathrm{C}$ & -5.139891000 & -0.064589000 & 0.843795000 \\
\hline $\mathrm{H}$ & -3.570634000 & 0.298529000 & 2.254747000 \\
\hline $\mathrm{C}$ & -4.543676000 & -1.291863000 & -1.108457000 \\
\hline $\mathrm{H}$ & -2.523440000 & -1.972360000 & -1.236999000 \\
\hline $\mathrm{C}$ & -5.525019000 & -0.620544000 & -0.380780000 \\
\hline $\mathrm{H}$ & -5.879739000 & 0.456454000 & 1.449244000 \\
\hline $\mathrm{H}$ & -4.809398000 & -1.760658000 & -2.054459000 \\
\hline $\mathrm{H}$ & 0.357696000 & -1.987357000 & 2.569725000 \\
\hline $\mathrm{C}$ & 0.549780000 & 1.549308000 & 2.750693000 \\
\hline $\mathrm{H}$ & -0.437557000 & 1.979335000 & 2.584958000 \\
\hline $\mathrm{H}$ & 0.451875000 & 0.505070000 & 3.058892000 \\
\hline $\mathrm{H}$ & 1.059326000 & 2.110595000 & 3.542566000 \\
\hline $\mathrm{C}$ & -6.936683000 & -0.483797000 & -0.894269000 \\
\hline
\end{tabular}




\begin{tabular}{lrrr}
$\mathrm{H}$ & -7.669978000 & -0.584924000 & -0.086449000 \\
$\mathrm{H}$ & -7.103081000 & 0.495432000 & -1.361789000 \\
$\mathrm{H}$ & $-\mathbf{7 . 1 6 2 2 2 8 0 0 0}$ & -1.246740000 & -1.646598000 \\
\hline
\end{tabular}

\begin{tabular}{|c|c|c|c|}
\hline \multicolumn{4}{|c|}{ A3 } \\
\hline & $\mathrm{X}$ & Y & Z \\
\hline $\bar{C}$ & 0.631817000 & -2.813976000 & -1.604056000 \\
\hline $\mathrm{C}$ & 1.572523000 & -1.972203000 & -2.448308000 \\
\hline $\mathrm{C}$ & 2.870323000 & -1.764334000 & -1.685311000 \\
\hline $\mathrm{N}$ & 0.366425000 & -2.111109000 & -0.367417000 \\
\hline $\mathrm{H}$ & 1.061622000 & -3.817237000 & -1.431204000 \\
\hline $\mathrm{H}$ & -0.321972000 & -2.955503000 & -2.121632000 \\
\hline $\mathrm{H}$ & 1.088498000 & -1.010846000 & -2.647497000 \\
\hline $\mathrm{H}$ & 1.781114000 & -2.453827000 & -3.408739000 \\
\hline $\mathrm{H}$ & 3.529544000 & -2.636057000 & -1.825979000 \\
\hline $\mathrm{H}$ & 3.406758000 & -0.892115000 & -2.084745000 \\
\hline $\mathrm{N}$ & 2.654543000 & -1.564662000 & -0.264430000 \\
\hline $\mathrm{C}$ & 3.811629000 & -1.048356000 & 0.447281000 \\
\hline $\mathrm{C}$ & 3.703563000 & -1.339645000 & 1.934615000 \\
\hline $\mathrm{C}$ & 2.337482000 & -0.864473000 & 2.418680000 \\
\hline $\mathrm{N}$ & 1.246387000 & -1.412284000 & 1.643491000 \\
\hline $\mathrm{C}$ & 1.445312000 & -1.676492000 & 0.398465000 \\
\hline $\mathrm{H}$ & 3.903146000 & 0.034728000 & 0.273002000 \\
\hline $\mathrm{H}$ & 4.705045000 & -1.526574000 & 0.024786000 \\
\hline $\mathrm{H}$ & 3.800018000 & -2.418600000 & 2.104737000 \\
\hline $\mathrm{H}$ & 4.516301000 & -0.839198000 & 2.471922000 \\
\hline $\mathrm{H}$ & 2.187556000 & -1.145323000 & 3.467687000 \\
\hline $\mathrm{H}$ & 2.307969000 & 0.236490000 & 2.382127000 \\
\hline $\mathrm{C}$ & -5.737676000 & 1.409562000 & -1.089718000 \\
\hline $\mathrm{C}$ & -4.563122000 & 1.559483000 & -0.362099000 \\
\hline $\mathrm{C}$ & -3.963428000 & 0.456898000 & 0.255627000 \\
\hline $\mathrm{C}$ & -4.578068000 & -0.796282000 & 0.139638000 \\
\hline $\mathrm{C}$ & -5.756256000 & -0.943800000 & -0.584728000 \\
\hline $\mathrm{C}$ & -6.339870000 & 0.158453000 & -1.202390000 \\
\hline $\mathrm{H}$ & -6.193143000 & 2.276566000 & -1.559095000 \\
\hline $\mathrm{H}$ & -4.110363000 & 2.540037000 & -0.250031000 \\
\hline $\mathrm{H}$ & -4.112593000 & -1.666683000 & 0.595476000 \\
\hline $\mathrm{H}$ & -6.215362000 & -1.924040000 & -0.673228000 \\
\hline $\mathrm{H}$ & -7.262456000 & 0.044826000 & -1.763563000 \\
\hline $\mathrm{C}$ & -2.704815000 & 0.595276000 & 1.024432000 \\
\hline $\mathrm{C}$ & -1.682626000 & 1.374670000 & 0.657255000 \\
\hline $\mathrm{H}$ & -1.784466000 & 1.936057000 & -0.267055000 \\
\hline
\end{tabular}




$\begin{array}{lrrr}\mathrm{O} & -2.586241000 & -0.193154000 & 2.140725000 \\ \mathrm{C} & -0.394814000 & 1.494815000 & 1.424048000 \\ \mathrm{H} & -0.186333000 & 0.539755000 & 1.924564000 \\ \mathrm{C} & 0.797763000 & 1.845759000 & 0.563065000 \\ \mathrm{C} & 1.819806000 & 2.660752000 & 1.054485000 \\ \mathrm{C} & 0.940374000 & 1.330840000 & -0.725781000 \\ \mathrm{C} & 2.947370000 & 2.944040000 & 0.291107000 \\ \mathrm{H} & 1.733189000 & 3.077553000 & 2.055614000 \\ \mathrm{C} & 2.062562000 & 1.623275000 & -1.493259000 \\ \mathrm{H} & 0.173612000 & 0.667867000 & -1.117525000 \\ \mathrm{C} & 3.087813000 & 2.431752000 & -1.000503000 \\ \mathrm{H} & 3.728899000 & 3.578475000 & 0.703603000 \\ \mathrm{H} & 2.147132000 & 1.208151000 & -2.496003000 \\ \mathrm{H} & -3.467252000 & -0.465654000 & 2.423375000 \\ \mathrm{H} & -0.499419000 & 2.249111000 & 2.216973000 \\ \mathrm{C} & -0.844697000 & -2.481180000 & 0.336736000 \\ \mathrm{H} & -0.781979000 & -3.492194000 & 0.768965000 \\ \mathrm{H} & -1.026556000 & -1.776152000 & 1.145485000 \\ \mathrm{H} & -1.680388000 & -2.447850000 & -0.371037000 \\ \mathrm{C} & 4.295662000 & 2.762201000 & -1.841658000 \\ \mathrm{H} & 4.174788000 & 3.728249000 & -2.346624000 \\ \mathrm{H} & 5.202919000 & 2.823820000 & -1.231724000 \\ \mathrm{H} & 4.460844000 & 2.005551000 & -2.615675000 \\ ------1--1\end{array}$

(8) $\mathrm{R}_{1}=\mathrm{H}, \mathrm{R}_{2}=\mathrm{CF}_{3}$

\begin{tabular}{|c|c|c|c|}
\hline \multicolumn{4}{|c|}{$\mathbf{A 1}_{\text {Кон }}$} \\
\hline & \multicolumn{3}{|c|}{ Coordinates (Angstroms) } \\
\hline & $X$ & $\mathrm{Y}$ & $\mathrm{Z}$ \\
\hline$\overline{\mathrm{C}}$ & 3.392930000 & 3.203885000 & -0.782426000 \\
\hline $\mathrm{C}$ & 2.610490000 & 2.056697000 & -0.682555000 \\
\hline $\mathrm{C}$ & 3.075743000 & 0.945228000 & 0.024800000 \\
\hline $\mathrm{C}$ & 4.329896000 & 0.990383000 & 0.642546000 \\
\hline $\mathrm{C}$ & 5.106276000 & 2.142141000 & 0.542012000 \\
\hline $\mathrm{C}$ & 4.643186000 & 3.247000000 & -0.169971000 \\
\hline $\mathrm{H}$ & 3.022825000 & 4.067330000 & -1.328086000 \\
\hline $\mathrm{H}$ & 1.624525000 & 2.029641000 & -1.143601000 \\
\hline $\mathrm{H}$ & 4.647569000 & 0.097699000 & 1.198364000 \\
\hline $\mathrm{H}$ & 6.076930000 & 2.181683000 & 1.029340000 \\
\hline $\mathrm{H}$ & 5.250618000 & 4.145131000 & -0.240540000 \\
\hline $\mathrm{C}$ & 2.271272000 & -0.341820000 & 0.087003000 \\
\hline $\mathrm{C}$ & 0.807472000 & -0.103063000 & 0.302441000 \\
\hline $\mathrm{H}$ & 0.584176000 & 0.548573000 & 1.145960000 \\
\hline
\end{tabular}




\begin{tabular}{|c|c|c|c|}
\hline $\mathrm{O}$ & 2.517013000 & -1.126909000 & -1.102450000 \\
\hline $\mathrm{C}$ & -0.175296000 & -0.616609000 & -0.442427000 \\
\hline $\mathrm{H}$ & 0.098531000 & -1.254698000 & -1.280968000 \\
\hline $\mathrm{C}$ & -1.619170000 & -0.404956000 & -0.249634000 \\
\hline $\mathrm{C}$ & -2.502923000 & -0.784729000 & -1.267309000 \\
\hline $\mathrm{C}$ & -2.161224000 & 0.161918000 & 0.912560000 \\
\hline $\mathrm{C}$ & -3.872512000 & -0.593577000 & -1.144048000 \\
\hline $\mathrm{H}$ & -2.106246000 & -1.231424000 & -2.175255000 \\
\hline $\mathrm{C}$ & -3.527360000 & 0.356818000 & 1.042597000 \\
\hline $\mathrm{H}$ & -1.509141000 & 0.445980000 & 1.732335000 \\
\hline $\mathrm{C}$ & -4.388992000 & -0.017403000 & 0.012335000 \\
\hline $\mathrm{H}$ & -4.538533000 & -0.887289000 & -1.948351000 \\
\hline $\mathrm{H}$ & -3.927738000 & 0.801276000 & 1.947696000 \\
\hline $\mathrm{H}$ & 2.700888000 & -0.955813000 & 0.908797000 \\
\hline $\mathrm{H}$ & 2.344895000 & -0.549450000 & -1.858342000 \\
\hline $\mathrm{O}$ & 4.249103000 & -1.862255000 & 1.770433000 \\
\hline $\mathrm{H}$ & 4.140450000 & -1.632311000 & 2.696840000 \\
\hline K & 4.562791000 & -2.702716000 & -0.344191000 \\
\hline $\mathrm{C}$ & -5.873002000 & 0.141990000 & 0.180572000 \\
\hline $\mathrm{F}$ & -6.424503000 & -0.928220000 & 0.783856000 \\
\hline $\mathrm{F}$ & -6.179485000 & 1.212687000 & 0.934159000 \\
\hline $\mathrm{F}$ & -6.499615000 & 0.288330000 & -1.000170000 \\
\hline
\end{tabular}

TSA1-2 кон

\begin{tabular}{lrrr} 
& & Coordinates (Angstroms) & Z \\
\hline $\mathrm{C}$ & -4.301011000 & -3.071624000 & -0.134243000 \\
$\mathrm{C}$ & -3.294363000 & -2.111841000 & -0.223520000 \\
$\mathrm{C}$ & -3.585203000 & -0.752593000 & -0.074489000 \\
$\mathrm{C}$ & -4.916933000 & -0.376739000 & 0.153489000 \\
$\mathrm{C}$ & -5.923323000 & -1.330231000 & 0.226322000 \\
$\mathrm{C}$ & -5.618377000 & -2.684380000 & 0.085823000 \\
$\mathrm{H}$ & -4.055286000 & -4.123658000 & -0.250302000 \\
$\mathrm{H}$ & -2.270403000 & -2.421723000 & -0.417967000 \\
$\mathrm{H}$ & -5.136423000 & 0.678281000 & 0.295067000 \\
$\mathrm{H}$ & -6.949639000 & -1.021431000 & 0.405551000 \\
$\mathrm{H}$ & -6.404221000 & -3.431495000 & 0.149075000 \\
$\mathrm{C}$ & -2.546745000 & 0.322397000 & -0.151523000 \\
$\mathrm{C}$ & -1.149121000 & -0.053216000 & 0.000142000 \\
$\mathrm{H}$ & -0.953900000 & -0.708346000 & 0.849981000 \\
$\mathrm{O}$ & -2.768953000 & 1.201321000 & -1.277790000 \\
$\mathrm{C}$ & -0.102374000 & 0.419389000 & -0.718050000 \\
$\mathrm{H}$ & -0.315076000 & 0.992696000 & -1.621521000
\end{tabular}




$\begin{array}{lrrr}\mathrm{C} & 1.314547000 & 0.168886000 & -0.444941000 \\ \mathrm{C} & 2.269455000 & 0.431193000 & -1.440259000 \\ \mathrm{C} & 1.783812000 & -0.311795000 & 0.789820000 \\ \mathrm{C} & 3.622027000 & 0.208862000 & -1.227258000 \\ \mathrm{H} & 1.937747000 & 0.808719000 & -2.404666000 \\ \mathrm{C} & 3.132887000 & -0.541849000 & 1.006186000 \\ \mathrm{H} & 1.083223000 & -0.493275000 & 1.599203000 \\ \mathrm{C} & 4.062158000 & -0.281679000 & -0.000568000 \\ \mathrm{H} & 4.337879000 & 0.420487000 & -2.014640000 \\ \mathrm{H} & 3.470019000 & -0.910648000 & 1.969646000 \\ \mathrm{H} & -2.829425000 & 1.145178000 & 0.868893000 \\ \mathrm{H} & -2.742864000 & 0.666229000 & -2.083603000 \\ \mathrm{O} & -3.109825000 & 2.144695000 & 1.630839000 \\ \mathrm{H} & -3.678412000 & 1.793484000 & 2.321487000 \\ \mathrm{~K} & -1.689577000 & 3.232371000 & 0.048853000 \\ \mathrm{C} & 5.515232000 & -0.575902000 & 0.225563000 \\ \mathrm{~F} & 6.310342000 & 0.179505000 & -0.553918000 \\ \mathrm{~F} & 5.879686000 & -0.354931000 & 1.502316000 \\ \mathrm{~F} & 5.816967000 & -1.861346000 & -0.043262000 \\ \text { - - - - - - - - }\end{array}$

\begin{tabular}{lrcc} 
A2 кон & & \\
& & \\
& Coordinates $($ Angstroms) & Z \\
\hline $\mathrm{C}$ & 4.682017000 & 2.384315000 & 0.786191000 \\
$\mathrm{C}$ & 3.529879000 & 1.769557000 & 0.322891000 \\
$\mathrm{C}$ & 3.587950000 & 0.636200000 & -0.526134000 \\
$\mathrm{C}$ & 4.879583000 & 0.159357000 & -0.856896000 \\
$\mathrm{C}$ & 6.028634000 & 0.780826000 & -0.375394000 \\
$\mathrm{C}$ & 5.947172000 & 1.896002000 & 0.451591000 \\
$\mathrm{H}$ & 4.591961000 & 3.258194000 & 1.426293000 \\
$\mathrm{H}$ & 2.567177000 & 2.175968000 & 0.618409000 \\
$\mathrm{H}$ & 4.965542000 & -0.686682000 & -1.530971000 \\
$\mathrm{H}$ & 7.000670000 & 0.388231000 & -0.664075000 \\
$\mathrm{H}$ & 6.844297000 & 2.380527000 & 0.823399000 \\
$\mathrm{C}$ & 2.410955000 & -0.083608000 & -0.958555000 \\
$\mathrm{C}$ & 1.093674000 & 0.197786000 & -0.661324000 \\
$\mathrm{H}$ & 0.909846000 & 1.171753000 & -0.213592000 \\
$\mathrm{O}$ & 2.700837000 & -1.345786000 & -1.532845000 \\
$\mathrm{C}$ & -0.007622000 & -0.645636000 & -0.852599000 \\
$\mathrm{H}$ & 0.152214000 & -1.606277000 & -1.341574000 \\
$\mathrm{C}$ & -1.391388000 & -0.297135000 & -0.616927000 \\
$\mathrm{C}$ & -2.403798000 & -1.248171000 & -0.887154000 \\
$\mathrm{C}$ & -1.823460000 & 0.942385000 & -0.093714000
\end{tabular}




$\begin{array}{lrrr}\mathrm{C} & -3.739623000 & -0.987082000 & -0.648031000 \\ \mathrm{H} & -2.117846000 & -2.211795000 & -1.303064000 \\ \mathrm{C} & -3.161667000 & 1.203391000 & 0.149867000 \\ \mathrm{H} & -1.099772000 & 1.723800000 & 0.117989000 \\ \mathrm{C} & -4.136536000 & 0.244909000 & -0.122770000 \\ \mathrm{H} & -4.486281000 & -1.741635000 & -0.877668000 \\ \mathrm{H} & -3.454225000 & 2.169520000 & 0.548590000 \\ \mathrm{H} & 2.131129000 & -1.455206000 & -2.304893000 \\ \mathrm{O} & 0.658764000 & -1.928595000 & 1.732776000 \\ \mathrm{H} & -0.090083000 & -2.011944000 & 2.331865000 \\ \mathrm{~K} & 3.120273000 & -1.898391000 & 1.058615000 \\ \mathrm{H} & 0.297704000 & -1.469356000 & 0.931393000 \\ \mathrm{C} & -5.576324000 & 0.495295000 & 0.185416000 \\ \mathrm{~F} & -5.963100000 & -0.088264000 & 1.342817000 \\ \mathrm{~F} & -6.392218000 & 0.002458000 & -0.768626000 \\ \mathrm{~F} & -5.854794000 & 1.806811000 & 0.308699000 \\ \text { - } & & \end{array}$

TSA2-3 кон

$\begin{array}{lrcc} & & \text { Coordinates (Angstroms) } \\ & \mathrm{X} & \mathrm{Y} & \mathrm{Z} \\ \mathrm{C} & 4.454080000 & 2.770925000 & 0.033507000 \\ \mathrm{C} & 3.392262000 & 1.977809000 & -0.376027000 \\ \mathrm{C} & 3.550975000 & 0.591077000 & -0.559280000 \\ \mathrm{C} & 4.836289000 & 0.051652000 & -0.360436000 \\ \mathrm{C} & 5.899951000 & 0.855500000 & 0.044939000 \\ \mathrm{C} & 5.715294000 & 2.216823000 & 0.256678000 \\ \mathrm{H} & 4.299928000 & 3.837988000 & 0.166295000 \\ \mathrm{H} & 2.432403000 & 2.442693000 & -0.577114000 \\ \mathrm{H} & 5.001440000 & -1.001245000 & -0.566453000 \\ \mathrm{H} & 6.882086000 & 0.410684000 & 0.181702000 \\ \mathrm{H} & 6.544311000 & 2.845188000 & 0.567292000 \\ \mathrm{C} & 2.425636000 & -0.299151000 & -0.881422000 \\ \mathrm{C} & 1.111837000 & 0.020461000 & -0.757683000 \\ \mathrm{H} & 0.888917000 & 1.024537000 & -0.398612000 \\ \mathrm{O} & 2.835432000 & -1.589682000 & -1.182741000 \\ \mathrm{C} & 0.001903000 & -0.892125000 & -0.988288000 \\ \mathrm{C} & -1.361299000 & -0.457571000 & -0.688868000 \\ \mathrm{C} & -1.662576000 & 0.450736000 & 0.347149000 \\ \mathrm{C} & -2.457830000 & -0.990418000 & -1.395328000 \\ \mathrm{C} & -2.966636000 & 0.813725000 & 0.642606000 \\ \mathrm{H} & -0.856670000 & 0.875365000 & 0.940945000 \\ \mathrm{C} & -3.763940000 & -0.642108000 & -1.094542000 \\ \mathrm{H} & -2.268063000 & -1.690075000 & -2.205853000 \\ & & & \\ & & 5474 & \end{array}$




$\begin{array}{lrrr}\mathrm{C} & -4.032054000 & 0.270716000 & -0.074585000 \\ \mathrm{H} & -3.161462000 & 1.525669000 & 1.439621000 \\ \mathrm{H} & -4.582216000 & -1.069494000 & -1.664990000 \\ \mathrm{H} & 2.110901000 & -2.206359000 & -0.949207000 \\ \mathrm{H} & 0.381058000 & -2.011178000 & -0.076146000 \\ \mathrm{O} & 0.987407000 & -2.819763000 & 0.481458000 \\ \mathrm{~K} & 2.769209000 & -1.525020000 & 1.687195000 \\ \mathrm{H} & 0.484299000 & -3.634341000 & 0.369913000 \\ \mathrm{H} & 0.083142000 & -1.442736000 & -1.932162000 \\ \mathrm{C} & -5.436171000 & 0.625388000 & 0.295616000 \\ \mathrm{~F} & -5.863892000 & -0.043943000 & 1.389453000 \\ \mathrm{~F} & -6.311738000 & 0.348386000 & -0.688430000 \\ \mathrm{~F} & -5.567046000 & 1.935960000 & 0.587210000 \\ -\end{array}$

\begin{tabular}{|c|c|c|c|}
\hline \multicolumn{4}{|c|}{ Coordinates (Angstroms) } \\
\hline & $\mathrm{X}$ & Y & Z \\
\hline $\bar{C}$ & 5.447561000 & -0.399263000 & -1.282122000 \\
\hline $\mathrm{C}$ & 4.474470000 & 0.565449000 & -1.035716000 \\
\hline $\mathrm{C}$ & 3.369715000 & 0.281052000 & -0.222119000 \\
\hline $\mathrm{C}$ & 3.270944000 & -0.988258000 & 0.371996000 \\
\hline $\mathrm{C}$ & 4.250495000 & -1.942228000 & 0.118796000 \\
\hline $\mathrm{C}$ & 5.334312000 & -1.661821000 & -0.712250000 \\
\hline $\mathrm{H}$ & 6.299206000 & -0.154787000 & -1.910557000 \\
\hline $\mathrm{H}$ & 4.614054000 & 1.561372000 & -1.450583000 \\
\hline $\mathrm{H}$ & 2.438338000 & -1.231088000 & 1.058634000 \\
\hline $\mathrm{H}$ & 4.163024000 & -2.920474000 & 0.584246000 \\
\hline $\mathrm{H}$ & 6.091061000 & -2.417400000 & -0.903099000 \\
\hline $\mathrm{C}$ & 2.323614000 & 1.314864000 & -0.002872000 \\
\hline $\mathrm{C}$ & 1.476905000 & 1.338982000 & 1.034819000 \\
\hline $\mathrm{H}$ & 1.472247000 & 0.509078000 & 1.750576000 \\
\hline $\mathrm{O}$ & 2.259998000 & 2.324132000 & -0.940712000 \\
\hline $\mathrm{C}$ & 0.388614000 & 2.377347000 & 1.180132000 \\
\hline $\mathrm{H}$ & 0.620647000 & 3.271170000 & 0.594552000 \\
\hline $\mathrm{C}$ & -0.914487000 & 1.771104000 & 0.709292000 \\
\hline $\mathrm{C}$ & -1.155433000 & 1.621830000 & -0.663878000 \\
\hline $\mathrm{C}$ & -1.834093000 & 1.229243000 & 1.610302000 \\
\hline $\mathrm{C}$ & -2.255840000 & 0.910037000 & -1.122679000 \\
\hline $\mathrm{H}$ & -0.443362000 & 2.042706000 & -1.368376000 \\
\hline $\mathrm{C}$ & -2.939149000 & 0.510520000 & 1.160992000 \\
\hline $\mathrm{H}$ & -1.659735000 & 1.331241000 & 2.676872000 \\
\hline $\mathrm{C}$ & -3.140249000 & 0.333911000 & -0.206393000 \\
\hline $\mathrm{H}$ & -2.424433000 & 0.788406000 & -2.188104000 \\
\hline
\end{tabular}




$\begin{array}{lrrr}\mathrm{H} & -3.628934000 & 0.070944000 & 1.874042000 \\ \mathrm{O} & 0.909761000 & -1.476168000 & 2.248195000 \\ \mathrm{H} & 0.297213000 & 2.676904000 & 2.229460000 \\ \mathrm{H} & 2.698510000 & 2.019406000 & -1.743784000 \\ \mathrm{H} & 1.407002000 & -1.611216000 & 3.059218000 \\ \mathrm{~K} & -0.419556000 & -1.450118000 & 0.368750000 \\ \mathrm{C} & -4.208338000 & -0.598650000 & -0.695060000 \\ \mathrm{~F} & -3.700959000 & -1.853596000 & -0.834094000 \\ \mathrm{~F} & -4.689210000 & -0.247553000 & -1.895138000 \\ \mathrm{~F} & -5.239340000 & -0.693566000 & 0.152714000\end{array}$

\begin{tabular}{|c|c|c|c|}
\hline \multicolumn{4}{|c|}{$\mathrm{A1}_{\mathrm{NaOH}}$} \\
\hline & $X$ & Y & Z \\
\hline $\bar{C}$ & 3.817280000 & 2.859255000 & -0.762589000 \\
\hline $\mathrm{C}$ & 2.956236000 & 1.769333000 & -0.668610000 \\
\hline $\mathrm{C}$ & 3.339659000 & 0.629480000 & 0.043212000 \\
\hline $\mathrm{C}$ & 4.588602000 & 0.586413000 & 0.671601000 \\
\hline $\mathrm{C}$ & 5.443533000 & 1.681558000 & 0.575343000 \\
\hline $\mathrm{C}$ & 5.062922000 & 2.814806000 & -0.140531000 \\
\hline $\mathrm{H}$ & 3.513978000 & 3.744925000 & -1.313711000 \\
\hline $\mathrm{H}$ & 1.975809000 & 1.809550000 & -1.140322000 \\
\hline $\mathrm{H}$ & 4.848178000 & -0.327524000 & 1.221980000 \\
\hline $\mathrm{H}$ & 6.411788000 & 1.652394000 & 1.067566000 \\
\hline $\mathrm{H}$ & 5.733657000 & 3.666825000 & -0.208824000 \\
\hline $\mathrm{C}$ & 2.454536000 & -0.602142000 & 0.092961000 \\
\hline $\mathrm{C}$ & 1.000876000 & -0.278495000 & 0.264121000 \\
\hline $\mathrm{H}$ & 0.787741000 & 0.385872000 & 1.100240000 \\
\hline $\mathrm{O}$ & 2.682898000 & -1.408828000 & -1.093118000 \\
\hline $\mathrm{C}$ & 0.009743000 & -0.755170000 & -0.493048000 \\
\hline $\mathrm{H}$ & 0.256313000 & -1.464006000 & -1.281473000 \\
\hline $\mathrm{C}$ & -1.420971000 & -0.451889000 & -0.320836000 \\
\hline $\mathrm{C}$ & -2.376198000 & -1.339314000 & -0.831401000 \\
\hline $\mathrm{C}$ & -1.873284000 & 0.702829000 & 0.330817000 \\
\hline $\mathrm{C}$ & -3.734537000 & -1.102176000 & -0.673713000 \\
\hline $\mathrm{H}$ & -2.046087000 & -2.234842000 & -1.350923000 \\
\hline $\mathrm{C}$ & -3.229212000 & 0.945281000 & 0.493724000 \\
\hline $\mathrm{H}$ & -1.156601000 & 1.430902000 & 0.698243000 \\
\hline $\mathrm{C}$ & -4.164453000 & 0.041828000 & -0.006668000 \\
\hline $\mathrm{H}$ & -4.460100000 & -1.803559000 & -1.072007000 \\
\hline $\mathrm{H}$ & -3.562762000 & 1.846621000 & 0.996521000 \\
\hline $\mathrm{H}$ & 2.818797000 & -1.241329000 & 0.919004000 \\
\hline $\mathrm{H}$ & 2.574931000 & -0.825155000 & -1.855968000 \\
\hline
\end{tabular}




$\begin{array}{lrcc}\mathrm{O} & 4.437500000 & -2.314400000 & 1.548348000 \\ \mathrm{H} & 4.589264000 & -2.629444000 & 2.441208000 \\ \mathrm{C} & -5.632152000 & 0.273388000 & 0.216237000 \\ \mathrm{~F} & -6.062848000 & -0.322588000 & 1.343843000 \\ \mathrm{~F} & -5.923216000 & 1.580788000 & 0.330349000 \\ \mathrm{~F} & -6.375535000 & -0.218106000 & -0.790711000 \\ \mathrm{Na} & \mathbf{4 . 4 1 3 7 1 0 0 0 0} & -2.748742000 & -0.414402000\end{array}$

TSA1-2 ${ }_{\mathrm{NaOH}}$

\begin{tabular}{|c|c|c|c|}
\hline \multicolumn{4}{|c|}{ Coordinates (Angstroms) } \\
\hline & $X$ & $\mathrm{Y}$ & $\mathrm{Z}$ \\
\hline $\mathrm{C}$ & -4.569255000 & -2.733236000 & 0.200759000 \\
\hline $\mathrm{C}$ & -3.506026000 & -1.836351000 & 0.150884000 \\
\hline $\mathrm{C}$ & -3.724613000 & -0.472660000 & -0.079878000 \\
\hline $\mathrm{C}$ & -5.045314000 & -0.043969000 & -0.280582000 \\
\hline $\mathrm{C}$ & -6.104194000 & -0.942352000 & -0.246698000 \\
\hline $\mathrm{C}$ & -5.873737000 & -2.293805000 & 0.000569000 \\
\hline $\mathrm{H}$ & -4.371946000 & -3.786073000 & 0.382437000 \\
\hline $\mathrm{H}$ & -2.495597000 & -2.212532000 & 0.280603000 \\
\hline $\mathrm{H}$ & -5.231234000 & 1.010352000 & -0.462821000 \\
\hline $\mathrm{H}$ & -7.117304000 & -0.583479000 & -0.406824000 \\
\hline $\mathrm{H}$ & -6.700684000 & -2.996882000 & 0.031465000 \\
\hline $\mathrm{C}$ & -2.631472000 & 0.541706000 & -0.076448000 \\
\hline $\mathrm{C}$ & -1.240484000 & 0.115383000 & 0.011844000 \\
\hline $\mathrm{H}$ & -1.034941000 & -0.601485000 & 0.805939000 \\
\hline $\mathrm{O}$ & -2.824039000 & 1.561967000 & -1.084257000 \\
\hline $\mathrm{C}$ & -0.196795000 & 0.632419000 & -0.679825000 \\
\hline $\mathrm{H}$ & -0.406933000 & 1.267655000 & -1.542593000 \\
\hline $\mathrm{C}$ & 1.219502000 & 0.336616000 & -0.438731000 \\
\hline $\mathrm{C}$ & 2.160964000 & 0.566093000 & -1.453116000 \\
\hline $\mathrm{C}$ & 1.691412000 & -0.157196000 & 0.788584000 \\
\hline $\mathrm{C}$ & 3.508687000 & 0.294852000 & -1.265143000 \\
\hline $\mathrm{H}$ & 1.824292000 & 0.954193000 & -2.411466000 \\
\hline $\mathrm{C}$ & 3.035441000 & -0.436478000 & 0.979354000 \\
\hline $\mathrm{H}$ & 0.999354000 & -0.303518000 & 1.612432000 \\
\hline $\mathrm{C}$ & 3.952421000 & -0.212003000 & -0.046682000 \\
\hline $\mathrm{H}$ & 4.217318000 & 0.479792000 & -2.065495000 \\
\hline $\mathrm{H}$ & 3.378429000 & -0.813628000 & 1.937354000 \\
\hline $\mathrm{H}$ & -2.754064000 & 1.298721000 & 1.024648000 \\
\hline $\mathrm{H}$ & -2.809838000 & 1.140139000 & -1.955108000 \\
\hline $\mathrm{O}$ & -2.666176000 & 2.292230000 & 1.850032000 \\
\hline $\mathrm{H}$ & -3.562541000 & 2.478320000 & 2.147269000 \\
\hline $\mathrm{C}$ & 5.398444000 & -0.560376000 & 0.153328000 \\
\hline
\end{tabular}




\begin{tabular}{lrrr} 
F & 6.204678000 & 0.145850000 & -0.659018000 \\
F & 5.799377000 & -0.329238000 & 1.416709000 \\
F & 5.638499000 & -1.862243000 & -0.095328000 \\
$\mathrm{Na}$ & -1.567771000 & 3.041242000 & 0.202307000 \\
\hline
\end{tabular}

\begin{tabular}{|c|c|c|c|}
\hline & $\mathrm{Co}$ & inates (Angstrom & \\
\hline & $X$ & $\mathrm{Y}$ & $\mathrm{Z}$ \\
\hline$\overline{\mathrm{C}}$ & 4.817181000 & 2.355868000 & 0.488095000 \\
\hline $\mathrm{C}$ & 3.672771000 & 1.692532000 & 0.080838000 \\
\hline $\mathrm{C}$ & 3.735853000 & 0.396311000 & -0.489464000 \\
\hline $\mathrm{C}$ & 5.022083000 & -0.185357000 & -0.603960000 \\
\hline $\mathrm{C}$ & 6.164508000 & 0.491903000 & -0.180214000 \\
\hline $\mathrm{C}$ & 6.078097000 & 1.763927000 & 0.370636000 \\
\hline $\mathrm{H}$ & 4.725221000 & 3.352172000 & 0.912353000 \\
\hline $\mathrm{H}$ & 2.713197000 & 2.186621000 & 0.197471000 \\
\hline $\mathrm{H}$ & 5.118676000 & -1.157325000 & -1.076836000 \\
\hline $\mathrm{H}$ & 7.134174000 & 0.015290000 & -0.299536000 \\
\hline $\mathrm{H}$ & 6.969104000 & 2.290493000 & 0.696836000 \\
\hline $\mathrm{C}$ & 2.556404000 & -0.361179000 & -0.847822000 \\
\hline $\mathrm{C}$ & 1.235903000 & -0.022441000 & -0.631621000 \\
\hline $\mathrm{H}$ & 1.057020000 & 1.022283000 & -0.388972000 \\
\hline $\mathrm{O}$ & 2.845873000 & -1.726665000 & -1.124291000 \\
\hline $\mathrm{C}$ & 0.129061000 & -0.878098000 & -0.654713000 \\
\hline $\mathrm{H}$ & 0.271547000 & -1.912440000 & -0.968752000 \\
\hline $\mathrm{C}$ & -1.253697000 & -0.471117000 & -0.497897000 \\
\hline $\mathrm{C}$ & -2.276838000 & -1.441908000 & -0.579525000 \\
\hline $\mathrm{C}$ & -1.666262000 & 0.853668000 & -0.236349000 \\
\hline $\mathrm{C}$ & -3.609859000 & -1.120115000 & -0.400371000 \\
\hline $\mathrm{H}$ & -2.005169000 & -2.473962000 & -0.789357000 \\
\hline $\mathrm{C}$ & -3.000691000 & 1.177359000 & -0.054323000 \\
\hline $\mathrm{H}$ & -0.931982000 & 1.651889000 & -0.183949000 \\
\hline $\mathrm{C}$ & -3.988258000 & 0.196632000 & -0.133062000 \\
\hline $\mathrm{H}$ & -4.366931000 & -1.894677000 & -0.474749000 \\
\hline $\mathrm{H}$ & -3.278659000 & 2.208461000 & 0.140049000 \\
\hline $\mathrm{H}$ & 2.244186000 & -2.025389000 & -1.817041000 \\
\hline $\mathrm{O}$ & 1.064302000 & -1.833263000 & 1.976143000 \\
\hline $\mathrm{H}$ & 0.588140000 & -1.466173000 & 2.728589000 \\
\hline $\mathrm{H}$ & 0.587612000 & -1.490771000 & 1.170812000 \\
\hline $\mathrm{C}$ & -5.421976000 & 0.528602000 & 0.128275000 \\
\hline $\mathrm{F}$ & -5.768761000 & 0.325346000 & 1.419002000 \\
\hline $\mathrm{F}$ & -6.259227000 & -0.227712000 & -0.608613000 \\
\hline $\mathrm{F}$ & -5.705674000 & 1.817173000 & -0.141803000 \\
\hline
\end{tabular}


TSA2-3 $\mathrm{NaOH}$

\begin{tabular}{|c|c|c|c|}
\hline \multicolumn{4}{|c|}{ Coordinates (Angstroms) } \\
\hline & $\mathrm{X}$ & Y & Z \\
\hline$\overline{\mathrm{C}}$ & 4.666282000 & 2.609752000 & -0.007935000 \\
\hline $\mathrm{C}$ & 3.584740000 & 1.818800000 & -0.363595000 \\
\hline $\mathrm{C}$ & 3.709944000 & 0.419879000 & -0.447825000 \\
\hline $\mathrm{C}$ & 4.976419000 & -0.141110000 & -0.200172000 \\
\hline $\mathrm{C}$ & 6.059622000 & 0.661630000 & 0.154733000 \\
\hline $\mathrm{C}$ & 5.910878000 & 2.038378000 & 0.262877000 \\
\hline $\mathrm{H}$ & 4.540323000 & 3.687010000 & 0.049698000 \\
\hline $\mathrm{H}$ & 2.636394000 & 2.292558000 & -0.595870000 \\
\hline $\mathrm{H}$ & 5.117742000 & -1.209789000 & -0.328149000 \\
\hline $\mathrm{H}$ & 7.027714000 & 0.201565000 & 0.331997000 \\
\hline $\mathrm{H}$ & 6.754583000 & 2.664796000 & 0.535027000 \\
\hline $\mathrm{C}$ & 2.559980000 & -0.454973000 & -0.715637000 \\
\hline $\mathrm{C}$ & 1.250581000 & -0.118182000 & -0.635157000 \\
\hline $\mathrm{H}$ & 1.024350000 & 0.920170000 & -0.400587000 \\
\hline $\mathrm{O}$ & 2.941267000 & -1.789361000 & -0.873318000 \\
\hline $\mathrm{C}$ & 0.149728000 & -1.070049000 & -0.733047000 \\
\hline $\mathrm{H}$ & 0.229774000 & -1.739934000 & -1.597686000 \\
\hline $\mathrm{C}$ & -1.222511000 & -0.585205000 & -0.530694000 \\
\hline $\mathrm{C}$ & -2.301959000 & -1.172109000 & -1.215173000 \\
\hline $\mathrm{C}$ & -1.533527000 & 0.428286000 & 0.395536000 \\
\hline $\mathrm{C}$ & -3.610481000 & -0.771658000 & -0.996266000 \\
\hline $\mathrm{H}$ & -2.100299000 & -1.954044000 & -1.943134000 \\
\hline $\mathrm{C}$ & -2.838928000 & 0.842518000 & 0.609034000 \\
\hline $\mathrm{H}$ & -0.736603000 & 0.893236000 & 0.970510000 \\
\hline $\mathrm{C}$ & -3.890053000 & 0.246010000 & -0.085545000 \\
\hline $\mathrm{H}$ & -4.419363000 & -1.241135000 & -1.546741000 \\
\hline $\mathrm{H}$ & -3.045567000 & 1.632485000 & 1.324868000 \\
\hline $\mathrm{H}$ & 2.146638000 & -2.343089000 & -0.789560000 \\
\hline $\mathrm{O}$ & 1.031623000 & -2.619957000 & 1.185517000 \\
\hline $\mathrm{H}$ & 0.367663000 & -3.204546000 & 1.563178000 \\
\hline $\mathrm{H}$ & 0.482256000 & -1.953609000 & 0.370062000 \\
\hline $\mathrm{C}$ & -5.300955000 & 0.653638000 & 0.201054000 \\
\hline $\mathrm{F}$ & -5.798092000 & 0.038701000 & 1.296022000 \\
\hline $\mathrm{F}$ & -6.133889000 & 0.359259000 & -0.814316000 \\
\hline $\mathrm{F}$ & -5.406777000 & 1.977317000 & 0.432914000 \\
\hline $\mathrm{Na}$ & 2.838321000 & -1.562024000 & 1.561282000 \\
\hline
\end{tabular}

$\mathrm{A3}_{\mathrm{NaOH}}$ 


\begin{tabular}{|c|c|c|c|}
\hline \multicolumn{4}{|c|}{ Coordinates (Angstroms) } \\
\hline & $X$ & $\mathrm{Y}$ & Z \\
\hline $\mathrm{C}$ & 5.524246000 & -0.570702000 & -1.021133000 \\
\hline $\mathrm{C}$ & 4.551450000 & 0.415088000 & -0.885217000 \\
\hline $\mathrm{C}$ & 3.367329000 & 0.161217000 & -0.180698000 \\
\hline $\mathrm{C}$ & 3.177054000 & -1.098536000 & 0.407862000 \\
\hline $\mathrm{C}$ & 4.158245000 & -2.073930000 & 0.266725000 \\
\hline $\mathrm{C}$ & 5.327699000 & -1.822624000 & -0.449059000 \\
\hline $\mathrm{H}$ & 6.440242000 & -0.353057000 & -1.562859000 \\
\hline $\mathrm{H}$ & 4.745199000 & 1.402929000 & -1.297853000 \\
\hline $\mathrm{H}$ & 2.263985000 & -1.310946000 & 0.987744000 \\
\hline $\mathrm{H}$ & 4.004114000 & -3.047109000 & 0.725262000 \\
\hline $\mathrm{H}$ & 6.084770000 & -2.594842000 & -0.552048000 \\
\hline $\mathrm{C}$ & 2.336873000 & 1.223720000 & -0.062282000 \\
\hline $\mathrm{C}$ & 1.463323000 & 1.331791000 & 0.946596000 \\
\hline $\mathrm{H}$ & 1.427091000 & 0.551109000 & 1.707975000 \\
\hline $\mathrm{O}$ & 2.326673000 & 2.173703000 & -1.061018000 \\
\hline $\mathrm{C}$ & 0.403577000 & 2.407127000 & 1.000494000 \\
\hline $\mathrm{H}$ & 0.663108000 & 3.237527000 & 0.337994000 \\
\hline $\mathrm{C}$ & -0.909497000 & 1.792717000 & 0.575509000 \\
\hline $\mathrm{C}$ & -1.129204000 & 1.501199000 & -0.779068000 \\
\hline $\mathrm{C}$ & -1.855317000 & 1.368666000 & 1.511563000 \\
\hline $\mathrm{C}$ & -2.225665000 & 0.748449000 & -1.178353000 \\
\hline $\mathrm{H}$ & -0.399416000 & 1.836170000 & -1.510692000 \\
\hline $\mathrm{C}$ & -2.957104000 & 0.609811000 & 1.123453000 \\
\hline $\mathrm{H}$ & -1.698209000 & 1.582683000 & 2.563860000 \\
\hline $\mathrm{C}$ & -3.128308000 & 0.277108000 & -0.219092000 \\
\hline $\mathrm{H}$ & -2.374273000 & 0.508325000 & -2.226424000 \\
\hline $\mathrm{H}$ & -3.658414000 & 0.246699000 & 1.867330000 \\
\hline $\mathrm{O}$ & 0.583792000 & -1.451527000 & 2.020851000 \\
\hline $\mathrm{H}$ & 0.316128000 & 2.802364000 & 2.018070000 \\
\hline $\mathrm{H}$ & 2.805565000 & 1.818060000 & -1.819131000 \\
\hline $\mathrm{H}$ & 0.801508000 & -2.100484000 & 2.694425000 \\
\hline $\mathrm{C}$ & -4.148236000 & -0.748791000 & -0.610198000 \\
\hline $\mathrm{F}$ & -3.576725000 & -1.986390000 & -0.562005000 \\
\hline $\mathrm{F}$ & -4.598984000 & -0.584454000 & -1.859438000 \\
\hline $\mathrm{F}$ & -5.201094000 & -0.778508000 & 0.212570000 \\
\hline $\mathrm{Na}$ & -0.732062000 & -1.213321000 & 0.507721000 \\
\hline
\end{tabular}

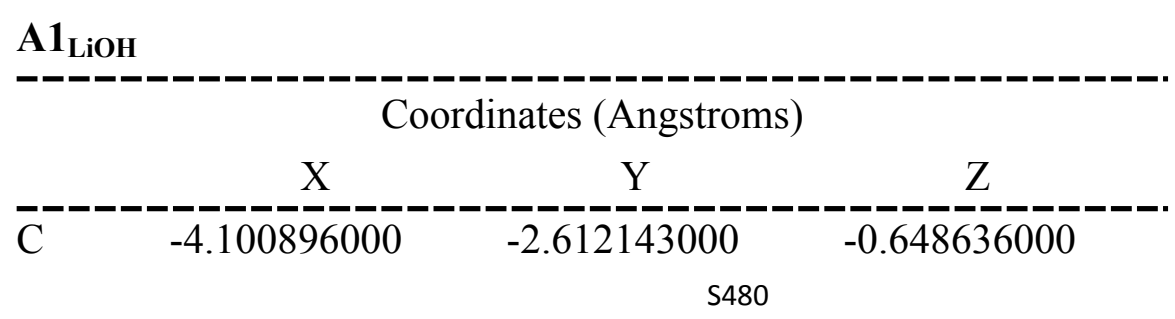




\begin{tabular}{lrrr}
$\mathrm{C}$ & -3.197840000 & -1.556744000 & -0.551782000 \\
$\mathrm{C}$ & -3.579619000 & -0.361129000 & 0.061380000 \\
$\mathrm{C}$ & -4.871605000 & -0.223300000 & 0.579521000 \\
$\mathrm{C}$ & -5.768096000 & -1.283053000 & 0.481730000 \\
$\mathrm{C}$ & -5.385850000 & -2.475501000 & -0.130128000 \\
$\mathrm{H}$ & -3.799668000 & -3.541451000 & -1.123236000 \\
$\mathrm{H}$ & -2.189592000 & -1.665875000 & -0.946228000 \\
$\mathrm{H}$ & -5.142788000 & 0.732502000 & 1.036149000 \\
$\mathrm{H}$ & -6.769559000 & -1.178572000 & 0.889240000 \\
$\mathrm{H}$ & -6.089699000 & -3.299931000 & -0.200774000 \\
$\mathrm{C}$ & -2.643746000 & 0.829747000 & 0.115866000 \\
$\mathrm{C}$ & -1.200763000 & 0.456776000 & 0.282986000 \\
$\mathrm{H}$ & -1.004692000 & -0.179379000 & 1.144782000 \\
$\mathrm{O}$ & -2.844527000 & 1.654858000 & -1.066863000 \\
$\mathrm{C}$ & -0.202634000 & 0.862537000 & -0.504163000 \\
$\mathrm{H}$ & -0.431589000 & 1.544543000 & -1.320762000 \\
$\mathrm{C}$ & 1.217734000 & 0.512489000 & -0.331342000 \\
$\mathrm{C}$ & 2.199585000 & 1.349368000 & -0.874641000 \\
$\mathrm{C}$ & 1.631625000 & -0.636001000 & 0.355360000 \\
$\mathrm{C}$ & 3.549693000 & 1.070581000 & -0.713337000 \\
$\mathrm{H}$ & 1.898387000 & 2.238491000 & -1.421914000 \\
$\mathrm{C}$ & 2.979170000 & -0.920299000 & 0.521019000 \\
$\mathrm{H}$ & 0.891745000 & -1.327005000 & 0.747814000 \\
$\mathrm{C}$ & 3.942352000 & -0.065364000 & -0.010657000 \\
$\mathrm{H}$ & 4.297148000 & 1.733368000 & -1.136158000 \\
$\mathrm{H}$ & 3.283828000 & -1.816488000 & 1.050716000 \\
$\mathrm{H}$ & -2.970852000 & 1.483441000 & 0.936869000 \\
$\mathrm{H}$ & -2.906669000 & 1.061151000 & -1.826588000 \\
$\mathrm{~F}$ & -4.680688000 & 2.840144000 & 1.138402000 \\
$\mathrm{H}$ & -5.093342000 & 3.364218000 & 1.824584000 \\
$\mathrm{C}$ & 5.402154000 & -0.340552000 & 0.217703000 \\
$\mathrm{~F}$ & 5.850986000 & 0.264035000 & 1.333210000 \\
$\mathrm{~F}$ & 5.648568000 & -1.654302000 & 0.359348000 \\
& 6.161020000 & 0.104686000 & -0.798631000 \\
-4.181229000 & 2.938910000 & -0.459141000 \\
\hline
\end{tabular}

TSA1-2

\begin{tabular}{lrrr} 
& & \multicolumn{3}{c}{ Coordinates (Angstroms) } & Z \\
\hline $\mathrm{C}$ & -4.838478000 & -2.460254000 & 0.019293000 \\
$\mathrm{C}$ & -3.720678000 & -1.631120000 & 0.039432000 \\
$\mathrm{C}$ & -3.854011000 & -0.240242000 & -0.063341000 \\
$\mathrm{C}$ & -5.145398000 & 0.287871000 & -0.210694000
\end{tabular}




$\begin{array}{lccc}\mathrm{C} & -6.257753000 & -0.543096000 & -0.249690000 \\ \mathrm{C} & -6.113002000 & -1.923326000 & -0.128354000 \\ \mathrm{H} & -4.706371000 & -3.535307000 & 0.103956000 \\ \mathrm{H} & -2.735765000 & -2.081337000 & 0.122043000 \\ \mathrm{H} & -5.267559000 & 1.363443000 & -0.290067000 \\ \mathrm{H} & -7.246243000 & -0.107634000 & -0.367111000 \\ \mathrm{H} & -6.983191000 & -2.572440000 & -0.152792000 \\ \mathrm{C} & -2.697833000 & 0.688228000 & 0.021249000 \\ \mathrm{C} & -1.330652000 & 0.209580000 & 0.035365000 \\ \mathrm{H} & -1.148674000 & -0.638751000 & 0.694016000 \\ \mathrm{O} & -2.840622000 & 1.852711000 & -0.836288000 \\ \mathrm{C} & -0.266056000 & 0.815202000 & -0.551475000 \\ \mathrm{H} & -0.451266000 & 1.589768000 & -1.299238000 \\ \mathrm{C} & 1.143577000 & 0.458538000 & -0.367920000 \\ \mathrm{C} & 2.100291000 & 0.945623000 & -1.270316000 \\ \mathrm{C} & 1.598485000 & -0.349841000 & 0.688099000 \\ \mathrm{C} & 3.447300000 & 0.636190000 & -1.139086000 \\ \mathrm{H} & 1.778097000 & 1.572026000 & -2.098720000 \\ \mathrm{C} & 2.939494000 & -0.669459000 & 0.818376000 \\ \mathrm{H} & 0.895553000 & -0.723522000 & 1.426344000 \\ \mathrm{C} & 3.872740000 & -0.177891000 & -0.094999000 \\ \mathrm{H} & 4.165845000 & 1.021832000 & -1.853902000 \\ \mathrm{H} & 3.267574000 & -1.300264000 & 1.638829000 \\ \mathrm{H} & -2.723358000 & 1.448479000 & 1.215778000 \\ \mathrm{H} & -2.777347000 & 1.574526000 & -1.761471000 \\ \mathrm{O} & -2.534625000 & 2.469616000 & 1.869546000 \\ \mathrm{H} & -2.150747000 & 2.204927000 & 2.711804000 \\ \mathrm{Li} & -1.664006000 & 2.896359000 & 0.344811000 \\ \mathrm{C} & 5.325568000 & -0.497898000 & 0.100694000 \\ \mathrm{~F} & 6.069338000 & -0.170202000 & -0.969103000 \\ \mathrm{~F} & 5.841729000 & 0.160101000 & 1.157233000 \\ \mathrm{~F} & 5.517365000 & -1.809018000 & 0.337451000 \\ -----2 .- & & \\ & & & \end{array}$

\begin{tabular}{|c|c|c|c|}
\hline \multicolumn{4}{|c|}{$\mathbf{A 2}_{\mathrm{LiOH}}$} \\
\hline & \multicolumn{3}{|c|}{ Coordinates (Angstroms) } \\
\hline & $\mathrm{X}$ & $\mathrm{Y}$ & Z \\
\hline$\overline{\mathrm{C}}$ & 4.978837000 & 2.294322000 & 0.175136000 \\
\hline $\mathrm{C}$ & 3.844302000 & 1.588352000 & -0.182214000 \\
\hline $\mathrm{C}$ & 3.892957000 & 0.193262000 & -0.416496000 \\
\hline $\mathrm{C}$ & 5.149849000 & -0.438340000 & -0.272038000 \\
\hline $\mathrm{C}$ & 6.283450000 & 0.286726000 & 0.095726000 \\
\hline $\mathrm{C}$ & 6.211812000 & 1.652982000 & 0.327282000 \\
\hline $\mathrm{H}$ & 4.902982000 & 3.366012000 & 0.337340000 \\
\hline
\end{tabular}




\begin{tabular}{lrrr}
$\mathrm{H}$ & 2.907797000 & 2.124563000 & -0.299805000 \\
$\mathrm{H}$ & 5.243687000 & -1.494934000 & -0.501762000 \\
$\mathrm{H}$ & 7.234009000 & -0.231774000 & 0.187791000 \\
$\mathrm{H}$ & 7.095921000 & 2.215469000 & 0.609273000 \\
$\mathrm{C}$ & 2.707130000 & -0.592132000 & -0.698283000 \\
$\mathrm{C}$ & 1.384672000 & -0.218006000 & -0.554443000 \\
$\mathrm{H}$ & 1.220097000 & 0.850789000 & -0.443230000 \\
$\mathrm{O}$ & 2.992672000 & -1.992305000 & -0.736313000 \\
$\mathrm{C}$ & 0.265651000 & -1.052821000 & -0.473209000 \\
$\mathrm{H}$ & 0.375521000 & -2.112057000 & -0.710407000 \\
$\mathrm{C}$ & -1.111349000 & -0.597765000 & -0.365948000 \\
$\mathrm{C}$ & -2.160786000 & -1.535840000 & -0.461722000 \\
$\mathrm{C}$ & -1.483752000 & 0.743021000 & -0.137996000 \\
$\mathrm{C}$ & -3.487923000 & -1.165617000 & -0.335466000 \\
$\mathrm{H}$ & -1.918008000 & -2.580109000 & -0.643691000 \\
$\mathrm{C}$ & -2.812177000 & 1.116848000 & -0.013002000 \\
$\mathrm{H}$ & -0.723739000 & 1.514548000 & -0.057148000 \\
$\mathrm{C}$ & -3.828474000 & 0.168450000 & -0.109932000 \\
$\mathrm{H}$ & -4.267687000 & -1.916113000 & -0.420375000 \\
$\mathrm{H}$ & -3.060926000 & 2.159450000 & 0.156992000 \\
$\mathrm{H}$ & 2.343503000 & -2.423702000 & -1.304712000 \\
$\mathrm{O}$ & 1.418708000 & -1.786689000 & 2.110352000 \\
$\mathrm{H}$ & 1.126729000 & -1.275595000 & 2.874136000 \\
$\mathrm{H}$ & 0.816279000 & -1.520176000 & 1.354921000 \\
$\mathrm{C}$ & -5.260092000 & 0.553982000 & 0.089181000 \\
$\mathrm{~F}$ & -5.671029000 & 0.353026000 & 1.360193000 \\
$\mathrm{~F}$ & -6.089609000 & -0.163950000 & -0.692634000 \\
$\mathrm{~F}$ & -5.480064000 & 1.854148000 & -0.182042000 \\
$\mathrm{Li}$ & 3.044824000 & -1.605999000 & 1.166412000 \\
\hline---1 & &
\end{tabular}

TSA2-3 ${ }_{\text {LiOH }}$

$\begin{array}{lrrr} & & & \text { Coordinates (Angstroms) } \\ \text { C } & -4.904252000 & -2.347081000 & 0.470265000 \\ \mathrm{C} & -3.791412000 & -1.524754000 & 0.364059000 \\ \mathrm{C} & -3.889381000 & -0.254134000 & -0.227509000 \\ \mathrm{C} & -5.147177000 & 0.170369000 & -0.679902000 \\ \mathrm{C} & -6.261151000 & -0.654839000 & -0.562230000 \\ \mathrm{C} & -6.147374000 & -1.917897000 & 0.008481000 \\ \mathrm{H} & -4.801587000 & -3.326399000 & 0.928418000 \\ \mathrm{H} & -2.838304000 & -1.870900000 & 0.753374000 \\ \mathrm{H} & -5.243746000 & 1.148364000 & -1.138087000 \\ \mathrm{H} & -7.223122000 & -0.306192000 & -0.926868000\end{array}$




\begin{tabular}{lrrr}
$\mathrm{H}$ & -7.017108000 & -2.561393000 & 0.097807000 \\
$\mathrm{C}$ & -2.719702000 & 0.626104000 & -0.347791000 \\
$\mathrm{C}$ & -1.406092000 & 0.307879000 & -0.252455000 \\
$\mathrm{H}$ & -1.185666000 & -0.747714000 & -0.110555000 \\
$\mathrm{O}$ & -3.080046000 & 1.991437000 & -0.507437000 \\
$\mathrm{C}$ & -0.293937000 & 1.241906000 & -0.266805000 \\
$\mathrm{H}$ & -0.399657000 & 2.069771000 & -0.979943000 \\
$\mathrm{C}$ & 1.079990000 & 0.712524000 & -0.243419000 \\
$\mathrm{C}$ & 2.119385000 & 1.382143000 & -0.909738000 \\
$\mathrm{C}$ & 1.429162000 & -0.434152000 & 0.494067000 \\
$\mathrm{C}$ & 3.430248000 & 0.932117000 & -0.856161000 \\
$\mathrm{H}$ & 1.887908000 & 2.273564000 & -1.487482000 \\
$\mathrm{C}$ & 2.734305000 & -0.896824000 & 0.539594000 \\
$\mathrm{H}$ & 0.666095000 & -0.969107000 & 1.053837000 \\
$\mathrm{C}$ & 3.746962000 & -0.217313000 & -0.136661000 \\
$\mathrm{H}$ & 4.208882000 & 1.469189000 & -1.387386000 \\
$\mathrm{H}$ & 2.971177000 & -1.791093000 & 1.108479000 \\
$\mathrm{H}$ & -2.417002000 & 2.424109000 & -1.063355000 \\
$\mathrm{O}$ & -0.998300000 & 2.589828000 & 1.868151000 \\
$\mathrm{H}$ & -0.456151000 & 2.423797000 & 2.646124000 \\
$\mathrm{H}$ & -0.525687000 & 1.993610000 & 0.967608000 \\
$\mathrm{C}$ & 5.158441000 & -0.706343000 & -0.032948000 \\
$\mathrm{~F}$ & 5.701350000 & -0.434153000 & 1.172282000 \\
$\mathrm{~F}$ & 5.962884000 & -0.149828000 & -0.955875000 \\
$\mathrm{~F}$ & 5.238632000 & -2.043007000 & -0.187474000 \\
$\mathrm{Li}$ & -2.651048000 & 2.081637000 & 1.408947000 \\
\hline$-----1-2$ &
\end{tabular}

\section{A3 $_{\text {LiOH }}$}

Coordinates (Angstroms) $\mathrm{X}$ Y Z

\begin{tabular}{lrrr}
\hline $\mathrm{C}$ & 5.607566000 & -0.440745000 & -0.854918000 \\
$\mathrm{C}$ & 4.554711000 & 0.463791000 & -0.782121000 \\
$\mathrm{C}$ & 3.360876000 & 0.111900000 & -0.136992000 \\
$\mathrm{C}$ & 3.226244000 & -1.158848000 & 0.434940000 \\
$\mathrm{C}$ & 4.289653000 & -2.054225000 & 0.355364000 \\
$\mathrm{C}$ & 5.475277000 & -1.704325000 & -0.285231000 \\
$\mathrm{H}$ & 6.532322000 & -0.155390000 & -1.347545000 \\
$\mathrm{H}$ & 4.678410000 & 1.462038000 & -1.197071000 \\
$\mathrm{H}$ & 2.282267000 & -1.469071000 & 0.903874000 \\
$\mathrm{H}$ & 4.179451000 & -3.042214000 & 0.792654000 \\
$\mathrm{H}$ & 6.294728000 & -2.414970000 & -0.342461000 \\
$\mathrm{C}$ & 2.257592000 & 1.102636000 & -0.080061000 \\
$\mathrm{C}$ & 1.489961000 & 1.340109000 & 0.995350000
\end{tabular}




\begin{tabular}{lrcc}
$\mathrm{H}$ & 1.671208000 & 0.755189000 & 1.891670000 \\
$\mathrm{O}$ & 2.068680000 & 1.859104000 & -1.206633000 \\
$\mathrm{C}$ & 0.375870000 & 2.364888000 & 1.015139000 \\
$\mathrm{H}$ & 0.594955000 & 3.174262000 & 0.312937000 \\
$\mathrm{C}$ & -0.927058000 & 1.691933000 & 0.637936000 \\
$\mathrm{C}$ & -1.232413000 & 1.469621000 & -0.714136000 \\
$\mathrm{C}$ & -1.758079000 & 1.129387000 & 1.610760000 \\
$\mathrm{C}$ & -2.325653000 & 0.692088000 & -1.077824000 \\
$\mathrm{H}$ & -0.585105000 & 1.892286000 & -1.476627000 \\
$\mathrm{C}$ & -2.855618000 & 0.350287000 & 1.251474000 \\
$\mathrm{H}$ & -1.523191000 & 1.268285000 & 2.661762000 \\
$\mathrm{C}$ & -3.136081000 & 0.125619000 & -0.092255000 \\
$\mathrm{H}$ & -2.548739000 & 0.523374000 & -2.126213000 \\
$\mathrm{H}$ & -3.478151000 & -0.096083000 & 2.018517000 \\
$\mathrm{O}$ & 0.388631000 & -1.915037000 & 1.360461000 \\
$\mathrm{H}$ & 0.294025000 & 2.803589000 & 2.014341000 \\
$\mathrm{H}$ & 2.577253000 & 1.462880000 & -1.925571000 \\
$\mathrm{H}$ & 0.109330000 & -2.815312000 & 1.528646000 \\
$\mathrm{C}$ & -4.266969000 & -0.782363000 & -0.494531000 \\
$\mathrm{~F}$ & -3.827794000 & -2.030067000 & -0.735571000 \\
$\mathrm{~F}$ & -4.866100000 & -0.353948000 & -1.618350000 \\
$\mathrm{~F}$ & -5.204836000 & -0.867482000 & 0.460300000 \\
$\mathrm{Li}$ & -0.154657000 & -0.616147000 & 0.468563000 \\
\hline-------1 &
\end{tabular}

\begin{tabular}{lccc} 
A1 & & \\
& & \\
Coordinates $($ Angstroms) & Y & \\
\hline $\mathrm{C}$ & -2.328864000 & 3.949352000 & -0.951069000 \\
$\mathrm{C}$ & -1.653011000 & 2.902000000 & -0.332480000 \\
$\mathrm{C}$ & -2.262159000 & 1.652114000 & -0.195505000 \\
$\mathrm{C}$ & -3.551063000 & 1.452159000 & -0.699164000 \\
$\mathrm{C}$ & -4.221598000 & 2.501981000 & -1.322706000 \\
$\mathrm{C}$ & -3.615873000 & 3.750242000 & -1.446274000 \\
$\mathrm{H}$ & -1.847496000 & 4.917157000 & -1.058517000 \\
$\mathrm{H}$ & -0.637619000 & 3.050403000 & 0.031517000 \\
$\mathrm{H}$ & -3.988829000 & 0.453440000 & -0.601419000 \\
$\mathrm{H}$ & -5.218075000 & 2.340602000 & -1.724973000 \\
$\mathrm{H}$ & -4.139900000 & 4.565597000 & -1.936882000 \\
$\mathrm{C}$ & -1.569848000 & 0.524130000 & 0.548961000 \\
$\mathrm{C}$ & -0.135074000 & 0.355131000 & 0.149512000 \\
$\mathrm{H}$ & 0.013143000 & 0.247172000 & -0.923671000 \\
$\mathrm{O}$ & -1.723934000 & 0.715970000 & 1.972850000 \\
$\mathrm{C}$ & 0.900671000 & 0.324875000 & 0.991253000
\end{tabular}




$\begin{array}{lrcc}\mathrm{H} & 0.706184000 & 0.447803000 & 2.055394000 \\ \mathrm{C} & 2.313847000 & 0.144513000 & 0.619158000 \\ \mathrm{C} & 3.310045000 & 0.499698000 & 1.535890000 \\ \mathrm{C} & 2.713023000 & -0.375017000 & -0.620309000 \\ \mathrm{C} & 4.657033000 & 0.364297000 & 1.226873000 \\ \mathrm{H} & 3.023054000 & 0.897998000 & 2.505583000 \\ \mathrm{C} & 4.055727000 & -0.511738000 & -0.936053000 \\ \mathrm{H} & 1.966084000 & -0.692255000 & -1.341094000 \\ \mathrm{C} & 5.033289000 & -0.138499000 & -0.014521000 \\ \mathrm{H} & 5.413133000 & 0.654690000 & 1.948140000 \\ \mathrm{H} & 4.347481000 & -0.913508000 & -1.900847000 \\ \mathrm{H} & -2.126713000 & -0.406773000 & 0.338612000 \\ \mathrm{H} & -1.398358000 & 1.603256000 & 2.175839000 \\ \mathrm{C} & -5.041146000 & -2.428072000 & -1.642764000 \\ \mathrm{C} & -3.749323000 & -2.454652000 & -0.797909000 \\ \mathrm{H} & -5.904705000 & -2.629939000 & -0.996999000 \\ \mathrm{H} & -5.175280000 & -1.433108000 & -2.085189000 \\ \mathrm{H} & -5.036417000 & -3.167848000 & -2.454196000 \\ \mathrm{C} & -2.551815000 & -2.142801000 & -1.726004000 \\ \mathrm{C} & -3.561711000 & -3.875350000 & -0.222995000 \\ \mathrm{H} & -1.614625000 & -2.192488000 & -1.156151000 \\ \mathrm{H} & -2.474126000 & -2.844126000 & -2.567185000 \\ \mathrm{H} & -2.651132000 & -1.128750000 & -2.133700000 \\ \mathrm{H} & -3.480368000 & -4.647474000 & -0.999676000 \\ \mathrm{H} & -2.651146000 & -3.902190000 & 0.388437000 \\ \mathrm{H} & -4.412823000 & -4.126767000 & 0.422823000 \\ \mathrm{O} & -3.825676000 & -1.536355000 & 0.221016000 \\ \mathrm{~K} & -4.092579000 & -0.530378000 & 2.306512000 \\ \mathrm{C} & 6.484191000 & -0.345922000 & -0.344262000 \\ \mathrm{~F} & 7.283422000 & 0.470940000 & 0.363408000 \\ \mathrm{~F} & 6.879872000 & -1.605497000 & -0.080753000 \\ \mathrm{~F} & 6.735191000 & -0.125804000 & -1.646909000 \\ ------ & \end{array}$

TSA1-2 tBuOK $_{\text {}}$

\begin{tabular}{llll} 
& \multicolumn{3}{c}{ Coordinates (Angstroms) } \\
C & -4.515501000 & -3.172293000 & -0.211967000 \\
$\mathrm{C}$ & -3.600712000 & -2.288847000 & -0.787192000 \\
$\mathrm{C}$ & -2.756957000 & -1.496725000 & 0.006202000 \\
$\mathrm{C}$ & -2.876184000 & -1.615098000 & 1.402173000 \\
$\mathrm{C}$ & -3.777349000 & -2.502731000 & 1.972015000 \\
$\mathrm{C}$ & -4.608930000 & -3.286603000 & 1.169787000 \\
$\mathrm{H}$ & -5.145646000 & -3.783877000 & -0.852996000
\end{tabular}




\begin{tabular}{lrrr}
$\mathrm{H}$ & -3.518369000 & -2.229881000 & -1.869913000 \\
$\mathrm{H}$ & -2.267711000 & -0.978650000 & 2.039710000 \\
$\mathrm{H}$ & -3.845648000 & -2.575626000 & 3.053986000 \\
$\mathrm{H}$ & -5.317384000 & -3.975507000 & 1.619489000 \\
$\mathrm{C}$ & -1.848157000 & -0.464799000 & -0.577157000 \\
$\mathrm{C}$ & -0.434072000 & -0.573208000 & -0.233284000 \\
$\mathrm{H}$ & -0.228530000 & -1.003441000 & 0.746194000 \\
$\mathrm{O}$ & -2.072261000 & -0.268642000 & -1.982527000 \\
$\mathrm{C}$ & 0.588956000 & -0.141934000 & -0.998351000 \\
$\mathrm{H}$ & 0.357352000 & 0.343392000 & -1.944930000 \\
$\mathrm{C}$ & 2.011186000 & -0.201504000 & -0.652471000 \\
$\mathrm{C}$ & 2.929444000 & 0.536559000 & -1.414656000 \\
$\mathrm{C}$ & 2.517991000 & -0.968302000 & 0.409253000 \\
$\mathrm{C}$ & 4.286481000 & 0.525359000 & -1.128019000 \\
$\mathrm{H}$ & 2.564583000 & 1.139750000 & -2.241969000 \\
$\mathrm{C}$ & 3.872057000 & -0.980231000 & 0.704081000 \\
$\mathrm{H}$ & 1.846555000 & -1.575331000 & 1.008710000 \\
$\mathrm{C}$ & 4.764884000 & -0.232039000 & -0.062196000 \\
$\mathrm{H}$ & 4.972813000 & 1.113109000 & -1.728660000 \\
$\mathrm{H}$ & 4.239747000 & -1.576095000 & 1.533205000 \\
$\mathrm{H}$ & -2.304246000 & 0.712358000 & -0.047989000 \\
$\mathrm{H}$ & -1.540664000 & -0.917877000 & -2.468578000 \\
$\mathrm{H}$ & -3.067324000 & 3.892191000 & 1.253630000 \\
$\mathrm{C}$ & -2.160922000 & 2.725449000 & 0.840993000 \\
$\mathrm{C}$ & -3.581255000 & 4.297285000 & 0.373142000 \\
$\mathrm{H}$ & -3.825219000 & 3.543742000 & 1.964269000 \\
$\mathrm{H}$ & -2.499675000 & 4.705300000 & 1.721916000 \\
$\mathrm{H}$ & -1.452771000 & 2.160200000 & 2.084127000 \\
$\mathrm{C}$ & -1.112681000 & 3.217072000 & -0.170808000 \\
$\mathrm{C}$ & -0.766914000 & 1.355096000 & 1.796654000 \\
$\mathrm{H}$ & -0.868420000 & 2.927148000 & 2.606459000 \\
$\mathrm{H}$ & -2.195914000 & 1.753490000 & 2.779250000 \\
$\mathrm{H}$ & -0.473851000 & 4.003590000 & 0.248913000 \\
$\mathrm{H}$ & -0.471158000 & 2.385380000 & -0.483224000 \\
$\mathrm{H}$ & -1.612488000 & 3.618158000 & -1.061083000 \\
$\mathrm{H}$ & -4.47530000 & 1.738984000 & 0.260633000 \\
$\mathrm{H}$ & 0.783029000 & -1.386426000 \\
$\mathrm{H}$ & -0.301498000 & 0.218409000 \\
$\mathrm{H}$ & -1.320181000 & -0.439130000 \\
$\mathrm{H}$ & -0.820975000 & -0.156255000 \\
$\mathrm{H}$ & -0.490562000 & 1.526193000 \\
\hline
\end{tabular}

\section{$\mathbf{A 2}_{\mathrm{tBuOK}}$}




\begin{tabular}{|c|c|c|c|}
\hline \multicolumn{4}{|c|}{ Coordinates (Angstroms) } \\
\hline & $X$ & $\mathrm{Y}$ & $\mathrm{Z}$ \\
\hline $\mathrm{C}$ & -6.037922000 & -1.060778000 & 0.443887000 \\
\hline $\mathrm{C}$ & -4.915929000 & -1.294285000 & -0.346855000 \\
\hline $\mathrm{C}$ & -3.608247000 & -1.241125000 & 0.193728000 \\
\hline $\mathrm{C}$ & -3.504737000 & -0.926007000 & 1.572585000 \\
\hline $\mathrm{C}$ & -4.631161000 & -0.707838000 & 2.349955000 \\
\hline $\mathrm{C}$ & -5.912687000 & -0.764984000 & 1.797385000 \\
\hline $\mathrm{H}$ & -7.024280000 & -1.125345000 & -0.009012000 \\
\hline $\mathrm{H}$ & -5.037961000 & -1.554907000 & -1.393103000 \\
\hline $\mathrm{H}$ & -2.526403000 & -0.849584000 & 2.037995000 \\
\hline $\mathrm{H}$ & -4.506166000 & -0.481205000 & 3.405666000 \\
\hline $\mathrm{H}$ & -6.789684000 & -0.587057000 & 2.411457000 \\
\hline $\mathrm{C}$ & -2.454605000 & -1.383758000 & -0.663605000 \\
\hline $\mathrm{C}$ & -1.127057000 & -1.302548000 & -0.297646000 \\
\hline $\mathrm{H}$ & -0.933292000 & -1.366413000 & 0.770687000 \\
\hline $\mathrm{O}$ & -2.764064000 & -1.318157000 & -2.044613000 \\
\hline $\mathrm{C}$ & -0.029560000 & -1.112284000 & -1.142468000 \\
\hline $\mathrm{H}$ & -0.201949000 & -1.022508000 & -2.214547000 \\
\hline $\mathrm{C}$ & 1.348621000 & -1.048673000 & -0.722663000 \\
\hline $\mathrm{C}$ & 2.345226000 & -0.661553000 & -1.651274000 \\
\hline $\mathrm{C}$ & 1.788243000 & -1.288801000 & 0.600286000 \\
\hline $\mathrm{C}$ & 3.663971000 & -0.483141000 & -1.280721000 \\
\hline $\mathrm{H}$ & 2.054391000 & -0.476309000 & -2.682702000 \\
\hline $\mathrm{C}$ & 3.109610000 & -1.102119000 & 0.973527000 \\
\hline $\mathrm{H}$ & 1.083666000 & -1.635954000 & 1.350318000 \\
\hline $\mathrm{C}$ & 4.061200000 & -0.686096000 & 0.044157000 \\
\hline $\mathrm{H}$ & 4.393810000 & -0.168122000 & -2.020439000 \\
\hline $\mathrm{H}$ & 3.404921000 & -1.283242000 & 2.002151000 \\
\hline $\mathrm{H}$ & -0.242584000 & 0.924730000 & -0.889753000 \\
\hline $\mathrm{H}$ & -2.216034000 & -1.971594000 & -2.497718000 \\
\hline $\mathrm{C}$ & -0.275119000 & 1.699951000 & 1.562605000 \\
\hline $\mathrm{C}$ & -0.016342000 & 2.503451000 & 0.285249000 \\
\hline $\mathrm{H}$ & -1.349640000 & 1.530518000 & 1.703601000 \\
\hline $\mathrm{H}$ & 0.228068000 & 0.728928000 & 1.507910000 \\
\hline $\mathrm{H}$ & 0.109177000 & 2.228101000 & 2.441632000 \\
\hline $\mathrm{C}$ & 1.485067000 & 2.654401000 & 0.037038000 \\
\hline $\mathrm{C}$ & -0.697955000 & 3.867112000 & 0.355077000 \\
\hline $\mathrm{H}$ & 1.660866000 & 3.200371000 & -0.895199000 \\
\hline $\mathrm{H}$ & 1.962517000 & 3.199723000 & 0.858174000 \\
\hline $\mathrm{H}$ & 1.965528000 & 1.673556000 & -0.042604000 \\
\hline $\mathrm{H}$ & -0.278908000 & 4.471192000 & 1.165426000 \\
\hline $\mathrm{H}$ & -0.561949000 & 4.408349000 & -0.587057000 \\
\hline $\mathrm{H}$ & -1.773624000 & 3.754328000 & 0.543938000 \\
\hline
\end{tabular}




$\begin{array}{lrrr}\mathrm{O} & -0.619857000 & 1.833406000 & -0.830568000 \\ \mathrm{~K} & -3.080058000 & 1.263703000 & -1.377112000 \\ \mathrm{C} & 5.495253000 & -0.521450000 & 0.427866000 \\ \mathrm{~F} & 5.650293000 & -0.328352000 & 1.752126000 \\ \mathrm{~F} & 6.066137000 & 0.530068000 & -0.196282000 \\ \mathrm{~F} & 6.245434000 & -1.598021000 & 0.107607000\end{array}$

TSA2-3 ${ }_{\text {tBuOK }}$

\begin{tabular}{|c|c|c|c|}
\hline \multicolumn{4}{|c|}{ Coordinates (Angstroms) } \\
\hline & $\mathrm{X}$ & $\mathrm{Y}$ & Z \\
\hline$\overline{\mathrm{C}}$ & -5.837890000 & -2.244096000 & 0.038744000 \\
\hline $\mathrm{C}$ & -4.700345000 & -1.920712000 & -0.693070000 \\
\hline $\mathrm{C}$ & -3.554813000 & -1.418931000 & -0.055780000 \\
\hline $\mathrm{C}$ & -3.604012000 & -1.220313000 & 1.335160000 \\
\hline $\mathrm{C}$ & -4.739198000 & -1.555812000 & 2.063068000 \\
\hline $\mathrm{C}$ & -5.863296000 & -2.069478000 & 1.419824000 \\
\hline $\mathrm{H}$ & -6.708489000 & -2.643169000 & -0.473931000 \\
\hline $\mathrm{H}$ & -4.681250000 & -2.067645000 & -1.767682000 \\
\hline $\mathrm{H}$ & -2.747448000 & -0.790151000 & 1.847406000 \\
\hline $\mathrm{H}$ & -4.749864000 & -1.401335000 & 3.138230000 \\
\hline $\mathrm{H}$ & -6.752057000 & -2.324643000 & 1.988818000 \\
\hline $\mathrm{C}$ & -2.366708000 & -1.048396000 & -0.838774000 \\
\hline $\mathrm{C}$ & -1.113368000 & -0.884583000 & -0.371062000 \\
\hline $\mathrm{H}$ & -0.952387000 & -1.177265000 & 0.662307000 \\
\hline $\mathrm{O}$ & -2.692160000 & -0.709252000 & -2.160301000 \\
\hline $\mathrm{C}$ & -0.020165000 & -0.205930000 & -1.064596000 \\
\hline $\mathrm{H}$ & -0.132671000 & -0.164304000 & -2.155156000 \\
\hline $\mathrm{C}$ & 1.387125000 & -0.480589000 & -0.702286000 \\
\hline $\mathrm{C}$ & 2.406771000 & -0.023733000 & -1.557444000 \\
\hline $\mathrm{C}$ & 1.788745000 & -1.095865000 & 0.493147000 \\
\hline $\mathrm{C}$ & 3.746160000 & -0.167723000 & -1.240031000 \\
\hline $\mathrm{H}$ & 2.130565000 & 0.469870000 & -2.485998000 \\
\hline $\mathrm{C}$ & 3.131354000 & -1.241565000 & 0.819394000 \\
\hline $\mathrm{H}$ & 1.048092000 & -1.477277000 & 1.189060000 \\
\hline $\mathrm{C}$ & 4.119808000 & -0.778567000 & -0.042347000 \\
\hline $\mathrm{H}$ & 4.507068000 & 0.200818000 & -1.920851000 \\
\hline $\mathrm{H}$ & 3.408765000 & -1.720184000 & 1.752894000 \\
\hline $\mathrm{H}$ & -0.293397000 & 1.058710000 & -0.742282000 \\
\hline $\mathrm{H}$ & -1.910789000 & -0.876614000 & -2.704414000 \\
\hline $\mathrm{C}$ & -0.213403000 & 1.900456000 & 1.835426000 \\
\hline $\mathrm{C}$ & -0.187924000 & 2.839704000 & 0.613276000 \\
\hline $\mathrm{H}$ & -1.248280000 & 1.606679000 & 2.059885000 \\
\hline $\mathrm{H}$ & 0.364538000 & 0.992491000 & 1.631540000 \\
\hline
\end{tabular}




$\begin{array}{lrrr}\mathrm{H} & 0.209075000 & 2.374647000 & 2.729333000 \\ \mathrm{C} & 1.269009000 & 3.190724000 & 0.270554000 \\ \mathrm{C} & -0.955716000 & 4.126716000 & 0.955056000 \\ \mathrm{H} & 1.293824000 & 3.849882000 & -0.604198000 \\ \mathrm{H} & 1.776648000 & 3.693959000 & 1.102726000 \\ \mathrm{H} & 1.829714000 & 2.281631000 & 0.027287000 \\ \mathrm{H} & -0.507981000 & 4.663790000 & 1.799755000 \\ \mathrm{H} & -0.968833000 & 4.796061000 & 0.087187000 \\ \mathrm{H} & -1.995420000 & 3.890572000 & 1.225860000 \\ \mathrm{O} & -0.812122000 & 2.233779000 & -0.479372000 \\ \mathrm{~K} & -3.161224000 & 1.741921000 & -0.978026000 \\ \mathrm{C} & 5.568585000 & -0.991034000 & 0.270289000 \\ \mathrm{~F} & 6.332982000 & 0.031231000 & -0.159789000 \\ \mathrm{~F} & 6.056880000 & -2.103341000 & -0.317868000 \\ \mathrm{~F} & \text { 5.789447000 } & -1.126875000 & 1.591079000 \\ -\end{array}$

\begin{tabular}{|c|c|c|c|}
\hline \multicolumn{4}{|c|}{$\mathrm{A3}_{\mathrm{tBuOK}}$} \\
\hline & $\mathrm{X}$ & Y & $\mathrm{Z}$ \\
\hline $\mathrm{C}$ & -5.145083000 & -1.676736000 & -1.277020000 \\
\hline $\mathrm{C}$ & -4.182396000 & -2.049037000 & -0.342953000 \\
\hline $\mathrm{C}$ & -3.124225000 & -1.189041000 & -0.022073000 \\
\hline $\mathrm{C}$ & -3.060992000 & 0.069980000 & -0.642674000 \\
\hline $\mathrm{C}$ & -4.029169000 & 0.432032000 & -1.572641000 \\
\hline $\mathrm{C}$ & -5.068452000 & -0.436683000 & -1.901385000 \\
\hline $\mathrm{H}$ & -5.961215000 & -2.355775000 & -1.505915000 \\
\hline $\mathrm{H}$ & -4.292975000 & -3.002215000 & 0.169561000 \\
\hline $\mathrm{H}$ & -2.260575000 & 0.785033000 & -0.398009000 \\
\hline $\mathrm{H}$ & -3.965001000 & 1.409882000 & -2.042262000 \\
\hline $\mathrm{H}$ & -5.818551000 & -0.144551000 & -2.630194000 \\
\hline $\mathrm{C}$ & -2.084512000 & -1.612124000 & 0.952698000 \\
\hline $\mathrm{C}$ & -1.335732000 & -0.763678000 & 1.668332000 \\
\hline $\mathrm{H}$ & -1.439960000 & 0.303257000 & 1.492167000 \\
\hline $\mathrm{O}$ & -1.913266000 & -2.966762000 & 1.124702000 \\
\hline $\mathrm{C}$ & -0.218102000 & -1.192062000 & 2.587187000 \\
\hline $\mathrm{H}$ & -0.355229000 & -2.224685000 & 2.917906000 \\
\hline $\mathrm{C}$ & 1.080968000 & -1.049159000 & 1.825582000 \\
\hline $\mathrm{C}$ & 1.587764000 & -2.125732000 & 1.088004000 \\
\hline $\mathrm{C}$ & 1.707836000 & 0.195653000 & 1.714985000 \\
\hline $\mathrm{C}$ & 2.663800000 & -1.955323000 & 0.223500000 \\
\hline $\mathrm{H}$ & 1.108053000 & -3.096740000 & 1.172455000 \\
\hline $\mathrm{C}$ & 2.785701000 & 0.375695000 & 0.855649000 \\
\hline $\mathrm{H}$ & 1.308673000 & 1.046934000 & 2.255549000 \\
\hline
\end{tabular}




$\begin{array}{lrcc}\mathrm{C} & 3.251917000 & -0.695649000 & 0.093906000 \\ \mathrm{H} & 3.041802000 & -2.793545000 & -0.353149000 \\ \mathrm{H} & 3.234423000 & 1.357891000 & 0.749534000 \\ \mathrm{C} & -1.388456000 & 3.088881000 & 1.817954000 \\ \mathrm{C} & -0.741435000 & 3.109818000 & 0.413882000 \\ \mathrm{H} & -2.364900000 & 2.591157000 & 1.767323000 \\ \mathrm{H} & -0.754267000 & 2.516185000 & 2.507967000 \\ \mathrm{H} & -1.534923000 & 4.091180000 & 2.241778000 \\ \mathrm{C} & 0.621347000 & 3.831562000 & 0.516094000 \\ \mathrm{C} & -1.656165000 & 3.920314000 & -0.531432000 \\ \mathrm{H} & 1.094682000 & 3.866449000 & -0.473260000 \\ \mathrm{H} & 0.539173000 & 4.858282000 & 0.896937000 \\ \mathrm{H} & 1.284772000 & 3.270496000 & 1.187209000 \\ \mathrm{H} & -1.816892000 & 4.953773000 & -0.196274000 \\ \mathrm{H} & -1.212483000 & 3.947258000 & -1.534391000 \\ \mathrm{H} & -2.634031000 & 3.428035000 & -0.606627000 \\ \mathrm{O} & -0.572538000 & 1.834689000 & -0.056146000 \\ \mathrm{H} & -0.204629000 & -0.551707000 & 3.475656000 \\ \mathrm{~K} & 0.294200000 & -0.031096000 & -1.179162000 \\ \mathrm{H} & -2.316654000 & -3.425512000 & 0.378640000 \\ \mathrm{C} & 4.274834000 & -0.458457000 & -0.976898000 \\ \mathrm{~F} & 4.982001000 & -1.559133000 & -1.267473000 \\ \mathrm{~F} & 5.135424000 & 0.516321000 & -0.664561000 \\ \mathrm{~F} & 3.658204000 & -0.079989000 & -2.130316000 \\ - & & & \end{array}$

\begin{tabular}{lrrr} 
A1 & & \\
& & & \\
Coordinates $($ Angstroms) & \\
\hline $\mathrm{C}$ & -2.408449000 & 4.031934000 & -0.609942000 \\
$\mathrm{C}$ & -1.716208000 & 2.912613000 & -0.155866000 \\
$\mathrm{C}$ & -2.366503000 & 1.681726000 & -0.043110000 \\
$\mathrm{C}$ & -3.716053000 & 1.574457000 & -0.394565000 \\
$\mathrm{C}$ & -4.402772000 & 2.696442000 & -0.849172000 \\
$\mathrm{C}$ & -3.753190000 & 3.924472000 & -0.955968000 \\
$\mathrm{H}$ & -1.896823000 & 4.985594000 & -0.701780000 \\
$\mathrm{H}$ & -0.660881000 & 2.992259000 & 0.098024000 \\
$\mathrm{H}$ & -4.194672000 & 0.594861000 & -0.312104000 \\
$\mathrm{H}$ & -5.448500000 & 2.608614000 & -1.130184000 \\
$\mathrm{H}$ & -4.291134000 & 4.796536000 & -1.316567000 \\
$\mathrm{C}$ & -1.660686000 & 0.466223000 & 0.524597000 \\
$\mathrm{C}$ & -0.222650000 & 0.356889000 & 0.113992000 \\
$\mathrm{H}$ & -0.063981000 & 0.434255000 & -0.960271000 \\
$\mathrm{O}$ & -1.809873000 & 0.455215000 & 1.968609000
\end{tabular}




\begin{tabular}{lrrr}
$\mathrm{C}$ & 0.803733000 & 0.173823000 & 0.947499000 \\
$\mathrm{H}$ & 0.597232000 & 0.117917000 & 2.014867000 \\
$\mathrm{C}$ & 2.219854000 & 0.038830000 & 0.565922000 \\
$\mathrm{C}$ & 3.205762000 & 0.190835000 & 1.548339000 \\
$\mathrm{C}$ & 2.631072000 & -0.244275000 & -0.743246000 \\
$\mathrm{C}$ & 4.554301000 & 0.083518000 & 1.238279000 \\
$\mathrm{H}$ & 2.909530000 & 0.402356000 & 2.572372000 \\
$\mathrm{C}$ & 3.976780000 & -0.352753000 & -1.060416000 \\
$\mathrm{H}$ & 1.891819000 & -0.399101000 & -1.522698000 \\
$\mathrm{C}$ & 4.943438000 & -0.185644000 & -0.070871000 \\
$\mathrm{H}$ & 5.303715000 & 0.212124000 & 2.012045000 \\
$\mathrm{H}$ & 4.276396000 & -0.570510000 & -2.079883000 \\
$\mathrm{H}$ & -2.219795000 & -0.427548000 & 0.201232000 \\
$\mathrm{H}$ & -1.537195000 & 1.325703000 & 2.287984000 \\
$\mathrm{C}$ & -5.681979000 & -3.047959000 & -0.401262000 \\
$\mathrm{C}$ & -4.279059000 & -2.429746000 & -0.561404000 \\
$\mathrm{H}$ & -5.774784000 & -3.501293000 & 0.593882000 \\
$\mathrm{H}$ & -6.440429000 & -2.261068000 & -0.486886000 \\
$\mathrm{H}$ & -5.895189000 & -3.820029000 & -1.152085000 \\
$\mathrm{C}$ & -4.159865000 & -1.828637000 & -1.978086000 \\
$\mathrm{C}$ & -3.220997000 & -3.541565000 & -0.406396000 \\
$\mathrm{H}$ & -3.173884000 & -1.364051000 & -2.103981000 \\
$\mathrm{H}$ & -4.289586000 & -2.579934000 & -2.767632000 \\
$\mathrm{H}$ & -4.917819000 & -1.048525000 & -2.117812000 \\
$\mathrm{H}$ & -3.331248000 & -4.343619000 & -1.147926000 \\
$\mathrm{H}$ & -2.217234000 & -3.111133000 & -0.510467000 \\
$\mathrm{H}$ & -3.297263000 & -3.986425000 & 0.594145000 \\
$\mathrm{O}$ & -4.076312000 & -1.451033000 & 0.383891000 \\
$\mathrm{Na}$ & -3.725276000 & -0.755991000 & 2.248251000 \\
$\mathrm{C}$ & 6.399831000 & -0.365162000 & -0.395259000 \\
$\mathrm{~F}$ & 7.180584000 & 0.421027000 & 0.366697000 \\
$\mathrm{~F}$ & 6.802421000 & -1.632415000 & -0.186109000 \\
$\mathrm{~F}-----6.68226000$ & -0.076463000 & -1.679858000 \\
\hline & & & \\
& 6 &
\end{tabular}

TSA1-2 tBuONa $_{\text {a }}$ 


\begin{tabular}{|c|c|c|c|}
\hline $\mathrm{H}$ & -5.524102000 & -3.448783000 & -1.150459000 \\
\hline $\mathrm{H}$ & -3.830072000 & -1.883168000 & -2.017289000 \\
\hline $\mathrm{H}$ & -2.317436000 & -1.320666000 & 1.964217000 \\
\hline $\mathrm{H}$ & -3.955555000 & -2.943364000 & 2.811564000 \\
\hline $\mathrm{H}$ & -5.594923000 & -4.006346000 & 1.270801000 \\
\hline $\mathrm{C}$ & -2.002544000 & -0.421385000 & -0.570769000 \\
\hline $\mathrm{C}$ & -0.588873000 & -0.559296000 & -0.250934000 \\
\hline $\mathrm{H}$ & -0.375105000 & -1.074830000 & 0.684509000 \\
\hline $\mathrm{O}$ & -2.240299000 & -0.062270000 & -1.946366000 \\
\hline $\mathrm{C}$ & 0.431061000 & -0.056915000 & -0.976052000 \\
\hline $\mathrm{H}$ & 0.198792000 & 0.511643000 & -1.874869000 \\
\hline $\mathrm{C}$ & 1.852662000 & -0.152878000 & -0.633959000 \\
\hline $\mathrm{C}$ & 2.766926000 & 0.715544000 & -1.249816000 \\
\hline $\mathrm{C}$ & 2.359947000 & -1.083481000 & 0.286590000 \\
\hline $\mathrm{C}$ & 4.120241000 & 0.674203000 & -0.949988000 \\
\hline $\mathrm{H}$ & 2.401520000 & 1.445363000 & -1.967570000 \\
\hline $\mathrm{C}$ & 3.711046000 & -1.126861000 & 0.594210000 \\
\hline $\mathrm{H}$ & 1.690613000 & -1.796493000 & 0.758300000 \\
\hline $\mathrm{C}$ & 4.599183000 & -0.245935000 & -0.020413000 \\
\hline $\mathrm{H}$ & 4.805012000 & 1.364092000 & -1.432232000 \\
\hline $\mathrm{H}$ & 4.079373000 & -1.851906000 & 1.312488000 \\
\hline $\mathrm{H}$ & -2.493755000 & 0.718717000 & 0.051895000 \\
\hline $\mathrm{H}$ & -1.780633000 & -0.694461000 & -2.519567000 \\
\hline $\mathrm{C}$ & -3.430708000 & 3.931381000 & 1.068002000 \\
\hline $\mathrm{C}$ & -2.456546000 & 2.810729000 & 0.693554000 \\
\hline $\mathrm{H}$ & -4.073118000 & 4.176091000 & 0.212611000 \\
\hline $\mathrm{H}$ & -4.071918000 & 3.610482000 & 1.895731000 \\
\hline $\mathrm{H}$ & -2.902194000 & 4.843561000 & 1.368903000 \\
\hline $\mathrm{C}$ & -1.565460000 & 2.464722000 & 1.895989000 \\
\hline $\mathrm{C}$ & -1.579826000 & 3.248946000 & -0.489546000 \\
\hline $\mathrm{H}$ & -0.851744000 & 1.679308000 & 1.622176000 \\
\hline $\mathrm{H}$ & -0.995448000 & 3.334882000 & 2.241897000 \\
\hline $\mathrm{H}$ & -2.183418000 & 2.099517000 & 2.723022000 \\
\hline $\mathrm{H}$ & -0.978957000 & 4.133073000 & -0.245670000 \\
\hline $\mathrm{H}$ & -0.899688000 & 2.439273000 & -0.775824000 \\
\hline $\mathrm{H}$ & -2.208977000 & 3.493361000 & -1.355626000 \\
\hline $\mathrm{O}$ & -3.215305000 & 1.681521000 & 0.337972000 \\
\hline $\mathrm{Na}$ & -4.265869000 & 0.839847000 & -1.321060000 \\
\hline $\mathrm{C}$ & 6.069759000 & -0.335314000 & 0.260354000 \\
\hline $\mathrm{F}$ & 6.676469000 & 0.860643000 & 0.150096000 \\
\hline $\mathrm{F}$ & 6.696647000 & -1.164952000 & -0.597894000 \\
\hline $\mathrm{F}$ & 6.320362000 & -0.799983000 & 1.497812000 \\
\hline
\end{tabular}

A $\boldsymbol{2}_{\text {tBuOna }}$ 


\begin{tabular}{|c|c|c|c|}
\hline \multicolumn{4}{|c|}{ Coordinates (Angstroms) } \\
\hline & $\mathrm{X}$ & $\mathrm{Y}$ & Z \\
\hline $\mathrm{C}$ & -6.053639000 & -0.695601000 & 0.341056000 \\
\hline $\mathrm{C}$ & -4.948613000 & -0.996901000 & -0.454037000 \\
\hline $\mathrm{C}$ & -3.660768000 & -1.174575000 & 0.109432000 \\
\hline $\mathrm{C}$ & -3.558984000 & -1.032315000 & 1.517103000 \\
\hline $\mathrm{C}$ & -4.667495000 & -0.742699000 & 2.293288000 \\
\hline $\mathrm{C}$ & -5.928755000 & -0.561207000 & 1.717763000 \\
\hline $\mathrm{H}$ & -7.025953000 & -0.578961000 & -0.130899000 \\
\hline $\mathrm{H}$ & -5.079381000 & -1.146230000 & -1.521038000 \\
\hline $\mathrm{H}$ & -2.597300000 & -1.155907000 & 2.005369000 \\
\hline $\mathrm{H}$ & -4.546021000 & -0.648876000 & 3.369133000 \\
\hline $\mathrm{H}$ & -6.790539000 & -0.328955000 & 2.335005000 \\
\hline $\mathrm{C}$ & -2.511206000 & -1.371033000 & -0.744229000 \\
\hline $\mathrm{C}$ & -1.181033000 & -1.331624000 & -0.374115000 \\
\hline $\mathrm{H}$ & -0.996569000 & -1.434482000 & 0.692161000 \\
\hline $\mathrm{O}$ & -2.813239000 & -1.179026000 & -2.121932000 \\
\hline $\mathrm{C}$ & -0.076113000 & -1.113119000 & -1.198536000 \\
\hline $\mathrm{H}$ & -0.224134000 & -1.027855000 & -2.274745000 \\
\hline $\mathrm{C}$ & 1.293782000 & -1.011230000 & -0.749868000 \\
\hline $\mathrm{C}$ & 2.298723000 & -0.616508000 & -1.662341000 \\
\hline $\mathrm{C}$ & 1.707603000 & -1.215535000 & 0.587267000 \\
\hline $\mathrm{C}$ & 3.608776000 & -0.408946000 & -1.268775000 \\
\hline $\mathrm{H}$ & 2.026520000 & -0.452541000 & -2.702363000 \\
\hline $\mathrm{C}$ & 3.016911000 & -1.001309000 & 0.982768000 \\
\hline $\mathrm{H}$ & 0.993043000 & -1.555138000 & 1.331266000 \\
\hline $\mathrm{C}$ & 3.981626000 & -0.587180000 & 0.064020000 \\
\hline $\mathrm{H}$ & 4.347244000 & -0.093340000 & -1.998548000 \\
\hline $\mathrm{H}$ & 3.293898000 & -1.158321000 & 2.021010000 \\
\hline $\mathrm{H}$ & -0.450013000 & 0.879478000 & -1.065115000 \\
\hline $\mathrm{H}$ & -2.253076000 & -1.772894000 & -2.635615000 \\
\hline $\mathrm{C}$ & -0.657983000 & 1.685015000 & 1.364579000 \\
\hline $\mathrm{C}$ & -0.417784000 & 2.495579000 & 0.088846000 \\
\hline $\mathrm{H}$ & -1.714060000 & 1.401733000 & 1.457926000 \\
\hline $\mathrm{H}$ & -0.052432000 & 0.772632000 & 1.356925000 \\
\hline $\mathrm{H}$ & -0.379089000 & 2.266058000 & 2.249924000 \\
\hline $\mathrm{C}$ & 1.072936000 & 2.772158000 & -0.104693000 \\
\hline $\mathrm{C}$ & -1.224816000 & 3.789430000 & 0.103478000 \\
\hline $\mathrm{H}$ & 1.238254000 & 3.327226000 & -1.033221000 \\
\hline $\mathrm{H}$ & 1.470789000 & 3.358392000 & 0.730198000 \\
\hline $\mathrm{H}$ & 1.634287000 & 1.832796000 & -0.158216000 \\
\hline $\mathrm{H}$ & -0.893635000 & 4.444322000 & 0.914946000 \\
\hline $\mathrm{H}$ & -1.104775000 & 4.323757000 & -0.844412000 \\
\hline $\mathrm{H}$ & -2.292115000 & 3.582450000 & 0.258667000 \\
\hline
\end{tabular}




$\begin{array}{lrrr}\mathrm{O} & -0.904248000 & 1.757708000 & -1.047661000 \\ \mathrm{Na} & -2.968579000 & 1.004945000 & -1.389753000 \\ \mathrm{C} & 5.399698000 & -0.405987000 & 0.499764000 \\ \mathrm{~F} & 6.068241000 & -1.576081000 & 0.581187000 \\ \mathrm{~F} & 6.096959000 & 0.375597000 & -0.346808000 \\ \mathrm{~F} & 5.482814000 & 0.158521000 & 1.722182000\end{array}$

TSA2-3

\begin{tabular}{|c|c|c|c|}
\hline & $\mathrm{Co}$ & nates (Angstrom & \\
\hline & $X$ & Y & Z \\
\hline$\overline{\mathrm{C}}$ & -6.116141000 & -1.792042000 & -0.129170000 \\
\hline $\mathrm{C}$ & -4.986044000 & -1.386815000 & -0.832495000 \\
\hline $\mathrm{C}$ & -3.755724000 & -1.219147000 & -0.178521000 \\
\hline $\mathrm{C}$ & -3.705992000 & -1.447603000 & 1.207616000 \\
\hline $\mathrm{C}$ & -4.833539000 & -1.864606000 & 1.901705000 \\
\hline $\mathrm{C}$ & -6.047001000 & -2.038170000 & 1.238528000 \\
\hline $\mathrm{H}$ & -7.056028000 & -1.923080000 & -0.657650000 \\
\hline $\mathrm{H}$ & -5.045211000 & -1.211667000 & -1.901224000 \\
\hline $\mathrm{H}$ & -2.777518000 & -1.286073000 & 1.747653000 \\
\hline $\mathrm{H}$ & -4.766652000 & -2.040898000 & 2.971273000 \\
\hline $\mathrm{H}$ & -6.929830000 & -2.355974000 & 1.784501000 \\
\hline $\mathrm{C}$ & -2.572462000 & -0.758086000 & -0.918387000 \\
\hline $\mathrm{C}$ & -1.282147000 & -0.813606000 & -0.526232000 \\
\hline $\mathrm{H}$ & -1.090789000 & -1.372408000 & 0.385180000 \\
\hline $\mathrm{O}$ & -2.924604000 & -0.055996000 & -2.089258000 \\
\hline $\mathrm{C}$ & -0.168974000 & -0.110125000 & -1.155496000 \\
\hline $\mathrm{H}$ & -0.255943000 & -0.004273000 & -2.244846000 \\
\hline $\mathrm{C}$ & 1.219965000 & -0.436054000 & -0.777627000 \\
\hline $\mathrm{C}$ & 2.267924000 & -0.101984000 & -1.653817000 \\
\hline $\mathrm{C}$ & 1.583181000 & -0.977395000 & 0.467377000 \\
\hline $\mathrm{C}$ & 3.596941000 & -0.293530000 & -1.313342000 \\
\hline $\mathrm{H}$ & 2.026292000 & 0.331275000 & -2.621171000 \\
\hline $\mathrm{C}$ & 2.912518000 & -1.174236000 & 0.813890000 \\
\hline $\mathrm{H}$ & 0.820281000 & -1.245979000 & 1.192098000 \\
\hline $\mathrm{C}$ & 3.930713000 & -0.832466000 & -0.071706000 \\
\hline $\mathrm{H}$ & 4.379304000 & -0.018145000 & -2.012954000 \\
\hline $\mathrm{H}$ & 3.159054000 & -1.590802000 & 1.785452000 \\
\hline $\mathrm{H}$ & -0.408877000 & 1.191131000 & -0.779319000 \\
\hline $\mathrm{H}$ & -2.177472000 & -0.103876000 & -2.701036000 \\
\hline $\mathrm{C}$ & -0.271128000 & 2.045555000 & 1.806732000 \\
\hline $\mathrm{C}$ & -0.195659000 & 2.947720000 & 0.563437000 \\
\hline $\mathrm{H}$ & -1.319923000 & 1.832992000 & 2.058727000 \\
\hline $\mathrm{H}$ & 0.236151000 & 1.093999000 & 1.617663000 \\
\hline
\end{tabular}




$\begin{array}{lrrr}\mathrm{H} & 0.198706000 & 2.512381000 & 2.680053000 \\ \mathrm{C} & 1.273693000 & 3.200204000 & 0.197051000 \\ \mathrm{C} & -0.894587000 & 4.281549000 & 0.853829000 \\ \mathrm{H} & 1.330230000 & 3.821354000 & -0.703067000 \\ \mathrm{H} & 1.810321000 & 3.707799000 & 1.007283000 \\ \mathrm{H} & 1.783060000 & 2.252913000 & -0.009603000 \\ \mathrm{H} & -0.419743000 & 4.820241000 & 1.682026000 \\ \mathrm{H} & -0.871610000 & 4.919671000 & -0.036099000 \\ \mathrm{H} & -1.946020000 & 4.110320000 & 1.127886000 \\ \mathrm{O} & -0.861172000 & 2.339932000 & -0.515644000 \\ \mathrm{Na} & -2.912723000 & 1.792735000 & -0.657686000 \\ \mathrm{C} & 5.360073000 & -1.098542000 & 0.286547000 \\ \mathrm{~F} & 5.584224000 & -0.949338000 & 1.606351000 \\ \mathrm{~F} & 5.737945000 & -2.356222000 & -0.023446000 \\ \mathrm{~F} & 6.206133000 & -0.274017000 & -0.358302000 \\ -\end{array}$

\begin{tabular}{|c|c|c|c|}
\hline \multicolumn{4}{|c|}{$\mathrm{A}_{\mathrm{tBuONa}}$} \\
\hline & $\mathrm{X}$ & Y & Z \\
\hline$\overline{\mathrm{C}}$ & -5.192267000 & -1.339579000 & -1.375377000 \\
\hline $\mathrm{C}$ & -4.257791000 & -1.819535000 & -0.462862000 \\
\hline $\mathrm{C}$ & -3.141233000 & -1.049870000 & -0.111213000 \\
\hline $\mathrm{C}$ & -2.986301000 & 0.225243000 & -0.676143000 \\
\hline $\mathrm{C}$ & -3.926743000 & 0.695035000 & -1.586488000 \\
\hline $\mathrm{C}$ & -5.025839000 & -0.082257000 & -1.946463000 \\
\hline $\mathrm{H}$ & -6.055910000 & -1.946730000 & -1.630520000 \\
\hline $\mathrm{H}$ & -4.431637000 & -2.784570000 & 0.008545000 \\
\hline $\mathrm{H}$ & -2.138231000 & 0.868347000 & -0.401435000 \\
\hline $\mathrm{H}$ & -3.791718000 & 1.682885000 & -2.018295000 \\
\hline $\mathrm{H}$ & -5.752647000 & 0.292311000 & -2.661237000 \\
\hline $\mathrm{C}$ & -2.136131000 & -1.592224000 & 0.839368000 \\
\hline $\mathrm{C}$ & -1.368296000 & -0.844703000 & 1.643922000 \\
\hline $\mathrm{H}$ & -1.432149000 & 0.237477000 & 1.570175000 \\
\hline $\mathrm{O}$ & -2.021097000 & -2.961293000 & 0.892395000 \\
\hline $\mathrm{C}$ & -0.285731000 & -1.413962000 & 2.531682000 \\
\hline $\mathrm{H}$ & -0.490753000 & -2.459717000 & 2.775034000 \\
\hline $\mathrm{C}$ & 1.016393000 & -1.291724000 & 1.772491000 \\
\hline $\mathrm{C}$ & 1.364121000 & -2.269094000 & 0.828670000 \\
\hline $\mathrm{C}$ & 1.786411000 & -0.128543000 & 1.847986000 \\
\hline $\mathrm{C}$ & 2.404030000 & -2.055148000 & -0.068000000 \\
\hline $\mathrm{H}$ & 0.774962000 & -3.179778000 & 0.771618000 \\
\hline $\mathrm{C}$ & 2.830631000 & 0.094864000 & 0.955256000 \\
\hline $\mathrm{H}$ & 1.518812000 & 0.645601000 & 2.559620000 \\
\hline
\end{tabular}




$\begin{array}{lrcc}\mathrm{C} & 3.120288000 & -0.855245000 & -0.021781000 \\ \mathrm{H} & 2.649874000 & -2.804919000 & -0.813363000 \\ \mathrm{H} & 3.378172000 & 1.030505000 & 0.985693000 \\ \mathrm{C} & -0.963829000 & 3.193972000 & 1.873784000 \\ \mathrm{C} & -0.404339000 & 3.095042000 & 0.438349000 \\ \mathrm{H} & -1.991733000 & 2.812017000 & 1.895537000 \\ \mathrm{H} & -0.358540000 & 2.570330000 & 2.544802000 \\ \mathrm{H} & -0.966715000 & 4.219287000 & 2.265926000 \\ \mathrm{C} & 1.038825000 & 3.642056000 & 0.432858000 \\ \mathrm{C} & -1.272171000 & 3.965810000 & -0.494051000 \\ \mathrm{H} & 1.458119000 & 3.562366000 & -0.578185000 \\ \mathrm{H} & 1.106010000 & 4.689733000 & 0.754086000 \\ \mathrm{H} & 1.660692000 & 3.036828000 & 1.105672000 \\ \mathrm{H} & -1.285518000 & 5.024539000 & -0.203422000 \\ \mathrm{H} & -0.891850000 & 3.891560000 & -1.520024000 \\ \mathrm{H} & -2.304077000 & 3.593458000 & -0.487177000 \\ \mathrm{O} & -0.422159000 & 1.789191000 & 0.021018000 \\ \mathrm{H} & -0.233544000 & -0.850758000 & 3.468793000 \\ \mathrm{H} & -2.452046000 & -3.334914000 & 0.114338000 \\ \mathrm{Na} & 0.409703000 & 0.111046000 & -0.737801000 \\ \mathrm{C} & 4.056420000 & -0.513632000 & -1.142440000 \\ \mathrm{~F} & 3.349788000 & 0.042270000 & -2.163997000 \\ \mathrm{~F} & 4.673887000 & -1.591030000 & -1.645466000 \\ \mathrm{~F} & 4.990887000 & 0.373249000 & -0.787755000 \\ \mathrm{H}------\end{array}$

\begin{tabular}{llcc} 
A1 & & \\
& & & \\
Coordinates $($ Angstroms $)$ & \\
\hline $\mathrm{C}$ & -2.391136000 & 4.022351000 & -0.383174000 \\
$\mathrm{C}$ & -1.717372000 & 2.849786000 & -0.051053000 \\
$\mathrm{C}$ & -2.411660000 & 1.641132000 & 0.028503000 \\
$\mathrm{C}$ & -3.787396000 & 1.611620000 & -0.223356000 \\
$\mathrm{C}$ & -4.455377000 & 2.785662000 & -0.554631000 \\
$\mathrm{C}$ & -3.759859000 & 3.990636000 & -0.635623000 \\
$\mathrm{H}$ & -1.846464000 & 4.959530000 & -0.449371000 \\
$\mathrm{H}$ & -0.646214000 & 2.872702000 & 0.136448000 \\
$\mathrm{H}$ & -4.309710000 & 0.656425000 & -0.147439000 \\
$\mathrm{H}$ & -5.522321000 & 2.758118000 & -0.755648000 \\
$\mathrm{H}$ & -4.283611000 & 4.905160000 & -0.898341000 \\
$\mathrm{C}$ & -1.726625000 & 0.360562000 & 0.460302000 \\
$\mathrm{C}$ & -0.279839000 & 0.275968000 & 0.074030000 \\
$\mathrm{H}$ & -0.097482000 & 0.436737000 & -0.987133000 \\
$\mathrm{O}$ & -1.910000000 & 0.181588000 & 1.892357000
\end{tabular}




\begin{tabular}{|c|c|c|c|}
\hline $\mathrm{C}$ & 0.727325000 & 0.026315000 & 0.912646000 \\
\hline $\mathrm{H}$ & 0.500678000 & -0.107576000 & 1.968691000 \\
\hline $\mathrm{C}$ & 2.150127000 & -0.081017000 & 0.545840000 \\
\hline $\mathrm{C}$ & 3.122195000 & 0.049730000 & 1.544739000 \\
\hline $\mathrm{C}$ & 2.577780000 & -0.316313000 & -0.767081000 \\
\hline $\mathrm{C}$ & 4.475112000 & -0.029010000 & 1.245645000 \\
\hline $\mathrm{H}$ & 2.811868000 & 0.223530000 & 2.571604000 \\
\hline $\mathrm{C}$ & 3.928232000 & -0.396905000 & -1.073007000 \\
\hline $\mathrm{H}$ & 1.847770000 & -0.457146000 & -1.557947000 \\
\hline $\mathrm{C}$ & 4.881252000 & -0.249360000 & -0.067633000 \\
\hline $\mathrm{H}$ & 5.215039000 & 0.083862000 & 2.030895000 \\
\hline $\mathrm{H}$ & 4.242310000 & -0.578484000 & -2.095137000 \\
\hline $\mathrm{H}$ & -2.280410000 & -0.485721000 & 0.031957000 \\
\hline $\mathrm{H}$ & -1.734927000 & 1.033083000 & 2.314577000 \\
\hline $\mathrm{C}$ & -6.124072000 & -2.963275000 & 0.242025000 \\
\hline $\mathrm{C}$ & -4.804127000 & -2.368665000 & -0.280976000 \\
\hline $\mathrm{H}$ & -5.949879000 & -3.461591000 & 1.203283000 \\
\hline $\mathrm{H}$ & -6.852739000 & -2.160540000 & 0.402857000 \\
\hline $\mathrm{H}$ & -6.558330000 & -3.693602000 & -0.452624000 \\
\hline $\mathrm{C}$ & -5.064063000 & -1.682795000 & -1.636449000 \\
\hline $\mathrm{C}$ & -3.782122000 & -3.503221000 & -0.483749000 \\
\hline $\mathrm{H}$ & -4.136951000 & -1.231759000 & -2.011067000 \\
\hline $\mathrm{H}$ & -5.436561000 & -2.384119000 & -2.393335000 \\
\hline $\mathrm{H}$ & -5.804495000 & -0.882896000 & -1.515577000 \\
\hline $\mathrm{H}$ & -4.129688000 & -4.261508000 & -1.196753000 \\
\hline $\mathrm{H}$ & -2.837206000 & -3.088216000 & -0.855635000 \\
\hline $\mathrm{H}$ & -3.582778000 & -3.996133000 & 0.475463000 \\
\hline $\mathrm{O}$ & -4.307988000 & -1.451762000 & 0.620171000 \\
\hline $\mathrm{C}$ & 6.344167000 & -0.397016000 & -0.380587000 \\
\hline $\mathrm{F}$ & 7.101961000 & 0.390217000 & 0.402698000 \\
\hline $\mathrm{F}$ & 6.766722000 & -1.660010000 & -0.188022000 \\
\hline $\mathrm{F}$ & 6.619419000 & -0.081558000 & -1.657173000 \\
\hline $\mathrm{Li}$ & -3.557379000 & -0.857799000 & 1.988012000 \\
\hline
\end{tabular}

TSA1-2 ${ }_{\text {tBuOLi }}$

\begin{tabular}{llll} 
& \multicolumn{3}{c}{ Coordinates (Angstroms) } \\
C & X & Y & Z \\
C & 3.4345649000 & -2.110762000 & -1.932016000 \\
$\mathrm{C}$ & 3.470705000 & -1.467466000 & -1.306108000 \\
$\mathrm{C}$ & 4.616437000 & -1.553947000 & 0.782704000 \\
$\mathrm{C}$ & 5.676088000 & -2.199886000 & 0.152212000 \\
$\mathrm{C}$ & 5.622886000 & -2.486691000 & -1.207072000
\end{tabular}




\begin{tabular}{lrcc}
$\mathrm{H}$ & 4.436442000 & -2.317823000 & -2.997034000 \\
$\mathrm{H}$ & 2.574762000 & -1.174500000 & -1.902457000 \\
$\mathrm{H}$ & 4.681940000 & -1.318989000 & 1.839753000 \\
$\mathrm{H}$ & 6.552140000 & -2.476693000 & 0.732521000 \\
$\mathrm{H}$ & 6.450742000 & -2.989018000 & -1.698037000 \\
$\mathrm{C}$ & 2.365499000 & -0.447976000 & 0.729485000 \\
$\mathrm{C}$ & 0.983645000 & -0.658381000 & 0.354303000 \\
$\mathrm{H}$ & 0.805707000 & -0.805871000 & -0.708926000 \\
$\mathrm{O}$ & 2.503309000 & -0.335554000 & 2.166713000 \\
$\mathrm{C}$ & -0.096090000 & -0.540054000 & 1.169411000 \\
$\mathrm{H}$ & 0.049565000 & -0.525230000 & 2.252102000 \\
$\mathrm{C}$ & -1.495208000 & -0.530395000 & 0.731551000 \\
$\mathrm{C}$ & -2.513916000 & -0.827974000 & 1.649284000 \\
$\mathrm{C}$ & -1.874719000 & -0.210374000 & -0.581857000 \\
$\mathrm{C}$ & -3.848947000 & -0.827974000 & 1.273418000 \\
$\mathrm{H}$ & -2.250338000 & -1.078645000 & 2.673953000 \\
$\mathrm{C}$ & -3.207297000 & -0.217268000 & -0.965369000 \\
$\mathrm{H}$ & -1.119298000 & 0.066251000 & -1.311391000 \\
$\mathrm{C}$ & -4.201613000 & -0.528401000 & -0.040349000 \\
$\mathrm{~F}$ & -5.824437000 & -0.756817000 & -1.738531000 \\
$\mathrm{H}$ & -4.617562000 & -1.072072000 & 1.999123000 \\
$\mathrm{H}$ & -3.475715000 & 0.026818000 & -1.987822000 \\
$\mathrm{H}$ & 2.487102000 & 0.933894000 & 0.483497000 \\
$\mathrm{H}$ & 2.278118000 & -1.186074000 & 2.573130000 \\
$\mathrm{C}$ & 2.639385000 & 4.249590000 & -0.258672000 \\
$\mathrm{C}$ & 1.867903000 & 2.927515000 & -0.264779000 \\
$\mathrm{H}$ & 2.538723000 & 4.738437000 & 0.716596000 \\
$\mathrm{H}$ & 3.702723000 & 4.061525000 & -0.435947000 \\
$\mathrm{H}$ & 2.269985000 & 4.935713000 & -1.029869000 \\
$\mathrm{C}$ & 2.047106000 & 2.224074000 & -1.617044000 \\
$\mathrm{H}$ & 0.376873000 & 3.181360000 & -0.001072000 \\
$\mathrm{H}$ & 1.453787000 & 1.303411000 & -1.659416000 \\
$\mathrm{H}$ & 1.726550000 & 2.869614000 & -2.441800000 \\
$\mathrm{H}$ & 3.098661000 & 1.959003000 & -1.768510000 \\
$\mathrm{H}$ & -0.077631000 & 3.814729000 & -0.771094000 \\
$\mathrm{H}$ & -0.173925000 & 2.232326000 & 0.024966000 \\
$\mathrm{H}$ & -6.246743000 & 3.689800000 & 0.964382000 \\
$\mathrm{H}$ & -6.390258000 & 2.118890000 & 0.771652000 \\
$\mathrm{H}$ & -0.469178000 & -0.437035000 \\
$\mathrm{H}$ & -171466000 & 0.756179000 & -0.235987000 \\
$\mathrm{H}$ & -366706000 & 2.15768000 \\
\hline
\end{tabular}

A2 tBuOLi $_{\text {in }}$ 


\begin{tabular}{|c|c|c|c|}
\hline \multicolumn{4}{|c|}{ Coordinates (Angstroms) } \\
\hline & $X$ & $\mathrm{Y}$ & $\mathrm{Z}$ \\
\hline $\mathrm{C}$ & -6.009558000 & -0.494169000 & 0.249820000 \\
\hline $\mathrm{C}$ & -4.924001000 & -0.745783000 & -0.590895000 \\
\hline $\mathrm{C}$ & -3.678972000 & -1.178814000 & -0.072226000 \\
\hline $\mathrm{C}$ & -3.601125000 & -1.364862000 & 1.330949000 \\
\hline $\mathrm{C}$ & -4.688487000 & -1.116655000 & 2.148084000 \\
\hline $\mathrm{C}$ & -5.904574000 & -0.667133000 & 1.622064000 \\
\hline $\mathrm{H}$ & -6.949954000 & -0.171031000 & -0.188705000 \\
\hline $\mathrm{H}$ & -5.052043000 & -0.661812000 & -1.665619000 \\
\hline $\mathrm{H}$ & -2.679162000 & -1.722348000 & 1.778704000 \\
\hline $\mathrm{H}$ & -4.589420000 & -1.277290000 & 3.218145000 \\
\hline $\mathrm{H}$ & -6.749932000 & -0.472710000 & 2.273905000 \\
\hline $\mathrm{C}$ & -2.529029000 & -1.299854000 & -0.941354000 \\
\hline $\mathrm{C}$ & -1.197110000 & -1.296470000 & -0.562823000 \\
\hline $\mathrm{H}$ & -1.026120000 & -1.501947000 & 0.490445000 \\
\hline $\mathrm{O}$ & -2.832053000 & -0.889723000 & -2.277323000 \\
\hline $\mathrm{C}$ & -0.086557000 & -0.970993000 & -1.337695000 \\
\hline $\mathrm{H}$ & -0.201366000 & -0.821458000 & -2.411478000 \\
\hline $\mathrm{C}$ & 1.272768000 & -0.869895000 & -0.845464000 \\
\hline $\mathrm{C}$ & 2.295257000 & -0.428160000 & -1.712729000 \\
\hline $\mathrm{C}$ & 1.651189000 & -1.130971000 & 0.490830000 \\
\hline $\mathrm{C}$ & 3.597292000 & -0.244111000 & -1.279165000 \\
\hline $\mathrm{H}$ & 2.049120000 & -0.218829000 & -2.751045000 \\
\hline $\mathrm{C}$ & 2.951688000 & -0.942891000 & 0.926509000 \\
\hline $\mathrm{H}$ & 0.917643000 & -1.494823000 & 1.204270000 \\
\hline $\mathrm{C}$ & 3.938451000 & -0.492208000 & 0.049619000 \\
\hline $\mathrm{H}$ & 4.353467000 & 0.102289000 & -1.975872000 \\
\hline $\mathrm{H}$ & 3.203286000 & -1.146660000 & 1.963088000 \\
\hline $\mathrm{H}$ & -0.663779000 & 0.962139000 & -1.155751000 \\
\hline $\mathrm{H}$ & -2.196522000 & -1.299950000 & -2.874946000 \\
\hline $\mathrm{C}$ & -0.945688000 & 1.581758000 & 1.314652000 \\
\hline $\mathrm{C}$ & -0.905166000 & 2.497963000 & 0.090165000 \\
\hline $\mathrm{H}$ & -1.924419000 & 1.095685000 & 1.412504000 \\
\hline $\mathrm{H}$ & -0.175692000 & 0.806730000 & 1.240918000 \\
\hline $\mathrm{H}$ & -0.757279000 & 2.157056000 & 2.226895000 \\
\hline $\mathrm{C}$ & 0.498206000 & 3.061691000 & -0.120893000 \\
\hline $\mathrm{C}$ & -1.947810000 & 3.604187000 & 0.198270000 \\
\hline $\mathrm{H}$ & 0.522347000 & 3.704800000 & -1.005945000 \\
\hline $\mathrm{H}$ & 0.813902000 & 3.648509000 & 0.747813000 \\
\hline $\mathrm{H}$ & 1.219591000 & 2.248091000 & -0.261150000 \\
\hline $\mathrm{H}$ & -1.727841000 & 4.257185000 & 1.048006000 \\
\hline $\mathrm{H}$ & -1.959718000 & 4.211275000 & -0.712577000 \\
\hline $\mathrm{H}$ & -2.949131000 & 3.181449000 & 0.353231000 \\
\hline
\end{tabular}




$\begin{array}{lrrr}\mathrm{O} & -1.269879000 & 1.743644000 & -1.088212000 \\ \mathrm{C} & 5.345761000 & -0.338131000 & 0.531993000 \\ \mathrm{~F} & 5.983820000 & -1.522468000 & 0.637508000 \\ \mathrm{~F} & 6.086049000 & 0.427778000 & -0.290741000 \\ \mathrm{~F} & 5.397799000 & 0.226140000 & 1.755976000 \\ \mathrm{Li} & -2.888582000 & 0.804075000 & -1.297836000\end{array}$

TSA2-3 tBuOLi $_{\text {i }}$

\begin{tabular}{|c|c|c|c|}
\hline \multicolumn{4}{|c|}{ Coordinates (Angstroms) } \\
\hline & $\mathrm{X}$ & $\mathrm{Y}$ & Z \\
\hline$\overline{\mathrm{C}}$ & -6.337342000 & -1.440798000 & -0.234637000 \\
\hline $\mathrm{C}$ & -5.201536000 & -0.897254000 & -0.826746000 \\
\hline $\mathrm{C}$ & -3.945611000 & -1.018731000 & -0.214495000 \\
\hline $\mathrm{C}$ & -3.870864000 & -1.689820000 & 1.017509000 \\
\hline $\mathrm{C}$ & -5.005351000 & -2.238449000 & 1.598083000 \\
\hline $\mathrm{C}$ & -6.247425000 & -2.116644000 & 0.977198000 \\
\hline $\mathrm{H}$ & -7.298189000 & -1.337303000 & -0.730386000 \\
\hline $\mathrm{H}$ & -5.280620000 & -0.385531000 & -1.779412000 \\
\hline $\mathrm{H}$ & -2.918310000 & -1.777237000 & 1.531530000 \\
\hline $\mathrm{H}$ & -4.920284000 & -2.754993000 & 2.549592000 \\
\hline $\mathrm{H}$ & -7.134162000 & -2.541703000 & 1.436968000 \\
\hline $\mathrm{C}$ & -2.753686000 & -0.429580000 & -0.838894000 \\
\hline $\mathrm{C}$ & -1.448997000 & -0.657330000 & -0.557939000 \\
\hline $\mathrm{H}$ & -1.252838000 & -1.410849000 & 0.200781000 \\
\hline $\mathrm{O}$ & -3.080945000 & 0.561612000 & -1.802795000 \\
\hline $\mathrm{C}$ & -0.318904000 & 0.051354000 & -1.134327000 \\
\hline $\mathrm{H}$ & -0.413319000 & 0.269909000 & -2.205924000 \\
\hline $\mathrm{C}$ & 1.051320000 & -0.347976000 & -0.782618000 \\
\hline $\mathrm{C}$ & 2.106877000 & -0.108965000 & -1.680897000 \\
\hline $\mathrm{C}$ & 1.393459000 & -0.879279000 & 0.474257000 \\
\hline $\mathrm{C}$ & 3.423767000 & -0.386313000 & -1.351073000 \\
\hline $\mathrm{H}$ & 1.882310000 & 0.311252000 & -2.658080000 \\
\hline $\mathrm{C}$ & 2.709430000 & -1.166033000 & 0.806761000 \\
\hline $\mathrm{H}$ & 0.620806000 & -1.055297000 & 1.217866000 \\
\hline $\mathrm{C}$ & 3.736104000 & -0.920050000 & -0.101757000 \\
\hline $\mathrm{H}$ & 4.213710000 & -0.185656000 & -2.067454000 \\
\hline $\mathrm{H}$ & 2.941067000 & -1.571804000 & 1.786513000 \\
\hline $\mathrm{H}$ & -0.522286000 & 1.383799000 & -0.630521000 \\
\hline $\mathrm{H}$ & -2.406589000 & 0.553231000 & -2.496282000 \\
\hline $\mathrm{C}$ & 0.055383000 & 2.384160000 & 1.881023000 \\
\hline $\mathrm{C}$ & -0.124111000 & 3.163872000 & 0.573122000 \\
\hline $\mathrm{H}$ & -0.917984000 & 2.207997000 & 2.356205000 \\
\hline $\mathrm{H}$ & 0.527673000 & 1.415665000 & 1.684770000 \\
\hline
\end{tabular}




\begin{tabular}{|c|c|c|c|}
\hline $\mathrm{H}$ & 0.686833000 & 2.932366000 & 2.589011000 \\
\hline $\mathrm{C}$ & 1.233117000 & 3.371117000 & -0.107905000 \\
\hline $\mathrm{C}$ & -0.794469000 & 4.511825000 & 0.844020000 \\
\hline $\mathrm{H}$ & 1.097521000 & 3.887598000 & -1.063799000 \\
\hline $\mathrm{H}$ & 1.902473000 & 3.969270000 & 0.520184000 \\
\hline $\mathrm{H}$ & 1.717260000 & 2.407808000 & -0.302149000 \\
\hline $\mathrm{H}$ & -0.181220000 & 5.137379000 & 1.501987000 \\
\hline $\mathrm{H}$ & -0.953496000 & 5.049138000 & -0.096842000 \\
\hline $\mathrm{H}$ & -1.768175000 & 4.365243000 & 1.329335000 \\
\hline $\mathrm{O}$ & -0.975221000 & 2.439286000 & -0.297457000 \\
\hline $\mathrm{C}$ & 5.149188000 & -1.275910000 & 0.243445000 \\
\hline $\mathrm{F}$ & 5.436868000 & -2.561377000 & -0.048208000 \\
\hline $\mathrm{F}$ & 6.039560000 & -0.521895000 & -0.427606000 \\
\hline $\mathrm{F}$ & 5.401390000 & -1.118695000 & 1.557169000 \\
\hline $\mathrm{Li}$ & -2.645103000 & 1.828351000 & -0.369619000 \\
\hline
\end{tabular}

\begin{tabular}{|c|c|c|c|}
\hline \multicolumn{4}{|c|}{$\mathrm{A}_{\mathrm{tBuOLi}}$} \\
\hline & $\mathrm{X}$ & Y & Z \\
\hline $\mathrm{C}$ & -5.609506000 & -0.361092000 & -0.989170000 \\
\hline $\mathrm{C}$ & -4.604200000 & -1.252115000 & -0.632006000 \\
\hline $\mathrm{C}$ & -3.370640000 & -0.776575000 & -0.167026000 \\
\hline $\mathrm{C}$ & -3.149799000 & 0.601672000 & -0.069938000 \\
\hline $\mathrm{C}$ & -4.163388000 & 1.484221000 & -0.431580000 \\
\hline $\mathrm{C}$ & -5.389966000 & 1.010552000 & -0.889166000 \\
\hline $\mathrm{H}$ & -6.565718000 & -0.738838000 & -1.338672000 \\
\hline $\mathrm{H}$ & -4.792863000 & -2.322437000 & -0.681502000 \\
\hline $\mathrm{H}$ & -2.174198000 & 0.992000000 & 0.238539000 \\
\hline $\mathrm{H}$ & -3.981518000 & 2.552975000 & -0.364341000 \\
\hline $\mathrm{H}$ & -6.172867000 & 1.707955000 & -1.172033000 \\
\hline $\mathrm{C}$ & -2.312567000 & -1.750099000 & 0.200961000 \\
\hline $\mathrm{C}$ & -1.543667000 & -1.668561000 & 1.299147000 \\
\hline $\mathrm{H}$ & -1.703768000 & -0.829087000 & 1.968834000 \\
\hline $\mathrm{O}$ & -2.162247000 & -2.823223000 & -0.635110000 \\
\hline $\mathrm{C}$ & -0.454481000 & -2.664093000 & 1.639508000 \\
\hline $\mathrm{H}$ & -0.689394000 & -3.643465000 & 1.213997000 \\
\hline $\mathrm{C}$ & 0.858538000 & -2.154101000 & 1.082053000 \\
\hline $\mathrm{C}$ & 1.206882000 & -2.427981000 & -0.249801000 \\
\hline $\mathrm{C}$ & 1.641223000 & -1.249801000 & 1.805307000 \\
\hline $\mathrm{C}$ & 2.290533000 & -1.791792000 & -0.844695000 \\
\hline $\mathrm{H}$ & 0.601358000 & -3.122901000 & -0.823992000 \\
\hline $\mathrm{C}$ & 2.724825000 & -0.606375000 & 1.212200000 \\
\hline $\mathrm{H}$ & 1.373196000 & -1.008820000 & 2.829730000 \\
\hline
\end{tabular}




$\begin{array}{lrcc}\mathrm{C} & 3.043106000 & -0.869704000 & -0.115179000 \\ \mathrm{H} & 2.546057000 & -2.003733000 & -1.877880000 \\ \mathrm{H} & 3.298114000 & 0.120965000 & 1.774713000 \\ \mathrm{C} & -0.809737000 & 3.488538000 & 1.492575000 \\ \mathrm{C} & 0.097848000 & 2.848413000 & 0.424275000 \\ \mathrm{H} & -1.863454000 & 3.352830000 & 1.219178000 \\ \mathrm{H} & -0.647881000 & 2.994604000 & 2.457689000 \\ \mathrm{H} & -0.622323000 & 4.562725000 & 1.615282000 \\ \mathrm{C} & 1.571139000 & 3.058173000 & 0.823255000 \\ \mathrm{C} & -0.161880000 & 3.529796000 & -0.932442000 \\ \mathrm{H} & 2.225909000 & 2.596887000 & 0.074667000 \\ \mathrm{H} & 1.838577000 & 4.118883000 & 0.912463000 \\ \mathrm{H} & 1.759628000 & 2.572480000 & 1.788917000 \\ \mathrm{H} & 0.022160000 & 4.611382000 & -0.902142000 \\ \mathrm{H} & 0.487176000 & 3.087802000 & -1.697504000 \\ \mathrm{H} & -1.202889000 & 3.362243000 & -1.234131000 \\ \mathrm{O} & -0.174437000 & 1.504130000 & 0.330428000 \\ \mathrm{H} & -0.383862000 & -2.779591000 & 2.725434000 \\ \mathrm{H} & -2.673863000 & -2.657968000 & -1.437417000 \\ \mathrm{C} & 4.150585000 & -0.119885000 & -0.805541000 \\ \mathrm{~F} & 3.659879000 & 0.851359000 & -1.595883000 \\ \mathrm{~F} & 4.874790000 & -0.934118000 & -1.593249000 \\ \mathrm{~F} & 4.989324000 & 0.456591000 & 0.065333000 \\ \mathrm{Li} & 0.070294000 & -0.104594000 & 0.048467000 \\ \mathrm{H}----- & \end{array}$

\begin{tabular}{lrrr} 
A1 & \multicolumn{3}{c}{ Coordinates $($ Angstroms) } \\
& X & Y & Z \\
\hline $\mathrm{C}$ & 3.929550000 & -1.029150000 & 2.416691000 \\
$\mathrm{C}$ & 4.608757000 & -2.395809000 & 2.420547000 \\
$\mathrm{C}$ & 5.776383000 & -2.360582000 & 1.445603000 \\
$\mathrm{~N}$ & 3.494223000 & -0.643701000 & 1.091480000 \\
$\mathrm{H}$ & 4.625950000 & -0.280418000 & 2.830788000 \\
$\mathrm{H}$ & 3.056836000 & -1.038118000 & 3.079267000 \\
$\mathrm{H}$ & 3.885475000 & -3.155319000 & 2.102022000 \\
$\mathrm{H}$ & 4.968238000 & -2.668312000 & 3.418249000 \\
$\mathrm{H}$ & 6.620350000 & -1.802616000 & 1.878873000 \\
$\mathrm{H}$ & 6.135372000 & -3.378562000 & 1.243220000 \\
$\mathrm{~N}$ & 5.386930000 & -1.757987000 & 0.179231000 \\
$\mathrm{C}$ & 6.190976000 & -2.163662000 & -0.962645000 \\
$\mathrm{C}$ & 5.903195000 & -1.326126000 & -2.199604000 \\
$\mathrm{C}$ & 4.396794000 & -1.193150000 & -2.356852000 \\
$\mathrm{~N}$ & 3.881923000 & -0.561799000 & -1.164186000
\end{tabular}




\begin{tabular}{|c|c|c|c|}
\hline $\mathrm{C}$ & 4.234015000 & -1.003131000 & 0.092129000 \\
\hline $\mathrm{H}$ & 6.004370000 & -3.227814000 & -1.182636000 \\
\hline $\mathrm{H}$ & 7.250078000 & -2.077482000 & -0.685451000 \\
\hline $\mathrm{H}$ & 6.337211000 & -0.326986000 & -2.088163000 \\
\hline $\mathrm{H}$ & 6.355041000 & -1.802325000 & -3.075145000 \\
\hline $\mathrm{H}$ & 4.146814000 & -0.559961000 & -3.213109000 \\
\hline $\mathrm{H}$ & 3.950017000 & -2.185810000 & -2.538357000 \\
\hline $\mathrm{H}$ & 2.932872000 & -0.199096000 & -1.210777000 \\
\hline $\mathrm{C}$ & 0.733810000 & 4.632575000 & -0.121485000 \\
\hline $\mathrm{C}$ & 0.297689000 & 3.315020000 & -0.235226000 \\
\hline $\mathrm{C}$ & 1.141746000 & 2.257937000 & 0.112988000 \\
\hline $\mathrm{C}$ & 2.431410000 & 2.532898000 & 0.574806000 \\
\hline $\mathrm{C}$ & 2.863859000 & 3.849882000 & 0.691022000 \\
\hline $\mathrm{C}$ & 2.017994000 & 4.901187000 & 0.343140000 \\
\hline $\mathrm{H}$ & 0.068284000 & 5.447760000 & -0.390667000 \\
\hline $\mathrm{H}$ & -0.710294000 & 3.105548000 & -0.586927000 \\
\hline $\mathrm{H}$ & 3.078943000 & 1.699138000 & 0.839808000 \\
\hline $\mathrm{H}$ & 3.865615000 & 4.057172000 & 1.056540000 \\
\hline $\mathrm{H}$ & 2.358362000 & 5.928470000 & 0.437892000 \\
\hline $\mathrm{C}$ & 0.711634000 & 0.813968000 & -0.085165000 \\
\hline $\mathrm{C}$ & -0.747259000 & 0.598019000 & 0.214319000 \\
\hline $\mathrm{H}$ & -1.062284000 & 0.956571000 & 1.193342000 \\
\hline $\mathrm{O}$ & 1.059824000 & 0.374741000 & -1.404192000 \\
\hline $\mathrm{C}$ & -1.614200000 & 0.025067000 & -0.622568000 \\
\hline $\mathrm{H}$ & -1.240316000 & -0.304912000 & -1.589855000 \\
\hline $\mathrm{C}$ & -3.047062000 & -0.206861000 & -0.375929000 \\
\hline $\mathrm{C}$ & -3.864192000 & -0.589407000 & -1.447086000 \\
\hline $\mathrm{C}$ & -3.641996000 & -0.061127000 & 0.884652000 \\
\hline $\mathrm{C}$ & -5.225067000 & -0.802828000 & -1.276791000 \\
\hline $\mathrm{H}$ & -3.422833000 & -0.714787000 & -2.432159000 \\
\hline $\mathrm{C}$ & -5.000557000 & -0.272774000 & 1.062767000 \\
\hline $\mathrm{H}$ & -3.036548000 & 0.212484000 & 1.742971000 \\
\hline $\mathrm{C}$ & -5.798611000 & -0.640636000 & -0.019127000 \\
\hline $\mathrm{H}$ & -5.841890000 & -1.089899000 & -2.121979000 \\
\hline $\mathrm{H}$ & -5.444040000 & -0.150330000 & 2.045074000 \\
\hline $\mathrm{H}$ & 1.318107000 & 0.182340000 & 0.576920000 \\
\hline $\mathrm{H}$ & 0.865466000 & 1.104956000 & -2.006531000 \\
\hline $\mathrm{C}$ & -7.259490000 & -0.926100000 & 0.182550000 \\
\hline $\mathrm{F}$ & -7.984858000 & -0.617650000 & -0.907149000 \\
\hline $\mathrm{F}$ & -7.769659000 & -0.232811000 & 1.215057000 \\
\hline $\mathrm{F}$ & -7.481215000 & -2.229148000 & 0.440118000 \\
\hline
\end{tabular}

TSA1-2 TBD 


\begin{tabular}{|c|c|c|c|}
\hline \multicolumn{4}{|c|}{ Coordinates (Angstroms) } \\
\hline & $X$ & $\mathrm{Y}$ & Z \\
\hline $\bar{C}$ & -1.675261000 & 0.833337000 & 2.384023000 \\
\hline $\mathrm{C}$ & -0.216362000 & 0.427873000 & 2.554698000 \\
\hline $\mathrm{C}$ & 0.663381000 & 1.650069000 & 2.346491000 \\
\hline $\mathrm{N}$ & -1.893725000 & 1.464424000 & 1.094848000 \\
\hline $\mathrm{H}$ & -1.980123000 & 1.514777000 & 3.189319000 \\
\hline $\mathrm{H}$ & -2.335474000 & -0.038779000 & 2.431046000 \\
\hline $\mathrm{H}$ & 0.040828000 & -0.331663000 & 1.809712000 \\
\hline $\mathrm{H}$ & -0.032463000 & 0.008626000 & 3.548234000 \\
\hline $\mathrm{H}$ & 0.623839000 & 2.315682000 & 3.219359000 \\
\hline $\mathrm{H}$ & 1.705779000 & 1.341907000 & 2.208739000 \\
\hline $\mathrm{N}$ & 0.269631000 & 2.401501000 & 1.154398000 \\
\hline $\mathrm{C}$ & 1.353771000 & 3.128099000 & 0.499832000 \\
\hline $\mathrm{C}$ & 0.829684000 & 4.070218000 & -0.571453000 \\
\hline $\mathrm{C}$ & -0.120985000 & 3.303532000 & -1.476713000 \\
\hline $\mathrm{N}$ & -1.177116000 & 2.744042000 & -0.658772000 \\
\hline $\mathrm{C}$ & -0.921659000 & 2.175625000 & 0.549595000 \\
\hline $\mathrm{H}$ & 2.054421000 & 2.402306000 & 0.061683000 \\
\hline $\mathrm{H}$ & 1.893864000 & 3.687602000 & 1.271974000 \\
\hline $\mathrm{H}$ & 0.297570000 & 4.909120000 & -0.110754000 \\
\hline $\mathrm{H}$ & 1.669747000 & 4.471992000 & -1.144567000 \\
\hline $\mathrm{H}$ & -0.578920000 & 3.965528000 & -2.216079000 \\
\hline $\mathrm{H}$ & 0.427303000 & 2.518864000 & -2.019247000 \\
\hline $\mathrm{H}$ & -1.957060000 & 2.305872000 & -1.147085000 \\
\hline $\mathrm{C}$ & -5.048496000 & -2.713115000 & 0.963080000 \\
\hline $\mathrm{C}$ & -3.893351000 & -2.034433000 & 0.599506000 \\
\hline $\mathrm{C}$ & -3.907497000 & -1.054750000 & -0.409296000 \\
\hline $\mathrm{C}$ & -5.138923000 & -0.776322000 & -1.018910000 \\
\hline $\mathrm{C}$ & -6.295699000 & -1.457008000 & -0.651855000 \\
\hline $\mathrm{C}$ & -6.262282000 & -2.432811000 & 0.338470000 \\
\hline $\mathrm{H}$ & -5.000422000 & -3.467681000 & 1.743767000 \\
\hline $\mathrm{H}$ & -2.966748000 & -2.269814000 & 1.117879000 \\
\hline $\mathrm{H}$ & -5.181316000 & -0.007046000 & -1.782130000 \\
\hline $\mathrm{H}$ & -7.233237000 & -1.218888000 & -1.147602000 \\
\hline $\mathrm{C}$ & -2.694642000 & -0.285207000 & -0.772100000 \\
\hline $\mathrm{C}$ & -1.385804000 & -0.875894000 & -0.712132000 \\
\hline $\mathrm{H}$ & -1.278115000 & -1.678750000 & 0.015344000 \\
\hline $\mathrm{O}$ & -2.880924000 & 0.647920000 & -1.832925000 \\
\hline $\mathrm{C}$ & -0.269571000 & -0.395904000 & -1.320680000 \\
\hline $\mathrm{H}$ & -0.390865000 & 0.400464000 & -2.051450000 \\
\hline $\mathrm{C}$ & 1.106116000 & -0.747310000 & -1.003621000 \\
\hline $\mathrm{C}$ & 2.154994000 & -0.048447000 & -1.631412000 \\
\hline $\mathrm{C}$ & 1.473730000 & -1.712877000 & -0.044670000 \\
\hline
\end{tabular}




\begin{tabular}{lrrr}
$\mathrm{C}$ & 3.484713000 & -0.271022000 & -1.307425000 \\
$\mathrm{H}$ & 1.912048000 & 0.689335000 & -2.392578000 \\
$\mathrm{C}$ & 2.799788000 & -1.937273000 & 0.284650000 \\
$\mathrm{H}$ & 0.708408000 & -2.299154000 & 0.455084000 \\
$\mathrm{C}$ & 3.817718000 & -1.211350000 & -0.335398000 \\
$\mathrm{H}$ & 4.266827000 & 0.290895000 & -1.807495000 \\
$\mathrm{H}$ & 3.049695000 & -2.680605000 & 1.035436000 \\
$\mathrm{H}$ & -2.411916000 & 0.687359000 & 0.302376000 \\
$\mathrm{H}$ & -2.779487000 & 0.179335000 & -2.674794000 \\
$\mathrm{H}$ & -7.165830000 & -2.962548000 & 0.624668000 \\
$\mathrm{C}$ & 5.247996000 & -1.494419000 & 0.006641000 \\
$\mathrm{~F}$ & 6.061650000 & -0.478114000 & -0.334912000 \\
$\mathrm{~F}$ & 5.714191000 & -2.592968000 & -0.618723000 \\
$\mathrm{~F}$ & 5.411865000 & -1.710200000 & 1.327114000 \\
\hline
\end{tabular}

\begin{tabular}{|c|c|c|c|}
\hline \multicolumn{4}{|c|}{ A2 TBD } \\
\hline & \multicolumn{3}{|c|}{ Coordinates (Angstroms) } \\
\hline & $\mathrm{X}$ & $\mathrm{Y}$ & Z \\
\hline$\overline{\mathrm{C}}$ & -3.675426000 & 1.187708000 & -1.546775000 \\
\hline $\mathrm{C}$ & -4.114813000 & 1.704185000 & -0.187385000 \\
\hline $\mathrm{C}$ & -3.277525000 & 2.923328000 & 0.167305000 \\
\hline $\mathrm{N}$ & -2.236907000 & 0.959745000 & -1.540918000 \\
\hline $\mathrm{H}$ & -3.934462000 & 1.903512000 & -2.337423000 \\
\hline $\mathrm{H}$ & -4.151972000 & 0.232629000 & -1.771412000 \\
\hline $\mathrm{H}$ & -3.979852000 & 0.916375000 & 0.561706000 \\
\hline $\mathrm{H}$ & -5.173041000 & 1.976998000 & -0.205659000 \\
\hline $\mathrm{H}$ & -3.545938000 & 3.783436000 & -0.461035000 \\
\hline $\mathrm{H}$ & -3.444052000 & 3.209563000 & 1.211136000 \\
\hline $\mathrm{N}$ & -1.848183000 & 2.643809000 & 0.020532000 \\
\hline $\mathrm{C}$ & -0.948863000 & 3.449831000 & 0.845589000 \\
\hline $\mathrm{C}$ & 0.501334000 & 3.309311000 & 0.404663000 \\
\hline $\mathrm{C}$ & 0.831715000 & 1.834982000 & 0.239413000 \\
\hline $\mathrm{N}$ & -0.114127000 & 1.261112000 & -0.704440000 \\
\hline $\mathrm{C}$ & -1.401951000 & 1.612992000 & -0.723798000 \\
\hline $\mathrm{H}$ & -1.063546000 & 3.145861000 & 1.894971000 \\
\hline $\mathrm{H}$ & -1.276096000 & 4.492446000 & 0.765839000 \\
\hline $\mathrm{H}$ & 0.656157000 & 3.825908000 & -0.548460000 \\
\hline $\mathrm{H}$ & 1.154214000 & 3.771084000 & 1.149648000 \\
\hline $\mathrm{H}$ & 1.834097000 & 1.680658000 & -0.165355000 \\
\hline $\mathrm{H}$ & 0.783019000 & 1.310114000 & 1.202418000 \\
\hline $\mathrm{H}$ & 0.107595000 & 0.301951000 & -1.032586000 \\
\hline $\mathrm{C}$ & -4.096395000 & -1.364079000 & 2.439428000 \\
\hline $\mathrm{C}$ & -2.966771000 & -1.509775000 & 1.646874000 \\
\hline
\end{tabular}




\begin{tabular}{lrrr}
$\mathrm{C}$ & -3.067198000 & -1.835678000 & 0.276160000 \\
$\mathrm{C}$ & -4.367162000 & -1.997019000 & -0.246711000 \\
$\mathrm{C}$ & -5.494141000 & -1.832438000 & 0.550050000 \\
$\mathrm{C}$ & -5.374477000 & -1.513418000 & 1.900130000 \\
$\mathrm{H}$ & -3.976646000 & -1.119953000 & 3.492025000 \\
$\mathrm{H}$ & -1.989956000 & -1.355621000 & 2.096014000 \\
$\mathrm{H}$ & -4.477473000 & -2.256447000 & -1.294237000 \\
$\mathrm{H}$ & -6.478888000 & -1.964961000 & 0.108837000 \\
$\mathrm{C}$ & -1.911054000 & -1.922336000 & -0.593404000 \\
$\mathrm{C}$ & -0.592476000 & -1.834340000 & -0.242550000 \\
$\mathrm{H}$ & -0.384784000 & -1.875834000 & 0.823777000 \\
$\mathrm{O}$ & -2.229911000 & -1.841510000 & -1.967760000 \\
$\mathrm{C}$ & 0.499134000 & -1.623708000 & -1.112233000 \\
$\mathrm{H}$ & 0.329210000 & -1.628331000 & -2.188361000 \\
$\mathrm{C}$ & 1.844247000 & -1.358092000 & -0.691606000 \\
$\mathrm{C}$ & 2.814110000 & -0.943096000 & -1.642033000 \\
$\mathrm{C}$ & 2.276377000 & -1.379696000 & 0.660814000 \\
$\mathrm{C}$ & 4.083557000 & -0.542931000 & -1.273114000 \\
$\mathrm{H}$ & 2.535833000 & -0.919666000 & -2.693402000 \\
$\mathrm{C}$ & 3.548138000 & -0.977899000 & 1.027308000 \\
$\mathrm{H}$ & 1.599566000 & -1.720823000 & 1.438400000 \\
$\mathrm{C}$ & 4.466286000 & -0.537275000 & 0.071964000 \\
$\mathrm{H}$ & 4.787264000 & -0.217530000 & -2.033378000 \\
$\mathrm{H}$ & 3.833744000 & -1.001671000 & 2.075148000 \\
$\mathrm{H}$ & -1.932666000 & 0.055388000 & -1.908239000 \\
$\mathrm{H}$ & -1.662448000 & -2.468680000 & -2.434563000 \\
$\mathrm{H}$ & -6.255909000 & -1.389152000 & 2.521605000 \\
$\mathrm{C}$ & 5.848502000 & -0.144779000 & 0.469638000 \\
$\mathrm{~F}$ & 6.389993000 & 0.746157000 & -0.387622000 \\
$\mathrm{~F}$ & 5.883673000 & 0.417406000 & 1.697251000 \\
$\mathrm{~F}$ & 6.702995000 & -1.190510000 & 0.513714000 \\
\hline-----
\end{tabular}

TSA2-3 TBD

\begin{tabular}{llcc} 
& \multicolumn{3}{c}{ Coordinates (Angstroms) } \\
C & -3.844890000 & 1.326448000 & -1.182520000 \\
$\mathrm{C}$ & -4.132758000 & 1.682536000 & 0.266267000 \\
$\mathrm{C}$ & -3.371473000 & 2.951457000 & 0.618134000 \\
$\mathrm{~N}$ & -2.408910000 & 1.295628000 & -1.412053000 \\
$\mathrm{H}$ & -4.315398000 & 2.057001000 & -1.854273000 \\
$\mathrm{H}$ & -4.241105000 & 0.337630000 & -1.424842000 \\
$\mathrm{H}$ & -3.815797000 & 0.858050000 & 0.914663000 \\
$\mathrm{H}$ & -5.204390000 & 1.839035000 & 0.416186000
\end{tabular}




\begin{tabular}{|c|c|c|c|}
\hline $\mathrm{H}$ & -3.794446000 & 3.817855000 & 0.089123000 \\
\hline $\mathrm{H}$ & -3.446286000 & 3.154954000 & 1.692394000 \\
\hline $\mathrm{N}$ & -1.955430000 & 2.813722000 & 0.298138000 \\
\hline $\mathrm{C}$ & -1.033950000 & 3.649389000 & 1.059649000 \\
\hline $\mathrm{C}$ & 0.339735000 & 3.686711000 & 0.406532000 \\
\hline $\mathrm{C}$ & 0.761742000 & 2.262443000 & 0.076270000 \\
\hline $\mathrm{N}$ & -0.226864000 & 1.639766000 & -0.787924000 \\
\hline $\mathrm{C}$ & -1.506938000 & 1.912779000 & -0.614831000 \\
\hline $\mathrm{H}$ & -0.958157000 & 3.263971000 & 2.086647000 \\
\hline $\mathrm{H}$ & -1.464369000 & 4.656302000 & 1.117861000 \\
\hline $\mathrm{H}$ & 0.300382000 & 4.277875000 & -0.515059000 \\
\hline $\mathrm{H}$ & 1.054732000 & 4.162077000 & 1.083956000 \\
\hline $\mathrm{H}$ & 1.722554000 & 2.239649000 & -0.444165000 \\
\hline $\mathrm{H}$ & 0.889878000 & 1.682134000 & 1.001784000 \\
\hline $\mathrm{H}$ & 0.076039000 & 0.490925000 & -1.095326000 \\
\hline $\mathrm{C}$ & -4.107736000 & -2.052207000 & 2.252291000 \\
\hline $\mathrm{C}$ & -2.991483000 & -1.806869000 & 1.463754000 \\
\hline $\mathrm{C}$ & -3.031133000 & -1.995793000 & 0.070937000 \\
\hline $\mathrm{C}$ & -4.245177000 & -2.412263000 & -0.497223000 \\
\hline $\mathrm{C}$ & -5.365207000 & -2.643014000 & 0.294783000 \\
\hline $\mathrm{C}$ & -5.305479000 & -2.469183000 & 1.674097000 \\
\hline $\mathrm{H}$ & -4.044866000 & -1.902274000 & 3.326639000 \\
\hline $\mathrm{H}$ & -2.081208000 & -1.445381000 & 1.932881000 \\
\hline $\mathrm{H}$ & -4.298990000 & -2.555687000 & -1.570859000 \\
\hline $\mathrm{H}$ & -6.290922000 & -2.967817000 & -0.172294000 \\
\hline $\mathrm{C}$ & -1.874428000 & -1.707186000 & -0.780207000 \\
\hline $\mathrm{C}$ & -0.599982000 & -1.506105000 & -0.384978000 \\
\hline $\mathrm{H}$ & -0.383305000 & -1.708958000 & 0.659720000 \\
\hline $\mathrm{O}$ & -2.221357000 & -1.493509000 & -2.121165000 \\
\hline $\mathrm{C}$ & 0.457784000 & -0.941600000 & -1.205037000 \\
\hline $\mathrm{H}$ & 0.370674000 & -1.097292000 & -2.284510000 \\
\hline $\mathrm{C}$ & 1.847347000 & -0.912485000 & -0.751453000 \\
\hline $\mathrm{C}$ & 2.885685000 & -0.704311000 & -1.683197000 \\
\hline $\mathrm{C}$ & 2.223991000 & -0.961845000 & 0.606945000 \\
\hline $\mathrm{C}$ & 4.203827000 & -0.547215000 & -1.291092000 \\
\hline $\mathrm{H}$ & 2.638738000 & -0.657388000 & -2.741161000 \\
\hline $\mathrm{C}$ & 3.543204000 & -0.808585000 & 1.003262000 \\
\hline $\mathrm{H}$ & 1.469921000 & -1.109558000 & 1.374469000 \\
\hline $\mathrm{C}$ & 4.547037000 & -0.593381000 & 0.060691000 \\
\hline $\mathrm{H}$ & 4.973523000 & -0.384125000 & -2.038943000 \\
\hline $\mathrm{H}$ & 3.794410000 & -0.848898000 & 2.058852000 \\
\hline $\mathrm{H}$ & -2.068545000 & 0.481239000 & -1.915918000 \\
\hline $\mathrm{H}$ & -1.502591000 & -1.840240000 & -2.665786000 \\
\hline $\mathrm{H}$ & -6.180115000 & -2.651827000 & 2.290986000 \\
\hline
\end{tabular}




\begin{tabular}{lrrr} 
C & 5.974632000 & -0.481045000 & 0.490270000 \\
F & 6.708774000 & 0.248878000 & -0.372308000 \\
F & 6.582136000 & -1.682781000 & 0.585540000 \\
F & 6.093850000 & 0.098674000 & 1.702225000 \\
\hline
\end{tabular}

\begin{tabular}{|c|c|c|c|}
\hline \multicolumn{4}{|c|}{$\mathrm{A3}$ TBD } \\
\hline & $X$ & Y & Z \\
\hline$\overline{\mathrm{C}}$ & -0.676009000 & 1.954311000 & 1.817619000 \\
\hline $\mathrm{C}$ & 0.346462000 & 1.116034000 & 2.571499000 \\
\hline $\mathrm{C}$ & 1.756710000 & 1.505318000 & 2.137552000 \\
\hline $\mathrm{N}$ & -0.451273000 & 1.755725000 & 0.406961000 \\
\hline $\mathrm{H}$ & -0.591722000 & 3.013537000 & 2.117640000 \\
\hline $\mathrm{H}$ & -1.692714000 & 1.623762000 & 2.051863000 \\
\hline $\mathrm{H}$ & 0.159477000 & 0.063245000 & 2.347283000 \\
\hline $\mathrm{H}$ & 0.250413000 & 1.253901000 & 3.653114000 \\
\hline $\mathrm{H}$ & 2.098672000 & 2.370463000 & 2.728013000 \\
\hline $\mathrm{H}$ & 2.452006000 & 0.680868000 & 2.348630000 \\
\hline $\mathrm{N}$ & 1.858622000 & 1.841478000 & 0.726437000 \\
\hline $\mathrm{C}$ & 3.213477000 & 2.039349000 & 0.241406000 \\
\hline $\mathrm{C}$ & 3.211367000 & 2.900101000 & -1.013447000 \\
\hline $\mathrm{C}$ & 2.180312000 & 2.345375000 & -1.994435000 \\
\hline $\mathrm{N}$ & 0.864436000 & 2.210482000 & -1.408615000 \\
\hline $\mathrm{C}$ & 0.797037000 & 1.959771000 & -0.144336000 \\
\hline $\mathrm{H}$ & 3.689479000 & 1.067562000 & 0.045770000 \\
\hline $\mathrm{H}$ & 3.791112000 & 2.526294000 & 1.038452000 \\
\hline $\mathrm{H}$ & 2.943632000 & 3.930046000 & -0.748394000 \\
\hline $\mathrm{H}$ & 4.213812000 & 2.916770000 & -1.454117000 \\
\hline $\mathrm{H}$ & 2.103641000 & 3.004436000 & -2.867507000 \\
\hline $\mathrm{H}$ & 2.535491000 & 1.371919000 & -2.369774000 \\
\hline $\mathrm{C}$ & -6.314305000 & -1.818537000 & 0.098161000 \\
\hline $\mathrm{C}$ & -5.147320000 & -1.504476000 & -0.588079000 \\
\hline $\mathrm{C}$ & -4.430853000 & -0.343414000 & -0.277831000 \\
\hline $\mathrm{C}$ & -4.923831000 & 0.505156000 & 0.721219000 \\
\hline $\mathrm{C}$ & -6.095291000 & 0.192717000 & 1.402806000 \\
\hline $\mathrm{C}$ & -6.793444000 & -0.970975000 & 1.094673000 \\
\hline $\mathrm{H}$ & -6.859842000 & -2.722492000 & -0.155836000 \\
\hline $\mathrm{H}$ & -4.793906000 & -2.151676000 & -1.385128000 \\
\hline $\mathrm{H}$ & -4.371281000 & 1.403515000 & 0.983837000 \\
\hline $\mathrm{H}$ & -6.459119000 & 0.857943000 & 2.180227000 \\
\hline $\mathrm{C}$ & -3.175068000 & 0.001201000 & -0.985460000 \\
\hline $\mathrm{C}$ & -2.263937000 & -0.892697000 & -1.383002000 \\
\hline $\mathrm{H}$ & -2.454865000 & -1.935091000 & -1.140333000 \\
\hline
\end{tabular}




$\begin{array}{lrrr}\mathrm{O} & -2.943185000 & 1.347118000 & -1.158409000 \\ \mathrm{C} & -0.984751000 & -0.573864000 & -2.095928000 \\ \mathrm{H} & -0.874017000 & 0.509387000 & -2.228982000 \\ \mathrm{C} & 0.267287000 & -1.054365000 & -1.391089000 \\ \mathrm{C} & 1.477034000 & -1.094074000 & -2.089624000 \\ \mathrm{C} & 0.275529000 & -1.380841000 & -0.036717000 \\ \mathrm{C} & 2.668393000 & -1.392304000 & -1.446262000 \\ \mathrm{H} & 1.488029000 & -0.845707000 & -3.147703000 \\ \mathrm{C} & 1.462284000 & -1.691695000 & 0.616669000 \\ \mathrm{H} & -0.654217000 & -1.347014000 & 0.522068000 \\ \mathrm{C} & 2.664680000 & -1.674121000 & -0.080551000 \\ \mathrm{H} & 3.603757000 & -1.381852000 & -1.996582000 \\ \mathrm{H} & 1.455402000 & -1.918542000 & 1.678041000 \\ \mathrm{H} & -1.203287000 & 1.970324000 & -0.235427000 \\ \mathrm{H} & -3.791429000 & 1.801470000 & -1.234069000 \\ \mathrm{H} & -1.010863000 & -1.016834000 & -3.101683000 \\ \mathrm{H} & -7.709661000 & -1.214487000 & 1.623913000 \\ \mathrm{C} & 3.953024000 & -1.980052000 & 0.621957000 \\ \mathrm{~F} & 4.975998000 & -1.257769000 & 0.121896000 \\ \mathrm{~F} & 4.305975000 & -3.273584000 & 0.510931000 \\ \mathrm{~F} & 3.886057000 & -1.707536000 & 1.940469000 \\ \mathrm{H}----- \text { - - - } & \end{array}$

\begin{tabular}{|c|c|c|c|}
\hline \multicolumn{4}{|c|}{$\mathbf{A} 1_{\text {DBU }}$} \\
\hline & \multicolumn{3}{|c|}{ Coordinates (Angstroms) } \\
\hline & $\mathrm{X}$ & Y & Z \\
\hline$\overline{\mathrm{C}}$ & 0.512468000 & 4.506825000 & -0.771228000 \\
\hline $\mathrm{C}$ & 0.038963000 & 3.196722000 & -0.749274000 \\
\hline $\mathrm{C}$ & 0.897067000 & 2.137312000 & -0.448734000 \\
\hline $\mathrm{C}$ & 2.240177000 & 2.408920000 & -0.175273000 \\
\hline $\mathrm{C}$ & 2.713628000 & 3.715856000 & -0.195031000 \\
\hline $\mathrm{C}$ & 1.850398000 & 4.768756000 & -0.493451000 \\
\hline $\mathrm{H}$ & -0.166402000 & 5.322438000 & -1.003364000 \\
\hline $\mathrm{H}$ & -1.009158000 & 2.995183000 & -0.958769000 \\
\hline $\mathrm{H}$ & 2.899884000 & 1.577481000 & 0.058229000 \\
\hline $\mathrm{H}$ & 3.759415000 & 3.915440000 & 0.022337000 \\
\hline $\mathrm{H}$ & 2.220407000 & 5.789991000 & -0.507887000 \\
\hline $\mathrm{C}$ & 0.424214000 & 0.692138000 & -0.499243000 \\
\hline $\mathrm{C}$ & -1.015411000 & 0.542519000 & -0.081373000 \\
\hline $\mathrm{H}$ & -1.257600000 & 0.987456000 & 0.883064000 \\
\hline $\mathrm{O}$ & 0.678837000 & 0.134081000 & -1.785974000 \\
\hline $\mathrm{C}$ & -1.945071000 & -0.082962000 & -0.805734000 \\
\hline $\mathrm{H}$ & -1.640239000 & -0.500125000 & -1.763590000 \\
\hline & & S510 & \\
\hline
\end{tabular}




\begin{tabular}{|c|c|c|c|}
\hline $\mathrm{C}$ & -3.359439000 & -0.271476000 & -0.444376000 \\
\hline $\mathrm{C}$ & -4.259537000 & -0.696963000 & -1.429833000 \\
\hline $\mathrm{C}$ & -3.856713000 & -0.049331000 & 0.846817000 \\
\hline $\mathrm{C}$ & -5.605823000 & -0.879119000 & -1.147950000 \\
\hline $\mathrm{H}$ & -3.894397000 & -0.881083000 & -2.436660000 \\
\hline $\mathrm{C}$ & -5.200925000 & -0.230044000 & 1.136601000 \\
\hline $\mathrm{H}$ & -3.183011000 & 0.256536000 & 1.640995000 \\
\hline $\mathrm{C}$ & -6.081827000 & -0.642462000 & 0.138915000 \\
\hline $\mathrm{H}$ & -6.287631000 & -1.201253000 & -1.928111000 \\
\hline $\mathrm{H}$ & -5.567936000 & -0.052086000 & 2.141711000 \\
\hline $\mathrm{H}$ & 1.053642000 & 0.105405000 & 0.184245000 \\
\hline $\mathrm{H}$ & 0.400425000 & 0.788507000 & -2.440058000 \\
\hline $\mathrm{C}$ & 6.420056000 & -1.875046000 & 0.300188000 \\
\hline $\mathrm{C}$ & 6.072102000 & -3.046772000 & -0.612299000 \\
\hline $\mathrm{C}$ & 3.611545000 & -1.593995000 & -0.903299000 \\
\hline $\mathrm{C}$ & 5.654689000 & -2.619107000 & -2.021613000 \\
\hline $\mathrm{C}$ & 4.673153000 & -1.444474000 & -1.995292000 \\
\hline $\mathrm{H}$ & 7.067023000 & -1.164311000 & -0.239939000 \\
\hline $\mathrm{H}$ & 3.299379000 & -2.643820000 & -0.823570000 \\
\hline $\mathrm{H}$ & 5.285379000 & -3.641765000 & -0.134710000 \\
\hline $\mathrm{H}$ & 5.195466000 & -3.475064000 & -2.531002000 \\
\hline $\mathrm{H}$ & 7.019611000 & -2.260889000 & 1.134895000 \\
\hline $\mathrm{H}$ & 6.951884000 & -3.697206000 & -0.673014000 \\
\hline $\mathrm{H}$ & 2.712101000 & -1.036138000 & -1.171732000 \\
\hline $\mathrm{H}$ & 6.544218000 & -2.347453000 & -2.603599000 \\
\hline $\mathrm{H}$ & 4.178603000 & -1.356416000 & -2.968091000 \\
\hline $\mathrm{H}$ & 5.214593000 & -0.501845000 & -1.844677000 \\
\hline $\mathrm{C}$ & 3.996216000 & -1.087093000 & 0.478043000 \\
\hline $\mathrm{N}$ & 3.026249000 & -0.578792000 & 1.159389000 \\
\hline $\mathrm{N}$ & 5.302545000 & -1.162164000 & 0.915609000 \\
\hline $\mathrm{C}$ & 5.715715000 & -0.438854000 & 2.116166000 \\
\hline $\mathrm{H}$ & 5.896541000 & -1.159273000 & 2.928611000 \\
\hline $\mathrm{H}$ & 6.675059000 & 0.052966000 & 1.906382000 \\
\hline $\mathrm{C}$ & 4.682049000 & 0.587807000 & 2.545727000 \\
\hline $\mathrm{H}$ & 4.918528000 & 0.953661000 & 3.549908000 \\
\hline $\mathrm{H}$ & 4.701441000 & 1.447848000 & 1.865365000 \\
\hline $\mathrm{C}$ & 3.305626000 & -0.063720000 & 2.484060000 \\
\hline $\mathrm{H}$ & 2.524183000 & 0.659426000 & 2.742207000 \\
\hline $\mathrm{H}$ & 3.246983000 & -0.874703000 & 3.226695000 \\
\hline $\mathrm{C}$ & -7.527026000 & -0.899614000 & 0.456585000 \\
\hline $\mathrm{F}$ & -8.330096000 & -0.571258000 & -0.571503000 \\
\hline $\mathrm{F}$ & -7.754365000 & -2.199795000 & 0.724276000 \\
\hline $\mathrm{F}$ & -7.940919000 & -0.202260000 & 1.528597000 \\
\hline
\end{tabular}

TSA1-2 DBU 


\begin{tabular}{|c|c|c|c|}
\hline \multicolumn{4}{|c|}{ Coordinates (Angstroms) } \\
\hline & $X$ & $\mathrm{Y}$ & $\mathrm{Z}$ \\
\hline $\mathrm{C}$ & 2.803675000 & 1.940609000 & 2.411762000 \\
\hline $\mathrm{C}$ & 1.812108000 & 1.493638000 & 1.547633000 \\
\hline $\mathrm{C}$ & 1.721752000 & 1.963175000 & 0.220432000 \\
\hline $\mathrm{C}$ & 2.693326000 & 2.894913000 & -0.191757000 \\
\hline $\mathrm{C}$ & 3.683991000 & 3.339318000 & 0.675927000 \\
\hline $\mathrm{C}$ & 3.755393000 & 2.865846000 & 1.984609000 \\
\hline $\mathrm{H}$ & 2.834753000 & 1.559850000 & 3.430037000 \\
\hline $\mathrm{H}$ & 1.086008000 & 0.767448000 & 1.907928000 \\
\hline $\mathrm{H}$ & 2.656261000 & 3.259537000 & -1.213182000 \\
\hline $\mathrm{H}$ & 4.410785000 & 4.067731000 & 0.324179000 \\
\hline $\mathrm{H}$ & 4.530946000 & 3.213694000 & 2.660181000 \\
\hline $\mathrm{C}$ & 0.750015000 & 1.405432000 & -0.726827000 \\
\hline $\mathrm{C}$ & -0.594532000 & 1.121834000 & -0.328141000 \\
\hline $\mathrm{H}$ & -0.761617000 & 1.070673000 & 0.748072000 \\
\hline $\mathrm{O}$ & 0.921964000 & 1.803453000 & -2.071308000 \\
\hline $\mathrm{C}$ & -1.640536000 & 0.875160000 & -1.164604000 \\
\hline $\mathrm{H}$ & -1.449061000 & 0.870913000 & -2.235662000 \\
\hline $\mathrm{C}$ & -2.997202000 & 0.546664000 & -0.757245000 \\
\hline $\mathrm{C}$ & -3.921653000 & 0.106660000 & -1.723928000 \\
\hline $\mathrm{C}$ & -3.461740000 & 0.635574000 & 0.570893000 \\
\hline $\mathrm{C}$ & -5.221459000 & -0.238636000 & -1.390613000 \\
\hline $\mathrm{H}$ & -3.599821000 & 0.028172000 & -2.759325000 \\
\hline $\mathrm{C}$ & -4.757750000 & 0.285472000 & 0.909750000 \\
\hline $\mathrm{H}$ & -2.800998000 & 1.000571000 & 1.351482000 \\
\hline $\mathrm{C}$ & -5.649610000 & -0.159421000 & -0.066690000 \\
\hline $\mathrm{H}$ & -5.905873000 & -0.580690000 & -2.160198000 \\
\hline $\mathrm{H}$ & -5.083987000 & 0.361883000 & 1.942589000 \\
\hline $\mathrm{H}$ & 1.286857000 & -0.107122000 & -0.749434000 \\
\hline $\mathrm{H}$ & 0.308208000 & 2.535032000 & -2.242553000 \\
\hline $\mathrm{C}$ & 5.104768000 & -2.392460000 & 0.326867000 \\
\hline $\mathrm{C}$ & 5.942396000 & -2.023157000 & -0.891413000 \\
\hline $\mathrm{C}$ & 3.753967000 & -0.250212000 & -1.387411000 \\
\hline $\mathrm{C}$ & 6.252000000 & -0.527975000 & -1.003509000 \\
\hline $\mathrm{C}$ & 5.017342000 & 0.345768000 & -0.764140000 \\
\hline $\mathrm{H}$ & 5.497337000 & -1.888928000 & 1.221759000 \\
\hline $\mathrm{H}$ & 3.978419000 & -0.743568000 & -2.341504000 \\
\hline $\mathrm{H}$ & 5.440782000 & -2.392455000 & -1.793075000 \\
\hline $\mathrm{H}$ & 6.660942000 & -0.331866000 & -2.001652000 \\
\hline $\mathrm{H}$ & 5.203118000 & -3.469794000 & 0.500337000 \\
\hline $\mathrm{H}$ & 6.880538000 & -2.584236000 & -0.819437000 \\
\hline $\mathrm{H}$ & 3.037697000 & 0.536906000 & -1.630127000 \\
\hline $\mathrm{H}$ & 7.036449000 & -0.259469000 & -0.286388000 \\
\hline
\end{tabular}




$\begin{array}{lrrr}\mathrm{H} & 5.176292000 & 1.343397000 & -1.181231000 \\ \mathrm{H} & 4.844781000 & 0.505126000 & 0.306767000 \\ \mathrm{C} & 3.021504000 & -1.236179000 & -0.508239000 \\ \mathrm{~N} & 1.712287000 & -1.148850000 & -0.543930000 \\ \mathrm{~N} & 3.655949000 & -2.147487000 & 0.252381000 \\ \mathrm{C} & 2.910131000 & -2.954383000 & 1.228818000 \\ \mathrm{H} & 2.782979000 & -3.967343000 & 0.824387000 \\ \mathrm{H} & 3.529722000 & -3.032882000 & 2.128102000 \\ \mathrm{C} & 1.567199000 & -2.333923000 & 1.570903000 \\ \mathrm{H} & 0.981130000 & -3.031420000 & 2.175007000 \\ \mathrm{H} & 1.723771000 & -1.421331000 & 2.155161000 \\ \mathrm{C} & 0.853184000 & -1.986149000 & 0.274417000 \\ \mathrm{H} & -0.070996000 & -1.428303000 & 0.447717000 \\ \mathrm{H} & 0.590446000 & -2.894027000 & -0.281785000 \\ \mathrm{C} & -7.064925000 & -0.478932000 & 0.298748000 \\ \mathrm{~F} & -7.848674000 & 0.619491000 & 0.326984000 \\ \mathrm{~F} & -7.156256000 & -1.038973000 & 1.521873000 \\ \mathrm{~F} & -7.634991000 & -1.333026000 & -0.572667000 \\ -\end{array}$

A2 DBU

\begin{tabular}{lrrr} 
& & \\
& & & \\
Coordinates (Angstroms) & Y & \\
\hline $\mathrm{C}$ & -3.715723000 & -1.847291000 & 2.202836000 \\
$\mathrm{C}$ & -2.595942000 & -1.794956000 & 1.385240000 \\
$\mathrm{C}$ & -2.700054000 & -1.881207000 & -0.021597000 \\
$\mathrm{C}$ & -3.997720000 & -2.067944000 & -0.545866000 \\
$\mathrm{C}$ & -5.116022000 & -2.108138000 & 0.277922000 \\
$\mathrm{C}$ & -4.993979000 & -1.984157000 & 1.660373000 \\
$\mathrm{H}$ & -3.587338000 & -1.782788000 & 3.281031000 \\
$\mathrm{H}$ & -1.620531000 & -1.696682000 & 1.851217000 \\
$\mathrm{H}$ & -4.110873000 & -2.169261000 & -1.619403000 \\
$\mathrm{H}$ & -6.097287000 & -2.244676000 & -0.169670000 \\
$\mathrm{H}$ & -5.868827000 & -2.016890000 & 2.302335000 \\
$\mathrm{C}$ & -1.570661000 & -1.709031000 & -0.914861000 \\
$\mathrm{C}$ & -0.264662000 & -1.499071000 & -0.552714000 \\
$\mathrm{H}$ & -0.043291000 & -1.626806000 & 0.503621000 \\
$\mathrm{O}$ & -1.905155000 & -1.646899000 & -2.271497000 \\
$\mathrm{C}$ & 0.792769000 & -1.083147000 & -1.382225000 \\
$\mathrm{H}$ & 0.600507000 & -0.928727000 & -2.443214000 \\
$\mathrm{C}$ & 2.139648000 & -0.859389000 & -0.941561000 \\
$\mathrm{C}$ & 3.086838000 & -0.264857000 & -1.817217000 \\
$\mathrm{C}$ & 2.607739000 & -1.146924000 & 0.368787000 \\
$\mathrm{C}$ & 4.368732000 & 0.048635000 & -1.412975000 \\
& & &
\end{tabular}




\begin{tabular}{lrcc}
$\mathrm{H}$ & 2.781726000 & -0.039008000 & -2.836634000 \\
$\mathrm{C}$ & 3.892844000 & -0.826185000 & 0.772480000 \\
$\mathrm{H}$ & 1.954839000 & -1.649768000 & 1.076650000 \\
$\mathrm{C}$ & 4.788509000 & -0.213439000 & -0.104387000 \\
$\mathrm{H}$ & 5.055304000 & 0.513172000 & -2.114684000 \\
$\mathrm{H}$ & 4.208479000 & -1.059455000 & 1.785144000 \\
$\mathrm{H}$ & 0.405601000 & 0.997485000 & -0.822377000 \\
$\mathrm{H}$ & -1.234881000 & -2.153918000 & -2.748067000 \\
$\mathrm{C}$ & -3.663703000 & 1.788752000 & 0.806432000 \\
$\mathrm{C}$ & -4.138537000 & 2.800891000 & -0.235944000 \\
$\mathrm{C}$ & -1.898460000 & 1.459990000 & -1.662648000 \\
$\mathrm{C}$ & -4.356319000 & 2.197984000 & -1.629087000 \\
$\mathrm{C}$ & -3.350341000 & 1.096327000 & -1.951810000 \\
$\mathrm{H}$ & -4.148449000 & 0.811361000 & 0.698038000 \\
$\mathrm{H}$ & -1.644539000 & 2.432082000 & -2.107143000 \\
$\mathrm{H}$ & -3.403786000 & 3.614711000 & -0.276495000 \\
$\mathrm{H}$ & -4.304878000 & 2.997500000 & -2.377856000 \\
$\mathrm{H}$ & -3.918845000 & 2.161804000 & 1.801636000 \\
$\mathrm{H}$ & -5.069750000 & 3.251190000 & 0.123052000 \\
$\mathrm{H}$ & -1.255727000 & 0.715940000 & -2.150691000 \\
$\mathrm{~F}$ & -5.364724000 & 1.773657000 & -1.693500000 \\
$\mathrm{H}$ & -3.415214000 & 0.831709000 & -3.011080000 \\
$\mathrm{H}$ & -3.603704000 & 0.183120000 & -1.411524000 \\
$\mathrm{C}$ & -1.420886000 & 1.510736000 & -0.231786000 \\
$\mathrm{H}$ & -0.092922000 & 1.547933000 & -0.111432000 \\
$\mathrm{H}$ & -2.200432000 & 1.602307000 & 0.839133000 \\
$\mathrm{C}$ & -1.662264000 & 1.479332000 & 2.204964000 \\
$\mathrm{H}$ & -1.732355000 & 2.465674000 & 2.681385000 \\
$\mathrm{H}$ & -2.323986000 & 0.794133000 & 2.742045000 \\
$\mathrm{H}$ & -0.233398000 & 0.966450000 & 2.239585000 \\
$\mathrm{H}$ & 0.188329000 & 1.136369000 & 3.233655000 \\
$\mathrm{H}$ & -0.212270000 & -0.107599000 & 2.044535000 \\
$\mathrm{H}$ & 0.583047000 & 1.675010000 & 1.171865000 \\
$\mathrm{H}$ & 1.575697000 & 1.234403000 & 1.066346000 \\
$\mathrm{H}$ & 0.698979000 & 2.739124000 & 1.409028000 \\
$\mathrm{H}$ & 6.189118000 & 0.083547000 & 0.312512000 \\
$\mathrm{H}$ & 7.052937000 & -0.906169000 & -0.004323000 \\
$\mathrm{H}$ & 0.264406000 & 1.645563000 \\
\hline-137000 & &
\end{tabular}

TSA2-3 DBU

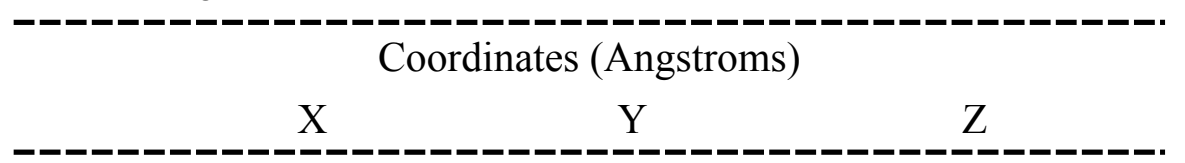




\begin{tabular}{|c|c|c|c|}
\hline $\mathrm{C}$ & -3.804490000 & -1.770365000 & 2.202446000 \\
\hline $\mathrm{C}$ & -2.710674000 & -1.524450000 & 1.382402000 \\
\hline $\mathrm{C}$ & -2.651032000 & -2.038207000 & 0.075157000 \\
\hline $\mathrm{C}$ & -3.748654000 & -2.781444000 & -0.384581000 \\
\hline $\mathrm{C}$ & -4.847460000 & -3.015609000 & 0.433927000 \\
\hline $\mathrm{C}$ & -4.885078000 & -2.514576000 & 1.732597000 \\
\hline $\mathrm{H}$ & -3.820414000 & -1.364250000 & 3.210683000 \\
\hline $\mathrm{H}$ & -1.897629000 & -0.906739000 & 1.752160000 \\
\hline $\mathrm{H}$ & -3.729096000 & -3.167501000 & -1.397660000 \\
\hline $\mathrm{H}$ & -5.683074000 & -3.595989000 & 0.052625000 \\
\hline $\mathrm{H}$ & -5.745739000 & -2.696989000 & 2.368970000 \\
\hline $\mathrm{C}$ & -1.527142000 & -1.740718000 & -0.818623000 \\
\hline $\mathrm{C}$ & -0.294400000 & -1.346278000 & -0.427093000 \\
\hline $\mathrm{H}$ & -0.107130000 & -1.395160000 & 0.642063000 \\
\hline $\mathrm{O}$ & -1.860085000 & -1.826289000 & -2.162805000 \\
\hline $\mathrm{C}$ & 0.725017000 & -0.735676000 & -1.250475000 \\
\hline $\mathrm{H}$ & 0.582780000 & -0.778242000 & -2.333101000 \\
\hline $\mathrm{C}$ & 2.122914000 & -0.668926000 & -0.844820000 \\
\hline $\mathrm{C}$ & 3.081399000 & -0.126628000 & -1.730345000 \\
\hline $\mathrm{C}$ & 2.589447000 & -1.032079000 & 0.437519000 \\
\hline $\mathrm{C}$ & 4.398036000 & 0.067331000 & -1.356891000 \\
\hline $\mathrm{H}$ & 2.764455000 & 0.160474000 & -2.730306000 \\
\hline $\mathrm{C}$ & 3.907956000 & -0.830009000 & 0.816691000 \\
\hline $\mathrm{H}$ & 1.912117000 & -1.491498000 & 1.150916000 \\
\hline $\mathrm{C}$ & 4.825330000 & -0.271307000 & -0.070520000 \\
\hline $\mathrm{H}$ & 5.102574000 & 0.494067000 & -2.064289000 \\
\hline $\mathrm{H}$ & 4.228287000 & -1.115032000 & 1.814222000 \\
\hline $\mathrm{H}$ & 0.253156000 & 0.742132000 & -0.988558000 \\
\hline $\mathrm{H}$ & -1.050913000 & -2.047351000 & -2.642164000 \\
\hline $\mathrm{C}$ & -3.617999000 & 2.233981000 & 0.854482000 \\
\hline $\mathrm{C}$ & -4.279823000 & 2.899301000 & -0.350549000 \\
\hline $\mathrm{C}$ & -2.198651000 & 1.390812000 & -1.713469000 \\
\hline $\mathrm{C}$ & -4.679727000 & 1.910897000 & -1.450524000 \\
\hline $\mathrm{C}$ & -3.618399000 & 0.832488000 & -1.659065000 \\
\hline $\mathrm{H}$ & -4.116309000 & 1.290208000 & 1.113452000 \\
\hline $\mathrm{H}$ & -2.161459000 & 2.303327000 & -2.324335000 \\
\hline $\mathrm{H}$ & -3.595338000 & 3.662219000 & -0.741206000 \\
\hline $\mathrm{H}$ & -4.849810000 & 2.462766000 & -2.382855000 \\
\hline $\mathrm{H}$ & -3.721605000 & 2.897934000 & 1.719079000 \\
\hline $\mathrm{H}$ & -5.165236000 & 3.437921000 & 0.003325000 \\
\hline $\mathrm{H}$ & -1.547091000 & 0.669915000 & -2.216935000 \\
\hline $\mathrm{H}$ & -5.631053000 & 1.432393000 & -1.190778000 \\
\hline $\mathrm{H}$ & -3.807518000 & 0.288476000 & -2.588500000 \\
\hline $\mathrm{H}$ & -3.679339000 & 0.081047000 & -0.867324000 \\
\hline
\end{tabular}




$\begin{array}{lrrr}\mathrm{C} & -1.510369000 & 1.701886000 & -0.404985000 \\ \mathrm{~N} & -0.192023000 & 1.696877000 & -0.469491000 \\ \mathrm{~N} & -2.171493000 & 1.997606000 & 0.729248000 \\ \mathrm{C} & -1.485018000 & 2.037833000 & 2.028103000 \\ \mathrm{H} & -1.502218000 & 3.074084000 & 2.391492000 \\ \mathrm{H} & -2.078079000 & 1.435404000 & 2.726173000 \\ \mathrm{C} & -0.056341000 & 1.525084000 & 1.959712000 \\ \mathrm{H} & 0.484451000 & 1.833658000 & 2.858672000 \\ \mathrm{H} & -0.037951000 & 0.431915000 & 1.920065000 \\ \mathrm{C} & 0.593009000 & 2.072601000 & 0.697130000 \\ \mathrm{H} & 1.599914000 & 1.674529000 & 0.559242000 \\ \mathrm{H} & 0.664183000 & 3.166584000 & 0.745303000 \\ \mathrm{C} & 6.258853000 & -0.103322000 & 0.316757000 \\ \mathrm{~F} & 6.410638000 & 0.056193000 & 1.646837000 \\ \mathrm{~F} & 7.017716000 & -1.165782000 & -0.027278000 \\ \mathrm{~F} & 6.820552000 & 0.968800000 & -0.27802 \\ \text { - } & & \end{array}$

\begin{tabular}{lrrr} 
A3 & \multicolumn{3}{c}{ Coordinates (Angstroms) } \\
& & Y & Z \\
\hline $\mathrm{C}$ & 3.402360000 & 1.745022000 & 2.335295000 \\
$\mathrm{C}$ & 2.441986000 & 1.482160000 & 1.366715000 \\
$\mathrm{C}$ & 2.512123000 & 2.089631000 & 0.107901000 \\
$\mathrm{C}$ & 3.580745000 & 2.948036000 & -0.167176000 \\
$\mathrm{C}$ & 4.543943000 & 3.207167000 & 0.802646000 \\
$\mathrm{C}$ & 4.458250000 & 2.610195000 & 2.056951000 \\
$\mathrm{H}$ & 3.332192000 & 1.262201000 & 3.305854000 \\
$\mathrm{H}$ & 1.647268000 & 0.773700000 & 1.574605000 \\
$\mathrm{H}$ & 3.654206000 & 3.407318000 & -1.146788000 \\
$\mathrm{H}$ & 5.365852000 & 3.879616000 & 0.575112000 \\
$\mathrm{H}$ & 5.213484000 & 2.811080000 & 2.810861000 \\
$\mathrm{C}$ & 1.502071000 & 1.790619000 & -0.930121000 \\
$\mathrm{C}$ & 0.249546000 & 1.391789000 & -0.677360000 \\
$\mathrm{H}$ & -0.073162000 & 1.337232000 & 0.355493000 \\
$\mathrm{O}$ & 2.000623000 & 1.941521000 & -2.194830000 \\
$\mathrm{C}$ & -0.735573000 & 0.963104000 & -1.735730000 \\
$\mathrm{H}$ & -0.391178000 & 0.030862000 & -2.201129000 \\
$\mathrm{C}$ & -2.120919000 & 0.730746000 & -1.180472000 \\
$\mathrm{C}$ & -2.741799000 & -0.509666000 & -1.316981000 \\
$\mathrm{C}$ & -2.801363000 & 1.755820000 & -0.517046000 \\
$\mathrm{C}$ & -4.014941000 & -0.727574000 & -0.799510000 \\
$\mathrm{H}$ & -2.206200000 & -1.316585000 & -1.809290000 \\
$\mathrm{C}$ & -4.070998000 & 1.546799000 & 0.001624000
\end{tabular}




\begin{tabular}{|c|c|c|c|}
\hline $\mathrm{H}$ & -2.326056000 & 2.726866000 & -0.403664000 \\
\hline $\mathrm{C}$ & -4.680305000 & 0.300525000 & -0.140213000 \\
\hline $\mathrm{H}$ & -4.487602000 & -1.697537000 & -0.910010000 \\
\hline $\mathrm{H}$ & -4.592153000 & 2.351102000 & 0.510841000 \\
\hline $\mathrm{H}$ & 1.290110000 & 1.794659000 & -2.831217000 \\
\hline $\mathrm{C}$ & 3.559095000 & -2.109576000 & 1.000478000 \\
\hline $\mathrm{C}$ & 4.378224000 & -2.769829000 & -0.106817000 \\
\hline $\mathrm{C}$ & 2.286983000 & -1.590466000 & -1.736372000 \\
\hline $\mathrm{C}$ & 4.772349000 & -1.800355000 & -1.224045000 \\
\hline $\mathrm{C}$ & 3.622050000 & -0.862460000 & -1.585882000 \\
\hline $\mathrm{H}$ & 3.979676000 & -1.123218000 & 1.252524000 \\
\hline $\mathrm{H}$ & 2.432007000 & -2.527523000 & -2.290937000 \\
\hline $\mathrm{H}$ & 3.801190000 & -3.611543000 & -0.507991000 \\
\hline $\mathrm{H}$ & 5.086262000 & -2.373202000 & -2.105703000 \\
\hline $\mathrm{H}$ & 3.650399000 & -2.724100000 & 1.905006000 \\
\hline $\mathrm{H}$ & 5.279652000 & -3.201988000 & 0.341599000 \\
\hline $\mathrm{H}$ & 1.606218000 & -0.991384000 & -2.345397000 \\
\hline $\mathrm{H}$ & 5.638774000 & -1.205716000 & -0.909584000 \\
\hline $\mathrm{H}$ & 3.848534000 & -0.337342000 & -2.519176000 \\
\hline $\mathrm{H}$ & 3.529090000 & -0.082019000 & -0.825238000 \\
\hline $\mathrm{C}$ & 1.491423000 & -1.912162000 & -0.477173000 \\
\hline $\mathrm{N}$ & 0.227350000 & -2.086768000 & -0.660699000 \\
\hline $\mathrm{N}$ & 2.123735000 & -1.999393000 & 0.747956000 \\
\hline $\mathrm{C}$ & 1.346285000 & -1.991525000 & 1.984212000 \\
\hline $\mathrm{H}$ & 1.447889000 & -2.970475000 & 2.477123000 \\
\hline $\mathrm{H}$ & 1.785426000 & -1.248378000 & 2.665940000 \\
\hline $\mathrm{C}$ & -0.120911000 & -1.690725000 & 1.732758000 \\
\hline $\mathrm{H}$ & -0.708929000 & -1.952842000 & 2.618149000 \\
\hline $\mathrm{H}$ & -0.271920000 & -0.623146000 & 1.537144000 \\
\hline $\mathrm{C}$ & -0.552820000 & -2.474433000 & 0.499163000 \\
\hline $\mathrm{H}$ & -1.612515000 & -2.304511000 & 0.288569000 \\
\hline $\mathrm{H}$ & -0.431540000 & -3.552747000 & 0.692393000 \\
\hline $\mathrm{H}$ & -0.811235000 & 1.723200000 & -2.531489000 \\
\hline $\mathrm{C}$ & -6.028043000 & 0.064582000 & 0.480171000 \\
\hline $\mathrm{F}$ & -5.928168000 & -0.187834000 & 1.799778000 \\
\hline $\mathrm{F}$ & -6.831615000 & 1.136033000 & 0.353295000 \\
\hline $\mathrm{F}$ & -6.664316000 & -0.982330000 & -0.071277000 \\
\hline
\end{tabular}

\section{A1 $1_{\text {MTBD }}$}

\begin{tabular}{|c|c|c|c|}
\hline \multicolumn{4}{|c|}{ Coordinates (Angstroms) } \\
\hline & $\mathrm{X}$ & Y & Z \\
\hline $\mathrm{C}$ & 2.987124000 & 1.724578000 & -1.971396000 \\
\hline 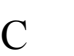 & 2.038750000 & 0.657577000 & -2.508485000 \\
\hline
\end{tabular}




\begin{tabular}{|c|c|c|c|}
\hline $\mathrm{C}$ & 0.616308000 & 1.196209000 & -2.452770000 \\
\hline $\mathrm{N}$ & 2.640872000 & 2.127887000 & -0.626530000 \\
\hline $\mathrm{H}$ & 2.978112000 & 2.592776000 & -2.651718000 \\
\hline $\mathrm{H}$ & 4.013462000 & 1.341605000 & -1.967702000 \\
\hline $\mathrm{H}$ & 2.139673000 & -0.244139000 & -1.894013000 \\
\hline $\mathrm{H}$ & 2.282098000 & 0.378479000 & -3.538984000 \\
\hline $\mathrm{H}$ & 0.422792000 & 1.846265000 & -3.319393000 \\
\hline $\mathrm{H}$ & -0.109592000 & 0.371388000 & -2.506947000 \\
\hline $\mathrm{N}$ & 0.354040000 & 1.946044000 & -1.231001000 \\
\hline $\mathrm{C}$ & -1.061583000 & 2.124592000 & -0.953561000 \\
\hline $\mathrm{C}$ & -1.297629000 & 3.094399000 & 0.187986000 \\
\hline $\mathrm{C}$ & -0.357759000 & 2.743452000 & 1.327170000 \\
\hline $\mathrm{N}$ & 1.010386000 & 2.802312000 & 0.859391000 \\
\hline $\mathrm{C}$ & 1.382152000 & 2.270110000 & -0.363716000 \\
\hline $\mathrm{H}$ & -1.516967000 & 1.151989000 & -0.714530000 \\
\hline $\mathrm{H}$ & -1.546324000 & 2.487981000 & -1.869908000 \\
\hline $\mathrm{H}$ & -1.100886000 & 4.122108000 & -0.135528000 \\
\hline $\mathrm{H}$ & -2.340896000 & 3.028215000 & 0.511383000 \\
\hline $\mathrm{H}$ & -0.460726000 & 3.468514000 & 2.141398000 \\
\hline $\mathrm{H}$ & -0.602596000 & 1.751308000 & 1.737680000 \\
\hline $\mathrm{C}$ & 3.943504000 & -3.494710000 & -0.499502000 \\
\hline $\mathrm{C}$ & 3.088672000 & -2.736908000 & 0.294599000 \\
\hline $\mathrm{C}$ & 3.360437000 & -1.390267000 & 0.546759000 \\
\hline $\mathrm{C}$ & 4.493926000 & -0.807609000 & -0.019870000 \\
\hline $\mathrm{C}$ & 5.345157000 & -1.561082000 & -0.823081000 \\
\hline $\mathrm{C}$ & 5.073848000 & -2.905971000 & -1.061269000 \\
\hline $\mathrm{H}$ & 3.723877000 & -4.541558000 & -0.688078000 \\
\hline $\mathrm{H}$ & 2.190571000 & -3.191193000 & 0.709992000 \\
\hline $\mathrm{H}$ & 4.688421000 & 0.246872000 & 0.156694000 \\
\hline $\mathrm{H}$ & 6.221735000 & -1.096433000 & -1.265065000 \\
\hline $\mathrm{H}$ & 5.737750000 & -3.493719000 & -1.688348000 \\
\hline $\mathrm{C}$ & 2.435249000 & -0.565528000 & 1.426261000 \\
\hline $\mathrm{C}$ & 1.013529000 & -0.681038000 & 0.947439000 \\
\hline $\mathrm{H}$ & 0.877044000 & -0.512108000 & -0.118238000 \\
\hline $\mathrm{O}$ & 2.577136000 & -0.865775000 & 2.804306000 \\
\hline $\mathrm{C}$ & -0.051548000 & -0.923368000 & 1.713079000 \\
\hline $\mathrm{H}$ & 0.074222000 & -1.012331000 & 2.790366000 \\
\hline $\mathrm{C}$ & -1.419252000 & -0.981777000 & 1.166658000 \\
\hline $\mathrm{C}$ & -2.493021000 & -0.449743000 & 1.888993000 \\
\hline $\mathrm{C}$ & -1.672247000 & -1.514565000 & -0.104727000 \\
\hline $\mathrm{C}$ & -3.769922000 & -0.404833000 & 1.343739000 \\
\hline $\mathrm{H}$ & -2.317890000 & -0.040246000 & 2.880192000 \\
\hline $\mathrm{C}$ & -2.945233000 & -1.474920000 & -0.653986000 \\
\hline $\mathrm{H}$ & -0.858496000 & -1.966983000 & -0.663634000 \\
\hline
\end{tabular}




$\begin{array}{lrcc}\mathrm{C} & -3.996467000 & -0.909988000 & 0.066255000 \\ \mathrm{H} & -4.588205000 & 0.029948000 & 1.907397000 \\ \mathrm{H} & -3.123965000 & -1.885002000 & -1.642612000 \\ \mathrm{H} & 2.734726000 & 0.480676000 & 1.316700000 \\ \mathrm{H} & 2.522537000 & -1.825585000 & 2.899268000 \\ \mathrm{C} & 2.023710000 & 3.052813000 & 1.861641000 \\ \mathrm{H} & 2.087461000 & 2.233888000 & 2.593738000 \\ \mathrm{H} & 2.987251000 & 3.160081000 & 1.366498000 \\ \mathrm{H} & 1.780631000 & 3.976576000 & 2.399656000 \\ \mathrm{C} & -5.381232000 & -0.916089000 & -0.517083000 \\ \mathrm{~F} & -5.361561000 & -0.699056000 & -1.844455000 \\ \mathrm{~F} & -6.164735000 & 0.026634000 & 0.033234000 \\ \mathrm{~F} & -5.994685000 & -2.098564000 & -0.326613000\end{array}$

TSA1-2 MTBD

\begin{tabular}{|c|c|c|c|}
\hline \multicolumn{4}{|c|}{ Coordinates (Angstroms) } \\
\hline & $X$ & Y & $\mathrm{Z}$ \\
\hline$\overline{\mathrm{C}}$ & -2.284548000 & 0.806968000 & 2.079125000 \\
\hline $\mathrm{C}$ & -1.207945000 & 1.028138000 & 3.131172000 \\
\hline $\mathrm{C}$ & 0.149253000 & 0.826835000 & 2.479900000 \\
\hline $\mathrm{N}$ & -1.979714000 & 1.454023000 & 0.806509000 \\
\hline $\mathrm{H}$ & -3.252963000 & 1.178946000 & 2.428726000 \\
\hline $\mathrm{H}$ & -2.411067000 & -0.268171000 & 1.900091000 \\
\hline $\mathrm{H}$ & -1.343034000 & 0.329268000 & 3.962086000 \\
\hline $\mathrm{H}$ & -1.262826000 & 2.045186000 & 3.534954000 \\
\hline $\mathrm{H}$ & 0.959876000 & 1.019799000 & 3.188695000 \\
\hline $\mathrm{H}$ & 0.264442000 & -0.204458000 & 2.116840000 \\
\hline $\mathrm{N}$ & 0.296413000 & 1.770989000 & 1.380647000 \\
\hline $\mathrm{C}$ & 1.667712000 & 2.061208000 & 0.972862000 \\
\hline $\mathrm{C}$ & 1.708495000 & 3.184258000 & -0.047141000 \\
\hline $\mathrm{C}$ & 0.696429000 & 2.905526000 & -1.144012000 \\
\hline $\mathrm{N}$ & -0.622479000 & 2.704800000 & -0.564576000 \\
\hline $\mathrm{C}$ & -0.772005000 & 1.946728000 & 0.549544000 \\
\hline $\mathrm{H}$ & 2.127731000 & 1.154152000 & 0.559770000 \\
\hline $\mathrm{H}$ & 2.230309000 & 2.339566000 & 1.870515000 \\
\hline $\mathrm{H}$ & 1.470744000 & 4.139520000 & 0.432786000 \\
\hline $\mathrm{H}$ & 2.714495000 & 3.255053000 & -0.470139000 \\
\hline $\mathrm{H}$ & 0.631996000 & 3.754887000 & -1.829379000 \\
\hline $\mathrm{H}$ & 0.989505000 & 2.023566000 & -1.729166000 \\
\hline $\mathrm{C}$ & -4.937035000 & -2.691297000 & 0.975338000 \\
\hline $\mathrm{C}$ & -3.771061000 & -2.151210000 & 0.445027000 \\
\hline $\mathrm{C}$ & -3.811725000 & -1.115377000 & -0.505157000 \\
\hline $\mathrm{C}$ & -5.080002000 & -0.668043000 & -0.912220000 \\
\hline
\end{tabular}




\begin{tabular}{|c|c|c|c|}
\hline $\mathrm{C}$ & -6.244010000 & -1.216672000 & -0.388013000 \\
\hline $\mathrm{C}$ & -6.184890000 & -2.229122000 & 0.565895000 \\
\hline $\mathrm{H}$ & -4.867584000 & -3.492263000 & 1.706872000 \\
\hline $\mathrm{H}$ & -2.817800000 & -2.560486000 & 0.768427000 \\
\hline $\mathrm{H}$ & -5.134843000 & 0.125892000 & -1.649566000 \\
\hline $\mathrm{H}$ & -7.207484000 & -0.844760000 & -0.727214000 \\
\hline $\mathrm{H}$ & -7.094018000 & -2.656486000 & 0.977984000 \\
\hline $\mathrm{C}$ & -2.605297000 & -0.440762000 & -1.039361000 \\
\hline $\mathrm{C}$ & -1.284267000 & -0.950863000 & -0.822980000 \\
\hline $\mathrm{H}$ & -1.153294000 & -1.500000000 & 0.108801000 \\
\hline $\mathrm{O}$ & -2.805206000 & 0.200912000 & -2.285684000 \\
\hline $\mathrm{C}$ & -0.172851000 & -0.635719000 & -1.541474000 \\
\hline $\mathrm{H}$ & -0.303307000 & -0.071834000 & -2.462796000 \\
\hline $\mathrm{C}$ & 1.199117000 & -0.895886000 & -1.137050000 \\
\hline $\mathrm{C}$ & 2.258763000 & -0.378147000 & -1.907674000 \\
\hline $\mathrm{C}$ & 1.555123000 & -1.594461000 & 0.036093000 \\
\hline $\mathrm{C}$ & 3.580805000 & -0.481833000 & -1.503266000 \\
\hline $\mathrm{H}$ & 2.027731000 & 0.134846000 & -2.838275000 \\
\hline $\mathrm{C}$ & 2.872914000 & -1.700548000 & 0.444974000 \\
\hline $\mathrm{H}$ & 0.784774000 & -2.056486000 & 0.645937000 \\
\hline $\mathrm{C}$ & 3.898150000 & -1.126161000 & -0.309406000 \\
\hline $\mathrm{H}$ & 4.369355000 & -0.052377000 & -2.112367000 \\
\hline $\mathrm{H}$ & 3.110405000 & -2.226716000 & 1.364809000 \\
\hline $\mathrm{H}$ & -2.376451000 & 0.723369000 & -0.080620000 \\
\hline $\mathrm{H}$ & -2.866552000 & -0.486128000 & -2.964954000 \\
\hline $\mathrm{C}$ & -1.766811000 & 2.988323000 & -1.418862000 \\
\hline $\mathrm{H}$ & -1.946052000 & 2.187543000 & -2.144470000 \\
\hline $\mathrm{H}$ & -2.658851000 & 3.093802000 & -0.801111000 \\
\hline $\mathrm{H}$ & -1.575124000 & 3.931743000 & -1.938245000 \\
\hline $\mathrm{C}$ & 5.317992000 & -1.262144000 & 0.145169000 \\
\hline $\mathrm{F}$ & 6.134086000 & -0.385722000 & -0.468100000 \\
\hline $\mathrm{F}$ & 5.436775000 & -1.055099000 & 1.472851000 \\
\hline $\mathrm{F}$ & 5.815601000 & -2.491556000 & -0.091693000 \\
\hline
\end{tabular}

\begin{tabular}{|c|c|c|c|}
\hline \multicolumn{4}{|c|}{ A2 2 MTBD } \\
\hline & \multicolumn{3}{|c|}{ Coordinates (Angstroms) } \\
\hline & $X$ & $\mathrm{Y}$ & $\mathrm{Z}$ \\
\hline$\overline{\mathrm{C}}$ & 3.651332000 & 0.601907000 & 1.988934000 \\
\hline $\mathrm{C}$ & 4.366675000 & 1.175663000 & 0.777387000 \\
\hline $\mathrm{C}$ & 3.701623000 & 2.478461000 & 0.368764000 \\
\hline $\mathrm{N}$ & 2.203566000 & 0.826192000 & 1.935592000 \\
\hline $\mathrm{H}$ & 4.020091000 & 1.059440000 & 2.915821000 \\
\hline $\mathrm{H}$ & 3.815975000 & -0.479587000 & 2.048691000 \\
\hline
\end{tabular}




\begin{tabular}{|c|c|c|c|}
\hline $\mathrm{H}$ & 4.320129000 & 0.476121000 & -0.058898000 \\
\hline $\mathrm{H}$ & 5.419771000 & 1.342421000 & 1.018658000 \\
\hline $\mathrm{H}$ & 3.801093000 & 3.244332000 & 1.150300000 \\
\hline $\mathrm{H}$ & 4.151982000 & 2.866203000 & -0.547165000 \\
\hline $\mathrm{N}$ & 2.289307000 & 2.236554000 & 0.093788000 \\
\hline $\mathrm{C}$ & 1.687645000 & 2.810866000 & -1.106964000 \\
\hline $\mathrm{C}$ & 0.205984000 & 3.067865000 & -0.877975000 \\
\hline $\mathrm{C}$ & -0.454784000 & 1.781851000 & -0.417279000 \\
\hline $\mathrm{N}$ & 0.274902000 & 1.220643000 & 0.714801000 \\
\hline $\mathrm{C}$ & 1.592383000 & 1.406063000 & 0.890125000 \\
\hline $\mathrm{H}$ & 1.849565000 & 2.136164000 & -1.957833000 \\
\hline $\mathrm{H}$ & 2.209353000 & 3.748447000 & -1.315379000 \\
\hline $\mathrm{H}$ & 0.083358000 & 3.849128000 & -0.119822000 \\
\hline $\mathrm{H}$ & -0.261355000 & 3.417881000 & -1.801985000 \\
\hline $\mathrm{H}$ & -1.488595000 & 1.942237000 & -0.103736000 \\
\hline $\mathrm{H}$ & -0.476238000 & 1.048084000 & -1.232468000 \\
\hline $\mathrm{H}$ & -0.084387000 & 0.291069000 & 0.989650000 \\
\hline $\mathrm{C}$ & 3.794607000 & -0.421437000 & -2.594500000 \\
\hline $\mathrm{C}$ & 2.691205000 & -0.754729000 & -1.822434000 \\
\hline $\mathrm{C}$ & 2.790527000 & -1.658248000 & -0.737800000 \\
\hline $\mathrm{C}$ & 4.072567000 & -2.184897000 & -0.468112000 \\
\hline $\mathrm{C}$ & 5.175037000 & -1.835229000 & -1.237909000 \\
\hline $\mathrm{C}$ & 5.054219000 & -0.949681000 & -2.308293000 \\
\hline $\mathrm{H}$ & 3.672528000 & 0.270401000 & -3.425094000 \\
\hline $\mathrm{H}$ & 1.731654000 & -0.301325000 & -2.057431000 \\
\hline $\mathrm{H}$ & 4.181565000 & -2.879906000 & 0.357302000 \\
\hline $\mathrm{H}$ & 6.144446000 & -2.266603000 & -1.001002000 \\
\hline $\mathrm{H}$ & 5.918447000 & -0.680217000 & -2.907461000 \\
\hline $\mathrm{C}$ & 1.663147000 & -1.986257000 & 0.105661000 \\
\hline $\mathrm{C}$ & 0.357342000 & -1.652621000 & -0.139920000 \\
\hline $\mathrm{H}$ & 0.160874000 & -1.261897000 & -1.136694000 \\
\hline $\mathrm{O}$ & 1.998619000 & -2.603461000 & 1.320115000 \\
\hline $\mathrm{C}$ & -0.728871000 & -1.678945000 & 0.754911000 \\
\hline $\mathrm{H}$ & -0.558937000 & -1.997688000 & 1.782138000 \\
\hline $\mathrm{C}$ & -2.068299000 & -1.290617000 & 0.420131000 \\
\hline $\mathrm{C}$ & -3.029785000 & -1.086205000 & 1.445304000 \\
\hline $\mathrm{C}$ & -2.511983000 & -1.028363000 & -0.902249000 \\
\hline $\mathrm{C}$ & -4.299552000 & -0.616089000 & 1.179749000 \\
\hline $\mathrm{H}$ & -2.743645000 & -1.290143000 & 2.474941000 \\
\hline $\mathrm{C}$ & -3.783847000 & -0.548982000 & -1.165205000 \\
\hline $\mathrm{H}$ & -1.850407000 & -1.226964000 & -1.740612000 \\
\hline $\mathrm{C}$ & -4.692096000 & -0.322051000 & -0.131359000 \\
\hline $\mathrm{H}$ & -4.998560000 & -0.460831000 & 1.996743000 \\
\hline $\mathrm{H}$ & -4.079731000 & -0.356769000 & -2.192154000 \\
\hline
\end{tabular}




$\begin{array}{lrcc}\mathrm{H} & 1.349246000 & -3.304906000 & 1.464361000 \\ \mathrm{C} & 1.449798000 & 0.113636000 & 2.959955000 \\ \mathrm{H} & 0.455146000 & 0.547875000 & 3.069189000 \\ \mathrm{H} & 1.380580000 & -0.949694000 & 2.713433000 \\ \mathrm{H} & 1.984121000 & 0.234530000 & 3.907290000 \\ \mathrm{C} & -6.079099000 & 0.148710000 & -0.408504000 \\ \mathrm{~F} & -6.181619000 & 0.765848000 & -1.603523000 \\ \mathrm{~F} & -6.513425000 & 1.023556000 & 0.525597000 \\ \mathrm{~F} & -6.983410000 & -0.855833000 & -0.421574000\end{array}$

TSA2-3 MTBD

\begin{tabular}{|c|c|c|c|}
\hline \multicolumn{4}{|c|}{ Coordinates (Angstroms) } \\
\hline & $X$ & $\mathrm{Y}$ & $\mathrm{Z}$ \\
\hline$\overline{\mathrm{C}}$ & 3.636600000 & 1.370208000 & 1.630754000 \\
\hline $\mathrm{C}$ & 4.366404000 & 1.236371000 & 0.302338000 \\
\hline $\mathrm{C}$ & 3.806517000 & 2.244340000 & -0.685914000 \\
\hline $\mathrm{N}$ & 2.223452000 & 1.702490000 & 1.462863000 \\
\hline $\mathrm{H}$ & 4.091598000 & 2.162732000 & 2.240701000 \\
\hline $\mathrm{H}$ & 3.698741000 & 0.430851000 & 2.191950000 \\
\hline $\mathrm{H}$ & 4.236079000 & 0.233431000 & -0.108941000 \\
\hline $\mathrm{H}$ & 5.436662000 & 1.400591000 & 0.455549000 \\
\hline $\mathrm{H}$ & 4.004095000 & 3.275644000 & -0.358091000 \\
\hline $\mathrm{H}$ & 4.266182000 & 2.108422000 & -1.667624000 \\
\hline $\mathrm{N}$ & 2.374577000 & 2.028106000 & -0.834110000 \\
\hline $\mathrm{C}$ & 1.765919000 & 2.120537000 & -2.155311000 \\
\hline $\mathrm{C}$ & 0.364455000 & 2.700491000 & -2.040213000 \\
\hline $\mathrm{C}$ & -0.429351000 & 1.865031000 & -1.049628000 \\
\hline $\mathrm{N}$ & 0.283428000 & 1.696309000 & 0.210084000 \\
\hline $\mathrm{C}$ & 1.606160000 & 1.789515000 & 0.258321000 \\
\hline $\mathrm{H}$ & 1.740363000 & 1.130103000 & -2.630411000 \\
\hline $\mathrm{H}$ & 2.401200000 & 2.765969000 & -2.768423000 \\
\hline $\mathrm{H}$ & 0.429557000 & 3.737956000 & -1.693013000 \\
\hline $\mathrm{H}$ & -0.126411000 & 2.701674000 & -3.017625000 \\
\hline $\mathrm{H}$ & -1.397969000 & 2.323840000 & -0.831921000 \\
\hline $\mathrm{H}$ & -0.640200000 & 0.875655000 & -1.479841000 \\
\hline $\mathrm{H}$ & -0.170417000 & 0.738146000 & 0.774727000 \\
\hline $\mathrm{C}$ & 3.911023000 & -1.868659000 & -2.190612000 \\
\hline $\mathrm{C}$ & 2.796956000 & -1.617760000 & -1.401363000 \\
\hline $\mathrm{C}$ & 2.795153000 & -1.919269000 & -0.027265000 \\
\hline $\mathrm{C}$ & 3.968103000 & -2.460149000 & 0.522736000 \\
\hline $\mathrm{C}$ & 5.087780000 & -2.695092000 & -0.267921000 \\
\hline $\mathrm{C}$ & 5.069664000 & -2.403517000 & -1.629185000 \\
\hline $\mathrm{H}$ & 3.880098000 & -1.629831000 & -3.250464000 \\
\hline
\end{tabular}




\begin{tabular}{lrrr}
$\mathrm{H}$ & 1.921581000 & -1.157866000 & -1.850784000 \\
$\mathrm{H}$ & 3.990825000 & -2.686486000 & 1.582835000 \\
$\mathrm{H}$ & 5.982451000 & -3.113494000 & 0.185058000 \\
$\mathrm{H}$ & 5.945654000 & -2.586839000 & -2.243963000 \\
$\mathrm{C}$ & 1.651529000 & -1.603555000 & 0.829195000 \\
$\mathrm{C}$ & 0.402176000 & -1.310840000 & 0.404367000 \\
$\mathrm{H}$ & 0.214722000 & -1.453592000 & -0.657072000 \\
$\mathrm{O}$ & 1.979888000 & -1.544421000 & 2.180467000 \\
$\mathrm{C}$ & -0.633273000 & -0.652359000 & 1.169273000 \\
$\mathrm{H}$ & -0.484469000 & -0.580338000 & 2.249242000 \\
$\mathrm{C}$ & -2.036296000 & -0.657346000 & 0.772288000 \\
$\mathrm{C}$ & -2.982931000 & 0.018162000 & 1.575191000 \\
$\mathrm{C}$ & -2.518531000 & -1.221015000 & -0.426132000 \\
$\mathrm{C}$ & -4.307218000 & 0.140446000 & 1.200831000 \\
$\mathrm{H}$ & -2.649425000 & 0.466543000 & 2.508421000 \\
$\mathrm{C}$ & -3.847026000 & -1.094822000 & -0.807595000 \\
$\mathrm{H}$ & -1.849272000 & -1.789432000 & -1.064880000 \\
$\mathrm{C}$ & -4.753669000 & -0.408292000 & -0.004770000 \\
$\mathrm{H}$ & -5.002751000 & 0.675166000 & 1.841048000 \\
$\mathrm{H}$ & -4.182664000 & -1.542807000 & -1.737442000 \\
$\mathrm{H}$ & 1.185621000 & -1.788854000 & 2.673600000 \\
$\mathrm{C}$ & 1.463646000 & 1.714636000 & 2.699370000 \\
$\mathrm{H}$ & 0.474857000 & 2.135885000 & 2.521664000 \\
$\mathrm{H}$ & 1.367512000 & 0.702723000 & 3.102586000 \\
$\mathrm{H}$ & 1.996574000 & 2.338030000 & 3.427071000 \\
$\mathrm{C}$ & -6.197328000 & -0.308618000 & -0.378318000 \\
$\mathrm{~F}$ & -6.965495000 & -1.197512000 & 0.287254000 \\
$\mathrm{~F}$ & -6.707810000 & 0.908905000 & -0.097968000 \\
$\mathrm{~F}$ & -6.403235000 & -0.531111000 & -1.690638000 \\
\hline---2 & & \\
& & &
\end{tabular}

\begin{tabular}{|c|c|c|c|}
\hline \multicolumn{4}{|c|}{ A3 3 MTBD } \\
\hline & $\mathrm{X}$ & $\mathrm{Y}$ & Z \\
\hline$\overline{\mathrm{C}}$ & -0.128640000 & 2.697014000 & 1.889855000 \\
\hline $\mathrm{C}$ & 1.021380000 & 1.957753000 & 2.549652000 \\
\hline $\mathrm{C}$ & 2.259823000 & 2.087951000 & 1.678068000 \\
\hline $\mathrm{N}$ & -0.372491000 & 2.113558000 & 0.587889000 \\
\hline $\mathrm{H}$ & 0.091035000 & 3.777954000 & 1.825172000 \\
\hline $\mathrm{H}$ & -1.042587000 & 2.588171000 & 2.482039000 \\
\hline $\mathrm{H}$ & 0.735702000 & 0.906962000 & 2.660547000 \\
\hline $\mathrm{H}$ & 1.229307000 & 2.358200000 & 3.546602000 \\
\hline $\mathrm{H}$ & 2.756298000 & 3.050066000 & 1.883687000 \\
\hline $\mathrm{H}$ & 2.984173000 & 1.298573000 & 1.921552000 \\
\hline
\end{tabular}




\begin{tabular}{|c|c|c|c|}
\hline $\mathrm{N}$ & 1.955574000 & 2.014606000 & 0.259929000 \\
\hline $\mathrm{C}$ & 3.131456000 & 1.924555000 & -0.591122000 \\
\hline $\mathrm{C}$ & 2.812427000 & 2.360357000 & -2.011846000 \\
\hline $\mathrm{C}$ & 1.539887000 & 1.651901000 & -2.464545000 \\
\hline $\mathrm{N}$ & 0.434391000 & 1.866053000 & -1.558243000 \\
\hline $\mathrm{C}$ & 0.692887000 & 1.998419000 & -0.303201000 \\
\hline $\mathrm{H}$ & 3.522423000 & 0.896554000 & -0.580794000 \\
\hline $\mathrm{H}$ & 3.909045000 & 2.568824000 & -0.159480000 \\
\hline $\mathrm{H}$ & 2.651658000 & 3.444769000 & -2.036422000 \\
\hline $\mathrm{H}$ & 3.657729000 & 2.129679000 & -2.668605000 \\
\hline $\mathrm{H}$ & 1.242739000 & 2.007399000 & -3.458097000 \\
\hline $\mathrm{H}$ & 1.743855000 & 0.573883000 & -2.565493000 \\
\hline $\mathrm{C}$ & -6.007729000 & -1.996917000 & 1.172412000 \\
\hline $\mathrm{C}$ & -4.909106000 & -1.948046000 & 0.322555000 \\
\hline $\mathrm{C}$ & -4.465962000 & -0.726941000 & -0.196327000 \\
\hline $\mathrm{C}$ & -5.161539000 & 0.440739000 & 0.141727000 \\
\hline $\mathrm{C}$ & -6.263751000 & 0.389400000 & 0.988456000 \\
\hline $\mathrm{C}$ & -6.689823000 & -0.829375000 & 1.507849000 \\
\hline $\mathrm{H}$ & -6.341459000 & -2.952722000 & 1.565416000 \\
\hline $\mathrm{H}$ & -4.396243000 & -2.862672000 & 0.040418000 \\
\hline $\mathrm{H}$ & -4.816523000 & 1.400074000 & -0.236047000 \\
\hline $\mathrm{H}$ & -6.785251000 & 1.305781000 & 1.248568000 \\
\hline $\mathrm{H}$ & -7.550458000 & -0.870482000 & 2.168575000 \\
\hline $\mathrm{C}$ & -3.282505000 & -0.648995000 & -1.084506000 \\
\hline $\mathrm{C}$ & -2.171064000 & -1.373257000 & -0.917475000 \\
\hline $\mathrm{H}$ & -2.142035000 & -2.066768000 & -0.082029000 \\
\hline $\mathrm{O}$ & -3.333344000 & 0.288941000 & -2.081860000 \\
\hline $\mathrm{C}$ & -0.956234000 & -1.268399000 & -1.794591000 \\
\hline $\mathrm{H}$ & -0.922420000 & -0.269594000 & -2.248840000 \\
\hline $\mathrm{C}$ & 0.353868000 & -1.475129000 & -1.069472000 \\
\hline $\mathrm{C}$ & 1.470134000 & -1.955287000 & -1.763509000 \\
\hline $\mathrm{C}$ & 0.507070000 & -1.134600000 & 0.273567000 \\
\hline $\mathrm{C}$ & 2.709557000 & -2.056411000 & -1.149445000 \\
\hline $\mathrm{H}$ & 1.366983000 & -2.231596000 & -2.809842000 \\
\hline $\mathrm{C}$ & 1.741458000 & -1.245793000 & 0.901622000 \\
\hline $\mathrm{H}$ & -0.337094000 & -0.727666000 & 0.821012000 \\
\hline $\mathrm{C}$ & 2.848638000 & -1.690488000 & 0.189071000 \\
\hline $\mathrm{H}$ & 3.570640000 & -2.407665000 & -1.709507000 \\
\hline $\mathrm{H}$ & 1.848706000 & -0.967575000 & 1.944319000 \\
\hline $\mathrm{H}$ & -4.254962000 & 0.523608000 & -2.242715000 \\
\hline $\mathrm{H}$ & -1.023240000 & -1.989171000 & -2.621939000 \\
\hline $\mathrm{C}$ & -1.689878000 & 2.343994000 & 0.030890000 \\
\hline $\mathrm{H}$ & -1.871830000 & 3.411708000 & -0.170302000 \\
\hline $\mathrm{H}$ & -1.790211000 & 1.799630000 & -0.905338000 \\
\hline
\end{tabular}




$\begin{array}{lrrr}\mathrm{H} & -2.440133000 & 1.986051000 & 0.745209000 \\ \mathrm{C} & 4.195307000 & -1.791260000 & 0.840094000 \\ \mathrm{~F} & 5.158340000 & -1.252967000 & 0.064480000 \\ \mathrm{~F} & 4.239582000 & -1.150037000 & 2.024089000 \\ \mathrm{~F} & 4.557924000 & -3.065898000 & 1.070488000\end{array}$

(9) $\mathrm{R}_{1}=\mathrm{H}, \mathrm{R}_{2}=\mathrm{NO}_{2}$

\begin{tabular}{|c|c|c|c|}
\hline \multicolumn{4}{|c|}{ A1 кон } \\
\hline & \multicolumn{3}{|c|}{ Coordinates (Angstroms) } \\
\hline & $\mathrm{X}$ & $\mathrm{Y}$ & Z \\
\hline$\overline{\mathrm{C}}$ & 2.983352000 & 3.181459000 & -0.873757000 \\
\hline $\mathrm{C}$ & 2.204535000 & 2.035542000 & -0.738794000 \\
\hline $\mathrm{C}$ & 2.675446000 & 0.945382000 & -0.002553000 \\
\hline $\mathrm{C}$ & 3.931765000 & 1.010844000 & 0.608769000 \\
\hline $\mathrm{C}$ & 4.704582000 & 2.161343000 & 0.473246000 \\
\hline $\mathrm{C}$ & 4.235709000 & 3.244727000 & -0.267407000 \\
\hline $\mathrm{H}$ & 2.609178000 & 4.028220000 & -1.442180000 \\
\hline $\mathrm{H}$ & 1.217261000 & 1.993233000 & -1.196016000 \\
\hline $\mathrm{H}$ & 4.253761000 & 0.134891000 & 1.187056000 \\
\hline $\mathrm{H}$ & 5.676879000 & 2.217104000 & 0.955543000 \\
\hline $\mathrm{H}$ & 4.840413000 & 4.142022000 & -0.365266000 \\
\hline $\mathrm{C}$ & 1.874012000 & -0.340855000 & 0.097602000 \\
\hline $\mathrm{C}$ & 0.413871000 & -0.096493000 & 0.322435000 \\
\hline $\mathrm{H}$ & 0.200738000 & 0.570938000 & 1.156032000 \\
\hline $\mathrm{O}$ & 2.107446000 & -1.154843000 & -1.074208000 \\
\hline $\mathrm{C}$ & -0.576869000 & -0.621853000 & -0.404699000 \\
\hline $\mathrm{H}$ & -0.311418000 & -1.276490000 & -1.232731000 \\
\hline $\mathrm{C}$ & -2.016312000 & -0.400916000 & -0.203168000 \\
\hline $\mathrm{C}$ & -2.911772000 & -0.828608000 & -1.194543000 \\
\hline $\mathrm{C}$ & -2.543392000 & 0.217931000 & 0.941691000 \\
\hline $\mathrm{C}$ & -4.279185000 & -0.636877000 & -1.067839000 \\
\hline $\mathrm{H}$ & -2.524495000 & -1.316671000 & -2.084504000 \\
\hline $\mathrm{C}$ & -3.905865000 & 0.419162000 & 1.085018000 \\
\hline $\mathrm{H}$ & -1.881452000 & 0.537004000 & 1.739738000 \\
\hline $\mathrm{C}$ & -4.758724000 & -0.010061000 & 0.074134000 \\
\hline $\mathrm{H}$ & -4.973130000 & -0.962830000 & -1.832652000 \\
\hline $\mathrm{H}$ & -4.317782000 & 0.894814000 & 1.966416000 \\
\hline $\mathrm{H}$ & 2.312188000 & -0.935027000 & 0.932161000 \\
\hline $\mathrm{H}$ & 1.946613000 & -0.591182000 & -1.842943000 \\
\hline $\mathrm{O}$ & 3.832222000 & -1.821515000 & 1.799695000 \\
\hline $\mathrm{H}$ & 3.733828000 & -1.572724000 & 2.722402000 \\
\hline K & 4.162127000 & -2.717184000 & -0.292019000 \\
\hline
\end{tabular}




$\begin{array}{lrcc}\mathrm{N} & -6.199955000 & 0.199124000 & 0.220651000 \\ \mathrm{O} & -6.926423000 & -0.190011000 & -0.682351000 \\ \mathrm{O} & -6.594521000 & 0.751990000 & 1.236857000\end{array}$

TSA1-2

\begin{tabular}{|c|c|c|c|}
\hline \multicolumn{4}{|c|}{ Coordinates (Angstroms) } \\
\hline & $\mathrm{X}$ & Y & Z \\
\hline$\overline{\mathrm{C}}$ & 4.589106000 & 1.796345000 & -1.364747000 \\
\hline $\mathrm{C}$ & 3.624763000 & 0.790320000 & -1.290261000 \\
\hline $\mathrm{C}$ & 2.767081000 & 0.689203000 & -0.187195000 \\
\hline $\mathrm{C}$ & 2.921694000 & 1.622639000 & 0.849538000 \\
\hline $\mathrm{C}$ & 3.871868000 & 2.631305000 & 0.769575000 \\
\hline $\mathrm{C}$ & 4.715754000 & 2.724235000 & -0.338029000 \\
\hline $\mathrm{H}$ & 5.234454000 & 1.857330000 & -2.237358000 \\
\hline $\mathrm{H}$ & 3.527354000 & 0.080867000 & -2.108200000 \\
\hline $\mathrm{H}$ & 2.305762000 & 1.529079000 & 1.740001000 \\
\hline $\mathrm{H}$ & 3.967829000 & 3.343030000 & 1.584752000 \\
\hline $\mathrm{H}$ & 5.463694000 & 3.509158000 & -0.393955000 \\
\hline $\mathrm{C}$ & 1.787370000 & -0.440344000 & -0.033408000 \\
\hline $\mathrm{C}$ & 0.380838000 & -0.043414000 & 0.081825000 \\
\hline $\mathrm{H}$ & 0.212083000 & 0.899306000 & 0.600141000 \\
\hline $\mathrm{O}$ & 1.997888000 & -1.470552000 & -1.010431000 \\
\hline $\mathrm{C}$ & -0.666005000 & -0.766786000 & -0.359432000 \\
\hline $\mathrm{H}$ & -0.463954000 & -1.723013000 & -0.838191000 \\
\hline $\mathrm{C}$ & -2.080704000 & -0.421331000 & -0.225652000 \\
\hline $\mathrm{C}$ & -3.045313000 & -1.333303000 & -0.688993000 \\
\hline $\mathrm{C}$ & -2.540029000 & 0.781303000 & 0.342574000 \\
\hline $\mathrm{C}$ & -4.401991000 & -1.073999000 & -0.588889000 \\
\hline $\mathrm{H}$ & -2.716228000 & -2.268150000 & -1.134097000 \\
\hline $\mathrm{C}$ & -3.891594000 & 1.055365000 & 0.453500000 \\
\hline $\mathrm{H}$ & -1.830246000 & 1.518367000 & 0.703188000 \\
\hline $\mathrm{C}$ & -4.812242000 & 0.122700000 & -0.014392000 \\
\hline $\mathrm{H}$ & -5.142303000 & -1.780014000 & -0.944497000 \\
\hline $\mathrm{H}$ & -4.245923000 & 1.979361000 & 0.893599000 \\
\hline $\mathrm{H}$ & 2.145286000 & -1.022295000 & 1.070454000 \\
\hline $\mathrm{H}$ & 1.559300000 & -1.202288000 & -1.832071000 \\
\hline $\mathrm{O}$ & 2.807192000 & -1.700909000 & 2.026964000 \\
\hline $\mathrm{H}$ & 2.153466000 & -1.911447000 & 2.699400000 \\
\hline K & 4.342420000 & -2.074558000 & 0.243140000 \\
\hline $\mathrm{N}$ & -6.238707000 & 0.408074000 & 0.099666000 \\
\hline $\mathrm{O}$ & -7.025002000 & -0.426022000 & -0.328739000 \\
\hline $\mathrm{O}$ & -6.571225000 & 1.465653000 & 0.617633000 \\
\hline
\end{tabular}




\begin{tabular}{|c|c|c|c|}
\hline \multicolumn{4}{|c|}{ A2 кон } \\
\hline & $\mathrm{X}$ & $\mathrm{Y}$ & Z \\
\hline $\mathrm{C}$ & 4.353188000 & 2.308172000 & 0.813171000 \\
\hline $\mathrm{C}$ & 3.183384000 & 1.705730000 & 0.375797000 \\
\hline $\mathrm{C}$ & 3.211201000 & 0.614744000 & -0.524838000 \\
\hline $\mathrm{C}$ & 4.484301000 & 0.155175000 & -0.931749000 \\
\hline $\mathrm{C}$ & 5.651959000 & 0.760860000 & -0.476121000 \\
\hline $\mathrm{C}$ & 5.601735000 & 1.840956000 & 0.398795000 \\
\hline $\mathrm{H}$ & 4.290157000 & 3.150136000 & 1.497342000 \\
\hline $\mathrm{H}$ & 2.232862000 & 2.086431000 & 0.737844000 \\
\hline $\mathrm{H}$ & 4.541360000 & -0.665431000 & -1.639033000 \\
\hline $\mathrm{H}$ & 6.611569000 & 0.385752000 & -0.822832000 \\
\hline $\mathrm{H}$ & 6.513083000 & 2.313693000 & 0.750650000 \\
\hline $\mathrm{C}$ & 2.012353000 & -0.088830000 & -0.941390000 \\
\hline $\mathrm{C}$ & 0.709059000 & 0.221981000 & -0.639207000 \\
\hline $\mathrm{H}$ & 0.546475000 & 1.202215000 & -0.197905000 \\
\hline $\mathrm{O}$ & 2.280337000 & -1.348419000 & -1.525060000 \\
\hline $\mathrm{C}$ & -0.413166000 & -0.607341000 & -0.820322000 \\
\hline $\mathrm{H}$ & -0.266504000 & -1.585202000 & -1.278290000 \\
\hline $\mathrm{C}$ & -1.778832000 & -0.237906000 & -0.582774000 \\
\hline $\mathrm{C}$ & -2.806852000 & -1.196546000 & -0.789577000 \\
\hline $\mathrm{C}$ & -2.192101000 & 1.036579000 & -0.112321000 \\
\hline $\mathrm{C}$ & -4.132462000 & -0.920720000 & -0.535015000 \\
\hline $\mathrm{H}$ & -2.533899000 & -2.181159000 & -1.162092000 \\
\hline $\mathrm{C}$ & -3.516605000 & 1.320985000 & 0.146591000 \\
\hline $\mathrm{H}$ & -1.458126000 & 1.821656000 & 0.039853000 \\
\hline $\mathrm{C}$ & -4.489770000 & 0.342151000 & -0.058444000 \\
\hline $\mathrm{H}$ & -4.903063000 & -1.664770000 & -0.697572000 \\
\hline $\mathrm{H}$ & -3.818808000 & 2.298400000 & 0.503744000 \\
\hline $\mathrm{H}$ & 1.674664000 & -1.463646000 & -2.268691000 \\
\hline $\mathrm{O}$ & 0.261212000 & -1.887114000 & 1.773950000 \\
\hline $\mathrm{H}$ & -0.474334000 & -1.987592000 & 2.386896000 \\
\hline $\mathrm{K}$ & 2.715108000 & -1.922553000 & 1.071860000 \\
\hline $\mathrm{H}$ & -0.119018000 & -1.422587000 & 0.987107000 \\
\hline $\mathrm{N}$ & -5.874571000 & 0.638806000 & 0.219739000 \\
\hline $\mathrm{O}$ & -6.156048000 & 1.759884000 & 0.634648000 \\
\hline $\mathrm{O}$ & -6.706229000 & -0.245223000 & 0.031949000 \\
\hline
\end{tabular}

TSA2-3 Кон

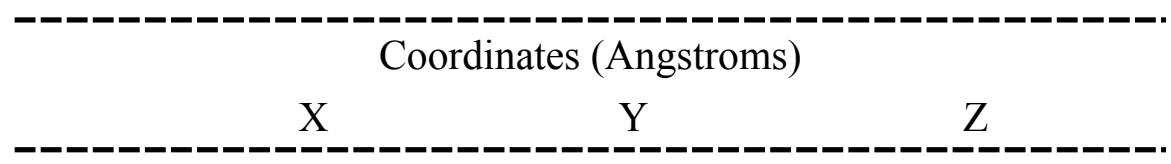




\begin{tabular}{lrrr}
$\mathrm{C}$ & -4.178781000 & -2.700743000 & -0.128703000 \\
$\mathrm{C}$ & -3.096842000 & -1.917607000 & -0.505213000 \\
$\mathrm{C}$ & -3.209592000 & -0.518159000 & -0.583294000 \\
$\mathrm{C}$ & -4.466799000 & 0.052649000 & -0.315881000 \\
$\mathrm{C}$ & -5.551385000 & -0.739518000 & 0.055054000 \\
$\mathrm{C}$ & -5.412384000 & -2.118589000 & 0.163033000 \\
$\mathrm{H}$ & -4.061996000 & -3.779397000 & -0.076857000 \\
$\mathrm{H}$ & -2.159617000 & -2.399982000 & -0.763596000 \\
$\mathrm{H}$ & -4.595958000 & 1.122986000 & -0.441281000 \\
$\mathrm{H}$ & -6.513496000 & -0.271927000 & 0.245877000 \\
$\mathrm{H}$ & -6.257816000 & -2.737447000 & 0.447460000 \\
$\mathrm{C}$ & -2.055178000 & 0.354717000 & -0.870198000 \\
$\mathrm{C}$ & -0.756708000 & -0.023361000 & -0.779326000 \\
$\mathrm{H}$ & -0.571777000 & -1.055213000 & -0.484182000 \\
$\mathrm{O}$ & -2.427190000 & 1.665002000 & -1.101540000 \\
$\mathrm{C}$ & 0.393990000 & 0.854996000 & -0.991171000 \\
$\mathrm{H}$ & 0.347178000 & 1.413278000 & -1.932611000 \\
$\mathrm{C}$ & 1.727417000 & 0.375601000 & -0.666662000 \\
$\mathrm{C}$ & 2.863327000 & 0.912061000 & -1.316774000 \\
$\mathrm{C}$ & 1.969618000 & -0.577688000 & 0.349396000 \\
$\mathrm{C}$ & 4.146802000 & 0.529466000 & -0.984430000 \\
$\mathrm{H}$ & 2.716120000 & 1.644454000 & -2.106360000 \\
$\mathrm{C}$ & 3.249148000 & -0.978553000 & 0.685907000 \\
$\mathrm{H}$ & 1.132095000 & -1.006017000 & 0.893543000 \\
$\mathrm{C}$ & 4.336719000 & -0.421110000 & 0.018979000 \\
$\mathrm{H}$ & 5.009129000 & 0.945407000 & -1.491530000 \\
$\mathrm{H}$ & 3.423665000 & -1.713712000 & 1.462578000 \\
$\mathrm{H}$ & -1.684504000 & 2.254943000 & -0.845574000 \\
$\mathrm{O}$ & -0.518081000 & 2.783408000 & 0.507803000 \\
$\mathrm{H}$ & -0.010895000 & 3.594893000 & 0.391705000 \\
$\mathrm{H}$ & -2.223950000 & 1.455039000 & 1.782121000 \\
$\mathrm{H}$ & 0.069534000 & 1.961898000 & -0.081513000 \\
$\mathrm{O}$ & 5.680002000 & -0.830626000 & 0.374161000 \\
$\mathrm{O}$ & 5.813903000 & -1.671200000 & 1.257954000 \\
---.618526000 & -0.316022000 & -0.224392000 \\
\hline
\end{tabular}

\begin{tabular}{|c|c|c|c|}
\hline \multicolumn{4}{|c|}{ A3 ${ }_{\text {KOH }}$} \\
\hline & \multicolumn{3}{|c|}{ Coordinates (Angstroms) } \\
\hline & $\mathrm{X}$ & Y & Z \\
\hline$\overline{\mathrm{C}}$ & 5.207105000 & -0.004008000 & -1.263807000 \\
\hline $\mathrm{C}$ & 4.165911000 & 0.843029000 & -0.894806000 \\
\hline $\mathrm{C}$ & 3.089879000 & 0.371237000 & -0.131431000 \\
\hline $\mathrm{C}$ & 3.094026000 & -0.969352000 & 0.291928000 \\
\hline
\end{tabular}




\begin{tabular}{lrcc}
$\mathrm{C}$ & 4.140388000 & -1.804891000 & -0.083632000 \\
$\mathrm{C}$ & 5.193332000 & -1.336248000 & -0.867893000 \\
$\mathrm{H}$ & 6.034031000 & 0.385318000 & -1.850516000 \\
$\mathrm{H}$ & 4.230949000 & 1.893394000 & -1.170360000 \\
$\mathrm{H}$ & 2.285401000 & -1.363305000 & 0.935665000 \\
$\mathrm{H}$ & 4.131584000 & -2.839534000 & 0.249107000 \\
$\mathrm{H}$ & 6.002583000 & -2.001131000 & -1.155532000 \\
$\mathrm{C}$ & 1.966787000 & 1.283334000 & 0.213990000 \\
$\mathrm{C}$ & 1.110485000 & 1.095327000 & 1.227450000 \\
$\mathrm{H}$ & 1.151796000 & 0.168397000 & 1.813153000 \\
$\mathrm{O}$ & 1.832765000 & 2.410187000 & -0.568593000 \\
$\mathrm{C}$ & -0.048809000 & 2.025638000 & 1.498856000 \\
$\mathrm{H}$ & 0.133246000 & 3.012902000 & 1.066167000 \\
$\mathrm{C}$ & -1.300351000 & 1.425817000 & 0.899517000 \\
$\mathrm{C}$ & -1.571251000 & 1.597864000 & -0.466356000 \\
$\mathrm{C}$ & -2.141272000 & 0.599410000 & 1.653567000 \\
$\mathrm{C}$ & -2.645856000 & 0.959318000 & -1.071015000 \\
$\mathrm{H}$ & -0.913186000 & 2.229538000 & -1.055936000 \\
$\mathrm{C}$ & -3.223661000 & -0.047773000 & 1.066561000 \\
$\mathrm{H}$ & -1.924829000 & 0.432092000 & 2.703518000 \\
$\mathrm{C}$ & -3.456487000 & 0.137304000 & -0.291696000 \\
$\mathrm{H}$ & -2.866465000 & 1.089257000 & -2.123781000 \\
$\mathrm{H}$ & -3.875274000 & -0.694290000 & 1.641915000 \\
$\mathrm{O}$ & 0.672438000 & -1.844313000 & 1.951470000 \\
$\mathrm{H}$ & -0.180600000 & 2.148912000 & 2.578699000 \\
$\mathrm{H}$ & 2.305147000 & 2.267039000 & -1.396950000 \\
$\mathrm{H}$ & 1.071589000 & -2.122169000 & 2.779991000 \\
$\mathrm{~K}$ & -0.360901000 & -1.523267000 & -0.080244000 \\
$\mathrm{~N}$ & -4.581221000 & -0.559592000 & -0.925767000 \\
$\mathrm{O}$ & -4.726433000 & -0.419413000 & -2.131149000 \\
$\mathrm{O}$ & -5.296478000 & -1.246865000 & -0.213919000 \\
\hline.----- &
\end{tabular}

\begin{tabular}{|c|c|c|c|}
\hline \multicolumn{4}{|c|}{$\mathbf{A} 1_{\mathrm{NaOH}}$} \\
\hline & \multicolumn{3}{|c|}{ Coordinates (Angstroms) } \\
\hline & $X$ & $\mathrm{Y}$ & $\mathrm{Z}$ \\
\hline$\overline{\mathrm{C}}$ & 3.463201000 & 2.824123000 & -0.838292000 \\
\hline $\mathrm{C}$ & 2.582838000 & 1.752880000 & -0.713210000 \\
\hline $\mathrm{C}$ & 2.951147000 & 0.622684000 & 0.021595000 \\
\hline $\mathrm{C}$ & 4.203816000 & 0.570725000 & 0.641712000 \\
\hline $\mathrm{C}$ & 5.078151000 & 1.646926000 & 0.514051000 \\
\hline $\mathrm{C}$ & 4.712668000 & 2.770385000 & -0.224841000 \\
\hline $\mathrm{H}$ & 3.172089000 & 3.702542000 & -1.407251000 \\
\hline $\mathrm{H}$ & 1.600092000 & 1.800802000 & -1.179496000 \\
\hline
\end{tabular}




$\begin{array}{lrcc}\mathrm{H} & 4.449855000 & -0.335257000 & 1.210453000 \\ \mathrm{H} & 6.049543000 & 1.610866000 & 0.999447000 \\ \mathrm{H} & 5.398815000 & 3.607544000 & -0.318239000 \\ \mathrm{C} & 2.046249000 & -0.591967000 & 0.105500000 \\ \mathrm{C} & 0.600316000 & -0.242240000 & 0.283699000 \\ \mathrm{H} & 0.406570000 & 0.442623000 & 1.107845000 \\ \mathrm{O} & 2.250023000 & -1.429910000 & -1.062893000 \\ \mathrm{C} & -0.405285000 & -0.724894000 & -0.451068000 \\ \mathrm{H} & -0.175487000 & -1.453404000 & -1.226075000 \\ \mathrm{C} & -1.829664000 & -0.407426000 & -0.265563000 \\ \mathrm{C} & -2.795654000 & -1.293743000 & -0.762607000 \\ \mathrm{C} & -2.265337000 & 0.755861000 & 0.386742000 \\ \mathrm{C} & -4.150657000 & -1.051964000 & -0.595140000 \\ \mathrm{H} & -2.475613000 & -2.195040000 & -1.277515000 \\ \mathrm{C} & -3.615170000 & 1.012868000 & 0.564627000 \\ \mathrm{H} & -1.539677000 & 1.478597000 & 0.745649000 \\ \mathrm{C} & -4.541608000 & 0.101009000 & 0.072487000 \\ \mathrm{H} & -4.900989000 & -1.738031000 & -0.967909000 \\ \mathrm{H} & -3.960093000 & 1.908216000 & 1.066723000 \\ \mathrm{H} & 2.406372000 & -1.218575000 & 0.944493000 \\ \mathrm{H} & 2.165937000 & -0.858967000 & -1.838287000 \\ \mathrm{O} & 3.976089000 & -2.307887000 & 1.587155000 \\ \mathrm{H} & 4.132729000 & -2.592189000 & 2.489442000 \\ \mathrm{~N} & -5.970214000 & 0.368428000 & 0.254706000 \\ \mathrm{O} & -6.762914000 & -0.446333000 & -0.194271000 \\ \mathrm{O} & -6.286693000 & 1.390297000 & 0.845604000 \\ \mathrm{Na} & 3.949023000 & -2.805402000 & -0.361376000 \\ ------ & & \end{array}$

TSA1-2 $\mathrm{NaOH}$

\begin{tabular}{|c|c|c|c|}
\hline \multicolumn{4}{|c|}{ Coordinates (Angstroms) } \\
\hline & $X$ & Y & Z \\
\hline $\mathrm{C}$ & -4.190499000 & -2.730616000 & -0.254093000 \\
\hline $\mathrm{C}$ & -3.123017000 & -1.835309000 & -0.285880000 \\
\hline $\mathrm{C}$ & -3.329280000 & -0.468609000 & -0.075423000 \\
\hline $\mathrm{C}$ & -4.636646000 & -0.016389000 & 0.152105000 \\
\hline $\mathrm{C}$ & -5.703045000 & -0.905073000 & 0.164448000 \\
\hline $\mathrm{C}$ & -5.483218000 & -2.268182000 & -0.034095000 \\
\hline $\mathrm{H}$ & -4.010504000 & -3.789761000 & -0.415320000 \\
\hline $\mathrm{H}$ & -2.119380000 & -2.203481000 & -0.484375000 \\
\hline $\mathrm{H}$ & -4.794669000 & 1.042249000 & 0.337679000 \\
\hline $\mathrm{H}$ & -6.709579000 & -0.537297000 & 0.343081000 \\
\hline $\mathrm{H}$ & -6.316258000 & -2.964863000 & -0.016210000 \\
\hline $\mathrm{C}$ & -2.219440000 & 0.530823000 & -0.082392000 \\
\hline
\end{tabular}




$\begin{array}{lrrr}\mathrm{C} & -0.847939000 & 0.075516000 & 0.037352000 \\ \mathrm{H} & -0.689684000 & -0.663362000 & 0.823336000 \\ \mathrm{O} & -2.381491000 & 1.521991000 & -1.127790000 \\ \mathrm{C} & 0.226547000 & 0.568933000 & -0.629489000 \\ \mathrm{H} & 0.046717000 & 1.218988000 & -1.487308000 \\ \mathrm{C} & 1.625750000 & 0.235231000 & -0.374506000 \\ \mathrm{C} & 2.597480000 & 0.553960000 & -1.340761000 \\ \mathrm{C} & 2.062847000 & -0.385808000 & 0.810846000 \\ \mathrm{C} & 3.936907000 & 0.259383000 & -1.150766000 \\ \mathrm{H} & 2.287016000 & 1.032684000 & -2.265870000 \\ \mathrm{C} & 3.396908000 & -0.693296000 & 1.012988000 \\ \mathrm{H} & 1.348978000 & -0.618995000 & 1.594258000 \\ \mathrm{C} & 4.323648000 & -0.366985000 & 0.028223000 \\ \mathrm{H} & 4.682331000 & 0.501787000 & -1.898107000 \\ \mathrm{H} & 3.734342000 & -1.171006000 & 1.924648000 \\ \mathrm{H} & -2.393936000 & 1.357963000 & 0.972913000 \\ \mathrm{H} & -2.375101000 & 1.070845000 & -1.983279000 \\ \mathrm{O} & -2.481943000 & 2.433022000 & 1.659209000 \\ \mathrm{H} & -2.433691000 & 2.179308000 & 2.585437000 \\ \mathrm{~N} & 5.733003000 & -0.679490000 & 0.242316000 \\ \mathrm{O} & 6.526902000 & -0.367220000 & -0.634734000 \\ \mathrm{O} & 6.042269000 & -1.235959000 & 1.286930000 \\ \mathrm{Na} & -1.259593000 & 3.105931000 & 0.084785000 \\ ---------~\end{array}$

\begin{tabular}{|c|c|c|c|}
\hline \multicolumn{4}{|c|}{$\mathrm{A} 2 \mathrm{NaOH}$} \\
\hline & $\mathrm{X}$ & $\mathrm{Y}$ & Z \\
\hline$\overline{\mathrm{C}}$ & 4.480278000 & 2.310085000 & 0.482256000 \\
\hline $\mathrm{C}$ & 3.317875000 & 1.663214000 & 0.097980000 \\
\hline $\mathrm{C}$ & 3.353748000 & 0.373648000 & -0.484046000 \\
\hline $\mathrm{C}$ & 4.625725000 & -0.224645000 & -0.635486000 \\
\hline $\mathrm{C}$ & 5.786996000 & 0.433544000 & -0.234300000 \\
\hline $\mathrm{C}$ & 5.728853000 & 1.702327000 & 0.328038000 \\
\hline $\mathrm{H}$ & 4.411809000 & 3.303349000 & 0.917307000 \\
\hline $\mathrm{H}$ & 2.367103000 & 2.166093000 & 0.244645000 \\
\hline $\mathrm{H}$ & 4.696621000 & -1.195913000 & -1.113974000 \\
\hline $\mathrm{H}$ & 6.747462000 & -0.053874000 & -0.379357000 \\
\hline $\mathrm{H}$ & 6.634293000 & 2.214838000 & 0.636397000 \\
\hline $\mathrm{C}$ & 2.151839000 & -0.366569000 & -0.823956000 \\
\hline $\mathrm{C}$ & 0.845023000 & 0.003534000 & -0.612435000 \\
\hline $\mathrm{H}$ & 0.688760000 & 1.051544000 & -0.370537000 \\
\hline $\mathrm{O}$ & 2.416503000 & -1.733312000 & -1.106739000 \\
\hline $\mathrm{C}$ & -0.285648000 & -0.830998000 & -0.640744000 \\
\hline
\end{tabular}




$\begin{array}{lrrr}\mathrm{H} & -0.163379000 & -1.868981000 & -0.950720000 \\ \mathrm{C} & -1.647876000 & -0.398392000 & -0.472643000 \\ \mathrm{C} & -2.696193000 & -1.350734000 & -0.551833000 \\ \mathrm{C} & -2.029054000 & 0.938757000 & -0.193851000 \\ \mathrm{C} & -4.017050000 & -1.008350000 & -0.354515000 \\ \mathrm{H} & -2.446696000 & -2.385953000 & -0.771496000 \\ \mathrm{C} & -3.348253000 & 1.291616000 & 0.006152000 \\ \mathrm{H} & -1.276288000 & 1.718837000 & -0.140592000 \\ \mathrm{C} & -4.343202000 & 0.317899000 & -0.069208000 \\ \mathrm{H} & -4.805740000 & -1.748521000 & -0.416315000 \\ \mathrm{H} & -3.626608000 & 2.317292000 & 0.217212000 \\ \mathrm{H} & 1.797091000 & -2.024878000 & -1.787131000 \\ \mathrm{O} & 0.615995000 & -1.829930000 & 1.987523000 \\ \mathrm{H} & 0.119224000 & -1.526950000 & 2.754780000 \\ \mathrm{H} & 0.135642000 & -1.465853000 & 1.196535000 \\ \mathrm{~N} & -5.724141000 & 0.686063000 & 0.148649000 \\ \mathrm{O} & -5.976869000 & 1.859537000 & 0.404695000 \\ \mathrm{O} & -6.576671000 & -0.193200000 & 0.069888000 \\ \mathrm{Na} & 2.674514000 & -1.750714000 & 1.184665000 \\ - & \end{array}$

TSA2-3 ${ }_{\mathrm{NaOH}}$

\begin{tabular}{lccc} 
& \multicolumn{3}{c}{ Coordinates (Angstroms) } \\
C & X & Y & Z \\
$\mathrm{C}$ & 3.336760000 & 2.555411000 & -0.219239000 \\
$\mathrm{C}$ & 3.333571000 & 0.357966000 & -0.470171000 \\
$\mathrm{C}$ & 4.567290000 & -0.207458000 & -0.100203000 \\
$\mathrm{C}$ & 5.656324000 & 0.599128000 & 0.226290000 \\
$\mathrm{C}$ & 5.545320000 & 1.983413000 & 0.180353000 \\
$\mathrm{H}$ & 4.244838000 & 3.635210000 & -0.290388000 \\
$\mathrm{H}$ & 2.332258000 & 2.227070000 & -0.889813000 \\
$\mathrm{H}$ & 4.680663000 & -1.287692000 & -0.112643000 \\
$\mathrm{H}$ & 6.599583000 & 0.136061000 & 0.501943000 \\
$\mathrm{H}$ & 6.393959000 & 2.612653000 & 0.429550000 \\
$\mathrm{C}$ & 2.165921000 & -0.509944000 & -0.697433000 \\
$\mathrm{C}$ & 0.868878000 & -0.123296000 & -0.634606000 \\
$\mathrm{H}$ & 0.685333000 & 0.930576000 & -0.433536000 \\
$\mathrm{O}$ & 2.518847000 & -1.851754000 & -0.801316000 \\
$\mathrm{C}$ & -0.272972000 & -1.029037000 & -0.723648000 \\
$\mathrm{H}$ & -0.218066000 & -1.720078000 & -1.572046000 \\
$\mathrm{C}$ & -1.616305000 & -0.505540000 & -0.497906000 \\
$\mathrm{C}$ & -2.732453000 & -1.089824000 & -1.135199000 \\
$\mathrm{C}$ & -1.875647000 & 0.544074000 & 0.410300000 \\
& & &
\end{tabular}




$\begin{array}{lrrr}\mathrm{C} & -4.021742000 & -0.656424000 & -0.893657000 \\ \mathrm{H} & -2.568640000 & -1.896542000 & -1.844778000 \\ \mathrm{C} & -3.159809000 & 0.994469000 & 0.656633000 \\ \mathrm{H} & -1.050827000 & 1.005498000 & 0.946469000 \\ \mathrm{C} & -4.229790000 & 0.389765000 & 0.003288000 \\ \mathrm{H} & -4.871599000 & -1.106557000 & -1.392417000 \\ \mathrm{H} & -3.350922000 & 1.800215000 & 1.355245000 \\ \mathrm{H} & 1.739370000 & -2.396595000 & -0.583678000 \\ \mathrm{O} & 0.635378000 & -2.606222000 & 1.148330000 \\ \mathrm{H} & 0.033967000 & -3.302294000 & 1.430747000 \\ \mathrm{H} & 0.052443000 & -1.932168000 & 0.381288000 \\ \mathrm{~N} & -5.580229000 & 0.853247000 & 0.263774000 \\ \mathrm{O} & -5.730790000 & 1.771691000 & 1.061931000 \\ \mathrm{O} & -6.502781000 & 0.302246000 & -0.325633000 \\ \mathrm{Na} & 2.373090000 & -1.452463000 & 1.656080000 \\ \text { - - - - - } & \end{array}$

\begin{tabular}{|c|c|c|c|}
\hline \multicolumn{4}{|c|}{$\mathbf{A 3}{ }_{\mathrm{NaOH}}$} \\
\hline & $X$ & Y & $\mathrm{Z}$ \\
\hline $\mathrm{C}$ & 5.257917000 & -0.030584000 & -1.156861000 \\
\hline $\mathrm{C}$ & 4.183471000 & 0.785960000 & -0.818677000 \\
\hline $\mathrm{C}$ & 3.076464000 & 0.266677000 & -0.134287000 \\
\hline $\mathrm{C}$ & 3.071433000 & -1.087546000 & 0.234902000 \\
\hline $\mathrm{C}$ & 4.152378000 & -1.892470000 & -0.110225000 \\
\hline $\mathrm{C}$ & 5.241374000 & -1.377212000 & -0.810258000 \\
\hline $\mathrm{H}$ & 6.111409000 & 0.391492000 & -1.679083000 \\
\hline $\mathrm{H}$ & 4.242208000 & 1.847306000 & -1.051344000 \\
\hline $\mathrm{H}$ & 2.227094000 & -1.514739000 & 0.805069000 \\
\hline $\mathrm{H}$ & 4.139892000 & -2.940857000 & 0.175095000 \\
\hline $\mathrm{H}$ & 6.076584000 & -2.019179000 & -1.075015000 \\
\hline $\mathrm{C}$ & 1.931374000 & 1.156536000 & 0.192216000 \\
\hline $\mathrm{C}$ & 1.095149000 & 0.986586000 & 1.227204000 \\
\hline $\mathrm{H}$ & 1.193196000 & 0.097373000 & 1.851851000 \\
\hline $\mathrm{O}$ & 1.760933000 & 2.251974000 & -0.621676000 \\
\hline $\mathrm{C}$ & -0.067662000 & 1.914034000 & 1.502242000 \\
\hline $\mathrm{H}$ & 0.119697000 & 2.903299000 & 1.075634000 \\
\hline $\mathrm{C}$ & -1.324251000 & 1.326779000 & 0.899068000 \\
\hline $\mathrm{C}$ & -1.566921000 & 1.466247000 & -0.477376000 \\
\hline $\mathrm{C}$ & -2.189974000 & 0.530452000 & 1.657135000 \\
\hline $\mathrm{C}$ & -2.635945000 & 0.820569000 & -1.085779000 \\
\hline $\mathrm{H}$ & -0.892034000 & 2.077075000 & -1.069868000 \\
\hline $\mathrm{C}$ & -3.266738000 & -0.122876000 & 1.065019000 \\
\hline $\mathrm{H}$ & -1.999708000 & 0.391623000 & 2.716241000 \\
\hline
\end{tabular}




$\begin{array}{lrrr}\mathrm{C} & -3.469925000 & 0.027673000 & -0.301052000 \\ \mathrm{H} & -2.835428000 & 0.924980000 & -2.145558000 \\ \mathrm{H} & -3.934188000 & -0.749959000 & 1.643338000 \\ \mathrm{O} & 0.566719000 & -2.003131000 & 1.699740000 \\ \mathrm{H} & -0.197913000 & 2.032277000 & 2.582376000 \\ \mathrm{H} & 2.282125000 & 2.121706000 & -1.423059000 \\ \mathrm{H} & 0.666180000 & -2.839215000 & 2.160825000 \\ \mathrm{~N} & -4.593240000 & -0.670903000 & -0.938732000 \\ \mathrm{O} & -4.710319000 & -0.562583000 & -2.149948000 \\ \mathrm{O} & -5.335491000 & -1.321426000 & -0.221132000 \\ \mathrm{Na} & -0.384548000 & -1.233680000 & 0.095373000\end{array}$

\begin{tabular}{|c|c|c|c|}
\hline \multicolumn{4}{|c|}{$\mathbf{A 1}_{\text {LiOH }}$} \\
\hline & $X$ & $\mathrm{Y}$ & Z \\
\hline $\mathrm{C}$ & -3.741719000 & -2.568320000 & -0.733820000 \\
\hline $\mathrm{C}$ & -2.819775000 & -1.533151000 & -0.603238000 \\
\hline $\mathrm{C}$ & -3.182921000 & -0.347964000 & 0.040932000 \\
\hline $\mathrm{C}$ & -4.474980000 & -0.200041000 & 0.555767000 \\
\hline $\mathrm{C}$ & -5.390552000 & -1.239587000 & 0.423942000 \\
\hline $\mathrm{C}$ & -5.026903000 & -2.421706000 & -0.218508000 \\
\hline $\mathrm{H}$ & -3.455322000 & -3.489988000 & -1.231834000 \\
\hline $\mathrm{H}$ & -1.811593000 & -1.650471000 & -0.995734000 \\
\hline $\mathrm{H}$ & -4.730329000 & 0.747561000 & 1.037347000 \\
\hline $\mathrm{H}$ & -6.392220000 & -1.127613000 & 0.828827000 \\
\hline $\mathrm{H}$ & -5.745454000 & -3.230547000 & -0.315600000 \\
\hline $\mathrm{C}$ & -2.225428000 & 0.822472000 & 0.134738000 \\
\hline $\mathrm{C}$ & -0.792100000 & 0.418469000 & 0.308853000 \\
\hline $\mathrm{H}$ & -0.619366000 & -0.245714000 & 1.154240000 \\
\hline $\mathrm{O}$ & -2.395900000 & 1.681731000 & -1.027901000 \\
\hline $\mathrm{C}$ & 0.222892000 & 0.833789000 & -0.451915000 \\
\hline $\mathrm{H}$ & 0.014478000 & 1.542482000 & -1.250674000 \\
\hline $\mathrm{C}$ & 1.635668000 & 0.464451000 & -0.268084000 \\
\hline $\mathrm{C}$ & 2.630483000 & 1.297081000 & -0.798839000 \\
\hline $\mathrm{C}$ & 2.029604000 & -0.694267000 & 0.417334000 \\
\hline $\mathrm{C}$ & 3.976391000 & 1.008007000 & -0.631455000 \\
\hline $\mathrm{H}$ & 2.342048000 & 2.193714000 & -1.339859000 \\
\hline $\mathrm{C}$ & 3.369858000 & -0.998418000 & 0.594759000 \\
\hline $\mathrm{H}$ & 1.279170000 & -1.377171000 & 0.802597000 \\
\hline $\mathrm{C}$ & 4.326498000 & -0.138155000 & 0.069350000 \\
\hline $\mathrm{H}$ & 4.750444000 & 1.652264000 & -1.029510000 \\
\hline $\mathrm{H}$ & 3.683559000 & -1.890527000 & 1.122448000 \\
\hline $\mathrm{H}$ & -2.548037000 & 1.461252000 & 0.969915000 \\
\hline
\end{tabular}




$\begin{array}{lccc}\mathrm{H} & -2.477994000 & 1.108908000 & -1.801728000 \\ \mathrm{O} & -4.197368000 & 2.843757000 & 1.199563000 \\ \mathrm{H} & -4.605056000 & 3.349479000 & 1.902333000 \\ \mathrm{~N} & 5.745752000 & -0.455114000 & 0.251772000 \\ \mathrm{O} & 6.565479000 & 0.317535000 & -0.221621000 \\ \mathrm{O} & 6.025870000 & -1.472977000 & 0.866990000 \\ \mathrm{Li} & -3.703780000 & 2.988613000 & -0.396771000\end{array}$

TSA1-2 ${ }_{\text {LiOH }}$

\begin{tabular}{|c|c|c|c|}
\hline \multicolumn{4}{|c|}{ Coordinates (Angstroms) } \\
\hline & $X$ & Y & Z \\
\hline $\mathrm{C}$ & -4.434248000 & -2.437603000 & -0.248961000 \\
\hline $\mathrm{C}$ & -3.313693000 & -1.610083000 & -0.232214000 \\
\hline $\mathrm{C}$ & -3.446466000 & -0.226595000 & -0.069924000 \\
\hline $\mathrm{C}$ & -4.737755000 & 0.307524000 & 0.050237000 \\
\hline $\mathrm{C}$ & -5.855109000 & -0.515434000 & 0.012236000 \\
\hline $\mathrm{C}$ & -5.708677000 & -1.894762000 & -0.130594000 \\
\hline $\mathrm{H}$ & -4.307070000 & -3.509107000 & -0.374268000 \\
\hline $\mathrm{H}$ & -2.327704000 & -2.047800000 & -0.363268000 \\
\hline $\mathrm{H}$ & -4.852476000 & 1.379348000 & 0.182433000 \\
\hline $\mathrm{H}$ & -6.845962000 & -0.080495000 & 0.108019000 \\
\hline $\mathrm{H}$ & -6.582568000 & -2.539125000 & -0.152367000 \\
\hline $\mathrm{C}$ & -2.278148000 & 0.690430000 & -0.001949000 \\
\hline $\mathrm{C}$ & -0.924884000 & 0.189015000 & 0.035667000 \\
\hline $\mathrm{H}$ & -0.772518000 & -0.649759000 & 0.714594000 \\
\hline $\mathrm{O}$ & -2.405549000 & 1.834662000 & -0.891976000 \\
\hline $\mathrm{C}$ & 0.158108000 & 0.759692000 & -0.556840000 \\
\hline $\mathrm{H}$ & -0.006200000 & 1.515028000 & -1.328091000 \\
\hline $\mathrm{C}$ & 1.553449000 & 0.383515000 & -0.342782000 \\
\hline $\mathrm{C}$ & 2.532102000 & 0.821331000 & -1.253484000 \\
\hline $\mathrm{C}$ & 1.978912000 & -0.392554000 & 0.751571000 \\
\hline $\mathrm{C}$ & 3.868608000 & 0.495432000 & -1.097179000 \\
\hline $\mathrm{H}$ & 2.230471000 & 1.421840000 & -2.107796000 \\
\hline $\mathrm{C}$ & 3.310093000 & -0.732946000 & 0.918140000 \\
\hline $\mathrm{H}$ & 1.259878000 & -0.721577000 & 1.494767000 \\
\hline $\mathrm{C}$ & 4.244219000 & -0.284059000 & -0.009513000 \\
\hline $\mathrm{H}$ & 4.619718000 & 0.829883000 & -1.802061000 \\
\hline $\mathrm{H}$ & 3.639197000 & -1.329560000 & 1.760168000 \\
\hline $\mathrm{H}$ & -2.299207000 & 1.485086000 & 1.167191000 \\
\hline $\mathrm{H}$ & -2.358054000 & 1.530391000 & -1.809387000 \\
\hline $\mathrm{O}$ & -2.129120000 & 2.530310000 & 1.786023000 \\
\hline $\mathrm{H}$ & -1.749401000 & 2.303140000 & 2.640949000 \\
\hline $\mathrm{Li}$ & -1.275031000 & 2.943605000 & 0.250675000 \\
\hline
\end{tabular}




\begin{tabular}{llll}
$\mathrm{N}$ & 5.651013000 & -0.630214000 & 0.169429000 \\
$\mathrm{O}$ & 5.951007000 & -1.319416000 & 1.134511000 \\
$\mathrm{O}$ & $\mathbf{6 . 4 5 1 2 6 6 0 0 0}$ & -0.211058000 & -0.655478000 \\
\hline
\end{tabular}

\begin{tabular}{|c|c|c|c|}
\hline \multicolumn{4}{|c|}{ A2 $2_{\text {LiOH }}$} \\
\hline & \multicolumn{3}{|c|}{ Coordinates (Angstroms) } \\
\hline & $X$ & $\mathrm{Y}$ & $\mathrm{Z}$ \\
\hline$\overline{\mathrm{C}}$ & 4.644149000 & 2.244328000 & 0.199059000 \\
\hline $\mathrm{C}$ & 3.483820000 & 1.563605000 & -0.126494000 \\
\hline $\mathrm{C}$ & 3.502521000 & 0.176188000 & -0.394583000 \\
\hline $\mathrm{C}$ & 4.749830000 & -0.479869000 & -0.317172000 \\
\hline $\mathrm{C}$ & 5.909600000 & 0.216893000 & 0.019525000 \\
\hline $\mathrm{C}$ & 5.869757000 & 1.579042000 & 0.283863000 \\
\hline $\mathrm{H}$ & 4.593473000 & 3.312732000 & 0.389261000 \\
\hline $\mathrm{H}$ & 2.551655000 & 2.116472000 & -0.188520000 \\
\hline $\mathrm{H}$ & 4.812604000 & -1.533941000 & -0.567243000 \\
\hline $\mathrm{H}$ & 6.854130000 & -0.318479000 & 0.061506000 \\
\hline $\mathrm{H}$ & 6.774432000 & 2.120134000 & 0.541477000 \\
\hline $\mathrm{C}$ & 2.289802000 & -0.583526000 & -0.655343000 \\
\hline $\mathrm{C}$ & 0.981633000 & -0.179978000 & -0.513457000 \\
\hline $\mathrm{H}$ & 0.840734000 & 0.889709000 & -0.383503000 \\
\hline $\mathrm{O}$ & 2.543770000 & -1.986691000 & -0.728749000 \\
\hline $\mathrm{C}$ & -0.161938000 & -0.993891000 & -0.460508000 \\
\hline $\mathrm{H}$ & -0.072438000 & -2.049741000 & -0.719281000 \\
\hline $\mathrm{C}$ & -1.519222000 & -0.513248000 & -0.344519000 \\
\hline $\mathrm{C}$ & -2.591582000 & -1.433052000 & -0.438944000 \\
\hline $\mathrm{C}$ & -1.862317000 & 0.839071000 & -0.103959000 \\
\hline $\mathrm{C}$ & -3.907723000 & -1.042747000 & -0.299781000 \\
\hline $\mathrm{H}$ & -2.369348000 & -2.480392000 & -0.627154000 \\
\hline $\mathrm{C}$ & -3.176140000 & 1.241597000 & 0.034870000 \\
\hline $\mathrm{H}$ & -1.085601000 & 1.593205000 & -0.024228000 \\
\hline $\mathrm{C}$ & -4.197951000 & 0.299310000 & -0.059884000 \\
\hline $\mathrm{H}$ & -4.717995000 & -1.757944000 & -0.374599000 \\
\hline $\mathrm{H}$ & -3.426318000 & 2.279734000 & 0.217442000 \\
\hline $\mathrm{H}$ & 1.894234000 & -2.390063000 & -1.317573000 \\
\hline $\mathrm{O}$ & 0.917864000 & -1.844505000 & 2.113309000 \\
\hline $\mathrm{H}$ & 0.624249000 & -1.378649000 & 2.904805000 \\
\hline $\mathrm{H}$ & 0.324667000 & -1.533020000 & 1.369998000 \\
\hline $\mathrm{Li}$ & 2.551044000 & -1.676140000 & 1.185006000 \\
\hline $\mathrm{N}$ & -5.576112000 & 0.718956000 & 0.093686000 \\
\hline $\mathrm{O}$ & -5.797485000 & 1.905834000 & 0.309231000 \\
\hline $\mathrm{O}$ & -6.452181000 & -0.134184000 & 0.002553000 \\
\hline
\end{tabular}


TSA2-3 ${ }_{\mathrm{LiOH}}$

\begin{tabular}{lccc} 
& Coordinates (Angstroms) & \\
& $\mathrm{X}$ & $\mathrm{Y}$ & $\mathrm{Z}$ \\
\hline $\mathrm{C}$ & -4.580673000 & -2.289771000 & 0.396368000 \\
$\mathrm{C}$ & -3.446456000 & -1.492436000 & 0.328778000 \\
$\mathrm{C}$ & -3.504663000 & -0.207940000 & -0.235221000 \\
$\mathrm{C}$ & -4.741978000 & 0.259416000 & -0.699622000 \\
$\mathrm{C}$ & -5.877562000 & -0.540520000 & -0.621031000 \\
$\mathrm{C}$ & -5.803574000 & -1.818715000 & -0.077696000 \\
$\mathrm{H}$ & -4.510915000 & -3.280919000 & 0.834550000 \\
$\mathrm{H}$ & -2.508627000 & -1.868447000 & 0.727616000 \\
$\mathrm{H}$ & -4.805866000 & 1.250033000 & -1.136162000 \\
$\mathrm{H}$ & -6.824152000 & -0.160716000 & -0.994238000 \\
$\mathrm{H}$ & -6.690460000 & -2.441950000 & -0.018405000 \\
$\mathrm{C}$ & -2.308640000 & 0.643802000 & -0.316769000 \\
$\mathrm{C}$ & -1.008887000 & 0.276590000 & -0.256142000 \\
$\mathrm{H}$ & -0.823071000 & -0.792406000 & -0.183129000 \\
$\mathrm{O}$ & -2.632988000 & 2.022629000 & -0.393388000 \\
$\mathrm{C}$ & 0.134861000 & 1.181743000 & -0.239189000 \\
$\mathrm{H}$ & 0.058233000 & 2.022841000 & -0.940482000 \\
$\mathrm{C}$ & 1.486852000 & 0.613431000 & -0.220666000 \\
$\mathrm{C}$ & 2.556462000 & 1.291700000 & -0.838481000 \\
$\mathrm{C}$ & 1.792654000 & -0.577820000 & 0.468432000 \\
$\mathrm{C}$ & 3.851406000 & 0.809554000 & -0.790371000 \\
$\mathrm{H}$ & 2.356161000 & 2.216796000 & -1.372284000 \\
$\mathrm{C}$ & 3.081586000 & -1.079278000 & 0.517008000 \\
$\mathrm{H}$ & 1.006770000 & -1.116115000 & 0.991002000 \\
$\mathrm{C}$ & 4.105133000 & -0.381322000 & -0.115481000 \\
$\mathrm{H}$ & 4.667557000 & 1.332726000 & -1.273698000 \\
$\mathrm{H}$ & 3.309965000 & -1.998364000 & 1.043067000 \\
$\mathrm{H}$ & -1.945724000 & 2.477204000 & -0.900338000 \\
$\mathrm{O}$ & -0.527975000 & 2.477941000 & 1.934474000 \\
$\mathrm{H}$ & 0.040664000 & 2.314672000 & 2.693608000 \\
$\mathrm{H}$ & -0.071111000 & 1.895179000 & 1.000240000 \\
$\mathrm{~N}$ & -2.201478000 & 2.016199000 & 1.530906000 \\
$\mathrm{O}$ & 5.462417000 & -0.901844000 & -0.065587000 \\
$\mathrm{H}$ & 6.343968000 & -0.262326000 & -0.625739000 \\
& 5.653115000 & -1.952846000 & 0.534097000 \\
\hline
\end{tabular}

A3 $3_{\text {LiOH }}$

Coordinates (Angstroms)

$\mathrm{X} \quad \mathrm{Y} \quad \mathrm{Z}$ 


\begin{tabular}{lrcc}
$\mathrm{C}$ & -5.374562000 & 0.197780000 & -0.782199000 \\
$\mathrm{C}$ & -4.269302000 & -0.636470000 & -0.663288000 \\
$\mathrm{C}$ & -3.075805000 & -0.156315000 & -0.106337000 \\
$\mathrm{C}$ & -2.994439000 & 1.172005000 & 0.328228000 \\
$\mathrm{C}$ & -4.109455000 & 1.996522000 & 0.202212000 \\
$\mathrm{C}$ & -5.295058000 & 1.518857000 & -0.349416000 \\
$\mathrm{H}$ & -6.298047000 & -0.187002000 & -1.204308000 \\
$\mathrm{H}$ & -4.350746000 & -1.677938000 & -0.967646000 \\
$\mathrm{H}$ & -2.056430000 & 1.580679000 & 0.727385000 \\
$\mathrm{H}$ & -4.040281000 & 3.029059000 & 0.531392000 \\
$\mathrm{H}$ & -6.156176000 & 2.174045000 & -0.443289000 \\
$\mathrm{C}$ & -1.917912000 & -1.078069000 & 0.007286000 \\
$\mathrm{C}$ & -1.112180000 & -1.168953000 & 1.078785000 \\
$\mathrm{H}$ & -1.309912000 & -0.513264000 & 1.921153000 \\
$\mathrm{O}$ & -1.713224000 & -1.925548000 & -1.046932000 \\
$\mathrm{C}$ & 0.051767000 & -2.133313000 & 1.170695000 \\
$\mathrm{H}$ & -0.141480000 & -3.016751000 & 0.555735000 \\
$\mathrm{C}$ & 1.320024000 & -1.448745000 & 0.704860000 \\
$\mathrm{C}$ & 1.607930000 & -1.369066000 & -0.667592000 \\
$\mathrm{C}$ & 2.134923000 & -0.753753000 & 1.605320000 \\
$\mathrm{C}$ & 2.679480000 & -0.614025000 & -1.129230000 \\
$\mathrm{H}$ & 0.970725000 & -1.894938000 & -1.372137000 \\
$\mathrm{C}$ & 3.214517000 & 0.001521000 & 1.159449000 \\
$\mathrm{H}$ & 1.904907000 & -0.778867000 & 2.665978000 \\
$\mathrm{C}$ & 3.468249000 & 0.059507000 & -0.203159000 \\
$\mathrm{H}$ & 2.909929000 & -0.539897000 & -2.184994000 \\
$\mathrm{H}$ & 3.841565000 & 0.555170000 & 1.846941000 \\
$\mathrm{O}$ & -0.151568000 & 2.149707000 & 1.053011000 \\
$\mathrm{H}$ & 0.169641000 & -2.468175000 & 2.205678000 \\
$\mathrm{H}$ & -2.271384000 & -1.638526000 & -1.781111000 \\
$\mathrm{H}$ & 0.122963000 & 3.060717000 & 1.153907000 \\
$\mathrm{~N}$ & 4.600326000 & 0.863260000 & -0.684433000 \\
$\mathrm{O}$ & 4.772178000 & 0.927196000 & -1.891483000 \\
& 5.298728000 & 1.412188000 & 0.151698000 \\
0.294822000 & 0.746542000 & 0.290775000 \\
\hline--- & & &
\end{tabular}

\begin{tabular}{|c|c|c|c|}
\hline \multirow{2}{*}{\multicolumn{4}{|c|}{$\mathrm{A1}_{\text {tBuOK }}$}} \\
\hline & & & \\
\hline & $\mathrm{X}$ & $\mathrm{Y}$ & $\mathrm{Z}$ \\
\hline$\overline{\mathrm{C}}$ & -1.960874000 & 4.001449000 & -0.835330000 \\
\hline $\mathrm{C}$ & -1.289314000 & 2.925924000 & -0.262045000 \\
\hline $\mathrm{C}$ & -1.904245000 & 1.674087000 & -0.176428000 \\
\hline $\mathrm{C}$ & -3.195423000 & 1.501574000 & -0.684740000 \\
\hline
\end{tabular}




\begin{tabular}{|c|c|c|c|}
\hline $\mathrm{C}$ & -3.861795000 & 2.580288000 & -1.261893000 \\
\hline $\mathrm{C}$ & -3.249902000 & 3.829413000 & -1.335394000 \\
\hline $\mathrm{H}$ & -1.474760000 & 4.970350000 & -0.903858000 \\
\hline $\mathrm{H}$ & -0.273038000 & 3.055685000 & 0.106993000 \\
\hline $\mathrm{H}$ & -3.639213000 & 0.502383000 & -0.630683000 \\
\hline $\mathrm{H}$ & -4.859700000 & 2.440693000 & -1.668690000 \\
\hline $\mathrm{H}$ & -3.770307000 & 4.666760000 & -1.791402000 \\
\hline $\mathrm{C}$ & -1.215185000 & 0.511480000 & 0.515932000 \\
\hline $\mathrm{C}$ & 0.213309000 & 0.346755000 & 0.095133000 \\
\hline $\mathrm{H}$ & 0.353202000 & 0.317715000 & -0.984093000 \\
\hline $\mathrm{O}$ & -1.351592000 & 0.644668000 & 1.947401000 \\
\hline $\mathrm{C}$ & 1.251216000 & 0.230705000 & 0.927919000 \\
\hline $\mathrm{H}$ & 1.057606000 & 0.267077000 & 1.998397000 \\
\hline $\mathrm{C}$ & 2.660192000 & 0.056642000 & 0.543174000 \\
\hline $\mathrm{C}$ & 3.649687000 & 0.180122000 & 1.528865000 \\
\hline $\mathrm{C}$ & 3.064375000 & -0.231088000 & -0.770040000 \\
\hline $\mathrm{C}$ & 4.995841000 & 0.040238000 & 1.226168000 \\
\hline $\mathrm{H}$ & 3.356769000 & 0.392162000 & 2.553310000 \\
\hline $\mathrm{C}$ & 4.403991000 & -0.372725000 & -1.091146000 \\
\hline $\mathrm{H}$ & 2.323542000 & -0.359782000 & -1.551947000 \\
\hline $\mathrm{C}$ & 5.354920000 & -0.231666000 & -0.086695000 \\
\hline $\mathrm{H}$ & 5.762037000 & 0.137519000 & 1.985104000 \\
\hline $\mathrm{H}$ & 4.722314000 & -0.594802000 & -2.102193000 \\
\hline $\mathrm{H}$ & -1.786856000 & -0.405273000 & 0.273807000 \\
\hline $\mathrm{H}$ & -1.008284000 & 1.516863000 & 2.184651000 \\
\hline $\mathrm{C}$ & -4.688912000 & -3.168289000 & -1.077578000 \\
\hline $\mathrm{C}$ & -3.335884000 & -2.480139000 & -0.800177000 \\
\hline $\mathrm{H}$ & -5.071024000 & -3.617601000 & -0.152151000 \\
\hline $\mathrm{H}$ & -5.415534000 & -2.419460000 & -1.414723000 \\
\hline $\mathrm{H}$ & -4.621958000 & -3.955499000 & -1.840209000 \\
\hline $\mathrm{C}$ & -2.808220000 & -1.887612000 & -2.126713000 \\
\hline $\mathrm{C}$ & -2.328290000 & -3.541303000 & -0.306391000 \\
\hline $\mathrm{H}$ & -1.842111000 & -1.393277000 & -1.960855000 \\
\hline $\mathrm{H}$ & -2.669860000 & -2.650062000 & -2.904469000 \\
\hline $\mathrm{H}$ & -3.509006000 & -1.133951000 & -2.505260000 \\
\hline $\mathrm{H}$ & -2.170044000 & -4.352951000 & -1.028972000 \\
\hline $\mathrm{H}$ & -1.359983000 & -3.065256000 & -0.106149000 \\
\hline $\mathrm{H}$ & -2.688679000 & -3.983098000 & 0.631531000 \\
\hline $\mathrm{O}$ & -3.488591000 & -1.496296000 & 0.147653000 \\
\hline K & -3.779549000 & -0.508350000 & 2.247779000 \\
\hline $\mathrm{N}$ & 6.772791000 & -0.380372000 & -0.421980000 \\
\hline $\mathrm{O}$ & 7.059506000 & -0.621477000 & -1.585232000 \\
\hline $\mathrm{O}$ & 7.587409000 & -0.253154000 & 0.480453000 \\
\hline
\end{tabular}


TSA1-2 ${ }_{\text {tBuOK }}$

\begin{tabular}{|c|c|c|c|}
\hline \multicolumn{4}{|c|}{ Coordinates (Angstroms) } \\
\hline & $\mathrm{X}$ & $\mathrm{Y}$ & $\mathrm{Z}$ \\
\hline $\mathrm{C}$ & -4.200460000 & -3.134095000 & -0.283592000 \\
\hline $\mathrm{C}$ & -3.268611000 & -2.255063000 & -0.837609000 \\
\hline $\mathrm{C}$ & -2.428823000 & -1.480818000 & -0.024144000 \\
\hline $\mathrm{C}$ & -2.569038000 & -1.607230000 & 1.368237000 \\
\hline $\mathrm{C}$ & -3.487399000 & -2.490729000 & 1.917512000 \\
\hline $\mathrm{C}$ & -4.313940000 & -3.259228000 & 1.095947000 \\
\hline $\mathrm{H}$ & -4.828240000 & -3.733217000 & -0.938303000 \\
\hline $\mathrm{H}$ & -3.172351000 & -2.184319000 & -1.918417000 \\
\hline $\mathrm{H}$ & -1.964064000 & -0.980849000 & 2.018923000 \\
\hline $\mathrm{H}$ & -3.573080000 & -2.571897000 & 2.997518000 \\
\hline $\mathrm{H}$ & -5.035437000 & -3.945147000 & 1.529106000 \\
\hline $\mathrm{C}$ & -1.494129000 & -0.456218000 & -0.585920000 \\
\hline $\mathrm{C}$ & -0.086847000 & -0.600052000 & -0.234928000 \\
\hline $\mathrm{H}$ & 0.101204000 & -1.047908000 & 0.739900000 \\
\hline $\mathrm{O}$ & -1.708965000 & -0.225977000 & -1.986086000 \\
\hline $\mathrm{C}$ & 0.950138000 & -0.173473000 & -0.985931000 \\
\hline $\mathrm{H}$ & 0.733111000 & 0.328211000 & -1.927045000 \\
\hline $\mathrm{C}$ & 2.364313000 & -0.258873000 & -0.628406000 \\
\hline $\mathrm{C}$ & 3.303260000 & 0.451047000 & -1.397647000 \\
\hline $\mathrm{C}$ & 2.847123000 & -1.024751000 & 0.448737000 \\
\hline $\mathrm{C}$ & 4.656655000 & 0.417929000 & -1.107459000 \\
\hline $\mathrm{H}$ & 2.955092000 & 1.047884000 & -2.236068000 \\
\hline $\mathrm{C}$ & 4.195918000 & -1.065853000 & 0.755201000 \\
\hline $\mathrm{H}$ & 2.159874000 & -1.610531000 & 1.050456000 \\
\hline $\mathrm{C}$ & 5.089928000 & -0.342126000 & -0.027653000 \\
\hline $\mathrm{H}$ & 5.377267000 & 0.969955000 & -1.698237000 \\
\hline $\mathrm{H}$ & 4.568226000 & -1.654881000 & 1.584363000 \\
\hline $\mathrm{H}$ & -1.922460000 & 0.701733000 & -0.043444000 \\
\hline $\mathrm{H}$ & -1.199076000 & -0.882238000 & -2.485114000 \\
\hline $\mathrm{C}$ & -2.657627000 & 3.890052000 & 1.308515000 \\
\hline $\mathrm{C}$ & -1.768018000 & 2.714190000 & 0.883992000 \\
\hline $\mathrm{H}$ & -3.160669000 & 4.314976000 & 0.431081000 \\
\hline $\mathrm{H}$ & -3.424460000 & 3.544024000 & 2.010698000 \\
\hline $\mathrm{H}$ & -2.079790000 & 4.687818000 & 1.790569000 \\
\hline $\mathrm{C}$ & -1.074692000 & 2.121750000 & 2.123190000 \\
\hline $\mathrm{C}$ & -0.706552000 & 3.204659000 & -0.115031000 \\
\hline $\mathrm{H}$ & -0.401641000 & 1.308069000 & 1.828834000 \\
\hline $\mathrm{H}$ & -0.478890000 & 2.871044000 & 2.657970000 \\
\hline $\mathrm{H}$ & -1.827588000 & 1.719218000 & 2.810197000 \\
\hline $\mathrm{H}$ & -0.059265000 & 3.976849000 & 0.318084000 \\
\hline
\end{tabular}




$\begin{array}{lrrr}\mathrm{H} & -0.074474000 & 2.368451000 & -0.434918000 \\ \mathrm{H} & -1.195431000 & 3.623643000 & -1.003084000 \\ \mathrm{O} & -2.582910000 & 1.748330000 & 0.286268000 \\ \mathrm{~K} & -4.106616000 & 0.856220000 & -1.373773000 \\ \mathrm{~N} & 6.513855000 & -0.387807000 & 0.287189000 \\ \mathrm{O} & 7.277684000 & 0.251224000 & -0.424034000 \\ \mathrm{O} & \mathbf{6 . 8 6 7 1 4 9 0 0 0} & -1.062468000 & 1.245132000\end{array}$

A2 ${ }_{\text {tBuOK }}$

$\begin{array}{lrcc} & & \text { Coordinates (Angstroms) } & \\ & \mathrm{X} & \mathrm{Y} & \mathrm{Z} \\ \mathrm{C} & -5.748298000 & -1.067424000 & 0.365098000 \\ \mathrm{C} & -4.614626000 & -1.267387000 & -0.418156000 \\ \mathrm{C} & -3.317397000 & -1.206051000 & 0.139220000 \\ \mathrm{C} & -3.228262000 & -0.915380000 & 1.521808000 \\ \mathrm{C} & -4.365321000 & -0.731327000 & 2.293720000 \\ \mathrm{C} & -5.638729000 & -0.798959000 & 1.725656000 \\ \mathrm{H} & -6.729351000 & -1.136897000 & -0.097737000 \\ \mathrm{H} & -4.720300000 & -1.504705000 & -1.471589000 \\ \mathrm{H} & -2.254744000 & -0.829082000 & 1.995403000 \\ \mathrm{H} & -4.254898000 & -0.522498000 & 3.354442000 \\ \mathrm{H} & -6.524559000 & -0.647776000 & 2.334172000 \\ \mathrm{C} & -2.147199000 & -1.322494000 & -0.709469000 \\ \mathrm{C} & -0.831602000 & -1.298084000 & -0.317789000 \\ \mathrm{H} & -0.658902000 & -1.410801000 & 0.749558000 \\ \mathrm{O} & -2.440978000 & -1.199712000 & -2.086909000 \\ \mathrm{C} & 0.291197000 & -1.108798000 & -1.141679000 \\ \mathrm{H} & 0.137438000 & -0.970416000 & -2.211359000 \\ \mathrm{C} & 1.651506000 & -1.094438000 & -0.700253000 \\ \mathrm{C} & 2.682317000 & -0.733701000 & -1.611152000 \\ \mathrm{C} & 2.059023000 & -1.363150000 & 0.635167000 \\ \mathrm{C} & 3.995719000 & -0.600191000 & -1.219477000 \\ \mathrm{H} & 2.417113000 & -0.538117000 & -2.647188000 \\ \mathrm{C} & 3.371559000 & -1.224477000 & 1.037130000 \\ \mathrm{H} & 1.330090000 & -1.697871000 & 1.366687000 \\ \mathrm{C} & 4.341693000 & -0.831959000 & 0.114659000 \\ \mathrm{H} & 4.766551000 & -0.310713000 & -1.923649000 \\ \mathrm{H} & 3.665824000 & -1.422804000 & 2.060927000 \\ \mathrm{H} & 0.109395000 & 0.930535000 & -0.849251000 \\ \mathrm{H} & -1.878837000 & -1.823617000 & -2.564047000 \\ \mathrm{C} & 0.072332000 & 1.659729000 & 1.621982000 \\ \mathrm{C} & 0.349367000 & 2.479547000 & 0.359058000 \\ \mathrm{H} & -1.005909000 & 1.511689000 & 1.759246000\end{array}$




\begin{tabular}{|c|c|c|c|}
\hline $\mathrm{H}$ & 0.554379000 & 0.679104000 & 1.550745000 \\
\hline $\mathrm{H}$ & 0.467178000 & 2.163376000 & 2.510474000 \\
\hline $\mathrm{C}$ & 1.853300000 & 2.605378000 & 0.114268000 \\
\hline $\mathrm{C}$ & -0.306444000 & 3.854392000 & 0.449823000 \\
\hline $\mathrm{H}$ & 2.041348000 & 3.165020000 & -0.807336000 \\
\hline $\mathrm{H}$ & 2.341118000 & 3.124898000 & 0.945726000 \\
\hline $\mathrm{H}$ & 2.314375000 & 1.616732000 & 0.016580000 \\
\hline $\mathrm{H}$ & 0.122661000 & 4.436418000 & 1.270787000 \\
\hline $\mathrm{H}$ & -0.158370000 & 4.408669000 & -0.482809000 \\
\hline $\mathrm{H}$ & -1.384473000 & 3.759545000 & 0.636133000 \\
\hline $\mathrm{O}$ & -0.265245000 & 1.837562000 & -0.768360000 \\
\hline K & -2.737946000 & 1.372515000 & -1.333353000 \\
\hline $\mathrm{N}$ & 5.710732000 & -0.671597000 & 0.538578000 \\
\hline $\mathrm{O}$ & 6.541231000 & -0.331575000 & -0.300035000 \\
\hline $\mathrm{O}$ & 5.980979000 & -0.877354000 & 1.719062000 \\
\hline
\end{tabular}

TSA2-3 ${ }_{\mathrm{tBuOK}}$

\begin{tabular}{|c|c|c|c|}
\hline \multicolumn{4}{|c|}{ Coordinates (Angstroms) } \\
\hline & $\mathrm{X}$ & Y & $\mathrm{Z}$ \\
\hline $\bar{C}$ & -5.602041000 & -2.088270000 & -0.028953000 \\
\hline $\mathrm{C}$ & -4.447505000 & -1.796213000 & -0.747178000 \\
\hline $\mathrm{C}$ & -3.289688000 & -1.350408000 & -0.092251000 \\
\hline $\mathrm{C}$ & -3.339649000 & -1.174892000 & 1.301036000 \\
\hline $\mathrm{C}$ & -4.492459000 & -1.478435000 & 2.015567000 \\
\hline $\mathrm{C}$ & -5.630250000 & -1.936326000 & 1.354821000 \\
\hline $\mathrm{H}$ & -6.483777000 & -2.443983000 & -0.554125000 \\
\hline $\mathrm{H}$ & -4.426054000 & -1.924340000 & -1.824147000 \\
\hline $\mathrm{H}$ & -2.470081000 & -0.787686000 & 1.825571000 \\
\hline $\mathrm{H}$ & -4.505639000 & -1.342339000 & 3.093082000 \\
\hline $\mathrm{H}$ & -6.532622000 & -2.166400000 & 1.912897000 \\
\hline $\mathrm{C}$ & -2.081395000 & -1.008903000 & -0.860828000 \\
\hline $\mathrm{C}$ & -0.825985000 & -0.926593000 & -0.383171000 \\
\hline $\mathrm{H}$ & -0.685079000 & -1.264073000 & 0.639058000 \\
\hline $\mathrm{O}$ & -2.389803000 & -0.609258000 & -2.166718000 \\
\hline $\mathrm{C}$ & 0.304799000 & -0.278114000 & -1.055899000 \\
\hline $\mathrm{H}$ & 0.209047000 & -0.222266000 & -2.147336000 \\
\hline $\mathrm{C}$ & 1.691721000 & -0.615265000 & -0.683118000 \\
\hline $\mathrm{C}$ & 2.738476000 & -0.206515000 & -1.533504000 \\
\hline $\mathrm{C}$ & 2.051894000 & -1.245486000 & 0.521371000 \\
\hline $\mathrm{C}$ & 4.066845000 & -0.405765000 & -1.208490000 \\
\hline $\mathrm{H}$ & 2.491576000 & 0.291655000 & -2.467279000 \\
\hline $\mathrm{C}$ & 3.378961000 & -1.451902000 & 0.864323000 \\
\hline $\mathrm{H}$ & 1.287252000 & -1.591128000 & 1.209138000 \\
\hline
\end{tabular}




$\begin{array}{lrcc}\mathrm{C} & 4.378215000 & -1.030688000 & -0.003847000 \\ \mathrm{H} & 4.864301000 & -0.086957000 & -1.868443000 \\ \mathrm{H} & 3.649560000 & -1.939992000 & 1.792690000 \\ \mathrm{H} & 0.085043000 & 0.971521000 & -0.728435000 \\ \mathrm{H} & -1.610057000 & -0.767860000 & -2.715434000 \\ \mathrm{C} & 0.188354000 & 1.834881000 & 1.861971000 \\ \mathrm{C} & 0.237050000 & 2.770640000 & 0.637482000 \\ \mathrm{H} & -0.854639000 & 1.578285000 & 2.094617000 \\ \mathrm{H} & 0.731117000 & 0.905966000 & 1.655226000 \\ \mathrm{H} & 0.634196000 & 2.293754000 & 2.752503000 \\ \mathrm{C} & 1.703000000 & 3.075087000 & 0.286910000 \\ \mathrm{C} & -0.484698000 & 4.083825000 & 0.983635000 \\ \mathrm{H} & 1.744124000 & 3.728723000 & -0.591295000 \\ \mathrm{H} & 2.230735000 & 3.565792000 & 1.113957000 \\ \mathrm{H} & 2.234553000 & 2.148115000 & 0.045013000 \\ \mathrm{H} & -0.012918000 & 4.608904000 & 1.822818000 \\ \mathrm{H} & -0.484137000 & 4.750841000 & 0.113859000 \\ \mathrm{H} & -1.529083000 & 3.882766000 & 1.265377000 \\ \mathrm{O} & -0.412213000 & 2.184092000 & -0.449118000 \\ \mathrm{~K} & -2.790204000 & 1.827983000 & -0.902526000 \\ \mathrm{~N} & 5.773215000 & -1.250350000 & 0.349196000 \\ \mathrm{O} & 6.630697000 & -0.875896000 & -0.440769000 \\ \mathrm{O} & 6.016328000 & -1.797997000 & 1.417235000 \\ ----------1\end{array}$

\begin{tabular}{|c|c|c|c|}
\hline \multicolumn{4}{|c|}{$\mathrm{A} 3_{\mathrm{tBuOK}}$} \\
\hline & $\mathrm{X}$ & Y & Z \\
\hline$\overline{\mathrm{C}}$ & -5.012083000 & -1.794559000 & -1.017910000 \\
\hline $\mathrm{C}$ & -3.965740000 & -2.116346000 & -0.158233000 \\
\hline $\mathrm{C}$ & -2.924227000 & -1.209397000 & 0.075192000 \\
\hline $\mathrm{C}$ & -2.965754000 & 0.046811000 & -0.553923000 \\
\hline $\mathrm{C}$ & -4.016543000 & 0.358359000 & -1.409796000 \\
\hline $\mathrm{C}$ & -5.037929000 & -0.558381000 & -1.653689000 \\
\hline $\mathrm{H}$ & -5.812805000 & -2.510296000 & -1.179051000 \\
\hline $\mathrm{H}$ & -3.998940000 & -3.068288000 & 0.367179000 \\
\hline $\mathrm{H}$ & -2.181062000 & 0.795982000 & -0.371245000 \\
\hline $\mathrm{H}$ & -4.033386000 & 1.334491000 & -1.886774000 \\
\hline $\mathrm{H}$ & -5.852846000 & -0.305565000 & -2.325404000 \\
\hline $\mathrm{C}$ & -1.794942000 & -1.581456000 & 0.968365000 \\
\hline $\mathrm{C}$ & -1.014184000 & -0.697595000 & 1.602866000 \\
\hline $\mathrm{H}$ & -1.151288000 & 0.363890000 & 1.410833000 \\
\hline $\mathrm{O}$ & -1.567134000 & -2.926138000 & 1.150707000 \\
\hline $\mathrm{C}$ & 0.174737000 & -1.082543000 & 2.448382000 \\
\hline
\end{tabular}




$\begin{array}{lrcc}\mathrm{H} & 0.067471000 & -2.097934000 & 2.838594000 \\ \mathrm{C} & 1.420520000 & -0.978609000 & 1.597126000 \\ \mathrm{C} & 1.881973000 & -2.091826000 & 0.881300000 \\ \mathrm{C} & 2.055095000 & 0.255654000 & 1.412139000 \\ \mathrm{C} & 2.947876000 & -1.982784000 & -0.004057000 \\ \mathrm{H} & 1.386276000 & -3.048901000 & 1.015047000 \\ \mathrm{C} & 3.126477000 & 0.383408000 & 0.536115000 \\ \mathrm{H} & 1.677768000 & 1.133899000 & 1.923919000 \\ \mathrm{C} & 3.553196000 & -0.739542000 & -0.165501000 \\ \mathrm{H} & 3.317746000 & -2.836571000 & -0.559058000 \\ \mathrm{H} & 3.613705000 & 1.338137000 & 0.378742000 \\ \mathrm{C} & -0.957116000 & 3.176433000 & 1.802298000 \\ \mathrm{C} & -0.554529000 & 3.136304000 & 0.310473000 \\ \mathrm{H} & -1.919308000 & 2.667495000 & 1.939296000 \\ \mathrm{H} & -0.207481000 & 2.645224000 & 2.403946000 \\ \mathrm{H} & -1.049690000 & 4.196828000 & 2.196938000 \\ \mathrm{C} & 0.792452000 & 3.874405000 & 0.145932000 \\ \mathrm{C} & -1.631937000 & 3.886648000 & -0.503287000 \\ \mathrm{H} & 1.089538000 & 3.866006000 & -0.910392000 \\ \mathrm{H} & 0.758508000 & 4.917713000 & 0.486446000 \\ \mathrm{H} & 1.571084000 & 3.353122000 & 0.718406000 \\ \mathrm{H} & -1.744159000 & 4.937705000 & -0.205711000 \\ \mathrm{H} & -1.373341000 & 3.854813000 & -1.569041000 \\ \mathrm{H} & -2.601749000 & 3.389307000 & -0.375194000 \\ \mathrm{O} & -0.443020000 & 1.839852000 & -0.121398000 \\ \mathrm{H} & 0.252206000 & -0.399460000 & 3.300540000 \\ \mathrm{~K} & 0.303421000 & -0.047474000 & -1.293510000 \\ \mathrm{H} & -2.031349000 & -3.416039000 & 0.462102000 \\ \mathrm{~N} & 4.664494000 & -0.609996000 & -1.115572000 \\ \mathrm{O} & 4.970632000 & -1.598967000 & -1.765788000 \\ \mathrm{O} & 5.207346000 & 0.479067000 & -1.208169000 \\ ----- & \end{array}$

\begin{tabular}{llll} 
& & \\
& & & \\
& Coordinates $($ Angstroms) & Z \\
\hline C & -2.002519000 & 4.037928000 & -0.611003000 \\
$\mathrm{C}$ & -1.320321000 & 2.911891000 & -0.158428000 \\
$\mathrm{C}$ & -1.983648000 & 1.688396000 & -0.040518000 \\
$\mathrm{C}$ & -3.336036000 & 1.595333000 & -0.384801000 \\
$\mathrm{C}$ & -4.012660000 & 2.723832000 & -0.838084000 \\
$\mathrm{C}$ & -3.350049000 & 3.944381000 & -0.950221000 \\
$\mathrm{H}$ & -1.481269000 & 4.985914000 & -0.706864000 \\
$\mathrm{H}$ & -0.262952000 & 2.981021000 & 0.090323000
\end{tabular}




\begin{tabular}{lrcc}
$\mathrm{H}$ & -3.824140000 & 0.621224000 & -0.298143000 \\
$\mathrm{H}$ & -5.060666000 & 2.647188000 & -1.113630000 \\
$\mathrm{H}$ & -3.880119000 & 4.821617000 & -1.309781000 \\
$\mathrm{C}$ & -1.289074000 & 0.465533000 & 0.524033000 \\
$\mathrm{C}$ & 0.140295000 & 0.331463000 & 0.094554000 \\
$\mathrm{H}$ & 0.283763000 & 0.398329000 & -0.982521000 \\
$\mathrm{O}$ & -1.420015000 & 0.459637000 & 1.969491000 \\
$\mathrm{C}$ & 1.175510000 & 0.136413000 & 0.915065000 \\
$\mathrm{H}$ & 0.983456000 & 0.091753000 & 1.985363000 \\
$\mathrm{C}$ & 2.581919000 & -0.023883000 & 0.512852000 \\
$\mathrm{C}$ & 3.582613000 & 0.098326000 & 1.486893000 \\
$\mathrm{C}$ & 2.968705000 & -0.304821000 & -0.806493000 \\
$\mathrm{C}$ & 4.925268000 & -0.033680000 & 1.165157000 \\
$\mathrm{H}$ & 3.302363000 & 0.303727000 & 2.516161000 \\
$\mathrm{C}$ & 4.304853000 & -0.438627000 & -1.146500000 \\
$\mathrm{H}$ & 2.216676000 & -0.439650000 & -1.576732000 \\
$\mathrm{C}$ & 5.267566000 & -0.297936000 & -0.153763000 \\
$\mathrm{H}$ & 5.701446000 & 0.062818000 & 1.914028000 \\
$\mathrm{H}$ & 4.610454000 & -0.657406000 & -2.162146000 \\
$\mathrm{H}$ & -1.865286000 & -0.422093000 & 0.209296000 \\
$\mathrm{H}$ & -1.153241000 & 1.334272000 & 2.282504000 \\
$\mathrm{C}$ & -5.289696000 & -3.029144000 & -0.380916000 \\
$\mathrm{C}$ & -3.886460000 & -2.413233000 & -0.544591000 \\
$\mathrm{H}$ & -5.380515000 & -3.483509000 & 0.613941000 \\
$\mathrm{H}$ & -6.047201000 & -2.241078000 & -0.463676000 \\
$\mathrm{H}$ & -5.506122000 & -3.800061000 & -1.131876000 \\
$\mathrm{C}$ & -3.770203000 & -1.810802000 & -1.960822000 \\
$\mathrm{C}$ & -2.829397000 & -3.526306000 & -0.393091000 \\
$\mathrm{H}$ & -2.783830000 & -1.347654000 & -2.089520000 \\
$\mathrm{H}$ & -3.903161000 & -2.561237000 & -2.750533000 \\
$\mathrm{H}$ & -4.527319000 & -1.029501000 & -2.098021000 \\
$\mathrm{H}$ & -2.942444000 & -4.327492000 & -1.135023000 \\
$\mathrm{H}$ & -1.825226000 & -3.097213000 & -0.499380000 \\
$\mathrm{H}$ & -2.903662000 & -3.972123000 & 0.607164000 \\
$\mathrm{H}$ & -3.679561000 & -1.435276000 & 0.401583000 \\
$\mathrm{H}$ & -3.334299000 & -0.757370000 & 2.274994000 \\
$\mathrm{H}$ & 7.582062000 & -0.442446000 & -0.507549000 \\
$\mathrm{H}$ & --954075000 & -0.316756000 & 0.385330000 \\
$\mathrm{H}$ & & & -1.675025000 \\
\hline
\end{tabular}

TSA1-2 ${ }_{\text {tBuONa }}$

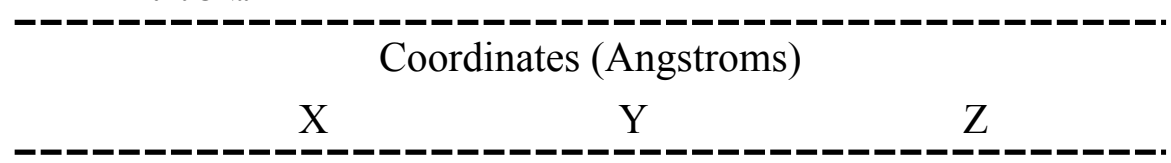




\begin{tabular}{|c|c|c|c|}
\hline $\mathrm{C}$ & -4.480345000 & -2.968206000 & -0.506941000 \\
\hline $\mathrm{C}$ & -3.510120000 & -2.081374000 & -0.979014000 \\
\hline $\mathrm{C}$ & -2.601682000 & -1.465821000 & -0.105003000 \\
\hline $\mathrm{C}$ & -2.712622000 & -1.762059000 & 1.264529000 \\
\hline $\mathrm{C}$ & -3.668359000 & -2.651957000 & 1.729447000 \\
\hline $\mathrm{C}$ & -4.564727000 & -3.259999000 & 0.847360000 \\
\hline $\mathrm{H}$ & -5.160996000 & -3.440540000 & -1.210432000 \\
\hline $\mathrm{H}$ & -3.433196000 & -1.896037000 & -2.048204000 \\
\hline $\mathrm{H}$ & -2.055190000 & -1.261951000 & 1.970908000 \\
\hline $\mathrm{H}$ & -3.730783000 & -2.861484000 & 2.793499000 \\
\hline $\mathrm{H}$ & -5.316578000 & -3.950222000 & 1.216800000 \\
\hline $\mathrm{C}$ & -1.639084000 & -0.423408000 & -0.569245000 \\
\hline $\mathrm{C}$ & -0.238184000 & -0.575540000 & -0.209141000 \\
\hline $\mathrm{H}$ & -0.063834000 & -1.075203000 & 0.742212000 \\
\hline $\mathrm{O}$ & -1.840579000 & -0.072182000 & -1.950963000 \\
\hline $\mathrm{C}$ & 0.808600000 & -0.111481000 & -0.923549000 \\
\hline $\mathrm{H}$ & 0.605243000 & 0.398429000 & -1.863131000 \\
\hline $\mathrm{C}$ & 2.219881000 & -0.208522000 & -0.557094000 \\
\hline $\mathrm{C}$ & 3.184548000 & 0.299462000 & -1.445303000 \\
\hline $\mathrm{C}$ & 2.677749000 & -0.785480000 & 0.642217000 \\
\hline $\mathrm{C}$ & 4.539149000 & 0.236148000 & -1.165342000 \\
\hline $\mathrm{H}$ & 2.856848000 & 0.754446000 & -2.375853000 \\
\hline $\mathrm{C}$ & 4.027289000 & -0.857115000 & 0.937785000 \\
\hline $\mathrm{H}$ & 1.968920000 & -1.183585000 & 1.360775000 \\
\hline $\mathrm{C}$ & 4.948027000 & -0.345908000 & 0.028252000 \\
\hline $\mathrm{H}$ & 5.279289000 & 0.626711000 & -1.852881000 \\
\hline $\mathrm{H}$ & 4.380070000 & -1.301302000 & 1.860415000 \\
\hline $\mathrm{H}$ & -2.118202000 & 0.708368000 & 0.026441000 \\
\hline $\mathrm{H}$ & -1.400116000 & -0.729376000 & -2.510450000 \\
\hline $\mathrm{C}$ & -3.057958000 & 3.943554000 & 1.040618000 \\
\hline $\mathrm{C}$ & -2.088506000 & 2.811954000 & 0.685912000 \\
\hline $\mathrm{H}$ & -3.668459000 & 4.205632000 & 0.167165000 \\
\hline $\mathrm{H}$ & -3.730401000 & 3.623485000 & 1.843486000 \\
\hline $\mathrm{H}$ & -2.527096000 & 4.845172000 & 1.368043000 \\
\hline $\mathrm{C}$ & -1.239283000 & 2.445261000 & 1.913022000 \\
\hline $\mathrm{C}$ & -1.169317000 & 3.248936000 & -0.465199000 \\
\hline $\mathrm{H}$ & -0.524313000 & 1.655535000 & 1.654387000 \\
\hline $\mathrm{H}$ & -0.672099000 & 3.305671000 & 2.286548000 \\
\hline $\mathrm{H}$ & -1.887440000 & 2.077740000 & 2.715596000 \\
\hline $\mathrm{H}$ & -0.561585000 & 4.120499000 & -0.194642000 \\
\hline $\mathrm{H}$ & -0.493416000 & 2.431654000 & -0.740828000 \\
\hline $\mathrm{H}$ & -1.768224000 & 3.512379000 & -1.347035000 \\
\hline $\mathrm{O}$ & -2.848954000 & 1.696383000 & 0.297398000 \\
\hline $\mathrm{Na}$ & -3.870433000 & 0.882196000 & -1.385170000 \\
\hline
\end{tabular}




$\begin{array}{llrr}\mathrm{N} & 6.372382000 & -0.422214000 & 0.334912000 \\ \mathrm{O} & 7.158664000 & 0.040199000 & -0.480831000 \\ \mathrm{O} & \mathbf{6 . 7 0 3 2 8 4 0 0 0} & -0.945071000 & 1.390623000\end{array}$

\begin{tabular}{|c|c|c|c|}
\hline \multicolumn{4}{|c|}{ A2 tBuONa $_{-}$} \\
\hline & \multicolumn{3}{|c|}{ Coordinates (Angstroms) } \\
\hline & $\mathrm{X}$ & $\mathrm{Y}$ & Z \\
\hline$\overline{\mathrm{C}}$ & -5.767207000 & -0.668317000 & 0.256645000 \\
\hline $\mathrm{C}$ & -4.646096000 & -0.956443000 & -0.520319000 \\
\hline $\mathrm{C}$ & -3.371037000 & -1.129923000 & 0.066244000 \\
\hline $\mathrm{C}$ & -3.291163000 & -0.996152000 & 1.473731000 \\
\hline $\mathrm{C}$ & -4.414993000 & -0.720805000 & 2.234188000 \\
\hline $\mathrm{C}$ & -5.665833000 & -0.544998000 & 1.636543000 \\
\hline $\mathrm{H}$ & -6.731934000 & -0.553256000 & -0.230390000 \\
\hline $\mathrm{H}$ & -4.755465000 & -1.093804000 & -1.591145000 \\
\hline $\mathrm{H}$ & -2.335933000 & -1.112213000 & 1.976067000 \\
\hline $\mathrm{H}$ & -4.313026000 & -0.632774000 & 3.312315000 \\
\hline $\mathrm{H}$ & -6.540206000 & -0.323290000 & 2.239799000 \\
\hline $\mathrm{C}$ & -2.200250000 & -1.318164000 & -0.769494000 \\
\hline $\mathrm{C}$ & -0.884918000 & -1.329684000 & -0.369579000 \\
\hline $\mathrm{H}$ & -0.725843000 & -1.461328000 & 0.697082000 \\
\hline $\mathrm{O}$ & -2.474043000 & -1.099838000 & -2.146213000 \\
\hline $\mathrm{C}$ & 0.248798000 & -1.128136000 & -1.170928000 \\
\hline $\mathrm{H}$ & 0.123422000 & -1.015046000 & -2.247334000 \\
\hline $\mathrm{C}$ & 1.599325000 & -1.066979000 & -0.693290000 \\
\hline $\mathrm{C}$ & 2.641343000 & -0.699709000 & -1.586143000 \\
\hline $\mathrm{C}$ & 1.976415000 & -1.288180000 & 0.657930000 \\
\hline $\mathrm{C}$ & 3.941820000 & -0.525594000 & -1.165325000 \\
\hline $\mathrm{H}$ & 2.398008000 & -0.533481000 & -2.632556000 \\
\hline $\mathrm{C}$ & 3.276030000 & -1.112958000 & 1.088703000 \\
\hline $\mathrm{H}$ & 1.236098000 & -1.614562000 & 1.381773000 \\
\hline $\mathrm{C}$ & 4.259035000 & -0.722425000 & 0.180318000 \\
\hline $\mathrm{H}$ & 4.722721000 & -0.233031000 & -1.856952000 \\
\hline $\mathrm{H}$ & 3.548224000 & -1.279577000 & 2.124100000 \\
\hline $\mathrm{H}$ & -0.064734000 & 0.885994000 & -1.023601000 \\
\hline $\mathrm{H}$ & -1.906198000 & -1.684806000 & -2.662133000 \\
\hline $\mathrm{C}$ & -0.304911000 & 1.676394000 & 1.410966000 \\
\hline $\mathrm{C}$ & -0.022513000 & 2.489630000 & 0.145891000 \\
\hline $\mathrm{H}$ & -1.370818000 & 1.426643000 & 1.487417000 \\
\hline $\mathrm{H}$ & 0.271959000 & 0.745619000 & 1.403611000 \\
\hline $\mathrm{H}$ & -0.020537000 & 2.240548000 & 2.305241000 \\
\hline $\mathrm{C}$ & 1.477044000 & 2.731663000 & -0.020880000 \\
\hline $\mathrm{C}$ & -0.799832000 & 3.801364000 & 0.153886000 \\
\hline
\end{tabular}




$\begin{array}{lrrr}\mathrm{H} & 1.671990000 & 3.290268000 & -0.941387000 \\ \mathrm{H} & 1.875128000 & 3.300393000 & 0.825685000 \\ \mathrm{H} & 2.016677000 & 1.779582000 & -0.073080000 \\ \mathrm{H} & -0.467605000 & 4.444090000 & 0.974468000 \\ \mathrm{H} & -0.651931000 & 4.338029000 & -0.788666000 \\ \mathrm{H} & -1.874122000 & 3.618226000 & 0.290673000 \\ \mathrm{O} & -0.507130000 & 1.768584000 & -1.003653000 \\ \mathrm{Na} & -2.584214000 & 1.084340000 & -1.392116000 \\ \mathrm{~N} & 5.616012000 & -0.524296000 & 0.633713000 \\ \mathrm{O} & 5.860911000 & -0.701067000 & 1.823630000 \\ \mathrm{O} & \mathbf{6 . 4 5 8 0 1 8 0 0 0} & -0.184353000 & -0.192292000\end{array}$

TSA2-3

\begin{tabular}{|c|c|c|c|}
\hline & $\mathrm{Co}$ & inates (Angstrom & \\
\hline & $X$ & $\mathrm{Y}$ & Z \\
\hline$\overline{\mathrm{C}}$ & 5.859627000 & -0.809576000 & -0.379323000 \\
\hline $\mathrm{C}$ & 4.725200000 & -0.235811000 & 0.191769000 \\
\hline $\mathrm{C}$ & 3.560753000 & -0.994744000 & 0.403562000 \\
\hline $\mathrm{C}$ & 3.592162000 & -2.357939000 & 0.063626000 \\
\hline $\mathrm{C}$ & 4.723805000 & -2.922016000 & -0.505984000 \\
\hline $\mathrm{C}$ & 5.862971000 & -2.151268000 & -0.741308000 \\
\hline $\mathrm{H}$ & 6.749384000 & -0.203688000 & -0.525223000 \\
\hline $\mathrm{H}$ & 4.750829000 & 0.799462000 & 0.519818000 \\
\hline $\mathrm{H}$ & 2.731043000 & -2.985597000 & 0.269501000 \\
\hline $\mathrm{H}$ & 4.723210000 & -3.978939000 & -0.755837000 \\
\hline $\mathrm{H}$ & 6.747907000 & -2.600685000 & -1.180538000 \\
\hline $\mathrm{C}$ & 2.339742000 & -0.344828000 & 0.908163000 \\
\hline $\mathrm{C}$ & 1.077114000 & -0.815449000 & 0.781195000 \\
\hline $\mathrm{H}$ & 0.957836000 & -1.775845000 & 0.283133000 \\
\hline $\mathrm{O}$ & 2.588930000 & 0.935023000 & 1.394697000 \\
\hline $\mathrm{C}$ & -0.115304000 & -0.071880000 & 1.185001000 \\
\hline $\mathrm{H}$ & -0.072684000 & 0.308809000 & 2.211418000 \\
\hline $\mathrm{C}$ & -1.434234000 & -0.557346000 & 0.802617000 \\
\hline $\mathrm{C}$ & -2.568572000 & -0.247896000 & 1.585033000 \\
\hline $\mathrm{C}$ & -1.660515000 & -1.246205000 & -0.409411000 \\
\hline $\mathrm{C}$ & -3.846007000 & -0.595120000 & 1.188838000 \\
\hline $\mathrm{H}$ & -2.428468000 & 0.272611000 & 2.528815000 \\
\hline $\mathrm{C}$ & -2.932393000 & -1.608836000 & -0.813877000 \\
\hline $\mathrm{H}$ & -0.821102000 & -1.479689000 & -1.058660000 \\
\hline $\mathrm{C}$ & -4.021932000 & -1.277887000 & -0.012992000 \\
\hline $\mathrm{H}$ & -4.710513000 & -0.353889000 & 1.795536000 \\
\hline $\mathrm{H}$ & -3.099321000 & -2.132524000 & -1.747459000 \\
\hline $\mathrm{H}$ & 0.062119000 & 1.123226000 & 0.413922000 \\
\hline
\end{tabular}




$\begin{array}{lrrr}\mathrm{H} & 1.747786000 & 1.426720000 & 1.424887000 \\ \mathrm{C} & -1.533534000 & 2.508453000 & -1.399062000 \\ \mathrm{C} & -0.508148000 & 3.044057000 & -0.393169000 \\ \mathrm{H} & -1.039997000 & 2.262852000 & -2.347220000 \\ \mathrm{H} & -2.007843000 & 1.598996000 & -1.015547000 \\ \mathrm{H} & -2.317985000 & 3.245946000 & -1.602712000 \\ \mathrm{C} & -1.196431000 & 3.368778000 & 0.938670000 \\ \mathrm{C} & 0.181017000 & 4.292944000 & -0.947181000 \\ \mathrm{H} & -0.455823000 & 3.696303000 & 1.676729000 \\ \mathrm{H} & -1.941547000 & 4.162734000 & 0.818619000 \\ \mathrm{H} & -1.707803000 & 2.482568000 & 1.329358000 \\ \mathrm{H} & -0.536518000 & 5.100400000 & -1.129663000 \\ \mathrm{H} & 0.935362000 & 4.654202000 & -0.238980000 \\ \mathrm{H} & 0.676524000 & 4.064763000 & -1.900401000 \\ \mathrm{O} & 0.494446000 & 2.067970000 & -0.176768000 \\ \mathrm{Na} & 2.321259000 & 1.325357000 & -1.031806000 \\ \mathrm{~N} & -5.359905000 & -1.644975000 & -0.439899000 \\ \mathrm{O} & -6.300092000 & -1.339440000 & 0.284260000 \\ \mathrm{O} & -5.481888000 & -2.240731000 & -1.504357000 \\ - \text { - - - - - - - - - }\end{array}$

\begin{tabular}{|c|c|c|c|}
\hline \multicolumn{4}{|c|}{$\mathrm{A}_{\mathrm{tBuONa}}$} \\
\hline & \multicolumn{3}{|c|}{ Coordinates (Angstroms) } \\
\hline & $X$ & $\mathrm{Y}$ & $\mathrm{Z}$ \\
\hline$\overline{\mathrm{C}}$ & -5.080534000 & -1.506727000 & -1.119079000 \\
\hline $\mathrm{C}$ & -4.053310000 & -1.936726000 & -0.284826000 \\
\hline $\mathrm{C}$ & -2.961025000 & -1.104114000 & -0.008497000 \\
\hline $\mathrm{C}$ & -2.926654000 & 0.183211000 & -0.566791000 \\
\hline $\mathrm{C}$ & -3.958806000 & 0.602559000 & -1.398879000 \\
\hline $\mathrm{C}$ & -5.032665000 & -0.237345000 & -1.685902000 \\
\hline $\mathrm{H}$ & -5.922326000 & -2.163556000 & -1.317003000 \\
\hline $\mathrm{H}$ & -4.137191000 & -2.913347000 & 0.187353000 \\
\hline $\mathrm{H}$ & -2.099428000 & 0.873809000 & -0.350987000 \\
\hline $\mathrm{H}$ & -3.914975000 & 1.599634000 & -1.827847000 \\
\hline $\mathrm{H}$ & -5.831111000 & 0.097914000 & -2.341049000 \\
\hline $\mathrm{C}$ & -1.852075000 & -1.591671000 & 0.853622000 \\
\hline $\mathrm{C}$ & -1.056844000 & -0.800205000 & 1.588422000 \\
\hline $\mathrm{H}$ & -1.185262000 & 0.277031000 & 1.523537000 \\
\hline $\mathrm{O}$ & -1.658067000 & -2.950749000 & 0.895138000 \\
\hline $\mathrm{C}$ & 0.122793000 & -1.305322000 & 2.386504000 \\
\hline $\mathrm{H}$ & -0.026038000 & -2.346769000 & 2.683639000 \\
\hline $\mathrm{C}$ & 1.362881000 & -1.181581000 & 1.527806000 \\
\hline $\mathrm{C}$ & 1.687955000 & -2.202490000 & 0.620729000 \\
\hline $\mathrm{C}$ & 2.109541000 & 0.002062000 & 1.507335000 \\
\hline
\end{tabular}




$\begin{array}{lrcc}\mathrm{C} & 2.720805000 & -2.043738000 & -0.295400000 \\ \mathrm{H} & 1.106092000 & -3.119498000 & 0.627747000 \\ \mathrm{C} & 3.151163000 & 0.176153000 & 0.601905000 \\ \mathrm{H} & 1.841677000 & 0.815857000 & 2.172720000 \\ \mathrm{C} & 3.435891000 & -0.848680000 & -0.292123000 \\ \mathrm{H} & 2.983273000 & -2.823008000 & -1.000873000 \\ \mathrm{H} & 3.719087000 & 1.097864000 & 0.565430000 \\ \mathrm{C} & -0.680113000 & 3.307123000 & 1.747253000 \\ \mathrm{C} & -0.276650000 & 3.131971000 & 0.267608000 \\ \mathrm{H} & -1.699405000 & 2.931138000 & 1.897898000 \\ \mathrm{H} & -0.006944000 & 2.717190000 & 2.383162000 \\ \mathrm{H} & -0.642763000 & 4.351573000 & 2.082569000 \\ \mathrm{C} & 1.155576000 & 3.673060000 & 0.078167000 \\ \mathrm{C} & -1.240840000 & 3.955593000 & -0.610979000 \\ \mathrm{H} & 1.462740000 & 3.539907000 & -0.966646000 \\ \mathrm{H} & 1.252697000 & 4.735914000 & 0.334632000 \\ \mathrm{H} & 1.849479000 & 3.103709000 & 0.710263000 \\ \mathrm{H} & -1.220430000 & 5.028474000 & -0.379452000 \\ \mathrm{H} & -0.977447000 & 3.822961000 & -1.667206000 \\ \mathrm{H} & -2.266478000 & 3.592540000 & -0.469647000 \\ \mathrm{O} & -0.337870000 & 1.805000000 & -0.074861000 \\ \mathrm{H} & 0.236935000 & -0.708053000 & 3.296184000 \\ \mathrm{H} & -2.151273000 & -3.355987000 & 0.171778000 \\ \mathrm{Na} & 0.312225000 & 0.048315000 & -0.820753000 \\ \mathrm{~N} & 4.513789000 & -0.664767000 & -1.272692000 \\ \mathrm{O} & 4.681598000 & -1.550347000 & -2.097908000 \\ \mathrm{O} & 5.168784000 & 0.362073000 & -1.207411000 \\ ------- & & \end{array}$

\begin{tabular}{llcc} 
A1 & & \\
& & \\
& Coordinates $($ Angstroms) & Z \\
\hline $\mathrm{C}$ & -1.952533000 & 4.027591000 & -0.385537000 \\
$\mathrm{C}$ & -1.296253000 & 2.845328000 & -0.053233000 \\
$\mathrm{C}$ & -2.009368000 & 1.647795000 & 0.030295000 \\
$\mathrm{C}$ & -3.385981000 & 1.638929000 & -0.217904000 \\
$\mathrm{C}$ & -4.036463000 & 2.822634000 & -0.549529000 \\
$\mathrm{C}$ & -3.322312000 & 4.016464000 & -0.634173000 \\
$\mathrm{H}$ & -1.393798000 & 4.956196000 & -0.454701000 \\
$\mathrm{H}$ & -0.224300000 & 2.852134000 & 0.131507000 \\
$\mathrm{H}$ & -3.921706000 & 0.691700000 & -0.139301000 \\
$\mathrm{H}$ & -5.104166000 & 2.811843000 & -0.747878000 \\
$\mathrm{H}$ & -3.832620000 & 4.938512000 & -0.896808000 \\
$\mathrm{C}$ & -1.343651000 & 0.357938000 & 0.463037000
\end{tabular}




\begin{tabular}{|c|c|c|c|}
\hline $\mathrm{C}$ & 0.095319000 & 0.243720000 & 0.058242000 \\
\hline $\mathrm{H}$ & 0.265334000 & 0.393648000 & -1.006496000 \\
\hline $\mathrm{O}$ & -1.511418000 & 0.190382000 & 1.898138000 \\
\hline $\mathrm{C}$ & 1.109202000 & -0.018755000 & 0.885296000 \\
\hline $\mathrm{H}$ & 0.895005000 & -0.140635000 & 1.945114000 \\
\hline $\mathrm{C}$ & 2.523351000 & -0.152072000 & 0.498061000 \\
\hline $\mathrm{C}$ & 3.510242000 & -0.046404000 & 1.487475000 \\
\hline $\mathrm{C}$ & 2.926788000 & -0.389958000 & -0.824289000 \\
\hline $\mathrm{C}$ & 4.858119000 & -0.149622000 & 1.176567000 \\
\hline $\mathrm{H}$ & 3.215726000 & 0.124992000 & 2.518907000 \\
\hline $\mathrm{C}$ & 4.268462000 & -0.495106000 & -1.153216000 \\
\hline $\mathrm{H}$ & 2.183595000 & -0.515055000 & -1.604817000 \\
\hline $\mathrm{C}$ & 5.217666000 & -0.369450000 & -0.145854000 \\
\hline $\mathrm{H}$ & 5.624714000 & -0.064449000 & 1.936489000 \\
\hline $\mathrm{H}$ & 4.589148000 & -0.681161000 & -2.170734000 \\
\hline $\mathrm{H}$ & -1.918460000 & -0.481029000 & 0.046721000 \\
\hline $\mathrm{H}$ & -1.334938000 & 1.045782000 & 2.311876000 \\
\hline $\mathrm{C}$ & -5.720307000 & -2.938944000 & 0.268155000 \\
\hline $\mathrm{C}$ & -4.401994000 & -2.347491000 & -0.261955000 \\
\hline $\mathrm{H}$ & -5.542721000 & -3.436153000 & 1.229402000 \\
\hline $\mathrm{H}$ & -6.447069000 & -2.134867000 & 0.430820000 \\
\hline $\mathrm{H}$ & -6.158807000 & -3.669725000 & -0.423232000 \\
\hline $\mathrm{C}$ & -4.666648000 & -1.664097000 & -1.617587000 \\
\hline $\mathrm{C}$ & -3.382154000 & -3.483598000 & -0.466159000 \\
\hline $\mathrm{H}$ & -3.740698000 & -1.214835000 & -1.997273000 \\
\hline $\mathrm{H}$ & -5.042852000 & -2.366619000 & -2.371441000 \\
\hline $\mathrm{H}$ & -5.405880000 & -0.863306000 & -1.495561000 \\
\hline $\mathrm{H}$ & -3.733612000 & -4.243102000 & -1.175851000 \\
\hline $\mathrm{H}$ & -2.438226000 & -3.070934000 & -0.843255000 \\
\hline $\mathrm{H}$ & -3.179317000 & -3.974621000 & 0.493287000 \\
\hline $\mathrm{O}$ & -3.900240000 & -1.428792000 & 0.635190000 \\
\hline $\mathrm{N}$ & 6.638492000 & -0.482572000 & -0.489077000 \\
\hline $\mathrm{O}$ & 7.451962000 & -0.369638000 & 0.415491000 \\
\hline $\mathrm{O}$ & 6.924758000 & -0.681620000 & -1.659929000 \\
\hline $\mathrm{Li}$ & -3.164403000 & -0.844132000 & 2.016501000 \\
\hline
\end{tabular}

TSA1-2 ${ }_{\text {tBuOLi }}$

\begin{tabular}{|c|c|c|c|}
\hline \multicolumn{4}{|c|}{ Coordinates (Angstroms) } \\
\hline & $X$ & $\mathrm{Y}$ & Z \\
\hline $\mathrm{C}$ & 4.136458000 & -2.480759000 & -1.695995000 \\
\hline $\mathrm{C}$ & 3.023419000 & -1.893698000 & -1.103701000 \\
\hline $\mathrm{C}$ & 3.138927000 & -1.182890000 & 0.098608000 \\
\hline $\mathrm{C}$ & 4.406457000 & -1.094363000 & 0.691407000 \\
\hline
\end{tabular}




\begin{tabular}{|c|c|c|c|}
\hline $\mathrm{C}$ & 5.513939000 & -1.694460000 & 0.104497000 \\
\hline $\mathrm{C}$ & 5.388505000 & -2.387772000 & -1.096194000 \\
\hline $\mathrm{H}$ & 4.018407000 & -3.024243000 & -2.629206000 \\
\hline $\mathrm{H}$ & 2.056652000 & -2.005994000 & -1.585090000 \\
\hline $\mathrm{H}$ & 4.516146000 & -0.542091000 & 1.619039000 \\
\hline $\mathrm{H}$ & 6.484190000 & -1.610977000 & 0.586107000 \\
\hline $\mathrm{H}$ & 6.254933000 & -2.852203000 & -1.556969000 \\
\hline $\mathrm{C}$ & 1.994504000 & -0.468610000 & 0.717979000 \\
\hline $\mathrm{C}$ & 0.625598000 & -0.697126000 & 0.321208000 \\
\hline $\mathrm{H}$ & 0.468748000 & -0.809855000 & -0.749813000 \\
\hline $\mathrm{O}$ & 2.115981000 & -0.311249000 & 2.153790000 \\
\hline $\mathrm{C}$ & -0.467794000 & -0.605274000 & 1.122781000 \\
\hline $\mathrm{H}$ & -0.332682000 & -0.615218000 & 2.206168000 \\
\hline $\mathrm{C}$ & -1.856122000 & -0.589437000 & 0.665686000 \\
\hline $\mathrm{C}$ & -2.889557000 & -0.859493000 & 1.580084000 \\
\hline $\mathrm{C}$ & -2.216579000 & -0.287948000 & -0.660770000 \\
\hline $\mathrm{C}$ & -4.219842000 & -0.852517000 & 1.195450000 \\
\hline $\mathrm{H}$ & -2.638957000 & -1.091029000 & 2.611981000 \\
\hline $\mathrm{C}$ & -3.541178000 & -0.286121000 & -1.063278000 \\
\hline $\mathrm{H}$ & -1.451303000 & -0.029429000 & -1.385864000 \\
\hline $\mathrm{C}$ & -4.531304000 & -0.570055000 & -0.129252000 \\
\hline $\mathrm{H}$ & -5.014390000 & -1.065651000 & 1.899848000 \\
\hline $\mathrm{H}$ & -3.819720000 & -0.055621000 & -2.084227000 \\
\hline $\mathrm{H}$ & 2.089332000 & 0.893082000 & 0.452260000 \\
\hline $\mathrm{H}$ & 1.992500000 & -1.170935000 & 2.581059000 \\
\hline $\mathrm{C}$ & 2.205237000 & 4.241108000 & -0.273072000 \\
\hline $\mathrm{C}$ & 1.471241000 & 2.898128000 & -0.308530000 \\
\hline $\mathrm{H}$ & 2.065168000 & 4.721165000 & 0.701838000 \\
\hline $\mathrm{H}$ & 3.277718000 & 4.083313000 & -0.422715000 \\
\hline $\mathrm{H}$ & 1.838447000 & 4.922060000 & -1.050021000 \\
\hline $\mathrm{C}$ & 1.708995000 & 2.209125000 & -1.659015000 \\
\hline $\mathrm{C}$ & -0.033286000 & 3.111042000 & -0.086826000 \\
\hline $\mathrm{H}$ & 1.156676000 & 1.264451000 & -1.719976000 \\
\hline $\mathrm{H}$ & 1.381882000 & 2.844799000 & -2.488851000 \\
\hline $\mathrm{H}$ & 2.773624000 & 1.988216000 & -1.787045000 \\
\hline $\mathrm{H}$ & -0.482543000 & 3.737538000 & -0.865459000 \\
\hline $\mathrm{H}$ & -0.559705000 & 2.148176000 & -0.080976000 \\
\hline $\mathrm{H}$ & -0.204310000 & 3.609612000 & 0.877629000 \\
\hline $\mathrm{O}$ & 1.985360000 & 2.096993000 & 0.736763000 \\
\hline $\mathrm{N}$ & -5.930724000 & -0.559426000 & -0.547123000 \\
\hline $\mathrm{O}$ & -6.780758000 & -0.797711000 & 0.299490000 \\
\hline $\mathrm{O}$ & -6.173631000 & -0.312960000 & -1.720184000 \\
\hline $\mathrm{Li}$ & 1.107648000 & 1.374323000 & 2.138287000 \\
\hline
\end{tabular}




\begin{tabular}{|c|c|c|c|}
\hline \multicolumn{4}{|c|}{ Coordinates (Angstroms) } \\
\hline & $\mathrm{X}$ & Y & Z \\
\hline$\overline{\mathrm{C}}$ & -5.699089000 & -0.444941000 & 0.152229000 \\
\hline $\mathrm{C}$ & -4.592197000 & -0.695976000 & -0.659982000 \\
\hline $\mathrm{C}$ & -3.367159000 & -1.139942000 & -0.109886000 \\
\hline $\mathrm{C}$ & -3.325226000 & -1.338061000 & 1.291085000 \\
\hline $\mathrm{C}$ & -4.433406000 & -1.091550000 & 2.081471000 \\
\hline $\mathrm{C}$ & -5.631195000 & -0.631593000 & 1.525253000 \\
\hline $\mathrm{H}$ & -6.625398000 & -0.111858000 & -0.307795000 \\
\hline $\mathrm{H}$ & -4.687244000 & -0.598924000 & -1.737074000 \\
\hline $\mathrm{H}$ & -2.416548000 & -1.702124000 & 1.760043000 \\
\hline $\mathrm{H}$ & -4.364569000 & -1.260832000 & 3.152391000 \\
\hline $\mathrm{H}$ & -6.493307000 & -0.438049000 & 2.155010000 \\
\hline $\mathrm{C}$ & -2.192073000 & -1.261852000 & -0.951966000 \\
\hline $\mathrm{C}$ & -0.876122000 & -1.299027000 & -0.542808000 \\
\hline $\mathrm{H}$ & -0.733115000 & -1.517966000 & 0.511540000 \\
\hline $\mathrm{O}$ & -2.458699000 & -0.840244000 & -2.289386000 \\
\hline $\mathrm{C}$ & 0.263898000 & -0.996556000 & -1.295115000 \\
\hline $\mathrm{H}$ & 0.174015000 & -0.836505000 & -2.369602000 \\
\hline $\mathrm{C}$ & 1.603195000 & -0.925084000 & -0.773665000 \\
\hline $\mathrm{C}$ & 2.659841000 & -0.504837000 & -1.621131000 \\
\hline $\mathrm{C}$ & 1.944903000 & -1.196784000 & 0.575416000 \\
\hline $\mathrm{C}$ & 3.949859000 & -0.343758000 & -1.160691000 \\
\hline $\mathrm{H}$ & 2.441978000 & -0.296466000 & -2.665546000 \\
\hline $\mathrm{C}$ & 3.233247000 & -1.036786000 & 1.046454000 \\
\hline $\mathrm{H}$ & 1.188271000 & -1.550790000 & 1.268721000 \\
\hline $\mathrm{C}$ & 4.234697000 & -0.604234000 & 0.179821000 \\
\hline $\mathrm{H}$ & 4.745518000 & -0.017089000 & -1.819326000 \\
\hline $\mathrm{H}$ & 3.479446000 & -1.244987000 & 2.080705000 \\
\hline $\mathrm{H}$ & -0.259446000 & 0.970321000 & -1.116779000 \\
\hline $\mathrm{H}$ & -1.816206000 & -1.253823000 & -2.877546000 \\
\hline $\mathrm{C}$ & -0.584989000 & 1.568215000 & 1.349756000 \\
\hline $\mathrm{C}$ & -0.519108000 & 2.497526000 & 0.136657000 \\
\hline $\mathrm{H}$ & -1.567413000 & 1.085321000 & 1.424454000 \\
\hline $\mathrm{H}$ & 0.183121000 & 0.790688000 & 1.281902000 \\
\hline $\mathrm{H}$ & -0.410994000 & 2.132395000 & 2.271556000 \\
\hline $\mathrm{C}$ & 0.887635000 & 3.063210000 & -0.041993000 \\
\hline $\mathrm{C}$ & -1.564339000 & 3.602158000 & 0.233985000 \\
\hline $\mathrm{H}$ & 0.929058000 & 3.714567000 & -0.920186000 \\
\hline $\mathrm{H}$ & 1.186566000 & 3.641512000 & 0.838153000 \\
\hline $\mathrm{H}$ & 1.612033000 & 2.251257000 & -0.175672000 \\
\hline $\mathrm{H}$ & -1.361746000 & 4.246889000 & 1.094138000 \\
\hline
\end{tabular}




$\begin{array}{lccc}\mathrm{H} & -1.558696000 & 4.218488000 & -0.670691000 \\ \mathrm{H} & -2.568597000 & 3.178086000 & 0.366449000 \\ \mathrm{O} & -0.860593000 & 1.754158000 & -1.057061000 \\ \mathrm{~N} & 5.582983000 & -0.425209000 & 0.674913000 \\ \mathrm{O} & 5.799617000 & -0.659993000 & 1.859316000 \\ \mathrm{O} & 6.441194000 & -0.044181000 & -0.114283000 \\ \mathrm{Li} & -2.494668000 & 0.859221000 & -1.315756000\end{array}$

TSA2-3 tBuOLi $_{\text {in }}$

\begin{tabular}{|c|c|c|c|}
\hline \multicolumn{4}{|c|}{ Coordinates (Angstroms) } \\
\hline & $\mathrm{X}$ & $\mathrm{Y}$ & Z \\
\hline $\mathrm{C}$ & 6.081734000 & -1.206489000 & 0.289045000 \\
\hline $\mathrm{C}$ & 4.913343000 & -0.716635000 & 0.864327000 \\
\hline $\mathrm{C}$ & 3.674591000 & -0.899347000 & 0.234059000 \\
\hline $\mathrm{C}$ & 3.647454000 & -1.572892000 & -0.997714000 \\
\hline $\mathrm{C}$ & 4.814474000 & -2.068430000 & -1.561666000 \\
\hline $\mathrm{C}$ & 6.039852000 & -1.887844000 & -0.922495000 \\
\hline $\mathrm{H}$ & 7.029989000 & -1.058145000 & 0.797257000 \\
\hline $\mathrm{H}$ & 4.953875000 & -0.199950000 & 1.816874000 \\
\hline $\mathrm{H}$ & 2.706590000 & -1.702708000 & -1.524385000 \\
\hline $\mathrm{H}$ & 4.767972000 & -2.588876000 & -2.513592000 \\
\hline $\mathrm{H}$ & 6.951793000 & -2.271710000 & -1.368960000 \\
\hline $\mathrm{C}$ & 2.444446000 & -0.370557000 & 0.841559000 \\
\hline $\mathrm{C}$ & 1.162057000 & -0.690391000 & 0.561220000 \\
\hline $\mathrm{H}$ & 1.019927000 & -1.478771000 & -0.173455000 \\
\hline $\mathrm{O}$ & 2.708642000 & 0.661335000 & 1.777931000 \\
\hline $\mathrm{C}$ & -0.015717000 & -0.036101000 & 1.121639000 \\
\hline $\mathrm{H}$ & 0.053317000 & 0.180030000 & 2.195774000 \\
\hline $\mathrm{C}$ & -1.352749000 & -0.509219000 & 0.755650000 \\
\hline $\mathrm{C}$ & -2.434824000 & -0.309948000 & 1.637687000 \\
\hline $\mathrm{C}$ & -1.643961000 & -1.078901000 & -0.500510000 \\
\hline $\mathrm{C}$ & -3.728432000 & -0.659522000 & 1.298266000 \\
\hline $\mathrm{H}$ & -2.245291000 & 0.133024000 & 2.611708000 \\
\hline $\mathrm{C}$ & -2.933228000 & -1.441297000 & -0.852306000 \\
\hline $\mathrm{H}$ & -0.849895000 & -1.226673000 & -1.226634000 \\
\hline $\mathrm{C}$ & -3.968876000 & -1.229446000 & 0.050874000 \\
\hline $\mathrm{H}$ & -4.552852000 & -0.505875000 & 1.983906000 \\
\hline $\mathrm{H}$ & -3.150154000 & -1.880901000 & -1.818267000 \\
\hline $\mathrm{H}$ & 0.117857000 & 1.284810000 & 0.619630000 \\
\hline $\mathrm{H}$ & 2.012401000 & 0.656661000 & 2.449737000 \\
\hline $\mathrm{C}$ & -0.514881000 & 2.255780000 & -1.897033000 \\
\hline $\mathrm{C}$ & -0.357171000 & 3.056679000 & -0.599081000 \\
\hline $\mathrm{H}$ & 0.462464000 & 2.105218000 & -2.373043000 \\
\hline
\end{tabular}




$\begin{array}{lrrr}\mathrm{H} & -0.954597000 & 1.274479000 & -1.688851000 \\ \mathrm{H} & -1.165495000 & 2.773796000 & -2.610293000 \\ \mathrm{C} & -1.717539000 & 3.226148000 & 0.085899000 \\ \mathrm{C} & 0.264664000 & 4.424079000 & -0.889967000 \\ \mathrm{H} & -1.594957000 & 3.755867000 & 1.036226000 \\ \mathrm{H} & -2.409561000 & 3.795352000 & -0.544363000 \\ \mathrm{H} & -2.169151000 & 2.249432000 & 0.291757000 \\ \mathrm{H} & -0.372063000 & 5.022038000 & -1.551175000 \\ \mathrm{H} & 0.411308000 & 4.976994000 & 0.043876000 \\ \mathrm{H} & 1.239956000 & 4.304713000 & -1.380137000 \\ \mathrm{O} & 0.523104000 & 2.373911000 & 0.275377000 \\ \mathrm{~N} & -5.325776000 & -1.604201000 & -0.315676000 \\ \mathrm{O} & -5.506050000 & -2.098637000 & -1.421713000 \\ \mathrm{O} & -6.216577000 & -1.405484000 & 0.500817000 \\ \mathrm{Li} & 2.230875000 & 1.889198000 & 0.320039000 \\ -\end{array}$

\begin{tabular}{|c|c|c|c|}
\hline \multicolumn{4}{|c|}{ A3 tBuOLi } \\
\hline & $X$ & Y & Z \\
\hline$\overline{\mathrm{C}}$ & -5.358341000 & -0.767678000 & -0.965134000 \\
\hline $\mathrm{C}$ & -4.304098000 & -1.506562000 & -0.440616000 \\
\hline $\mathrm{C}$ & -3.094558000 & -0.879386000 & -0.113776000 \\
\hline $\mathrm{C}$ & -2.949201000 & 0.497276000 & -0.321307000 \\
\hline $\mathrm{C}$ & -4.010506000 & 1.226432000 & -0.848990000 \\
\hline $\mathrm{C}$ & -5.212153000 & 0.601681000 & -1.171513000 \\
\hline $\mathrm{H}$ & -6.296039000 & -1.260188000 & -1.203886000 \\
\hline $\mathrm{H}$ & -4.439095000 & -2.569049000 & -0.249456000 \\
\hline $\mathrm{H}$ & -1.999436000 & 0.998352000 & -0.105781000 \\
\hline $\mathrm{H}$ & -3.886093000 & 2.292034000 & -1.017498000 \\
\hline $\mathrm{H}$ & -6.033917000 & 1.178676000 & -1.584979000 \\
\hline $\mathrm{C}$ & -1.980420000 & -1.691771000 & 0.435963000 \\
\hline $\mathrm{C}$ & -1.175939000 & -1.306633000 & 1.440810000 \\
\hline $\mathrm{H}$ & -1.346330000 & -0.330377000 & 1.883856000 \\
\hline $\mathrm{O}$ & -1.806710000 & -2.932803000 & -0.113267000 \\
\hline $\mathrm{C}$ & -0.024777000 & -2.141791000 & 1.961093000 \\
\hline $\mathrm{H}$ & -0.235163000 & -3.205701000 & 1.820940000 \\
\hline $\mathrm{C}$ & 1.240445000 & -1.764155000 & 1.217425000 \\
\hline $\mathrm{C}$ & 1.510371000 & -2.342166000 & -0.034071000 \\
\hline $\mathrm{C}$ & 2.061614000 & -0.725860000 & 1.670796000 \\
\hline $\mathrm{C}$ & 2.567963000 & -1.891565000 & -0.815059000 \\
\hline $\mathrm{H}$ & 0.868805000 & -3.139036000 & -0.397893000 \\
\hline $\mathrm{C}$ & 3.125101000 & -0.264702000 & 0.901557000 \\
\hline $\mathrm{H}$ & 1.845918000 & -0.246119000 & 2.620313000 \\
\hline
\end{tabular}




$\begin{array}{lccc}\mathrm{C} & 3.358737000 & -0.854870000 & -0.331447000 \\ \mathrm{H} & 2.785316000 & -2.326341000 & -1.783020000 \\ \mathrm{H} & 3.749459000 & 0.555901000 & 1.232121000 \\ \mathrm{C} & -0.717208000 & 3.318192000 & 1.536527000 \\ \mathrm{C} & 0.156309000 & 2.942964000 & 0.323145000 \\ \mathrm{H} & -1.774223000 & 3.131651000 & 1.310577000 \\ \mathrm{H} & -0.440654000 & 2.694605000 & 2.395736000 \\ \mathrm{H} & -0.607435000 & 4.371487000 & 1.823436000 \\ \mathrm{C} & 1.632917000 & 3.216131000 & 0.665907000 \\ \mathrm{C} & -0.253755000 & 3.809041000 & -0.882195000 \\ \mathrm{H} & 2.270314000 & 2.918170000 & -0.175333000 \\ \mathrm{H} & 1.826203000 & 4.272612000 & 0.890811000 \\ \mathrm{H} & 1.922937000 & 2.620359000 & 1.541032000 \\ \mathrm{H} & -0.129614000 & 4.882053000 & -0.689312000 \\ \mathrm{H} & 0.352573000 & 3.538592000 & -1.754043000 \\ \mathrm{H} & -1.305259000 & 3.621225000 & -1.131112000 \\ \mathrm{O} & -0.018319000 & 1.612402000 & 0.023468000 \\ \mathrm{H} & 0.104575000 & -1.964839000 & 3.032854000 \\ \mathrm{H} & -2.352579000 & -2.995668000 & -0.907557000 \\ \mathrm{~N} & 4.467844000 & -0.361157000 & -1.159499000 \\ \mathrm{O} & 4.620789000 & -0.872258000 & -2.257870000 \\ \mathrm{O} & 5.166001000 & 0.524999000 & -0.696243000 \\ \mathrm{Li} & 0.238573000 & -0.005495000 & -0.152900000 \\ - & & & \end{array}$

\begin{tabular}{|c|c|c|c|}
\hline \multicolumn{4}{|c|}{$\mathbf{A} \mathbf{1}_{\mathrm{TBD}}$} \\
\hline & \multicolumn{3}{|c|}{ Coordinates (Angstroms) } \\
\hline & $X$ & $\mathrm{Y}$ & $\mathrm{Z}$ \\
\hline$\overline{\mathrm{C}}$ & 3.551942000 & -0.862978000 & 2.419197000 \\
\hline $\mathrm{C}$ & 4.218385000 & -2.233961000 & 2.494782000 \\
\hline $\mathrm{C}$ & 5.389665000 & -2.258401000 & 1.524017000 \\
\hline $\mathrm{N}$ & 3.127172000 & -0.539277000 & 1.074235000 \\
\hline $\mathrm{H}$ & 4.252623000 & -0.101015000 & 2.800229000 \\
\hline $\mathrm{H}$ & 2.675569000 & -0.831856000 & 3.076367000 \\
\hline $\mathrm{H}$ & 3.489606000 & -3.002263000 & 2.211953000 \\
\hline $\mathrm{H}$ & 4.571523000 & -2.458558000 & 3.506501000 \\
\hline $\mathrm{H}$ & 6.236164000 & -1.684318000 & 1.930322000 \\
\hline $\mathrm{H}$ & 5.741740000 & -3.287920000 & 1.375721000 \\
\hline $\mathrm{N}$ & 5.008605000 & -1.720070000 & 0.226318000 \\
\hline $\mathrm{C}$ & 5.813621000 & -2.188609000 & -0.890567000 \\
\hline $\mathrm{C}$ & 5.541716000 & -1.406744000 & -2.166864000 \\
\hline $\mathrm{C}$ & 4.037553000 & -1.266747000 & -2.339976000 \\
\hline $\mathrm{N}$ & 3.521756000 & -0.575946000 & -1.181125000 \\
\hline $\mathrm{C}$ & 3.865953000 & -0.957112000 & 0.096896000 \\
\hline
\end{tabular}




\begin{tabular}{lrcc}
$\mathrm{H}$ & 5.616070000 & -3.259582000 & -1.061962000 \\
$\mathrm{H}$ & 6.872094000 & -2.101148000 & -0.611718000 \\
$\mathrm{H}$ & 5.985092000 & -0.407833000 & -2.099029000 \\
$\mathrm{H}$ & 5.993846000 & -1.927016000 & -3.016750000 \\
$\mathrm{H}$ & 3.799446000 & -0.671930000 & -3.226608000 \\
$\mathrm{H}$ & 3.582341000 & -2.262469000 & -2.478654000 \\
$\mathrm{H}$ & 2.576755000 & -0.206256000 & -1.247492000 \\
$\mathrm{C}$ & 0.187002000 & 4.624312000 & -0.175767000 \\
$\mathrm{C}$ & -0.190717000 & 3.286977000 & -0.265105000 \\
$\mathrm{C}$ & 0.713730000 & 2.273385000 & 0.059344000 \\
$\mathrm{C}$ & 2.005777000 & 2.611409000 & 0.470753000 \\
$\mathrm{C}$ & 2.380514000 & 3.948008000 & 0.560734000 \\
$\mathrm{C}$ & 1.473829000 & 4.955877000 & 0.237989000 \\
$\mathrm{H}$ & -0.525265000 & 5.405410000 & -0.425188000 \\
$\mathrm{H}$ & -1.199859000 & 3.028170000 & -0.578617000 \\
$\mathrm{H}$ & 2.699595000 & 1.811017000 & 0.720374000 \\
$\mathrm{H}$ & 3.384784000 & 4.204747000 & 0.885716000 \\
$\mathrm{H}$ & 1.768504000 & 5.998716000 & 0.313063000 \\
$\mathrm{C}$ & 0.345338000 & 0.809674000 & -0.112631000 \\
$\mathrm{C}$ & -1.097653000 & 0.535247000 & 0.211391000 \\
$\mathrm{H}$ & -1.410308000 & 0.882324000 & 1.195265000 \\
$\mathrm{O}$ & 0.692928000 & 0.365392000 & -1.429846000 \\
$\mathrm{C}$ & -1.953700000 & -0.074005000 & -0.611626000 \\
$\mathrm{H}$ & -1.580972000 & -0.390068000 & -1.583704000 \\
$\mathrm{C}$ & -3.371624000 & -0.358101000 & -0.343471000 \\
$\mathrm{C}$ & -4.181378000 & -0.796251000 & -1.401271000 \\
$\mathrm{C}$ & -3.958902000 & -0.210205000 & 0.923024000 \\
$\mathrm{H}$ & -5.529821000 & -1.062827000 & -1.218735000 \\
$\mathrm{H}$ & -3.742620000 & -0.925703000 & -2.386457000 \\
$\mathrm{C}$ & -5.304067000 & -0.470850000 & 1.124227000 \\
$\mathrm{H}$ & -3.356170000 & 0.102990000 & 1.769020000 \\
$\mathrm{H}$ & -6.074905000 & -0.892192000 & 0.046370000 \\
$\mathrm{H}$ & -6.159269000 & -1.396732000 & -2.034227000 \\
$\mathrm{H}$ & -5.763049000 & -0.357924000 & 2.098588000 \\
$\mathrm{H}$ & 0.986381000 & 0.214209000 & 0.551353000 \\
$\mathrm{H}$ & -7.489586000 & 1.088971000 & -2.037073000 \\
$\mathrm{H}$ & -8.149699000 & -1.546132000 & -0.709518000 \\
\hline
\end{tabular}

TSA1-2 TBD

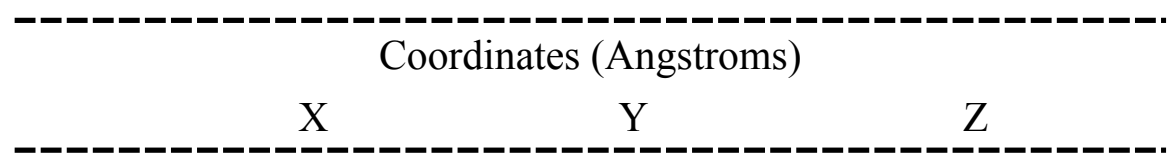




$\begin{array}{lrrr}\mathrm{C} & -1.362279000 & 0.823657000 & 2.392929000 \\ \mathrm{C} & 0.065197000 & 0.320223000 & 2.569305000 \\ \mathrm{C} & 1.025745000 & 1.483172000 & 2.377446000 \\ \mathrm{~N} & -1.530913000 & 1.473281000 & 1.105746000 \\ \mathrm{H} & -1.624973000 & 1.519966000 & 3.200470000 \\ \mathrm{H} & -2.079663000 & -0.002500000 & 2.434218000 \\ \mathrm{H} & 0.275587000 & -0.449042000 & 1.819155000 \\ \mathrm{H} & 0.215201000 & -0.119316000 & 3.559775000 \\ \mathrm{H} & 1.024357000 & 2.141263000 & 3.256857000 \\ \mathrm{H} & 2.046581000 & 1.107892000 & 2.243633000 \\ \mathrm{~N} & 0.688862000 & 2.269482000 & 1.190645000 \\ \mathrm{C} & 1.818516000 & 2.946114000 & 0.560237000 \\ \mathrm{C} & 1.361938000 & 3.924519000 & -0.509277000 \\ \mathrm{C} & 0.376721000 & 3.222094000 & -1.429683000 \\ \mathrm{~N} & -0.721087000 & 2.727695000 & -0.624982000 \\ \mathrm{C} & -0.512447000 & 2.128338000 & 0.578402000 \\ \mathrm{H} & 2.488031000 & 2.189877000 & 0.124972000 \\ \mathrm{H} & 2.375567000 & 3.468875000 & 1.346045000 \\ \mathrm{H} & 0.874300000 & 4.789718000 & -0.048160000 \\ \mathrm{H} & 2.230369000 & 4.280646000 & -1.070126000 \\ \mathrm{H} & -1.630594000 & -1.922507000 & -0.061720000 \\ \mathrm{H} & -0.029877000 & 3.913879000 & -2.171688000 \\ \mathrm{H} & 0.881798000 & 2.406184000 & -1.968673000 \\ \mathrm{H} & -1.529749000 & 2.356139000 & -1.119769000 \\ \mathrm{C} & -4.950818000 & -2.490543000 & 0.919030000 \\ \mathrm{C} & -3.748650000 & -1.890051000 & 0.569508000 \\ \mathrm{C} & -3.690985000 & -0.896566000 & -0.422670000 \\ \mathrm{C} & -4.895932000 & -0.518972000 & -1.029817000 \\ \mathrm{C} & -6.099879000 & -1.121074000 & -0.677222000 \\ \mathrm{C} & -6.138781000 & -2.113406000 & 0.296052000 \\ \mathrm{H} & -4.959471000 & -3.259102000 & 1.687114000 \\ \mathrm{H} & -2.843806000 & -2.200335000 & 1.086582000 \\ \mathrm{H} & -4.881454000 & 0.264410000 & -1.779470000 \\ \mathrm{H} & -7.016215000 & -0.807229000 & -1.170058000 \\ \mathrm{H} & -2.423550000 & -0.207894000 & -0.775876000 \\ \mathrm{H} & -1.160296000 & -0.890036000 & -0.720860000 \\ \mathrm{H} & -1.107092000 & -1.702751000 & 0.001234000 \\ \mathrm{H} & -0.013556000 & -0.477878000 & -1.325811000 \\ \mathrm{H} & 1.330252000 & -0.921000000 & -1.011067000 \\ \mathrm{H} & 2.424184000 & -0.282313000 & -1.635402000 \\ \mathrm{H} & & 0.474310000 & -2.390844000\end{array}$




$\begin{array}{lrrr}\mathrm{C} & 2.934991000 & -2.238920000 & 0.269699000 \\ \mathrm{H} & 0.827037000 & -2.464189000 & 0.427143000 \\ \mathrm{C} & 3.981340000 & -1.561990000 & -0.351971000 \\ \mathrm{H} & 4.567034000 & -0.089321000 & -1.795868000 \\ \mathrm{H} & 3.159240000 & -3.002616000 & 1.004576000 \\ \mathrm{H} & -2.091248000 & 0.712724000 & 0.286828000 \\ \mathrm{H} & -2.502681000 & 0.273032000 & -2.674181000 \\ \mathrm{H} & -7.078357000 & -2.583195000 & 0.570361000 \\ \mathrm{~N} & 5.353794000 & -1.877814000 & 0.009176000 \\ \mathrm{O} & 6.251048000 & -1.261338000 & -0.552524000 \\ \mathrm{O} & 5.539747000 & -2.739014000 & 0.859882000\end{array}$

\begin{tabular}{|c|c|c|c|}
\hline \multicolumn{4}{|c|}{ A2 TBD } \\
\hline & \multicolumn{3}{|c|}{ Coordinates (Angstroms) } \\
\hline & $X$ & Y & Z \\
\hline$\overline{\mathrm{C}}$ & -3.299989000 & 1.229232000 & -1.569806000 \\
\hline $\mathrm{C}$ & -3.740850000 & 1.779869000 & -0.224363000 \\
\hline $\mathrm{C}$ & -2.874433000 & 2.981357000 & 0.121383000 \\
\hline $\mathrm{N}$ & -1.867744000 & 0.965305000 & -1.541603000 \\
\hline $\mathrm{H}$ & -3.530962000 & 1.937706000 & -2.375402000 \\
\hline $\mathrm{H}$ & -3.797894000 & 0.282730000 & -1.784723000 \\
\hline $\mathrm{H}$ & -3.637246000 & 1.000694000 & 0.538644000 \\
\hline $\mathrm{H}$ & -4.790541000 & 2.082014000 & -0.260651000 \\
\hline $\mathrm{H}$ & -3.110135000 & 3.836807000 & -0.525839000 \\
\hline $\mathrm{H}$ & -3.046898000 & 3.290992000 & 1.157390000 \\
\hline $\mathrm{N}$ & -1.451342000 & 2.659828000 & 0.000090000 \\
\hline $\mathrm{C}$ & -0.538312000 & 3.456744000 & 0.820027000 \\
\hline $\mathrm{C}$ & 0.909327000 & 3.282622000 & 0.383182000 \\
\hline $\mathrm{C}$ & 1.214844000 & 1.800713000 & 0.241550000 \\
\hline $\mathrm{N}$ & 0.254148000 & 1.225676000 & -0.687682000 \\
\hline $\mathrm{C}$ & -1.024213000 & 1.609360000 & -0.725792000 \\
\hline $\mathrm{H}$ & -0.662009000 & 3.166835000 & 1.872138000 \\
\hline $\mathrm{H}$ & -0.844168000 & 4.504486000 & 0.726942000 \\
\hline $\mathrm{H}$ & 1.074300000 & 3.782694000 & -0.577006000 \\
\hline $\mathrm{H}$ & 1.570333000 & 3.742897000 & 1.121682000 \\
\hline $\mathrm{H}$ & 2.212189000 & 1.625878000 & -0.167130000 \\
\hline $\mathrm{H}$ & 1.163972000 & 1.291523000 & 1.212296000 \\
\hline $\mathrm{H}$ & 0.461651000 & 0.268147000 & -1.014297000 \\
\hline $\mathrm{C}$ & -3.846010000 & -1.268540000 & 2.408451000 \\
\hline $\mathrm{C}$ & -2.709442000 & -1.446544000 & 1.632038000 \\
\hline $\mathrm{C}$ & -2.801391000 & -1.799892000 & 0.269746000 \\
\hline $\mathrm{C}$ & -4.093355000 & -1.952032000 & -0.268839000 \\
\hline $\mathrm{C}$ & -5.228127000 & -1.754583000 & 0.510005000 \\
\hline
\end{tabular}




$\begin{array}{lrrr}\mathrm{C} & -5.118188000 & -1.411165000 & 1.854554000 \\ \mathrm{H} & -3.736360000 & -1.004296000 & 3.456987000 \\ \mathrm{H} & -1.735334000 & -1.295089000 & 2.087581000 \\ \mathrm{H} & -4.193790000 & -2.230026000 & -1.312520000 \\ \mathrm{H} & -6.209481000 & -1.880796000 & 0.060215000 \\ \mathrm{C} & -1.630015000 & -1.923133000 & -0.584018000 \\ \mathrm{C} & -0.322178000 & -1.880999000 & -0.205684000 \\ \mathrm{H} & -0.137363000 & -1.925357000 & 0.863907000 \\ \mathrm{O} & -1.929765000 & -1.849089000 & -1.959161000 \\ \mathrm{C} & 0.798842000 & -1.712349000 & -1.058975000 \\ \mathrm{H} & 0.648651000 & -1.722752000 & -2.137975000 \\ \mathrm{C} & 2.122249000 & -1.451212000 & -0.614453000 \\ \mathrm{C} & 3.130624000 & -1.081113000 & -1.555342000 \\ \mathrm{C} & 2.515046000 & -1.431162000 & 0.758225000 \\ \mathrm{C} & 4.382877000 & -0.667239000 & -1.166753000 \\ \mathrm{H} & 2.886477000 & -1.107934000 & -2.614722000 \\ \mathrm{C} & 3.768372000 & -1.019158000 & 1.151731000 \\ \mathrm{H} & 1.814443000 & -1.749978000 & 1.523536000 \\ \mathrm{C} & 4.704917000 & -0.614851000 & 0.195406000 \\ \mathrm{H} & 5.128143000 & -0.372476000 & -1.896088000 \\ \mathrm{H} & 4.047096000 & -1.001174000 & 2.199090000 \\ \mathrm{H} & -1.578062000 & 0.057086000 & -1.907035000 \\ \mathrm{H} & -1.361756000 & -2.481935000 & -2.417325000 \\ \mathrm{H} & -6.005377000 & -1.261093000 & 2.461924000 \\ \mathrm{~N} & 5.997911000 & -0.150344000 & 0.610594000 \\ \mathrm{O} & 6.801239000 & 0.192211000 & -0.256116000 \\ \mathrm{O} & 6.242993000 & -0.105169000 & 1.815895000 \\ -----0 .-0\end{array}$

TSA2-3 TBD

$\begin{array}{llll} & & & \text { Coordinates (Angstroms) } \\ \text { C } & -3.473654000 & 1.419455000 & -1.148790000 \\ \mathrm{C} & -3.738196000 & 1.835571000 & 0.288385000 \\ \mathrm{C} & -2.923860000 & 3.083019000 & 0.595565000 \\ \mathrm{~N} & -2.041265000 & 1.331528000 & -1.383906000 \\ \mathrm{H} & -3.923850000 & 2.141990000 & -1.842747000 \\ \mathrm{H} & -3.906337000 & 0.437122000 & -1.353832000 \\ \mathrm{H} & -3.450185000 & 1.021074000 & 0.962464000 \\ \mathrm{H} & -4.802006000 & 2.038700000 & 0.438000000 \\ \mathrm{H} & -3.308485000 & 3.946368000 & 0.033112000 \\ \mathrm{H} & -2.991386000 & 3.329416000 & 1.661263000 \\ \mathrm{~N} & -1.515369000 & 2.870291000 & 0.285811000 \\ \mathrm{C} & -0.558735000 & 3.704099000 & 1.005624000\end{array}$




\begin{tabular}{|c|c|c|c|}
\hline $\mathrm{C}$ & 0.812625000 & 3.658751000 & 0.347775000 \\
\hline $\mathrm{C}$ & 1.171808000 & 2.207524000 & 0.064006000 \\
\hline $\mathrm{N}$ & 0.157265000 & 1.599046000 & -0.779903000 \\
\hline $\mathrm{C}$ & -1.107890000 & 1.930489000 & -0.607865000 \\
\hline $\mathrm{H}$ & -0.494297000 & 3.364571000 & 2.049285000 \\
\hline $\mathrm{H}$ & -0.948883000 & 4.729003000 & 1.017591000 \\
\hline $\mathrm{H}$ & 0.795041000 & 4.218805000 & -0.593607000 \\
\hline $\mathrm{H}$ & 1.550269000 & 4.126006000 & 1.006250000 \\
\hline $\mathrm{H}$ & 2.130973000 & 2.128079000 & -0.454255000 \\
\hline $\mathrm{H}$ & 1.276124000 & 1.653485000 & 1.008729000 \\
\hline $\mathrm{H}$ & 0.427949000 & 0.408440000 & -1.062487000 \\
\hline $\mathrm{C}$ & -3.933589000 & -1.954488000 & 2.199811000 \\
\hline $\mathrm{C}$ & -2.797649000 & -1.723068000 & 1.434872000 \\
\hline $\mathrm{C}$ & -2.797635000 & -1.960989000 & 0.050474000 \\
\hline $\mathrm{C}$ & -3.988758000 & -2.407072000 & -0.540383000 \\
\hline $\mathrm{C}$ & -5.128473000 & -2.624713000 & 0.226685000 \\
\hline $\mathrm{C}$ & -5.108387000 & -2.404895000 & 1.600752000 \\
\hline $\mathrm{H}$ & -3.904713000 & -1.768155000 & 3.269778000 \\
\hline $\mathrm{H}$ & -1.903737000 & -1.334158000 & 1.913687000 \\
\hline $\mathrm{H}$ & -4.009614000 & -2.583537000 & -1.610277000 \\
\hline $\mathrm{H}$ & -6.037508000 & -2.974907000 & -0.254170000 \\
\hline $\mathrm{C}$ & -1.613016000 & -1.695576000 & -0.774248000 \\
\hline $\mathrm{C}$ & -0.346206000 & -1.539518000 & -0.344770000 \\
\hline $\mathrm{H}$ & -0.162591000 & -1.755557000 & 0.703158000 \\
\hline $\mathrm{O}$ & -1.927814000 & -1.472030000 & -2.118090000 \\
\hline $\mathrm{C}$ & 0.751760000 & -1.006031000 & -1.145010000 \\
\hline $\mathrm{H}$ & 0.686437000 & -1.173789000 & -2.224470000 \\
\hline $\mathrm{C}$ & 2.126423000 & -1.020951000 & -0.664533000 \\
\hline $\mathrm{C}$ & 3.189026000 & -0.843091000 & -1.581170000 \\
\hline $\mathrm{C}$ & 2.472638000 & -1.082242000 & 0.704809000 \\
\hline $\mathrm{C}$ & 4.500836000 & -0.721242000 & -1.169635000 \\
\hline $\mathrm{H}$ & 2.961783000 & -0.794538000 & -2.642955000 \\
\hline $\mathrm{C}$ & 3.782941000 & -0.965147000 & 1.130701000 \\
\hline $\mathrm{H}$ & 1.699555000 & -1.213690000 & 1.455261000 \\
\hline $\mathrm{C}$ & 4.794433000 & -0.779832000 & 0.192276000 \\
\hline $\mathrm{H}$ & 5.305361000 & -0.584854000 & -1.882361000 \\
\hline $\mathrm{H}$ & 4.037133000 & -1.013816000 & 2.182803000 \\
\hline $\mathrm{H}$ & -1.732492000 & 0.507703000 & -1.890559000 \\
\hline $\mathrm{H}$ & -1.197225000 & -1.810976000 & -2.651684000 \\
\hline $\mathrm{H}$ & -5.998682000 & -2.576952000 & 2.197913000 \\
\hline $\mathrm{N}$ & 6.167536000 & -0.645504000 & 0.636205000 \\
\hline $\mathrm{O}$ & 7.036094000 & -0.487775000 & -0.214833000 \\
\hline $\mathrm{O}$ & 6.392415000 & -0.691906000 & 1.841010000 \\
\hline
\end{tabular}




\begin{tabular}{|c|c|c|c|}
\hline \multicolumn{4}{|c|}{$\mathbf{A 3}$ TBD } \\
\hline & $X$ & $\mathrm{Y}$ & $\mathrm{Z}$ \\
\hline $\mathrm{C}$ & -0.217051000 & 2.035471000 & 1.753957000 \\
\hline $\mathrm{C}$ & 0.739789000 & 1.165415000 & 2.555328000 \\
\hline $\mathrm{C}$ & 2.169345000 & 1.381382000 & 2.066678000 \\
\hline $\mathrm{N}$ & -0.047894000 & 1.700787000 & 0.360078000 \\
\hline $\mathrm{H}$ & -0.025361000 & 3.102576000 & 1.961518000 \\
\hline $\mathrm{H}$ & -1.254483000 & 1.825834000 & 2.031865000 \\
\hline $\mathrm{H}$ & 0.448319000 & 0.121604000 & 2.418263000 \\
\hline $\mathrm{H}$ & 0.685701000 & 1.393971000 & 3.624223000 \\
\hline $\mathrm{H}$ & 2.604744000 & 2.256458000 & 2.574652000 \\
\hline $\mathrm{H}$ & 2.788651000 & 0.514688000 & 2.339273000 \\
\hline $\mathrm{N}$ & 2.268412000 & 1.587215000 & 0.629216000 \\
\hline $\mathrm{C}$ & 3.624304000 & 1.654107000 & 0.110173000 \\
\hline $\mathrm{C}$ & 3.661969000 & 2.440072000 & -1.192530000 \\
\hline $\mathrm{C}$ & 2.569272000 & 1.913520000 & -2.120357000 \\
\hline $\mathrm{N}$ & 1.259862000 & 1.924718000 & -1.504690000 \\
\hline $\mathrm{C}$ & 1.201017000 & 1.757433000 & -0.226024000 \\
\hline $\mathrm{H}$ & 4.023641000 & 0.639651000 & -0.040993000 \\
\hline $\mathrm{H}$ & 4.255546000 & 2.136839000 & 0.867472000 \\
\hline $\mathrm{H}$ & 3.480453000 & 3.501107000 & -0.983836000 \\
\hline $\mathrm{H}$ & 4.652682000 & 2.351277000 & -1.650354000 \\
\hline $\mathrm{H}$ & 2.526781000 & 2.520455000 & -3.032461000 \\
\hline $\mathrm{H}$ & 2.835979000 & 0.892765000 & -2.439287000 \\
\hline $\mathrm{C}$ & -6.294821000 & -1.488252000 & 0.028803000 \\
\hline $\mathrm{C}$ & -5.081590000 & -1.276348000 & -0.614876000 \\
\hline $\mathrm{C}$ & -4.278406000 & -0.182342000 & -0.275321000 \\
\hline $\mathrm{C}$ & -4.729586000 & 0.705239000 & 0.709281000 \\
\hline $\mathrm{C}$ & -5.947011000 & 0.495171000 & 1.348370000 \\
\hline $\mathrm{C}$ & -6.732558000 & -0.603083000 & 1.011345000 \\
\hline $\mathrm{H}$ & -6.908425000 & -2.340359000 & -0.247961000 \\
\hline $\mathrm{H}$ & -4.758053000 & -1.951240000 & -1.401526000 \\
\hline $\mathrm{H}$ & -4.108945000 & 1.550422000 & 0.995408000 \\
\hline $\mathrm{H}$ & -6.278598000 & 1.188300000 & 2.115679000 \\
\hline $\mathrm{C}$ & -2.974364000 & 0.049211000 & -0.940817000 \\
\hline $\mathrm{C}$ & -2.134347000 & -0.924455000 & -1.307279000 \\
\hline $\mathrm{H}$ & -2.419782000 & -1.943589000 & -1.058929000 \\
\hline $\mathrm{O}$ & -2.624164000 & 1.367859000 & -1.116312000 \\
\hline $\mathrm{C}$ & -0.817493000 & -0.725372000 & -1.994208000 \\
\hline $\mathrm{H}$ & -0.626152000 & 0.340910000 & -2.169075000 \\
\hline $\mathrm{C}$ & 0.379899000 & -1.255508000 & -1.235289000 \\
\hline $\mathrm{C}$ & 1.596398000 & -1.428583000 & -1.903646000 \\
\hline
\end{tabular}




\begin{tabular}{|c|c|c|c|}
\hline $\mathrm{C}$ & 0.332338000 & -1.501235000 & 0.137492000 \\
\hline $\mathrm{C}$ & 2.752692000 & -1.766877000 & -1.219542000 \\
\hline $\mathrm{H}$ & 1.644687000 & -1.251543000 & -2.974428000 \\
\hline $\mathrm{C}$ & 1.475171000 & -1.854182000 & 0.840941000 \\
\hline $\mathrm{H}$ & -0.605146000 & -1.369490000 & 0.667361000 \\
\hline $\mathrm{C}$ & 2.677108000 & -1.955380000 & 0.155943000 \\
\hline $\mathrm{H}$ & 3.704961000 & -1.866003000 & -1.725700000 \\
\hline $\mathrm{H}$ & 1.453660000 & -2.024293000 & 1.910558000 \\
\hline $\mathrm{H}$ & -0.789844000 & 1.946665000 & -0.282636000 \\
\hline $\mathrm{H}$ & -3.428338000 & 1.892141000 & -1.217947000 \\
\hline $\mathrm{H}$ & -0.845918000 & -1.207326000 & -2.981502000 \\
\hline $\mathrm{H}$ & -7.684097000 & -0.766248000 & 1.507987000 \\
\hline $\mathrm{N}$ & 3.901281000 & -2.224689000 & 0.907122000 \\
\hline $\mathrm{O}$ & 3.812136000 & -2.353013000 & 2.121215000 \\
\hline $\mathrm{O}$ & 4.952437000 & -2.287871000 & 0.285066000 \\
\hline
\end{tabular}

\begin{tabular}{|c|c|c|c|}
\hline \multicolumn{4}{|c|}{$\mathbf{A} 1_{\text {DBU }}$} \\
\hline & \multicolumn{3}{|c|}{ Coordinates (Angstroms) } \\
\hline & $\mathrm{X}$ & $\mathrm{Y}$ & $\mathrm{Z}$ \\
\hline$\overline{\mathrm{C}}$ & -0.043116000 & 4.503274000 & -0.808675000 \\
\hline $\mathrm{C}$ & -0.454673000 & 3.172609000 & -0.784478000 \\
\hline $\mathrm{C}$ & 0.454163000 & 2.154272000 & -0.490939000 \\
\hline $\mathrm{C}$ & 1.785586000 & 2.486762000 & -0.227407000 \\
\hline $\mathrm{C}$ & 2.197024000 & 3.814722000 & -0.249637000 \\
\hline $\mathrm{C}$ & 1.283404000 & 4.826335000 & -0.540095000 \\
\hline $\mathrm{H}$ & -0.760790000 & 5.286751000 & -1.034160000 \\
\hline $\mathrm{H}$ & -1.494229000 & 2.923838000 & -0.986095000 \\
\hline $\mathrm{H}$ & 2.484716000 & 1.686546000 & 0.001209000 \\
\hline $\mathrm{H}$ & 3.233802000 & 4.062430000 & -0.039154000 \\
\hline $\mathrm{H}$ & 1.604735000 & 5.863851000 & -0.555301000 \\
\hline $\mathrm{C}$ & 0.044949000 & 0.689871000 & -0.532576000 \\
\hline $\mathrm{C}$ & -1.375393000 & 0.479339000 & -0.079306000 \\
\hline $\mathrm{H}$ & -1.609604000 & 0.911918000 & 0.892562000 \\
\hline $\mathrm{O}$ & 0.291609000 & 0.138838000 & -1.823297000 \\
\hline $\mathrm{C}$ & -2.297172000 & -0.182443000 & -0.782159000 \\
\hline $\mathrm{H}$ & -2.000904000 & -0.584160000 & -1.749021000 \\
\hline $\mathrm{C}$ & -3.691529000 & -0.424830000 & -0.383148000 \\
\hline $\mathrm{C}$ & -4.596392000 & -0.901787000 & -1.342620000 \\
\hline $\mathrm{C}$ & -4.163837000 & -0.205094000 & 0.920524000 \\
\hline $\mathrm{C}$ & -5.926974000 & -1.137110000 & -1.030707000 \\
\hline $\mathrm{H}$ & -4.247141000 & -1.086674000 & -2.354446000 \\
\hline $\mathrm{C}$ & -5.489117000 & -0.433818000 & 1.250485000 \\
\hline $\mathrm{H}$ & -3.483423000 & 0.136314000 & 1.693495000 \\
\hline
\end{tabular}




\begin{tabular}{|c|c|c|c|}
\hline $\mathrm{C}$ & -6.356767000 & -0.895733000 & 0.267115000 \\
\hline $\mathrm{H}$ & -6.628737000 & -1.501036000 & -1.770840000 \\
\hline $\mathrm{H}$ & -5.859639000 & -0.267758000 & 2.254470000 \\
\hline $\mathrm{H}$ & 0.712773000 & 0.133235000 & 0.140161000 \\
\hline $\mathrm{H}$ & -0.003323000 & 0.790323000 & -2.472941000 \\
\hline $\mathrm{C}$ & 6.041617000 & -1.893597000 & 0.346207000 \\
\hline $\mathrm{C}$ & 5.661899000 & -3.080979000 & -0.532949000 \\
\hline $\mathrm{C}$ & 3.265433000 & -1.546983000 & -0.913807000 \\
\hline $\mathrm{C}$ & 5.286281000 & -2.684329000 & -1.962754000 \\
\hline $\mathrm{C}$ & 4.349869000 & -1.473464000 & -1.991305000 \\
\hline $\mathrm{H}$ & 6.721949000 & -1.225099000 & -0.206430000 \\
\hline $\mathrm{H}$ & 2.908767000 & -2.580215000 & -0.808434000 \\
\hline $\mathrm{H}$ & 4.842262000 & -3.625778000 & -0.050740000 \\
\hline $\mathrm{H}$ & 4.804260000 & -3.538463000 & -2.453738000 \\
\hline $\mathrm{H}$ & 6.613543000 & -2.273439000 & 1.202663000 \\
\hline $\mathrm{H}$ & 6.514218000 & -3.769135000 & -0.555817000 \\
\hline $\mathrm{H}$ & 2.393046000 & -0.962812000 & -1.214399000 \\
\hline $\mathrm{H}$ & 6.195617000 & -2.464512000 & -2.535741000 \\
\hline $\mathrm{H}$ & 3.874653000 & -1.398947000 & -2.974821000 \\
\hline $\mathrm{H}$ & 4.924137000 & -0.547360000 & -1.861581000 \\
\hline $\mathrm{C}$ & 3.646501000 & -1.014369000 & 0.459038000 \\
\hline $\mathrm{N}$ & 2.683854000 & -0.455602000 & 1.110986000 \\
\hline $\mathrm{N}$ & 4.941940000 & -1.120556000 & 0.920010000 \\
\hline $\mathrm{C}$ & 5.361673000 & -0.374737000 & 2.104663000 \\
\hline $\mathrm{H}$ & 5.507615000 & -1.075590000 & 2.940713000 \\
\hline $\mathrm{H}$ & 6.338974000 & 0.080096000 & 1.894512000 \\
\hline $\mathrm{C}$ & 4.355215000 & 0.696728000 & 2.487293000 \\
\hline $\mathrm{H}$ & 4.589724000 & 1.087150000 & 3.482570000 \\
\hline $\mathrm{H}$ & 4.410191000 & 1.533000000 & 1.779822000 \\
\hline $\mathrm{C}$ & 2.960522000 & 0.085443000 & 2.425901000 \\
\hline $\mathrm{H}$ & 2.197322000 & 0.837676000 & 2.654140000 \\
\hline $\mathrm{H}$ & 2.868728000 & -0.703842000 & 3.188326000 \\
\hline $\mathrm{N}$ & -7.758198000 & -1.141212000 & 0.611725000 \\
\hline $\mathrm{O}$ & -8.499438000 & -1.547337000 & -0.271373000 \\
\hline $\mathrm{O}$ & -8.107346000 & -0.924902000 & 1.763168000 \\
\hline
\end{tabular}

TSA1-2 DBU

\begin{tabular}{|c|c|c|c|}
\hline & \multicolumn{3}{|c|}{ Coordinates (Angstroms) } \\
\hline & $\mathrm{X}$ & Y & Z \\
\hline$\overline{\mathrm{C}}$ & 2.175520000 & 2.517375000 & 2.228883000 \\
\hline $\mathrm{C}$ & 1.215545000 & 1.870399000 & 1.461775000 \\
\hline $\mathrm{C}$ & 1.239636000 & 1.921892000 & 0.053249000 \\
\hline $\mathrm{C}$ & 2.287276000 & 2.648729000 & -0.537940000 \\
\hline
\end{tabular}




\begin{tabular}{|c|c|c|c|}
\hline $\mathrm{C}$ & 3.250772000 & 3.291421000 & 0.233253000 \\
\hline $\mathrm{C}$ & 3.209041000 & 3.230924000 & 1.623140000 \\
\hline $\mathrm{H}$ & 2.115202000 & 2.461931000 & 3.313134000 \\
\hline $\mathrm{H}$ & 0.432155000 & 1.312078000 & 1.969704000 \\
\hline $\mathrm{H}$ & 2.337062000 & 2.698380000 & -1.620802000 \\
\hline $\mathrm{H}$ & 4.042593000 & 3.848027000 & -0.262204000 \\
\hline $\mathrm{H}$ & 3.960373000 & 3.734158000 & 2.223984000 \\
\hline $\mathrm{C}$ & 0.307366000 & 1.131016000 & -0.771015000 \\
\hline $\mathrm{C}$ & -1.052474000 & 0.931062000 & -0.365965000 \\
\hline $\mathrm{H}$ & -1.255975000 & 1.096306000 & 0.691193000 \\
\hline $\mathrm{O}$ & 0.515557000 & 1.224590000 & -2.167458000 \\
\hline $\mathrm{C}$ & -2.069978000 & 0.517432000 & -1.169652000 \\
\hline $\mathrm{H}$ & -1.846874000 & 0.323676000 & -2.216299000 \\
\hline $\mathrm{C}$ & -3.433501000 & 0.258450000 & -0.750378000 \\
\hline $\mathrm{C}$ & -4.353231000 & -0.259183000 & -1.687790000 \\
\hline $\mathrm{C}$ & -3.913855000 & 0.489043000 & 0.557871000 \\
\hline $\mathrm{C}$ & -5.663560000 & -0.542546000 & -1.350746000 \\
\hline $\mathrm{H}$ & -4.016261000 & -0.442950000 & -2.704308000 \\
\hline $\mathrm{C}$ & -5.219482000 & 0.207591000 & 0.910798000 \\
\hline $\mathrm{H}$ & -3.254446000 & 0.905385000 & 1.312676000 \\
\hline $\mathrm{C}$ & -6.089917000 & -0.309896000 & -0.046002000 \\
\hline $\mathrm{H}$ & -6.361077000 & -0.940889000 & -2.077571000 \\
\hline $\mathrm{H}$ & -5.582044000 & 0.385884000 & 1.915966000 \\
\hline $\mathrm{H}$ & 0.912771000 & -0.255053000 & -0.475386000 \\
\hline $\mathrm{H}$ & -0.022594000 & 1.959146000 & -2.500965000 \\
\hline $\mathrm{C}$ & 5.049289000 & -2.073992000 & 0.458542000 \\
\hline $\mathrm{C}$ & 5.596108000 & -2.140493000 & -0.962417000 \\
\hline $\mathrm{C}$ & 3.260398000 & -0.570394000 & -1.516635000 \\
\hline $\mathrm{C}$ & 5.795099000 & -0.768377000 & -1.611886000 \\
\hline $\mathrm{C}$ & 4.598354000 & 0.160065000 & -1.386667000 \\
\hline $\mathrm{H}$ & 5.584564000 & -1.306318000 & 1.035451000 \\
\hline $\mathrm{H}$ & 3.304707000 & -1.326061000 & -2.311308000 \\
\hline $\mathrm{H}$ & 4.936741000 & -2.772808000 & -1.567880000 \\
\hline $\mathrm{H}$ & 5.960657000 & -0.910111000 & -2.686281000 \\
\hline $\mathrm{H}$ & 5.245144000 & -3.033372000 & 0.950231000 \\
\hline $\mathrm{H}$ & 6.556552000 & -2.665061000 & -0.916648000 \\
\hline $\mathrm{H}$ & 2.472442000 & 0.121403000 & -1.822263000 \\
\hline $\mathrm{H}$ & 6.703278000 & -0.300671000 & -1.214009000 \\
\hline $\mathrm{H}$ & 4.622259000 & 0.979174000 & -2.111303000 \\
\hline $\mathrm{H}$ & 4.650955000 & 0.638451000 & -0.401809000 \\
\hline $\mathrm{C}$ & 2.772278000 & -1.245993000 & -0.255516000 \\
\hline $\mathrm{N}$ & 1.475641000 & -1.204080000 & -0.066526000 \\
\hline $\mathrm{N}$ & 3.603301000 & -1.855516000 & 0.612494000 \\
\hline $\mathrm{C}$ & 3.117069000 & -2.304755000 & 1.924695000 \\
\hline
\end{tabular}




$\begin{array}{lrrr}\mathrm{H} & 3.020174000 & -3.398683000 & 1.908318000 \\ \mathrm{H} & 3.887237000 & -2.052396000 & 2.661133000 \\ \mathrm{C} & 1.799930000 & -1.648553000 & 2.298426000 \\ \mathrm{H} & 1.390458000 & -2.127141000 & 3.192011000 \\ \mathrm{H} & 1.963149000 & -0.587973000 & 2.516698000 \\ \mathrm{C} & 0.853779000 & -1.773060000 & 1.114008000 \\ \mathrm{H} & -0.084180000 & -1.233594000 & 1.277537000 \\ \mathrm{H} & 0.599387000 & -2.823310000 & 0.925619000 \\ \mathrm{~N} & -7.462256000 & -0.608190000 & 0.323625000 \\ \mathrm{O} & -8.207373000 & -1.057525000 & -0.539440000 \\ \mathrm{O} & -7.805681000 & -0.397319000 & 1.481405000\end{array}$

\begin{tabular}{|c|c|c|c|}
\hline \multicolumn{4}{|c|}{$\mathrm{A} 2$ DBU } \\
\hline & $\mathrm{X}$ & Y & Z \\
\hline$\overline{\mathrm{C}}$ & -3.469083000 & -1.753058000 & 2.206491000 \\
\hline $\mathrm{C}$ & -2.340907000 & -1.749935000 & 1.398525000 \\
\hline $\mathrm{C}$ & -2.437145000 & -1.866005000 & -0.004572000 \\
\hline $\mathrm{C}$ & -3.731309000 & -2.022568000 & -0.540638000 \\
\hline $\mathrm{C}$ & -4.859049000 & -2.013478000 & 0.272473000 \\
\hline $\mathrm{C}$ & -4.744530000 & -1.866359000 & 1.652575000 \\
\hline $\mathrm{H}$ & -3.349892000 & -1.669747000 & 3.284163000 \\
\hline $\mathrm{H}$ & -1.367200000 & -1.668771000 & 1.870963000 \\
\hline $\mathrm{H}$ & -3.836891000 & -2.142368000 & -1.612937000 \\
\hline $\mathrm{H}$ & -5.839698000 & -2.130351000 & -0.181290000 \\
\hline $\mathrm{H}$ & -5.625903000 & -1.862137000 & 2.286368000 \\
\hline $\mathrm{C}$ & -1.288385000 & -1.752328000 & -0.891694000 \\
\hline $\mathrm{C}$ & 0.009746000 & -1.554038000 & -0.515408000 \\
\hline $\mathrm{H}$ & 0.214141000 & -1.644530000 & 0.547230000 \\
\hline $\mathrm{O}$ & -1.611856000 & -1.729948000 & -2.247804000 \\
\hline $\mathrm{C}$ & 1.091104000 & -1.189473000 & -1.351032000 \\
\hline $\mathrm{H}$ & 0.906277000 & -1.047427000 & -2.415112000 \\
\hline $\mathrm{C}$ & 2.414270000 & -0.945964000 & -0.900556000 \\
\hline $\mathrm{C}$ & 3.385060000 & -0.383336000 & -1.785227000 \\
\hline $\mathrm{C}$ & 2.858989000 & -1.182703000 & 0.438039000 \\
\hline $\mathrm{C}$ & 4.648585000 & -0.038221000 & -1.369416000 \\
\hline $\mathrm{H}$ & 3.103128000 & -0.211548000 & -2.821415000 \\
\hline $\mathrm{C}$ & 4.123532000 & -0.834534000 & 0.859071000 \\
\hline $\mathrm{H}$ & 2.197675000 & -1.674146000 & 1.145474000 \\
\hline $\mathrm{C}$ & 5.022322000 & -0.245653000 & -0.035163000 \\
\hline $\mathrm{H}$ & 5.364775000 & 0.398571000 & -2.055479000 \\
\hline $\mathrm{H}$ & 4.444323000 & -1.020685000 & 1.877673000 \\
\hline $\mathrm{H}$ & 0.831780000 & 1.020679000 & -0.779811000 \\
\hline
\end{tabular}




\begin{tabular}{|c|c|c|c|}
\hline $\mathrm{H}$ & -0.923744000 & -2.225250000 & -2.710513000 \\
\hline $\mathrm{C}$ & -3.281978000 & 1.850599000 & 0.737000000 \\
\hline $\mathrm{C}$ & -3.704373000 & 2.853010000 & -0.336533000 \\
\hline $\mathrm{C}$ & -1.450370000 & 1.449041000 & -1.674253000 \\
\hline $\mathrm{C}$ & -3.893986000 & 2.229829000 & -1.724671000 \\
\hline $\mathrm{C}$ & -2.899900000 & 1.104476000 & -1.998075000 \\
\hline $\mathrm{H}$ & -3.785414000 & 0.882348000 & 0.632858000 \\
\hline $\mathrm{H}$ & -1.168087000 & 2.409313000 & -2.126953000 \\
\hline $\mathrm{H}$ & -2.953352000 & 3.652139000 & -0.368896000 \\
\hline $\mathrm{H}$ & -3.806560000 & 3.014665000 & -2.485411000 \\
\hline $\mathrm{H}$ & -3.555311000 & 2.248240000 & 1.717655000 \\
\hline $\mathrm{H}$ & -4.636493000 & 3.327532000 & -0.013186000 \\
\hline $\mathrm{H}$ & -0.804140000 & 0.687907000 & -2.129713000 \\
\hline $\mathrm{H}$ & -4.907607000 & 1.822303000 & -1.810700000 \\
\hline $\mathrm{H}$ & -2.939551000 & 0.819125000 & -3.053056000 \\
\hline $\mathrm{H}$ & -3.185927000 & 0.207635000 & -1.446919000 \\
\hline $\mathrm{C}$ & -1.014514000 & 1.515678000 & -0.229980000 \\
\hline $\mathrm{N}$ & 0.307524000 & 1.529669000 & -0.065145000 \\
\hline $\mathrm{N}$ & -1.824092000 & 1.630873000 & 0.814619000 \\
\hline $\mathrm{C}$ & -1.329484000 & 1.511745000 & 2.197770000 \\
\hline $\mathrm{H}$ & -1.396131000 & 2.503354000 & 2.662878000 \\
\hline $\mathrm{H}$ & -2.020416000 & 0.843630000 & 2.719589000 \\
\hline $\mathrm{C}$ & 0.087937000 & 0.973048000 & 2.280184000 \\
\hline $\mathrm{H}$ & 0.481804000 & 1.141899000 & 3.285549000 \\
\hline $\mathrm{H}$ & 0.096774000 & -0.102770000 & 2.092144000 \\
\hline $\mathrm{C}$ & 0.950426000 & 1.660270000 & 1.235081000 \\
\hline $\mathrm{H}$ & 1.937564000 & 1.201662000 & 1.162893000 \\
\hline $\mathrm{H}$ & 1.076021000 & 2.724442000 & 1.466145000 \\
\hline $\mathrm{N}$ & 6.329935000 & 0.137442000 & 0.414223000 \\
\hline $\mathrm{O}$ & 7.105388000 & 0.634373000 & -0.401308000 \\
\hline $\mathrm{O}$ & 6.615560000 & -0.040909000 & 1.598362000 \\
\hline
\end{tabular}

TSA2-3 DBU

\begin{tabular}{llcc} 
& \multicolumn{3}{c}{ Coordinates (Angstroms) } \\
C & 3.588843000 & 1.800420000 & 2.110882000 \\
$\mathrm{C}$ & 2.488066000 & 1.503651000 & 1.316553000 \\
$\mathrm{C}$ & 2.343259000 & 2.070667000 & 0.040015000 \\
$\mathrm{C}$ & 3.359888000 & 2.915221000 & -0.425526000 \\
$\mathrm{C}$ & 4.464500000 & 3.204140000 & 0.367283000 \\
$\mathrm{C}$ & 4.585845000 & 2.652250000 & 1.640008000 \\
$\mathrm{H}$ & 3.676799000 & 1.352254000 & 3.097100000 \\
$\mathrm{H}$ & 1.740413000 & 0.802553000 & 1.676479000
\end{tabular}




\begin{tabular}{|c|c|c|c|}
\hline $\mathrm{H}$ & 3.271238000 & 3.341706000 & -1.418615000 \\
\hline $\mathrm{H}$ & 5.236424000 & 3.867993000 & -0.011584000 \\
\hline $\mathrm{H}$ & 5.451079000 & 2.877589000 & 2.256166000 \\
\hline $\mathrm{C}$ & 1.200376000 & 1.736236000 & -0.821331000 \\
\hline $\mathrm{C}$ & -0.006260000 & 1.317413000 & -0.386907000 \\
\hline $\mathrm{H}$ & -0.158282000 & 1.358193000 & 0.688057000 \\
\hline $\mathrm{O}$ & 1.495294000 & 1.832098000 & -2.169791000 \\
\hline $\mathrm{C}$ & -1.042075000 & 0.684771000 & -1.188208000 \\
\hline $\mathrm{H}$ & -0.907784000 & 0.710531000 & -2.272787000 \\
\hline $\mathrm{C}$ & -2.434383000 & 0.652602000 & -0.781130000 \\
\hline $\mathrm{C}$ & -3.407938000 & 0.127425000 & -1.666310000 \\
\hline $\mathrm{C}$ & -2.887115000 & 1.038206000 & 0.504108000 \\
\hline $\mathrm{C}$ & -4.726459000 & -0.031695000 & -1.295876000 \\
\hline $\mathrm{H}$ & -3.098655000 & -0.168359000 & -2.665644000 \\
\hline $\mathrm{C}$ & -4.204863000 & 0.875725000 & 0.889174000 \\
\hline $\mathrm{H}$ & -2.195363000 & 1.488238000 & 1.208835000 \\
\hline $\mathrm{C}$ & -5.121956000 & 0.334548000 & -0.008450000 \\
\hline $\mathrm{H}$ & -5.460406000 & -0.436172000 & -1.982596000 \\
\hline $\mathrm{H}$ & -4.542459000 & 1.173781000 & 1.874873000 \\
\hline $\mathrm{H}$ & -0.599326000 & -0.750934000 & -0.914707000 \\
\hline $\mathrm{H}$ & 0.664228000 & 1.994945000 & -2.634980000 \\
\hline $\mathrm{C}$ & 3.316486000 & -2.326074000 & 0.828541000 \\
\hline $\mathrm{C}$ & 3.947744000 & -2.961602000 & -0.407929000 \\
\hline $\mathrm{C}$ & 1.836403000 & -1.436535000 & -1.690276000 \\
\hline $\mathrm{C}$ & 4.324038000 & -1.946276000 & -1.491199000 \\
\hline $\mathrm{C}$ & 3.253781000 & -0.869234000 & -1.658757000 \\
\hline $\mathrm{H}$ & 3.831367000 & -1.394881000 & 1.101495000 \\
\hline $\mathrm{H}$ & 1.794823000 & -2.346875000 & -2.303806000 \\
\hline $\mathrm{H}$ & 3.254064000 & -3.714714000 & -0.801196000 \\
\hline $\mathrm{H}$ & 4.480816000 & -2.476162000 & -2.438393000 \\
\hline $\mathrm{H}$ & 3.433223000 & -3.015102000 & 1.671650000 \\
\hline $\mathrm{H}$ & 4.840520000 & -3.509279000 & -0.088059000 \\
\hline $\mathrm{H}$ & 1.169751000 & -0.720198000 & -2.179059000 \\
\hline $\mathrm{H}$ & 5.277552000 & -1.469296000 & -1.236850000 \\
\hline $\mathrm{H}$ & 3.420522000 & -0.309679000 & -2.583116000 \\
\hline $\mathrm{H}$ & 3.328284000 & -0.130493000 & -0.855591000 \\
\hline $\mathrm{C}$ & 1.177573000 & -1.756163000 & -0.367610000 \\
\hline $\mathrm{N}$ & -0.139148000 & -1.729348000 & -0.394050000 \\
\hline $\mathrm{N}$ & 1.870265000 & -2.070687000 & 0.744286000 \\
\hline $\mathrm{C}$ & 1.219203000 & -2.121551000 & 2.060553000 \\
\hline $\mathrm{H}$ & 1.220281000 & -3.164540000 & 2.404475000 \\
\hline $\mathrm{H}$ & 1.844176000 & -1.546844000 & 2.754320000 \\
\hline $\mathrm{C}$ & -0.197265000 & -1.572123000 & 2.039662000 \\
\hline $\mathrm{H}$ & -0.718693000 & -1.873801000 & 2.952184000 \\
\hline
\end{tabular}




$\begin{array}{llcc}\mathrm{H} & -0.186234000 & -0.478044000 & 2.010191000 \\ \mathrm{C} & -0.897780000 & -2.093015000 & 0.793169000 \\ \mathrm{H} & -1.898113000 & -1.668173000 & 0.688160000 \\ \mathrm{H} & -0.998575000 & -3.184821000 & 0.837188000 \\ \mathrm{~N} & -6.501068000 & 0.160348000 & 0.394767000 \\ \mathrm{O} & -7.288516000 & -0.302751000 & -0.423952000 \\ \mathrm{O} & -6.814465000 & 0.479946000 & 1.537262000\end{array}$

A3 DbU

\begin{tabular}{|c|c|c|c|}
\hline \multicolumn{4}{|c|}{ Coordinates (Angstroms) } \\
\hline & $\mathrm{X}$ & Y & Z \\
\hline$\overline{\mathrm{C}}$ & -3.183263000 & -1.756690000 & 2.277540000 \\
\hline $\mathrm{C}$ & -2.197197000 & -1.489071000 & 1.336535000 \\
\hline $\mathrm{C}$ & -2.236856000 & -2.086021000 & 0.071470000 \\
\hline $\mathrm{C}$ & -3.301050000 & -2.937782000 & -0.239074000 \\
\hline $\mathrm{C}$ & -4.289762000 & -3.201761000 & 0.703251000 \\
\hline $\mathrm{C}$ & -4.234394000 & -2.615645000 & 1.964393000 \\
\hline $\mathrm{H}$ & -3.137162000 & -1.282016000 & 3.253486000 \\
\hline $\mathrm{H}$ & -1.406221000 & -0.784722000 & 1.571013000 \\
\hline $\mathrm{H}$ & -3.350484000 & -3.388709000 & -1.224088000 \\
\hline $\mathrm{H}$ & -5.107805000 & -3.869158000 & 0.448752000 \\
\hline $\mathrm{H}$ & -5.009071000 & -2.820540000 & 2.697140000 \\
\hline $\mathrm{C}$ & -1.198848000 & -1.782146000 & -0.937091000 \\
\hline $\mathrm{C}$ & 0.050084000 & -1.396983000 & -0.646926000 \\
\hline $\mathrm{H}$ & 0.346091000 & -1.358292000 & 0.394538000 \\
\hline $\mathrm{O}$ & -1.666622000 & -1.911995000 & -2.215005000 \\
\hline $\mathrm{C}$ & 1.061740000 & -0.959694000 & -1.676283000 \\
\hline $\mathrm{H}$ & 0.729758000 & -0.024866000 & -2.144964000 \\
\hline $\mathrm{C}$ & 2.432480000 & -0.731041000 & -1.086391000 \\
\hline $\mathrm{C}$ & 3.059650000 & 0.509687000 & -1.214020000 \\
\hline $\mathrm{C}$ & 3.090852000 & -1.758668000 & -0.402909000 \\
\hline $\mathrm{C}$ & 4.320960000 & 0.727857000 & -0.673402000 \\
\hline $\mathrm{H}$ & 2.534271000 & 1.317494000 & -1.715030000 \\
\hline $\mathrm{C}$ & 4.349245000 & -1.559591000 & 0.145301000 \\
\hline $\mathrm{H}$ & 2.606405000 & -2.725317000 & -0.294866000 \\
\hline $\mathrm{C}$ & 4.946223000 & -0.313104000 & -0.001075000 \\
\hline $\mathrm{H}$ & 4.817230000 & 1.686638000 & -0.760083000 \\
\hline $\mathrm{H}$ & 4.870189000 & -2.345740000 & 0.677670000 \\
\hline $\mathrm{H}$ & -0.940576000 & -1.764675000 & -2.833466000 \\
\hline $\mathrm{C}$ & -3.261489000 & 2.121218000 & 0.989554000 \\
\hline $\mathrm{C}$ & -4.042755000 & 2.803766000 & -0.131487000 \\
\hline $\mathrm{C}$ & -1.925433000 & 1.617403000 & -1.718652000 \\
\hline $\mathrm{C}$ & -4.419889000 & 1.850939000 & -1.268789000 \\
\hline
\end{tabular}




$\begin{array}{lrrr}\mathrm{C} & -3.271975000 & 0.902918000 & -1.611373000 \\ \mathrm{H} & -3.699606000 & 1.136933000 & 1.218593000 \\ \mathrm{H} & -2.045908000 & 2.563001000 & -2.264447000 \\ \mathrm{H} & -3.444247000 & 3.641767000 & -0.508221000 \\ \mathrm{H} & -4.704130000 & 2.436766000 & -2.151991000 \\ \mathrm{H} & -3.369571000 & 2.726017000 & 1.898621000 \\ \mathrm{H} & -4.949877000 & 3.243153000 & 0.297875000 \\ \mathrm{H} & -1.235973000 & 1.018861000 & -2.318382000 \\ \mathrm{H} & -5.301332000 & 1.263977000 & -0.982977000 \\ \mathrm{H} & -3.480024000 & 0.391323000 & -2.556331000 \\ \mathrm{H} & -3.207353000 & 0.113133000 & -0.857521000 \\ \mathrm{C} & -1.158819000 & 1.914526000 & -0.435668000 \\ \mathrm{~N} & 0.112053000 & 2.075391000 & -0.584393000 \\ \mathrm{~N} & -1.821087000 & 1.997084000 & 0.773339000 \\ \mathrm{C} & -1.076646000 & 1.967295000 & 2.029752000 \\ \mathrm{H} & -1.181667000 & 2.941637000 & 2.530650000 \\ \mathrm{H} & -1.541390000 & 1.221248000 & 2.690863000 \\ \mathrm{C} & 0.393678000 & 1.654814000 & 1.813645000 \\ \mathrm{H} & 0.960500000 & 1.902747000 & 2.716682000 \\ \mathrm{H} & 0.539240000 & 0.587500000 & 1.611730000 \\ \mathrm{C} & 0.863857000 & 2.446729000 & 0.599412000 \\ \mathrm{H} & 1.927841000 & 2.272760000 & 0.415139000 \\ \mathrm{H} & 0.744910000 & 3.523879000 & 0.799685000 \\ \mathrm{H} & 1.161524000 & -1.714775000 & -2.474171000 \\ \mathrm{~N} & 6.274078000 & -0.090346000 & 0.579354000 \\ \mathrm{O} & 6.801000000 & -1.022180000 & 1.169095000 \\ \mathrm{O} & 6.777719000 & 1.014343000 & 0.440875000 \\ -------0 .-1\end{array}$

\begin{tabular}{|c|c|c|c|}
\hline \multicolumn{4}{|c|}{ A1 $1_{\text {MTBD }}$} \\
\hline & \multicolumn{3}{|c|}{ Coordinates (Angstroms) } \\
\hline & $X$ & $\mathrm{Y}$ & Z \\
\hline$\overline{\mathrm{C}}$ & -2.803902000 & 1.986223000 & 1.751728000 \\
\hline $\mathrm{C}$ & -1.954658000 & 0.921850000 & 2.438523000 \\
\hline $\mathrm{C}$ & -0.509286000 & 1.397594000 & 2.461264000 \\
\hline $\mathrm{N}$ & -2.337576000 & 2.265612000 & 0.411664000 \\
\hline $\mathrm{H}$ & -2.799397000 & 2.903919000 & 2.363603000 \\
\hline $\mathrm{H}$ & -3.846483000 & 1.654559000 & 1.696339000 \\
\hline $\mathrm{H}$ & -2.048204000 & -0.017590000 & 1.881624000 \\
\hline $\mathrm{H}$ & -2.291819000 & 0.729144000 & 3.462211000 \\
\hline $\mathrm{H}$ & -0.358302000 & 2.111213000 & 3.285083000 \\
\hline $\mathrm{H}$ & 0.171274000 & 0.553030000 & 2.644750000 \\
\hline $\mathrm{N}$ & -0.115712000 & 2.029675000 & 1.208092000 \\
\hline $\mathrm{C}$ & 1.323095000 & 2.125817000 & 1.031547000 \\
\hline
\end{tabular}




\begin{tabular}{|c|c|c|c|}
\hline $\mathrm{C}$ & 1.691076000 & 2.989026000 & -0.159732000 \\
\hline $\mathrm{C}$ & 0.828276000 & 2.582000000 & -1.340277000 \\
\hline $\mathrm{N}$ & -0.568130000 & 2.732681000 & -0.992467000 \\
\hline $\mathrm{C}$ & -1.056982000 & 2.327563000 & 0.238284000 \\
\hline $\mathrm{H}$ & 1.748271000 & 1.118279000 & 0.903263000 \\
\hline $\mathrm{H}$ & 1.755334000 & 2.540014000 & 1.952511000 \\
\hline $\mathrm{H}$ & 1.515312000 & 4.046314000 & 0.065345000 \\
\hline $\mathrm{H}$ & 2.752457000 & 2.857825000 & -0.390778000 \\
\hline $\mathrm{H}$ & 1.028747000 & 3.231252000 & -2.199011000 \\
\hline $\mathrm{H}$ & 1.060460000 & 1.550463000 & -1.648974000 \\
\hline $\mathrm{C}$ & -3.819172000 & -3.439503000 & 0.520839000 \\
\hline $\mathrm{C}$ & -2.895268000 & -2.714939000 & -0.225564000 \\
\hline $\mathrm{C}$ & -3.120808000 & -1.368246000 & -0.519821000 \\
\hline $\mathrm{C}$ & -4.278442000 & -0.752049000 & -0.044308000 \\
\hline $\mathrm{C}$ & -5.198785000 & -1.472149000 & 0.711713000 \\
\hline $\mathrm{C}$ & -4.972539000 & -2.817394000 & 0.992160000 \\
\hline $\mathrm{H}$ & -3.635401000 & -4.486820000 & 0.742040000 \\
\hline $\mathrm{H}$ & -1.981646000 & -3.195331000 & -0.571620000 \\
\hline $\mathrm{H}$ & -4.441851000 & 0.300584000 & -0.259729000 \\
\hline $\mathrm{H}$ & -6.094045000 & -0.982309000 & 1.083203000 \\
\hline $\mathrm{H}$ & -5.690461000 & -3.379488000 & 1.582074000 \\
\hline $\mathrm{C}$ & -2.128040000 & -0.582048000 & -1.359087000 \\
\hline $\mathrm{C}$ & -0.732551000 & -0.738851000 & -0.818249000 \\
\hline $\mathrm{H}$ & -0.626932000 & -0.526803000 & 0.243883000 \\
\hline $\mathrm{O}$ & -2.217639000 & -0.891618000 & -2.739536000 \\
\hline $\mathrm{C}$ & 0.342507000 & -1.061374000 & -1.540050000 \\
\hline $\mathrm{H}$ & 0.236063000 & -1.189371000 & -2.615244000 \\
\hline $\mathrm{C}$ & 1.696735000 & -1.153553000 & -0.971758000 \\
\hline $\mathrm{C}$ & 2.805281000 & -0.788570000 & -1.748503000 \\
\hline $\mathrm{C}$ & 1.913745000 & -1.562967000 & 0.352581000 \\
\hline $\mathrm{C}$ & 4.085230000 & -0.782599000 & -1.214699000 \\
\hline $\mathrm{H}$ & 2.654398000 & -0.484483000 & -2.780350000 \\
\hline $\mathrm{C}$ & 3.186459000 & -1.564447000 & 0.901960000 \\
\hline $\mathrm{H}$ & 1.072504000 & -1.891345000 & 0.955103000 \\
\hline $\mathrm{C}$ & 4.256105000 & -1.164249000 & 0.110038000 \\
\hline $\mathrm{H}$ & 4.945010000 & -0.486406000 & -1.802906000 \\
\hline $\mathrm{H}$ & 3.363417000 & -1.874005000 & 1.924637000 \\
\hline $\mathrm{H}$ & -2.394867000 & 0.475497000 & -1.268167000 \\
\hline $\mathrm{H}$ & -2.243534000 & -1.853759000 & -2.821596000 \\
\hline $\mathrm{C}$ & -1.490682000 & 2.921069000 & -2.092493000 \\
\hline $\mathrm{H}$ & -1.552420000 & 2.028505000 & -2.732838000 \\
\hline $\mathrm{H}$ & -2.478913000 & 3.134295000 & -1.689198000 \\
\hline $\mathrm{H}$ & -1.152964000 & 3.763818000 & -2.706484000 \\
\hline $\mathrm{N}$ & 5.602982000 & -1.153097000 & 0.686284000 \\
\hline
\end{tabular}




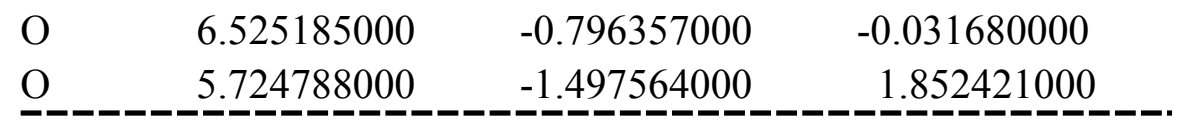

TSA1-2

\begin{tabular}{lrrr} 
& Coordinates (Angstroms) & \\
& $\mathrm{X}$ & $\mathrm{Y}$ & $\mathrm{Z}$ \\
\hline $\mathrm{C}$ & -2.048365000 & 0.841599000 & 2.033232000 \\
$\mathrm{C}$ & -0.999128000 & 1.026031000 & 3.119758000 \\
$\mathrm{C}$ & 0.369578000 & 0.764529000 & 2.515705000 \\
$\mathrm{~N}$ & -1.676886000 & 1.469008000 & 0.768446000 \\
$\mathrm{H}$ & -3.012542000 & 1.253046000 & 2.348306000 \\
$\mathrm{H}$ & -2.211330000 & -0.229648000 & 1.856038000 \\
$\mathrm{H}$ & -1.189983000 & 0.340147000 & 3.950495000 \\
$\mathrm{H}$ & -1.025237000 & 2.047738000 & 3.514406000 \\
$\mathrm{H}$ & 1.163200000 & 0.930522000 & 3.250150000 \\
$\mathrm{H}$ & 0.454574000 & -0.274862000 & 2.166531000 \\
$\mathrm{~N}$ & 0.592104000 & 1.690739000 & 1.414177000 \\
$\mathrm{C}$ & 1.986515000 & 1.914083000 & 1.045813000 \\
$\mathrm{C}$ & 2.111925000 & 3.025194000 & 0.019949000 \\
$\mathrm{C}$ & 1.122158000 & 2.782490000 & -1.105351000 \\
$\mathrm{~N}$ & -0.221852000 & 2.648177000 & -0.565795000 \\
$\mathrm{C}$ & -0.442646000 & 1.907849000 & 0.549481000 \\
$\mathrm{H}$ & 2.415041000 & 0.983133000 & 0.651094000 \\
$\mathrm{H}$ & 2.535771000 & 2.170981000 & 1.957820000 \\
$\mathrm{H}$ & 1.903944000 & 3.994408000 & 0.485308000 \\
$\mathrm{H}$ & 3.132874000 & 3.045500000 & -0.371355000 \\
$\mathrm{H}$ & 1.117952000 & 3.626461000 & -1.800176000 \\
$\mathrm{H}$ & 1.392190000 & 1.881836000 & -1.673706000 \\
$\mathrm{C}$ & -4.764887000 & -2.628042000 & 0.841178000 \\
$\mathrm{C}$ & -3.578132000 & -2.145949000 & 0.299460000 \\
$\mathrm{C}$ & -3.566635000 & -1.019527000 & -0.539143000 \\
$\mathrm{C}$ & -4.800841000 & -0.417098000 & -0.831753000 \\
$\mathrm{C}$ & -5.986554000 & -0.907605000 & -0.299029000 \\
$\mathrm{C}$ & -5.978559000 & -2.012949000 & 0.548481000 \\
$\mathrm{H}$ & -4.740445000 & -3.503998000 & 1.484095000 \\
$\mathrm{H}$ & -2.653062000 & -2.671586000 & 0.518947000 \\
$\mathrm{H}$ & -4.811899000 & 0.448442000 & -1.486636000 \\
$\mathrm{H}$ & -6.925472000 & -0.418098000 & -0.543767000 \\
$\mathrm{H}$ & -6.904583000 & -2.395350000 & 0.967042000 \\
$\mathrm{C}$ & -2.325064000 & -0.406267000 & -1.075580000 \\
$\mathrm{C}$ & -1.034378000 & -0.974268000 & -0.839695000 \\
$\mathrm{H}$ & -0.940384000 & -1.502942000 & 0.108277000 \\
$\mathrm{O}$ & -2.490692000 & 0.240650000 & -2.322675000 \\
& & 5572 & \\
& & & \\
& & &
\end{tabular}




$\begin{array}{lrrr}\mathrm{C} & 0.101579000 & -0.724061000 & -1.549579000 \\ \mathrm{H} & 0.008310000 & -0.178574000 & -2.486095000 \\ \mathrm{C} & 1.446125000 & -1.037110000 & -1.114202000 \\ \mathrm{C} & 2.545364000 & -0.584571000 & -1.879278000 \\ \mathrm{C} & 1.746386000 & -1.735345000 & 0.078458000 \\ \mathrm{C} & 3.850988000 & -0.746095000 & -1.456239000 \\ \mathrm{H} & 2.353371000 & -0.078991000 & -2.822192000 \\ \mathrm{C} & 3.046256000 & -1.905775000 & 0.513985000 \\ \mathrm{H} & 0.943518000 & -2.150590000 & 0.679186000 \\ \mathrm{C} & 4.095058000 & -1.391141000 & -0.245926000 \\ \mathrm{H} & 4.684715000 & -0.378663000 & -2.042306000 \\ \mathrm{H} & 3.266110000 & -2.428186000 & 1.437340000 \\ \mathrm{H} & -2.069607000 & 0.724473000 & -0.140945000 \\ \mathrm{H} & -2.654677000 & -0.440719000 & -2.989894000 \\ \mathrm{C} & -1.323844000 & 2.977844000 & -1.457633000 \\ \mathrm{H} & -1.511413000 & 2.185237000 & -2.189775000 \\ \mathrm{H} & -2.231378000 & 3.120162000 & -0.870966000 \\ \mathrm{H} & -1.076518000 & 3.911618000 & -1.970996000 \\ \mathrm{~N} & 5.460219000 & -1.537119000 & 0.226817000 \\ \mathrm{O} & 6.360248000 & -1.077413000 & -0.465924000 \\ \mathrm{O} & 5.639753000 & -2.104491000 & 1.298063000 \\ -----------~\end{array}$

\section{A2 ${ }_{\text {MTBD }}$}

\begin{tabular}{lrrr} 
& \multicolumn{3}{c}{ Coordinates (Angstroms) } \\
& $\mathrm{X}$ & $\mathrm{Y}$ & $\mathrm{Z}$ \\
$\mathrm{C}$ & 3.280956000 & 0.550163000 & 2.055894000 \\
$\mathrm{C}$ & 4.026116000 & 1.160195000 & 0.880664000 \\
$\mathrm{C}$ & 3.367536000 & 2.471058000 & 0.487761000 \\
$\mathrm{~N}$ & 1.833476000 & 0.762156000 & 1.964300000 \\
$\mathrm{H}$ & 3.617885000 & 0.987774000 & 3.004165000 \\
$\mathrm{H}$ & 3.454320000 & -0.530981000 & 2.094127000 \\
$\mathrm{H}$ & 4.006864000 & 0.483020000 & 0.025132000 \\
$\mathrm{H}$ & 5.071116000 & 1.325034000 & 1.155773000 \\
$\mathrm{H}$ & 3.447242000 & 3.219068000 & 1.288418000 \\
$\mathrm{H}$ & 3.838611000 & 2.880051000 & -0.408301000 \\
$\mathrm{~N}$ & 1.962506000 & 2.234107000 & 0.172679000 \\
$\mathrm{C}$ & 1.383156000 & 2.864834000 & -1.011177000 \\
$\mathrm{C}$ & -0.103229000 & 3.110302000 & -0.802338000 \\
$\mathrm{C}$ & -0.771810000 & 1.805306000 & -0.412396000 \\
$\mathrm{~N}$ & -0.064940000 & 1.200491000 & 0.711849000 \\
$\mathrm{C}$ & 1.247917000 & 1.379414000 & 0.925776000 \\
$\mathrm{H}$ & 1.563944000 & 2.232807000 & -1.890564000 \\
$\mathrm{H}$ & 1.907116000 & 3.811796000 & -1.164132000
\end{tabular}




\begin{tabular}{lrrr}
$\mathrm{H}$ & -0.243877000 & 3.858110000 & -0.014301000 \\
$\mathrm{H}$ & -0.551294000 & 3.499706000 & -1.719994000 \\
$\mathrm{H}$ & -1.812439000 & 1.951553000 & -0.115567000 \\
$\mathrm{H}$ & -0.773192000 & 1.106289000 & -1.257863000 \\
$\mathrm{H}$ & -0.435084000 & 0.272427000 & 0.961420000 \\
$\mathrm{C}$ & 3.535082000 & -0.311494000 & -2.551702000 \\
$\mathrm{C}$ & 2.418738000 & -0.676903000 & -1.812970000 \\
$\mathrm{C}$ & 2.502010000 & -1.628533000 & -0.772613000 \\
$\mathrm{C}$ & 3.774293000 & -2.170650000 & -0.502304000 \\
$\mathrm{C}$ & 4.891321000 & -1.789336000 & -1.236503000 \\
$\mathrm{C}$ & 4.787279000 & -0.857290000 & -2.267581000 \\
$\mathrm{H}$ & 3.429431000 & 0.417170000 & -3.352056000 \\
$\mathrm{H}$ & 1.464064000 & -0.209449000 & -2.039248000 \\
$\mathrm{H}$ & 3.867039000 & -2.902141000 & 0.292827000 \\
$\mathrm{H}$ & 5.856100000 & -2.232310000 & -1.003629000 \\
$\mathrm{H}$ & 5.661849000 & -0.563944000 & -2.839873000 \\
$\mathrm{C}$ & 1.353290000 & -1.995208000 & 0.036006000 \\
$\mathrm{C}$ & 0.057087000 & -1.663719000 & -0.231373000 \\
$\mathrm{H}$ & -0.119207000 & -1.235068000 & -1.215210000 \\
$\mathrm{O}$ & 1.673763000 & -2.652094000 & 1.228379000 \\
$\mathrm{C}$ & -1.053975000 & -1.734993000 & 0.642846000 \\
$\mathrm{H}$ & -0.897703000 & -2.083586000 & 1.662602000 \\
$\mathrm{C}$ & -2.372037000 & -1.327436000 & 0.306177000 \\
$\mathrm{C}$ & -3.364640000 & -1.190758000 & 1.322834000 \\
$\mathrm{C}$ & -2.783966000 & -0.976086000 & -1.015321000 \\
$\mathrm{C}$ & -4.618596000 & -0.690973000 & 1.064724000 \\
$\mathrm{H}$ & -3.106393000 & -1.474228000 & 2.340639000 \\
$\mathrm{C}$ & -4.036664000 & -0.468348000 & -1.277723000 \\
$\mathrm{H}$ & -2.105549000 & -1.132614000 & -1.848591000 \\
$\mathrm{C}$ & -4.958052000 & -0.306191000 & -0.239066000 \\
$\mathrm{H}$ & -5.352363000 & -0.580697000 & 1.854413000 \\
$\mathrm{H}$ & -4.330937000 & -0.204112000 & -2.286893000 \\
$\mathrm{H}$ & 1.002167000 & -3.334039000 & 1.362952000 \\
$\mathrm{H}$ & 1.055456000 & 0.024891000 & 2.952134000 \\
$\mathrm{H}$ & 0.058323000 & 0.456131000 & 3.049801000 \\
$\mathrm{H}$ & 0.992441000 & -1.032072000 & 2.679373000 \\
$\mathrm{H}$ & -6.25484500000000000 \\
$\mathrm{H}$ & -7.049779000 & 0.121384000 & 3.914650000 \\
$\mathrm{H}$ & 0.244072000 & -0.511937000 \\
$\mathrm{H}$ & 0.358245000 & 0.420340000 \\
$\mathrm{H}$ & 0.586057000 & -1.666069000 \\
\hline
\end{tabular}

TSA2-3 MTBD 


\begin{tabular}{|c|c|c|c|}
\hline & $X$ & $\mathrm{Y}$ & Z \\
\hline $\mathrm{C}$ & 3.273656000 & 1.429590000 & 1.640251000 \\
\hline $\mathrm{C}$ & 4.030740000 & 1.269605000 & 0.330227000 \\
\hline $\mathrm{C}$ & 3.490843000 & 2.257798000 & -0.688624000 \\
\hline $\mathrm{N}$ & 1.862374000 & 1.747790000 & 1.435778000 \\
\hline $\mathrm{H}$ & 3.712230000 & 2.238927000 & 2.240133000 \\
\hline $\mathrm{H}$ & 3.330613000 & 0.504013000 & 2.224755000 \\
\hline $\mathrm{H}$ & 3.909473000 & 0.258238000 & -0.063364000 \\
\hline $\mathrm{H}$ & 5.097534000 & 1.437791000 & 0.502037000 \\
\hline $\mathrm{H}$ & 3.682078000 & 3.295242000 & -0.376795000 \\
\hline $\mathrm{H}$ & 3.970803000 & 2.103766000 & -1.657946000 \\
\hline $\mathrm{N}$ & 2.062584000 & 2.037466000 & -0.862903000 \\
\hline $\mathrm{C}$ & 1.481655000 & 2.119960000 & -2.197242000 \\
\hline $\mathrm{C}$ & 0.077532000 & 2.698882000 & -2.116761000 \\
\hline $\mathrm{C}$ & -0.734451000 & 1.872281000 & -1.133290000 \\
\hline $\mathrm{N}$ & -0.050477000 & 1.719508000 & 0.143617000 \\
\hline $\mathrm{C}$ & 1.269188000 & 1.817381000 & 0.216695000 \\
\hline $\mathrm{H}$ & 1.466614000 & 1.126196000 & -2.666277000 \\
\hline $\mathrm{H}$ & 2.128766000 & 2.761411000 & -2.802148000 \\
\hline $\mathrm{H}$ & 0.133725000 & 3.739866000 & -1.778887000 \\
\hline $\mathrm{H}$ & -0.393461000 & 2.689157000 & -3.103840000 \\
\hline $\mathrm{H}$ & -1.709167000 & 2.330999000 & -0.943603000 \\
\hline $\mathrm{H}$ & -0.932738000 & 0.877941000 & -1.559145000 \\
\hline $\mathrm{H}$ & -0.523335000 & 0.739677000 & 0.716121000 \\
\hline $\mathrm{C}$ & 3.681463000 & -1.909232000 & -2.107225000 \\
\hline $\mathrm{C}$ & 2.549603000 & -1.629771000 & -1.353205000 \\
\hline $\mathrm{C}$ & 2.504366000 & -1.918862000 & 0.021361000 \\
\hline $\mathrm{C}$ & 3.647928000 & -2.474938000 & 0.612804000 \\
\hline $\mathrm{C}$ & 4.785723000 & -2.739401000 & -0.142014000 \\
\hline $\mathrm{C}$ & 4.811365000 & -2.461087000 & -1.505733000 \\
\hline $\mathrm{H}$ & 3.687846000 & -1.679789000 & -3.169372000 \\
\hline $\mathrm{H}$ & 1.696675000 & -1.156046000 & -1.830398000 \\
\hline $\mathrm{H}$ & 3.634408000 & -2.690992000 & 1.675166000 \\
\hline $\mathrm{H}$ & 5.658928000 & -3.169877000 & 0.340150000 \\
\hline $\mathrm{H}$ & 5.701381000 & -2.667234000 & -2.092484000 \\
\hline $\mathrm{C}$ & 1.337730000 & -1.576170000 & 0.841943000 \\
\hline $\mathrm{C}$ & 0.103968000 & -1.293001000 & 0.376313000 \\
\hline $\mathrm{H}$ & -0.055763000 & -1.454322000 & -0.686229000 \\
\hline $\mathrm{O}$ & 1.636304000 & -1.491001000 & 2.194871000 \\
\hline $\mathrm{C}$ & -0.953676000 & -0.617739000 & 1.111141000 \\
\hline $\mathrm{H}$ & -0.821242000 & -0.532501000 & 2.192581000 \\
\hline $\mathrm{C}$ & -2.348638000 & -0.665147000 & 0.708052000 \\
\hline $\mathrm{C}$ & -3.326833000 & -0.057476000 & 1.531787000 \\
\hline $\mathrm{C}$ & -2.799339000 & -1.208672000 & -0.517030000 \\
\hline
\end{tabular}




$\begin{array}{lrrr}\mathrm{C} & -4.652320000 & 0.023666000 & 1.160044000 \\ \mathrm{H} & -3.016559000 & 0.366200000 & 2.483785000 \\ \mathrm{C} & -4.124711000 & -1.129319000 & -0.903971000 \\ \mathrm{H} & -2.102000000 & -1.723430000 & -1.170137000 \\ \mathrm{C} & -5.048593000 & -0.508700000 & -0.067422000 \\ \mathrm{H} & -5.389820000 & 0.492796000 & 1.800096000 \\ \mathrm{H} & -4.461861000 & -1.553664000 & -1.842232000 \\ \mathrm{H} & 0.824837000 & -1.691676000 & 2.679423000 \\ \mathrm{C} & 1.077177000 & 1.786325000 & 2.655063000 \\ \mathrm{H} & 0.091673000 & 2.201434000 & 2.447426000 \\ \mathrm{H} & 0.973522000 & 0.783976000 & 3.080475000 \\ \mathrm{H} & 1.593011000 & 2.426035000 & 3.380854000 \\ \mathrm{~N} & -6.436765000 & -0.423666000 & -0.471466000 \\ \mathrm{O} & -7.228427000 & 0.118927000 & 0.292393000 \\ \mathrm{O} & -6.752680000 & -0.895135000 & -1.559054000 \\ -- \text { - } & - \text { - } & \end{array}$

\begin{tabular}{|c|c|c|c|}
\hline \multicolumn{4}{|c|}{ A3 3 мTBD } \\
\hline & $X$ & $\mathrm{Y}$ & Z \\
\hline $\bar{C}$ & 0.223200000 & 2.901310000 & 1.607980000 \\
\hline $\mathrm{C}$ & 1.327497000 & 2.191288000 & 2.370640000 \\
\hline $\mathrm{C}$ & 2.575306000 & 2.144723000 & 1.505304000 \\
\hline $\mathrm{N}$ & -0.049356000 & 2.166242000 & 0.390332000 \\
\hline $\mathrm{H}$ & 0.504365000 & 3.950347000 & 1.405904000 \\
\hline $\mathrm{H}$ & -0.697339000 & 2.922457000 & 2.199675000 \\
\hline $\mathrm{H}$ & 0.983712000 & 1.180061000 & 2.611188000 \\
\hline $\mathrm{H}$ & 1.551372000 & 2.702591000 & 3.311687000 \\
\hline $\mathrm{H}$ & 3.109006000 & 3.105918000 & 1.576676000 \\
\hline $\mathrm{H}$ & 3.264537000 & 1.369210000 & 1.866915000 \\
\hline $\mathrm{N}$ & 2.270871000 & 1.877645000 & 0.109379000 \\
\hline $\mathrm{C}$ & 3.435179000 & 1.572752000 & -0.706564000 \\
\hline $\mathrm{C}$ & 3.154310000 & 1.839922000 & -2.175587000 \\
\hline $\mathrm{C}$ & 1.840462000 & 1.159329000 & -2.544457000 \\
\hline $\mathrm{N}$ & 0.747965000 & 1.559496000 & -1.685812000 \\
\hline $\mathrm{C}$ & 1.011398000 & 1.859165000 & -0.460114000 \\
\hline $\mathrm{H}$ & 3.728806000 & 0.522242000 & -0.558689000 \\
\hline $\mathrm{H}$ & 4.267250000 & 2.194411000 & -0.352276000 \\
\hline $\mathrm{H}$ & 3.063861000 & 2.919827000 & -2.341888000 \\
\hline $\mathrm{H}$ & 3.985112000 & 1.471965000 & -2.786454000 \\
\hline $\mathrm{H}$ & 1.568347000 & 1.395190000 & -3.579767000 \\
\hline $\mathrm{H}$ & 1.978477000 & 0.066481000 & -2.499841000 \\
\hline $\mathrm{C}$ & -5.922199000 & -1.690103000 & 1.262971000 \\
\hline $\mathrm{C}$ & -4.795419000 & -1.774778000 & 0.453665000 \\
\hline
\end{tabular}




\begin{tabular}{lrrr}
$\mathrm{C}$ & -4.273808000 & -0.631182000 & -0.159420000 \\
$\mathrm{C}$ & -4.916870000 & 0.596462000 & 0.042732000 \\
$\mathrm{C}$ & -6.047330000 & 0.678448000 & 0.848916000 \\
$\mathrm{C}$ & -6.553013000 & -0.464107000 & 1.461785000 \\
$\mathrm{H}$ & -6.318591000 & -2.587338000 & 1.728754000 \\
$\mathrm{H}$ & -4.321898000 & -2.735636000 & 0.276295000 \\
$\mathrm{H}$ & -4.509840000 & 1.498053000 & -0.408472000 \\
$\mathrm{H}$ & -6.529309000 & 1.639157000 & 1.003777000 \\
$\mathrm{H}$ & -7.437223000 & -0.401136000 & 2.088771000 \\
$\mathrm{C}$ & -3.063445000 & -0.697997000 & -1.011674000 \\
$\mathrm{C}$ & -1.996527000 & -1.457066000 & -0.739547000 \\
$\mathrm{H}$ & -2.020002000 & -2.057958000 & 0.164922000 \\
$\mathrm{O}$ & -3.039754000 & 0.132443000 & -2.100054000 \\
$\mathrm{C}$ & -0.759135000 & -1.500986000 & -1.591669000 \\
$\mathrm{H}$ & -0.657574000 & -0.547729000 & -2.127712000 \\
$\mathrm{C}$ & 0.516050000 & -1.705359000 & -0.809936000 \\
$\mathrm{C}$ & 1.584318000 & -2.417517000 & -1.366679000 \\
$\mathrm{C}$ & 0.681235000 & -1.131188000 & 0.453350000 \\
$\mathrm{C}$ & 2.798076000 & -2.532711000 & -0.703958000 \\
$\mathrm{H}$ & 1.467225000 & -2.877170000 & -2.344228000 \\
$\mathrm{C}$ & 1.884928000 & -1.234876000 & 1.132008000 \\
$\mathrm{H}$ & -0.128783000 & -0.551984000 & 0.885445000 \\
$\mathrm{C}$ & 2.933028000 & -1.925845000 & 0.538654000 \\
$\mathrm{H}$ & 3.634089000 & -3.070458000 & -1.133965000 \\
$\mathrm{H}$ & 2.028659000 & -0.780697000 & 2.104273000 \\
$\mathrm{H}$ & -3.942745000 & 0.399528000 & -2.309220000 \\
$\mathrm{H}$ & -0.845757000 & -2.287371000 & -2.354249000 \\
$\mathrm{C}$ & -1.344297000 & 2.399017000 & -0.217261000 \\
$\mathrm{H}$ & -1.439987000 & 3.422722000 & -0.611930000 \\
$\mathrm{H}$ & -1.490940000 & 1.703424000 & -1.040888000 \\
$\mathrm{H}$ & -2.119128000 & 2.237741000 & 0.540733000 \\
$\mathrm{O}$ & 4.218877000 & -1.992504000 & 1.231581000 \\
$\mathrm{H}$ & 4.357093000 & -1.318748000 & 2.245004000 \\
$0--$ & 5.088677000 & -2.707721000 & 0.757471000 \\
\hline
\end{tabular}

